Florida International University FIU Digital Commons

FIU Electronic Theses and Dissertations

University Graduate School

3-8-2013

\title{
Pseudomonas Aeruginosa AmpR Transcriptional Regulatory Network
}

Deepak Balasubramanian

Florida International University, deeps66@gmail.com

DOI: $10.25148 /$ etd.FI13042330

Follow this and additional works at: https:// digitalcommons.fiu.edu/etd

Part of the Bacteria Commons, Bacterial Infections and Mycoses Commons, Bacteriology Commons, Bioinformatics Commons, Genomics Commons, Molecular Genetics Commons, Organismal Biological Physiology Commons, Other Genetics and Genomics Commons, Pathogenic Microbiology Commons, and the Respiratory Tract Diseases Commons

\section{Recommended Citation}

Balasubramanian, Deepak, "Pseudomonas Aeruginosa AmpR Transcriptional Regulatory Network" (2013). FIU Electronic Theses and Dissertations. 863.

https://digitalcommons.fiu.edu/etd/863

This work is brought to you for free and open access by the University Graduate School at FIU Digital Commons. It has been accepted for inclusion in FIU Electronic Theses and Dissertations by an authorized administrator of FIU Digital Commons. For more information, please contact dcc@fiu.edu. 


\section{FLORIDA INTERNATIONAL UNIVERSITY}

Miami, Florida

PSEUDOMONAS AERUGINOSA AMPR

TRANSCRIPTIONAL REGULATORY NETWORK

A dissertation submitted in partial fulfillment of

the requirements for the degree of

DOCTOR OF PHILOSOPHY

in

BIOLOGY

by

Deepak Balasubramanian

2013 
To: Dean Kenneth G. Furton

College of Arts and Sciences

This dissertation, written by Deepak Balasubramanian, and entitled Pseudomonas aeruginosa AmpR transcriptional regulatory network, having been approved in respect to style and intellectual content, is referred to you for judgment.

We have read this dissertation and recommend that it be approved.

Stephen Lory

John Makemson

Giri Narasimhan

Fernando Noriega

Kalai Mathee, Major Professor

Date of Defense: March 08, 2013

The dissertation of Deepak Balasubramanian is approved.

Dean Kenneth G. Furton
College of Arts and Sciences

Florida International University, 2013 


\section{DEDICATION}

To appa, amma and anna for the unconditional love and support 


\section{ACKNOWLEDGMENTS}

This dissertation is the result of the support of many people. It is difficult to overstate my gratitude to my mentor, Dr. Kalai Mathee. With her enthusiasm, her inspiration, she helped to make science even more fun than it already is. I'm forever in debt! Throughout my stay here, she provided encouragement, good teaching, great company, and lots of ideas. I would have been lost without her. Thanks Kalai!

I would like to thank my committee members, Dr. Stephen Lory, Dr. John Makemson, Dr. Giri Narasimhan and Dr. Fernando Noriega for their helpful ideas, guidance, and help with my project. Special thanks to Dr. Lory for allowing me to work in his lab. I learned a lot from Steve and his group of talented postdocs. And I had a lot of fun in Boston! I'm very thankful to Dr. Narasimhan for all the bioinformatics help. I relied heavily on computational analyses for my project, which could not have been possible without the help of Giri and his talented students, Camilo Valdes and Melita Jaric. One of my first classes at FIU was with Dr. Noriega on genomics and proteomics way back when. I thoroughly enjoyed Fernando's class but little did I know then that my project would be so genomically-skewed (I just made that word up!). Dr. Makemson, thank you for making me study biochemistry for my quals! I'm not a big fan of biochemistry and would've never read Lehninger but your quals questions were so much fun to answer (I know I sound like a nerd but whatever!).

I am indebted to the Mathee Crew, past and present, for their support. Working in the lab was fun, thanks to them. There are so many people in the Mathee Lab that made my journey through here smooth. The lab 'adopted' me as it's own. I have many fond 
memories with people in the lab, some with the lab members and some with visiting researchers. I especially want to thank Oli, Lisa, Cami, Marios, Ciraj, Senthil, Nat, Mario and the many, many others (that's what happens when you stay in one place for six years!) for making my stay such a pleasant experience. I had a wonderful time outside the lab, thanks to Wim, Hideaki, Saeed, Senthil, (our camping, canoeing and kayaking trips were awesome!) Ziad, Gorakh, and Jitesh. Ever since she joined the lab, Hansi has been a major positive influence on my research and me. She has been a wonderful friend and my sounding board for science and non-science issues. Her unbiased and outright honest opinions have guided me through some rough patches in research. Thanks re! She and Bhupesh took me into their family and their hearts, and have made me feel at home away from home. We've had so much fun on our trips and it is going to continue!

Money helps when you are doing science! The Biological Sciences Department and the Herbert Werthiem College of Medicine have supported me through these years and I am grateful for that. I'm also thankful to the Cystic Fibrosis Foundation for the two Student Traineeship awards and to the National Institutes of Health-MRBS RISE program for a Summer Student award. My special thanks to FIU University Graduate School for supporting me at the final critical writing phase with the Dissertation Year Fellowship.

Lastly, and most importantly, I would not be where I am today if it were not for the constant support and encouragement of my parents, Balasubramanian and Vasantha, and my brother Satish. My parents put our education and well being above all else through some tough times and made us into what we are. My family supported my decisions and loved me unconditionally. I'm eternally grateful for everything. 


\author{
ABSTRACT OF THE DISSERTATION \\ PSEUDOMONAS AERUGINOSA AMPR \\ TRANSCRIPTIONAL REGULATORY NETWORK
}

by

Deepak Balasubramanian

Florida International University, 2013

Miami, Florida

\title{
Professor Kalai Mathee, Major Professor
}

In Enterobacteriaceae, the transcriptional regulator AmpR, a member of the LysR family, regulates the expression of a chromosomal $\beta$-lactamase AmpC. The regulatory repertoire of AmpR is broader in Pseudomonas aeruginosa, an opportunistic pathogen responsible for numerous acute and chronic infections including cystic fibrosis. Previous studies showed that in addition to regulating ampC, $P$. aeruginosa AmpR regulates the sigma factor $\mathrm{AlgT} / \mathrm{U}$ and production of some quorum sensing (QS)regulated virulence factors. In order to better understand the $a m p R$ regulon, the transcriptional profiles generated using DNA microarrays and RNA-Seq of the prototypic $P$. aeruginosa PAO1 strain with its isogenic ampR deletion mutant, PAO $\triangle a m p R$ were analyzed. Transcriptome analysis demonstrates that the AmpR regulon is much more extensive than previously thought influencing the differential expression of over 500 genes. In addition to regulating resistance to $\beta$-lactam antibiotics via AmpC, AmpR also regulates non- $\beta$-lactam antibiotic resistance by modulating the MexEF-OprN efflux pump. Virulence mechanisms including biofilm formation, QS-regulated acute virulence, and diverse physiological processes such as oxidative stress response, heat- 
shock response and iron uptake are AmpR-regulated. Real-time PCR and phenotypic assays confirmed the transcriptome data. Further, Caenorhabditis elegans model demonstrates that a functional AmpR is required for full pathogenicity of $P$. aeruginosa. AmpR, a member of the core genome, also regulates genes in the regions of genome plasticity that are acquired by horizontal gene transfer. The extensive AmpR regulon included other transcriptional regulators and sigma factors, accounting for the extensive AmpR regulon. Gene expression studies demonstrate AmpR-dependent expression of the QS master regulator LasR that controls expression of many virulence factors. Using a chromosomally tagged AmpR, ChIP-Seq studies show direct AmpR binding to the lasR promoter. The data demonstrates that AmpR functions as a global regulator in $P$. aeruginosa and is a positive regulator of acute virulence while negatively regulating chronic infection phenotypes. In summary, my dissertation sheds light on the complex regulatory circuit in $P$. aeruginosa to provide a better understanding of the bacterial response to antibiotics and how the organism coordinately regulates a myriad of virulence factors. 


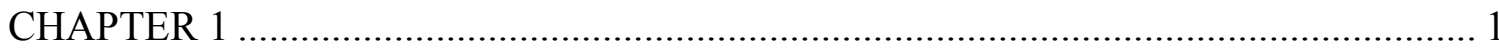

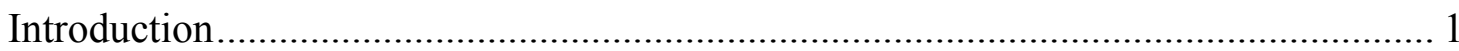

The model bacterium, Pseudomonas aeruginosa ................................................... 2

Antibiotics, antibiotic resistance and AmpR …………......................................... 5

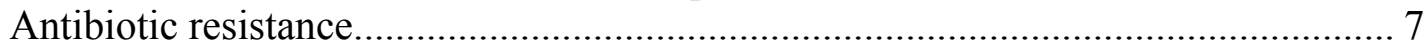

Antibiotic resistance in $P$. aeruginosa ............................................................. 9

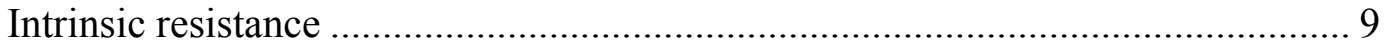

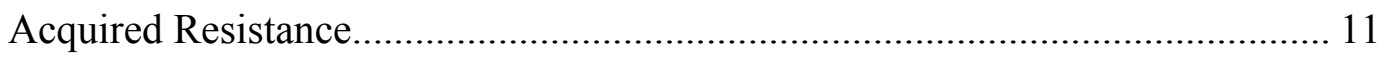

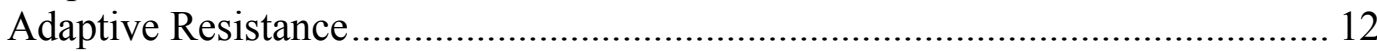

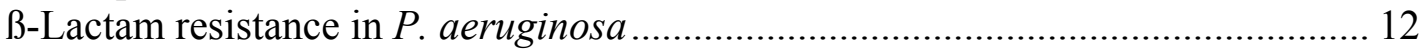

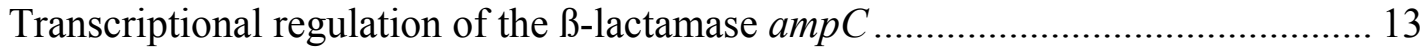

P. aeruginosa AmpR regulates other genes.......................................................... 14

Role of AmpR in peptidoglycan recycling .......................................................... 16

Transcriptional regulation: How does it work? ........................................................ 21

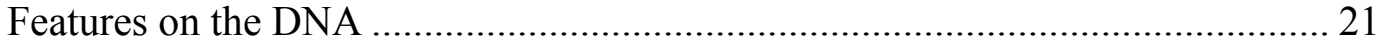

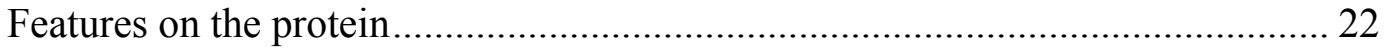

DNA-binding motifs in proteins ………………………................................ 22

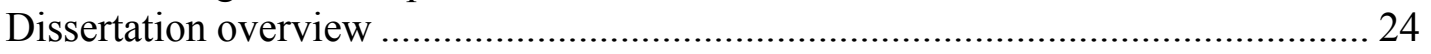

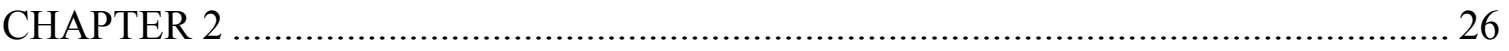

Comparative transcriptomic analyses of Pseudomonas aeruginosa ............................. 26

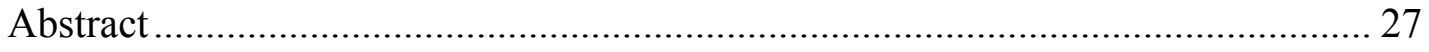

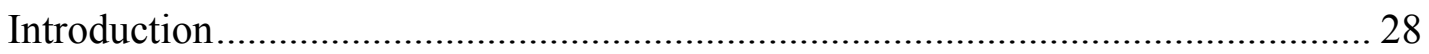

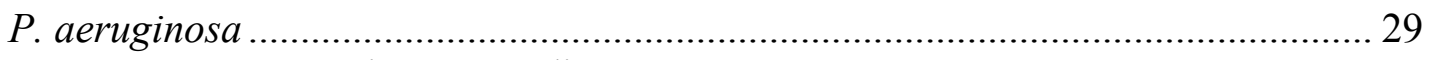

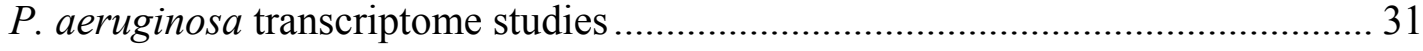

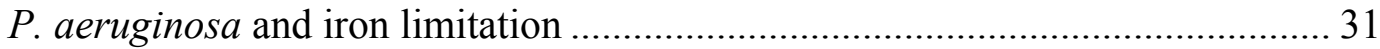

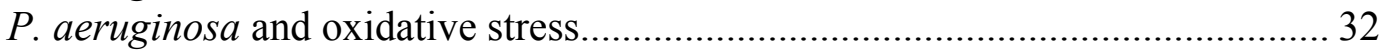

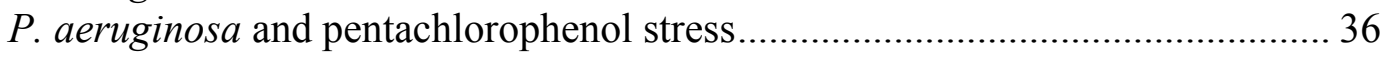

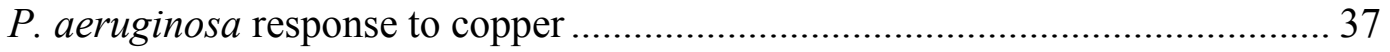

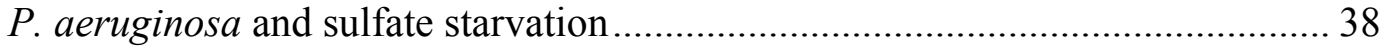

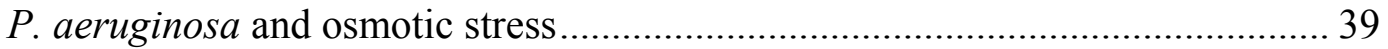

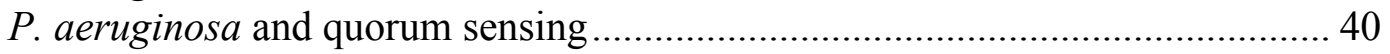

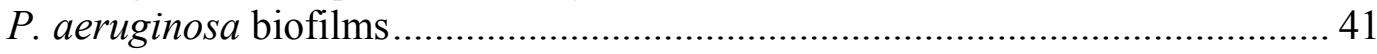

Transcriptomics of alginate production in P. aeruginosa ..................................... 43

Lipopolysaccharides and lipoproteins as virulence determinants........................... 44

Transcriptome of a post transcriptional regulator .............................................. 45

P. aeruginosa interactions with human cells ..................................................... 45

Comparative analysis of all transcriptomes ........................................................ 48

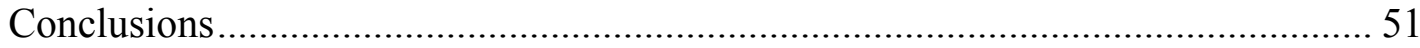




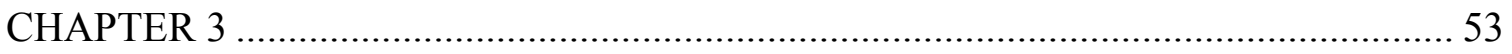

Transcriptional regulatory network in Pseudomonas aeruginosa ………………........ 53

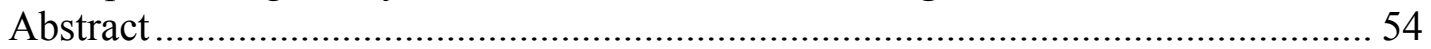

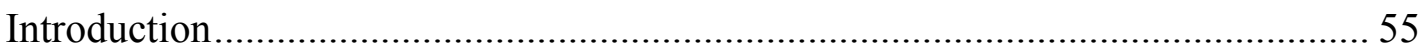

Families of transcriptional regulators in P. aeruginosa …………...........................59

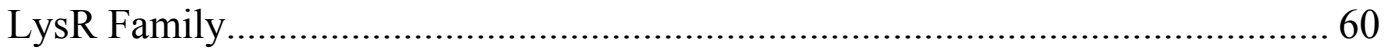

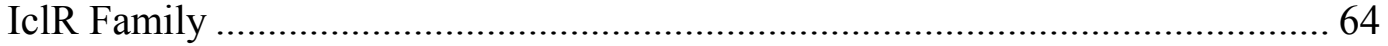

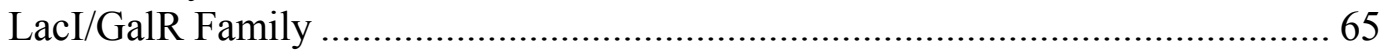

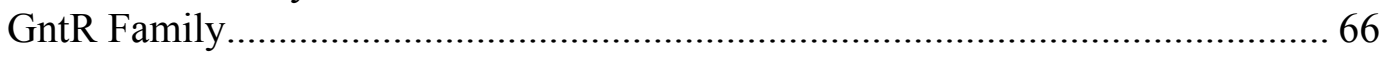

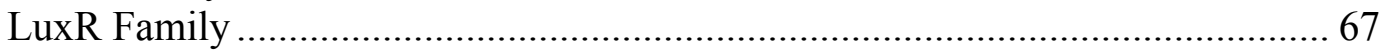

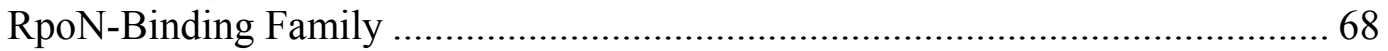

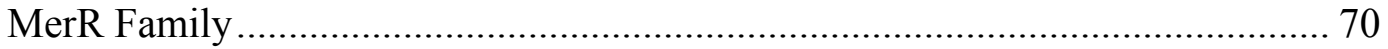

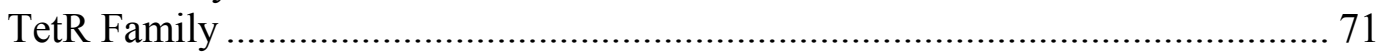

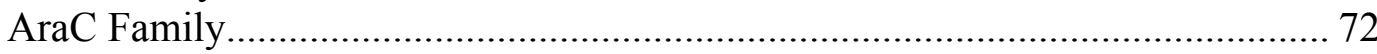

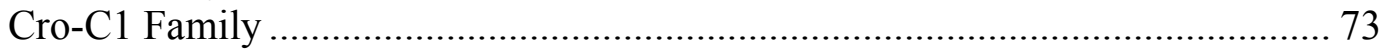

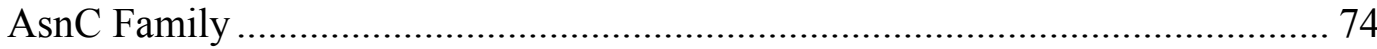

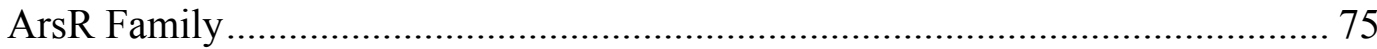

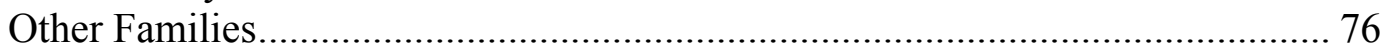

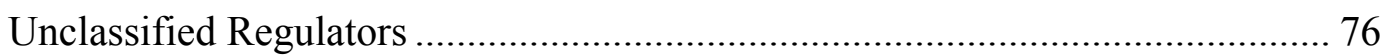

P. aeruginosa transcriptional regulatory network .................................................. 77

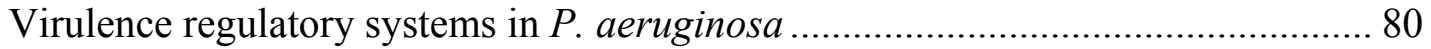

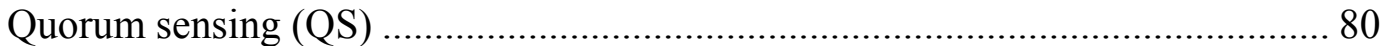

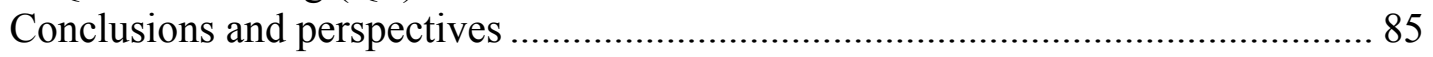

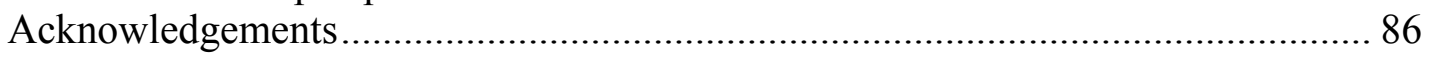

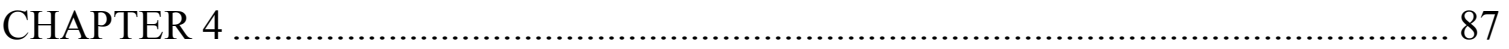

The regulatory repertoire of Pseudomonas aeruginosa AmpC B-lactamase regulator

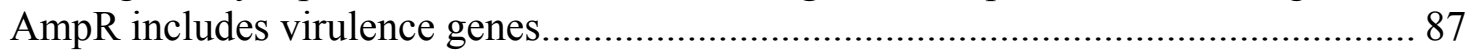

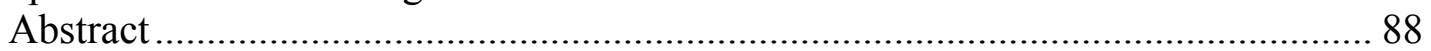

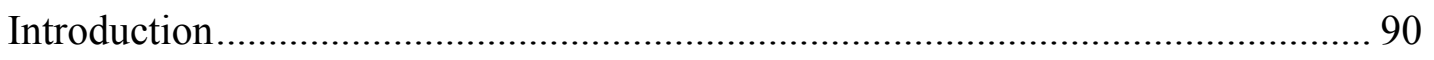

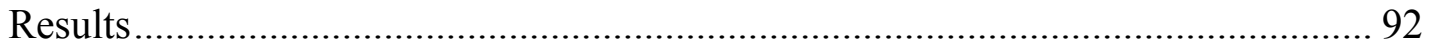

Deletion of $a m p R$ reduces $\beta$-lactam resistance of PAO1 .................................... 92

Loss of $a m p R$ affects ability of PAO1 to kill C. elegans ..................................... 95

AmpR regulates numerous genes in P. aeruginosa ............................................. 97

AmpR regulates genes both in the absence and presence of $\beta$-lactam stress...... 100

Functional categorization of AmpR-regulated genes ......................................... 103

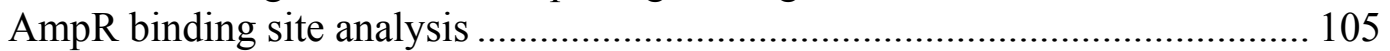

Regulation of the amp genes by AmpR .......................................................... 106

AmpR regulates the expression of antibiotic resistance and virulence systems. 109

Resistance-Nodulation-Division (RND) efflux systems................................. 109

QS-regulated virulence factors .............................................................. 112

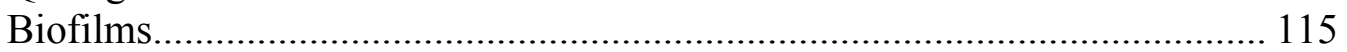

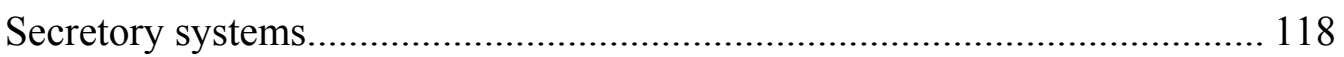

AmpR regulates genes found in regions of genome plasticity .......................... 121 
AmpR regulates other transcriptional regulators ................................................ 122

Subtractive transcriptomics of the AmpR regulon............................................ 127

Phenotypic microarray analysis of PAO $\triangle a m p R$............................................ 129

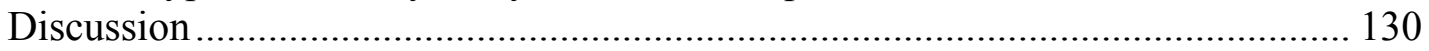

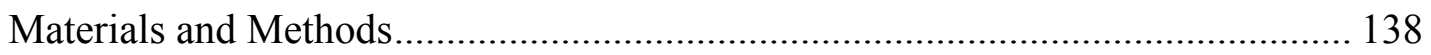

Bacterial strains, nematodes, media and primers............................................ 138

Construction of deletion mutants, complementation clones ................................. 141

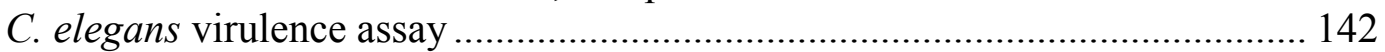

RNA isolation, generation of cDNA probes, microarray experiments and data

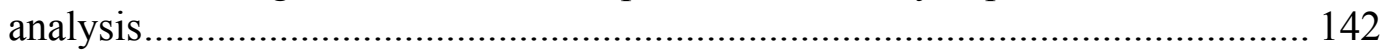

Bioinformatics analysis of the AmpR-binding site........................................... 143

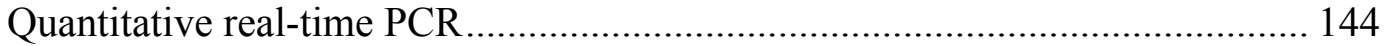

Determination of minimum inhibitory concentration (MIC)............................. 145

Quantifying $\beta$-lactamase activity ................................................................ 145

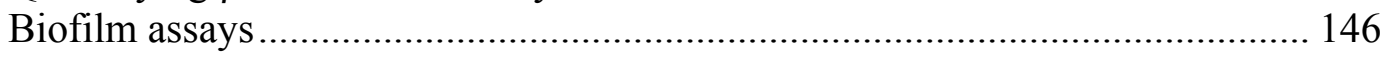

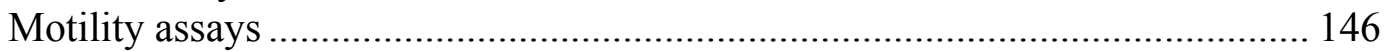

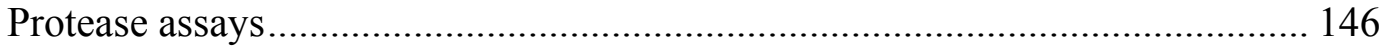

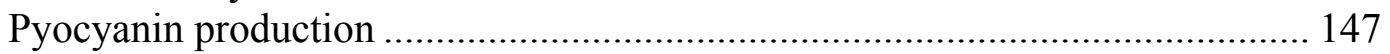

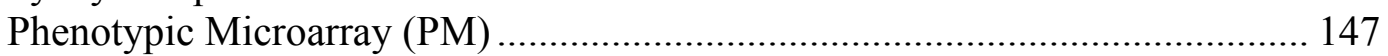

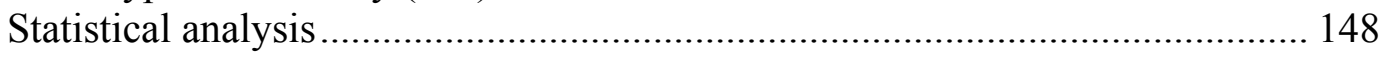

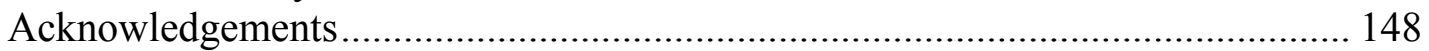

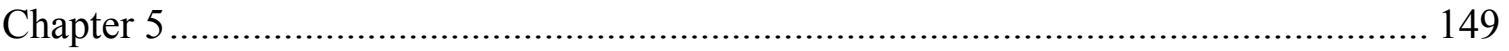

Deep Sequencing Expands the Pseudomonas aeruginosa ....................................... 149

AmpR Regulon to Include Regulatory RNAs ...................................................... 149

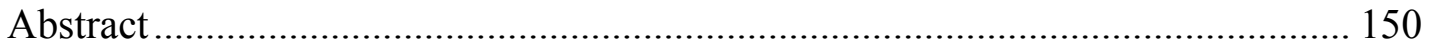

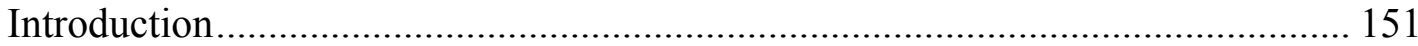

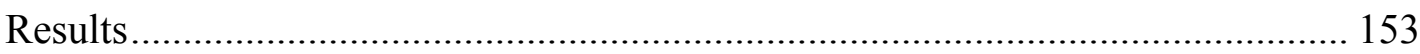

RNA-Seq analysis expands the AmpR regulon .............................................. 153

Identification of AmpR- and AmpR-B-lactam-dependent gene sets.................... 156

AmpR-regulated genes are enriched in specific functional categories ................ 159

Regulation of small RNAs (sRNAs) by AmpR .............................................. 162

AmpR regulates iron uptake positively............................................................. 163

P. aeruginosa AmpR regulates heat-shock response by modulating $\operatorname{rgP} 32$

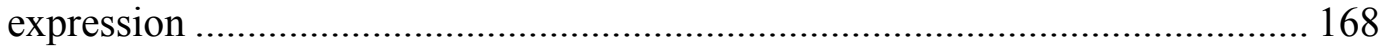

AmpR positively regulates $P$. aeruginosa oxidative stress response ................ 171

AmpR regulates phenazine production by modulating expression of $p h z A 1-G 1$

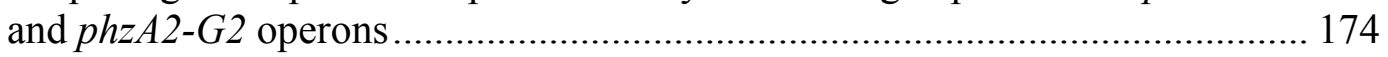

The PQS system is also positively regulated by AmpR .................................... 176

V5-tagged AmpR is functional in vivo ……………….................................. 178

Identifying direct AmpR targets by ChIP-Seq............................................... 179

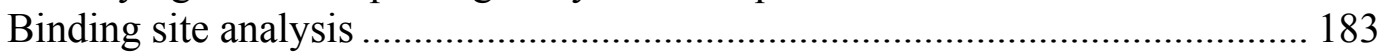

LasR is a direct target of AmpR ................................................................ 185

Comparison of microarray, RNA-Seq and proteome data................................. 186 


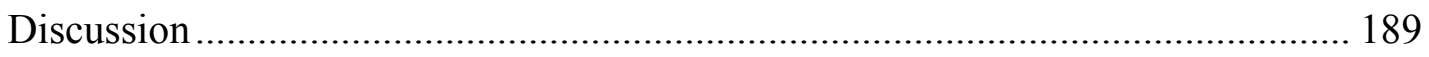

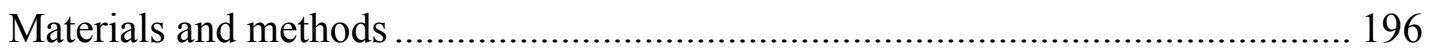

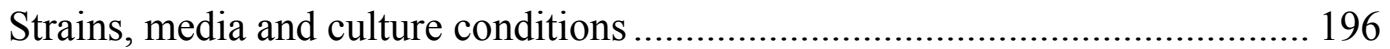

Library preparation for RNA-Seq analysis ..................................................... 198

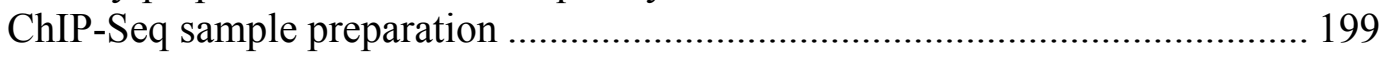

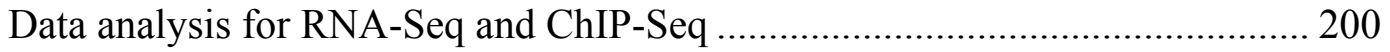

Enrichment of functional categories ............................................................... 200

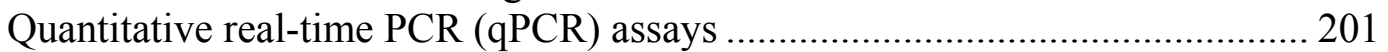

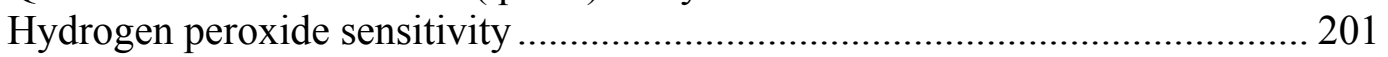

Growth in iron-limited media ........................................................................ 202

Temperature sensitivity assays ………………………............................. 202

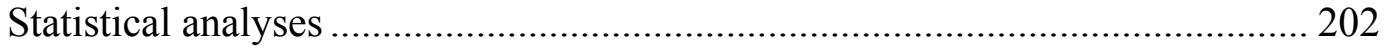

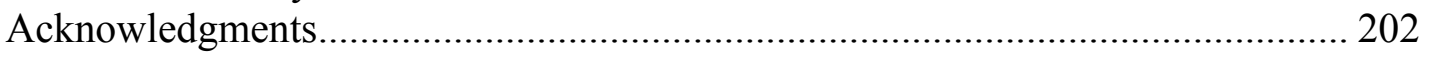

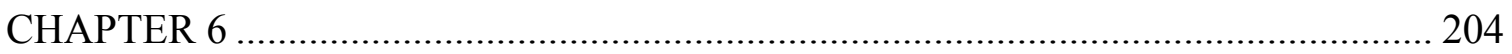

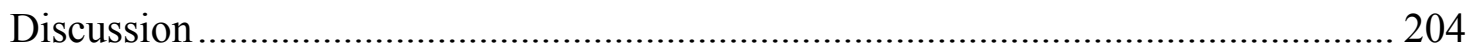

P. aeruginosa AmpR is a major regulator of antibiotic resistance ........................ 207

AmpR regulates quorum sensing in P. aeruginosa ............................................. 209

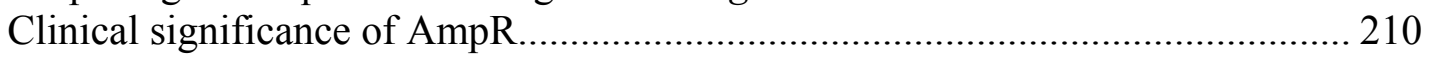

Major virulence regulatory systems in P. aeruginosa .......................................... 212

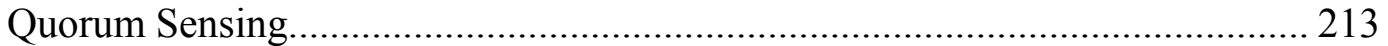

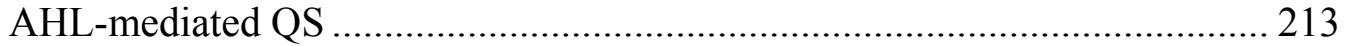

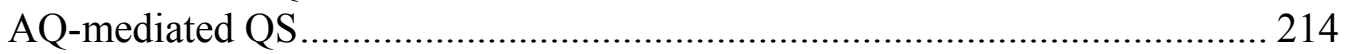

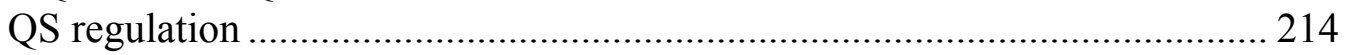

Two component regulatory systems (TCSs) ..................................................... 217

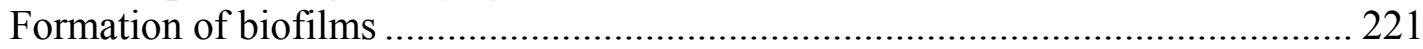

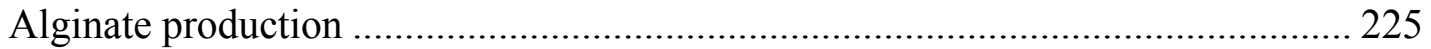

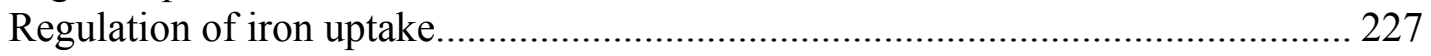

Toxins and exoproteins .................................................................................. 230

Regulatory RNAs in P. aeruginosa virulence ................................................... 232

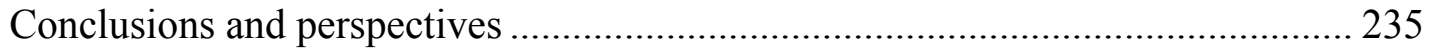

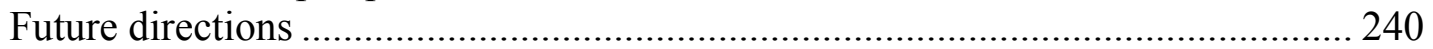

Identifying effectors that modulate AmpR activity ....................................... 241

Identification of small molecule inhibitors of AmpR .......................................... 245

Known, putative and novel small regulatory RNAs in the AmpR regulon ......... 246

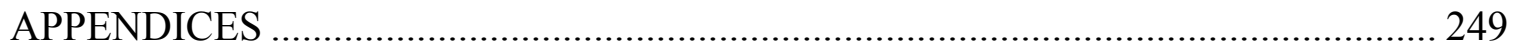

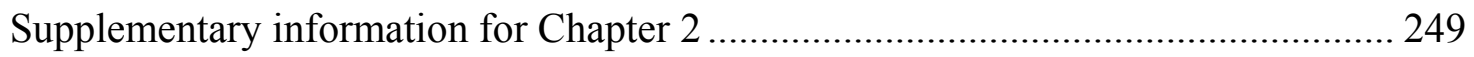

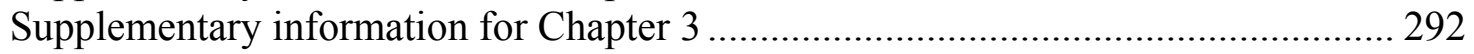

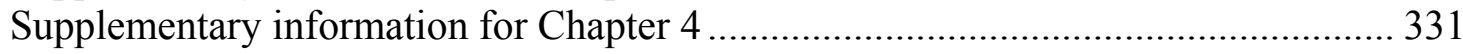

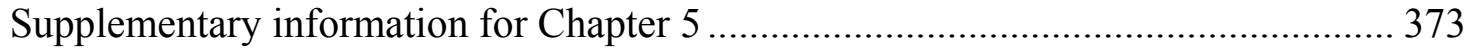


BIBLIOGRAPHY.

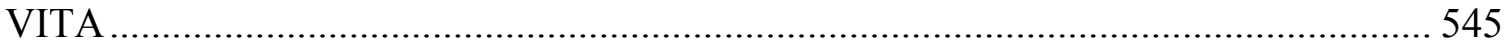




\section{LIST OF TABLES}

TABLE TITLE PAGE

2.1 Comparative analysis of significantly differentially $\quad 49$ regulated genes from various studies within each condition

3.1 Characteristics of the major families of transcriptional 61 regulators in P. aeruginosa

$\begin{array}{lll}4.1 & \text { Microarray vs. qPCR }\end{array}$

4.2 $a m p R$ deletion affects susceptibility to MexEF-OprN 111 substrates

4.3 Effect of deletion of $a m p R$ on QS-regulated virulence $\quad 114$ phenotypes

4.4 Transcriptional regulators and sigma factors regulated by 123 AmpR

4.5 Strains and plasmids used in this study 139

5.1 AmpR ChIP peaks 180

$\begin{array}{lll}5.2 & \text { Strains and plasmids } & 197\end{array}$ 


\section{LIST OF FIGURES}

FIGURE TITLE

PAGE

1.1 Antibiotic resistance mechanisms in P. aeruginosa

10

1.2 Components of the bacterial peptidoglycan function as effectors in modulating AmpR activity

20

3.1 P. aeruginosa transcriptional regulatory protein families

58

3.2 Interaction among transcriptional regulators

78

3.3 Association between QS regulators in P. aeruginosa

82

4.1 Antibiotic resistance profile of PAO $\Delta a m p R$

4.2 Effect of $\operatorname{ampR}$ deletion on pathogenicity to C. elegans 96

4.3 Scatter plots of significantly regulated genes 98

4.4 Venn diagram of differentially regulated genes 101

$\begin{array}{lll}\text { 4.5 Enrichment of functional categories } & 104\end{array}$

$\begin{array}{lll}\text { 4.6 AmpR binding site analysis } & 107\end{array}$

4.7 Expression of amp genes in PAO $\triangle a m p R \quad 108$

$\begin{array}{lll}4.8 & \text { AmpR affects biofilm formation } & 117\end{array}$

$\begin{array}{ll}4.9 & \text { Regulation of secretion genes by AmpR } \\ \end{array}$

4.10 Gene expression in PAO $\triangle a m p R$ at 40 minutes and two hours post 126 B-lactam exposure

4.11 Comparison of the AmpR transcriptome with other transcriptomes 128 
4.12 AmpR is a master regulator of gene expression in P. aeruginosa PAO1

5.1 Gene expression in PAO $\triangle a m p R$

155

5.2 Venn diagram of differentially regulated genes

157

5.3 Functionally categorization of AmpR-regulated genes

160

5.4 AmpR regulates iron uptake

166

5.5 Regulation of heat-shock response by AmpR

5.6 AmpR regulates resistance to oxidative stress

5.7 Regulation of phenazines genes by AmpR

5.8 AmpR positively regulates critical QS regulators of the Las, Rhl and PQS systems

5.9 Quantitative PCR analysis of Gac-Rsm pathway genes

182

5.10 ChIP-Seq data based AmpR binding site analysis

184

5.11 Comparative analyses of AmpR microarray, RNA-Seq and

187 proteome datasets

5.12 AmpR-mediated regulation of virulence and physiological processes in $P$. aeruginosa

6.1 P. aeruginosa virulence determinants

6.2 The P. aeruginosa virulence regulatory network 


\section{LIST OF SYMBOLS}

$\begin{array}{cl}\text { SYMBOL } & \text { TERM/ UNIT } \\ { }^{\circ} \mathrm{C} & \text { Beta } \\ \Delta & \text { Degree celsius } \\ \text { et al. } & \text { Delta (deletion) } \\ \mu \mathrm{g} & \text { Microgram } \\ \mu \mathrm{l} & \text { Microliter } \\ \% & \end{array}$




\section{LIST OF ACRONYMS AND ABBREVIATIONS}

\section{ACRONYM/ \\ ABBREVIATION}

ADP

AHL

AQ

ATP

$\mathrm{bp}$

cDNA

CFU

ChIP

C. elegans

DNA

$\mathrm{ECF}$

E. coli

FDR

GC

GlcNAc

GOID

HLH

$\mathrm{HTH}$
TERM

Adenosine diphosphate

$\mathrm{N}$-acyl homoserine lactone

2-alkyl-4 quinolone

Adenosine triphosphate

Base pairs

Complementary deoxyribonucleic acid

Colony forming unit

Chromatin immunoprecipitation

Caenorhabditis elegans

Deoxyribonucleic acid

Extracytoplasmic function

Escherichia coli

False discovery rate

Guanine-cytosine

$\mathrm{N}$-acetyl glucosamine

Gene ontology identification

Helix-loop-helix

Helix-turn-helix 


\begin{tabular}{|c|c|}
\hline IclR & Isocitrate lyase regulator \\
\hline LB & Luria Bertani \\
\hline LPS & Lipopolysaccharide \\
\hline LTTR & LysR-type transcriptional regulator \\
\hline MB & Mega base pairs \\
\hline MDR & Multi drug resistant \\
\hline MFS & Major facilitator superfamily \\
\hline MIC & Minimum inhibitory concentration \\
\hline $\mathrm{ml}$ & Milliliter \\
\hline $\mathrm{mm}$ & Millimeter \\
\hline $\mathrm{mM}$ & Millimolar \\
\hline MurNAc & $\mathrm{N}$-acetyl muramic acid \\
\hline $\mathrm{NaCl}$ & Sodium chloride \\
\hline NADH & Nicotiamide adenine dinucleotide \\
\hline $\mathrm{NaOCl}$ & Sodium hypochloride \\
\hline $\mathrm{OM}$ & Outer membrane \\
\hline ORF & Open reading frame \\
\hline P. aeruginosa & Pseudomonas aeruginosa \\
\hline PBP & Penicillin binding protein \\
\hline
\end{tabular}




\begin{tabular}{|c|c|}
\hline PCP & Penta chlorophenol \\
\hline PCR & Polymerase chain reaction \\
\hline PFAM & Protein family \\
\hline PQS & Pseudomonas quinolone signaling \\
\hline qPCR & Quantitative real-time polymerase chain reaction \\
\hline QS & Quorum sensing \\
\hline RGP & Regions of genome plasticity \\
\hline RNA & Ribonucleic acid \\
\hline RND & Resistance nodulation division \\
\hline$r r n$ & Ribosomal RNA locus \\
\hline SAGE & Serial analysis of gene expression \\
\hline SSM & Synthetic succinate medium \\
\hline T3SS & Type III secretion system \\
\hline T6SS & Type VI secretion system \\
\hline TCS & Two component system \\
\hline TFP & Type IV pili \\
\hline wHTH & Winged helix-turn-helix \\
\hline XDR & Extremely drug resistant \\
\hline
\end{tabular}




\section{CHAPTER 1}

\section{Introduction}




\section{The model bacterium, Pseudomonas aeruginosa}

The genus Pseudomonas encompasses metabolically versatile bacteria that can utilize a wide range of organic and inorganic substrates. It comes as no surprise then that members of this genus are found in a variety of habitats in soil and water and are also important pathogens of plants, animals and humans (1). Walter Migula of the Karlsruhe Institute in Germany named the genus Pseudomonas in the 1894 . He defined the genus based on cell morphology as,

'Cells with polar organs of motility. Endospore formation of spores occurs in some species but infrequently (for instance, Pseudomonas violacea)' (2).

The definition was brief and somewhat vague. We now know that none of the pseudomonads produce spores and what Migula observed were probably refractile granules. In Greek, Pseudes means false, Monas means units, and the genus was described as such in the Bergey's Manual of Determinative Bacteriology and later in the Bergey's Manual of Systematic Bacteriology $(3,4)$. However, it has been hypothesized that Migula was actually referring to the nanoflagellate Monas whose cells bear a close resemblance to Pseudomonas cells and did not define the genus as 'false units' (5). Pseudomonads have now been studied extensively and the genus is now described as follows in the Bergey's Manual of Systematic Bacteriology:

Straight or slightly curved rods but not helical, 0.5-1.0 $\mu \mathrm{m}$ in diameter by 1.5-5.0 $\mu m$ in length. Most of the species do not accumulate granules of poly- $\beta$-hydroxybutyrate, but the accumulation of poly-hydroxyalkanoates of monomer lengths higher than C4 may occur when growing on alkanes or gluconate. Do not produce prosthecae and are not surrounded by sheaths. No resting stages are known. Gram-negative. Motile by one or 
several polar flagella; rarely non-motile. In some species, lateral flagella of short wavelength may also be formed. Aerobic, having a strictly respiratory type of metabolism with oxygen as the terminal electron acceptor; in some cases, nitrate can be used as an alternate electron acceptor, allowing growth to occur anaerobically. Xanthomonadins are not produced. Most, if not all, species fail to grow under acid conditions (pH 4.5 or lower). Most species do not require organic growth factors. Oxidase positive or negative. Catalase positive. Chemoorganotrophic. Strains of the species include in their composition the hydroxylated fatty acids 3-OH $10: 0$ and 12:0, and 2-OH 12:0, and ubiquinone Q-9. Widely distributed in nature. Some species are pathogenic for humans, animals or plants. The mol\% $G+C$ content of the DNA is 58-69.

Type species: Pseudomonas aeruginosa (Schroeter 1872) Migula 1900

Classification of strains into this genus now requires extensive polyphasic data, including molecular and biochemical characterization. Many strains produce pili and fimbriae. The cells are non-capsulated though they produce at least three different extracellular polysaccharides (alginate, Pel and Psl) under some growth conditions. The cells are aerobic but can grow anaerobically using nitrate as the terminal electron acceptor. This makes some species function as denitrifiers in the soil environment, though all species can use nitrate as a carbon source (6). Metabolic versatility is a hallmark of this genus and can utilize a wide range of organic and inorganic substrates as carbon source.

The type species of this genus is $P$. aeruginosa. The species name aeruginosa is Latin for verdigris meaning copper rust, referring to the blue-green pigment seen in lab cultures. The coloration is due to production of two extracellular pigments, pyocyanin and pyoverdin. $P$. aeruginosa can switch lifestyles from being an environmental isolate, found 
in the soil and water, to a human pathogen (7). It is an epitome of opportunistic human pathogens, being implicated in a wide range of infections of the pulmonary tract $(8,9)$, urinary tract (10-13), burns (14-16), wounds $(17,18)$, lung cancer patients (19), chronic obstructive pulmonary disease (20), bronchiectasis (21) and in pediatric and adult AIDS patients (22). It causes persistent nosocomial infections $(23,24)$ and is also a major pathogen in cystic fibrosis patients. One of the major weapons in its arsenal is its resistance to antibiotics. It is both inherently resistant and can also acquire resistance genes from other bacteria making it a notorious pathogen. Understandably, numerous studies over the years have focused on elucidating the mechanisms of pathogenesis and antibiotic resistance of $P$. aeruginosa. Since the sequencing of the genome (25), the emphasis of these studies has shifted from conventional pathogenesis research, where the focus was on individual virulence determinants, to a much larger scale involving whole genome analyses.

The prototypic reference strain of $P$. aeruginosa is PAO1, isolated from a burn wound (26), and was the first $P$. aeruginosa strain to be sequenced (25). The most prominent feature of the PAO1 genome is its size. It has one of the largest bacterial genomes with 6.26 million base pairs comprising of almost $90 \%$ protein coding genes $(25,27)$. The genome complexity of $P$. aeruginosa explains its versatile nature to adapt and thrive in a wide range of environments. The genome has 5,570 open reading frames (ORFs), a number that is close to simple eukaryotes like Saccharomyces cerevisiae. Other features of the PAO1 genome include the presence of four ribosomal RNA loci (rrn) that are the regions of the longest repeats and a high GC ratio at $66.6 \%$ (25). Following the sequencing of the genome, high-density DNA microarrays were developed for $P$. 
aeruginosa (28) that offered us a chance to take snapshots of the dynamic responses of this versatile genome to various stimuli. An important study in this context, which analyzed the genomes of both environmental and clinical isolates of $P$. aeruginosa, suggested the presence of a core genome in different strains and expression of different sets of genes to facilitate growth in particular environments (29). This calls for a robust control over gene expression mediated by transcriptional regulators. About $10 \%$ of the PAO1 genome is comprised of transcriptional regulators suggesting a tight control over the way genes are expressed in $P$. aeruginosa (25).

Subsequent to PAO1, other P. aeruginosa strains such as PA14 (30), PA2192 (a CF isolate) (31), C3719 (the Manchester epidemic strain) (32), and PACS2 (33), have been sequenced. Comparative genome analysis of the five sequenced $P$. aeruginosa strains has led to the identification of a set of core shared genes that all the genomes contain, the majority of which are housekeeping genes (33). In addition, there are accessory genomes comprising of mostly hypothetical ORFs or those related to transposons and bacteriophages, and genes involved in adaptation specific to each of the five strains (33). The accessory gene set also contains transcriptional regulators that may control expression of ORFs in the core genome. In order to better manage $P$. aeruginosa infections, it is imperative for us to understand the workings of the bacterium, what are the environmental signals it recognizes, how are these signals translated into a transcriptional response and how can we interfere with any of these processes.

\section{Antibiotics, antibiotic resistance and AmpR}

Antibiotics are antimicrobials that are produced by certain soil bacteria and actinomycetes as secondary metabolites. They kill other bacteria at low concentrations 
and have been used to treat bacterial infections, in human and veterinary medicine, and in agriculture. The antibiotic era was ushered in by the serendipitous discovery of penicillin by Alexander Fleming. Subsequently, the 'father of antibiotics' Selman Waksman, identified over 20 different antibiotics that have revolutionized the way we manage bacterial infections. The mode of action of antibiotics varies but fall into two main categories: bactericidal and bacteriostatic. Bactericidal antibiotics kill bacteria while bacteriostatics only inhibit growth.

Antibiotics are also classified based on their chemical structure and mode of action. For example, DNA synthesis is inhibited directly by fluoroquinolones (nalidixic acid and ciprofloxacin) by affecting the topoisomerases, and indirectly by trimethoprimsulfamethaxazole by inhibiting tetrahydrofolic acid synthesis. Rifamycins (rifamycin, rifampicin, rifapentine) inhibit RNA synthesis by acting on the DNA-dependent RNA polymerase. Protein synthesis inhibitors (aminoglycosides, macrolides, tetracyclines, ketolides, phenicols) act primarily by interfering with the activity of the $30 \mathrm{~S}$ and $50 \mathrm{~S}$ ribosomes affecting translation. Cell wall synthesis inhibitors include B-lactams (penicillins, cephalosporins, carbapenems), glycopeptides (vancomycin, teicoplanin) and lipopeptides (daptomycin, polymixin B) that inhibit various stages of the peptidoglycan synthesis process.

Antibiotics affect disparate cellular functions and four groups of genes have been identified based on their expression response to antibiotic treatment: genes that are directly affected by target inhibition (group 1); genes affected indirectly by antibiotic action (group 2); genes affected due to secondary effects of antibiotics (group 3); those that are differentially regulated in response to antibiotics but are not related to the drug 
target (group 4) (34). Determining the transcriptional response of novel antimicrobials and comparing it to the transcriptional profiles of known antibiotics can potentially also provide information on the mechanism of action (35).

The role of antibiotics in nature is much debated. The contention is that if the initial intent of antibiotics production by bacteria was to wipe out the competition, one should find antibiotics at inhibitory concentrations in, for example, soil but this is not the case. Moreover, antibiotics are produced as secondary metabolites, during the late-log to stationary phase. If the primary role of antibiotics was to provide a growth advantage by wiping out competition, they should be produced in the log phase when the producer cell needs the most nutrients. Recent studies have shown that antibiotics at low concentration can in fact enhance biofilm growth and motility (36). It has therefore been proposed that antibiotics could function as signaling molecules in the environment (37). In Streptomyces spp., antibiotic production is under the control of a cell-cell communication mechanism called quorum sensing (QS) (38). In P. aeruginosa, PQS, the signaling molecule of one of the three QS systems, possess antibacterial activity at very high concentrations $(39,40)$. In addition, some antibiotics have immunomodulatory effects; macrolides have anti-inflammatory properties and are used in CF patients to exploit this property rather than as an antibacterial (41). Rifampicin, on the other hand has proinflammatory activity (42).

\section{Antibiotic resistance}

ß-Lactamases, enzymes that degrade $\beta$-lactam antibiotics, are presumed to have evolved around 2 million years ago (43). However, the race to evolve resistance to the newer antimicrobials has been accelerated in the recent decades with the widespread use 
of antibiotics. Bacteria have quickly become resistant to almost all natural, synthetic and semi-synthetic antibiotics used to eradicate them (44). In fact, microbes seem to have the upper hand in the arms race. In spite of their evolutionary simplicity, bacteria possess extreme adaptability that has helped them survive over millions of years.

Bacteria resist antibiotics in a variety of mechanisms that include reduced membrane permeability, target alteration, efflux pumps, and hydrolytic enzymes (Figure 1.1). Some bacteria, such as $P$. aeruginosa, are intrinsically resistant to antibiotics while most of them can acquire antibiotic resistance genes from other organisms via horizontal gene transfer. Transfer can occur on mobile elements such as transposons, uptake of free DNA, be mediated by bacteriophages or by conjugation between the donor and recipient bacteria. Acquisition of such genes can lead to enhanced resistance to antimicrobials for the recipient bacteria. On the other hand, some mutations can reduce resistance, though typically the reduction in levels of resistance is not dramatic. Such low level increases in resistance are important in the clinical setting (45).

Development of resistance to an antibiotic is seen to follow soon after its introduction. Fleming noted this soon after he discovered penicillin. Penicillin had a MIC of $0.04 \mu \mathrm{g} / \mathrm{ml}$ when first introduced in 1940 against Streptococcus pneumonia (46), increased to $0.12-1 \mu \mathrm{g} / \mathrm{ml}$ in 1980 (47) and by the $1990 \mathrm{~s}$, the strains became completely resistant prompting discontinued usage. With other bacteria, the development of resistance is even more drastic. Quinolones, such as ciprofloxacin, were first used to treat P. aeruginosa in 1990 when the MIC was $<0.5 \mu \mathrm{g} / \mathrm{ml}$ (48). In less than 8 years, clinical isolates started displaying intermediate resistance ( $8 \mu \mathrm{g} / \mathrm{ml}$ in 1998) (49) and in 2009 , the resistance has increased to $32-64 \mu \mathrm{g} / \mathrm{ml}$ (50). The same trend is also seen with the 
aminoglycoside, tobramycin for $P$. aeruginosa. The clinical isolates started displaying resistance ( $<2 \mu \mathrm{g} / \mathrm{ml}$ in $1980,8 \mu \mathrm{g} / \mathrm{ml}$ in 1987 and $\geq 16 \mu \mathrm{g} / \mathrm{ml}$ in 2000$)$ in a very short time span (51-53).

\section{Antibiotic resistance in $P$. aeruginosa}

P. aeruginosa displays a high level of resistance to many of the clinically relevant antibiotics. Resistance is a cumulative measure of three main types mechanisms: intrinsic, acquired and adaptive. Whereas intrinsic and acquired resistances are stable and heritable, adaptive resistance is dependent on the environment and is inducible. The antibiotic resistance mechanisms of $P$. aeruginos $a$ are summarized in Figure 1.1.

\section{Intrinsic resistance}

Intrinsic resistance is a characteristic feature of $P$. aeruginosa and is principally mediated by low outer membrane permeability. The outer membrane in Gram-negative bacteria act as molecular sieves, and uptake of hydrophilic molecules is by diffusion through water-filled porins. The major porin in P. aeruginosa is OprF and most OprF channels are small similar to OprD and OprB. 


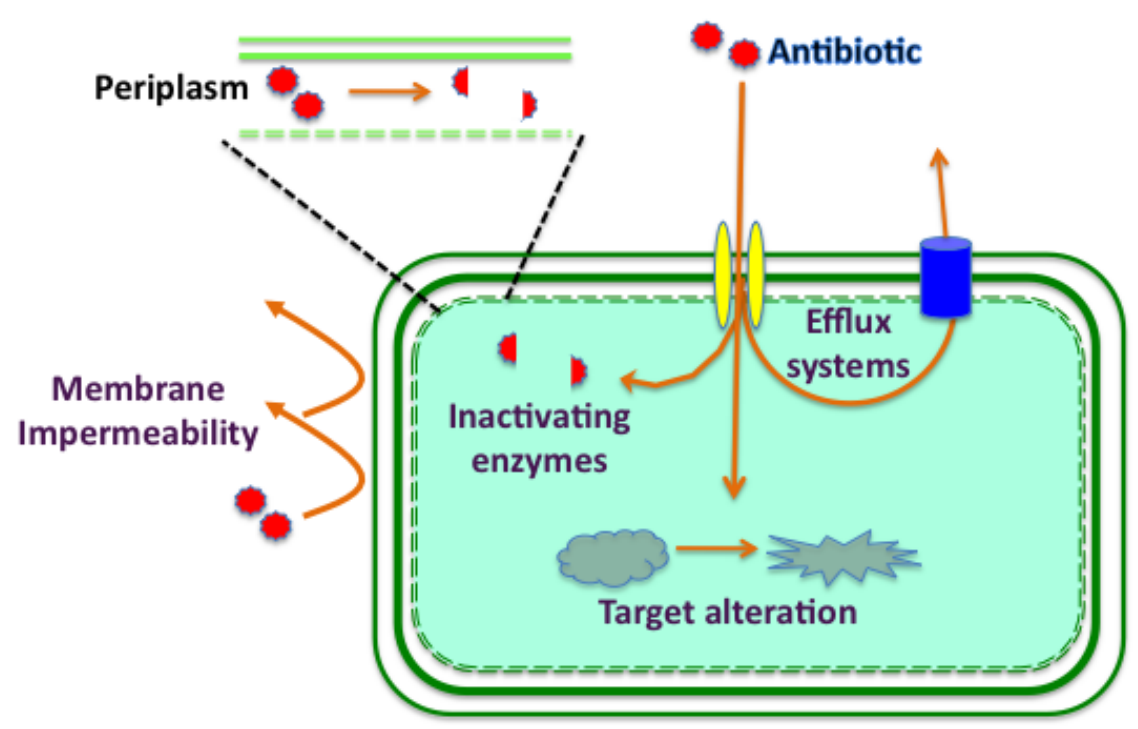

Figure 1.1: Antibiotic resistance mechanisms in P. aeruginosa. Schematic representation of the multiple mechanisms of antibiotic resistance in P. aeruginosa. Cellular appendages and intracellular components are not shown for simplicity.

The $P$. aeruginosa outer membrane is thus 12 to 100 times less permeable compared to that of E. coli (54). Antibiotics enter P. aeruginosa through the porins (for example, imipenem through OprD), self-promoted uptake (polycationic antibiotics) or by diffusion through the membrane (hydrophobic molecules). Hydrophilic molecules, however, still equilibrate across the outer membrane quickly and this is where the other mechanisms of intrinsic resistance play a role. Efflux pumps such as MexAB-OprM, MexXY-OprM (55-57) and induction of the B-lactamase AmpC (58) effectively deal with the antibiotics by either pumping them out of the cell thereby reducing cytoplasmic retention time or by hydrolyzing them, respectively. These secondary resistance mechanisms rely on the reduced permeability for enhanced effectiveness. In addition, recent studies have identified many mechanisms that contribute to $\beta$-lactam resistance in P. aeruginosa (59-61). P. aeruginosa adaptively regulates resistance mechanisms in response to antibiotics contributing to the resistance profile seen with this pathogen 
$(34,62)$. Intrinsic resistance is thus multifactorial and a major contributing factor to the high level of clinical resistance displayed by P. aeruginosa.

\section{Acquired Resistance}

Acquired resistance of $P$. aeruginosa to antibiotics is achieved by horizontal gene transfer or by mutations. Resistance genes can be acquired on plasmids, pathogenicity islands, prophages, transposons or integrons, sometimes harboring resistance against multiple antibiotics. Typically, horizontal gene transfer affects resistance to aminoglycosides and $\beta$-lactams $(63,64)$. Many plasmids that harbor genes encoding resistance to extended spectrum ß-lactams and metallo ß-lactams have now been identified (65).

Mutations can also lead to acquired resistance. Natural resistance frequencies for individual antibiotics vary between $10^{-6}$ to $10^{-9}$, which can increase under conditions such as biofilm growth, DNA damage or in hypermutator strains (66-68). Mutations in the porin (69) or in their regulators (70) can lead to reduced uptake of antibiotics. Enhanced expression/activity of efflux pumps can also occur due to regulator mutations $(71,72)$. Mutations can also lead to overexpression of hydrolytic enzymes that inactivate antibiotics conferring resistance to specific classes of antibiotics such as ß-lactamases (73-76). Strains develop resistance to fluoroquinolones due to target alterations, a consequence of mutations (77-79). Recent work has also defined the phenomenon of 'creeping baselines', where resistance is acquired in seemingly non-significant increases in MIC but the cumulative resistance is clinically significant (35). 


\section{Adaptive Resistance}

In response to specific environmental cues, cells display enhanced resistance to one or more antibiotics. When the stressor/signal is removed, the cells lose the resistance phenotype, though 'normal' levels of resistance are typically not restored (80). This phenomenon is termed 'adaptive resistance'. Though the phenomenon was observed in the 1960s (81), it has not been studied in detail. The four main defining features of adaptive resistance are (i) it is transient, (ii) not heritable, (iii) generally reverts upon removal of the inducing signal and (iv) is dependent on the environment (35). In addition to sub-MIC antibiotics, other identified triggers of adaptive resistance are environmental cues (anaerobiosis, ions and ionic binding, $\mathrm{pH}$, carbon sources, polyamines), biofilm formation and swarming motility (35). Two-component systems such as ParRS and CprRS sense cationic peptides in the environment and trigger adaptive resistance $(72,82)$.

In many cases, $P$. aeruginosa infections can be effectively dealt with. However, when $P$. aeruginosa forms biofilms, for example in the cystic fibrosis lung, eradication of infection becomes very difficult due to antibiotic resistance. The presence of persister cells further compounds this problem (83). This calls for urgent steps to not only find new treatment options but also to devise measures for more prudent use of antibiotics.

\section{B-Lactam resistance in $P$. aeruginosa}

ß-Lactam antibiotics, in combination with aminoglycosides, are part of the current treatment regimen for $P$. aeruginosa infections (84). However, the phenomenon of $\beta$ lactam resistance is a major issue with $P$. aeruginosa acute and chronic infections. Antibiotic resistant isolates of $P$. aeruginosa are selectively favored in vivo in CF patients $(85,86)$. A major mechanism of B-lactam resistance in $P$. aeruginosa is the 
overproduction of the chromosomal ß-lactamase, AmpC. Hyperexpression and presence of B-lactamases in the biofilm matrix, either free or in membrane vesicles, has been linked to the reduced effectiveness of $\beta$-lactam treatment (87-89). In the 90 s, many $P$. aeruginosa $\mathrm{CF}$ isolates were found to either express high inducible or constitutively overexpress $\beta$-lactamase $(90,91)$. One of the molecular mechanisms of overproduction of B-lactamases was attributed to insertional inactivation of the $a m p D$ gene in P. aeruginosa $(73,92)$. Studying the regulation of amp genes will aid in understanding the molecular mechanisms of $\beta$-lactamase overexpression in P. aeruginosa.

\section{Transcriptional regulation of the $B$-lactamase ampC}

The LysR-type transcriptional regulator AmpR regulates inducible ampC $\beta$ lactamase expression. The genes $a m p R$ and $\operatorname{ampC}$ have been found in many organisms (93-97). In systems with inducible $\operatorname{ampC}$, the $\operatorname{ampR}$ gene is always present adjacent to ampC but is divergently transcribed (98-100). The ampR-ampC arrangement in $P$. aeruginosa is similar to that seen in other organisms including Citrobacter freundii and Enterobacter cloacae. AmpR of C. freundii and E. cloacae can cross-complement each other (101) and sequence analysis revealed that $P$. aeruginosa AmpR is similar to that found in C. freundii (58\%) and E. cloacae (62\%) (102). In C. freundii, AmpR recognizes a 15-bp sequence 5' TCTGCTGCTAAATTT 3' in $\mathrm{P}_{a m p C}(98,102)$. There is a putative AmpR binding site 5' TCTGCTCCAAATTT 3' in the ampR-ampC intergenic region in $P$. aeruginosa. AmpR has a helix-turn-helix ( $\mathrm{HTH})$ motif that is typical of DNA binding proteins (102). C. freundii AmpR has been shown to bind DNA through its HTH motif (98). In P. aeruginosa, the third helix of the AmpR HTH motif is critical for DNA 
binding and amino acid residues important for DNA binding have been identified (O. Caille, unpublished data).

AmpR belongs to the LysR family of transcriptional regulators that typically autorepress their own expression $(103,104)$. This has been suggested to be the case with C. freundii (98). The ampR-ampC intergenic region in $P$. aeruginosa is only $149 \mathrm{bp}$ suggesting that ampR expression is autoregulated (102). Previous studies from our lab using $\mathrm{P}_{\text {ampR }}$-lacZ fusion have shown that there is no difference in the expression from the $a m p R$ promoter between the wild-type and $\operatorname{ampR}$ mutant strains suggesting that $a m p R$ is not autoregulated in P. aeruginosa (105).

\section{P. aeruginosa AmpR regulates other genes}

The regulatory role of AmpR was determined by comparing the properties of $P$. aeruginosa PAO1 with its isogenic ampR insertion mutant, PAOampR::aacCl, henceforth referred to as PAOampR (105). PAOampR expressed high basal levels of ßlactamase even in the absence of antibiotic stress (105). Inducibility was restored by transcomplementation of $a m p R$. There was no expression from $\mathrm{P}_{\text {amp }}$ in PAOampR (105). These data indicate that AmpR positively regulates ampC similar to data from other bacterial genera.

Interestingly, loss of ampC reduced but did not completely abolish $B$-lactamase activity. This, and previous observations with $\mathrm{P}_{\text {ampc }}$-lacZ, led to the discovery of a second chromosomal B-lactamase, an oxacillinase PoxB (106). The poxB gene is part of a twogene operon (poxAB). Introduction of this operon on a plasmid into E. coli, in the presence and absence of $P$. aeruginosa ampR, resulted in higher levels of B-lactamase activity only in the absence of $a m p R$. Reduction of activity in the presence of $a m p R$ 
suggested that $A m p R$ negatively regulates poxB (106). Ongoing work in the Mathee lab is aimed at better understanding AmpR-regulation of poxB.

The PAOampR strain also produced higher levels of pigments when cultured on plates or in broth suggesting that ampR may be involved in regulating pigment production (105). Pseudomonas produces green pigments that act as siderophores, chelating iron from the environment, offering the bacteria a survival advantage and enhancing virulence $(107,108)$. Pyocyanin production was significantly higher in PAOampR compared to PAO1, suggesting that AmpR negatively regulates pyocyanin production (105).

Pyocyanin production in $P$. aeruginosa is under the regulation of the las quorumsensing (QS) system that along with the $r h l$ system controls expression of many virulence genes in P. aeruginosa (109). Thus, AmpR dependence of two other virulence factors under QS control, LasA protease and LasB elastase were analyzed (105). LasA is a zinc metalloprotease of the B-lytic endopeptidase family (110). PAOampR produced more LasA compared to PAO1, and the levels were restored on ampR complementation suggesting that AmpR negatively regulates LasA (105). This was also shown with $\mathrm{P}_{\text {lasA }}{ }^{-}$ lacZ fusions in PAO1 and PAOampR. LasB elastase is a zinc metalloprotease that can inactivate a wide range of biological tissues and immunological agents (111). In PAOampR, there was a two-fold induction in LasB production that was B-lactamindependent. The inducible phenotype was restored on complementing ampR in trans (105). This suggests that AmpR positively regulates LasB. $\mathrm{P}_{\text {lasB }}$-lacZ fusions however, did not show a significant change in lacZ expression between PAO1 and PAOampR indicating that $\mathrm{AmpR}$ does not regulate $\mathrm{P}_{\text {lasB }}$ directly. Expression of lasI and lasR, two 
other QS genes, were shown to be higher in PAOampR than in PAO1 (105) that demonstrates negative regulation of LasI and LasR by AmpR. These studies suggest that AmpR plays a central role in a regulon.

\section{Role of AmpR in peptidoglycan recycling}

The purported 'natural' role of AmpR in the cell is in the recycling of peptidoglycan. The peptidoglycan layer provides rigidity and shape to the bacterial cell. Not only is the peptidoglycan layer strong enough to withstand the internal pressure of the cell $(\sim 25 \mathrm{~atm})$, it is also flexible and porous enough to allow diffusion of large molecules (112). The bacterial peptidoglycan is a complex polysaccharide of $\mathrm{N}$-acetyl muramic acid (MurNAc) and N-acetyl glucosamine (GlcNAc) linked by $\beta-1,4$ glycosidic linkages (113). Multiple GlcNAc-MurNAc polymer chains are crosslinked by pentapeptide bridges (L-alanine-D-glutamine-meso diaminopimelic acid-D-alanine-Dalanine) forming a complex network of peptidoglycan $(113,114)$.

Most of the peptidoglycan required for the growing cell is synthesized de novo (115). Biosynthesis of peptidoglycan is a complex process involving over 20 steps that take place in the cytoplasm, and on the inner and outer sides of the cytoplasmic membrane (115-117). However, not all of the peptidoglycan incorporated in the growing cell wall is newly synthesized. Both Gram-positive and Gram-negative bacteria are known to recycle peptidoglycan during cell division $(118,119)$.

In $P$. aeruginosa and other bacteria, this recycling process is tied in with ß-lactam resistance. The continuous breakdown and regeneration of peptidoglycan as part of remodeling in a growing cell is called turnover (120). The turnover process releases the disaccharide GlcNAc-anhydrous MurNAc (GlcNAc-AnhMurNAc) still attached to the 
peptide chain. The chain may be three, four or five peptides long (GlcNAc-AnhMurNAc tri-, tetra-, and pentapeptides, respectively). These are transported into the cytoplasm by the AmpG permease (121). In E. coli, the acetylglucosaminidase NagZ acts on these products to cleave off GlcNAc resulting in tri-, tetra- and penta-peptides of AnhMurNAc (122). The NagZ homolog in P. aeruginosa (PA3005) has not been functionally demonstrated to be a glucosaminidase but its role in B-lactam resistance is established $(123,124)$. Following NagZ, the amidase AmpD breaks the amide bond between the sugar and amino acid (121). The AmpD amidase was identified as being important in $\beta$ lactamase induction in Citrobacter freundii and Enterobacter cloacae $(101,125)$. In addition to AmpD, P. aeruginosa has two other homologs, AmpDh2 and AmpDh3, and all three play a role in B-lactam resistance (73).

The recycled peptides and sugars then enter the de novo synthesis pathway where uridyl diphosphate (UDP)-GlcNAc is first converted to UDP-MurNAc by the enzymes MurA and MurB followed by addition of the pentapeptide side chain, forming UDPMurNAc pentapeptide (115). The enzyme MraY then links UDP-MurNAc pentapeptide to an undecaprenyl attached on the inner side of the cytoplasmic membrane forming lipid I (116). Addition of a molecule of GlcNAc to lipid I results in the formation of lipid II (116). A flippase enzyme, presumably MurJ in E. coli, then flips lipid II to the periplasmic side of the cytoplasmic membrane (126). Penicillin binding proteins (PBPs) then take over the process of incorporating the newly synthesized sugar-peptide complex to the growing peptidoglycan chain (117).

Studies show that though this system is similar in P. aeruginosa, there are important differences. Details of peptidoglycan recycling and their ties with ß-lactam 
resistance have been studied in Enterobacteriaceae members and due to the homology of the corresponding proteins in P. aeruginosa, the proteins are presumed to be orthologous. The role of many players including AmpR, AmpC, AmpD and AmpG has been demonstrated in P. aeruginosa and are similar to their Enterobacteriaceae counterparts. In addition, new players have been identified in $P$. aeruginosa, such as the AmpD homologs AmpDh2 and AmpDh3 (73), and AmpP (127) adding to the complexity of the process. Further, studies in the Mathee lab have demonstrated that AmpR is an inner membrane protein (Caille et al., personal communication), which leads to unique questions in how the effector molecules are transported to their binding sites on AmpR. In Figure 1.2, we propose a model that links cell wall recycling and B-lactam resistance in P. aeruginosa based on our current understanding of this process.

B-Lactam antibiotics target the PBPs, thus interfering with cell wall synthesis. When there are no ß-lactams in the milieu, the concentration of the GlcNAc-UDPMurNAc pentapeptide is high and this binds to the effector binding sites in AmpR, allowing AmpR to repress ampC $\beta$-lactamase expression. In the face of $\beta$-lactam attack, however, inhibition of PBP activity by the antibiotics leads to accumulation of the peptidoglycan degradation products (GlcNAc-AnhMurNAc tri-, tetra peptides), which serve as effectors by binding AmpR and activating ampC expression. Enhanced production of AmpC results in degradation of the antibiotics inactivating them, thus providing resistance. Recently, a second permease AmpP has been shown to be important in ß-lactamase induction in P. aeruginosa (127). Further, AmpR has been demonstrated to be a membrane-bound regulator, with the HTH domain in the cytoplasm and effectorbinding domain in the periplasm (Caille et al., manuscript in preparation). We 
hypothesize that AmpP siphons off some of the UDP-MurNAc pentapeptide from the cytoplasm for it to bind to the effector-binding domain of AmpR in the periplasm, repressing ampC expression.

Fig 1.2. Components of the bacterial peptidoglycan function as effectors in modulating AmpR activity. As part of the cell wall recycling process, the GlcNAc-anhyd-MurNAc-tri and tetrapeptides released are transported into the cytoplasm by AmpG. NagZ activity results in anhyd-MurNAc-peptides (potential positive regulators). They are recycled to UDP-MurNAc-pentapeptide (a negative regulator of AmpR activity) that subsequently forms lipid I. MurG adds GlcNAc to this molecule to form lipid II. Lipid II is transported to the periplasm by flippase activity utilizing energy from UDP hydrolysis and transglycosylases add it to the growing peptidoglycan chain. In our model, AmpR is an integral membrane protein and we propose a second transport protein, AmpP, transports the negative regulator (UDPMurNAc-pentapeptide) to the periplasm to affect AmpR regulation. In the presence of $\beta$-lactam antibiotics, a different positive regulator (GlcNAc-anhyd-MurNAc pentapeptide) is formed due to antibiotic action on transglycosylases and this has been proposed to positively regulate AmpR action, leading to production of B-lactamases. 


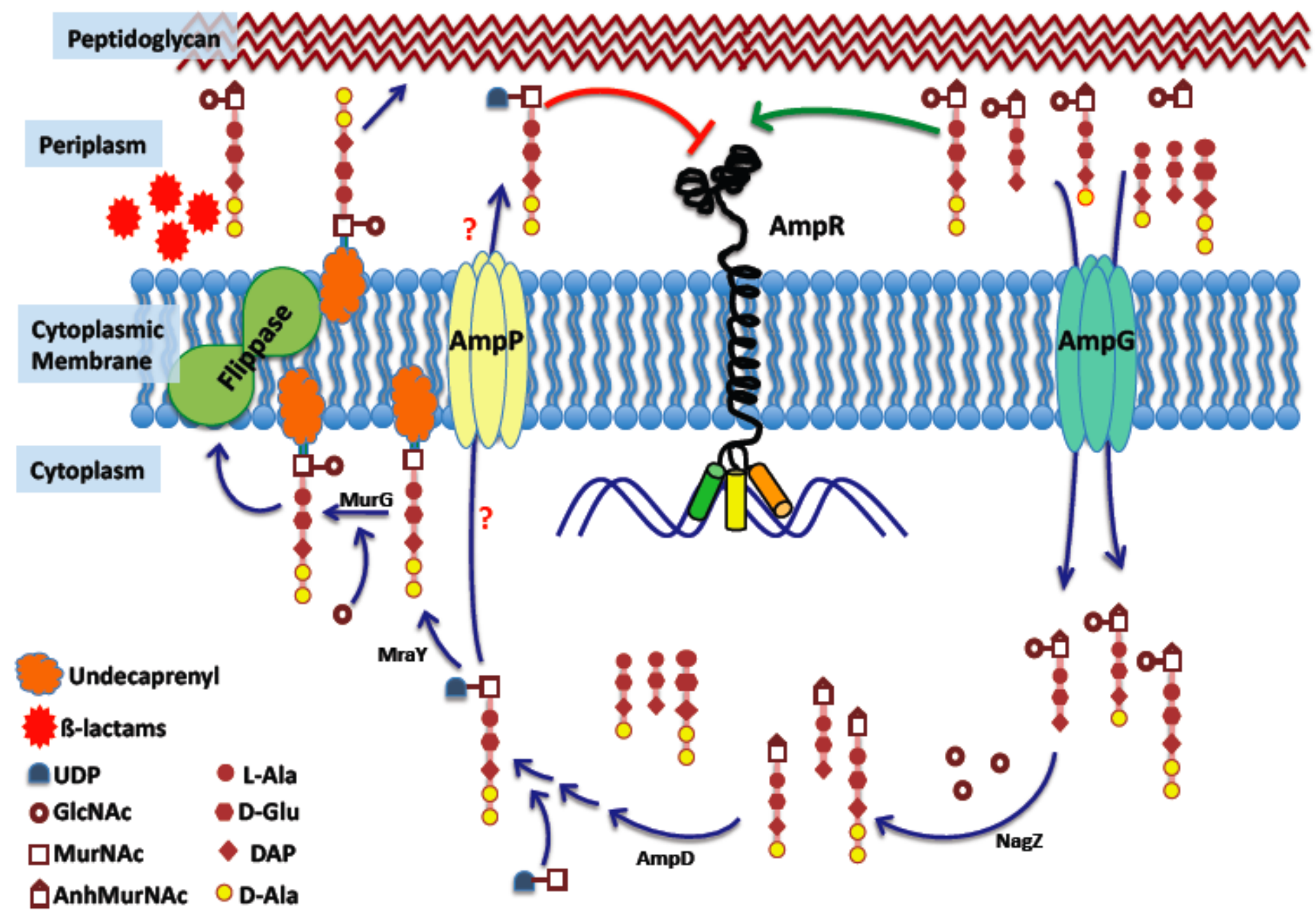




\section{Transcriptional regulation: How does it work?}

All cells, prokaryotes and eukaryotes, contain hundreds of thousands of genes that define and dictate everything about the organism. However, not the entire complement of the genome is expressed at any given time. Gene expression is both condition- and timespecific. Synthesis of proteins, the workhorses of the cell, is regulated at several stages and regulation of transcription is the first. One of the first steps in transcription is the binding of transcription factors to DNA. There are specific features in both DNA and proteins that are critical to this process. This section deals with the basics of protein-DNA interaction.

\section{Features on the DNA}

The outer surface of the DNA double helix is studded with sequence information that proteins recognize. On the helix, the edges of the base pairs are exposed and have hydrogen bond donors ( $\mathrm{H}$ attached to $\mathrm{N})$, hydrogen bond acceptors $(\mathrm{N}, \mathrm{O})$ and hydrophobic patches (methyl group on thymidine). The pattern of arrangement of these features is distinct for different bases only in the major groove of the DNA. In addition to these, transcriptional regulators also recognize the 'distortions' that are generated by the specific arrangement of nucelotides that alter the geometry of the double helix. For example, the repeat 5' AAAANNN 3' at 10 nucleotide intervals causes a slight local bending in the DNA. Such natural curves (bends) help form a tight fit between transcriptional regulators and DNA. 


\section{Features on the protein}

DNA-binding proteins recognize specific sequences on the DNA because the surface features of the protein and the DNA they bind to bear extensive complementarity. DNA-protein interactions involve hydrogen bonds, ionic bonds and hydrophobic interactions. Though these binds are individually weak, there are typically about 20 interactions per recognition site, which is strong enough to bring about binding. The weakness of the individual interactions is also critical for reversal of the process, allowing control of regulation. Features on both DNA and protein determine binding specificity. Specific amino acids bond with specific bases on the DNA. Asparagine, for

example, binds with adenine and arginine binds with guanine. Given that there are only four types of nucleotide bases while there are 20 amino acids, some amino acids can bind multiple bases. In addition to arginine, guanine can also bind with serine, lysine and histidine; adenine binds serine and asparagine; thymine interacts with alanine and serine while cytosine binds aspartic acid. Most regulatory proteins bind DNA as dimers or multimers because it is a simple way to achieve strong specific binding.

\section{DNA-binding motifs in proteins}

The regions of the protein that bind DNA can be classified on a structural basis into different types.

Helix-turn-helix (HTH) motif: HTH was the first DNA-binding motif to be discovered. It is the most common motif found in DNA-binding proteins and is prevalent in both prokaryotes and eukaryotes. The HTH motif is composed of two $\alpha$-helices connected by a short extended chain of amino acids, which form the turn. The helices are held at a fixed angle of $120^{\circ}$ by interactions between the helices. Typically, the helix at the C-terminal 
end of the protein (the second helix) is the recognition helix that interacts with the major groove of the DNA. The way that this helix is presented to the exposed bases in the major groove depends on the structure of the rest of the protein and how it contacts the DNA. The side chain amino acids vary between proteins and confer specificity. While the HTH motif is more or less conserved, barring the variation that confers specificity, the proteins can differ enormously outside of the HTH motif. The regions outside the HTH also, sometimes, interact with the DNA to fine tune recognition. Typically, HTH motifcontaining proteins bind DNA as symmetrical dimers to two similar/identical half-sites.

Homeodomains are a special class of HTH motifs that are present in eukaryotes. An important difference between regulators that contain HTH motif or homeodomain is that the latter can bind DNA as a monomer whereas the former binds DNA as a dimer or multimer.

Another variation of the HTH motif is the helix-loop-helix (HLH) motif. HLH motifs consist of a short $\alpha$-helix connected by a loop to a longer $\alpha$-helix. The loop connecting the two helices is flexible such that one helix folds back and packs against the other. The loop is also the site of dimerization (homo or heterodimers). Many HLH proteins lack a $\alpha$-helical extension needed to bind DNA. When these truncated proteins form homo or heterodimers with other full-length proteins, they form partially active dimers that make only half of the necessary contacts. This is one way of keeping such regulatory proteins in check.

Zinc-finger motifs: These motifs are composed of amino acids and zinc atoms as part of the structure. All groups of zinc-finger motif-containing proteins have zinc as a structural 
element and bind DNA via a $\alpha$-helix. Several groups have been identified based on structural studies.

In the first type, a zinc molecule holds together a $\alpha$-helix and a $\beta$-sheet. This type of arrangement is typically found in a cluster with other zinc fingers in tandem. Each $\alpha$ helix contacts the major groove of the DNA. The specificity and strength of the interactions are enhanced by the presence of tandem repeats of $\alpha$-helices. A second type of a zinc-finger motif is where two $\alpha$-helices are packed together with zinc atoms in an arrangement that is similar to a HTH motif. They form dimers, which allows one of the two $\alpha$-helices to bind DNA.

Leucine zipper coiled-coil: Originally identified as a dimerization motif, leucine zippers are $\alpha$-helical segments. Leucine zippers mediate DNA binding and protein dimerization. Secondary $\alpha$-helices, one from each monomer, join to form a short coiled-coil. The helices are held together by interactions between hydrophobic side chains, often at a leucine. The two helices separate just beyond the dimerization interface to form a Yshaped structure, thus allowing the side chains to interact with the DNA.

B-ribbon proteins: $\beta$-ribbons can also bind DNA. The major groove of the DNA is recognized by a two-stranded $\beta$-sheet with side chains of amino acids extending from the sheet toward the DNA. DNA recognition depends on the amino acid sequence, like with $\alpha$-helices.

\section{Dissertation overview}

P. aeruginosa is a bacterium that has fascinated researchers for decades and has been extensively studied. Numerous studies have addressed different regulatory aspects 
of metabolism, physiology and pathogenesis. This has led to a great deal of understanding of the molecular mechanisms of gene regulatory processes in $P$. aeruginosa. However, with the advent of high-throughput genomics, our traditional understanding of regulatory repertoires is changing.

With this in mind, my study has two main objectives:

To better understand gene regulation in $P$. aeruginosa: This was achieved by performing global analyses of published data, specifically metaanalysis of transcriptome studies, and analyses of putative and established regulatory networks in P. aeruginosa. This objective is the basis of chapters 2 and 3 of this dissertation.

To exemplify the regulatory complexity in P. aeruginosa using AmpR: Previous studies in the lab have established that AmpR regulates some non- $\beta$-lactam resistance genes, in addition to regulating its primary target, $\operatorname{ampC}$ that encodes a $\beta$-lactamase. I explore the regulatory repertoire of the ß-lactamase regulator AmpR adopting global approaches such as microarrays, RNA-Seq and ChIP-Seq. Chapters 4 and 5 of this dissertation address this objective. 


\section{CHAPTER 2}

\section{Comparative transcriptomic analyses of Pseudomonas aeruginosa}

This chapter has been published:

Balasubramanian D, and K Mathee. 2009. Human Genomics. 3(4): 349-361.

DOI: 10.1186/1479-7364-3-4-361 


\begin{abstract}
One of the hallmarks of bacterial survival is their ability to adapt rapidly to changing environmental conditions. Niche adaptation is a response to the signals received that are relayed, often to regulators that modulate gene expression. In the post-genomic era, DNA microarrays are used to study dynamics of gene expression on a global scale. Numerous studies have used Pseudomonas aeruginosa, a Gram-negative environmental and opportunistic human pathogenic bacterium, as the model organism in whole genome transcriptome analysis. Here, we review the transcriptome studies that have led to immense advances in our understanding of the biology of this intractable human pathogen. Comparative analysis of $23 P$. aeruginosa transcriptome studies has led to the identification of a unique set of genes that are signal-specific and a core set that was differentially regulated. The 303 genes in the core set are involved in bacterial homeostasis making them attractive therapeutic targets.
\end{abstract}




\section{Introduction}

Bacteria rapidly adapt to changing conditions by altering gene expression. The process of bacterial adaptation has fascinated researchers as it provides important insights into their physiology. This information is especially useful for human pathogens because it can be applied to better combat infections. This premise has led to studies that examine gene expression on a global scale. An entire set of RNA transcripts produced by a population of cells at any given time during gene expression is referred to as a transcriptome (128). Variations in the external environment or genome perturbations, such as mutations, can alter the transcriptome of a cell. Transcriptome studies thus provide us with a snapshot of the organisms' response based on the genes that are expressed. Though there is not always a linear relationship between the expressed genes and the proteins synthesized, such studies give us a reasonable insight into the state of the cell.

In the pre-genomic era, transcriptome analysis relied on techniques such as differential display and RNA arbitrary primed PCR (129-131), cDNA-amplified restriction fragment length polymorphism (132-134), random expressed sequence tag sequencing (135), serial analysis of gene expression [SAGE; (136)], LongSAGE (137), SuperSAGE $(138,139)$, and massively parallel signature sequencing (140). Prior sequence information is not required for these methods but they are both labor and timeintensive. Bacterial studies entered the genomic era with the sequencing of the Hemophilus influenzae genome in 1995 (141). Since then, the genomes of over 1000 bacteria have been fully sequenced. Large-scale sequencing has ushered the development of high throughput genome-wide expression techniques such as DNA microarrays. This 
powerful technology has been harnessed in numerous studies, under diverse conditions to elucidate whole genome expression in both prokaryotes and eukaryotes. One such prokaryote is Pseudomonas aeruginosa, where DNA microarrays have been of immense help in understanding its complex biology.

\section{P. aeruginosa}

P. aeruginosa is a Gram-negative human pathogen of the family Pseudomonadaceae and can switch from being an environmental isolate, found in the soil and water, to a human pathogen (7). It is an epitome of opportunistic human pathogens, being implicated in a wide range of infections of the pulmonary tract $(8,9)$, urinary tract $(10,11,13)$, burns $(14-16)$ and wounds $(17,18)$ among others. It causes persistent nosocomial infections $(23,24)$ and is also a major pathogen in cystic fibrosis patients. One of the major weapons in its arsenal is its resistance to antibiotics. It is both inherently resistant and can also acquire resistance genes from other bacteria making it a notorious pathogen. Understandably, numerous studies over the years have focused on elucidating the mechanisms of pathogenesis and antibiotic resistance of $P$. aeruginosa. Since the sequencing of the genome (25), the emphasis of these studies has shifted from conventional pathogenesis research, where the focus was on individual virulence determinants, to a much larger scale involving whole genome analyses.

The prototypic reference strain of $P$. aeruginosa is PAO1, isolated from a burn wound, and was the first $P$. aeruginosa strain to be sequenced (25). The most prominent feature of the PAO1 genome is its size. It has one of the largest bacterial genomes with 6.26 million base pairs comprising of almost $90 \%$ protein coding genes. The genome complexity of $P$. aeruginosa explains its versatile nature to adapt and thrive in a wide 
range of environments. The genome has 5,570 open reading frames (ORFs), a number that is close to simple eukaryotes like Saccharomyces cerevisiae. Other features of the PAO1 genome include the presence of four ribosomal RNA loci $(r r n)$ that are the regions of the longest repeats and a high GC ratio at $66.6 \%$ (25). Following the sequencing of the genome, high-density DNA microarrays were developed for $P$. aeruginosa (28) that offered us a chance to take snapshots of the dynamic responses of this versatile genome to various stimuli. An important study in this context (29), which analyzed the genomes of both environmental and clinical isolates of $P$. aeruginosa, suggested the presence of a core genome in different strains and expression of different sets of genes to facilitate growth in particular environments. This calls for a robust control over transcription mediated by transcriptional regulators. Analysis revealed that about $10 \%$ of the PAO1 genome is comprised of transcriptional regulators (25). This suggests the existence of a tight control over the way genes are expressed in P.aeruginosa.

Subsequent to PAO1, other $P$. aeruginosa strains such as PA14 (142), PA2192 [a CF isolate, (31)], C3719 [the Manchester epidemic strain, (32)] and PACS2 (33), have been sequenced. Comparative genome analysis of the five sequenced $P$. aeruginosa strains has led to the identification of a set of core shared genes that all the genomes contain, the majority of which are housekeeping genes (33). In addition, there is an accessory genome comprising of mostly hypothetical ORFs or those related to transposons and bacteriophages, and genes involved in niche adaptation specific to each of the five strains (33). The accessory set also contains transcriptional regulators that may control expression of ORFs in the core genome. Since the currently available microarray chips are based on the PAO1 genome, performing microarray studies with non-PAO1 
strains would seriously underestimate the transcriptional profile. Though GeneChip (Affymetrix) contains 117 probe sets for ORFs from non-PAO1 P. aeruginosa, it is not sufficient to cover the accessory genomes from all the other sequenced strains. Analysis of five $P$. aeruginosa genomes revealed that 5021 genes are conserved between the strains (core gene set) and that the genome of each strain has anywhere between 79 to 507 unique genes that are part of the accessory genome for that strain (33). There is thus a gross under-representation of non-PAO1 genes on the Affymetrix GeneChip that will undoubtedly affect data interpretation. Thus, the next major advance in $P$. aeruginosa microarrays would be the development of a pangenome array, comprised of probe sets for the core and accessory genomes from all sequenced strains. This will greatly advance our understanding of the system by allowing researchers to study non-PAO1 strains and help appreciate better the biology of this versatile bacterium.

\section{$P$. aeruginosa transcriptome studies}

\section{P. aeruginosa and iron limitation}

Iron is an important cofactor that plays a critical role in many cellular processes. Low iron availability in host tissues is a major impediment that the bacteria have to overcome in establishing an infection. Bacteria have evolved an elaborate system of acquiring iron from the surroundings. P. aeruginosa secretes siderophores (pyochelin and pyoverdine) that chelate iron and bring it back into the cell via specific cell-surface receptors (108). Fur is a major repressor that tightly regulates this uptake process by controlling expression of the regulators, PvdS and PchR (143).

In one of the initial transcriptome studies of the response of $P$. aeruginosa to iron (144), stationary phase $P$. aeruginosa cells were grown in the presence and absence of 
iron at $32{ }^{\circ} \mathrm{C}$ followed by gene expression analysis. Results indicated that 137 genes were upregulated on iron depletion (144). A more systematic study was performed by Palma et al., which addressed how the transcriptome changes when iron-starved P. aeruginosa cells are fed with iron (145). Differential regulation was seen in mRNA levels of 177 genes. Most of the genes were down regulated, especially genes involved in transport of small molecules, transcriptional regulators and hypothetical proteins. It was interesting to note that genes involved in virulence were upregulated, some of which probably help the cells in overcoming the host innate immune system (145). An important finding was that 38 genes, including those coding for siderophore receptors, iron transport, regulators, sensors, sigma factors were identified whose transcription decreased on iron treatment. These 38 genes were proposed to be part of a regulon involved in iron response in $P$. aeruginosa. In another study, Zheng and coworkers identified a hypothetical protein, PA2384, that regulates over 70 genes involved in the iron uptake process in a Furindependent manner (146). Moreover, deleting PA2384 also led to differential expression of many genes involved in cellular physiology, like translation, metabolism and electron transfer indicating a broader role for this protein.

\section{$P$. aeruginosa and oxidative stress}

For bacteria to successfully infect a human host, they should be able to overcome many barriers. One primary defense mechanism is production of reactive oxygen species 
(ROS) by the host cells that leads to oxidative stress in bacteria. Palma et al., studied the response of $P$. aeruginosa to a natural oxidant in human systems, hydrogen peroxide, as an indication of the global transcriptional status of the cell's early response (147). The study confirmed that some of the genes that were earlier found to be important in a cell's response to oxidants were upregulated in addition to identifying 520 genes with a significant (>5-fold) change in mRNA levels (147). Of these, 216 genes were upregulated, seven of which were upregulated more than 50-fold, primarily hypothetical proteins of unknown function. Further, upregulated genes included cytotoxin genes such as exoenzymes S and T, and PvdS that control PrpL proteinase and exotoxin A expression and could potentially inhibit the cells of the innate immune system. Most of the genes that were down regulated are involved in metabolic pathways and ribosomal synthesis, which makes sense, for the cell under oxidative stress would not want to actively metabolize or grow but deal with the stress first (147).

In another study looking at response to hydrogen peroxide, stationary phase cells of three strains belonging to two clonal types were tested (148). Two of the strains, TB and 892 were cystic fibrosis (CF) isolates and their response was compared with PAO1, which belongs to a different clonal type. The expression profiles of the $\mathrm{CF}$ isolates were more similar to each other than to PAO1, sharing more than $80 \%$ of the differentially expressed genes with each other. This study highlighted the importance of clone-specific transcriptome profiles, variations seen in both intraclonal (between the two CF isolates) and interclonal (between $\mathrm{CF}$ isolates and PAO1) strains.

Genome-wide transcriptomes of the three strains, P. aeruginosa PAO1 and the two CF isolates TB and 892 were also analyzed for their response to superoxide- 
generating paraquat (149). Less than 30 genes were differentially expressed in each of the three strains, of which only six were upregulated in all three. Upregulated genes belonged to oxidative stress response, transport and acetyl-CoA and acetoin metabolism. Four genes of a single operon coding for the superoxide dismutase SodB, catalase KatA and alkylhydroperoxide reductase $\mathrm{AhpC}$ were upregulated in three strains, highlighting their importance in $P$. aeruginosa superoxide stress response. Energy metabolism genes were downregulated to counter the superoxide stress (149).

Two recent papers by Small et al., looked at the oxidative stress response of $P$. aeruginosa. Both of these articles focused on the effect of oxidizing agents routinely used in hospitals for surface sterilization and their effect on $P$. aeruginosa. The first is a transcriptome analysis of sodium hypochlorite $(\mathrm{NaOCl})$-induced oxidative stress (150). In this study, Luria-Bertani (LB) broth grown mid-log phase P. aeruginosa PAO1 cells were treated for 20 minutes with sub-lethal concentrations of bleach. RNA extraction and microarray analysis followed. Results were compared with similarly grown untreated cells. About $20 \%$ of the 5570 genes of $P$. aeruginosa were differentially expressed in response to the treatment, approximately $10 \%$ each being up- and downregulated. Responses similar to that seen with hydrogen peroxide were generated, with upregulation of catalase, alkyl hydroperoxide reductase, and glutathione peroxide/reductase genes indicating that the mode of action of bleach is similar to that of hydrogen peroxide in inducing the oxidant defense system genes. Genes involved in transport of sulfur compounds were highly upregulated. A possible explanation for this observation is that the free electrons in the sulfhydryl groups in the biological molecules react with the hypochlorous acid formed by exposure to $\mathrm{NaOCl}$, a known strong oxidizer, countering its 
effect. This would increase chances for cell survival (150). Genes that are repressed are primarily the ones involved in oxidative phosphorylation and electron transport indicating that the cells under $\mathrm{NaOCl}$-induced stress would have lowered energy due to impaired metabolic processes.

Another study compared and analyzed the transcription patterns of three different oxidative damage-inducing agents: $\mathrm{NaOCl}$, peracetic acid and hydrogen peroxide on $P$. aeruginosa (151). Over 550, 300 and 200 genes were differentially regulated as a result of $\mathrm{NaOCl}$, peracetic acid and hydrogen peroxide, respectively. Of these, 63 genes were common to all three agents, 40 upregulated and 23 downregulated. About $25 \%$ of the common upregulated genes were 'related to phage, transposon or plasmid' and eight were to membrane protein. The primary downregulated genes were those involved in transport of small molecules. Other prominent gene categories included membrane proteins and putative enzymes. It is surprising to note that membrane protein genes were both up and downregulated. $\mathrm{NaOCl}$ resulted in the most downregulation of genes involved in energy metabolism suggesting that $\mathrm{NaOCl}$ may be more effective in limiting energy production than the other two agents. Peracetic acid exposure resulted in stimulating expression of genes involved in adaptation and protection and downregulation of transcriptional regulators. Several protective mechanisms were triggered after exposure of cells to hydrogen peroxide. A significant finding of this study was positive expression of the pyocin genes of the F-, R- and S- types after hydrogen peroxide treatment but was downregulated with the other two treatments. This has been linked to DNA damage and RecA activation that has been known to induce pyocins. RecA has been demonstrated to cleave single stranded DNA with the help of hydroxyl radicals that are formed by 
exposure of cells to hydrogen peroxide. The induction of pyocin genes in response to hydrogen peroxide treatment, published separately (152), demonstrates that $\mathrm{NaOCl}$ exposure leads to significant differences in the transcription pattern of $P$. aeruginosa and is more lethal to the cell compared to either hydrogen peroxide or peracetic acid (151).

Nde et al., tested the response of $P$. aeruginosa to ortho-phenylphenol (OPP) (153), a widely used disinfectant and antimicrobial agent whose mode of action on bacteria has not yet been elucidated. Using two different exposure times (15 minutes and 60 minutes), they identified genes coding for virulence, membrane and ribosomal proteins upregulated at both time points. Forty-five genes of the translation, posttranslation modification and degradation were upregulated, primarily initiation, elongation and release factors (153). Genes encoding components of the SecY system, involved in export of various proteins, toxins and enzymes, showed enhanced expression as were some membrane associated proteins involved in small molecule transport, possibly indicating that the OPP-treated bacteria were transporting proteins across the cell membrane as part of an adaptive process. Also upregulated were genes of type IV pili assembly proteins, fatty acid biosynthesis and energy metabolism, all of which indicate adaptation (153).

\section{$P$. aeruginosa and pentachlorophenol stress}

Response of $P$. aeruginosa to pentachlorophenol (PCP) was carried out recently (154). PCP is thought to embed in the membrane where it acts as a proton shuttle 
uncoupling oxidative phosphorylation (155). In addition, since RpoS is the stationary phase sigma factor thought to be an important factor in the cell's stress response, rpoS mutants were also examined under the same conditions. Sixty genes showed two-fold or greater increase in transcription. RpoS did not play a protective role in the cell's response to PCP-induced stress. The highlight of this study is the induction of multiple operons involved in multidrug efflux, the first anthropogenic induction of the MexAB-OprM system with chemicals. Notably, two operons of the resistance-nodulation-cell division (RND) pumps were upregulated in addition to one operon that positively regulates MexAB-OprM. These are involved in antibiotic and solvent efflux. Putative major facilitator superfamily (MFS) proteins were also upregulated in response to $\mathrm{PCP}$-induced stress. Selective downregulation of genes involved in central metabolism, fatty acid metabolism, energy metabolism and adaptation/ protection was observed (154).

\section{$P$. aeruginosa response to copper}

Copper is one of the essential micronutrients playing an important role in cellular respiration, as part of electron transport and many biological reactions (156). Bacteria have to tightly control cellular levels of copper to prevent toxicity or starvation. Two studies looked at how $P$. aeruginosa responds to varying levels of copper.

Tietzal et al., identified 41 genes that form a core response of $P$. aeruginosa to copper, comparing transcriptomes of cells exposed to high levels of copper either for a short time (copper-shocked, 405 genes) to those grown in copper [copper-adapted, 331 genes; (157)]. Genes involved in active efflux of copper included transporters, copper 
oxidase, periplasmic thiol-disulfide interchange proteins, lyases, and transcriptional regulators.

Copper starvation of $P$. aeruginosa under aerobic conditions (158) led to downregulation of 132 genes while only 10 genes were upregulated. The most strongly downregulated genes were the iron acquisition genes, mostly those repressed by Fur, the iron-uptake regulator. One of the downregulated transcriptional regulators, PA3410, encodes a probable extracellular sigma factor (157). The upregulated genes included mostly hypothetical genes, and a bacterioferritin involved in iron storage. In fact, copper starvation and iron depletion $(144,145)$ led to the expression of similar sets of genes but have opposite effects on them.

\section{$P$. aeruginosa and sulfate starvation}

The CF lung mucin is highly sulfated, probably a defense mechanism to prevent degradation by bacterial enzymes $(159,160)$. The metabolically versatile $P$. aeruginosa can utilize a variety of organic and inorganic compounds for growth. Under limiting conditions of inorganic sulfate, the cells can uptake organosulfur compounds for growth without affecting the growth rate (161). Global response to sulfate starvation on a nonmucoid CF isolate of $P$. aeruginosa E601 and PAO1 was addressed (162). The study was aimed at determining the genes regulated by sulfur supply and whether this was altered during CF infection, a condition simulated by exposure to mucin. Three groups of responses were observed: response to sulfur starvation, to mucin as a sulfur source and to mucin exposure (162). Functional analysis of differentially expressed genes revealed that both strains showed similar responses in the different functional classes, the maximally 
regulated genes belonging to hypothetical proteins, transport of small molecules and putative enzymes. Unlike PAO1, the CF isolate E601 was shown to utilize mucin as a sulfur source. Moreover, several genes involved in the type III secretion system and exoenzyme $\mathrm{S}$ were induced, suggesting that cells recognize mucin not only as a sulfur source but also as a host component: a dual response (162).

\section{$P$. aeruginosa and osmotic stress}

P. aeruginosa, being a versatile pathogen, can survive in a wide range of environments, some of which can be osmotically stressful. Transcriptional profiling of osmotically-stressed P. aeruginosa was studied (163). P. aeruginosa PA14 cells, grown in glucose minimal media, were exposed to osmotic stress, either under steady-state conditions $(0.3 \mathrm{M} \mathrm{NaCl}$ and $0.7 \mathrm{M}$ sucrose) or osmotically up-shocked $(0.3 \mathrm{M} \mathrm{NaCl}$ for a short time). A major difference in the expression pattern between these two conditions is the number of genes that are differentially regulated. While 431 genes were differentially regulated on osmotic up shocking, only about 100 genes were more than 3 -fold regulated under either condition of steady-state osmotic shock. These studies led to the identification of a steady-state osmotic shock regulon comprised of 66 genes. Forty of these are virulence-associated, primarily genes of the type III secretion system, two type III exotoxins and two chaperones. In contrast, the osmotically up-shocked cells showed repression of the type III secretion system genes that the authors hypothesize to be an energy-saving step. In addition, the sigma factor $\mathrm{AlgT} / \mathrm{U}$, previously known to be induced in varied stress conditions, and genes known to be involved in osmoprotection, 
including hydrophilins whose exact role in osmoprotection has not yet been conclusively proven in bacteria (164) were induced in all the conditions of osmotic shocking (163).

\section{$P$. aeruginosa and quorum sensing}

$P$. aeruginosa senses the cell density for coordinated expression of virulence factors using a global regulatory system called quorum sensing (QS). Sensing is achieved using small diffusible molecules that in $P$. aeruginosa and in other Gram-negative bacteria are the N-acylated homoserine lactones (165). P. aeruginosa has two Nacylated homoserine lactone systems, las and $r h l$ (166). Transcriptome analyses of lasI rhlI mutants were performed by three groups $(167-169)$. Two of these groups $(167,168)$ identified about 300 genes that were induced by quorum sensing while the other group got only 163 differentially expressed genes as a result of use of more stringent criteria. The three studies had approximately 100 common genes and most belonged to the functional class of secreted factors. This group included toxins and extracellular enzymes. In addition, above average expression changes were seen in genes involved in metabolism, biosynthesis of cofactors and fatty acid metabolism, implying that QS signals mediated by $\mathrm{N}$ - acylated homoserine lactones were involved in regulation of many genes other than those involved in virulence. The third group, Hentzer et al., targeted the QS system in an attempt to mitigate $P$. aeruginosa virulence (169) using a furanone isolated from the Australian seaweed Delisea pulchra. The authors elegantly show using transcriptome analyses that about $80 \%$ of the genes that were downregulated by the furanone were also repressed in an $\mathrm{N}$ - acylated homoserine lactones mutant. The furanone compound also affected biofilm formation and persistence of infection in a 
mouse lung model. The three studies together led to he identification of a core set of 77 genes of the QS regulon that are positively regulated (169). Schuster and Greenberg (170) review many other studies of regulators that further elucidate the complex QS system and the intricate interconnections. They propose a complex network that involves 13 different regulators that interact with LasI and RhII and form the QS regulon.

\section{P. aeruginosa biofilms}

Quorum sensing has been implicated to be an important step in the formation of ordered, antibiotic-resistant bacterial communities called biofilms (171,172). Planktonic cells, in response to external stimuli, form biofilms in varied habitats, like in the CF lung, surgical implants and in various environmental situations exhibiting drastically altered physiology that lead to the hypothesis that the gene expression between these two states of cells are very different. One of the earliest studies that employed microarrays to understand differences in gene expression patterns between planktonic and biofilm cells, in addition to antibiotic resistance mechanisms in biofilms, was by Whiteley et al (173). Using chemostats, it was found that a surprisingly small number (1\%) of genes were differentially regulated between planktonic and biofilm cells. The most highly upregulated genes were those of the Pfl-like prophage with nine of the 11 genes on the chromosome induced over 100-1000 fold in the biofilm cells. This could imply higher rates of gene transfer in biofilms via transduction. Genes involved in bacterial motility were repressed, as was $r p o S$, the stationary phase sigma factor. Strains harboring a mutant $r p o S$ were shown to form larger more drug-resistant biofilms. Exposure of the biofilm to the antibiotic tobramycin followed by transcriptional analysis showed tolA was 
upregulated, which can serve as a barrier to aminoglycoside uptake. Cytochrome C oxidase genes were repressed, probably slowing growth, negating antibiotic effect. In addition, antibiotic exposure also induced two probable efflux systems and many hypothetical genes.

Effect of the $\beta$-lactam imipenem on gene expression in planktonic and biofilm cells of $P$. aeruginosa was examined by Bagge et al (174). Microarray analysis of imipenem-exposed planktonic and biofilm cells revealed that the chromosomal B-

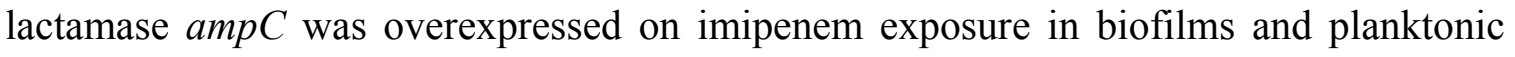
cells. Thirty-four genes were more than five-fold differentially regulated and included genes involved alginate production, flagella biosynthesis, cell division, peptidoglycanrelated and type 4 pili biogenesis. In addition, biofilm cells also showed enhanced production of alginate, unlike in the Whiteley study with tobramycin. Bagge et al could not ascribe higher antibiotic resistance of biofilm cells to a specific set of genes due to similar expression patterns in planktonic and biofilm cells (174).

A more recent study makes a comprehensive analysis of transcriptomes of planktonic cultures (in $\log$ and stationary phase) and biofilms at 8, 14, 24 and 48 hours (microcolony and mature biofilms) using a static biofilm model (173). This study shows a similar gene expression pattern between developing biofilms and log phase planktonic cultures while mature biofilms have expression patterns similar to stationary phase cells. The most highly upregulated genes in biofilm cells were those of a Pf1-related temperate bacteriophage suggesting an important role for phage-mediated gene transfer in biofilm cells. Downregulated genes include those coding for pili that suggests that though pili are required for establishment of biofilms, they are not essential in mature biofilms. Also, 
expression is relatively stable in developed biofilms with only $1 \%$ of the genes differentially expressed. This was the first study to look at global gene expression for two planktonic and two biofilm states of the cells and the authors have generated a comprehensive dataset for reference that compares transcriptomes of planktonic, developing and mature biofilms.

\section{Transcriptomics of alginate production in $P$. aeruginosa}

Alginate gene upregulation in $P$. aeruginosa is one of the primary modes of survival in the cystic fibrosis lung and converts the non-mucoid cells to a mucoid phenotype (175) as a result of over-production of the extracellular polysaccharide alginate. Production of alginate is part of a stress response in $P$. aeruginosa and is mediated by the alternate sigma factor $\mathrm{AlgT} / \mathrm{U}$, a sigma $\mathrm{E}$ homolog. AlgT/U is held sequestered by the anti-sigma factor MucA. Release of AlgT/U leads to alginate production and is part of a much larger response to help the cells adapt (176). Transcriptome analysis of the mucA, algT/U-mucA mutants and the wild type PAO1 showed that alginate was upregulated in the тисA mutant where there was no control on AlgT/U production. This control was lost in the double mutant as expected (177). What was exciting was that many genes responsible for toxin production, enzymes and transport were also upregulated indicating that $\mathrm{AlgT} / \mathrm{U}$ was part of a regulon that coordinated expression of many genes, ultimately enhancing $P$. aeruginosa survival in the harsh CF lung environment.

P. aeruginosa, like other bacteria, produces flagella only when needed, for example in biofilm formation and has been implicated in establishing pulmonary 
infections (178). AlgT/U was shown to negatively regulate flagella production in $P$. aeruginosa by downregulating an essential flagella regulator, FleQ (179). There are many regulators in flagella biosynthesis and a systematic study of several regulator mutants was performed by Dasgupta et al (180). Using transcriptome analysis, they found that flagella are assembled in a four-step process that is controlled by the sequential expression of different regulators.

\section{Lipopolysaccharides and lipoproteins as virulence determinants}

Lipopolysaccharides play a major role in P. aeruginosa virulence (181). The bacteria are known to modify their lipopolysaccharide layer in response to the milieu. Lipid A, a major constituent of the bacterial liposaccharide is hepta- or hexa-acetylated in strains causing acute infections while it is penta-acetylated in chronic infections like in the cystic fibrosis lung (182). Lipopolysaccharide changes with alginate production (183) and production of both of these important virulence factors are influenced by oxygen concentration $(184,185,186)$. Though microarray studies that compare lipopolysaccharide mutants have not been published yet, Firoved et al., have demonstrated that lipoprotein genes are the most highly upregulated when $P$. aeruginosa switches over to the mucoid phenotype by comparing the expression patterns of a mucoid (PAO578II) and the isogenic non-mucoid parent [PAO6865; (187)]. Eight of the nine genes that were upregulated over 30-fold encoded lipoproteins, which can have toxic effects in environs like the cystic fibrosis lung by disrupting host Toll-like receptor 2 signaling and are also antigenic, activating host defense mechanisms. Citing these reasons, some of these lipoproteins have been termed lipotoxins (187). Elucidating mechanisms of coregulation 
of LPS production/ modification and conversion of the strains to mucoidy would help us better understand the adaptation/ pathogenesis of this versatile bacterium in chronic infections, like in the cystic fibrosis lung.

\section{Transcriptome of a post transcriptional regulator}

Burrowes et al., studied the role of a small posttranscriptional regulatory protein RsmA (188). RsmB, a small untranslated regulatory RNA, binds RsmA limiting its activity (189). RsmA controls expression of many virulence genes and QS signaling molecule synthesis $(189,190)$ and thus plays an important role in $P$. aeruginosa pathogenesis. Though some of the targets of RsmA were known this study focused on determining the direct and indirect regulatory control exerted by RsmA in P. aeruginosa (188). Transcriptional analysis of PAO1 and the $r_{s m A}$ mutant revealed that of the 506 genes that had significantly altered expression, genes involved in iron acquisition and antibiotic resistance, especially genes involved in the MexEF-OprN pump were upregulated and genes involved in Pseudomonas quinolone signal biosynthesis, and motility were downregulated. The data obtained suggests that RsmA is a global regulator (188).

\section{P. aeruginosa interactions with human cells}

When a bacterium comes into contact with a host cell for the first time, there is a drastic change in the lifestyle of the bacteria that aid in colonization. The host, on the other hand, recognizes this threat and mounts an immune response. As one can predict, this race between the pathogen and host to outdo each other will undoubtedly be accompanied by changes in the gene expression profile in both organisms. Numerous 
studies have looked at the response of the human immune system to $P$. aeruginosa exposure and we have a fairly good idea of the immune signals and molecules involved. One of the first studies to employ transcriptomics to investigate host responses to initial contact with $P$. aeruginosa was carried out by Ichikawa and Lory (191). Using a time course microarray experiment with lung epithelial cells exposed to P. aeruginosa and custom made cDNA microarrays containing about 1500 human clones with both characterized and uncharacterized genes, they determined that about $25 \%$ of the human genes represented on the array were differentially regulated. Of interest was a lipopolysaccharide-independent two-fold activation of the interferon regulatory factor-1 gene with no activation of other downstream genes that are normally interferon-activated. This indicated that $P$. aeruginos a activation of interferon regulatory factor- 1 is different from the normal mechanism of interferon regulatory factor-1 activation mediated by cognate receptor binding (191). Using a type IV pili mutant, they demonstrate that PilA is the ligand responsible for LPS-independent activation of interferon regulatory factor-1. This study demonstrates the power of microarrays in understanding host-pathogen interactions by elucidating potentially new signaling pathways and by characterizing roles of different virulence factors involved in pathogen recognition and mounting an immune response.

Two other studies have employed DNA microarrays to study the interactions of $P$. aeruginosa to human epithelial cells but the results are contradictory with respect to the regulation of iron-responsive genes. In the first study by Frisk et al (192), P. aeruginosa was exposed to primary human airway epithelial cells for 4 and 12 hours in vitro and analyzed transcription post-exposure. Two major groups of genes were differentially 
regulated: phosphate uptake genes were upregulated whereas iron acquisition genes were downregulated. This was contrary to the notion that iron is a very important limiting factor in the human host and one would have expected upregulation of the iron acquisition genes. The authors concluded that $P$. aeruginosa was able to acquire enough iron for infection that led to deregulation of those genes. The reverse was true for phosphate.

In an attempt to better understand the initial adaptation processes of $P$. aeruginosa, especially on interaction with human epithelia, a second study from the Greenberg lab (193) compared transcriptomes of $P$. aeruginosa PAO1 wild-type with a double mutant that was deficient in Type III secretion system and rhamnolipid biosynthesis. These two bacterial systems are key factors contributing to human airway epithelial cell damage. Strain-specific responses were seen. Whereas virulence genes like PA-1 galactophilic lectin were induced in the wild-type strain, alginate regulatory genes were induced in the mutant along with gacA (193), which is known to be upregulated in chronic $P$. aeruginosa infections (28). Also, Vfr, a known upregulated regulator in acute infections was downregulated indicating early stages of a chronic infection.

In contrast to the results obtained by Frisk et al (192), the Chugani study showed that iron starvation genes were positively regulated indicating that the infecting cells had little iron to utilize. This is in concordance with the belief that infecting bacterial cells have little access to iron in the human body. One possible explanation for these discordant results, as noted by the authors, is that the epithelial cell integrity in the previous study could have been compromised leading to leakage of intracellular iron resulting in iron-replete conditions. This would explain the increased expression of iron 
dependent genes over time seen in the Frisk study. Epithelial cell integrity was monitored in the Chugani study by monitoring the transepithelial electrical resistance (Rt). An interesting finding by Chugani et al. is that the general pattern of gene expression is similar to that seen in CF isolates from chronic human infections.

Many bacteria use Type III secretion systems to inject proteins directly into host cells. An interesting whole genome array study by Wolfgang et al (29) looked at the conditions and regulatory mechanisms that control expression of the Type III secretion systems. They utilized the observation that Type III secretion system is induced by either growth in low-calcium medium or by cell contact and found eleven calcium dependent regulators that were involved in the process by growing cells in low calcium. Analysis of these proteins led to the discovery that a membrane-associated adenylate cyclase initiates a signaling pathway that controls expression of Type III secretion system genes (29).

The transcriptome studies of $P$. aeruginosa with human cells indicate the presence of as yet unidentified signals, from both the host and the bacteria during interactions. Unraveling these signals could provide us with valuable information to effectively counter infections by $P$. aeruginosa and microarrays will undoubtedly prove vital in this endeavor.

\section{Comparative analysis of all transcriptomes}

In order to better understand the response of $P$. aeruginos $a$ to the different stress conditions, we compared significantly differentially regulated genes from the studies within each stress condition. This analysis was performed only for those conditions that had multiple studies and had publicly available gene lists. Our comparative analysis led 
to the identification of common sets of genes that are regulated in response to each particular stress condition across the different studies. Additionally, we also identified unique genes that are specifically regulated only in individual studies. This data has been summarized in Table 2.1.

Table 2.1: Comparative analysis of significantly differentially regulated genes from various studies within each condition.

\begin{tabular}{|c|c|c|c|}
\hline \multirow{2}{*}{ Stress condition } & \multirow{2}{*}{ \# of studies } & \multicolumn{2}{|c|}{$\begin{array}{c}\text { Differentially regulated } \\
\text { genes }\end{array}$} \\
\cline { 3 - 4 } & & 69 & Common \\
\hline Iron & 3 & 121 & Unique \\
\hline Quorum sensing & 3 & 26 & 862 \\
\hline Copper & 2 & 83 & 386 \\
\hline Oxidative stress & 7 & 55 & 650 \\
\hline Biofilms & 3 & \multicolumn{2}{|c}{} \\
\hline
\end{tabular}

Analysis of significantly differentially regulated genes from all of the above studies led to the identification of 303 genes that form a core set and are differentially expressed in all the different conditions (Appendix; Supplementary information for Chapter 2, Table 1). This suggests that this set of core genes is important for maintaining homeostasis in P. aeruginosa. Functional categorization of the core set genes (Appendix; Supplementary information for Chapter 2, Figure 1) reveals that apart from the hypothetical genes $(27 \%)$, the most highly differentially regulated genes encode membrane proteins $(15 \%)$ followed by genes involved in transport of small molecules (11\%), adaptation and protection (7\%) and transcriptional regulators (6\%) clearly indicating an adaptive response of $P$. aeruginosa to the changing environment. We then 
compared this microarray core set of 303 genes with the core genome of $P$. aeruginosa that contains genes found in all $P$. aeruginosa sequenced strains (33). Most of these genes (90\%) belong to the P. aeruginosa core genome, but we identified 29 genes belonging to the PAO1 accessory genome (Appendix; Supplementary information for Chapter 2, Table 1). This set of 29 genes contains two type 4 pili-related genes, genes involved in adaptation and protection, membrane proteins, transport of small molecules, metabolism and hypothetical genes, among others. A caveat to this analysis is that unlike PAO1, the other sequenced $P$. aeruginosa genomes do not have $100 \%$ coverage.

After subtracting the core set, we compiled sets of unique genes that are specifically differentially regulated in each of the conditions examined. Functional categorization of all the genes in the unique gene sets (Appendix; Supplementary information for Chapter 2, Table 2) revealed that overall, as expected, hypothetical genes were the most differentially regulated. In addition, genes involved in adaptation (membrane proteins, transport of small molecules, putative enzymes, transcriptional regulators and energy metabolism), showed the highest percentage of differential regulation.

Table 3 (Appendix; Supplementary information for Chapter2) lists all the unique genes identified under the various conditions used in these studies. In the iron studies, for example, genes of the secreted factors group (toxins, enzymes, alginate) constitute $7 \%$ of the differentially regulated genes. This supports the view that $P$. aeruginosa upregulates virulence genes in response to iron limitation to help survival in the host system (145). Analysis of the data from response of $P$. aeruginosa to copper reveals that $33 \%$ of the differentially regulated genes are membrane proteins, much more than the 
small molecule transport genes (19\%) or hypothetical genes (14\%) suggesting that copper stress primarily affects the membrane. By far, the most interesting observation was from the osmotic shock study. A stark contrast to the other studies, there were only a very small percentage of hypothetical genes (3\%) that were differentially regulated. Transcriptional regulators (37\%), protein secretion/ export apparatus (33\%) and twocomponent regulatory systems $(10 \%)$ form over $70 \%$ of the genes differentially regulated under osmotic stress. The osmotic study was performed using P. aeruginosa PA14, which is known to be more virulent than $P$. aeruginosa PAO1 in a wide range of hosts (194-196). Since the chips used were designed for PAO1 that has 5571 ORFs as compared to 5905 ORFs in PA14, there could be an underrepresentation of the genes that are differentially regulated.

\section{Conclusions}

The transcriptome analysis studies that have been reviewed here provide evidence of the immense power of the DNA microarray. Microarray profiling generates an enormous amount of data that adds to our knowledge database. Genes that are differentially regulated by transcription factors, by host environment, by changing physiological conditions including response to external stimuli that could range from nutrients to other factors affecting growth, interactions with host cells all have been studied by microarrays. Analysis of transcriptome data generated in $P$. aeruginosa has been performed previously by Goodman and Lory (28), who show that about a quarter of the $P$. aeruginosa genome is differentially regulated in response to various stimuli. Our comparative analysis of the significantly differentially regulated genes from all these various studies show that about $35 \%$ of the $P$. aeruginosa genome (1932 genes) is 
regulated overall. To arrive at more comprehensive understanding on how bacteria react to the ever-changing environment, we would need to recognize the importance of physiological experiments before the analysis of data, protocol standardization and appropriate controls.

The next major advances in DNA microarrays would be to develop technologies to analyze the changing transcriptome of the host-pathogen interaction process in vivo that would provide us with vital clues to better understanding the organism, and the development of pangenome arrays that would give us insights into the workings of this versatile bacterium. Together, these will help devise ways to better counter P. aeruginosa infections.

Acknowledgements I thank Lisa Schneper for critical reading of the manuscript and Camilo Valdes for assistance with the PAO1 core genome analysis. 


\section{CHAPTER 3}

\section{Transcriptional regulatory network in Pseudomonas aeruginosa}

This chapter has been published:

Balasubramanian, D., S.K. Murugapiran, E. Silva-Herzog, L. Schneper,

X. Yang, G. Tatke, G. Narasimhan, and K. Mathee. 2013.

In M, M. Babu. (ed.), Bacterial Gene Regulation and Transcriptional Networks.

Caiser Academic Press, United Kingdom.

ISBN: 978-1-908230-14-0 


\begin{abstract}
Pseudomonas aeruginosa is found in a wide range of habitats, primarily in soil and water and is the epitome of opportunistic human pathogens. A myriad of virulence factors produced by the bacterium ensure its success as a pathogen. $P$. aeruginosa has one of the largest genomes among eubacteria and transcriptional regulators comprise about $8 \%$ of the genome. Sequence analysis of the regulators belonging to different families shows clustering while network analysis shows extensive crosstalk, and reveals empirically identified and novel interactions between regulators. Gene expression in P. aeruginosa is an intricately interlinked process and is exemplified in the regulation of virulence factor expression. Major regulatory processes such as quorum sensing involving multiple regulators translate external signals perceived by the bacterium into gene expression/repression via regulatory cascades. Many global regulators have been identified that serve to link different virulence systems. Understanding the role of the as yet uncharacterized transcriptional regulatory proteins will provide important insights into the physiology of this important human pathogen and has potential therapeutic implications.
\end{abstract}




\section{Introduction}

Pseudomonas aeruginosa is an environmental saprophyte that can cause a number of opportunistic infections in humans including infections of the pulmonary and urinary tracts, and skin $(9,11,13)$. It is notably known for its ability to cause acute $(15,18,24,197)$ and chronic (198-200) infections. P. aeruginosa is inherently resistant to antibiotics and can also acquire resistance genes by horizontal gene transfer (201). The ability of $P$. aeruginosa to infect animals, nematodes, plants and insects is due to the expression of a wide range of virulence factors that are either cell-associated or secreted. Cell-associated factors are involved primarily in adherence and colonization (pili, LPS, flagella) and motility (type IV pili, flagella). In addition, some components have multiple functions. The LPS, for example induces the immune system and has been linked to dysregulation of the host response and sepsis (202). In chronic infections, however, LPS modifications have been identified that make the bacteria less 'visible' to the immune system (203). In addition, $P$. aeruginosa secretes a plethora of extracellular virulence factors; a majority of them (LasA, LasB, protease IV, exotoxinA, lipases, phospholipase C) secreted via the Xcp Type II secretion system $(204,205)$. P. aeruginosa can also specifically deliver toxins directly into the host cell using the sophisticated type III and type VI secretion systems [T3SS and T6SS, respectively; $(206,207)]$.

Pathogenesis of $P$. aeruginosa is thus due to the concerted action of multiple virulence mechanisms and is typically not due to the specific action of a single virulence factor. An intricate network of regulation involving multiple transcriptional regulators (transcriptional control), and small RNA-binding proteins and regulatory RNA (posttranscriptional control) orchestrate the timed co-expression of multiple virulence 
mechanisms. At the heart of the $P$. aeruginosa pathogenesis network is quorum sensing (QS), a system of interconnected sensors and regulators that control expression of virulence and other bacterial traits in a cell-density dependent manner (208). Key to the QS regulatory network are autoinducers (also known as quoromones): small diffusible chemical signals that help bacteria sense self and non-self population densities and coordinate expression of virulence factors under high cell numbers thus increasing the chances of a successful infection (208). To further enhance survival P. aeruginosa, like other bacteria, has evolved to combine signal recognition with transcriptional regulation for a quick and efficient response to changes in the environment. Critical to this process are the two-component systems (TCS) comprised of an inner membrane-bound sensor kinase that transduces the signal to a cytoplasmic response regulator, either directly or via a phosphorelay. In some cases, the two components are part of the same protein, the socalled hybrid sensor kinases that are known to be critical for P. aeruginosa pathogenesis (209-212). Auxiliary regulators connect histidine kinases with regulatory processes forming three-component regulatory signaling systems (213).

If genome sizes are any indication of complexity, $P$. aeruginosa is indeed a highly complex bacterium. With strains harboring 6-7 MB chromosomes (33,214), $P$. aeruginosa has one of the largest genomes in bacteria closely approaching that of lower eukaryotes such as Saccharomyces. In bacteria like $P$. aeruginosa, where the genome repertoire far exceeds many others, regulatory genes are acquired through horizontal gene transfer or evolution of paralogs that have new specificities for new sets of target genes. This is underscored by the comparative analyses of transcription factors from 175 prokaryotic genomes showing reduced conservation as compared to their target genes 
(215). However, such extensive analysis still precludes our ability to predict the new lineage-specific transcription factors and the new interactions that continue to evolve as the organisms fight for their survival in a myriad of environments. Thus it is not surprising that a single species, such as $P$. aeruginosa with its ability to thrive in diverse environments, has a mosaic genome consisting of a conserved core component interrupted in each strain by combinations of specific blocks of genes (33). These strainspecific segments of the genome are found in limited chromosomal locations, referred to as regions of genomic plasticity (RGP), and favors survival by enhancing the bacteria's metabolic and/or virulence capabilities (33).

Transcriptional regulation in $P$. aeruginosa is a complex and intricately interlinked process. Many 'global' regulators have been identified (Vfr, MvfR, AmpR, Rsm, LasR, RhlR) that have a profound effect on the gene expression pattern. Though many transcriptomic studies have looked at global scale expression of genes, understanding the role of transcription factors, structure-function and interaction analyses and the associated networks in P. aeruginosa still lags behind Escherichia coli. However, certain networks such as QS and alginate regulation are much better characterized in $P$. aeruginosa than in others. 


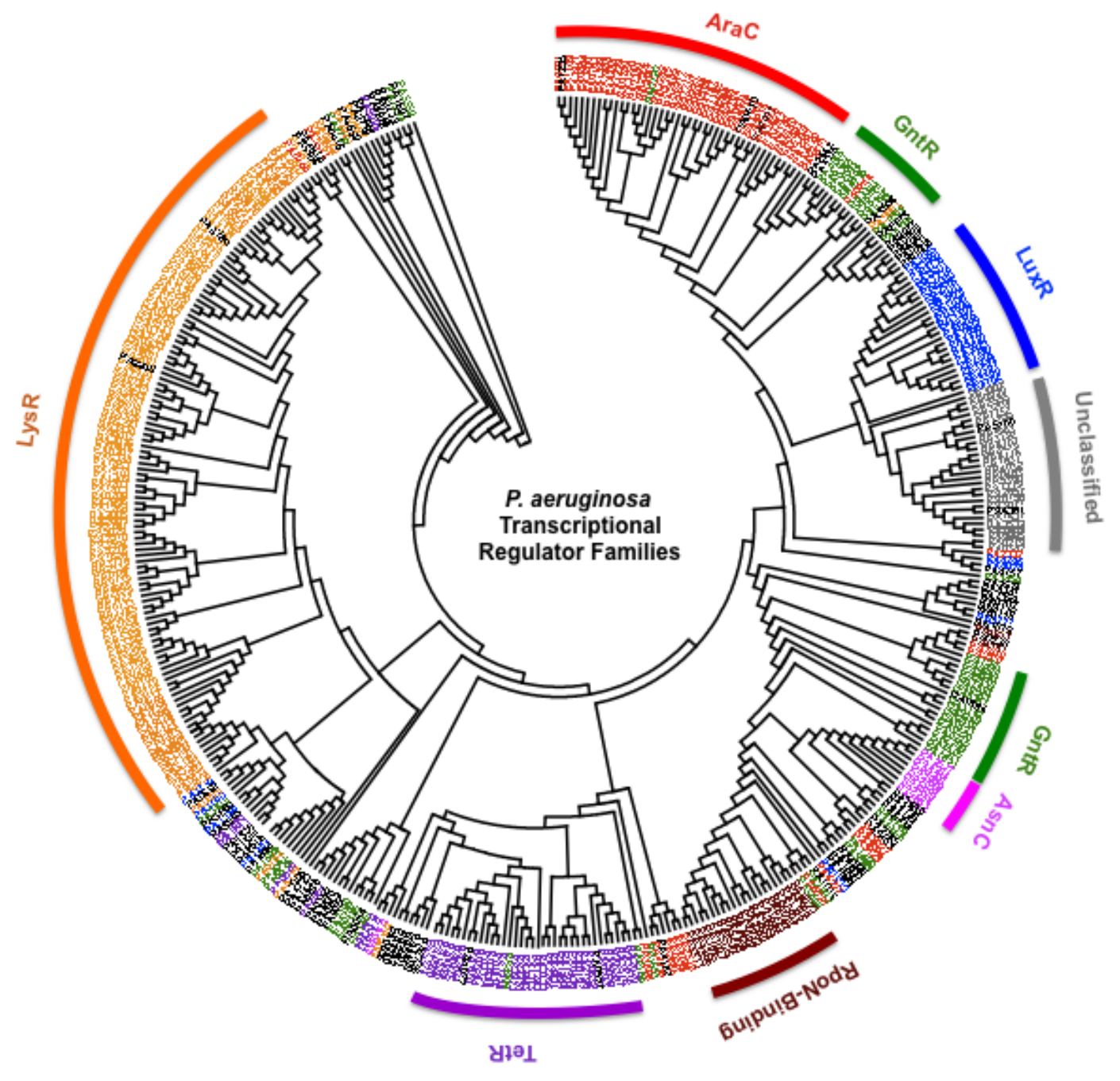


Figure 3.1: $P$. aeruginosa transcriptional regulatory protein families. A total of 434 amino acid sequences of transcription factors (Appendix; Supplementary information for Chapter 3, Table 1) were aligned using ClustalW (Version 2.1.10) (216). The first tree out of 31 most parsimonious trees (length $=$ $69259)$ is shown. The consistency index is $0.155474(0.147175)$, the retention index is 0.298198 (0.218198), and the composite index is $0.046362(0.043887)$ for all the sites and parsimony-informative sites (in parentheses). The maximum parsimony tree was obtained using the Close-Neighbor-Interchange algorithm (217) with search level 2 (217,218) in which the initial trees were obtained with the random addition of sequences (10 replicates). All alignment gaps were treated as missing data. There were a total of 1137 positions in the final dataset, out of which 743 were parsimony informative. Phylogenetic analyses were conducted in MEGA4 (219). The different families that cluster together are colored. Members of families that do not form tight clusters (IclR, LacI/GalR, MerR, Cro-C1, CRP, ArsR, H-NS, OmpC, RpiR, and Dks/TraR) are colored black.

The key to understanding $P$. aeruginosa pathogenesis is to have a unified view of how the different regulators and regulatory networks are linked. This review will focus on the genome composition of the transcription factors in P. aeruginosa and attempt to glean meaningful interactions.

\section{Families of transcriptional regulators in $P$. aeruginosa}

Transcriptional regulators are grouped into numerous families based on their functional domains, particularly DNA and effector binding domains. Even though many regulators have been studied in $P$. aeruginosa and in silico analyses group them into different families (Figure 1), experimental evidence assigning function for most of the 434 regulators is still lacking. Phylogenetic analysis shows that not all members fall into tight groups indicating that sequence homology is not sufficient to cluster all the members (Figure 3.1). Some families, like MerR and Cro-cI (labeled in black font in Figure 3.1), are scattered all over the tree. Others, like the GntR family, form multiple clusters, while some members of well-formed clusters fall in other clusters. For example, PA5261 of the LysR family is grouped with one of the GntR clusters (Figure 3.1). Interestingly, the unclassified proteins, which do not have a known signature sequence, 
cluster together (Figure 3.1). Experimental data such as structure analysis will help classify these proteins as well as the outliers into families.

This section deals with the major transcriptional regulator families in $P$. aeruginosa PAO1 and their prominent members. The characteristics of the major families are listed in Table 3.1.

\section{LysR Family}

LysR-type transcriptional regulators (LTTRs) are the largest family of prokaryotic transcriptional regulators $(103,104)$. Many LTTRs are global activators/repressors of target gene expression. The targets are either divergently transcribed from them or are part of unlinked regulons at different locations on the chromosome (220,221). Divergent transcription from the target gene allows for autoregulation, though there are exceptions $(104,105)$. This family is the namesake for the prototypic member LysR that activates lys $A$ transcription involved in lysine biosynthesis in E. coli (222). LTTR members typically have a N-terminal winged helix-turn-helix (wHTH)-DNA binding domain (residues 1-65), C-terminus effector-binding domain (residues 100-173 and 196-206) and a domain that is needed for both functions (residues 227-253) (103). DNA binding, either as dimers or tetramers, occurs at the target promoters even in the absence of the effector signals. In most cases, co-inducer binding causes a change in footprint resulting in DNA bending and transcription activation (104). 
Table 3.1: Characteristics of the major families of transcriptional regulators in P. aeruginosa

\begin{tabular}{|c|c|c|c|c|c|c|c|}
\hline Family & $\begin{array}{c}\text { \# in } \\
\text { P.aeruginosa }\end{array}$ & $\begin{array}{c}\# \text { in } \\
\text { E.coli }\end{array}$ & $\begin{array}{c}\text { DNA } \\
\text { binding } \\
\text { motif } \\
\end{array}$ & Other domains & Regulation & Oligomerization & Prototype \\
\hline LysR & 127 & 45 & $\begin{array}{l}\text { N-term } \\
\text { wHTH }\end{array}$ & $\begin{array}{l}\text { C-term effector-binding; } \\
\text { a DNA/effector-binding }\end{array}$ & $\begin{array}{l}\text { Activator/ } \\
\text { repressor }\end{array}$ & Dimer/tetramer & E. coli LysR \\
\hline AraC/XylS & 61 & 21 & $\begin{array}{l}\text { C-term } \\
\text { HTH }\end{array}$ & $\begin{array}{l}\mathrm{N} \text {-term } \\
\text { dimerization/sugar- } \\
\text { binding }\end{array}$ & Activator & Dimer & E. coli AraC \\
\hline TetR & 39 & 11 & $\begin{array}{l}\text { N-term } \\
\text { HTH }\end{array}$ & $\begin{array}{l}\text { C-term effector- } \\
\text { binding/oligomerization }\end{array}$ & Repressor & Dimer/octamer & $\begin{array}{l}\text { Enterobacte } \\
\text { rial TetR }\end{array}$ \\
\hline LuxR & 32 & 17 & $\begin{array}{l}\text { C-term } \\
\text { HTH }\end{array}$ & $\begin{array}{l}\mathrm{N} \text {-term regulatory } \\
\text { domain }\end{array}$ & Activator & Mutimer & $\begin{array}{l}\text { V. fisheri } \\
\text { LuxR }\end{array}$ \\
\hline GntR & 31 & 3 & $\begin{array}{l}\text { N-term } \\
\text { HTH }\end{array}$ & $\begin{array}{l}\text { C-term effector- } \\
\text { binding/oligomerization }\end{array}$ & $\begin{array}{l}\text { Repressor/ } \\
\text { activator }\end{array}$ & Homodimer & $\begin{array}{l}\text { B. subtilis } \\
\text { GntR }\end{array}$ \\
\hline $\begin{array}{l}\text { RpoN- } \\
\text { binding }\end{array}$ & 22 & 2 & $\begin{array}{l}\text { C-term } \\
\text { HTH }\end{array}$ & $\begin{array}{l}\text { Central RpoN- } \\
\text { interacting; N-term } \\
\text { effector-binding }\end{array}$ & Activator & Dimer/multimer & E. coli $\mathrm{NtrC}$ \\
\hline Cro-C1 & 17 & 3 & $\begin{array}{c}\text { N-term } \\
\text { HTH }\end{array}$ & C-term oligomerization & Repressor & Homodimer & $\begin{array}{l}\text { Phage } 434 \\
\text { (Cro); } \\
\text { Phage } \\
\text { lambda (C1) }\end{array}$ \\
\hline IclR & 9 & 8 & $\begin{array}{l}\text { N-term } \\
\text { wHTH }\end{array}$ & $\begin{array}{l}\text { C-term effector- } \\
\text { binding/oligomerization }\end{array}$ & $\begin{array}{l}\text { Repressor/ } \\
\text { activator }\end{array}$ & Dimer/tetramer & E. coli IclR \\
\hline
\end{tabular}




\begin{tabular}{|c|c|c|c|c|c|c|c|}
\hline Family & $\begin{array}{c}\text { \# in } \\
\text { P.aeruginosa }\end{array}$ & $\begin{array}{l}\text { \# in } \\
\text { E.coli }\end{array}$ & $\begin{array}{c}\text { DNA } \\
\text { binding } \\
\text { motif }\end{array}$ & Other domains & Regulation & Oligomerization & Prototype \\
\hline AsnC & 9 & 3 & $\begin{array}{c}\text { N-term } \\
\text { HTH }\end{array}$ & $\begin{array}{l}\text { C-term effector- } \\
\text { binding/oligomerization }\end{array}$ & $\begin{array}{l}\text { Repressor/ } \\
\text { activator }\end{array}$ & Multimer & $\begin{array}{l}\text { E. coli } \\
\text { AsnC }\end{array}$ \\
\hline LacI & 7 & 15 & $\begin{array}{l}\text { N-term } \\
\text { HTH }\end{array}$ & $\begin{array}{l}\text { C-term effector- } \\
\text { binding/oligomerization }\end{array}$ & Repressor & Dimer/tetramer & E. coli LacI \\
\hline MerR & 7 & 5 & $\begin{array}{l}\text { N-term } \\
\text { HTH }\end{array}$ & C-term effector-binding & $\begin{array}{l}\text { Activator/ } \\
\text { repressor }\end{array}$ & Dimer & $\begin{array}{l}\text { E. coli } \\
\text { MerR }\end{array}$ \\
\hline ArsR & 4 & 2 & $\begin{array}{l}\text { N-term } \\
\text { HTH }\end{array}$ & $\begin{array}{l}\text { C-term effector- } \\
\text { binding/dimerization }\end{array}$ & Repressor & Homodimer & E. coli ArsR \\
\hline
\end{tabular}


P. aeruginosa PAO1 has 127 LTTR members that despite controlling diverse functions (Appendix; Supplementary information for Chapter 3, Table 1) group together on amino acid sequence analysis (Figure 3.1). Though the functions of many members remain unknown, the few that are known are regulators of critical processes including virulence in $P$. aeruginosa. MvfR (PA1003), for example regulates QS in a LasIR/RhlIRindependent manner by modulating PqsE (PA1000), the quinolone response signal protein $(223,224)$. The chromosomal B-lactamase regulator AmpR (PA4109) is a global regulator of virulence in $P$. aeruginosa. In addition to regulating $\beta$-lactam and non- $\beta$ lactam resistance, AmpR also positively modulates expression of many acute virulence factors and negatively regulates chronic infection phenotypes $(105,225,226)$. OxyR (PA5344) is an example of a regulator controlling expression of multiple related phenotypes. Under oxidative stress, OxyR enhances expression of antioxidant genes like $k a t B, a h p C F$ and $a h p B$ (227). In addition, OxyR also plays a role in virulence (228), cytotoxicity (229), pyoverdine utilization, and pyocyanin and rhamnolipid production $(230,231)$. Turner et al. recently identified the bistable regulon of a LysR-type regulator BexR (PA2432) that regulates virulence factors like AprA (232). Other characterized LysR-type members include an efflux pump regulator, MexT (PA2492) that has been recently shown to regulate other genes (233) and MetR (PA3587), a regulator of genes involved in methionine synthesis (234). Except for PA0207 (RGP1), PA1223 (RGP11), PA2054 (RGP20), PA2056 (RGP20), and PA2220 (RGP53), all LysR members are part of the core genome (Appendix; Supplementary information for Chapter 3, Table 1). 


\section{IcIR Family}

The isocitrate lyase regulator (IclR) family controls gene expression in prokaryotes in a signal-dependent manner (235). IclR members are distributed in over 46 species of eubacteria and archea and typically regulate genes and operons involved in carbon metabolism or virulence. In the absence of specific substrates, IclR regulators typically repress specific catabolic genes, and in the presence of excess signaling molecules, de-repress expression (236). Two domains in the protein bring about regulation: a winged $\mathrm{HTH}$ domain at the $\mathrm{N}$-terminus that binds DNA, and an effectorbinding domain at the C-terminus. IclR-like regulators form dimers or tetramers and recognize palindromic sequences on the DNA (235). Some of the well-studied members of this family include the family namesake in E. coli IclR, a glyoxalate shunt repressor of the aceBAK operon involved in acetate utilization, the glycerol catabolism pathway repressor GlyR in Streptomyces coelicolor and KdgR, the pectin degradation pathway repressor in Erwinia chrysanthemi. A Thermatoga maritima IclR member TM0065, involved in xylulose metabolism was the first member of this family to be crystallized (237).

P. aeruginosa PAO1 has nine IclR homologs and all of them are yet to be characterized. A PA0155 (PcaR) homolog in P. putida has been demonstrated to regulate the pca operon involved in p-hydroxybenzoate degradation by binding both the -35 and 10 regions in the promoter (238). PA1015 is $42 \%$ similar to GlyR, a glycerol catabolic operon $(g y l A B X)$ regulatory protein in $S$. coelicolor and is present immediately upstream of the gylABX promoter (239). In $P$. aeruginosa however, there is no gylABX operon in the immediate vicinity and the role of PA1015 remains unclear. All IclR members are 
part of the core genome with the exception of PA3508 (RGP34) (Appendix; Supplementary information for Chapter 3, Table 1).

\section{LacI/GaIR Family}

The LacI/GalR family members are metabolic regulators in prokaryotes that recognize sugar inducers. The prototypic members are the E. coli lactose repressor LacI and the galactose operon repressor GalR (240). They are typically localized in the cytoplasm and possess a N-terminal HTH motif of about 50-60 residues. The C-terminus contains regions that are involved in oligomerization and signal molecule recognition (241). LacI members function as either dimers or tetramers (242). Well-studied proteins with a LacI-type HTH include the Bacillus subtilis CcpA and CcpB involved in repression of several operons (243), the Hemophilus influenzae galactose repressor GalR (244), the E. coli lactose operon repressor LacI (245), and the Salmonella typhimurium fructose repressor FruR (246).

Five of the seven LacI homologs in P. aeruginosa, all of which are part of the core genome, are involved in carbohydrate transport/metabolism (Appendix; Supplementary information for Chapter 3, Table 1). A fructose transport repressor (FruR) homolog is found in P. aeruginosa PAO1 (PA3563). However, others are involved in virulence. NfxB (PA4600), a regulator of the MexCD-OprJ efflux pump has also been shown to affect motility, probably through its effect on the motility regulator MorA (PA4601), and QS-dependent phenotypes by inappropriate MexCD-OprJ efflux expression (247). Interestingly, there is another LacI-like regulator in the same locus, 
PA4596, upstream of the MexCD-OprJ operon that is $74 \%$ similar to $\mathrm{NfxB}$. Whether this is also involved in regulating MexCD-OprJ remains to be determined.

PtxS (PA2259) is an example of a transcriptional regulator controlling two very different pathways in $P$. aeruginosa. It is a repressor of the exotoxin A regulator PtxR (PA2258) (248) not by direct binding at $\mathrm{P}_{p t x R}$ from which it is divergently transcribed but through an unknown mechanism (249). PtxS is also part of a 5-gene $\mathrm{kgu}$ operon (PA2259-PA2263) involved in 2-ketoglutonate utilization, which it regulates by binding to two different operator sequences (250).

\section{GntR Family}

The prototypic member of this family is Bacillus subtilis GntR, a repressor of the gluconate operon $(251,252)$. Many of the members of this family have been shown experimentally to be autoregulatory, enabling the prediction of operator sites and the discovery of cis/trans relationships (253). This family is further subdivided into four major (FadR, HutC, PlmA, MocR) and two minor (YtrA, AraR) subfamilies, which regulate various biological processes and important bacterial metabolic pathways (254). DNA binding by GntR members is inducer-independent. For example, GntR binds to the promoter of the operon in the absence of gluconate but not in the presence (255). This family of proteins has a wHTH domain about $60-70$ residues at the $\mathrm{N}$-terminus whereas the C-terminus contains a subfamily-specific effector-binding domain and/or an oligomerization domain (252). The GntR family of regulators binds as homodimers to two-fold symmetric DNA elements $(256,257)$. 
P. aeruginosa PAO1 genome harbors 31 regulators belonging to the GntR family, of which many are grouped together by sequence analysis (Figure 1) and only two are part of RGPs (PA2032 and PA2100 in RGP20 and RGP21, respectively). Based on sequence analysis, six of the transcriptional regulators are presumed to be involved in glycolate (PA5356), lactate (PA4769), gluconate (PA2320), histidine (PA5105), phosphate (PA3381) and N-acetyl-D-glucosamine (PA3757) catabolism. Of the 31 only

the role of PA5499 (Np20) has been experimentally elucidated. Expression of $n p 20$ is inducible in vitro by respiratory mucus, in vivo during infection of mice and was shown to be important in bacterial virulence in a neutropenic-mouse infection model (258). However, no structure-function studies are available.

\section{LuxR Family}

The LuxR-type transcription regulator superfamily derives its name from the Vibrio fisheri LuxR protein, which alters transcription in response to QS to produce bioluminescence (259-262). LuxR proteins contain two functional domains: a C-terminus HTH DNA binding domain and an N-terminal regulatory domain (263). The DNA binding domain consists of a four-helix bundle. The LuxR proteins bind promoters as multimers (264). Based upon the N-terminus, the LuxR family may be further subdivided into at least four groups: the FixJ group (265-268), the LuxR group [reviewed in (263)], the large ATP-binding regulators of the LuxR superfamily (LAL) group $(269,270)$, and the autonomous effector domain LuxR-type regulators $(264,271)$. Many proteins that have been classified as LuxR superfamily members do not belong to any of these groups. Moreover, new groups are still being identified (272). 
P. aeruginosa PAO1 harbors 32 members of the LuxR superfamily (Appendix; Supplementary information for Chapter 3, Table 1) that group together in neighborjoining trees (Figure 3.1). Half of these appear to belong to the FixJ group. Of these, six have been demonstrated to function as TCS response regulators and 10 are predicted to be response regulators based upon PFAM analysis (Appendix; Supplementary information for Chapter 3, Table 1). A LuxR member, NarL (PA3879), acting in concert with the sensor kinase NarX (PA3878), represses expression of proteins involved in the arginine fermentation pathway for ATP generation. In addition, NarL also activates proteins in the nitrate reductase operon (NarH, PA3874), the porin OprE (PA0291) and the class II ribonucleotide reductase components NrdJa (PA5497) and NrdJb [PA5496, (273)]. RocA1 (PA3948), and its cognate sensor kinase RocS1 (PA3946), were identified as regulators of fimbril cup genes [cupB and cupC, (274)]. Other studied members of this family include BfiR [PA4196, (275,276)], ErdR [PA3604, (277)], GacA [PA2586, (278)], and the QS regulators LasR (PA1430), RhlR (PA3477), QscR (PA1898), and VqsR (PA2591) [see section on QS below; (279-282)]. All LuxR superfamily members are encoded by the $P$. aeruginosa core genome (Appendix; Supplementary information for Chapter 3, Table 1).

\section{RpoN-Binding Family}

This family of transcriptional regulators activates the expression of genes from promoters recognized by core RNA polymerase associated with the alternative sigma-54 factor (RpoN). RpoN enhancer binding proteins share a conserved domain of about 230 residues involved in the ATP-dependent interaction with RpoN (283). The RpoN-binding 
domain, which has ATPase activity, contains an atypical ATP-binding motif A (P-loop), and a motif B (284). These proteins are part of the ATPases associated with diverse cellular activities clan: proteins that perform chaperone-like functions and assist in the assembly, operation, or disassembly of protein complexes (285).

In $P$. aeruginosa, there are 22 RpoN-binding proteins, encoded by the core genome, which are involved in the regulation of metabolism, production of the exopolysaccharide alginate, virulence as well as biofilm formation (Appendix; Supplementary information for Chapter 3, Table 1). Nine of the 22 RpoN interacting proteins belong to signal transduction TCS and contain an N-terminal domain that can be phosphorylated by a sensor-kinase protein. Almost all of these proteins possess a HTH DNA binding domain in their C-terminus. At least six are NtrC-like RpoN enhancer binding proteins (PilR-PA4547, CbrB-PA4726, NtrC-PA5125, AlgB-PA5483, MifRPA5511 and FleQ-PA1097). NtrC transcriptional activators, first described in the regulation of nitrogen metabolism of enteric bacteria, possess three primary domains: a RpoN-interacting conserved central domain, a C-terminal domain which contains the HTH DNA binding motif and a homologous receiver N-terminal domain (286).

The transcriptional regulator CbrB is part of the TCS CbrAB (PA4725-PA4726), involved in regulating the carbon-nitrogen balance in $P$. aeruginosa. Phosphorylated CbrB activates the expression of the aot-argR operon (arginine catabolism) and the hiu operon (histidine catabolism) (287). Two biofilm regulators, MifR (PA5511), part of a signal transduction network in microcolony formation (275), and RocR of the RocSAR (SadARS) three-component signaling system involved in biofilm maturation (288), have been defined. RocSAR consists of the histidine kinase RocS1 and two response regulators, 
RocA1 (PA3948) and RocR (PA3947). The RocSAR system controls bacterial biofilm formation and virulence gene expression by regulating the transcription of various genes, including the cup fimbrial-gene clusters and Type III secretion system genes (288). AlgB (PA5483), also a part of TCS, activates alginate production in mucoid P. aeruginosa. Alginate has been implicated in the pathogenesis of $P$. aeruginosa including resistance to phagocytosis and adherence mechanisms (289).

\section{MerR Family}

The classical MerR proteins are dimeric transcriptional regulators of Gramnegative mercury resistance operons present in transposons $\operatorname{Tn} 21$ and $\operatorname{Tn} 50$ (290). Like many of the regulators already discussed, these regulators consist of two domains, a 70amino acid N-terminal DNA-binding domain and a C-terminal effector-binding domain. The carboxy-terminus contains specific effector binding regions to respond to environmental stimuli such as heavy metals, oxidative stress, antibiotics or metal ions $(290,291)$. The MerR proteins differ from many other classes of regulators in that they function as both an activator and repressor of the same gene. They achieve this by binding to the same cis element between -10 and -35 promoter regions containing a critical 19 bp region essential for MerR-dependent activation (290). MerR acts as a weak repressor in the absence of ligand $(\mathrm{Hg}(\mathrm{II}))$ and as an activator when bound to $\mathrm{Hg}$ (II) (292).

P. aeruginosa contains seven MerR regulators, two of which, SoxR (PA2273) and CueR (PA4778) are characterized. Like its E. coli homologue, the P. aeruginosa SoxR contains a [2Fe-2S] center (293). In E. coli, SoxR regulates genes involved in the 
oxidative stress response to superoxide anions or nitric oxide by activating an AraC-type regulator SoxS $(294,295)$, which is not conserved in P. aeruginosa. SoxR, however, binds weakly and activates transcription of the promoter from its divergently transcribed neighbor, PA2274, encoding a hypothetical protein (293). CueR controls a copper resistance regulon consisting of five operons and 11 genes (296), and is under regulation of LasR (PA1430), a LuxR member (296). PA3689 is similar to CadR, a MerR family member from $P$. putida involved in cadmium resistance (297). All MerR proteins are encoded as part of the $P$. aeruginosa core genome (Appendix; Supplementary information for Chapter 3, Table 1).

\section{TetR Family}

The TetR family is named after the enterobacterial TetR repressor protein that confers resistance to tetracycline (298) and subsequently a family profile was developed (299). TetR members are typically repressors that contain a 60 residue N-terminal HTH DNA binding domain and a C-terminus effector-binding/oligomerization domain that bind DNA either as dimers or octamers (300-302). TetR, a well-studied example, is a repressor of the enterobacterial tetracycline exporter, TetA and binds to the operator sites in $\mathrm{P}_{\text {teta }}$. Binding of the tetracycline-Mg complex to DNA-bound TetR leads to depression of tetA and confer tetracycline resistance (303). TetR members primarily control multidrug efflux pumps, biosynthesis of antibiotics, catabolic pathways and virulence in both Gram-positive and Gram-negative bacteria.

There are 39 TetR members in $P$. aeruginosa PA01, all of which are encoded by the core genome (Appendix; Supplementary information for Chapter 3, Table 1). The 
expression of the MexAB-OprM efflux pump (PA0425-PA0427) that confers resistance to $\beta$-lactams, in addition to diverse substrates, is under the control of two TetR members NalC (PA3721) and NalD (PA3574), of which NalC is thought to act via the primary repressor MexR (PA0424) (304-306). PsrA (PA3006) has been demonstrated to control expression of the $P$. aeruginosa T3SS (307), probably through its regulatory effect on RpoS (308). A TetR member, CifR (PA2931) represses expression of a toxin, CFTR inhibitory factor (Cif, PA2934) (309). Cif has been demonstrated to reduce expression of the CFTR protein in cell lines and proposed to play a role in aiding initial colonization by P. aeruginosa (310). CifR regulation of Cif thus aids in the later stages on infection when Cif function is no longer required (309). Other characterized $P$. aeruginosa TetR members (AguR-PA0294, AtuR-PA2885, DesT-PA4890, BetI-PA5374) are primarily metabolic repressors [Appendix; Supplementary information for Chapter 3, Table 1; (311-314)].

\section{AraC Family}

The E. coli L-arabinose operon activator, AraC exemplifies this group of transcriptional regulators with which members share a 99-residue C-terminus homology (315-317). AraC proteins harbor a C-terminus HTH motif, a N-terminus dimerization/sugar-binding domain and primarily activate expression of genes involved in sugar metabolism, stress response and virulence (299,318). Most AraC members, together with cAMP-receptor protein (CRP), activate promoters, either as monomers or dimers $(319,320)$. 
P. aeruginosa PAO1 has 61 AraC members (Appendix; Supplementary information for Chapter 3, Table 1). PchR (PA4227), an AraC member, is a regulator of the biosynthesis of an iron uptake protein, pyochelin, and acts both as a positive and negative regulator of fptA (PA4221) and pchR expression (321). PchR binds to a $32 \mathrm{bp}$ PchR box in the promoter of genes that it regulates (322). ExsA (PA1713), a Yersinia enterocolitica VirF homolog, was initially identified as part of a trans-regulatory locus involved in the expression of the T3SS effector exoenzyme S $(323,324)$ and has since been shown to regulate expression of the T3SS genes (reviewed in (325). MmsR (PA3571) regulates the mmsAB operon (PA3569-PA3570) that encodes enzymes involved in valine metabolism (326). Other than PA1380 (RGP15) and PA2047 (RGP20), AraC protein-encoding genes belong to the core genome of $P$. aeruginosa (Appendix; Supplementary information for Chapter 3, Table 1).

\section{Cro-C1 Family}

This superfamily is named after the Cro and $\mathrm{C} 1$ repressors of the lysogenic phages 434 and lambda, respectively, where they are part of a binary switch that regulates lytic/lysogenic growth of the phage by differential binding to the operator sites $(327,328)$. They generally have a 50-60 residue HTH domain at the N-terminus of the protein while oligomerization occurs at the C-terminus. The lambda Cro repressor is a 66 amino acid protein and binds DNA as a homodimer (329).

P. aeruginosa has 17 regulators belonging to this family, all of which are encoded by the core genome (Appendix; Supplementary information for Chapter 3, Table 1). Pyocin production in P. aeruginosa is under the tight regulation of PrtR (PA0611). As 
part of the SOS response, RecA (PA3617) degrades PrtR, which inhibits transcription of the pyocin gene activator, PrtN [PA0610, (330)]. PrtR also modulates activity of a Dks/TraR member PtrB (PA0612), a repressor of the T3SS (331), linking secretion and pyocin synthesis in response to DNA damage. Two other hypothetical Cro/C1 members, PA0906 and PA4077 are 66\% similar to PrtR, and PA4077 is co-transcribed with a hypothetical inner membrane protein PA4076 (332). Whether these are also involved in pyocin regulation is unclear. PA1359, a hypothetical regulator, bears $49 \%$ similarity to $E$. coli $\mathrm{HipB}$, a known regulator of persister formation by modulation of HipA activity (333335). Interestingly, PA1359 is part of a two-gene operon (332), similar to the hipBA operon in E. coli. None of the other members of this family have been characterized in PAO1.

\section{AsnC Family}

This family of transcriptional regulators is typified by aspargine synthase $\mathrm{C}$ of $E$. coli and is part of the feast/famine regulatory proteins (FFRP) that includes Lrp and Asn (336). Members of this family are distributed in both archea and eubacteria. They typically contain a HTH DNA binding domain at the N-terminus and a dimerization/effector-binding domain at the C-terminus, which also plays a role in assembly of the protein. FFRP members bind DNA as dimers, tetramers, octamers or hexamers $(337,338)$ and regulate genes in response to diverse signals. Identification of FFRPs is often complicated by the fact that the amino acid homologies between members are often only about $30 \%(336)$. 
P. aeruginosa has nine AsnC members (Appendix; Supplementary information for Chapter 3, Table 1), all of which except PA2028 (RGP20) are encoded by the core genome. PA0513, an as yet uncharacterized regulator, is part of an 11-gene nir operon that is involved in the denitrification pathway (339). PA0515 is also part of the same operon and is listed as a probable AsnC-type transcriptional regulator in the Pseudomonas database (332) but has been excluded from our list (Appendix; Supplementary information for Chapter 3, Table 1) because it lacked a DNA binding motif in our analysis. BkdR (PA2246) is divergently transcribed from the $b k d$ operon that is involved in valine, leucine, isoleucine degradation and has been characterized in $P$. putida (340). The P. aeruginosa homolog of Lrp (PA5308), which regulates the global leucine response in E. coli, regulates the dadA (PA5304) and dadX (PA5302) genes that are required for L-alanine utilization (341).

\section{ArsR Family}

The E. coli ArsR that represses the ars operon in response to arsenic is the prototype for this family of transcriptional repressors (342). This family of proteins binds the promoter region of genes that they regulate (primarily metal transport and detoxification) and dissociate upon effector binding due to allosteric changes, allowing transcription by derepression (343). The metal-binding site is at the C-terminus of the protein whereas the DNA binding domain is at the N-terminus. ArsR members typically function as homodimers (344).

There are four members in $P$. aeruginosa encoded by the core genome (Appendix; Supplementary information for Chapter 3, Table 1). arsR (PA2277) is part of 
a four-gene cluster that has been demonstrated to confer resistance to arsenic and antimony when expressed in a heterologous E. coli ars mutant (345). PA0547 is an uncharacterized ArsR-type regulator that is part of a two-gene operon with the Sadenosylmethionine synthetase gene, metK (PA0546). The other two ArsR members in $P$. aeruginosa are also uncharacterized (Appendix; Supplementary information for Chapter 3, Table 1).

\section{Other Families}

Other $P$. aeruginosa regulatory families include the Dks/TraR family (PtrB (PA0612) and DksA (PA4723)), H-NS silencing proteins (MvaT (PA4315) and MvaU (PA2667)), CRP family (Vfr- PA0652 and Anr- PA1544), OmpC family (AmgRPA5200), OmpR-PhoP class (PA4381) and the RpiR family (PA5506).

\section{Unclassified Regulators}

In addition to the families listed above, 37 regulators do not contain the signature motif for any family but still are significant contributors to the regulatory processes in $P$. aeruginosa (Appendix 2; Supplementary Table 1). Of these, 27 have been characterized. CreB (PA0463), the regulator of the CreBC TCS operon, along with CreD (PA0465), encoded immediately downstream, are involved in colicin resistance in E. coli (346). The CreBC TCS is involved in regulating ß-lactam resistance in Aeromonas spp. (347) while in $P$. aeruginosa it contributes to resistance only in a $\triangle P B P 4$ background (348). LexA (PA3007) is a repressor of RecA and is induced upon DNA damage, as part of the SOS response (349). IrlR (PA4885) is part of a two-component system, a homolog of which has been shown to be involved in heavy metal homeostasis in Alcaligenes eutrophus 
(350). A few other members [KdpE (PA1637), DauR (PA3864), GltR (PA3192)] are metabolic regulators.

\section{P. aeruginosa transcriptional regulatory network}

Studying regulatory networks aid in elucidating interactions between the different transcriptional regulators and provide important insights into processes and pathways. To better understand regulatory networks and identify network motifs, analysis of network structure has proved useful. Two major network motifs that aid in bacterial response to environmental signals have been described: feed-forward motifs, and single-input and multiple-input motifs (215). Using published data on bacterial regulatory networks (215) and STRING analysis (351) of $P$. aeruginosa PAO1 regulators, a transcriptional regulatory network was obtained (Figure 3.2a) consisting of 288 regulators and 593 target genes. As seen in regulatory networks in other bacteria (215), the $P$. aeruginosa network also consists of feed-forward motifs, and single- and multiple-input motifs (Figure 3.2a). Vfr (PA0652), the P. aeruginosa homolog of E. coli cyclic AMP receptor protein and a regulator of virulence and QS, has the highest number of interacting partners, followed by the anaerobic growth and cyanide synthesis regulator Anr, an E. coli Fnr homolog (Figure 3.2a). Interestingly, both these regulators belong to the diverse Crp family of transcriptional regulators that have a $\mathrm{N}$-terminal nucleotide-binding domain similar to $\mathrm{CRP}$, in addition to a C-terminus HTH (352). 


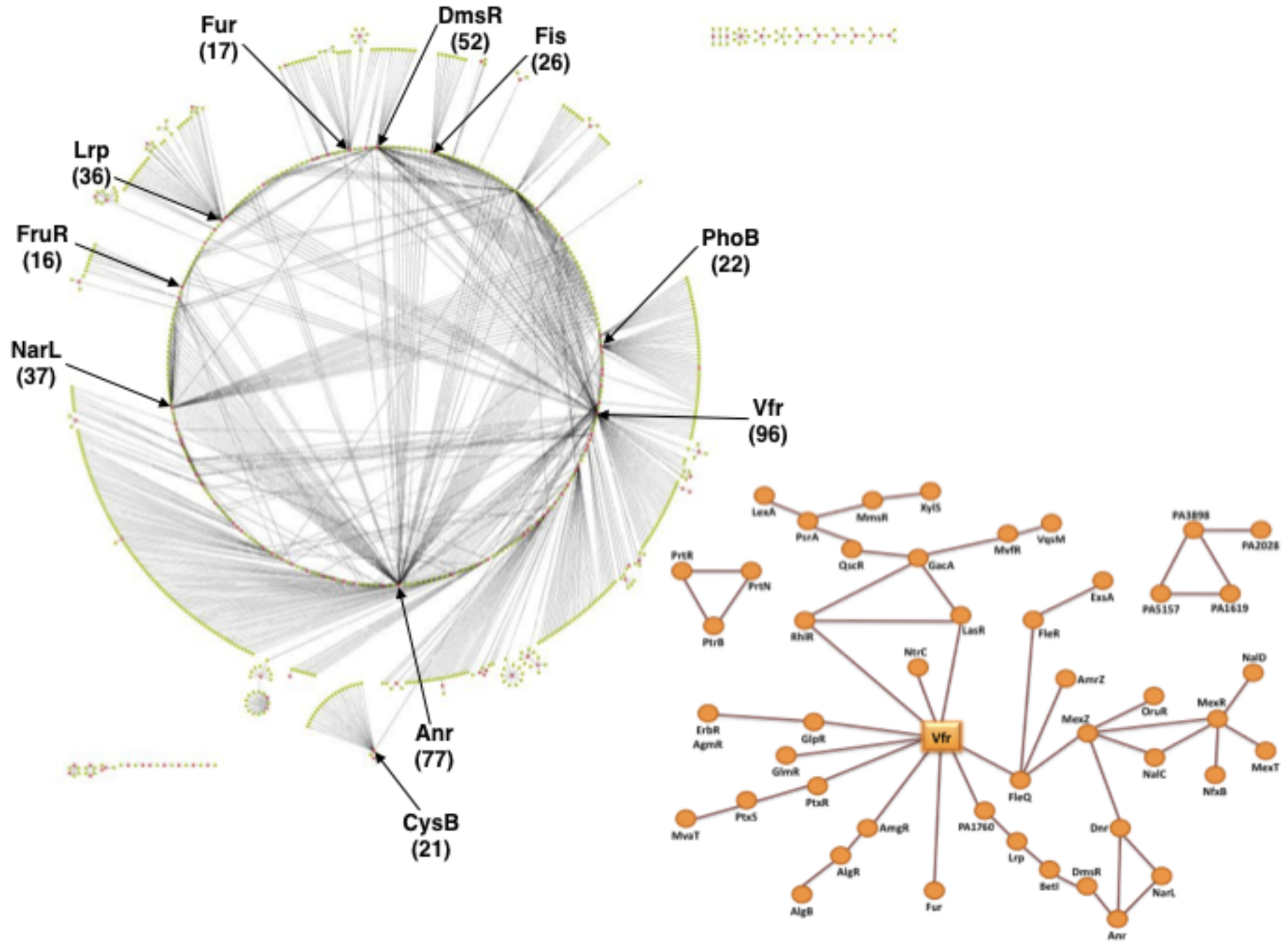


Figure 3.2a: Transcriptional regulators and interacting partners. Previously inferred regulators of $P$. aeruginosa (215) and those obtained by text mining using STRING (351) analysis, were used to construct the transcriptional regulatory network using Cytoscape (Version 2.7.0) (353). The network consists of 288 regulators and 593 target genes resulting in 1095 interactions. The transcriptional regulators with the highest interactions are shown in the figure (See also Appendix; Supplementary information for Chapter 3, Table 2).

Figure 3.2b: Interactions among transcriptional regulators. VisANT [Version 3.86; (354)] was used to visualize a total of 63 interactions between the regulators from the 1095 interactions of Fig 2a. Vfr emerges as the key regulator. Other interactions such as the pyocin induction network and an uncharacterized network appear isolated from these interactions.

Conclusions about hierarchy among the regulators are difficult to draw from this network but we used the data to identify interactions between the regulators alone without their corresponding target genes. The Vfr regulatory hub stood out in this analysis (Figure 3.2b). Vfr has been demonstrated experimentally to regulate multiple virulence mechanisms in $P$. aeruginosa. Network analysis shows that Vfr is linked to various other critical pathogenesis networks including alginate production, efflux systems, quorum sensing, iron uptake, flagella, T3SS, and metabolic regulons (Figure 3.2b). While some of these links have already been established (355-357), it would be interesting to see whether the other interactions pan out. Our Cytoscape analysis also revealed the established interactions between PrtR, PrtN and PtrB to regulate pyocin production and T3SS in P. aeruginosa [Figure 3.2b; (330,331)]. Moreover, PA5157, an uncharacterized regulator that bears $60 \%$ similarity to the E. coli MarR protein involved in regulating multiple antibiotic resistance and oxidative stress (358), interacts with three other hypothetical regulators PA1619, PA3898 and PA2028 (Figure 3.2b). Whether these genes are part of a network contributing to the antibiotic resistance profile of $P$. aeruginosa is not known. 


\section{Virulence regulatory systems in $P$. aeruginosa}

Network modeling, while providing us with a framework to study, has its limitations in requiring confirmation. The absolute proof of a regulatory interaction comes from empirical data, which serves to either confirm or negate findings from the bioinformatics analyses. We chose the QS system in $P$. aeruginosa, to highlight the similarities in complexity between modeled and established regulatory networks.

\section{Quorum sensing (QS)}

QS, a cell population density-dependent signaling system important in hostpathogen interaction, is involved in regulating virulence factor production, swarming motility, biofilm maturation, and efflux pump expression (reviewed in (208)). Two types of QS signals are produced by $P$. aeruginosa, $N$-acylhomoserine lactones and 4quinolones (359-361). Central to $N$-acylhomoserine lactone QS signaling are LasR (PA1430) and RhlR (PA3477), two LuxR-type regulators that respectively bind $\mathrm{N}$-(3oxododecanoylhomoserine lactone (3-oxo-C12-HSL) and $\mathrm{N}$-butanoylhomoserine lactone (C4-HSL) $(359,360)$. These HSLs are synthesized by the cognate synthetases, LasI and RhlI (279,362). Expression of lasI and rhlI is regulated by positive feedback loops mediated by 3-oxo-C12-HSL:LasR and C4-HSL:RhlR. LasR is higher up in the $P$. aeruginosa QS hierarchy and 3-oxo-C12-HSL:LasR activates rhlR and rhlI expression $(363,364)$. The binding site for LasR and RhlR has been termed the las-rhl box [CT- $\mathrm{N}_{12-}$ AG; $(365,366)]$ and LasR binding sites have been identified in the promoters of many genes including known and putative regulators such as MvfR (PA1003), RsaL (PA1431), VqsR (PA2591), RhlR (PA3477), PvdS (PA2426), AmrZ (PA3385), PA2588, PA4778, 
and PA1760 (365). In addition, three microarray studies identified a common set of 102 genes in the LasR/RhlR regulons (167-170,367).

Most QS-regulated genes are expressed during stationary phase and are dependent on the stationary phase sigma factor RpoS (PA3622, (366,368)). Several additional regulators also play a role in QS (Figure 3.3). Vfr binds and activates transcription of $\mathrm{P}_{\text {lasR }}$ (369) and $\mathrm{P}_{\text {rhlR }}$ (370), and represses the 4-hydroxy-2-quinolone-dependent QS pathway (371). Transcriptome analysis indicates that Vfr affects the expression of more than 100 genes in P. aeruginosa including T3SS genes (29). RsaL (PA1431), which is divergently transcribed from the lasI gene, encodes an autoregulating repressor that inhibits not only lasI transcription, but also genes involved in pyocyanin and cyanide synthesis (372,373). Interestingly, RsaL repression is dominant over 3-oxo-C12HSL:LasR activation of lasI to counteract the positive feedback loop (372). RsaL binds as a dimer (373) and regulates expression of 130 genes, including those involved in virulence, independent of its QS effect (373). Another transcription factor that appears to co-regulate QS genes with LasR and RhlR is the anaerobic regulator Anr (PA1544). Together with LasR and RhlR, Anr directly activates the hydrogen cyanide biosynthetic hcnABC genes [PA2193-PA2195, (374)]. Bioinformatic analysis identified putative Anr consensus binding sites, the Fnr/Anr box, in about $25 \%$ of QS regulated genes, suggesting that Anr may regulate these genes under oxygen limiting conditions (170). Interestingly, studies suggest that the growth conditions affect the las-rhl hierarchy $(375,376)$. 


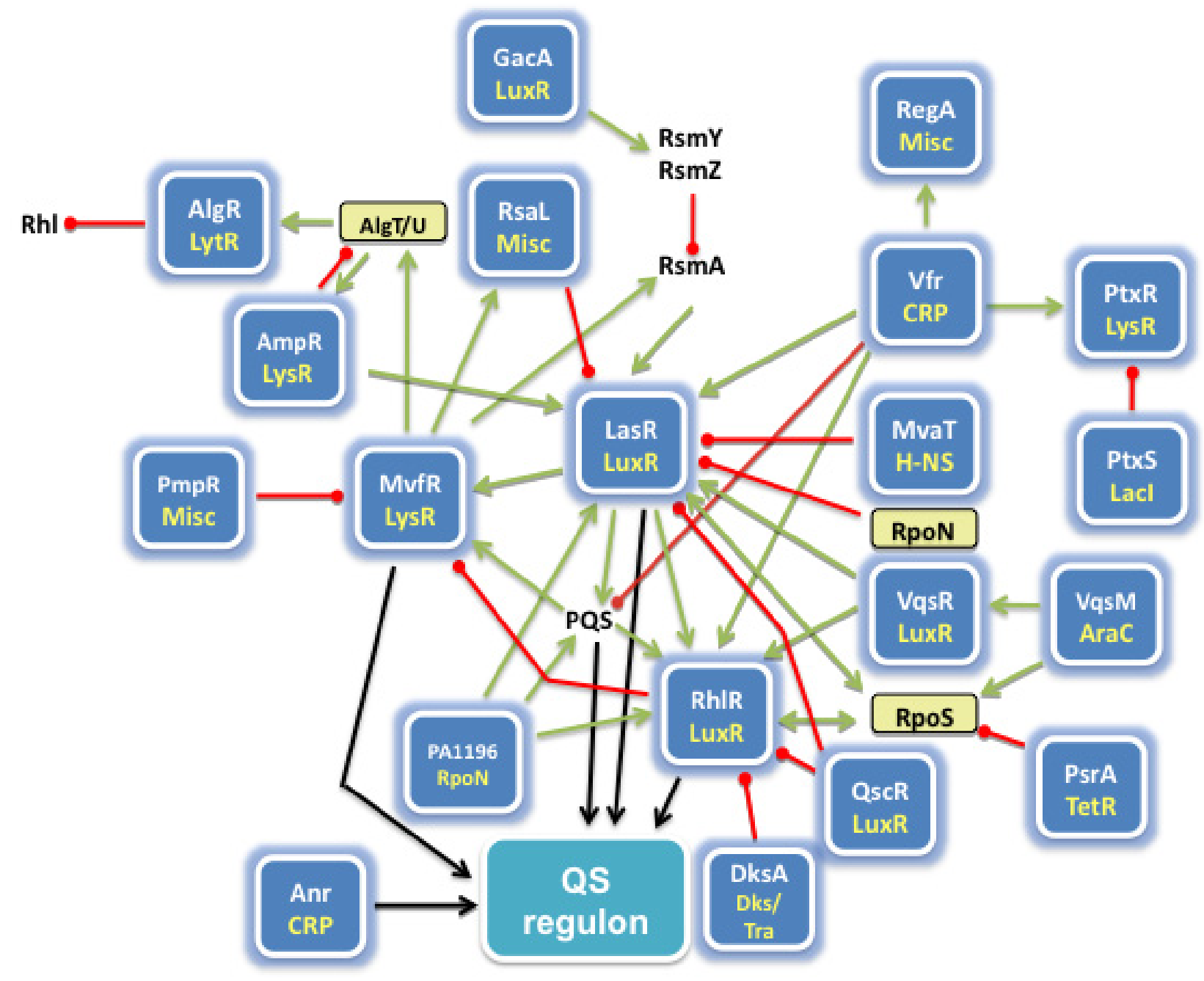


Figure 3.3: Association between QS regulators in P. aeruginosa. Interactions between the different transcriptional regulators (blue boxes) and sigma factors (yellow boxes) to control QS gene expression are depicted. The regulation is positive (green lines), negative (red lines), or both (black lines) and can be direct or indirect. PQS refers to the Pseudomonas quinolone signaling molecule. See text for details and references of individual interactions.

P. aeruginosa synthesizes over 50 4-quinolones, however, two, 2-heptyl-4quinolone (HHQ) and 2-heptyl-3-hydroxy-4-quinolone (PQS) have been shown to act as QS signals (361,377,378). Synthesis of these 4-quinolones is dependent upon the pqsABCDE (PA0996-PA1000) and phnAB (PA1001-PA1002) operons (377). The pqsE gene is dispensible for 4-quinolone synthesis but is required for virulence $(379,380)$. A LTTR member, MvfR (also known as PqsR; PA1003), regulates the pqs operon (381). MvfR expression is positively regulated by LasR:3-oxo-C12-HSL, and negatively regulated by RhlR (382) and the PqsR-mediated PQS regulator, PmpR (PA0964, (383)). Transcriptome analysis revealed an extensive MvfR regulon that includes RsaL (PA1431), AlgT/U (PA0762), RsmA (PA0905), and LasR (PA1430) (224). Promoter binding of MvfR is enhanced by either HHQ or PQS (381). HHQ is converted to PQS by PqsH (PA2587) whose expression is LasR:3-oxo-C12-HSL-dependent $(377,380)$. PQS differs from $\mathrm{HHQ}$ in that it can sequester iron and induce an iron starvation response by activating genes involved in siderophore biosynthesis and iron scavenging $(378,384)$. Thus PQS affects gene expression in multiple ways: $m v f R$ - and $p q s E$-dependent expression, $m v f R$-dependent and $p q s E$-independent expression, and through iron sequestration in a $m v f R$ - and $p q s E$-independent manner (378).

QscR (PA1898) and VqsR (PA2591) are two additional LuxR-type QS regulators in $P$. aeruginosa (Figure 3.3). QscR closely resembles the HSL-responsive LuxR 
homologues and has been proposed to control the timing of QS-regulated genes by repressing lasI expression (280). Transcriptome analysis identified 400 genes as being part of the QscR regulon (385). Additional experiments suggest that QscR can also act by mediating a response to 3-oxo-C10-HSL produced by other bacteria or form heterodimers with LasR or RhlR (386). The vqsR promoter contains a las-rhl box and in vivo occupancy of LasR has been demonstrated $(282,365)$. Consistent with this finding, vqsR mutants failed to produce C4-HSL or 3-oxo-C12-HSL, showed reduced pyocyanin and protease production, and reduced virulence in a $C$. elegans model system due to a negative impact on expression of the QS regulon (282). VqsR functions downstream of VqsM (PA2227), an AraC type regulator (282,387). Mutation in $v q s M$ reduced expression of $r h l R, r s a L, v q s R, m v f R, p p r B, r p o S$, lasI and $r h l I(387)$.

Other regulators have also been implicated in QS signaling. Mutations in the gene encoding the FIS regulator, PA1196, downregulate expression of $r h l I$ and $r h l R$ in addition to $p q s A$, which encodes the enzyme that modifies anthranilate for entry into the Pseudomonas quinolone signal (PQS) biosynthetic pathway (388). Transposon insertional mutants of PA5499 (np20) reduce rhlI expression as well as phenazine and cyanide production (380). Mutations in a LTTR AmpR have also been shown to modulate QS activity, linking QS with ß-lactam resistance in $P$. aeruginosa $(105,225,226)$.

QS is also regulated post-transcriptionally through the action of the small regulatory RNAs, rsmZ (PA3621.1) and rsmY (PA0527.1) (389,390). rsmYZ antagonize the RNA-binding protein RsmA (PA0905), which negatively regulates QS genes. The GacSA two-component system is activated at high cell densities and most of the effects 
of the Gac/Rsm pathway on QS can be attributed to increased C4-HSL levels $(389,391)$. QteE (PA2593), though not a transcriptional regulator, controls the QS threshold by lowering LasR protein stability without affecting las $R$ transcription or translation, and by reducing RhlR levels (392). The stringent response also affects the QS regulon. Under starvation conditions, RelA (PA0934) enhances production of guanosine tetraphosphate (ppGpp) (393). Overexpression of RelA, also caused by decreased membrane fluidity (394), resulted in premature expression of $r p o S$, lasR and $r h l R$ and premature synthesis of 3-oxo-C12-HSL and C4-HSL (395). Both expression and stringent response of expression of 3-oxo-C12-HSL is induced by a serine animo acid analogue (395).

Although there have been many advances, much remains unknown in our understanding of QS regulation in P. aeruginosa. Recent studies by Chugani and Greenberg showed that a $l a s R, r h l R$, and $q s c R$ triple mutant retained the ability to regulate 37 genes, including those in the kynurenine pathway, in response to acylhomoserine lactones (396). The kynurenine pathway serves as the source of anthranilate for the synthesis of the PQS $(396,397)$.

\section{Conclusions and perspectives}

Computational analyses and mathematical models have greatly advanced our understanding of regulatory networks $(215,398,399)$, some of which have been confirmed by empirical data. Our enhanced understanding of gene regulation in bacteria has come a far way from transcriptional regulators alone, as traditionally thought, to include posttranscriptional and post-translational control, including regulatory RNAs, RNA-binding proteins, sigma factors, DNA supercoiling, proteolysis and others. In P. aeruginosa, as with numerous other bacteria, a majority of the transcriptional regulators and open 
reading frames have yet to be characterized in terms of gene function and mode of regulation, which in itself undermines our efforts in predicting gene networks. Nevertheless, such models provide us with insight into potential interactions and give us a framework to design experiments to elucidate complex networks, and further reinforce empirical data. In addition, they also help identify paralogs and orthologs between genera. The wealth of data that we now possess from numerous $P$. aeruginosa whole genome transcriptome analyses [reviewed in $(28,400)$ ], though critical in our understanding of the system and in identifying potential therapeutic targets, further expose the gap in our knowledge in accurately determining links between signals recognized by the regulators and how they are transduced to their target genes. Analysis of regulator-mutant transcriptomes helps fill in the gap, but warrant further studies, especially in linking metabolic regulation with virulence. It is exciting to know that complementing data from traditional molecular biological experiments with data from high-throughput, whole genome analyses such as microarray, RNA deep sequencing, and network modeling will undoubtedly advance our understanding of how bacteria regulate gene expression, as individuals and as communities in response to environmental signals.

\section{Acknowledgements}

This work is partly supported by NIH SC1 GM083677 (K.M.) and Florida International University Teaching Assistantship (D.B.). I thank Lisa Schneper and Giri Narasimhan for critical reading of the manuscript and Camilo Valdes for assistance with the PAO1 core genome analysis. 


\section{CHAPTER 4}

The regulatory repertoire of Pseudomonas aeruginosa AmpC B-lactamase regulator AmpR includes virulence genes

This chapter has been published:

Balasubramanian D, L. Schneper, M. Merighi, R. Smith, G. Narasimhan, S. Lory, K. Mathee. 2012. PLoS ONE. 7(3): e34067

DOI: $10.1371 /$ journal.pone. 0034067 


\begin{abstract}
In Enterobacteriaceae, the transcriptional regulator $A m p R$, a member of the LysR family, regulates the expression of a chromosomal $\beta$-lactamase AmpC. The regulatory repertoire of AmpR is broader in Pseudomonas aeruginosa, an opportunistic pathogen responsible for numerous acute and chronic infections including cystic fibrosis. In addition to regulating $\operatorname{amp} C, P$. aeruginosa $\mathrm{AmpR}$ regulates the sigma factor $\mathrm{AlgT} / \mathrm{U}$ and production of some quorum sensing (QS)-regulated virulence factors. In order to better understand the $a m p R$ regulon, we compared the transcriptional profile generated using DNA microarrays of the prototypic $P$. aeruginosa PAO1 strain with its isogenic ampR deletion mutant, PAO $\triangle a m p R$. Transcriptome analysis demonstrates that the AmpR regulon is much more extensive than previously thought, with the deletion of $a m p R$ influencing the differential expression of over 500 genes. In addition to regulating resistance to $\beta$-lactam antibiotics via $\mathrm{AmpC}$, AmpR also regulates non- $\beta$ lactam antibiotic resistance by modulating the MexEF-OprN efflux pump. Other virulence mechanisms including biofilm formation and QS-regulated acute virulence factors are AmpR-regulated. Real-time PCR and phenotypic assays confirmed the microarray data. Further, using a Caenorhabditis elegans model, we demonstrate that a functional AmpR is required for $P$. aeruginosa pathogenicity. AmpR, a member of the core genome, also regulates genes in the regions of genome plasticity that are acquired by horizontal gene transfer. Further, we show differential regulation of other transcriptional regulators and sigma factors by AmpR, accounting for the extensive AmpR regulon. The data demonstrates that AmpR functions as a global regulator in $P$. aeruginosa and is a positive regulator of acute virulence while negatively regulating biofilm formation, a
\end{abstract}


chronic infection phenotype. Unraveling this complex regulatory circuit will provide a better understanding of the bacterial response to antibiotics and how the organism coordinately regulates a myriad of virulence factors in response to antibiotic exposure. 


\section{Introduction}

Pseudomonas aeruginosa is one of the leading opportunistic Gram-negative nosocomial pathogens. This is particularly true in critically ill patients, where multi-drug resistant $P$. aeruginosa is a severe problem. It is the leading pathogen in ventilatorassociated pneumonia with a mortality rate of $40-60 \%(401)$. P. aeruginosa is also a primary cause of urinary tract infections in the US and Europe (402), wound infections leading to bacteremia with one-third to two-thirds mortality rate $(403,404)$, pulmonary infections including cystic fibrosis (CF) (405), lung cancer patients (19) and in pediatric and adult AIDS patients (22). Inability to eradicate the infection is partly due to intrinsic and acquired antibiotic resistance of $P$. aeruginosa. Antibiotic resistant isolates of $P$. aeruginosa are selectively favored in vivo in CF patients $(85,86)$. Resistance of $P$. aeruginosa to the $\beta$-lactam class of antibiotics, currently used to treat $P$. aeruginosa infections, is partly mediated by a group of genes belonging to the amp system.

The amp genes were first discovered in Enterobacter cloacae to confer resistance to $\beta$-lactams (93) and later in other members of Enterobacteriaceae (94-97). The products of amp genes in E. cloacae and other organisms include the AmpC $\beta$-lactamase, the AmpG permease, a putative AmpE permease, the AmpD cytoplasmic amidase, and the transcriptional regulator AmpR (93-97). Recent studies have identified another permease, AmpP that is required for $\beta$-lactamase induction in $P$. aeruginosa (406). Expression of $a m p C$ is regulated by AmpR. The $a m p R$ gene is located adjacent to ampC and is divergently transcribed in $C$. freundii and E. cloacae, as well as in P. aeruginosa (98-100). AmpR of C. freundii and E. cloacae can cross-complement each other (101) and $P$. aeruginosa AmpR is similar to that found in C. freundii (58\%) and E. cloacae 
(62\%) (102). In C. freundii, AmpR binds to a 15 bp sequence 5' TCTGCTGCAAATTT 3' $(98,102)$ and there is a nearly identical putative AmpR binding site (5', TCTGCTCCAAATTT 3') in the ampR-ampC intergenic region in P. aeruginosa (105). AmpR has a helix-turn-helix motif that is typical of DNA-binding proteins and the $C$. freundii AmpR binds DNA using this motif (98). The AmpR-AmpC system is also conserved in many other pathogens including Burkholderia cenocepacia (407), Yersinia enterocolitica (408), and Stenotrophomonas maltophilia (409).

AmpR belongs to the LysR family of transcriptional regulators that typically autorepress their own expression (103) which has been demonstrated in C. freundii (98). In $P$. aeruginosa, however, there is no evidence of autoregulation (105). It has been postulated that the signals mediating ampC regulation by AmpR are peptidoglycan degradation products that function as effector molecules and are brought into the cell cytoplasm from their point of origin in the periplasm via the AmpG permease (79). In vitro studies have demonstrated that $C$. freundii AmpR can both activate and repress ampC expression depending on its interaction with specific peptidoglycan degradation products (410). Thus the levels of these cell wall intermediates dictate AmpR regulation of $a m p C$ and the known regulatory repertoire of $\mathrm{AmpR}$ in Enterobacteriaceae have been limited to regulating ampC expression $(79,95,410)$. Previous studies comparing the properties of $P$. aeruginosa $\mathrm{PAO}$ with its isogenic ampR insertion mutant, PAOampR::aacCl, have shown that AmpR regulates $\operatorname{ampC}$ as well as some quorum sensing (QS) genes (105). This led us to hypothesize that the regulatory role of $P$. aeruginosa AmpR is more extensive than previously thought. 
To test the hypothesis that AmpR regulates different pathways in P. aeruginosa and to identify the AmpR regulon, we compared the expression profile of wild-type $\mathrm{PAO} 1$ and that of an in-frame $a m p R$ deletion mutant, $\mathrm{PAO} \triangle a m p R$, with and without subMIC $\beta$-lactam stress. Our data suggests that $P$. aeruginosa AmpR is a master regulator affecting the expression of over 500 genes. Functional analyses demonstrate the negative regulatory role of AmpR of multiple virulence mechanisms including biofilm formation and the MexEF-OprN multidrug efflux pump. Further, we demonstrate that AmpR positively regulates multiple acute virulence factors. Using a C. elegans model, we demonstrate that AmpR is required for pathogenesis in P. aeruginosa. This study establishes the critical regulatory role that AmpR plays in antibiotic resistance, virulence and general metabolism in P. aeruginosa.

\section{Results}

\section{Deletion of ampR reduces $\beta$-lactam resistance of PAO1}

In contrast to previous studies that looked at the role of $P$. aeruginosa AmpR using an insertion mutant, this study employed PAO $\triangle a m p R$, an in-frame deletion mutant in the prototypic $P$. aeruginosa PAO1. The presence of the $a m p R$ deletion was confirmed by PCR and restriction digestion of the amplicons (data not shown). AmpR is a known positive regulator of the chromosomal AmpC $\beta$-lactamase in different bacterial species $(96,102,411)$. Consequently, after constructing PAO $\triangle a m p R$, the strains were tested for altered production of $\beta$-lactamase. The resistance profile of $\beta$-lactam antibiotics for $\mathrm{PAO}$, PAO $\triangle a m p R$ and $\mathrm{PAO} \triangle a m p R$ (pAmpR) shows that, as expected, loss of $a m p R$ enhances strain sensitivity to $\beta$-lactams and expressing ampR in trans on a low-copy plasmid can restore this defect with multiple $\beta$-lactam antibiotics (Fig 4.1a). Loss of 
$a m p R$ seems to have a stronger effect on penicillins (ampicillin, amoxicillin and piperacillin, with and without $\beta$-lactamase inhibitors), imipenem and tazobactam than the cephalosporins tested. This finding is interesting because $\mathrm{AmpC}$ is a cephalosporinase. Overexpression of $a m p C$ under $\mathrm{P}_{\text {tac }}$ control, however, results in enhanced resistance to the cephalosporin ceftazidime (D. Zincke, personal communication). $\beta$-Lactamase quantification showed that $\mathrm{PAO} \triangle a m p R$ produced significantly lower amounts in response to $\beta$-lactam stress compared to PAO1 (PAO1: $11.27 \mathrm{mU}$ vs. PAO $\Delta a m p R$ : $6.5 \mathrm{mU}, p$ value 0.0003 ; Fig $4.1 \mathrm{~b}$ ), which is in agreement with the E-test data. The loss of induction was recovered by expressing ampR from a low-copy plasmid (PAO $\triangle a m p R$ : $6.5 \mathrm{mU}$ vs. PAO $\triangle a m p R(\mathrm{pAmpR}): 11.35 \mathrm{mU}, p$ value 0.004 ; Fig $4.1 \mathrm{~b}$ ). These data clearly reiterate the role of AmpR in $\beta$-lactam resistance in $P$. aeruginosa as previously described (105). The PAO $\triangle a m p R$ strain was used for all further assays. 
A

\begin{tabular}{|c|c|c|c|c|}
\hline & & \multicolumn{3}{|c|}{$\mathrm{MIC}(\mu \mathrm{g} / \mathrm{ml})^{*}$} \\
\hline Class & Antibiotic & $\begin{array}{c}\text { PAO1 } \\
\left.\text { (vector }^{\S}\right)\end{array}$ & $\begin{array}{c}\text { PAO } \triangle a m p R \\
\left(\text { vector }^{\S}\right)\end{array}$ & $\begin{array}{c}\text { PAO } \Delta a m p R \\
\text { (pAmpR) }\end{array}$ \\
\hline \multirow{7}{*}{ Penicillins } & Ampicillin & $>256$ & 12 & $>256$ \\
\hline & Ampicillin/sulbactam & 96 & 6 & 64 \\
\hline & Amoxicillin & $>256$ & 12 & $>256$ \\
\hline & Amoxicillin/clavulanic acid & $>256$ & 6 & $>256$ \\
\hline & Oxacillin & $>256$ & $>256$ & $>256$ \\
\hline & Piperacillin & 3 & 1.5 & 3 \\
\hline & Piperacillin/tazobactam & 2 & 1 & 1.5 \\
\hline \multirow{3}{*}{ Cephalosporins } & Cefaclor & $>256$ & $>256$ & $>256$ \\
\hline & Ceftazidime & 1.5 & 1 & 1 \\
\hline & Cefepime & 2 & 1 & 1 \\
\hline \multirow{2}{*}{ Penems } & Imipenem & 2 & 0.38 & 1 \\
\hline & Meropenem & 1 & 1.5 & 1.5 \\
\hline Monobactam & Aztreonam & 3 & 0.75 & 2 \\
\hline
\end{tabular}

* MIC was determined by the E-test, as described in the methods section

${ }^{\S}$ Vector-pMMB67EH-Gm

B

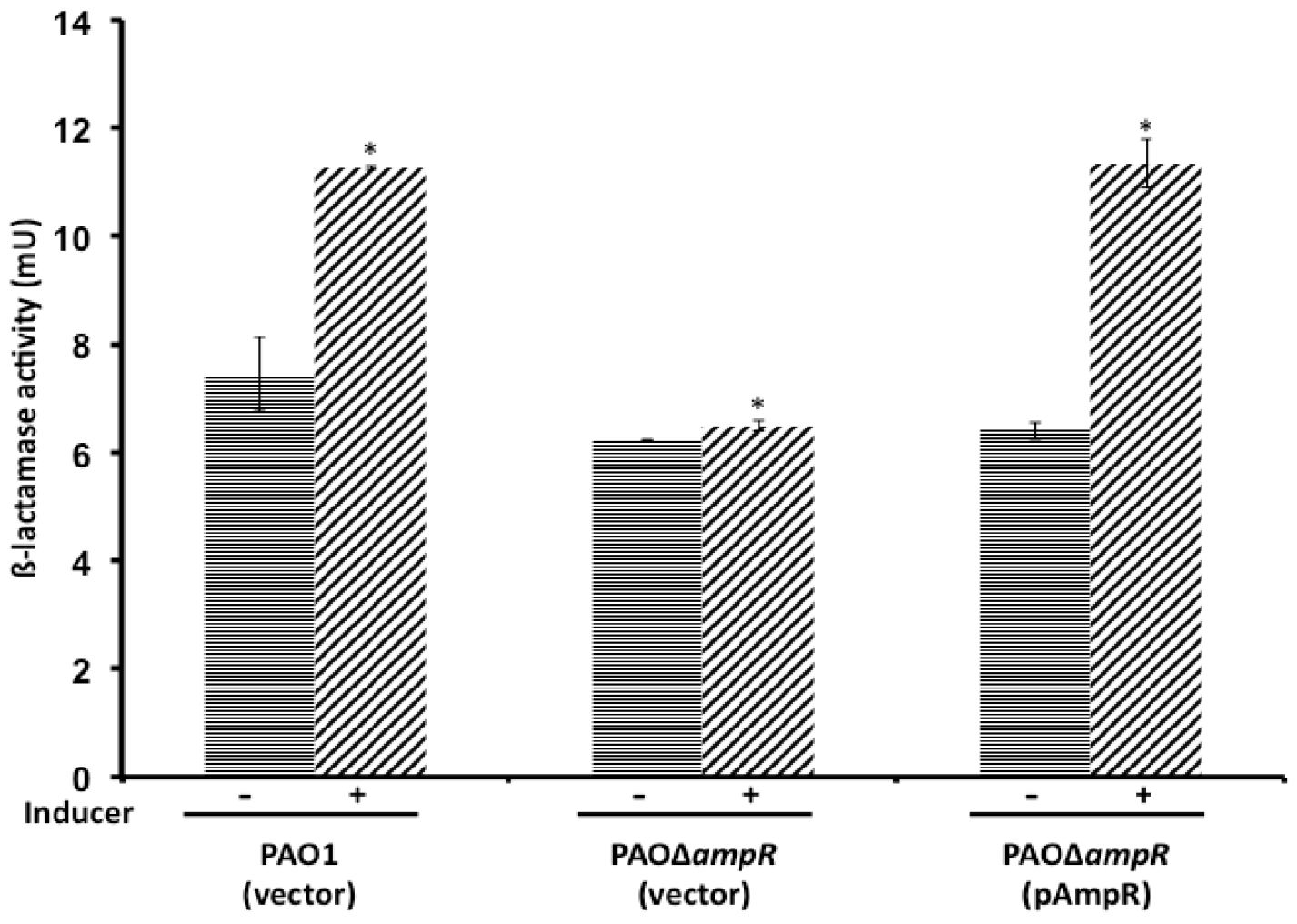


Figure 4.1. Antibiotic resistance profile of PAO $\triangle a m p R$. A clean in-frame deletion of ampR was generated in $P$. aeruginosa PAO1 as described in the methods section to generate PAO $\triangle a m p R$. Fig 4.1a shows the resistance profile of the strains to the four major classes of $\beta$-lactam antibiotics. Representative data from three different biological replicate trials are shown. The amount of $\beta$-lactamase produced was quantified (Fig 4.1b) in the presence (+) and absence (-) of sub-MIC concentration of a $\beta$-lactam inducer.

\section{Loss of ampR affects ability of PAO1 to kill C. elegans}

The importance of $\operatorname{ampR}$ in virulence was determined in a $C$. elegans model, as reported previously $(412,413)$. Using the fast killing (paralytic) assay, we monitored the ability of PAO1 and its isogenic ampR mutant, PAO $\triangle a m p R$ to kill C. elegans over eight hours. PAO $\triangle a m p R$ showed reduced pathogenicity, killing only $15 \%$ of the nematodes compared to the $38 \%$ killed by the wild-type PAO1 at the end of the study period ( $p$ value $<0.05$ at all time points; Fig 4.2). The results indicate that a functional AmpR is required for full pathogenicity of $P$. aeruginosa in the nematode model. To characterize the full extent of AmpR-mediated regulation of $P$. aeruginosa pathogenesis, we analyzed PAO $\triangle a m p R$ further. 


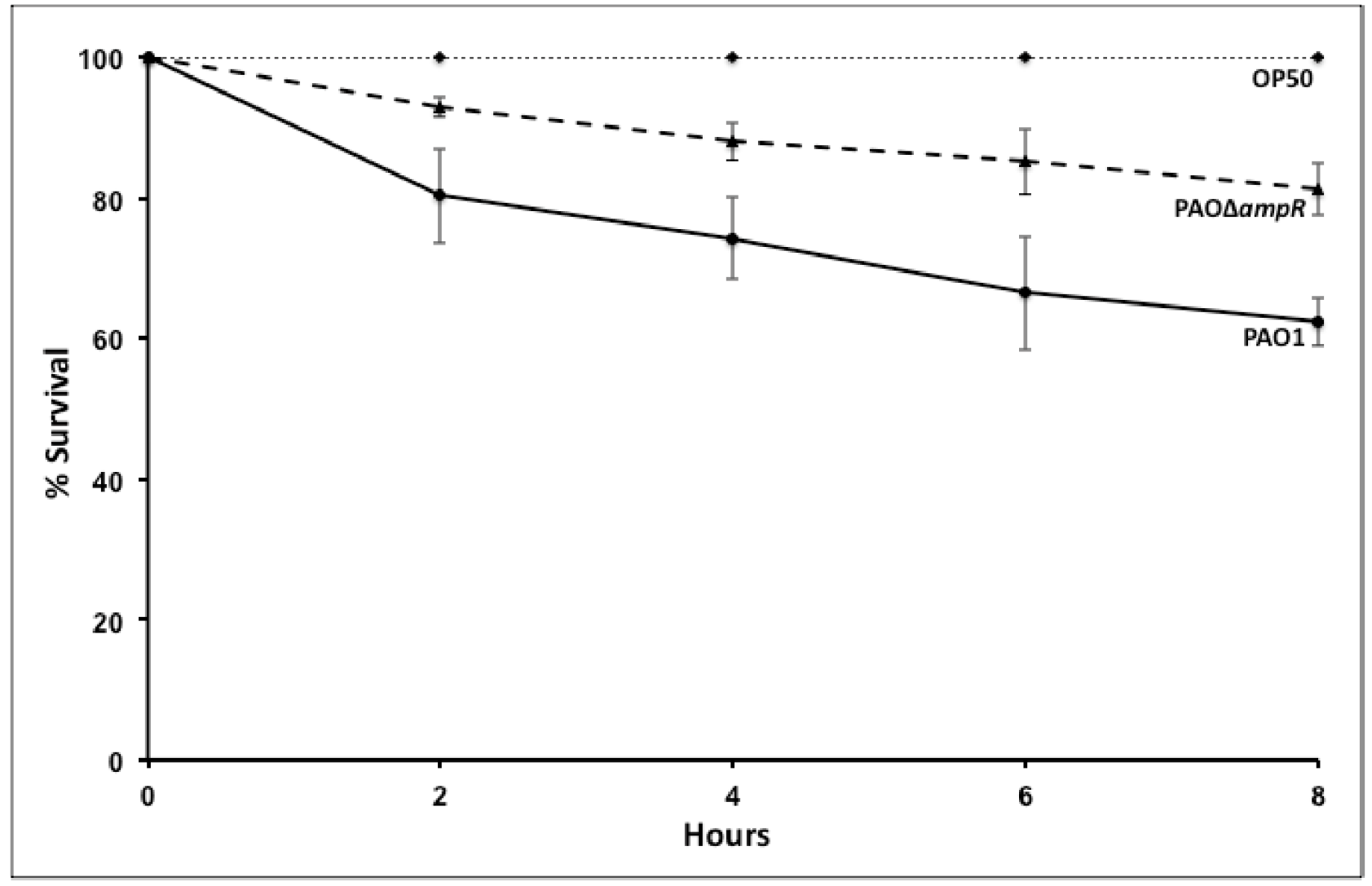

Figure 4.2. Effect of ampR deletion on pathogenicity to C. elegans. The fast killing assay was used to test the effect of loss of ampR on the $C$. elegans killing ability of PAO1. $p$-value $<0.05$ at all time points. 


\section{AmpR regulates numerous genes in $P$. aeruginosa}

Using DNA microarrays, we compared the expression profiles of PAO1 and PAO $\triangle a m p R$, without (uninduced) and with (induced) sub-MIC $\beta$-lactam stress to identify genes that are regulated under the different conditions. Pair-wise comparisons of the datasets of significantly differentially regulated genes ( $p$ value $\leq 0.01, \geq$ two-fold) either with or without sub-MIC $\beta$-lactam stress led to the identification of 32 genes (PAO1 uninduced vs. PAO1 induced; Condition A), 258 genes (PAO $\triangle a m p R$ uninduced vs. PAO $\triangle a m p R$ induced; Condition B), 345 genes (PAO1 uninduced vs. PAO $\triangle a m p R$ uninduced; Condition C) and 338 genes (PAO1 induced vs. PAO $\triangle a m p R$ induced; Condition D) (Fig 4.3). As seen in Fig 4.3, the expression of 345 genes is altered in the absence of AmpR (Condition C), clearly indicating that AmpR influences the expression of numerous genes in $P$. aeruginosa.

Quantitative real-time PCR (qPCR) was used to verify the microarray results. Genes for the verification analysis were selected across the spectrum of regulation, based on the raw microarray reads after normalization, including both up and downregulated genes. Twelve genes were selected for initial qPCR analysis, six each from the up and downregulated sets, using $\operatorname{clp} X(P A 1802)$ as the reference control gene since $\operatorname{clp} X$ expression did not change in our microarray data between the strains and conditions tested. qPCR data showed the same trend of either up- or downregulation of the genes as in the microarray, validating our microarray observations, notwithstanding the variations expected due to differences in the sensitivity of the two assays (Table 4.1). 

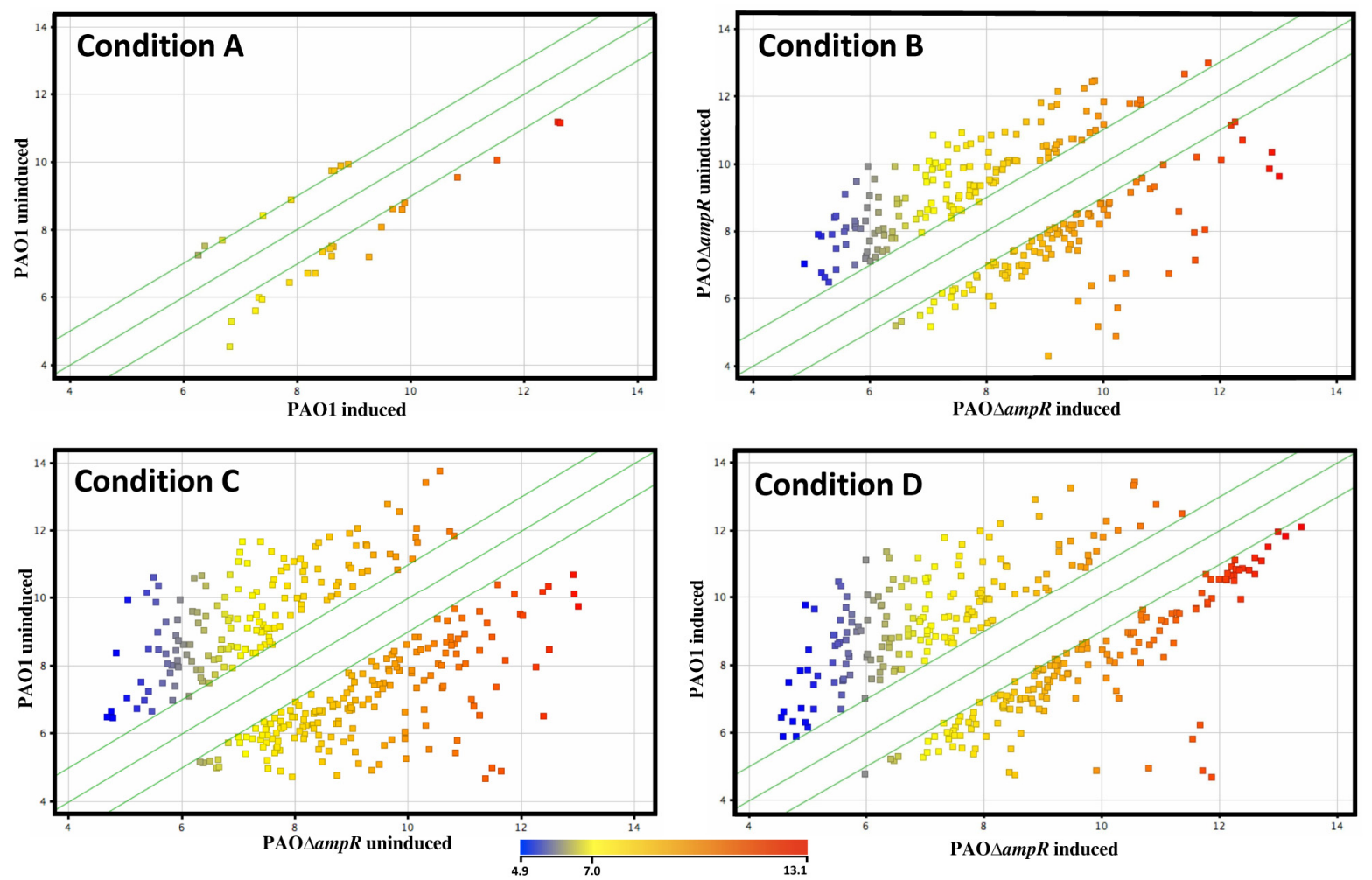

Figure 4.3. Scatter plots of significantly regulated genes. Only genes that showed significant $(p \leq 0.01)$ differential regulation under the various conditions are depicted as colored squares. The colors represent the extent of gene expression from low (blue) to high (red) in either condition, as depicted in the color scale. The two outer green diagonal lines in each plot represent the two-fold cutoff. Each sub-plot depicts the differential gene expression between two strains/conditions (shown along the plot axes): Condition A- PAO1 uninduced vs. PAO1 induced; Condition B- PAO $\triangle a m p R$ uninduced vs. PAO $\triangle a m p R$ induced; Condition C- PAO1 uninduced vs. PAO $\triangle a m p R$ uninduced; Condition D- PAO1 induced vs. PAO $\triangle a m p R$ induced. 
Table 4.1: Microarray vs. qPCR. Confirmation of microarray data was performed by qPCR, using RNA isolated from cells as explained in the text. Data shown is gene expression in penicillin non-treated PAO $\triangle a m p R$ cells, normalized to expression in PAO1.

\begin{tabular}{|c|c|c|}
\hline \multirow{2}{*}{ Locus ID/ Gene } & \multicolumn{2}{|c|}{ Fold change } \\
\cline { 2 - 3 } & Microarray & qPCR \\
\hline PA0610/ prtN & 12 & 6 \\
\hline PA0762/ algU & 5 & 3 \\
\hline PA3602 & 5 & 10 \\
\hline PA2493/mexE & 108 & 8089 \\
\hline PA4121 & 7 & 2 \\
\hline PA1708/ popB & -8 & -4 \\
\hline PA2193/ hcnA & -7 & -3 \\
\hline PA1078/ flgC & -5 & -2 \\
\hline PA2069 & -38 & -93 \\
\hline PA2331 & -7 & -40 \\
\hline
\end{tabular}




\section{AmpR regulates genes both in the absence and presence of $\beta$-lactam stress}

Subsets of genes that are differentially regulated either due to loss of $a m p R$ or due to $\beta$-lactam antibiotic exposure or both (Fig 4.3) could potentially be regulated under more than one condition and this overlap would be misinterpreted in the total number of genes regulated in each condition. To address this issue, the 973 differentially regulated genes $(p \leq 0.01, \mathrm{FC} \geq 2$-fold) in the four pair-wise comparisons (Conditions A-D in Fig 4.3) were plotted in 4-way Venn diagrams and separated into upregulated (Fig 4.4a) and downregulated (Fig 4.4b) genes.

From these two Venn diagrams, genes that were dependent exclusively on either AmpR or $\beta$-lactams or on both were identified. Comparison of Conditions A and B yield genes unique to each condition (U1, D1 and U2, D2 respectively in Fig 4.4a-4.4b). There are 18 upregulated genes (U1 in Fig 4.4a) that are unique to Condition A (response of PAO1 to $\beta$-lactam stress). This includes AmpR-dependent genes (there is a functional AmpR in these strains that helps in the response to $\beta$-lactams) and AmpR-independent genes (some of these genes may be regulated in an AmpR-independent manner). In contrast, in Condition B (response of PAO $\triangle a m p R$ to $\beta$-lactam stress), 61 genes are upregulated in response to antibiotics (U2 in Fig 4.4a). AmpR may negatively regulate these genes, since their expression is enhanced in the absence of AmpR, or their expression is AmpR-independent. The observation that loss of ampR leads altered gene expression in PAO1 in response to sub-MIC $\beta$-lactam stress (similar to that seen in the absence of $\beta$-lactam stress) further hints at a global regulatory role for AmpR in $P$. aeruginosa. 
A

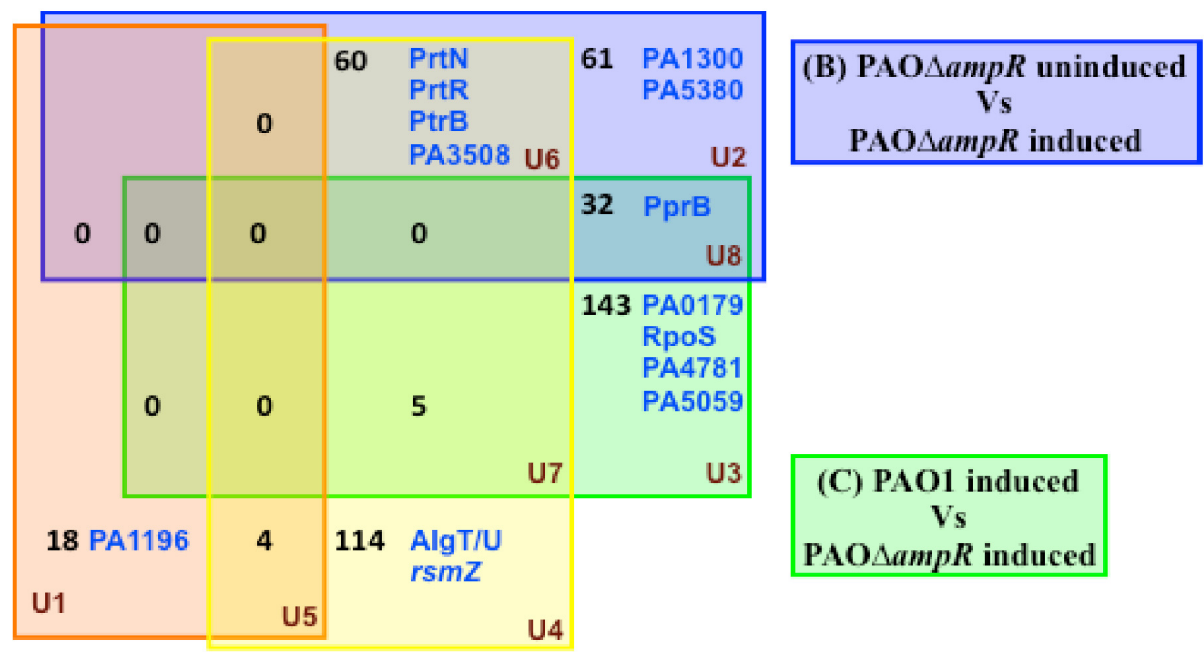

(A) PAO1 uninduced

vs

(D) PAO1 uninduced

Vs

PAO1 induced

PAO $\triangle a m p R$ uninduced

B

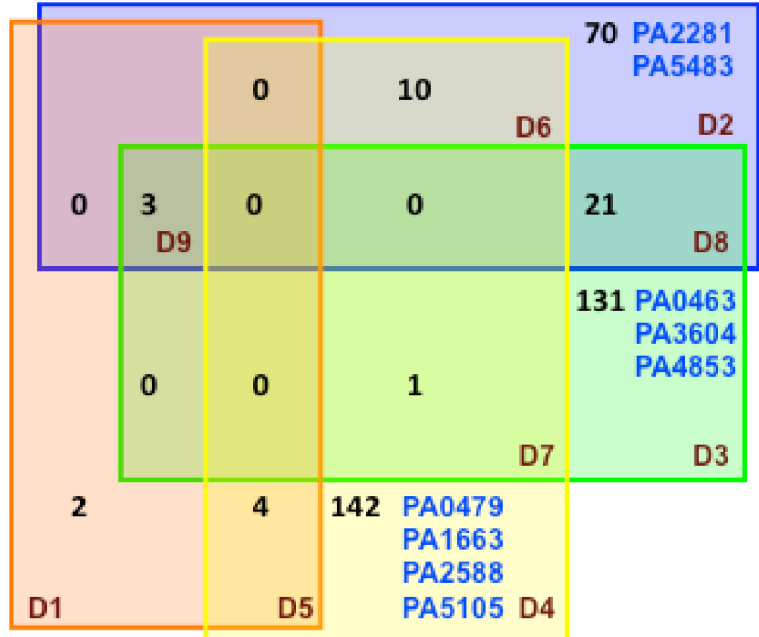
(B) PAO $\triangle a m p R$ uninduced Vs
PAOMampR induced

(C) PAO1 induced

Vs

PAOAampR induced

\section{(D) PAO1 uninduced \\ Vs \\ PAOAampR uninduced}

Figure 4.4. Venn diagram of the differentially regulated genes. Distribution of significantly $(p \leq 0.01)$ differentially regulated ( $>2$-fold) genes in PAO1 and PAO $\triangle a m p R$ with (induced) and without (uninduced) $\beta$-lactam treatment showing upregulated (Fig 4.4a) and downregulated (Fig 4.4b) genes. The transcriptional regulators, sigma factors and small RNAs in each group are identified either by their gene names or PA numbers. Annotations are from the Pseudomonas Genome database (332). 
Of the 18 upregulated genes in U1 (PAO1 induced), there could also be a subset of genes that are AmpR- and $\beta$-lactam dependent due to a functional AmpR in PAO1. In order to identify and separate exclusively $\beta$-lactam regulated genes, we compared these 18 upregulated genes to see if they were downregulated in Condition B (in D2) and identified PA0465 (creD), PA0466 (hypothetical) and PA3889 (hypothetical). So, of the 18 genes in U1, only these three genes are specifically AmpR-regulated. Using a similar logic (for key, see (Appendix; Supplementary information for Chapter 4, Table S1), we identified $\beta$-lactam dependent genes (Appendix; Supplementary information for Chapter 4, Tables S2A, S2B) to fall in regions U6, D6, U7, D7, U8 and D8 in addition to U1 and D1 of the Venn diagram (Fig 4.4a, 4.4b). The genes that were regulated exclusively in response to $\beta$-lactam antibiotics include $m r c B$, a penicillin-binding protein 1 (PBP1) that is involved in peptidoglycan synthesis and is upregulated 3.5-fold (corrected $p$-value 3.71E-03). Genes of putative RND efflux and $\mathrm{ABC}$ transporters are also upregulated (Appendix; Supplementary information for Chapter 4, Table S2A), as are genes involved in flagellar biosynthesis and the sox operon (PA5416-PA5419; involved in amino acid metabolism). Genes that are downregulated in response to $\beta$-lactam exposure include 10 membrane proteins suggesting adaptation to stress, in addition to genes of the $p h z 2$ phenazine biosynthetic operon.

The genes that are AmpR-dependent (U2, D2, U4 and D4: 387 genes) and AmpR$\beta$-lactam dependent (U3, D3, U5 and D5: 282 genes) (Fig 4a, 4b) are of interest to us, since AmpR influences both these gene sets. To further eliminate $\beta$-lactam specific genes, we compared the AmpR-dependent and AmpR- $\beta$-lactam dependent gene lists between themselves as well as to the $206 \beta$-lactam-specific genes (Appendix; Supplementary 
information for Chapter 4, Table S2A). This led to the identification of 520 genes whose expression is influenced by AmpR, of which 313 are AmpR-dependent (Appendix; Supplementary information for Chapter 4, Table S3A, Table S3B) and a further 207 that are AmpR- $\beta$-lactam dependent (Appendix; Supplementary information for Chapter 4, Table S4A, Table S4B).

\section{Functional categorization of AmpR-regulated genes}

Functional categorization of the 520 AmpR-regulated genes was then performed and compared to the distribution in the $P$. aeruginosa PAO1 genome from the Pseudomonas Genome database (332) to look for enrichment of specific classes of genes in the different conditions (Fig 4.5). Category $\mathrm{N}$ genes (secreted factors, toxins, enzymes, alginate) were found to be downregulated in an AmpR-dependent manner and this correlated with decreased production of some secreted virulence factors (elaborated in the following sections). Similarly, genes in category G (cell wall, LPS, capsule) were downregulated, indicating that AmpR positively regulates these genes. Moreover, metabolic genes (amino acid biosynthesis - category E; central intermediary metabolism category Q) show marginal downregulation in the $\operatorname{ampR}$ mutant. Genes involved in carbon compound catabolism (category S), adaptation and protection (category K), and those related to phage/transposon/plasmid (category V) were upregulated in the PAO $\triangle a m p R$, indicating negative AmpR regulation in an AmpR-dependent manner (Fig 4.5). Interestingly, genes encoding transcriptional regulators (category L) show differential regulation indicating that $A m p R$ could potentially regulate other genes indirectly via intermediate transcriptional regulators. 


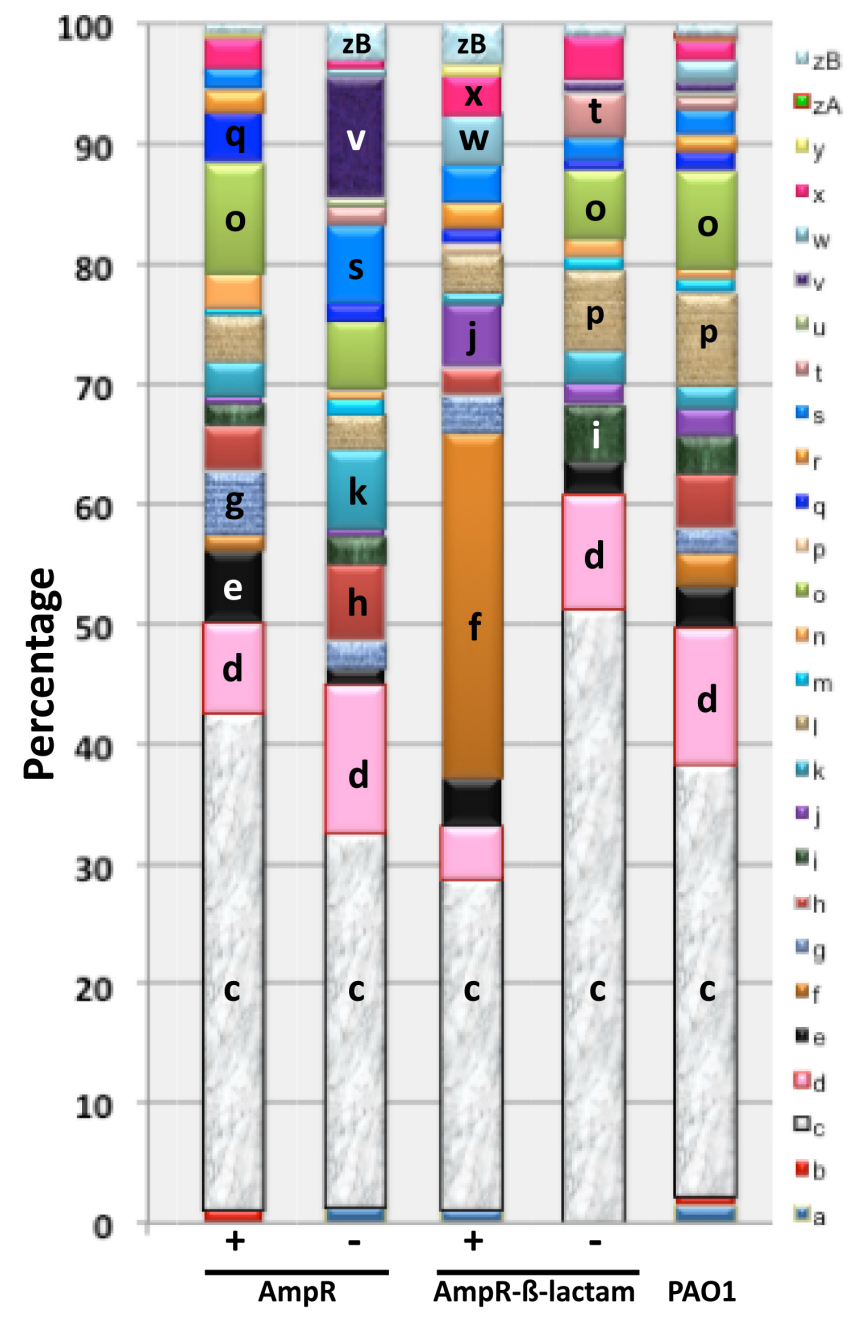

Code Category (\% of genes in PAO1)

zB Transcription, RNA processing and degradation (1)

zA Antibiotic resistance and susceptibility (0.2)

$y \quad$ Cell division (0.4)

$\mathrm{x}$ Protein secretion/export apparatus (2)

w Non-coding RNA genes (2)

v Related to phage, transposon, or plasmid (1)

4 Chemotaxis (0.4)

t Motility \& Attachment (1)

s Carbon compound catabolism (2)

$r \quad$ Nucleotide biosynthesis and metabolism (1)

q Central intermediary metabolism (1)

p Chaperones \& heat shock proteins $(0.3)$

o Putative enzymes (8)

n Secreted Factors- toxins, enzymes, alginate (1)

m Two-component regulatory systems (1)

I Transcriptional regulators (8)

k Adaptation, Protection (2)

j Biosynthesis of cofactors, prosthetic groups and carriers (2)

Energy metabolism (3)

h Transport of small molecules (5)

g Cell wall / LPS / capsule (2)

f Translation, post-translational modification, degradation (3)

e Amino acid biosynthesis and metabolism (4)

d Membrane proteins (11)

c Hypothetical, unclassified, unknown (36)

b Fatty acid and phospholipid metabolism (1)

a DNA replication, recombination, modification and repair (1)

AmpR

AmpR-ß-lactam 
Figure 4.5. Enrichment of functional categories. Functional categorization of the AmpR-dependent and AmpR- $\beta$-lactam dependent genes was performed to identify enrichment of specific classes of genes relative to their distribution in PAO1. '+' and '-' refers to upregulation and downregulation of genes, respectively in either an AmpR-dependent or AmpR- $\beta$-lactam-dependent manner. The most differentially regulated categories are labeled in the figure using the corresponding code, mentioned in the figure table. Functional categories, and their codes and percentages in PAO1 are from the Pseudomonas Genome database (332).

In the presence of $\beta$-lactam stress, loss of $a m p R$ leads to downregulation of membrane proteins (category D), putative enzymes (category O) and those involved in translation (category F), while motility and attachment genes (category $\mathrm{T}$ ) were upregulated (Fig 4.5). In addition to membrane proteins (category D), the most differential regulation across all conditions was of the hypothetical proteins (category C), but until the functions of these proteins are determined, no significant conclusions can be drawn.

\section{AmpR binding site analysis}

In an attempt to identify the genes that are directly regulated by AmpR, a bioinformatics approach was adopted. IEM and RSA analyses were performed to identify, refine and scan the $P$. aeruginosa genomes as well as the AmpR-regulated genes from the microarray data for potential AmpR-binding sites. The consensus sequences derived from the AmpR- and AmpR- $\beta$-lactam dependent genes are similar, yet distinct (Fig 4.6 A, C). Using these consensus sequences to search the upstream regulatory regions of the PAO1 genome led to the identification of the AmpR-dependent element in the upstream region of 244 genes and the AmpR- $\beta$-lactam-dependent element in the upstream region of 207 genes. The motifs derived from the IEM and RSA analysis for both the AmpR-dependent and AmpR- $\beta$-lactam-dependent genes are almost identical (Fig 6). Of the genes identified in the microarray analysis, only $11.9 \%$ of AmpR-dependent and $14.5 \%$ of AmpR- $\beta$ - 
lactam-dependent upstream regulatory regions were identified by IEM or RSA analysis as having an AmpR binding site. This suggests that perhaps AmpR is exerting its effect by either directly or indirectly altering expression of a global transcription regulator. Studies aimed at identifying the direct targets of AmpR are needed to identify genes that are directly regulated by AmpR.

\section{Regulation of the amp genes by AmpR}

$\beta$-Lactam resistance in P. aeruginosa is mediated, in part, by the amp genes that are also tied in with the cell wall recycling process (414). The genes involved in this process include the regulator AmpR $(105,415)$, the chromosomal $\beta$-lactamase AmpC (95,96,411,416), the permeases AmpG and AmpP $(406,417)$ and the amidases AmpD, AmpDh2 and AmpDh3 (73,418). In addition, the hydrolase NagZ also plays a role in $\beta$ lactam resistance $(419,420)$. Since $A m p R$ is known to positively regulate $\mathrm{AmpC}$ expression, we hypothesized that AmpR also regulates the other amp genes. qPCR analysis revealed downregulation of the amp genes in PAO $\triangle a m p R$, normalized to expression in PAO1 (Fig 4.7), indicating a positive regulatory role for AmpR in the expression of $\operatorname{amp} G$ (RQ - uninduced: $0.59 \pm 0.01, p$-value 0.006 ; induced: $0.63 \pm 0.01$, $p$-value 0.04$), \operatorname{ampP}$ (RQ - uninduced: $0.1 \pm 0.01, p$-value 0.004 ; induced- $0.86 \pm 0.01, p$ value NS) , $a m p D$ (RQ - uninduced: $0.82 \pm 0.01, p$-value NS; induced $0.67 \pm 0.01, p$ value 0.04$)$, ampDh2 (RQ - uninduced: $1.01 \pm 0.05, p$-value NS; induced- $0.56 \pm 0.01, p$ value 0.002 ), $a m p D h 3$ (RQ - uninduced: $0.98 \pm 0.007, p$-value NS; induced: $0.66 \pm 0.04$, $p$-value 0.02 ) and nagZ (RQ - uninduced: $0.27 \pm 0.06, p$-value 0.002 ; induced: $0.51 \pm$ $0.01, p$-value 0.0002$)$. 

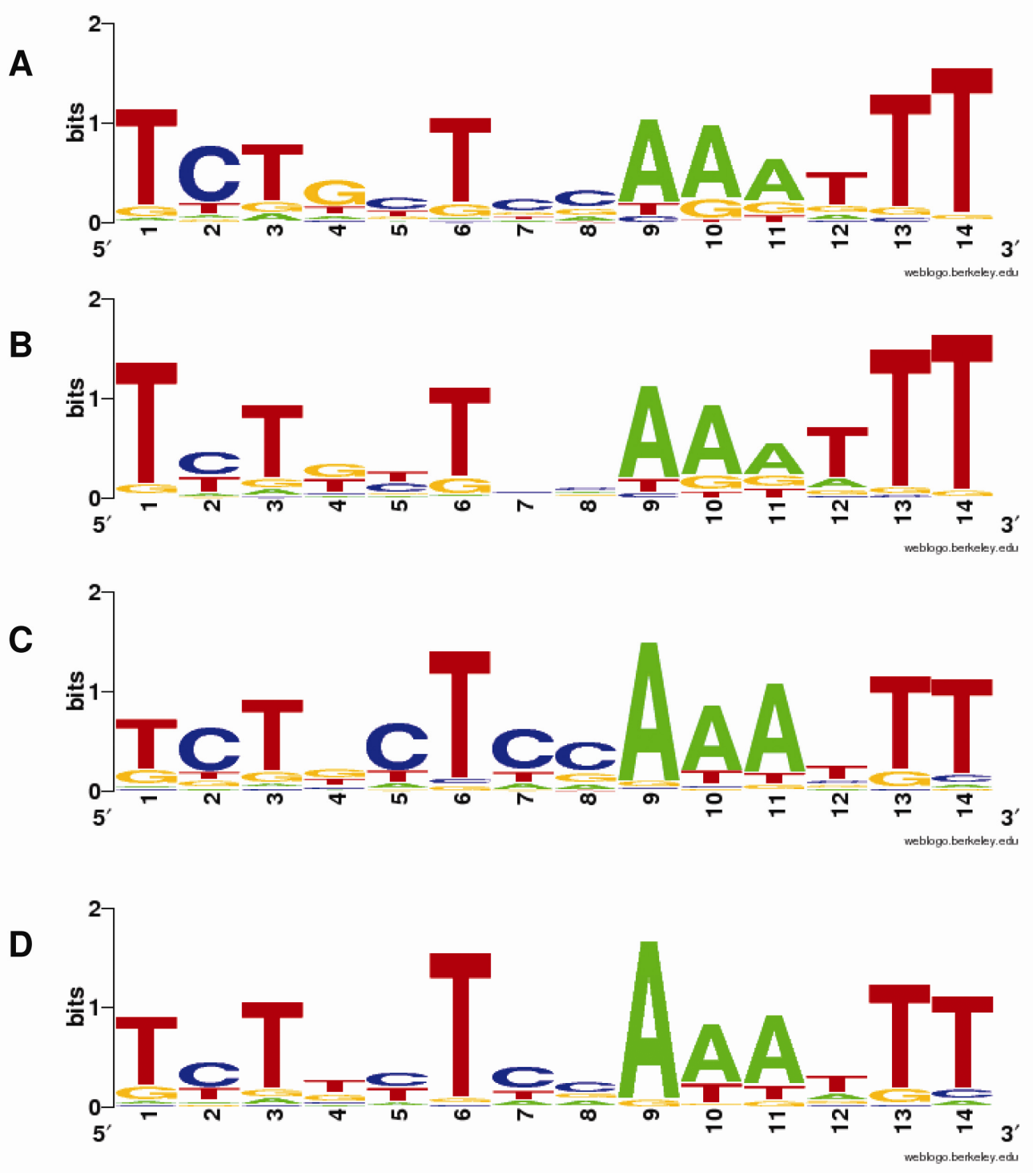

Figure 4.6: AmpR binding site analysis. The putative AmpR binding site was refined using IEM algorithm and used to search the upstream promoter elements of AmpR-regulated and AmpR- $\beta$-lactam regulated genes (listed in Appendix, Supplementary information for Chapter 4, Tables S3, S4) in PAO1 and other $P$. aeruginosa strains. DOOR database $(421,422)$ was used to identify the operons. The RSAT tool (423) was used to identify PAO1 promoter sequences containing the identified AmpR- and AmpR- $\beta$ lactam-regulated genes. The output is represented using WebLogo (424) for the AmpR-dependent genes from IEM (A) and RSA (B), and AmpR- $\beta$-lactam dependent genes from IEM (C) and RSA (D) 


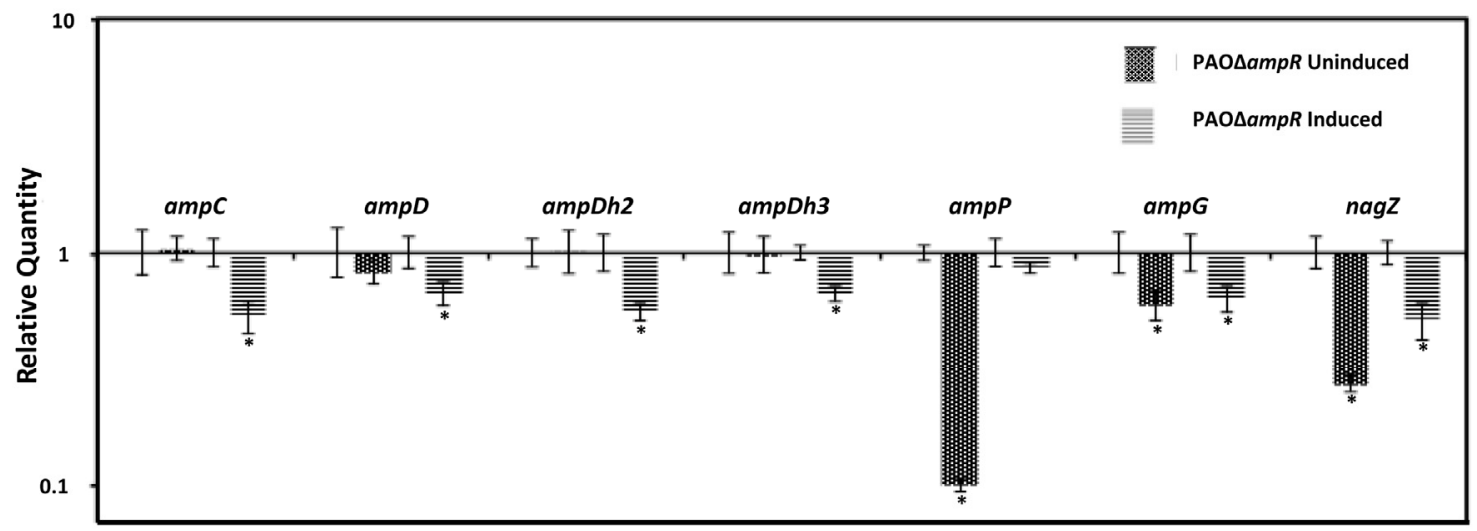

Figure 4.7. Expression of the amp genes in PAO $\triangle a m p R$. RNA was isolated from cells exposed to $\beta$ lactam antibiotic, reverse transcribed to cDNA and tested in triplicate by qPCR with gene-specific primers, as described in the text. The expression of $\operatorname{ampC}$ ( $\beta$-lactamase), ampD, ampDh2, and ampDh3 (amidases), amp $G$ and $\operatorname{amp} P$ (permeases), and $n a g Z$ (glycoside hydrolase) were tested in the ampR mutant relative to PAO1. Values have been normalized to expression in PAO1 under the same conditions $\left(\log _{10} R Q=1\right)$ and bars above or below the line represent up- and down-regulation, respectively. ${ }^{*} p$ value $<0.05$

Specifically, when the cells are exposed to $\beta$-lactams, they need amidase activity to help in the peptidoglycan recycling process. This is reflected in upregulation of the amidases, (AmpD, AmpDh2, and AmpDh3) and AmpC only when the cells are exposed to $\beta$-lactams. Simultaneously, AmpG, which functions to transport the degraded peptidoglycan material into the cytoplasm (406) is upregulated by AmpR (downregulated in $\mathrm{PAO} \triangle a m p R$ ) in an inducer-independent manner. This shows that AmpR upregulates the amidases and AmpC $\beta$-lactamase in response to $\beta$-lactams while upregulating AmpG (Fig 4.7), and agrees with the proposed model for peptidoglycan recycling in $P$. aeruginosa (406). The data, thus, demonstrates the central role of AmpR in influencing expression of the cell wall recycling/AmpC-mediated $\beta$-lactam resistance machinery in $P$. aeruginosa. 


\section{AmpR regulates the expression of antibiotic resistance and virulence systems}

It has previously been shown that AmpR regulates the expression of genes related to QS and protease production (425). Microarray analyses from this study show that AmpR affects the expression of multiple virulence systems in $P$. aeruginosa, as explained further in this section.

\section{Resistance-Nodulation-Division (RND) efflux systems}

RND transporters are tripartite pumps present in Gram-negative bacteria that are involved in the efflux of antibiotics and several other compounds decreasing cytoplasmic retention and thus conferring resistance (426). All of the $P$. aeruginosa strains sequenced so far carry 12 known and putative RND efflux pumps, suggesting that the efflux pumps are an integral part of the $P$. aeruginosa genome $(25,33)$. The MexEF-OprN efflux system that is primarily concerned with resistance to fluoroquinolones, chloramphenicol and trimethoprim (427) was significantly upregulated in PAO $\triangle a m p R$. The genes mexE (seven-fold), mexF (89-fold) and $\operatorname{opr} N$ (103-fold) are overexpressed in the ampR mutant in the absence of antibiotic stress in microarray studies (Appendix; Supplementary information for Chapter 4, Table S3A) and overexpression of the first gene of the operon, mexE, was confirmed by qPCR (Table 4.1). MexT, an activator of this efflux system $(427,428)$, is not significantly differentially regulated (1-fold) in the microarray studies, but is upregulated in an inducer-independent manner when tested by qPCR (RQ: uninduced $7.5 \pm 0.11, p$-value 0.02 ; induced $7.0 \pm 0.25, p$-value 0.02$)$. However, the negative regulator MexS shows no differential regulation either in microarray or $\mathrm{qPCR}$ analysis (data not shown). Carbapenems use the outer membrane porin OprD to gain 
entry into the cell (429) and MexT negatively regulates this porin both at the transcriptional and post-transcriptional level $(79,430,431)$. Indeed, qPCR analysis shows that with upregulation of mexT, there is a downregulation of $\operatorname{opr} D$ expression in the $\operatorname{ampR}$ mutant compared to PAO1 (RQ: uninduced $0.31 \pm 0.004, p$-value 0.001 ; induced $0.08 \pm 0.000002, p$-value $<0.0001)$. Surprisingly, OprD downregulation did not lead to increased resistance to imipenem and meropenem (Fig 1a). In addition, we did not see differential regulation of the other known MexT regulator, MvaT (432) in our microarray analysis.

To further investigate whether upregulation of this pump translates into a resistance phenotype, we determined the MICs for MexEF-OprN substrate antibiotics by the standard broth microdilution method (433). PAO $\triangle a m p R$ showed enhanced resistance to four of the antibiotics tested when compared to the resistance profile of wild-type PAO1, correlating microarray and qPCR data with the phenotype (Table 4.2). This suggests that AmpR is involved in resistance to $\beta$-lactam antibiotics by regulating AmpC as shown earlier, and non- $\beta$-lactam antibiotics via the MexEF-OprN efflux pump. Analysis of the promoter regions of the genes of this pump and their regulators (MexS and MexT) using the putative AmpR-binding site (434) as a query sequence did not reveal signs of AmpR binding, suggesting indirect regulation by AmpR.

MexAB-OprM was the first RND-type efflux pump to be reported in $P$. aeruginosa and has very broad substrate specificity including $\beta$-lactam antibiotics and non-antibiotic substrates (435). In fact, it has been implicated to play a more significant role in resistance to $\beta$-lactam antibiotics than $\beta$-lactamases $(58,436)$. Using the putative $P$. aeruginosa AmpR binding site (434), we identified a potential AmpR binding site 
Table 4.2: ampR deletion affects susceptibility to MexEF-OprN substrates. The minimum inhibitory concentrations (in $\mu \mathrm{g} / \mathrm{ml}$ ) of MexEF-OprN substrate antibiotics to PAO1 and PAO $\triangle a m p R$ were determined by broth microdilution method (see text for details).

\begin{tabular}{lcc}
\hline Antibiotic & \multicolumn{2}{c}{ MIC $(\boldsymbol{\mu g} / \mathrm{ml})$} \\
\hline & PAO1 & PAO $\triangle$ ampR \\
\cline { 2 - 3 } Ofloxacin & 4 & 32 \\
Ciprofloxacin & 0.25 & 2 \\
Chloramphenicol & 128 & 512 \\
Trimethoprim & 200 & $>200$ \\
\hline
\end{tabular}

upstream of the MexR repressor of this pump in the MexR-MexA intergenic region (5' AAGCCTGCAAATGT 3') indicating possible regulation of this pump by AmpR. qPCR analysis of mexR expression revealed downregulation of this gene in PAO $\triangle a m p R$ compared to PAO1 (RQ: uninduced $0.46 \pm 0.006, p$-value 0.002 ; induced $0.4 \pm 0.007, p$ value 0.01 ). It is thus interesting to note that $\mathrm{AmpR}$ not only positively regulates $\mathrm{AmpC}$ $\beta$-lactamase but potentially also MexAB-OprM, two different mechanisms of resistance to $\beta$-lactams. The MexAB-OprM efflux can also pump out fluoroquinolones (79) and the enhanced quinolone resistance of PAO $\triangle a m p R$ seen in the MIC studies is potentially due to a combined effect of upregulation of the MexEF-OprN and the MexAB-OprM efflux pumps. In addition, AmpR negatively regulates a two-gene putative RND efflux operon PA1435-PA1436 (nine-fold and four-fold respectively in microarray) that codes for a 
membrane fusion protein and efflux transporter, respectively. Potential AmpR regulation of the MexGHI-OpmD efflux pump is discussed in the QS section.

\section{QS-regulated virulence factors}

Many of $P$. aeruginosa virulence factors are QS-regulated and form a critical component of pathogenesis $(208,437)$. In our previous meta-analysis of published $P$. aeruginosa transcriptomes, we identified differentially regulated sets of system-specific and condition-specific genes, including QS-regulated genes (400). Using this as our knowledge base, the $\operatorname{ampR}$ microarray profile was compared to identify differentially regulated QS-specific genes. The microarray data shows that AmpR influences expression of many QS-regulated genes (Appendix; Supplementary information for Chapter 4, Table S5).

To further verify AmpR-mediated regulation of QS virulence factors, we quantified the production of pyocyanin, LasA protease, and LasB elastase. Pyocyanin is a redox active exotoxin pigment that contributes to lung pathophysiology of chronic $P$. aeruginosa infections (438) and interferes with multiple host cellular functions (439). Genes in the locus of the phzl operon that is involved in QS-regulated phenazine synthesis, including phzAl (PA4210, 4-fold down), phzB1 (PA4211, 21-fold down), phzS (PA4217, 28-fold down), phzM (PA4209, 4-fold down), and the MexGHI-OpmD efflux pump (PA4205-PA4208, 11-30-fold down) that plays a role in pumping out the pigments (440), show decreased expression in PAO $\triangle a m p R$. In agreement with this data, there was a statistically significant $(p$-value $<0.0001)$ reduction in pyocyanin production by the ampR mutant, compared to PAO1 and this effect was independent of $\beta$-lactam stress on 
the cells (Table 4.3). The data indicates that AmpR influences pyocyanin production which is in agreement with the C. elegans killing data (Fig 4.2), since phenazines are major players in C. elegans mortality in the fast-killing assay (413).

Elastases (pseudolysins) are highly toxic zinc metalloproteases that play a critical role in immunomodulation $(441,442)$, host tissue damage aiding invasion (443) and biofilm formation (444). The LasB elastase production was also severely affected due to the loss of $\operatorname{ampR}(p$-value $\leq 0.02)$ in an inducer-independent manner (Table 4.3). Along with LasB, a zinc metalloendopeptidase, LasA plays a major role in P. aeruginosainduced keratitis (445). Reduction in LasA protease production, however, was significantly lower $(p$-value $<0.05)$ in PAO $\triangle a m p R$ only when the strains were exposed to sub-MIC $\beta$-lactam stress (Table 4.3), and this is in agreement with data from microarray (8-fold downregulated) and qPCR (RQ: uninduced- NS; induced $0.21 \pm 0.07$, $p$-value $<0.0001)$ analysis.

Microarray data shows that loss of ampR also affects other QS-regulated virulence genes, such as the $h c n$ operon PA2193-PA2195 (5- to 7-fold downregulated) that is involved in the production of hydrogen cyanide. Cyanide toxicity is the primary mode of fast killing of C. elegans by P. aeruginosa PAO1 (446). The downregulation of the $h c n$ operon concurs with reduced killing by PAO $\triangle a m p R$. 
Table 4.3: Effect of deletion of ampR on QS-regulated virulence phenotypes

\begin{tabular}{|c|c|c|c|c|c|c|}
\hline \multirow{2}{*}{ Strain } & \multicolumn{2}{|c|}{ Pyocyanin production } & \multicolumn{2}{c|}{ LasA activity $^{\text {b }}$} & \multicolumn{2}{c|}{ LasB activity $^{\text {c }}$} \\
\cline { 2 - 7 } & UI & IN $^{\mathrm{d}}$ & UI & IN $^{\mathrm{d}}$ & UI & IN $^{\mathrm{d}}$ \\
\hline PAO1 & $22.31 \pm 0.18$ & $24.30 \pm 1.10$ & $0.37 \pm 0.005$ & $0.39 \pm 0.004$ & $1.65 \pm 0.25$ & $1.54 \pm 0.15$ \\
\hline PAO $\triangle$ ampR & $2.29 \pm 0.11^{\mathrm{e}}$ & $5.74 \pm 0.56^{\mathrm{f}}$ & $0.26 \pm 0.002$ & $0.19 \pm 0.002^{\mathrm{g}}$ & $0.5 \pm 0.03^{\mathrm{h}}$ & $0.57 \pm 0.08^{\mathrm{i}}$ \\
\hline
\end{tabular}

${ }^{a}$ Pyocyanin concentrations were expressed as $\mu \mathrm{g}$ pyocyanin produced per $\mu \mathrm{g}$ protein.

${ }^{b}$ LasA activity was expressed as change in OD600 per hour per $\mu \mathrm{g}$ of protein.

${ }^{c}$ LasB elastase activity was expressed as change in OD495 per $\mu \mathrm{g}$ of protein.

${ }^{\mathrm{d}}$ Induction was carried out with $100 \mu \mathrm{g} / \mathrm{ml}$ penicillinG.

e, $p$-value $<0.0001$ when comparing PAO1 and PAO $\Delta a m p R$

g,h $p$-value $<0.02$ when comparing PAO1 and PAO $\triangle a m p R$

${ }^{\mathrm{i}} p$-value $<0.01$ when comparing PAO1 and $\mathrm{PAO} \Delta a m p R$ 
The expression of the galactophilic lectin lecA (PA2570) is also downregulated in PAO $\triangle a m p R$ (RQ: uninduced- $0.22 \pm 0.0005, p$-value 0.0001 ; induced: $0.16 \pm 0.003, p$ value 0.0005 ) indicating positive AmpR regulation. LecA facilitates bacterial entry into host cells by aiding in adhesion to endothelia and epithelia (447) and is involved in biofilm formation (448). It has previously been shown that lec $A$ expression is regulated by the sigma factor RpoS (449) and by the QS regulator RhlR (449). Thus, the effect of AmpR on lecA expression could be mediated indirectly via RpoS and/or RhlR. As predicted, RpoS was downregulated (RQ: uninduced- $0.55 \pm 0.08, p$-value 0.01 ; induced $0.51 \pm 0.02, p$-value 0.0003), indicating AmpR positive regulation in a $\beta$-lactamindependent manner. Also, since we see downregulation of multiple QS phenotypes, RhlR is also potentially involved in AmpR-mediated lecA regulation.

QS activates the operon PA2327-PA2331 (168) coding for a probable ABC transporter. Wolfgang et al. found this operon to be repressed when P. aeruginosa was grown in CF respiratory liquid containing media (450). Genes of the operon are significantly upregulated (6- to 18 -fold) in $\mathrm{PAO} \triangle a m p R$ indicating that AmpR negatively regulates this operon and further connects AmpR to QS regulation, adding another regulatory player in this complex regulatory network.

\section{Biofilms}

Successful biofilm formation is dependent on nutrient availability, motility and QS (451). Comparison of gene expression profiles from this study with the biofilmspecific gene list generated from our previous analysis of $P$. aeruginosa transcriptomes (400) revealed the differential regulation of many biofilm genes in PAO $\triangle a m p R$ 
(Appendix; Supplementary information for Chapter 4, Table S6). This suggests a role for AmpR in biofilm regulation, either directly or indirectly. Testing the tube biofilmforming ability of the strains revealed that PAO $\triangle a m p R$ formed better biofilms, compared to PAO1 (Fig 4.8). The difference was significant at all time points tested ( $p$-value $\leq$ 0.03 ) over a period of 72 hours indicating that $A m p R$ is a negative regulator of biofilm formation.

Microarray data also revealed upregulation of PA4651 (encoding a probable pili assembly protein; 11.6-fold), and PA4306 (coding for Flp, Type IVb pilin, 36-fold) in PAO $\triangle a m p R$, in the absence of antibiotics. Since type IV pili-mediated twitching and flagella-mediated swimming motilities are known proponents for biofilm formation at different stages (452), we tested for these phenotypes with PAO1 and PAO $\triangle a m p R$. In the absence of $\beta$-lactam antibiotic stress, the PAO $\triangle a m p R$ strain demonstrated enhanced twitching ability (PAO $\triangle a m p R: 15.1 \mathrm{~mm} \pm 1.1 \mathrm{~mm} ; \mathrm{PAO}: 2 \mathrm{~mm} \pm 0.1 \mathrm{~mm} ; p$ value $<$ 0.0001) potentially explaining its ability to form better biofilms. Moreover, unlike in PAO1, under sub-MIC $\beta$-lactam stress, PAO $\triangle a m p R$ showed a marginal but statistically significant increase in twitching zones (uninduced: $15.1 \mathrm{~mm} \pm 1.1 \mathrm{~mm}$; induced: 16.5 $\mathrm{mm} \pm 0.8 \mathrm{~mm} ; p$-value 0.04$)$. This observation is in agreement with the enrichment of the gene set for the 'motility and attachment' functional category under $\beta$-lactam stress in PAO $\triangle a m p R$ (see section above). There was, however, no difference in the swimming motility of the strains (data not shown), although fleR of the FleSR two-component system that is involved in the flagella biosynthesis regulatory pathway (180) was differentially regulated in the $a m p R$ mutant (RQ: uninduced- $0.45 \pm 0.1, p$-value 0.0007 ; induced- $0.32 \pm 0.01, p$-value 0.0006$)$. 


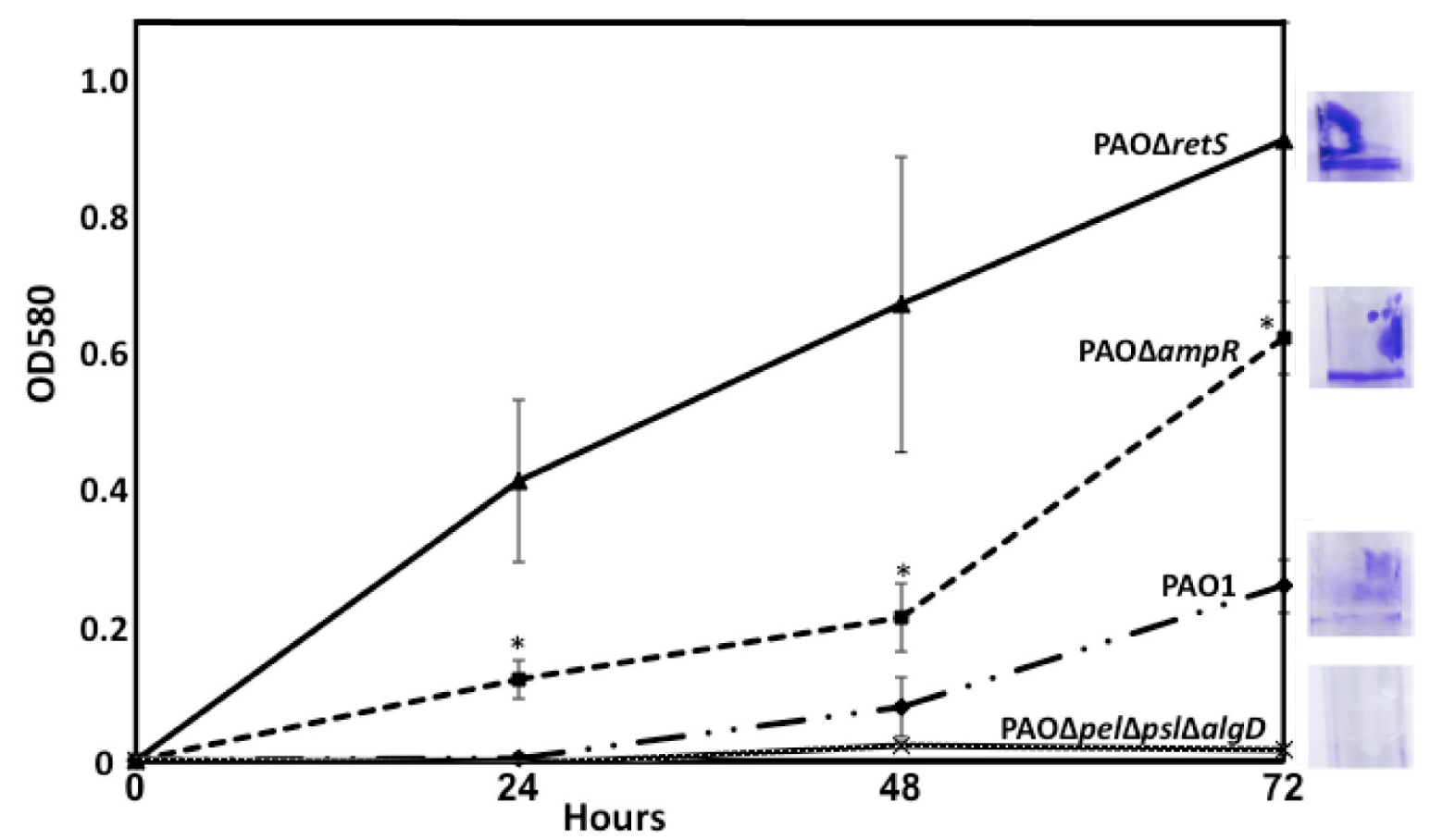

Figure 4.8. AmpR affects biofilm formation. AmpR regulates many genes involved in biofilm formation (Appendix, Supplementary information for Chapter 4, Table S6) that is reflected in the tube biofilm assay monitored for 72 hours. The strains tested were wild type PAO1, PAO $\triangle a m p R$, PAO $\Delta$ retS (positive control), and PAO $\Delta$ pel $\Delta$ psl $\Delta a l g D$ (negative control). The inset, taken at 72 hours, demonstrates the superior biofilm formation capacity of PAO $\triangle a m p R$ compared to PAO1. $p$-values comparing PAO $\Delta a m p R$ with PAO1: 24 hrs- 0.002; 48 hrs- 0.03; 72 hrs- 0.007. 
The Pel polysaccharide is a glucose-rich exopolymer, encoded by the pel operon (PA3058-PA3064) that along with the mannose-rich Psl polysaccharide plays a major role in pellicle formation $(453,454)$. PAO $\triangle a m p R$ forms darker red colonies on TryptoneCongo red agar plates compared to PAO1 (data not shown) signifying higher Pel production (453). This is consistent with the observation that the ampR mutant produces better biofilms. Collectively, these data suggest that AmpR negatively influences biofilm formation in P. aeruginosa either directly or indirectly.

Recently, a novel efflux pump that confers antibiotic resistance in $P$. aeruginosa biofilms has been identified (455). Deletion of the operon (PA1874-1877) encoding this pump in PAO1 enhances sensitivity to gentamycin, tobramycin and ciprofloxacin, especially in biofilm cells. Genes in this operon are downregulated four-fold in the $a m p R$ mutant strain. This observation, though not verified separately, suggests that AmpR positively regulates this operon, thus possibly contributing to non- $\beta$-lactam antibiotic resistance in biofilms.

\section{Secretory systems}

The type III secretion system (T3SS) is essential for P. aeruginosa not only for contact-dependent toxin delivery to host cells but also for phagocyte evasion $(206,456)$. Using a burn mouse model, it was shown that loss of T3SS results in reduced virulence (457). The genes encoding the regulatory and structural components of the T3SS in $P$. aeruginosa are concentrated in one locus (PA1690-PA1725) whereas the effectors and their chaperones are scattered in the genome (332). Deletion of ampR from PAO1 led to the downregulation of a few T3SS genes in the regulatory-structural gene cluster (Fig 
4.9) indicating positive AmpR-regulation. These include the genes encoding the regulators PcrH (involved in regulating ExoS synthesis) and ExsE (a secreted regulator of the ExsCED regulatory cascade), and the translocator proteins PopB and PopD. qPCR confirmation of the microarray findings for the T3SS genes was done using RNA isolated from $\mathrm{Ca}^{2+}$ depleted inducing media (MinS-NTA), since $\mathrm{Ca}^{2+}$ is a known inhibitor of T3SS (325). Since $p c r H, p o p B$ and $p o p D$ are the last three genes of a 12-gene operon, we tested expression of only $p c r H$ by qPCR, which showed a downregulation in $\mathrm{PAO} \triangle a m p R$ compared to PAO1 (RQ: uninduced- $0.71 \pm 0.06, p$-value 0.02 ; induced- $0.88 \pm 0.03, p$ value 0.003). Similarly, exsE, the second gene of the exsCEB operon was also downregulated in PAO $\triangle a m p R$ (RQ: uninduced- $0.57 \pm 0.01, p$-value 0.001 ; induced$0.69 \pm 0.18, p$-value NS) indicating positive regulation of these genes by AmpR.

Transcriptional regulation of T3SS in $P$. aeruginosa is a complex process and involves multiple tiers of regulation (325). One of the mechanisms of control involves a small RNA-binding protein RsmA and non-coding small RNAs (sRNA), rsm $Y$ and $r s m Z$. Sequestration of RsmA by the sRNAs inhibits its activity. RsmA has an extensive virulence regulon that is tied in with the GacSA two-component system regulatory cascade $(390,458)$. In PAO $\triangle a m p R, r s m Z$ is downregulated (RQ: uninduced- $0.21 \pm 0.03$, $p$-value 0.01 ; induced- $0.78 \pm 0.09, p$-value NS) and corresponds with an upregulation of RsmA (in microarray, uninduced - 2.3 fold; corrected $p$-value 0.009; induced- NS). Some of the T3SS genes are, however, downregulated in the $\operatorname{ampR}$ mutant (Fig 4.9). RsmA is a positive regulator of T3SS. This suggests that the effect of AmpR on T3SS is probably not via RsmA, or involves an additional step of post-transcriptional regulation. PtrB 


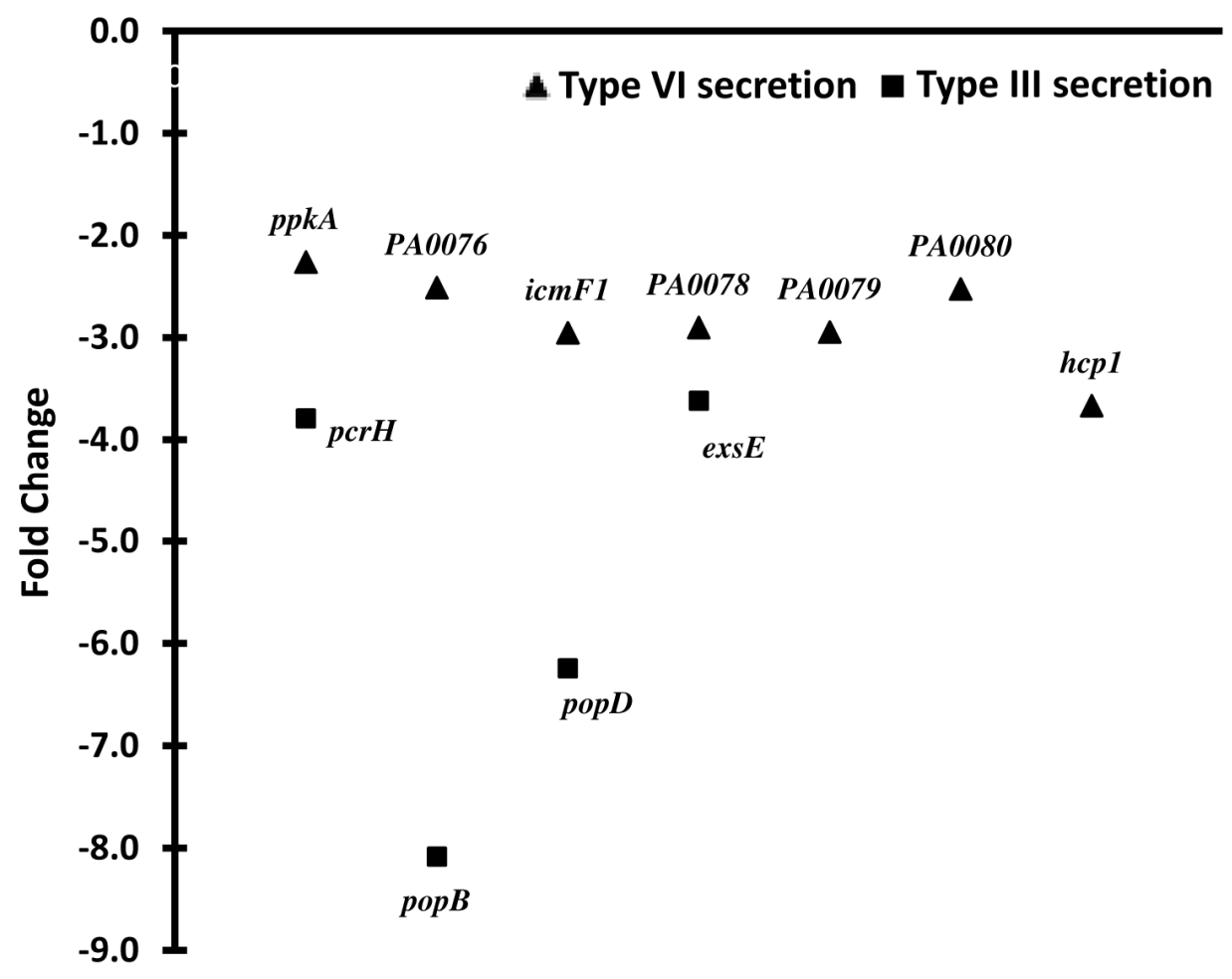

Figure 4.9. Regulation of secretion genes by AmpR. Fold changes (FC) as determined by microarray experiments in the absence of $\beta$-lactam stress of the Type VI secretion ( $\boldsymbol{\Delta}$, HSI-I) and Type III secretion (-) genes in the $\operatorname{ampR}$ mutant strain, normalized to expression in the wild-type strain PAO1. Gene annotations are from the Pseudomonas Genome database (332). 
represses T3SS gene expression (331) and since AmpR regulates PtrB expression (see section below), AmpR regulation of T3SS is potentially via PtrB.

P. aeruginosa T6SS is involved in chronic CF infections (459). RsmA also negatively regulates genes of the Type 6 secretion system (T6SS), particularly of the HSI-I system (458). Thus, downregulation of genes of the T6SS in PAOAampR (Fig 4.9) is possibly through an indirect effect of AmpR on RsmA.

\section{AmpR regulates genes found in regions of genome plasticity}

Comparative analysis of five $P$. aeruginosa chromosomes identified RGPs that are strain-specific (33). These are genome segments that can be acquired by horizontal gene transfer, bacteriophages or transposons (33). We wanted to determine whether an endogenous transcriptional regulator, such as AmpR, could regulate expression of genes acquired by the strain, such as RGP genes. Microarray analysis revealed that in the PAO $\triangle a m p R$ mutant, 31 RGP genes are regulated in an AmpR-dependent manner and an additional eight RGP genes under sub-MIC $\beta$-lactam stress (Appendix; Supplementary information for Chapter 4, Table S7).

Most of the RGP03 (PA0612-PA0628) and RGP04 (PA0641-PA0648) ORFs belong to functional category $\mathrm{V}$ (related to phage, transposon and plasmid) and code for the two classes of high molecular weight pyocins, types $\mathrm{R}$ and $\mathrm{F}$. Pyocins $\mathrm{R}$ and $\mathrm{F}$ are related to bacteriophage tails and kill susceptible cells thus conferring a survival advantage on the producing strain (460). Thirty-two percent of the RGP genes regulated in an AmpR-dependent manner are clustered and localized to RGP03. The expression of 10 RGP03 genes and two RGP04 genes (59\% and 20\% of genes in RGP03 and RGP04, 
respectively) are significantly upregulated (6-8-fold) in PAO $\triangle a m p R$ as seen in microarray data (Appendix; Supplementary information for Chapter 4, Table S7). This suggests that AmpR is involved in negatively regulating these genes, either directly or indirectly.

PrtN (PA0610), and the product of the first gene in RGP03, ptrB (PA0612), is a positive regulator of pyocin production, both of which are repressed by PrtR (PA0611) (330). Upon DNA damage, RecA (PA3617) degrades PrtR, thus inducing pyocin production (330). In PAO $\triangle a m p R$ subjected to sub-MIC $\beta$-lactam stress compared to PAO1, prtN (12-fold), recA (3-fold) and $\operatorname{ptrB}$ (20-fold) are upregulated, while the negative regulator PrtR is downregulated (qPCR relative quantity: uninduced- $0.88 \pm 0.4$, $p$-value $p$-value 0.004; induced- $0.76 \pm 0.109 p$-value 0.0002$)$ implying that AmpR is a negative regulator of pyocin production.

\section{AmpR regulates other transcriptional regulators}

The AmpR-regulation of hundreds of genes (Fig 4.4a, 4.4b) could be by direct binding to the promoters or in a subset of genes, indirectly through intermediate transcriptional regulators, sigma factors or regulatory RNAs. In a preliminary attempt to further elucidate the AmpR regulon, we looked at the transcriptional regulators and sigma factors that are AmpR-regulated. Of the 430 transcriptional regulators in $P$. aeruginosa PAO1 (332), 19 met the selection criteria in our microarray analysis (see materials and methods) and are over 2-fold significantly differentially regulated in the ampR mutant (Table 4.4). This suggests that AmpR regulates a proportion of the genes through intermediate transcriptional regulators. 
Table 4.4: Transcriptional regulators and sigma factors regulated by AmpR

\section{Transcriptional regulators}

\begin{tabular}{|c|c|c|c|c|}
\hline $\begin{array}{l}\text { Locus } \\
\text { Tag }\end{array}$ & $\begin{array}{l}\text { Gene } \\
\text { Name }\end{array}$ & Product Name & $\begin{array}{l}\text { Corrected } p \text { - } \\
\text { value }\end{array}$ & $\begin{array}{l}\text { Fold } \\
\text { change }\end{array}$ \\
\hline PA0463 & $\operatorname{creB}$ & $\begin{array}{l}\text { two-component response regulator } \\
\text { CreB }\end{array}$ & 8.39E-03 & -2.1 \\
\hline PA0479 & & probable transcriptional regulator & $9.90 \mathrm{E}-03$ & -2.1 \\
\hline PA0610 & $\operatorname{prt} N$ & transcriptional regulator PrtN & 3.81E-03 & 11.6 \\
\hline PA0611 & prtR & transcriptional regulator PrtR & $6.10 \mathrm{E}-03$ & -3.9 \\
\hline PA0612 & $\operatorname{ptr} B$ & repressor, PtrB & $3.44 \mathrm{E}-03$ & 19.5 \\
\hline PA1196 & & probable transcriptional regulator & $9.89 \mathrm{E}-03$ & 2.6 \\
\hline PA1663 & & probable transcriptional regulator & $5.13 \mathrm{E}-03$ & -3.9 \\
\hline PA1707 & pcrH & regulatory protein $\mathrm{PcrH}$ & $3.98 \mathrm{E}-03$ & -3.8 \\
\hline PA2281 & & probable transcriptional regulator & $9.89 \mathrm{E}-03$ & -2.1 \\
\hline PA2588 & & probable transcriptional regulator & $3.77 \mathrm{E}-03$ & -27.9 \\
\hline PA3508 & & probable transcriptional regulator & $1.60 \mathrm{E}-03$ & 3.5 \\
\hline PA3604 & & $\begin{array}{l}\text { probable two-component response } \\
\text { regulator }\end{array}$ & $6.96 \mathrm{E}-03$ & -2.2 \\
\hline PA4296 & $\operatorname{ppr} B$ & $\begin{array}{l}\text { two-component response regulator, } \\
\text { PprB }\end{array}$ & $6.01 \mathrm{E}-03$ & 7.4 \\
\hline PA4781 & & $\begin{array}{l}\text { probable two-component response } \\
\text { regulator }\end{array}$ & $9.10 \mathrm{E}-03$ & 2.8 \\
\hline PA4853 & fis & DNA-binding protein Fis & $6.65 \mathrm{E}-03$ & -3.3 \\
\hline PA5059 & & probable transcriptional regulator & $6.33 \mathrm{E}-03$ & 4.0 \\
\hline PA5105 & hutC & histidine utilization repressor & 8.27E-03 & -3.1 \\
\hline PA5380 & $g b d R$ & GbdR & $5.75 \mathrm{E}-03$ & 2.6 \\
\hline PA5483 & $\operatorname{alg} B$ & $\begin{array}{l}\text { two-component response regulator } \\
\text { AlgB }\end{array}$ & 7.83E-03 & 2.4 \\
\hline
\end{tabular}




\section{Sigma factors}

\begin{tabular}{|c|c|c|c|c|}
\hline Locus Tag & $\begin{array}{l}\text { Gene } \\
\text { Name }\end{array}$ & Product Name & $\begin{array}{l}\text { Corrected } p \text { - } \\
\text { value }\end{array}$ & $\begin{array}{c}\text { Fold } \\
\text { change }\end{array}$ \\
\hline PA0762 & $\operatorname{alg} T$ & sigma factor AlgU & $3.35 \mathrm{E}-03$ & 5.4 \\
\hline PA1300 & & $\begin{array}{l}\text { probable sigma- } 70 \text { factor, ECF } \\
\text { subfamily }\end{array}$ & $9.59 \mathrm{E}-03$ & 2.5 \\
\hline PA3622 & rpos & sigma factor RpoS & $6.65 \mathrm{E}-03$ & 2.2 \\
\hline
\end{tabular}

Three of the 24-known/putative sigma factors (461) are also over 2-fold differentially regulated, including RpoS and AlgT/U (Table 4.4). The stationary sigma factor, RpoS controls virulence in different bacteria including P. aeruginosa (462-464). Since $r p o S$ expression is upregulated in the stationary phase $(461,463)$ and the RNA used for microarray analysis was harvested two hours post- $\beta$-lactam induction (at OD 3.0), some of the phenotypic changes seen in PAO $\triangle a m p R$ is likely to be RpoS-mediated.

To investigate this possibility, RNA was harvested 40 minutes post-induction (OD600 of 1.0) and the expression of known RpoS-dependent and RpoS-independent genes was compared between PAO1 and PAO $\triangle a m p R$. As expected, RpoS expression was significantly higher at 2 hours than at 40 minutes in PAO $\triangle a m p R$ compared to PAO1 ( $p$ value: uninduced 0.0049, induced 0.0023; Fig 4.10), and this increase in RpoS expression corresponded with a growth phase-dependent increase in the expression of two of the RpoS-dependent genes, lecA ( $p$-value: uninduced 0.0061; induced 0.0043) and lecB ( $p$ value: uninduced NS; induced 0.0002) (Fig 4.10). However, expression of the MexEFOprN activator, MexT, which is regulated in an RpoS-independent manner, did not change at the different time points tested (Fig 4.10). This suggests that AmpR, via RpoS, 
regulates genes of the RpoS regulon in $P$. aeruginosa in a growth phase- and stressdependent manner, which is in agreement with previous studies $(463,464)$. Moreover, the MexT data suggests that harvesting the cells either after 40 minutes or 2 hours of $\beta$ lactam treatment does not affect AmpR-mediated gene expression for non-RpoSdependent genes (Fig 10).

The sigma factor $\mathrm{AlgT} / \mathrm{U}$ is a master regulator of alginate biosynthesis $(465,466)$ and we have shown previously with an $\operatorname{ampR}$ insertion mutant that there is crosstalk between AmpR and AlgT/U (425). Our microarray data shows upregulation of $A \lg T / \mathrm{U}$ in PAO $\triangle a m p R$ (5.4-fold, corrected $p$-value 3.35E-03), indicating negative AmpR regulation (Table 4.4) in agreement with previous findings (425). 


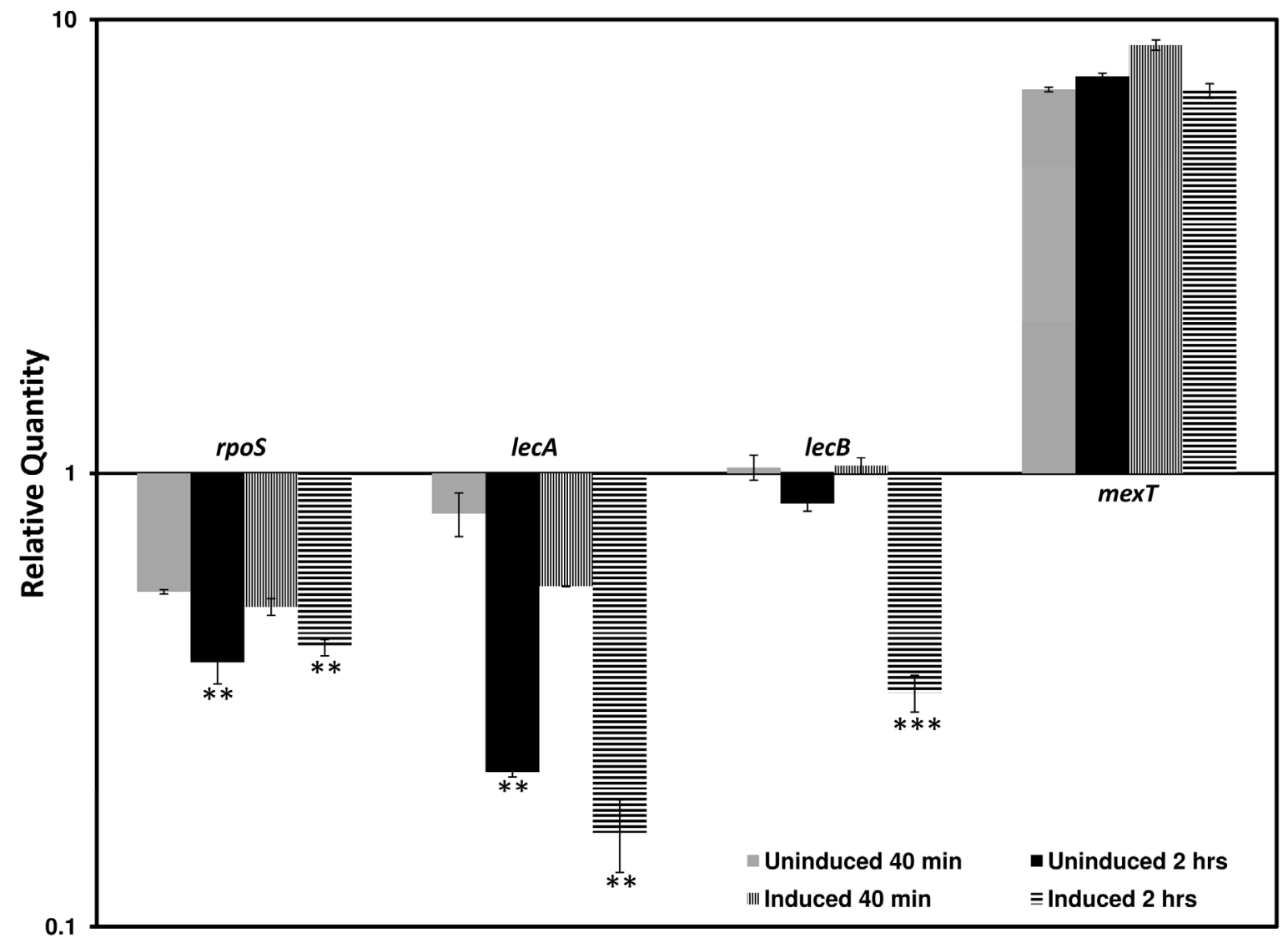

Figure 4.10. Gene expression in PAO $\triangle a m p R$ at 40 minutes and 2 hrs post- $\beta$-lactam exposure. RNA was isolated from PAO1 and PAO $\triangle a m p R$ cells exposed to $\beta$-lactams for either 40 minutes or 2 hours and reverse transcribed to cDNA. The expression of the sigma factor $r p o S$, lecA and lecB (galactophilic lectin genes known to be RpoS-regulated), and mexT (MexEF-OprN efflux pump regulator that is not RpoS-regulated) were tested by qPCR with gene-specific primers, as described in the text. Values have been normalized to expression in PAO1 under the respective conditions. ${ }^{* *} p$-value $<0.006$; *** $p$-value 0.0002 as determined by unpaired $t$ test. 


\section{Subtractive transcriptomics of the AmpR regulon}

Meta analysis of 18 condition-specific $P$. aeruginosa transcriptomes led to the identification of an expression core gene set of 303 genes that are significantly differentially regulated under all the different conditions analyzed and were proposed to be involved in maintaining cell homeostasis (400). Comparing the 520 genes whose regulation is AmpR-dependent to the core led to the identification of 57 genes (of the 520) that were part of the expression core genes (Fig 4.11). Further, we wanted to identify genes that are specifically under AmpR-regulation and those that are not involved in other pathways. To derive this list, we compared the 463 AmpR-dependent genes (520 minus 57 core genes) with the 1726 genes that are regulated in other conditions/by other regulators [1598 condition-specific genes (400), and 128 genes that are either RpoS (368) or AlgT/U-regulated (467)]. This comparison reduced the number of AmpR-dependent genes to 133 (from 313) and the AmpR- $\beta$-lactam-dependent genes to 86 (from 207) (Fig 4.11; Appendix; Supplementary information for Chapter 4, Table S8). 


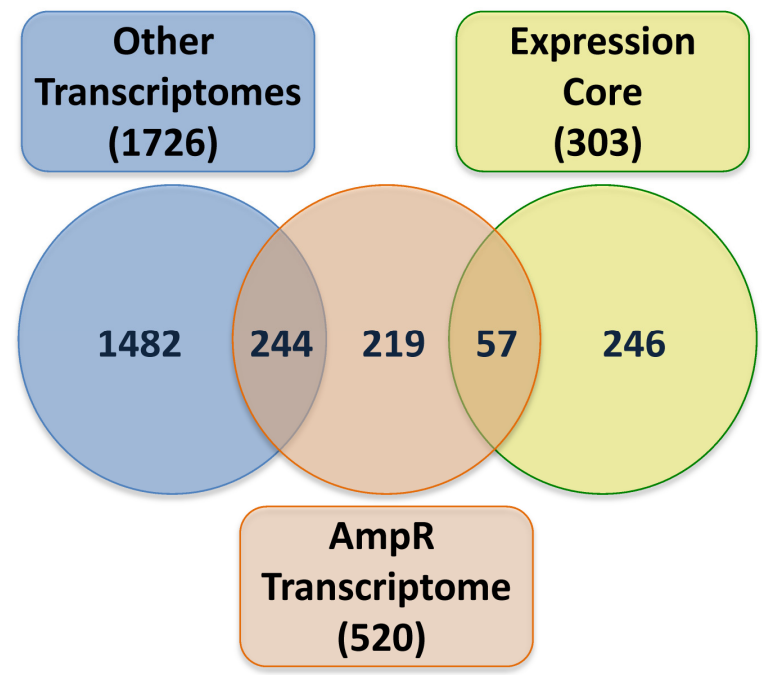

Figure 4.11. Comparison of the AmpR transcriptome with other transcriptomes. AmpR-dependent and AmpR- $\beta$-lactam-dependent genes were compared with the 303 genes of the expression core and the 1726 condition-specific genes identified previously as part of a meta-analysis of 18 P. aeruginosa transcriptomes (400).

The exclusively AmpR-dependent genes include the $\mathrm{O}$ antigen chain regulator $w z z$, the $p c a$ genes involved in carbon compound catabolism and $c r e B$, which codes for the response regulator of the CreBC TCS (Table S8). The CreBC TCS has been demonstrated to be involved in $\beta$-lactam resistance in Aeromonas spp. (468). CreBC positively regulates expression of an inner membrane protein CreD in E. coli (469) and in P. aeruginosa the CreBCD system contributes to $\beta$-lactam resistance only in a $\triangle \mathrm{PBP} 4$ background (348). Microarray analysis of PAO $\triangle a m p R$ in the presence $\beta$-lactam stress shows reduced creB expression (-2.1, corrected $p$-value 8.4E-03). Expression of creD, however, is significantly increased in PAO $\triangle a m p R$ compared to PAO1 under $\beta$-lactam stress as determined by qPCR (RQ: induced $11.04 \pm 0.0001, p$-value $<0.0001$ ). This is in agreement with a previous report that also showed $c r e D$ overexpression in cefoxitintreated PAO $\triangle a m p R$ cells (348). AmpR thus positively regulates $c r e B$ while negatively 
regulating $c r e D$ expression suggesting potential $A m p R$ regulation of $c r e D$ in a CreBindependent manner.

\section{Phenotypic microarray analysis of PAO $\triangle a m p R$}

Since loss of $\operatorname{ampR}$ led to dysregulation of over 500 genes as seen in DNA microarrays, we decided to characterize its metabolic effect using phenotypic microarrays. Biolog analysis was performed with PAO1 and PAO $\triangle a m p R$ in the absence of antibiotic stress. In all, seven phenotypes were gained and 47 phenotypes were lost (Appendix; Supplementary information for Chapter 4, Table S9). PAO $\triangle a m p R$ grew marginally better in media containing nutritional supplements including citrulline, histidine, shikimic acid, leucine, serine, spermidine and pyridoxal. This indicates that AmpR is a negative regulator of utilization of these agents. Of the 47 observed phenotypes that were lost in PAO $\triangle a m p R, 45$ belong to the sensitivity panel. Fourteen of the 45 observed phenotypes were associated with antibiotics, further supporting the role of AmpR as a major regulator of antibiotic resistance in $P$. aeruginosa. 


\section{Discussion}

P. aeruginosa AmpR, a LysR-type transcriptional regulator and a positive regulator of the chromosomal $\operatorname{amp} C \beta$-lactamase expression, has been shown previously to play a role in regulating a few QS-dependent phenotypes and the alginate master regulator, $\mathrm{AlgT} / \mathrm{U}(105,425)$. In this study, we determined the whole genome expression profiles of a clean in-frame deletion mutant of ampR in $P$. aeruginosa PAO1 under normal conditions and under sub-MIC $\beta$-lactam stress, using DNA microarrays. The results demonstrate that $A m p R$ influences the expression of 313 genes in the absence of $\beta$-lactam stress and an additional 207 genes when exposed to sub-MIC $\beta$-lactam stress. The AmpR regulon is thus much more extensive than previously thought including virulence, antibiotic-resistance and metabolic genes.

Multi-drug resistant $P$. aeruginosa isolates are a frequent occurrence in many acute and chronic infections (201). $\beta$-lactamases and efflux pumps are major mediators of antibiotic resistance in $P$. aeruginosa (79). We show that in addition to positively regulating the $a m p C$ and potentially the MexAB-OprM efflux pump by modulating expression of the MexR repressor, AmpR also mediates non- $\beta$-lactam resistance by negatively regulating the MexEF-OprN efflux pump. The PAO1 strain used for constructing PAO $\triangle a m p R$ was the strain used in the genome-sequencing project (25), which has an 8 bp insertion in the MexEF-OprN activator mexT leading to premature mexT termination (470) and consequent non-inducibility of the MexEF efflux pump. Strains with a $n f x C$ mutation, however, have different ways to overcome this, including secondary mutations and deletion of the $8 \mathrm{bp}$ insertion $(428,470)$, leading to activation of the MexEF-OprN efflux. This was also observed in $n f x C$ mutants isolated in a mouse 
model (471). However, there was no differential expression of the MexT activator in both these studies $(470,471)$. With PAO $\triangle a m p R$, even though mexT expression is upregulated in a $\beta$-lactam-independent manner, this will still not be able to overcome the effect of the 8 bp deletion. It has, however been suggested that there is a putative LacI-like repressor binding site in the mexT-mexE intergenic region (472) and that there is a second repressor that binds this site regulating expression of mexEF-oprN (473). This suggests that the LTTR AmpR potentially regulates this LacI-type repressor, leading to induction of the MexEF-OprN pump in a MexT-independent manner. The outer membrane porin OprD serves as a conduit for the entry of carbapenems into the cell (429). Although we see decreased expression of $\operatorname{opr} D$, the strain is still sensitive to imipenem and meropenem, which is contrary to expectation. However, our finding is in agreement with a previous observation where mexEF-oprN overexpressing strains showed no altered imipenem susceptibility (474), the associated mechanism remains to be elucidated. Previous studies have also demonstrated an inverse correlation between $\beta$-lactam resistance and biofilm formation, both in vitro and in CF isolates (475-477). Our data supports these findings as far as $\beta$-lactam antibiotics are concerned, since $A m p R$ positively regulates production of AmpC $\beta$-lactamase while negatively regulating biofilm formation. However, negative regulation of the MexEF-OprN efflux (providing resistance to fluoroquinolones, chloramphenicol and trimethoprim) by AmpR (Table 4.2, Fig 4.12) suggests that the antagonistic regulation of antibiotic resistance and biofilm formation is dependent on the class of antibiotics. The physiological advantage to the bacteria in this context is unclear. Co-regulation of $\beta$-lactam and fluoroquinolone resistance by $A m p R$ is significant in itself, since this puts AmpR among one of the few proteins that regulates resistance to multiple 
classes of antibiotic (478). In addition, since fluoroquinolones are part of the current systemic antibiotic treatment regimen for $P$. aeruginosa infections (201), this finding could potentially have important therapeutic implications.

QS is at the heart of the virulence regulatory network in P. aeruginosa with multiple regulators feeding into the regulation process (170). We have shown previously that AmpR is also part of the QS regulatory process and regulates production of proteases and pyocyanin $(105,425)$, but the determined extent of the regulation was limited due to the experimental approaches adopted. Using whole genome transcriptome, we show here that the AmpR-influenced QS regulon is much more extensive than previously thought (Appendix; Supplementary information for Chapter 4, Table S5). QS regulated phenotypes, such as pyocyanin and protease production are positively regulated by AmpR in the current study (Fig 4.12), which is in contrast to that seen in our previous analyses (105). We believe that this difference stems from the fact that in our previous studies, we used an insertion mutant (aacCl cassette inserted into the PstI site of ampR) whereas in this study, we have used a clean in-frame deletion mutant of ampR. One potential reason for the discrepancy may be that the gentamycin cassette insertion at the PstI site (554 bases into the ampR coding region) (105) did not disrupt the N-terminus HTH motif of AmpR. Since LysR members are known to bind DNA even in the absence of inducer signals (104), this intact HTH motif might have somehow interfered with the regulatory process. The P. aeruginosa PAO1 insertion ampR mutant in the previous study produced more pyocyanin compared to the wild-type PAO1 (105). In contrast, in this study, we find that PAO $\triangle a m p R$ produces significantly lower amounts of phenazines as compared to PAO1 (Table 4.3). The differences in phenazine production were also 
translated into differential susceptibilities in the C. elegans paralytic assays [Fig 4.2; (425)] since phenazines are one of the major contributors to C. elegans toxicity in this assay (195) explaining the reduced killing of C. elegans with this strain (Fig 4.2). Furthermore, with PAO $\triangle a m p R$, the microarray, $\mathrm{qPCR}$ and phenotypic data concur, and support our current findings.

The MexGHI-OpmD (PA4205-PA4208) efflux pump is involved in the efflux of acriflavin, ethidium bromide, novobiocin, rhodamine, and vanadium, and in maintaining QS homeostasis (440). Mutants of mexI and opmD have been demonstrated to be impaired in QS-related phenotypes including swarming motility, production of elastase, rhamnolipids, pyocyanin and pyoverdine (440). This pump is downregulated in PAO $\triangle a m p R$ indicating positive AmpR regulation and correlates with some of the observed phenotypes, such as decreased production of elastase and pyocyanin. Furthermore, SoxR is known to regulate this pump (479), but is not differentially regulated in the microarray data.

AlgR regulates the $h c n A B C$ operon genes (PA2193-PA2195) involved in hydrogen cyanide synthesis in $P$. aeruginosa (480). In the ampR mutant, these genes are downregulated five- to seven-fold without a corresponding significant differential regulation of algR (< two-fold). AmpR, however, negatively regulates AlgT/U (5.4-fold upregulated in PAO $\triangle a m p R$ in the absence of antibiotics) in agreement with previous findings (425), and $\mathrm{AlgT} / \mathrm{U}$ regulates algR $(481,482)$. Thus the regulation of the $h c n A B C$ operon in $\mathrm{PAO} \triangle a m p R$ is potentially through $\mathrm{AlgT} / \mathrm{U}$-mediated regulation of $\mathrm{AlgR}$ (Fig 4.12). Moreover, RpoS, in conjunction with the Gac-Rsm regulatory system, has been shown to regulate oxidative stress-mediated resistance in P. fluorescens (483). In our 
study, both RpoS and the sRNA, $r s m Z$ are regulated in an AmpR- $\beta$-lactam-dependent and AmpR-dependent manner, respectively. This could also be a potential mode of regulation of the hcn operon in PAO $\triangle a m p R$, provided the regulatory mechanism is conserved in $P$. aeruginosa. AmpR also negatively influences expression of the galactophilic lectin LecA (PA2570) (Fig 4.10) that is RpoS and QS-regulated. LecA has been shown to be critical to the gut pathogenicity of $P$. aeruginosa and to enhance cytotoxic effects of exotoxins by inducing a permeability defect (484). These results further support the hypothesis that AmpR regulates virulence in $P$. aeruginosa.

P. aeruginosa produces bacteriocins called pyocins that kill susceptible cells through either pore-formation and subsequent leakage of cytoplasmic contents, or by endonuclease activity (460). The two high molecular weight pyocins, types R and F, are proposed to be remnants of lysogenic phages and resemble phage tails. Like lysogenic phages, they are induced in response to DNA damage, linked to the RecA-mediated SOS response (485) and other stress conditions such as exposure to hydrogen peroxide (152) or ciprofloxacin (486). AmpR influences the expression of many of these genes including the regulators PrtR, PtrN, PtrB, and the SOS response mediator RecA (Table 4.4; section in results). Specific and significant differential regulation of these genes under $\beta$-lactam stress in PAO $\triangle a m p R$ implies a role for AmpR in influencing expression of these genes under stress conditions. Moreover, pyocin production confers a survival advantage by killing neighboring susceptible cells and $97 \%$ of the $P$. aeruginosa $\mathrm{CF}$ isolates tested showed bacteriocin-like killing activity (487). 


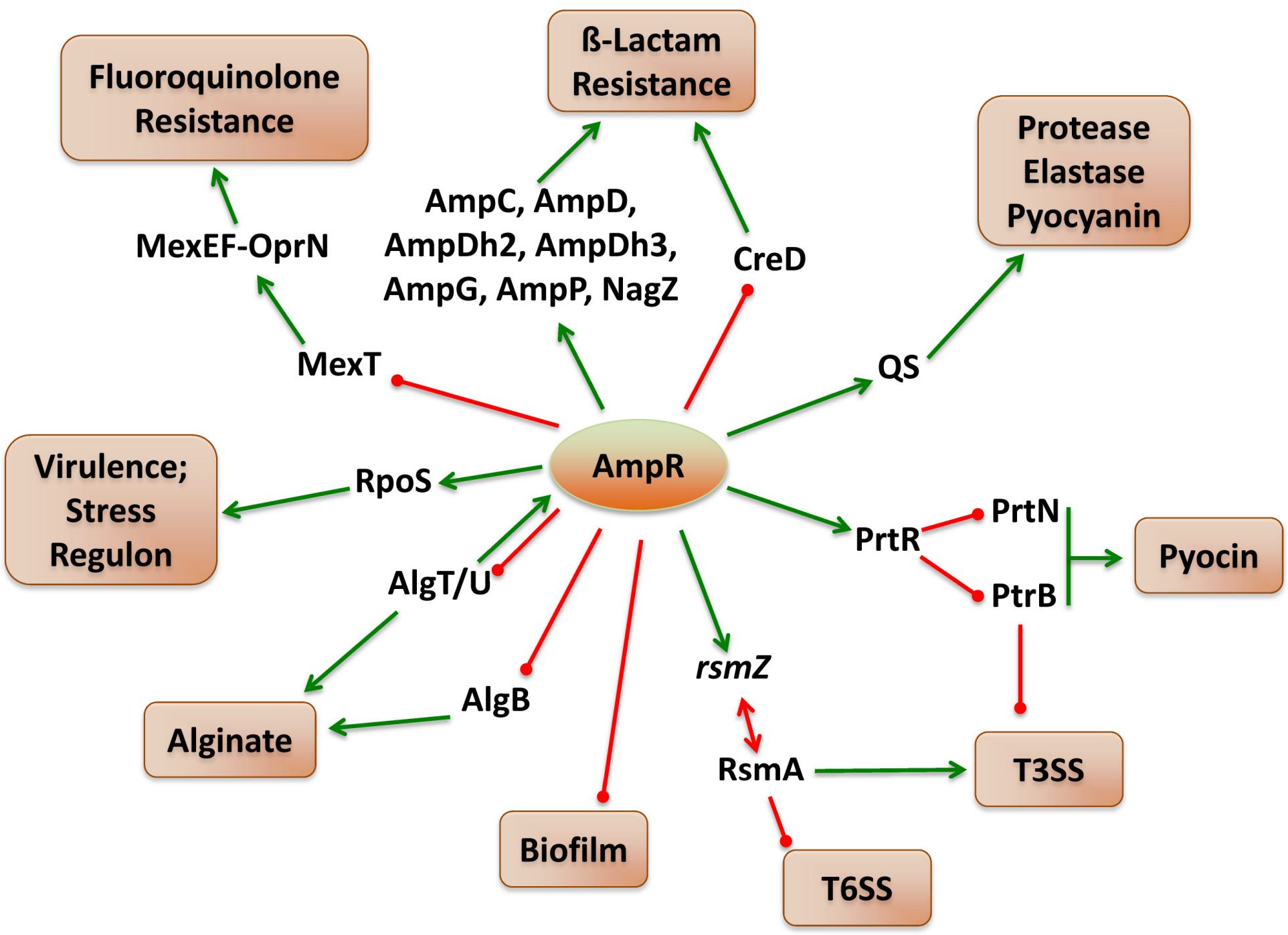


Figure 4.12. AmpR is a master regulator of gene expression in $P$. aeruginosa PAO1. AmpR positively regulates resistance to $\beta$-lactam antibiotics by upregulating expression of the amp genes, nagZ and downregulating $c r e D$. In addition, AmpR affects fluoroquinolone resistance by negatively regulating expression of mexT, the positive regulator of the MexEF-OprN efflux pump. Expression of the virulence and stress response sigma factor, RpoS and QS-regulated acute virulence factors is downregulated in PAO $\triangle a m p R$, indicating positive AmpR regulation. AmpR also negatively regulates biofilm formation via an unknown mechanism. AmpR modulates levels of the small RNA rsmZ, whose levels are lower in PAO $\triangle a m p R$ with a corresponding enhanced expression of RsmA. Downregulation of some of the T3SS genes in the $a m p R$ mutant is possibly by regulating $p \operatorname{tr} B$ expression, via PrtR. Further, two major regulators of the alginate biosynthetic pathway, $\mathrm{AlgT} / \mathrm{U}$ and $\mathrm{AlgB}$ are negatively regulated by $\mathrm{AmpR}$, thereby potentially also regulating alginate production. Whether these AmpR interactions are direct or indirect needs to be investigated.

The pyocin genes are located in RGP03 and RGP04 and are acquired by $P$. aeruginosa (33). Though some RGP loci contain regulators that control expression of RGP genes (488), it is interesting to note that an endogenous regulator such as AmpR is able to regulate acquired genes and highlights the transcriptional versatility of $P$. aeruginosa.

Negative regulation of the genes involved in biofilm formation and pyocin biosynthesis by AmpR (Fig 4.12) fits with the profile of AmpR as a negative regulator of chronic infection phenotypes and positive regulator of acute infection, as seen with the QS-regulated phenotypes. RsmA, a small RNA-binding protein, is a global regulator of virulence in $P$. aeruginosa and is intricately tied in with the GacSA TCS $(390,458)$. Two small RNAs, rsmZ and $r \operatorname{sm} Y$, which bind and sequester RsmA, keep RsmA activity in check in Pseudomonas and other bacteria (389,458,489-491). In P. aeruginosa, RsmA positively regulates T3SS and negatively regulates biofilm formation $(492,493)$. AmpR positively regulates $r \operatorname{smZ}$ (downregulated in PAO $\triangle a m p R$ ), resulting in downregulation of RsmA (upregulated in PAO $\triangle a m p R$ ). PAO $\triangle a m p R$ forms enhanced biofilms (Fig 4.8) and shows decreased production of QS regulated phenotypes compared to PAO1 (Table 4.3). 
These data suggest that regulation of some of the phenotypes seen in an ampR mutant is not via modulating the activity of $r s m Z$ and consequently RsmA. However, analysis of the $r s m Z$ promoter for potential AmpR binding sites using a previously identified putative AmpR binding site (434) revealed a weak AmpR consensus (5' CCCGCGCCTTTTGT 3'). The possibility of direct AmpR regulation of $r s m Z$ remains to be elucidated.

In conclusion, unlike AmpR found in other Enterobacteriaceae members, the targets of $P$. aeruginosa AmpR are widely distributed in the genome and consist of over 500 genes. Since the genes regulated include transcriptional regulators, sigma factors and non-coding RNAs, a subset of these genes are possibly regulated indirectly by AmpR via intermediate regulators. It is also interesting to note that AmpR regulates genes that have been acquired by horizontal gene transfer, which reflects on the transcriptional versatility of $P$. aeruginosa. Although the exact AmpR regulatory mechanisms are as yet unclear, this study highlights the complexity and multi-tiered regulatory processes by which $P$. aeruginosa controls expression of genes of diverse functions. Teasing apart the AmpR regulatory network will involve differentiating direct and indirect AmpR-regulated genes that will advance our understanding of how this bacterium regulates multiple different pathogenesis and metabolic mechanisms. This understanding will potentially lead to identifying mechanistic targets that can help in dealing with intractable $P$. aeruginosa infections, and other bacterial pathogens that harbor similar systems. 


\section{Materials and Methods}

\section{Bacterial strains, nematodes, media and primers}

All experiments were performed with either the wild type or derivatives of $P$. aeruginosa PAO1 (25) and Escherichia coli DH5 $\alpha$ cultivated in Luria Bertani (LB) broth (Difco, USA) incubated at $37{ }^{\circ} \mathrm{C}$ unless specified otherwise. The strains and plasmids used in this study are listed in Table 4.5. Nematode assays were performed with Caenorhabditis elegans strain N2 (Bristol) and E. coli OP50 obtained from the Caenorhabditis Genetics Center, which is funded by the NIH National Center for Research Resources (NCRR). E. coli OP50 was grown in nematode growth medium (1.7 \% agar, $0.35 \%$ peptone, $0.34 \% \mathrm{~K}_{2} \mathrm{HPO}_{3}, 0.3 \% \mathrm{NaCl}, 0.012 \% \mathrm{MgSO}_{4}, 0.011 \%$ $\mathrm{CaCl}_{2}, 0.0005 \%$ cholesterol) whereas the $P$. aeruginosa strains used in the nematode assays were grown on brain-heart infusion agar (Difco, USA). Biofilm assays were performed in T-broth (tryptone $10.0 \mathrm{~g} / \mathrm{L}, \mathrm{NaCl} 5.0 \mathrm{~g} / \mathrm{L}$ ). T-agar plates were prepared by addition of $1 \%$ agar to T-broth. MinS-NTA minimal media (494) was used for RNA isolation to determine expression of T3SS genes. Cation-adjusted Mueller Hinton broth and agar (Difco, USA) was used in MIC assays. For pyocyanin production, strains were cultivated in King A medium (Difco, USA). Antibiotics were supplemented, when needed, at the following concentrations for E. coli: ampicillin (Ap) $100 \mu \mathrm{g} / \mathrm{ml}$, gentamycin (Gm) $15 \mu \mathrm{g} / \mathrm{ml}$, tetracycline (Tc) $15 \mu \mathrm{g} / \mathrm{ml}$; for P. aeruginosa: $\mathrm{Gm} 75 \mu \mathrm{g} / \mathrm{ml}$, Tc $60 \mu \mathrm{g} / \mathrm{ml}$. Primers used in this study are listed in Appendix; Supplementary information for Chapter 4, Table S10. 
Table 4.5: Strains and plasmids used in this study

\begin{tabular}{|c|c|c|}
\hline Strain/ plasmid & Relevant characteristics & Source \\
\hline Strains & & \\
\hline \multicolumn{3}{|l|}{ Escherichia coli } \\
\hline DH5 $\alpha$ & General purpose cloning strain; $\Delta($ lacZ) $M 15$ & $\begin{array}{l}\text { New } \\
\text { England } \\
\text { Biolabs }\end{array}$ \\
\hline OP50 & Food source for culturing C. elegans & $\begin{array}{l}\text { C. elegans } \\
\text { Genetics } \\
\text { Center }\end{array}$ \\
\hline DBS7 & $\begin{array}{l}\mathrm{DH} 5 \alpha \text { harboring } \mathrm{pEXG} 2 \text { carrying the } 1520 \mathrm{bp} \\
\Delta a m p R \text { fragment (pDBS7) }\end{array}$ & This study \\
\hline DBS220 & $\begin{array}{l}\text { DH5 } \alpha \text { harboring the } 944 \text { bp promoterless ampR } \\
\text { ORF cloned into pCR } 2.1 \text { TOPO (pDBS220) }\end{array}$ & This study \\
\hline DBS271 & $\begin{array}{l}\text { DH5 } \alpha \text { containing the } 944 \text { bp } a m p R \text { ORF subcloned } \\
\text { as a EcoRI-SacI fragment from pDBS } 220 \text { into } \\
\text { pMMB67EHGm }\end{array}$ & This study \\
\hline
\end{tabular}

\begin{tabular}{|c|c|c|}
\hline Strain/ plasmid & Relevant characteristics & Source \\
\hline \multicolumn{3}{|c|}{ Pseudomonas aeruginosa } \\
\hline PAO1 & Wild-type & $(25)$ \\
\hline PKM314 & PAO1 with pMMB67EH-Gm (empty vector) & This study \\
\hline PKM315 & PAO $\triangle a m p R ;$ In-frame deletion of $a m p R(P A 4109)$ & This study \\
\hline PKM316 & PAO $\Delta a m p R$ with pMMB67EH-Gm (empty vector) & This study \\
\hline PKM317 & $\begin{array}{l}\text { PAO } \triangle a m p R(\mathrm{pAmpR}) ; a m p R \text { ORF on } \\
\text { pMMB67EH-Gm (pDBS271) moved into } \\
\text { PAO } \Delta a m p R ; \text { IPTG-inducible; Gm }{ }^{\mathrm{R}} \text {; ampR } \\
\text { complementing clone }\end{array}$ & This study \\
\hline PAOretS::aacC1 & Gm insertion mutant of ret $S$; hyper biofilm-former & S. Lory \\
\hline $\begin{array}{l}\text { PAO } \Delta p e l \Delta p s l \\
\quad \Delta a \lg D\end{array}$ & $\begin{array}{l}\text { In-frame triple deletion of the } \operatorname{alg} D \mathrm{ORF} \text { and pel } \\
\text { and } p s l \text { operons; biofilm non-former }\end{array}$ & S. Lory \\
\hline \multicolumn{3}{|c|}{ Staphylococcus aureus } \\
\hline DBS116 & Wild type $S$. aureus used for LasA assays & $\begin{array}{c}\text { Lab } \\
\text { collection } \\
\end{array}$ \\
\hline \multicolumn{3}{|l|}{ Plasmids } \\
\hline pCR2.1 TOPO & $\begin{array}{l}\text { TA cloning vector for PCR products; } \mathrm{Ap}^{\mathrm{R}}, \mathrm{Km}^{\mathrm{R}} \text {; } \\
\text { ColE1 f1 ori lacZ } \alpha\end{array}$ & Invitrogen \\
\hline pRK600 & Helper plasmid for conjugation & {$[133]$} \\
\hline
\end{tabular}




\begin{tabular}{|c|c|c|}
\hline $\mathrm{pEXG} 2$ & $\begin{array}{l}\text { Allelic exchange vector; } \mathrm{Gm}^{\mathrm{R}} \text {, colE1 ori, oriT, } \\
\text { sacB }\end{array}$ & $(495)$ \\
\hline pMMB67EH-Gm & $\mathrm{Gm}^{\mathrm{R}}$; IncQ, RSF1010, $\operatorname{lacI}_{\mathrm{q}} \mathrm{P}_{t a c}$ expression vector & $(496)$ \\
\hline pDBS7 & $\begin{array}{l}\mathrm{pEXG} 2 \text { with the } \Delta a m p R \text { fragment, flanked by } \\
\text { EcoRI and BamHI sites }\end{array}$ & This study \\
\hline pDBS220 & $\begin{array}{l}\text { pCR2.1 TOPO containing the } 944 \text { bp ampR ORF, } \\
\text { PCR amplified from the PAO1 genome using } \\
\text { DBS_ampRF2 and DBS_ampRR2 }\end{array}$ & This study \\
\hline pDBS271 & $\begin{array}{l}\text { pMMB67EH-Gm containing the } a m p R \text { ORF, } \\
\text { subcloned as an EcoRI-SacI fragment from } \\
\text { pDBS220 }\end{array}$ & This study \\
\hline
\end{tabular}




\section{Construction of deletion mutants, complementation clones}

An unmarked ampR null mutant of $P$. aeruginosa was generated by gene splicing (497) using primers KMampRUF1 and KMampRUR1 (to generate the upstream product P1, flanked by EcoRI and NheI sites), and primers KMampRDF1 and KMampRDR2 (to generate the downstream product $\mathrm{P} 2$, flanked by NheI and BamHI sites). After sequencing to ensure absence of mutations, P1 and P2 were spliced together to obtain a $1520 \mathrm{bp}$ deletion fragment of $a m p R$ containing stop codons in all three reading frames at their junction (inserted as part of the NheI sites in the primer). This was then sequenced and subcloned into a $P$. aeruginosa non-replicative plasmid pEXG2 (495) as an EcoRIBamHI fragment and moved into PAO1 by allelic exchange (498) using pRK600 (499) as the helper plasmid. Double crossover mutants were selected for the loss of plasmid (gentamycin-sensitive, sucrose counter-selection). The presence of deletion in PAO $\triangle a m p R$ was confirmed using standard molecular methods (PCR and restriction analysis of amplicons) and biological assays (antibiotic sensitivity pattern and $\beta$ lactamase assays). Complementation of the $a m p R$ deletion was achieved by amplifying

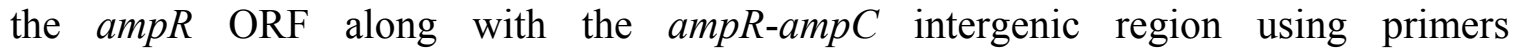
DBS_ampRF2 and DBS_ampRR2 and cloning into pCR2.1 TOPO using TA cloning technique. After confirming absence of mutations by sequencing, the $944 \mathrm{bp} a m p R$ ORF was moved into pMMB67EH-Gm (496) as an EcoRI-SacI fragment. The plasmid was then moved into PAO $\triangle a m p R$ by electroporation (500), selecting for gentamycin-resistant colonies. 


\section{C. elegans virulence assay}

The P. aeruginosa - C. elegans standard paralysis assay was modified from previous protocols (446). Overnight bacterial cultures were diluted 1:1000 and plated onto brain heart infusion agar plates. The plates were incubated for $18-24$ hours at $37{ }^{\circ} \mathrm{C}$ for the formation of bacterial lawns. Meanwhile, a synchronized culture of L4 stage larvae hermaphrodite Bristol N2 C. elegans was washed off E. coli OP50-seeded nematode growth medium plates using M9 media. The nematodes were centrifuged at $1300 \mathrm{x} g$ for two minutes and washed twice with M9 medium to remove residual E. coli bacteria. Thirty to 35 nematodes were then added to each of the $P$. aeruginosa bacterial lawns. Both live and paralyzed nematodes were scored at two-hour intervals for eight hours via microscopic observation. Nematodes were considered dead when they did not respond to physical stimuli. Each strain was tested in triplicate.

\section{RNA isolation, generation of cDNA probes, microarray experiments and data analysis}

P. aeruginosa strains PAO1 and PAO $\triangle a m p R$, with and without $\beta$-lactam antibiotic treatment were used for RNA extraction. The cells were subcultured at $37^{\circ} \mathrm{C}$, $300 \mathrm{rpm}$ from overnight cultures grown in LB broth to an OD600 of 0.01 . When the cells reached an OD600 of 0.5-0.6, they were split into two flasks. One of the flasks was treated with sub-MIC concentration of penicillin $(100 \mu \mathrm{g} / \mathrm{ml}$ penicillin G Potassium salt, Fisher Bioreagents) for two hours. The RNA was stabilized by addition of phenol-ethanol mixture (390) prior to extraction (RNeasy Mini Kit, Qiagen) following manufacturer protocols. Residual DNA was removed using RQ1 RNase-free DNase (Promega) and the 
RNA repurified using the kit. After quantification by measuring absorbance at $260 \mathrm{~nm}$ (Beckman DU640, Beckman Coulter), and preliminary quality check on a denaturing agarose gel (NorthernMax Gly, Ambion), the RNA integrity was analyzed using RNA Nano chips in an Agilent Bioanalyzer 2100. cDNA was then synthesized by annealing NS5 random primers to total purified RNA, spiked with Bacillus subtilis internal control RNAs and subsequent extension carried out using SuperScript III reverse transcriptase (Invitrogen). Fragmentation of cDNA was performed using RQ1 DNase (Promega), and the fragments were biotin labeled. Hybridization with GeneChip P. aeruginosa genome array (Affymetrix) and scanning were performed according to manufacturer specifications.

Data analyses from three biological replicates for each of the conditions tested were performed after normalizing and summarizing probe level measurements using Guanine Cytosine - Robust Multiarray Average (GC-RMA). Only genes that fit stringent criteria (expression cutoff: $50-100 \%$ stringency; $p$-value $\leq 0.01$ of one-way ANOVA data corrected by Benjamini Hochberg FDR; fold-change $\geq 2.0$ ) were selected for further analysis. All microarray data analysis was performed using GeneSpring GX 10.0 Expression Analysis software (Silicon Genetics). The microarray data is MIAME compliant and has been deposited in NCBI GEO (accession number GSE33188).

\section{Bioinformatics analysis of the AmpR-binding site}

The promoters of AmpR-dependent and AmpR- $\beta$-lactam-dependent genes (listed in Appendix; Supplementary information for Chapter 4, Tables S3 and S4) were used to refine the AmpR binding motif using the Iterative Enhancement of Motifs (IEM) 
algorithm (434). The AmpR binding site in the promoter of $\operatorname{ampC}$ (5'TCTGCTCCAAATTT-3') was used to search the AmpR-dependent or AmpR- $\beta$-lactamdependent promoters from $P$. aeruginosa PAO1 and their orthologs from $P$. aeruginosa strains PA14, PA2192, C3719 and PACS2. The output of IEM was a motif matrix. WebLogo (424) was used to graphically represent the multiple sequence alignment of the output. The Regulatory Sequence Analysis Matrix Scan Tool (RSA) (423) was then used to identify PAO1 promoter sequences containing the identified AmpR-dependent or AmpR- $\beta$-lactam-dependent motifs in the respective gene sets. The RSA output was then used to generate a WebLogo for the gene sets.

\section{Quantitative real-time PCR}

Specific genes that were significantly, over two-fold up or down regulated between $P$. aeruginosa PAO1 and PAO $\triangle a m p R$ as seen in microarray experiments were verified by qPCR. Total RNA isolation and reverse transcription into cDNA was as described for the microarrays without addition of spike transcripts, fragmentation or labeling. For qPCR, the ABI Step One (Applied Biosystems) cycler was used with PowerSYBR Green PCR MasterMix with ROX (Applied Biosystems). Expression was normalized to $\operatorname{clpX}$ (PA1802), whose expression was determined to remain constant between the samples and conditions tested. Assays were performed at least in biological triplicate, each with technical triplicates, for every gene analyzed. Melt curves were determined to ensure primer specificity. The cycling conditions used were $95{ }^{\circ} \mathrm{C} / 2$ minutes (holding); 40 cycles of $95{ }^{\circ} \mathrm{C} / 15 \mathrm{sec}, 60{ }^{\circ} \mathrm{C} / 1 \mathrm{~min}$ (cycling); $95{ }^{\circ} \mathrm{C} / 15 \mathrm{sec}, 60$ ${ }^{\circ} \mathrm{C} / 1 \mathrm{~min}, 95{ }^{\circ} \mathrm{C} / 15 \mathrm{sec}$ (melt curve). Differential regulation of the T3SS genes, 
including $r s m Z$ was determined using RNA isolated from cells grown in MinS-NTA inducing media (494) from PAO1 and PAO $\triangle a m p R$, essentially as described above.

Gene expression in PAO $\triangle a m p R$ were normalized to the corresponding PAO1 values, for both the $\beta$-lactam uninduced and induced conditions and is presented as relative expression in $\mathrm{PAO} \triangle a m p R \pm$ standard error.

\section{Determination of minimum inhibitory concentration (MIC)}

MICs were determined one of two ways. For testing the MIC of $\beta$-lactams, E-test was used following manufacturer protocols (BioMerieux). The MICs of the MexEFOprN efflux pump substrates (ofloxacin, chloramphenicol, ciprofloxacin, and trimethoprim) were determined by standard broth microdilution method (433). Briefly, serial two-fold dilutions of the antibiotics were incubated at $37{ }^{\circ} \mathrm{C}$ for $16-18 \mathrm{hrs}$ with $5 \mathrm{x}$ $10^{5} \mathrm{CFU} / \mathrm{ml}$ of bacteria in a total volume of $200 \mu \mathrm{l}$ in 96 -well flat bottom polystyrene plates. The highest dilution of antibiotic that prevented bacterial growth was considered as the MIC. The assays were performed at least in triplicate, each with technical triplicate, for each antibiotic in cation-adjusted Mueller Hinton broth.

\section{Quantifying $\beta$-lactamase activity}

$\beta$-lactamase activity was quantified as described previously (105). Briefly, cells in LB broth at an OD600 of 0.5-0.6 were treated with $100 \mu \mathrm{g} / \mathrm{ml}$ Penicillin G for two hours at $37{ }^{\circ} \mathrm{C}$. The cells were then harvested, OD normalized, and lysed with BugBuster Protein Extraction Reagent (Novagen) and r-Lysozyme (Novagen), and treated with Benzonase nuclease (Novagen). The amount of $\beta$-lactamase was quantified in the soluble fraction by determining hydrolyzing activity on nitrocefin (Oxoid, England). Protein 
concentrations in the samples were determined by Bradford assay. Enzyme activity was expressed as milliunits of $\beta$-lactamase (nanomoles of nitrocefin hydrolyzed per minute per microgram of protein).

\section{Biofilm assays}

Time course biofilm assays were performed in $12 \times 75 \mathrm{~mm}$ round-bottom glass culture tubes (VWR) as described previously (453). Briefly, fresh overnight T-agar plate cultures of the test strains were scraped into $1 \mathrm{ml} \mathrm{T}$-broth and diluted to a final OD600 of 0.0025. Aliquots of $1 \mathrm{ml}$ per tube were made at time zero and incubated static at room temperature for 24, 48, 72 and 96 hours. To assay for pellicle formation, the tubes were washed with running tap water five times, after discarding the cultures and stained with $1 \%$ crystal violet for 20 minutes. After pouring off the dye, the tubes were washed thoroughly with running tap water 10 to 15 times. Quantification of the attached and stained cells was done at $590 \mathrm{~nm}$ after solubilization of the dye with absolute ethanol.

\section{Motility assays}

Twitching and swimming assays were performed on $1 \%$ and $0.3 \%$ agar plates, respectively, as described previously (501).

\section{Protease assays}

LasA protease activity was measured by the ability of the strain supernatant to lyse boiled Staphylococcus aureus cells as described (502). Overnight culture supernatants of the test strains $(100 \mu \mathrm{l})$ were mixed with $900 \mu \mathrm{l}$ of a Tris- $\mathrm{HCl}(\mathrm{pH} 8.5)$ suspension of 
boiled $S$. aureus culture diluted to a final OD600 of 0.8 . The lysis was monitored over an hour and LasA activity was expressed as the change in OD600 per hour per $\mu \mathrm{g}$ protein.

LasB elastolytic assay was performed with an elastin-congo red (ECR, Sigma) conjugate (503). The overnight culture supernatants $(100 \mu 1)$ were mixed with $900 \mu \mathrm{l}$ of ECR buffer (100 mM Tris, $1 \mathrm{mM} \mathrm{CaCl}$, pH 7.5) containing $20 \mu \mathrm{g}$ of ECR. Tubes were incubated shaking for one hour at $37{ }^{\circ} \mathrm{C}$, and the supernatant was read at $495 \mathrm{~nm}$. LasB activity was expressed as change in A495 per $\mu$ g protein compared to an LB control.

\section{Pyocyanin production}

The amount of pyocyanin produced was determined by extracting the pigment from overnight King A culture supernatants. A $5 \mathrm{ml}$ 18-hour supernatant was mixed with $3 \mathrm{ml}$ of chloroform to extract pyocyanin into the chloroform phase. Pyocyanin was then extracted with $0.2 \mathrm{~N} \mathrm{HCl}$, the absorbance measured at $520 \mathrm{~nm}$, and the pyocyanin concentration expressed as $\mu \mathrm{g}$ pyocyanin produced per $\mu \mathrm{g}$ of protein (504).

\section{Phenotypic Microarray (PM)}

PM profiles of PAO $\triangle a m p R$ were compared to that of wild-type PAO1 in the absence of antibiotic stress to test the effect of ampR deletion. PM arrays (Biolog Inc., Hayward, CA, USA) comprise of about 2000 tests spanning 20 96-well plates and include $\sim 800$ tests for carbon, nitrogen, phosphorous and sulfur utilization, $\sim 100$ tests each for $\mathrm{pH}$ growth range and osmotic sensitivity, and $\sim 1000$ tests for chemical sensitivity. Suspensions of control and test strains, in duplicate, were prepared in inoculating fluid containing $0.01 \%$ tetrazolium violet and transferred to the PM plates. After incubation for 24 hours, growth differences between the strains were determined 
from the kinetic response curves obtained by measuring changes in the color of the redox dye in each well in the OmniLog ${ }^{\circledR}$ incubator reader. Better growth of PAO $\triangle a m p R$ in the presence of a specific test compound compared to PAO1 indicates gain of the phenotype (AmpR negatively regulates the phenotype) whereas poorer growth of the mutant compared to the wild type in a specific well indicates loss of phenotype due to ampR deletion (AmpR positively regulates the phenotype). OmniLog ${ }^{\circledR}$ PM software was used for data analysis.

\section{Statistical analysis}

All data were analyzed for statistical significance using $t$-test on GraphPad statistical analysis software, except for the microarray data, which was performed on GeneSpring GX 10.0 as mentioned earlier.

\section{Acknowledgements}

We thank Melita Jaric (Narasimhan Lab) for help with the IEM analysis, Keith Turner (Dove Lab, Children's Hospital Boston) for help with the initial qPCR analysis, Hassan Sakhtah and Lars Dietrich (Columbia University) for helpful discussions about phenazine assays. We are grateful to Hansi Kumari (Mathee Lab) for critical reading of the manuscript. 
Chapter 5

Deep Sequencing Expands the Pseudomonas aeruginosa AmpR Regulon to Include Regulatory RNAs 


\begin{abstract}
A Gram-negative opportunistic bacterium, Pseudomonas aeruginosa is a major human pathogen implicated in a number of acute and chronic infections. Of particular concern is the wide prevalence of $P$. aeruginosa in hospitals. Multiple virulence factors determine pathogenicity in $P$. aeruginosa. Virulence factor expression is a tightly regulated process and about $8-10 \%$ of the $P$. aeruginosa genome encodes transcriptional regulators. Some regulators control the expression of different pathogenic determinants in response to largely unidentified signals. Previously, using DNA microarrays we identified that AmpR, a transcriptional regulator of the LysR family and a positive regulator of the AmpC B-lactamase, also regulates other virulence phenotypes in $P$. aeruginosa. Specifically, AmpR turns on acute virulence factor production and represses chronic ones. Using RNA-Seq, this study further expands the regulatory role of $P$. aeruginosa AmpR. Further, quantitative real-time PCRs and phenotypic assays establish the role of AmpR in regulating diverse processes such as oxidative stress response, heatshock response and iron uptake. The Gac-Rsm pathway is a major regulatory switch in determining expression of acute versus chronic virulence factors in $P$. aeruginosa. Gene expression studies demonstrate AmpR-dependent expression of the QS master regulator, LasR. ChIP-Seq studies with a chromosomally tagged AmpR show direct AmpR binding to the lasR promoter. Our studies establish the global regulatory role of $P$. aeruginosa AmpR in pathogenesis by contributing to regulation of quorum sensing and the quorumsensing pathway. This is the first study that analyzes the response of $P$. aeruginosa to antibiotics using RNA-Seq and ChIP-Seq.
\end{abstract}




\section{Introduction}

Pseudomonas aeruginosa is a ubiquitous bacterium and can be isolated from diverse sources, including plants, animals and humans. A high degree of nutritional versatility and adaptability ensures that $P$. aeruginosa is able to colonize a wide range of natural and man-made habitats. In humans, $P$. aeruginosa is seldom part of the microbial flora and is found in less than $2 \%-6 \%$ of individuals in the skin, throat and nasal mucosa (505). An opportunistic, nosocomial pathogen, $P$. aeruginosa colonization rates in hospitalized patients, however, can be over $50 \%$ and especially so in cases of mucosal or cutaneous breach, or in immunocompromised individuals (506). P. aeruginosa is also the leading cause of mortality in cystic fibrosis patients (84).

The pathogenic potential of $P$. aeruginosa is multifactorial and can be broadly classified into cell-associated and secreted virulence factors. The cell-associated virulence factors are typically structural components of the cell, such as the lipopolysaccharide (LPS), pili and flagella (507-509). The process of quorum sensing (QS) regulates expression of many of the major secreted virulence factors. QS is a mechanism of coordinating gene expression based on the population density, employed by both non-pathogenic and pathogenic bacteria (165). Quoromones (acyl homoserine lactones) are small diffusible molecules that mediate QS communication between cells to synchronize virulence gene expression (208). Precise signaling is ensured by the speciesspecific nature of quoromones, although crosstalk between related bacteria is known to occur (510). P. aeruginosa employs three interdependent mechanisms of QS namely the Las, Rhl and Pseudomonas quinolone system (PQS). The Las system is at the top of the regulatory hierarchy, above the Rhl system, while PQS interacts with both Las and Rhl 
[reviewed in $(511,512)]$. In P. aeruginosa, the QS process controls production of secreted enzymes and toxins such as LasA, LasB and ToxA, redox-active compounds such as phenazines (512), and in the case of chronic infections, the formation of bacterial communities called biofilms (451). In addition, some efflux pumps, such as MexGHIOpmD, which play a role in pumping out quoromones from the cytoplasm to the cell exterior, are also QS-regulated $(440,513)$. A majority of the 12 putative and established RND efflux pumps in $P$. aeruginosa, however, are involved in antibiotic resistance $(79,514)$.

Antibiotic resistance is a major problem in dealing with $P$. aeruginosa infections. The current treatment regimen for $P$. aeruginosa is typically a combination therapy of $\beta$ lactams, aminoglycosides and quinolones $(84,515)$. However, a six-year survey by the National Nosocomial Infections Surveillance System of the CDC revealed that $P$. aeruginosa isolates were resistant to many commonly used antibiotics in both ICU and non-ICU patients (516). The infection rates with antibiotic-resistant $P$. aeruginosa were as high as $36 \%(516)$.

P. aeruginosa has multiple mechanisms of antibiotic resistance (79). Resistance to the $\beta$-lactam class of antibiotics is primarily conferred by the chromosomally encoded $\beta$ lactamase AmpC, whereas the MexEF-OprN efflux pump mediates quinolone resistance $(79,427)$. Our recent study demonstrated that the LysR-type transcriptional regulator AmpR that positively regulates $a m p C$ expression, also regulates the MexEF-OprN efflux pump (225). In addition, $P$. aeruginosa AmpR has a global regulatory effect on gene regulation and is a regulator of many virulence determinants and transcriptional regulators $(225,425)$. Using DNA microarrays and complementary assays, we have 
demonstrated that the AmpR regulon consists of over 500 genes, involved in virulence and metabolism (225). AmpR thus activates expression of many acute virulence phenotypes while chronic infection phenotypes were repressed. Interestingly, the AmpR regulon included the small regulatory RNA rgRsmZ (225). Given the extensive nature of the AmpR regulon, we hypothesized that other small regulatory RNAs could also be AmpR-regulated. The microarray platform, however, is not designed to detect small RNAs effectively. Moreover, given the limited sensitivity of microarrays, genes that were not identified in the study could also potentially be AmpR-regulated. In addition, proteomic analyses identified targets that were not identified in the microarray studies (unpublished data). This prompted use of RNA-Seq and ChIP-Seq to identify some noncoding RNAs to be part of the AmpR regulon and determine direct targets of AmpR regulation. Furthermore, we assign a role for AmpR in previously unidentified critical cellular processes such as iron uptake, and in response to oxidative stress and heat shock. This study establishes AmpR as a critical regulator of $P$. aeruginosa virulence and physiology.

\section{Results}

\section{RNA-Seq analysis expands the AmpR regulon}

In order to study the function of AmpR in $P$. aeruginosa, a clean in-frame deletion strain (PAO $\triangle a m p R)$ was constructed in PAO1 in a previous study (225). PAO1 and its isogenic ampR mutant, $\mathrm{PAO} \triangle a m p R$ were used for gene expression profiling using RNA-Seq. In order to be able to compare results from the two studies, the experimental conditions for RNA-Seq was similar to the microarray experiments that were performed 
earlier (225). PAO1 and PAO $\triangle a m p R$ were subjected to sub-MIC B-lactam stress before RNA isolation and cDNA synthesis as described in the methods section.

Using RNA-Seq, the transcription profiles of PAO1 and PAO $\triangle a m p R$ were compared in the presence (induced) and absence (uninduced) of sub-MIC ß-lactam stress. After data normalization between the replicates under each condition, the expression values across the entire genome for PAO $\triangle a m p R$ was normalized to PAO1. The PAO $\triangle a m p R$ data in the uninduced (Panel A, Figure 5.1) and induced (Panel B, Figure 5.1) were plotted. The expression profiles were markedly different in $\mathrm{PAO} \triangle a m p R$ compared to PAO1, both in the absence and presence of antibiotic exposure, attesting to the global regulatory role of AmpR in $P$. aeruginosa. As expected, the B-lactamase ampC, which is under positive AmpR regulation $(105,225)$, is significantly downregulated under ß-lactam stress in PAO $\triangle a m p R$ (Panel B, Figure 5.1). Expression of genes identified in a previous study to be positively regulated by AmpR such as the elastase lasB (225) were also differentially regulated here (Panels A, B; Figure 5.1). The MexEF-OprN efflux pump that provides resistance against fluoroquinolones and chloramphenicol is negatively regulated by AmpR [Panels A, B; Figure 5.1, (225)]. We had demonstrated AmpR to be a positive regulator of the QS system and in agreement, genes such as pqsA and $r h l A$, which are key players in QS-mediated gene regulation, are downregulated in the $\operatorname{ampR}$ mutant (Panel A, Figure 5.1). These findings, in addition to others discussed in the following sections, add credence to the current study.

In addition to the genes that were identified in the microarray, the normalized data identified two transcriptional regulators (PA1015 and PA2588) and one ECF sigma factor 


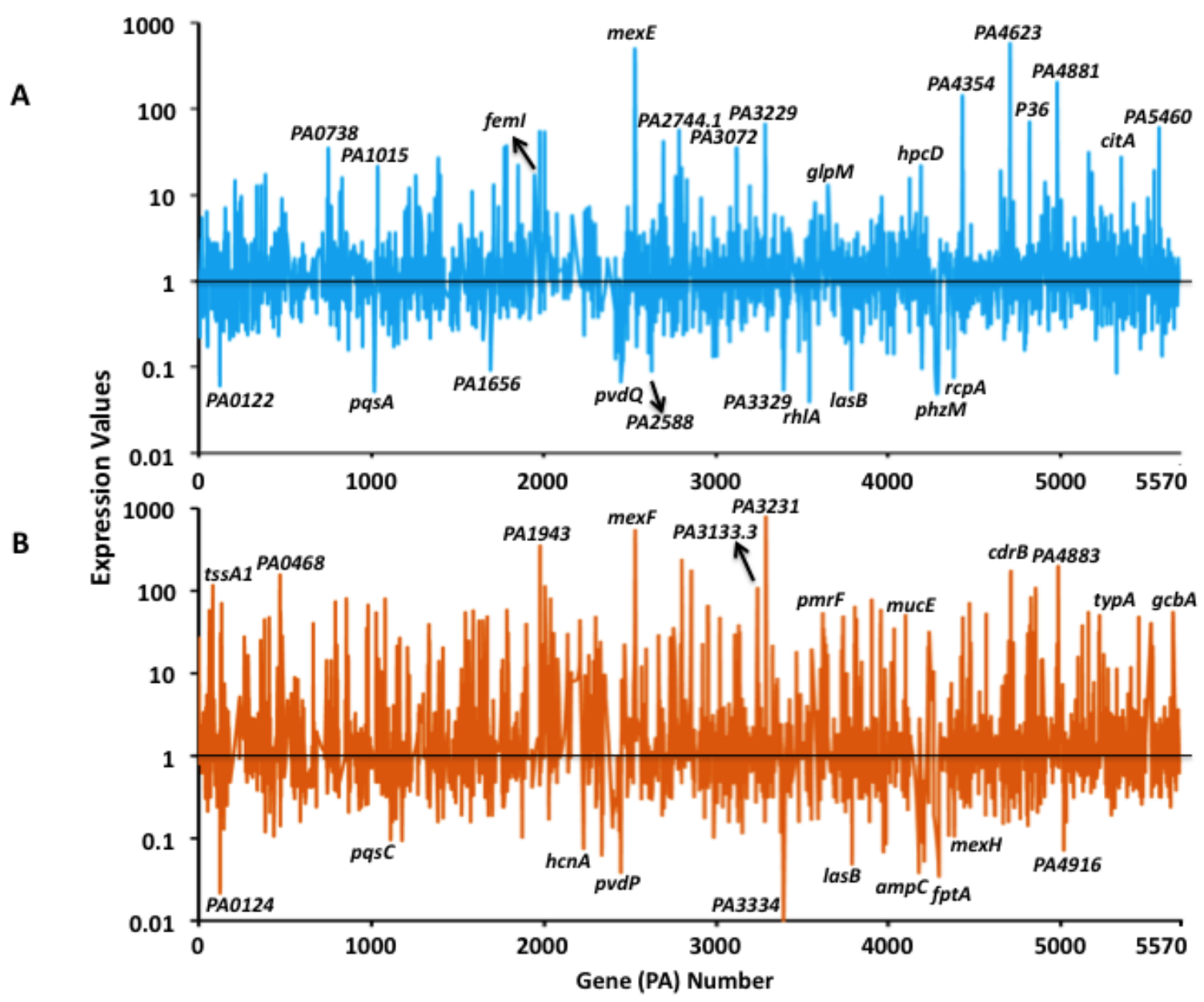

Figure 5.1: Gene expression in PAOAampR. Relative gene expression in PAO $\triangle a m p R$ compared to PAO1 (normalized to one), based on RNA-Seq data, is shown in the absence (top panel) and presence (bottom panel) of sub-MIC ß-lactam stress. Some significantly regulated genes are named. Gene annotations are from the Pseudomonas Genome Database (27). 
(FemI, PA1912) to be differentially regulated (Panel A, Figure 5.1). PA2588 is a putative transcriptional regulator of the AraC family (27). Interestingly, it is located downstream of $p q s H$, which is an important component of the PQS system (380). The role of AmpR in regulating the PQS system is discussed in a later section. The ECF sigma factor FemI, upregulated in PAO $\triangle a m p R$, is part of a two-gene operon with femR (PA1911). FemR, along with FemA (PA1910), are involved in uptake of the mycobacterial siderophore mycobactin (517). Differential regulation of femI, along with the gene encoding the pyochelin receptor fptA (Panel B in Figure 5.1), suggests a role for AmpR in iron uptake regulation and is further explored in the later sections of this study. In addition, noncoding RNAs such as P36, PA2744.1 and PA3133.1 are also differentially regulated (Figure 5.1).

\section{Identification of AmpR-and AmpR-B-lactam-dependent gene sets}

The RNA-Seq data was normalized and pairwise comparisons were performed to determine differential gene expression (fold change $\geq 2.0$, Bonferroni correction of $p$ value $\leq 0.05$ ). The four pairwise comparisons performed were PAO1 uninduced vs. PAO1 induced (Condition I), PAO $\triangle a m p R$ uninduced vs. PAO $\triangle a m p R$ induced (Condition II), PAO1 uninduced vs. PAO $\triangle a m p R$ uninduced (Condition III), and PAO1 induced vs. PAO $\triangle a m p R$ induced (Condition IV). This led to the identification of a total of 2568 genes that were differentially expressed across all four pair-wise comparisons: Condition I-384 genes, Condition II- 672 genes, Condition III- 532 genes, and Condition IV- 980 genes. Although these numbers are indicative, they are not a true measure of the AmpR 


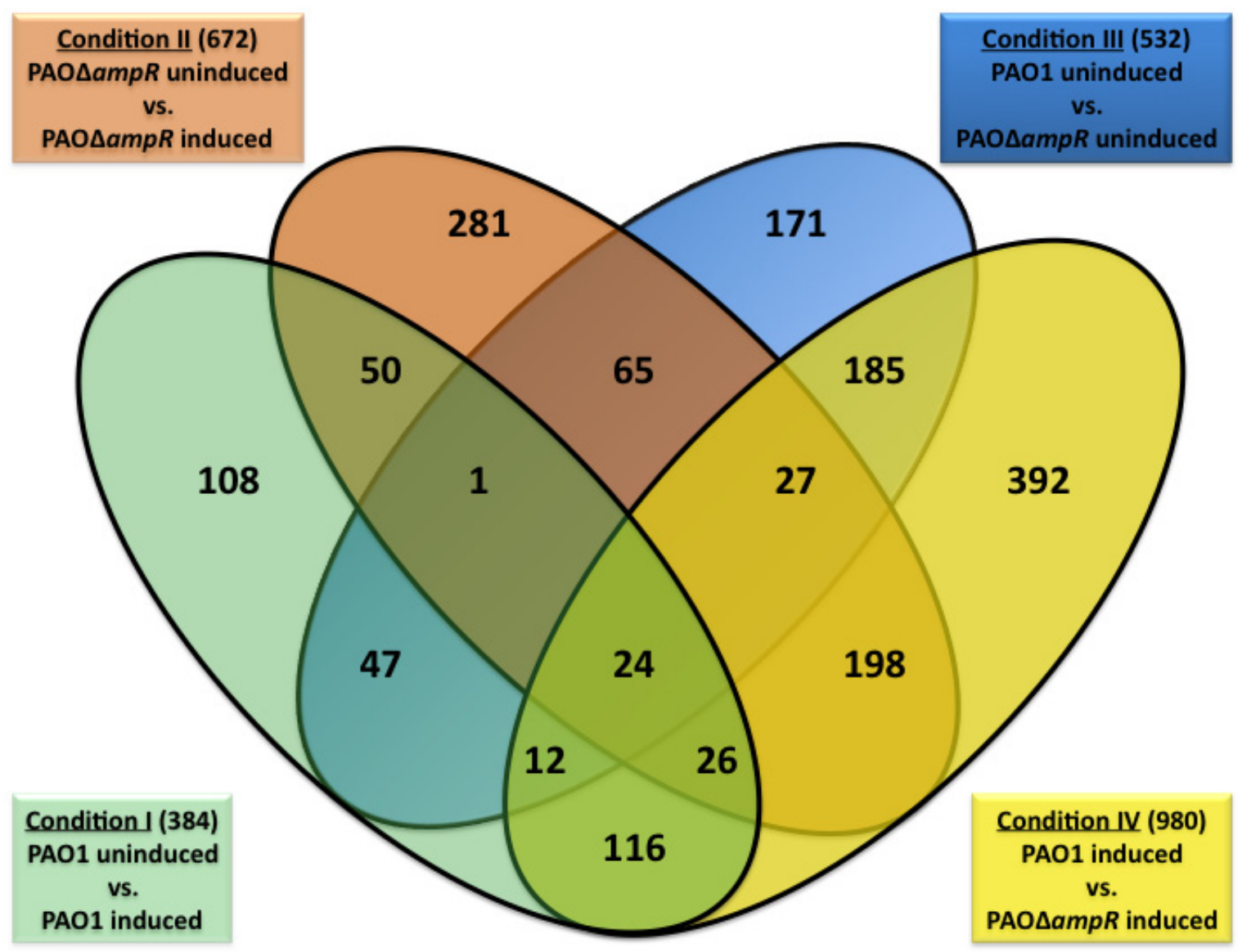

Figure 5.2: Venn diagram of differentially regulated genes. Distribution of significantly ( $p$-value $\leq 0.01)$ regulated genes $(\geq 2.0$-fold) in PA01 and PAO $\triangle a m p R$ without (uninduced) and with (induced) sub-MIC ß-lactam stress. 
regulatory repertoire due to potential overlaps between the conditions, i.e., individual genes could be differentially expressed under more than one condition. In order to address this issue, the pair-wise comparison data was plotted using a four-way Venn diagram (Figure 5.2). Of the 2568 genes that were identified in the original pair-wise analysis under all four conditions, 865 genes were found to be redundant. From Figure 5.2, 1703 non-redundant genes were identified across the four conditions, representing $31 \%$ of the PAO1 genome. Further, genes that were dependent on AmpR, AmpR-ßlactam, and ß-lactam were identified in accordance with the expression criteria listed in the Appendix (Supplementary information for Chapter 5, Table 1). For example, genes in category B (Figure 5.2) are regulated in an AmpR-dependent manner, independent of ßlactam stress.

Twenty-four genes (category $\mathrm{O}$ ) were omitted from further analysis since they were differentially regulated under all four pair-wise analysis conditions, irrespective of AmpR and/or antibiotic stress. A further 56 genes could not be assigned unambiguously for analysis [category H (44 genes), category K (1 gene), category M (4 genes), category $\mathrm{N}$ (7 genes)] and were omitted. Thus, of the remaining 1623 genes, we identified a total of 654 AmpR-dependent genes (Appendix; Supplementary information for Chapter 5, Table 2), 483 genes that were AmpR-dependent only under ß-lactam stress (Appendix; Supplementary information for Chapter 5, Table 3), and 486 genes that are differentially expressed in response to ß-lactam stress independent of AmpR (Appendix; Supplementary information for Chapter 5, Table 4). 


\section{AmpR-regulated genes are enriched in specific functional categories}

The positively and negatively AmpR-regulated genes, both in the presence and absence of sub-MIC ß-lactam exposure, were functionally categorized based on the Pseudomonas Genome Database [(27), Figure 5.3]. The AmpR-regulated genes were expressed as a percentage of the distribution of each functional category in the PAO1 genome (taken as 100\%). This revealed a gross upregulation of genes related to phage, transposon or plasmid (45\% genes of category ' $v$ ' genes in AmpR-positive regulated gene set; Figure 5.3). Functional class 'v' was shown to be positively regulated by AmpR in a previous transcriptome study as well (225). Similarly, genes of functional class ' $\mathrm{n}$ ' (secreted factors- toxins, enzymes, alginate) were also shown to be under AmpR positive regulation ( $46 \%$ of genes of the category ' $n$ ' genes in the AmpR-positive regulated gene set; Figure 5.3). We have previously demonstrated that ampR mutants are impaired in production of extracellular enzymes such as LasA and LasB, and some of the type III secretion genes (225). Similarly, genes belonging to functional categories ' $k$ ' (adaptation and protection; 19\%), 'zA' (antibiotic resistance and susceptibility; 10\%) and 'q' (central intermediary metabolism; 11\%) were also positively regulated by AmpR (Figure 5.3).

This finding agrees with our previous observations from DNA microarrays and phenotypic microarrays that AmpR is an important regulator of antibiotic resistance, cell wall recycling enzymes and metabolism in P. aeruginosa (225). 


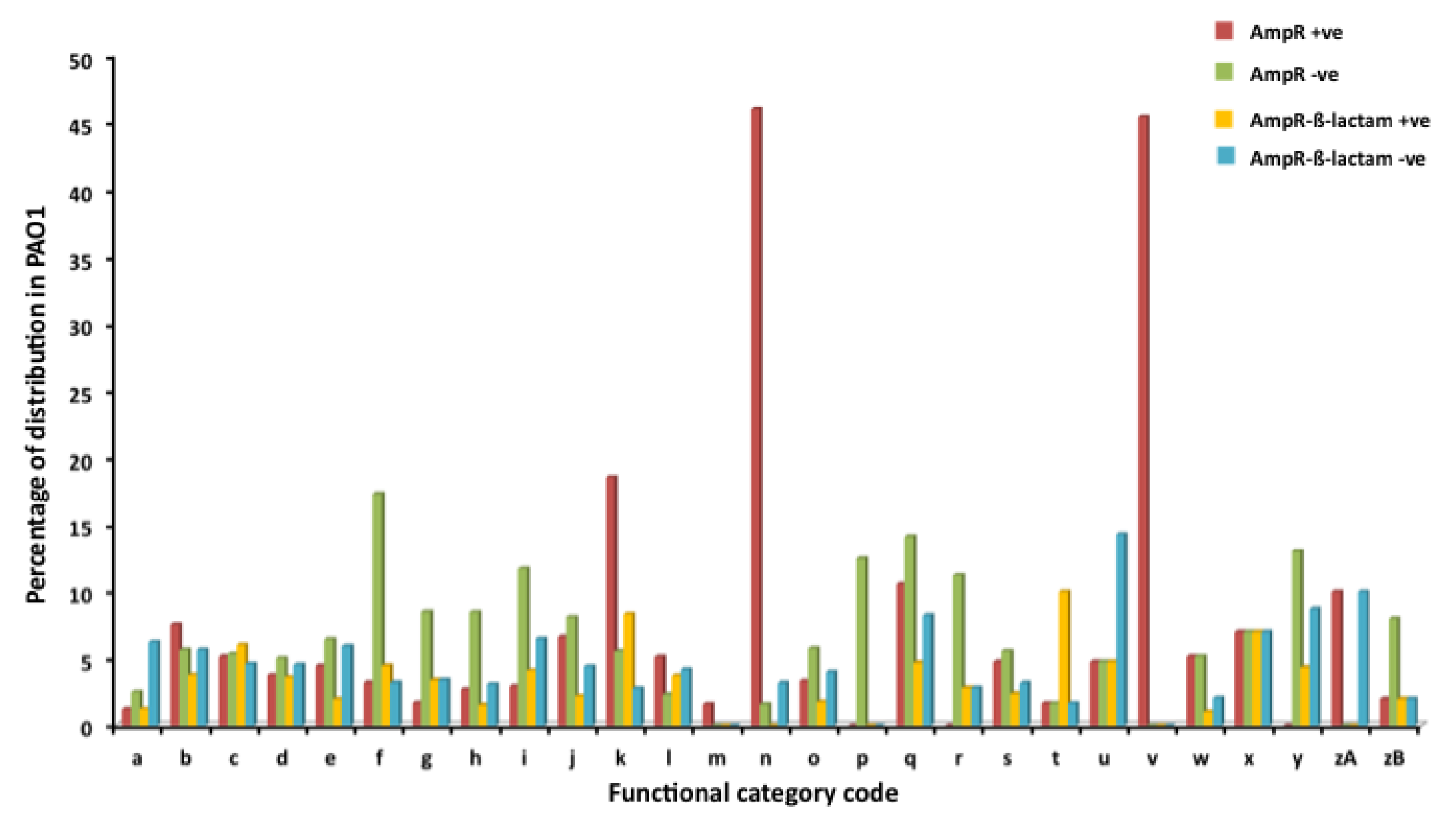


Figure 5.3: Functional categorization of AmpR-regulated genes. AmpR differentially regulated genes are functionally categorized and expressed as a percentage of the respective category in the PAO1 genome (27). The functional categories are (a) DNA replication, recombination, modification and repair; (b) fatty acid and phospholipid metabolism; (c) hypothetical; (d) membrane proteins; (e) amino acid biosynthesis, metabolism; (f) translation, post-translational modification, degradation; (g) cell wall/LPS/capsule; (h) transport of small molecules; (i) energy metabolism; (j) biosynthesis of cofactors, prosthetic groups, carriers; (k) adaptation, protection; (l) transcriptional regulators; (m) two-component regulatory systems; (n) secreted factors- toxins, enzymes, alginate; (o) putative enzymes; (p) chaperones, heat-shock proteins; (q) central intermediary metabolism; (r) nucleotide biosynthesis and metabolism; (s) carbon compound catabolism; $(\mathrm{t})$ motility and attachment; $(\mathrm{u})$ chemotaxis; (v) related to phage, transposon, plasmid; ( $w$ ) non-coding RNA genes; (x) protein secretion, export apparatus; (y) cell division; (zA) antibiotic resistance, susceptibility; (zB) transcription, RNA processing, degradation.

The AmpR negatively regulated genes are involved in translation (category $\mathrm{f}$ 17\%), energy metabolism (category $\mathrm{i}-12 \%$ ), nucleotide metabolism (category $\mathrm{r}-11 \%$ ), chaperones and heat shock (category $\mathrm{p}-13 \%$ ), and cell division (category y - 13\%). Genes involved in motility and attachment (category $\mathrm{t}-10 \%$ ) are positively regulated by AmpR under ß-lactam stress. Similarly, AmpR negatively regulates genes involved in chemotaxis under ß-lactam stress (Figure 5.3).

To determine whether the functional categorization in the different gene sets is significant, enrichment analysis was performed using GOEAST (518). Primarily, gene ontology identifications (GOIDs) belonging to biological processes and cellular components were enriched in the AmpR positively regulated and negatively regulated gene sets, respectively (Appendix; Supplementary information for Chapter 5, Table 5). GOIDs belonging to ribosomal protein biosynthesis and oxidative phosphorylation were statistically significantly enriched in the AmpR-dependent negatively regulated gene set $[\log$ odd ratio $>1.5 ; p$-value $<0.05$; (Appendix; Supplementary information for Chapter 5, Table 5)]. There was no enrichment in the AmpR-ß-lactam-dependent gene sets. 
In the AmpR-positively regulated gene set, many genes involved in pyoverdine biosynthesis were significantly enriched $[\log$ odd ratio $>2.5$, $p$-value $<0.03$; (Appendix; Supplementary information for Chapter 5, Table 5)]. Also enriched in the same gene set are the genes encoding the major catalase KatA, and the superoxide dismutase SodA. The physiological effects of enrichment of these genes on iron acquisition and oxidative stress response, and the role of $\mathrm{AmpR}$ in their regulation are discussed in later sections. Furthermore, genes such as the QS regulator $r h l R$, the stress phase sigma factor rpoS, which were identified to be AmpR-regulated previously (225), also showed significant enrichment (Appendix; Supplementary information for Chapter 5, Table 5).

\section{Regulation of small RNAs (sRNAs) by AmpR}

RNA-Seq allows detection of expression profiles of sRNAs. Previous microarray studies with PAO $\triangle a m p R$ showed dysregulation of the small regulatory RNA rgRsmZ (225). This led us to hypothesize that other sRNAs may also be AmpR-regulated but were not detected in the microarray studies due to technical limitations. RNA-Seq analysis of the $\operatorname{ampR}$ mutant revealed dysregulation of many non-coding RNAs (ncRNAs), both in the absence and presence of sub-MIC B-lactam stress (Appendix; Supplementary information for Chapter 5, Tables 2, 3). Some of these were tRNAs, which is expected given their abundance. Downregulated ncRNAs in PAO $\triangle a m p R$ (AmpR positive regulation) include PA1030.1 (P8; uninduced -30-fold, $p$-value 2.14E06; induced -92-fold, $p$-value 1.03E-14), and prF1 (PA4704.1; uninduced -4-fold, $p$ value $0.00 \mathrm{E}+00$; induced NS), whereas expression of $P 7$ (PA0887.1; uninduced 6.3-fold,

$p$-value 0.00E +00 ; induced 3.1-fold, $p$-value 5.35E-14) and amiL (PA3366.1; uninduced 
2.5-fold, $p$-value 1.82E-13; induced NS) were upregulated (AmpR negative regulation). Interestingly, rgRsmZ, which was detected in the microarray analysis, was not detected in RNA-Seq but dysregulation was confirmed by qPCR (discussed in the following sections).

AmpR mediated regulation of some of the sRNAs was determined by qPCR. AmpR was found to positively regulate expression of PA5181.1 (P34; RQ: uninduced $0.39 \pm 0.06, p$-value 0.0003 ; induced $0.38 \pm 0.016, p$-value 0.002). Expression of $P 34$ requires RpoS (519). Since AmpR positively regulates RpoS (225), AmpR regulation of P34 is likely via RpoS. Positive regulation of P32 (PA4758.1) by AmpR and its physiological effects on the AmpR mutant strain are discussed in the section on heatshock response. Further, AmpR was also found to positively regulate the antisense RNA asPrrF1 (Appendix; Supplementary information for Chapter 5, Table 2) and is discussed further in the following section.

\section{AmpR regulates iron uptake positively}

Iron is critical in many biological reactions across kingdoms and $P$. aeruginosa is no exception. However, freely available iron is in a poorly soluble and biologically unusable ferric $\left(\mathrm{Fe}^{3+}\right)$ form at neutral $\mathrm{pH}$ in aerobic conditions (107). In order to circumvent this issue, $P$. aeruginosa has evolved high affinity iron uptake systems mediated by siderophores. Siderophores are iron chelators that bind extracellular iron and transport it to receptors on the cell surface [reviewed in (107)]. P. aeruginosa can produce and take up heme- or iron-siderophore complexes (520). In addition, $P$. aeruginosa also synthesizes outer membrane receptors for siderophores produced by 
other bacteria including pyoverdines produced by other pseudomonads (521), aerobactin (522) and enterobactin $(522,523)$. P. aeruginosa produces two main types of siderophores, pyoverdine and pyochelin (107). Pyoverdine is the green-yellow fluorescent pigment that is produced typically under conditions of iron limitation (524). The $p v d$ genes encode pyoverdine and are clustered (PA2385-PA2426) on the PAO1 genome (27). RNA-Seq analysis of the $\operatorname{ampR}$ mutant revealed downregulation (3-fold to 103-fold) of many $p v d$ genes (Appendix; Supplementary information for Chapter 5, Table 2). This includes $p v d S$ (PA2426), the ECF sigma factor (uninduced -7.6, $p$-value 3.49E-09, induced -38.2. $p$ value $1.93 \mathrm{E}-08)$ that is known to regulate expression of the $p v d$ genes (525). Genes encoding the second siderophore system, pyochelin, are part of a gene cluster (PA4220PA4231) and consist of three operons (PA4220-PA4221, PA4222-PA4226, PA4228PA4231). Genes of all these operons (PA4224-PA4226, PA4228-PA4231) are significantly downregulated (14-fold to 193-fold) in PAO $\triangle a m p R$ in a ß-lactamindependent manner (Appendix; Supplementary information for Chapter 5, Table 2). This is also reflected in the GOEAST analysis of the AmpR-positively regulated gene set revealing a significant enrichment (log odds ratio $\geq 1.0, p$-value $\leq 0.05)$ of the $p v d$ genes (Appendix; Supplementary information for Chapter 5, Table 5). These findings suggest that AmpR is potentially involved in iron uptake.

Further, comparison of AmpR-regulated genes from this study with the ironrelated genes identified as part of a previous transcriptome metaanalysis study (400) revealed overlaps (Figure 5.4A). Genes involved in pyochelin biosynthesis are part of the 19 genes that are shared between the iron-regulated gene set and AmpR-positively regulated genes (Figure 5.4A). The overlapping genes also include prpL, a PvdS- 
regulated protease that has been implicated in virulence (526) and $p f e R$ encoding the transcriptional regulator of the PfeSR two-component system that positively regulates the enterobactin outer membrane receptor [PfeA; (527)]. Moreover, asPrrF1, which is a positive regulator of iron uptake, is also downregulated in PAO $\triangle a m p R$ in the RNA-Seq (uninduced: 4-fold, $p$-value $<0.001$; induced: NS) and qPCR (uninduced: $0.47 \pm 0.04, p$ value 0.004; induced: NS) assays. However, the master repressor of iron uptake Fur (143) is not significantly differentially regulated in the ampR RNA-Seq analyses. Downregulation of genes involved in siderophore biosynthesis, and comparison with previous metaanalysis studies led us to hypothesize that AmpR plays a positive regulatory role in iron uptake.

To further study the function of AmpR in iron uptake, growth curves were performed in iron-limited synthetic succinate media [SSM, (528)]. Deletion of AmpR resulted in impaired growth in SSM compared to PAO1 (orange lines; Figure 5.4B). Maximum growth difference between the strains was seen in the log and early stationary phases ( $p$-value $<0.0001$ at all time points between 4 and 14 hours) of growth (orange lines; Figure 5.4B). The reduced growth of PAO $\triangle a m p R$ in iron-deficient media can be a result of impairment in either uptake or utilization of iron. To address this question, growth curves were performed with exogenously added $\mathrm{FeCl}_{3}$, making the media ironreplete (blue lines; Figure 5.4B). Addition of iron to the media enhances the growth rate of both PAO1 and PAO $\triangle a m p R$ (blue lines; Figure 5.4B), compared to growth in SSM without exogenously added iron (orange lines; Figure 5.4B). Moreover, under ironreplete conditions, growth of the two strains is very similar till about 10 hours after which the $\operatorname{ampR}$ mutant again shows significantly reduced growth (blue lines; Figure 5.4B). 
A

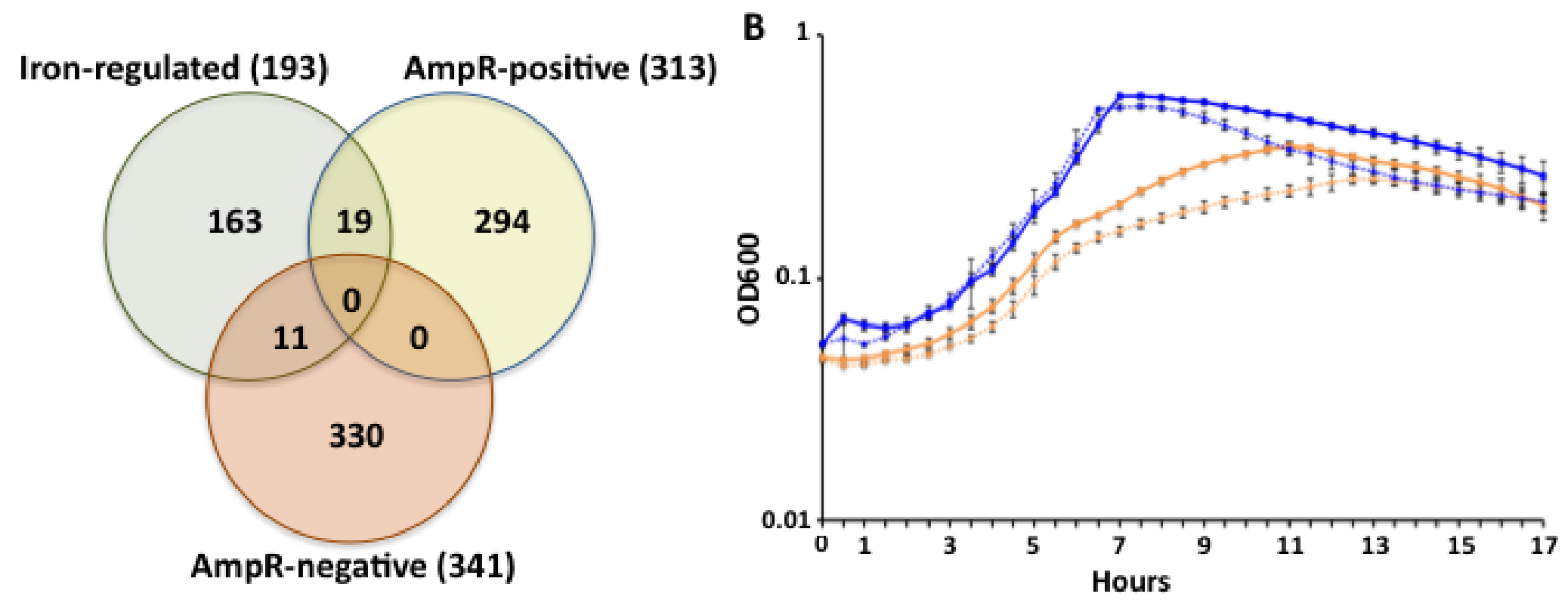

Figure 5.4: AmpR regulates iron uptake. (A) Comparing the AmpR positively and negatively regulated genes against the iron-regulated gene set shows overlaps between the datasets. (B) Growth in iron-limited media of PA01 (solid lines) and PAO $\triangle a m p R$ (dashed lines) in the absence (orange lines) and presence (blue lines) of exogenously added $\mathrm{FeCl}_{3}$. 
Thus, in the presence of excess iron in the log phase, PAO $\triangle a m p R$ shows no growth deficiency.

This observation, and the transcriptome data, strongly suggests that AmpR plays a role in iron uptake, and not iron utilization. The growth impairment seen under ironreplete conditions with PAO $\triangle a m p R$ between 10 and 16 hours is significant (blue lines, Figure 5.4B; $p$-value $\leq 0.0003$ at all points). This is possibly due to the fact that PAO $\triangle a m p R$ uses up all the freely available iron to maintain growth rates similar to PAO1 for the first 10 hours of the experiment. When conditions start to become iron limiting (after 10 hours, Figure 5.4B), continued growth of PAO $\triangle a m p R$ is hampered due to impaired iron uptake. Moreover, under iron-replete conditions, neither strain produced pyoverdine, seen visually as a lack of yellow-green color of the cultures (data not shown), suggesting pyoverdine-independent iron uptake. $P$. aeruginosa also has an uncharacterized low affinity iron uptake system that functions under iron replete conditions (P. Cornelis, personal communication) and a citrate-mediated iron uptake system (529), potentially explaining growth.

In addition to the siderophore-mediated uptake, expression of the heme acquisition protein HasAp (PA3407) is downregulated in an AmpR-ß-lactam-dependent manner [34.9-fold downregulated, $p$-value 8.5E-08; (Appendix; Supplementary information for Chapter 5, Table 3)]. This further attests to the role of AmpR in iron uptake in P. aeruginosa. The $\mathrm{pH}$ of the media is known to influence growth in SSM (528) but there was no difference in the $\mathrm{pH}$ of the media between the strains (data not shown). Thus, the gene expression and phenotypic data clearly indicate a positive regulatory role for AmpR in iron uptake in P. aeruginosa. 


\section{P. aeruginosa AmpR regulates heat-shock response by modulating rgP32 expression}

RNA-Seq analysis revealed that AmpR positively regulates the small RNA rgP32 [20.8-fold downregulated in PAO $\triangle a m p R, p$-value 7.77E-14; (Appendix; Supplementary information for Chapter 5, Table 2)]. P32 is the last gene of a three-gene operon with $d n a J$ and $d a p B$ (27). DnaJ is part of the Hsp70 heat-shock response system (530). The DnaJ-DnaK-GrpE (PA4760-PA4762) chaperone Hsp70 system prevents premature folding of nascent polypeptides and, along with the GroEL (Hsp60) system, helps in the heat-shock response in bacteria $(530,531)$. DnaK is the $P$. aeruginosa homolog of E. coli Hsp70 (27). Conversion between the ATP- or ADP-bound forms of DnaK are controlled by DnaJ and GrpE, which function as a co-chaperone and a nucleotide exchange factor, respectively (532). Given that the small RNA is part of the operon encoding for the Hsp70 system, we hypothesized differential regulation of the Hsp70 heat shock system in PAO $\triangle a m p R$. qPCR analysis showed that the expression of $g r p E$ (RQ: uninduced $0.51 \pm$ $0.004, p$-value 0.0017 ; induced $0.38 \pm 0.003, p$-value 0.0003 ), dnaJ (RQ: uninduced 0.65 $\pm 0.045, p$-value 0.015 ; induced $0.6 \pm 0.005, p$-value 0.0004$)$, and $d n a K$ (RQ: uninduced $0.45 \pm 0.02, p$-value 0.0019 ; induced $0.62 \pm 0.007, p$-value 0.0028$)$ are downregulated in PAO $\triangle a m p R$ (Figure 5.5A). The positive regulation is independent of sub-MIC ß-lactam in the system (Figure 5.5A). This suggests that the cell wall intermediate products, shown previously to be probable AmpR effector molecules in inducing ß-lactam resistance $(121,410)$, play no role in AmpR-mediated regulation of Hsp70-encoding genes.

Downregulation of the Hsp70 heat-shock system genes in the ampR mutant led us to hypothesize that, compared to PAO1, PAO $\triangle a m p R$ would behave differently at higher temperatures. However, growth curves of the $a m p R$ mutant were not different from 
PAO1 at $30^{\circ} \mathrm{C}, 37^{\circ} \mathrm{C}$ or $43^{\circ} \mathrm{C}$ (data not shown). The heat tolerance of the two strains was then examined by enumerating CFUs after exposure of both log- and stationary-phase cells to $50^{\circ} \mathrm{C}$, inducing the cellular heat-shock response. There was no significant difference between the input cells and the control cells (grown at $30^{\circ} \mathrm{C}$ ) for either the log or stationary phases. However, PAO $\triangle a m p R \log$-phase cells were more susceptible compared to PAO1, with $>90 \%$ of cells killed after three hours of $50^{\circ} \mathrm{C}$ exposure ( $p$-value 0.0018 ; Figure 5.5B). The stationary phase cells also followed a similar trend as the log phase cells. Loss of $\operatorname{ampR}$ led to a $99.9 \%$ loss in cell viability compared to PAO1 when stationary phase cells were exposed to $50^{\circ} \mathrm{C}$ for one hour ( $p$-value 0.0014 ; Figure 5.5B).

The stationary phase cells are more sensitive to the elevated temperature compared to the log phase cells, and show a 2-log greater drop in CFU even after a brief exposure (Figure $5.5 \mathrm{~B}$ ). This is counterintuitive since stationary phase cells are thought to be more resistant to changing conditions compared to log phase cells. Downregulation of the stationary phase sigma factor RpoS in PAO $\triangle a m p R$ (225) potentially plays a role in the enhanced sensitivity of PAO $\triangle a m p R$ cells. Regulation of the heat-shock sigma factor RpoH was also not significantly different between the strains in the RNA-Seq analysis (data not shown). This, however, is not surprising since expression of transcriptional regulators are typically not highly differentially regulated to achieve effective function. Dysregulation of the $h s p 70$ genes, confirmed by reduced temperature tolerance of $\mathrm{PAO} \triangle a m p R$, suggests a positive regulatory role for AmpR in the heat-shock response of P. aeruginosa. 

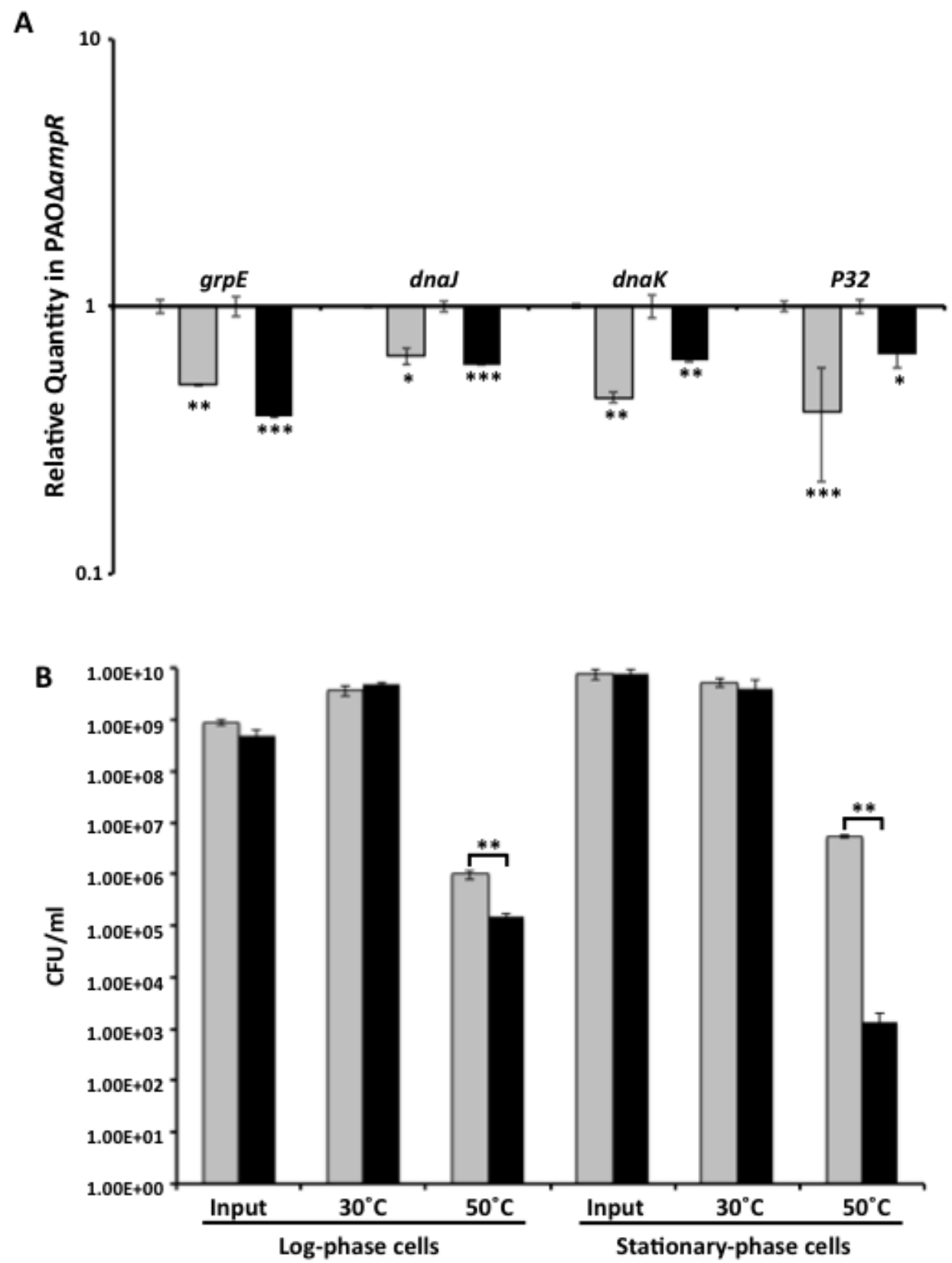

Figure 5.5: Regulation of heat-shock response by AmpR. (A) qPCR of $h s p 70$ genes: RNA was isolated from PAO1 and PAO $\triangle a m p R$ cells, without and with sub-MIC B-lactam stress, reverse transcribed to cDNA, and tested by qPCR with gene-specific primers, as described in the text. Relative gene expression in PAO $\triangle a m p R$ is shown without (grey bars) and with (black bars) sub-MIC ß-lactam exposure. Values have been normalized to expression in PA01 under the same conditions $(\log 10$ $\mathrm{RQ}=1$ ) and bars above and below the threshold represent up- and down-regulation, respectively. (B) CFU counts of heat-shock exposed and unexposed cells in the log and stationary growth phases: Cells grown at $30^{\circ} \mathrm{C}$ were $0 D 600$ normalized, split into two aliquots and maintained at $30^{\circ} \mathrm{C}$ and $50^{\circ} \mathrm{C}$ for varying periods of time ( 3 hours for log phase, 1 hour for stationary phase) before enumeration. Data of PAO1 (grey bars) and PAO $\triangle a m p R$ (black bars) are represented. $p$-values $*<0.02, * *<0.003, * * *$ $<0.0005$. 


\section{AmpR positively regulates $P$. aeruginosa oxidative stress response}

Hydrogen peroxide $\left(\mathrm{H}_{2} \mathrm{O}_{2}\right)$ is a byproduct of $\mathrm{O}_{2}$ metabolism whose deleterious effects on cells include altered membrane potential (533) and DNA mutation caused by single-stranded nicks (534). Intracellular $\mathrm{H}_{2} \mathrm{O}_{2}$ detoxification is achieved by the enzyme catalase and $P$. aeruginosa has four homologs: KatA (PA4236), KatB (PA4613), KatE (PA2147) and KatN [PA2185; (535)]. Of these, KatA is the major catalase and is expressed in all stages of cell growth but is produced more in the stationary phase (535). RNA-Seq analysis of PAO $\triangle a m p R$ revealed downregulation of katA expression (-2.1-fold) compared to PAO1 in the absence of antibiotic stress, suggesting AmpR-dependent expression (Appendix; Supplementary information for Chapter 5, Table 2). Differential expression of katA was validated using qPCR (RQ uninduced: $0.12 \pm 0.01, p$-value 0.0012). The small RNA rgRgsA (PA2958.1), which requires GacA and RpoS for its expression, contributes to hydrogen peroxide resistance (536). Expression of rgRgsA is downregulated over 2-fold in PAO $\triangle a m p R$ (Appendix; Supplementary information for Chapter 5, Table 2), indicating positive AmpR regulation.

Previous meta-analysis studies of $P$. aeruginosa transcriptomes led to the identification of genes that were specifically differentially regulated under oxidative stress conditions (400). The differential regulation of katA and rgRgsA in PAO $\triangle a m p R$ prompted comparison of the AmpR-regulated genes with the oxidative stress gene set. Seventy genes were shared between the two conditions, 48 and 22 of which are positively and negatively regulated by $\mathrm{AmpR}$, respectively (Figure 5.6A). The 48 positively regulated genes include the major $P$. aeruginosa catalase katA, and four genes involved in PQS signal biogenesis [pqsA (PA0996), pqsE (PA1000), phnA (PA1001) and phnB 
(PA1002)]. Interestingly, 22 of the 48 genes are clustered in a single locus on the genome that is involved in the production of R-and F-type pyocins $(460,537)$, and are located in RGPs, RGP03 and RGP04 (33). These genes were also identified in a previous transcriptome study to be AmpR-regulated (225). Most of these $22 \mathrm{AmpR}$-downregulated genes that are shared with the oxidative stress gene set are involved in metabolism including six of the nuo genes, which synthesize components of NADH dehydrogenase I (538). Another member of the LTTR family of transcriptional regulators, OxyR regulates katA expression in response to oxidative stress (539). Although ampR deletion in PAO1 did not affect $\operatorname{oxy} R$ expression in the RNA-Seq analysis (data not shown), qPCR analysis revealed that AmpR positively regulates $\operatorname{oxy} R$ expression (RQ uninduced: $0.31 \pm 0.012$, $p$-value 0.001).

To determine whether reduced expression of katA and other oxidative stressresponse genes translates into an observable phenotype, the $\mathrm{H}_{2} \mathrm{O}_{2}$ sensitivity of PAO1 and PAO $\triangle a m p R$ was compared using the gradient plate method (540). PAO $\triangle a m p R$ demonstrates a concentration-dependent reduced growth compared to PAO1 on the $\mathrm{H}_{2} \mathrm{O}_{2}$ gradient (Figure 5.6B), suggesting an impaired resistance to oxidative stress. This finding is in agreement with downregulation of oxidative stress response genes in PAO $\triangle a m p R$.

Thus, the transcriptomic and phenotypic data demonstrate a role for AmpR in positively regulating oxidative stress response in $P$. aeruginosa. 
A

Oxidative stress (288) AmpR-positive (313)

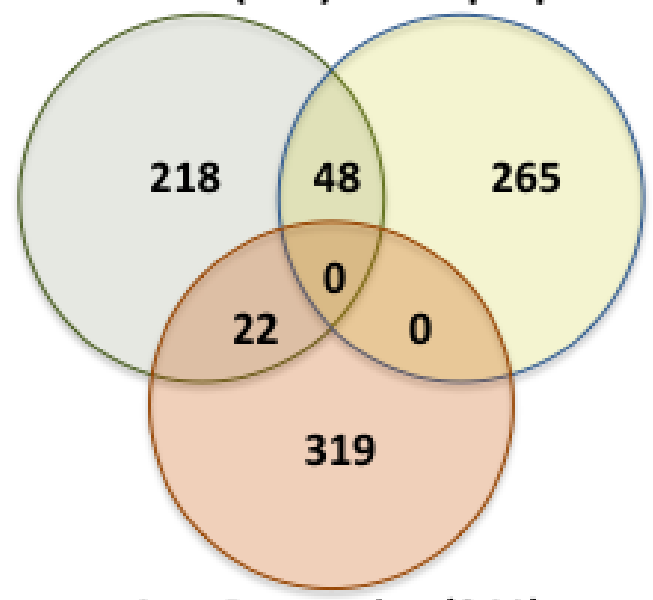

AmpR-negative (341)
B

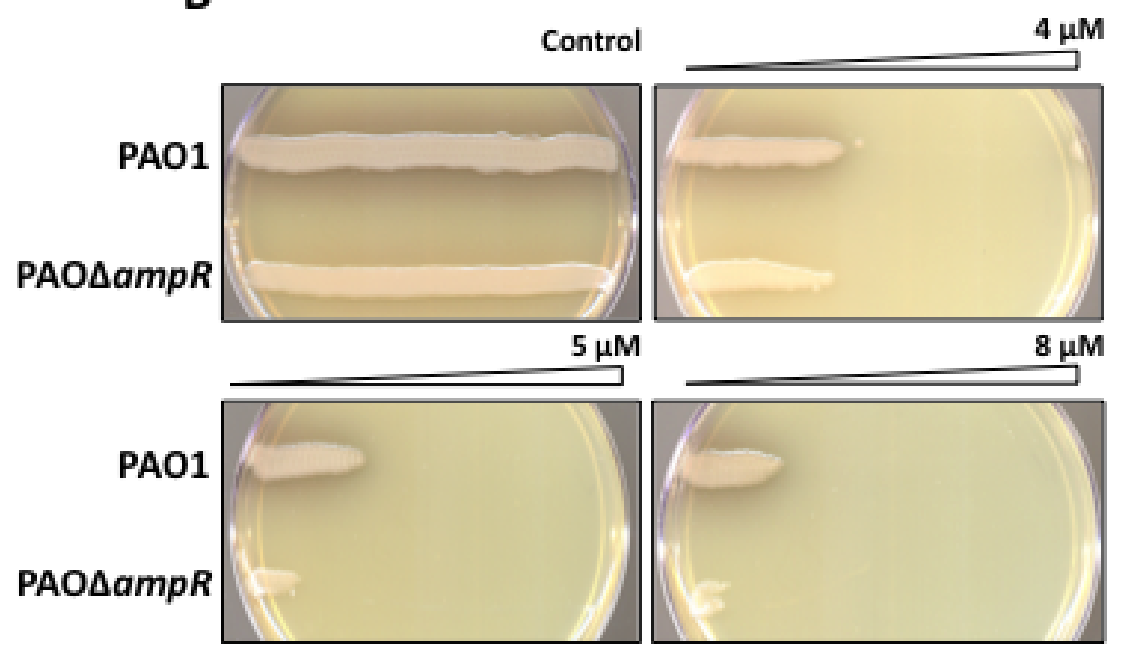

Figure 5.6: AmpR regulates resistance to oxidative stress. (A) Comparing the AmpR positively and negatively regulated genes against the oxidative stress gene set shows overlaps between the datasets. Overlapping genes include those identified previously to play a role in the oxidative stress response. (B) Gradient plates demonstrate decreased resistance of $\mathrm{PAO} \Delta a m p R$ to $\mathrm{H}_{2} \mathrm{O}_{2}$, compared to the control plate without $\mathrm{H}_{2} \mathrm{O}_{2}$. Representative data from four independent experiments is shown. 


\section{AmpR regulates phenazine production by modulating expression of phzA1-G1 and}

\section{phzA2-G2 operons}

The PAO $\triangle a m p R$ strain is impaired in producing pyocyanin (225). P. aeruginosa PAO1 and PA14 have two redundant operons phzA1-G1 (PA4210-PA4216; phz1 operon) and phzA2-G2 (PA1899-PA1905; phz2 operon) that are involved in biosynthesis of the phenazine precursor, phenazine-1-carboxylic acid (27). This precursor is then sequentially modified by a methyltransferase (PhzM, PA4209) and a monooxygenase (PhzS, PA4217) to form pyocyanin (541). All the genes involved in pyocyanin biosynthesis and export (phzl and phz2 operons, phzM, phzS, mexGHI-opmD) was significantly downregulated in the $\operatorname{ampR}$ mutant (positive AmpR regulation) in the RNASeq analysis [Figure 5.7A; (Appendix; Supplementary information for Chapter 5, Table 2)]. Positive AmpR regulation of $p h z A 1, p h z A 2$ (the first genes of $p h z$ operons), $p h z H$ and $p h z M$ was also confirmed by qPCR (Figure 5.7B). The GOIDs under which $p h z M$ and $p h z S$ are classified (antibiotic metabolic processes, antibiotic biosynthetic processes, drug metabolic processes) also showed functional enrichment [log-odd ratio 3.46, $p$-value 0.03; (Appendix; Supplementary information for Chapter 5, Table 5)]. Recently, it has been demonstrated that, in P. aeruginosa PA14, the phzl operon is expressed in liquid cultures whereas the $\mathrm{phz} 2$ operon is responsible for phenazine production in solid cultures (541). It is therefore interesting to note that in PAO $\triangle a m p R$, both operons are downregulated in liquid cultures, indicating positive AmpR regulation. The findings are possibly attributable to strain differences but are worth further investigation. 
A

\begin{tabular}{|lllcc|}
\hline Gene ID & $\begin{array}{c}\text { Gene } \\
\text { Name }\end{array}$ & $\begin{array}{c}\text { Pold } \\
\text { Change }\end{array}$ & $\begin{array}{c}\text { Corrected } \\
\text { p-value }\end{array}$ \\
\hline PA1899 & phzA2 & probable phenazine biosynthesis protein & -203 & $0.00 \mathrm{E}+00$ \\
\hline PA1900 & phzB2 & probable phenazine biosynthesis protein & -270.6 & $0.00 \mathrm{E}+00$ \\
\hline PA1901 & phzC2 & phenazine biosynthesis protein PhzC & -8.4 & $9.74 \mathrm{E}-14$ \\
\hline PA1902 & phzD2 & phenazine biosynthesis protein PhzD & -117.4 & $0.00 \mathrm{E}+00$ \\
\hline PA1903 & phzE2 & phenazine biosynthesis protein PhzE & -91.6 & $0.00 \mathrm{E}+00$ \\
\hline PA1904 & phzF2 & probable phenazine biosynthesis protein & -13.8 & $4.10 \mathrm{E}-13$ \\
\hline PA1905 & phzG2 & probable pyridoxamine 5'-phosphate oxidase & -113.1 & $0.00 \mathrm{E}+00$ \\
\hline PA4209 & phzM & probable phenazine-specific methyltransferase & -18.5 & $3.99 \mathrm{E}-15$ \\
\hline PA4210 & phzA1 & probable phenazine biosynthesis protein & -169 & $0.00 \mathrm{E}+00$ \\
\hline PA4211 & phzB1 & probable phenazine biosynthesis protein & -367.3 & $2.88 \mathrm{E}-13$ \\
\hline PA4212 & phzC1 & phenazine biosynthesis protein PhzC & -41.3 & $0.00 \mathrm{E}+00$ \\
\hline PA4213 & phzD1 & phenazine biosynthesis protein PhzD & -109.8 & $5.95 \mathrm{E}-14$ \\
\hline PA4214 & phzE1 & phenazine biosynthesis protein PhzE & -77.8 & $4.83 \mathrm{E}-14$ \\
\hline PA4215 & phzF1 & probable phenazine biosynthesis protein & -21.9 & $4.29 \mathrm{E}-14$ \\
\hline PA4216 & phzG1 & probable pyridoxamine 5'-phosphate oxidase & -10.9 & $9.50 \mathrm{E}-13$ \\
\hline PA4217 & phzS flavin-containing monooxygenase & -7.7 & $0.00 \mathrm{E}+00$ \\
\hline
\end{tabular}

B

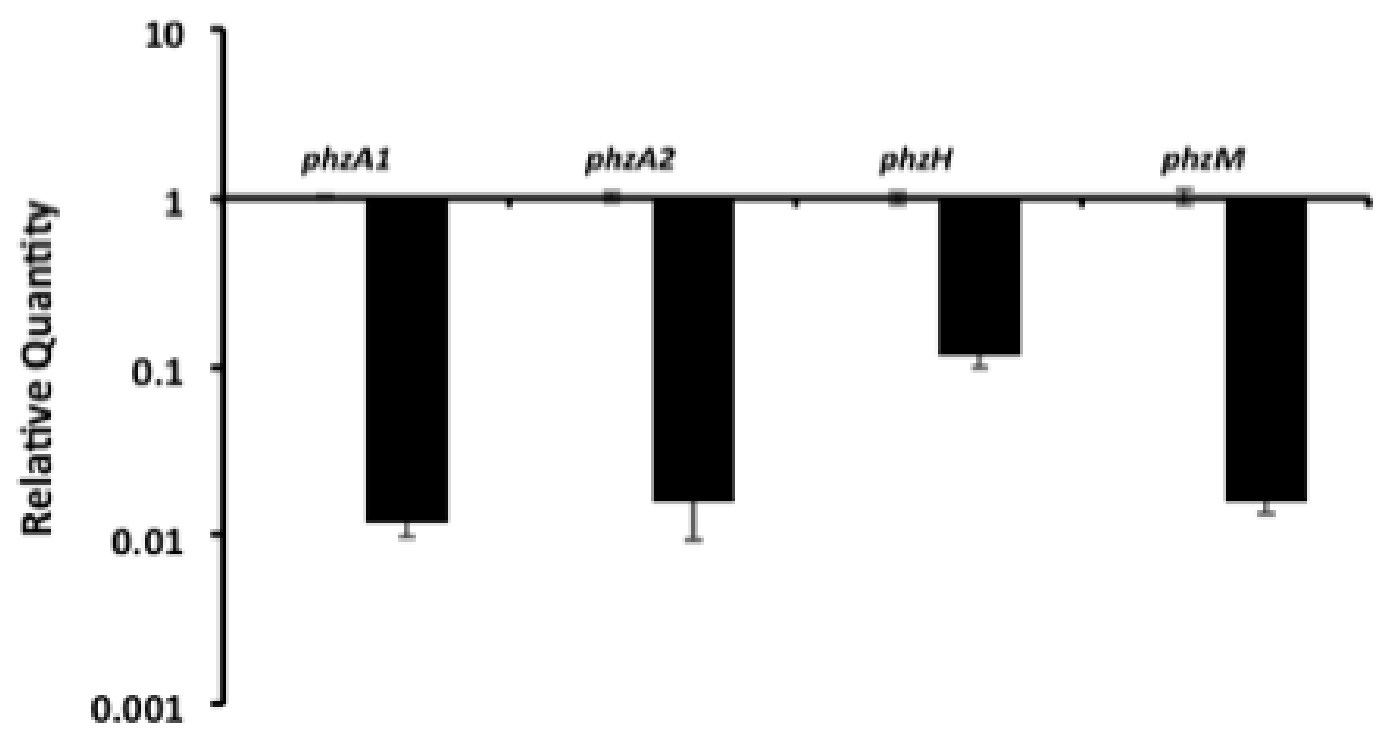

Figure 5.7: Regulation of phenazine genes by AmpR. (A) Genes involved in phenazine biosynthesis are significantly downregulated in PAO $\triangle a m p R$ as seen in RNA-Seq analysis. (B) Differential regulation of the first genes of the phenazine biosynthetic operons $p h z A 1$ and $p h z A 2$, and the modifying enzymes $p h z H$ and $p h z M$ were validated by qPCR. 
The phzl operon is known to be QS regulated while the regulators of the phz2 operon are not known $(388,541)$. Downregulation of the $p h z 2$ operon in PAO $\triangle a m p R$ suggests a potential positive regulatory role for AmpR of this operon and is a novel finding.

\section{The PQS system is also positively regulated by AmpR}

Of the 654 AmpR-dependent genes, 313 and 341 genes are positively and negatively regulated, respectively. Genes that are positively regulated include the QSregulated genes lasA, lasB, rhlAB and rhlR (Appendix; Supplementary information for Chapter 5, Table 2). In addition, AmpR also positively regulates the $h c n A B C$ operon, which is QS-regulated and is responsible for hydrogen cyanide biosynthesis (373). These concur with our previous findings that AmpR is a positive regulator of some QS phenotypes (225) and positive regulation of las $R$ and $r h l R$ expression by AmpR was confirmed by qPCR (Figure 5.8). Furthermore, even under sub-MIC ß-lactam stress, AmpR positively regulated expression of $l a s R$ (RQ induced: $0.84 \pm 0.03, p$-value 0.001 ) and $r h l R$ (RQ induced: $0.71 \pm 0.007, p$-value 0.0001$)$.

The PQS system is a critical part of QS signaling in P. aeruginosa, and complements the las and $r h l$ systems $(361,542)$. Genes of the two operons pqsABCDE (PA0996-PA1000) and phnAB (PA1001-PA1002), and pqsH (PA2587) are involved in PQS biosynthesis (543). PhnAB converts the PQS precursor chorismate to anthralinate, which is further converted to the signaling molecule PQS by PqsA-D and PqsH $(380,543)$. The RNA-Seq data shows that AmpR positively regulates all these genes except for $p q s H$ (Appendix; Supplementary information for Chapter 5, Table 2). 


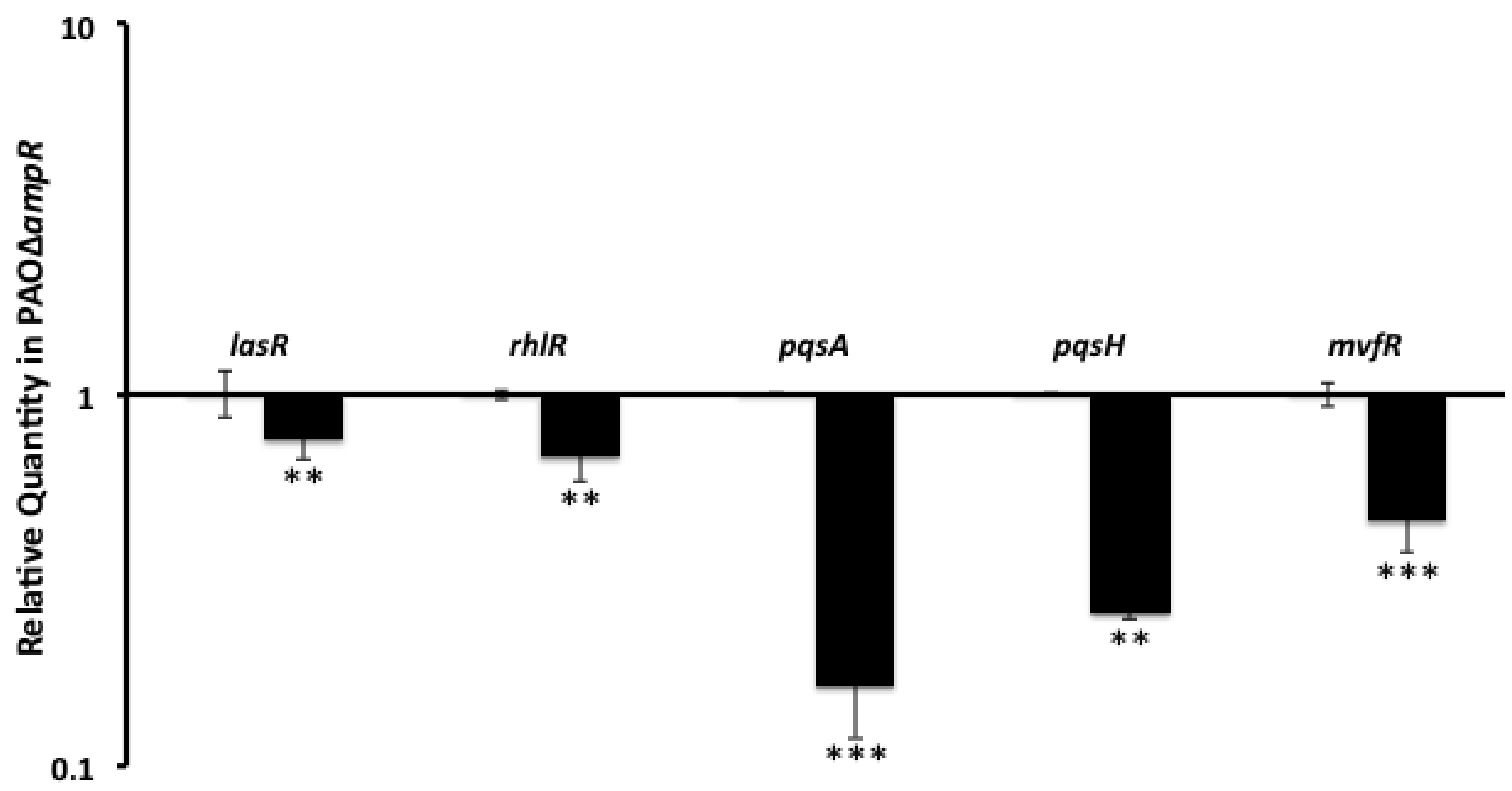

Figure 5.8: AmpR positively regulates critical QS regulators of the Las, Rhl and PQS systems. Expression of the genes was determined by qPCR. The expression levels in PAO $\triangle a m p R$ are shown, normalized to expression in PAO1. $p$-values $*<0.02, * *<0.003, * * *<0.0005$. 
We validated the RNA-Seq data using qPCR and demonstrate positive regulation of the first gene of the pqs operon ( $p q s A$ ), in addition to $p q s H$ (Figure 5.8). MvfR (PA1003) lies downstream of the pqs genes and positively regulates genes in this cluster (224,544). qPCR analysis reveals that AmpR positively regulates $m v f R$ expression (Figure 5.8). Thus, it is likely that AmpR-mediated regulation of the PQS system in $P$. aeruginosa is via MvfR.

In addition, QscR (PA1898), which is a QS regulator in P. aeruginosa (280) and is in the same locus as and upstream of the phzA2 operon, was also downregulated in the $\operatorname{ampR}$ mutant, as determined by qPCR (RQ: uninduced- $0.38 \pm 0.17, p$-value 0.0057 ). This further supports the role of AmpR as a QS regulator.

\section{V5-tagged AmpR is functional in vivo}

The previous and current study suggests that the AmpR regulon in $P$. aeruginosa is extensive $(225,425)$. However, it is highly unlikely that all the genes are under direct AmpR regulation. AmpR possibly indirectly controls a subset of genes. Accordingly, we identified transcriptional regulators in the AmpR regulon that could be potential targets of AmpR direct regulation [Appendix; Supplementary information for Chapter 5, Tables 2, 3, (225)]. Moreover, using the putative AmpR binding site (434), in silico analysis of the P. aeruginosa PAO1 genome identified potential genes that have an AmpR-binding site in their promoter (225). Some of these targets were confirmed to be differentially regulated using DNA microarrays (225). However, direct AmpR targets have not been demonstrated as yet. 
In order to identify the direct targets of AmpR, ChIP-Seq studies were performed using a 3x-V5-tagged AmpR integrated as a single copy on the chromosome. Since AmpR has a positive regulatory role in B-lactam resistance, the functionality of the tagged AmpR was verified by determining the MIC. Amoxicillin had an MIC of $4 \mu \mathrm{g} / \mathrm{ml}$ for PAO $\triangle a m p R$ whereas the wild-type PAO1 is resistant $(>256 \mu \mathrm{g} / \mathrm{ml})$. The MIC of amoxicillin on PAO $\triangle a m p R:: a m p R$-V5 was $>256 \mu \mathrm{g} / \mathrm{ml}$, similar to PAO1 indicating that tagging AmpR at the C-terminus did not inhibit function. Chromatin immunoprecipitation was then performed both in the presence and absence of sub-MIC Blactam stress. Before proceeding with the high-throughput sequencing, validity of the pulldown was tested by qPCR for $\mathrm{P}_{a m p C}$. AmpR occupancy data for the $a m p C$ promoter revealed that compared to the input DNA, the ChIP DNA showed a $17.1 \pm 1.2$-fold and $21.5 \pm 4.3$-fold higher occupancy, in the absence and presence of $\beta$-lactam stress, respectively. This demonstrated that the tagged AmpR protein was able to effectively bind the ampC promoter in vivo. Thus, the MIC and ChIP-qPCR studies confirmed in vivo functionality of the V5-tagged AmpR protein.

\section{Identifying direct AmpR targets by ChIP-Seq}

After confirming functionality of the tagged AmpR protein in vivo, ChIP and input DNA samples were processed on the Helicos sequencer. Data analysis was performed on the CLC Genomics Workbench as described in the methods section. The target regions on the PAO1 genome that AmpR binds to are shown in Table 1. All the loci in the table have Wilcoxon $p$-values $<3.0 \mathrm{E}-05$ and, thus, are significant. In agreement with the ChIP-qPCR data (previous section), ChIP-Seq data showed that 
Table 5.1: AmpR ChIP peaks. ChIP-Seq studies were performed on P. aeruginosa PAO $\triangle a m p R$ strain harboring V5-tagged AmpR in the absence (uninduced) and presence (induced) of sub-MIC $\beta$-lactam stress. Regions on the chromosome that were enriched in the ChIP DNA samples compared to the input DNA are shown. All readings have a Wilcoxon Filter $p$-value $\leq 3.0 \mathrm{E}-05$.

\section{Uninduced}

\begin{tabular}{|c|c|c|c|c|c|c|}
\hline \multirow[b]{2}{*}{ Chromosomal Locus } & \multirow{2}{*}{$\begin{array}{c}\text { Average } \\
\text { Read } \\
\text { Length }\end{array}$} & \multirow{2}{*}{$\begin{array}{l}\text { \% Reads } \\
\text { mapping } \\
\text { to locus }\end{array}$} & \multirow[b]{2}{*}{ Strand } & \multirow{2}{*}{$\begin{array}{c}\text { FDR } \\
(\%)\end{array}$} & \multicolumn{2}{|c|}{ Flanking Genes } \\
\hline & & & & & $5^{\prime}$ & $3^{\prime}$ \\
\hline $586888-586930$ & 42 & 88 & + & $2.0 \mathrm{E}-01$ & $\operatorname{rsm} Y$ & PA0528 \\
\hline 797294-797374 & 80 & 53 & + & $7.5 \mathrm{E}-01$ & PA0728 & PA0729 \\
\hline $901840-901853$ & 13 & 95 & - & $1.9 \mathrm{E}-01$ & $P A 0826$ & $\operatorname{ssr} A$ \\
\hline $1668963-1669003$ & 40 & 95 & + & $1.0 \mathrm{E}+00$ & $f f s$ & PA1531 \\
\hline $1921430-1921543$ & 113 & 95 & + & $1.2 \mathrm{E}+00$ & oprF & $\operatorname{cob} A$ \\
\hline $3123393-3123411$ & 18 & 83 & - & $5.3 \mathrm{E}-02$ & $P A 2763$ & $P A 2764$ \\
\hline $4057616-4057641$ & 25 & 89 & - & $1.6 \mathrm{E}-09$ & $f d x A$ & $r s m Z$ \\
\hline $4592895-4593005$ & 110 & 97 & - & $1.3 \mathrm{E}-04$ & PA4108 & $a m p R$ \\
\hline $4362457-4362649$ & 192 & 88 & + & $1.6 \mathrm{E}+00$ & $P A 4140$ & $P A 4141$ \\
\hline $4782725-4783036$ & 311 & 89 & - & $3.4 \mathrm{E}+00$ & rplA & $r p l K$ \\
\hline $4956459-4956671$ & 212 & 91 & - & $1.9 \mathrm{E}+00$ & $r n p B$ & $P A 4422$ \\
\hline $5387789-5387840$ & 51 & 95 & - & $6.8 \mathrm{E}-02$ & PA4802 & PA4802.1 \\
\hline $5884393-5884467$ & 74 & 94 & + & $1.3 \mathrm{E}+00$ & $s s r S$ & PA5228 \\
\hline $5986032-5986133$ & 101 & 90 & - & $1.7 \mathrm{E}+00$ & $r p m G$ & $r p m B$ \\
\hline $6183549-6183590$ & 41 & 88 & - & $2.6 \mathrm{E}+00$ & PA5492 & polA \\
\hline
\end{tabular}

Induced

\begin{tabular}{|c|c|c|c|c|c|c|}
\hline \multirow[b]{2}{*}{ Chromosomal Locus } & \multirow{2}{*}{$\begin{array}{c}\text { Average } \\
\text { Read } \\
\text { Length }\end{array}$} & \multirow{2}{*}{$\begin{array}{c}\text { \% Reads } \\
\text { mapping } \\
\text { to locus }\end{array}$} & \multirow[b]{2}{*}{ Strand } & \multirow{2}{*}{$\begin{array}{c}\text { FDR } \\
(\%)\end{array}$} & \multicolumn{2}{|c|}{ Flanking Genes } \\
\hline & & & & & $5^{\prime}$ & $3^{\prime}$ \\
\hline 586884-586929 & 45 & 96 & + & $2.3 \mathrm{E}-03$ & $\operatorname{rsm} Y$ & PA0528 \\
\hline $901794-901852$ & 58 & 92 & - & $3.1 \mathrm{E}-03$ & PA0826 & $s s r A$ \\
\hline $1668962-1669008$ & 46 & 98 & + & $6.3 \mathrm{E}-02$ & $f f s$ & PA1531 \\
\hline $3206872-3207029$ & 157 & 96 & + & $1.0 \mathrm{E}-02$ & PA2852.1 & oprI \\
\hline $4057616-4057641$ & 25 & 88 & - & $3.2 \mathrm{E}-05$ & $f d x A$ & $r s m Z$ \\
\hline $4592896-4593007$ & 111 & 73 & - & $2.5 \mathrm{E}-03$ & PA4108 & $a m p R$ \\
\hline $4956491-4956668$ & 177 & 91 & - & $1.9 \mathrm{E}-03$ & $r n p B$ & $P A 4422$ \\
\hline $5308608-5308852$ & 244 & 93 & + & $3.8 \mathrm{E}-02$ & $\operatorname{crcZ}$ & $P A 4726.2$ \\
\hline $5387787-5387840$ & 53 & 94 & - & $7.2 \mathrm{E}-02$ & PA4802 & PA4802.1 \\
\hline $5884382-5884467$ & 85 & 93 & + & $3.7 \mathrm{E}-01$ & $s s r S$ & PA5228 \\
\hline $6183549-6183593$ & 44 & 90 & - & $1.3 \mathrm{E}+00$ & PA5492 & polA \\
\hline
\end{tabular}


AmpR binds to promoter DNA upstream of ampC under both induced and uninduced conditions (Table 1). This is typical of LysR-type transcriptional regulators, which bind their target sequences irrespective of effector binding $(103,104)$ and is also seen in the $\mathrm{P}_{\text {ampC }}$ ChIP-qPCR (previous section).

The region with the least $\%$ false discovery rate (FDR) value, irrespective of inducer presence, is within rgRsmZ (PA3621.1). The locus that was pulled down in ChIP is on the negative strand (Table 5.1) and corresponds to a 25 bp region within the rsmZ gene. AmpR-dependent regulation of the $r \sin Z$ gene was also seen previously in transcriptome studies using microarrays (225) and in the current RNA-Seq analysis (Appendix; Supplementary information for Chapter 5, Table 2). The $\operatorname{rgRNAs} r s m Y$ and rsm Z are thought to be functionally redundant (545) and play a major role in the acute to chronic lifestyle transition of $P$. aeruginosa $(209,210,390)$. Transcription of $r s m Y$ and rsmZ is repressed by NarL [PA3879, (546)], CafA [PA4477, (547)], and MvaT/U [PA4315, (390)] and is activated by GacA [PA2586, (548,549)].

In an attempt to understand the role of AmpR this process, qPCR assays were performed with the other players of this well-established regulatory cascade (Figure 5.9). Two hybrid sensor kinases LadS (PA3974) and RetS (PA4856) have a positive and negative effect, respectively on the sensor kinase GacS $(209,210)$. GacS activates transcription of $\operatorname{rgRsmZ}$ and $\operatorname{rgRsmY}$ indirectly through GacA (390). The $\operatorname{rgRNAs} r s m Y$ and $r s m Z$ sequester the RNA-binding protein RsmA (PA0905), which plays a key role in regulating over 500 genes, controlling the acute versus chronic lifestyle transition (458). qPCR analysis revealed that in the $\operatorname{ampR}$ mutant, expression of ladS (RQ: uninduced 0.35 $\pm 0.018, p$-value $<0.0001$; induced $0.42 \pm 0.012, p$-value $<0.0001$ ), and $\operatorname{rgRsmZ}$ (RQ: 


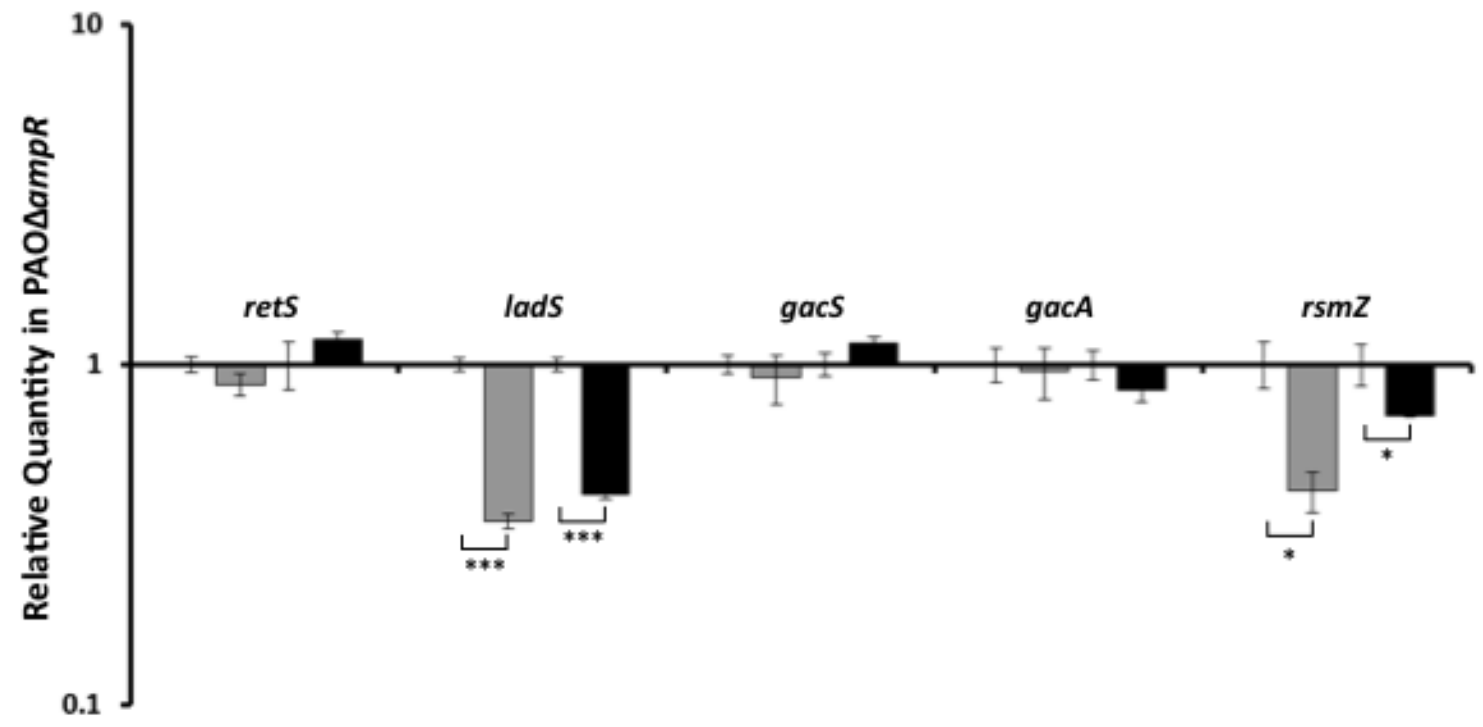

Figure 5.9: Quantitative PCR analysis of Gac-Rsm pathway genes. Relative expression of genes of the RetS-LadS-GacSA-Rsm pathway in PAOLampR compared to PAO1 was analyzed in the absence (grey bars) and presence (black bars) of sub-MIC b-lactam stress. Gene expression in the ampR mutant has been normalized to the corresponding condition in the wild-type strain and expressed as relative numbers of gene-specific transcripts. $p$-values: ${ }^{*} \leq 0.02,{ }^{* * *}<0.0001$.

uninduced $0.43 \pm 0.059, p$-value $=0.01$; induced $0.71 \pm 0.006, p$-value $=0.02$ ) was downregulated, indicating that $A m p R$ is required for their expression (Figure 5.9). No dysregulation of $\operatorname{ret} S, \operatorname{gacS}$ or gacA was observed (Figure 5.9). Our previous microarray studies (225), and our current RNA-Seq, qPCR and ChIP-Seq studies show that AmpR activates transcription of $\operatorname{rgRsmZ}$. Furthermore, $P$. aeruginosa AmpR is a positive regulator of acute virulence factors, many of which were QS-regulated, while negatively regulating chronic infection phenotypes such as biofilm formation (225). The current ChIP-Seq data seems to suggest that this regulation by AmpR is mediated by the regulatory RNA rgRsmZ. 


\section{Binding site analysis}

The ChIP data was used to identify the AmpR binding site. A region of about 200 bp upstream of the genes that were identified to be AmpR-bound (Table 5.1) was used as input for regulatory sequence analysis tools [RSAT; rsat.ulb.ac.be; (423)]. These regions were queried using the putative AmpR-binding site identified earlier (434) and the matrix derived as part of genome-wide analysis of AmpR-binding (225). The AmpR binding sites that were identified in the promoter regions of the AmpR-regulated genes were then used as input to generate a WebLogo under both uninduced and induced conditions (Figure 5.10). The DNA motif that AmpR seems to bind, both in the presence and absence of effectors, is almost identical except for minor changes at positions 1, 2, 3 and 5 (Panels A and B, Figure 5.10). The AmpR motif, like the LTTR box (104), is AT-rich and the bases that are critical for AmpR-binding seems to be A's and T's (at positions 1, 6, 9, 10, 13 and 14; Figure 5.10). 

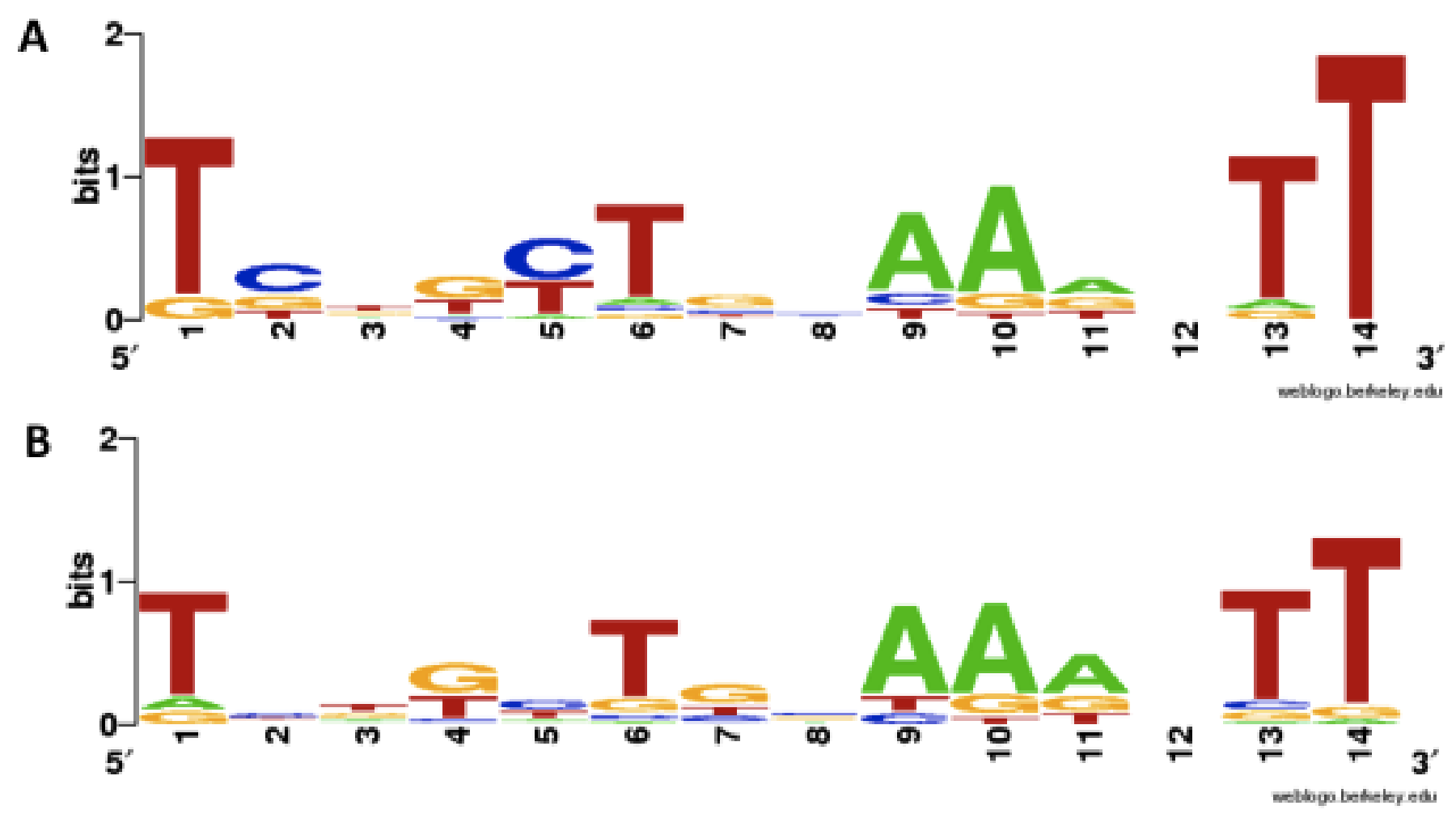

Figure 5.10: ChIP-Seq data based AmpR binding site analysis. Promoters of the geens that were identified by ChIP-Seq to be AmpR-regulated, either in the absence or presence of ß-lactam stress, were scanned for the presence of the putative AmpR-binding motif using RSAT. The binding sites upstream of each gene were then used as input to generate a WebLogo for the uninduced (top panel) and induced (bottom panel). $p$-value 0.001 


\section{LasR is a direct target of AmpR}

The regulator LasR is at the top of the QS regulatory hierarchy in P. aeruginosa $(363,364,370)$. Other regulators, in addition to LasR, regulate the Rhl and PQS systems [reviewed in (511)]. Our findings have identified that AmpR is one of these regulators [Figure 5.8; (225)]. One of these regulators is AmpR, which has been demonstrated previously to positively regulate the Las and Rhl systems transcriptionally (PQS section above) and phenotypically (225). ChIP-Seq data suggested that AmpR regulates the QS master regulator LasR directly (Table 5.1), and the presence of a putative AmpR binding motif upstream of the LasR ORF strengthens the finding. In order to validate the ChIPSeq data, ChIP-qPCR was performed for selected targets using the V5-tagged AmpR strain.

As expected, the $\operatorname{ampC}$ promoter showed 79-fold enrichment in the ChIP DNA compared to the control DNA, demonstrating strong AmpR binding. AmpR pulldown of $\mathrm{P}_{a m p C}$ was also confirmed using a VSVG-tagged AmpR (data not shown), and agrees with previous observations that $\mathrm{AmpR}$ is a positive regulator of $\operatorname{ampC}$ expression by direct binding to $\mathrm{P}_{a m p C}(550)$. ChIP-qPCR data also showed that the lasR promoter showed 3fold enrichment compared to the input DNA indicating direct binding of AmpR to $\mathrm{P}_{\text {lasR }}$. This binding, though not as strong as AmpR binding at $\mathrm{P}_{a m p C}$, is significant. Transcriptome and phenotypic data show that even at this reduced binding AmpR is able to bring about significant changes in the QS system [Figure 5.8, (225)]. 


\section{Comparison of microarray, RNA-Seq and proteome data}

In order to get a comprehensive picture of AmpR regulation in $P$. aeruginosa, we have performed transcriptomics studies, using DNA microarrays (225) and RNA-Seq (this study), and proteomics analysis (unpublished data). The transcriptomics and proteomics studies were performed using the same two strains (PAO1 and PAO $\triangle a m p R$ ), under identical conditions (without and with sub-MIC B-lactam stress). Comparing the microarray and RNA-Seq datasets revealed little overlap in the number of genes regulated (Figure 5.11). This seems contrary to previous findings that demonstrate a better overlap between data from the two techniques (551-553). However, several genes that were identified in the microarray studies to be AmpR-regulated (225) were also identified in the RNA-Seq study. Examples have been discussed in the previous sections and include both positively regulated (numerous QS-regulated genes, ampC) and negatively regulated (mexEF-oprN, alginate regulators) genes. Thus, though the total number of genes showed poor overlap between the transcriptome assays, the phenotypes regulated are the same. Further, similar to previous studies with other systems $(554,555)$, there is a poor overlap between the AmpR transcriptome and proteome studies (Figure 5.11). This can be attributed to a variety of reasons that may be technical (sample preparation, sensitivities of the techniques) or functional (post-transcriptional and/or posttranslational regulation). For example, in Desulfovibrio vulgaricans, variations between mRNA and protein levels were attributed to differences in expression (34-44\%), stability of protein (5\%) and mRNA (2\%) (556). Codon usage, amino acid composition, and the Shine-Dalgarno sequence contributed to an additional $15-26 \%$ variation (555). 
A

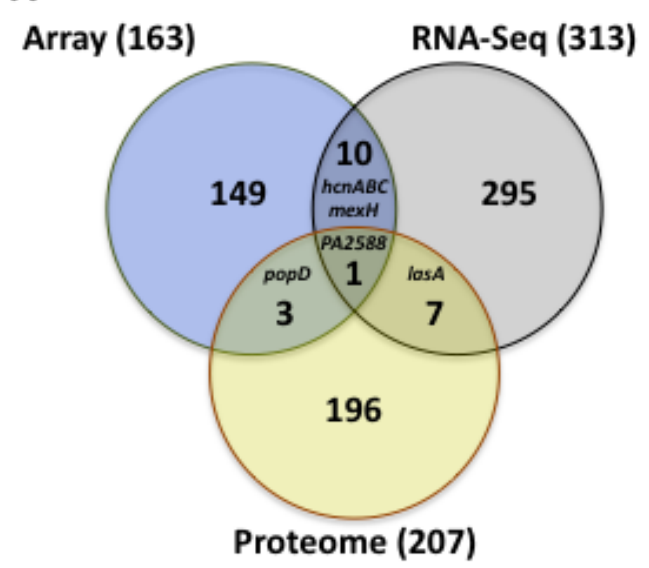

C

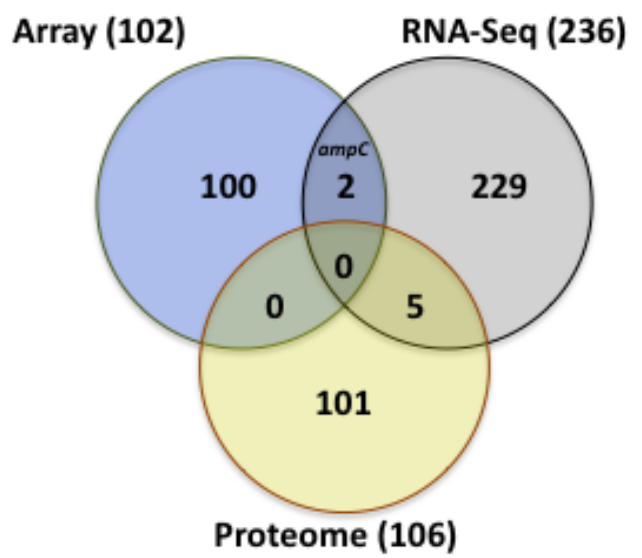

B

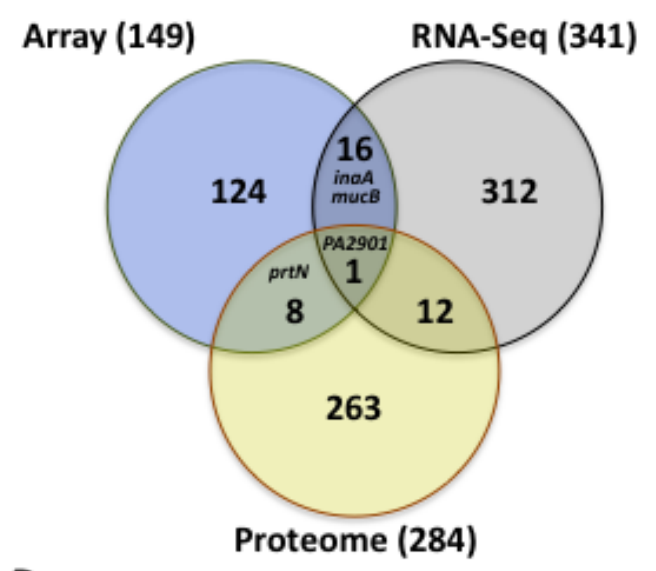

D
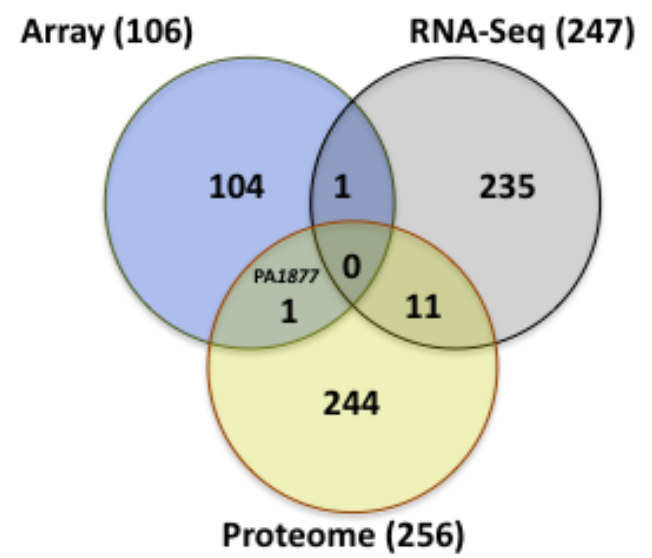

Figure 5.11: Comparative analyses of AmpR microarray, RNA-Seq and proteome datasets. The AmpR positive (A), AmpR-negative (B), AmpR$ß$-lactam positive (C) and AmpR- $ß$-lactam negative (D) gene sets from our previous microarray (225) and the proteome (unpublished) studies were compared with the RNA-Seq data from the current study. 
With the AmpR-positively regulated gene set (Figure 5.11A), genes of the type VI secretion system (PA1657, PA1662, PA1664), the hydrogen cyanide biosynthetic operon hcnABC (PA2193-PA2195), and the membrane fusion component of the MexGHI-OpmD efflux pump (MexH, PA4206) are positively regulated in both the microarray and RNASeq datasets. In agreement with our previous phenotypic assays (225), LasA (PA1871), a QS-regulated protease is also differentially expressed at the protein level (Figure 5.11A). PopD (PA1709), which along with PopB (PA1708) forms the translocation pore on the eukaryotic cell surface for injection of T3SS effectors (557), is AmpR-positively regulated in both the microarray and proteome analyses.

Using an insertion mutant of $a m p R$ and transcriptional fusions, we have previously demonstrated crosstalk between $\mathrm{AmpR}$ and the alginate master regulator $\mathrm{AlgT} / \mathrm{U}$, where AmpR negatively regulates $\mathrm{AlgT} / \mathrm{U}$, which in turn positively regulates ampR expression (425). In addition, AmpR also negatively regulates the anti-anti-sigma factor MucB (PA0764), as seen in both microarray and RNA-Seq analyses (Figure 5.11 B). The genes encoding $\mathrm{AlgT} / \mathrm{U}$ and MucB are part of the same operon (algT/U-mucAтисВ-тис (-тисD) and the apparent contradiction in AmpR-mediated regulation of algT/U and тисв is most likely resolved at the posttranscriptional level. Genes of the mexEF-oprN efflux pump are also downregulated in both the transcriptome analyses (Figure 5.11B), and concurs with data from MIC studies (225). The positive regulator of pyocin production PrtN (PA0610) was identified in both the microarray (AmpR negatively regulated) and proteome studies (AmpR negatively regulated under ß-lactam stress; Panels B, D, Figure 5.11). This concurs with the upregulation of about 39 genes of the pyocin cluster PAO $\triangle a m p R$ in the microarray analysis (225). 
AmpR negatively regulates PA4378 in the microarray, and RNA-Seq analyses. PA4378 is annotated as InaA that has a $61 \%$ similarity to the $\mathrm{pH}$-inducible protein InaA in E. coli (27). It is part of a three-gene operon (PA4377-PA4379) and both the array and RNA-Seq data show that in addition to PA4378, PA4379 is also upregulated (AmpR negative regulation). In $P$. aeruginosa PA7, the gene PA4378 is annotated as a lipopolysaccharide kinase (27). BLASTP analysis reveals that the PA4378 has homologs in other species of Pseudomonas in addition to strains of $P$. aeruginosa (data not shown) and the operon is part of the core genome in P. aeruginosa strains (33). InaA has also been identified previously in Erwinia ananas to be involved in ice nucleation (558). In $E$. coli, inaA expression is regulated by SoxRS, the superoxide stress response system, and marRAB the multiple antibiotic resistance (Mar) operon (559). Downregulation of inaA expression by AmpR in both transcriptome studies, along with positive regulation of oxidative stress (Figure 5.6), suggests that $\mathrm{AmpR}$ might regulate inaA and oxidative stress via SoxRS. However, there was no differential regulation of $\operatorname{soxRS}$ in the transcriptome studies.

\section{Discussion}

In the opportunistic human pathogen $P$. aeruginosa, gene expression is a tightly controlled process and many regulators act in concert to control virulence traits $(511,512)$. In silico analyses and empirical evidence have identified critical regulators such as Vfr, the $P$. aeruginosa homolog of the $E$. coli cAMP receptor protein (CRP), to be central to $P$. aeruginosa pathogenicity $(355,560-562)$. This study establishes the role of $P$. aeruginosa AmpR as not only a regulator of virulence factors but also important physiological processes such as response to oxidative stress and heat shock. AmpR positively regulates 
the stress sigma factor RpoS and a growth phase-dependent expression of some RpoSregulated virulence factors has been demonstrated in PAO $\triangle a m p R$ (225). So, a subset of the AmpR-regulated genes is possibly regulated via RpoS. In addition, subtractive transcriptomics of the AmpR regulon identified AmpR-regulated genes that were not shared with other transcriptomes (225). RNA-Seq has a better coverage than microarrays in detecting transcript levels (563). Expression of some genes that were not identified in the microarrays was differentially regulated in the AmpR RNA-Seq study. In this study, we identified some sRNAs to be targets of AmpR regulation. Selected sRNAs were pursued further to establish a phenotypic difference arising due to AmpR-mediated regulation.

Iron acquisition is a critical determinant of $P$. aeruginosa pathogenicity and has been proposed as a potential target to counter infections (564). In the healthy lung, iron is typically bound by ferritin and transferrin, and free iron is available only in very low quantities (565). In the CF lung, however, higher iron concentration is a potential reason that allows bacteria such as $P$. aeruginosa to colonize (565). The primary mode of sequestration of extracellular iron in $P$. aeruginosa is by use of the siderophores pyoverdine and pyochelin produced under iron-limiting conditions $(566,567)$. AmpR positively regulates iron uptake, as evidenced by a reduced expression of both siderophore gene expression and PQS in PAO $\triangle a m p R$, and growth impairment under ironlimited conditions (Figure 5.4). However, since iron is not a limiting factor in the CF lung, as evidenced by iron-rich CF sputa $(565,568)$, AmpR is likely not important in iron uptake in this setting. 
In the CF lung, $P$. aeruginosa turns mucoid by overproducing the extracellular polysaccharide alginate in response to the $\mathrm{H}_{2} \mathrm{O}_{2}$ released by polymorphonuclear leukocytes (569). It has also been established that the ECF sigma factor AlgT/U is the master regulator of alginate production by turning on expression of the $\operatorname{alg} D$ operon (481). Thus, the enhanced sensitivity of $\mathrm{PAO} \triangle a m p R$ to $\mathrm{H}_{2} \mathrm{O}_{2}$ is interesting in the light of the fact that AmpR negatively regulates algT/U expression in response to unidentified signals (425). Taken together, these studies suggest that, on one hand, AmpR negatively regulates alginate production by repressing $\operatorname{alg} T / U$ transcription, while on the other hand, is required for resistance against $\mathrm{H}_{2} \mathrm{O}_{2}$, which turns on alginate production. Thus, when the bacterium is in the CF lung, the role of AmpR in establishing chronic infections seems conflicting. One possible explanation for this seemingly contradictory data is the role of other regulators. Even though loss of AmpR leads to enhanced algT/U transcription, posttranslational control by the anti-sigma factor MucA prevents AlgT/Umediated $\operatorname{alg} D$ transcription (570). Moreover, gene regulation is a complex interlinked process in $P$. aeruginosa and multiple tiers of regulation for critical pathways is common $(511,560,571)$.

Positive regulation of iron uptake, PQS and oxidative stress by AmpR seem to be disparate phenotypes but they are, in fact, interlinked [Figure 5.12, (511)]. The relationship between iron uptake and QS is complex and some transcriptional regulators involved in QS regulation also modulate iron response $(168,572,573)$. MvfR is one such 


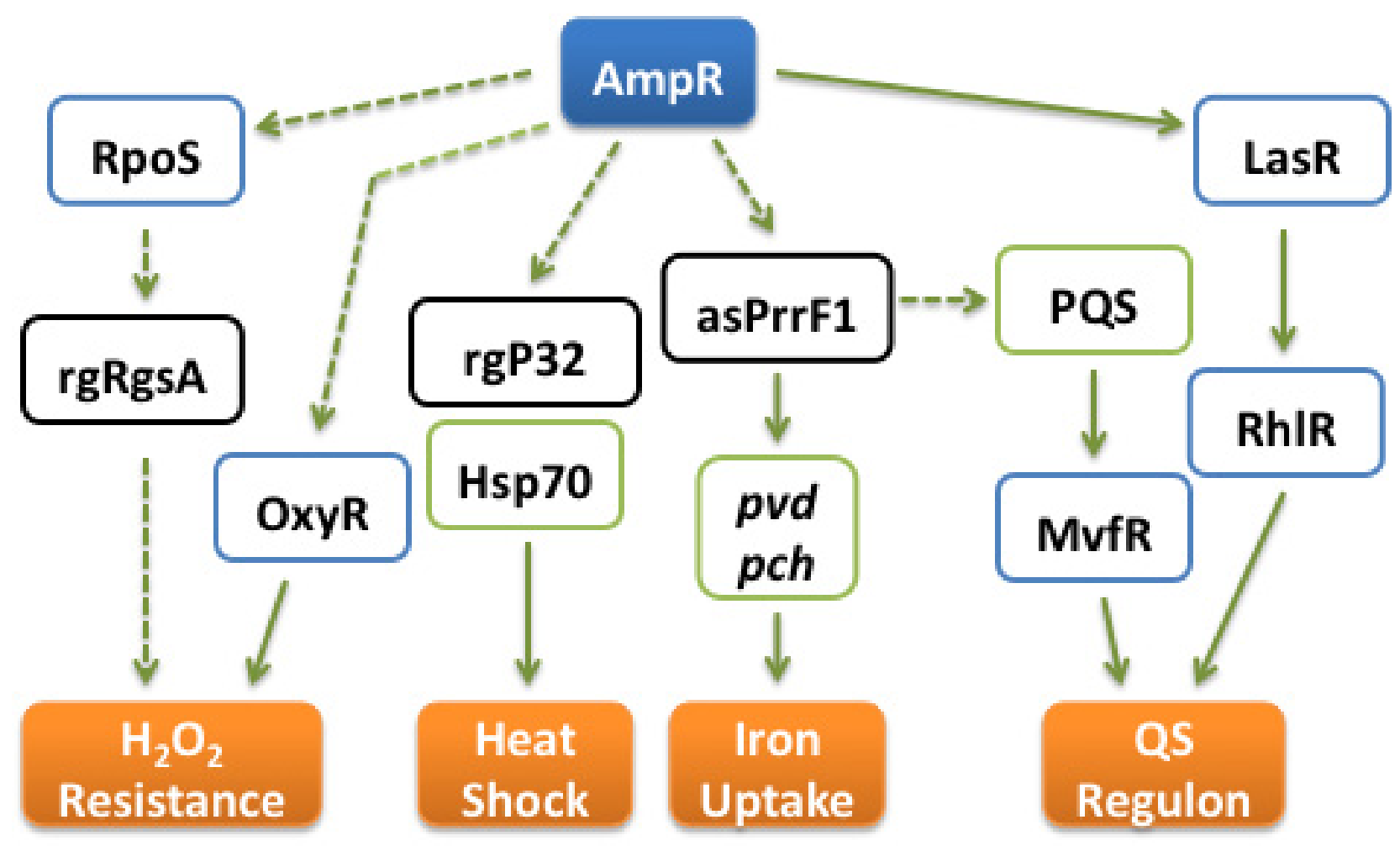

Figure 5.12: AmpR-mediated regulation of virulence and physiological processes in P. aeruginosa. AmpR affects expression of QS genes by directly binding to $\mathrm{P}_{\text {lasR }}$ and modulating lasR expression. AmpR also positively regulates the PQS system by modulating levels of the antisense RNA asPrrF1, thus also affecting iron uptake. By regulating expression of the stationary phase sigma factor RpoS (225), oxyR and the small RNA rgRgsA AmpR positively regulates the oxidative stress response. The genes encoding the Hsp70 heat-shock response system are also positively regulated by AmpR. This, and previous findings (225) demonstrate that AmpR is a major regulator of virulence and physiological processes in P. aeruginosa. 
example. The iron-responsive sigma factor PvdS (PA2426) turns on $m v f R$ transcription in response to iron starvation $(144,574)$. Our data demonstrates that AmpR positively regulates $m v f R$ expression (Figure 5.8). Furthermore, the antisense RNAs asPrrF1 and asPrrF2 positively regulate production of the signaling molecule PQS (575), which helps in iron chelation, facilitating the activity of pyoverdine and pyochelin $(378,384,576)$. We show here that AmpR positively regulates asPrrF1, but not asPrrF2, in addition to the $p v d$ and pch genes, thus affecting iron uptake and PQS synthesis. AmpR also positively regulates the stationary phase sigma factor RpoS and some RpoS-regulated genes such as the galactophilic lectins LecA and LecB (225). Since the link between QS and RpoS is established (366,368), AmpR regulation of both these processes is not surprising (Figure 5.12). RpoS also positively regulates expression of the sRNA $\operatorname{rgs} A$ (PA2958.1), which is also regulated by GacA, and contributes to hydrogen peroxide resistance in P. aeruginosa (536). AmpR also plays a role in this process, as demonstrated here (Figure 5.12). PAO $\triangle a m p R$ shows dysregulation of not only $r p o S$ and the sRNA $r g s A$ but also OxyR, the major regulator of the catalase KatA that ultimately affects resistance to hydrogen peroxide (Figure 5.12).

CF lung isolates are known to be heterogeneous genotypically and phenotypically [reviewed in (577)]. Higher mutation rates are the driving force for the P. aeruginosa population heterogeneity in the CF lung (578-580). Specifically, mutations in $m u c A$ and las $R$ arise early in the colonization process, followed by mutations in anti-mutator genes such as mutS, mutT, mut $Y$ and mutM (581). MucA mutations trigger the regulated intramembrane proteolytic cascade, freeing $\mathrm{AlgT} / \mathrm{U}$ and allowing for overexpression of alginate [reviewed in (582)]. Mutations in lasR would abolish expression of QS-regulated 
acute virulence factors [reviewed in (583)]. In the CF lung, $P$. aeruginosa loses the ability to produce acute virulence phenotypes after initial colonization and starts to overexpress chronic infection traits (584). AmpR negatively regulates algT/U expression, which is a chronic infection phenotype (425). AmpR mutant strains display some characteristics reminiscent of $\mathrm{CF}$ isolates, including acquisition of fluoroquinolone resistance, reduced production of QS-regulated virulence factors such as proteases and pyocyanin, and enhanced biofilm formation [this study, (225)]. Furthermore, PAO $\triangle a m p R$ RNA-Seq studies show upregulation of the genes mutY (PA5147; 2.4-fold, $p$-value 9.25E06) and mutM (PA0357; 2.6-fold, $p$-value 5.28E-06) under B-lactam stress (Appendix; Supplementary information for Chapter 5, Table 3), indicating negative AmpR regulation. So, one would expect the mutation frequencies of PAO $\triangle a m p R$ to be lower than PAO1, given that some anti-mutators are overexpressed. However, the mutation frequencies for rifampicin and streptomycin were not significantly different between PAO1 and PAO $\triangle a m p R$ (data not shown). This seeming contradiction is not surprising since mutY and $m u t M$ are known to be weak anti-mutators in $P$. aeruginosa and potentially have a lower effect than strong anti-mutators such as MutS (581). The data suggests that inactivating ampR in the CF lung, in addition to other mutations, will help P. aeruginosa colonize better. However, the occurrence and frequency of $\operatorname{ampR}$ mutations in CF isolates needs to be determined.

Our previous and current analyses showed that AmpR positively regulates QS by modulating expression of major QS regulators such as LasR [Figure 5.8, (225)]. ChIPSeq and complementary data demonstrate that AmpR directly binds to and activates las $R$ expression, thus modulating QS phenotypes. AmpR binding site analysis using the ChIP- 
Seq data revealed a motif that was very similar to that identified in our previous studies using microarray studies (225). Comparatively however, the motif in Figure 5.10 appears to be more refined and is likely to resemble the AmpR binding site closely because it is based on the promoters of genes identified in the ChIP study. Minor motif variations from the consensus sequence are expected. For this reason, identifying consensus sequences for transcription factor binding motifs using data from multiple promoters is likely to be more accurate compared to footprinting studies that look at individual promoters.

In conclusion, the data presented here, and previously by our lab, demonstrate that P. aeruginosa AmpR is a critical component of not only virulence and metabolic regulation but also physiological processes. The clinical significance of AmpR is highlighted in a recent study, which demonstrated that constitutive AmpR-activating mutations were responsible for $\mathrm{AmpC}$ overproduction in extremely drug-resistant highrisk $P$. aeruginosa clinical isolates (585). Moreover, given the global effect that AmpR has on the regulatory repertoire of $P$. aeruginosa, it is likely that other virulence traits exhibited by high-risk clinical isolates (585) are also AmpR-mediated. Small molecule inhibitors targeting specific proteins such as P. aeruginosa NagZ (123) and Vibrio cholera LuxO (586) show therapeutic promise. Inhibitors of AmpR function will render the strain sensitive to $\beta$-lactam antibiotics and reduce the production of acute virulence factors. Thus, combination therapies using AmpR inhibitors and antibiotics will potentially provide us with means to counter $P$. aeruginosa infections, and warrants further investigation. 


\section{Materials and methods}

\section{Strains, media and culture conditions}

The strains used in this study are listed in Table 5.2. The wild-type P. aeruginosa PAO1 and its isogenic in-frame $a m p R$ deletion strain, PAO $\triangle a m p R$ used in this study are described earlier $(25,225)$. These strains were used for the RNA-Seq study, with and without sub-MIC ß-lactam stress, essentially as described previously (225).

For ChIP-Seq studies, AmpR was tagged at the 3' end with a 3x-V5 epitope tag. Briefly, the 3x-V5 epitope was PCR amplified from plasmid ZM474 (587) using primers DBS_V5F and DBS_V5R containing KpnI and NheI sites, respectively. The NheI site also contains termination codons in all three reading frames to prevent runoff transcription into the plasmid. The $3 \mathrm{x}-\mathrm{V} 5$ amplicon was cloned into pCR2.1 TOPO (Invitrogen) to generate plasmid pDBS206 and sequenced to ensure absence of mutations. The $\operatorname{ampR}$ ORF with the native promoter but without the stop codon was PCR amplified using primers DBS_ampRF1 (with a $K p n I$ site) and DBS_ampRR (with a SacI site), cloned into pCR 2.1 TOPO (pDBS215) and sequenced. The 1112-bp ampR ORF with the native promoter was then moved as a KpnI-SacI fragment from pDBS215 upstream of and in-frame with the $3 x-V 5$ tag in pDBS206 to obtain plasmid pDBS222. The $3 x$ V5tagged $a m p R$ was then moved into mini-CTX2 [pDBS227; (588)] as a KpnI-NheI fragment, generating plasmid pDBS234. After confirmation by PCR and restriction digestion, the plasmid pDBS234 was moved into PAO $\triangle a m p R$ by electroporation (500). This resulted in strain DBS248 that was used for the ChIP-Seq studies. Functionality of the tagged AmpR in DBS248 was verified by determining the MIC of the ß-lactams ampicillin-sulbactam and amoxicillin, and by ChIP-qPCR. 
Table 5.2: Strains and plasmids used in this study

\begin{tabular}{|c|c|c|}
\hline Strain/ plasmid & Relevant characteristics & Source \\
\hline \multicolumn{3}{|l|}{ Strains } \\
\hline \multicolumn{3}{|l|}{ Escherichia coli } \\
\hline DH5 $\alpha$ & General purpose cloning strain; $\Delta$ (lacZ)M15 & $\begin{array}{l}\text { New England } \\
\text { Biolabs }\end{array}$ \\
\hline DBS206 & E. coli $\mathrm{DH} 5 \alpha$ harboring $3 \mathrm{x}-\mathrm{V} 5$ tag on $\mathrm{pCR} 2.1 \mathrm{TOPO}$ & This study \\
\hline DBS215 & $\begin{array}{l}\text { DH5 } \alpha \text { with } a m p R \text { ORF and the } a m p R-a m p C \\
\text { intergenic region PCR cloned into pCR } 2.1 \text { TOPO }\end{array}$ & This study \\
\hline DBS222 & $\begin{array}{l}\text { DH5 } \alpha \text { harboring ampR ORF tagged with } 3 x \text { V5-tag } \\
\text { on pCR } 2.1 \text { TOPO }\end{array}$ & This study \\
\hline DBS234 & $\begin{array}{l}\text { DH5 } \alpha \text { with mini CTX2, containing } 3 \times \text { V5-tagged } \\
a m p R\end{array}$ & This study \\
\hline \multicolumn{3}{|c|}{ Pseudomonas aeruginosa } \\
\hline PAO1 & Wild-type & $(25)$ \\
\hline PKM315 & PAO $\triangle a m p R ;$ In-frame deletion of $a m p R(P A 4109)$ & $(225)$ \\
\hline DBS248 & $\begin{array}{l}\text { PAO } \triangle a m p R:: a m p R-V 5 ; 3 \mathrm{x}-\mathrm{V} 5 \text {-tagged } a m p R \\
\text { cloned onto mini-CTX2 and moved into PKM315 }\end{array}$ & This study \\
\hline \multicolumn{3}{|l|}{ Plasmids } \\
\hline pCR2.1 TOPO & $\begin{array}{l}\text { TA cloning vector for PCR products; } \mathrm{Ap}^{\mathrm{R}}, \mathrm{Km}^{\mathrm{R}} \text {; } \\
\text { ColE1 f1 ori lacZ } \alpha\end{array}$ & Invitrogen \\
\hline ZM747-V5 & $\begin{array}{l}\text { 3x V5 tag C-term and N-term of yeast URA-3 in } \\
\text { pBlueScript; pDBS193 }\end{array}$ & $(587)$ \\
\hline Mini CTX2 & $\begin{array}{l}\text { Plasmid for single-copy gene integration in } P \text {. } \\
\text { aeruginosa; } \mathrm{Tc}^{\mathrm{R}}\end{array}$ & $(588)$ \\
\hline pDBS206 & $\begin{array}{l}\text { 3x V5 tag PCR amplified from ZM747-V5 and } \\
\text { cloned into pCR2.1 TOPO }\end{array}$ & This study \\
\hline pDBS215 & $\begin{array}{l}1112 \text { bp ampR ORF along with the } a m p R \text { - } a m p C \\
\text { intergenic region, PCR amplified with primers } \\
\text { DBS_ampRF1 and DBSampR; cloned into pCR } 2.1 \\
\text { TOPO }\end{array}$ & This study \\
\hline pDBS222 & $\begin{array}{l}\text { ampR ORF from pDBS } 215 \text { subcloned as KpnI-SacI } \\
\text { upstream of and inframe with } 3 \times \text { V } 5 \text { tag in } \\
\text { pDBS206 }\end{array}$ & This study \\
\hline pDBS234 & $\begin{array}{l}3 \mathrm{x} \text { V5-tagged } a m p R \text { ORF from pDBS } 222 \\
\text { subcloned as a KpnI-NotI fragment into mini-CTX2 }\end{array}$ & This study \\
\hline
\end{tabular}


All strains were grown in standard LB media with aeration, unless otherwise specified. Synthetic succinate medium (SSM) was used as the iron-limited media (528) and contained $(\mathrm{g} / \mathrm{l}) \mathrm{K}_{2} \mathrm{HPO}_{4} 6.0, \mathrm{KH}_{2} \mathrm{PO}_{4} 3.0,\left(\mathrm{NH}_{4}\right)_{2} \mathrm{SO}_{4} 1.0, \mathrm{MgSO}_{4} .7 \mathrm{H}_{2} \mathrm{O}$ 0.2, sodium succinate 4.0, $\mathrm{pH}$ 7.0. Antibiotics were used at the following concentrations: for E. coli gentamycin $15 \mu \mathrm{g} / \mathrm{ml}$, tetracycline $15 \mu \mathrm{g} / \mathrm{ml}$, ampicillin $100 \mu \mathrm{g} / \mathrm{ml}$; for P. aeruginosa gentamycin $75 \mu \mathrm{g} / \mathrm{ml}$, tetracycline $60 \mu \mathrm{g} / \mathrm{ml}$, carbenicillin $150 \mu \mathrm{g} / \mathrm{ml}$.

\section{Library preparation for RNA-Seq analysis}

Total RNA was isolated from PAO1 and PAO $\triangle a m p R$ with and without sub-MIC ßlactam stress as described previously (225) using the hot-phenol extraction protocol (589). RNA quality was analyzed on the Agilent Bioanalyzer and those with RNA Integrity Numbers of 8.0 or above was used for rRNA depletion using the MICROBExpress Kit (Ambion). After rRNA depletion, cDNA synthesis was performed using the SuperScript III First-Strand Synthesis System (Invitrogen) as per manufacturer protocols. Terminal transferase (New England Biolabs) and dATP (New England Biolabs) were used to polyA tail the cDNA samples to obtain tail lengths of 100-300 bases following manufacturer instructions. The 3' ends were then blocked with biotinylated ddATP (Perkin Elmer) using terminal transferase (New England Biolabs), flowed by cleanup with the MinElute cleanup kit (Qiagen). Tailed and blocked samples were then processed on the HeliScope Single Molecule Sequencer at the Molecular Biology Core Facility, Dana Farber Cancer Institute, Boston, MA. 


\section{ChIP-Seq sample preparation}

For ChIP-Seq studies, the 3x-V5-tagged AmpR containing strain (DBS248) was grown and exposed to sub-MIC ß-lactam stress as described previously (225). ProteinDNA interactions were then crosslinked in vivo with formaldehyde (final concentration of $1 \%$ ) at room temperature for 20 minutes and quenched for 15 minutes with $0.25 \mathrm{M}$ glycine. After three washes with $1 \mathrm{X}$ PBS, the cells were resuspended in $1 \mathrm{ml}$ lysis buffer (10 mM Tris pH 8.0, $100 \mathrm{mM} \mathrm{NaCl}, 1 \mathrm{mM}$ EDTA, 0.5 mM EGTA, 0.1\% deoxycholic acid and $0.5 \% \mathrm{~N}$-laurylsarcosine) containing a protease inhibitor cocktail (Roche). After chilling on ice, the cells were sonicated to shear the DNA to a size range of $0.5-1 \mathrm{~kb}$. Cellular debris was removed by centrifugation and a $3 \mu 1$ aliquot of the supernatant was checked on an agarose gel. A $50 \mu 1$ aliquot of the supernatant was stored as the input DNA and the rest was immunoprecipitated using DynaBeads Protein G (Life Technologies), which was previously equilibrated and bound with anti-V5 monoclonal antibody (Sigma) as per manufacturer instructions. After immunoprecipitation overnight, the beads were washed 5x with RIPA buffer (50 mM HEPES pH 7.5, $500 \mathrm{mM} \mathrm{LiCl,} 1$ mM EDTA, 1\% NP40, 0.7\% deoxycholic acid, $50 \mathrm{mM} \mathrm{NaCl}$ in 1xTE). The beads were resuspended in $100 \mu$ l elution buffer $\left(50 \mathrm{mM}\right.$ Tris-HCl, $10 \mathrm{mM}$ EDTA, $1 \% \mathrm{SDS}$ ) at $65^{\circ} \mathrm{C}$ for 30 minutes, centrifuged to remove residual beads and incubated at $65^{\circ} \mathrm{C}$ overnight to reverse the crosslink. TE buffer was then added $(100 \mu \mathrm{l})$ and the samples were treated with RNase $\left(37^{\circ} \mathrm{C}\right.$ for 2 hours). The immunoprecipitated proteins were removed with proteinase $\mathrm{K}\left(55^{\circ} \mathrm{C}\right.$ for 2 hours $)$, and the DNA cleanup performed using the Qiagen Mini Reaction Cleanup kit. RNase and proteinase $\mathrm{K}$ treatment was also performed for the input DNA. DNA concentrations were determined using Quant-iT PicoGreen dsDNA Kit (Life 
Technologies). Before proceeding further, AmpR occupancy of the ampC promoter was determined using qPCR as described previously (225).

The DNA samples were then poly-A tailed, blocked with biotinylated ddATP, purified and processed on the Helicos sequencer as described in the RNA-Seq section.

\section{Data analysis for RNA-Seq and ChIP-Seq}

The raw data files for both RNA-Seq and ChIP-Seq studies from the Helicos sequencing runs were converted to the FASTA format using Helisphere Open Source project. The sequence reads were then mapped to the $P$. aeruginosa PAO1 genome (NCBI reference sequence NC_002516.2) using CLC Genomics Workbench, version 5 (CLC Bio). For RNA-Seq analysis, replicates of each sample were merged and pairwise comparisons were performed to obtain comparative expression. The total number of reads per gene between samples was normalized using RPKM ([reads/kb gene]/[million reads aligning to the genome]). Bonferroni correction was performed and FDR $p$-values were calculated. For RNA-Seq, only genes with a proportions fold-change of $\geq 2.0$ and with Bonferroni corrected $p$-value of $\leq 0.05$ were considered for further analysis. For ChIP-Seq, in addition to calculating FDR, peaks were shifted based on read length and filtered using a maximum Wilcoxon $p$-value of 1.0E-04.

\section{Enrichment of functional categories}

The gene sets that were positively and negatively regulated by AmpR without and with ß-lactam stress were functionally categorized based on the Pseudomonas Genome Database (27). Gene distribution under individual categories in PAO1 was considered as $100 \%$ and the relative distributions in each of the four gene sets were plotted. Enrichment 
of specific functional categories in the individual datasets were determined using GOEAST (518). The Log odds-ratio was calculated for the GOEAST enrichment. The bigger the value of the ratio, the higher the relative abundance of the GO term is compared to a random condition.

\section{Quantitative real-time PCR (qPCR) assays}

Genes that were differentially regulated in the RNA-Seq analysis, and those that had not been tested in our previous microarray analysis (225) were selected for qPCR confirmation. RNA isolation and cDNA synthesis were performed as described previously (225). Ten nanograms of cDNA were used per reaction well in the qPCR assays. Expression of the test genes was normalized to $\operatorname{clp} X(P A 1802)$.

\section{Hydrogen peroxide sensitivity}

To determine differences in $\mathrm{H}_{2} \mathrm{O}_{2}$ sensitivities of PAOl and PAO $\Delta a m p R$, gradient-plate assay was used (540). Briefly, $37 \mathrm{ml}$ of LB agar held at $50^{\circ} \mathrm{C}$ was supplemented with 4-8 $\mu \mathrm{M} \mathrm{H}_{2} \mathrm{O}_{2}$ and poured onto tilted $90 \mathrm{~mm}$ petri dishes. After solidification, $37 \mathrm{ml}$ of $\mathrm{LB}$ agar without $\mathrm{H}_{2} \mathrm{O}_{2}$ was poured onto the plates on a flat surface to generate a gradient. OD600 normalized overnight LB cultures of the strains were then used to form a $75 \mathrm{~mm}$ streak across the gradient using a cotton swab. $\mathrm{H}_{2} \mathrm{O}_{2}$ sensitivity was scored as the extent of growth into the gradient, compared to the control (on LB plates without $\mathrm{H}_{2} \mathrm{O}_{2}$ ). The assay was performed in triplicate and representative results are shown. 


\section{Growth in iron-limited media}

The differential growth abilities of the $a m p R$ mutant vis-à-vis the wild-type strain were determined on SSM (528). Input cells for the growth curve assays were grown overnight in SSM from fresh LB plates. For the assay, the OD600 of the SSM cultures were normalized to 0.02 in SSM and the OD 600 of $200 \mu 1$ aliquots in 96-well flat bottom tissue culture plates (Nunc) was monitored for 17 hours at $37{ }^{\circ} \mathrm{C}$. Conditions were made iron-replete conditions by supplementing SSM with $100 \mu \mathrm{M} \mathrm{FeCl}_{3}$ (528).

\section{Temperature sensitivity assays}

The ability of PAO1 and PAO $\triangle a m p R$ to resist elevated temperatures was examined. Briefly, cells grown in LB broth to either the $\log$ (OD600 0.6-0.8) or stationary $(\mathrm{OD} 600>2.0)$ phase at $30^{\circ} \mathrm{C}$ were exposed to $50^{\circ} \mathrm{C}$ for $1-3$ hours. Control aliquots were maintained at $30^{\circ} \mathrm{C}$ as controls. Cell counts were determined for test and control samples before and after exposure by plating for CFUs.

\section{Statistical analyses}

All the data from qPCR and phenotypic assays were examined for statistical significance using the unpaired two-tailed $t$-test on GraphPad analysis software (www.graphpad.com). RNA-Seq and ChIP-Seq data were analyzed for significance on CLC Genomics Workbench as described above.

\section{Acknowledgments}

I thank Hansi Kumari (Mathee lab, FIU) for help with the experiments and critical reading of the manuscript, Deborah Yoder-Himes (University of Louisville), Bryan 
Davies and William Robins (Mekalanos lab, Harvard Medical School) for guidance and helpful discussions with RNA-Seq and ChIP-Seq. This work was funded by National Institutes of Health-Minority Biomedical Research Support SCORE grants (S06 GM08205 and 5SC1AI081376, to K.M.), Florida International University (FIU) Research Assistantship (Herbert Werthiem College of Medicine, to D.B.) and FIU University Graduate School Dissertation Year Fellowship (to D.B.). 


\section{CHAPTER 6}

\section{Discussion}

Parts of this chapter have been published:

Balasubramanian D, L. Schneper, H. Kumari and K Mathee. 2013.

A dynamic and intricate regulatory network determines Pseudomonas aeruginosa virulence. Nucleic Acids Research. 41(1): 1-20.

DOI: $10.1093 /$ nar/gks1039. 
Metabolic versatility, intrinsic and acquired antibiotic resistance, biofilm formation, and production of multiple virulence factors make $P$. aeruginosa a formidable pathogen. The virulence machinery of $P$. aeruginosa is comprised of both cell-associated determinants (such as lipopolysaccharides, pili, flagella) and numerous secreted factors (such as elastases, proteases, exotoxins, pyocyanin, extracellular polysaccharides; Figure 6.1). One of the mechanisms by which $P$. aeruginosa senses external signals is using sensor proteins that, via phosphotransfer or phosphorelay, activate specific transcriptional regulators. These sensor-regulator protein pairs are called two-component systems (TCS). The $P$. aeruginosa PAO1 genome encodes about 127 TCS members, compared to 60 in Escherichia coli (590) and 70 in Bacillus subtilis (591), reflecting the adaptability of $P$. aeruginosa. TCS and their modifications also feed into major regulatory pathways and play a critical role in allowing cells to modulate gene expression in response to environmental conditions $(213,592)$. Many of the secreted virulence factors and phenotypes such as biofilm formation are under the control of a cell density recognition mechanism called quorum sensing (QS) that aids in the coordinated expression of genes $(208,451)$. QS is key to virulence gene expression in many bacteria and serves as an attractive target for antibacterial chemotherapy (593).

In humans, acute $P$. aeruginosa infections in specific sites such as the CF lung eventually lead to chronic infections. This is caused by adaptive modifications in the infecting clonal type resulting in diverse morphotypes (594). The transition to a chronic 


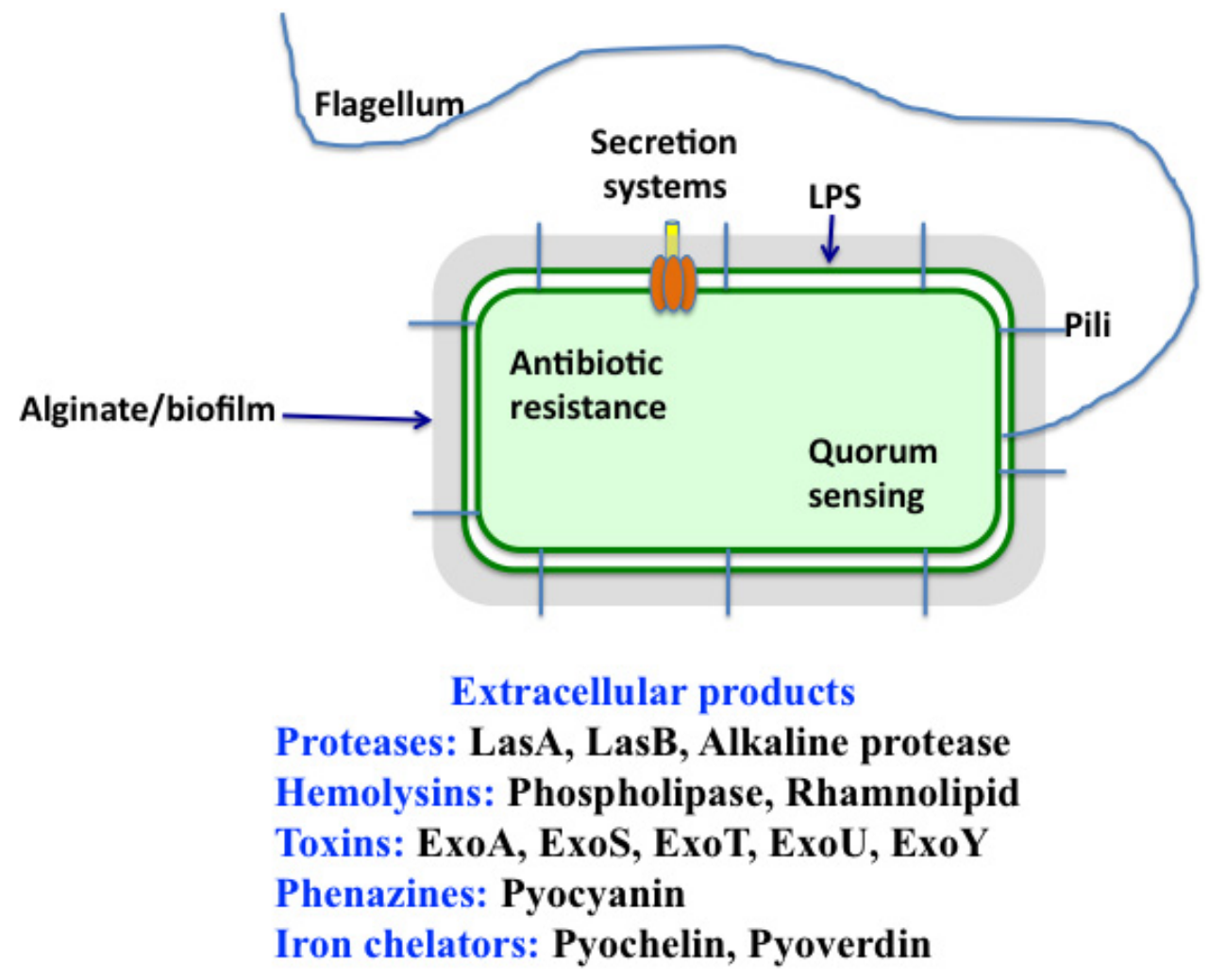

Fig 6.1: $P$. aeruginosa virulence determinants. The major cell-associated and secreted virulence factors of $P$. aeruginosa are shown.

infection phase is the result of numerous changes in cellular physiology in response to external stimuli. The changes include downregulation of acute virulence genes with a concomitant upregulation of chronic infection phenotypes and antibiotic resistance, facilitating recalcitrant infections [reviewed in (595)]. Successful establishment of chronic infection (host invasion, establishment of acute infection and the subsequent transition to the chronic phase) involves tightly regulated expression of many genes associated with metabolism, virulence and antibiotic resistance. Several key players in these transition processes have been identified and include transcriptional and posttranscriptional regulators $(209,210,225)$. 
Gene regulation in $P$. aeruginosa is a complex process involving numerous transcriptional regulators, regulatory RNAs (rgRNA) and sigma factors. The $P$. aeruginosa genome is over $6 \mathrm{MB}$ (25), approaching that of lower eukaryotes. The genome is plastic and has acquired genes and undergone extensive rearrangements in order to adapt to specific niches (33). The large genome of $P$. aeruginosa supports a multitude of regulatory networks with approximately $8 \%$ of the total genome dedicated to the regulatory proteins (27). P. aeruginosa PAO1 encodes 434 putative and identified transcriptional regulators, 24 putative sigma factors and 34 predicted small RNAs $(25,519,560,596)$. Moreover, predicted regulatory networks indicate that there is an extensive crosstalk between the different transcriptional regulators $(560,597)$. These networks, however, are based in part on in silico analyses and their validity needs to be established. Understanding the virulence regulatory networks in P. aeruginosa will provide a framework for future studies to better understand pathogenic processes in $P$. aeruginosa and in related bacteria.

\section{P. aeruginosa AmpR is a major regulator of antibiotic resistance}

Antibiotic resistance is a major concern when dealing with $P$. aeruginosa infections in the hospitals. The current treatment regimen for $P$. aeruginosa infections is a combination therapy of aminoglycosides, quinolones and B-lactam antibiotics (84). The major chromosomally encoded $\beta$-lactamase that determines $B$-lactam resistance in $P$. aeruginosa is AmpC. Previous studies have established that mutations leading to loss of AmpR function render the strain sensitive to B-lactam antibiotics (105). Also, transcriptional fusions and mutation studies of $\mathrm{P}_{a m p C}$ in $a m p R$ knockout strains have been used to demonstrate the positive regulatory role of AmpR at the ampC promoter [O. 
Caille, unpublished data, (105)]. AmpR, a member of the LysR family of transcriptional regulators, is thus a critical player in determining resistance to the $\beta$-lactam class of antibiotics. AmpR also positively regulates MexR (Chapter 4), a positive regulator of the MexAB-OprM efflux pump, which contributes to ß-lactam resistance. The CreBCD system is known to be involved in regulating ß-lactam resistance $(124,348)$ and AmpR regulates $\mathrm{CreD}$ in a $\mathrm{CreB}$-independent manner (Chapter 4). Thus, AmpR regulates $B$ lactam resistance in more ways than one.

Furthermore, AmpR functions as a negative regulator of a second chromosomal ßlactamase, an oxacillinase termed PoxB, whose activity spectrum is limited primarily to carbapenems [D. Zincke, unpublished data; (106)]. The physiological significance of negative regulation of $\operatorname{pox} B$ B-lactamase expression by $A m p R$ is not yet clear. EMSA studies suggest indirect regulation of $\operatorname{poxB}$ by AmpR (O. Caille, unpublished data), although it is possible that recognition and binding of $\mathrm{P}_{p o x}$ by AmpR requires as yet unidentified signals. Interestingly, although PoxB is classified as an oxacillinase based on sequence homology with other oxacillinases, it demonstrates no significant activity against oxacillin (D. Zincke, personal communication).

In addition to regulating $\beta$-lactam resistance positively, AmpR also regulates quinolone resistance (Chapter 4). Gene expression and phenotypic assays indicate that AmpR negatively regulates transcription and ultimately function of the mexEF-oprF efflux system, by modulating expression of the positive regulator MexT (Chapter 4). Repression of mexT expression by AmpR is B-lactam independent (Chapter 4). It is interesting that AmpR positively and negatively regulates resistance to $\beta$-lactams and quinolones, respectively. Furthermore, phenotypic microarray data suggests that AmpR 
influences resistance to many multiple antibiotics (Chapter 4), and is thus among the very few identified transcriptional regulators in $P$. aeruginosa that regulate resistance to different classes of antibiotics.

\section{AmpR regulates quorum sensing in $P$. aeruginosa}

Quorum sensing (QS) is a mechanism that bacterial cells use to gauge population densities that allow them to coordinate gene expression (165). Intercellular communication is particularly useful in the case of opportunistic pathogens to coordinate expression of virulence factors, and when the cells are part of microbial communities called biofilms (208). In P. aeruginosa, expression of many of the acute virulence factors is under QS regulation [reviewed in (598)]. Also, the decision to form biofilms in planktonic cultures is QS-influenced (599). Previous studies using an insertion mutant of ampR indicated that AmpR is a negative regulator of some QS-regulated virulence factors, such as the production of pyocyanin, and extracellular proteases such as LasA and LasB (105). However, using a clean in-frame deletion strain, gene expression and phenotypic studies, this dissertation shows that AmpR is really a positive regulator of QS-regulated acute virulence factors that are under Las-Rhl regulation (Chapter 4). The discrepancy between the insertion and deletion mutants is probably because in the insertion mutant,

the AmpR HTH domain remains unaffected [Chapter 4; (106)]. Furthermore, RNA-Seq data also shows that AmpR positively regulates the PQS signaling system (Chapter 5). Thus, AmpR positively regulates all three the QS systems, and consequently, the associated virulence mechanisms in $P$. aeruginosa. 


\section{Clinical significance of AmpR}

The infecting clonal types of $P$. aeruginosa undergo many changes at the site of infection in order to adapt and colonize, a process driven by mutations $(577,600,601)$. Several recent studies have identified genes that are mutated in either clinical isolates of P. aeruginosa or in strains that have been subjected to CF-like growth conditions (600,602-604). Mechanisms that contribute to $\beta$-lactam resistance are also affected in the adaptation process including overexpression of efflux pumps, downregulation of the carbapenem porin OprD, and hyperproduction of $\mathrm{AmpC}(76,348,605)$. Some strains that have been implicated in outbreaks harbor more than one mutation resulting in multidrugresistant (MDR) and extensively drug-resistant (XDR) clones $(606,607)$. The incidence of MDR and XDR clones of $P$. aeruginosa in patients is on the rise and undermines treatment strategies $(608,609)$. Some MDR/XDR clones that disseminate to multiple hospitals worldwide posing a grave threat to human health have been termed 'high-risk clones' (610). Three such clones ST235, ST111 and ST175 are more prevalent than the rest. A recent study analyzing 190 bloodstream isolates from 10 hospitals determined that about $57 \%$ of them were antibiotic-resistant, of which $33 \%$ of isolates were MDR/XDR (585). Genetic analysis of the molecular mechanisms contributing to enhanced resistance of the XDR clones revealed combinations of resistance to ß-lactams (AmpC overproduction, inactivation of $\mathrm{OprD}$ ), fluoroquinolone resistance (point mutations in GyrA), resistance to gentamycin and tobramycin ( $a a d B$ gene acquired on a class I integron), and upregulation of aminoglycoside resistance (mutation in the mexZ repressor of the MexXY-OprM efflux pump) (585). 
Specifically, in a majority of the most-prevalent ST175 high-risk XDR/MDR isolates analyzed, a novel mutation in AmpR (Glycine 154 Arginine) was the reason for constitutive activation of $a m p C$ expression (585). The G154R mutation was found only in strains that overproduce AmpC. In the sporadic XDR/MDR and moderately resistant strains, other $a m p R$ mutations were detected (E114A, G283E, M288R, A51T) but these polymorphisms are also found in wild-type strains such as PA14 $(27,585)$. Complementing an $\operatorname{ampR}$ deletion strain in trans with a plasmid harboring $a m p R$-G154R enhanced ampC expression and resistance to ceftazidime (585). In the light of studies in dissertation that demonstrates AmpR to be a positive regulator of acute virulence factors and antibiotic resistance (Chapters 4, 5), it is very possible that AmpR overexpression contributes to the success of high-risk XDR clones such as ST175. This, however, remains to be examined.

In $C$ freundii, AmpR becomes a constitutive activator of ampC expression due to amino acid substitutions R86C, G102E and D135N (611,612), of which only the D135N mutation has been found in a clinical isolate (92). Studies in our lab have demonstrated that mutating the aspartic acid residue at position 135 to asparagine (D135N) in $P$. aeruginosa AmpR locks it in the constitutively active conformation (O. Caille, unpublished data). However, the G102E mutation in P. aeruginosa AmpR seems to affect AmpR stability more than activity (O. Caille, unpublished data). These studies from the Mathee lab and elsewhere demonstrate that AmpR plays an important role in regulating antibiotic resistance in P. aeruginosa. Moreover, as demonstrated in Chapters 4 and 5, AmpR is also a critical regulator of $P$. aeruginosa virulence and metabolism. 
AmpR is one of 434 transcriptional regulators identified in the PAO1 genome, many of which remain uncharacterized. With such a large complement of regulatory proteins and accessory metabolic genes, it is no surprise that $P$. aeruginosa is able to adapt and thrive in a wide range of habitats. Understanding how AmpR and other regulators orchestrate the virulence and metabolic processes in $P$. aeruginosa in response to external signals is critical to dealing with infections caused by this successful opportunistic pathogen.

\section{Major virulence regulatory systems in $P$. aeruginosa}

The experimentally established virulence regulatory network in $P$. aeruginosa is depicted in Figure 6.2. Our lab and others have previously performed in silico analyses of the $P$. aeruginosa transcriptional regulatory network $(560,597,613)$. Comparing those networks with the network depicted in Figure 6.2 clearly demonstrates the gap in knowledge between predicted networks and established ones. An important contributing factor to this discrepancy is the fact that the functions of the majority of the genes in the PAO1 genome remain unknown. Deep sequencing, transcriptome metaanalysis $(28,400)$, and complementary studies will aid in assigning functions to the hypothetical genes and undoubtedly narrow this knowledge gap.

Cis regulatory elements (CREs) form a critical part of transcription. CREs are non-coding DNA sequences present in or near a gene, and often contain binding sites for transcription factors and/or other regulators of transcription (614). The two major CREs are promoters and enhancers $(615,616)$. The promoters contain the binding sites for transcription factors and other regulatory molecules such as sigma factors and regulatory RNAs (617-619). Enhancers, once thought to be part of only eukaryotes, are found 
widely in prokaryotes also and function in conjunction with the $\sigma^{54}$-RNA polymerase (620-622). This section will focus on the transcriptional and post-transcriptional regulation of critical pathways that determine $P$. aeruginosa pathogenesis.

\section{Quorum Sensing}

QS is a signaling mechanism that bacteria use to regulate gene expression in a population density-dependent manner and was first demonstrated in Vibrio fischeri (623). In QS, the bacteria produce and secrete small molecules called autoinducers or quoromones. When these molecules reach a concentration threshold, they diffuse back into the cell to elicit a coordinated response promoting group survival (624). $P$. aeruginosa uses QS to regulate production of various virulence determinants such as extracellular proteases, iron chelators, efflux pump expression, biofilm formation, motility, and the response to host immune signals (625). This is achieved using two types of autoinducers, $\mathrm{N}$-acyl-homoserine lactones (AHLs) and 2-alkyl-4 quinolones (AQs) (512).

\section{AHL-mediated QS}

P. aeruginosa has two canonical AHL QS signaling pathways, the las and rhl systems. Together, these pathways affect expression of approximately $10 \%$ of the $P$. aeruginosa transcriptome (170). The lasI (PA1432) and rhlI (PA3476) genes encode the $\mathrm{N}$-3-oxododecanoylhomoserine lactone (3-oxo-C12-AHL) synthetase $(359,626)$ and $\mathrm{N}$ butyrylhomoserine lactone (C4-AHL) synthetase, respectively $(360,362,627,628)$. The resulting AHLs then bind and activate their cognate LuxR family regulators, LasR 
(PA1430) (359) or RhlR (PA3477) (362). LasR and RhlR multimerize in the presence of their cognate AHL $(629,630)$. In in vitro studies, LasR-DNA interaction is cooperative and non-cooperative in the presence or absence of a dyad symmetery in the binding sites, respectively (631). Rhl-regulated promoters have binding sites with a dyad symmetry (366).

\section{AQ-mediated QS}

P. aeruginosa synthesizes two AQ QS signals, 2-heptyl-3-hydroxy-4-quinolone (PQS) and its precursor, 2-heptyl-4-quinolone (HHQ) (378). Both PQS and HHQ enhance in vitro binding of the LysR-type transcription regulator, MvfR (also known as PqsR, PA1003), to the promoter of the pqsABCDE operon (PA0996-PA1000), suggesting that they function as MvfR effectors (381). Microarray analysis identified 141 genes differentially expressed in a $m v f R$ mutant strain, including $\operatorname{las} R, \operatorname{alg} T / U$ (PA0762), rsmA (PA0905), and rsaL (PA1431) (224). PQS also acts independently of MvfR to induce expression of the Fur regulon through its ability to bind iron $(378,384)$ and membrane vessicle formation by inducing membrane curvature $(576,632)$. PmpR (PA0964), a YebC member, negatively regulates MvfR (Figure 6.2) (383).

\section{QS regulation}

The las, rhl, and PQS/HHQ/MvfR systems all exhibit positive feed-forward autoregulation $(544,633)$. In addition, the $P$. aeruginosa AHL systems function in a hierarchical manner, as the 3-oxo-C12-AHL-LasR complex positively regulates $r h l I, r h l R$ and $m v f R$ expression as well as lasI $(363,364,634)$. Exceptions to this have been noted. 
RhlR has been shown to regulate LasR-dependent genes in strains lacking lasR (375), and timing of lasI, las $R$, rhlI and $r h l R$ expression can vary drastically depending upon growth conditions (376).

Many global regulators have been shown to modulate QS-dependent genes. RpoS (PA3622), the stationary phase sigma factor affects approximately $40 \%$ of the QS regulon (366,368). RpoS binding sites have been identifed in several of the QS-dependent promoters. RpoS also affects las $R$ and $r h l R$ expression and LasR binding sites have been identified in promoters of other transcriptional regulators in the QS regulon, including PA2588, PA4778, pvdS (PA2426), vqsR (PA2591) and rsaL (365). Chromatin immunoprecipitation studies have shown occupancy by histone-like silencers MvaT (PA4315) and MvaU (PA2667) on lasI, lasR, $m v f R, r p o S$ and $r s a L$ (635). RsaL plays an important role in las signaling homeostasis, by binding to the lasI promoter and preventing LasR-mediated activation (373). In addition to affecting gene expression through las regulation, microarray analyses indicate that RsaL affects expression of 130 genes, including direct regulation of pyocyanin and hydrogen cyanide genes (373). RsaL also appears to be important in regulating the transition from planktonic to a sessile state as $r s a L$ mutants exhibit increased swarming motility and fail to form biofilms (636). RsaL expression is under the control of LysR-type regulator OxyR (PA5344) (637). The lasI promoter region has also been shown to be bound by CzcR (PA2523), which is required for expression of $r h l I$ and $r h l R$ in addition to lasI (Figure 6.2) (638). CzcR is part of the CzcRS TCS, shown to be involved in carbapenem and heavy metal resistance (639). 
VqsR (PA2591), which is induced by $\mathrm{H}_{2} \mathrm{O}_{2}$ or human serum (282) and is under LasR regulation (640), regulates QS through inhibition of the LuxR-type regulator, QscR (PA1898, Figure 6.2) (641). Though QscR binds 3-oxo-C12-AHL, its specificity is not as stringent as LasR (642). The QscR regulon partially overlaps that ascribed to the las and $r h l$ systems, but also has unique targets (385). In the absence of AHL, QscR can multimerize and form heterodimers with LasR and RhlR (643). QscR also plays a role in LasI homeostasis as mutations in $q s c R$ result in premature lasI expression (280). An AraC family member VqsM (PA2227) regulates VqsR in addition to numerous genes involved in QS including RsaL, PprB (PA4296), MvfR, RpoS as well as AlgT/U and MexR (PA0424) (644).

Additionally, pqsH (PA2587), which encodes the enzyme responsible for oxidation of HHQ to form PQS, is positively regulated by the las system $(377,380)$ and negatively regulated by the $r h l$ system (544). PQS is derived from anthranilate, which is synthesized by the kynurenine pathway (645). Kynurenine pathway anthranilate is also required for $\mathrm{N}$-decanoyl-homoserine lactone (C10-AHL) dependent signaling that is indepdendent of las, $r h l$, and $q s c R$ (645). The receptor for this signaling is yet to be identified (396). Besides potential heterodimerization with QscR, additional posttranscriptional regulation of QS has been described. In one such mechanism, QteE (PA2593) destabilizes LasR and RhlR and in the absence of qteE the quorum thresholdrequirement for activation of QS-dependent genes is lost (646). RsmA negatively regulates $r h l$ and las signaling, resulting in reduced AHL levels (190). Moreover, it has been shown the RNA chaperone Hfq (PA4944) positively regulates rhlI translation via rsm $Y$ and $\operatorname{RsmA~(647).~}$ 
Recently, our lab has established a role for the ß-lactamase regulator AmpR (PA4109) in activating QS-regulated genes [Chapter 4, (225)]. The production of QSregulated secreted virulence factors such as LasA (PA1871) and LasB (PA3724) proteases, and pyocyanin production is significantly impaired in AmpR-deficient strains. Further, loss of ampR reduced virulence in the Caenorhabditis elegans toxicity assay [Chapter 4, (225)]. In addition, AmpR regulates non- $\beta$-lactam resistance by repressing activity of the MexEF-OprN (PA2493-PA2495) efflux pump, the alginate master regulator $\mathrm{AlgT} / \mathrm{U}(226)$ and biofilm formation [Chapter 4, (225)], suggesting that it plays a role in maintaining the acute mode of infection.

\section{Two component regulatory systems (TCSs)}

TCS are sophisticated signaling mechanisms marked by a highly modular design that have been adapted and integrated into a wide variety of cellular signaling circuits. The archetypical TCS is composed of a membrane integrated sensory histidine kinase (HK) and a cytoplasmic response regulator (RR) (648). The HK contains a periplasmic

$\mathrm{N}$-terminal domain that detects specific stimuli (sensing domain) and a C-terminal cytoplasmic transmitter domain that is comprised of a dimerization domain, a conserved histidine, and an ATP catalytic domain (649). HKs can have two or more transmembrane domains with little or no periplasmic domain while others are completely cytoplasmic. The cognate response regulator (RR) contains a conserved receiver domain and a variable output domain (650). 


\section{Figure 6.2: The $P$. aeruginosa virulence regulatory network}

The pathogenic potential of $P$. aeruginosa is dictated by multiple virulence systems that are regulated transcriptionally, post-transcriptionally and post-translationally. The central mechanism for $P$. aeruginosa virulence regulation is QS, which controls expression of many virulence factors in a population density-dependent manner. Key activators of this system are LasR, RhlR, MvfR, VqsR, the cAMP receptor protein Vfr, and the stationary phase sigma factor RpoS. Las system repressors include RsaL, the H-NS protein MvaT, the sigma factor RpoN, and the small RNA binding protein RsmA, while others like QscR repress both the Las and Rhl systems. Other regulators such as AmpR affect QS genes by an unknown mechanism. QS plays a role in regulating critical pathogenic mechanisms including biofilm formation, secretion systems, production of numerous virulence factors, efflux pumps, antibiotic resistance and motility.

Acute $P$. aeruginosa infections can lead to chronic infections in response to largely unidentified signals. A key regulatory pathway that controls this lifestyle switch is the RetSLadS-GacSA-RsmA pathway. RetS and LadS are hybrid sensor proteins that, in response to external signals, either activate or repress the GacSA TCS. The GacA regulator then activates transcription of two rgRNAs, $\operatorname{rgRsmZ}$ and $\operatorname{rgRsmY}$ that sequester and inhibit activity of the small RNA binding protein, RsmA. RsmA is a key activator/repressor that post-transcriptionally regulates numerous acute and chronic infection phenotypes including multiple QS-regulated virulence factors, biofilm formation, type 2, type 3 and type 6 secretion systems, and motility.

Another major phenotypic change associated with the switch from acute to chronic phases of infection is the formation of biofilm. This is associated with extensive changes in transcription. Three key TCS involved in activating biofilm formation are BfiSR, MifR and BfmSR. Cyclic-di-GMP is another major player influencing this process, whose levels are controlled by diguanylate cyclases and phosphodiesterases. QS and the cup genes enhance biofilms, while regulators like AmpR repress it. An important component of $P$. aeruginosa biofilms are extracellular polysaccharides such as alginate, Pel and Psl. Alginate production is under the control of the master regulator ECF $\mathrm{AlgT} / \mathrm{U}$, whose activity is regulated transcriptionally by AmpR, post-translationally by $\mathrm{MucA}$ and $\mathrm{MucB}$, and by regulated intermembrane proteolysis involving $\mathrm{MucP}, \mathrm{AlgW}, \mathrm{ClpXP}$ and others. AlgT/U activates the alginate biosynthetic operon via $\mathrm{AlgR}, \mathrm{AlgB}$ and $\mathrm{AmrZ}$. In addition, biofilm formation is also affected by iron concentration, a process governed by the master repressor of iron uptake, Fur. Fur controls uptake of iron by regulating the sigma factor PvdS, thereby modulating sidephore levels. Fur also modulates transcription of two key regulatory RNAs, asPrrF1 and asPrrF2. These two sRNAs are involved not only in regulating iron-uptake related genes, but also enzymes of the TCA cycle, and genes involved in anthranilate synthesis. Anthralinate, a precursor for synthesis of PQS, is a key regulatory molecule of the PQS signaling system in P. aeruginosa that is involved in expression of QS-regulated virulence factors.

Details on the individual interactions and the appropriate references can be found in the text. Some of the interactions labeled as indirect are regulated by unknown mechanisms and warrant further investigation. In the figure, some regulators and phenotypes have been mentioned more than once. 


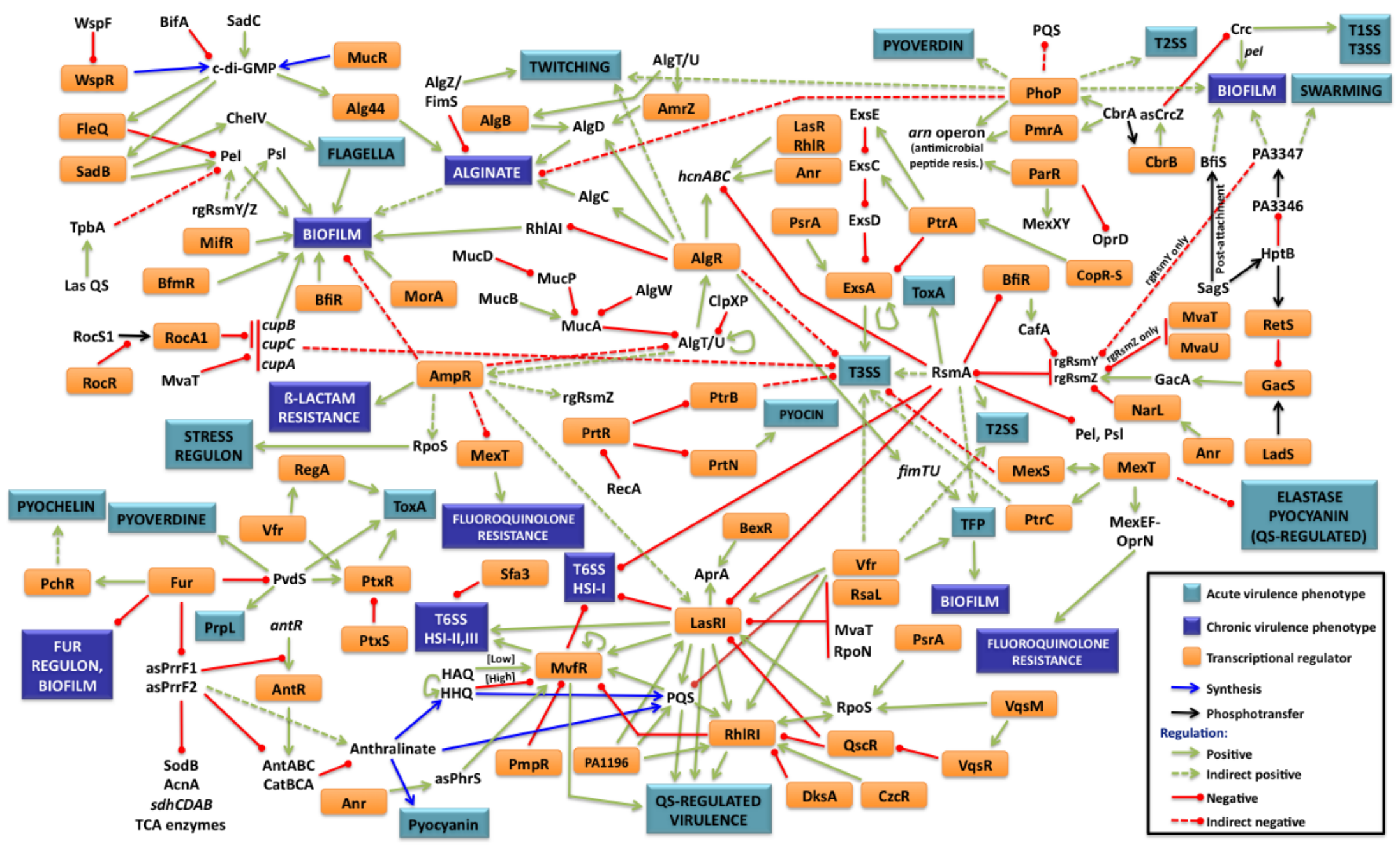


Upon receiving a signal, two HK monomers dimerize and cross-phosphorylate at the conserved histidine residue, and the phosphate is subsequently transferred to an aspartate residue in the receiver domain of the cognate RR (651).The phosphotransfer is catalyzed by the receiver domain and results in a conformational change that activates the output domain, which often binds DNA and modulates gene expression or enzymatic activity $(592,650,652)$. Variations to this model occur in phosphorelays where a sensor kinase first transfers the phosphoryl group to a $R R$ that has no output domain. This $P \sim R R$ then transfers the phosphoryl group to a histidine-containing phosphotransfer protein (HPT), and this in turn serves as a phosphate donor to a terminal RR which has an output domain mediating a cellular response (213). In other cases, the sensor kinase and the RR lacking an output domain are fused into one protein (hybrid sensor kinase) (653). Other variations include the TCS connectors, a group of proteins that modulate the phosphorylation state and activity of sensor HK and RR and establish regulatory links between otherwise independent signal transduction pathways (654).

P. aeruginosa, equipped with $55 \mathrm{HKs}, 89$ RRs and 14 HK-RR hybrids, possesses one of the largest pool of TCS proteins identified in any microorganism thus far analyzed (25). This provides the bacterium with a sophisticated capability to regulate diverse metabolic adaptations, virulence and antibiotic resistance processes that are hallmark of $P$. aeruginosa infections. One of the critical TCSs is GacSA (GacS-PA0928, GacAPA2586) that is central to expression of virulence factors, secondary metabolites, biofilm formation and QS (190,655), and the switch between acute and chronic infections (656,657). GacS is a hybrid sensor HK that contains a HK domain, a RR domain and a histidine phosphotransfer (Hpt) domain $(210,658)$. GacS phosphorylation is under the 
control of two hybrid sensor kinases, RetS (PA4856) (210) and LadS (PA3974) (209) (Figure 6.2). RetS can directly interact with GacS and prevent GacS phosphorylation $(209,659)$ while LadS phosphorylates GacS (209). Phosphorylated GacA positively regulates the transcription of two small regulatory RNAs, rgRsmZ (PA3621.1) and rgRsmY (PA0527.1), which block the negative regulator RNA-binding protein RsmA (PA0905). RsmA positively regulates genes of the Type 3 secretion system, type IV pili formation and iron homeostasis while repressing QS, Type 6 secretion, and potentially other transcription factors $(29,391,458)$. The GacSA TCS is also involved in antibiotic resistance to three different families of antibiotics: tobramycin, ciprofloxacin and tetracycline (660) apparently through RsmA/rgRsmZ.

In $P$. aeruginosa, PhoPQ (PA1179-PA1180) together with PmrAB (PA4776PA4777) are two TCSs that respond to limiting concentrations of cations, and regulate resistance to polymyxin B and cationic antimicrobial peptides through the regulation of the arnBCADTEF-pmrE (PA3552-PA3559) LPS modification operon $(661,662)$. PhoQ is involved in swarming and twitching motility as well as in biofilm formation and is required for virulence without affecting the T3SS or QS systems (Figure 6.2) (663). The HK PhoQ activates the RR PmrA independently of PmrB suggesting an interaction between these TCSs (664). In addition, increased resistance to antibiotics including polymyxin $\mathrm{B}$, aminoglycosides and quinolones in $p h o Q$ mutants suggests crosstalk between PhoPQ and other TCSs $(665,666)$.

\section{Formation of biofilms}

Biofilms are surface-associated multicellular bacterial communities encapsulated in a self-produced extracellular matrix composed of polysaccharides, proteins and nucleic 
acids that mediate cell-to-cell and cell-to-surface interactions (667). P. aeruginosa biofilms, typically associated with poor patient prognosis, signify the switch from an acute to a chronic infection. Biofilms can be formed on abiotic (environment), or biotic (wounds, surgical implants, cystic fibrosis lung) surfaces (668). Biofilm formation and maintenance is tightly regulated in response to environmental cues, conferring enhanced resistance against antimicrobial agents and immune defense mechanisms on the biofilm bacteria (451). Formation of biofilms is a multi-stage process that is initiated by the surface attachment of planktonic bacteria to form a monolayer, clonal growth/aggregation leading to the formation of microcolonies, maturation to form mushroom-shaped structures, and dispersal $(171,669,670)$. As can be imagined, this complex transition in the bacterial lifestyle is accompanied by drastic changes in gene regulation.

Surface attachment by $P$. aeruginosa to form microcolonies has been attributed to Type IV pili, flagella, free DNA, alginate, and Pel and Psl polysaccharides, though pili, alginate and flagella mutants also form biofilms $(670,671)$. Attachment is a reversible process and the commitment to form biofilms is partly under positive SadB (PA5346) regulation (672). SadB upregulates both Pel polysaccharide production, and the chemotaxis-like cluster CheIV (PA0408-PA0417) that is thought to regulate flagellar motion by an unknown mechanism (673).

The Cup fimbriae, encoded by three distinct gene clusters cupA (PA2128PA2133), cupB (PA4081-PA4086), and cupC (PA0992, PA0993, PA0994) in $P$. aeruginosa PAO1, have been demonstrated to play a role in different stages of biofilm formation on biotic and abiotic surfaces (674). Regulation of the cup genes is complex involving a phase variation-dependent repression of cupA expression by a H-NS member 
MvaT $(675,676)$. MvaT also regulates the $\operatorname{cupB}$ and сupC loci to a lesser extent $(675)$. The сирB and сupC clusters are under the primary regulation of the RocS1-RocR-RocA1 (PA3946-PA3948) three-component system (274). This system is similar to the Bordetella pertussis BvgASR system (677) and consists of the hybrid sensor kinase RocS1, the response regulator RocA1, and the RocA1-repressor RocR $(274,288)$. RocR has been hypothesized to bind c-di-GMP via its EAL (diguanylate phosphodiestrerase) domain and prevent phosphotransfer from RocS1 to RocA1, thus preventing RocA1 activation $(274,288)$. P. aeruginosa PA14 has a fourth cup cluster (cupD) on the pathogenicity island PAPI-I that is controlled positively by the response regulator RcsB (PA4080), and negatively by the EAL-domain containing response regulator PvrR (678). In addition, diguanylate cyclases and phosphodiesterases of the wsp gene cluster (PA3702-PA3708) (679,680) MorA (PA4601) (681) and TpbA-TpbB (PA3885, PA1120) (682) modulate intracellular levels of c-di-GMP to exert a regulatory effect on the cup gene clusters (Figure 6.2).

The main components of of the extracellular polymeric substance matrix of biofilms are Pel and Psl polysaccharides, alginate, and free DNA $(451,670)$. Both pel and psl gene loci are post-transcriptionally regulated by the RetS-LadS (PA4856 and PA3974, respectively) system via $r s m Y$ and $r z m Z(209,210,659)$ and by c-di-GMP levels, either directly (679) or by binding the transcriptional regulator FleQ (PA1097).(683) The pel operon is also repressed by the las QS system via the tyrosine phosphatase TpbA (PA3885) (682). A membrane-bound sensor, PpyR (PA2663) enhances biofilm formation via the $p s l$ operon, and virulence via modulating QS (684). Although alginate is a major component of biofilms and affects biofilm structure, it is not essential for biofilm 
formation (670). Alginate regulation is discussed in a separate section (see below). QS regulates cell lysis in biofilms (685-687) thereby controlling the release of extracellular DNA, a major component of the biofilm matrix $(688,689)$. The QS system also regulates rhamnolipid production (362) that promotes motility and hence formation of the cap in the mushroom structure of mature biofilms (690), and maintenance of biofilm channels (691). BfiRS (PA4196-PA4197), BfmRS (PA4101-PA4102) and MifR (PA5511) are TCSs shown to regulate biofilm development and maturation by sequential phosphorylation (275). They activate biofilm formation at different transition stages: reversible to irreversible attachment (BfiRS), irreversible attachment to maturation stage1 (BfmRS), and maturation stage-1 to mushroom structure formation (MifR) (Figure 6.2) (275). The BfiRS system may function in conjunction with the GacSA TCS and feed into the Rsm loop of regulation to control biofilm formation (275). SagS (PA2824), the cognate sensor of HptB (PA3345), modulates biofilm development (by controlling BifS phosphorylation) and other virulence phenotypes (by modulating rgsRmZ levels) depending on whether the cells are in the planktonic or biofilm phase (692).

Analyses of clinical isolates reveal a positive correlation between expression of lasR, rhlR and acute virulence factors $(693,694)$ suggesting that QS is required for virulence in vivo. QS is also important when $P$. aeruginosa grows as biofilms in the CF lung (695). In vivo studies show that lasI and rhlI mutants produce milder chronic lung infections compared to their wild-type counterparts (696), and form more susceptible biofilms (697). However, in some in vitro studies, there was no apparent difference in the biofilms formed by the QS mutants and the wild-type strains $(698,699)$. This discrepancy in the requirement of QS for biofilm formation and establishment of a successful chronic 
infection is probably not surprising since QS regulates many different functions. Further, it has been demonstrated that the CF environment selects for strains with las $R$ mutations, though the $r h l$ system is intact (700). Although lasR is higher up in the QS hierarchy, studies have shown that secondary mutations can reestablish $r h l$ expression in las mutants (701). This suggests that in CF biofilms, the rhl system is more important and las $R$ inactivation serves to downregulate the acute virulence factors (702).

A major cause of antibiotic resistance in biofilms has recently been attributed to the phenomenon of persistence. Persister cells are small subpopulations of antibioticsensitive cells that have acquired transient antibiotic tolerance (703). When the antibiotic levels drop, the persisters grow into a population of sensitive cells, again with a small sub-population of persisters $(704,705)$. Many genes involved in the formation of persisters have been identified in $P$. aeruginosa PA14, including two transcriptional regulators AlgR (PA5261) and PilH (PA0409) (706). However, this topic is outside the scope of this dissertation but has been extensively reviewed elsewhere $(83,707)$.

\section{Alginate production}

In the lungs, especially of patients with cystic fibrosis, $P$. aeruginosa can convert from a non-mucoid to an alginate-overproducing mucoid phenotype signaling chronic infection (708). Chronic $P$. aeruginosa infection seems to be localized to foci within the anaerobic mucus environment in the lung's respiratory zone (709-712). These foci lead to tissue damage decreasing lung function and the appearance of the mucoid phenotype correlates with poor patient prognosis $(713,714)$. The exopolysaccharide alginate is a linear polymer of $\beta$-D-mannuronic acid and $\alpha$-L-guluronic acid (715), which stimulates production of $\operatorname{IgG}$ and $\operatorname{IgA}$ antibodies (716). Although production of alginate is 
metabolically taxing, it protects the bacteria from phagocytosis and antibodies thus conferring a survival advantage $(717,718)$. Conversion to mucoidy occurs when biofilms are treated with activated polymorphonuclear leukocytes (569), hydrogen peroxide (569), antibiotics (719), and nutrient starvation (720,721).

A complex regulatory pathway controls alginate biosynthesis. The central player is the $\sigma^{\mathrm{E}}$ family extracytoplasmic function sigma factor $\mathrm{AlgT} / \mathrm{U}$ (PA0762) $(465,722)$ whose activity is inhibited post-transcriptionally by the anti-sigma factor MucA (PA0763), and by MucB (PA0764) $(570,723,724)$. Loss of function mutations in mucA or mucB result in a mucoid phenotype $(466,723,725)$ due to release of $A \lg \mathrm{T} / \mathrm{U}$ from MucA by a regulated intramembrane proteolytic pathway (reviewed in $(582,726)$ ). It was recently demonstrated that MucA proteolysis is regulated not only by AlgW (PA4446) but also by MucD (PA0766) by activating the MucP protease (PA3649) (727). In addition, AmpR links alginate production with antibiotic resistance and QS by negatively regulating algT/U expression (Figure 6.2) (226). AlgT/U regulates alginate production at least in part by autoregulation (465), controlling expression of the transcriptional regulators $\operatorname{alg} R$ (481,482), $\operatorname{alg} B(481,725), \operatorname{amrZ}(728)$, and the $\operatorname{alg} D(P A 3540)$ alginate biosynthetic operon (481,729,730). AlgB (731), AlgR (732-734), and AmrZ (735) all directly bind to the $\operatorname{alg} D$ operon to activate transcription. The alternative sigma factor RpoN (PA4462) is also required for high levels of $a \lg T / U$ and $\operatorname{alg} D$ expression (736).

The $\operatorname{algB}(P A 5483)$ and $\operatorname{algR}$ (PA5261) genes respectively encode NtrC and LytR subfamily of TCS RRs (737). Interestingly, aspartic acid phosphorylation in the regulatory domain is not essential for alginate production (738). Transcriptome analysis of a PAOтисA22 mucoid strain (PDO300) (570) identified seven predicted 
transcriptional regulators, PA1235, PA1261, PA1637 (KdpE), PA2881, PA3420, PA3771, and PA5431, and one sensor kinase, EraS (PA1979), whose expression was downregulated in an $\operatorname{alg} B$ mutant but not in a strain containing a mutation in its cognate TCS sensor, KinB (PA5484) (731). In addition to regulating the algD operon, AlgR directly activates transcription of algC (PA5322), which encodes a phosphomannomutase/phosphoglucomutase essential for Psl, alginate and rhamnolipid synthesis (Figure 6.2) (739-742). AlgR also is important for mature biofilm formation, possibly by directly repressing $r h l$-QS (743), type IV pilus formation by binding to the fimTU-pilVWXY1Y2E promoter $(744,745)$, and hydrogen cyanide $(\mathrm{HCN})$ production by binding to the hcnA (PA2193) promoter (480). Interestingly, in contrast to alginate production, the phosphorylation site is required for regulating cyanide production and twitching motility $(744,746)$. AlgR has also been shown to indirectly regulate the cyclic AMP/Vfr-dependent pathway (747). The AlgR regulon has been characterized by several transcriptome studies $(480,743,748)$. Two other regulators of alginate production in $P$. aeruginosa are Alg44 (PA3542) (749) and a diguanylate cyclase, MucR (PA1727) (750). MucR produces a pool of c-di-GMP in the vicinity of the PilZ domain of Alg44 (PA3542), which then positively regulates alginate production (Figure 6.2) (749-751).

\section{Regulation of iron uptake}

Iron is critical for growth of all organisms and $P$. aeruginosa is no exception. Transcriptome studies reveal that a large number of genes are regulated in response to iron $(144,145)$. Biologically useful iron $\left(\mathrm{Fe}^{2+}\right)$ in the environment is scarce and is available mostly in the insoluble $\mathrm{Fe}^{3+}$ form. To help scavenge this free iron, bacteria produce siderophores that bind extracellular iron and transport them back into the cell via 
TonB-dependent receptors on the cell surface (752). P. aeruginosa produces two siderophores, pyoverdine and pyochelin, and can also subvert siderophores produced by other organisms to take up heme $(753,754)$. However, excess free iron in the cell leads to formation of toxic reactive oxygen species and so cells tightly regulate the uptake (755). The ferric uptake regulator (Fur, PA4769) is a conserved protein in P. aeruginosa and other Gram-negative bacteria, and is a major iron acquisition regulator (756). Fur dimerizes rapidly after synthesis and it takes a minimum of two dimers to bind promoters of genes under Fur regulation in P. aeruginosa (757). Fur control the iron regulon directly by binding the Fur box (758), and indirectly by modulating expression of other regulators including the pyochelin uptake regulator PchR (PA4227), ECF sigma factors like PvdS, TCS regulators and small regulatory RNAs (asPrrF1 (PA4704.1), asPrrF2 (PA4704.2; Figure 6.2) (758-760).

Iron concentrations in the cell also influence expression of virulence factors in $P$. aeruginosa. PvdS, for example, is critical in linking iron and virulence by controlling the production of pyoverdine, an outer membrane pyoverdine receptor (FpvA (PA2398)) and two important extracellular virulence factors (PrpL (PA4175) and exotoxin A (PA1148); Figure 6.2) (761-763). Also, $p v d S$ mutants showed reduced virulence in a rabbit endocarditis model (764). Human lactoferrin inhibits P. aeruginosa biofilm formation indicating a role for iron in the process (765). Iron chelation by lactoferrin induces twitching motility in $P$. aeruginosa negating colonization and ultimately, biofilm formation $(765,766)$. Intracellular iron concentrations are one of the signals for biofilm development in a process involving Fur but not the iron uptake regulatory RNAs asPrrF1 
and asPrF2 (767). Further, high levels of iron suppress the PQS system, release of extracellular DNA and biofilm formation (768).

The link between iron and QS systems in P. aeruginosa is complex. QS systems are enhanced under limiting iron concentrations $(376,769,770)$ and major QS regulators are also involved in regulating iron responsive genes $(168,224,573)$. MvfR, for example, has been demonstrated to control transcription of iron-related genes and itself has an ironstarvation (IS) box in its promoter, a site recognized by PvdS (PA2426) to turn on transcription under low iron conditions $(144,771)$. It was recently demonstrated that iron levels affect activity of the MvfR signaling molecule HAQ, adding another layer of complexity to the role of iron in QS (772). Another major QS and virulence regulator, VqsR regulates phenazine production by modulating phnAB expression (282). Moreover, the small regulatory RNAs asPrFF1 and asPrrF2, which are negatively regulated by Fur, positively regulate PQS production (Figure 6.2) (773). PQS has been shown to accumulate in the outer membrane and in membrane vesicles (576). PQS chelates iron and this facilitates pyochelin and pyoverdin in scavenging iron $(378,384)$.

Thus, iron uptake regulation in $P$. aeruginosa is a complex affair and involves multiple regulators that affect expression of numerous genes either by themselves or through other regulators. In addition, the interconnections between iron uptake mechanisms and other virulence systems such as QS and biofilm formation demonstrates the versatility of this bacterium in being able to pragmatically read environmental signals to accordingly modulate gene expression. 


\section{Toxins and exoproteins}

Exoproteins are an important component of bacterial survival because not only do they allow the bacteria to interact with their immediate environment and other organisms in the vicinity, but also because they play a critical role in virulence. $P$. aeruginosa has a large complement of secreted proteins and five (type I, II, III, V and VI) of the seven secretion systems characterized in bacteria (774). A majority of the secreted proteins are toxins that aid in $P$. aeruginosa virulence, most of which, including LasA, LasB, PrpL, ToxA, and phospholipases (PlcH (PA0844), PlcN (PA3319), PlcB (PA0026)), are secreted via the Xcp type II secretion system (T2SS) (774). Effector molecules that are crucial for evading the host phagocytic response are secreted via a dedicated T3SS (206), whereas the type I system (T1SS) secretes the alkaline protease AprA $(445,775)$ and the hemophore HasAp (PA3407) (776). Substrates of the recently identified T6SS are just being discovered (777). In addition, c-di-GMP levels, modulated by the diguanylate cyclase WspR (PA3702) is involved in the switch between T3SS and T6SS independent of RetS but dependent on rgRsmY and rgRsmZ (Figure 6.2) (778).

HasAp, a T1SS-secreted heme-uptake protein in $P$. aeruginosa, is under QS control (168). QS is also known to regulate PrpL that targets the human lactoferrin (see section above) (761). Thus, QS in P. aeruginosa not only regulates enzymes to degrade the human lactoferrin but also produces proteins to retrieve the iron from the degraded lactoferrin. The other known T1SS substrate, AprA, is regulated by a novel LTTR named BexR (PA2432) that controls bistability in P. aeruginosa (Figure 6.2) (232). Inactivation of QS has been demonstrated to reduce expression of T2SS-secreted proteases, chitinases and lipases $(626,779)$ due to downregulation of the Xcp T2SS $(780,781)$. The TCS 
PhoBR (PA5360-PA5361) regulates other T2SS-secreted exoproteins such as PlcH, PlcC, PlcN and the Hxc T2SS secreted alkaline phosphatase LapA (782,783). Microarray analysis revealed that a novel cell-surface signaling system PUMA3 regulates Hxc T2SS genes (784). The three T6SS systems (HSI-I, HSI-II and HSI-III) in P. aeruginosa are differentially regulated by the QS systems (785). Whereas LasR and MvfR negatively regulate the HSI-I system, they positively regulate expression of the functionally redundant HSI-II and HSI-III (Figure 6.2) (785). In addition, a putative regulator Sfa3 (SfnR) in P. aeruginosa PA14 (an ortholog of PA2359 in PAO1) potentially regulates the HSI-III cluster (785). HSI-I expression is also regulated by RetS via RsmA (459).

P. aeruginosa T3SS is regulated in a complex and multi-tiered process, and is probably the most well understood (325). Expression of T3SS is regulated transcriptionally and post-transcriptionally in response to host cell contact and environmental $\mathrm{Ca}^{2+}$ levels $(786,787)$. ExsA (PA1713), an AraC member regulates expression of the 43 genes that form the T3SS in $P$. aeruginosa by binding as a monomer to an A-rich 8-bp region upstream of the -35 in the promoter of genes under its regulation (786,788). ExsA autoregulates its own expression and is also activated by PsrA (PA3006), a member of the TetR family $(307,308)$. Two anti-activators (ExsD (PA1714) and PtrA (PA2808)) also regulate ExsA-mediated activation (Figure 6.2). T3SS transcription is coupled to secretion and involves the anti-activator ExsD and the anti-anti-activator ExsC (PA1710) that regulate ExsA function. Under non-inducing conditions (high $\mathrm{Ca}^{2+}$ ), ExsE (PA1711) binds the anti-anti-activator ExsC, allowing the anti-activator ExsD to bind ExsA and inhibit transcription. Under $\mathrm{Ca}^{2+}$ delimiting conditions, ExsE is secreted freeing ExsC to bind ExsD. Free ExsA then activates T3SS expression (Figure 6.2) (789). 
Though this is the primary mode of control, T3SS can also be triggered by stress due to DNA damage (RecA-mediated activation of PtrB) (331), high salt $(790,791)$, metabolic stress $(29,791,792)$, alginate regulators $\mathrm{AlgT} / \mathrm{U}, \mathrm{AlgR}$ and MucA (793), the MexEFOprN efflux pump regulator MexT (PA2492) via PtrC (794), and the RetS/LadS/GacRsm TCSs $(209,210,493)$. Under low oxygen conditions, the anaerobic regulator Anr activates the response regulator NarL (PA3879), which in turn represses rgRsmY and rgRsmZ expression, allowing RsmA to activate T3SS (546). Expression of T3SS, however, happens only in a subset of the population even under inducing conditions (791,795). Multiple levels of control allow fine-tuning of T3SS expression allowing $P$. aeruginosa to sense various environmental conditions and regulate expression in conjunction with other virulence factors.

\section{Regulatory RNAs in P. aeruginosa virulence}

RNAs other than messenger RNAs, transfer RNAs or ribosomal RNAs are termed

small RNAs (sRNAs) and affect all steps in gene expression pathways in both prokaryotes and eukaryotes (796). In general, sRNA-mediated regulation occurs in one of two ways: base pairing with DNA or mRNA, or by affecting the activity of a protein or protein complex (796). Not surprisingly, virulence gene expression in P. aeruginosa also relies on small regulatory RNA (rgRNA)-mediated post-transcriptional regulation. The importance of $\operatorname{rgRNAs}$ in regulation of bacterial virulence is well established $(619,797,798)$. Most of the $P$. aeruginosa rgRNAs that have been characterized play a role in virulence gene regulation (discussed below). A recent study identified about 150 novel sRNAs using sRNA-Seq in $P$. aeruginosa PAO1 and PA14, which includes both strain-specific and shared ones (799). 
Perhaps the most well characterized system involves the rgRNAs, rgRsmY and $\operatorname{rgRsmZ}$ (whose roles in virulence regulation have been discussed in the TCS, and toxins and exoproteins sections). These are two functionally redundant rgRNAs in $P$. aeruginosa that play a critical role in the switch between acute and chronic infections $(210,800)$. GacA of the GacSA TCS positively regulates expression of rgRsmY and rgRsmZ, which then bind to and sequester the small RNA binding protein RsmA via the GGA motif $(548,549,801)$, leading to derepression of the genes that RsmA represses $(190,458,545,802)$. The consequences of RsmA sequestration results in dysregulation of the expression of numerous virulence factors, as discussed in the TCS section. Given the importance of this regulatory process in $P$. aeruginosa pathogenesis, regulation of expression of $\operatorname{rgRsmY}$ and $\operatorname{rgRsmZ}$ is multi-tiered. The histidine phosphotransfer protein HptB is phosphorylated by a phosphorelay involving the three sensor kinases PA2824, PA1611 and PA1976 (803). Phosphorylated HptB then transfers the phosphate to an antianti-sigma factor PA3374, to negatively regulate expression of $\operatorname{rsm} Y(803,804)$. In another mode of regulation, the BfiSR TCS activates expression of the ribonuclease CafA (PA4477), which specifically targets rgRsmZ (547). Further regulation is achieved by the global regulators of the H-NS family of proteins MvaT and MvaU, which bind to AT-rich regions upstream of the $r s m Z$ gene repressing their expression (390). In addition to all this, there is a negative autoregulatory feedback mechanism, the details of which have not been elucidated yet (805). Upon synthesis, rgRsmY is stabilized by Hfq binding, either alone or in conjunction with RsmA $(647,806)$.

Another example of post-transcriptional regulation by sequestering a RNAbinding protein links virulence with metabolism. P. aeruginosa Crc (PA5332) is a RNA- 
binding protein that recognizes CA-motifs around the ribosome binding sites of the mRNA of carbon compound catabolism genes. Crc thus represses genes whose products help utilize less preferred carbon sources (807-809). When less preferred substrates such as mannitol have to be utilized, expression of catabolic genes is achieved by sequestration of $\mathrm{Crc}$ by the $\operatorname{rgRNA}, \operatorname{rgCrcZ}(810)$. Expression of $\operatorname{rgCrcZ}$ is under the control of the TCS CbrAB (PA4725-PA4726), which in conjunction with Crc plays a role in carbon compound catabolism, biofilm formation, antibiotic resistance, secretion systems and swarming (811-817).

P. aeruginosa antisense small RNAs (asRNAs) can also act by base-pairing with target mRNAs, thus inhibiting translation (818). One such example is asPhrS (PA3305.1) that plays a role in PQS and pyocyanin expression (819). Transcriptome studies indicate an extensive overlap between the genes that are positively regulated by the transcriptional regulator PqsR (also known as MvfR, PA1003) and asPhrS suggesting that asPhrS regulates $p q s R$ mRNA $(224,819)$. Interestingly, it was shown that asPhrS specifically targets a region in the RBS of a small ORF (uof) that is present upstream of PqsR (819). Since translation of $p q s R$ and $u o f$ are coupled, asPhrS regulates $p q s R$ translation by modulating translation of uof (819). Expression of asPhrS is under the control of the oxygen responsive regulator Anr (819). Hfq controls asPhrS expression indirectly by regulating Anr expression, whose mechanism of action is yet to be elucidated $(819,820)$.

Small asRNAs also play a role in regulation of iron uptake and involves base pairing by the sRNAs asPrrF1 and asPrrF2, which are the P. aeruginosa orthologs of $E$. coli $\mathrm{RyhB}(758,821)$. Expression of asPrrF1 and asPrF2 is repressed by Fur when iron concentrations are high (758). Under iron starvation conditions, asPrrF1 and asPrrF2 are 
expressed and base pair with the mRNA of target genes, which include the superoxide dismutase $\operatorname{sodB}$ (PA4366), genes involved in the TCA cycle, and anthranilate and cathechol degradation (107). Thus, asPrrF1 and asPrrF2 link carbon metabolism, iron uptake and QS-mediated virulence. Another asRNA gene, asPrrH is located in the same locus as asPrrF1 and asPrrF2. The asPrrH asRNA (at $325 \mathrm{nt}$ ) is longer than asPrrF1 (116 nt) and asPrrF2 (114 nt), and the coding region of asPrrH overlaps with the asPrrF1 terminator, the intergenic region between asPrrF1 and asPrrF2, and the 5' end of the asPrrF2 ORF (822). The expression of asPrrH is maximal in the stationary phase of growth, similar to asPrrF1 and asPrrF2, and under iron-deplete conditions (822). Heme represses asPrrH expression and this involves the outer membrane heme receptors PhuR (PA4710) and HasR (PA3408) (822). Interestingly, under conditions of heme starvation, asPrrH expression leads to the repression of $a \operatorname{ch} A B$ and $s d h C D A B$, which are also targets of the PrrF asRNAs (822). In addition to these targets, asPrrH also represses NirL, a protein involved in biosynthesis of heme, under heme and iron limitation (822).

\section{Conclusions and perspectives}

P. aeruginosa is a very versatile bacterium that can thrive in a wide range of habitats. This is achieved by an intricately interlinked regulatory system of transcriptional regulators, sigma factors, small RNAs, and their regulons. The exquisite control of gene expression is exemplified in the virulence regulatory network (Figure 6.2), which demonstrates that none of the virulence mechanisms are isolated. Expression of individual virulence networks is under transcriptional and post-transcriptional regulation

of multiple regulatory systems, either directly or indirectly. Furthermore, some signaling cascades inversely regulate the acute and chronic virulence phenotypes depending on the 
signals sensed. The next critical phase of research should focus on the signals that the bacteria recognizes in order to achieve gene regulation.

The extent of crossregulation between the transcriptional regulators highlights the global nature of the regulation where individual subnetworks (such as the QS network, alginate network, etc.,) are interlinked to form a hyperconnected network (Figure 6.2). Given the complexity of the connections, one can expect the response of a cell to be elaborate even when faced with a simple stress condition. A fundamental point in a network setting is that one should evaluate the role of individual players (such as a regulator) not in isolation, but with the knowledge that the entire network will react to what it does. In other words, local changes can have global effects. This, in turn, results in very subtle cause-effect relationships. Studying the functions of a regulator by generating deletion or overexpression strains is often performed under the assumption that other regulators will remain static. In reality, however, such modifications can lead to abberant changes across the network in ways that were initially unintended. Moreover, there is a possibility that such changes can occur because the network connections might not always be obvious. This can be attributed, in part, to as yet uneludicated implicit players in the network that dictate or otherwise influence cellular response. This is a likely explanation for the many 'global' regulators in P. aeruginosa and in similar bacteria that have complex regulatory networks. In such cases, many of the phenotypes observed with single regulator mutant strains can be part of a ripple effect that propagates through the network affecting disparate phenotypes.

A simplified model of gene regulatory network treats genes as being on or off i.e., taking binary values. It is therefore no surprise that Boolean networks (discrete 
dynamical network models) have been used to model and study gene regulatory circuits (823-825). Probabilistic Boolean networks, which take into account molecular and genetic noise $(826,827)$ and stochastic Boolean networks, which permit the modeling of gene perturbations (828), provide important insights into the dynamical behavior of the system. Though they are computationally complex, they are a valuable addition to the numerous other programs that are available to analyze gene regulatory networks $(613,829,830)$.

Dynamical systems theory helps us to analyze the behavior of complex systems that can frequently be expressed by time-differential equations. When the behavior of a dynamical system depends very sensitively on small changes in initial conditions, then the system is said to be chaotic, i.e., capable of exhibiting chaotic behavior. Researchers have investigated whether regulatory networks can have subsystems that are capable of exhibiting chaotic behavior (831). It has been shown that competition between two or more subnetworks of comparable importance can lead to chaos (832-834). In fact, chaos has been shown to be possible in biochemical systems with only two feedback loops and positive feedback is known to be necessary for chaotic behavior (835). So, one would expect chaotic subsystems in a regulatory network as complex as the one that controls $P$. aeruginosa virulence (Figure 6.2). In spite of this predisposition, gene regulatory networks seldom exhibit chaotic behavior. This could be because the competitions between opposing nodes are not strong enough (836), or that chaotic behaviors are shortlived due to triggering of other pathways such as cell-cell communication (837). Another possibility is that the natural random variability of biochemical systems masks the chaotic 
behavior (836). However, maintaining a low level of chaos in such a complex network is probably a combination of the above mentioned and, potentially, as yet unknown factors.

In gene regulatory networks, a particular dynamical system is characterized by time-evolving variables (chemical concentrations, gene expression, etc.) and by parameters (temperature, ambient chemical concentrations, etc). A network can exhibit chaotic or non-chaotic behavior depending on the parameters that influence it (838). Environmental factors such as the temperature or the nutritional status of the cells parameterize the relationship between transcription factors and the genes that they regulate. While it is understood that some choices of parameters can induce chaotic behaviors, the parameter may not even be achievable, such as very high temperatures (838). Mutations can also alter relationships in regulatory networks by causing changes in existing links or forming new ones. In a dynamically robust (non-chaotic) system, small, finite changes in the parameters lead to only qualitative changes in the dynamical behavior. However, there are boundaries in the parameter space where the behavior of the system changes qualitatively, and may include the possibility of chaotic dynamics. Predicting whether a network will be stable or chaos-prone under some conditions has proven to be difficult and remains poorly characterized. Recent work has identified the minimum number, types and interactions among three and four nodes/subnetworks that can lead to chaos in a gene regulatory network (836). Such minimal subnetworks have been termed 'chaotic motifs' and networks with these motifs can exhibit chaotic behavior under the right parameters (836). Analysis of the network in Figure 6.2 does not readily show such chaotic motifs. This could be because the network is incomplete (lack of data on the interactions among the $P$. aeruginosa regulators) or due to errors in the inferred 
interactions. Though $P$. aeruginosa virulence regulation has been extensively studied, there is yet much to learn. Thus, absence of empirical evidence does not preclude a propensity to chaos and is worth further investigation.

Depending on an elaborate network to achieve gene regulation is likely an adaptive mechanism by $P$. aeruginosa. Possessing alternate pathways to regulate the same phenotype ensures a rapid response to stimuli even if one of the pathways is affected, thus enhancing survival. Such examples can be seen throughout the network. As discussed in the section on toxins and exoproteins, expression of T3SS genes can be regulated at multiple levels, in response to various different signals and stress conditions, and is not entirely dependent on any one signal. However, the extent of contributions of the individual regulators and consequently, the fine balance that exists in some regulatory cascades within the network, are sometimes not easily apparent. Network dependence is also a probable reason for regulator genes being non-essential, in the sense that deleting a transcriptional regulator gene typically does not affect cell viability due to the presence alternate regulatory mechanisms. Having key regulators modulate different related phenotypes has the added advantage in allowing the cells to adapt to external signals by modulating one or a few regulators instead of individually regulating different virulence systems. A case in point is AmpR that positively regulates acute virulence factors while downregulating chronic infection phenotypes (225). Also of importance is the coregulation of metabolism and virulence. Studies have identified regulators like CbrB that, with its cognate sensor CbrA, not only regulate carbon metabolism but also virulence phenotypes via the $\operatorname{rgRNA}, \operatorname{rgCrcZ}$ and the RNA-binding protein $\mathrm{Crc}(811,812)$. 
Moreover, there is crosstalk between $\mathrm{CbrA}$ and regulators other than $\mathrm{CbrB}$, highlighting the complexity of the system (811).

The plethora of transcriptome studies using microarrays, or deep sequencing will add to the database of genes that are differentially expressed in response to regulator mutations or specific growth conditions. Differentiating the direct effect of a change from a ripple effect can, at least partly, be achieved by metaanalysis studies that look at multiple transcriptomes, identifying effects unique to each condition and differentiating them from the so-called ripple $(28,400)$. Network analyses can help us understand the relationship between different regulators, group them based on function and, more importantly, help identify critical nodes and prominent players. This can serve as a means of target identification in attempting to deal with P. aeruginosa infections. In Figure 6.2, we see that some parts of the network are more densely connected than others, with central cores containing most of the links. A case in point is LasR of the QS subnetwork. It is well known that QS is central to virulence regulation in $P$. aeruginosa and targeting key regulators will have a better chance of therapeutic success. Recently, inhibitors of a key QS regulator were shown to reduce pathogenicity in Vibrio cholera (586).

\section{Future directions}

Taking the AmpR project forward will involve working on two main aspects: mechanistic and application-oriented.

The mechanistic basis of the regulatory process, such as the effectors recognized by $P$. aeruginosa AmpR, is not known. This will be aided by resolving the AmpR structure. Further, the details of the AmpR regulatory cascade remains largely unsolved. For example, data from my dissertation identified the QS master regulator LasR to be a 
direct target of AmpR. However, this does not fully explain the extensive AmpR regulon. The role of sRNAs in the AmpR regulon needs to be elucidated.

Data from my dissertation has identified AmpR to be a major regulator of pathogenesis and metabolism in P. aeruginosa. So, an application-oriented extension of my project will be to identify inhibitors of AmpR function that will help manage $P$. aeruginosa infections in the clinical setting. These have been discussed in more detail in the following section.

\section{Identifying effectors that modulate AmpR activity}

It has been well established that LTTRs regulate expression of target genes in response to effectors $(103,104)$. Analysis of the P. aeruginosa AmpR protein sequence reveals a predicted N-terminal DNA-binding HTH domain (amino acids 1-63), and Cterminal effector-binding domain (amino acids 90-290). In vitro analyses have shown that the anhydro-MurNAc tripeptide activates $C$. freundii AmpR whereas in the presence of antibiotics, anhydro-MurNAc-pentapeptides positively activate E. cloacae AmpR $(121,410,839)$. In C. freundii, UDP-MurNAc-pentapeptide represses AmpR activity $(121,410)$. In $P$. aeruginosa, although these effectors have been suggested to play a role in AmpC B-lactamase induction $(73,348)$, there is no supporting experimental evidence.

To date, AmpR is believed to be a soluble cytoplasmic protein (410). In the purification of AmpR from C. freundii, most of the protein was found to be in the insoluble fraction but this was attributed to protein aggregation (410). However, research in the Mathee lab shows that $P$. aeruginosa AmpR is an inner membrane protein, with a single predicted transmembrane domain between the HTH and effector-binding domains 
(O. Caille, personal communication). Since the effector-binding domain is in the periplasm, the activating and repressing effectors molecules have to be found in periplasm. Based on what is known about E. coli cell-wall synthesis and recycling, one could predict the putative effectors to be one of the following molecules: GlcNAcanhydroMurNAc tri- or tetra-peptides, GlcNAc-anhydroMurNAc, anhydroMurNAc-trior tetra-peptides, free peptides (tri- and tetrapeptides) GlcNAc-anhydroMurNAcpentapeptide or anhydroMurNAc-pentapeptide (pentapeptides only in the presence of antibiotics) (Chapter 1, Figure 3). Identification of AmpR effector molecules can therefore be addressed with three specific aims:

a. Analysis of specific cellular fractions by HPLC to identify the muramyl peptide effectors.

b. Determine the role of effectors in activation and repression of AmpR using in vitro transcription assays.

c. Crystallizing the AmpR protein in the presence and absence of the effector molecules.

\section{Experimental Design to identify effectors of $\mathrm{P}$. aeruginosa AmpR}

Construction of $\operatorname{lys} \boldsymbol{A}$ strain of PAO1 Previous work in the lab to label $P$. aeruginosa diaminopimelate (DAP) peptidoglycan showed that PAO1 was very refractory to labeling (840). This is due to the enzymatic activity of LysA (PA5277 encoding a diaminopimelate decarboxylase) that converts DAP to L-lysine. In the presence of LysA, DAP cannot be labeled with ${ }^{3}[\mathrm{H}]$. Labeling was achieved in E. coli only in the absence of lys $A$ (840). In order to label the P. aeruginosa peptidoglycan 
precursors, the $y_{s} A$ gene will have to be deleted in PAO1. The permease AmpG has been demonstrated to transport the muramyl degradation products (GlcNAc-anhydroMurNAcpeptides) into the cytoplasm for recycling (121). We propose that an AmpG homolog, AmpP, is also involved in this process in P. aeruginosa and transports UDP-MurNAcpentapeptide into the periplasm (Chapter 1, Figure 2). These genes should also be deleted in the lys $A$ mutant strain background (PAO $\Delta y_{s} A \Delta a m p G$ and $\left.\mathrm{PAO} \Delta l y s A \Delta a m p P\right)$ to ensure isolation of a high concentration of peptidoglycan degradation products from the periplasm and cytoplasm.

Labeling, fractionation and HPLC analysis To isolate labeled muropeptides, $\mathrm{PAO} \Delta l y s A, \mathrm{PAO} \Delta l y s A \Delta a m p G$ and $\mathrm{PAO} \Delta l y s A \Delta a m p P$ should be grown in LB broth containing ${ }^{3}[\mathrm{H}]$, thus labeling the DAP in the peptidoglycan layer. The periplasmic and cytoplasmic contents can then be isolated using commercially available kits (Epicentre Biotechnologies). Log/late-log phase cells, with and without exposure to sub-MIC ßlactams, should be used for the fractionation process. Spheroplasts can be generated by treating the cells with lysozyme and EDTA followed by osmotic shocking. This will release the periplasmic contents to form spheroplasts (841), which can be purified and lysed to obtain the cytoplasmic contents. The cytoplasmic, periplasmic and whole cell fractions from PAO1 and the mutant strains can then be analyzed by HPLC to determine the fractions that contain the putative effectors. As controls to ensure proper fractionation, the different fractions can be subjected to Western blot analysis using antibodies against ß-lactamase (periplasmic fraction) and $\sigma 70$ (cytoplasmic fraction). 
HPLC analysis of the different cellular fractions will identify the fractions containing the putative effectors. Fractions that contain radioactivity (due to DAP) and absorb UV (due to uridine) are the ones that contain the compounds of interest.

Purification of AmpR For the in vitro transcription assays, purified $A m p R$ is required. For purification of $\mathrm{AmpR}$, expression clones (pMMB67EH-Gm, $\mathrm{P}_{\mathrm{tac}}$ ) containing a C-terminal 6-His tag of the whole ORF (290 amino acids) should be used. This overexpression construct in PAO $\triangle a m p R$ has already been generated (DBS39, DBS176). The overexpressed protein can be purified by affinity chromatography on NiNTA matrix. We already have data from radioactive mobility-shift assays of the $\operatorname{ampC}$ promoter with AmpR-containing membrane fractions of overexpression strains (O. Caille, unpublished data).

In vitro transcription assay The $а m p C$ promoter from $\mathrm{PAO} 1$ can be cloned into pUC19-spf' and used as the template for the in vitro transcription assay. This plasmid is a derivative of pUC19 that contains a spf transcription terminator downstream of the multiple cloning site (842). Transcription termination will occur at spf to produce discrete-sized transcripts. Purified E. coli RNA polymerase (USB-Affymetrix) can be used for the assay. This approach should work because AmpR activation of ampC occurs in E. coli (105).

Testing effector molecules to influence B-lactamase production Purified AmpR with the template (target promoter cloned in pUC19-spf') can be incubated in a Tris- $\mathrm{HCl}$ buffer containing DTT, $\mathrm{KCl}, \mathrm{MgCl}_{2}$ and glycerol. Following this, RNA polymerase and NTPs (with $\propto^{32}$-labeled UTP, other bases unlabeled) should be added 
and incubated to allow in vitro transcription. The samples must then be DNase-treated, spiked with unlabeled tRNA followed by the phenol-chloroform protocol to isolate nucleic acids. The samples should then be run on a denaturing polyacrylamide gel, and exposed to film to locate the bands.

Once this assay has been standardized for AmpR concentration and conditions, effector molecule fractions can be tested for activation or repression of ampC expression (incubated in the first step with AmpR). Activation of ampC expression by the effectors will lead to a higher concentration of mRNA, whereas repression will have the opposite effect. Pure effectors can be synthesized for this experiment.

Crystallization of AmpR with and without effectors This aspect of the project can be performed in collaboration with Emerald Biostructures. The non-effector bound AmpR can be crystallized to determine the pocket where the effector molecule would bind. Modeling and docking studies can then be done to correlate our in vitro data on effector molecules and compare with the in vivo and in vitro analysis. Subsequently, cocrystallization can be done. Emerald Biostructures also provides analysis of potential inhibitors that are likely to bind. The process to crystallize AmpR in collaboration with Emerald Biostructures has already been initiated.

\section{Identification of small molecule inhibitors of AmpR}

Once the AmpR effectors are determined, the next logical step would be to design/identify inhibitors of AmpR function. Small molecule inhibitors of AmpR can be potential therapeutic agents against $P$. aeruginosa virulence and reduce resistance to $B$ lactams. The screening can be performed at the National Screening Laboratory for the 
Regional Centers for Biodefense and Emerging Infectious Diseases (NSRB; nsrb.med.harvard.edu), which has a library of over 100,000 structurally diverse compounds. Reporter strains that harbor promoter fusions of ampC (AmpR-positively regulated) or mexE (AmpR negatively-regulated) can be used to determine small molecule potentiators or inhibitors of AmpR function. Following the initial screen to identify compounds of interest, further assays such as qPCRs, MICs, in vitro production of various virulence factors, cytotoxicity, virulence in C. elegans can be performed to validate the efficacy of the inhibitor compounds. After the compounds have been identified and their inhibitory roles confirmed, one could model the compound with AmpR to identify potential interaction domains.

The studies that have identified AmpR effectors in Enterobacteriaceae members $(121,410,839)$ consider AmpR to be only a positive regulator of ampC expression. However, data from my dissertation demonstrates that AmpR is a major transcriptional regulator that affects expression of diverse phenotypes. Whether AmpR-mediated regulation of the non-AmpC phenotypes is in response to the cell wall intermediates or other, as yet unidentified, effectors is a major question that needs to be answered. Answering this question will also help synthesize effector mimics that can potentially be used to inactivate AmpR function in the clinical setting, thus reducing virulence and rendering the cells sensitive to ß-lactam antibiotics.

\section{Known, putative and novel small regulatory RNAs in the AmpR regulon}

Microarrays, RNA-Seq, qPCR and complementary studies have identified some known sRNAs (rgRsmZ, asPrrF1, rgRgsA) to be under AmpR regulation (Chapters 4, 5). 
This clearly shows that the AmpR regulon includes sRNAs. However, recent studies have identified over 500 novel sRNAs in $P$. aeruginosa PAO1 $(843,844)$ whose function and regulation have not been elucidated. Given the extensive nature of the AmpR regulon, it is very likely that many more sRNAs are AmpR-regulated. The next phase of research will involve determining the sRNAs that are regulated by AmpR. Further, detailing the mode of action of the AmpR-regulated sRNAs will make for exciting research.

Moreover, it is possible that there are novel sRNAs that have not been identified yet. Quantitative analysis of the RNA-Seq data will provide insights into novel ncRNAs. These can be regulatory RNAs or antisense RNAs and are explained below.

a. Regulatory RNAs: RNA-Seq reads that map to intergenic regions, or even within ORFs, need to be analyzed further in silico (look for putative promoters and terminators, secondary structures of the reads, differential expression between the conditions) and in vitro (deletion and overexpression phenotypic analyses, Northern blot analysis) in PAO1 and the ampR mutant strains to confirm presence, and AmpR-mediated regulation.

b. Antisense RNAs (asRNAs): These are RNA-Seq reads that map to within the preannotated ORFs in the Pseudomonas Genome Database (27), but in the opposite strand to the ORF in the RNA-Seq data. In addition to the in silico and in vitro analyses mentioned in the previous section, potential targets of asRNAs can be determined by BLAST analysis of the asRNA sequence against the PAO1 genome.

With the extensive use of high-throughput transcriptomics, gene regulation studies are now focusing on the role of non-coding RNAs in bacteria. rgRNAs have been 
shown to be extensively involved in gene regulation in P. aeruginosa and other bacteria $(390,458,758,810,819,845)$. Moreover, the interplay of rgRNAs and transcriptional regulators in controlling critical functions in bacteria is being increasingly appreciated. Given the important regulatory role of AmpR in P. aeruginosa virulence and metabolism, it is not surprising that rgRNAs such as rgRsmZ are AmpR-regulated (Chapter 5). Elucidating the mechanism of regulation of AmpR, the regulatory interaction of AmpR with $\operatorname{rgRNAs}$ and the signals that determine AmpR-mediated regulation would be the natural extension of my work. Other groups, by crystallizing the effector-binding domain, have initiated biochemical studies detailing AmpR (612). Moreover, techniques such as RNA-Seq allow for the entire transcriptome to be sequenced, giving us an unprecedented insight into non-coding RNAs, asRNAs and sRNAs involved in regulation. Preliminary studies using prediction software and complementary experiments have already advanced our understanding $(390,458,519,647,819,846)$. Given the many different ways in which small RNAs can modulate gene expression (818) and potentially undiscovered ones, we can look forward to exciting new discoveries in bacterial gene regulation in the coming years. 


\section{APPENDICES}

Supplementary information for Chapter 2 


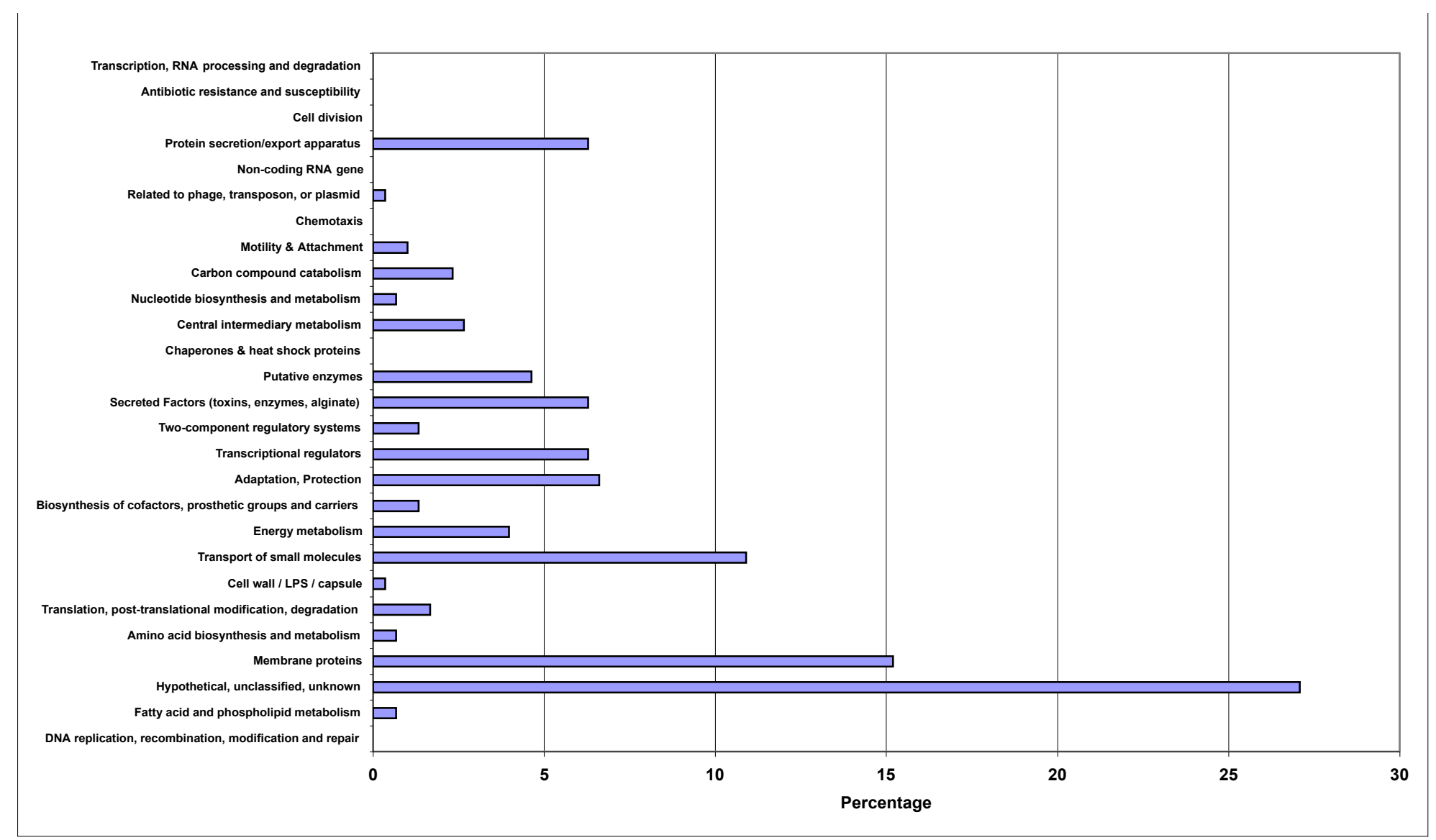

Supplementary Fig 1: Core set of gene differentially regulated in all the studies 
Supplementary Table 1: Comparison of all the genes that are differentially regulated in the $\mathbf{1 8}$ transcriptomes. The list of ORFs are divided into core and accessory gene set, as defined in Mathee et al (PMID 18287045).

\begin{tabular}{|c|c|c|}
\hline ORF & Product name & Function class \\
\hline \multicolumn{3}{|c|}{ Transcriptional core set ORFs present in the $P$. aeruginosa core genome } \\
\hline PA0044 & exoenzyme T & Secreted Factors (toxins, enzymes, alginate) \\
\hline PA0045 & hypothetical protein & Hypothetical, unclassified, unknown \\
\hline PA0059 & osmotically inducible protein $\mathrm{OsmC}$ & Adaptation, Protection \\
\hline PA0070 & hypothetical protein & Membrane proteins \\
\hline PA0078 & hypothetical protein & Hypothetical, unclassified, unknown \\
\hline PA0082 & hypothetical protein & Hypothetical, unclassified, unknown \\
\hline PA0084 & conserved hypothetical protein & Hypothetical, unclassified, unknown \\
\hline PA0085 & conserved hypothetical protein & Hypothetical, unclassified, unknown \\
\hline PA0140 & alkyl hydroperoxide reductase subunit $F$ & Adaptation, Protection \\
\hline PA0141 & conserved hypothetical protein & Hypothetical, unclassified, unknown \\
\hline PA0162 & histidine porin OpdC & Membrane proteins \\
\hline PA0183 & arylsulfatase & Central intermediary metabolism \\
\hline PA0184 & probable ATP-binding component of $\mathrm{ABC}$ transporter & Transport of small molecules \\
\hline PA0185 & probable permease of $\mathrm{ABC}$ transporter & Membrane proteins \\
\hline PA0186 & probable binding protein component of $\mathrm{ABC}$ transporter & Transport of small molecules \\
\hline PA0229 & dicarboxylic acid transporter PcaT & Membrane proteins \\
\hline PA0284 & hypothetical protein & Hypothetical, unclassified, unknown \\
\hline PA0290 & hypothetical protein & Hypothetical, unclassified, unknown \\
\hline PA0291 & Anaerobically-induced outer membrane porin OprE precursor & Membrane proteins \\
\hline PA0424 & multidrug resistance operon repressor MexR & Transcriptional regulators \\
\hline PA0447 & glutaryl-CoA dehydrogenase & Fatty acid and phospholipid metabolism \\
\hline PA0460 & hypothetical protein & Hypothetical, unclassified, unknown \\
\hline PA0471 & probable transmembrane sensor & Two-component regulatory systems \\
\hline PA0472 & probable sigma- 70 factor, ECF subfamily & Transcriptional regulators \\
\hline PA0514 & heme $\mathrm{d} l$ biosynthesis protein $\mathrm{NirL}$ & Hypothetical, unclassified, unknown \\
\hline PA0517 & probable c-type cytochrome precursor & Biosynthesis of cofactors, prosthetic groups and carriers \\
\hline PA0518 & cytochrome c-551 precursor & Biosynthesis of cofactors, prosthetic groups and carriers \\
\hline PA0519 & nitrite reductase precursor & Energy metabolism \\
\hline PA0524 & nitric-oxide reductase subunit $B$ & Energy metabolism \\
\hline PA0572 & hypothetical protein & Hypothetical, unclassified, unknown \\
\hline PA0604 & probable binding protein component of $\mathrm{ABC}$ transporter & Transport of small molecules \\
\hline PA0605 & probable permease of $\mathrm{ABC}$ transporter & Membrane proteins \\
\hline PA0672 & heme oxygenase & Biosynthesis of cofactors, prosthetic groups and carriers \\
\hline PA0688 & probable binding protein component of $\mathrm{ABC}$ transporter & Transport of small molecules \\
\hline PA0762 & sigma factor $\mathrm{Alg} \mathrm{U}$ & Transcriptional regulators \\
\hline PA0763 & anti-sigma factor MucA & Transcriptional regulators \\
\hline PA0764 & negative regulator for alginate biosynthesis MucB & Transcriptional regulators \\
\hline PA0775 & conserved hypothetical protein & Hypothetical, unclassified, unknown \\
\hline PA0848 & probable alkyl hydroperoxide reductase & Adaptation, Protection \\
\hline PA0849 & thioredoxin reductase 2 & Nucleotide biosynthesis and metabolism \\
\hline PA0852 & chitin-binding protein $\mathrm{CbpD}$ precursor & Secreted Factors (toxins, enzymes, alginate) \\
\hline PA0866 & aromatic amino acid transport protein AroP2 & Transport of small molecules \\
\hline PA0874 & hypothetical protein & Hypothetical, unclassified, unknown \\
\hline PA0929 & two-component response regulator & Transport of small molecules \\
\hline PA0938 & hypothetical protein & Hypothetical, unclassified, unknown \\
\hline PA0958 & Outer membrane porin OprD precursor & Transport of small molecules \\
\hline PA0962 & probable dna-binding stress protein & Adaptation, Protection \\
\hline PA1071 & branched-chain amino acid transport protein $\mathrm{BraF}$ & Transport of small molecules \\
\hline PA1156 & NrdA, catalytic component of class Ia ribonucleotide reductase & Nucleotide biosynthesis and metabolism \\
\hline PA1179 & two-component response regulator $\mathrm{PhoP}$ & Transcriptional regulators \\
\hline PA1180 & two-component sensor PhoQ & Two-component regulatory systems \\
\hline PA1230 & hypothetical protein & Membrane proteins \\
\hline PA1245 & hypothetical protein & Membrane proteins \\
\hline PA1246 & alkaline protease secretion protein $\mathrm{AprD}$ & Secreted Factors (toxins, enzymes, alginate) \\
\hline PA1249 & alkaline metalloproteinase precursor & Secreted Factors (toxins, enzymes, alginate) \\
\hline
\end{tabular}




\begin{tabular}{|c|c|c|}
\hline ORF & Product name & Function class \\
\hline PA1289 & hypothetical protein & Hypothetical, unclassified, unknown \\
\hline PA1300 & probable sigma-70 factor, ECF subfamily & Transcriptional regulators \\
\hline PA1317 & cytochrome 0 ubiquinol oxidase subunit II & Energy metabolism \\
\hline PA1318 & cytochrome 0 ubiquinol oxidase subunit I & Energy metabolism \\
\hline PA1319 & cytochrome o ubiquinol oxidase subunit III & Energy metabolism \\
\hline PA1323 & hypothetical protein & Hypothetical, unclassified, unknown \\
\hline PA1324 & hypothetical protein & Hypothetical, unclassified, unknown \\
\hline PA1346 & hypothetical protein & Hypothetical, unclassified, unknown \\
\hline PA1408 & hypothetical protein & Membrane proteins \\
\hline PA1541 & probable drug efflux transporter & Membrane proteins \\
\hline PA1544 & transcriptional regulator Anr & Transcriptional regulators \\
\hline PA1546 & oxygen-independent coproporphyrinogen III oxidase & Biosynthesis of cofactors, prosthetic groups and carriers \\
\hline PA1555 & probable cytochrome c & Energy metabolism \\
\hline PA1556 & probable cytochrome c oxidase subunit & Energy metabolism \\
\hline PA1557 & probable cytochrome oxidase subunit (cbb3-type) & Energy metabolism \\
\hline PA1657 & conserved hypothetical protein & Hypothetical, unclassified, unknown \\
\hline PA1673 & hypothetical protein & Hypothetical, unclassified, unknown \\
\hline PA1691 & translocation protein in type III secretion & Protein secretion/export apparatus \\
\hline PA1696 & translocation protein in type III secretion & Protein secretion/export apparatus \\
\hline PA1698 & Type III secretion outer membrane protein PopN precursor & Membrane proteins \\
\hline PA1699 & conserved hypothetical protein in type III secretion & Protein secretion/export apparatus \\
\hline PA1700 & conserved hypothetical protein in type III secretion & Protein secretion/export apparatus \\
\hline PA1701 & conserved hypothetical protein in type III secretion & Protein secretion/export apparatus \\
\hline PA1705 & regulator in type III secretion & Protein secretion/export apparatus \\
\hline PA1706 & type III secretion protein $\mathrm{PcrV}$ & Protein secretion/export apparatus \\
\hline PA1707 & regulatory protein $\mathrm{PcrH}$ & Secreted Factors (toxins, enzymes, alginate) \\
\hline PA1708 & translocator protein PopB & Protein secretion/export apparatus \\
\hline PA1709 & Translocator outer membrane protein PopD precursor & Protein secretion/export apparatus \\
\hline PA1710 & ExsC, exoenzyme $\mathrm{S}$ synthesis protein $\mathrm{C}$ precursor. & Translation, post-translational modification, degradation \\
\hline PA1711 & ExsE & Hypothetical, unclassified, unknown \\
\hline PA1712 & exoenzyme $\mathrm{S}$ synthesis protein $\mathrm{B}$ & Translation, post-translational modification, degradation \\
\hline PA1713 & transcriptional regulator ExsA & Protein secretion/export apparatus \\
\hline PA1714 & ExsD & Hypothetical, unclassified, unknown \\
\hline PA1715 & type III export apparatus protein & Protein secretion/export apparatus \\
\hline PA1716 & Type III secretion outer membrane protein PscC precursor & Protein secretion/export apparatus \\
\hline PA1717 & type III export protein PscD & Protein secretion/export apparatus \\
\hline PA1719 & type III export protein PscF & Protein secretion/export apparatus \\
\hline PA1720 & type III export protein PscG & Protein secretion/export apparatus \\
\hline PA1721 & type III export protein PscH & Protein secretion/export apparatus \\
\hline PA1722 & type III export protein PscI & Protein secretion/export apparatus \\
\hline PA1723 & type III export protein PscJ & Protein secretion/export apparatus \\
\hline PA1761 & hypothetical protein & Hypothetical, unclassified, unknown \\
\hline PA1793 & peptidyl-prolyl cis-trans isomerase B & Translation, post-translational modification, degradation \\
\hline PA1869 & probable acyl carrier protein & Fatty acid and phospholipid metabolism \\
\hline PA1894 & hypothetical protein & Hypothetical, unclassified, unknown \\
\hline PA1902 & phenazine biosynthesis protein $\mathrm{PhzD}$ & Secreted Factors (toxins, enzymes, alginate) \\
\hline PA1904 & probable phenazine biosynthesis protein & Secreted Factors (toxins, enzymes, alginate) \\
\hline PA1982 & quinoprotein alcohol dehydrogenase & Carbon compound catabolism \\
\hline PA2080 & hypothetical protein & Hypothetical, unclassified, unknown \\
\hline PA2081 & kynurenine formamidase, KynB & Hypothetical, unclassified, unknown \\
\hline PA2087 & hypothetical protein & Hypothetical, unclassified, unknown \\
\hline PA2088 & hypothetical protein & Hypothetical, unclassified, unknown \\
\hline PA2111 & hypothetical protein & Hypothetical, unclassified, unknown \\
\hline PA2112 & conserved hypothetical protein & Hypothetical, unclassified, unknown \\
\hline PA2113 & pyroglutamate porin 0 pdO & Transport of small molecules \\
\hline PA2114 & probable major facilitator superfamily (MFS) transporter & Membrane proteins \\
\hline PA2143 & hypothetical protein & Hypothetical, unclassified, unknown \\
\hline
\end{tabular}




\begin{tabular}{|c|c|c|}
\hline ORF & Product name & Function class \\
\hline PA2146 & conserved hypothetical protein & Hypothetical, unclassified, unknown \\
\hline PA2158 & probable alcohol dehydrogenase (Zn-dependent) & Putative enzymes \\
\hline PA2159 & conserved hypothetical protein & Hypothetical, unclassified, unknown \\
\hline PA2166 & hypothetical protein & Hypothetical, unclassified, unknown \\
\hline PA2171 & hypothetical protein & Hypothetical, unclassified, unknown \\
\hline PA2173 & hypothetical protein & Hypothetical, unclassified, unknown \\
\hline PA2177 & probable sensor/response regulator hybrid & Two-component regulatory systems \\
\hline PA2191 & adenylate cyclase ExoY & Secreted Factors (toxins, enzymes, alginate) \\
\hline PA2193 & hydrogen cyanide synthase $\mathrm{HcnA}$ & Central intermediary metabolism \\
\hline PA2194 & hydrogen cyanide synthase $\mathrm{HcnB}$ & Central intermediary metabolism \\
\hline PA2195 & hydrogen cyanide synthase $\mathrm{HcnC}$ & Central intermediary metabolism \\
\hline PA2247 & 2-oxoisovalerate dehydrogenase (alpha subunit) & Amino acid biosynthesis and metabolism \\
\hline PA2248 & 2-oxoisovalerate dehydrogenase (beta subunit) & Amino acid biosynthesis and metabolism \\
\hline PA2260 & hypothetical protein & Hypothetical, unclassified, unknown \\
\hline PA2261 & probable 2-ketogluconate kinase & Carbon compound catabolism \\
\hline PA2262 & probable 2-ketogluconate transporter & Membrane proteins \\
\hline PA2263 & probable 2-hydroxyacid dehydrogenase & Putative enzymes \\
\hline PA2264 & conserved hypothetical protein & Hypothetical, unclassified, unknown \\
\hline PA2265 & gluconate dehydrogenase & Carbon compound catabolism \\
\hline PA2266 & probable cytochrome $\mathrm{c}$ precursor & Carbon compound catabolism \\
\hline PA2299 & probable transcriptional regulator & Transcriptional regulators \\
\hline PA2302 & probable non-ribosomal peptide synthetase & Putative enzymes \\
\hline PA2304 & hypothetical protein & Hypothetical, unclassified, unknown \\
\hline PA2305 & probable non-ribosomal peptide synthetase & Putative enzymes \\
\hline PA2307 & probable permease of $\mathrm{ABC}$ transporter & Membrane proteins \\
\hline PA2309 & hypothetical protein & Hypothetical, unclassified, unknown \\
\hline PA2310 & hypothetical protein & Putative enzymes \\
\hline PA2331 & hypothetical protein & Membrane proteins \\
\hline PA2385 & PvdQ & Adaptation, Protection \\
\hline PA2386 & L-ornithine N5-oxygenase & Adaptation, Protection \\
\hline PA2389 & conserved hypothetical protein & Hypothetical, unclassified, unknown \\
\hline PA2392 & PvdP & Adaptation, Protection \\
\hline PA2393 & probable dipeptidase precursor & Central intermediary metabolism \\
\hline PA2394 & $\operatorname{PvdN}$ & Adaptation, Protection \\
\hline PA2396 & pyoverdine synthetase $F$ & Secreted Factors (toxins, enzymes, alginate) \\
\hline PA2397 & pyoverdine biosynthesis protein PvdE & Membrane proteins \\
\hline PA2403 & hypothetical protein & Membrane proteins \\
\hline PA2404 & hypothetical protein & Membrane proteins \\
\hline PA2405 & hypothetical protein & Hypothetical, unclassified, unknown \\
\hline PA2407 & probable adhesion protein & Motility \& Attachment \\
\hline PA2408 & probable ATP-binding component of $\mathrm{ABC}$ transporter & Transport of small molecules \\
\hline PA2409 & probable permease of $A B C$ transporter & Membrane proteins \\
\hline PA2410 & hypothetical protein & Hypothetical, unclassified, unknown \\
\hline PA2411 & probable thioesterase & Adaptation, Protection \\
\hline PA2412 & conserved hypothetical protein & Hypothetical, unclassified, unknown \\
\hline PA2413 & L-2,4-diaminobutyrate:2-ketoglutarate 4-aminotransferase, $\mathrm{PvdH}$ & Adaptation, Protection \\
\hline PA2414 & L-sorbosone dehydrogenase & Carbon compound catabolism \\
\hline PA2415 & hypothetical protein & Membrane proteins \\
\hline PA2424 & PvdL & Adaptation, Protection \\
\hline PA2425 & PvdG & Adaptation, Protection \\
\hline PA2426 & sigma factor PvdS & Transcriptional regulators \\
\hline PA2433 & hypothetical protein & Hypothetical, unclassified, unknown \\
\hline PA2468 & ECF sigma factor FoxI & Transcriptional regulators \\
\hline PA2505 & tyrosine porin $0 \mathrm{pdT}$ & Transport of small molecules \\
\hline PA2523 & probable two-component response regulator & Transcriptional regulators \\
\hline PA2548 & hypothetical protein & Hypothetical, unclassified, unknown \\
\hline PA2588 & probable transcriptional regulator & Transcriptional regulators \\
\hline
\end{tabular}




\begin{tabular}{|c|c|c|}
\hline ORF & Product name & Function class \\
\hline PA2592 & probable periplasmic spermidine/putrescine-binding protein & Transport of small molecules \\
\hline PA2630 & conserved hypothetical protein & Hypothetical, unclassified, unknown \\
\hline PA2634 & isocitrate lyase & Putative enzymes \\
\hline PA2687 & two-component sensor PfeS & Two-component regulatory systems \\
\hline PA2747 & hypothetical protein & Hypothetical, unclassified, unknown \\
\hline PA2760 & probable outer membrane protein precursor & Transport of small molecules \\
\hline PA2808 & two-component response repressor, $\operatorname{PtrA}$ & Transcriptional regulators \\
\hline PA2850 & organic hydroperoxide resistance protein & Adaptation, Protection \\
\hline PA2896 & probable sigma-70 factor, ECF subfamily & Transcriptional regulators \\
\hline PA3235 & conserved hypothetical protein & Membrane proteins \\
\hline PA3278 & hypothetical protein & Membrane proteins \\
\hline PA3281 & hypothetical protein & Membrane proteins \\
\hline PA3283 & conserved hypothetical protein & Hypothetical, unclassified, unknown \\
\hline PA3284 & hypothetical protein & Hypothetical, unclassified, unknown \\
\hline PA3287 & conserved hypothetical protein & Hypothetical, unclassified, unknown \\
\hline PA3328 & probable FAD-dependent monooxygenase & Putative enzymes \\
\hline PA3369 & hypothetical protein & Membrane proteins \\
\hline PA3371 & hypothetical protein & Hypothetical, unclassified, unknown \\
\hline PA3413 & conserved hypothetical protein & Hypothetical, unclassified, unknown \\
\hline PA3414 & hypothetical protein & Hypothetical, unclassified, unknown \\
\hline PA3416 & probable pyruvate dehydrogenase E1 component, beta chain & Energy metabolism \\
\hline PA3446 & conserved hypothetical protein & Hypothetical, unclassified, unknown \\
\hline PA3447 & probable ATP-binding component of $\mathrm{ABC}$ transporter & Transport of small molecules \\
\hline PA3448 & probable permease of $\mathrm{ABC}$ transporter & Membrane proteins \\
\hline PA3450 & probable antioxidant protein & Adaptation, Protection \\
\hline PA3460 & probable acetyltransferase & Putative enzymes \\
\hline PA3461 & conserved hypothetical protein & Hypothetical, unclassified, unknown \\
\hline PA3515 & hypothetical protein & Hypothetical, unclassified, unknown \\
\hline PA3516 & probable lyase & Putative enzymes \\
\hline PA3517 & probable lyase & Carbon compound catabolism \\
\hline PA3518 & hypothetical protein & Hypothetical, unclassified, unknown \\
\hline PA3519 & hypothetical protein & Hypothetical, unclassified, unknown \\
\hline PA3520 & hypothetical protein & Hypothetical, unclassified, unknown \\
\hline PA3523 & probable RND efflux membrane fusion protein precursor & Transport of small molecules \\
\hline PA3531 & bacterioferritin & Transport of small molecules \\
\hline PA3573 & probable major facilitator superfamily (MFS) transporter & Adaptation, Protection \\
\hline PA3600 & conserved hypothetical protein & Translation, post-translational modification, degradation \\
\hline PA3690 & probable metal-transporting P-type ATPase & Membrane proteins \\
\hline PA3691 & hypothetical protein & Hypothetical, unclassified, unknown \\
\hline PA3709 & probable major facilitator superfamily (MFS) transporter & Membrane proteins \\
\hline PA3790 & Putative copper transport outer membrane porin OprC precursor & Transport of small molecules \\
\hline PA3842 & probable chaperone & Secreted Factors (toxins, enzymes, alginate) \\
\hline PA3899 & probable sigma-70 factor, ECF subfamily & Transcriptional regulators \\
\hline PA3900 & probable transmembrane sensor & Membrane proteins \\
\hline PA3904 & hypothetical protein & Hypothetical, unclassified, unknown \\
\hline PA3936 & probable permease of $\mathrm{ABC}$ taurine transporter & Membrane proteins \\
\hline PA3937 & probable ATP-binding component of $\mathrm{ABC}$ taurine transporter & Transport of small molecules \\
\hline PA3938 & probable periplasmic taurine-binding protein precursor & Transport of small molecules \\
\hline PA3954 & hypothetical protein & Hypothetical, unclassified, unknown \\
\hline PA4067 & Outer membrane protein OprG precursor & Membrane proteins \\
\hline PA4139 & hypothetical protein & Hypothetical, unclassified, unknown \\
\hline PA4156 & probable TonB-dependent receptor & Transport of small molecules \\
\hline PA4168 & second ferric pyoverdine receptor FpvB & Transport of small molecules \\
\hline PA4171 & probable protease & Putative enzymes \\
\hline PA4179 & probable porin & Membrane proteins \\
\hline PA4217 & flavin-containing monooxygenase & Putative enzymes \\
\hline PA4218 & probable transporter & Membrane proteins \\
\hline
\end{tabular}




\begin{tabular}{|c|c|c|}
\hline$\overline{\mathrm{ORF}}$ & Product name & Function class \\
\hline PA4219 & hypothetical protein & Membrane proteins \\
\hline PA4220 & hypothetical protein & Hypothetical, unclassified, unknown \\
\hline PA4221 & $\mathrm{Fe}(\mathrm{III})$-pyochelin outer membrane receptor precursor & Transport of small molecules \\
\hline PA4222 & probable ATP-binding component of $\mathrm{ABC}$ transporter & Transport of small molecules \\
\hline PA4224 & pyochelin biosynthetic protein PchG & Transport of small molecules \\
\hline PA4225 & pyochelin synthetase & Secreted Factors (toxins, enzymes, alginate) \\
\hline PA4226 & dihydroaeruginoic acid synthetase & Secreted Factors (toxins, enzymes, alginate) \\
\hline PA4227 & transcriptional regulator $P c h R$ & Transcriptional regulators \\
\hline PA4228 & pyochelin biosynthesis protein PchD & Secreted Factors (toxins, enzymes, alginate) \\
\hline PA4229 & pyochelin biosynthetic protein PchC & Secreted Factors (toxins, enzymes, alginate) \\
\hline PA4230 & salicylate biosynthesis protein $\mathrm{PchB}$ & Secreted Factors (toxins, enzymes, alginate) \\
\hline PA4231 & salicylate biosynthesis isochorismate synthase & Secreted Factors (toxins, enzymes, alginate) \\
\hline PA4296 & two-component response regulator, $\mathrm{PprB}$ & Transcriptional regulators \\
\hline PA4359 & conserved hypothetical protein & Hypothetical, unclassified, unknown \\
\hline PA4429 & probable cytochrome $\mathrm{cl}$ precursor & Energy metabolism \\
\hline PA4430 & probable cytochrome b & Energy metabolism \\
\hline PA4431 & probable iron-sulfur protein & Putative enzymes \\
\hline PA4467 & hypothetical protein & Membrane proteins \\
\hline PA4468 & superoxide dismutase & Adaptation, Protection \\
\hline PA4469 & hypothetical protein & Hypothetical, unclassified, unknown \\
\hline PA4470 & fumarate hydratase & Energy metabolism \\
\hline PA4471 & hypothetical protein & Hypothetical, unclassified, unknown \\
\hline PA4480 & rod shape-determining protein $\mathrm{MreC}$ & Cell wall / LPS / capsule \\
\hline PA4501 & Glycine-glutamate dipeptide porin OpdP & Transport of small molecules \\
\hline PA4563 & 30 S ribosomal protein $\mathrm{S} 20$ & Central intermediary metabolism \\
\hline PA4570 & hypothetical protein & Hypothetical, unclassified, unknown \\
\hline PA4590 & protein activator & Transport of small molecules \\
\hline PA4613 & catalase & Adaptation, Protection \\
\hline PA4670 & ribose-phosphate pyrophosphokinase & Carbon compound catabolism \\
\hline PA4673 & conserved hypothetical protein & Hypothetical, unclassified, unknown \\
\hline PA4708 & Heme-transport protein, PhuT & Transport of small molecules \\
\hline PA4709 & probable hemin degrading factor & Putative enzymes \\
\hline PA4710 & Haem/Haemoglobin uptake outer membrane receptor PhuR precursor & Transport of small molecules \\
\hline PA4719 & probable transporter & Membrane proteins \\
\hline PA4738 & conserved hypothetical protein & Hypothetical, unclassified, unknown \\
\hline PA4739 & conserved hypothetical protein & Hypothetical, unclassified, unknown \\
\hline PA4876 & osmotically inducible lipoprotein $0 \mathrm{smE}$ & Membrane proteins \\
\hline PA4880 & probable bacterioferritin & Central intermediary metabolism \\
\hline PA4896 & probable sigma- 70 factor, ECF subfamily & Transcriptional regulators \\
\hline PA4909 & probable ATP-binding component of $\mathrm{ABC}$ transporter & Transport of small molecules \\
\hline PA4981 & probable amino acid permease & Membrane proteins \\
\hline PA5261 & alginate biosynthesis regulatory protein $\mathrm{AlgR}$ & Secreted Factors (toxins, enzymes, alginate) \\
\hline PA5311 & probable major facilitator superfamily (MFS) transporter & Membrane proteins \\
\hline PA5315 & 50 S ribosomal protein $\mathrm{L} 33$ & Translation, post-translational modification, degradation \\
\hline PA5407 & hypothetical protein & Hypothetical, unclassified, unknown \\
\hline PA5469 & conserved hypothetical protein & Membrane proteins \\
\hline PA5482 & hypothetical protein & Membrane proteins \\
\hline PA5530 & probable MFS dicarboxylate transporter & Membrane proteins \\
\hline \multicolumn{3}{|c|}{ Transcriptional core set ORFs present in the $P$. aeruginosa PA01 accessory genome } \\
\hline PA0203 & probable binding protein component of $\mathrm{ABC}$ transporter & Transport of small molecules \\
\hline PA0204 & probable permease of $\mathrm{ABC}$ transporter & Membrane proteins \\
\hline PA0205 & probable permease of $\mathrm{ABC}$ transporter & Membrane proteins \\
\hline PA0206 & probable ATP-binding component of $\mathrm{ABC}$ transporter & Transport of small molecules \\
\hline PA0633 & hypothetical protein & Related to phage, transposon, or plasmid \\
\hline PA0691 & hypothetical protein & Hypothetical, unclassified, unknown \\
\hline
\end{tabular}




\begin{tabular}{lll}
\hline ORF & Product name & Function class \\
\hline & & \\
PA1414 & hypothetical protein & Hypothetical, unclassified, unknown \\
PA1592 & hypothetical protein & Hypothetical, unclassified, unknown \\
PA1718 & type III export protein PscE & Protein secretion/export apparatus \\
PA2033 & hypothetical protein & Hypothetical, unclassified, unknown \\
PA2034 & hypothetical protein & Hypothetical, unclassified, unknown \\
PA2184 & conserved hypothetical protein & Hypothetical, unclassified, unknown \\
PA2190 & conserved hypothetical protein & Hypothetical, unclassified, unknown \\
PA2308 & probable ATP-binding component of ABC transporter & Transport of small molecules \\
PA2398 & ferripyoverdine receptor & Transport of small molecules \\
PA2399 & pyoverdine synthetase D & Secreted Factors (toxins, enzymes, alginate) \\
PA2400 & PvdJ & Adaptation, Protection \\
PA2402 & probable non-ribosomal peptide synthetase & Putative enzymes \\
PA2452 & hypothetical protein & Hypothetical, unclassified, unknown \\
PA3370 & hypothetical protein & Membrane proteins \\
PA3477 & transcriptional regulator RhlR & Adaptation, Protection \\
PA3841 & exoenzyme S & Secreted Factors (toxins, enzymes, alginate) \\
PA3866 & pyocin protein & Adaptation, Protection \\
PA3923 & hypothetical protein & Hypothetical, unclassified, unknown \\
PA4193 & probable permease of ABC transporter & Membrane proteins \\
PA4223 & probable ATP-binding component of ABC transporter & Membrane proteins \\
PA4306 & Type IVb pilin, Flp & Motility \& Attachment \\
PA4555 & type 4 fimbrial biogenesis protein PilY2 & Motility \& Attachment \\
PA4748 & triosephosphate isomerase & Central intermediary metabolism \\
& &
\end{tabular}


Supplementary Table 2: Functional categorization of genes that are uniquely expressed under the different study conditions.

\begin{tabular}{|c|c|c|c|c|c|c|c|c|c|c|c|}
\hline \multirow[b]{2}{*}{ Code } & \multirow[b]{2}{*}{ Class } & \multicolumn{10}{|c|}{ Percentage of differentially regulated unique genes (minus the core set) } \\
\hline & & Iron & QS & Oxi. Stress & PCP & Copper & Sulfate & Osmotic & Biofilms & RsmA & Total \% \\
\hline $\mathbf{a}$ & DNA replication, recombination, modification and repair & 0 & 1 & 1 & 0 & 0 & 0 & 0 & 0 & 0 & 0.4 \\
\hline b & Fatty acid and phospholipid metabolism & 0 & 2 & 0 & 1 & 0 & 0 & 0 & 2 & 1 & 1.2 \\
\hline c & Hypothetical, unclassified, unknown & 27 & 32 & 26 & 31 & 14 & 14 & 3 & 40 & 1 & 29.7 \\
\hline d & Membrane proteins & 22 & 12 & 8 & 11 & 33 & 14 & 0 & 12 & 8 & 13.3 \\
\hline e & Amino acid biosynthesis and metabolism & 3 & 3 & 2 & 4 & 0 & 0 & 3 & 2 & 6 & 2.5 \\
\hline f & Translation, post-translational modification, degradation & 1 & 1 & 3 & 5 & 2 & 0 & 3 & 4 & 1 & 2.4 \\
\hline g & Cell wall / LPS / capsule & 1 & 1 & 1 & 3 & 0 & 0 & 0 & 5 & 6 & 2.2 \\
\hline h & Transport of small molecules & 10 & 7 & 9 & 12 & 19 & 17 & 0 & 4 & 8 & 7.7 \\
\hline i & Energy metabolism & 4 & 3 & 15 & 3 & 7 & 3 & 0 & 3 & 7 & 5.1 \\
\hline $\mathbf{j}$ & Biosynthesis of cofactors, prosthetic groups and carriers & 4 & 2 & 2 & 3 & 1 & 0 & 0 & 0 & 3 & 1.7 \\
\hline k & Adaptation, Protection & 3 & 2 & 4 & 0 & 5 & 0 & 3 & 3 & 14 & 3.1 \\
\hline 1 & Transcriptional regulators & 7 & 6 & 3 & 7 & 10 & 3 & 37 & 5 & 3 & 5.8 \\
\hline $\mathbf{m}$ & Two-component regulatory systems & 1 & 1 & 0 & 0 & 4 & 0 & 10 & 2 & 1 & 1.1 \\
\hline n & Secreted Factors (toxins, enzymes, alginate) & 7 & 2 & 0 & 1 & 1 & 0 & 0 & 1 & 7 & 2.2 \\
\hline $\mathbf{0}$ & Putative enzymes & 7 & 10 & 7 & 9 & 3 & 34 & 3 & 7 & 0 & 8.0 \\
\hline p & Chaperones \& heat shock proteins & 1 & 0 & 0 & 1 & 0 & 0 & 0 & 0 & 0 & 0.2 \\
\hline$q$ & Central intermediary metabolism & 1 & 3 & 3 & 4 & 0 & 0 & 0 & 1 & 0 & 2.1 \\
\hline $\mathbf{r}$ & Nucleotide biosynthesis and metabolism & 0 & 1 & 0 & 0 & 0 & 14 & 0 & 1 & 1 & 0.7 \\
\hline s & Carbon compound catabolism & 1 & 6 & 4 & 0 & 0 & 14 & 0 & 1 & 11 & 3.9 \\
\hline $\mathrm{t}$ & Motility \& Attachment & 1 & 0 & 1 & 0 & 0 & 0 & 0 & 2 & 13 & 1.2 \\
\hline u & Chemotaxis & 1 & 0 & 1 & 1 & 0 & 0 & 0 & 1 & 0 & 0.4 \\
\hline $\mathbf{v}$ & Related to phage, transposon, or plasmid & 0 & 5 & 10 & 1 & 0 & 0 & 0 & 0 & 1 & 3.2 \\
\hline $\mathbf{w}$ & Non-coding RNA gene & 0 & 0 & 0 & 0 & 0 & 0 & 0 & 0 & 0 & 0.1 \\
\hline $\mathbf{x}$ & Protein secretion/export apparatus & 1 & 1 & 0 & 0 & 0 & 0 & 33 & 1 & 1 & 1.1 \\
\hline $\mathbf{y}$ & Cell division & 0 & 0 & 0 & 0 & 0 & 0 & 0 & 1 & 0 & 0.3 \\
\hline $\mathrm{zA}$ & Antibiotic resistance and susceptibility & 0 & 0 & 0 & 1 & 0 & 0 & 0 & 0 & 1 & 0.2 \\
\hline $\mathrm{zB}$ & Transcription, RNA processing and degradation & 0 & 0 & 0 & 0 & 0 & 0 & 3 & 1 & 0 & 0.4 \\
\hline
\end{tabular}

Note: QS- quorum sensing, Oxi. Stress- oxidative stress, PCP- pentachlorophenol stress 
Supplementary Table 3: List of genes that are uniquely expressed in the various transcriptome studies. The various conditions include iron response, quorum sensing, oxidative stress, pentachlorophenol stress, copper stress, sulfate starvation, osmotic shock, biofilm formation, alginate production and loss of the post-transcriptional regulator RsmA.

\begin{tabular}{|c|c|c|}
\hline ORF & Product name & Function class \\
\hline PA0013 & conserved hypothetical protein & Membrane proteins \\
\hline PA0027 & hypothetical protein & Hypothetical, unclassified, unknown \\
\hline PA0128 & conserved hypothetical protein & Hypothetical, unclassified, unknown \\
\hline PA0203 & probable binding protein component of $\mathrm{ABC}$ transporter & Transport of small molecules \\
\hline PA0223 & probable dihydrodipicolinate synthetase & Cell wall / LPS / capsule \\
\hline PA0224 & probable aldolase & Putative enzymes \\
\hline PA0251 & hypothetical protein & Hypothetical, unclassified, unknown \\
\hline PA0252 & hypothetical protein & Hypothetical, unclassified, unknown \\
\hline PA0265 & succinate-semialdehyde dehydrogenase & Central intermediary metabolism \\
\hline PA0266 & 4-aminobutyrate aminotransferase & Central intermediary metabolism \\
\hline PA0296 & probable glutamine synthetase & Putative enzymes \\
\hline PA0346 & hypothetical protein & Hypothetical, unclassified, unknown \\
\hline PA0417 & probable chemotaxis protein & Chemotaxis \\
\hline PA0433 & hypothetical protein & Hypothetical, unclassified, unknown \\
\hline PA0460 & hypothetical protein & Hypothetical, unclassified, unknown \\
\hline PA0470 & Ferrichrome receptor FiuA & Transport of small molecules \\
\hline PA0471 & probable transmembrane sensor & Two-component regulatory systems \\
\hline PA0472 & probable sigma- 70 factor, ECF subfamily & Transcriptional regulators \\
\hline PA0500 & biotin synthase & Biosynthesis of cofactors, prosthetic groups and carriers \\
\hline PA0527 & transcriptional regulator Dnr & Transcriptional regulators \\
\hline PA0539 & hypothetical protein & Membrane proteins \\
\hline PA0605 & probable permease of $\mathrm{ABC}$ transporter & Membrane proteins \\
\hline PA0672 & heme oxygenase & Biosynthesis of cofactors, prosthetic groups and carriers \\
\hline PA0707 & transcriptional regulator ToxR & Transcriptional regulators \\
\hline PA0725 & hypothetical protein of bacteriophage Pfl & Hypothetical, unclassified, unknown \\
\hline PA0774 & conserved hypothetical protein & Hypothetical, unclassified, unknown \\
\hline PA0801 & hypothetical protein & Membrane proteins \\
\hline PA0802 & hypothetical protein & Membrane proteins \\
\hline PA0818 & hypothetical protein & Hypothetical, unclassified, unknown \\
\hline PA0842 & probable glycosyl transferase & Putative enzymes \\
\hline PA0844 & hemolytic phospholipase $\mathrm{C}$ precursor & Secreted Factors (toxins, enzymes, alginate) \\
\hline PA0848 & probable alkyl hydroperoxide reductase & Adaptation, Protection \\
\hline PA0930 & two-component sensor & Transport of small molecules \\
\hline PA0931 & ferric enterobactin receptor PirA & Transport of small molecules \\
\hline PA0968 & conserved hypothetical protein & Hypothetical, unclassified, unknown \\
\hline PA1003 & Transcriptional regulator MvfR & Biosynthesis of cofactors, prosthetic groups and carriers \\
\hline PA1108 & probable major facilitator superfamily (MFS) transporter & Membrane proteins \\
\hline PA1134 & hypothetical protein & Hypothetical, unclassified, unknown \\
\hline PAll 148 & exotoxin A precursor & Secreted Factors (toxins, enzymes, alginate) \\
\hline PA1211 & hypothetical protein & Hypothetical, unclassified, unknown \\
\hline PA1247 & alkaline protease secretion protein $\mathrm{AprE}$ & Secreted Factors (toxins, enzymes, alginate) \\
\hline PA1248 & Alkaline protease secretion outer membrane protein AprF precursor & Secreted Factors (toxins, enzymes, alginate) \\
\hline PA1249 & alkaline metalloproteinase precursor & Secreted Factors (toxins, enzymes, alginate) \\
\hline PA1300 & probable sigma- 70 factor, ECF subfamily & Transcriptional regulators \\
\hline PA1301 & probable transmembrane sensor & Membrane proteins \\
\hline PA1302 & probable heme utilization protein precursor & Membrane proteins \\
\hline PA1316 & probable major facilitator superfamily (MFS) transporter & Membrane proteins \\
\hline PA1317 & cytochrome 0 ubiquinol oxidase subunit II & Energy metabolism \\
\hline PA1318 & cytochrome o ubiquinol oxidase subunit I & Energy metabolism \\
\hline PA1319 & cytochrome o ubiquinol oxidase subunit III & Energy metabolism \\
\hline PA1320 & cytochrome o ubiquinol oxidase subunit IV & Energy metabolism \\
\hline PA1321 & cytochrome o ubiquinol oxidase protein $\mathrm{CyoE}$ & Energy metabolism \\
\hline PA1363 & probable sigma- 70 factor, ECF subfamily & Transcriptional regulators \\
\hline PAl365 & probable siderophore receptor & Transport of small molecules \\
\hline PA1431 & regulatory protein RsaL & Secreted Factors (toxins, enzymes, alginate) \\
\hline PA1556 & probable cytochrome c oxidase subunit & Energy metabolism \\
\hline PA1559 & hypothetical protein & Hypothetical, unclassified, unknown \\
\hline PAl 654 & probable aminotransferase & Putative enzymes \\
\hline PAl655 & probable glutathione S-transferase & Putative enzymes \\
\hline PAl687 & spermidine synthase & Amino acid biosynthesis and metabolism \\
\hline PA1908 & probable major facilitator superfamily (MFS) transporter & Membrane proteins \\
\hline PA1911 & probable transmembrane sensor & Membrane proteins \\
\hline PA1917 & hypothetical protein & Hypothetical, unclassified, unknown \\
\hline PA1972 & conserved hypothetical protein & Membrane proteins \\
\hline PA2002 & conserved hypothetical protein & Hypothetical, unclassified, unknown \\
\hline PA2021 & hypothetical protein & Hypothetical, unclassified, unknown \\
\hline
\end{tabular}




\begin{tabular}{|c|c|c|}
\hline ORF & Product name & Function class \\
\hline PA2034 & hypothetical protein & Hypothetical, unclassified, unknown \\
\hline PA2038 & hypothetical protein & Hypothetical, unclassified, unknown \\
\hline PA2039 & hypothetical protein & Membrane proteins \\
\hline PA2041 & probable amino acid permease & Transport of small molecules \\
\hline PA2131 & fimbrial subunit CupA4 & Motility \& Attachment \\
\hline PA2181 & hypothetical protein & Hypothetical, unclassified, unknown \\
\hline PA2258 & transcriptional regulator PtxR & Secreted Factors (toxins, enzymes, alginate) \\
\hline PA2383 & probable transcriptional regulator & Transcriptional regulators \\
\hline PA2384 & hypothetical protein & Hypothetical, unclassified, unknown \\
\hline PA2387 & probable sigma-70 factor, ECF subfamily & Transcriptional regulators \\
\hline PA2388 & probable transmembrane sensor & Membrane proteins \\
\hline PA2390 & probable ATP-binding/permease fusion $\mathrm{ABC}$ transporter & Membrane proteins \\
\hline PA2391 & probable outer membrane protein precursor & Membrane proteins \\
\hline PA2395 & Pvd0 & Adaptation, Protection \\
\hline PA2406 & hypothetical protein & Hypothetical, unclassified, unknown \\
\hline PA2420 & probable porin & Membrane proteins \\
\hline PA2427 & hypothetical protein & Hypothetical, unclassified, unknown \\
\hline PA2437 & hypothetical protein & Membrane proteins \\
\hline PA2441 & hypothetical protein & Hypothetical, unclassified, unknown \\
\hline PA2451 & hypothetical protein & Hypothetical, unclassified, unknown \\
\hline PA2467 & Anti-sigma factor FoxR & Membrane proteins \\
\hline PA2490 & conserved hypothetical protein & Hypothetical, unclassified, unknown \\
\hline PA2531 & probable aminotransferase & Amino acid biosynthesis and metabolism \\
\hline PA2637 & NADH dehydrogenase I chain A & Energy metabolism \\
\hline PA2638 & NADH dehydrogenase I chain B & Energy metabolism \\
\hline PA2686 & two-component response regulator PfeR & Transcriptional regulators \\
\hline PA2688 & Ferric enterobactin receptor, outer membrane protein PfeA precursor & Membrane proteins \\
\hline PA2691 & conserved hypothetical protein & Hypothetical, unclassified, unknown \\
\hline PA2753 & hypothetical protein & Hypothetical, unclassified, unknown \\
\hline PA2761 & hypothetical protein & Membrane proteins \\
\hline PA2767 & probable enoyl-CoA hydratase/isomerase & Putative enzymes \\
\hline PA2776 & conserved hypothetical protein & Hypothetical, unclassified, unknown \\
\hline PA2910 & conserved hypothetical protein & Hypothetical, unclassified, unknown \\
\hline PA2916 & hypothetical protein & Membrane proteins \\
\hline PA2917 & probable transcriptional regulator & Transcriptional regulators \\
\hline PA3049 & ribosome modulation factor & Translation, post-translational modification, degradation \\
\hline PA3165 & histidinol-phosphate aminotransferase & Amino acid biosynthesis and metabolism \\
\hline PA3221 & CsaA protein & Protein secretion/export apparatus \\
\hline PA3222 & hypothetical protein & Membrane proteins \\
\hline РА3233 & hypothetical protein & Hypothetical, unclassified, unknown \\
\hline PA3355 & hypothetical protein & Membrane proteins \\
\hline PA3397 & ferredoxin--NADP+ reductase & Biosynthesis of cofactors, prosthetic groups and carriers \\
\hline PA3407 & heme acquisition protein HasAp & Transport of small molecules \\
\hline PA3408 & Haem uptake outer membrane receptor HasR precursor & Transport of small molecules \\
\hline PA3409 & probable transmembrane sensor & Membrane proteins \\
\hline PA3410 & probable sigma- 70 factor, ECF subfamily & Transcriptional regulators \\
\hline PA3436 & hypothetical protein & Hypothetical, unclassified, unknown \\
\hline PA3530 & conserved hypothetical protein & Hypothetical, unclassified, unknown \\
\hline PA3553 & $\mathrm{ArnC}$ & Adaptation, Protection \\
\hline PA3556 & inner membrane L-Ara $4 \mathrm{~N}$ transferase ArnT & Adaptation, Protection \\
\hline PA3558 & $\operatorname{ArnF}$ & Membrane proteins \\
\hline PA3608 & polyamine transport protein $\mathrm{Pot} B$ & Membrane proteins \\
\hline PA3749 & probable major facilitator superfamily (MFS) transporter & Membrane proteins \\
\hline PA3767 & conserved hypothetical protein & Hypothetical, unclassified, unknown \\
\hline PA3768 & probable metallo-oxidoreductase & Putative enzymes \\
\hline PA3775 & hypothetical protein & Membrane proteins \\
\hline PA3811 & heat shock protein $\mathrm{HscB}$ & Chaperones \& heat shock proteins \\
\hline PA3812 & probable iron-binding protein IscA & Biosynthesis of cofactors, prosthetic groups and carriers \\
\hline PA3814 & L-cysteine desulfurase (pyridoxal phosphate-dependent) & Amino acid biosynthesis and metabolism \\
\hline PA3885 & hypothetical protein & Hypothetical, unclassified, unknown \\
\hline PA3901 & $\mathrm{Fe}$ (III) dicitrate transport protein $\mathrm{FecA}$ & Membrane proteins \\
\hline PA4090 & hypothetical protein & Hypothetical, unclassified, unknown \\
\hline PA4091 & 4-hydroxyphenylacetate 3-monooxygenase lar ge chain & Carbon compound catabolism \\
\hline PA4158 & ferric enterobactin transport protein $\mathrm{FepC}$ & Transport of small molecules \\
\hline PA4175 & protease IV & Secreted Factors (toxins, enzymes, alginate) \\
\hline PA4223 & probable ATP-binding component of $\mathrm{ABC}$ transporter & Membrane proteins \\
\hline PA4224 & pyochelin biosynthetic protein PchG & Transport of small molecules \\
\hline PA4225 & pyochelin synthetase & Secreted Factors (toxins, enzymes, alginate) \\
\hline
\end{tabular}




\begin{tabular}{|c|c|c|}
\hline ORF & Product name & Function class \\
\hline PA4226 & dihydroaeruginoic acid synthetase & Secreted Factors (toxins, enzymes, alginate) \\
\hline PA4227 & transcriptional regulator PchR & Transcriptional regulators \\
\hline PA4228 & pyochelin biosynthesis protein $\mathrm{PchD}$ & Secreted Factors (toxins, enzymes, alginate) \\
\hline PA4229 & pyochelin biosynthetic protein PchC & Secreted Factors (toxins, enzymes, alginate) \\
\hline PA4230 & salicylate biosynthesis protein $\mathrm{PchB}$ & Secreted Factors (toxins, enzymes, alginate) \\
\hline PA4231 & salicylate biosynthesis isochorismate synthase & Secreted Factors (toxins, enzymes, alginate) \\
\hline PA4288 & probable transcriptional regulator & Transcriptional regulators \\
\hline PA4357 & conserved hypothetical protein & Hypothetical, unclassified, unknown \\
\hline PA4358 & probable ferrous iron transport protein & Membrane proteins \\
\hline PA4359 & conserved hypothetical protein & Hypothetical, unclassified, unknown \\
\hline PA4370 & Insulin-cleaving metalloproteinase outer membrane protein precursor & Membrane proteins \\
\hline PA4371 & hypothetical protein & Hypothetical, unclassified, unknown \\
\hline PA4372 & hypothetical protein & Hypothetical, unclassified, unknown \\
\hline PA4500 & probable binding protein component of $\mathrm{ABC}$ transporter & Transport of small molecules \\
\hline PA4503 & probable permease of $\mathrm{ABC}$ transporter & Membrane proteins \\
\hline PA4513 & probable oxidoreductase & Putative enzymes \\
\hline PA4514 & probable outer membrane receptor for iron transport & Transport of small molecules \\
\hline PA4515 & conserved hypothetical protein & Hypothetical, unclassified, unknown \\
\hline PA4516 & hypothetical protein & Hypothetical, unclassified, unknown \\
\hline PA4517 & conserved hypothetical protein & Hypothetical, unclassified, unknown \\
\hline PA4519 & ornithine decarboxylase & Amino acid biosynthesis and metabolism \\
\hline PA4629 & hypothetical protein & Hypothetical, unclassified, unknown \\
\hline PA4630 & hypothetical protein & Hypothetical, unclassified, unknown \\
\hline PA4633 & probable chemotaxis transducer & Adaptation, Protection \\
\hline PA4654 & probable major facilitator superfamily (MFS) transporter & Membrane proteins \\
\hline PA4675 & probable TonB-dependent receptor & Transport of small molecules \\
\hline PA4687 & ferric iron-binding periplasmic protein HitA & Transport of small molecules \\
\hline PA4688 & iron (III)-transport system permease HitB & Membrane proteins \\
\hline PA4706 & probable ATP-binding component of $\mathrm{ABC}$ transporter & Transport of small molecules \\
\hline PA4773 & hypothetical protein & Hypothetical, unclassified, unknown \\
\hline PA4785 & probable acyl-CoA thiolase & Putative enzymes \\
\hline PA4826 & hypothetical protein & Hypothetical, unclassified, unknown \\
\hline PA4833 & conserved hypothetical protein & Membrane proteins \\
\hline PA4838 & hypothetical protein & Hypothetical, unclassified, unknown \\
\hline PA4858 & conserved hypothetical protein & Hypothetical, unclassified, unknown \\
\hline PA4860 & probable permease of $\mathrm{ABC}$ transporter & Membrane proteins \\
\hline PA4861 & probable ATP-binding component of $\mathrm{ABC}$ transporter & Transport of small molecules \\
\hline PA4862 & probable ATP-binding component of $\mathrm{ABC}$ transporter & Transport of small molecules \\
\hline PA4883 & hypothetical protein & Hypothetical, unclassified, unknown \\
\hline PA4892 & urease accessory protein UreF & Biosynthesis of cofactors, prosthetic groups and carriers \\
\hline PA4893 & urease accessory protein UreG & Biosynthesis of cofactors, prosthetic groups and carriers \\
\hline PA4894 & hypothetical protein & Membrane proteins \\
\hline PA4895 & probable transmembrane sensor & Membrane proteins \\
\hline PA4897 & hypothetical protein & Hypothetical, unclassified, unknown \\
\hline PA4973 & thiamin biosynthesis protein $\mathrm{ThiC}$ & Biosynthesis of cofactors, prosthetic groups and carriers \\
\hline PA5119 & glutamine synthetase & Amino acid biosynthesis and metabolism \\
\hline PA5124 & two-component sensor NtrB & Two-component regulatory systems \\
\hline PA5125 & two-component response regulator $\mathrm{NtrC}$ & Transcriptional regulators \\
\hline PA5150 & probable short-chain dehydrogenase & Putative enzymes \\
\hline PA5217 & probable binding protein component of $\mathrm{ABC}$ iron transporter & Transport of small molecules \\
\hline PA5287 & ammonium transporter $A m+B$ & Membrane proteins \\
\hline PA5309 & probable oxidoreductase & Putative enzymes \\
\hline PA5312 & probable aldehyde dehydrogenase & Putative enzymes \\
\hline PA5313 & probable pyridoxal-dependent aminotransferase & Putative enzymes \\
\hline PA5314 & hypothetical protein & Hypothetical, unclassified, unknown \\
\hline PA5386 & probable 3-hydroxyacyl-CoA dehydrogenase & Putative enzymes \\
\hline PA5479 & proton-glutamate symporter & Membrane proteins \\
\hline PA5526 & hypothetical protein & Hypothetical, unclassified, unknown \\
\hline PA5531 & TonB protein & Transport of small molecules \\
\hline
\end{tabular}




\begin{tabular}{|c|c|c|}
\hline ORF & Product name & Function class \\
\hline \multicolumn{3}{|c|}{ Quorum Sensing } \\
\hline PA0006 & conserved hypothetical protein & Hypothetical, unclassified, unknown \\
\hline PA0048 & probable transcriptional regulator & Transcriptional regulators \\
\hline PA0049 & hypothetical protein & Hypothetical, unclassified, unknown \\
\hline PA0050 & hypothetical protein & Hypothetical, unclassified, unknown \\
\hline PA0051 & potential phenazine-modifying enzyme & Putative enzymes \\
\hline PA0052 & hypothetical protein & Hypothetical, unclassified, unknown \\
\hline PA0055 & hypothetical protein & Hypothetical, unclassified, unknown \\
\hline PA0079 & hypothetical protein & Hypothetical, unclassified, unknown \\
\hline PA0080 & hypothetical protein & Hypothetical, unclassified, unknown \\
\hline PA0097 & hypothetical protein & Hypothetical, unclassified, unknown \\
\hline PA0105 & cytochrome c oxidase, subunit II & Energy metabolism \\
\hline PA0106 & cytochrome c oxidase, subunit I & Energy metabolism \\
\hline PA0107 & conserved hypothetical protein & Energy metabolism \\
\hline PA0108 & cytochrome c oxidase, subunit III & Energy metabolism \\
\hline PA0112 & hypothetical protein & Membrane proteins \\
\hline PA0113 & probable cytochrome c oxidase assembly factor & Energy metabolism \\
\hline PA0119 & probable dicarboxylate transporter & Transport of small molecules \\
\hline PA0122 & conserved hypothetical protein & Hypothetical, unclassified, unknown \\
\hline PA0129 & gamma-aminobutyrate permease & Transport of small molecules \\
\hline PA0130 & probable aldehyde dehydrogenase & Putative enzymes \\
\hline PA0131 & hypothetical protein & Hypothetical, unclassified, unknown \\
\hline PA0132 & beta-alanine--pyruvate transaminase & Amino acid biosynthesis and metabolism \\
\hline PA0144 & hypothetical protein & Hypothetical, unclassified, unknown \\
\hline PA0145 & hypothetical protein & Hypothetical, unclassified, unknown \\
\hline PA0153 & protocatechuate 3,4-dioxygenase, beta subunit & Carbon compound catabolism \\
\hline PA0154 & protocatechuate 3,4-dioxygenase, alpha subunit & Carbon compound catabolism \\
\hline PA0160 & hypothetical protein & Hypothetical, unclassified, unknown \\
\hline PA0167 & probable transcriptional regulator & Transcriptional regulators \\
\hline PA0191 & probable transcriptional regulator & Transcriptional regulators \\
\hline PA0200 & hypothetical protein & Hypothetical, unclassified, unknown \\
\hline PA0209 & conserved hypothetical protein & Putative enzymes \\
\hline PA0210 & malonate decarboxylase delta subunit & Carbon compound catabolism \\
\hline PA0211 & malonate decarboxylase beta subunit & Carbon compound catabolism \\
\hline PA0212 & malonate decarboxylase gamma subunit & Carbon compound catabolism \\
\hline PA0213 & hypothetical protein & Carbon compound catabolism \\
\hline PA0214 & probable acyl transferase & Carbon compound catabolism \\
\hline PA0221 & probable aminotransferase & Putative enzymes \\
\hline PA0226 & probable $\mathrm{CoA}$ transferase, subunit A & Carbon compound catabolism \\
\hline PA0227 & probable $\mathrm{C} 0 \mathrm{~A}$ transferase, subunit $\mathrm{B}$ & Carbon compound catabolism \\
\hline PA0228 & beta-ketoadipyl $\mathrm{CoA}$ thiolase $\mathrm{PcaF}$ & Fatty acid and phospholipid metabolism \\
\hline PA0234 & hypothetical protein & Hypothetical, unclassified, unknown \\
\hline PA0235 & 4-hydroxybenzoate transporter PcaK & Membrane proteins \\
\hline PA0239 & hypothetical protein & Membrane proteins \\
\hline PA0241 & probable major facilitator superfamily (MFS) transporter & Membrane proteins \\
\hline PA0251 & hypothetical protein & Hypothetical, unclassified, unknown \\
\hline PA0264 & hypothetical protein & Hypothetical, unclassified, unknown \\
\hline PA0265 & succinate-semialdehyde dehydrogenase & Central intermediary metabolism \\
\hline PA0266 & 4-aminobutyrate aminotransferase & Central intermediary metabolism \\
\hline PA0269 & conserved hypothetical protein & Hypothetical, unclassified, unknown \\
\hline PA0270 & hypothetical protein & Hypothetical, unclassified, unknown \\
\hline PA0271 & hypothetical protein & Hypothetical, unclassified, unknown \\
\hline PA0274 & hypothetical protein & Hypothetical, unclassified, unknown \\
\hline PA0287 & 3-guanidinopropionate transport protein & Transport of small molecules \\
\hline PA0297 & probable glutamine amidotransferase & Amino acid biosynthesis and metabolism \\
\hline PA0298 & probable glutamine synthetase & Putative enzymes \\
\hline PA0299 & putrescine aminotransferase & Putative enzymes \\
\hline PA0300 & polyamine transport protein & Transport of small molecules \\
\hline PA0301 & polyamine transport protein & Transport of small molecules \\
\hline PA0302 & polyamine transport protein $\mathrm{PotG}$ & Transport of small molecules \\
\hline PA0303 & polyamine transport protein $\mathrm{PotH}$ & Membrane proteins \\
\hline PA0304 & polyamine transport protein PotI & Membrane proteins \\
\hline PA0305 & hypothetical protein & Hypothetical, unclassified, unknown \\
\hline PA0321 & probable acetylpolyamine aminohydrolase & Putative enzymes \\
\hline PA0363 & phosphopantetheine adenylyltransferase & Central intermediary metabolism \\
\hline PA0364 & probable oxidoreductase & Putative enzymes \\
\hline PA0385 & hypothetical protein & Hypothetical, unclassified, unknown \\
\hline
\end{tabular}




\begin{tabular}{|c|c|c|}
\hline ORF & Product name & Function class \\
\hline PA0399 & cystathionine beta-synthase & Amino acid biosynthesis and metabolism \\
\hline PA0436 & probable transcriptional regulator & Transcriptional regulators \\
\hline PA0439 & probable oxidoreductase & Putative enzymes \\
\hline PA0440 & probable oxidoreductase & Putative enzymes \\
\hline PA0441 & dihydropyrimidinase & Nucleotide biosynthesis and metabolism \\
\hline PA0443 & probable transporter & Membrane proteins \\
\hline PA0444 & $\mathrm{N}$-carbamoyl-beta-alanine amidohydrolase & Nucleotide biosynthesis and metabolism \\
\hline PA0453 & hypothetical protein & Hypothetical, unclassified, unknown \\
\hline PA0456 & probable cold-shock protein & Transcriptional regulators \\
\hline PA0459 & probable $\mathrm{ClpA} / \mathrm{B}$ protease ATP binding subunit & Translation, post-translational modification, degradation \\
\hline PA0467 & conserved hypothetical protein & Hypothetical, unclassified, unknown \\
\hline PA0468 & hypothetical protein & Hypothetical, unclassified, unknown \\
\hline PA0469 & hypothetical protein & Hypothetical, unclassified, unknown \\
\hline PA0473 & probable glutathione S-transferase & Putative enzymes \\
\hline PA0474 & hypothetical protein & Hypothetical, unclassified, unknown \\
\hline PA0480 & probable hydrolase & Putative enzymes \\
\hline PA0482 & malate synthase $\mathrm{G}$ & Central intermediary metabolism \\
\hline PA0489 & probable phosphoribosyl transferase & Putative enzymes \\
\hline PA0497 & hypothetical protein & Hypothetical, unclassified, unknown \\
\hline PA0498 & hypothetical protein & Hypothetical, unclassified, unknown \\
\hline PA0512 & conserved hypothetical protein & Biosynthesis of cofactors, prosthetic groups and carriers \\
\hline PA0513 & probable transcriptional regulator & Biosynthesis of cofactors, prosthetic groups and carriers \\
\hline PA0527 & transcriptional regulator Dnr & Transcriptional regulators \\
\hline PA0542 & conserved hypothetical protein & Hypothetical, unclassified, unknown \\
\hline PA0610 & transcriptional regulator PrtN & Transcriptional regulators \\
\hline PA0614 & hypothetical protein & Hypothetical, unclassified, unknown \\
\hline PA0615 & hypothetical protein & Hypothetical, unclassified, unknown \\
\hline PA0616 & hypothetical protein & Related to phage, transposon, or plasmid \\
\hline PA0617 & probable bacteriophage protein & Related to phage, transposon, or plasmid \\
\hline PA0618 & probable bacteriophage protein & Related to phage, transposon, or plasmid \\
\hline PA0619 & probable bacteriophage protein & Related to phage, transposon, or plasmid \\
\hline PA0620 & probable bacteriophage protein & Related to phage, transposon, or plasmid \\
\hline PA0621 & conserved hypothetical protein & Related to phage, transposon, or plasmid \\
\hline PA0622 & probable bacteriophage protein & Related to phage, transposon, or plasmid \\
\hline PA0623 & probable bacteriophage protein & Related to phage, transposon, or plasmid \\
\hline PA0624 & hypothetical protein & Related to phage, transposon, or plasmid \\
\hline PA0625 & hypothetical protein & Related to phage, transposon, or plasmid \\
\hline PA0626 & hypothetical protein & Related to phage, transposon, or plasmid \\
\hline PA0627 & conserved hypothetical protein & Related to phage, transposon, or plasmid \\
\hline PA0628 & conserved hypothetical protein & Related to phage, transposon, or plasmid \\
\hline PA0629 & conserved hypothetical protein & Related to phage, transposon, or plasmid \\
\hline PA0630 & hypothetical protein & Related to phage, transposon, or plasmid \\
\hline PA0631 & hypothetical protein & Related to phage, transposon, or plasmid \\
\hline PA0632 & hypothetical protein & Related to phage, transposon, or plasmid \\
\hline PA0635 & hypothetical protein & Related to phage, transposon, or plasmid \\
\hline PA0636 & hypothetical protein & Related to phage, transposon, or plasmid \\
\hline PA0637 & conserved hypothetical protein & Related to phage, transposon, or plasmid \\
\hline PA0638 & probable bacteriophage protein & Related to phage, transposon, or plasmid \\
\hline PA0639 & conserved hypothetical protein & Related to phage, transposon, or plasmid \\
\hline PA0640 & probable bacteriophage protein & Related to phage, transposon, or plasmid \\
\hline PA0641 & probable bacteriophage protein & Related to phage, transposon, or plasmid \\
\hline PA0642 & hypothetical protein & Related to phage, transposon, or plasmid \\
\hline PA0643 & hypothetical protein & Related to phage, transposon, or plasmid \\
\hline PA0644 & hypothetical protein & Related to phage, transposon, or plasmid \\
\hline PA0645 & hypothetical protein & Related to phage, transposon, or plasmid \\
\hline PA0646 & hypothetical protein & Related to phage, transposon, or plasmid \\
\hline PA0647 & hypothetical protein & Related to phage, transposon, or plasmid \\
\hline PA0648 & hypothetical protein & Related to phage, transposon, or plasmid \\
\hline PA0671 & hypothetical protein & Hypothetical, unclassified, unknown \\
\hline PA0683 & probable type II secretion system protein & Protein secretion/export apparatus \\
\hline PA0701 & probable transcriptional regulator & Transcriptional regulators \\
\hline PA0717 & hypothetical protein of bacteriophage Pfl & Hypothetical, unclassified, unknown \\
\hline PA0718 & hypothetical protein of bacteriophage Pfl & Hypothetical, unclassified, unknown \\
\hline PA0719 & hypothetical protein of bacteriophage Pfl & Hypothetical, unclassified, unknown \\
\hline PA0720 & helix destabilizing protein of bacteriophage Pfl & DNA replication, recombination, modification and repair \\
\hline PA0721 & hypothetical protein of bacteriophage Pfl & Hypothetical, unclassified, unknown \\
\hline PA0723 & coat protein $B$ of bacteriophage $\mathrm{Pfl}$ & Related to phage, transposon, or plasmid \\
\hline PA0736 & hypothetical protein & Membrane proteins \\
\hline
\end{tabular}




\begin{tabular}{|c|c|c|}
\hline ORF & Product name & Function class \\
\hline PA0740 & SDS hydrolase SdsAl & Putative enzymes \\
\hline PA0745 & probable enoyl-CoA hydratase/isomerase & Putative enzymes \\
\hline PA0746 & probable acyl-CoA dehydrogenase & Putative enzymes \\
\hline PA0747 & probable aldehyde dehydrogenase & Putative enzymes \\
\hline PA0776 & hypothetical protein & Hypothetical, unclassified, unknown \\
\hline PA0783 & sodium/proline symporter PutP & Membrane proteins \\
\hline PA0826 & hypothetical protein & Hypothetical, unclassified, unknown \\
\hline PA0841 & hypothetical protein & Hypothetical, unclassified, unknown \\
\hline PA0845 & conserved hypothetical protein & Hypothetical, unclassified, unknown \\
\hline PA0854 & fumarate hydratase & Energy metabolism \\
\hline PA0867 & hypothetical protein & Hypothetical, unclassified, unknown \\
\hline PA0870 & aromatic amino acid aminotransferase & Amino acid biosynthesis and metabolism \\
\hline PA0871 & pterin-4-alpha-carbinolamine dehydratase & Amino acid biosynthesis and metabolism \\
\hline PA0885 & probable C4-dicarboxylate transporter & Transport of small molecules \\
\hline PA0910 & hypothetical protein & Hypothetical, unclassified, unknown \\
\hline PA0938 & hypothetical protein & Hypothetical, unclassified, unknown \\
\hline PA0943 & hypothetical protein & Hypothetical, unclassified, unknown \\
\hline PA0997 & Homologous to beta-keto-acyl-acyl-carrier protein synthase & Biosynthesis of cofactors, prosthetic groups and carriers \\
\hline PA0998 & Homologous to beta-keto-acyl-acyl-carrier protein synthase & Biosynthesis of cofactors, prosthetic groups and carriers \\
\hline PA0999 & 3-oxoacyl-[acyl-carrier-protein] synthase III & Biosynthesis of cofactors, prosthetic groups and carriers \\
\hline PA1018 & hypothetical protein & Hypothetical, unclassified, unknown \\
\hline PA1019 & cis,cis-muconate transporter MucK & Membrane proteins \\
\hline PA1025 & probable porin & Membrane proteins \\
\hline PA1049 & pyridoxine 5'-phosphate oxidase & Biosynthesis of cofactors, prosthetic groups and carriers \\
\hline PA1051 & probable transporter & Membrane proteins \\
\hline PA1070 & branched-chain amino acid transport protein $\mathrm{BraG}$ & Transport of small molecules \\
\hline PA1072 & branched-chain amino acid transport protein $\mathrm{BraE}$ & Membrane proteins \\
\hline PA1073 & branched-chain amino acid transport protein BraD & Membrane proteins \\
\hline PA1074 & branched-chain amino acid transport protein $\mathrm{BraC}$ & Transport of small molecules \\
\hline PAl106 & hypothetical protein & Hypothetical, unclassified, unknown \\
\hline PA1113 & probable ATP-binding/permease fusion $\mathrm{ABC}$ transporter & Membrane proteins \\
\hline PA1123 & hypothetical protein & Hypothetical, unclassified, unknown \\
\hline PA1127 & probable oxidoreductase & Adaptation, Protection \\
\hline PA1130 & rhamnosyltransferase 2 & Cell wall / LPS / capsule \\
\hline PAl131 & probable major facilitator superfamily (MFS) transporter & Membrane proteins \\
\hline PA1137 & probable oxidoreductase & Putative enzymes \\
\hline PA1143 & hypothetical protein & Hypothetical, unclassified, unknown \\
\hline PA1144 & probable major facilitator superfamily (MFS) transporter & Membrane proteins \\
\hline PA1150 & pyocin S2 & Adaptation, Protection \\
\hline PAl166 & hypothetical protein & Hypothetical, unclassified, unknown \\
\hline PAl183 & C4-dicarboxylate transport protein & Transport of small molecules \\
\hline PAl186 & hypothetical protein & Hypothetical, unclassified, unknown \\
\hline PAl190 & conserved hypothetical protein & Membrane proteins \\
\hline PAl197 & hypothetical protein & Hypothetical, unclassified, unknown \\
\hline PA1206 & hypothetical protein & Hypothetical, unclassified, unknown \\
\hline PA1207 & glutathione-regulated potassium-ef flux system protein KefB & Transport of small molecules \\
\hline PA1220 & hypothetical protein & Hypothetical, unclassified, unknown \\
\hline PA1223 & probable transcriptional regulator & Transcriptional regulators \\
\hline PA1228 & hypothetical protein & Hypothetical, unclassified, unknown \\
\hline PA1236 & probable major facilitator superfamily (MFS) transporter & Membrane proteins \\
\hline PA1239 & hypothetical protein & Hypothetical, unclassified, unknown \\
\hline PA1242 & hypothetical protein & Hypothetical, unclassified, unknown \\
\hline PA1250 & alkaline proteinase inhibitor AprI & Secreted Factors (toxins, enzymes, alginate) \\
\hline PA1262 & probable major facilitator superfamily (MFS) transporter & Membrane proteins \\
\hline PA1266 & probable oxidoreductase & Putative enzymes \\
\hline PA1338 & gamma-glutamyltranspeptidase precursor & Amino acid biosynthesis and metabolism \\
\hline PA1340 & probable permease of $\mathrm{ABC}$ transporter & Transport of small molecules \\
\hline PA1341 & probable permease of $\mathrm{ABC}$ transporter & Membrane proteins \\
\hline PA1342 & probable binding protein component of $\mathrm{ABC}$ transporter & Transport of small molecules \\
\hline PA1344 & probable short-chain dehydrogenase & Putative enzymes \\
\hline PA1367 & hypothetical protein & Hypothetical, unclassified, unknown \\
\hline PA1377 & conserved hypothetical protein & Hypothetical, unclassified, unknown \\
\hline PA1379 & probable short-chain dehydrogenase & Putative enzymes \\
\hline PA1386 & probable ATP-binding component of $\mathrm{ABC}$ transporter & Transport of small molecules \\
\hline PA1395 & hypothetical protein & Hypothetical, unclassified, unknown \\
\hline PA1399 & probable transcriptional regulator & Transcriptional regulators \\
\hline PA1400 & probable pyruvate carboxylase & Energy metabolism \\
\hline PA1416 & conserved hypothetical protein & Hypothetical, unclassified, unknown \\
\hline
\end{tabular}




\begin{tabular}{|c|c|c|}
\hline ORF & Product name & Function class \\
\hline PA1418 & probable sodium:solute symport protein & Transport of small molecules \\
\hline PA1419 & probable transporter & Membrane proteins \\
\hline PA1426 & hypothetical protein & Hypothetical, unclassified, unknown \\
\hline PA1431 & regulatory protein RsaL & Secreted Factors (toxins, enzymes, alginate) \\
\hline PA1440 & hypothetical protein & Hypothetical, unclassified, unknown \\
\hline PA1519 & probable transporter & Membrane proteins \\
\hline PA1537 & probable short-chain dehydrogenase & Putative enzymes \\
\hline PA1538 & probable flavin-containing monooxygenase & Putative enzymes \\
\hline PA1540 & conserved hypothetical protein & Membrane proteins \\
\hline PA1542 & hypothetical protein & Hypothetical, unclassified, unknown \\
\hline PA1568 & conserved hypothetical protein & Hypothetical, unclassified, unknown \\
\hline PA1569 & probable major facilitator superfamily (MFS) transporter & Membrane proteins \\
\hline PA1575 & hypothetical protein & Hypothetical, unclassified, unknown \\
\hline PA1576 & probable 3-hydroxyisobutyrate dehydrogenase & Putative enzymes \\
\hline PA1608 & probable chemotaxis transducer & Adaptation, Protection \\
\hline PA1609 & beta-ketoacyl-ACP synthase I & Fatty acid and phospholipid metabolism \\
\hline PA1610 & beta-hydroxydecanoyl-ACP dehydrase & Fatty acid and phospholipid metabolism \\
\hline PA1617 & probable AMP-binding enzyme & Putative enzymes \\
\hline PA 1650 & probable transporter & Membrane proteins \\
\hline PA1746 & hypothetical protein & Hypothetical, unclassified, unknown \\
\hline PA1759 & probable transcriptional regulator & Transcriptional regulators \\
\hline PA1763 & hypothetical protein & Hypothetical, unclassified, unknown \\
\hline PA1768 & hypothetical protein & Hypothetical, unclassified, unknown \\
\hline PA1772 & probable methyltransferase & Biosynthesis of cofactors, prosthetic groups and carriers \\
\hline PA1774 & CfrX protein & Hypothetical, unclassified, unknown \\
\hline PA1775 & conserved cytoplasmic membrane protein, $\mathrm{CmpX}$ protein & Membrane proteins \\
\hline PA1776 & ECF sigma factor $\operatorname{Sig} X$ & Transcriptional regulators \\
\hline PA1781 & assimilatory nitrite reductase lar ge subunit & Central intermediary metabolism \\
\hline PA1784 & hypothetical protein & Hypothetical, unclassified, unknown \\
\hline PA1789 & hypothetical protein & Hypothetical, unclassified, unknown \\
\hline PA1790 & hypothetical protein & Hypothetical, unclassified, unknown \\
\hline PA1812 & membrane-bound lytic murein transglycosylase $\mathrm{D}$ precursor & Amino acid biosynthesis and metabolism \\
\hline PA1824 & conserved hypothetical protein & Membrane proteins \\
\hline PA1852 & hypothetical protein & Hypothetical, unclassified, unknown \\
\hline PA1853 & probable transcriptional regulator & Transcriptional regulators \\
\hline PA1871 & LasA protease precursor & Secreted Factors (toxins, enzymes, alginate) \\
\hline PA1875 & probable outer membrane protein precursor & Protein secretion/export apparatus \\
\hline PA1891 & hypothetical protein & Membrane proteins \\
\hline PA1892 & hypothetical protein & Hypothetical, unclassified, unknown \\
\hline PA1893 & hypothetical protein & Putative enzymes \\
\hline PA1895 & hypothetical protein & Membrane proteins \\
\hline PA1896 & hypothetical protein & Hypothetical, unclassified, unknown \\
\hline PA1897 & hypothetical protein & Hypothetical, unclassified, unknown \\
\hline PA1898 & quorum-sensing control repressor & Transcriptional regulators \\
\hline PA1901 & phenazine biosynthesis protein $\mathrm{PhzC}$ & Secreted Factors (toxins, enzymes, alginate) \\
\hline PA1903 & phenazine biosynthesis protein $\mathrm{PhzE}$ & Secreted Factors (toxins, enzymes, alginate) \\
\hline PA1905 & probable pyridoxamine $5^{\prime}$-phosphate oxidase & Secreted Factors (toxins, enzymes, alginate) \\
\hline PA1922 & probable TonB-dependent receptor & Transport of small molecules \\
\hline PA1924 & hypothetical protein & Hypothetical, unclassified, unknown \\
\hline PA1927 & 5-methyltetrahydropteroyltriglutamate-homocysteine S-methyltransferase & Amino acid biosynthesis and metabolism \\
\hline PA1935 & hypothetical protein & Hypothetical, unclassified, unknown \\
\hline PA1942 & hypothetical protein & Hypothetical, unclassified, unknown \\
\hline PA1946 & binding protein component precursor of $\mathrm{ABC}$ ribose transporter & Transport of small molecules \\
\hline PA1947 & ribose transport protein $\mathrm{RbsA}$ & Transport of small molecules \\
\hline PA1955 & hypothetical protein & Hypothetical, unclassified, unknown \\
\hline PA1974 & hypothetical protein & Hypothetical, unclassified, unknown \\
\hline PA1976 & probable two-component sensor & Two-component regulatory systems \\
\hline PA1977 & hypothetical protein & Membrane proteins \\
\hline PA1978 & probable transcriptional regulator & Transcriptional regulators \\
\hline PA1979 & probable two-component sensor & Two-component regulatory systems \\
\hline PA1980 & probable two-component response regulator & Transcriptional regulators \\
\hline PA1981 & hypothetical protein & Hypothetical, unclassified, unknown \\
\hline PA1983 & cytochrome c550 & Energy metabolism \\
\hline PA1984 & probable aldehyde dehydrogenase & Putative enzymes \\
\hline PA1991 & probable iron-containing alcohol dehydrogenase & Putative enzymes \\
\hline PA1992 & probable two-component sensor & Two-component regulatory systems \\
\hline PA2000 & probable $\mathrm{C} 0 \mathrm{~A}$ transferase, subunit $\mathrm{B}$ & Amino acid biosynthesis and metabolism \\
\hline PA2001 & acetyl-CoA acetyltransferase & Central intermediary metabolism \\
\hline
\end{tabular}




\begin{tabular}{|c|c|c|}
\hline ORF & Product name & Function class \\
\hline PA2002 & conserved hypothetical protein & Hypothetical, unclassified, unknown \\
\hline PA2003 & 3-hydroxybutyrate dehydrogenase & Carbon compound catabolism \\
\hline PA2004 & conserved hypothetical protein & Membrane proteins \\
\hline PA2006 & probable major facilitator superfamily (MFS) transporter & Membrane proteins \\
\hline PA2012 & methylcrotonyl-CoA carboxylase, alpha-subunit (biotin-containing) & Carbon compound catabolism \\
\hline PA2013 & putative 3-methylglutaconyl-CoA hydratase & Carbon compound catabolism \\
\hline PA2014 & methylcrotonyl-CoA carboxylase, beta-subunit & Carbon compound catabolism \\
\hline PA2015 & putative isovaleryl-CoA dehydrogenase & Carbon compound catabolism \\
\hline PA2018 & Resistance-Nodulation-Cell Division (RND) multidrug ef flux transporter & Transport of small molecules \\
\hline PA2030 & hypothetical protein & Hypothetical, unclassified, unknown \\
\hline PA2031 & hypothetical protein & Hypothetical, unclassified, unknown \\
\hline PA2041 & probable amino acid permease & Transport of small molecules \\
\hline PA2060 & probable permease of $\mathrm{ABC}$ transporter & Transport of small molecules \\
\hline PA2066 & hypothetical protein & Hypothetical, unclassified, unknown \\
\hline PA2067 & probable hydrolase & Putative enzymes \\
\hline PA2068 & probable major facilitator superfamily (MFS) transporter & Membrane proteins \\
\hline PA2069 & probable carbamoyl transferase & Putative enzymes \\
\hline PA2079 & probable amino acid permease & Transport of small molecules \\
\hline PA2091 & hypothetical protein & Membrane proteins \\
\hline PA2109 & hypothetical protein & Hypothetical, unclassified, unknown \\
\hline PA2110 & hypothetical protein & Hypothetical, unclassified, unknown \\
\hline PA2116 & conserved hypothetical protein & Hypothetical, unclassified, unknown \\
\hline PA2119 & alcohol dehydrogenase (Zn-dependent) & Putative enzymes \\
\hline PA2122 & hypothetical protein & Hypothetical, unclassified, unknown \\
\hline PA2129 & chaperone CupA2 & Motility \& Attachment \\
\hline PA2134 & hypothetical protein & Hypothetical, unclassified, unknown \\
\hline PA2160 & probable glycosyl hydrolase & Putative enzymes \\
\hline PA2161 & hypothetical protein & Hypothetical, unclassified, unknown \\
\hline PA2169 & hypothetical protein & Hypothetical, unclassified, unknown \\
\hline PA2172 & hypothetical protein & Hypothetical, unclassified, unknown \\
\hline PA2185 & non-heme catalase KatN & Adaptation, Protection \\
\hline PA2198 & hypothetical protein & Hypothetical, unclassified, unknown \\
\hline PA2201 & hypothetical protein & Hypothetical, unclassified, unknown \\
\hline PA2209 & hypothetical protein & Hypothetical, unclassified, unknown \\
\hline PA2210 & probable major facilitator superfamily (MFS) transporter & Membrane proteins \\
\hline PA2211 & conserved hypothetical protein & Hypothetical, unclassified, unknown \\
\hline PA2212 & conserved hypothetical protein & Hypothetical, unclassified, unknown \\
\hline PA2213 & probable porin & Membrane proteins \\
\hline PA2214 & probable major facilitator superfamily (MFS) transporter & Membrane proteins \\
\hline PA2217 & probable aldehyde dehydrogenase & Putative enzymes \\
\hline PA2225 & hypothetical protein & Hypothetical, unclassified, unknown \\
\hline PA2228 & hypothetical protein & Hypothetical, unclassified, unknown \\
\hline PA2249 & branched-chain alpha-keto acid dehydrogenase (lipoamide component) & Amino acid biosynthesis and metabolism \\
\hline PA2250 & lipoamide dehydrogenase-Val & Amino acid biosynthesis and metabolism \\
\hline PA2258 & transcriptional regulator PtxR & Secreted Factors (toxins, enzymes, alginate) \\
\hline PA2274 & hypothetical protein & Hypothetical, unclassified, unknown \\
\hline PA2300 & chitinase & Carbon compound catabolism \\
\hline PA2303 & hypothetical protein & Hypothetical, unclassified, unknown \\
\hline PA2313 & hypothetical protein & Membrane proteins \\
\hline PA2322 & gluconate permease & Transport of small molecules \\
\hline PA2328 & hypothetical protein & Hypothetical, unclassified, unknown \\
\hline PA2329 & probable ATP-binding component of $\mathrm{ABC}$ transporter & Transport of small molecules \\
\hline PA2330 & hypothetical protein & Hypothetical, unclassified, unknown \\
\hline PA2334 & probable transcriptional regulator & Transcriptional regulators \\
\hline PA2359 & probable transcriptional regulator & Transcriptional regulators \\
\hline PA2423 & hypothetical protein & Hypothetical, unclassified, unknown \\
\hline PA2439 & hypothetical protein & Membrane proteins \\
\hline PA2453 & hypothetical protein & Hypothetical, unclassified, unknown \\
\hline PA2474 & hypothetical protein & Hypothetical, unclassified, unknown \\
\hline PA2499 & probable deaminase & Putative enzymes \\
\hline PA2507 & catechol 1,2-dioxygenase & Carbon compound catabolism \\
\hline PA2508 & muconolactone delta-isomerase & Carbon compound catabolism \\
\hline PA2509 & muconate cycloisomerase I & Carbon compound catabolism \\
\hline PA2511 & probable transcriptional regulator & Transcriptional regulators \\
\hline PA2512 & anthranilate dioxygenase lar ge subunit & Carbon compound catabolism \\
\hline PA2513 & anthranilate dioxygenase small subunit & Carbon compound catabolism \\
\hline PA2514 & anthranilate dioxygenase reductase & Carbon compound catabolism \\
\hline PA2515 & cis-1,2-dihydroxycyclohexa-3,4-diene carboxylate dehydrogenase & Carbon compound catabolism \\
\hline
\end{tabular}




\begin{tabular}{|c|c|c|}
\hline ORF & Product name & Function class \\
\hline PA2516 & toluate 1,2-dioxygenase electron transfer component & Energy metabolism \\
\hline PA2517 & toluate 1,2-dioxygenase beta subunit & Carbon compound catabolism \\
\hline PA2518 & toluate 1,2-dioxygenase alpha subunit & Carbon compound catabolism \\
\hline PA2519 & transcriptional regulator XylS & Transcriptional regulators \\
\hline PA2529 & hypothetical protein & Hypothetical, unclassified, unknown \\
\hline PA2554 & probable short-chain dehydrogenase & Putative enzymes \\
\hline PA2555 & probable AMP-binding enzyme & Putative enzymes \\
\hline PA2557 & probable AMP-binding enzyme & Fatty acid and phospholipid metabolism \\
\hline PA2564 & hypothetical protein & Hypothetical, unclassified, unknown \\
\hline PA2566 & conserved hypothetical protein & Hypothetical, unclassified, unknown \\
\hline PA2567 & hypothetical protein & Hypothetical, unclassified, unknown \\
\hline PA2570 & LecA & Adaptation, Protection \\
\hline PA2587 & probable FAD-dependent monooxygenase & Biosynthesis of cofactors, prosthetic groups and carriers \\
\hline PA2591 & probable transcriptional regulator & Transcriptional regulators \\
\hline PA2595 & conserved hypothetical protein & Hypothetical, unclassified, unknown \\
\hline PA2625 & conserved hypothetical protein & Hypothetical, unclassified, unknown \\
\hline PA2629 & adenylosuccinate lyase & Amino acid biosynthesis and metabolism \\
\hline PA2654 & probable chemotaxis transducer & Adaptation, Protection \\
\hline PA2660 & hypothetical protein & Hypothetical, unclassified, unknown \\
\hline PA2667 & conserved hypothetical protein & Hypothetical, unclassified, unknown \\
\hline PA2682 & conserved hypothetical protein & Putative enzymes \\
\hline PA2711 & probable periplasmic spermidine/putrescine-binding protein & Transport of small molecules \\
\hline PA2717 & chloroperoxidase precursor & Central intermediary metabolism \\
\hline PA2719 & hypothetical protein & Hypothetical, unclassified, unknown \\
\hline PA2753 & hypothetical protein & Hypothetical, unclassified, unknown \\
\hline PA2754 & conserved hypothetical protein & Hypothetical, unclassified, unknown \\
\hline PA2769 & hypothetical protein & Hypothetical, unclassified, unknown \\
\hline PA2776 & conserved hypothetical protein & Hypothetical, unclassified, unknown \\
\hline PA2778 & hypothetical protein & Hypothetical, unclassified, unknown \\
\hline PA2779 & hypothetical protein & Hypothetical, unclassified, unknown \\
\hline PA2780 & hypothetical protein & Hypothetical, unclassified, unknown \\
\hline PA2781 & hypothetical protein & Hypothetical, unclassified, unknown \\
\hline PA2801 & hypothetical protein & Hypothetical, unclassified, unknown \\
\hline PA2805 & hypothetical protein & Hypothetical, unclassified, unknown \\
\hline PA2819 & hypothetical protein & Hypothetical, unclassified, unknown \\
\hline PA2838 & probable transcriptional regulator & Transcriptional regulators \\
\hline PA2847 & conserved hypothetical protein & Hypothetical, unclassified, unknown \\
\hline PA2851 & translation elongation factor $\mathrm{P}$ & Translation, post-translational modification, degradation \\
\hline PA2876 & orotidine 5'-phosphate decarboxylase & Nucleotide biosynthesis and metabolism \\
\hline PA2892 & GCase, alpha-subunit (biotin-containing) & Putative enzymes \\
\hline PA2905 & precorrin isomerase $\mathrm{CobH}$ & Biosynthesis of cofactors, prosthetic groups and carriers \\
\hline PA2915 & hypothetical protein & Hypothetical, unclassified, unknown \\
\hline PA2922 & probable hydrolase & Putative enzymes \\
\hline PA2924 & histidine transport system permease His $Q$ & Membrane proteins \\
\hline PA2927 & hypothetical protein & Hypothetical, unclassified, unknown \\
\hline PA2938 & probable transporter & Membrane proteins \\
\hline PA2939 & probable aminopeptidase & Secreted Factors (toxins, enzymes, alginate) \\
\hline PA2942 & probable magnesium chelatase & Biosynthesis of cofactors, prosthetic groups and carriers \\
\hline PA2950 & hypothetical protein & Hypothetical, unclassified, unknown \\
\hline PA2960 & type 4 fimbrial biogenesis protein PilZ & Motility \& Attachment \\
\hline PA2966 & acyl carrier protein & Fatty acid and phospholipid metabolism \\
\hline PA2967 & 3-oxoacyl-[acyl-carrier-protein] reductase & Fatty acid and phospholipid metabolism \\
\hline PA2968 & malonyl-CoA-[acyl-carrier-protein] transacylase & Fatty acid and phospholipid metabolism \\
\hline PA3032 & cytochrome c Snrl & Energy metabolism \\
\hline PA3035 & probable glutathione S-transferase & Putative enzymes \\
\hline PA3036 & hypothetical protein & Hypothetical, unclassified, unknown \\
\hline PA3062 & PelC & Cell wall / LPS / capsule \\
\hline PA3066 & hypothetical protein & Hypothetical, unclassified, unknown \\
\hline PA3079 & hypothetical protein & Membrane proteins \\
\hline PA3131 & probable aldolase & Central intermediary metabolism \\
\hline PA3139 & probable amino acid aminotransferase & Amino acid biosynthesis and metabolism \\
\hline PA3186 & Glucose/carbohydrate outer membrane porin 0 prB precursor & Transport of small molecules \\
\hline PA3188 & probable permease of $\mathrm{ABC}$ sugar transporter & Transport of small molecules \\
\hline PA3189 & probable permease of $\mathrm{ABC}$ sugar transporter & Transport of small molecules \\
\hline PA3208 & conserved hypothetical protein & Hypothetical, unclassified, unknown \\
\hline PA3209 & conserved hypothetical protein & Hypothetical, unclassified, unknown \\
\hline PA3213 & hypothetical protein & Hypothetical, unclassified, unknown \\
\hline PA3221 & CsaA protein & Protein secretion/export apparatus \\
\hline
\end{tabular}




\begin{tabular}{|c|c|c|}
\hline ORF & Product name & Function class \\
\hline PA3234 & probable sodium:solute symporter & Membrane proteins \\
\hline PA3236 & probable glycine betaine-binding protein precursor & Adaptation, Protection \\
\hline PA3237 & hypothetical protein & Hypothetical, unclassified, unknown \\
\hline PA3249 & probable transcriptional regulator & Transcriptional regulators \\
\hline PA3270 & hypothetical protein & Hypothetical, unclassified, unknown \\
\hline PA3283 & conserved hypothetical protein & Hypothetical, unclassified, unknown \\
\hline PA3295 & probable HIT family protein & Putative enzymes \\
\hline PA3301 & hypothetical protein & Putative enzymes \\
\hline PA3309 & conserved hypothetical protein & Hypothetical, unclassified, unknown \\
\hline PA3313 & hypothetical protein & Transport of small molecules \\
\hline PA3326 & probable Clp-family ATP-dependent protease & Translation, post-translational modification, degradation \\
\hline PA3329 & hypothetical protein & Hypothetical, unclassified, unknown \\
\hline PA3330 & probable short chain dehydrogenase & Putative enzymes \\
\hline PA3331 & cytochrome P450 & Adaptation, Protection \\
\hline PA3332 & conserved hypothetical protein & Hypothetical, unclassified, unknown \\
\hline PA3333 & 3-oxoacyl-[acyl-carrier-protein] synthase III & Fatty acid and phospholipid metabolism \\
\hline PA3334 & probable acyl carrier protein & Fatty acid and phospholipid metabolism \\
\hline PA3335 & hypothetical protein & Hypothetical, unclassified, unknown \\
\hline PA3336 & probable major facilitator superfamily (MFS) transporter & Membrane proteins \\
\hline PA3337 & ADP-L-glycero-D-mannoheptose 6-epimerase & Cell wall / LPS / capsule \\
\hline PA3361 & fucose-binding lectin PA-IIL & Motility \& Attachment \\
\hline PA3362 & hypothetical protein & Membrane proteins \\
\hline PA3363 & aliphatic amidase regulator & Carbon compound catabolism \\
\hline PA3364 & aliphatic amidase expression-regulating protein & Transcriptional regulators \\
\hline PA3365 & probable chaperone & Chaperones \& heat shock proteins \\
\hline PA3366 & aliphatic amidase & Carbon compound catabolism \\
\hline PA3394 & NosF protein & Energy metabolism \\
\hline PA3422 & hypothetical protein & Hypothetical, unclassified, unknown \\
\hline PA3431 & conserved hypothetical protein & Membrane proteins \\
\hline PA3432 & hypothetical protein & Membrane proteins \\
\hline PA3458 & probable transcriptional regulator & Transcriptional regulators \\
\hline PA3466 & probable ATP-dependent RNA helicase & Transcription, RNA processing and degradation \\
\hline PA3467 & probable major facilitator superfamily (MFS) transporter & Membrane proteins \\
\hline PA3478 & rhamnosyltransferase chain B & Secreted Factors (toxins, enzymes, alginate) \\
\hline PA3479 & rhamnosyltransferase chain A & Secreted Factors (toxins, enzymes, alginate) \\
\hline PA3480 & probable deoxycytidine triphosphate deaminase & Nucleotide biosynthesis and metabolism \\
\hline PA3507 & probable short-chain dehydrogenase & Putative enzymes \\
\hline PA3524 & lactoylglutathione lyase & Central intermediary metabolism \\
\hline PA3529 & probable peroxidase & Adaptation, Protection \\
\hline PA3535 & probable serine protease & Putative enzymes \\
\hline PA3542 & alginate biosynthesis protein Alg44 & Cell wall / LPS / capsule \\
\hline PA3555 & ArnD & Adaptation, Protection \\
\hline PA3559 & probable nucleotide sugar dehydrogenase & Putative enzymes \\
\hline PA3560 & phosphotransferase system, fructose-specific IIBC component & Carbon compound catabolism \\
\hline PA3562 & probable phosphotransferase system enzyme I & Central intermediary metabolism \\
\hline PA3564 & conserved hypothetical protein & Hypothetical, unclassified, unknown \\
\hline PA3565 & probable transcriptional regulator & Transcriptional regulators \\
\hline PA3568 & probable acetyl-coa synthetase & Putative enzymes \\
\hline PA3569 & 3-hydroxyisobutyrate dehydrogenase & Carbon compound catabolism \\
\hline PA3570 & methylmalonate-semialdehyde dehydrogenase & Amino acid biosynthesis and metabolism \\
\hline PA3572 & hypothetical protein & Hypothetical, unclassified, unknown \\
\hline PA3581 & glycerol uptake facilitator protein & Transport of small molecules \\
\hline PA3582 & glycerol kinase & Central intermediary metabolism \\
\hline PA3586 & probable hydrolase & Putative enzymes \\
\hline PA3595 & probable major facilitator superfamily (MFS) transporter & Membrane proteins \\
\hline PA3608 & polyamine transport protein $\mathrm{PotB}$ & Membrane proteins \\
\hline PA3613 & hypothetical protein & Hypothetical, unclassified, unknown \\
\hline PA3614 & hypothetical protein & Hypothetical, unclassified, unknown \\
\hline PA3618 & conserved hypothetical protein & Hypothetical, unclassified, unknown \\
\hline PA3621 & ferredoxin I & Energy metabolism \\
\hline PA3661 & hypothetical protein & Hypothetical, unclassified, unknown \\
\hline PA3662 & hypothetical protein & Hypothetical, unclassified, unknown \\
\hline PA3674 & hypothetical protein & Hypothetical, unclassified, unknown \\
\hline PA3682 & hypothetical protein & Hypothetical, unclassified, unknown \\
\hline PA3686 & adenylate kinase & Nucleotide biosynthesis and metabolism \\
\hline PA3692 & probable outer membrane protein precursor & Membrane proteins \\
\hline PA3699 & probable transcriptional regulator & Transcriptional regulators \\
\hline PA3710 & probable GMC-type oxidoreductase & Carbon compound catabolism \\
\hline
\end{tabular}




\begin{tabular}{|c|c|c|}
\hline ORF & Product name & Function class \\
\hline PA3718 & probable major facilitator superfamily (MFS) transporter & Membrane proteins \\
\hline PA3724 & elastase LasB & Secreted Factors (toxins, enzymes, alginate) \\
\hline PA3737 & thiol:disulfide interchange protein $\mathrm{DsbC}$ & Translation, post-translational modification, degradation \\
\hline PA3779 & hypothetical protein & Hypothetical, unclassified, unknown \\
\hline PA3780 & hypothetical protein & Membrane proteins \\
\hline PA3781 & probable transporter & Membrane proteins \\
\hline PA3789 & hypothetical protein & Membrane proteins \\
\hline PA3795 & probable oxidoreductase & Putative enzymes \\
\hline PA3836 & hypothetical protein & Hypothetical, unclassified, unknown \\
\hline PA3837 & probable permease of $\mathrm{ABC}$ transporter & Membrane proteins \\
\hline PA3838 & probable ATP-binding component of $\mathrm{ABC}$ transporter & Transport of small molecules \\
\hline PA3839 & probable sodium:sulfate symporter & Membrane proteins \\
\hline PA3840 & conserved hypothetical protein & Hypothetical, unclassified, unknown \\
\hline PA3865 & probable amino acid binding protein & Transport of small molecules \\
\hline PA3880 & conserved hypothetical protein & Hypothetical, unclassified, unknown \\
\hline PA3887 & $\mathrm{Na}+/ \mathrm{H}+$ antiporter $\mathrm{NhaP}$ & Membrane proteins \\
\hline PA3906 & hypothetical protein & Hypothetical, unclassified, unknown \\
\hline PA3908 & hypothetical protein & Hypothetical, unclassified, unknown \\
\hline PA3913 & probable protease & Putative enzymes \\
\hline PA3940 & probable DNA binding protein & DNA replication, recombination, modification and repair \\
\hline PA3959 & hypothetical protein & Hypothetical, unclassified, unknown \\
\hline PA3960 & hypothetical protein & Hypothetical, unclassified, unknown \\
\hline PA3966 & hypothetical protein & Membrane proteins \\
\hline PA3967 & hypothetical protein & Hypothetical, unclassified, unknown \\
\hline PA3984 & apolipoprotein $\mathrm{N}$-acyltransferase & Cell wall / LPS / capsule \\
\hline PA4021 & probable transcriptional regulator & Transcriptional regulators \\
\hline PA4023 & probable transport protein & Transport of small molecules \\
\hline PA4024 & ethanolamine ammonia-lyase lar ge subunit & Central intermediary metabolism \\
\hline PA4025 & probable ethanolamine ammonia-lyase light chain & Carbon compound catabolism \\
\hline PA4031 & inorganic pyrophosphatase & Central intermediary metabolism \\
\hline PA4055 & riboflavin synthase alpha chain & Biosynthesis of cofactors, prosthetic groups and carriers \\
\hline PA4057 & $\mathrm{NrdR}$ & Nucleotide biosynthesis and metabolism \\
\hline PA4058 & hypothetical protein & Hypothetical, unclassified, unknown \\
\hline PA4070 & probable transcriptional regulator & Transcriptional regulators \\
\hline PA4072 & probable amino acid permease & Transport of small molecules \\
\hline PA4073 & probable aldehyde dehydrogenase & Putative enzymes \\
\hline PA4078 & probable nonribosomal peptide synthetase & Adaptation, Protection \\
\hline PA4079 & probable dehydrogenase & Putative enzymes \\
\hline PA4091 & 4-hydroxyphenylacetate 3-monooxygenase lar ge chain & Carbon compound catabolism \\
\hline PA4092 & 4-hydroxyphenylacetate 3-monooxygenase small chain & Carbon compound catabolism \\
\hline PA4093 & hypothetical protein & Hypothetical, unclassified, unknown \\
\hline PA4094 & probable transcriptional regulator & Transcriptional regulators \\
\hline PA4120 & probable transcriptional regulator & Transcriptional regulators \\
\hline PA4121 & conserved hypothetical protein & Hypothetical, unclassified, unknown \\
\hline PA4122 & conserved hypothetical protein & Hypothetical, unclassified, unknown \\
\hline PA4123 & 5-carboxy-2-hydroxymuconate semialdehyde dehydrogenase & Carbon compound catabolism \\
\hline PA4124 & homoprotocatechuate 2,3-dioxygenase & Carbon compound catabolism \\
\hline PA4125 & 5-carboxymethyl-2-hydroxymuconate isomerase & Carbon compound catabolism \\
\hline PA4126 & probable major facilitator superfamily (MFS) transporter & Membrane proteins \\
\hline PA4127 & 2-oxo-hept-3-ene-1,7-dioate hydratase & Carbon compound catabolism \\
\hline PA4128 & conserved hypothetical protein & Putative enzymes \\
\hline PA4129 & hypothetical protein & Hypothetical, unclassified, unknown \\
\hline PA4130 & probable sulfite or nitrite reductase & Central intermediary metabolism \\
\hline PA4131 & probable iron-sulfur protein & Putative enzymes \\
\hline PA4132 & conserved hypothetical protein & Hypothetical, unclassified, unknown \\
\hline PA4133 & cytochrome c oxidase subunit (cbb3-type) & Energy metabolism \\
\hline PA4134 & hypothetical protein & Hypothetical, unclassified, unknown \\
\hline PA4136 & probable major facilitator superfamily (MFS) transporter & Membrane proteins \\
\hline PA4137 & probable porin & Transport of small molecules \\
\hline PA4140 & hypothetical protein & Hypothetical, unclassified, unknown \\
\hline PA4141 & hypothetical protein & Hypothetical, unclassified, unknown \\
\hline PA4142 & probable secretion protein & Protein secretion/export apparatus \\
\hline PA4147 & transcriptional regulator $\mathrm{AcoR}$ & Transcriptional regulators \\
\hline PA4148 & probable short-chain dehydrogenase & Putative enzymes \\
\hline PA4150 & probable dehydrogenase El component & Carbon compound catabolism \\
\hline PA4151 & acetoin catabolism protein $\mathrm{AcoB}$ & Carbon compound catabolism \\
\hline PA4152 & probable hydrolase & Carbon compound catabolism \\
\hline PA4153 & 2,3-butanediol dehydrogenase & Carbon compound catabolism \\
\hline
\end{tabular}




\begin{tabular}{|c|c|c|}
\hline ORF & Product name & Function class \\
\hline PA4175 & protease IV & Secreted Factors (toxins, enzymes, alginate) \\
\hline PA4180 & probable acetolactate synthase lar ge subunit & Amino acid biosynthesis and metabolism \\
\hline PA4205 & hypothetical protein & Membrane proteins \\
\hline PA4206 & probable Resistance-Nodulation-Cell Division (RND) ef flux membrane fusion protein & Transport of small molecules \\
\hline PA4207 & probable Resistance-Nodulation-Cell Division (RND) ef flux transporter & Membrane proteins \\
\hline PA4208 & probable outer membrane protein precursor & Membrane proteins \\
\hline PA4209 & probable phenazine-specific methyltransferase & Putative enzymes \\
\hline PA4210 & probable phenazine biosynthesis protein & Secreted Factors (toxins, enzymes, alginate) \\
\hline PA4211 & probable phenazine biosynthesis protein & Secreted Factors (toxins, enzymes, alginate) \\
\hline PA4288 & probable transcriptional regulator & Transcriptional regulators \\
\hline PA4293 & two-component sensor PprA & Two-component regulatory systems \\
\hline PA4294 & hypothetical protein & Hypothetical, unclassified, unknown \\
\hline PA4297 & TadG & Membrane proteins \\
\hline PA4298 & hypothetical protein & Hypothetical, unclassified, unknown \\
\hline PA4316 & exodeoxyribonuclease I & DNA replication, recombination, modification and repair \\
\hline PA4324 & hypothetical protein & Hypothetical, unclassified, unknown \\
\hline PA4328 & hypothetical protein & Hypothetical, unclassified, unknown \\
\hline PA4329 & pyruvate kinase II & Energy metabolism \\
\hline PA4348 & conserved hypothetical protein & Hypothetical, unclassified, unknown \\
\hline PA4351 & probable acyltransferase & Fatty acid and phospholipid metabolism \\
\hline PA4352 & conserved hypothetical protein & Hypothetical, unclassified, unknown \\
\hline PA4357 & conserved hypothetical protein & Hypothetical, unclassified, unknown \\
\hline PA4358 & probable ferrous iron transport protein & Membrane proteins \\
\hline PA4377 & hypothetical protein & Hypothetical, unclassified, unknown \\
\hline PA4394 & conserved hypothetical protein & Hypothetical, unclassified, unknown \\
\hline PA4433 & $50 \mathrm{~S}$ ribosomal protein $\mathrm{L} 13$ & Translation, post-translational modification, degradation \\
\hline PA4436 & probable transcriptional regulator & Transcriptional regulators \\
\hline PA4496 & probable binding protein component of $\mathrm{ABC}$ transporter & Transport of small molecules \\
\hline PA4497 & probable binding protein component of $\mathrm{ABC}$ transporter & Transport of small molecules \\
\hline PA4498 & probable metallopeptidase & Translation, post-translational modification, degradation \\
\hline PA4500 & probable binding protein component of $\mathrm{ABC}$ transporter & Transport of small molecules \\
\hline PA4502 & probable binding protein component of $\mathrm{ABC}$ transporter & Transport of small molecules \\
\hline PA4503 & probable permease of $\mathrm{ABC}$ transporter & Membrane proteins \\
\hline PA4504 & probable permease of $\mathrm{ABC}$ transporter & Membrane proteins \\
\hline PA4505 & probable ATP-binding component of $\mathrm{ABC}$ transporter & Transport of small molecules \\
\hline PA4506 & probable ATP-binding component of ABC dipeptide transporter & Transport of small molecules \\
\hline PA4520 & probable chemotaxis transducer & Adaptation, Protection \\
\hline PA4569 & octaprenyl-diphosphate synthase & Energy metabolism \\
\hline PA4577 & hypothetical protein & Hypothetical, unclassified, unknown \\
\hline PA4588 & glutamate dehydrogenase & Amino acid biosynthesis and metabolism \\
\hline PA4596 & probable transcriptional regulator & Transcriptional regulators \\
\hline PA4606 & conserved hypothetical protein & Adaptation, Protection \\
\hline PA4611 & hypothetical protein & Hypothetical, unclassified, unknown \\
\hline PA4632 & hypothetical protein & Hypothetical, unclassified, unknown \\
\hline PA4633 & probable chemotaxis transducer & Adaptation, Protection \\
\hline PA4654 & probable major facilitator superfamily (MFS) transporter & Membrane proteins \\
\hline PA4676 & probable carbonic anhydrase & Putative enzymes \\
\hline PA4677 & hypothetical protein & Hypothetical, unclassified, unknown \\
\hline PA4681 & hypothetical protein & Hypothetical, unclassified, unknown \\
\hline PA4682 & hypothetical protein & Hypothetical, unclassified, unknown \\
\hline PA4683 & hypothetical protein & Hypothetical, unclassified, unknown \\
\hline PA4695 & acetolactate synthase isozyme III small subunit & Biosynthesis of cofactors, prosthetic groups and carriers \\
\hline PA4715 & probable aminotransferase & Putative enzymes \\
\hline PA4733 & acetyl-coenzyme A synthetase & Carbon compound catabolism \\
\hline PA4757 & conserved hypothetical protein & Membrane proteins \\
\hline PA4758 & carbamoyl-phosphate synthase small chain & Amino acid biosynthesis and metabolism \\
\hline PA4759 & dihydrodipicolinate reductase & Amino acid biosynthesis and metabolism \\
\hline PA4770 & L-lactate permease & Transport of small molecules \\
\hline PA4773 & hypothetical protein & Hypothetical, unclassified, unknown \\
\hline PA4779 & hypothetical protein & Membrane proteins \\
\hline PA4782 & hypothetical protein & Hypothetical, unclassified, unknown \\
\hline PA4808 & L-seryl-tRNA(ser) selenium transferase & Translation, post-translational modification, degradation \\
\hline PA4817 & hypothetical protein & Hypothetical, unclassified, unknown \\
\hline PA4851 & hypothetical protein & Hypothetical, unclassified, unknown \\
\hline PA4852 & conserved hypothetical protein & Hypothetical, unclassified, unknown \\
\hline PA4860 & probable permease of $\mathrm{ABC}$ transporter & Membrane proteins \\
\hline PA4862 & probable ATP-binding component of $\mathrm{ABC}$ transporter & Transport of small molecules \\
\hline PA4867 & urease beta subunit & Central intermediary metabolism \\
\hline
\end{tabular}




\begin{tabular}{|c|c|c|}
\hline ORF & Product name & Function class \\
\hline PA4884 & hypothetical protein & Hypothetical, unclassified, unknown \\
\hline PA4893 & urease accessory protein UreG & Biosynthesis of cofactors, prosthetic groups and carriers \\
\hline PA4894 & hypothetical protein & Membrane proteins \\
\hline PA4899 & probable aldehyde dehydrogenase & Putative enzymes \\
\hline PA4901 & benzoylformate decarboxylase & Carbon compound catabolism \\
\hline PA4908 & hypothetical protein & Hypothetical, unclassified, unknown \\
\hline PA4910 & probable ATP-binding component of $\mathrm{ABC}$ transporter & Transport of small molecules \\
\hline PA4911 & probable permease of $\mathrm{ABC}$ branched-chain amino acid transporter & Membrane proteins \\
\hline PA4912 & probable permease of $\mathrm{ABC}$ branched chain amino acid transporter & Membrane proteins \\
\hline PA4913 & probable binding protein component of $\mathrm{ABC}$ transporter & Transport of small molecules \\
\hline PA4943 & probable GTP-binding protein & Putative enzymes \\
\hline PA4979 & probable acyl-CoA dehydrogenase & Putative enzymes \\
\hline PA4983 & probable two-component response regulator & Transcriptional regulators \\
\hline PA4984 & probable transcriptional regulator & Transcriptional regulators \\
\hline PA4985 & hypothetical protein & Hypothetical, unclassified, unknown \\
\hline PA4986 & probable oxidoreductase & Putative enzymes \\
\hline PA4997 & transport protein MsbA & Fatty acid and phospholipid metabolism \\
\hline PA5020 & probable acyl-CoA dehydrogenase & Putative enzymes \\
\hline PA5025 & homocysteine synthase & Amino acid biosynthesis and metabolism \\
\hline PA5027 & hypothetical protein & Hypothetical, unclassified, unknown \\
\hline PA5028 & conserved hypothetical protein & Hypothetical, unclassified, unknown \\
\hline PA5030 & probable major facilitator superfamily (MFS) transporter & Membrane proteins \\
\hline PA5051 & arginyl-tRNA synthetase & Translation, post-translational modification, degradation \\
\hline PA5059 & probable transcriptional regulator & Transcriptional regulators \\
\hline PA5082 & probable binding protein component of $\mathrm{ABC}$ transporter & Transport of small molecules \\
\hline PA5096 & probable binding protein component of $\mathrm{ABC}$ transporter & Transport of small molecules \\
\hline PA5097 & probable amino acid permease & Membrane proteins \\
\hline PA5098 & histidine ammonia-lyase & Amino acid biosynthesis and metabolism \\
\hline PA5106 & conserved hypothetical protein & Hypothetical, unclassified, unknown \\
\hline PA5112 & esterase EstA & Secreted Factors (toxins, enzymes, alginate) \\
\hline PA5129 & glutaredoxin & Energy metabolism \\
\hline PA5130 & conserved hypothetical protein & Hypothetical, unclassified, unknown \\
\hline PA5137 & hypothetical protein & Hypothetical, unclassified, unknown \\
\hline PA5149 & conserved hypothetical protein & Hypothetical, unclassified, unknown \\
\hline PA5152 & probable ATP-binding component of $\mathrm{ABC}$ transporter & Transport of small molecules \\
\hline PA5153 & probable periplasmic binding protein & Transport of small molecules \\
\hline PA5154 & probable permease of $\mathrm{ABC}$ transporter & Membrane proteins \\
\hline PA5155 & probable permease of $\mathrm{ABC}$ transporter & Membrane proteins \\
\hline PA5161 & dTDP-D-glucose 4,6-dehydratase & Carbon compound catabolism \\
\hline PA5162 & dTDP-4-dehydrorhamnose reductase & Cell wall / LPS / capsule \\
\hline PA5163 & glucose-1-phosphate thymidylyltransferase & Cell wall / LPS / capsule \\
\hline PA5164 & dTDP-4-dehydrorhamnose 3,5-epimerase & Cell wall / LPS / capsule \\
\hline PA5168 & probable dicarboxylate transporter & Membrane proteins \\
\hline PA5169 & probable C4-dicarboxylate transporter & Membrane proteins \\
\hline PA5174 & probable beta-ketoacyl synthase & Fatty acid and phospholipid metabolism \\
\hline PA5180 & conserved hypothetical protein & Hypothetical, unclassified, unknown \\
\hline PA5220 & hypothetical protein & Hypothetical, unclassified, unknown \\
\hline PA5237 & conserved hypothetical protein & Hypothetical, unclassified, unknown \\
\hline PA5240 & thioredoxin & Translation, post-translational modification, degradation \\
\hline PA5249 & hypothetical protein & Membrane proteins \\
\hline PA5275 & conserved hypothetical protein & Hypothetical, unclassified, unknown \\
\hline PA5285 & hypothetical protein & Hypothetical, unclassified, unknown \\
\hline PA5286 & conserved hypothetical protein & Hypothetical, unclassified, unknown \\
\hline PA5288 & nitrogen regulatory protein $\mathrm{P}$-II 2 & Central intermediary metabolism \\
\hline PA5289 & hypothetical protein & Hypothetical, unclassified, unknown \\
\hline PA5297 & pyruvate dehydrogenase (cytochrome) & Central intermediary metabolism \\
\hline PA5303 & conserved hypothetical protein & Hypothetical, unclassified, unknown \\
\hline PA5309 & probable oxidoreductase & Putative enzymes \\
\hline PA5324 & probable transcriptional regulator & Transcriptional regulators \\
\hline PA5325 & hypothetical protein & Hypothetical, unclassified, unknown \\
\hline PA5328 & probable cytochrome c(mono-heme type) & Energy metabolism \\
\hline PA5329 & conserved hypothetical protein & Hypothetical, unclassified, unknown \\
\hline PA5338 & guanosine-3',5'-bis(diphosphate) 3'-pyrophosphohydrolase & Nucleotide biosynthesis and metabolism \\
\hline PA5344 & probable transcriptional regulator & Transcriptional regulators \\
\hline PA5348 & probable DNA-binding protein & DNA replication, recombination, modification and repair \\
\hline PA5351 & Rubredoxin 1 & Carbon compound catabolism \\
\hline PA5353 & glycolate oxidase subunit GlcF & Central intermediary metabolism \\
\hline PA5355 & glycolate oxidase subunit GlcD & Central intermediary metabolism \\
\hline
\end{tabular}




\begin{tabular}{|c|c|c|}
\hline ORF & Product name & Function class \\
\hline PA5380 & GbdR & Transcriptional regulators \\
\hline PA5381 & hypothetical protein & Hypothetical, unclassified, unknown \\
\hline PA5400 & probable electron transfer flavoprotein alpha subunit & Energy metabolism \\
\hline PA5409 & hypothetical protein & Membrane proteins \\
\hline PA5427 & alcohol dehydrogenase & Energy metabolism \\
\hline PA5434 & tryptophan permease & Membrane proteins \\
\hline PA5435 & probable transcarboxylase subunit & Central intermediary metabolism \\
\hline PA5436 & probable biotin carboxylase subunit of a transcarboxylase & Central intermediary metabolism \\
\hline PA5442 & conserved hypothetical protein & Membrane proteins \\
\hline PA5445 & probable coenzyme A transferase & Putative enzymes \\
\hline PA5451 & membrane subunit of A-band LPS efflux transporter & Cell wall / LPS / capsule \\
\hline PA5452 & phosphomannose isomerase/GDP-mannose WbpW & Cell wall / LPS / capsule \\
\hline PA5461 & hypothetical protein & Hypothetical, unclassified, unknown \\
\hline PA5475 & hypothetical protein & Hypothetical, unclassified, unknown \\
\hline PA5491 & probable cytochrome & Energy metabolism \\
\hline PA5506 & hypothetical protein & Hypothetical, unclassified, unknown \\
\hline PA5507 & hypothetical protein & Hypothetical, unclassified, unknown \\
\hline PA5508 & probable glutamine synthetase & Putative enzymes \\
\hline PA5509 & hypothetical protein & Hypothetical, unclassified, unknown \\
\hline PA5510 & probable transporter & Membrane proteins \\
\hline PA5522 & probable glutamine synthetase & Putative enzymes \\
\hline PA5523 & probable aminotransferase & Putative enzymes \\
\hline PA5533 & hypothetical protein & Hypothetical, unclassified, unknown \\
\hline PA5536 & conserved hypothetical protein & Hypothetical, unclassified, unknown \\
\hline PA5542 & hypothetical protein & Putative enzymes \\
\hline PA5543 & hypothetical protein & Hypothetical, unclassified, unknown \\
\hline PA5544 & conserved hypothetical protein & Membrane proteins \\
\hline PA5545 & conserved hypothetical protein & Hypothetical, unclassified, unknown \\
\hline PA5546 & conserved hypothetical protein & Putative enzymes \\
\hline
\end{tabular}


Oxidative stress

\section{PA0007 hypothetical protein}

PA0069 conserved hypothetical protein

PA0105 cytochrome c oxidase, subunit II

PA0106 cytochrome c oxidase, subunit I

PA0107 conserved hypothetical protein

PA0109 hypothetical protein

PA0122 conserved hypothetical protein

PA0128 conserved hypothetical protein

PA0158 RND; triclosan efflux transporter, TriC

PA0171 hypothetical protein

PA0173 probable methylesterase

PA0174 conserved hypothetical protein

PA0176 aerotaxis transducer Aer2

PA0177 probable purine-binding chemotaxis protein

PA0178 probable two-component sensor

PA0179 probable two-component response regulator

PA0182 probable short-chain dehydrogenase

PA0250 conserved hypothetical protein

PA0280 sulfate transport protein CysA

PA0281 sulfate transport protein CysW

PA0282 sulfate transport protein CysT

PA0283 sulfate-binding protein precursor

PA0335 hypothetical protein

PA0337 phosphoenolpyruvate-protein phosphotransferase PtsP

PA0352 probable transporter

PA0382 DNA mismatch repair protein MicA

PA0385 hypothetical protein

PA0459 probable ClpA/B protease ATP binding subunit

PA0492 conserved hypothetical protein

PA0493 probable biotin-requiring enzyme

PA0494 probable acyl-CoA carboxylase subunit

PA0495 hypothetical protein

PA0555 fructose-1,6-bisphosphate aldolase

PA0582 dihydroneopterin aldolase

PA0586 conserved hypothetical protein

PA0587 conserved hypothetical protein

PA0612 repressor, PtrB

PA0613 hypothetical protein

PA0614 hypothetical protein

PA0615 hypothetical protein

PA0616 hypothetical protein

PA0617 probable bacteriophage protein

PA0618 probable bacteriophage protein

PA0619 probable bacteriophage protein

PA0620 probable bacteriophage protein

PA0621 conserved hypothetical protein

PA0622 probable bacteriophage protein

PA0623 probable bacteriophage protein

PA0624 hypothetical protein

PA0625 hypothetical protein

PA0626 hypothetical protein

PA0627 conserved hypothetical protein

PA0628 conserved hypothetical protein

PA0629 conserved hypothetical protein

PA0630 hypothetical protein

PA0631 hypothetical protein

PA0634 hypothetical protein

PA0635 hypothetical protein

PA0636 hypothetical protein

PA0637 conserved hypothetical protein

PA0638 probable bacteriophage protein

PA0639 conserved hypothetical protein

PA0640 probable bacteriophage protein

PA0641 probable bacteriophage protein

PA0642 hypothetical protein

PA0643 hypothetical protein
Membrane proteins

Hypothetical, unclassified, unknown

Energy metabolism

Energy metabolism

Energy metabolism

Membrane proteins

Hypothetical, unclassified, unknown

Hypothetical, unclassified, unknown

Antibiotic resistance and susceptibility

Motility \& Attachment

Adaptation, Protection

Hypothetical, unclassified, unknown

Adaptation, Protection

Adaptation, Protection

Chemotaxis

Chemotaxis

Putative enzymes

Hypothetical, unclassified, unknown

Transport of small molecules

Membrane proteins

Membrane proteins

Transport of small molecules

Hypothetical, unclassified, unknown

Transport of small molecules

Membrane proteins

DNA replication, recombination, modification and repair

Hypothetical, unclassified, unknown

Translation, post-translational modification, degradation

Hypothetical, unclassified, unknown

Putative enzymes

Putative enzymes

Hypothetical, unclassified, unknown

Central intermediary metabolism

Biosynthesis of cofactors, prosthetic groups and carriers

Hypothetical, unclassified, unknown

Hypothetical, unclassified, unknown

Transcriptional regulators

Hypothetical, unclassified, unknown

Hypothetical, unclassified, unknown

Hypothetical, unclassified, unknown

Related to phage, transposon, or plasmid

Related to phage, transposon, or plasmid

Related to phage, transposon, or plasmid

Related to phage, transposon, or plasmid

Related to phage, transposon, or plasmid

Related to phage, transposon, or plasmid

Related to phage, transposon, or plasmid

Related to phage, transposon, or plasmid

Related to phage, transposon, or plasmid

Related to phage, transposon, or plasmid

Related to phage, transposon, or plasmid

Related to phage, transposon, or plasmid

Related to phage, transposon, or plasmid

Related to phage, transposon, or plasmid

Related to phage, transposon, or plasmid

Related to phage, transposon, or plasmid

Related to phage, transposon, or plasmid

Related to phage, transposon, or plasmid

Related to phage, transposon, or plasmid

Related to phage, transposon, or plasmid

Related to phage, transposon, or plasmid

Related to phage, transposon, or plasmid

Related to phage, transposon, or plasmid

Related to phage, transposon, or plasmid

Related to phage, transposon, or plasmic

Related to phage, transposon, or plasmid 


\begin{tabular}{|c|c|c|}
\hline ORF & Product name & Function class \\
\hline PA0645 & hypothetical protein & Related to phage, transposon, or plasmid \\
\hline PA0646 & hypothetical protein & Related to phage, transposon, or plasmid \\
\hline PA0669 & probable DNA polymerase alpha chain & Putative enzymes \\
\hline PA0670 & hypothetical protein & Hypothetical, unclassified, unknown \\
\hline PA0671 & hypothetical protein & Hypothetical, unclassified, unknown \\
\hline PA0743 & probable 3-hydroxyisobutyrate dehydrogenase & Carbon compound catabolism \\
\hline PA0745 & probable enoyl-CoA hydratase/isomerase & Putative enzymes \\
\hline PA0746 & probable acyl-CoA dehydrogenase & Putative enzymes \\
\hline PA0747 & probable aldehyde dehydrogenase & Putative enzymes \\
\hline PA0873 & transcriptional regulator PhhR & Transcriptional regulators \\
\hline PA0887 & acetyl-coenzyme A synthetase & Carbon compound catabolism \\
\hline PA0887.1 & aaa & Non-coding RNA gene \\
\hline PA0889 & arginine/ornithine transport protein AotQ & Membrane proteins \\
\hline PA0905 & RsmA, regulator of secondary metabolites & Translation, post-translational modification, degradation \\
\hline PA0915 & conserved hypothetical protein & Hypothetical, unclassified, unknown \\
\hline PA0916 & conserved hypothetical protein & Hypothetical, unclassified, unknown \\
\hline PA0922 & hypothetical protein & Hypothetical, unclassified, unknown \\
\hline PA0975 & probable radical activating enzyme & Putative enzymes \\
\hline PA0976 & conserved hypothetical protein & Hypothetical, unclassified, unknown \\
\hline PA0983 & conserved hypothetical protein & Hypothetical, unclassified, unknown \\
\hline PA0996 & probable coenzyme A ligase & Biosynthesis of cofactors, prosthetic groups and carriers \\
\hline PA1000 & Quinolone signal response protein & Biosynthesis of cofactors, prosthetic groups and carriers \\
\hline PA1001 & anthranilate synthase component I & Adaptation, Protection \\
\hline PA1002 & anthranilate synthase component II & Amino acid biosynthesis and metabolism \\
\hline PA1041 & probable outer membrane protein precursor & Membrane proteins \\
\hline PA1112 & conserved hypothetical protein & Hypothetical, unclassified, unknown \\
\hline PA1150 & pyocin S2 & Adaptation, Protection \\
\hline PA1151 & pyocin S2 immunity protein & Adaptation, Protection \\
\hline PA1172 & cytochrome c-type protein NapC & Energy metabolism \\
\hline PA1173 & cytochrome c-type protein NapB precursor & Energy metabolism \\
\hline PA1174 & periplasmic nitrate reductase protein NapA & Energy metabolism \\
\hline PA1175 & NapD protein of periplasmic nitrate reductase & Energy metabolism \\
\hline PA1176 & ferredoxin protein $\mathrm{NapF}$ & Energy metabolism \\
\hline PA1177 & periplasmic nitrate reductase protein $\mathrm{NapE}$ & Energy metabolism \\
\hline PA1228 & hypothetical protein & Hypothetical, unclassified, unknown \\
\hline PA1320 & cytochrome 0 ubiquinol oxidase subunit IV & Energy metabolism \\
\hline PA1321 & cytochrome o ubiquinol oxidase protein $\mathrm{CyoE}$ & Energy metabolism \\
\hline PA1372 & hypothetical protein & Hypothetical, unclassified, unknown \\
\hline PA1432 & autoinducer synthesis protein LasI & Adaptation, Protection \\
\hline PA1466 & hypothetical protein & Hypothetical, unclassified, unknown \\
\hline PA1552 & probable cytochrome c & Energy metabolism \\
\hline PA1553 & probable cytochrome c oxidase subunit & Energy metabolism \\
\hline PA1554 & probable cytochrome oxidase subunit (cbb3-type) & Energy metabolism \\
\hline PA1574 & conserved hypothetical protein & Hypothetical, unclassified, unknown \\
\hline PA1581 & succinate dehydrogenase (C subunit) & Energy metabolism \\
\hline PA1582 & succinate dehydrogenase (D subunit) & Energy metabolism \\
\hline PA1583 & succinate dehydrogenase (A subunit) & Energy metabolism \\
\hline PA1584 & succinate dehydrogenase (B subunit) & Energy metabolism \\
\hline PA1634 & potassium-transporting ATPase, B chain & Transport of small molecules \\
\hline PA1687 & spermidine synthase & Amino acid biosynthesis and metabolism \\
\hline PA1759 & probable transcriptional regulator & Transcriptional regulators \\
\hline PA1760 & probable transcriptional regulator & Transcriptional regulators \\
\hline PA1776 & ECF sigma factor SigX & Transcriptional regulators \\
\hline PA1837 & hypothetical protein & Hypothetical, unclassified, unknown \\
\hline PA1845 & hypothetical protein & Hypothetical, unclassified, unknown \\
\hline PA1852 & hypothetical protein & Hypothetical, unclassified, unknown \\
\hline PA1865 & hypothetical protein & Hypothetical, unclassified, unknown \\
\hline PA1930 & probable chemotaxis transducer & Adaptation, Protection \\
\hline PA1946 & binding protein component precursor of $\mathrm{ABC}$ ribose transporter & Transport of small molecules \\
\hline PA1951 & hypothetical protein & Hypothetical, unclassified, unknown \\
\hline PA2006 & probable major facilitator superfamily (MFS) transporter & Membrane proteins \\
\hline PA2007 & maleylacetoacetate isomerase & Carbon compound catabolism \\
\hline PA2008 & fumarylacetoacetase & Carbon compound catabolism \\
\hline PA2009 & homogentisate 1,2-dioxygenase & Carbon compound catabolism \\
\hline PA2012 & methylcrotonyl-CoA carboxylase, alpha-subunit (biotin-containing) & Carbon compound catabolism \\
\hline PA2288 & hypothetical protein & Hypothetical, unclassified, unknown \\
\hline PA2290 & glucose dehydrogenase & Carbon compound catabolism \\
\hline PA2321 & gluconokinase & Energy metabolism \\
\hline
\end{tabular}




\begin{tabular}{|c|c|c|}
\hline ORF & Product name & Function class \\
\hline PA2322 & gluconate permease & Transport of small molecules \\
\hline PA2338 & probable binding protein component of $\mathrm{ABC}$ maltose/mannitol transporter & Transport of small molecules \\
\hline PA2339 & probable binding-protein-dependent maltose/mannitol transport protein & Transport of small molecules \\
\hline PA2340 & probable binding-protein-dependent maltose/mannitol transport protein & Transport of small molecules \\
\hline PA2341 & probable ATP-binding component of $\mathrm{ABC}$ maltose/mannitol transporter & Transport of small molecules \\
\hline PA2342 & mannitol dehydrogenase & Carbon compound catabolism \\
\hline PA2344 & fructokinase & Carbon compound catabolism \\
\hline PA2453 & hypothetical protein & Hypothetical, unclassified, unknown \\
\hline PA2552 & probable acyl-CoA dehydrogenase & Putative enzymes \\
\hline PA2553 & probable acyl- $\mathrm{CoA}$ thiolase & Putative enzymes \\
\hline PA2555 & probable AMP-binding enzyme & Putative enzymes \\
\hline PA2557 & probable AMP-binding enzyme & Fatty acid and phospholipid metabolism \\
\hline PA2573 & probable chemotaxis transducer & Adaptation, Protection \\
\hline PA2579 & hypothetical protein & Putative enzymes \\
\hline PA2619 & initiation factor & Translation, post-translational modification, degradation \\
\hline PA2637 & NADH dehydrogenase I chain A & Energy metabolism \\
\hline PA2638 & NADH dehydrogenase I chain B & Energy metabolism \\
\hline PA2639 & $\mathrm{NADH}$ dehydrogenase I chain C,D & Energy metabolism \\
\hline PA2640 & NADH dehydrogenase I chain E & Energy metabolism \\
\hline PA2641 & NADH dehydrogenase I chain F & Energy metabolism \\
\hline PA2642 & NADH dehydrogenase I chain G & Energy metabolism \\
\hline PA2643 & $\mathrm{NADH}$ dehydrogenase I chain $\mathrm{H}$ & Energy metabolism \\
\hline PA2644 & NADH Dehydrogenase I chain I & Energy metabolism \\
\hline PA2645 & NADH dehydrogenase I chain J & Energy metabolism \\
\hline PA2646 & NADH dehydrogenase I chain $\mathrm{K}$ & Energy metabolism \\
\hline PA2647 & NADH dehydrogenase I chain $\mathrm{L}$ & Energy metabolism \\
\hline PA2648 & NADH dehydrogenase I chain M & Energy metabolism \\
\hline PA2649 & NADH dehydrogenase I chain N & Energy metabolism \\
\hline PA2653 & probable transporter & Membrane proteins \\
\hline PA2667 & conserved hypothetical protein & Hypothetical, unclassified, unknown \\
\hline PA2730 & hypothetical protein & Hypothetical, unclassified, unknown \\
\hline PA2746 & hypothetical protein & Membrane proteins \\
\hline PA2911 & probable TonB-dependent receptor & Membrane proteins \\
\hline PA2937 & hypothetical protein & Hypothetical, unclassified, unknown \\
\hline PA3007 & repressor protein LexA & Adaptation, Protection \\
\hline PA3008 & hypothetical protein & Hypothetical, unclassified, unknown \\
\hline PA3009 & hypothetical protein & Hypothetical, unclassified, unknown \\
\hline PA3080 & hypothetical protein & Hypothetical, unclassified, unknown \\
\hline PA3147 & probable glycosyl transferase $\mathrm{WbpJ}$ & Putative enzymes \\
\hline PA3149 & probable glycosyltransferase $\mathrm{WbpH}$ & Putative enzymes \\
\hline PA3150 & LPS biosynthesis protein $\mathrm{WbpG}$ & Cell wall / LPS / capsule \\
\hline PA3181 & 2-keto-3-deoxy-6-phosphogluconate aldolase & Central intermediary metabolism \\
\hline PA3183 & glucose-6-phosphate 1-dehydrogenase & Energy metabolism \\
\hline PA3186 & Glucose/carbohydrate outer membrane porin OprB precursor & Transport of small molecules \\
\hline PA3187 & probable ATP-binding component of ABC transporter & Transport of small molecules \\
\hline PA3188 & probable permease of $\mathrm{ABC}$ sugar transporter & Transport of small molecules \\
\hline PA3189 & probable permease of $A B C$ sugar transporter & Transport of small molecules \\
\hline PA3190 & probable binding protein component of $\mathrm{ABC}$ sugar transporter & Transport of small molecules \\
\hline PA3194 & phosphogluconate dehydratase & Energy metabolism \\
\hline PA3237 & hypothetical protein & Hypothetical, unclassified, unknown \\
\hline PA3282 & hypothetical protein & Hypothetical, unclassified, unknown \\
\hline PA3311 & conserved hypothetical protein & Membrane proteins \\
\hline PA3404 & probable outer membrane protein precursor & Protein secretion/export apparatus \\
\hline PA3415 & probable dihydrolipoamide acetyltransferase & Energy metabolism \\
\hline PA3417 & probable pyruvate dehydrogenase E1 component, alpha subunit & Energy metabolism \\
\hline PA3418 & leucine dehydrogenase & Amino acid biosynthesis and metabolism \\
\hline PA3441 & probable molybdopterin-binding protein & Transport of small molecules \\
\hline PA3442 & probable ATP-binding component of $\mathrm{ABC}$ transporter & Transport of small molecules \\
\hline PA3443 & probable permease of $\mathrm{ABC}$ transporter & Membrane proteins \\
\hline PA3444 & conserved hypothetical protein & Putative enzymes \\
\hline PA3445 & conserved hypothetical protein & Hypothetical, unclassified, unknown \\
\hline PA3446 & conserved hypothetical protein & Hypothetical, unclassified, unknown \\
\hline PA3449 & conserved hypothetical protein & Hypothetical, unclassified, unknown \\
\hline PA3496 & hypothetical protein & Hypothetical, unclassified, unknown \\
\hline PA3561 & 1-phosphofructokinase & Central intermediary metabolism \\
\hline PA3574 & probable transcriptional regulator & Transcriptional regulators \\
\hline PA3581 & glycerol uptake facilitator protein & Transport of small molecules \\
\hline PA3582 & glycerol kinase & Central intermediary metabolism \\
\hline
\end{tabular}




\begin{tabular}{|c|c|c|}
\hline ORF & Product name & Function class \\
\hline PA3584 & glycerol-3-phosphate dehydrogenase & Central intermediary metabolism \\
\hline PA3600 & conserved hypothetical protein & Translation, post-translational modification, degradation \\
\hline PA3607 & polyamine transport protein PotA & Transport of small molecules \\
\hline PA3608 & polyamine transport protein PotB & Membrane proteins \\
\hline PA3609 & polyamine transport protein $\mathrm{PotC}$ & Membrane proteins \\
\hline PA3610 & polyamine transport protein PotD & Transport of small molecules \\
\hline PA3616 & conserved hypothetical protein & Hypothetical, unclassified, unknown \\
\hline PA3617 & RecA protein & DNA replication, recombination, modification and repair \\
\hline PA3621 & ferredoxin I & Energy metabolism \\
\hline PA3672 & probable ATP-binding component of $\mathrm{ABC}$ transporter & Transport of small molecules \\
\hline PA3712 & hypothetical protein & Hypothetical, unclassified, unknown \\
\hline PA3713 & spermidine dehydrogenase, $\mathrm{SpdH}$ & Hypothetical, unclassified, unknown \\
\hline PA3723 & probable FMN oxidoreductase & Putative enzymes \\
\hline PA3753 & conserved hypothetical protein & Transport of small molecules \\
\hline PA3767 & conserved hypothetical protein & Hypothetical, unclassified, unknown \\
\hline PA3787 & conserved hypothetical protein & Hypothetical, unclassified, unknown \\
\hline PA3808 & conserved hypothetical protein & Hypothetical, unclassified, unknown \\
\hline PA3870 & molybdopterin biosynthetic protein $\mathrm{Al}$ & Biosynthesis of cofactors, prosthetic groups and carriers \\
\hline PA3871 & probable peptidyl-prolyl cis-trans isomerase, PpiC-type & Translation, post-translational modification, degradation \\
\hline PA3872 & respiratory nitrate reductase gamma chain & Energy metabolism \\
\hline PA3873 & respiratory nitrate reductase delta chain & Energy metabolism \\
\hline PA3874 & respiratory nitrate reductase beta chain & Energy metabolism \\
\hline PA3875 & respiratory nitrate reductase alpha chain & Energy metabolism \\
\hline PA3876 & nitrite extrusion protein 2 & Membrane proteins \\
\hline PA3877 & nitrite extrusion protein 1 & Membrane proteins \\
\hline PA3901 & $\mathrm{Fe}(\mathrm{III})$ dicitrate transport protein $\mathrm{FecA}$ & Membrane proteins \\
\hline PA3913 & probable protease & Putative enzymes \\
\hline PA3922 & conserved hypothetical protein & Hypothetical, unclassified, unknown \\
\hline PA3935 & taurine dioxygenase & Carbon compound catabolism \\
\hline PA3967 & hypothetical protein & Hypothetical, unclassified, unknown \\
\hline PA3986 & hypothetical protein & Hypothetical, unclassified, unknown \\
\hline PA4055 & riboflavin synthase alpha chain & Biosynthesis of cofactors, prosthetic groups and carriers \\
\hline PA4131 & probable iron-sulfur protein & Putative enzymes \\
\hline PA4132 & conserved hypothetical protein & Hypothetical, unclassified, unknown \\
\hline PA4133 & cytochrome c oxidase subunit (cbb3-type) & Energy metabolism \\
\hline PA4141 & hypothetical protein & Hypothetical, unclassified, unknown \\
\hline PA4199 & probable acyl- $\mathrm{CoA}$ dehydrogenase & Putative enzymes \\
\hline PA4236 & catalase & Adaptation, Protection \\
\hline PA4390 & hypothetical protein & Hypothetical, unclassified, unknown \\
\hline PA4427 & stringent starvation protein B & Adaptation, Protection \\
\hline PA4432 & $30 \mathrm{~S}$ ribosomal protein $\mathrm{S} 9$ & Translation, post-translational modification, degradation \\
\hline PA4496 & probable binding protein component of $\mathrm{ABC}$ transporter & Transport of small molecules \\
\hline PA4553 & type 4 fimbrial biogenesis protein PilX & Motility \& Attachment \\
\hline PA4563 & 30 S ribosomal protein $\mathrm{S} 20$ & Central intermediary metabolism \\
\hline PA4573 & hypothetical protein & Hypothetical, unclassified, unknown \\
\hline PA4582 & conserved hypothetical protein & Hypothetical, unclassified, unknown \\
\hline PA4583 & conserved hypothetical protein & Hypothetical, unclassified, unknown \\
\hline PA4584 & conserved hypothetical protein & Hypothetical, unclassified, unknown \\
\hline PA4585 & RNA 3'-terminal phosphate cyclase & Transcription, RNA processing and degradation \\
\hline PA4607 & hypothetical protein & Hypothetical, unclassified, unknown \\
\hline PA4612 & conserved hypothetical protein & Hypothetical, unclassified, unknown \\
\hline PA4642 & hypothetical protein & Hypothetical, unclassified, unknown \\
\hline PA4645 & probable purine/pyrimidine phosphoribosyl transferase & Nucleotide biosynthesis and metabolism \\
\hline PA4648 & hypothetical protein & Hypothetical, unclassified, unknown \\
\hline PA4651 & probable pili assembly chaperone & Motility \& Attachment \\
\hline PA4672 & peptidyl-tRNA hydrolase & Translation, post-translational modification, degradation \\
\hline PA4687 & ferric iron-binding periplasmic protein HitA & Transport of small molecules \\
\hline PA4688 & iron (III)-transport system permease HitB & Membrane proteins \\
\hline PA4702 & hypothetical protein & Hypothetical, unclassified, unknown \\
\hline PA4703 & hypothetical protein & Hypothetical, unclassified, unknown \\
\hline PA4732 & glucose-6-phosphate isomerase & Carbon compound catabolism \\
\hline PA4746 & conserved hypothetical protein & Hypothetical, unclassified, unknown \\
\hline PA4747 & secretion protein $\mathrm{SecG}$ & Membrane proteins \\
\hline PA4748 & triosephosphate isomerase & Central intermediary metabolism \\
\hline PA4753 & conserved hypothetical protein & Hypothetical, unclassified, unknown \\
\hline PA4759 & dihydrodipicolinate reductase & Amino acid biosynthesis and metabolism \\
\hline PA4763 & DNA repair protein $\mathrm{RecN}$ & DNA replication, recombination, modification and repair \\
\hline PA4839 & biosynthetic arginine decarboxylase & Amino acid biosynthesis and metabolism \\
\hline
\end{tabular}




$\begin{array}{ll}\text { PA4840 } & \text { conserved hypothetical protein } \\ \text { PA4843 } & \text { probable two-component response regulator } \\ \text { PA5024 } & \text { conserved hypothetical protein } \\ \text { PA5049 } & \text { 50S ribosomal protein L31 } \\ \text { PA5074 } & \text { probable ATP-binding component of ABC transporter } \\ \text { PA5167 } & \text { probable c4-dicarboxylate-binding protein } \\ \text { PA5235 } & \text { glycerol-3-phosphate transporter } \\ \text { PA5351 } & \text { Rubredoxin 1 } \\ \text { PA5380 } & \text { GbdR } \\ \text { PA5401 } & \text { hypothetical protein } \\ \text { PA5436 } & \text { probable biotin carboxylase subunit of a transcarboxylase } \\ \text { PA5446 } & \text { hypothetical protein } \\ \text { PA5452 } & \text { phosphomannose isomerase/GDP-mannose WbpW } \\ \text { PA5453 } & \text { GDP-mannose 4,6-dehydratase } \\ \text { PA5465 } & \text { hypothetical protein } \\ \text { PA5470 } & \text { probable peptide chain release factor } \\ \text { PA5471 } & \text { hypothetical protein } \\ \text { PA5479 } & \text { proton-glutamate symporter }\end{array}$

Hypothetical, unclassified, unknown

Transcriptional regulators

Hypothetical, unclassified, unknown

Translation, post-translational modification, degradation

Transport of small molecules

Membrane proteins

Membrane proteins

Carbon compound catabolism

Transcriptional regulators

Hypothetical, unclassified, unknown

Central intermediary metabolism

Hypothetical, unclassified, unknown

Cell wall / LPS / capsule

Cell wall / LPS / capsule

Hypothetical, unclassified, unknown

Translation, post-translational modification, degradation

Hypothetical, unclassified, unknown

Membrane proteins 


\begin{tabular}{|c|c|c|}
\hline PA0224 & probable aldolase & Putative enzymes \\
\hline PA0246 & probable major facilitator superfamily (MFS) transporter & Membrane proteins \\
\hline PA0263 & secreted protein $\mathrm{Hcp}$ & Secreted Factors (toxins, enzymes, alginate) \\
\hline PA0355 & protease PfpI & Translation, post-translational modification, degradation \\
\hline PA0417 & probable chemotaxis protein & Chemotaxis \\
\hline PA0425 & RND multidrug efflux membrane fusion protein MexA precursor & Transport of small molecules \\
\hline PA0426 & RND multidrug efflux transporter MexB & Transport of small molecules \\
\hline PA0427 & Major intrinsic multiple antibiotic resistance ef flux outer membrane protein OprM prec & c Antibiotic resistance and susceptibility \\
\hline PA0445 & probable transposase & Related to phage, transposon, or plasmid \\
\hline PA0526 & hypothetical protein & Hypothetical, unclassified, unknown \\
\hline PA0579 & $30 \mathrm{~S}$ ribosomal protein $\mathrm{S} 21$ & Translation, post-translational modification, degradation \\
\hline PA0726 & hypothetical protein of bacteriophage $\mathrm{Pfl}$ & Hypothetical, unclassified, unknown \\
\hline PA0868 & conserved hypothetical protein & Hypothetical, unclassified, unknown \\
\hline PA1178 & $\mathrm{PhoP} / \mathrm{Q}$ and low $\mathrm{Mg} 2+$ inducible outer membrane protein $\mathrm{H} 1$ precursor & Membrane proteins \\
\hline PA1198 & conserved hypothetical protein & Hypothetical, unclassified, unknown \\
\hline PA1281 & cobalamin (5'-phosphate) synthase & Biosynthesis of cofactors, prosthetic groups and carriers \\
\hline PA1393 & adenosine 5'-phosphosulfate (APS) kinase & Amino acid biosynthesis and metabolism \\
\hline PA1493 & sulfate-binding protein of $\mathrm{ABC}$ transporter & Transport of small molecules \\
\hline PA1588 & succinyl-CoA synthetase beta chain & Energy metabolism \\
\hline PA1656 & hypothetical protein & Hypothetical, unclassified, unknown \\
\hline PA1658 & conserved hypothetical protein & Hypothetical, unclassified, unknown \\
\hline PAl660 & hypothetical protein & Hypothetical, unclassified, unknown \\
\hline PA1664 & hypothetical protein & Hypothetical, unclassified, unknown \\
\hline PA1780 & assimilatory nitrite reductase small subunit & Central intermediary metabolism \\
\hline PA2062 & probable pyridoxal-phosphate dependent enzyme & Putative enzymes \\
\hline PA2562 & hypothetical protein & Hypothetical, unclassified, unknown \\
\hline PA2658 & hypothetical protein & Hypothetical, unclassified, unknown \\
\hline PA2669 & hypothetical protein & Hypothetical, unclassified, unknown \\
\hline PA2906 & probable oxidoreductase & Putative enzymes \\
\hline PA2941 & hypothetical protein & Hypothetical, unclassified, unknown \\
\hline PA2951 & electron transfer flavoprotein alpha-subunit & Energy metabolism \\
\hline PA3136 & probable secretion protein & Transport of small molecules \\
\hline PA3137 & probable major facilitator superfamily (MFS) transporter & Membrane proteins \\
\hline PA3159 & probable UDP-glucose/GDP-mannose dehydrogenase WbpA & Putative enzymes \\
\hline PA3162 & $30 \mathrm{~S}$ ribosomal protein $\mathrm{S} 1$ & Translation, post-translational modification, degradation \\
\hline PA3266 & cold acclimation protein $B$ & Transcriptional regulators \\
\hline PA3547 & poly(beta-d-mannuronate) lyase precursor AlgL & Cell wall / LPS / capsule \\
\hline PA3552 & ArnB & Cell wall / LPS / capsule \\
\hline PA3676 & probable Resistance-Nodulation-Cell Division (RND) ef flux transporter & Membrane proteins \\
\hline PA3677 & probable RND ef flux membrane fusion protein precursor & Transport of small molecules \\
\hline PA3678 & probable transcriptional regulator & Transcriptional regulators \\
\hline PA3679 & hypothetical protein & Hypothetical, unclassified, unknown \\
\hline PA3719 & hypothetical protein & Hypothetical, unclassified, unknown \\
\hline PA3720 & hypothetical protein & Hypothetical, unclassified, unknown \\
\hline PA3721 & probable transcriptional regulator & Transcriptional regulators \\
\hline PA3814 & L-cysteine desulfurase (pyridoxal phosphate-dependent) & Amino acid biosynthesis and metabolism \\
\hline PA3815 & conserved hypothetical protein & Hypothetical, unclassified, unknown \\
\hline PA3819 & conserved hypothetical protein & Membrane proteins \\
\hline PA3905 & hypothetical protein & Hypothetical, unclassified, unknown \\
\hline PA4033 & hypothetical protein & Hypothetical, unclassified, unknown \\
\hline PA4037 & probable ATP-binding component of $\mathrm{ABC}$ transporter & Transport of small molecules \\
\hline PA4053 & 6,7-dimethyl-8-ribityllumazine synthase & Biosynthesis of cofactors, prosthetic groups and carriers \\
\hline PA4178 & hypothetical protein & Hypothetical, unclassified, unknown \\
\hline PA4344 & probable hydrolase & Putative enzymes \\
\hline PA4386 & GroES protein & Chaperones \& heat shock proteins \\
\hline PA4443 & ATP sulfurylase small subunit & Central intermediary metabolism \\
\hline PA4568 & $50 \mathrm{~S}$ ribosomal protein $\mathrm{L} 21$ & Translation, post-translational modification, degradation \\
\hline PA4816 & hypothetical protein & Hypothetical, unclassified, unknown \\
\hline PA4847 & biotin carboxyl carrier protein (BCCP) & Fatty acid and phospholipid metabolism \\
\hline PA5013 & branched-chain amino acid transferase & Amino acid biosynthesis and metabolism \\
\hline PA5 5157 & probable transcriptional regulator & Transcriptional regulators \\
\hline PA5158 & probable outer membrane protein precursor & Transport of small molecules \\
\hline PA5159 & multidrug resistance protein & Transport of small molecules \\
\hline PA5160 & drug efflux transporter & Membrane proteins \\
\hline PA5182 & hypothetical protein & Membrane proteins \\
\hline
\end{tabular}


PA5212 hypothetical protein

PA5216 probable permease of $\mathrm{ABC}$ iron transporter

Hypothetical, unclassified, unknown

PA5253 alginate regulatory protein AlgP

PA5312 probable aldehyde dehydrogenase

PA5326 hypothetical protein

PA5369 phosphate $\mathrm{ABC}$ transporter, periplasmic phosphate-binding protein, PstS

PA5390 probable peptidic bond hydrolase

PA5481 hypothetical protein

PA5570 50S ribosomal protein L34

Membrane proteins

Transcriptional regulators

Putative enzymes

Hypothetical, unclassified, unknown

Transport of small molecules

Putative enzymes

Hypothetical, unclassified, unknown

Central intermediary metabolism 
Copper

\begin{tabular}{|c|c|}
\hline PA0149 & probable sigma-70 factor, ECF subfamily \\
\hline PA0150 & probable transmembrane sensor \\
\hline PA0397 & probable cation efflux system protein \\
\hline PA0423 & PasP \\
\hline PA0450 & probable phosphate transporter \\
\hline PA0802 & hypothetical protein \\
\hline PA0913 & probable $\mathrm{Mg}$ transporter $\mathrm{MgtE}$ \\
\hline PA0918 & cytochrome b561 \\
\hline PA0931 & ferric enterobactin receptor PirA \\
\hline PA1134 & hypothetical protein \\
\hline PA1286 & probable major facilitator superfamily (MFS) transporter \\
\hline PA1297 & probable metal transporter \\
\hline PA1301 & probable transmembrane sensor \\
\hline PA1302 & probable heme utilization protein precursor \\
\hline PA1363 & probable sigma- 70 factor, ECF subfamily \\
\hline PA1364 & probable transmembrane sensor \\
\hline PA1365 & probable siderophore receptor \\
\hline PA1390 & probable glycosyl transferase \\
\hline PA1435 & RND efflux membrane fusion protein precursor \\
\hline PA1436 & probable RND efflux transporter \\
\hline PA1437 & probable two-component response regulator \\
\hline PA1438 & probable two-component sensor \\
\hline PA1562 & aconitate hydratase 1 \\
\hline PA1909 & hypothetical protein \\
\hline PA1910 & probable tonB-dependent receptor protein \\
\hline PA1911 & probable transmembrane sensor \\
\hline PA1912 & probable sigma-70 factor, ECF subfamily \\
\hline PA2064 & copper resistance protein $B$ precursor \\
\hline PA2065 & copper resistance protein A precursor \\
\hline PA2277 & ArsR protein \\
\hline PA2278 & ArsB protein \\
\hline PA2279 & ArsC protein \\
\hline PA2384 & hypothetical protein \\
\hline PA2390 & probable ATP-binding/permease fusion $\mathrm{ABC}$ transporter \\
\hline PA2391 & probable outer membrane protein precursor \\
\hline PA2395 & PvdO \\
\hline PA2406 & hypothetical protein \\
\hline PA2451 & hypothetical protein \\
\hline PA2466 & Ferrioxamine receptor FoxA \\
\hline PA2467 & Anti-sigma factor FoxR \\
\hline PA2476 & thiol:disulfide interchange protein DsbG \\
\hline PA2477 & probable thiol:disulfide interchange protein \\
\hline PA2478 & probable thiol:disulfide interchange protein \\
\hline PA2479 & probable two-component response regulator \\
\hline PA2480 & probable two-component sensor \\
\hline PA2520 & RND divalent metal cation ef flux transporter CzcA \\
\hline PA2521 & RND divalent metal cation ef flux membrane fusion protein $\mathrm{CzcB}$ precursor \\
\hline PA2522 & outer membrane protein precursor $\mathrm{CzcC}$ \\
\hline PA2524 & probable two-component sensor \\
\hline PA2664 & flavohemoprotein \\
\hline PA2686 & two-component response regulator PfeR \\
\hline PA2688 & Ferric enterobactin receptor, outer membrane protein PfeA precursor \\
\hline PA2807 & hypothetical protein \\
\hline PA2809 & two-component response regulator, $\mathrm{CopR}$ \\
\hline PA2810 & two-component sensor, CopS \\
\hline PA2826 & probable glutathione peroxidase \\
\hline PA2953 & electron transfer flavoprotein-ubiquinone oxidoreductase \\
\hline PA3392 & nitrous-oxide reductase precursor \\
\hline PA3407 & heme acquisition protein HasAp \\
\hline PA3408 & Haem uptake outer membrane receptor HasR precursor \\
\hline PA3409 & probable transmembrane sensor \\
\hline PA3410 & probable sigma- 70 factor, ECF subfamily \\
\hline PA3411 & hypothetical protein \\
\hline PA3412 & hypothetical protein \\
\hline PA3521 & probable outer membrane protein precursor \\
\hline
\end{tabular}

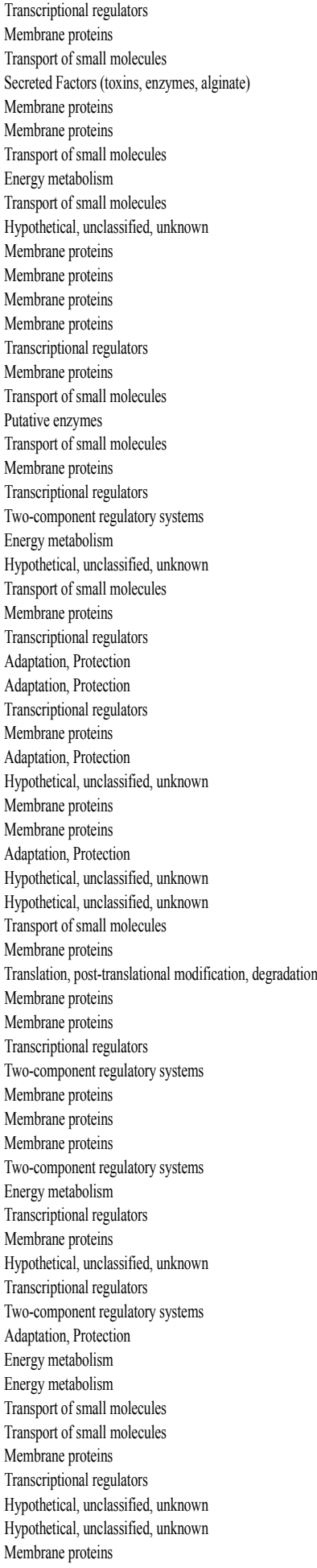




\begin{tabular}{|c|c|c|}
\hline ORF & Product name & Function class \\
\hline PA3522 & probable Resistance-Nodulation-Cell Division (RND) ef flux transporter & Membrane proteins \\
\hline PA3530 & conserved hypothetical protein & Hypothetical, unclassified, unknown \\
\hline PA3601 & conserved hypothetical protein & Translation, post-translational modification, degradation \\
\hline PA3602 & conserved hypothetical protein & Membrane proteins \\
\hline PA3749 & probable major facilitator superfamily (MFS) transporter & Membrane proteins \\
\hline PA3920 & probable metal transporting P-type ATPase & Membrane proteins \\
\hline PA3921 & probable transcriptional regulator & Transcriptional regulators \\
\hline PA4 458 & ferric enterobactin transport protein $\mathrm{FepC}$ & Transport of small molecules \\
\hline PA4159 & ferrienterobactin-binding periplasmic protein precursor FepB & Transport of small molecules \\
\hline PA4160 & ferric enterobactin transport protein FepD & Membrane proteins \\
\hline PA4192 & probable ATP-binding component of $\mathrm{ABC}$ transporter & Transport of small molecules \\
\hline PA4194 & probable permease of $\mathrm{ABC}$ transporter & Membrane proteins \\
\hline PA4195 & probable binding protein component of $\mathrm{ABC}$ transporter & Transport of small molecules \\
\hline PA4513 & probable oxidoreductase & Putative enzymes \\
\hline PA4514 & probable outer membrane receptor for iron transport & Transport of small molecules \\
\hline PA4515 & conserved hypothetical protein & Hypothetical, unclassified, unknown \\
\hline PA4655 & ferrochelatase & Biosynthesis of cofactors, prosthetic groups and carriers \\
\hline PA4675 & probable TonB-dependent receptor & Transport of small molecules \\
\hline PA4705 & hypothetical protein & Hypothetical, unclassified, unknown \\
\hline PA4706 & probable ATP-binding component of $\mathrm{ABC}$ transporter & Transport of small molecules \\
\hline PA4707 & probable permease of $\mathrm{ABC}$ transporter & Membrane proteins \\
\hline PA4711 & hypothetical protein & Hypothetical, unclassified, unknown \\
\hline PA4818 & conserved hypothetical protein & Membrane proteins \\
\hline PA4819 & probable glycosyl transferase & Putative enzymes \\
\hline PA4820 & hypothetical protein & Hypothetical, unclassified, unknown \\
\hline PA4825 & $\operatorname{Mg}(2+)$ transport ATPase, P-type 2 & Transport of small molecules \\
\hline PA5230 & probable permease of $\mathrm{ABC}$ transporter & Membrane proteins \\
\hline PA5231 & probable ATP-binding/permease fusion $\mathrm{ABC}$ transporter & Membrane proteins \\
\hline PA5300 & cytochrome c5 & Energy metabolism \\
\hline PA5304 & D-amino acid dehydrogenase, small subunit & Energy metabolism \\
\hline PA5531 & TonB protein & Transport of small molecules \\
\hline
\end{tabular}




$\begin{array}{ll}\text { Sulfate } & \\ & \\ \text { PA0192 } & \text { probable TonB-dependent receptor } \\ \text { PA0193 } & \text { hypothetical protein } \\ \text { PA0194 } & \text { hypothetical protein } \\ \text { PA0197 } & \text { hypothetical protein } \\ \text { PA0198 } & \text { transport protein ExbB } \\ \text { PA0199 } & \text { transport protein ExbD } \\ \text { PA2085 } & \text { probable ring-hydroxylating dioxygenase small subunit } \\ \text { PA2086 } & \text { probable epoxide hydrolase } \\ \text { PA2092 } & \text { probable major facilitator superfamily (MFS) transporter } \\ \text { PA2292 } & \text { hypothetical protein } \\ \text { PA2293 } & \text { hypothetical protein } \\ \text { PA2294 } & \text { probable ATP-binding component of ABC transporter } \\ \text { PA2295 } & \text { probable permease of ABC transporter } \\ \text { PA2296 } & \text { hypothetical protein } \\ \text { PA2297 } & \text { probable ferredoxin } \\ \text { PA2298 } & \text { probable oxidoreductase } \\ \text { PA2324 } & \text { hypothetical protein } \\ \text { PA2325 } & \text { hypothetical protein } \\ \text { PA2326 } & \text { hypothetical protein } \\ \text { PA2346 } & \text { conserved hypothetical protein } \\ \text { PA2347 } & \text { hypothetical protein } \\ \text { PA2348 } & \text { conserved hypothetical protein } \\ \text { PA2349 } & \text { conserved hypothetical protein } \\ \text { PA2350 } & \text { probable ATP-binding component of ABC transporter } \\ \text { PA2351 } & \text { probable permease of ABC transporter } \\ \text { PA2354 } & \text { probable transcriptional regulator } \\ \text { PA2355 } & \text { probable FMNH2-dependent monooxygenase } \\ \text { PA2356 } & \text { methanesulfonate sulfonatase MsuD } \\ \text { PA2357 } & \text { NADH-dependent FMN reductase MsuE } \\ & \\ & \end{array}$

\author{
Membrane proteins \\ Putative enzymes \\ Putative enzymes \\ Transport of small molecules \\ Transport of small molecules \\ Transport of small molecules \\ Carbon compound catabolism \\ Carbon compound catabolism \\ Membrane proteins \\ Hypothetical, unclassified, unknown \\ Hypothetical, unclassified, unknown \\ Transport of small molecules \\ Membrane proteins \\ Hypothetical, unclassified, unknown \\ Energy metabolism \\ Putative enzymes \\ Putative enzymes \\ Putative enzymes \\ Putative enzymes \\ Putative enzymes \\ Putative enzymes \\ Putative enzymes \\ Hypothetical, unclassified, unknown \\ Transport of small molecules \\ Membrane proteins \\ Transcriptional regulators \\ Putative enzymes \\ Carbon compound catabolism \\ Carbon compound catabolism
}


Osmotic shock

$\begin{array}{ll}\text { PA0236 } & \text { probable transcriptional regulator } \\ \text { PA0448 } & \text { probable transcriptional regulator } \\ \text { PA1159 } & \text { probable cold-shock protein } \\ \text { PA1226 } & \text { probable transcriptional regulator } \\ \text { PA1243 } & \text { probable sensor/response regulator hybrid } \\ \text { PA1690 } & \text { translocation protein in type III secretion } \\ \text { PA1692 } & \text { probable translocation protein in type III secretion } \\ \text { PA1693 } & \text { translocation protein in type III secretion } \\ \text { PA1694 } & \text { translocation protein in type III secretion } \\ \text { PA1695 } & \text { translocation protein in type III secretion } \\ \text { PA1697 } & \text { ATP synthase in type III secretion system } \\ \text { PA1702 } & \text { conserved hypothetical protein in type III secretion } \\ \text { PA1703 } & \text { type III secretory apparatus protein PcrD } \\ \text { PA1704 } & \text { transcriptional regulator protein PcrR } \\ \text { PA1724 } & \text { type III export protein PscK } \\ \text { PA1725 } & \text { type III export protein PscL } \\ \text { PA2147 } & \text { catalase HPII } \\ \text { PA2152 } & \text { probable trehalose synthase } \\ \text { PA2417 } & \text { probable transcriptional regulator } \\ \text { PA2665 } & \text { probable transcriptional regulator } \\ \text { PA3049 } & \text { ribosome modulation factor } \\ \text { PA3459 } & \text { probable glutamine amidotransferase } \\ \text { PA3462 } & \text { probable sensor/response regulator hybrid } \\ \text { PA3689 } & \text { probable transcriptional regulator } \\ \text { PA3831 } & \text { leucine aminopeptidase } \\ \text { PA3843 } & \text { hypothetical protein } \\ \text { PA4135 } & \text { probable transcriptional regulator } \\ \text { PA4581 } & \text { transcriptional regulator RtcR } \\ \text { PA5483 } & \text { two-component response regulator AlgB } \\ \text { PA5484 } & \text { probable two-component sensor } \\ & \\ & \end{array}$

Transcriptional regulators
Transcriptional regulators
Transcriptional regulators
Transcriptional regulators
Two-component regulatory systems
Protein secretion/export apparatus
Protein secretion/export apparatus
Protein secretion/export apparatus
Protein secretion/export apparatus
Protein secretion/export apparatus
Protein secretion/export apparatus
Protein secretion/export apparatus
Protein secretion/export apparatus
Transcriptional regulators
Protein secretion/export apparatus
Protein secretion/export apparatus
Adaptation, Protection
Putative enzymes
Transcriptional regulators
Transcriptional regulators
Translation, post-translational modification, degradation
Amino acid biosynthesis and metabolism
Two-component regulatory systems
Transcriptional regulators
Transcription, RNA processing and degradation
Hypothetical, unclassified, unknown
Transcriptional regulators
Transcriptional regulators
Transcriptional regulators
Two-component regulatory systems




\section{Biofilm}

$\mathrm{PA} 002$

hypothetical protein

PA0038 hypothetical protein

PA0040 conserved hypothetical protein

PA0046 hypothetical protein

PA0047 hypothetical protein

PA0061 hypothetical protein

PA0062 hypothetical protein

PA0074 serine/threonine protein kinase PpkA

PA0075 probable phosphoprotein phosphatase

PA0076 hypothetical protein

PA0083 conserved hypothetical protein

PA0086 hypothetical protein

PA0087 hypothetical protein

PA0088 hypothetical protein

PA0089 hypothetical protein

PA0090 probable ClpA/B-type chaperone
PA0091 conserved hypothetical protein

PA0092 hypothetical protein

PA0094 hypothetical protein

PA0102 probable carbonic anhydrase

PA0126 hypothetical protein

PA0134 probable guanine deaminase

PA0170 hypothetical protein

PA0224 probable aldolase

PA0237 probable oxidoreductase

PA0238 hypothetical protein

PA0252 hypothetical protein

PA0276 hypothetical protein

PA0320 conserved hypothetical protein

PA0327 hypothetical protein

PA0346 hypothetical protein

PA0347 glycerophosphoryl diester phosphodiesterase, periplasmic

PA0355 protease PfpI

$\mathrm{PA} 0376$ sigma factor $\mathrm{RpoH}$

PA0409 twitching motility protein PilH

PA0410 twitching motility protein Pill

PA0411 twitching motility protein PilJ

PA0413 still frameshift probable component of chemotactic signal transduction system

PA0414 probable methylesterase

PA0430 5,10-methylenetetrahydrofolate reductase

PA0431 hypothetical protein

PA0432 S-adenosyl-L-homocysteine hydrolase

PA0446 conserved hypothetical protein

PA0451 conserved hypothetical protein

PA0452 probable stomatin-like protein

PA0462 hypothetical protein

PA0464 two-component sensor $\mathrm{CreC}$

PA0465 inner membrane protein $\mathrm{CreD}$

PA0466 hypothetical protein

PA0490 hypothetical protein

PA0515 probable transcriptional regulato

PA0516 heme dl biosynthesis protein NirF

PA0523 nitric-oxide reductase subunit $C$

PA0526 hypothetical protein

PA0529 conserved hypothetical protein

PA0530 probable class III pyridoxal phosphate-dependent aminotransferase

PA0531 probable glutamine amidotransferase

PA0543 hypothetical protein

PA0547 probable transcriptional regulator

PA0557 hypothetical protein

PA0585 hypothetical protein

PA0588 conserved hypothetical protein

PA0601 probable two-component response regulator
Hypothetical, unclassified, unknown Hypothetical, unclassified, unknown Hypothetical, unclassified, unknown

Hypothetical, unclassified, unknown Hypothetical, unclassified, unknown Hypothetical, unclassified, unknown Hypothetical, unclassified, unknown Hypothetical, unclassified, unknown Hypothetical, unclassified, unknown Adaptation, Protection

Putative enzymes

Hypothetical, unclassified, unknown Hypothetical, unclassified, unknown Hypothetical, unclassified, unknown

Hypothetical, unclassified, unknown Hypothetical, unclassified, unknown Hypothetical, unclassified, unknown

Translation, post-translational modification, degradation

Hypothetical, unclassified, unknown

Hypothetical, unclassified, unknown

Hypothetical, unclassified, unknown

Central intermediary metabolism

Hypothetical, unclassified, unknown

Nucleotide biosynthesis and metabolism

Hypothetical, unclassified, unknown

Putative enzymes

Putative enzymes

Hypothetical, unclassified, unknown

Hypothetical, unclassified, unknown

Membrane proteins

Hypothetical, unclassified, unknown

Hypothetical, unclassified, unknown

Hypothetical, unclassified, unknown

Fatty acid and phospholipid metabolism

Translation, post-translational modification, degradation

Transcriptional regulators

Two-component regulatory systems

Chemotaxis

Chemotaxis

Two-component regulatory systems

Chemotaxis

Amino acid biosynthesis and metabolism

Hypothetical, unclassified, unknown

Amino acid biosynthesis and metabolism

Hypothetical, unclassified, unknown

Membrane proteins

Membrane proteins

Hypothetical, unclassified, unknown

Two-component regulatory systems

Membrane proteins

Hypothetical, unclassified, unknown

Hypothetical, unclassified, unknown

Biosynthesis of cofactors, prosthetic groups and carriers

Energy metabolism

Energy metabolism

Hypothetical, unclassified, unknown

Hypothetical, unclassified, unknown

Putative enzymes

Putative enzymes

Hypothetical, unclassified, unknown

Transcriptional regulators

Hypothetical, unclassified, unknown

Hypothetical, unclassified, unknown

Hypothetical, unclassified, unknown

Transcriptional regulators 


\begin{tabular}{|c|c|c|}
\hline ORF & Product name & Function class \\
\hline PA0603 & probable ATP-binding component of $\mathrm{ABC}$ transporter & Transport of small molecules \\
\hline PA0674 & hypothetical protein & Hypothetical, unclassified, unknown \\
\hline PA0675 & probable sigma-70 factor, ECF subfamily & Transcriptional regulators \\
\hline PA0676 & probable transmembrane sensor & Membrane proteins \\
\hline PA0693 & transport protein ExbB2 & Membrane proteins \\
\hline PA0696 & hypothetical protein & Hypothetical, unclassified, unknown \\
\hline PA0697 & hypothetical protein & Hypothetical, unclassified, unknown \\
\hline PA0698 & hypothetical protein & Hypothetical, unclassified, unknown \\
\hline PA0699 & probable peptidyl-prolyl cis-trans isomerase, PpiC-type & Translation, post-translational modification, degradation \\
\hline PA0707 & transcriptional regulator ToxR & Transcriptional regulators \\
\hline РА0732 & hypothetical protein & Hypothetical, unclassified, unknown \\
\hline PA0737 & hypothetical protein & Hypothetical, unclassified, unknown \\
\hline РА0738 & conserved hypothetical protein & Membrane proteins \\
\hline PA0752 & conserved hypothetical protein & Membrane proteins \\
\hline РА0753 & hypothetical protein & Membrane proteins \\
\hline PA0755 & cis-aconitate porin OpdH & Membrane proteins \\
\hline PA0765 & positive regulator for alginate biosynthesis MucC & Transcriptional regulators \\
\hline РА0788 & hypothetical protein & Hypothetical, unclassified, unknown \\
\hline PA0842 & probable glycosyl transferase & Putative enzymes \\
\hline PA0853 & probable oxidoreductase & Putative enzymes \\
\hline РА0877 & probable transcriptional regulator & Transcriptional regulators \\
\hline PA0878 & hypothetical protein & Hypothetical, unclassified, unknown \\
\hline PA0879 & probable acyl-CoA dehydrogenase & Putative enzymes \\
\hline PA0880 & probable ring-cleaving dioxygenase & Putative enzymes \\
\hline PA0882 & hypothetical protein & Putative enzymes \\
\hline PA0884 & probable C4-dicarboxylate-binding periplasmic protein & Transport of small molecules \\
\hline PA0886 & probable C4-dicarboxylate transporter & Transport of small molecules \\
\hline PA0921 & hypothetical protein & Membrane proteins \\
\hline PA0952 & hypothetical protein & Hypothetical, unclassified, unknown \\
\hline PA0985 & pyocin S5 & Membrane proteins \\
\hline PA1029 & hypothetical protein & Hypothetical, unclassified, unknown \\
\hline PA1065 & conserved hypothetical protein & Hypothetical, unclassified, unknown \\
\hline PA1066 & probable short-chain dehydrogenase & Putative enzymes \\
\hline PA1078 & flagellar basal-body rod protein $\mathrm{FlgC}$ & Cell wall / LPS / capsule \\
\hline PA1079 & flagellar basal-body rod modification protein FlgD & Cell wall / LPS / capsule \\
\hline PA1080 & flagellar hook protein $\mathrm{FlgE}$ & Cell wall / LPS / capsule \\
\hline PA1081 & flagellar basal-body rod protein $\mathrm{FlgF}$ & Cell wall / LPS / capsule \\
\hline PA1084 & flagellar P-ring protein precursor FlgI & Cell wall / LPS / capsule \\
\hline PA1111 & hypothetical protein & Hypothetical, unclassified, unknown \\
\hline PA1114 & hypothetical protein & Hypothetical, unclassified, unknown \\
\hline PA1115 & hypothetical protein & Membrane proteins \\
\hline PA1118 & hypothetical protein & Membrane proteins \\
\hline PA1155 & NrdB, tyrosyl radical-harboring component of class Ia ribonucleotide reductase & Nucleotide biosynthesis and metabolism \\
\hline PA1168 & hypothetical protein & Hypothetical, unclassified, unknown \\
\hline PA1178 & $\mathrm{PhoP} / \mathrm{Q}$ and low $\mathrm{Mg} 2+$ inducible outer membrane protein $\mathrm{H} 1$ precursor & Membrane proteins \\
\hline PA1195 & hypothetical protein & Hypothetical, unclassified, unknown \\
\hline PA1216 & hypothetical protein & Hypothetical, unclassified, unknown \\
\hline PA1217 & probable 2-isopropylmalate synthase & Amino acid biosynthesis and metabolism \\
\hline PA1247 & alkaline protease secretion protein AprE & Secreted Factors (toxins, enzymes, alginate) \\
\hline PA1285 & probable transcriptional regulator & Transcriptional regulators \\
\hline PA1333 & hypothetical protein & Hypothetical, unclassified, unknown \\
\hline PA1345 & hypothetical protein & Hypothetical, unclassified, unknown \\
\hline PA1404 & hypothetical protein & Hypothetical, unclassified, unknown \\
\hline PA1470 & probable short-chain dehydrogenase & Putative enzymes \\
\hline PA1471 & hypothetical protein & Hypothetical, unclassified, unknown \\
\hline PA1514 & conserved hypothetical protein & Hypothetical, unclassified, unknown \\
\hline PA1517 & conserved hypothetical protein & Hypothetical, unclassified, unknown \\
\hline PA1518 & conserved hypothetical protein & Hypothetical, unclassified, unknown \\
\hline PA1523 & xanthine dehydrogenase & Nucleotide biosynthesis and metabolism \\
\hline PA1524 & xanthine dehydrogenase & Nucleotide biosynthesis and metabolism \\
\hline PA1560 & hypothetical protein & Hypothetical, unclassified, unknown \\
\hline PA1600 & probable cytochrome c & Energy metabolism \\
\hline PA1601 & probable aldehyde dehydrogenase & Putative enzymes \\
\hline PA1602 & probable oxidoreductase & Carbon compound catabolism \\
\hline PA1603 & probable transcriptional regulator & Transcriptional regulators \\
\hline PA1606 & hypothetical protein & Hypothetical, unclassified, unknown \\
\hline PA1625 & conserved hypothetical protein & Membrane proteins \\
\hline PA1659 & hypothetical protein & Hypothetical, unclassified, unknown \\
\hline
\end{tabular}




\begin{tabular}{|c|c|c|}
\hline ORF & Product name & Function class \\
\hline PA1730 & conserved hypothetical protein & Hypothetical, unclassified, unknown \\
\hline PA1780 & assimilatory nitrite reductase small subunit & Central intermediary metabolism \\
\hline PA1786 & conserved hypothetical protein & Hypothetical, unclassified, unknown \\
\hline PA1791 & hypothetical protein & Hypothetical, unclassified, unknown \\
\hline PA1847 & conserved hypothetical protein & Hypothetical, unclassified, unknown \\
\hline PA1870 & hypothetical protein & Hypothetical, unclassified, unknown \\
\hline PA1889 & hypothetical protein & Hypothetical, unclassified, unknown \\
\hline PA1931 & probable ferredoxin & Energy metabolism \\
\hline PA1932 & probable hydroxylase molybdopterin-containing subunit & Carbon compound catabolism \\
\hline PA1933 & probable hydroxylase lar ge subunit & Carbon compound catabolism \\
\hline PA2017 & hypothetical protein & Hypothetical, unclassified, unknown \\
\hline PA2021 & hypothetical protein & Hypothetical, unclassified, unknown \\
\hline PA2022 & probable nucleotide sugar dehydrogenase & Putative enzymes \\
\hline PA2024 & probable ring-cleaving dioxygenase & Putative enzymes \\
\hline PA2042 & probable transporter (membrane subunit) & Transport of small molecules \\
\hline PA2071 & elongation factor $\mathrm{G}$ & Translation, post-translational modification, degradation \\
\hline PA2076 & probable transcriptional regulator & Transcriptional regulators \\
\hline PA2107 & hypothetical protein & Hypothetical, unclassified, unknown \\
\hline PA2136 & hypothetical protein & Hypothetical, unclassified, unknown \\
\hline PA2140 & probable metallothionein & Central intermediary metabolism \\
\hline PA2149 & hypothetical protein & Hypothetical, unclassified, unknown \\
\hline PA2155 & probable phospholipase & Putative enzymes \\
\hline PA2167 & hypothetical protein & Hypothetical, unclassified, unknown \\
\hline PA2168 & hypothetical protein & Hypothetical, unclassified, unknown \\
\hline PA2170 & hypothetical protein & Hypothetical, unclassified, unknown \\
\hline PA2174 & hypothetical protein & Hypothetical, unclassified, unknown \\
\hline PA2175 & hypothetical protein & Hypothetical, unclassified, unknown \\
\hline PA2176 & hypothetical protein & Hypothetical, unclassified, unknown \\
\hline PA2178 & hypothetical protein & Hypothetical, unclassified, unknown \\
\hline PA2179 & hypothetical protein & Hypothetical, unclassified, unknown \\
\hline PA2181 & hypothetical protein & Hypothetical, unclassified, unknown \\
\hline PA2186 & hypothetical protein & Hypothetical, unclassified, unknown \\
\hline PA2188 & probable alcohol dehydrogenase (Zn-dependent) & Putative enzymes \\
\hline PA2243 & hypothetical protein & Hypothetical, unclassified, unknown \\
\hline PA2365 & conserved hypothetical protein & Hypothetical, unclassified, unknown \\
\hline PA2366 & conserved hypothetical protein & Hypothetical, unclassified, unknown \\
\hline PA2367 & hypothetical protein & Hypothetical, unclassified, unknown \\
\hline PA2369 & hypothetical protein & Membrane proteins \\
\hline PA2441 & hypothetical protein & Hypothetical, unclassified, unknown \\
\hline PA2444 & serine hydroxymethyltransferase & Amino acid biosynthesis and metabolism \\
\hline PA2445 & glycine cleavage system protein $\mathrm{P} 2$ & Central intermediary metabolism \\
\hline PA2446 & glycine cleavage system protein $\mathrm{H} 2$ & Amino acid biosynthesis and metabolism \\
\hline PA2459 & hypothetical protein & Hypothetical, unclassified, unknown \\
\hline PA2462 & hypothetical protein & Hypothetical, unclassified, unknown \\
\hline PA2485 & hypothetical protein & Hypothetical, unclassified, unknown \\
\hline PA2486 & hypothetical protein & Hypothetical, unclassified, unknown \\
\hline PA2491 & probable oxidoreductase & Putative enzymes \\
\hline PA2493 & RND multidrug efflux membrane fusion protein MexE precursor & Transport of small molecules \\
\hline PA2494 & RND multidrug efflux transporter MexF & Transport of small molecules \\
\hline PA2495 & Multidrug efflux outer membrane protein OprN precursor & Membrane proteins \\
\hline PA2533 & probable sodium:alanine symporter & Membrane proteins \\
\hline PA2536 & probable phosphatidate cytidylyltransferase & Fatty acid and phospholipid metabolism \\
\hline PA2562 & hypothetical protein & Hypothetical, unclassified, unknown \\
\hline PA2569 & hypothetical protein & Hypothetical, unclassified, unknown \\
\hline PA2618 & hypothetical protein & Translation, post-translational modification, degradation \\
\hline PA2635 & hypothetical protein & Hypothetical, unclassified, unknown \\
\hline PA2658 & hypothetical protein & Hypothetical, unclassified, unknown \\
\hline PA2659 & hypothetical protein & Hypothetical, unclassified, unknown \\
\hline PA2708 & hypothetical protein & Hypothetical, unclassified, unknown \\
\hline PA2718 & probable transcriptional regulator & Transcriptional regulators \\
\hline PA2761 & hypothetical protein & Membrane proteins \\
\hline PA2777 & conserved hypothetical protein & Membrane proteins \\
\hline PA2782 & hypothetical protein & Hypothetical, unclassified, unknown \\
\hline PA2799 & hypothetical protein & Hypothetical, unclassified, unknown \\
\hline PA2803 & hypothetical protein & Hypothetical, unclassified, unknown \\
\hline PA2804 & hypothetical protein & Hypothetical, unclassified, unknown \\
\hline PA2815 & probable acyl-CoA dehydrogenase & Putative enzymes \\
\hline PA2816 & hypothetical protein & Hypothetical, unclassified, unknown \\
\hline
\end{tabular}




\begin{tabular}{|c|c|c|}
\hline ORF & Product name & Function class \\
\hline PA2827 & conserved hypothetical protein & Hypothetical, unclassified, unknown \\
\hline PA2880 & hypothetical protein & Hypothetical, unclassified, unknown \\
\hline PA2881 & probable two-component response regulator & Transcriptional regulators \\
\hline PA2882 & probable two-component sensor & Two-component regulatory systems \\
\hline PA2883 & hypothetical protein & Hypothetical, unclassified, unknown \\
\hline PA2895 & hypothetical protein & Hypothetical, unclassified, unknown \\
\hline PA2931 & probable transcriptional regulator & Transcriptional regulators \\
\hline PA2939 & probable aminopeptidase & Secreted Factors (toxins, enzymes, alginate) \\
\hline PA2969 & fatty acid biosynthesis protein PlsX & Fatty acid and phospholipid metabolism \\
\hline PA2970 & $50 \mathrm{~S}$ ribosomal protein $\mathrm{L} 32$ & Translation, post-translational modification, degradation \\
\hline PA3023 & conserved hypothetical protein & Hypothetical, unclassified, unknown \\
\hline PA3031 & hypothetical protein & Hypothetical, unclassified, unknown \\
\hline PA3079 & hypothetical protein & Membrane proteins \\
\hline PA3082 & glycine betaine transmethylase & Amino acid biosynthesis and metabolism \\
\hline PA3105 & general secretion pathway protein $\mathrm{D}$ & Protein secretion/export apparatus \\
\hline PA3121 & 3-isopropylmalate dehydratase lar ge subunit & Amino acid biosynthesis and metabolism \\
\hline PA3205 & hypothetical protein & Hypothetical, unclassified, unknown \\
\hline PA3218 & hypothetical protein & Hypothetical, unclassified, unknown \\
\hline PA3219 & hypothetical protein & Hypothetical, unclassified, unknown \\
\hline PA3221 & CsaA protein & Protein secretion/export apparatus \\
\hline PA3229 & hypothetical protein & Hypothetical, unclassified, unknown \\
\hline PA3249 & probable transcriptional regulator & Transcriptional regulators \\
\hline PA3273 & hypothetical protein & Hypothetical, unclassified, unknown \\
\hline PA3274 & hypothetical protein & Hypothetical, unclassified, unknown \\
\hline PA3280 & Pyrophosphate-specific outer membrane porin $\mathrm{OprO}$ precursor & Transport of small molecules \\
\hline PA3296 & alkaline phosphatase & Central intermediary metabolism \\
\hline PA3309 & conserved hypothetical protein & Hypothetical, unclassified, unknown \\
\hline PA3319 & non-hemolytic phospholipase $\mathrm{C}$ precursor & Secreted Factors (toxins, enzymes, alginate) \\
\hline PA3326 & probable Clp-family ATP-dependent protease & Translation, post-translational modification, degradation \\
\hline PA3327 & probable non-ribosomal peptide synthetase & Adaptation, Protection \\
\hline PA3329 & hypothetical protein & Hypothetical, unclassified, unknown \\
\hline PA3330 & probable short chain dehydrogenase & Putative enzymes \\
\hline PA3331 & cytochrome P450 & Adaptation, Protection \\
\hline PA3332 & conserved hypothetical protein & Hypothetical, unclassified, unknown \\
\hline PA3333 & 3-oxoacyl-[acyl-carrier-protein] synthase III & Fatty acid and phospholipid metabolism \\
\hline PA3334 & probable acyl carrier protein & Fatty acid and phospholipid metabolism \\
\hline PA3335 & hypothetical protein & Hypothetical, unclassified, unknown \\
\hline PA3336 & probable major facilitator superfamily (MFS) transporter & Membrane proteins \\
\hline PA3337 & ADP-L-glycero-D-mannoheptose 6-epimerase & Cell wall / LPS / capsule \\
\hline PA3368 & probable acetyltransferase & Putative enzymes \\
\hline PA3376 & probable ATP-binding component of $\mathrm{ABC}$ transporter & Transport of small molecules \\
\hline PA3377 & conserved hypothetical protein & Transport of small molecules \\
\hline PA3380 & conserved hypothetical protein & Transport of small molecules \\
\hline PA3381 & probable transcriptional regulator & Transcriptional regulators \\
\hline PA3382 & phosphonate transport protein PhnE & Membrane proteins \\
\hline PA3383 & binding protein component of $\mathrm{ABC}$ phosphonate transporter & Transport of small molecules \\
\hline PA3384 & ATP-binding component of $\mathrm{ABC}$ phosphonate transporter & Transport of small molecules \\
\hline PA3389 & probable ring-cleaving dioxygenase & Putative enzymes \\
\hline PA3397 & ferredoxin--NADP+ reductase & Biosynthesis of cofactors, prosthetic groups and carriers \\
\hline PA3431 & conserved hypothetical protein & Membrane proteins \\
\hline PA3432 & hypothetical protein & Membrane proteins \\
\hline PA3436 & hypothetical protein & Hypothetical, unclassified, unknown \\
\hline PA3476 & autoinducer synthesis protein RhII & Adaptation, Protection \\
\hline PA3478 & rhamnosyltransferase chain B & Secreted Factors (toxins, enzymes, alginate) \\
\hline PA3484 & hypothetical protein & Hypothetical, unclassified, unknown \\
\hline PA3540 & GDP-mannose 6-dehydrogenase AlgD & Cell wall / LPS / capsule \\
\hline PA3541 & alginate biosynthesis protein Alg8 & Cell wall / LPS / capsule \\
\hline PA3542 & alginate biosynthesis protein Alg44 & Cell wall / LPS / capsule \\
\hline PA3543 & alginate biosynthetic protein AlgK precursor & Cell wall / LPS / capsule \\
\hline PA3544 & Alginate production outer membrane protein AlgE precursor & Membrane proteins \\
\hline PA3545 & alginate-c5-mannuronan-epimerase AlgG & Cell wall / LPS / capsule \\
\hline PA3546 & alginate biosynthesis protein $\mathrm{AlgX}$ & Cell wall / LPS / capsule \\
\hline PA3547 & poly(beta-d-mannuronate) lyase precursor AlgL & Cell wall / LPS / capsule \\
\hline PA3548 & alginate 0 -acetyltransferase AlgI & Cell wall / LPS / capsule \\
\hline PA3549 & alginate 0 -acetyltransferase $\mathrm{AlgJ}$ & Cell wall / LPS / capsule \\
\hline PA3550 & alginate 0 -acetyltransferase AlgF & Cell wall / LPS / capsule \\
\hline PA3551 & phosphomannose isomerase / guanosine 5'-diphospho-D-mannose pyrophosphorylase & Cell wall / LPS / capsule \\
\hline PA3553 & ArnC & Adaptation, Protection \\
\hline
\end{tabular}




\begin{tabular}{|c|c|c|}
\hline ORF & Product name & Function class \\
\hline PA3554 & ArnA & Putative enzymes \\
\hline PA3555 & ArnD & Adaptation, Protection \\
\hline PA3556 & inner membrane L-Ara4N transferase ArnT & Adaptation, Protection \\
\hline PA3557 & ArnE & Adaptation, Protection \\
\hline PA3559 & probable nucleotide sugar dehydrogenase & Putative enzymes \\
\hline PA3569 & 3-hydroxyisobutyrate dehydrogenase & Carbon compound catabolism \\
\hline PA3577 & hypothetical protein & Hypothetical, unclassified, unknown \\
\hline PA3578 & conserved hypothetical protein & Hypothetical, unclassified, unknown \\
\hline PA3598 & conserved hypothetical protein & Hypothetical, unclassified, unknown \\
\hline PA3651 & phosphatidate cytidylyltransferase & Fatty acid and phospholipid metabolism \\
\hline PA3655 & elongation factor $\mathrm{Ts}$ & Translation, post-translational modification, degradation \\
\hline PA3688 & hypothetical protein & Hypothetical, unclassified, unknown \\
\hline PA3692 & probable outer membrane protein precursor & Membrane proteins \\
\hline PA3724 & elastase LasB & Secreted Factors (toxins, enzymes, alginate) \\
\hline PA3743 & tRNA (guanine-N1)-methyltransferase & Transcription, RNA processing and degradation \\
\hline PA3744 & 16S rRNA processing protein & Transcription, RNA processing and degradation \\
\hline PA3819 & conserved hypothetical protein & Membrane proteins \\
\hline PA3820 & secretion protein $\mathrm{SecF}$ & Protein secretion/export apparatus \\
\hline PA3821 & secretion protein $\mathrm{SecD}$ & Membrane proteins \\
\hline PA3840 & conserved hypothetical protein & Hypothetical, unclassified, unknown \\
\hline PA3890 & probable permease of $A B C$ transporter & Membrane proteins \\
\hline PA3905 & hypothetical protein & Hypothetical, unclassified, unknown \\
\hline PA3906 & hypothetical protein & Hypothetical, unclassified, unknown \\
\hline PA3907 & hypothetical protein & Hypothetical, unclassified, unknown \\
\hline PA3908 & hypothetical protein & Hypothetical, unclassified, unknown \\
\hline PA3909 & hypothetical protein & Hypothetical, unclassified, unknown \\
\hline PA3910 & hypothetical protein & Hypothetical, unclassified, unknown \\
\hline PA3929 & cyanide insensitive terminal oxidase & Energy metabolism \\
\hline PA3944 & conserved hypothetical protein & Hypothetical, unclassified, unknown \\
\hline PA3952 & hypothetical protein & Hypothetical, unclassified, unknown \\
\hline PA3957 & probable short-chain dehydrogenase & Putative enzymes \\
\hline PA3964 & hypothetical protein & Hypothetical, unclassified, unknown \\
\hline PA4017 & conserved hypothetical protein & Hypothetical, unclassified, unknown \\
\hline PA4040 & hypothetical protein & Hypothetical, unclassified, unknown \\
\hline PA4063 & hypothetical protein & Hypothetical, unclassified, unknown \\
\hline PA4110 & beta-lactamase precursor & Adaptation, Protection \\
\hline PA4111 & hypothetical protein & Hypothetical, unclassified, unknown \\
\hline PA4112 & probable sensor/response regulator hybrid & Two-component regulatory systems \\
\hline PA4154 & conserved hypothetical protein & Hypothetical, unclassified, unknown \\
\hline PA4155 & hypothetical protein & Hypothetical, unclassified, unknown \\
\hline PA4190 & probable FAD-dependent monooxygenase & Putative enzymes \\
\hline PA4238 & DNA-directed RNA polymerase alpha chain & Transcription, RNA processing and degradation \\
\hline PA4243 & secretion protein $\mathrm{SecY}$ & Membrane proteins \\
\hline PA4244 & 50 S ribosomal protein $\mathrm{L} 15$ & Translation, post-translational modification, degradation \\
\hline PA4246 & 30 S ribosomal protein S5 & Translation, post-translational modification, degradation \\
\hline PA4247 & 50S ribosomal protein $\mathrm{L} 18$ & Translation, post-translational modification, degradation \\
\hline PA4248 & 50 S ribosomal protein L6 & Translation, post-translational modification, degradation \\
\hline PA4255 & 50 S ribosomal protein $\mathrm{L} 29$ & Translation, post-translational modification, degradation \\
\hline PA4261 & 50 S ribosomal protein $\mathrm{L} 23$ & Translation, post-translational modification, degradation \\
\hline PA4262 & 50 S ribosomal protein L4 & Transcription, RNA processing and degradation \\
\hline PA4272 & $50 \mathrm{~S}$ ribosomal protein $\mathrm{L} 10$ & Translation, post-translational modification, degradation \\
\hline PA4293 & two-component sensor PprA & Two-component regulatory systems \\
\hline PA4317 & hypothetical protein & Membrane proteins \\
\hline PA4344 & probable hydrolase & Putative enzymes \\
\hline PA4345 & hypothetical protein & Hypothetical, unclassified, unknown \\
\hline PA4348 & conserved hypothetical protein & Hypothetical, unclassified, unknown \\
\hline PA4350 & conserved hypothetical protein & Hypothetical, unclassified, unknown \\
\hline PA4351 & probable acyltransferase & Fatty acid and phospholipid metabolism \\
\hline PA4354 & conserved hypothetical protein & Hypothetical, unclassified, unknown \\
\hline PA4355 & probable major facilitator superfamily (MFS) transporter & Membrane proteins \\
\hline PA4356 & xenobiotic reductase & Adaptation, Protection \\
\hline PA4357 & conserved hypothetical protein & Hypothetical, unclassified, unknown \\
\hline PA4371 & hypothetical protein & Hypothetical, unclassified, unknown \\
\hline PA4407 & cell division protein FtsZ & Cell division \\
\hline PA4408 & cell division protein FtsA & Cell division \\
\hline PA4409 & cell division protein $\mathrm{Fts} Q$ & Cell division \\
\hline PA4410 & D-alanine--D-alanine ligase & Cell wall / LPS / capsule \\
\hline PA4411 & UDP-N-acetylmuramate--alanine ligase & Cell wall / LPS / capsule \\
\hline
\end{tabular}




\begin{tabular}{|c|c|c|}
\hline ORF & Product name & Function class \\
\hline PA4412 & \multicolumn{2}{|c|}{ UDP-N-acetylglucosamine--N-acetylmuramyl undecaprenol N-acetylglucosamine trans Carbon compound catabolism } \\
\hline PA4413 & cell division protein $\mathrm{Fts} \mathrm{W}$ & Cell division \\
\hline PA4414 & UDP-N-acetylmuramoylalanine--D-glutamate ligase & Cell wall / LPS / capsule \\
\hline PA4415 & phospho-N-acetylmuramoyl-pentapeptide-transferase & Cell wall / LPS / capsule \\
\hline PA4416 & \multicolumn{2}{|c|}{ UDP-N-acetylmuramoylalanyl-D-glutamyl-2, 6-diaminopimelate--D-alanyl-D-alanyl lį̧Cell wall / LPS / capsule } \\
\hline PA4419 & cell division protein FtsL & Cell division \\
\hline PA4421 & conserved hypothetical protein & Hypothetical, unclassified, unknown \\
\hline PA4498 & probable metallopeptidase & Translation, post-translational modification, degradation \\
\hline PA4500 & probable binding protein component of $\mathrm{ABC}$ transporter & Transport of small molecules \\
\hline PA4502 & probable binding protein component of $\mathrm{ABC}$ transporter & Transport of small molecules \\
\hline PA4503 & probable permease of $\mathrm{ABC}$ transporter & Membrane proteins \\
\hline PA4504 & probable permease of $\mathrm{ABC}$ transporter & Membrane proteins \\
\hline PA4505 & probable ATP-binding component of $\mathrm{ABC}$ transporter & Transport of small molecules \\
\hline PA4506 & probable ATP-binding component of $\mathrm{ABC}$ dipeptide transporter & Transport of small molecules \\
\hline PA4507 & hypothetical protein & Membrane proteins \\
\hline PA4525 & type 4 fimbrial precursor PilA & Motility \& Attachment \\
\hline PA4526 & type 4 fimbrial biogenesis protein PilB & Motility \& Attachment \\
\hline PA4569 & octaprenyl-diphosphate synthase & Energy metabolism \\
\hline PA4575 & hypothetical protein & Hypothetical, unclassified, unknown \\
\hline PA4588 & glutamate dehydrogenase & Amino acid biosynthesis and metabolism \\
\hline PA4589 & probable outer membrane protein precursor & Membrane proteins \\
\hline PA4621 & probable oxidoreductase & Putative enzymes \\
\hline PA4623 & hypothetical protein & Hypothetical, unclassified, unknown \\
\hline PA4632 & hypothetical protein & Hypothetical, unclassified, unknown \\
\hline PA4657 & hypothetical protein & Hypothetical, unclassified, unknown \\
\hline PA4658 & hypothetical protein & Hypothetical, unclassified, unknown \\
\hline PA4671 & probable ribosomal protein $\mathrm{L} 25$ & Adaptation, Protection \\
\hline PA4674 & conserved hypothetical protein & Hypothetical, unclassified, unknown \\
\hline PA4691 & hypothetical protein & Hypothetical, unclassified, unknown \\
\hline PA4697 & hypothetical protein & Hypothetical, unclassified, unknown \\
\hline PA4713 & hypothetical protein & Hypothetical, unclassified, unknown \\
\hline PA4742 & tRNA pseudouridine 55 synthase & Translation, post-translational modification, degradation \\
\hline PA4743 & ribosome-binding factor $\mathrm{A}$ & Translation, post-translational modification, degradation \\
\hline PA4744 & translation initiation factor IF-2 & Translation, post-translational modification, degradation \\
\hline PA4773 & hypothetical protein & Hypothetical, unclassified, unknown \\
\hline PA4781 & probable two-component response regulator & Transcriptional regulators \\
\hline PA4782 & hypothetical protein & Hypothetical, unclassified, unknown \\
\hline PA4785 & probable acyl-CoA thiolase & Putative enzymes \\
\hline PA4786 & probable short-chain dehydrogenase & Putative enzymes \\
\hline PA4811 & nitrate-inducible formate dehydrogenase, beta subunit & Energy metabolism \\
\hline PA4813 & lipase LipC & Fatty acid and phospholipid metabolism \\
\hline PA4844 & probable chemotaxis transducer & Adaptation, Protection \\
\hline PA4858 & conserved hypothetical protein & Hypothetical, unclassified, unknown \\
\hline PA4862 & probable ATP-binding component of $\mathrm{ABC}$ transporter & Transport of small molecules \\
\hline PA4870 & conserved hypothetical protein & Hypothetical, unclassified, unknown \\
\hline PA4877 & hypothetical protein & Hypothetical, unclassified, unknown \\
\hline PA4879 & conserved hypothetical protein & Hypothetical, unclassified, unknown \\
\hline PA4881 & hypothetical protein & Hypothetical, unclassified, unknown \\
\hline PA4896 & probable sigma- 70 factor, ECF subfamily & Transcriptional regulators \\
\hline PA4933 & hypothetical protein & Membrane proteins \\
\hline PA5033 & hypothetical protein & Hypothetical, unclassified, unknown \\
\hline PA5040 & Type 4 fimbrial biogenesis outer membrane protein PilQ precursor & Motility \& Attachment \\
\hline PA5041 & type 4 fimbrial biogenesis protein PilP & Motility \& Attachment \\
\hline PA5042 & type 4 fimbrial biogenesis protein PilO & Motility \& Attachment \\
\hline PA5043 & type 4 fimbrial biogenesis protein PilN & Motility \& Attachment \\
\hline PA5044 & type 4 fimbrial biogenesis protein PilM & Motility \& Attachment \\
\hline PA5075 & probable permease of $\mathrm{ABC}$ transporter & Membrane proteins \\
\hline PA5097 & probable amino acid permease & Membrane proteins \\
\hline PA5099 & probable transporter & Membrane proteins \\
\hline PA5116 & probable transcriptional regulator & Transcriptional regulators \\
\hline PA5124 & two-component sensor NtrB & Two-component regulatory systems \\
\hline PA5125 & two-component response regulator $\mathrm{NtrC}$ & Transcriptional regulators \\
\hline PA5128 & secretion protein $\mathrm{SecB}$ & Protein secretion/export apparatus \\
\hline PA5129 & glutaredoxin & Energy metabolism \\
\hline PA5139 & hypothetical protein & Hypothetical, unclassified, unknown \\
\hline PA5169 & probable C4-dicarboxylate transporter & Membrane proteins \\
\hline PA5170 & arginine/ornithine antiporter & Membrane proteins \\
\hline PA5180 & conserved hypothetical protein & Hypothetical, unclassified, unknown \\
\hline
\end{tabular}




\begin{tabular}{|c|c|c|}
\hline ORF & Product name & Function class \\
\hline PA5182 & hypothetical protein & Membrane proteins \\
\hline PA5183 & hypothetical protein & Membrane proteins \\
\hline PA5192 & phosphoenolpyruvate carboxykinase & Carbon compound catabolism \\
\hline PA5202 & hypothetical protein & Hypothetical, unclassified, unknown \\
\hline PA5209 & hypothetical protein & Hypothetical, unclassified, unknown \\
\hline PA5212 & hypothetical protein & Hypothetical, unclassified, unknown \\
\hline PA5220 & hypothetical protein & Hypothetical, unclassified, unknown \\
\hline PA5232 & conserved hypothetical protein & Hypothetical, unclassified, unknown \\
\hline PA5262 & alginate biosynthesis protein $\mathrm{AlgZ/FimS}$ & Secreted Factors (toxins, enzymes, alginate) \\
\hline PA5275 & conserved hypothetical protein & Hypothetical, unclassified, unknown \\
\hline PA5287 & ammonium transporter $\mathrm{AmtB}$ & Membrane proteins \\
\hline PA5299 & hypothetical protein & Hypothetical, unclassified, unknown \\
\hline PA5322 & phosphomannomutase $\mathrm{Alg} \mathrm{C}$ & Cell wall / LPS / capsule \\
\hline PA5360 & two-component response regulator $\mathrm{PhoB}$ & Transcriptional regulators \\
\hline PA5361 & two-component sensor PhoR & Two-component regulatory systems \\
\hline PA5366 & ATP-binding component of $\mathrm{ABC}$ phosphate transporter & Membrane proteins \\
\hline PA5367 & membrane protein component of $\mathrm{ABC}$ phosphate transporter & Membrane proteins \\
\hline PA5368 & membrane protein component of $\mathrm{ABC}$ phosphate transporter & Membrane proteins \\
\hline PA5415 & serine hydroxymethyltransferase & Amino acid biosynthesis and metabolism \\
\hline PA5422 & hypothetical protein & Hypothetical, unclassified, unknown \\
\hline PA5441 & hypothetical protein & Hypothetical, unclassified, unknown \\
\hline PA5460 & hypothetical protein & Hypothetical, unclassified, unknown \\
\hline PA5473 & conserved hypothetical protein & Membrane proteins \\
\hline PA5475 & hypothetical protein & Hypothetical, unclassified, unknown \\
\hline PA5476 & citrate transporter & Membrane proteins \\
\hline PA5498 & probable adhesin & Motility \& Attachment \\
\hline PA5499 & transcriptional regulator np20 & Transcriptional regulators \\
\hline PA5524 & probable short-chain dehydrogenase & Putative enzymes \\
\hline PA5525 & probable transcriptional regulator & Transcriptional regulators \\
\hline PA5526 & hypothetical protein & Hypothetical, unclassified, unknown \\
\hline PA5549 & glucosamine--fructose-6-phosphate aminotransferase & Central intermediary metabolism \\
\hline PA5555 & ATP synthase gamma chain & Energy metabolism \\
\hline PA5556 & ATP synthase alpha chain & Energy metabolism \\
\hline PA5557 & ATP synthase delta chain & Energy metabolism \\
\hline PA5558 & ATP synthase B chain & Energy metabolism \\
\hline PA5559 & atp synthase $\mathrm{C}$ chain & Energy metabolism \\
\hline PA5560 & ATP synthase A chain & Energy metabolism \\
\hline PA5568 & conserved hypothetical protein & Membrane proteins \\
\hline
\end{tabular}


Alginate

PA2867

probable chemotaxis transducer

PA3819 conserved hypothetical protein

PA5182 hypothetical protein

RsmA

PA0105 cytochrome c oxidase, subunit II

PA0108 cytochrome c oxidase, subunit III

PA0129 gamma-aminobutyrate permease

PA0173 probable methylesterase

PA0210 malonate decarboxylase delta subunit

PA0212 malonate decarboxylase gamma subunit

PA0235 4-hydroxybenzoate transporter PcaK

PA0396 twitching motility protein PilU

PA0652 transcriptional regulator Vfr

PA0673 hypothetical protein

PA0706 chloramphenicol acetyltransferase

PA0710 lactoylglutathione lyase

PA0723 coat protein B of bacteriophage Pfl)

PA0843 phospholipase accessory protein PlcR precursor

PA0844 hemolytic phospholipase $\mathrm{C}$ precursor

PA0905 RsmA, regulator of secondary metabolites

$\mathrm{PA} 0994$ usher $\mathrm{CupC} 3$

PA1001 anthranilate synthase component I

PA1092 flagellin type B

PA1148 exotoxin A precurso

PA1151 pyocin S2 immunity protein

PAl173 cytochrome c-type protein NapB precurso

PA1247 alkaline protease secretion protein AprE

PA1251 probable chemotaxis transducer

PA1338 gamma-glutamyltranspeptidase precurso

PA1393 adenosine 5'-phosphosulfate (APS) kinase

PA1409 acetylpolyamine aminohydrolase

PA1635 potassium-transporting ATPase, C chain

PA1695 translocation protein in type III secretion

PA1758 para-aminobenzoate synthase component 1

PA1770 phosphoenolpyruvate synthase

PA1781 assimilatory nitrite reductase lar ge subunit

PA1947 ribose transport protein RbsA

PA1949 ribose operon repressor RbsR

PA1983 cytochrome c550

PA2007 maleylacetoacetate isomerase

PA2009 homogentisate 1,2-dioxygenase

PA2064 copper resistance protein B precursor

PA2129 chaperone CupA2

PA2337 transcriptional regulator MtIR

PA2493 RND multidrug efflux membrane fusion protein MexE precursor

PA2494 RND multidrug efflux transporter MexF

PA2495 Multidrug efflux outer membrane protein OprN precursor

PA2509 muconate cycloisomerase I

PA2926 histidine transport protein HisP

PA3540 GDP-mannose 6-dehydrogenase AlgD

PA3543 alginate biosynthetic protein AlgK precursor

PA3544 Alginate production outer membrane protein AlgE precurso

PA3549 alginate 0-acetyltransferase Alg

PA3603 diacylglycerol kinase

PA4110 beta-lactamase precursor

PA4127 2-oxo-hept-3-ene-1,7-dioate hydratase

PA4138 tyrosyl-tRNA synthetase

PA4158 ferric enterobactin transport protein FepC

PA4205 hypothetical protein

PA4210 probable phenazine biosynthesis protein

PA4307 chemotactic transducer PctC

PA4310 chemotactic transducer PctB

PA4356 xenobiotic reductase
Adaptation, Protection

Membrane proteins

Membrane proteins

Energy metabolism

Energy metabolism

Transport of small molecules

Adaptation, Protection

Carbon compound catabolism

Carbon compound catabolism

Membrane proteins

Motility \& Attachment

Transcriptional regulators

Hypothetical, unclassified, unknown

Antibiotic resistance and susceptibility

Central intermediary metabolism

Related to phage, transposon, or plasmid

Secreted Factors (toxins, enzymes, alginate)

Secreted Factors (toxins, enzymes, alginate)

Translation, post-translational modification, degradation

Motility \& Attachment

Adaptation, Protection

Motility \& Attachmen

Secreted Factors (toxins, enzymes, alginate)

Adaptation, Protection

Energy metabolism

Secreted Factors (toxins, enzymes, alginate)

Adaptation, Protection

Amino acid biosynthesis and metabolism

Amino acid biosynthesis and metabolism

Carbon compound catabolism

Transport of small molecules

Protein secretion/export apparatus

Biosynthesis of cofactors, prosthetic groups and carriers

Energy metabolism

Central intermediary metabolism

Transport of small molecules

Carbon compound catabolism

Energy metabolism

Carbon compound catabolism

Carbon compound catabolism

Adaptation, Protection

Motility \& Attachment

Transcriptional regulators

Transport of small molecules

Transport of small molecules

Membrane proteins

Carbon compound catabolism

Amino acid biosynthesis and metabolism

Cell wall / LPS / capsule

Cell wall / LPS / capsule

Membrane proteins

Cell wall / LPS / capsule

Fatty acid and phospholipid metabolism

Adaptation, Protection

Carbon compound catabolism

Amino acid biosynthesis and metabolism

Transport of small molecules

Membrane proteins

Secreted Factors (toxins, enzymes, alginate)

Adaptation, Protection

Adaptation, Protection

Adaptation, Protection 


\begin{tabular}{lll}
\hline ORF & Product name & Function class \\
\hline & & \\
PA4522 & beta-lactamase expression regulator AmpD & Cell wall / LPS / capsule \\
PA4527 & still frameshift type 4 fimbrial biogenesis protein PilC & Motility \& Attachment \\
PA4855 & phosphoribosylamine--glycine ligase & Nucleotide biosynthesis and metabolism \\
PA4953 & chemotaxis protein MotB & Membrane proteins \\
PA4954 & chemotaxis protein MotA & Adaptation, Protection \\
PA5041 & type 4 fimbrial biogenesis protein PilP & Motility \& Attachment \\
PA5042 & type 4 fimbrial biogenesis protein PilO & Motility \& Attachment \\
PA5043 & type 4 fimbrial biogenesis protein PilN & Motility \& Attachment \\
PA5044 & type 4 fimbrial biogenesis protein PilM & Motility \& Attachment \\
PA5243 & delta-aminolevulinic acid dehydratase & Biosynthesis of cofactors, prosthetic groups and carriers \\
PA5355 & glycolate oxidase subunit GlcD & Central intermediary metabolism \\
PA5361 & two-component sensor PhoR & Two-component regulatory systems \\
PA5501 & permease of ABC zinc transporter ZnuB & Membrane proteins
\end{tabular}


Supplementary information for Chapter 3 
Supplementary Table 1: Family-wise list of transcription factors found in $\boldsymbol{P}$. aeruginosa PAO1. The table shows the genomic location of the protein (core/RGP) (Mathee et al. 2008) and the different PFAM domains (Winzor et al. 2011, http://pfam.sanger.ac.uk/ and http://expasy.org/prosite/). ORF (PA) numbers, protein name, homolog and amino acid information are from the Pseudomonas database (Winzor et al. 2011).

\begin{tabular}{|c|c|c|c|c|c|c|c|c|c|c|c|}
\hline PA \# & $\begin{array}{c}\text { Protein } \\
\text { name }\end{array}$ & $\begin{array}{l}\# \text { of } \\
\text { amino } \\
\text { acids }\end{array}$ & Subfamily & Nearest Homolog & $\begin{array}{l}\text { Core/ } \\
\text { RGP }\end{array}$ & $\begin{array}{c}\text { Domain } \\
1\end{array}$ & $\begin{array}{l}\text { Domain } 1 \\
\text { Details }\end{array}$ & $\begin{array}{l}\text { Domain } \\
2\end{array}$ & $\begin{array}{c}\text { Domain } 2 \\
\text { Details }\end{array}$ & $\begin{array}{c}\text { Other } \\
\text { Domains }\end{array}$ & $\begin{array}{c}\text { Domain } \\
\text { Details }\end{array}$ \\
\hline \multicolumn{12}{|l|}{ AraC } \\
\hline PA0306 & & & & & Core & & & & & & \\
\hline PA0163 & & 265 & & $\begin{array}{l}52 \% \text { similar to putative regulatory protein } \\
\text { YeaM [Escherichia coli] }\end{array}$ & Core & PF00165 & HTH_AraC & & & & \\
\hline PA0248 & PobR & 288 & & $\begin{array}{l}\text { 87\% similar to putative regulator PobR } \\
\text { [Pseudomonas aeruginosa] }\end{array}$ & Core & PF00165 & HTH_AraC & & & & \\
\hline PA0416 & ChpD & 264 & & $\begin{array}{l}40 \% \text { similar to regulatory protein mmsR which } \\
\text { is a member of the AraC/XylS family of } \\
\text { transcriptional regulators [Pseudomonas } \\
\text { aeruginosa] }\end{array}$ & Core & PF02311 & AraC_binding & PF00165 & HTH_AraC & & \\
\hline PA0564 & & 303 & & $\begin{array}{l}57 \% \text { similarity to hypothetical transcriptional } \\
\text { regulator HI1052 from [[Haemophilus } \\
\text { influenzae]] }\end{array}$ & Core & PF00165 & HTH_AraC & & & & \\
\hline PA0748 & & 306 & & $\begin{array}{l}55 \% \text { similar to regulatory protein } \mathrm{mmsR} \\
\text { [Pseudomonas aeruginosa] }\end{array}$ & Core & PF00165 & HTH_AraC & & & & \\
\hline PA0780 & PruR & 250 & & $\begin{array}{l}54 \% \text { similar to lumQ gene product } \\
\text { [Photobacterium leiognathi] }\end{array}$ & Core & PF00165 & HTH_AraC & & & & \\
\hline PA0791 & & 262 & & $\begin{array}{l}50 \% \text { similarity to hypothetical yeaM gene } \\
\text { product of [Escherichia coli], a putative } \\
\text { regulatory protein. }\end{array}$ & Core & PF00165 & HTH_AraC & & & & \\
\hline PA0831 & OruR & 339 & & $\begin{array}{l}100 \% \text { identical to OruR, transcriptional } \\
\text { regulator required for growth on ornithine as } \\
\text { the sole carbon source [Pseudomonas } \\
\text { aeruginosa] }\end{array}$ & Core & PF00165 & HTH_AraC & & & & \\
\hline PA0864 & & 262 & & $\begin{array}{l}50 \% \text { similar to putative regulatory protein } \\
\text { YeaM [Escherichia coli] }\end{array}$ & Core & PF00165 & HTH_AraC & & & & \\
\hline PA0893 & $\operatorname{ArgR}$ & 329 & & 0 & Core & PF00165 & HTH_AraC & & & & \\
\hline PA1109 & & 259 & & $\begin{array}{l}52 \% \text { similar to putative regulatory protein } \\
\text { YeaM [Escherichia coli] }\end{array}$ & Core & PF00165 & HTH_AraC & & & & \\
\hline
\end{tabular}




\begin{tabular}{|c|c|c|c|c|c|c|}
\hline PA1182 & & 333 & $\begin{array}{l}47 \% \text { similar to transcriptional regulator OruR } \\
\text { [Pseudomonas aeruginosa] }\end{array}$ & Core & PF00165 & HTH_AraC \\
\hline PA1229 & YeaM & 268 & $\begin{array}{l}67 \% \text { similar to putative regulatory protein } \\
\text { YeaM [Escherichia coli] }\end{array}$ & Core & PF00165 & HTH_AraC \\
\hline PA1235 & & 260 & $\begin{array}{l}51 \% \text { similar to putative regulatory protein } \\
\text { YeaM [Escherichia coli] }\end{array}$ & Core & PF00165 & HTH_AraC \\
\hline PA1261 & & 224 & $\begin{array}{l}62 \% \text { similar to a region of probable } \\
\text { transcriptional regulator LumQ } \\
\text { [Photobacterium leiognathi] }\end{array}$ & Core & PF00165 & HTH_AraC \\
\hline PA1380 & & 321 & $\begin{array}{l}60 \% \text { similar to putative regulatory protein } \\
\text { YqhC [Escherichia coli] }\end{array}$ & $\begin{array}{l}\text { RGP } \\
15\end{array}$ & PF00165 & HTH_AraC \\
\hline PA1599 & & 260 & $\begin{array}{l}56 \% \text { similar to putative regulatory protein } \\
\text { YeaM [Escherichia coli] }\end{array}$ & Core & PF00165 & HTH_AraC \\
\hline PA1619 & & 274 & $\begin{array}{l}45 \% \text { similar to putative transcriptional } \\
\text { regulator [Streptomyces coelicolor] }\end{array}$ & Core & PF00165 & HTH_AraC \\
\hline PA1713 & ExsA & 278 & $\begin{array}{l}71 \% \text { similar to thermoregulatory transcription } \\
\text { factor LcrF [Yersinia pestis] ; } 72 \% \text { similar to } \\
\text { transcriptional activator VirF [Yersinia } \\
\text { enterocolitica]; } 99 \% \text { similar to exoenzyme S } \\
\text { synthesis regulatory protein ExsA } \\
\text { [Pseudomonas aeruginosa] }\end{array}$ & Core & PF00165 & HTH_AraC \\
\hline PA1850 & & 334 & $\begin{array}{l}57 \% \text { similar to AraC-like protein } \\
\text { [Azorhizobium caulinodans] }\end{array}$ & Core & PF00165 & HTH_AraC \\
\hline PA2047 & & 329 & $\begin{array}{l}51 \% \text { similar to putative regulatory } \\
\text { proteinYqhC [Escherichia coli] }\end{array}$ & $\begin{array}{l}\text { RGP } \\
20\end{array}$ & PF00165 & HTH_AraC \\
\hline PA2096 & & 344 & $\begin{array}{l}51 \% \text { similar to hypothetical transcriptional } \\
\text { regulator CY21B4.12 [Mycobacterium } \\
\text { tuberculosis] }\end{array}$ & Core & PF00165 & HTH_AraC \\
\hline PA2227 & VqsM & 325 & $\begin{array}{l}47 \% \text { similar to regulator OruR [Pseudomonas } \\
\text { aeruginosa] }\end{array}$ & Core & PF00165 & HTH_AraC \\
\hline PA2276 & & 296 & $\begin{array}{l}62 \% \text { similar (over C-terminal } 3 / 4 \text { ths of ORF) } \\
\text { to yqhC gene product of [Escherichia coli], a } \\
\text { putative transcriptional regulator. }\end{array}$ & Core & PF00165 & HTH_AraC \\
\hline PA2281 & & 284 & $\begin{array}{l}64 \% \text { similar to the C-terminal end of } \\
\text { regulatory protein PchR Synechocystis sp. }\end{array}$ & Core & PF00165 & HTH_AraC \\
\hline PA2332 & & 301 & $\begin{array}{l}46 \% \text { similar to putative regulator HI } 1052 \\
\text { [Haemophilus influenzae } \mathrm{Rd} \text { ] }\end{array}$ & Core & PF00165 & HTH_AraC \\
\hline PA2337 & MtlR & 301 & $\begin{array}{l}84 \% \text { similar to putative activator protein } \mathrm{MtlR} \\
{[\text { Pseudomonas fluorescens }]}\end{array}$ & Core & PF00165 & HTH_AraC \\
\hline PA2488 & & 254 & $\begin{array}{l}47 \% \text { similar to putative regulatory protein } \\
\text { YeaM [Escherichia coli] }\end{array}$ & Core & PF00165 & HTH_AraC \\
\hline
\end{tabular}




\begin{tabular}{|c|c|c|c|c|c|c|c|c|c|c|}
\hline PA2489 & & 271 & $\begin{array}{l}46 \% \text { similar to putative transcriptional } \\
\text { regulator [Streptomyces coelicolor] }\end{array}$ & Core & PF00165 & HTH_AraC & & & & \\
\hline PA2511 & & 333 & $\begin{array}{l}50 \% \text { similar to putative regulatory protein } \\
\text { OxoS Pseudomonas putida }\end{array}$ & Core & PF00165 & HTH_AraC & & & & \\
\hline PA2519 & XylS & 318 & $\begin{array}{l}70 \% \text { similar to positive regulatory protein } \\
\text { XylS [TOL plasmid] }\end{array}$ & Core & PF00165 & HTH_AraC & & & & \\
\hline PA2556 & & 361 & $\begin{array}{l}42 \% \text { similar to regulator OruR [Pseudomonas } \\
\text { aeruginosa] }\end{array}$ & Core & PF00165 & HTH_AraC & & & & \\
\hline PA2588 & & 342 & $\begin{array}{l}48 \% \text { similar to regulator OruR [Pseudomonas } \\
\text { aeruginosa] }\end{array}$ & Core & PF00165 & HTH_AraC & & & & \\
\hline PA2696 & & 297 & $\begin{array}{l}52 \% \text { similar to regulatory protein } \mathrm{GdhBR} \\
{[\text { Pantoea citrea }]}\end{array}$ & Core & PF00165 & HTH_AraC & & & & \\
\hline PA2704 & & 339 & $\begin{array}{l}54 \% \text { similar to transcriptional regulator OruR } \\
\text { [Pseudomonas aeruginosa] }\end{array}$ & Core & PF00165 & HTH_AraC & & & & \\
\hline PA2917 & & 278 & $\begin{array}{l}47 \% \text { similar to putative transcriptional } \\
\text { regulator [Streptomyces coelicolor] }\end{array}$ & Core & PF00165 & HTH_AraC & & & & \\
\hline PA3027 & & 343 & $\begin{array}{l}49 \% \text { similar to transcriptional regulator OruR } \\
\text { [Pseudomonas aeruginosa] }\end{array}$ & Core & PF00165 & HTH_AraC & PF08281 & $\underset{2}{\operatorname{Sigma} 70 \mathrm{r} 4}$ & PF09312 & SurA_N \\
\hline PA3094 & & 345 & $\begin{array}{l}80 \% \text { similar to putative virulence regulator } \\
{[\text { Pseudomonas alcaligenes }]}\end{array}$ & Core & PF00165 & HTH_AraC & & & & \\
\hline PA3215 & & 337 & $\begin{array}{l}52 \% \text { similar to transcriptional regulator PhbR } \\
\text { [Pseudomonas sp. 61-3] }\end{array}$ & Core & PF00165 & HTH_AraC & & & & \\
\hline PA3220 & & 254 & $\begin{array}{l}48 \% \text { similar to probable transcriptional } \\
\text { regulator LumQ [Photobacterium leiognathi] }\end{array}$ & Core & PF00165 & HTH_AraC & PF07883 & Cupin_2 & & \\
\hline PA3269 & & 284 & $\begin{array}{l}49 \% \text { similar to putative transcriptional } \\
\text { regulator [Streptomyces coelicolor] }\end{array}$ & Core & PF00165 & HTH_AraC & PF02311 & $\begin{array}{l}\text { AraC_bindi } \\
\text { ng }\end{array}$ & & \\
\hline PA3423 & & 247 & $\begin{array}{l}52 \% \text { similar to a region of putative araC-type } \\
\text { regulatory protein [Escherichia coli] }\end{array}$ & Core & PF00165 & HTH_AraC & PF07366 & SnoaL & & \\
\hline PA3571 & MmsR & 307 & & Core & PF00165 & HTH_AraC & PF00165 & HTH_AraC & $\begin{array}{l}\text { PF07883; } \\
\text { PF03079 }\end{array}$ & $\begin{array}{l}\text { Cupin } 2 \text {; } \\
\text { ARD }\end{array}$ \\
\hline PA3782 & & 317 & $\begin{array}{l}49 \% \text { similar to a region of ArgR regulatory } \\
\text { protein [Pseudomonas aeruginosa] }\end{array}$ & Core & PF00165 & HTH_AraC & PF01965 & DJ-1_PfpI & & \\
\hline PA3830 & & 270 & $\begin{array}{l}51 \% \text { similar to putative regulatory protein } \\
\text { YeaM [Escherichia coli] }\end{array}$ & Core & PF00165 & HTH_AraC & PF07883 & Cupin_2 & PF02311 & $\begin{array}{l}\text { AraC_bin } \\
\text { ding }\end{array}$ \\
\hline PA3898 & & 293 & $\begin{array}{l}54 \% \text { similar to regulatory protein } \mathrm{GdhBR} \\
\text { [Pantoea citrea }]\end{array}$ & Core & PF00165 & HTH_AraC & & & & \\
\hline PA3927 & & 262 & $\begin{array}{l}53 \% \text { similar to putative regulatory protein } \\
\text { YeaM [Escherichia coli] }\end{array}$ & Core & PF00165 & HTH_AraC & PF02311 & $\underset{\text { ng }}{\text { AraC_bindi }}$ & PF07883 & Cupin_2 \\
\hline PA4070 & & 316 & $\begin{array}{l}45 \% \text { similar to the regulatory protein for } 2- \\
\text { phenylethylamine catabolism FeaR } \\
\text { [Escherichia coli] }\end{array}$ & Core & PF00165 & HTH_AraC & & & & \\
\hline PA4094 & & 319 & $42 \%$ similar to nitrilase regulator & Core & PF00165 & HTH_AraC & & & & \\
\hline
\end{tabular}




\begin{tabular}{|c|c|c|c|c|c|c|c|c|c|c|}
\hline & & & [[Rhodococcus rhodochrous]] & & & & & & & \\
\hline PA4120 & & 303 & $\begin{array}{l}63 \% \text { similar to the HpaA gene product of } \\
\text { [Escherichia coli] }\end{array}$ & Core & PF00165 & HTH_AraC & PF02311 & $\underset{\text { ng }}{\text { Arac bindi }}$ & PF07883 & Cupin_2 \\
\hline PA4184 & & 340 & $\begin{array}{l}48 \% \text { similar to ArgR regulatory protein } \\
\text { [Pseudomonas aeruginosa] }\end{array}$ & Core & PF00165 & HTH_AraC & PF01965 & DJ-1_PfpI & & \\
\hline PA4227 & $\operatorname{PchR}$ & 296 & $\begin{array}{l}100 \% \text { similar to regulatory protein PchR } \\
\text { [Pseudomonas aeruginosa] }\end{array}$ & Core & PF00165 & HTH_AraC & & & & \\
\hline PA4288 & & 267 & $\begin{array}{l}51 \% \text { similar to putative regulatory protein } \\
\text { YeaM [Escherichia coli] }\end{array}$ & Core & PF00165 & HTH_AraC & PF07883 & Cupin_2 & PF02311 & $\begin{array}{l}\text { AraC_bin } \\
\text { ding }\end{array}$ \\
\hline PA4436 & & 299 & $\begin{array}{l}45 \% \text { similar to ArgR regulatory protein } \\
\text { [Pseudomonas aeruginosa] }\end{array}$ & Core & PF00165 & HTH_AraC & & & & \\
\hline PA4787 & & 333 & $\begin{array}{l}42 \% \text { similar to regulator OruR [Pseudomonas } \\
\text { aeruginosa] }\end{array}$ & Core & PF00165 & HTH_AraC & & & & \\
\hline PA5032 & & 357 & $\begin{array}{l}53 \% \text { similar to regulator OruR [Pseudomonas } \\
\text { aeruginosa] }\end{array}$ & Core & PF00165 & HTH_AraC & & & & \\
\hline PA5324 & & 356 & $\begin{array}{l}46 \% \text { similar to hypothetical transcriptional } \\
\text { regulator [Mycobacterium tuberculosis] }\end{array}$ & Core & PF00165 & HTH_AraC & PF09312 & SurA_N & & \\
\hline PA5342 & & 266 & $\begin{array}{l}46 \% \text { similar to putative transcriptional } \\
\text { regulator [Streptomyces coelicolor] }\end{array}$ & Core & PF00165 & HTH_AraC & PF02311 & $\underset{\text { ng }}{\text { AraC_bindi }}$ & PF07883 & Cupin_2 \\
\hline PA5380 & GbdR & 367 & $\begin{array}{l}59 \% \text { similar to ArgR regulatory protein } \\
\text { [Pseudomonas aeruginosa] }\end{array}$ & Core & PF00165 & HTH_AraC & PF01965 & DJ-1_PfpI & & \\
\hline PA5389 & CdhR & 336 & $\begin{array}{l}58 \% \text { similar to ArgR regulatory protein } \\
\text { [Pseudomonas aeruginosa] }\end{array}$ & Core & PF00165 & HTH_AraC & PF01965 & DJ-1_PfpI & & \\
\hline \multicolumn{11}{|l|}{$\overline{A r s R}$} \\
\hline PA0279 & YdfF & 232 & $\begin{array}{l}\text { 59\% similar to hypothetical protein } \mathrm{YdfF} \\
\text { [[Bacillus subtilis]] }\end{array}$ & Core & PS00846 & $\begin{array}{l}\text { Bacterial } \\
\text { regulatory } \\
\text { proteins, arsR } \\
\text { family } \\
\text { signature }\end{array}$ & & & & \\
\hline PA0547 & & 333 & $\begin{array}{l}49 \% \text { similar to a region of unknown protein } \\
\text { [Streptomyces fradiae Transposon Tn } 4556 \text { ] }\end{array}$ & Core & PS00846 & $\begin{array}{l}\text { Bacterial } \\
\text { regulatory } \\
\text { proteins, arsR } \\
\text { family } \\
\text { signature }\end{array}$ & PF01022 & $\begin{array}{l}\text { HTH_5, } \\
\text { Bacterial } \\
\text { regulatory } \\
\text { protein, } \\
\text { arsR family. }\end{array}$ & & \\
\hline PA2277 & ArsR & 116 & $\begin{array}{l}82 \% \text { identical to arsR gene product of }[P \text {. } \\
\text { aeruginosa]; } 73 \% \text { similar (over } 3 / 4 \text { ths of ORF } \\
\text { length) to arsR gene product of }[\text { Escherichia } \\
\text { coli] }\end{array}$ & Core & & & & & & \\
\hline PA4354 & & 100 & $\begin{array}{l}76 \% \text { similar to a region of hypothetical protein } \\
\text { [Agrobacterium radiobacter] }\end{array}$ & Core & PS00846 & $\begin{array}{l}\text { Bacterial } \\
\text { regulatory } \\
\text { proteins, ArsR } \\
\text { family }\end{array}$ & & & & \\
\hline
\end{tabular}




\begin{tabular}{|c|c|c|c|c|c|c|c|}
\hline AsnC & & & & & & & \\
\hline PA0513 & NirG & 147 & $\begin{array}{l}77 \% \text { similar to NirG protein [Pseudomonas } \\
\text { stutzeri] }\end{array}$ & Core & PF01037 & AsnC family & \\
\hline PA2028 & & 157 & $\begin{array}{l}62 \% \text { similar to AzlB protein [[Bacillus } \\
\text { subtilis]] }\end{array}$ & RGP20 & PF01037 & AsnC family & \\
\hline PA2082 & & 158 & $\begin{array}{l}57 \% \text { similar to leucine-responsive regulatory } \\
\text { protein Lrp [Escherichia coli] }\end{array}$ & Core & PF01037 & AsnC family & \\
\hline PA2246 & BkdR & 153 & $64 \%$ similar to BkdR protein $[P$. putida $]$ & Core & PF01037 & AsnC family & \\
\hline PA2577 & & 145 & $\begin{array}{l}\text { 55\% similar to hypothetical protein Rv3291c } \\
\text { [Mycobacterium tuberculosis] }\end{array}$ & Core & PF01037 & AsnC family & \\
\hline PA3965 & & 169 & $\begin{array}{l}63 \% \text { similar to Leucine-responsive regulatory } \\
\text { protein Lrp [Escherichia coli] }\end{array}$ & Core & PF01037 & AsnC family & $\begin{array}{lr}\text { PF01047 } & \begin{array}{r}\text { MarR } \\
\text { family }\end{array}\end{array}$ \\
\hline PA4508 & & 157 & $\begin{array}{l}70 \% \text { similar to transcription regulatory protein } \\
\text { PdhR [Ralstonia eutropha }]\end{array}$ & Core & PF01037 & AsnC family & \\
\hline PA4784 & & 155 & $\begin{array}{l}57 \% \text { similar to putative transcriptional } \\
\text { regulator [[Bacillus subtilis]] }\end{array}$ & Core & PF01037 & AsnC family & \\
\hline PA5308 & $\begin{array}{l}\text { Lrp, } \\
\text { DadR }\end{array}$ & 162 & $\begin{array}{l}\text { 79\% similar to global response regulator Lrp } \\
\text { [Escherichia coli] }\end{array}$ & Core & PF01037 & AsnC family & \\
\hline $\mathrm{Cro} / \mathrm{cI}$ & & & & & & & \\
\hline PA0048 & & 127 & $\begin{array}{l}55 \% \text { similar to a region of TrbA [plasmid } \\
\mathrm{RK} 2, \text { [Escherichia coli]] }\end{array}$ & Core & & & \\
\hline PA0225 & & 179 & $\begin{array}{l}51 \% \text { similar to putative epoxidase } \\
\text { [Methanobacterium thermoautotrophicum] }\end{array}$ & Core & & & \\
\hline PA0535 & & 184 & $\begin{array}{l}48 \% \text { similar to putative aldehyde } \\
\text { dehydrogenase [Azotobacter vinelandii] }\end{array}$ & Core & & & \\
\hline PA0611 & PrtR & 256 & 0 & Core & & & \\
\hline PA0906 & & 68 & $\begin{array}{l}\text { 70\% similar to hypothetical repressor protein } \\
\text { AF1793 [Archaeoglobus fulgidus] }\end{array}$ & Core & & & \\
\hline PA1359 & & 103 & $\begin{array}{lll}2 & 2 & 0\end{array}$ & Core & & & \\
\hline PA1879 & & 238 & $\begin{array}{l}60 \% \text { similar to regulator PhnR [Salmonella } \\
\text { typhimurium] }\end{array}$ & Core & & & \\
\hline PA1884 & & 72 & $\begin{array}{l}73 \% \text { similar to hypothetical protein YozG } \\
\text { [Bacillus subtilis] }\end{array}$ & Core & & & \\
\hline PA2312 & & 114 & & Core & & & \\
\hline PA2780 & & 193 & $\begin{array}{l}54 \% \text { similar to hypothetical protein [Bacillus } \\
\text { sp.] }\end{array}$ & Core & & & \\
\hline PA2785 & & 183 & $\begin{array}{l}51 \% \text { similar to putative transcriptional } \\
\text { regulator protein [Bacillus sp.] }\end{array}$ & Core & & & \\
\hline
\end{tabular}




\begin{tabular}{|c|c|c|c|c|c|c|c|c|}
\hline PA3260 & & 218 & $\begin{array}{l}\text { Small portion } 64 \% \text { similar to } P v u I I \text { restriction } \\
\text { endonuclease regulator } p v u I I C \text { of [Proteus } \\
\text { vulgaris] ; } 49 \% \text { similar to hypothetical hipB } \\
\text { protein of [Escherichia coli] }\end{array}$ & Core & & & & \\
\hline PA4077 & & 237 & $\begin{array}{l}66 \% \text { similar to pyocin synthesis negative } \\
\text { regulatory protein PrtR [Pseudomonas } \\
\text { aeruginosa] }\end{array}$ & Core & & & & \\
\hline PA4499 & & 187 & $\begin{array}{l}44 \% \text { similar to hypothetical protein } \mathrm{YcjC} \\
\text { [Escherichia coli] }\end{array}$ & Core & & & & \\
\hline PA4987 & & 199 & $\begin{array}{l}53 \% \text { similar to aldehyde dehydrogenase } \\
\text { [Azotobacter vinelandii] } ; 48 \% \text { similar to } \\
\text { hypothetical ycjC gene product of } \\
\text { [Escherichia coli] }\end{array}$ & Core & & & & \\
\hline PA5301 & YcjC & 182 & $\begin{array}{l}62 \% \text { similar to hypothetical protein } \mathrm{YcjC} \\
\text { [Escherichia coli] }\end{array}$ & Core & & & & \\
\hline PA5403 & & 68 & $\begin{array}{l}77 \% \text { similar to putative transcriptional } \\
\text { regulator YozG [Bacillus subtilis] }\end{array}$ & Core & & & & \\
\hline \multicolumn{9}{|c|}{ DksA/TraR } \\
\hline PA0612 & PtrB & 66 & $\begin{array}{l}59 \% \text { similar to hypothetical protein YbiI (over } \\
\text { N-terminal } 75 \% \text { of ORF) [Escherichia coli] }\end{array}$ & RGP 3 & PF01258 & $\begin{array}{l}\text { zf-dskA_traR, } \\
\text { Prokaryotic } \\
\text { dksA/traR C4- } \\
\text { type zinc } \\
\text { finger }\end{array}$ & & \\
\hline PA4723 & DksA & 148 & $\begin{array}{l}86 \% \text { similar to dosage-dependent dnaK } \\
\text { suppressor protein DksA [Escherichia coli] }\end{array}$ & Core & PF01258 & $\begin{array}{l}\text { zf-dskA_traR, } \\
\text { Prokaryotic } \\
\text { dksA/traR C4- } \\
\text { type zinc } \\
\text { finger }\end{array}$ & & \\
\hline PA4870 & YbiI & 88 & $\begin{array}{l}\text { 84\% similar to hypothetical protein YbiI } \\
\text { [Escherichia coli] }\end{array}$ & Core & PF01258 & $\begin{array}{l}\text { zf-dskA_traR, } \\
\text { Prokaryotic } \\
\text { dksA/traR C4- } \\
\text { type zinc } \\
\text { finger }\end{array}$ & & \\
\hline PA5536 & & 134 & $\begin{array}{l}59 \% \text { similar to dnaK suppressor protein DksA } \\
\text { [Escherichia coli] }\end{array}$ & Core & PF01258 & $\begin{array}{l}\text { zf-dskA_traR, } \\
\text { Prokaryotic } \\
\text { dksA/traR C4- } \\
\text { type zinc } \\
\text { finger }\end{array}$ & & \\
\hline \multicolumn{9}{|c|}{ RpoN-binding } \\
\hline PA0873 & PhhR & 519 & & Core & PF00158 & $\begin{array}{l}\text { Sigma-54 } \\
\text { interaction } \\
\text { domain }\end{array}$ & PF00989 & $\begin{array}{l}\text { PAS, PAS } \\
\quad \text { fold }\end{array}$ \\
\hline
\end{tabular}




\begin{tabular}{|c|c|c|c|c|c|c|c|c|c|c|}
\hline PA1097 & FleQ & 490 & $\begin{array}{l}66 \% \text { similar to sigma } 54 \text { transcriptional } \\
\text { activator [Vibrio cholerae] }\end{array}$ & Core & PF02954 & $\begin{array}{l}\mathrm{HTH} 8 \text {, } \\
\text { Bacterial } \\
\text { regulatory } \\
\text { protein, Fis } \\
\text { family }\end{array}$ & PF06490 & $\begin{array}{l}\text { FleQ, } \\
\text { Flagellar } \\
\text { regulatory } \\
\text { protein FleQ }\end{array}$ & PF00158 & $\begin{array}{l}\text { Sigma-54 } \\
\text { interactio } \\
\mathrm{n} \text { domain }\end{array}$ \\
\hline PA1099 & FleR & 473 & $\begin{array}{l}68 \% \text { similar to two-component response } \\
\text { regulator [Vibrio cholerae] }\end{array}$ & Core & PF00158 & $\begin{array}{l}\text { Sigma-54 } \\
\text { interaction } \\
\text { domain }\end{array}$ & PF00072 & $\begin{array}{l}\text { Response } \\
\text { regulator } \\
\text { receiver } \\
\text { domain }\end{array}$ & PF02954 & $\begin{array}{l}\text { HTH_8, } \\
\text { Bacterial } \\
\text { regulatory } \\
\text { protein, } \\
\text { Fis family }\end{array}$ \\
\hline PA1196 & & 466 & $\begin{array}{l}53 \% \text { similar to arginine catabolism positive } \\
\text { regulator RocR [Bacillus subtilis] }\end{array}$ & Core & PF07728 & $\begin{array}{l}\text { AAA_5, } \\
\text { ATPase } \\
\text { family } \\
\text { associated } \\
\text { with various } \\
\text { cellular } \\
\text { activities } \\
\text { (AAA) }\end{array}$ & PF08448 & $\begin{array}{l}\text { PAS } 4, \\
\text { PAS fold }\end{array}$ & $\begin{array}{l}\text { PF02954; } \\
\text { PF00158 }\end{array}$ & $\begin{array}{c}\text { HTH_8, } \\
\text { Bacterial } \\
\text { regulatory } \\
\text { protein, } \\
\text { Fis } \\
\text { family; } \\
\text { Sigma-54 } \\
\text { interactio } \\
\text { n domain }\end{array}$ \\
\hline PA1335 & & 425 & $\begin{array}{l}67 \% \text { similar to C4-dicarboxylate transport } \\
\text { transcription regulator DctD [Rhizobium } \\
\text { meliloti }]\end{array}$ & Core & PF02954 & $\begin{array}{l}\text { HTH_8, Fis } \\
\text { family }\end{array}$ & PF00158 & $\begin{array}{l}\text { Sigma54_ac } \\
\text { tivat, }\end{array}$ & PF00072 & $\begin{array}{l}\text { Response } \\
\text { regulator }\end{array}$ \\
\hline PA1663 & & 503 & $\begin{array}{l}55 \% \text { similar to response regulator of } \\
\text { hydrogenase } 3 \text { activity HydG [Escherichia } \\
\text { coli] }\end{array}$ & Core & PF00158 & $\begin{array}{l}\text { Sigma-54 } \\
\text { interaction } \\
\text { domain }\end{array}$ & PF02954 & $\begin{array}{l}\text { HTH_8, } \\
\text { Bacterial } \\
\text { regulatory } \\
\text { protein, Fis } \\
\text { family }\end{array}$ & & \\
\hline PA1945 & & 442 & $\begin{array}{l}57 \% \text { similar to hydG gene product of } \\
\text { [Escherichia coli] }\end{array}$ & Core & PF02954 & $\begin{array}{l}\mathrm{HTH} \_8, \\
\text { Bacterial } \\
\text { regulatory } \\
\text { protein, Fis } \\
\text { family }\end{array}$ & PF00158 & $\begin{array}{l}\text { Sigma-54 } \\
\text { interaction } \\
\text { domain }\end{array}$ & & \\
\hline PA2005 & & 481 & $\begin{array}{l}58 \% \text { similar to Acetoacetate metabolism } \\
\text { regulatory protein AtoC [Escherichia coli] } \\
53 \% \text { similar to nifA gene product of } \\
\text { [Azotobacter vinelandii] }\end{array}$ & Core & PF00158 & $\begin{array}{l}\text { Sigma-54 } \\
\text { interaction } \\
\text { domain }\end{array}$ & PF02954 & $\begin{array}{l}\text { HTH_8, } \\
\text { Bacterial } \\
\text { regulatory } \\
\text { protein, Fis } \\
\text { family }\end{array}$ & PF08448 & $\begin{array}{l}\text { PAS 4, } \\
\text { PAS fold }\end{array}$ \\
\hline PA2354 & & 376 & $\begin{array}{l}58 \% \text { similar to transcriptional protein } \mathrm{FtrC} \\
\text { [Caulobacter crescentus] }\end{array}$ & Core & PF00158 & $\begin{array}{l}\text { Sigma-54 } \\
\text { interaction } \\
\text { domain }\end{array}$ & PF07728 & $\begin{array}{c}\text { AAA_5, } \\
\text { ATPase } \\
\text { family } \\
\text { associated } \\
\text { with various } \\
\text { cellular }\end{array}$ & & \\
\hline
\end{tabular}




\begin{tabular}{|c|c|c|c|c|c|c|c|c|c|c|}
\hline PA2359 & & 361 & $\begin{array}{l}60 \% \text { similar to a region of transcriptional } \\
\text { protein FtrC [Caulobacter crescentus] }\end{array}$ & Core & PF07728 & $\begin{array}{c}\text { AAA_5, } \\
\text { ATPase } \\
\text { family } \\
\text { associated } \\
\text { with various } \\
\text { cellular } \\
\text { activities } \\
\text { (AAA) }\end{array}$ & PF00158 & $\begin{array}{l}\text { Sigma-5 } 4 \\
\text { interaction } \\
\text { domain }\end{array}$ & & \\
\hline PA2449 & & 511 & $\begin{array}{l}58 \% \text { similar phenylalanine hydroxylase gene } \\
\text { cluster transcription activator PhhR } \\
\text { [Pseudomonas aeruginosa] }\end{array}$ & Core & PF00158 & $\begin{array}{l}\text { Sigma-54 } \\
\text { interaction } \\
\text { domain }\end{array}$ & & & & \\
\hline PA2665 & & 517 & $\begin{array}{l}63 \% \text { similar to putative } 2 \text {-component } \\
\text { transcriptional regulator YgaA [Escherichia } \\
\text { coli] }\end{array}$ & Core & PF00158 & $\begin{array}{l}\text { Sigma-54 } \\
\text { interaction } \\
\text { domain }\end{array}$ & PF01590 & $\begin{array}{l}\text { GAF, GAF } \\
\text { domain }\end{array}$ & & \\
\hline PA3932 & & 324 & $\begin{array}{l}55 \% \text { similar to regulator for prp operon PrpR } \\
\text { [Escherichia coli] }\end{array}$ & Core & PF00158 & $\begin{array}{l}\text { Sigma-54 } \\
\text { interaction } \\
\text { domain }\end{array}$ & & & & \\
\hline PA4021 & & 643 & $\begin{array}{l}57 \% \text { similar to transcription factor AcoR } \\
\text { [Alcaligenes eutrophus] }\end{array}$ & Core & PF00158 & $\begin{array}{l}\text { Sigma-54 } \\
\text { interaction } \\
\text { domain }\end{array}$ & PF07728 & $\begin{array}{c}\text { AAA_5, } \\
\text { ATPase } \\
\text { family } \\
\text { associated } \\
\text { with various } \\
\text { cellular } \\
\text { activities } \\
\text { (AAA) }\end{array}$ & PF02954 & $\begin{array}{l}\text { HTH_8, } \\
\text { Bacterial } \\
\text { regulatory } \\
\text { protein, } \\
\text { Fis family }\end{array}$ \\
\hline PA4147 & AcoR & 625 & $\begin{array}{l}59 \% \text { similar to the acoR gene product of } \\
\text { [Alcaligenes eutrophus }]\end{array}$ & Core & PF00158 & $\begin{array}{l}\text { Sigma-54 } \\
\text { interaction } \\
\text { domain }\end{array}$ & PF02954 & $\begin{array}{l}\text { HTH_8, } \\
\text { Bacterial } \\
\text { regulatory } \\
\text { protein, Fis } \\
\text { family }\end{array}$ & & \\
\hline PA4493 & RoxR & 186 & $\begin{array}{l}67 \% \text { similar to putative photosynthetic } \\
\text { response regulator PrrA [Rhodobacter } \\
\text { sphaeroides]; } 66 \% \text { similar to RegA } \\
{[\text { Rhodovulum sulfidophilum] }}\end{array}$ & Core & PF02954 & $\begin{array}{l}\text { HTH_8, } \\
\text { Bacterial } \\
\text { regulatory } \\
\text { protein, Fis } \\
\text { family }\end{array}$ & PF00072 & $\begin{array}{l}\text { Response } \\
\text { regulator } \\
\text { receiver } \\
\text { domain }\end{array}$ & & \\
\hline PA4547 & PilR & 445 & & Core & PF02954 & $\begin{array}{l}\text { HTH_8, } \\
\text { Bacterial } \\
\text { regulatory } \\
\text { protein, Fis } \\
\text { family }\end{array}$ & PF00072 & $\begin{array}{l}\text { Response } \\
\text { regulator } \\
\text { receiver } \\
\text { domain }\end{array}$ & PF00158 & $\begin{array}{l}\text { Sigma-54 } \\
\text { interactio } \\
\text { n domain }\end{array}$ \\
\hline
\end{tabular}




\begin{tabular}{|c|c|c|c|c|c|c|c|c|c|c|c|}
\hline PA4581 & RtcR & 531 & & $\begin{array}{l}79 \% \text { similar to regulator RtcR [Escherichia } \\
\text { coli] }\end{array}$ & Core & PF00158 & $\begin{array}{l}\text { Sigma-54 } \\
\text { interaction } \\
\text { domain }\end{array}$ & PF06956 & $\begin{array}{c}\text { RtcR, } \\
\text { Regulator of } \\
\text { RNA } \\
\text { terminal } \\
\text { phosphate } \\
\text { cyclase }\end{array}$ & & \\
\hline PA4726 & $\mathrm{CbrB}$ & 478 & & $\begin{array}{l}99 \% \text { similar to regulator } \mathrm{CbrB}[\text { Azotobacter } \\
\text { vinelandii } \mathrm{DJ} ; 90 \% \text { similar to NifA } \\
{[\text { Alcaligenes faecalis] }}\end{array}$ & Core & PF07728 & $\begin{array}{c}\text { AAA_5, } \\
\text { ATPase } \\
\text { family } \\
\text { associated } \\
\text { with various } \\
\text { cellular } \\
\text { activities } \\
\text { (AAA) }\end{array}$ & PF00158 & $\begin{array}{l}\text { Sigma-54 } \\
\text { interaction } \\
\text { domain }\end{array}$ & PF00072 & $\begin{array}{c}\text { Response } \\
\text { regulator } \\
\text { receiver } \\
\text { domain }\end{array}$ \\
\hline PA4853 & Fis & 107 & & $80 \%$ similar to Fis protein [Escherichia coli] & Core & PF02954 & $\begin{array}{l}\text { HTH_8, } \\
\text { Bacterial } \\
\text { regulatory } \\
\text { protein, Fis } \\
\text { family }\end{array}$ & & & & \\
\hline PA5125 & $\mathrm{NtrC}$ & 476 & & $79 \%$ similar to S.typhimurium $\mathrm{NtrC}$ & Core & PF02954 & $\begin{array}{c}\text { HTH_8, } \\
\text { Bacterial } \\
\text { regulatory } \\
\text { protein, Fis } \\
\text { family }\end{array}$ & PF07728 & $\begin{array}{c}\text { AAA_5, } \\
\text { ATPase } \\
\text { family } \\
\text { associated } \\
\text { with various } \\
\text { cellular } \\
\text { activities } \\
\text { (AAA) }\end{array}$ & $\begin{array}{l}\text { PF00158; } \\
\text { PF00072 }\end{array}$ & $\begin{array}{c}\text { Sigma-54 } \\
\text { interactio } \\
\text { n domain; } \\
\text { Response } \\
\text { regulator } \\
\text { receiver } \\
\text { domain }\end{array}$ \\
\hline PA5166 & & 462 & & $\begin{array}{l}\text { 67\% similar to C4-dicarboxylate transport } \\
\text { system regulatory protein DctD }[\text { Rhizobium } \\
\text { meliloti }]\end{array}$ & Core & PF00072 & $\begin{array}{l}\text { Response } \\
\text { regulator } \\
\text { receiver } \\
\text { domain }\end{array}$ & PF00158 & $\begin{array}{l}\text { Sigma-54 } \\
\text { interaction } \\
\text { domain }\end{array}$ & & \\
\hline PA5483 & $\mathrm{AlgB}$ & 449 & & $\begin{array}{l}100 \% \text { similar to } \mathrm{AlgB} \text { [Azotobacter vinelandi]; } \\
97 \% \text { response regulator Desulfomicrobium } \\
\text { baculatum DSM } 4028]\end{array}$ & Core & PF02954 & $\begin{array}{l}\text { HTH_8, } \\
\text { Bacterial } \\
\text { regulatory } \\
\text { protein, Fis } \\
\text { family }\end{array}$ & PF00158 & $\begin{array}{l}\text { Sigma-54 } \\
\text { interaction } \\
\text { domain }\end{array}$ & PF00072 & $\begin{array}{c}\text { Response } \\
\text { regulator } \\
\text { receiver } \\
\text { domain }\end{array}$ \\
\hline PA5511 & MifR & 447 & & $69 \%$ similar to DctD gene product $[R$. meliloti $]$ & Core & PF00158 & $\begin{array}{l}\text { Sigma-54 } \\
\text { interaction } \\
\text { domain }\end{array}$ & PF00072 & $\begin{array}{l}\text { Response } \\
\text { regulator } \\
\text { receiver } \\
\text { domain }\end{array}$ & & \\
\hline \multicolumn{12}{|l|}{ GntR } \\
\hline PA0120 & & 228 & FadR & $\begin{array}{l}45 \% \text { similar to putative transcriptional } \\
\text { regulator LldR [Escherichia coli]; Part of two } \\
\text { gene operon }\end{array}$ & Core & PF07729 & $\begin{array}{l}\text { FCD, FCD } \\
\text { domain. }\end{array}$ & & & & \\
\hline
\end{tabular}




\begin{tabular}{|c|c|c|c|c|c|c|c|c|c|c|c|}
\hline PA0121 & & 244 & FadR & $\begin{array}{l}44 \% \text { similar to hypothetical transcriptional } \\
\text { regulator [Streptomyces ambofaciens] }\end{array}$ & Core & PF07729 & $\begin{array}{l}\text { FCD, FCD } \\
\text { domain. }\end{array}$ & & & & \\
\hline PA0253 & & 158 & MarR & $\begin{array}{l}55 \% \text { similar to MarR regulator [Salmonella } \\
\text { typhimurium] }\end{array}$ & Core & PF01047 & MarR family & & & & \\
\hline PA0275 & & 228 & GntR & $\begin{array}{l}48 \% \text { similar to hypothetical protein Rv } 3676 \\
\text { [Mycobacterium tuberculosis] }\end{array}$ & Core & PF00027 & $\begin{array}{l}\text { cNMP_bindin } \\
\text { g, Cyclic } \\
\text { nucleotide- } \\
\text { binding } \\
\text { domain. }\end{array}$ & & & & \\
\hline PA0424 & MexR & 147 & MarR & $\begin{array}{l}48 \% \text { similar to HosA protein [Escherichia } \\
\text { coli] }\end{array}$ & Core & PF01047 & MarR family & & & & \\
\hline PA0487 & & 252 & & $\begin{array}{l}54 \% \text { similar to molybdate uptake regulatory } \\
\text { protein ModE [Escherichia coli] }\end{array}$ & Core & PF03459 & $\begin{array}{l}\text { TOBE } \\
\text { domain to } \\
\text { bind ligands }\end{array}$ & PF00126 & HTH_1 & PF03459 & TOBE \\
\hline PA0797 & & 240 & FadR & $\begin{array}{l}48 \% \text { similar YdhC protein [[Bacillus } \\
\text { subtilis]]; } 3 \text { gene operon, Ist gene, the other } \\
\text { two genes are involved in citrate synthase } \\
\text { activity }\end{array}$ & Core & PF07729 & $\begin{array}{l}\text { FCD, FCD } \\
\text { domain. }\end{array}$ & & & & \\
\hline PA0942 & & 186 & MarR & $\begin{array}{l}51 \% \text { similar to a region of regulatory protein } \\
\text { PecS [Erwinia chrysanthemi }]\end{array}$ & Core & PF01047 & MarR family & & & & \\
\hline PA1142 & & 238 & HutC & $\begin{array}{l}65 \% \text { similar to PhnR, probable repressor } \\
\text { protein in phnXWRSTUV locus for uptake and } \\
\text { metabolism of } 2 \text {-aminoethylphosphonate } \\
\text { [Salmonella typhimurium }]\end{array}$ & Core & PF07702 & $\begin{array}{l}\text { UTRA, UTRA } \\
\text { domain. }\end{array}$ & & & & \\
\hline PA1269 & & 222 & FadR & $\begin{array}{l}44 \% \text { similar to putative transcriptional } \\
\text { regulator [Streptomyces coelicolor] }\end{array}$ & Core & PF07729 & $\begin{array}{l}\text { FCD, FCD } \\
\text { domain. }\end{array}$ & & & & \\
\hline PA1285 & & 149 & MarR & $\begin{array}{l}56 \% \text { similar to hypothetical protein } \\
\text { Rv1049[Mycobacterium tuberculosis] }\end{array}$ & Core & PF01047 & MarR family & & & & \\
\hline PA1374 & & 168 & $\begin{array}{l}\text { GntR } \\
\text { superfamily }\end{array}$ & $\begin{array}{l}51 \% \text { similar to hypothetical protein Rv3095 } \\
\text { [Mycobacterium tuberculosis] }\end{array}$ & Core & PF01638 & $\begin{array}{l}\text { HxlR-like } \\
\text { HTH }\end{array}$ & $\begin{array}{l}\text { Weak } \\
\text { MarR }\end{array}$ & & & \\
\hline PA1467 & & 323 & & $\begin{array}{l}52 \% \text { similar to putative repressor } \\
\text { [Streptomyces peucetius] }\end{array}$ & Core & & & & & & \\
\hline PA1490 & & 258 & & $\begin{array}{l}59 \% \text { similar to repressor GlpR [Pseudomonas } \\
\text { aeruginosa] }\end{array}$ & Core & & & & & & \\
\hline PA1520 & & 259 & FadR & $\begin{array}{l}48 \% \text { similar to putative transcriptional } \\
\text { regulator [Streptomyces coelicolor] }\end{array}$ & Core & PF07729 & $\begin{array}{l}\mathrm{FCD}, \mathrm{FCD} \\
\text { domain. }\end{array}$ & & & & \\
\hline PA1526 & & 219 & $\begin{array}{l}\text { GntR } \\
\text { superfamily }\end{array}$ & $\begin{array}{l}46 \% \text { similar to hypothetical protein } \\
\text { [Escherichia coli]; } 1 \text { st gene in a } 2 \text {-gene operon } \\
\text { (2nd gene - Hypo) }\end{array}$ & Core & & $\begin{array}{l}\text { No discernible } \\
\text { second } \\
\text { domain }\end{array}$ & & & & \\
\hline PA1603 & & 140 & MarR & $\begin{array}{l}50 \% \text { similar to HosA protein [Escherichia } \\
\text { coli] }\end{array}$ & Core & PF01047 & MarR family & & & & \\
\hline
\end{tabular}




\begin{tabular}{|c|c|c|c|c|c|c|c|}
\hline PA1627 & & 215 & FadR & $\begin{array}{l}70 \% \text { similar to unknown ORF [Pseudomonas } \\
\text { marginalis pv. alfalfae]; } 1 \text { st gene in a } 2 \text {-gene } \\
\text { operon; the other gene is probable major } \\
\text { facilitator superfamily (MFS) transporter }\end{array}$ & Core & PF07729 & $\begin{array}{l}\text { FCD, FCD } \\
\text { domain. }\end{array}$ \\
\hline PA1653 & & 158 & MarR & $\begin{array}{l}61 \% \text { similar to PetP protein Rhodobacter } \\
\text { capsulatus }\end{array}$ & Core & PF01047 & MarR family \\
\hline PA2032 & YjiR & 474 & MocR & $\begin{array}{l}72 \% \text { similar to putative regulator } \mathrm{YjiR} \\
\text { [Escherichia coli] }\end{array}$ & $\begin{array}{l}\text { RGP } \\
20\end{array}$ & PF00155 & $\begin{array}{l}\text { Aminotransfer } \\
\text { ase class I and } \\
\text { II. }\end{array}$ \\
\hline PA2100 & & 477 & MocR & $\begin{array}{l}49 \% \text { similar to hypothetical transcriptional } \\
\text { regulator [Rhodobacter sphaeroides]; Human } \\
\text { homolog }\end{array}$ & $\begin{array}{l}\text { RGP } \\
21\end{array}$ & PF00155 & $\begin{array}{l}\text { Aminotransfer } \\
\text { ase class I and } \\
\text { II. }\end{array}$ \\
\hline PA2299 & & 249 & HutC & $\begin{array}{l}53 \% \text { similar to putative transcriptional } \\
\text { regulator YvoA [[Bacillus subtilis]]; 3rd gene } \\
\text { in a 3-gene operon; The other two code for } \\
\text { probable ferridoxin and oxidoreductase }\end{array}$ & Core & PF07702 & $\begin{array}{l}\text { UTRA, UTRA } \\
\text { domain. }\end{array}$ \\
\hline PA2320 & $\begin{array}{l}\text { GntR, } \\
\text { GnuR }\end{array}$ & 343 & & $56 \%$ similarity to GntR [Escherchia coli] & Core & PF00532 & $\begin{array}{c}\text { Peripla_BP_1, } \\
\text { Periplasmic } \\
\text { binding } \\
\text { proteins and } \\
\text { sugar binding } \\
\text { domain of the } \\
\text { LacI family. }\end{array}$ \\
\hline PA2692 & & 174 & $\begin{array}{c}\text { Rrf2 } \\
\text { subfamily }\end{array}$ & $\begin{array}{l}55 \% \text { similar to hypothetical protein [Bacillus } \\
\text { halodurans]; Homologous to NsrR } \\
\text { [Pectobacterium carotovorum subsp. } \\
\text { carotovorum PC1] }\end{array}$ & Core & PF02082 & $\begin{array}{l}\text { Rrf2, } \\
\text { Transcriptiona } \\
1 \text { regulator. }\end{array}$ \\
\hline PA2802 & & 239 & HutC & $\begin{array}{l}56 \% \text { similar to probable repressor protein } \\
\text { PhnR [Salmonella typhimurium] }\end{array}$ & Core & PF07702 & $\begin{array}{l}\text { UTRA, UTRA } \\
\text { domain. }\end{array}$ \\
\hline PA2825 & OspR & 163 & MarR & $\begin{array}{l}69 \% \text { similar to hypothetical protein } \\
{[\text { Acinetobacter } \mathrm{sp} . \text { ADP } 1]}\end{array}$ & Core & PF01047 & MarR family \\
\hline PA2849 & OhrR & 151 & MarR & $\begin{array}{l}74 \% \text { similar to hypothetical protein } \\
{[\text { Acinetobacter } \text { sp. ADP1] }}\end{array}$ & Core & PF01047 & MarR family \\
\hline PA2897 & & 479 & MocR & $\begin{array}{l}\text { 59\% similar to putative regulator YjiR } \\
\text { [Escherichia coli]; Human Homolog; }\end{array}$ & Core & PF00155 & $\begin{array}{l}\text { Aminotransfer } \\
\text { ase class I and } \\
\text { II. }\end{array}$ \\
\hline PA3067 & & 147 & MarR & $\begin{array}{l}\text { 50\% similar to hypothetical protein Rv0880 } \\
\text { [Mycobacterium tuberculosis] }\end{array}$ & & PF01047 & MarR family \\
\hline PA3249 & & 298 & HutC & $\begin{array}{l}60 \% \text { similar to regulator PhnR [Salmonella } \\
\text { typhimurium }]\end{array}$ & Core & PF07702 & $\begin{array}{l}\text { UTRA, UTRA } \\
\text { domain. }\end{array}$ \\
\hline PA3341 & & 144 & MarR & $\begin{array}{l}62 \% \text { simialr to transcriptional regulator SlyA } \\
\text { [Salmonella typhimurium] }\end{array}$ & Core & PF01047 & MarR family \\
\hline PA3381 & $\mathrm{PhnF}$ & 240 & HutC & $\begin{array}{l}58 \% \text { similar to PhnF protein [Escherichia } \\
\text { coli]; 10th gene in a 13-gene operon }\end{array}$ & Core & PF07702 & $\begin{array}{l}\text { UTRA, UTRA } \\
\text { domain. }\end{array}$ \\
\hline
\end{tabular}




\begin{tabular}{|c|c|c|c|c|c|c|c|}
\hline PA3458 & & 157 & MarR & $\begin{array}{l}48 \% \text { similar to transcriptional regulator } \mathrm{YkoM} \\
\text { [[Bacillus subtilis]] }\end{array}$ & Core & PF01047 & MarR family \\
\hline PA3583 & GlpR & 251 & & & Core & & \\
\hline PA3757 & & 247 & HutC & $\begin{array}{l}55 \% \text { similar to putative transcriptional } \\
\text { regulator YvoA [[Bacillus subtilis]]; First gene } \\
\text { in a 5-gene operon involved in N- } \\
\text { acetylglucosamine catabolism }\end{array}$ & Core & PF07702 & $\begin{array}{l}\text { UTRA, UTRA } \\
\text { domain. }\end{array}$ \\
\hline PA3815 & IscR & 163 & $\begin{array}{c}\text { Rrf2 } \\
\text { subfamily }\end{array}$ & $\begin{array}{l}84 \% \text { similar to unknown hypothetical protein } \\
{[\text { A. vinelandii }]}\end{array}$ & Core & PF02082 & $\begin{array}{c}\text { Rrf2, } \\
\text { Transcriptiona } \\
1 \text { regulator }\end{array}$ \\
\hline PA4132 & & 471 & MocR & $\begin{array}{l}64 \% \text { similar to putative transcriptional } \\
\text { regulator [Escherichia coli] }\end{array}$ & Core & PF00155 & $\begin{array}{l}\text { Aminotransfer } \\
\text { ase class I and } \\
\text { II. }\end{array}$ \\
\hline PA4135 & & 140 & MarR & $\begin{array}{l}73 \% \text { similar to homoprotocatechuate } \\
\text { degradation operon regulator HpcR } \\
\text { [Escherichia coli] }\end{array}$ & Core & PF01047 & MarR family \\
\hline PA4165 & & 496 & MocR & $\begin{array}{l}53 \% \text { similar to putative regulatory protein } \\
\text { MocR [Rhizobium leguminosarum bv. viciae]; } \\
\text { Human Homolog }\end{array}$ & Core & PF00155 & $\begin{array}{l}\text { Aminotransfer } \\
\text { ase class I and } \\
\text { II. }\end{array}$ \\
\hline PA4169 & & 142 & $\begin{array}{l}\text { Rrf2 } \\
\text { subfamily }\end{array}$ & $\begin{array}{l}\text { 57\% similar to hypothetical protein YwnA } \\
\text { [Bacillus subtilis] }\end{array}$ & Core & PF02082 & $\begin{array}{c}\text { Rrf2, } \\
\text { Transcriptiona } \\
1 \text { regulator }\end{array}$ \\
\hline PA4185 & & 242 & FadR & $\begin{array}{l}47 \% \text { similar to putative transcriptional } \\
\text { regulator [Streptomyces coelicolor] }\end{array}$ & Core & PF07729 & $\begin{array}{l}\text { FCD, FCD } \\
\text { domain. }\end{array}$ \\
\hline PA4764 & Fur & 134 & $\begin{array}{c}\text { GntR } \\
\text { superfamily }\end{array}$ & $\begin{array}{l}77 \% \text { similar to Regulatory protein Fur } \\
\text { [Escherichia coli] }\end{array}$ & Core & PF01475 & $\begin{array}{l}\text { FUR, Ferric } \\
\text { uptake } \\
\text { regulator } \\
\text { family }\end{array}$ \\
\hline PA4769 & LldR & 257 & FadR & $\begin{array}{l}66 \% \text { similar to transcriptional regulator for } \\
\text { pyruvate dehydrogenase complex PdhR } \\
\text { [Escherichia coli] }\end{array}$ & Core & PF07729 & $\begin{array}{l}\text { FCD, FCD } \\
\text { domain. }\end{array}$ \\
\hline PA4906 & VanR & 237 & FadR & $\begin{array}{l}66 \% \text { similar to hypothetical protein AF009672 } \\
\text { [Acinetobacter sp. ADP } 1] ; \text { Part of a 2-gene } \\
\text { operon, upstream gene is a probable } \\
\text { dehydogenase }\end{array}$ & Core & PF07729 & $\begin{array}{l}\text { FCD, FCD } \\
\text { domain. }\end{array}$ \\
\hline PA5105 & HutC & 250 & HutC & $\begin{array}{l}94 \% \text { similar to hutC gene product of } \\
\text { Pseudomonas putida; } 1 \text { st gene in a } 2 \text {-gene } \\
\text { operon (2nd gene - Hypo) }\end{array}$ & Core & PF07702 & $\begin{array}{l}\text { UTRA, UTRA } \\
\text { domain. }\end{array}$ \\
\hline PA5157 & MarR & 156 & MarR & $\begin{array}{l}60 \% \text { similar to MarR protein [Escherichia } \\
\text { coli] }\end{array}$ & Core & PF01047 & MarR family \\
\hline PA5283 & & 458 & MocR & $\begin{array}{l}48 \% \text { similar to putative transcriptional } \\
\text { regulator [Bacillus subtilis] (Human homolog) }\end{array}$ & Core & PF00155 & $\begin{array}{l}\text { Aminotransfer } \\
\text { ase class I and } \\
\text { II. }\end{array}$ \\
\hline
\end{tabular}




\begin{tabular}{|c|c|c|c|c|c|c|c|c|}
\hline PA5356 & GlcC & 251 & FadR & $\begin{array}{l}78 \% \text { similar to GlcC protein [Escherichia } \\
\text { coli]; 1st gene in a 3-gene operon; 2nd-Hyp } \\
\text { and 3rd- 4-hydroxybenzoate-octaprenyl } \\
\text { transferase }\end{array}$ & Core & PF07729 & $\begin{array}{l}\mathrm{FCD}, \mathrm{FCD} \\
\text { domain. }\end{array}$ & \\
\hline PA5431 & & 491 & MocR & $\begin{array}{l}52 \% \text { similar to hypothetical regulator MocR } \\
\text { [Rhizobium meliloti]; 1st gene in a 2-gene } \\
\text { operon (2nd gene - Hypo) }\end{array}$ & Core & PF00155 & $\begin{array}{l}\text { Aminotransfer } \\
\text { ase class I and } \\
\text { II. }\end{array}$ & \\
\hline PA5499 & $\mathrm{Np} 20$ & 167 & & $\begin{array}{l}\text { 54\% similar to zinc-uptake regulator Zur } \\
\text { [Escherichia coli]; PMID: } 8971720 ; 1 \text { st gene } \\
\text { in a 3-gene operon; 2nd \& 3rd involved in Zn } \\
\text { tranport }\end{array}$ & Core & PF01475 & $\begin{array}{l}\text { FUR, Ferric } \\
\text { uptake } \\
\text { regulator } \\
\text { family. }\end{array}$ & \\
\hline PA5525 & & 246 & FadR & $\begin{array}{l}49 \% \text { similar to } \text { nta operon transcriptional } \\
\text { regulator [Escherichia coli]; 2nd gene in a part } \\
\text { of 2-gene operon; downstream gene is a } \\
\text { probable short-chain dehydrogenase }\end{array}$ & Core & PF07729 & $\begin{array}{l}\text { FCD, FCD } \\
\text { domain. }\end{array}$ & \\
\hline PA5550 & GlmR & 257 & & $\begin{array}{l}60 \% \text { similar to regulator for glucitol operon, } \\
\text { SrlR [Escherichia coli] }\end{array}$ & Core & & & \\
\hline PA0268 & & 473 & MocR & $\begin{array}{l}55 \% \text { similar to putative regulatory protein } \\
\text { MocR [Rhizobium leguminosarum bv. viciae]: } \\
\text { Has a human homolog }\end{array}$ & Core & PF00155 & $\begin{array}{l}\text { Aminotransfer } \\
\text { ase class I and } \\
\text { II. }\end{array}$ & \\
\hline \multicolumn{9}{|c|}{ H-NS (silencing) } \\
\hline PA2667 & $\mathrm{MvaU}$ & 117 & & $\begin{array}{l}64 \% \text { similar to P16 subunit of heteromeric } \\
\text { transcriptional activator MvaT [Pseudomonas } \\
\text { mevalonii] }\end{array}$ & Core & & & \\
\hline PA4315 & MvaT & 124 & & $\begin{array}{l}82 \% \text { similar to heteromeric transcriptional } \\
\text { activator MvaT P16 subunit [Pseudomonas } \\
\text { mevalonii] }\end{array}$ & Core & $\begin{array}{l}\mathrm{PB} 00330 \\
6\end{array}$ & Pfam-B_3306 & $\begin{array}{cc}\text { PF00816 } & \text { Histone_HN } \\
.14 & \mathrm{~S}\end{array}$ \\
\hline \multicolumn{9}{|l|}{$\overline{\text { IcIR }}$} \\
\hline PA0155 & PcaR & 279 & & $\begin{array}{l}\text { 88\% similar to regulatory protein PcaR } \\
{[\text { Pseudomonas putida }]}\end{array}$ & other & & & \\
\hline PA0236 & & 259 & & $\begin{array}{l}49 \% \text { similar to repressor protein IclR } \\
\text { [Escherichia coli] }\end{array}$ & Core & & & \\
\hline PA1015 & & 266 & & $\begin{array}{l}42 \% \text { similar to glycerol operon regulatory } \\
\text { protein GylR [Streptomyces coelicolor] }\end{array}$ & Core & & & \\
\hline PA1630 & & 288 & & $\begin{array}{l}58 \% \text { similar to hypothetical protein } \\
\text { Bordetella pertussis] }\end{array}$ & Core & & & \\
\hline PA2010 & & 267 & & $\begin{array}{l}49 \% \text { similar to putative transcriptional } \\
\text { regulatory protein OhbR [Pseudomonas } \\
\text { aeruginosa] }\end{array}$ & Core & & & \\
\hline PA3174 & & 242 & & 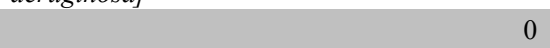 & Core & & & \\
\hline PA3508 & & 277 & & $\begin{array}{l}57 \% \text { similar to hypothetical protein } \\
\text { [Acinetobacter } \mathrm{sp} . \text { ADP1] }\end{array}$ & RGP34 & & & \\
\hline
\end{tabular}




\begin{tabular}{|c|c|c|c|c|c|c|c|c|c|c|}
\hline PA4157 & & 262 & $\begin{array}{l}51 \% \text { similar to putative transcriptional } \\
\text { regulatory protein OhbR [Pseudomonas } \\
\text { aeruginosa] }\end{array}$ & Core & & & & & & \\
\hline PA4341 & & 256 & $\begin{array}{l}45 \% \text { similar to transcriptional regulator for } \\
\text { mhp operon [Escherichia coli] }\end{array}$ & Core & & & & & & \\
\hline \multicolumn{11}{|c|}{ LacI/GalR } \\
\hline PA1949 & RbsR & 337 & $\begin{array}{l}62 \% \text { similar to regulator for rbs operon RbsR } \\
\text { [Escherichia coli] }\end{array}$ & Core & & & & & & \\
\hline PA2259 & PtxS & 340 & 0 & Core & & & & & & \\
\hline PA3184 & HexR & 285 & $\begin{array}{l}76 \% \text { similar to HexR protein [Escherichia } \\
\text { coli] }\end{array}$ & Core & PF01418 & RpiR family & PF01380 & $\begin{array}{l}\text { SIS (Sugar } \\
\text { ISomerase) } \\
\text { domain }\end{array}$ & & \\
\hline PA3563 & FruR & 329 & $\begin{array}{l}62 \% \text { similar to transcriptional repressor of fru } \\
\text { operon FruR [Escherichia coli] }\end{array}$ & Core & & & & & & \\
\hline PA4596 & & 172 & $\begin{array}{l}74 \% \text { similar to } \mathrm{nf} \times \mathrm{B} \text { gene product of } \\
\text { [Pseudomonas aeruginosa] }\end{array}$ & Core & & & & & & \\
\hline PA4600 & $\mathrm{NfxB}$ & 187 & $\begin{array}{l}95 \% \text { similar to } \mathrm{nfxB} \text { (excluding frameshift) }[P \text {. } \\
\text { aeruginosa }]\end{array}$ & Core & & & & & & \\
\hline PA5438 & & 293 & $\begin{array}{l}63 \% \text { similar to putative regulator HexR } \\
\text { [Pseudomonas aeruginosa] }\end{array}$ & Core & PF01418 & RpiR family & PF01380 & $\begin{array}{l}\text { SIS (Sugar } \\
\text { ISomerase) } \\
\text { domain }\end{array}$ & & \\
\hline \multicolumn{11}{|l|}{$\overline{\text { LuxR }}$} \\
\hline PA0034 & & 207 & $\begin{array}{l}75 \% \text { similar to BvgA positive transcription } \\
\text { regulator, putative [Bordetella pertussis]; }\end{array}$ & Core & PF00072 & $\begin{array}{l}\text { Response } \\
\text { regulator }\end{array}$ & PF00196 & GerE & & \\
\hline PA0533 & & 496 & $\begin{array}{l}44 \% \text { similar to a region of putative sensory } \\
\text { transduction histidine kinase } \\
\text { [Methanobacterium thermoautotrophicum] }\end{array}$ & Core & PF00196 & GerE & PF00989 & PAS & $\begin{array}{c}\text { PF08448; } \\
\text { PB00136 } \\
0 ; \\
\text { PB00254 } \\
6\end{array}$ & $\begin{array}{l}\text { PAS_4; } \\
\text { Pfam- } \\
\text { B_1360; } \\
\text { Pfam- } \\
\text { B_2546 }\end{array}$ \\
\hline PA0601 & & 210 & $\begin{array}{l}66 \% \text { similar to VsrD protein [Burkholderia } \\
\text { solanacearum] }\end{array}$ & Core & PF00072 & $\begin{array}{l}\text { Response } \\
\text { regulator }\end{array}$ & PF08266 & GerE & $\begin{array}{c}\text { PB00104 } \\
1\end{array}$ & $\begin{array}{l}\text { Pfam- } \\
\text { B_1041 }\end{array}$ \\
\hline PA1136 & & 243 & $\begin{array}{l}42 \% \text { similar to transcriptional activator LasR } \\
\text { [Pseudomonas aeruginosa] }\end{array}$ & Core & PF03472 & $\begin{array}{l}\text { Autoinducer } \\
\text { binding }\end{array}$ & PF00196 & GerE & & \\
\hline PA1347 & & 230 & $\begin{array}{l}65 \% \text { similar to a region of putative regulator } \\
\text { FimZ [Salmonella typhimurium }]\end{array}$ & Core & PF00196 & GerE & PF08448 & PAS_4 & & \\
\hline PA1397 & & 210 & $\begin{array}{l}56 \% \text { similar to nitrate/nitrite response } \\
\text { regulator NarP (sensor NarQ) [Escherichia } \\
\text { coli] }\end{array}$ & Core & PF00072 & $\begin{array}{l}\text { Response } \\
\text { regulator }\end{array}$ & PF00196 & GerE & & \\
\hline PA1430 & LasR & 239 & $\begin{array}{l}100 \% \text { identical to transcription activator LasR } \\
\text { [Pseudomonas aeruginosa] }\end{array}$ & Core & PF03472 & $\begin{array}{l}\text { Autoinducer } \\
\text { binding }\end{array}$ & PF00196 & GerE & & \\
\hline
\end{tabular}




\begin{tabular}{|c|c|c|c|c|c|c|c|c|c|c|}
\hline PA1484 & & 268 & $\begin{array}{l}50 \% \text { similar to DMSO reductase regulatory } \\
\text { protein DorX [Rhodobacter sphaeroides] }\end{array}$ & Core & PF00196 & GerE & $\begin{array}{l}\mathrm{PB} 0001 \\
46\end{array}$ & Pfam-B_146 & & \\
\hline PA1759 & & 901 & $\begin{array}{l}44 \% \text { similar to regulator AcoK }[\text { Klebsiella } \\
\text { pneumoniae }]\end{array}$ & Core & PF00196 & GerE & $\begin{array}{l}\text { PB0082 } \\
87\end{array}$ & $\begin{array}{l}\text { Pfam- } \\
\text { B_8287 }\end{array}$ & & \\
\hline PA1760 & & 907 & $\begin{array}{l}44 \% \text { similar to regulator AcoK [Klebsiella } \\
\text { pneumoniae }]\end{array}$ & Core & PF00196 & GerE & $\begin{array}{l}\text { PB0014 } \\
29\end{array}$ & $\begin{array}{l}\text { Pfam- } \\
\text { B_1429 }\end{array}$ & $\begin{array}{c}\mathrm{PB} 00828 \\
7\end{array}$ & $\begin{array}{l}\text { Pfam- } \\
\text { B_8287 }\end{array}$ \\
\hline PA1898 & $\begin{array}{l}\text { QscR, } \\
\text { PhzR }\end{array}$ & 237 & $\begin{array}{l}29 \% \text { identity, } 46 \% \text { similarity to LasR } \\
\text { [Pseudomonas aeruginosa] ; } 27 \% \text { identity, } \\
45 \% \text { similarity to LuxR [Vibrio fischeri] } ; 33 \% \\
\text { identity, } 49 \% \text { similarity to SolR [Ralstonia } \\
\text { solanacearum] ; } 49 \% \text { similar to positive } \\
\text { regulatory protein PhzR }[\text { Pseudomonas } \\
\text { aureofaciens]; } 53 \% \text { similar, } 32 \% \text { identity to } \\
\text { regulatory protein RhlR }[\text { Pseudomonas } \\
\text { aeruginosa] }\end{array}$ & Core & PF03472 & $\begin{array}{l}\text { Autoinducer } \\
\text { binding }\end{array}$ & PF00196 & GerE & & \\
\hline PA1978 & $\begin{array}{l}\text { ErbR, } \\
\text { AgmR }\end{array}$ & 221 & $\begin{array}{l}100 \% \text { identical to agm } R \text { gene product of }[P \text {. } \\
\text { aeruginosa }]\end{array}$ & Core & PF00072 & $\begin{array}{l}\text { Response } \\
\text { regulator }\end{array}$ & PF00196 & GerE & & \\
\hline PA1980 & $\begin{array}{l}\text { EraR, } \\
\text { ExaE }\end{array}$ & 225 & $\begin{array}{l}57 \% \text { similar to uhpA gene product of } \\
{[\text { Salmonella typhimurium }]}\end{array}$ & Core & PF00072 & $\begin{array}{l}\text { Response } \\
\text { regulator }\end{array}$ & PF00196 & GerE & & \\
\hline PA2376 & & 213 & $\begin{array}{l}54 \% \text { similar to nitrate/nitrite regulatory protein } \\
\text { NarL }[\text { Pseudomonas aeruginosa }]\end{array}$ & Core & PF00072 & $\begin{array}{l}\text { Response } \\
\text { regulator }\end{array}$ & PF00196 & GerE & & \\
\hline PA2586 & GacA & 214 & $\begin{array}{l}99 \% \text { similar to response regulator GacA } \\
\text { [Pseudomonas aeruginosa] }\end{array}$ & Core & PF00072 & $\begin{array}{l}\text { Response } \\
\text { regulator }\end{array}$ & PF00196 & GerE & $\begin{array}{l}\mathrm{PB} 00064 \\
5\end{array}$ & $\begin{array}{l}\text { Pfam- } \\
\text { B_645 }\end{array}$ \\
\hline PA2591 & VqsR & 268 & $\begin{array}{l}46 \% \text { similar to DMSO reductase regulatory } \\
\text { protein DorX [Rhodobacter sphaeroides] }\end{array}$ & Core & PF00196 & GerE & $\begin{array}{l}\text { PB0001 } \\
46\end{array}$ & Pfam-B_146 & & \\
\hline PA2899 & & 212 & $\begin{array}{l}53 \% \text { similar to biosynthesis positive regulator } \\
\text { RcsB [Escherichia coli] }\end{array}$ & Core & PF00072 & $\begin{array}{l}\text { Response } \\
\text { regulator }\end{array}$ & PF00196 & GerE & & \\
\hline PA3045 & & 207 & $\begin{array}{l}77 \% \text { similar to putative transcription regulator } \\
\text { BvgA [Bordetella pertussis] }\end{array}$ & Core & PF00072 & $\begin{array}{l}\text { Response } \\
\text { regulator }\end{array}$ & PF00196 & GerE & & \\
\hline PA3420 & & 827 & & Core & PF00196 & GerE & $\begin{array}{l}\mathrm{PB} 0103 \\
63\end{array}$ & $\begin{array}{c}\text { Pfam- } \\
\text { B_10363 }\end{array}$ & & \\
\hline PA3477 & RhlR & 241 & & Core & PF03472 & $\begin{array}{l}\text { Autoinducer } \\
\text { binding }\end{array}$ & PF00196 & GerE & & \\
\hline PA3599 & & 261 & $\begin{array}{l}47 \% \text { similar to dorX gene product of } \\
\text { [Rhodobacter sphaeroides] }\end{array}$ & Core & PF00196 & GerE & $\begin{array}{l}\text { PB0001 } \\
46\end{array}$ & Pfam-B_146 & $\begin{array}{c}\mathrm{PB} 00064 \\
5\end{array}$ & $\begin{array}{l}\text { Pfam- } \\
\text { B_645 }\end{array}$ \\
\hline PA3604 & ErdR & 217 & $\begin{array}{l}61 \% \text { similar to glycerol metabolism activator } \\
\text { (AGMR protein) }[P \text {. aeruginosa }]\end{array}$ & Core & PF00072 & $\begin{array}{l}\text { Response } \\
\text { regulator }\end{array}$ & PF00196 & GerE & & \\
\hline PA3714 & & 213 & $\begin{array}{l}62 \% \text { similar to RcsB protein [Erwinia } \\
\text { amylovora] }\end{array}$ & Core & PF00072 & $\begin{array}{l}\text { Response } \\
\text { regulator }\end{array}$ & PF00196 & GerE & & \\
\hline PA3771 & & 325 & $\begin{array}{l}54 \% \text { similar to a region of putative regulatory } \\
\text { protein [Streptomyces coelicolor] }\end{array}$ & Core & PF00196 & GerE & $\begin{array}{l}\text { PB0050 } \\
70\end{array}$ & $\begin{array}{l}\text { Pfam- } \\
\text { B_5070 }\end{array}$ & & \\
\hline PA3879 & NarL & 219 & $74 \%$ similar to E. coli NarL protein. & Core & PF00072 & Response & PF00196 & GerE & & \\
\hline
\end{tabular}


regulator

\begin{tabular}{|c|c|c|c|c|c|c|c|c|c|c|}
\hline PA3921 & & 906 & $\begin{array}{l}42 \% \text { similar to regulator AcoK [Klebsiella } \\
\text { pneumoniae }]\end{array}$ & Core & PF00196 & GerE & $\begin{array}{l}\text { PB0014 } \\
29\end{array}$ & $\begin{array}{l}\text { Pfam- } \\
\text { B_1429 }\end{array}$ & $\begin{array}{c}\text { PB00142 } \\
9 ; \\
\text { PB00455 } \\
0 ; \\
\text { PB00952 } \\
5 ; \\
\text { PB01036 } \\
3\end{array}$ & $\begin{array}{c}\text { Pfam- } \\
\text { B_1429; } \\
\text { Pfam- } \\
\text { B_4550; } \\
\text { Pfam- } \\
\text { B_9525; } \\
\text { Pfam- } \\
\text { B_10363 }\end{array}$ \\
\hline PA3948 & RocA1 & 209 & $\begin{array}{l}74 \% \text { similar to } b v g A \text { gene product of } \\
\text { Bordetella pertussis] }\end{array}$ & Core & PF00072 & $\begin{array}{l}\text { Response } \\
\text { regulator }\end{array}$ & PF00196 & GerE & & \\
\hline PA4074 & & 222 & $\begin{array}{l}54 \% \text { similar to regulator protein MoaR } \\
\text { [Klebsiella aerogenes] }\end{array}$ & Core & PF03472 & $\begin{array}{l}\text { Autoinducer } \\
\text { binding }\end{array}$ & PF00196 & GerE & $\begin{array}{l}\text { PB00014 } \\
6\end{array}$ & $\begin{array}{l}\text { Pfam- } \\
\text { B_146 }\end{array}$ \\
\hline PA4080 & RcsB & 214 & $\begin{array}{l}63 \% \text { similar to rcsB gene product of } \\
\text { [Escherichia coli] }\end{array}$ & Core & PF00072 & $\begin{array}{l}\text { Response } \\
\text { regulator }\end{array}$ & PF00196 & GerE & & \\
\hline PA4196 & bfiR & 214 & $\begin{array}{l}62 \% \text { similar to transcriptional regulatory } \\
\text { protein FixJ }[\text { Azorhizobium caulinodans }]\end{array}$ & Core & PF00196 & $\begin{array}{l}\text { GerE, } \\
\text { Bacterial } \\
\text { regulatory } \\
\text { proteins, luxR } \\
\text { family }\end{array}$ & PF08281 & $\begin{array}{l}\text { Sigma70_r4 } \\
2, \text { Sigma- } \\
70, \text { region } 4\end{array}$ & PF00072 & $\begin{array}{l}\text { Response } \\
\text { regulator } \\
\text { receiver } \\
\text { domain }\end{array}$ \\
\hline PA4296 & PprB & 275 & $\begin{array}{l}54 \% \text { similar to NtrC-type response regulator } \\
\text { [Eubacterium acidaminophilum]; } 43 \% \text { similar } \\
\text { to regulatory components ofsensory } \\
\text { transduction system Synechocystis } \mathrm{sp} \text {. }\end{array}$ & Core & PF00072 & $\begin{array}{l}\text { Response } \\
\text { regulator }\end{array}$ & PF00196 & $\begin{array}{l}\text { GerE, } \\
\text { Bacterial } \\
\text { regulatory } \\
\text { proteins, } \\
\text { LuxR } \\
\text { family }\end{array}$ & & \\
\hline PA4806 & & 227 & $\begin{array}{l}46 \% \text { similar to DMSO reductase regulatory } \\
\text { protein DorX [Rhodobacter sphaeroides] }\end{array}$ & Core & PF00196 & GerE & & & & \\
\hline \multicolumn{11}{|l|}{ LysR } \\
\hline PA0032 & & 304 & $\begin{array}{l}49 \% \text { similar to glycine cleavage system } \\
\text { transcriptional activator protein GcvA } \\
\text { [Escherichia coli] }\end{array}$ & Core & PF00126 & HTH_1 & PF03466 & $\begin{array}{l}\text { LysR_substr } \\
\text { ate }\end{array}$ & & \\
\hline PA0037 & TrpI & 295 & $\begin{array}{l}97 \% \text { similarity to TrpI protein [Pseudomonas } \\
\text { aeruginosa] }\end{array}$ & Core & PF00126 & HTH_1 & PF03466 & $\begin{array}{l}\text { LysR_substr } \\
\text { ate }\end{array}$ & & \\
\hline PA0056 & & 306 & $\begin{array}{l}55 \% \text { similar to regulator GstR [Rhizobium } \\
\text { leguminosarum] }\end{array}$ & Core & PF00126 & HTH_1 & PF03466 & $\begin{array}{c}\text { LysR_substr } \\
\text { ate }\end{array}$ & & \\
\hline PA0123 & & 302 & $\begin{array}{l}51 \% \text { similar to transcriptional activator PtxR } \\
\text { [Pseudomonas aeruginosa] }\end{array}$ & Core & PF00126 & HTH_1 & PF03466 & $\begin{array}{l}\text { LysR_substr } \\
\text { ate }\end{array}$ & & \\
\hline PA0133 & & 305 & $\begin{array}{l}47 \% \text { similar to transcriptional regulator OxyR } \\
{[\text { Mycobacterium xenopi] }}\end{array}$ & Core & PF00126 & HTH_1 & PF03466 & $\begin{array}{l}\text { LysR_substr } \\
\text { ate }\end{array}$ & & \\
\hline PA0152 & PcaQ & 275 & $\begin{array}{l}56 \% \text { similar to regulatory protein } \mathrm{PcaQ} \\
\text { [Agrobacterium tumefaciens] }\end{array}$ & Core & PF00126 & HTH_1 & PF03466 & $\begin{array}{l}\text { LysR_substr } \\
\text { ate }\end{array}$ & & \\
\hline
\end{tabular}




\begin{tabular}{|c|c|c|c|c|c|c|c|c|}
\hline PA0159 & & 312 & $\begin{array}{l}52 \% \text { similar to regulator GstR } \\
{[\text { Bradyrhizobium japonicum }]}\end{array}$ & Core & PF00126 & HTH_1 & PF03466 & $\begin{array}{c}\text { LysR_substr } \\
\text { ate }\end{array}$ \\
\hline PA0181 & & 310 & $\begin{array}{l}48 \% \text { similar to putative transcriptional } \\
\text { regulator YeaT [Escherichia coli] }\end{array}$ & Core & PF00126 & HTH_1 & PF03466 & $\begin{array}{c}\text { LysR_substr } \\
\text { ate }\end{array}$ \\
\hline PA0191 & & 305 & $\begin{array}{l}59 \% \text { similar to transcriptional control factor } \\
\text { SdsB Pseudomonas sp. }\end{array}$ & Core & PF00126 & HTH_1 & PF03466 & $\underset{\text { ate }}{\text { LysR_substr }}$ \\
\hline PA0207 & & 297 & $\begin{array}{l}51 \% \text { similar to glycine cleavage activator } \\
\text { protein GcvA [Escherichia coli] }\end{array}$ & RGP 1 & PF00126 & HTH_1 & PF03466 & $\underset{\substack{\text { ate } \\
\text { LysR_substr }}}{ }$ \\
\hline PA0217 & MdcR & 309 & $\begin{array}{l}65 \% \text { similar to malonate decarboxylase operon } \\
\text { regulator MdcR [Klebsiella pneumoniae] }\end{array}$ & Core & PF00126 & HTH_1 & PF03466 & $\underset{\text { ate }}{\text { LysR_substr }}$ \\
\hline PA0218 & & 306 & $\begin{array}{l}49 \% \text { similar to putative transcriptional } \\
\text { regulator YvbU [[Bacillus subtilis]] }\end{array}$ & Core & PF00126 & HTH_1 & PF03466 & $\begin{array}{c}\text { LysR_substr } \\
\text { ate }\end{array}$ \\
\hline PA0233 & & 313 & $\begin{array}{l}61 \% \text { similar to putative transcriptional } \\
\text { regulator [Rhizobium leguminosarum bv. } \\
\text { trifolii] }\end{array}$ & Core & PF00126 & HTH_1 & PF03466 & $\begin{array}{l}\text { LysR_substr } \\
\text { ate }\end{array}$ \\
\hline PA0272 & & 310 & $\begin{array}{l}44 \% \text { similar to MexT protein [Pseudomonas } \\
\text { aeruginosa] }\end{array}$ & Core & PF00126 & HTH_1 & PF03466 & $\begin{array}{l}\text { LysR_substr } \\
\text { ate }\end{array}$ \\
\hline PA0289 & GpuR & 320 & $\begin{array}{l}\text { 39\% similar to LysR-type regulatory protein } \\
\text { [Comamonas sp. JS765] }\end{array}$ & Core & PF00126 & HTH_1 & PF03466 & $\begin{array}{l}\text { LysR_substr } \\
\text { ate }\end{array}$ \\
\hline PA0448 & & 302 & $55 \%$ similar to GcvA gene product of $E$. coli. & Core & PF00126 & HTH_1 & PF03466 & $\begin{array}{l}\text { LysR_substr } \\
\text { ate }\end{array}$ \\
\hline PA0477 & & 308 & $\begin{array}{l}47 \% \text { similar to DNA-binding protein NahR } \\
\text { Pseudomonas putida } ; 48 \% \text { similar to MexT } \\
\text { protein [Pseudomonas aeruginosa] }\end{array}$ & Core & PF00126 & HTH_1 & PF03466 & $\underset{\text { ate }}{\text { LysR_substr }}$ \\
\hline PA0479 & & 316 & $\begin{array}{l}46 \% \text { similar to putative transcriptional } \\
\text { regulator YhaJ [Escherichia coli] }\end{array}$ & Core & PF00126 & HTH_1 & PF03466 & $\begin{array}{c}\text { LysR_substr } \\
\text { ate }\end{array}$ \\
\hline PA0491 & & 308 & $\begin{array}{l}46 \% \text { similar to oxidative stress transcriptional } \\
\text { regulator OxyR [Xanthomonas campestris] }\end{array}$ & Core & PF00126 & HTH_1 & PF03466 & $\begin{array}{l}\text { LysR_substr } \\
\text { ate }\end{array}$ \\
\hline PA0528 & & 292 & $\begin{array}{l}49 \% \text { similar to positive regulator CynR } \\
\text { [Escherichia coli] }\end{array}$ & Core & PF00126 & HTH_1 & PF03466 & $\begin{array}{l}\text { LysR_substr } \\
\text { ate }\end{array}$ \\
\hline PA0701 & & 328 & $\begin{array}{l}43 \% \text { similar to iron-regulated virulence } \\
\text { regulator [Vibrio cholerae] }\end{array}$ & Core & PF00126 & HTH_1 & PF03466 & $\begin{array}{l}\text { LysR_substr } \\
\text { ate }\end{array}$ \\
\hline PA0708 & & 284 & $\begin{array}{l}53 \% \text { similar to putative transcriptional } \\
\text { regulator YneJ [Escherichia coli] }\end{array}$ & Core & PF00126 & HTH_1 & PF03466 & $\begin{array}{l}\text { LysR_substr } \\
\text { ate }\end{array}$ \\
\hline PA0739 & & 304 & $\begin{array}{l}75 \% \text { similar to } \mathrm{SdsB} \text { (regulator of sdsA) } \\
\text { Pseudomonas } \mathrm{sp} \text {. }\end{array}$ & Core & PF00126 & HTH_1 & PF03466 & $\begin{array}{c}\text { LysR_substr } \\
\text { ate }\end{array}$ \\
\hline PA0784 & & 309 & $\begin{array}{l}50 \% \text { similar to glycine cleavage system } \\
\text { transcriptional activator GcvA [Haemophilus } \\
\text { influenzae } \mathrm{Rd} \text { ] }\end{array}$ & Core & PF00126 & HTH_1 & PF03466 & $\underset{\text { ate }}{\text { LysR_substr }}$ \\
\hline PA0815 & & 317 & $\begin{array}{l}46 \% \text { similar to putative transcriptional } \\
\text { regulator YjiE [Escherichia coli] }\end{array}$ & Core & PF00126 & HTH_1 & PF03466 & $\begin{array}{l}\text { LysR_substr } \\
\text { ate }\end{array}$ \\
\hline
\end{tabular}




\begin{tabular}{|c|c|c|c|c|c|c|c|c|}
\hline PA0816 & & 294 & $\begin{array}{l}60 \% \text { similar to Brg1 [Bordetella pertussis], a } \\
\text { transcriptional regulator that is controlled by } \\
\text { the oxygen-responsive transcriptional regulator } \\
\text { Btr under anaerobic conditions. }\end{array}$ & Core & PF00126 & HTH_1 & PF03466 & $\begin{array}{c}\text { LysR_substr } \\
\text { ate }\end{array}$ \\
\hline PA0876 & & 314 & $\begin{array}{l}54 \% \text { similar to putative transcriptional } \\
\text { regulator Synechocystis sp. }\end{array}$ & Core & PF00126 & HTH_1 & PF03466 & $\begin{array}{l}\text { LysR_substr } \\
\text { ate }\end{array}$ \\
\hline PA0877 & & 298 & $\begin{array}{l}60 \% \text { similar to hypothetical protein } \\
\text { [Acinetobacter lwoffii } \mathrm{K} 24]\end{array}$ & Core & PF00126 & HTH_1 & PF03466 & $\begin{array}{l}\text { LysR_substr } \\
\text { ate }\end{array}$ \\
\hline PA1003 & $\begin{array}{l}\text { MvfR, } \\
\text { PqsR }\end{array}$ & 332 & $\begin{array}{l}99 \% \text { identical to previously identified } \\
\text { hypothetical protein [Pseudomonas } \\
\text { aeruginosa] }\end{array}$ & Core & PF00126 & HTH_1 & PF03466 & $\begin{array}{l}\text { LysR_substr } \\
\text { ate }\end{array}$ \\
\hline PA1067 & & 304 & $\begin{array}{l}54 \% \text { similar to regulator GstR } \\
\text { [Bradyrhizobium japonicum] }\end{array}$ & Core & PF00126 & HTH_1 & PF03466 & $\begin{array}{l}\text { LysR_substr } \\
\text { ate }\end{array}$ \\
\hline PA1128 & & 303 & $\begin{array}{l}60 \% \text { similar to YcaN, hypothetical } \\
\text { transcriptional regulator [Escherichia coli] }\end{array}$ & Core & PF00126 & HTH_1 & PF03466 & $\begin{array}{l}\text { LysR_substr } \\
\text { ate }\end{array}$ \\
\hline PA1138 & & 291 & $\begin{array}{l}49 \% \text { similar to transcription activator } \\
\text { [Azospirillum brasilense] }\end{array}$ & Core & PF00126 & HTH_1 & PF03466 & $\begin{array}{c}\text { LysR_substr } \\
\text { ate }\end{array}$ \\
\hline PA1141 & & 300 & $\begin{array}{l}51 \% \text { similar to probable transcriptional } \\
\text { regulator LrhA [Escherichia coli] }\end{array}$ & Core & PF00126 & HTH_1 & PF03466 & $\underset{\text { ate }}{\text { LysR_substr }}$ \\
\hline PA1145 & & 300 & $\begin{array}{l}40 \% \text { similar to positive transcriptional control } \\
\text { factor SdsB Pseudomonas sp. }\end{array}$ & Core & PF00126 & HTH_1 & PF03466 & $\begin{array}{l}\text { LysR_substr } \\
\text { ate }\end{array}$ \\
\hline PA1184 & & 296 & $\begin{array}{l}59 \% \text { similar to glycine cleavage activator } \\
\text { protein GcvA [Escherichia coli] }\end{array}$ & Core & PF00126 & HTH_1 & PF03466 & $\underset{\text { ate }}{\text { LysR_substr }}$ \\
\hline PA1201 & & 304 & $\begin{array}{l}60 \% \text { similar to putative transcriptional } \\
\text { regulator Synechocystis sp. }\end{array}$ & Core & PF00126 & HTH_1 & PF03466 & $\begin{array}{l}\text { LysR_substr } \\
\text { ate }\end{array}$ \\
\hline PA1223 & & 297 & $\begin{array}{l}53 \% \text { similar to regulator SyrM1 [Rhizobium } \\
\text { sp. NGR234] }\end{array}$ & $\begin{array}{l}\text { RGP } \\
11\end{array}$ & PF00126 & HTH_1 & PF03466 & $\underset{\text { ate }}{\text { LysR_substr }}$ \\
\hline PA1264 & & 288 & $\begin{array}{l}47 \% \text { similar to regulator HexA [Erwinia } \\
\text { carotovora subsp. carotovora] }\end{array}$ & Core & PF00126 & HTH_1 & PF03466 & $\begin{array}{l}\text { LysR_substr } \\
\text { ate }\end{array}$ \\
\hline PA1309 & & 286 & $\begin{array}{l}52 \% \text { similar to putative transcriptional } \\
\text { regulator YwfK [[Bacillus subtilis]] }\end{array}$ & Core & PF00126 & HTH_1 & PF03466 & $\begin{array}{l}\text { LysR_substr } \\
\text { ate }\end{array}$ \\
\hline PA1312 & & 303 & $\begin{array}{l}57 \% \text { similar to PtxR [Pseudomonas } \\
\text { aeruginosa] }\end{array}$ & Core & PF00126 & HTH_1 & PF03466 & $\begin{array}{l}\text { LysR_substr } \\
\text { ate }\end{array}$ \\
\hline PA1328 & & 302 & $\begin{array}{l}55 \% \text { similar to a region of putative } \\
\text { transcriptional regulator YcaN [Escherichia } \\
\text { coli] }\end{array}$ & Core & PF00126 & HTH_1 & PF03466 & $\begin{array}{l}\text { LysR_substr } \\
\text { ate }\end{array}$ \\
\hline PA1399 & & 306 & $\begin{array}{l}49 \% \text { similar to putative regulatory protein } \\
\text { [Pseudomonas aeruginosa] }\end{array}$ & Core & PF00126 & HTH_1 & PF03466 & $\begin{array}{l}\text { LysR_substr } \\
\text { ate }\end{array}$ \\
\hline PA1413 & YneJ & 290 & $\begin{array}{l}69 \% \text { similar to putative transcriptional } \\
\text { regulator YneJ [Escherichia coli] }\end{array}$ & Core & PF00126 & HTH_1 & PF03466 & $\begin{array}{l}\text { LysR_substr } \\
\text { ate }\end{array}$ \\
\hline PA1422 & GbuR & 297 & $\begin{array}{l}49 \% \text { similar to regulatory protein } \mathrm{cfxR} \\
\text { [Alcaligenes eutrophus] }\end{array}$ & Core & PF00126 & HTH_1 & PF03466 & $\begin{array}{c}\text { LysR_substr } \\
\text { ate }\end{array}$ \\
\hline
\end{tabular}




\begin{tabular}{|c|c|c|c|c|c|c|c|c|}
\hline PA1570 & & 293 & $\begin{array}{l}54 \% \text { similar to transcriptional regulator protein } \\
\text { RbcR [Chromatium vinosum] }\end{array}$ & Core & PF00126 & HTH_1 & PF03466 & $\begin{array}{l}\text { LysR_substr } \\
\text { ate }\end{array}$ \\
\hline PA1738 & & 304 & $\begin{array}{l}62 \% \text { similar to putative transcriptional } \\
\text { regulator YcaN [Escherichia coli] }\end{array}$ & Core & PF00126 & HTH_1 & PF03466 & $\begin{array}{l}\text { LysR_substr } \\
\text { ate }\end{array}$ \\
\hline PA1754 & CysB & 324 & & Core & PF00126 & HTH_1 & PF03466 & $\underset{\text { ate }}{\text { LysR_substr }}$ \\
\hline PA1826 & & 301 & $\begin{array}{l}50 \% \text { similar to regulator of catechol } \\
\text { degradation }(\text { CatM) }[\text { Acinetobacter } \\
\text { calcoaceticus] }\end{array}$ & & PF00126 & HTH_1 & PF03466 & $\begin{array}{l}\text { LysR_substr } \\
\text { ate }\end{array}$ \\
\hline PA1853 & & 287 & $\begin{array}{l}80 \% \text { similar to ORF } 286 \text {, hypothetical protein } \\
\text { in the } 30 \text {-kb denitrification gene cluster } \\
\text { [Pseudomonas stutzeri] }\end{array}$ & Core & PF00126 & HTH_1 & PF03466 & $\begin{array}{l}\text { LysR_substr } \\
\text { ate }\end{array}$ \\
\hline PA1859 & & 295 & $\begin{array}{l}69 \% \text { similar to putative transcriptional } \\
\text { regulator YafC [Escherichia coli] }\end{array}$ & Core & PF00126 & HTH_1 & PF03466 & $\begin{array}{l}\text { LysR_substr } \\
\text { ate }\end{array}$ \\
\hline PA1915 & & 515 & & Core & PF07905 & PucR & & \\
\hline PA1961 & & 311 & $\begin{array}{l}50 \% \text { similar to putative transcriptional } \\
\text { regulator YafC [Escherichia coli] }\end{array}$ & Core & PF00126 & HTH_1 & PF03466 & $\begin{array}{l}\text { LysR_substr } \\
\text { ate }\end{array}$ \\
\hline PA1998 & DhcR & 306 & $\begin{array}{l}52 \% \text { similar to ybhD, yfeR, and yfhT } \\
\text { hypothetical transcriptional regulatory proteins } \\
\text { [Escherichia coli] }\end{array}$ & Core & PF00126 & HTH_1 & PF03466 & $\underset{\text { ate }}{\text { LysR_substr }}$ \\
\hline PA2054 & CynR & 295 & $\begin{array}{l}66 \% \text { similar to cynR gene product of } \\
\text { [Escherichia coli] }\end{array}$ & $\begin{array}{l}\text { RGP } \\
20\end{array}$ & PF00126 & HTH_1 & PF03466 & $\underset{\text { ate }}{\text { LysR_substr }}$ \\
\hline PA2056 & & 300 & $\begin{array}{l}54 \% \text { similar to regulator GstR } \\
\text { [Bradyrhizobium japonicum] }\end{array}$ & $\begin{array}{l}\text { RGP } \\
20\end{array}$ & PF00126 & HTH_1 & PF03466 & $\begin{array}{c}\text { LysR_substr } \\
\text { ate }\end{array}$ \\
\hline PA2076 & & 300 & $\begin{array}{l}44 \% \text { similar to putative LysR-type } \\
\text { transcriptional regulator Pseudomonas putida }\end{array}$ & Core & PF00126 & HTH_1 & PF03466 & $\begin{array}{l}\text { LysR_substr } \\
\text { ate }\end{array}$ \\
\hline PA2115 & & 317 & $\begin{array}{l}47 \% \text { similar to putative transcriptional } \\
\text { regulator YrdL [[Bacillus subtilis]] }\end{array}$ & Core & PF00126 & HTH_1 & PF03466 & $\underset{\text { ate }}{\text { LysR_substr }}$ \\
\hline PA2121 & & 301 & $\begin{array}{l}50 \% \text { similar to transcriptional activator nahR } \\
\text { of [Pseudomonas putida-plasmid] }\end{array}$ & Core & PF00126 & HTH_1 & PF03466 & $\begin{array}{l}\text { LysR_substr } \\
\text { ate }\end{array}$ \\
\hline PA2123 & & 310 & $\begin{array}{l}55 \% \text { similar to putative transcriptional } \\
\text { regulator YhcS [Haemophilus influenzae } \mathrm{Rd} \text { ] }\end{array}$ & Core & PF00126 & HTH_1 & PF03466 & $\begin{array}{l}\text { LysR_substr } \\
\text { ate }\end{array}$ \\
\hline PA2206 & & 315 & $\begin{array}{l}54 \% \text { similar to galactose binding protein } \\
\text { regulator [Azospirillum brasilense] }\end{array}$ & Core & PF00126 & HTH_1 & PF03466 & $\begin{array}{l}\text { LysR_substr } \\
\text { ate }\end{array}$ \\
\hline PA2220 & OprR & 306 & $\begin{array}{l}\text { Identical to putative transcriptional regulator } \\
\text { OprR [Pseudomonas aeruginosa] }\end{array}$ & $\begin{array}{l}\text { RGP } \\
23\end{array}$ & PF00126 & HTH_1 & PF03466 & $\begin{array}{l}\text { LysR_substr } \\
\text { ate }\end{array}$ \\
\hline PA2258 & PtxR & 312 & & Core & PF00126 & HTH_1 & PF03466 & $\begin{array}{l}\text { LysR_substr } \\
\text { ate }\end{array}$ \\
\hline PA2267 & & 298 & $\begin{array}{l}45 \% \text { similar to putative transcriptional } \\
\text { activator MauR [Paracoccus denitrificans] }\end{array}$ & Core & PF00126 & HTH_1 & PF03466 & $\underset{\text { ate }}{\text { LysR_substr }}$ \\
\hline PA2316 & & 297 & $\begin{array}{l}55 \% \text { similar to hypothetical yafC gene product } \\
\text { of [Escherichia coli] }\end{array}$ & Core & PF00126 & HTH_1 & PF03466 & $\begin{array}{l}\text { LysR_substr } \\
\text { ate }\end{array}$ \\
\hline
\end{tabular}




\begin{tabular}{|c|c|c|c|c|c|c|c|c|}
\hline PA2334 & & 303 & $\begin{array}{l}56 \% \text { similar to positive transcriptional control } \\
\text { factor SdsB Pseudomonas } \mathrm{sp} \text {. }\end{array}$ & $\begin{array}{l}\text { RGP } \\
53\end{array}$ & PF00126 & HTH_1 & PF03466 & $\underset{\text { ate }}{\text { LysR_substr }}$ \\
\hline PA2383 & & 306 & $\begin{array}{l}50 \% \text { similar to positive regulator of } \mathrm{gcv} \\
\text { operon GcvA [Escherichia coli] }\end{array}$ & Core & PF00126 & HTH_1 & PF03466 & $\begin{array}{l}\text { LysR_substr } \\
\text { ate }\end{array}$ \\
\hline PA2417 & & 312 & $\begin{array}{l}\text { 53\% similar to transcriptional regulator PtxR } \\
\text { [Pseudomonas aeruginosa] }\end{array}$ & Core & PF00126 & HTH_1 & PF03466 & $\begin{array}{c}\text { LysR_substr } \\
\text { ate }\end{array}$ \\
\hline PA2432 & BexR & 304 & $\begin{array}{l}55 \% \text { similar to putative transcriptional } \\
\text { regulator Synechocystis sp. }\end{array}$ & Core & PF00126 & HTH_1 & PF03466 & $\begin{array}{l}\text { LysR_substr } \\
\text { ate }\end{array}$ \\
\hline PA2447 & & 307 & $\begin{array}{l}51 \% \text { similar to hypothetical protein } \\
\text { [Haemophilus influenzae] }\end{array}$ & Core & PF00126 & HTH_1 & PF03466 & $\begin{array}{c}\text { LysR_substr } \\
\text { ate }\end{array}$ \\
\hline PA2469 & & 304 & $\begin{array}{l}52 \% \text { similar to galactose binding protein } \\
\text { regulator [Azospirillum brasilense] }\end{array}$ & Core & PF00126 & HTH_1 & PF03466 & $\begin{array}{l}\text { LysR_substr } \\
\text { ate }\end{array}$ \\
\hline PA2492 & MexT & 347 & $\begin{array}{l}100 \% \text { similar to a region of MexT protein } \\
\text { [Pseudomonas aeruginosa] }\end{array}$ & Core & PF00126 & HTH_1 & PF03466 & $\begin{array}{c}\text { LysR_substr } \\
\text { ate }\end{array}$ \\
\hline PA2497 & & 292 & $\begin{array}{l}52 \% \text { similar to transcriptional regulator HexA } \\
\text { [Erwinia carotovora subsp. atroseptica] }\end{array}$ & Core & PF00126 & HTH_1 & PF03466 & $\begin{array}{l}\text { LysR_substr } \\
\text { ate }\end{array}$ \\
\hline PA2510 & CatR & 290 & $\begin{array}{l}81 \% \text { similar to catR regulatory protein } \\
\text { Pseudomonas putida }\end{array}$ & Core & PF00126 & HTH_1 & PF03466 & $\begin{array}{l}\text { LysR_substr } \\
\text { ate }\end{array}$ \\
\hline PA2534 & & 303 & $\begin{array}{l}58 \% \text { similar to putative transcriptional } \\
\text { regulator YhjC [Escherichia coli] }\end{array}$ & Core & PF00126 & HTH_1 & PF03466 & $\underset{\text { ate }}{\text { LysR_substr }}$ \\
\hline PA2547 & & 305 & $\begin{array}{l}56 \% \text { similar to regulator GstR } \\
{[\text { Bradyrhizobium japonicum }]}\end{array}$ & Core & PF00126 & HTH_1 & PF03466 & $\begin{array}{c}\text { LysR_substr } \\
\text { ate }\end{array}$ \\
\hline PA2551 & & 310 & $\begin{array}{l}54 \% \text { similar to transcriptional activator NahR } \\
\text { [Pseudomonas putida plasmid NAH7] }\end{array}$ & Core & PF00126 & HTH_1 & PF03466 & $\begin{array}{l}\text { LysR_substr } \\
\text { ate }\end{array}$ \\
\hline PA2601 & & 298 & $\begin{array}{l}46 \% \text { similar to activator, hydrogen peroxide- } \\
\text { inducible genes, OxyR [Escherichia coli] }\end{array}$ & Core & PF00126 & HTH_1 & PF03466 & $\begin{array}{c}\text { LysR_substr } \\
\text { ate }\end{array}$ \\
\hline PA2681 & & 309 & $\begin{array}{l}53 \% \text { similar to regulator PtxR [Pseudomonas } \\
\text { aeruginosa] }\end{array}$ & Core & PF00126 & HTH_1 & PF03466 & $\begin{array}{l}\text { LysR_substr } \\
\text { ate }\end{array}$ \\
\hline PA2758 & & 295 & $\begin{array}{l}46 \% \text { similar to DNA binding protein HpkR } \\
{[\text { Pseudomonas syringae pv. syringae }]}\end{array}$ & Core & PF00126 & HTH_1 & PF03466 & $\begin{array}{c}\text { LysR_substr } \\
\text { ate }\end{array}$ \\
\hline PA2834 & & 319 & $\begin{array}{l}47 \% \text { similar to putative regulatory protein } \\
\text { [Pseudomonas aeruginosa] }\end{array}$ & Core & PF00126 & HTH_1 & PF03466 & $\begin{array}{c}\text { LysR_substr } \\
\text { ate }\end{array}$ \\
\hline PA2838 & & 323 & $\begin{array}{l}53 \% \text { similar to regulator GstR } \\
\text { [Bradyrhizobium japonicum }]\end{array}$ & Core & PF00126 & HTH_1 & PF03466 & $\begin{array}{l}\text { LysR_substr } \\
\text { ate }\end{array}$ \\
\hline PA2846 & & 284 & $\begin{array}{l}56 \% \text { similar to } 2,2 \text {-dialkylglycine } \\
\text { decarboxylase repressor protein [Burkholderia } \\
\text { cepacia] }\end{array}$ & Core & PF00126 & HTH_1 & PF03466 & $\begin{array}{l}\text { LysR_substr } \\
\text { ate }\end{array}$ \\
\hline PA2848 & & 339 & $\begin{array}{l}49 \% \text { similar to regulator GstR } \\
\text { [Bradyrhizobium japonicum] }\end{array}$ & Core & PF00126 & HTH_1 & PF03466 & $\underset{\text { ate }}{\text { LysR_substr }}$ \\
\hline PA2877 & & 297 & $\begin{array}{l}62 \% \text { similar to putative transcriptional } \\
\text { regulator YhjC [Escherichia coli] }\end{array}$ & Core & PF00126 & HTH_1 & PF03466 & $\underset{\text { ate }}{\text { LysR_substr }}$ \\
\hline
\end{tabular}




\begin{tabular}{|c|c|c|c|c|c|c|c|c|}
\hline PA2879 & HpkR & 296 & $\begin{array}{l}74 \% \text { similar to putative DNA binding protein } \\
\text { HpkR [Pseudomonas syringae pv. syringae] }\end{array}$ & Core & PF00126 & HTH_1 & PF03466 & $\begin{array}{c}\text { LysR_substr } \\
\text { ate }\end{array}$ \\
\hline PA2921 & & 329 & $\begin{array}{l}50 \% \text { similar to regulator TsaR [Comamonas } \\
\text { testosteroni] }\end{array}$ & Core & PF00126 & HTH_1 & PF03466 & $\begin{array}{l}\text { LysR_substr } \\
\text { ate }\end{array}$ \\
\hline PA2930 & & 308 & $\begin{array}{l}52 \% \text { similar to putative regulatory protein } \operatorname{TrpI} \\
\text { Pseudomonas syringae }\end{array}$ & Core & PF00126 & HTH_1 & PF03466 & $\begin{array}{l}\text { LysR_substr } \\
\text { ate }\end{array}$ \\
\hline PA3122 & YibL & 297 & $\begin{array}{l}\text { 93\% similar to hypothetical gene product YibL } \\
\text { [Azotobacter vinelandii] }\end{array}$ & Core & PF00126 & HTH_1 & PF03466 & $\begin{array}{l}\text { LysR_substr } \\
\text { ate }\end{array}$ \\
\hline PA3124 & & 303 & $\begin{array}{l}59 \% \text { similar to hypothetical gene product YafC } \\
\text { [Escherichia coli] }\end{array}$ & Core & PF00126 & HTH_1 & PF03466 & $\begin{array}{l}\text { LysR_substr } \\
\text { ate }\end{array}$ \\
\hline PA3135 & & 306 & $\begin{array}{l}55 \% \text { similar to putative transcriptional } \\
\text { regulator Synechocystis sp. }\end{array}$ & Core & PF00126 & HTH_1 & PF03466 & $\begin{array}{l}\text { LysR_substr } \\
\text { ate }\end{array}$ \\
\hline PA3225 & & 309 & $\begin{array}{l}48 \% \text { similar to putative transcriptional } \\
\text { regulator Yeey [Escherichia coli] }\end{array}$ & Core & PF00126 & HTH_1 & PF03466 & $\underset{\text { ate }}{\text { LysR_substr }}$ \\
\hline PA3321 & & 304 & $\begin{array}{l}52 \% \text { similar to regulatory protein MexT } \\
\text { [Pseudomonas aeruginosa] }\end{array}$ & Core & PF00126 & HTH_1 & PF03466 & $\begin{array}{l}\text { LysR_substr } \\
\text { ate }\end{array}$ \\
\hline PA3398 & & 308 & $\begin{array}{l}59 \% \text { similar to putative transcriptional } \\
\text { regulator YeiE [Escherichia coli] }\end{array}$ & Core & PF00126 & HTH_1 & PF03466 & $\begin{array}{l}\text { LysR_substr } \\
\text { ate }\end{array}$ \\
\hline PA3433 & YwbI & 297 & $\begin{array}{l}59 \% \text { similar to putative transcriptional } \\
\text { regulator YwbI [[Bacillus subtilis]] }\end{array}$ & Core & PF00126 & HTH_1 & PF03466 & $\underset{\text { ate }}{\text { LysR_substr }}$ \\
\hline PA3565 & & 306 & $\begin{array}{l}50 \% \text { similar to GstR }[\text { Rhizobium } \\
\text { leguminosarum }]\end{array}$ & Core & PF00126 & HTH_1 & PF03466 & $\begin{array}{l}\text { LysR_substr } \\
\text { ate }\end{array}$ \\
\hline PA3587 & MetR & 306 & $\begin{array}{l}63 \% \text { similar to regulator MetR [Escherichia } \\
\text { coli] }\end{array}$ & Core & PF00126 & HTH_1 & PF03466 & $\begin{array}{c}\text { LysR_substr } \\
\text { ate }\end{array}$ \\
\hline PA3594 & & 295 & $\begin{array}{l}55 \% \text { similar to putative transcriptional } \\
\text { regulator YnfL [Escherichia coli] }\end{array}$ & Core & PF00126 & HTH_1 & PF03466 & $\underset{\text { ate }}{\text { LysR_substr }}$ \\
\hline PA3630 & & 302 & $\begin{array}{l}53-56 \% \text { similar to numerous transcriptional } \\
\text { regulators }\end{array}$ & Core & PF00126 & HTH_1 & PF03466 & $\begin{array}{l}\text { LysR_substr } \\
\text { ate }\end{array}$ \\
\hline PA3711 & & 301 & $\begin{array}{l}43 \% \text { similar to putative transcriptional } \\
\text { regulator YgiP [Escherichia coli] }\end{array}$ & Core & PF00126 & HTH_1 & PF03466 & $\begin{array}{l}\text { LysR_substr } \\
\text { ate }\end{array}$ \\
\hline PA3776 & & 302 & $\begin{array}{l}52 \% \text { similar to regulator BudR [Klebsiella } \\
\text { terrigena }]\end{array}$ & Core & PF00126 & HTH_1 & PF03466 & $\begin{array}{c}\text { LysR_substr } \\
\text { ate }\end{array}$ \\
\hline PA3778 & & 311 & $\begin{array}{l}46 \% \text { similar to regulator CbbRI [Rhodobacter } \\
\text { capsulatus] }\end{array}$ & Core & PF00126 & HTH_1 & PF03466 & $\begin{array}{l}\text { LysR_substr } \\
\text { ate }\end{array}$ \\
\hline PA3845 & & 298 & $\begin{array}{l}52 \% \text { similar to positive regulator GcvA } \\
\text { [Escherichia coli] }\end{array}$ & Core & PF00126 & HTH_1 & PF03466 & $\begin{array}{l}\text { LysR_substr } \\
\text { ate }\end{array}$ \\
\hline PA3895 & & 317 & $\begin{array}{l}55 \% \text { similar to putative transcriptional } \\
\text { regulator Synechocystis sp. }\end{array}$ & Core & PF00126 & HTH_1 & PF03466 & $\begin{array}{c}\text { LysR_substr } \\
\text { ate }\end{array}$ \\
\hline PA3995 & & 297 & $\begin{array}{l}51 \% \text { similar to the HexA gene product of } \\
\text { [Erwinia carotovora subsp. atroseptica] }\end{array}$ & Core & PF00126 & HTH_1 & PF03466 & $\begin{array}{c}\text { LysR_substr } \\
\text { ate }\end{array}$ \\
\hline PA4109 & AmpR & 296 & & Core & PF00126 & HTH_1 & PF03466 & $\begin{array}{c}\text { LysR_substr } \\
\text { ate }\end{array}$ \\
\hline
\end{tabular}




\begin{tabular}{|c|c|c|c|c|c|c|c|c|}
\hline PA4145 & & 296 & $\begin{array}{l}48 \% \text { similar to transcriptional activator PtxR } \\
\text { [Pseudomonas aeruginosa] }\end{array}$ & Core & PF00126 & HTH_1 & PF03466 & $\begin{array}{c}\text { LysR_substr } \\
\text { ate }\end{array}$ \\
\hline PA4174 & & 307 & $\begin{array}{l}55 \% \text { similar to putative transcriptional } \\
\text { regulatorYhjC [Escherichia coli] }\end{array}$ & Core & PF00126 & HTH_1 & PF03466 & $\begin{array}{l}\text { LysR_substr } \\
\text { ate }\end{array}$ \\
\hline PA4203 & & 284 & $\begin{array}{l}61 \% \text { similar to putative transcriptional } \\
\text { regulator YneJ [Escherichia coli] }\end{array}$ & Core & PF00126 & HTH_1 & PF03466 & $\underset{\substack{\text { Lete } \\
\text { LysR_substr }}}{ }$ \\
\hline PA4363 & IciA & 300 & $\begin{array}{l}56 \% \text { similar to inhibitor of chromosome } \\
\text { initiaton IciA [Escherichia coli] }\end{array}$ & Core & PF00126 & HTH_1 & PF03466 & $\underset{\text { ate }}{\text { LysR_substr }}$ \\
\hline PA4902 & & 298 & $\begin{array}{l}51 \% \text { similar to putative regulatory protein } \\
\text { Pseudomonas stutzeri] }\end{array}$ & Core & PF00126 & HTH_1 & PF03466 & $\begin{array}{c}\text { LysR_substr } \\
\text { ate }\end{array}$ \\
\hline PA4914 & & 312 & $\begin{array}{l}53 \% \text { similar to positive regulator GcvA } \\
\text { [Escherichia coli] }\end{array}$ & Core & PF00126 & HTH_1 & PF03466 & $\begin{array}{l}\text { LysR_substr } \\
\text { ate }\end{array}$ \\
\hline PA4989 & & 294 & $\begin{array}{l}46 \% \text { similar to DNA binding protein HpkR } \\
{[\text { Pseudomonas syringae pv. syringae }]}\end{array}$ & Core & PF00126 & HTH_1 & PF03466 & $\begin{array}{c}\text { LysR_substr } \\
\text { ate }\end{array}$ \\
\hline PA5029 & YnfL & 301 & $\begin{array}{l}72 \% \text { similar to putative transcriptional } \\
\text { regulator YnfL [Escherichia coli] }\end{array}$ & Core & PF00126 & HTH_1 & PF03466 & $\begin{array}{l}\text { LysR_substr } \\
\text { ate }\end{array}$ \\
\hline PA5085 & & 318 & $\begin{array}{l}47 \% \text { similar to regulatory protein LysR } \\
\text { [Escherichia coli] }\end{array}$ & Core & PF00126 & HTH_1 & PF03466 & $\underset{\substack{\text { Lete } \\
\text { LysR_substr }}}{ }$ \\
\hline PA5179 & & 293 & $\begin{array}{l}47 \% \text { similar to putative LysR-type } \\
\text { transcriptional regulator [Mycobacterium } \\
\text { leprae] }\end{array}$ & Core & PF00126 & HTH_1 & PF03466 & $\begin{array}{l}\text { LysR_substr } \\
\text { ate }\end{array}$ \\
\hline PA5189 & & 302 & $\begin{array}{l}51 \% \text { similar to putative regulatory protein } \\
\text { [Pseudomonas aeruginosa] }\end{array}$ & Core & PF00126 & HTH_1 & PF03466 & $\begin{array}{l}\text { LysR_substr } \\
\text { ate }\end{array}$ \\
\hline PA5218 & & 304 & $\begin{array}{l}57 \% \text { similar to hypothetical ynfL gene product } \\
\text { of [Escherichia coli] }\end{array}$ & Core & PF00126 & HTH_1 & PF03466 & $\begin{array}{l}\text { LysR_substr } \\
\text { ate }\end{array}$ \\
\hline PA5293 & & 305 & $\begin{array}{l}53 \% \text { similar to positive regulator GcvA } \\
\text { [Escherichia coli] }\end{array}$ & Core & PF00126 & HTH_1 & PF03466 & $\begin{array}{c}\text { LysR_substr } \\
\text { ate }\end{array}$ \\
\hline PA5344 & OxyR & 310 & $\begin{array}{l}60 \% \text { similar to oxyR gene product of } \\
\text { [Escherichia coli] }\end{array}$ & Core & PF00126 & HTH_1 & PF03466 & $\begin{array}{l}\text { LysR_substr } \\
\text { ate }\end{array}$ \\
\hline PA5382 & YeiE & 297 & $\begin{array}{l}64 \% \text { similarity to putative transcriptional } \\
\text { regulator YeiE [Escherichia coli] }\end{array}$ & Core & PF00126 & HTH_1 & PF03466 & $\begin{array}{c}\text { LysR_substr } \\
\text { ate }\end{array}$ \\
\hline PA5428 & & 302 & $\begin{array}{l}56 \% \text { similar to putative transcriptional } \\
\text { regulator YjiE [Escherichia coli] }\end{array}$ & Core & PF00126 & HTH_1 & PF03466 & $\begin{array}{c}\text { LysR_substr } \\
\text { ate }\end{array}$ \\
\hline PA5437 & & 311 & $\begin{array}{l}62 \% \text { similar to regulator CbbRI [Rhodobacter } \\
\text { capsulatus] }\end{array}$ & Core & PF00126 & HTH_1 & PF03466 & $\begin{array}{c}\text { LysR_substr } \\
\text { ate }\end{array}$ \\
\hline PA5261 & AlgR & 248 & $\begin{array}{l}88 \% \text { similar to encystment and alginate } \\
\text { biosynthesis regulatory protein [Azotobacter } \\
\text { vinelandii] }\end{array}$ & Core & PF00072 & $\begin{array}{l}\text { Response } \\
\text { regulator } \\
\text { receiver } \\
\text { domain } \\
\end{array}$ & PF04397 & $\begin{array}{l}\text { LytTR, } \\
\text { LytT DNA- } \\
\text { binding } \\
\text { domain } \\
\end{array}$ \\
\hline
\end{tabular}




\begin{tabular}{|c|c|c|c|c|c|c|c|c|c|c|}
\hline PA2016 & $\begin{array}{l}\text { LiuR, } \\
\text { GnyR }\end{array}$ & 134 & $\begin{array}{l}85 \% \text { identical to transcriptional regulator, } \\
\text { putative [P. putida KT2440]; } 59 \% \text { similar to } \\
\text { putative transcriptional regulator YbbI } \\
\text { [Escherichia coli] }\end{array}$ & Core & PF00376 & MerR & PF09278 & $\begin{array}{l}\text { MerR- } \\
\text { DNA-bind }\end{array}$ & & \\
\hline PA2273 & SoxR & 156 & $\begin{array}{l}\text { Identical to unpublished SoxR of } P \text {. } \\
\text { aeruginosa (gi|2495412|sp|Q51506|);77\% } \\
\text { similar to SoxR gene product of [Escherichia } \\
\text { coli] }\end{array}$ & Core & $\begin{array}{l}\mathrm{PB} 00020 \\
0\end{array}$ & Pfam-B_200 & PF00376 & MerR & $\begin{array}{l}\text { PB01600 } \\
9 ; \\
\text { PF09278 }\end{array}$ & $\begin{array}{l}\text { Pfam- } \\
\text { B_16009; } \\
\text { MerR- } \\
\text { DNA- } \\
\text { binding }\end{array}$ \\
\hline PA2718 & & 162 & $\begin{array}{l}52 \% \text { similar to putative transcriptional } \\
\text { regulator YbbI [Escherichia coli] }\end{array}$ & Core & PF00376 & MerR & PF09278 & $\begin{array}{l}\text { MerR- } \\
\text { DNA-bind }\end{array}$ & & \\
\hline PA3689 & YhdM & 156 & $\begin{array}{l}66 \% \text { similar to putative regulatory protein } \\
\text { YhdM [Haemophilus influenzae] }\end{array}$ & Core & PF00376 & MerR & PF09278 & $\begin{array}{l}\text { MerR- } \\
\text { DNA-bind }\end{array}$ & & \\
\hline PA4659 & & 299 & $\begin{array}{l}47 \% \text { similar to putative transcriptional } \\
\text { regulator YcgE [Escherichia coli] }\end{array}$ & Core & $\begin{array}{l}\text { PB00020 } \\
0\end{array}$ & Pfam-B_200 & PF00376 & MerR & & \\
\hline PA4778 & $\begin{array}{l}\text { CueR, } \\
\text { YbbI }\end{array}$ & 132 & $\begin{array}{l}65 \% \text { similar to putative transcriptional } \\
\text { regulator YbbI [Escherichia coli] }\end{array}$ & Core & PF00376 & MerR & PF05103 & DivIVA & PF09278 & $\begin{array}{l}\text { MerR- } \\
\text { DNA- } \\
\text { binding }\end{array}$ \\
\hline PA4878 & & 270 & $\begin{array}{l}47 \% \text { similar to a region of multi-drug efflux } \\
\text { transporter regulator TipA [Streptomyces } \\
\text { lividans] }\end{array}$ & Core & PF00376 & MerR & PF06445 & $\begin{array}{l}\text { AraC- } \\
\text { binding } \\
\text { domain }\end{array}$ & & \\
\hline PA5116 & & 141 & $\begin{array}{l}49 \% \text { similar to activator SoxR [Escherichia } \\
\text { coli] }\end{array}$ & Core & PF00376 & MerR & PF09278 & $\begin{array}{l}\text { MerR- } \\
\text { DNA-bind }\end{array}$ & & \\
\hline \multicolumn{11}{|c|}{ OmpC family } \\
\hline PA5200 & $\mathrm{AmgR}$ & 247 & OmpR similar E.coli & Core & PF00486 & $\begin{array}{l}\text { Transcriptiona } \\
1 \text { regulatory } \\
\text { protein, } \mathrm{C} \\
\text { terminal }\end{array}$ & PF00072 & $\begin{array}{l}\text { Response } \\
\text { regulator } \\
\text { receiver } \\
\text { domain }\end{array}$ & & \\
\hline \multicolumn{11}{|c|}{ OmpR-PhoP class } \\
\hline PA4032 & & 238 & $\begin{array}{l}52 \% \text { similar to hypothetical protein Rv1033c } \\
\text { (response regulator homolog TrcR) } \\
\text { [Mycobacterium tuberculosis]; } 51 \% \text { similar to } \\
\text { phosphate regulatory protein PhoB } \\
{[\text { Bradyrhizobium japonicum }]}\end{array}$ & & PF0072 & $\begin{array}{l}\text { Response } \\
\text { regulator } \\
\text { receiver } \\
\text { domain }\end{array}$ & PF00486 & $\begin{array}{l}\text { Transcriptio } \\
\text { nal } \\
\text { regulatory } \\
\text { protein, C } \\
\text { terminal }\end{array}$ & & \\
\hline PA4381 & & 227 & $\begin{array}{l}91 \% \text { similar to ColR [Pseudomonas } \\
\text { fluorescens] }\end{array}$ & Core & PF00072 & $\begin{array}{l}\text { Response } \\
\text { regulator } \\
\text { receiver } \\
\text { domain }\end{array}$ & PF00486 & $\begin{array}{l}\text { Transcriptio } \\
\text { nal } \\
\text { regulatory } \\
\text { protein, C } \\
\text { terminal }\end{array}$ & & \\
\hline
\end{tabular}




\begin{tabular}{|c|c|c|c|c|c|c|c|}
\hline PA5506 & & 285 & $44 \%$ similar to RpiR protein [Escherichia coli] & Core & PF01418 & RpiR family & $\begin{array}{cc}\text { PF01380 } & \begin{array}{c}\text { SIS (Sugar } \\
\text { ISomerase) } \\
\text { domain }\end{array}\end{array}$ \\
\hline \multicolumn{8}{|l|}{ TetR } \\
\hline PA0167 & & 221 & $\begin{array}{l}60 \% \text { similar to putative regulator YcdC } \\
\text { [Escherichia coli] }\end{array}$ & Core & PF08362 & $\begin{array}{l}\text { TetR_C_3, } \\
\text { YcdC-like } \\
\text { protein, C- } \\
\text { terminal } \\
\text { region }\end{array}$ & \\
\hline PA0243 & & 222 & $\begin{array}{l}44 \% \text { similar to putative transcriptional } \\
\text { regulator [Methanobacterium } \\
\text { thermoautotrophicum] }\end{array}$ & Core & PF00440 & $\begin{array}{l}\text { TetR_N, } \\
\text { Bacterial } \\
\text { regulatory } \\
\text { proteins, tetR } \\
\text { family }\end{array}$ & \\
\hline PA0294 & AguR & 221 & $\begin{array}{l}49 \% \text { similar to putative transcriptional } \\
\text { regulator [Streptomyces coelicolor] }\end{array}$ & Core & PF00440 & $\begin{array}{l}\text { TetR_N, } \\
\text { Bacterial } \\
\text { regulatory } \\
\text { proteins, tetR } \\
\text { family }\end{array}$ & \\
\hline PA0367 & & 215 & $\begin{array}{l}53 \% \text { similar to hypothetical protein } \\
\text { Pseudomonas putida }\end{array}$ & Core & & & \\
\hline PA0436 & & 206 & $\begin{array}{l}54 \% \text { similar to putative tet operon regulator } \\
\text { YcdC [Escherichia coli] }\end{array}$ & Core & PF08362 & $\begin{array}{l}\text { TetR_C_3, } \\
\text { YcdC-like } \\
\text { protein, C- } \\
\text { terminal } \\
\text { region }\end{array}$ & \\
\hline PA0475 & & 189 & $\begin{array}{l}\text { 47\% similar to hypothetical protein Rv0196 } \\
\text { [Mycobacterium tuberculosis] }\end{array}$ & Core & & & \\
\hline PA0828 & & 227 & $\begin{array}{l}50 \% \text { similar to a region of putative } \\
\text { transcriptional regulator [Aquifex aeolicus] }\end{array}$ & Core & & & \\
\hline PA0839 & & 196 & $\begin{array}{l}48 \% \text { similar to hypothetical protein } \\
\text { [Escherichia coli] }\end{array}$ & Core & & & \\
\hline PA1226 & & 202 & $\begin{array}{l}45 \% \text { similar to putative transcriptional } \\
\text { regulator [Streptomyces coelicolor] }\end{array}$ & Core & & & \\
\hline PA1241 & & 186 & $\begin{array}{l}46 \% \text { similar to hypothetical protein } \mathrm{YxaF} \\
\text { [Bacillus subtilis] }\end{array}$ & Core & & & \\
\hline PA1283 & & 186 & $\begin{array}{l}48 \% \text { similar to hypothetical protein } \\
\text { Synechocystis sp. }\end{array}$ & Core & & & \\
\hline PA1290 & & 197 & $\begin{array}{l}54 \% \text { similar to unidentified reading frame } \mathrm{L} \\
\text { (ORFL TetC protein) in transposon Tn10 } \\
\text { [Escherichia coli] }\end{array}$ & Core & & & \\
\hline PA1315 & & 204 & $\begin{array}{l}49 \% \text { similar to MtrR protein [Neisseria } \\
\text { gonorrhoeae] }\end{array}$ & Core & & & \\
\hline
\end{tabular}




\begin{tabular}{|c|c|c|c|c|c|c|}
\hline \multicolumn{2}{|l|}{ PA1403 } & \multirow{2}{*}{$\begin{array}{l}210 \\
216\end{array}$} & \multirow{2}{*}{$\begin{array}{l}\text { 43\% similar to a region of hypothetical protein } \\
\text { Rv1963c [Mycobacterium tuberculosis] } \\
\text { 57\% similar to putative tet operon regulator } \\
\text { YcdC [Escherichia coli] }\end{array}$} & \multicolumn{2}{|l|}{ Core } & \multirow[b]{2}{*}{$\begin{array}{l}\text { TetR_C_3, } \\
\text { YcdC-like } \\
\text { protein, C- } \\
\text { terminal } \\
\text { region }\end{array}$} \\
\hline PA1504 & & & & Core & PF08362 & \\
\hline PA1539 & & 282 & $\begin{array}{l}53 \% \text { similar to a region of hypothetical protein } \\
\text { YijC [Escherichia coli] }\end{array}$ & & PF00440 & \\
\hline PA1836 & & 193 & $\begin{array}{l}45 \% \text { similar to putative repressor [Bacillus } \\
\text { megaterium] }\end{array}$ & Core & & \\
\hline PA1864 & & 216 & $\begin{array}{l}54 \% \text { similar to putative tet operon regulator } \\
\text { YcdC [Escherichia coli] }\end{array}$ & Core & PF08362 & $\begin{array}{l}\text { TetR_C_3, } \\
\text { YcdC-like } \\
\text { protein, C- } \\
\text { terminal } \\
\text { region }\end{array}$ \\
\hline PA2020 & & 210 & $\begin{array}{l}72 \% \text { similar to transcriptional regulator AmrR } \\
\text { [Burkholderia pseudomallei }]\end{array}$ & Core & PF08361 & $\begin{array}{l}\text { TetR_C_2, } \\
\text { MAATS-type } \\
\text { transcriptional } \\
\text { repressor, C- } \\
\text { terminal } \\
\text { region }\end{array}$ \\
\hline PA2 196 & & 194 & $\begin{array}{l}63 \% \text { similar to hypothetical protein } \\
\text { [Escherichia coli] }\end{array}$ & Core & & \\
\hline PA2270 & & 196 & $\begin{array}{l}52 \% \text { similar to hypothetical protein } \\
\text { Synechocystis } \mathrm{sp} \text {. }\end{array}$ & Core & & \\
\hline PA2484 & & 204 & $\begin{array}{l}65 \% \text { similar to SocA } 3 \text { protein Myxococcus } \\
\text { xanthus }\end{array}$ & Core & & \\
\hline PA2766 & & 193 & & Core & & \\
\hline PA2885 & AtuR & 198 & $\begin{array}{l}50 \% \text { similar to hypothetical protein Rv3557c } \\
\text { [Mycobacterium tuberculosis] }\end{array}$ & Core & & \\
\hline PA2931 & CifR & 196 & $\begin{array}{l}63 \% \text { similar to socA3 protein } \text { Myxococcus } \\
\text { xanthus }\end{array}$ & Core & & \\
\hline PA2957 & & 212 & $\begin{array}{l}44 \% \text { similar to putative transcriptional } \\
\text { regulator [Streptomyces coelicolor] }\end{array}$ & Core & & \\
\hline PA3006 & PsrA & 233 & $90 \%$ similar to PsrA Pseudomonas putida & Core & & \\
\hline PA3034 & & 185 & $\begin{array}{l}54 \% \text { similar to putative transcriptional } \\
\text { regulator Streptomyces lividans }\end{array}$ & Core & & \\
\hline PA3133 & & 180 & & Core & & \\
\hline
\end{tabular}




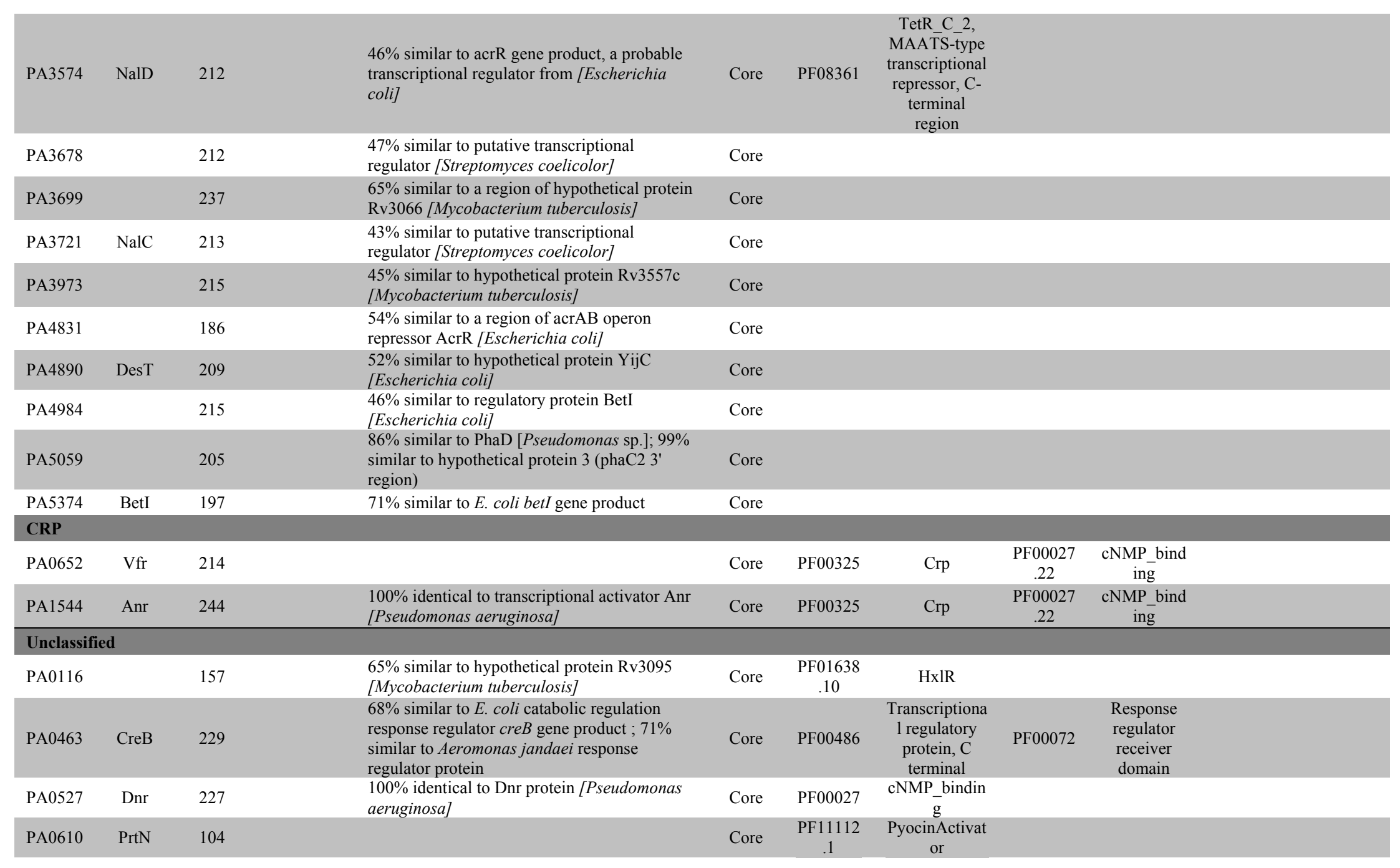




\begin{tabular}{|c|c|c|c|c|c|c|c|c|c|}
\hline PA0707 & $\begin{array}{l}\text { ToxR, } \\
\text { RegA }\end{array}$ & 259 & & $\begin{array}{l}99 \% \text { identity to ToxR (exotoxin A regulatory } \\
\text { protein) }[P \text {. aeruginosa }]\end{array}$ & Core & PF07720 & $\begin{array}{l}\text { TPR_3, } \\
\text { tetratricopepti } \\
\text { de repeat }\end{array}$ & & \\
\hline PA0756 & & 223 & & $\begin{array}{l}72 \% \text { similarity to trans-activating } \\
\text { transcriptional regulatory protein tctD } \\
{[\text { Salmonella typhimurium }]}\end{array}$ & Core & PF00486 & & PF00072 & $\begin{array}{l}\text { Response } \\
\text { regulator } \\
\text { receiver } \\
\text { domain }\end{array}$ \\
\hline PA0929 & & 239 & & $\begin{array}{l}53 \% \text { similar to Response-regulator BaeR } \\
\text { protein [Escherichia coli]; } 64 \% \text { similar to PfeR } \\
\text { protein [Pseudomonas aeruginosa] }\end{array}$ & Core & PF00486 & $\begin{array}{l}\text { Transcriptiona } \\
1 \text { regulatory } \\
\text { protein, C } \\
\text { terminal }\end{array}$ & PF00072 & $\begin{array}{l}\text { Response } \\
\text { regulator } \\
\text { receiver } \\
\text { domain }\end{array}$ \\
\hline PA1157 & & 236 & & $\begin{array}{l}63 \% \text { similar to transcriptional regulatory } \\
\text { protein RstA (RstB sensor) [Escherichia coli] } \\
64 \% \text { similar to response regulator OmpR } \\
\text { (sensor, EnvZ) }\end{array}$ & Core & PF00072 & $\begin{array}{l}\text { Response } \\
\text { regulator } \\
\text { receiver } \\
\text { domain }\end{array}$ & PF00486 & $\begin{array}{l}\text { Transcriptio } \\
\text { nal } \\
\text { regulatory } \\
\text { protein, C } \\
\text { terminal }\end{array}$ \\
\hline PA1179 & PhoP & 225 & & $\begin{array}{l}71 \% \text { similar to regulatory protein PhoP } \\
{[\text { Salmonella typhimurium }] ; 62 \% \text { similar to }} \\
\text { trans-activating protein tctD }[\text { Salmonella } \\
\text { typhimurium }]\end{array}$ & Core & PF00486 & $\begin{array}{l}\text { Transcriptiona } \\
1 \text { regulatory } \\
\text { protein, C } \\
\text { terminal }\end{array}$ & PF00072 & $\begin{array}{l}\text { Response } \\
\text { regulator } \\
\text { receiver } \\
\text { domain }\end{array}$ \\
\hline PA1431 & RsaL & 80 & & & $? ?$ & & & & \\
\hline PA1437 & & 229 & & $\begin{array}{l}81 \% \text { similar to transcriptional activator protein } \\
\text { CzcR }[\text { Ralstonia eutropha }] ; 74 \% \text { similar to } \\
\text { transcriptional activator protein CopR } \\
{[\text { Pseudomonas syringae }]}\end{array}$ & Core & PF00486 & $\begin{array}{l}\text { Transcriptiona } \\
1 \text { regulatory } \\
\text { protein, C } \\
\text { terminal }\end{array}$ & PF00072 & $\begin{array}{l}\text { Response } \\
\text { regulator } \\
\text { receiver } \\
\text { domain }\end{array}$ \\
\hline PA1607 & & 146 & & $\begin{array}{l}60 \% \text { similar to hypothetical protein } \\
\text { Rv3095[Mycobacterium tuberculosis] }\end{array}$ & Core & $\begin{array}{c}\text { PF01638 } \\
.10\end{array}$ & HxIR & & \\
\hline PA1637 & $\mathrm{KdpE}$ & & 230 & $69 \%$ similar to $k d p E$ gene product of $[E$. coli] & & PF00486 & $\begin{array}{l}\text { Transcriptiona } \\
1 \text { regulatory } \\
\text { protein, C } \\
\text { terminal }\end{array}$ & PF00072 & $\begin{array}{l}\text { Response } \\
\text { regulator } \\
\text { receiver } \\
\text { domain }\end{array}$ \\
\hline PA1704 & PcrR & 144 & & $\begin{array}{l}\text { 64\% similar to LcrR [Yersinia enterocolitica] } \\
99 \% \text { similar to PcrR [Pseudomonas } \\
\text { aeruginosa]; } 64 \% \text { similar to low calcium } \\
\text { response locusprotein LcrR [Yersinia pestis] }\end{array}$ & Core & $\begin{array}{l}\text { PF09621 } \\
.3\end{array}$ & LcrR & & \\
\hline PA1799 & ParR & 235 & & $64 \%$ similar to RstA [Escherichia coli] & Core & PF00072 & $\begin{array}{l}\text { Response } \\
\text { regulator } \\
\text { receiver } \\
\text { domain }\end{array}$ & PF00486 & $\begin{array}{l}\text { Transcriptio } \\
\quad \text { nal } \\
\text { regulatory } \\
\text { protein, C } \\
\text { terminal }\end{array}$ \\
\hline PA2479 & & 226 & & $\begin{array}{l}64 \% \text { similar to putative } 2 \text {-component } \\
\text { transcriptional regulator YgiX [Escherichia } \\
\text { coli] }\end{array}$ & Core & PF00486 & $\begin{array}{l}\text { Transcriptiona } \\
1 \text { regulatory } \\
\text { protein, C } \\
\text { terminal }\end{array}$ & PF00072 & $\begin{array}{l}\text { Response } \\
\text { regulator } \\
\text { receiver } \\
\text { domain }\end{array}$ \\
\hline
\end{tabular}




\begin{tabular}{|c|c|c|c|c|c|c|c|c|}
\hline PA2523 & & 224 & $\begin{array}{l}75 \% \text { similar to regulator protein } \mathrm{CzcR} \\
{[\text { Ralstonia eutropha }]}\end{array}$ & Core & PF00486 & $\begin{array}{l}\text { Transcriptiona } \\
1 \text { regulatory } \\
\text { protein, C } \\
\text { terminal }\end{array}$ & PF00072 & $\begin{array}{l}\text { Response } \\
\text { regulator } \\
\text { receiver } \\
\text { domain }\end{array}$ \\
\hline PA2572 & & 447 & $\begin{array}{l}57 \% \text { similar to a region of putative DNA- } \\
\text { binding protein HupR1 Rhodobacter } \\
\text { capsulatus }\end{array}$ & Core & PF00072 & $\begin{array}{l}\text { Response } \\
\text { regulator } \\
\text { receiver } \\
\text { domain }\end{array}$ & & \\
\hline PA2657 & & 223 & $\begin{array}{l}62 \% \text { similar to putative 2-component } \\
\text { transcriptional regulator YgiX [Escherichia } \\
\text { coli] }\end{array}$ & Core & PF00072 & $\begin{array}{l}\text { Response } \\
\text { regulator } \\
\text { receiver } \\
\text { domain }\end{array}$ & PF00486 & $\begin{array}{l}\text { Transcriptio } \\
\text { nal } \\
\text { regulatory } \\
\text { protein, C } \\
\text { terminal }\end{array}$ \\
\hline PA2686 & PfeR & 305 & $\begin{array}{l}\text { PfeR activator of the ferric enterobactin } \\
\text { receptor (PfeA) }\end{array}$ & Core & PF00486 & $\begin{array}{l}\text { Transcriptiona } \\
1 \text { regulatory } \\
\text { protein, C } \\
\text { terminal }\end{array}$ & PF00072 & $\begin{array}{l}\text { Response } \\
\text { regulator } \\
\text { receiver } \\
\text { domain }\end{array}$ \\
\hline PA2713 & & 159 & $\begin{array}{l}\text { 61\% similar to hypothetical protein Rv3095 } \\
\text { [Mycobacterium tuberculosis] }\end{array}$ & Core & $\begin{array}{l}\text { PF01638 } \\
.10\end{array}$ & HxlR & & \\
\hline PA2736 & & 117 & & $\begin{array}{l}\text { RGP } \\
28\end{array}$ & $\begin{array}{l}\text { PF01047 } \\
.15\end{array}$ & MarR & & \\
\hline PA2809 & CopR & 226 & $\begin{array}{l}82 \% \text { similar to transcriptional activator CopR } \\
\text { Pseudomonas syringae }\end{array}$ & Core & PF00072 & $\begin{array}{l}\text { Response } \\
\text { regulator } \\
\text { receiver } \\
\text { domain }\end{array}$ & PF00486 & $\begin{array}{c}\text { Transcriptio } \\
\text { nal } \\
\text { regulatory } \\
\text { protein, C } \\
\text { terminal }\end{array}$ \\
\hline \multicolumn{9}{|l|}{ PA2881 } \\
\hline PA3007 & LexA & 204 & $\begin{array}{l}77 \% \text { similar to LexA protein [Escherichia } \\
\text { coli] }\end{array}$ & Core & PF01726 & $\underset{\text { ind }}{\text { LexA_DNA_b }}$ & $\begin{array}{l}\text { PF00717 } \\
.16\end{array}$ & $\begin{array}{c}\text { Peptidase_S } \\
24\end{array}$ \\
\hline PA3077 & & 223 & $\begin{array}{l}68 \% \text { similar to ColR [Pseudomonas } \\
\text { fluorescens] }\end{array}$ & Core & PF00486 & $\begin{array}{l}\text { Transcriptiona } \\
1 \text { regulatory } \\
\text { protein, C } \\
\text { terminal }\end{array}$ & PF00072 & $\begin{array}{l}\text { Response } \\
\text { regulator } \\
\text { receiver } \\
\text { domain }\end{array}$ \\
\hline PA3192 & GltR & 242 & $\begin{array}{l}99 \% \text { similar to GltR [Pseudomonas } \\
\text { aeruginosa] }\end{array}$ & Core & PF00486 & $\begin{array}{l}\text { Transcriptiona } \\
1 \text { regulatory } \\
\text { protein, C } \\
\text { terminal }\end{array}$ & PF00072 & $\begin{array}{l}\text { Response } \\
\text { regulator } \\
\text { receiver } \\
\text { domain }\end{array}$ \\
\hline PA3204 & & 225 & $65 \%$ similar to $E$. coli cpxR gene product. & Core & PF00486 & $\begin{array}{l}\text { Transcriptiona } \\
1 \text { regulatory } \\
\text { protein, C } \\
\text { terminal }\end{array}$ & PF00072 & $\begin{array}{l}\text { Response } \\
\text { regulator } \\
\text { receiver } \\
\text { domain }\end{array}$ \\
\hline PA3385 & AmrZ & 108 & & Core & PF03869 & Arc & & \\
\hline
\end{tabular}




\begin{tabular}{|c|c|c|c|c|c|c|c|c|}
\hline PA3864 & DauR & 210 & $\begin{array}{l}44 \% \text { similar to hypothetical protein } \\
\text { [[Streptomyces coelicolor] A3(2)] }\end{array}$ & Core & $\begin{array}{l}\text { PF08348 } \\
.4\end{array}$ & PAS_6 & & \\
\hline PA4057 & $\begin{array}{l}\text { NrdR, } \\
\text { YbaD }\end{array}$ & 154 & $\begin{array}{l}82 \% \text { similar to hypothetical ybaD gene product } \\
\text { of [Escherichia coli] }\end{array}$ & Core & $\begin{array}{c}\text { PF03477 } \\
.9\end{array}$ & ATP-cone & & \\
\hline PA4101 & $\mathrm{BfmR}$ & 246 & $\begin{array}{l}\text { 63\% similar to glucose uptake regulatory gene } \\
\text { glrR [Pseudomonas aeruginosa] }\end{array}$ & $\begin{array}{c}\text { RGP } \\
44\end{array}$ & PF00072 & $\begin{array}{l}\text { Response } \\
\text { regulator } \\
\text { receiver } \\
\text { domain }\end{array}$ & PF00486 & $\begin{array}{l}\text { Transcriptio } \\
\quad \text { nal } \\
\text { regulatory } \\
\text { protein, C } \\
\text { terminal }\end{array}$ \\
\hline PA4182 & & 212 & $\begin{array}{l}51 \% \text { similar to hypothetical protein YKL070w } \\
\text { [Saccharomyces cerevisiae] }\end{array}$ & Core & $\begin{array}{l}\text { PF04299 } \\
.5\end{array}$ & FMN_bind_2 & & \\
\hline PA4776 & PmrA & 221 & $\begin{array}{l}70 \% \text { similar to putative } 2 \text {-component } \\
\text { transcriptional regulator YgiX [Escherichia } \\
\text { coli] }\end{array}$ & Core & PF00486 & $\begin{array}{l}\text { Transcriptiona } \\
1 \text { regulatory } \\
\text { protein, C } \\
\text { terminal }\end{array}$ & PF00072 & $\begin{array}{l}\text { Response } \\
\text { regulator } \\
\text { receiver } \\
\text { domain }\end{array}$ \\
\hline PA4885 & IrlR & 229 & $\begin{array}{l}79 \% \text { similar to IrlR gene product of } \\
\text { Burkholderia pseudomallei; } 81 \% \text { similar to } \\
\text { czcR }[\text { Ralstonia eutropha]; } 78 \% \text { similar to } \\
\text { transcriptional activatorprotein CopR } \\
\text { [Pseudomonas syringae] }\end{array}$ & Core & PF00072 & $\begin{array}{l}\text { Response } \\
\text { regulator } \\
\text { receiver } \\
\text { domain }\end{array}$ & PF00486 & $\begin{array}{l}\text { Transcriptio } \\
\quad \text { nal } \\
\text { regulatory } \\
\text { protein, C } \\
\text { terminal }\end{array}$ \\
\hline PA4983 & & 244 & $\begin{array}{l}63 \% \text { similar to DmsR [Rhodobacter } \\
\text { sphaeroides] }\end{array}$ & Core & PF00072 & $\begin{array}{l}\text { Response } \\
\text { regulator } \\
\text { receiver } \\
\text { domain }\end{array}$ & PF00486 & $\begin{array}{l}\text { Transcriptio } \\
\text { nal } \\
\text { regulatory } \\
\text { protein, C } \\
\text { terminal }\end{array}$ \\
\hline PA5360 & PhoB & 229 & $\begin{array}{l}96 \% \text { similar to } p h o B \text { gene product of }[P . \\
\text { aeruginosa }]\end{array}$ & Core & PF00072 & $\begin{array}{l}\text { Response } \\
\text { regulator } \\
\text { receiver } \\
\text { domain }\end{array}$ & PF00486 & $\begin{array}{l}\text { Transcriptio } \\
\text { nal } \\
\text { regulatory } \\
\text { protein, C } \\
\text { terminal }\end{array}$ \\
\hline
\end{tabular}


Supplementary Table 2: Transcription factor interacting partners. Genes that are differentially regulated by each transcriptional regulator were identified using the Pseudomonas database (Winsor et al., 2009), published data (Madan Babu et al., 2006) and data mining using STRING 8.3 (http://string.embl.de/). The data presented in this table was used as the input data for Cytoscape (www.cytoscape.org) to generate Fig 3.2.

\begin{tabular}{|c|c|c|c|}
\hline ORF \# & $\begin{array}{l}\text { Gene } \\
\text { Name }\end{array}$ & $\begin{array}{l}\text { No. of } \\
\text { regulated } \\
\text { genes }\end{array}$ & Genes regulated \\
\hline PA0652 & $v f r$ & 96 & $\begin{array}{l}\text { PA0066, PA0165, PA0224, PA0265, PA0347, PA0376, PA0551, PA0552, PA0589, PA0653, PA0689, PA0783, PA0836, PA1092, PA1097, PA1183, } \\
\text { PA1288, PA1337, PA1384, PA1430, PA1562, PA1580, PA1581, PA1582, PA1583, PA1584, PA1585, PA1586, PA1587, PA1588, PA1589, PA1629, }\end{array}$ \\
\hline PA1544 & $a n r$ & 77 & $\begin{array}{l}\text { PA0836, PA1172, PA1173, PA1174, PA1175, PA1176, PA1317, PA1318, PA1319, PA1320, PA1321, PA1337, PA1475, PA1476, PA1477, PA1479, } \\
\text { PA1480, PA1481, PA1482, PA1483, PA1546, PA1561, PA1562, PA1581, PA1582, PA1583, PA1584, PA1585, PA1586, PA1588, PA1589, PA1780, }\end{array}$ \\
\hline PA2738 & $\operatorname{him} A$ & 57 & $\begin{array}{l}\text { PA0066, PA0353, PA0482, PA0836, PA0888, PA0962, PA0993, PA0994, PA1326, PA1339, PA1376, PA1580, PA1585, PA1586, PA1588, PA1589, } \\
\text { PA1629, PA1631, PA1818, PA2634, PA2637, PA2638, PA2639, PA2640, PA2641, PA2642, PA2643, PA2644, PA2645, PA2646, PA2647, PA2648, }\end{array}$ \\
\hline PA4983 & & 52 & $\begin{array}{l}\text { PA0265, PA0482, PA0599, PA0854, PA1183, PA1317, PA1318, PA1319, PA1320, PA1321, PA1376, PA1562, PA1580, PA1581, PA1582, PA1583, } \\
\text { PA1584, PA1585, PA1586, PA1587, PA1588, PA1589, PA1787, PA2623, PA2634, PA2637, PA2638, PA2639, PA2640, PA2641, PA2642, PA2643, }\end{array}$ \\
\hline PA3879 & narL & 37 & $\begin{array}{l}\text { PA1172, PA1173, PA1174, PA1175, PA1176, PA1475, PA1476, PA1477, PA1479, PA1480, PA1481, PA1482, PA1483, PA1780, PA1781, PA2611, } \\
\text { PA2637, PA2638, PA2639, PA2640, PA2641, PA2642, PA2643, PA2644, PA2645, PA2646, PA2647, PA2648, PA2649, PA3724, PA3872, PA3873, }\end{array}$ \\
\hline PA5308 & $\operatorname{lrp}$ & 36 & $\begin{array}{l}\text { PA0059, PA0316, PA0353, PA0993, PA0994, PA1070, PA1071, PA1072, PA1073, PA1074, PA1217, PA1326, PA1760, PA2443, PA3118, PA3120, } \\
\text { PA3121, PA3167, PA3700, PA3972, PA4097, PA4503, PA4504, PA4505, PA4506, PA4695, PA4696, PA5013, PA5035, PA5036, PA5213, PA5214, }\end{array}$ \\
\hline PA4853 & fis & 26 & $\begin{array}{l}\text { PA1155, PA1156, PA1581, PA1582, PA1583, PA1584, PA1585, PA1586, PA1587, PA1588, PA1589, PA1787, PA1804, PA3769, PA3770, PA4022, } \\
\text { PA4343, PA4538, PA4740, PA4741, PA4742, PA4743, PA4744, PA4745, PA4746, PA4852 }\end{array}$ \\
\hline PA5360 & phoB & 22 & $\begin{array}{l}\text { PA0688, PA3127, PA3296, PA3372, PA3373, PA3374, PA3375, PA3376, PA3377, PA3378, PA3379, PA3380, PA3381, PA3382, PA3383, PA3384, } \\
\text { PA3981, PA4874, PA5361, PA5365, PA5366, PA5367 }\end{array}$ \\
\hline PA1754 & $c y s B$ & 21 & $\begin{array}{l}\text { PA0280, PA0281, PA0282, PA0932, PA1393, PA1493, PA1756, PA1838, PA2356, PA2357, PA2709, PA3444, PA3540, PA3935, PA3936, PA3937, } \\
\text { PA3938, PA4130, PA4442, PA4443, PA4513 }\end{array}$ \\
\hline PA4764 & fur & 17 & $\begin{array}{l}\text { PA1165, PA1618, PA1902, PA1922, PA2466, PA2688, PA3899, PA3900, PA4158, PA4159, PA4160, PA4161, PA4228, PA4231, PA4468, PA5521, } \\
\text { PA5531 }\end{array}$ \\
\hline PA3563 & fruR & 16 & PA0551, PA0552, PA1376, PA1498, PA1770, PA1780, PA1781, PA2611, PA2623, PA2634, PA3181, PA3194, PA3560, PA3561, PA3562, PA5192 \\
\hline PA0893 & $\arg R$ & 14 & PA0895, PA0896, PA0897, PA0898, PA0899, PA0901, PA2042, PA3068, PA3537, PA3934, PA4976, PA4977, PA5152, PA5170 \\
\hline PA3007 & $\operatorname{lex} A$ & 14 & PA0576, PA0577, PA0579, PA1886, PA2585, PA3008, PA3138, PA3617, PA4232, PA4234, PA4660, PA4763, PA4821, PA5443 \\
\hline PA0253 & & 12 & PA0254, PA0272, PA1928, PA1929, PA2002, PA2077, PA2113, PA2965, PA3703, PA3805, PA5237, PA5489 \\
\hline PA2510 & catR & 12 & PA2513, PA2518, PA2740, PA3279, PA3280, PA3656, PA4408, PA4417, PA4418, PA4759, PA5045, PA5259 \\
\hline PA3898 & & 12 & PA0305, PA0854, PA1384, PA1619, PA2028, PA2854, PA3183, PA3764, PA4378, PA4468, PA4997, PA5157 \\
\hline
\end{tabular}




\begin{tabular}{|c|c|c|c|}
\hline PA0424 & $\operatorname{mex} R$ & 11 & PA0425, PA0426, PA2020, PA2492, PA2495, PA3168, PA3574, PA3720, PA3721, PA4600, PA4964 \\
\hline PA1097 & fleQ & 11 & PA1098, PA1099, PA1100, PA1443, PA1452, PA1453, PA1454, PA1455, PA2020, PA3385, PA4462 \\
\hline PA1179 & phoP & 11 & PA1180, PA1343, PA1559, PA1560, PA1979, PA3552, PA3559, PA4773, PA4777, PA5199, PA5361 \\
\hline PA0272 & & 10 & PA1928, PA1929, PA2002, PA2077, PA2113, PA2965, PA3703, PA3805, PA5237, PA5489 \\
\hline PA0762 & $\operatorname{alg} U$ & 10 & PA0376, PA0576, PA0766, PA3337, PA4572, PA5011, PA5012, PA5077, PA5078, PA5261 \\
\hline PA1430 & las $R$ & 9 & PA1432, PA1871, PA2586, PA2587, PA3476, PA3477, PA3622, PA3724, PA3861 \\
\hline PA3204 & & 9 & PA0766, PA3644, PA3645, PA3646, PA3725, PA3737, PA4953, PA4954, PA5489 \\
\hline PA0527 & $d n r$ & 8 & PA1544, PA1546, PA1781, PA1920, PA2020, PA3391, PA3392, PA3879 \\
\hline PA0547 & & 8 & PA0960, PA0962, PA0963, PA2326, PA4943, PA4946, PA4967, PA5471 \\
\hline PA0873 & $\operatorname{phh} R$ & 8 & PA0292, PA1750, PA3000, PA3139, PA3166, PA4462, PA5039, PA5434 \\
\hline PA3477 & $r h l R$ & 8 & PA3478, PA3479, PA3622, PA3724, PA3861, PA4407, PA4408, PA4409 \\
\hline PA5125 & $n t r C$ & 8 & PA0888, PA1339, PA5075, PA5119, PA5124, PA5287, PA5288, PA5484 \\
\hline PA0001 & dnaA & 7 & PA0002, PA0003, PA0376, PA1155, PA1156, PA3769, PA3770 \\
\hline PA1003 & $m v f R$ & 7 & PA1430, PA1690, PA1725, PA2227, PA2587, PA3477, PA3861 \\
\hline PA1099 & fleR & 7 & PA1100, PA1101, PA1430, PA1454, PA1713, PA1714, PA4462 \\
\hline PA2273 & $\operatorname{sox} R$ & 7 & PA2740, PA3280, PA3656, PA3718, PA4417, PA4759, PA5259 \\
\hline PA1619 & & 6 & PA0305, PA0854, PA3183, PA4468, PA4615, PA5157 \\
\hline PA1998 & $d h c R$ & 5 & PA1999, PA2000, PA5384, PA5388, PA5389 \\
\hline PA2016 & $\operatorname{liuR}$ & 5 & PA2320, PA2886, PA2888, PA2891, PA2893 \\
\hline PA2020 & & 5 & PA2642, PA3391, PA3721, PA4671, PA5471 \\
\hline PA2259 & $p t x S$ & 5 & PA2260, PA2261, PA2262, PA2263, PA4315 \\
\hline PA3583 & $g l p R$ & 5 & PA0347, PA3581, PA3582, PA3584, PA5235 \\
\hline PA4280 & birA & 5 & PA0420, PA0500, PA0501, PA0503, PA0504 \\
\hline PA0487 & & 4 & PA1861, PA1863, PA4210, PA4811 \\
\hline PA0610 & $\operatorname{prtN}$ & 4 & PA0611, PA0612, PA0651, PA4590 \\
\hline PA0780 & pruR & 4 & PA0781, PA0782, PA0783, PA0982 \\
\hline PA1432 & lasI & 4 & PA1898, PA2227, PA3477, PA4296 \\
\hline PA1760 & & 4 & PA2163, PA2339, PA2340, PA2341 \\
\hline PA1949 & $r b s R$ & 4 & PA1946, PA1947, PA1948, PA1950 \\
\hline PA1979 & eras & 4 & PA1980, PA2586, PA3948, PA5483 \\
\hline PA2551 & & 4 & PA1217, PA3118, PA3120, PA3121 \\
\hline PA2586 & gacA & 4 & PA3476, PA3477, PA3622, PA4856 \\
\hline PA2665 & & 4 & PA1779, PA3697, PA4811, PA4893 \\
\hline PA3540 & $\operatorname{alg} D$ & 4 & PA5125, PA5200, PA5261, PA5483 \\
\hline
\end{tabular}




\begin{tabular}{|c|c|c|c|}
\hline PA5200 & $\operatorname{amgR}$ & 4 & PA0045, PA1288, PA5261, PA5361 \\
\hline PA5261 & $\operatorname{alg} R$ & 4 & PA5262, PA5322, PA5483, PA5484 \\
\hline PA5356 & $g l c C$ & 4 & PA0482, PA5352, PA5353, PA5355 \\
\hline PA5380 & $g b d R$ & 4 & PA5398, PA5399, PA5410, PA5411 \\
\hline PA0037 & $\operatorname{trpI}$ & 3 & PA0609, PA3166, PA4590 \\
\hline PA0152 & $p c a Q$ & 3 & PA0153, PA0154, PA0247 \\
\hline PA0928 & $\operatorname{gacS}$ & 3 & PA1898, PA2586, PA3006 \\
\hline PA0996 & $p q s A$ & 3 & PA1003, PA1430, PA3477 \\
\hline PA1184 & & 3 & PA5213, PA5214, PA5215 \\
\hline PA1637 & $k d p E$ & 3 & PA1633, PA1634, PA1635 \\
\hline PA1713 & $\operatorname{exs} A$ & 3 & PA1714, PA1716, PA3841 \\
\hline PA1898 & $q s c R$ & 3 & PA2586, PA3006, PA3476 \\
\hline PA2054 & cynR & 3 & PA2052, PA2053, PA2500 \\
\hline PA2118 & $a d a$ & 3 & PA1686, PA3306, PA3972 \\
\hline PA2258 & $p t x R$ & 3 & PA2259, PA2426, PA3479 \\
\hline PA2320 & gntR & 3 & PA2321, PA3181, PA3194 \\
\hline PA2809 & $\operatorname{cop} R$ & 3 & PA2810, PA5199, PA5361 \\
\hline PA2885 & atuR & 3 & PA2886, PA2888, PA2891 \\
\hline PA3006 & psrA & 3 & PA3007, PA3571, PA3622 \\
\hline PA3184 & & 3 & PA3193, PA3194, PA5036 \\
\hline PA3476 & rhlI & 3 & PA3477, PA4723, PA5261 \\
\hline PA3587 & metR & 3 & PA1843, PA4602, PA5124 \\
\hline PA3899 & & 3 & PA2914, PA3900, PA3901 \\
\hline PA4080 & & 3 & PA2978, PA3816, PA5563 \\
\hline PA4109 & $a m p R$ & 3 & PA4110, PA4393, PA4522 \\
\hline PA4341 & & 3 & PA0247, PA4127, PA4152 \\
\hline PA4363 & $i c i A$ & 3 & PA0001, PA0002, PA0003 \\
\hline PA4462 & rpoN & 3 & PA4547, PA5125, PA5483 \\
\hline PA4547 & pilR & 3 & PA4549, PA4556, PA5124 \\
\hline PA4600 & $n f x B$ & 3 & PA4964, PA5263, PA5348 \\
\hline PA4769 & & 3 & PA1587, PA5015, PA5016 \\
\hline PA5085 & & 3 & PA0836, PA2683, PA5277 \\
\hline PA5344 & $\operatorname{oxy} R$ & 3 & PA0139, PA0140, PA0962 \\
\hline PA5374 & betI & 3 & PA3933, PA5372, PA5373 \\
\hline
\end{tabular}




\begin{tabular}{|c|c|c|c|}
\hline PA5550 & $\operatorname{glm} R$ & 3 & PA2274, PA4457, PA5552 \\
\hline PA0036 & $\operatorname{trp} B$ & 2 & PA0037, PA3587 \\
\hline PA0155 & pcaR & 2 & PA0159, PA4974 \\
\hline PA0159 & & 2 & PA1294, PA4974 \\
\hline PA0287 & gриP & 2 & PA0289, PA1422 \\
\hline PA0288 & gриA & 2 & PA0289, PA1422 \\
\hline PA0289 & gpuR & 2 & PA1418, PA1421 \\
\hline PA0370 & & 2 & PA2273, PA2510 \\
\hline PA0409 & pilH & 2 & PA0416, PA3587 \\
\hline PA0416 & $\operatorname{chpD}$ & 2 & PA0417, PA1456 \\
\hline PA0425 & $\operatorname{mexA}$ & 2 & PA2020, PA3721 \\
\hline PA0482 & $g l c B$ & 2 & PA2273, PA2809 \\
\hline PA0506 & & 2 & PA3006, PA3571 \\
\hline PA0519 & nirs & 2 & PA0527, PA1544 \\
\hline PA0520 & $\operatorname{nir} Q$ & 2 & PA0527, PA1544 \\
\hline PA0576 & $r p o D$ & 2 & PA0652, PA1544 \\
\hline PA0611 & $p r t R$ & 2 & PA0612, PA4590 \\
\hline PA0831 & oruR & 2 & PA2020, PA4402 \\
\hline PA0888 & aotJ & 2 & PA0893, PA4315 \\
\hline PA1085 & $f l g J$ & 2 & PA1097, PA1099 \\
\hline PA1588 & $\operatorname{sucC}$ & 2 & PA2273, PA2510 \\
\hline PA1589 & sucD & 2 & PA2273, PA2510 \\
\hline PA1710 & $\operatorname{exs} C$ & 2 & PA1713, PA3006 \\
\hline PA1957 & & 2 & PA0761, PA4919 \\
\hline PA1978 & $e r b R$ & 2 & PA1982, PA3583 \\
\hline PA2012 & $\operatorname{liu} D$ & 2 & PA2016, PA2885 \\
\hline PA2014 & $\operatorname{liu} B$ & 2 & PA2016, PA2885 \\
\hline PA2219 & opdE & 2 & PA2273, PA2510 \\
\hline PA2227 & $v q s M$ & 2 & PA3476, PA4296 \\
\hline PA2272 & $p b p C$ & 2 & PA2273, PA2510 \\
\hline PA2492 & $\operatorname{mex} T$ & 2 & PA2493, PA2495 \\
\hline PA2523 & & 2 & PA2809, PA2810 \\
\hline PA2570 & $\operatorname{lec} A$ & 2 & PA3477, PA4315 \\
\hline PA2571 & & 2 & PA3587, PA5261 \\
\hline
\end{tabular}




\begin{tabular}{|c|c|c|c|}
\hline PA2686 & $p f e R$ & 2 & PA2687, PA2688 \\
\hline PA2810 & cops & 2 & PA5200, PA5360 \\
\hline PA2951 & etfA & 2 & PA3006, PA3571 \\
\hline PA2952 & etfB & 2 & PA3006, PA3571 \\
\hline PA2953 & & 2 & PA3006, PA3571 \\
\hline PA3574 & nalD & 2 & PA0425, PA0426 \\
\hline PA4227 & $p c h R$ & 2 & PA4228, PA4229 \\
\hline PA4581 & $r t c R$ & 2 & PA4583, PA4585 \\
\hline PA4726 & $c b r B$ & 2 & PA5105, PA5124 \\
\hline PA5199 & $\operatorname{amgS}$ & 2 & PA5200, PA5360 \\
\hline PA0017 & & 1 & PA2586 \\
\hline PA0024 & hemF & 1 & PA0527 \\
\hline PA0041 & & 1 & PA0652 \\
\hline PA0044 & exoT & 1 & PA1713 \\
\hline PA0176 & aer2 & 1 & PA1544 \\
\hline PA0186 & & 1 & PA1754 \\
\hline PA0240 & & 1 & PA3782 \\
\hline PA0254 & & 1 & PA0272 \\
\hline PA0286 & $\operatorname{des} A$ & 1 & PA4890 \\
\hline PA0292 & $\operatorname{aguA}$ & 1 & PA0294 \\
\hline PA0293 & $\operatorname{agu} B$ & 1 & PA0294 \\
\hline PA0296 & spuI & 1 & PA4726 \\
\hline PA0297 & spuA & 1 & PA4726 \\
\hline PA0298 & spuB & 1 & PA4726 \\
\hline PA0299 & spuC & 1 & PA4726 \\
\hline PA0328 & & 1 & PA0893 \\
\hline PA0408 & pilG & 1 & PA0416 \\
\hline PA0410 & pill & 1 & PA0416 \\
\hline PA0411 & pilJ & 1 & PA0416 \\
\hline PA0412 & pilK & 1 & PA0416 \\
\hline PA0413 & $\operatorname{chp} A$ & 1 & PA0416 \\
\hline PA0414 & $\operatorname{chp} B$ & 1 & PA0416 \\
\hline PA0415 & $\operatorname{chpC}$ & 1 & PA0416 \\
\hline PA0523 & nor $C$ & 1 & PA0527 \\
\hline
\end{tabular}




\begin{tabular}{|c|c|c|c|}
\hline \multicolumn{2}{|l|}{ PA0572 } & \multirow{2}{*}{$\begin{array}{l}1 \\
1\end{array}$} & \multirow{2}{*}{$\begin{array}{l}\text { PA0652 } \\
\text { PA5499 }\end{array}$} \\
\hline PA0705 & $\operatorname{mig} A$ & & \\
\hline PA0755 & opdH & 1 & PA0756 \\
\hline PA0756 & & 1 & PA0757 \\
\hline PA0763 & тисA & 1 & PA5483 \\
\hline PA0779 & & 1 & PA2665 \\
\hline PA0844 & plcH & 1 & PA5380 \\
\hline PA0870 & $p h h C$ & 1 & PA0873 \\
\hline PA0871 & $p h h B$ & 1 & PA0873 \\
\hline PA0872 & $p h h A$ & 1 & PA0873 \\
\hline PA0887 & $\operatorname{acs} A$ & 1 & PA1980 \\
\hline PA0889 & aot $Q$ & 1 & PA0893 \\
\hline PA0890 & aotM & 1 & PA0893 \\
\hline PA0891 & & 1 & PA0893 \\
\hline PA0892 & $\operatorname{aot} P$ & 1 & PA0893 \\
\hline PA0905 & $r \operatorname{sm} A$ & 1 & PA2586 \\
\hline PA0932 & cys $M$ & 1 & PA2354 \\
\hline PA0969 & tolQ & 1 & PA2686 \\
\hline PA1000 & $p q s E$ & 1 & PA1003 \\
\hline PA1002 & $p h n B$ & 1 & PA5499 \\
\hline PA1077 & $\operatorname{flg} B$ & 1 & PA1097 \\
\hline PA1078 & $f \lg C$ & 1 & PA1097 \\
\hline PA1082 & $f l g G$ & 1 & PA1097 \\
\hline PA1084 & flgI & 1 & PA1097 \\
\hline PA1093 & & 1 & PA1097 \\
\hline PA1094 & $f l i D$ & 1 & PA1097 \\
\hline PA1098 & fleS & 1 & PA1099 \\
\hline PA1174 & nарA & 1 & PA3879 \\
\hline PA1178 & oprH & 1 & PA1179 \\
\hline PA1196 & & 1 & PA3861 \\
\hline PA1418 & & 1 & PA1422 \\
\hline PA1421 & gbuA & 1 & PA1422 \\
\hline PA1458 & & 1 & PA5261 \\
\hline PA1461 & motD & 1 & PA2259 \\
\hline
\end{tabular}




\begin{tabular}{|c|c|c|c|}
\hline PA1543 & apt & 1 & PA1544 \\
\hline PA1546 & hemN & 1 & PA3879 \\
\hline PA1557 & $\operatorname{ccoN} 2$ & 1 & PA5261 \\
\hline PA1609 & $f a b B$ & 1 & PA4890 \\
\hline PA1610 & $f a b A$ & 1 & PA4890 \\
\hline PA1690 & $p s c U$ & 1 & PA4109 \\
\hline PA1712 & $\operatorname{exs} B$ & 1 & PA1713 \\
\hline PA1714 & exs $D$ & 1 & PA2519 \\
\hline PA1725 & $p s c L$ & 1 & PA4109 \\
\hline PA1796 & fold & 1 & PA4600 \\
\hline PA1850 & & 1 & PA2063 \\
\hline PA1920 & $n r d D$ & 1 & PA3879 \\
\hline PA1977 & & 1 & PA1978 \\
\hline PA1980 & eraR & 1 & PA1982 \\
\hline PA1999 & $d h c A$ & 1 & PA5389 \\
\hline PA2000 & $d h c B$ & 1 & PA5389 \\
\hline PA2011 & liuE & 1 & PA2016 \\
\hline PA2013 & $\operatorname{liu} C$ & 1 & PA2016 \\
\hline PA2015 & liuA & 1 & PA2016 \\
\hline PA2018 & & 1 & PA2020 \\
\hline PA2193 & hcnA & 1 & PA5261 \\
\hline PA2354 & & 1 & PA3444 \\
\hline PA2491 & & 1 & PA2492 \\
\hline PA2495 & $o p r N$ & 1 & PA4600 \\
\hline PA2507 & catA & 1 & PA2510 \\
\hline PA2508 & catC & 1 & PA2510 \\
\hline PA2511 & & 1 & PA2512 \\
\hline PA2515 & $x y l L$ & 1 & PA2519 \\
\hline PA2519 & $x y l S$ & 1 & PA3571 \\
\hline PA2522 & $c z c C$ & 1 & PA2809 \\
\hline PA2561 & & 1 & PA5360 \\
\hline PA2585 & $u v r C$ & 1 & PA2586 \\
\hline PA2587 & $p q s H$ & 1 & PA5499 \\
\hline PA2611 & cys $G$ & 1 & PA4723 \\
\hline
\end{tabular}




\begin{tabular}{|c|c|c|c|}
\hline PA2637 & пио A & 1 & PA2686 \\
\hline PA2664 & $f h p$ & 1 & PA2665 \\
\hline PA2696 & & 1 & PA1850 \\
\hline PA3082 & $g b t$ & 1 & PA5380 \\
\hline PA3094 & & 1 & PA5322 \\
\hline PA3168 & gyrA & 1 & PA4600 \\
\hline PA3175 & & 1 & PA5105 \\
\hline PA3176 & gltS & 1 & PA3184 \\
\hline PA3182 & $p g l$ & 1 & PA3184 \\
\hline PA3183 & $z w f$ & 1 & PA3184 \\
\hline PA3257 & prc & 1 & PA5483 \\
\hline PA3266 & $c a p B$ & 1 & PA3168 \\
\hline PA3385 & $a m r Z$ & 1 & PA5262 \\
\hline PA3391 & $n o s R$ & 1 & PA3587 \\
\hline PA3479 & rhlA & 1 & PA5261 \\
\hline PA3545 & $\operatorname{alg} G$ & 1 & PA5483 \\
\hline PA3547 & $\operatorname{alg} L$ & 1 & PA5483 \\
\hline PA3569 & $m m s B$ & 1 & PA3571 \\
\hline PA3570 & $m m s A$ & 1 & PA3571 \\
\hline PA3581 & glpF & 1 & PA3583 \\
\hline PA3594 & & 1 & PA3004 \\
\hline PA3689 & & 1 & PA3690 \\
\hline PA3710 & & 1 & PA3782 \\
\hline PA3720 & & 1 & PA3721 \\
\hline PA3721 & nalC & 1 & PA4598 \\
\hline PA3724 & las $B$ & 1 & PA4723 \\
\hline PA3782 & & 1 & PA5065 \\
\hline PA3815 & $i s c R$ & 1 & PA4236 \\
\hline PA3875 & $\operatorname{nar} G$ & 1 & PA3879 \\
\hline PA3878 & $n a r X$ & 1 & PA3879 \\
\hline PA3946 & $\begin{array}{l}\text { two- } \\
\text { compo }\end{array}$ & 1 & PA3948 \\
\hline PA3947 & rock & 1 & PA3948 \\
\hline PA4101 & bfmR & 1 & PA4208 \\
\hline
\end{tabular}




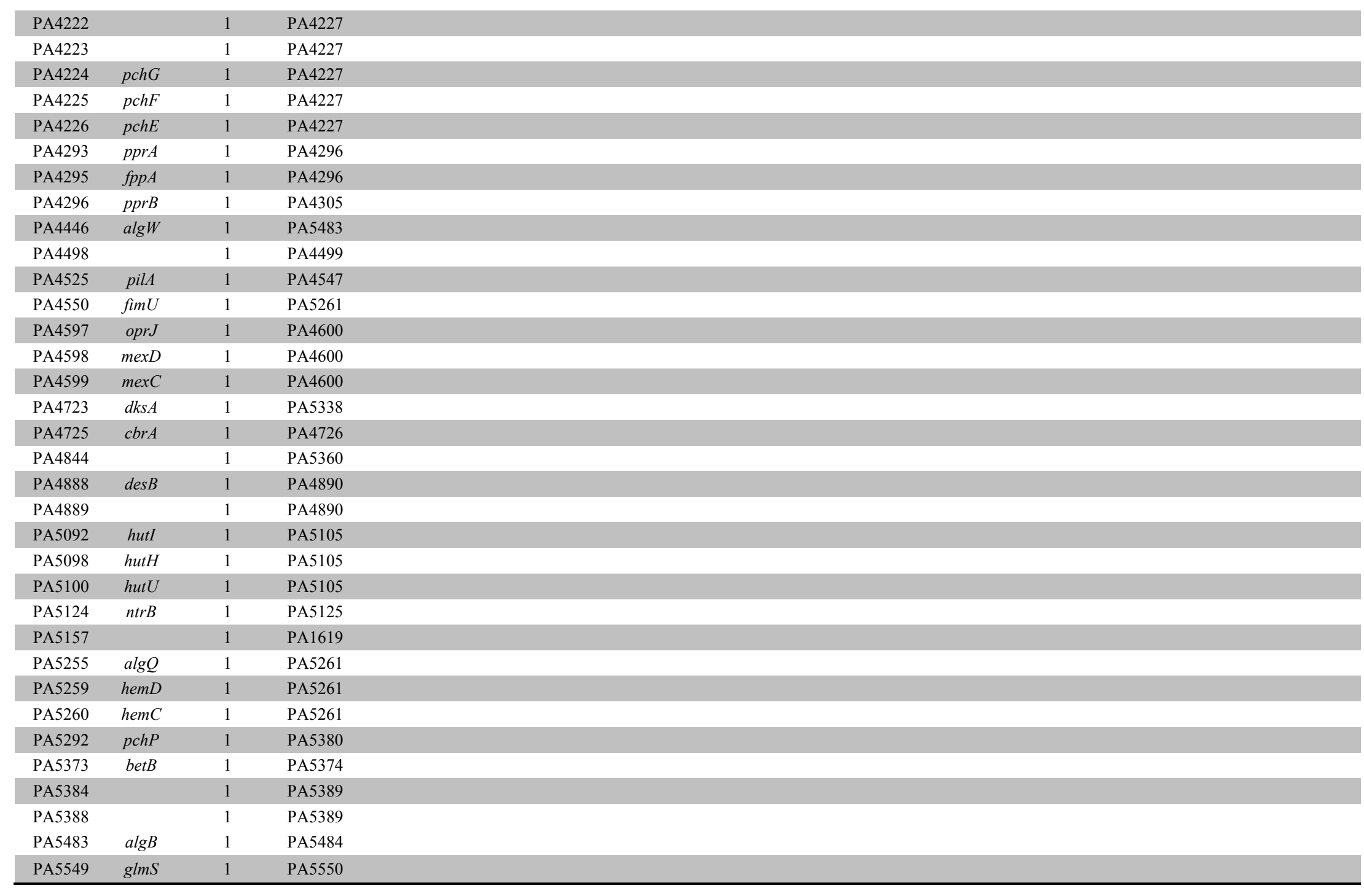


Supplementary information for Chapter 4 
Table S1: Key to identifying the AmpR-dependent, and AmpR- and B-lactam dependent genes from the venn diagram (Fig 4.4). Condition A: PAO1 uninduced vs PAO1 induced; Condition B: PAO $\triangle a m p R$ uninduced vs PAO $\triangle a m p R$ induced; Condition C: PAO1 uninduced vs PAO $\triangle a m p R$ uninduced; Condition D: PAO1 induced vs PAO $\triangle a m p R$ induced. NA- not applicable.

\begin{tabular}{|c|c|c|c|c|}
\hline Regulation & Condition & Condition & Condition & $\begin{array}{c}\text { AmpR } \\
\text { Regulation }\end{array}$ \\
\hline \multirow{6}{*}{$\begin{array}{c}\text { AmpR dependent, } \\
\text { B-lactam } \\
\text { independent }\end{array}$} & C- up & D- up & -- & Negative \\
\hline & C- down & D- down & -- & Positive \\
\hline & C- up & B- up & -- & Negative \\
\hline & C- down & B- down & -- & Positive \\
\hline & A- up & B- down & C- down & Positive \\
\hline & A- down & C- up & D- up & Negative \\
\hline \multirow{4}{*}{$\begin{array}{l}\text { AmpR dependent, } \\
\text { B-lactam dependent }\end{array}$} & A- down & B-up & -- & Negative \\
\hline & A- up & B- down & -- & Positive \\
\hline & A- up & D- down & -- & Positive \\
\hline & A- down & D- up & B- up & Negative \\
\hline \multirow{7}{*}{$\begin{array}{c}\text { AmpR } \\
\text { independent, B- } \\
\text { lactam dependent }\end{array}$} & C- down & D-up & -- & $\mathrm{NA}$ \\
\hline & C- up & D- down & -- & NA \\
\hline & A- down & B-down & -- & NA \\
\hline & A- up & B-up & -- & NA \\
\hline & A- up & D- up & -- & NA \\
\hline & A- up & C- down & D-up & NA \\
\hline & B- up & D- down & C- up & NA \\
\hline
\end{tabular}


Table S2: ß-lactam-dependent genes. The 206 ß-lactam stress-dependent genes are separated into upregulated and downregulated genes with the corresponding corrected $p$ values for the fold change (FC) observed. The genes are arranged based on either functional categorization. Locus tag annotations are from the Pseudomonas Genome database (31).

A

\begin{tabular}{|c|c|c|c|c|}
\hline \multicolumn{5}{|c|}{ Downregulated genes } \\
\hline Locus Tag & $\begin{array}{c}\text { Gene } \\
\text { Name }\end{array}$ & Product Name & FC & $\begin{array}{c}\text { Corrected } \\
p \text {-value }\end{array}$ \\
\hline \multicolumn{5}{|c|}{ Adaptation, Protection } \\
\hline PA4078 & & probable nonribosomal peptide synthetase & -3.6 & 4.07E-03 \\
\hline \multicolumn{5}{|c|}{ Amino acid biosynthesis and metabolism } \\
\hline PA5098 & hutH & histidine ammonia-lyase & -21.4 & $2.15 \mathrm{E}-03$ \\
\hline PA5100 & hut $U$ & urocanase & -10.5 & $2.61 \mathrm{E}-03$ \\
\hline PA3700 & $\operatorname{lys} S$ & lysyl-tRNA synthetase & -2.1 & $5.78 \mathrm{E}-03$ \\
\hline \multicolumn{5}{|c|}{ Carbon compound catabolism } \\
\hline PA0792 & $\operatorname{prpD}$ & propionate catabolic protein $\operatorname{PrpD}$ & -3.8 & $8.44 \mathrm{E}-03$ \\
\hline PA2300 & $\operatorname{chiC}$ & chitinase & -20.2 & 4.17E-04 \\
\hline \multicolumn{5}{|c|}{ Cell wall / LPS / capsule ; Adaptation, Protection } \\
\hline PA1130 & $r h l C$ & rhamnosyltransferase 2 & -5.4 & $5.32 \mathrm{E}-03$ \\
\hline \multicolumn{5}{|c|}{ Central intermediary metabolism } \\
\hline PA5549 & $\operatorname{glm} S$ & $\begin{array}{l}\text { glucosamine--fructose-6-phosphate } \\
\text { aminotransferase }\end{array}$ & -2.5 & $2.28 \mathrm{E}-03$ \\
\hline \multicolumn{5}{|c|}{ Energy metabolism } \\
\hline PA3930 & $\operatorname{cioA}$ & cyanide insensitive terminal oxidase & -3.8 & $6.69 \mathrm{E}-03$ \\
\hline \multicolumn{5}{|c|}{ Hypothetical, unclassified, unknown } \\
\hline PA0088 & & hypothetical protein & -3.2 & 4.39E-03 \\
\hline PA0122 & & conserved hypothetical protein & -9.2 & $6.61 \mathrm{E}-03$ \\
\hline PA0271 & & hypothetical protein & -3.0 & $3.10 \mathrm{E}-03$ \\
\hline PA1494 & & conserved hypothetical protein & -2.5 & 4.94E-03 \\
\hline PA1896 & & hypothetical protein & -2.7 & $1.76 \mathrm{E}-03$ \\
\hline PA2066 & & hypothetical protein & -4.8 & $5.07 \mathrm{E}-03$ \\
\hline PA2111 & & hypothetical protein & -2.3 & $2.78 \mathrm{E}-03$ \\
\hline PA2223 & & hypothetical protein & -3.4 & $9.59 \mathrm{E}-03$ \\
\hline PA2274 & & hypothetical protein & -8.7 & $3.92 \mathrm{E}-03$ \\
\hline PA2441 & & hypothetical protein & -3.4 & $5.33 \mathrm{E}-03$ \\
\hline PA2448 & & hypothetical protein & -7.9 & 5.07E-03 \\
\hline PA2684 & & conserved hypothetical protein & -2.2 & $2.78 \mathrm{E}-03$ \\
\hline PA3229 & & hypothetical protein & -2.1 & $4.66 \mathrm{E}-03$ \\
\hline PA3734 & & hypothetical protein & -4.1 & $8.70 \mathrm{E}-03$ \\
\hline PA3928 & & hypothetical protein & -3.5 & $7.28 \mathrm{E}-03$ \\
\hline PA4035 & & hypothetical protein & -2.6 & $2.61 \mathrm{E}-03$ \\
\hline PA4141 & & hypothetical protein & -8.7 & $3.29 \mathrm{E}-03$ \\
\hline PA5106 & & conserved hypothetical protein & -10.4 & $4.45 \mathrm{E}-03$ \\
\hline PA5201 & & conserved hypothetical protein & -2.5 & $2.15 \mathrm{E}-03$ \\
\hline PA5220 & & hypothetical protein & -9.4 & 4.71E-03 \\
\hline
\end{tabular}




\begin{tabular}{|c|c|c|c|c|}
\hline \multicolumn{5}{|c|}{ Membrane proteins } \\
\hline PA0070 & & hypothetical protein & -2.0 & 7.24E-03 \\
\hline PA1895 & & hypothetical protein & -3.3 & $3.71 \mathrm{E}-03$ \\
\hline PA5183 & & hypothetical protein & -4.3 & 4.45E-03 \\
\hline PA1212 & & $\begin{array}{l}\text { probable major facilitator superfamily } \\
\text { (MFS) transporter }\end{array}$ & -2.9 & $7.31 \mathrm{E}-03$ \\
\hline PA2068 & & $\begin{array}{l}\text { probable major facilitator superfamily } \\
\text { (MFS) transporter }\end{array}$ & -11.7 & $3.42 \mathrm{E}-03$ \\
\hline PA2327 & & probable permease of $\mathrm{ABC}$ transporter & -5.8 & $6.57 \mathrm{E}-03$ \\
\hline PA4034 & $a q p Z$ & aquaporin $\mathrm{Z}$ & -26.1 & $2.15 \mathrm{E}-03$ \\
\hline PA5095 & & probable permease of $\mathrm{ABC}$ transporter & -12.0 & $2.15 \mathrm{E}-03$ \\
\hline PA5097 & & probable amino acid permease & -20.7 & $2.78 \mathrm{E}-03$ \\
\hline PA5099 & & probable transporter & -12.4 & $4.21 \mathrm{E}-03$ \\
\hline \multicolumn{5}{|c|}{ Motility \& Attachment } \\
\hline PA0171 & & hypothetical protein & -3.0 & $3.68 \mathrm{E}-03$ \\
\hline PA3361 & $\operatorname{lec} B$ & fucose-binding lectin PA-IIL & -2.1 & $9.12 \mathrm{E}-03$ \\
\hline \multicolumn{5}{|c|}{ Protein secretion/export apparatus } \\
\hline PA1718 & $p s c E$ & type III export protein PscE & -3.2 & $3.71 \mathrm{E}-03$ \\
\hline PA3820 & $\sec F$ & secretion protein $\mathrm{SecF}$ & -2.4 & $9.63 \mathrm{E}-03$ \\
\hline \multicolumn{5}{|l|}{$\begin{array}{l}\text { Putative } \\
\text { enzymes }\end{array}$} \\
\hline PA0130 & & probable aldehyde dehydrogenase & -2.1 & $9.12 \mathrm{E}-03$ \\
\hline PA0372 & & probable zinc protease & -2.3 & $2.91 \mathrm{E}-03$ \\
\hline PA1914 & & conserved hypothetical protein & -14.8 & $7.12 \mathrm{E}-03$ \\
\hline PA2067 & & probable hydrolase & -12.0 & $1.76 \mathrm{E}-03$ \\
\hline PA2069 & & probable carbamoyl transferase & -35.4 & $2.66 \mathrm{E}-03$ \\
\hline PA4715 & & probable aminotransferase & -4.7 & $6.10 \mathrm{E}-03$ \\
\hline PA5093 & & $\begin{array}{l}\text { probable histidine/phenylalanine ammonia- } \\
\text { lyase }\end{array}$ & -12.5 & $3.73 \mathrm{E}-03$ \\
\hline PA0075 & pppA & PppA & -2.9 & $5.96 \mathrm{E}-03$ \\
\hline \multicolumn{5}{|c|}{ Secreted Factors (toxins, enzymes, alginate) } \\
\hline PA0852 & $\operatorname{cbpD}$ & chitin-binding protein $\mathrm{CbpD}$ precursor & -6.7 & $3.41 \mathrm{E}-03$ \\
\hline PA1901 & $p h z C 2$ & phenazine biosynthesis protein $\mathrm{PhzC}$ & -9.2 & $5.62 \mathrm{E}-03$ \\
\hline PA1902 & phzD2 & phenazine biosynthesis protein $\mathrm{PhzD}$ & -10.7 & 4.07E-03 \\
\hline PA1903 & $p h z E 2$ & phenazine biosynthesis protein $\mathrm{PhzE}$ & -17.1 & $5.81 \mathrm{E}-03$ \\
\hline PA1904 & $p h z F 2$ & probable phenazine biosynthesis protein & -15.8 & $3.98 \mathrm{E}-03$ \\
\hline PA1905 & $p h z G 2$ & $\begin{array}{l}\text { probable pyridoxamine } 5 \text { '-phosphate } \\
\text { oxidase }\end{array}$ & -19.8 & $3.44 \mathrm{E}-03$ \\
\hline PA1871 & $\operatorname{las} A$ & LasA protease precursor & -7.9 & $6.80 \mathrm{E}-03$ \\
\hline \multicolumn{5}{|c|}{ Transcription, RNA processing and degradation } \\
\hline PA4238 & rpoA & $\begin{array}{l}\text { DNA-directed RNA polymerase alpha } \\
\text { chain }\end{array}$ & -2.0 & $3.91 \mathrm{E}-03$ \\
\hline \multicolumn{5}{|c|}{ Translation, post-translational modification, degradation } \\
\hline PA2619 & $\inf A$ & initiation factor & -3.0 & $4.45 \mathrm{E}-03$ \\
\hline PA4241 & rps $M$ & 30S ribosomal protein $\mathrm{S} 13$ & -2.6 & $9.03 \mathrm{E}-03$ \\
\hline PA0090 & $\operatorname{clp} V 1$ & ClpV1 & -3.6 & $4.02 \mathrm{E}-03$ \\
\hline \multicolumn{5}{|c|}{ Transport of small molecules } \\
\hline PA0603 & & $\begin{array}{l}\text { probable ATP-binding component of ABC } \\
\text { transporter }\end{array}$ & -2.0 & 7.74E-03 \\
\hline
\end{tabular}




\section{Upregulated genes}

\begin{tabular}{|c|c|c|c|c|}
\hline Locus Tag & $\begin{array}{l}\text { Gene } \\
\text { Name }\end{array}$ & Product Name & FC & $\begin{array}{c}\text { Corrected } \\
p \text {-value }\end{array}$ \\
\hline \multicolumn{5}{|c|}{ Adaptation, Protection } \\
\hline PA0176 & aer2 & aerotaxis transducer Aer2 & 4.9 & 7.74E-03 \\
\hline PA0177 & & $\begin{array}{l}\text { probable purine-binding chemotaxis } \\
\text { protein }\end{array}$ & 3.1 & $9.87 \mathrm{E}-03$ \\
\hline PA1646 & & probable chemotaxis transducer & 2.6 & $7.41 \mathrm{E}-03$ \\
\hline PA2570 & $\operatorname{lec} A$ & LecA & 41.7 & $1.60 \mathrm{E}-03$ \\
\hline PA2654 & & probable chemotaxis transducer & 3.2 & $2.78 \mathrm{E}-03$ \\
\hline PA2788 & & probable chemotaxis transducer & 2.1 & 4.77E-03 \\
\hline PA3236 & & $\begin{array}{l}\text { probable glycine betaine-binding protein } \\
\text { precursor }\end{array}$ & 11.3 & $2.78 \mathrm{E}-03$ \\
\hline PA3349 & & probable chemotaxis protein & 2.4 & $5.78 \mathrm{E}-03$ \\
\hline PA4310 & $p c t B$ & chemotactic transducer PctB & 2.6 & $3.40 \mathrm{E}-03$ \\
\hline PA4566 & obg & GTP-binding protein Obg & 2.6 & 4.12E-03 \\
\hline PA4633 & & probable chemotaxis transducer & 3.0 & $3.54 \mathrm{E}-03$ \\
\hline PA5072 & & probable chemotaxis transducer & 2.2 & $4.36 \mathrm{E}-03$ \\
\hline \multicolumn{5}{|c|}{ Amino acid biosynthesis and metabolism } \\
\hline PA4759 & $d a p B$ & dihydrodipicolinate reductase & 2.6 & $9.12 \mathrm{E}-03$ \\
\hline PA5379 & $s d a B$ & L-serine dehydratase & 4.1 & $5.30 \mathrm{E}-03$ \\
\hline PA5398 & $d g c A$ & DgcA, Dimethylglycine catabolism & 8.4 & $2.15 \mathrm{E}-03$ \\
\hline PA5399 & $d g c B$ & DgcB, Dimethylglycine catabolism & 7.3 & $2.78 \mathrm{E}-03$ \\
\hline PA5410 & $g b c A$ & $\mathrm{GbcA}$ & 13.8 & $2.96 \mathrm{E}-03$ \\
\hline PA5411 & $g b c B$ & $\mathrm{GbcB}$ & 7.1 & $2.78 \mathrm{E}-03$ \\
\hline PA5416 & $\operatorname{sox} B$ & sarcosine oxidase beta subunit & 11.6 & $2.95 \mathrm{E}-03$ \\
\hline PA5417 & $\operatorname{sox} D$ & sarcosine oxidase delta subunit & 15.8 & $2.78 \mathrm{E}-03$ \\
\hline PA5419 & $\operatorname{sox} G$ & sarcosine oxidase gamma subunit & 6.5 & 7.74E-03 \\
\hline PA5429 & $\operatorname{asp} A$ & aspartate ammonia-lyase & 2.4 & $9.12 \mathrm{E}-03$ \\
\hline \multicolumn{5}{|c|}{ Carbon compound catabolism } \\
\hline PA4124 & $h p c B$ & homoprotocatechuate 2,3-dioxygenase & 4.0 & $8.81 \mathrm{E}-03$ \\
\hline PA5418 & $\operatorname{sox} A$ & sarcosine oxidase alpha subunit & 7.4 & $3.46 \mathrm{E}-03$ \\
\hline \multicolumn{5}{|c|}{ Cell wall / LPS / capsule } \\
\hline PA1078 & $f \lg C$ & flagellar basal-body rod protein $\mathrm{FlgC}$ & 3.4 & $4.45 \mathrm{E}-03$ \\
\hline PA1079 & $f \lg D$ & $\begin{array}{l}\text { flagellar basal-body rod modification } \\
\text { protein FlgD }\end{array}$ & 3.0 & $5.78 \mathrm{E}-03$ \\
\hline PA1080 & $f \lg E$ & flagellar hook protein FlgE & 3.3 & 7.19E-03 \\
\hline PA1087 & $f \lg L$ & $\begin{array}{l}\text { flagellar hook-associated protein type } 3 \\
\text { FlgL }\end{array}$ & 2.8 & 7.41E-03 \\
\hline PA1094 & fliD & flagellar capping protein FliD & 2.2 & $9.23 \mathrm{E}-03$ \\
\hline PA1959 & bacA & bacitracin resistance protein & 4.2 & $7.71 \mathrm{E}-03$ \\
\hline PA4700 & $m r c B$ & penicillin-binding protein $1 \mathrm{~B}$ & 3.5 & $3.71 \mathrm{E}-03$ \\
\hline
\end{tabular}


$\begin{array}{lllll}\text { PA4640 } & m q o B & \text { malate:quinone oxidoreductase } & 2.5 & 4.32 \mathrm{E}-03 \\ \text { PA4880 } & & \begin{array}{l}\text { probable bacterioferritin } \\ \text { glutathione-independent formaldehyde }\end{array} & 4.2 & 9.12 \mathrm{E}-03 \\ \text { PA5421 } & f d h A & 5.2 & 9.59 \mathrm{E}-03\end{array}$

Chaperones \& heat shock proteins

$\begin{array}{lllll}\text { PA3126 } & i b p A & \text { heat-shock protein IbpA } & 2.7 & 9.90 \mathrm{E}-03\end{array}$

Chemotaxis ; Adaptation, Protection ; Motility \& Attachment

$\begin{array}{lllll}\text { PA1452 flhA } & \text { flagellar biosynthesis protein FlhA } & 2.7 & 8.47 \mathrm{E}-03\end{array}$

DNA replication, recombination, modification and repair

$\begin{array}{lllll}\text { PA3745 } & r p s P & \text { 30S ribosomal protein S16 } & 4.3 & 9.29 \mathrm{E}-03 \\ \text { PA4763 } & \text { recN } & \text { DNA repair protein RecN } & 2.5 & 6.57 \mathrm{E}-03\end{array}$

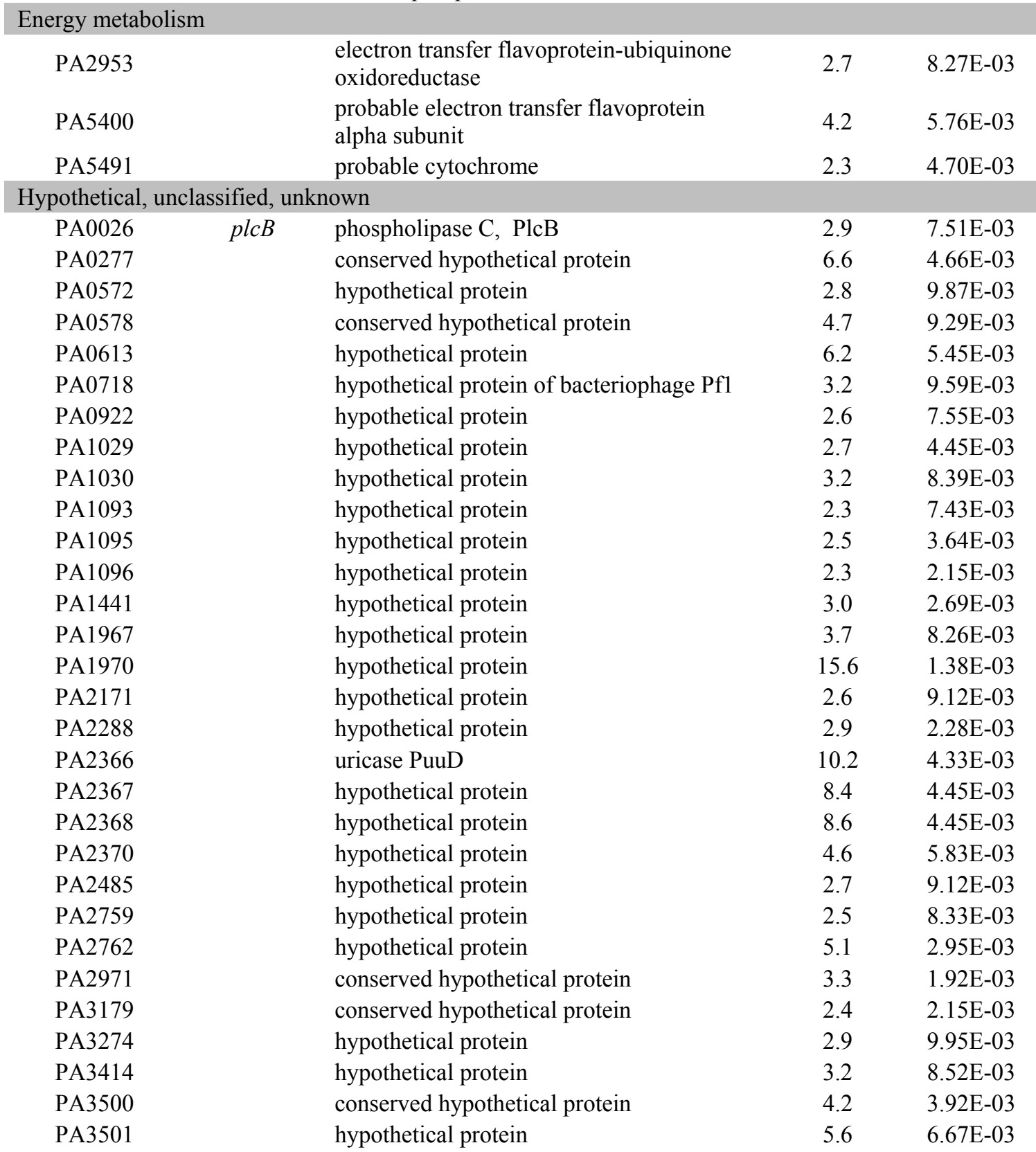




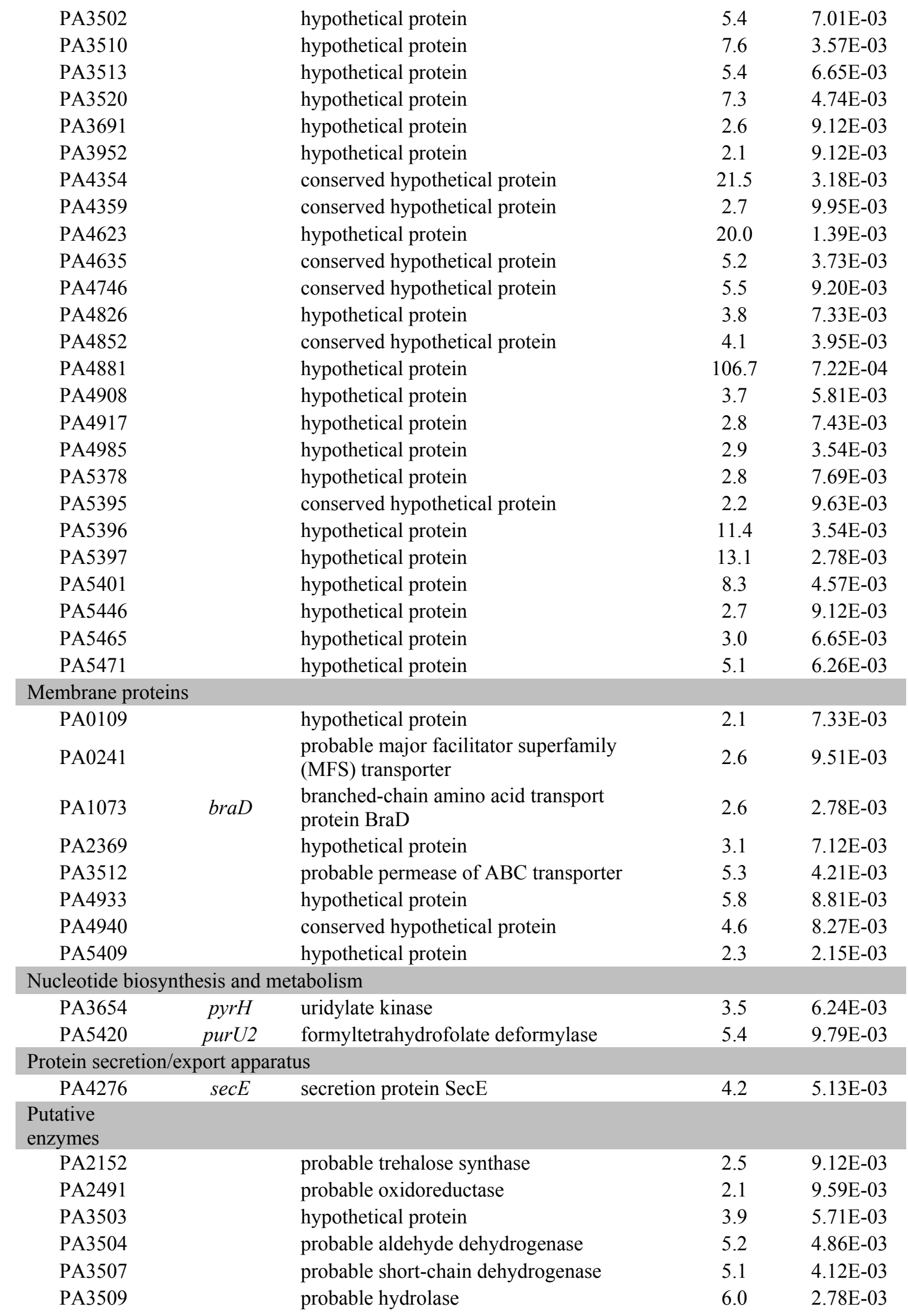




\begin{tabular}{|c|c|c|c|c|}
\hline PA3511 & & probable short-chain dehydrogenase & 6.8 & $4.02 \mathrm{E}-03$ \\
\hline PA4986 & & probable oxidoreductase & 2.7 & $5.71 \mathrm{E}-03$ \\
\hline \multicolumn{5}{|c|}{ Secreted Factors (toxins, enzymes, alginate) } \\
\hline PA3478 & $\operatorname{rhlB}$ & rhamnosyltransferase chain B & 6.0 & $3.71 \mathrm{E}-03$ \\
\hline PA3479 & rhlA & rhamnosyltransferase chain $\mathrm{A}$ & 20.5 & $1.60 \mathrm{E}-03$ \\
\hline PA5112 & estA & esterase EstA & 2.1 & $3.73 \mathrm{E}-03$ \\
\hline \multicolumn{5}{|c|}{ Transcription, RNA processing and degradation } \\
\hline PA3743 & trmD & tRNA (guanine-N1)-methyltransferase & 17.9 & $3.35 \mathrm{E}-03$ \\
\hline PA4264 & $r p s J$ & $30 \mathrm{~S}$ ribosomal protein $\mathrm{S} 10$ & 3.3 & $5.38 \mathrm{E}-03$ \\
\hline \multicolumn{5}{|c|}{ Transcriptional regulators } \\
\hline PA1196 & & probable transcriptional regulator & 2.6 & $9.89 \mathrm{E}-03$ \\
\hline PA3508 & & probable transcriptional regulator & 5.8 & $5.71 \mathrm{E}-03$ \\
\hline PA4296 & $\operatorname{ppr} B$ & two-component response regulator, $\mathrm{PprB}$ & 3.3 & $8.60 \mathrm{E}-03$ \\
\hline \multicolumn{5}{|c|}{ Translation, post-translational modification, degradation } \\
\hline PA0580 & $g c p$ & O-sialoglycoprotein endopeptidase & 3.3 & $8.70 \mathrm{E}-03$ \\
\hline PA0905 & $r \sin A$ & RsmA, regulator of secondary metabolites & 2.3 & $9.63 \mathrm{E}-03$ \\
\hline PA2741 & rplT & $50 \mathrm{~S}$ ribosomal protein $\mathrm{L} 20$ & 2.2 & $7.71 \mathrm{E}-03$ \\
\hline PA4542 & $\operatorname{clp} B$ & ClpB protein & 2.1 & $9.90 \mathrm{E}-03$ \\
\hline PA4567 & rрmA & 50 S ribosomal protein $\mathrm{L} 27$ & 4.5 & $5.62 \mathrm{E}-03$ \\
\hline PA4568 & $r p l U$ & 50 S ribosomal protein $\mathrm{L} 21$ & 7.0 & $4.81 \mathrm{E}-03$ \\
\hline PA4742 & truB & tRNA pseudouridine 55 synthase & 3.5 & $9.10 \mathrm{E}-03$ \\
\hline PA4932 & rplI & $50 \mathrm{~S}$ ribosomal protein $\mathrm{L} 9$ & 4.6 & $8.70 \mathrm{E}-03$ \\
\hline PA4934 & $r p s R$ & 30 S ribosomal protein $\mathrm{S} 18$ & 4.2 & $9.29 \mathrm{E}-03$ \\
\hline PA5316 & $\operatorname{rpm} B$ & 50 S ribosomal protein L28 & 2.3 & $5.97 \mathrm{E}-03$ \\
\hline PA5470 & & probable peptide chain release factor & 5.0 & $6.86 \mathrm{E}-03$ \\
\hline \multicolumn{5}{|c|}{ Transport of small molecules } \\
\hline PA1074 & braC & $\begin{array}{l}\text { branched-chain amino acid transport } \\
\text { protein } \mathrm{BraC}\end{array}$ & 2.9 & $4.45 \mathrm{E}-03$ \\
\hline PA1260 & & $\begin{array}{l}\text { amino acid } \mathrm{ABC} \text { transporter periplasmic } \\
\text { binding protein }\end{array}$ & 3.3 & $3.73 \mathrm{E}-03$ \\
\hline PA1435 & & $\begin{array}{l}\text { probable Resistance-Nodulation-Cell } \\
\text { Division (RND) efflux membrane fusion } \\
\text { protein precursor }\end{array}$ & 5.2 & $4.50 \mathrm{E}-03$ \\
\hline PA2019 & & $\begin{array}{l}\text { Resistance-Nodulation-Cell Division } \\
\text { (RND) multidrug efflux membrane fusion } \\
\text { protein precursor }\end{array}$ & 2.0 & $9.89 \mathrm{E}-03$ \\
\hline PA2812 & & $\begin{array}{l}\text { probable ATP-binding component of } \mathrm{ABC} \\
\text { transporter }\end{array}$ & 3.7 & $2.40 \mathrm{E}-03$ \\
\hline PA3514 & & $\begin{array}{l}\text { probable ATP-binding component of } \mathrm{ABC} \\
\text { transporter }\end{array}$ & 8.1 & $4.20 \mathrm{E}-03$ \\
\hline PA5468 & & probable citrate transporter & 2.7 & 4.37E-03 \\
\hline
\end{tabular}


Table S3: AmpR-dependent genes. The 313 AmpR-dependent genes are separated into upregulated and downregulated genes with the corresponding corrected $p$-values for the fold change (FC) observed. The genes are arranged based on either (A) functional categorization, or (B) fold change. Locus tag annotations are from the Pseudomonas Genome database (31).

\begin{tabular}{|c|c|c|c|c|}
\hline \multicolumn{5}{|c|}{ Downregulated genes } \\
\hline Locus Tag & $\begin{array}{l}\text { Gene } \\
\text { Name }\end{array}$ & Product Name & FC & $\begin{array}{c}\text { Corrected } \\
p \text {-value }\end{array}$ \\
\hline \multicolumn{5}{|c|}{ Adaptation, Protection } \\
\hline PA0074 & $p p k A$ & serine/threonine protein kinase PpkA & -2.3 & $8.41 \mathrm{E}-03$ \\
\hline PA1127 & & probable oxidoreductase & -2.0 & 4.74E-03 \\
\hline PA2826 & & probable glutathione peroxidase & -2.2 & $5.96 \mathrm{E}-03$ \\
\hline PA3109 & & hypothetical protein & -2.6 & $9.59 \mathrm{E}-03$ \\
\hline PA5117 & typ $A$ & regulatory protein TypA & -3.6 & $8.06 \mathrm{E}-03$ \\
\hline \multicolumn{5}{|c|}{ Amino acid biosynthesis and metabolism } \\
\hline PA0132 & & beta-alanine--pyruvate transaminase & -3.9 & $9.29 \mathrm{E}-03$ \\
\hline PA1337 & ans $B$ & $\begin{array}{l}\text { glutaminase-asparaginase } \\
\text { membrane-bound lytic murein }\end{array}$ & -2.4 & $3.35 \mathrm{E}-03$ \\
\hline PA1812 & $m l t D$ & transglycosylase $\mathrm{D}$ precursor & -2.1 & $5.32 \mathrm{E}-03$ \\
\hline PA2443 & $s d a A$ & L-serine dehydratase & -10.6 & $4.02 \mathrm{E}-03$ \\
\hline PA2444 & glyA2 & serine hydroxymethyltransferase & -7.1 & $1.76 \mathrm{E}-03$ \\
\hline PA2629 & purB & adenylosuccinate lyase & -2.3 & $9.89 \mathrm{E}-03$ \\
\hline PA5323 & $\arg B$ & acetylglutamate kinase & -2.6 & 4.45E-03 \\
\hline PA5415 & glyA1 & serine hydroxymethyltransferase & -2.1 & $5.91 \mathrm{E}-03$ \\
\hline \multicolumn{5}{|c|}{ Biosynthesis of cofactors, prosthetic groups and carriers } \\
\hline PA4664 & hemK & probable methyl transferase & -2.0 & $9.51 \mathrm{E}-03$ \\
\hline \multicolumn{5}{|c|}{ Carbon compound catabolism } \\
\hline PA0208 & $m d c A$ & malonate decarboxylase alpha subunit & -2.4 & $3.91 \mathrm{E}-03$ \\
\hline PA2414 & & L-sorbosone dehydrogenase & -2.2 & $8.90 \mathrm{E}-03$ \\
\hline \multicolumn{5}{|l|}{ Cell division } \\
\hline PA0857 & bolA & morphogene protein BolA & -2.3 & $2.78 \mathrm{E}-03$ \\
\hline \multicolumn{5}{|c|}{ Cell wall / LPS / capsule } \\
\hline PA2232 & $p s l B$ & $\begin{array}{l}\text { probable phosphomannose isomerase/GDP- } \\
\text { mannose pyrophosphorylase }\end{array}$ & -3.2 & $8.70 \mathrm{E}-03$ \\
\hline PA2234 & pslD & PslD & -3.0 & $8.27 \mathrm{E}-03$ \\
\hline PA3646 & $\operatorname{lpxD}$ & $\begin{array}{l}\text { UDP-3-O-[3-hydroxylauroyl] glucosamine N- } \\
\text { acyltransferase } \\
\text { periplasmic glucans biosynthesis protein }\end{array}$ & -2.1 & $4.78 \mathrm{E}-03$ \\
\hline PA5077 & $m d o H$ & $\mathrm{MdoH}$ & -2.2 & $4.86 \mathrm{E}-03$ \\
\hline PA5163 & $r m l A$ & glucose-1-phosphate thymidylyltransferase & -2.1 & $4.66 \mathrm{E}-03$ \\
\hline PA5322 & $\operatorname{alg} C$ & phosphomannomutase $\mathrm{Alg} \mathrm{C}$ & -2.3 & $4.45 \mathrm{E}-03$ \\
\hline \multicolumn{5}{|c|}{ Central intermediary metabolism } \\
\hline PA2023 & galU & UTP--glucose-1-phosphate uridylyltransferase & -2.5 & 4.75E-03 \\
\hline PA2193 & hcnA & hydrogen cyanide synthase HcnA & -7.3 & 4.68E-03 \\
\hline PA2194 & $h c n B$ & hydrogen cyanide synthase $\mathrm{HcnB}$ & -5.3 & $7.08 \mathrm{E}-03$ \\
\hline PA2195 & hcnC & hydrogen cyanide synthase HcnC & -4.6 & $4.02 \mathrm{E}-03$ \\
\hline PA2442 & gcvT2 & glycine cleavage system protein $\mathrm{T} 2$ & -4.8 & $3.36 \mathrm{E}-03$ \\
\hline
\end{tabular}




\begin{tabular}{|c|c|c|c|c|}
\hline $\begin{array}{l}\text { PA2445 } \\
\text { PA4100 }\end{array}$ & $g c v P 2$ & $\begin{array}{l}\text { glycine cleavage system protein } \mathrm{P} 2 \\
\text { probable dehydrogenase }\end{array}$ & $\begin{array}{l}-14.3 \\
-2.7\end{array}$ & $\begin{array}{l}5.03 \mathrm{E}-03 \\
6.81 \mathrm{E}-03\end{array}$ \\
\hline \multicolumn{5}{|c|}{ Energy metabolism } \\
\hline PA0854 & fumC2 & fumarate hydratase & -2.4 & $9.59 \mathrm{E}-03$ \\
\hline PA4470 & fumCl & fumarate hydratase & -17.3 & $5.97 \mathrm{E}-03$ \\
\hline PA5553 & atpC & ATP synthase epsilon chain & -2.2 & $5.71 \mathrm{E}-03$ \\
\hline \multicolumn{5}{|c|}{ Fatty acid and phospholipid metabolism } \\
\hline PA0798 & pmtA & phospholipid methyltransferase & -2.3 & $5.91 \mathrm{E}-03$ \\
\hline PA1869 & & probable acyl carrier protein & -6.0 & $4.66 \mathrm{E}-03$ \\
\hline \multicolumn{5}{|c|}{ Hypothetical, unclassified, unknown } \\
\hline PA0062 & & hypothetical protein & -4.9 & $2.91 \mathrm{E}-03$ \\
\hline PA0076 & & hypothetical protein & -2.5 & $3.34 \mathrm{E}-03$ \\
\hline PA0078 & & hypothetical protein & -2.9 & $8.66 \mathrm{E}-03$ \\
\hline PA0079 & & hypothetical protein & -2.9 & $5.97 \mathrm{E}-03$ \\
\hline PA0080 & & hypothetical protein & -2.5 & 4.94E-03 \\
\hline PA0169 & & hypothetical protein & -3.3 & $2.95 \mathrm{E}-03$ \\
\hline PA0170 & & hypothetical protein & -3.5 & $4.20 \mathrm{E}-03$ \\
\hline PA0269 & & conserved hypothetical protein & -3.4 & $3.18 \mathrm{E}-03$ \\
\hline PA0270 & & hypothetical protein & -3.0 & $8.85 \mathrm{E}-03$ \\
\hline PA0370 & & conserved hypothetical protein & -2.5 & $3.73 \mathrm{E}-03$ \\
\hline PA0371 & & hypothetical protein & -2.5 & $3.68 \mathrm{E}-03$ \\
\hline PA0468 & & hypothetical protein & -2.2 & $6.12 \mathrm{E}-03$ \\
\hline PA0567 & & conserved hypothetical protein & -3.2 & $9.87 \mathrm{E}-03$ \\
\hline PA0713 & & hypothetical protein & -3.1 & $7.40 \mathrm{E}-03$ \\
\hline PA0732 & & hypothetical protein & -2.9 & $4.45 \mathrm{E}-03$ \\
\hline PA0737 & & hypothetical protein & -2.0 & $9.86 \mathrm{E}-03$ \\
\hline PA0943 & & hypothetical protein & -2.0 & $3.65 \mathrm{E}-03$ \\
\hline PA1471 & & hypothetical protein & -6.5 & $2.78 \mathrm{E}-03$ \\
\hline PA1605 & & hypothetical protein & -2.3 & $7.41 \mathrm{E}-03$ \\
\hline PA1657 & & conserved hypothetical protein & -8.4 & $3.19 \mathrm{E}-03$ \\
\hline PA1658 & & conserved hypothetical protein & -9.7 & $1.60 \mathrm{E}-03$ \\
\hline PA1659 & & hypothetical protein & -7.9 & $1.91 \mathrm{E}-03$ \\
\hline PA1660 & & hypothetical protein & -4.7 & $8.44 \mathrm{E}-03$ \\
\hline PA1661 & & hypothetical protein & -3.6 & $3.95 \mathrm{E}-03$ \\
\hline PA1664 & & hypothetical protein & -6.3 & $4.73 \mathrm{E}-03$ \\
\hline PA1665 & & hypothetical protein & -7.6 & $2.00 \mathrm{E}-03$ \\
\hline PA1666 & & hypothetical protein & -6.1 & $3.68 \mathrm{E}-03$ \\
\hline PA1667 & & hypothetical protein & -6.0 & $2.90 \mathrm{E}-03$ \\
\hline PA1668 & & hypothetical protein & -4.1 & $3.98 \mathrm{E}-03$ \\
\hline PA1669 & & hypothetical protein & -4.0 & $5.46 \mathrm{E}-03$ \\
\hline PA1732 & & conserved hypothetical protein & -2.6 & $6.70 \mathrm{E}-03$ \\
\hline PA1892 & & hypothetical protein & -2.6 & $9.29 \mathrm{E}-03$ \\
\hline PA1894 & & hypothetical protein & -3.8 & $2.55 \mathrm{E}-03$ \\
\hline PA1897 & & hypothetical protein & -2.8 & $6.00 \mathrm{E}-03$ \\
\hline PA1951 & & hypothetical protein & -2.1 & $9.36 \mathrm{E}-03$ \\
\hline PA1968 & & hypothetical protein & -2.5 & $6.60 \mathrm{E}-03$ \\
\hline PA1969 & & hypothetical protein & -2.5 & $7.41 \mathrm{E}-03$ \\
\hline PA2030 & & hypothetical protein & -4.1 & $8.89 \mathrm{E}-03$ \\
\hline
\end{tabular}




\begin{tabular}{|c|c|c|c|c|}
\hline PA2031 & & hypothetical protein & -3.2 & $8.70 \mathrm{E}-03$ \\
\hline PA2034 & & hypothetical protein & -4.8 & $5.91 \mathrm{E}-03$ \\
\hline PA2080 & $\operatorname{kyn} U$ & kynureninase $\mathrm{KynU}$ & -7.2 & $2.29 \mathrm{E}-03$ \\
\hline PA2081 & kynB & kynurenine formamidase, $\mathrm{KynB}$ & -5.9 & $1.73 \mathrm{E}-03$ \\
\hline PA2222 & & hypothetical protein & -3.1 & 4.94E-03 \\
\hline PA2235 & pslE & hypothetical protein & -3.0 & $3.18 \mathrm{E}-03$ \\
\hline PA2236 & pslF & hypothetical protein & -2.8 & $6.78 \mathrm{E}-03$ \\
\hline PA2238 & pslH & hypothetical protein & -2.5 & $7.89 \mathrm{E}-03$ \\
\hline PA2242 & pslL & hypothetical protein & -2.7 & $3.41 \mathrm{E}-03$ \\
\hline PA2289 & & conserved hypothetical protein & -2.3 & $7.15 \mathrm{E}-03$ \\
\hline PA2384 & & hypothetical protein & -5.7 & $9.36 \mathrm{E}-03$ \\
\hline PA2412 & & conserved hypothetical protein & -2.2 & $3.95 \mathrm{E}-03$ \\
\hline PA2452 & & hypothetical protein & -5.1 & $3.95 \mathrm{E}-03$ \\
\hline PA2630 & & conserved hypothetical protein & -2.8 & $9.59 \mathrm{E}-03$ \\
\hline PA2702 & & hypothetical protein & -2.0 & $4.45 \mathrm{E}-03$ \\
\hline PA2703 & & hypothetical protein & -2.2 & $9.03 \mathrm{E}-03$ \\
\hline PA3042 & & hypothetical protein & -2.9 & $8.70 \mathrm{E}-03$ \\
\hline PA3413 & & conserved hypothetical protein & -3.6 & $7.60 \mathrm{E}-03$ \\
\hline PA3484 & & hypothetical protein & -2.1 & $7.63 \mathrm{E}-03$ \\
\hline PA3572 & & hypothetical protein & -4.7 & $1.86 \mathrm{E}-03$ \\
\hline PA3791 & & hypothetical protein & -2.1 & $9.75 \mathrm{E}-03$ \\
\hline PA4033 & & hypothetical protein & -26.9 & 4.02E-03 \\
\hline PA4154 & & conserved hypothetical protein & -3.8 & $5.96 \mathrm{E}-03$ \\
\hline PA4182 & & hypothetical protein & -2.6 & $5.46 \mathrm{E}-03$ \\
\hline PA4204 & $\operatorname{ppg} L$ & periplasmic gluconolactonase, $\mathrm{PpgL}$ & -3.3 & $2.15 \mathrm{E}-03$ \\
\hline PA4384 & & hypothetical protein & -3.0 & $4.66 \mathrm{E}-03$ \\
\hline PA4458 & & conserved hypothetical protein & -2.0 & $2.15 \mathrm{E}-03$ \\
\hline PA4459 & & conserved hypothetical protein & -2.7 & $6.00 \mathrm{E}-03$ \\
\hline PA4469 & & hypothetical protein & -9.7 & $9.29 \mathrm{E}-03$ \\
\hline PA4625 & & hypothetical protein & -2.9 & $8.25 \mathrm{E}-03$ \\
\hline PA5023 & & conserved hypothetical protein & -2.2 & $4.66 \mathrm{E}-03$ \\
\hline PA5052 & & hypothetical protein & -2.0 & $8.60 \mathrm{E}-03$ \\
\hline PA5104 & & conserved hypothetical protein & -2.5 & $4.45 \mathrm{E}-03$ \\
\hline PA5180 & & conserved hypothetical protein & -6.4 & $3.95 \mathrm{E}-03$ \\
\hline PA5202 & & hypothetical protein & -2.3 & $6.86 \mathrm{E}-03$ \\
\hline PA5463 & & hypothetical protein & -2.0 & $8.59 \mathrm{E}-03$ \\
\hline \multicolumn{5}{|c|}{ Membrane proteins } \\
\hline PA0605 & & probable permease of $\mathrm{ABC}$ transporter & -3.0 & 4.74E-03 \\
\hline PA0606 & & probable permease of $\mathrm{ABC}$ transporter & -2.4 & $4.43 \mathrm{E}-03$ \\
\hline PA2403 & & hypothetical protein & -3.3 & $7.28 \mathrm{E}-03$ \\
\hline PA2415 & & hypothetical protein & -2.3 & $2.78 \mathrm{E}-03$ \\
\hline PA2763 & & hypothetical protein & -3.6 & $8.47 \mathrm{E}-03$ \\
\hline PA2811 & & probable permease of $\mathrm{ABC}-2$ transporter & -4.6 & $6.12 \mathrm{E}-03$ \\
\hline PA3692 & & probable outer membrane protein precursor & -7.8 & $3.46 \mathrm{E}-03$ \\
\hline PA3821 & $\sec D$ & secretion protein SecD & -2.0 & $6.09 \mathrm{E}-03$ \\
\hline PA4467 & & hypothetical protein & -9.9 & $6.69 \mathrm{E}-03$ \\
\hline PA4503 & & probable permease of $\mathrm{ABC}$ transporter & -4.0 & $5.32 \mathrm{E}-03$ \\
\hline PA4504 & & probable permease of $\mathrm{ABC}$ transporter & -4.2 & $4.76 \mathrm{E}-03$ \\
\hline
\end{tabular}


PA5182

hypothetical protein

$-3.2 \quad 3.34 \mathrm{E}-03$

Nucleotide biosynthesis and metabolism

$\begin{array}{lll}\text { PA4855 purD } & \begin{array}{l}\text { phosphoribosylamine--glycine ligase } \\ \text { class II ribonucleotide-diphosphate reductase }\end{array}\end{array}$

$\begin{array}{lll}\text { PA5496 } n r d J b & \begin{array}{l}\text { subunit, NrdJb } \\ \text { class II ribonucleotide-diphosphate reductase }\end{array}\end{array}$

PA5497 nrdJa subunit, NrdJa

Protein secretion/export apparatus

$\begin{array}{lllll}\text { PA0077 icmF1 IcmF1 } & -3.0 & 8.66 \mathrm{E}-03\end{array}$

Translocator outer membrane protein PopD

$\begin{array}{lllll}\text { PA1709 popD } & \text { precursor } & -6.2 & 1.38 \mathrm{E}-03\end{array}$

PA1711 exsE ExsE $\quad-3.6 \quad 3.35 \mathrm{E}-03$

$\begin{array}{llll}\text { PA5210 } & \text { probable secretion pathway ATPase } & -2.3 & 3.31 \mathrm{E}-03\end{array}$

Putative enzymes

PA0507

PA0534

PA0853

PA1662

PA1893

PA2237

PA2239

$-2.0 \quad 8.66 \mathrm{E}-03$

$-3.0 \quad 5.62 \mathrm{E}-03$

$-3.3 \quad 8.44 \mathrm{E}-03$

PA2263

PA2317

PA2682

PA3427

PA3454

PA4171

PA5150

probable acyl-CoA dehydrogenase

$-3.8 \quad 6.26 \mathrm{E}-03$

conserved hypothetical protein

$-3.1 \quad 7.91 \mathrm{E}-03$

probable oxidoreductase

$-2.6 \quad 4.45 \mathrm{E}-03$

probable ClpA/B-type protease

$-5.0 \quad 3.41 \mathrm{E}-03$

hypothetical protein

$-2.9 \quad 4.77 \mathrm{E}-03$

$p s l G \quad$ probable glycosyl hydrolase

$-2.4 \quad 6.82 \mathrm{E}-03$

pslI

probable transferase

$-3.0 \quad 4.02 \mathrm{E}-03$

probable 2-hydroxyacid dehydrogenase $\quad-2.2 \quad 3.95 \mathrm{E}-03$

probable oxidoreductase $\quad 2.9 \quad 2.78 \mathrm{E}-03$

$\begin{array}{lll}\text { conserved hypothetical protein } & -3.0 & 7.58 \mathrm{E}-03\end{array}$

probable short-chain dehydrogenases $\quad-3.8 \quad 8.59 \mathrm{E}-03$

probable acyl-CoA thiolase

$-3.9 \quad 4.33 \mathrm{E}-03$

probable protease

$-2.4 \quad 4.86 \mathrm{E}-03$

probable short-chain dehydrogenase

$-2.0 \quad 5.46 \mathrm{E}-03$

PA5181

probable oxidoreductase

$-8.6 \quad 5.96 \mathrm{E}-03$

Secreted Factors (toxins, enzymes, alginate)

\begin{tabular}{|c|c|c|c|c|}
\hline PA0085 & hcpl & Hep1 & -3.7 & $3.09 \mathrm{E}-03$ \\
\hline PA0263 & $h c p C$ & secreted protein Hcp & -7.7 & $4.66 \mathrm{E}-03$ \\
\hline PA1707 & pcrH & regulatory protein PcrH & -3.8 & $3.98 \mathrm{E}-03$ \\
\hline PA3841 & $\operatorname{exoS}$ & exoenzyme S & -3.5 & $1.16 \mathrm{E}-03$ \\
\hline PA3842 & & probable chaperone & -2.9 & $5.73 \mathrm{E}-03$ \\
\hline \multicolumn{5}{|c|}{ Transcription, RNA processing and degradation } \\
\hline PA4740 & pnp & polyribonucleotide nucleotidyltransferase & -2.1 & 5.49E-03 \\
\hline \multicolumn{5}{|c|}{ Transcriptional regulators } \\
\hline PA0479 & & probable transcriptional regulator & -2.1 & $9.90 \mathrm{E}-03$ \\
\hline PA1663 & & probable transcriptional regulator & -3.9 & $5.13 \mathrm{E}-03$ \\
\hline PA2281 & & probable transcriptional regulator & -2.1 & $9.89 \mathrm{E}-03$ \\
\hline PA2588 & & probable transcriptional regulator & -27.9 & $3.77 \mathrm{E}-03$ \\
\hline PA5105 & hutC & histidine utilization repressor $\mathrm{HutC}$ & -3.1 & 8.27E-03 \\
\hline PA5483 & $\operatorname{alg} B$ & two-component response regulator $\mathrm{AlgB}$ & -2.4 & 7.83E-03 \\
\hline \multicolumn{5}{|c|}{ Translation, post-translational modification, degradation } \\
\hline PA0594 & surA & $\begin{array}{l}\text { peptidyl-prolyl cis-trans isomerase SurA } \\
\text { serine/threonine phosphoprotein phosphatase }\end{array}$ & -2.4 & $6.26 \mathrm{E}-03$ \\
\hline PA1670 & stpl & Stp1 & -2.3 & $3.81 \mathrm{E}-03$ \\
\hline
\end{tabular}




\begin{tabular}{|c|c|c|c|c|}
\hline \multicolumn{5}{|c|}{ Transport of small molecules } \\
\hline PA2987 & & $\begin{array}{l}\text { probable ATP-binding component of ABC } \\
\text { transporter }\end{array}$ & -2.1 & $3.68 \mathrm{E}-03$ \\
\hline PA4206 & $\operatorname{mexH}$ & $\begin{array}{l}\text { probable RND efflux membrane fusion } \\
\text { protein precursor }\end{array}$ & -21.4 & $2.54 \mathrm{E}-03$ \\
\hline PA4500 & & $\begin{array}{l}\text { probable binding protein component of } \mathrm{ABC} \\
\text { transporter }\end{array}$ & -5.2 & $3.73 \mathrm{E}-03$ \\
\hline PA4502 & & $\begin{array}{l}\text { transporter } \\
\text { probable ATP-binding component of } \mathrm{ABC}\end{array}$ & -4.9 & $3.41 \mathrm{E}-03$ \\
\hline PA4505 & & $\begin{array}{l}\text { transporter } \\
\text { probable ATP-binding component of } A B C\end{array}$ & -3.3 & $3.71 \mathrm{E}-03$ \\
\hline PA4506 & & dipeptide transporter & -3.8 & $2.99 \mathrm{E}-03$ \\
\hline \multicolumn{5}{|c|}{ Two-component regulatory systems } \\
\hline PA5484 & & probable two-component sensor & -3.0 & $7.01 \mathrm{E}-03$ \\
\hline
\end{tabular}

\begin{tabular}{|c|c|c|c|c|}
\hline \multicolumn{5}{|c|}{ Upregulated genes } \\
\hline Locus Tag & $\begin{array}{l}\text { Gene } \\
\text { Name }\end{array}$ & Product Name & FC & $\begin{array}{c}\text { Corrected } \\
p \text {-value }\end{array}$ \\
\hline \multicolumn{5}{|l|}{$\begin{array}{l}\text { Adaptation, } \\
\text { Protection }\end{array}$} \\
\hline PA0180 & & probable chemotaxis transducer & 2.1 & $5.78 \mathrm{E}-03$ \\
\hline PA0923 & $\operatorname{din} B$ & DNA Polymerase IV, DinB & 4.5 & $6.05 \mathrm{E}-03$ \\
\hline PA2652 & & probable chemotaxis transducer & 2.6 & $8.67 \mathrm{E}-03$ \\
\hline PA3007 & $\operatorname{lex} A$ & $\begin{array}{l}\text { repressor protein LexA } \\
\text { probable chemotaxis protein }\end{array}$ & 2.7 & $7.28 \mathrm{E}-03$ \\
\hline PA3348 & & methyltransferase & 2.2 & $3.64 \mathrm{E}-03$ \\
\hline PA4309 & pctA & chemotactic transducer PctA & 3.2 & $7.83 \mathrm{E}-03$ \\
\hline PA4356 & $x e n B$ & xenobiotic reductase & 3.2 & $5.96 \mathrm{E}-03$ \\
\hline PA4378 & inaA & InaA protein & 3.0 & $6.68 \mathrm{E}-03$ \\
\hline PA4520 & & probable chemotaxis transducer & 2.1 & $3.69 \mathrm{E}-03$ \\
\hline \multicolumn{5}{|c|}{ Amino acid biosynthesis and metabolism } \\
\hline PA0245 & $\operatorname{aroQ2}$ & 3-dehydroquinate dehydratase & 2.9 & $3.71 \mathrm{E}-03$ \\
\hline PA4565 & $\operatorname{proB}$ & glutamate 5-kinase & 2.9 & $5.03 \mathrm{E}-03$ \\
\hline \multicolumn{5}{|c|}{ Biosynthesis of cofactors, prosthetic groups and carriers } \\
\hline PA4919 & pncB1 & nicotinate phosphoribosyltransferase & 6.3 & 3.95E-03 \\
\hline \multicolumn{5}{|c|}{ Carbon compound catabolism } \\
\hline PA0153 & pcaH & $\begin{array}{l}\text { protocatechuate 3,4-dioxygenase, beta subunit } \\
\text { protocatechuate 3,4-dioxygenase, alpha }\end{array}$ & 3.3 & $3.42 \mathrm{E}-03$ \\
\hline PA0154 & pcaG & subunit & 2.2 & $6.25 \mathrm{E}-03$ \\
\hline PA0226 & & probable CoA transferase, subunit A & 4.4 & $9.97 \mathrm{E}-03$ \\
\hline PA0230 & $p c a B$ & 3-carboxy-cis,cis-muconate cycloisomerase & 3.3 & 7.08E-03 \\
\hline PA0231 & pcaD & beta-ketoadipate enol-lactone hydrolase & 3.7 & $5.16 \mathrm{E}-03$ \\
\hline PA0232 & pcaC & gamma-carboxymuconolactone decarboxylase & 4.1 & $8.44 \mathrm{E}-03$ \\
\hline PA2862 & $\operatorname{lip} A$ & lactonizing lipase precursor & 3.6 & 5.97E-03 \\
\hline PA4123 & $h p c C$ & $\begin{array}{l}\text { 5-carboxy-2-hydroxymuconate semialdehyde } \\
\text { dehydrogenase } \\
\text { 5-carboxymethyl-2-hydroxymuconate }\end{array}$ & 6.7 & $3.18 \mathrm{E}-03$ \\
\hline PA4125 & $h p c D$ & isomerase & 5.8 & $6.12 \mathrm{E}-03$ \\
\hline
\end{tabular}




\begin{tabular}{|c|c|c|c|c|}
\hline \multicolumn{5}{|c|}{ Cell wall / LPS / capsule } \\
\hline PA1082 & $\operatorname{flg} G$ & flagellar basal-body rod protein FlgG & 2.9 & $8.52 \mathrm{E}-03$ \\
\hline PA3160 & $w z z$ & O-antigen chain length regulator & 3.7 & $8.81 \mathrm{E}-03$ \\
\hline PA4480 & mreC & rod shape-determining protein $\mathrm{MreC}$ & 4.8 & $6.34 \mathrm{E}-03$ \\
\hline \multicolumn{5}{|c|}{ Central intermediary metabolism } \\
\hline PA2813 & & probable glutathione S-transferase & 2.6 & $9.10 \mathrm{E}-03$ \\
\hline PA3629 & $a d h C$ & alcohol dehydrogenase class III & 3.2 & $4.98 \mathrm{E}-03$ \\
\hline \multicolumn{5}{|c|}{ Chemotaxis } \\
\hline PA0179 & & probable two-component response regulator & 3.2 & $9.57 \mathrm{E}-03$ \\
\hline \multicolumn{5}{|c|}{ DNA replication, recombination, modification and repair } \\
\hline PA0577 & dnaG & DNA primase & 4.7 & $8.39 \mathrm{E}-03$ \\
\hline PA3617 & recA & RecA protein & 3.3 & $5.46 \mathrm{E}-03$ \\
\hline \multicolumn{5}{|c|}{ Energy metabolism } \\
\hline PA0105 & $\operatorname{cox} B$ & cytochrome c oxidase, subunit II & 2.5 & $8.21 \mathrm{E}-03$ \\
\hline PA3621 & $f d x A$ & ferredoxin I & 3.1 & $5.32 \mathrm{E}-03$ \\
\hline PA4430 & & probable cytochrome b & 2.2 & $2.00 \mathrm{E}-03$ \\
\hline \multicolumn{5}{|c|}{ Hypothetical, unclassified, unknown } \\
\hline PA0124 & & hypothetical protein & 2.8 & $3.81 \mathrm{E}-03$ \\
\hline PA0128 & & conserved hypothetical protein & 3.3 & $7.28 \mathrm{E}-03$ \\
\hline PA0466 & & hypothetical protein & 3.1 & $9.12 \mathrm{E}-03$ \\
\hline PA0529 & & conserved hypothetical protein & 3.0 & $8.58 \mathrm{E}-03$ \\
\hline PA0614 & & hypothetical protein & 6.7 & $8.26 \mathrm{E}-03$ \\
\hline PA0615 & & hypothetical protein & 6.5 & $5.78 \mathrm{E}-03$ \\
\hline PA0717 & & hypothetical protein of bacteriophage Pf1 & 3.5 & $3.44 \mathrm{E}-03$ \\
\hline PA0729 & & hypothetical protein & 9.4 & $3.41 \mathrm{E}-03$ \\
\hline PA0910 & & hypothetical protein & 8.5 & $6.26 \mathrm{E}-03$ \\
\hline PA0911 & & hypothetical protein & 6.8 & $7.83 \mathrm{E}-03$ \\
\hline PA1016 & & hypothetical protein & 2.6 & $7.58 \mathrm{E}-03$ \\
\hline PA1164 & & conserved hypothetical protein & 2.1 & $3.66 \mathrm{E}-03$ \\
\hline PA1299 & & conserved hypothetical protein & 3.0 & $3.41 \mathrm{E}-03$ \\
\hline PA1333 & & hypothetical protein & 2.6 & $5.97 \mathrm{E}-03$ \\
\hline PA1439 & & conserved hypothetical protein & 2.2 & $7.28 \mathrm{E}-03$ \\
\hline PA1545 & & hypothetical protein & 2.7 & $6.65 \mathrm{E}-03$ \\
\hline PA1744 & & hypothetical protein & 3.1 & $5.62 \mathrm{E}-03$ \\
\hline PA2362 & & hypothetical protein & 2.4 & $9.87 \mathrm{E}-03$ \\
\hline PA2365 & & conserved hypothetical protein & 9.4 & $4.50 \mathrm{E}-03$ \\
\hline PA2487 & & hypothetical protein & 11.6 & $2.26 \mathrm{E}-03$ \\
\hline PA2765 & & hypothetical protein & 14.6 & $1.60 \mathrm{E}-03$ \\
\hline PA2780 & & hypothetical protein & 2.6 & $4.66 \mathrm{E}-03$ \\
\hline PA2901 & & hypothetical protein & 16.5 & 4.43E-03 \\
\hline PA3008 & & hypothetical protein & 4.1 & $7.71 \mathrm{E}-03$ \\
\hline PA3306 & & hypothetical protein & 14.9 & $2.90 \mathrm{E}-03$ \\
\hline PA3353 & & hypothetical protein & 2.3 & $6.40 \mathrm{E}-03$ \\
\hline PA3371 & & hypothetical protein & 2.5 & $9.87 \mathrm{E}-03$ \\
\hline PA3499 & & hypothetical protein & 2.4 & $6.97 \mathrm{E}-03$ \\
\hline PA3505 & & hypothetical protein & 5.7 & $3.92 \mathrm{E}-03$ \\
\hline
\end{tabular}




\begin{tabular}{|c|c|c|c|c|}
\hline \multirow{2}{*}{\multicolumn{2}{|c|}{ PA3649 }} & & & \\
\hline & & conserved hypothetical protein & 2.1 & $6.31 \mathrm{E}-03$ \\
\hline \multicolumn{2}{|l|}{ PA3741 } & hypothetical protein & 7.1 & $6.52 \mathrm{E}-03$ \\
\hline \multicolumn{2}{|l|}{ PA3880 } & conserved hypothetical protein & 2.4 & $5.62 \mathrm{E}-03$ \\
\hline \multicolumn{2}{|l|}{ PA3922 } & conserved hypothetical protein & 2.4 & 8.59E-03 \\
\hline \multicolumn{2}{|l|}{ PA3923 } & hypothetical protein & 3.6 & $6.26 \mathrm{E}-03$ \\
\hline \multicolumn{2}{|l|}{ PA3951 } & conserved hypothetical protein & 2.1 & 8.33E-03 \\
\hline \multicolumn{2}{|l|}{ PA4121 } & conserved hypothetical protein & 4.9 & $6.75 \mathrm{E}-03$ \\
\hline \multicolumn{2}{|l|}{ PA4326 } & hypothetical protein & 3.1 & 7.63E-03 \\
\hline \multicolumn{2}{|l|}{ PA4379 } & conserved hypothetical protein & 5.0 & $3.41 \mathrm{E}-03$ \\
\hline \multicolumn{2}{|l|}{ PA4582 } & conserved hypothetical protein & 7.6 & 3.19E-03 \\
\hline \multicolumn{2}{|l|}{ PA4584 } & conserved hypothetical protein & 3.7 & 7.29E-03 \\
\hline \multicolumn{2}{|l|}{ PA4918 } & hypothetical protein & 16.9 & $2.59 \mathrm{E}-03$ \\
\hline \multicolumn{2}{|l|}{ PA4921 } & hypothetical protein & 4.2 & $3.77 \mathrm{E}-03$ \\
\hline PA5292 & $p \operatorname{ch} P$ & phosphorylcholine phosphatase & 2.3 & $9.89 \mathrm{E}-03$ \\
\hline \multicolumn{2}{|l|}{ PA5543 } & hypothetical protein & 2.0 & $5.81 \mathrm{E}-03$ \\
\hline \multicolumn{5}{|c|}{ Membrane proteins } \\
\hline PA0229 & pcaT & dicarboxylic acid transporter PcaT & 4.6 & $6.78 \mathrm{E}-03$ \\
\hline PA0240 & & probable porin & 2.3 & 8.70E-03 \\
\hline PA0465 & creD & $\begin{array}{l}\text { inner membrane protein } \mathrm{CreD} \\
\text { branched-chain amino acid transport protein }\end{array}$ & 4.7 & $9.95 \mathrm{E}-03$ \\
\hline \multirow{2}{*}{$\begin{array}{l}\text { PA1072 } \\
\text { PA1436 }\end{array}$} & braE & $\mathrm{BraE}$ & 2.8 & $4.45 \mathrm{E}-03$ \\
\hline & & $\begin{array}{l}\text { probable RND efflux transporter } \\
\text { Multidrug efflux outer membrane protein }\end{array}$ & 3.9 & $6.31 \mathrm{E}-03$ \\
\hline PA2495 & $\operatorname{opr} N$ & OprN precursor & 102.6 & $1.05 \mathrm{E}-04$ \\
\hline PA2988 & & conserved hypothetical protein & 2.2 & 5.73E-03 \\
\hline \multicolumn{2}{|l|}{ PA3041 } & $\begin{array}{l}\text { hypothetical protein } \\
\text { probable ATP-binding/permease fusion } \mathrm{ABC}\end{array}$ & 4.1 & $5.13 \mathrm{E}-03$ \\
\hline \multicolumn{2}{|l|}{ PA3228 } & transporter & 3.5 & $1.38 \mathrm{E}-03$ \\
\hline \multicolumn{2}{|l|}{ PA3234 } & probable sodium:solute symporter & 3.3 & $8.62 \mathrm{E}-03$ \\
\hline \multicolumn{2}{|l|}{ PA3235 } & $\begin{array}{l}\text { conserved hypothetical protein } \\
\text { probable major facilitator superfamily (MFS) }\end{array}$ & 3.0 & $5.47 \mathrm{E}-03$ \\
\hline \multicolumn{2}{|l|}{ PA3336 } & transporter & 2.3 & 3.19E-03 \\
\hline PA3526 & & probable outer membrane protein precursor & 2.3 & 3.64E-03 \\
\hline PA3575 & & hypothetical protein & 2.2 & $6.11 \mathrm{E}-03$ \\
\hline PA3602 & & $\begin{array}{l}\text { conserved hypothetical protein } \\
\text { probable major facilitator superfamily (MFS) }\end{array}$ & 2.3 & $6.70 \mathrm{E}-03$ \\
\hline PA4126 & & transporter & 2.4 & $9.20 \mathrm{E}-03$ \\
\hline PA4911 & & $\begin{array}{l}\text { probable permease-ABC branched-chain } \\
\text { amino acid transporter } \\
\text { branched chain amino acid } \mathrm{ABC} \text { transporter }\end{array}$ & 5.1 & $2.78 \mathrm{E}-03$ \\
\hline PA4912 & & membrane protein & 6.6 & $2.15 \mathrm{E}-03$ \\
\hline PA5561 & atpI & ATP synthase protein I & 2.6 & $5.05 \mathrm{E}-03$ \\
\hline Motility \& & achmer & & & \\
\hline PA1092 & $f l i C$ & flagellin type B & 2.5 & $9.63 \mathrm{E}-03$ \\
\hline PA1099 & fleR & two-component response regulator & 3.0 & $7.63 \mathrm{E}-03$ \\
\hline $\begin{array}{l}\text { Non-coding } \\
\text { gene }\end{array}$ & & & & \\
\hline PA3621.1 & $r s m Z$ & regulatory RNA RsmZ & 4.8 & $9.25 \mathrm{E}-03$ \\
\hline
\end{tabular}




\begin{tabular}{|c|c|c|c|c|}
\hline \multicolumn{5}{|c|}{ Protein secretion/export apparatus } \\
\hline PA2863 & lipH & lipase modulator protein & 2.2 & $4.85 \mathrm{E}-03$ \\
\hline \multicolumn{5}{|c|}{ Putative enzymes } \\
\hline PA0242 & & hypothetical protein & 2.6 & $7.19 \mathrm{E}-03$ \\
\hline PA0244 & & hypothetical protein & 2.9 & $4.52 \mathrm{E}-03$ \\
\hline PA1296 & & probable 2-hydroxyacid dehydrogenase & 3.2 & $3.44 \mathrm{E}-03$ \\
\hline PA3498 & & probable oxidoreductase & 2.4 & $7.51 \mathrm{E}-03$ \\
\hline PA3506 & & probable decarboxylase & 4.8 & $5.76 \mathrm{E}-03$ \\
\hline PA3628 & & probable esterase & 2.9 & $5.71 \mathrm{E}-03$ \\
\hline PA3817 & & probable methyltransferase & 4.2 & $5.32 \mathrm{E}-03$ \\
\hline PA5048 & & probable nuclease & 3.2 & $9.01 \mathrm{E}-03$ \\
\hline \multicolumn{5}{|c|}{ Related to phage, transposon, or plasmid } \\
\hline PA0616 & & hypothetical protein & 6.9 & $7.25 \mathrm{E}-03$ \\
\hline PA0617 & & probable bacteriophage protein & 7.8 & $8.66 \mathrm{E}-03$ \\
\hline PA0618 & & probable bacteriophage protein & 7.2 & 7.74E-03 \\
\hline PA0619 & & probable bacteriophage protein & 5.9 & $9.87 \mathrm{E}-03$ \\
\hline PA0620 & & probable bacteriophage protein & 7.5 & $5.76 \mathrm{E}-03$ \\
\hline PA0621 & & conserved hypothetical protein & 7.7 & $6.53 \mathrm{E}-03$ \\
\hline PA0622 & & probable bacteriophage protein & 6.0 & 4.45E-03 \\
\hline PA0623 & & probable bacteriophage protein & 6.3 & $7.01 \mathrm{E}-03$ \\
\hline PA0633 & & hypothetical protein & 7.5 & $5.76 \mathrm{E}-03$ \\
\hline PA0634 & & hypothetical protein & 6.6 & $7.15 \mathrm{E}-03$ \\
\hline PA0637 & & conserved hypothetical protein & 7.0 & $8.11 \mathrm{E}-03$ \\
\hline PA0638 & & probable bacteriophage protein & 6.3 & $9.02 \mathrm{E}-03$ \\
\hline PA0641 & & probable bacteriophage protein & 5.6 & $9.59 \mathrm{E}-03$ \\
\hline PA0643 & & hypothetical protein & 6.0 & $8.59 \mathrm{E}-03$ \\
\hline \multicolumn{5}{|c|}{ Secreted Factors (toxins, enzymes, alginate) } \\
\hline PA0843 & plcR & $\begin{array}{l}\text { phospholipase accessory protein PlcR } \\
\text { precursor }\end{array}$ & 2.2 & $2.16 \mathrm{E}-03$ \\
\hline \multicolumn{5}{|c|}{ Transcription, RNA processing and degradation } \\
\hline PA2840 & & $\begin{array}{l}\text { probable ATP-dependent RNA helicase } \\
\text { ribosomal large subunit pseudouridine }\end{array}$ & 3.5 & 4.17E-04 \\
\hline PA2975 & rluC & synthase $\mathrm{C}$ & 6.5 & $6.17 \mathrm{E}-03$ \\
\hline PA3744 & $\operatorname{rim} M$ & 16S rRNA processing protein & 6.2 & $4.66 \mathrm{E}-03$ \\
\hline PA4275 & $n u s G$ & transcription antitermination protein NusG & 2.7 & $9.69 \mathrm{E}-03$ \\
\hline \multicolumn{5}{|c|}{ Transcriptional regulators } \\
\hline PA0762 & $\operatorname{alg} U$ & sigma factor $\mathrm{AlgU}$ & 5.4 & $3.35 \mathrm{E}-03$ \\
\hline PA1300 & & probable sigma-70 factor, ECF subfamily & 2.5 & $9.59 \mathrm{E}-03$ \\
\hline PA5380 & $g b d R$ & $\begin{array}{l}\text { GbdR } \\
\text { negative regulator for alginate biosynthesis }\end{array}$ & 2.6 & $5.75 \mathrm{E}-03$ \\
\hline PA0764 & тисв & MucB & 2.5 & $8.79 \mathrm{E}-03$ \\
\hline PA0610 & $\operatorname{prt} N$ & transcriptional regulator PrtN & 6.1 & $5.58 \mathrm{E}-03$ \\
\hline PA0611 & $\operatorname{prt} R$ & transcriptional regulator PrtR & 2.9 & $8.52 \mathrm{E}-03$ \\
\hline PA0612 & $p t r B$ & repressor, PtrB & 6.0 & $2.78 \mathrm{E}-03$ \\
\hline \multicolumn{5}{|c|}{ Transport of small molecules } \\
\hline PA0913 & $m g t E$ & $\begin{array}{l}\text { probable } \mathrm{Mg} \text { transporter } \mathrm{MgtE} \\
\text { branched-chain amino acid transport protein }\end{array}$ & 3.1 & $6.25 \mathrm{E}-03$ \\
\hline PA1070 & braG & BraG & 2.5 & $4.02 \mathrm{E}-03$ \\
\hline
\end{tabular}




\begin{tabular}{|c|c|c|c|c|}
\hline & & branched-chain amino acid transport protein & & \\
\hline PA1071 & braF & $\begin{array}{l}\text { BraF } \\
\text { amino acid } A B C \text { transporter ATP binding }\end{array}$ & 2.2 & $7.55 \mathrm{E}-03$ \\
\hline PA1256 & & protein & 2.3 & $8.26 \mathrm{E}-03$ \\
\hline PA3038 & & probable porin & 2.3 & $4.85 \mathrm{E}-03$ \\
\hline & & probable binding protein component of $\mathrm{ABC}$ & & \\
\hline PA3889 & & $\begin{array}{l}\text { transporter } \\
\text { probable ATP-binding component of } \mathrm{ABC}\end{array}$ & 2.2 & $9.95 \mathrm{E}-03$ \\
\hline PA4909 & & transporter & 3.7 & $3.92 \mathrm{E}-03$ \\
\hline PA4910 & & $\begin{array}{l}\text { branched chain amino acid } \mathrm{ABC} \text { transporter } \\
\text { ATP binding protein } \\
\text { probable binding protein component of } \mathrm{ABC}\end{array}$ & 5.7 & $4.16 \mathrm{E}-03$ \\
\hline PA4913 & & transporter & 4.7 & $5.71 \mathrm{E}-03$ \\
\hline & & $\begin{array}{l}\text { Resistance-Nodulation-Cell Division (RND) } \\
\text { multidrug efflux membrane fusion protein }\end{array}$ & & \\
\hline PA2493 & $\operatorname{mex} E$ & MexE precursor & 6.9 & $2.72 \mathrm{E}-03$ \\
\hline PA2494 & $\operatorname{mex} F$ & $\begin{array}{l}\text { Resistance-Nodulation-Cell Division (RND) } \\
\text { multidrug efflux transporter MexF }\end{array}$ & 88.7 & $7.22 \mathrm{E}-04$ \\
\hline PA0958 & oprD & $\begin{array}{l}\text { Basic amino acid, basic peptide and imipenem } \\
\text { outer membrane porin OprD precursor } \\
\text { probable ATP-binding component of ABC }\end{array}$ & 2.2 & $3.49 \mathrm{E}-03$ \\
\hline PA5376 & & transporter & 2.2 & $6.12 \mathrm{E}-03$ \\
\hline \multicolumn{5}{|c|}{ Two-component regulatory systems } \\
\hline PA0757 & & probable two-component sensor & 2.3 & $3.77 \mathrm{E}-03$ \\
\hline PA1098 & fles & two-component sensor & 2.5 & $6.65 \mathrm{E}-03$ \\
\hline
\end{tabular}


Table S4: AmpR, B-lactam-dependent genes. The 207 genes that are specifically dependent on AmpR, and B-lactam stress are separated into upregulated and downregulated genes with the corresponding corrected $p$-values for the fold change (FC) observed. The genes are arranged based on either (A) functional categorization, or (B) fold change. Locus tag annotations are from the Pseudomonas Genome database (31).

\begin{tabular}{|c|c|c|c|c|}
\hline \multicolumn{5}{|c|}{ Downregulated genes } \\
\hline Locus Tag & Gene Name & Product Name & FC & $\begin{array}{c}\text { Corrected } \\
p \text {-value }\end{array}$ \\
\hline \multicolumn{5}{|c|}{ Adaptation, Protection } \\
\hline PA4671 & & probable ribosomal protein L25 & -2.6 & $9.07 \mathrm{E}-03$ \\
\hline \multicolumn{5}{|c|}{ Amino acid biosynthesis and metabolism } \\
\hline PA0390 & met $X$ & homoserine O-acetyltransferase & -2.5 & $6.29 \mathrm{E}-03$ \\
\hline PA0932 & cys $M$ & cysteine synthase B & -2.0 & $2.90 \mathrm{E}-03$ \\
\hline PA1750 & & phospho-2-dehydro-3-deoxyheptonate aldolase & -2.4 & $8.53 \mathrm{E}-03$ \\
\hline PA4846 & $\operatorname{aroQ1}$ & 3-dehydroquinate dehydratase & -2.3 & $6.79 \mathrm{E}-03$ \\
\hline \multicolumn{5}{|c|}{ Biosynthesis of cofactors, prosthetic groups and carriers } \\
\hline PA0024 & hemF & coproporphyrinogen III oxidase, aerobic & -3.2 & $6.65 \mathrm{E}-03$ \\
\hline PA4280 & $\operatorname{bir} A$ & BirA bifunctional protein & -3.0 & $9.14 \mathrm{E}-03$ \\
\hline PA5118 & thiI & thiazole biosynthesis protein ThiI & -2.9 & $7.95 \mathrm{E}-03$ \\
\hline PA3652 & uppS & undecaprenyl pyrophosphate synthetase & -2.3 & $6.65 \mathrm{E}-03$ \\
\hline PA0342 & thy $A$ & thymidylate synthase & -2.1 & $8.99 \mathrm{E}-03$ \\
\hline \multicolumn{5}{|c|}{$\begin{array}{l}\text { Carbon compound } \\
\text { catabolism }\end{array}$} \\
\hline PA0227 & & probable CoA transferase, subunit B & -3.1 & $6.65 \mathrm{E}-03$ \\
\hline PA5192 & $p c k A$ & phosphoenolpyruvate carboxykinase & -3.0 & $6.04 \mathrm{E}-04$ \\
\hline PA4670 & prs & ribose-phosphate pyrophosphokinase & -4.0 & $9.10 \mathrm{E}-03$ \\
\hline \multicolumn{5}{|l|}{ Cell division } \\
\hline PA5563 & soj & chromosome partitioning protein Soj & -2.1 & $9.71 \mathrm{E}-03$ \\
\hline \multicolumn{5}{|c|}{ Cell wall / LPS / capsule } \\
\hline PA4450 & murA & UDP-N-acetylglucosamine 1-carboxyvinyltransferase & -2.1 & $9.73 \mathrm{E}-03$ \\
\hline PA4545 & $\operatorname{com} L$ & competence protein $\mathrm{ComL}$ & -2.1 & $7.95 \mathrm{E}-03$ \\
\hline PA4481 & $m r e B$ & rod shape-determining protein $\mathrm{MreB}$ & -4.4 & $6.51 \mathrm{E}-03$ \\
\hline \multicolumn{5}{|c|}{ Central intermediary metabolism } \\
\hline PA4563 & $\operatorname{rps} T$ & 30 S ribosomal protein $\mathrm{S} 20$ & -5.9 & $9.92 \mathrm{E}-03$ \\
\hline \multicolumn{5}{|c|}{ Chaperones \& heat shock proteins } \\
\hline PA5193 & $y r f I$ & heat shock protein HSP33 & -2.5 & $6.65 \mathrm{E}-03$ \\
\hline \multicolumn{5}{|c|}{ DNA replication, recombination, modification and repair } \\
\hline PA3620 & mutS & DNA mismatch repair protein MutS & -2.6 & $9.22 \mathrm{E}-03$ \\
\hline \multicolumn{5}{|c|}{ Hypothetical, unclassified, unknown } \\
\hline PA0045 & & hypothetical protein & -4.2 & $7.48 \mathrm{E}-03$ \\
\hline PA0046 & & hypothetical protein & -3.4 & $9.10 \mathrm{E}-03$ \\
\hline PA1768 & & hypothetical protein & -3.4 & $7.86 \mathrm{E}-03$ \\
\hline PA2318 & & hypothetical protein & -3.1 & $8.53 \mathrm{E}-03$ \\
\hline PA2427 & & hypothetical protein & -2.2 & $9.12 \mathrm{E}-03$ \\
\hline PA2451 & & hypothetical protein & -2.2 & $9.58 \mathrm{E}-03$ \\
\hline PA2486 & & hypothetical protein & -4.0 & $7.00 \mathrm{E}-03$ \\
\hline
\end{tabular}




\begin{tabular}{|c|c|c|c|c|}
\hline PA2660 & & hypothetical protein & -2.6 & $9.73 \mathrm{E}-03$ \\
\hline PA2823 & & conserved hypothetical protein & -2.0 & $9.10 \mathrm{E}-03$ \\
\hline PA3453 & & conserved hypothetical protein & -2.8 & $9.34 \mathrm{E}-03$ \\
\hline PA3472 & & hypothetical protein & -5.4 & $8.53 \mathrm{E}-03$ \\
\hline PA3483 & & hypothetical protein & -2.0 & $7.09 \mathrm{E}-03$ \\
\hline PA3664 & & conserved hypothetical protein & -2.1 & $7.95 \mathrm{E}-03$ \\
\hline PA3906 & & hypothetical protein & -2.4 & $9.39 \mathrm{E}-03$ \\
\hline PA3908 & & hypothetical protein & -2.2 & $7.86 \mathrm{E}-03$ \\
\hline PA4004 & & conserved hypothetical protein & -2.7 & $6.65 \mathrm{E}-03$ \\
\hline PA4122 & & conserved hypothetical protein & -3.0 & 7.53E-03 \\
\hline PA4438 & & conserved hypothetical protein & -3.4 & $6.65 \mathrm{E}-03$ \\
\hline PA4451 & & conserved hypothetical protein & -3.7 & 7.09E-03 \\
\hline PA4460 & & conserved hypothetical protein & -2.1 & $8.44 \mathrm{E}-03$ \\
\hline PA4492 & & conserved hypothetical protein & -2.2 & 8.57E-03 \\
\hline PA4574 & & conserved hypothetical protein & -2.2 & $6.33 \mathrm{E}-03$ \\
\hline PA4673 & & conserved hypothetical protein & -2.8 & $7.09 \mathrm{E}-03$ \\
\hline PA4753 & & conserved hypothetical protein & -2.3 & $8.09 \mathrm{E}-03$ \\
\hline PA4817 & & hypothetical protein & -2.0 & 7.48E-03 \\
\hline PA5441 & & hypothetical protein & -2.0 & 7.09E-03 \\
\hline \multicolumn{5}{|c|}{ Membrane proteins } \\
\hline PA3794 & & hypothetical protein & -2.3 & 4.37E-03 \\
\hline PA3828 & & conserved hypothetical protein & -2.7 & $9.24 \mathrm{E}-03$ \\
\hline PA5568 & & conserved hypothetical protein & -3.2 & $6.35 \mathrm{E}-03$ \\
\hline PA4205 & $\operatorname{mex} G$ & $\begin{array}{l}\text { hypothetical protein } \\
\text { probable Resistance-Nodulation-Cell Division (RND) efflux }\end{array}$ & $\begin{array}{c}- \\
30.2 \\
-\end{array}$ & $1.18 \mathrm{E}-03$ \\
\hline PA4207 & mexI & transporter & $\begin{array}{c}10.8 \\
-\end{array}$ & $2.29 \mathrm{E}-03$ \\
\hline PA4208 & opmD & probable outer membrane protein precursor & 10.8 & $1.60 \mathrm{E}-03$ \\
\hline PA4292 & & probable phosphate transporter & -2.4 & $9.10 \mathrm{E}-03$ \\
\hline \multicolumn{5}{|c|}{ Nucleotide biosynthesis and metabolism } \\
\hline PA0944 & $\operatorname{purN}$ & phosphoribosylaminoimidazole synthetase & -2.2 & $9.73 \mathrm{E}-03$ \\
\hline PA3527 & pyrC & dihydroorotase & -2.5 & 8.09E-03 \\
\hline PA5298 & & xanthine phosphoribosyltransferase & -3.2 & $9.75 \mathrm{E}-03$ \\
\hline PA5336 & gmk & guanylate kinase & -3.0 & $8.86 \mathrm{E}-03$ \\
\hline \multicolumn{5}{|c|}{ Protein secretion/export apparatus } \\
\hline PA1708 & popB & translocator protein PopB & -2.0 & $9.63 \mathrm{E}-03$ \\
\hline PA5128 & $\sec B$ & secretion protein $\mathrm{SecB}$ & -2.5 & 7.43E-03 \\
\hline \multicolumn{5}{|c|}{ Putative enzymes } \\
\hline PA0386 & & probable oxidase & -2.4 & 7.33E-03 \\
\hline PA4073 & & probable aldehyde dehydrogenase & -3.3 & 8.33E-03 \\
\hline PA4209 & $p h z M$ & probable phenazine-specific methyltransferase & -3.8 & $3.95 \mathrm{E}-03$ \\
\hline PA4217 & $p h z S$ & flavin-containing monooxygenase & 28.0 & $2.90 \mathrm{E}-03$ \\
\hline PA4389 & & probable short-chain dehydrogenase & -2.1 & $7.86 \mathrm{E}-03$ \\
\hline \multicolumn{5}{|c|}{ Secreted Factors (toxins, enzymes, alginate) } \\
\hline PA4210 & $p h z A 1$ & probable phenazine biosynthesis protein & -3.5 & $3.81 \mathrm{E}-03$ \\
\hline PA4211 & $\operatorname{phzB1}$ & probable phenazine biosynthesis protein & 20.7 & $5.11 \mathrm{E}-03$ \\
\hline \multicolumn{5}{|c|}{ Transcription, RNA processing and degradation } \\
\hline PA0592 & $k \operatorname{sg} A$ & rRNA (adenine-N6,N6)-dimethyltransferase & -2.6 & $8.56 \mathrm{E}-03$ \\
\hline
\end{tabular}




\begin{tabular}{|c|c|c|c|c|}
\hline PA4745 & nusA & $\mathrm{N}$ utilization substance protein $\mathrm{A}$ & -2.3 & $8.39 \mathrm{E}-03$ \\
\hline PA4262 & $r p l D$ & $50 \mathrm{~S}$ ribosomal protein $\mathrm{L} 4$ & -3.0 & $6.33 \mathrm{E}-03$ \\
\hline \multicolumn{5}{|c|}{ Transcriptional regulators } \\
\hline PA4853 & fis & DNA-binding protein Fis & -3.3 & $6.65 \mathrm{E}-03$ \\
\hline PA0463 & creB & two-component response regulator $\mathrm{CreB}$ & -2.1 & $8.39 \mathrm{E}-03$ \\
\hline PA3604 & & probable two-component response regulator & -2.2 & $6.96 \mathrm{E}-03$ \\
\hline \multicolumn{5}{|c|}{ Translation, post-translational modification, degradation } \\
\hline PA0579 & $r p s U$ & $30 \mathrm{~S}$ ribosomal protein $\mathrm{S} 21$ & -3.5 & $9.32 \mathrm{E}-03$ \\
\hline PA2851 & $e f p$ & translation elongation factor $\mathrm{P}$ & -4.3 & $6.96 \mathrm{E}-03$ \\
\hline PA2970 & rpmF & $50 \mathrm{~S}$ ribosomal protein $\mathrm{L} 32$ & -3.8 & 7.34E-03 \\
\hline PA3656 & $r p s B$ & $30 \mathrm{~S}$ ribosomal protein $\mathrm{S} 2$ & -4.0 & $9.34 \mathrm{E}-03$ \\
\hline PA3742 & $r p l S$ & 50S ribosomal protein L19 & -2.7 & $6.83 \mathrm{E}-03$ \\
\hline PA4239 & $r p s D$ & $30 \mathrm{~S}$ ribosomal protein $\mathrm{S} 4$ & -2.4 & $6.51 \mathrm{E}-03$ \\
\hline PA4244 & rplO & 50S ribosomal protein L15 & -2.6 & $6.01 \mathrm{E}-03$ \\
\hline PA4246 & $r p s E$ & $30 \mathrm{~S}$ ribosomal protein $\mathrm{S} 5$ & -2.4 & $4.37 \mathrm{E}-03$ \\
\hline PA4247 & $r p l R$ & 50S ribosomal protein L18 & -2.9 & $7.53 \mathrm{E}-03$ \\
\hline PA4248 & $r p l F$ & 50S ribosomal protein L6 & -2.7 & $8.90 \mathrm{E}-03$ \\
\hline PA4249 & $r p s H$ & $30 \mathrm{~S}$ ribosomal protein $\mathrm{S} 8$ & -2.8 & $6.58 \mathrm{E}-03$ \\
\hline PA4251 & rple & 50S ribosomal protein L5 & -2.4 & $6.33 \mathrm{E}-03$ \\
\hline PA4253 & $r p l N$ & 50S ribosomal protein L14 & -3.0 & $9.10 \mathrm{E}-03$ \\
\hline PA4254 & $r p s Q$ & $30 \mathrm{~S}$ ribosomal protein $\mathrm{S} 17$ & -2.4 & $4.37 \mathrm{E}-03$ \\
\hline PA4257 & $r p s C$ & 30 S ribosomal protein $\mathrm{S} 3$ & -2.7 & $8.90 \mathrm{E}-03$ \\
\hline PA4261 & $r p l W$ & $50 \mathrm{~S}$ ribosomal protein $\mathrm{L} 23$ & -2.9 & $3.81 \mathrm{E}-03$ \\
\hline PA4263 & rplC & $50 \mathrm{~S}$ ribosomal protein $\mathrm{L} 3$ & -2.8 & $6.94 \mathrm{E}-03$ \\
\hline PA4266 & fusAl & elongation factor $\mathrm{G}$ & -2.1 & $9.73 \mathrm{E}-03$ \\
\hline PA4272 & $r p l J$ & $50 \mathrm{~S}$ ribosomal protein $\mathrm{L} 10$ & -3.7 & $6.33 \mathrm{E}-03$ \\
\hline PA4274 & $r p l K$ & 50S ribosomal protein L11 & -2.0 & $5.03 \mathrm{E}-03$ \\
\hline PA4665 & $\operatorname{prfA}$ & peptide chain release factor 1 & -2.2 & $6.65 \mathrm{E}-03$ \\
\hline PA4672 & & peptidyl-tRNA hydrolase & -3.3 & $9.10 \mathrm{E}-03$ \\
\hline PA4935 & $r p s F$ & 30S ribosomal protein S6 & -3.7 & $6.74 \mathrm{E}-03$ \\
\hline PA5049 & rpmE & $50 \mathrm{~S}$ ribosomal protein $\mathrm{L} 31$ & -4.0 & $8.29 \mathrm{E}-03$ \\
\hline PA5569 & rnpA & ribonuclease $\mathrm{P}$ protein component & -3.5 & $5.03 \mathrm{E}-03$ \\
\hline PA3818 & & extragenic suppressor protein SuhB & -2.7 & $9.10 \mathrm{E}-03$ \\
\hline PA4743 & $r b f A$ & ribosome-binding factor $\mathrm{A}$ & -2.8 & $9.34 \mathrm{E}-03$ \\
\hline \multicolumn{5}{|c|}{ Transport of small molecules } \\
\hline PA2983 & & probable tolQ-type transport protein & -2.7 & $6.74 \mathrm{E}-03$ \\
\hline PA3407 & hasAp & heme acquisition protein HasAp & 15.8 & $1.39 \mathrm{E}-03$ \\
\hline
\end{tabular}

\section{Upregulated genes}

\begin{tabular}{cllll} 
Locus Tag & Gene Name & Product Name & FC & $\begin{array}{c}\text { Corrected } \\
\boldsymbol{p} \text {-value }\end{array}$ \\
\hline \multicolumn{2}{|l}{$\begin{array}{cl}\text { Adaptation, Protection } \\
\text { PA1001 }\end{array} \quad$ phnA } & anthranilate synthase component I & 5.7 & $7.31 \mathrm{E}-03$ \\
PA2573 & & probable chemotaxis transducer & 4.3 & $5.19 \mathrm{E}-03$ \\
PA3866 & & pyocin protein & 2.1 & $9.34 \mathrm{E}-03$ \\
PA4110 & \multirow{2}{*}{ ampC } & beta-lactamase precursor & 2.0 & $3.84 \mathrm{E}-02$
\end{tabular}




\begin{tabular}{|c|c|c|c|c|}
\hline \multicolumn{5}{|c|}{ Amino acid biosynthesis and metabolism } \\
\hline PA1217 & & probable 2-isopropylmalate synthase & 13.8 & $6.51 \mathrm{E}-03$ \\
\hline PA3418 & $l d h$ & leucine dehydrogenase & 5.1 & $8.29 \mathrm{E}-03$ \\
\hline PA1002 & $\operatorname{phn} B$ & anthranilate synthase component II & 4.7 & 5.30E-03 \\
\hline \multicolumn{5}{|c|}{ Biosynthesis of cofactors, prosthetic groups and carriers } \\
\hline PA0502 & & probable biotin biosynthesis protein bioH & 2.3 & 7.53E-03 \\
\hline PA1000 & $p q s E$ & Quinolone signal response protein & 3.6 & 6.94E-03 \\
\hline \multicolumn{5}{|c|}{$\begin{array}{l}\text { Carbon compound } \\
\text { catabolism }\end{array}$} \\
\hline PA0743 & & probable 3-hydroxyisobutyrate dehydrogenase & 6.1 & $8.10 \mathrm{E}-03$ \\
\hline PA2290 & $g c d$ & glucose dehydrogenase & 3.9 & 4.89E-03 \\
\hline \multicolumn{5}{|c|}{ Central intermediary metabolism } \\
\hline PA4130 & & probable sulfite or nitrite reductase & 3.4 & $9.10 \mathrm{E}-03$ \\
\hline \multicolumn{5}{|c|}{ Energy metabolism } \\
\hline PA1177 & napE & periplasmic nitrate reductase protein $\mathrm{NapE}$ & 5.5 & $8.53 \mathrm{E}-03$ \\
\hline PA1317 & cyoA & cytochrome o ubiquinol oxidase subunit II & 3.9 & $8.51 \mathrm{E}-03$ \\
\hline PA3032 & snrl & cytochrome c Snr1 & 5.2 & $3.81 \mathrm{E}-03$ \\
\hline PA3415 & & probable dihydrolipoamide acetyltransferase & 8.1 & $6.51 \mathrm{E}-03$ \\
\hline PA3416 & & probable pyruvate dehydrogenase E1 component, beta chain & 8.2 & $6.96 \mathrm{E}-03$ \\
\hline \multicolumn{5}{|c|}{ Hypothetical, unclassified, unknown } \\
\hline PA0028 & & hypothetical protein & 2.5 & $8.55 \mathrm{E}-03$ \\
\hline PA0484 & & conserved hypothetical protein & 3.9 & $6.79 \mathrm{E}-03$ \\
\hline PA0586 & & conserved hypothetical protein & 4.7 & $6.33 \mathrm{E}-03$ \\
\hline PA0587 & & conserved hypothetical protein & 4.5 & $9.10 \mathrm{E}-03$ \\
\hline PA0588 & & conserved hypothetical protein & 3.6 & $8.61 \mathrm{E}-03$ \\
\hline PA0959 & & hypothetical protein & 2.3 & 8.93E-03 \\
\hline PA1043 & & hypothetical protein & 2.3 & $6.65 \mathrm{E}-03$ \\
\hline PA1088 & & hypothetical protein & 3.1 & $9.73 \mathrm{E}-03$ \\
\hline PA1090 & & hypothetical protein & 2.6 & $5.03 \mathrm{E}-03$ \\
\hline PA1091 & fgtA & flagellar glycosyl transferase, FgtA & 3.1 & $3.60 \mathrm{E}-03$ \\
\hline PA1135 & & conserved hypothetical protein & 3.8 & $5.57 \mathrm{E}-03$ \\
\hline PA1213 & & hypothetical protein & 5.5 & $5.30 \mathrm{E}-03$ \\
\hline PA1214 & & hypothetical protein & 4.9 & $8.55 \mathrm{E}-03$ \\
\hline PA1216 & & hypothetical protein & 15.9 & $6.99 \mathrm{E}-03$ \\
\hline PA1218 & & hypothetical protein & 10.8 & $7.00 \mathrm{E}-03$ \\
\hline PA1219 & & hypothetical protein & 5.0 & 4.37E-03 \\
\hline PA1221 & & hypothetical protein & 4.9 & $6.15 \mathrm{E}-03$ \\
\hline PA1356 & & hypothetical protein & 2.6 & 7.87E-03 \\
\hline PA1728 & & hypothetical protein & 5.0 & 8.53E-03 \\
\hline PA1784 & & hypothetical protein & 6.3 & $6.83 \mathrm{E}-03$ \\
\hline PA1887 & & hypothetical protein & 9.8 & $7.53 \mathrm{E}-03$ \\
\hline PA1888 & & hypothetical protein & 12.0 & $7.86 \mathrm{E}-03$ \\
\hline PA2166 & & hypothetical protein & 8.9 & $5.57 \mathrm{E}-03$ \\
\hline PA2174 & & hypothetical protein & 5.3 & $9.10 \mathrm{E}-03$ \\
\hline PA2226 & & hypothetical protein & 2.5 & $8.16 \mathrm{E}-03$ \\
\hline PA2328 & & hypothetical protein & 8.7 & $1.04 \mathrm{E}-03$ \\
\hline PA2330 & & hypothetical protein & 17.5 & $8.29 \mathrm{E}-03$ \\
\hline PA2374 & & hypothetical protein & 4.1 & $6.96 \mathrm{E}-03$ \\
\hline
\end{tabular}




\begin{tabular}{|c|c|c|c|c|}
\hline \multicolumn{2}{|l|}{ PA2381 } & hypothetical protein & 7.5 & $8.44 \mathrm{E}-03$ \\
\hline PA2566 & & conserved hypothetical protein & 4.0 & $9.34 \mathrm{E}-03$ \\
\hline PA2699 & & hypothetical protein & 3.9 & $9.10 \mathrm{E}-03$ \\
\hline PA2747 & & hypothetical protein & 12.1 & $7.31 \mathrm{E}-03$ \\
\hline PA2937 & & hypothetical protein & 4.3 & $8.53 \mathrm{E}-03$ \\
\hline PA3089 & & hypothetical protein & 2.9 & $9.50 \mathrm{E}-03$ \\
\hline PA3688 & & hypothetical protein & 4.9 & $9.10 \mathrm{E}-03$ \\
\hline PA4132 & & conserved hypothetical protein & 2.4 & $7.81 \mathrm{E}-03$ \\
\hline PA4298 & & hypothetical protein & 14.1 & $8.29 \mathrm{E}-03$ \\
\hline PA4362 & & hypothetical protein & 3.1 & $8.56 \mathrm{E}-03$ \\
\hline PA4421 & & conserved hypothetical protein & 2.1 & 7.37E-03 \\
\hline PA4573 & & hypothetical protein & 4.2 & $9.51 \mathrm{E}-03$ \\
\hline PA4583 & & conserved hypothetical protein & 2.1 & $9.12 \mathrm{E}-03$ \\
\hline PA4608 & & hypothetical protein & 2.8 & $9.73 \mathrm{E}-03$ \\
\hline PA4648 & & hypothetical protein & 13.5 & $4.37 \mathrm{E}-03$ \\
\hline PA4650 & & hypothetical protein & 2.1 & $6.65 \mathrm{E}-03$ \\
\hline PA4653 & & hypothetical protein & 3.8 & $3.60 \mathrm{E}-03$ \\
\hline PA4685 & & hypothetical protein & 2.6 & $5.03 \mathrm{E}-03$ \\
\hline PA4702 & & hypothetical protein & 6.3 & 4.37E-03 \\
\hline PA4738 & & conserved hypothetical protein & 15.6 & $7.30 \mathrm{E}-04$ \\
\hline PA4739 & & conserved hypothetical protein & 16.8 & $6.65 \mathrm{E}-03$ \\
\hline PA5061 & & conserved hypothetical protein & 4.2 & $6.51 \mathrm{E}-03$ \\
\hline PA5101 & & hypothetical protein & 3.2 & $8.55 \mathrm{E}-03$ \\
\hline PA5191 & & hypothetical protein & 2.2 & $8.23 \mathrm{E}-03$ \\
\hline PA5481 & & hypothetical protein & 5.9 & $7.42 \mathrm{E}-03$ \\
\hline PA1874 & & hypothetical protein & 22.1 & $5.09 \mathrm{E}-03$ \\
\hline \multicolumn{5}{|c|}{ Membrane proteins } \\
\hline PA0007 & & hypothetical protein & 2.6 & $9.73 \mathrm{E}-03$ \\
\hline PA1042 & & conserved hypothetical protein & 2.7 & $9.05 \mathrm{E}-03$ \\
\hline PA1119 & & probable outer membrane protein precursor & 2.2 & $9.22 \mathrm{E}-03$ \\
\hline PA2072 & & conserved hypothetical protein & 2.2 & $9.80 \mathrm{E}-03$ \\
\hline PA3370 & & hypothetical protein & 3.7 & $7.86 \mathrm{E}-03$ \\
\hline PA3789 & & hypothetical protein & 2.9 & $7.09 \mathrm{E}-03$ \\
\hline PA5482 & & hypothetical protein & 11.1 & $6.65 \mathrm{E}-03$ \\
\hline PA4614 & $m s c L$ & conductance mechanosensitive channel & 2.3 & $5.57 \mathrm{E}-03$ \\
\hline PA4297 & $\operatorname{tad} G$ & TadG & 3.5 & 7.64E-03 \\
\hline PA4301 & $\operatorname{tad} B$ & TadB & 4.0 & $6.33 \mathrm{E}-03$ \\
\hline \multicolumn{5}{|c|}{ Motility \& Attachment } \\
\hline PA4299 & $\operatorname{tad} D$ & TadD & 16.9 & $5.03 \mathrm{E}-03$ \\
\hline PA4305 & $r c p C$ & $\mathrm{RcpC}$ & 4.6 & $9.73 \mathrm{E}-03$ \\
\hline PA4306 & $f l p$ & Type IVb pilin, Flp & 35.7 & $3.60 \mathrm{E}-03$ \\
\hline PA4651 & & probable pili assembly chaperone & 11.6 & $1.42 \mathrm{E}-03$ \\
\hline \multicolumn{5}{|c|}{ Protein secretion/export apparatus } \\
\hline PA1877 & & probable secretion protein & 8.6 & $9.73 \mathrm{E}-03$ \\
\hline PA1875 & & probable outer membrane protein precursor & 11.5 & $8.53 \mathrm{E}-03$ \\
\hline PA4302 & $\operatorname{tad} A$ & TadA ATPase & 7.1 & $6.51 \mathrm{E}-03$ \\
\hline PA4304 & rсpA & RcpA & 8.7 & 7.33E-03 \\
\hline
\end{tabular}




\begin{tabular}{|c|c|c|c|}
\hline PA0704 & probable amidase & 3.6 & $8.56 \mathrm{E}-03$ \\
\hline PA0804 & probable oxidoreductase & 2.5 & $9.10 \mathrm{E}-03$ \\
\hline PA0838 & probable glutathione peroxidase & 2.4 & $9.34 \mathrm{E}-03$ \\
\hline PA1880 & probable oxidoreductase & 4.1 & $9.50 \mathrm{E}-03$ \\
\hline PA2024 & probable ring-cleaving dioxygenase & 6.1 & $2.46 \mathrm{E}-03$ \\
\hline PA5546 & conserved hypothetical protein & 4.6 & $7.09 \mathrm{E}-03$ \\
\hline \multicolumn{4}{|c|}{ Related to phage, transposon, or plasmid } \\
\hline PA0644 & hypothetical protein & 5.0 & $9.73 \mathrm{E}-03$ \\
\hline \multicolumn{4}{|c|}{ Secreted Factors (toxins, enzymes, alginate) } \\
\hline PA4175 & protease IV & 3.8 & $9.50 \mathrm{E}-03$ \\
\hline PA1876 & probable ATP-binding/permease fusion $\mathrm{ABC}$ transporter & 9.0 & $5.57 \mathrm{E}-03$ \\
\hline \multicolumn{4}{|c|}{ Transcription, RNA processing and degradation } \\
\hline PA4585 & RNA 3'-terminal phosphate cyclase & 2.1 & $9.12 \mathrm{E}-03$ \\
\hline \multicolumn{4}{|l|}{ Transcriptional regulators } \\
\hline PA3622 & sigma factor RpoS & 2.2 & $6.65 \mathrm{E}-03$ \\
\hline PA5059 & probable transcriptional regulator & 4.0 & $6.33 \mathrm{E}-03$ \\
\hline PA0175 & probable chemotaxis protein methyltransferase & 5.9 & $5.03 \mathrm{E}-03$ \\
\hline PA4781 & probable two-component response regulator & 2.8 & $9.10 \mathrm{E}-03$ \\
\hline PA2329 & probable ATP-binding component of $\mathrm{ABC}$ transporter & 14.7 & $7.53 \mathrm{E}-03$ \\
\hline PA3858 & probable amino acid-binding protein & 2.3 & $5.19 \mathrm{E}-03$ \\
\hline PA4497 & probable binding protein component of $\mathrm{ABC}$ transporter & 2.3 & $9.78 \mathrm{E}-03$ \\
\hline \multicolumn{4}{|c|}{ Two-component regulatory systems } \\
\hline PA4293 & two-component sensor PprA & 4.1 & $8.90 \mathrm{E}-03$ \\
\hline
\end{tabular}


Table S5: Regulation of QS-related genes by AmpR. List of genes that are significantly differentially regulated in PAO1 and PAO $\triangle a m p R$ in the presence and absence of B-lactam stress. For sake of clarity, only significant fold changes (FC) and their corresponding corrected $p$-values in the various conditions are shown. Annotations for the locus tags are from the Pseudomonas Genome database (31).

\begin{tabular}{cccccc}
\hline $\begin{array}{c}\text { Locus } \\
\text { Tag }\end{array}$ & $\begin{array}{c}\text { Gene } \\
\text { Name }\end{array}$ & Product Name & AmpR & $\begin{array}{c}\text { AmpR, B- } \\
\text { lactam } \\
\text { dependent }\end{array}$ \\
\hline & & FC & $\begin{array}{c}p- \\
\text { value }\end{array}$ FC & $\begin{array}{c}p- \\
\text { value }\end{array}$ \\
\cline { 3 - 6 }
\end{tabular}

Adaptation, protection

\begin{tabular}{|c|c|c|}
\hline \multicolumn{2}{|l|}{ PA4520 } & probable chemotaxis transducer \\
\hline \multicolumn{2}{|l|}{ PA1 127} & probable oxidoreductase \\
\hline \multicolumn{3}{|c|}{ Amino acid biosynthesis and metabolism } \\
\hline \multicolumn{2}{|l|}{ PA0132 } & beta-alanine--pyruvate transaminase \\
\hline PA1812 & $m l t D$ & $\begin{array}{l}\text { membrane-bound lytic murein transglycosylase } \\
\text { D precursor }\end{array}$ \\
\hline PA2629 & purB & adenylosuccinate lyase \\
\hline \multicolumn{3}{|c|}{ Carbon compound catabolism } \\
\hline PA0153 & pcaH & protocatechuate 3,4-dioxygenase, beta subunit \\
\hline PA0154 & $p c a G$ & protocatechuate 3,4-dioxygenase, alpha subunit \\
\hline PA0226 & & probable CoA transferase, subunit A \\
\hline PA4123 & $h p c C$ & $\begin{array}{l}\text { 5-carboxy-2-hydroxymuconate semialdehyde } \\
\text { dehydrogenase }\end{array}$ \\
\hline PA4125 & $h p c D$ & 5-carboxymethyl-2-hydroxymuconate isomerase \\
\hline PA0227 & & probable CoA transferase, subunit B \\
\hline
\end{tabular}

$\begin{array}{cc} & 3.7 \mathrm{E}- \\ 2.1 & 03 \\ - & 4.7 \mathrm{E}- \\ 2.0 & 03 \\ & \\ - & 9.3 \mathrm{E}- \\ 3.9 & 03 \\ - & 5.3 \mathrm{E}- \\ 2.1 & 03 \\ - & 9.9 \mathrm{E}- \\ 2.3 & 03\end{array}$

Cell wall / LPS / capsule

PA5163 rmlA glucose-1-phosphate thymidylyltransferase

$-\quad 4.7 \mathrm{E}-$

Central intermediary metabolism

PA4130 probable sulfite or nitrite reductase

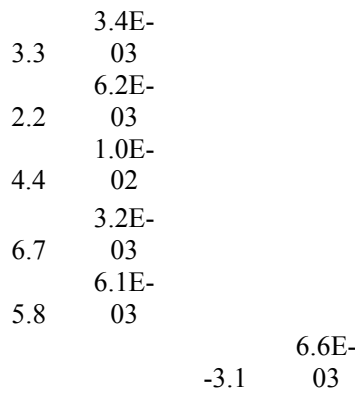

$2.1 \quad 03$

Energy metabolism

$\begin{array}{lll}\text { PA0105 } & \text { coxB } & \text { cytochrome c oxidase, subunit II } \\ \text { PA3621 } & f d x A & \text { ferredoxin I } \\ \text { PA3032 } & \text { snrl } & \text { cytochrome c Snr1 } \\ \text { PA0854 } & \text { fumC2 } & \text { fumarate hydratase }\end{array}$

Hypothetical, unclassified, unknown

$\begin{array}{ll}\text { PA0079 } & \begin{array}{l}\text { hypothetical protein } \\ \text { PA0080 }\end{array} \\ \text { PA0269 } & \text { conserved hypothetical protein } \\ \text { PA0270 } & \text { hypothetical protein } \\ \text { PA0468 } & \text { hypothetical protein }\end{array}$




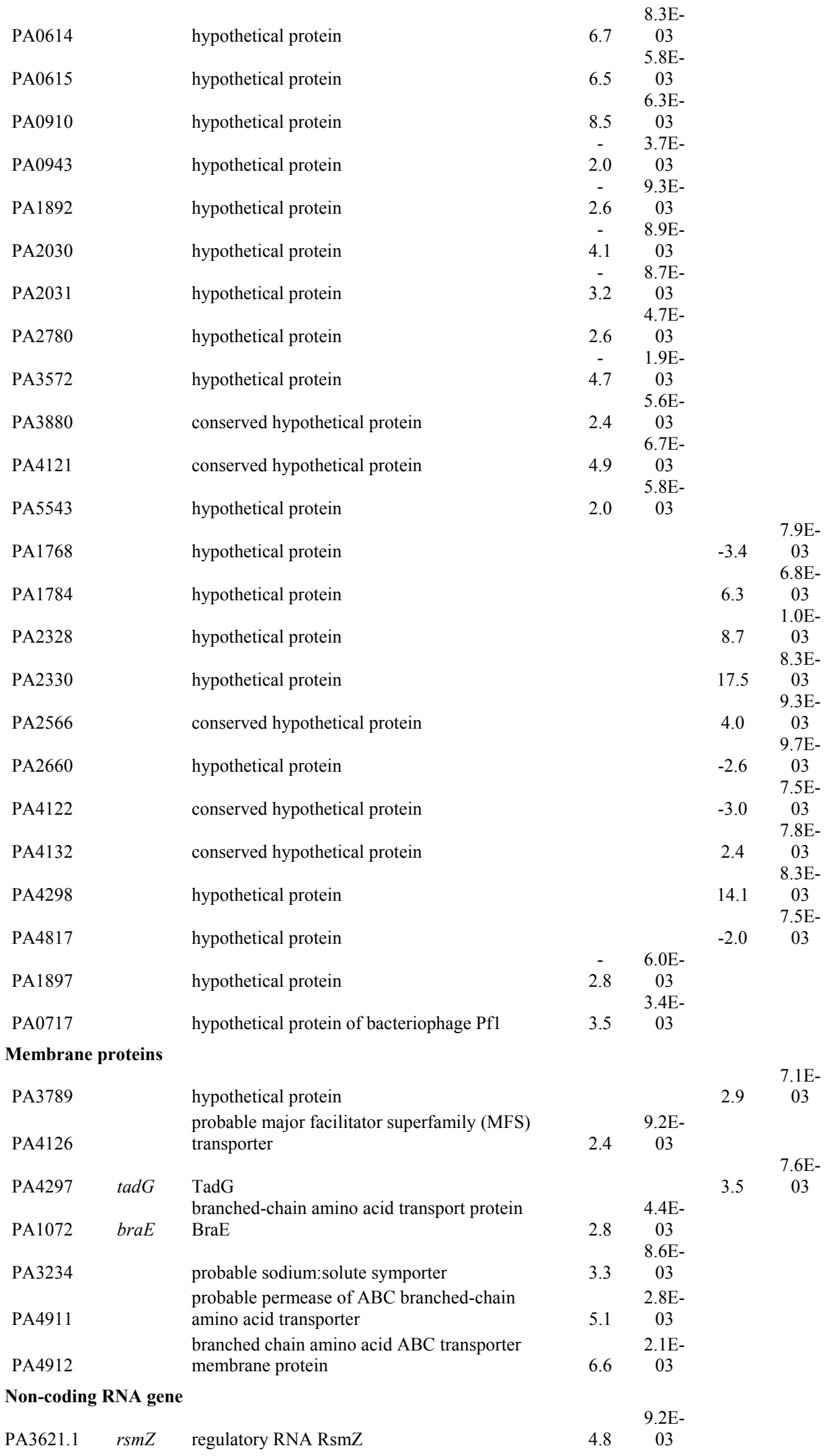


PA1875 probable outer membrane protein precursor $8.5 \mathrm{E}-$

Putative enzymes

$\begin{array}{ll}\text { PA1893 } & \begin{array}{l}\text { hypothetical protein } \\ \text { PA2682 }\end{array} \\ \text { PA4073 } & \text { probable aldehyde dehydrogenase } \\ \text { PA5546 } & \text { conserved hypothetical protein }\end{array}$

$\begin{array}{cc} & 8.3 \mathrm{E}- \\ -3.3 & 03 \\ & 7.1 \mathrm{E}- \\ 4.6 & 03\end{array}$

Related to phage, transposon, or plasmid

$\begin{array}{ll}\text { PA0616 } & \text { hypothetical protein } \\ \text { PA0617 } & \text { probable bacteriophage protein } \\ \text { PA0618 } & \text { probable bacteriophage protein } \\ \text { PA0619 } & \text { probable bacteriophage protein } \\ \text { PA0620 } & \text { conserved hypothetical protein } \\ \text { PA0621 } & \text { probable bacteriophage protein } \\ \text { PA0622 } & \text { probable bacteriophage protein } \\ \text { PA0623 } & \text { conserved hypothetical protein } \\ \text { PA0637 } & \text { probable bacteriophage protein } \\ \text { PA0638 } & \text { probable bacteriophage protein } \\ \text { PA0641 } & \text { hypothetical protein } \\ \text { PA0643 } & \text { hy0644 }\end{array}$

Secreted Factors (toxins, enzymes, alginate)

$\begin{array}{lll}\text { PA4175 } & \text { piv } & \text { protease IV } \\ \text { PA5380 } & \text { gbdR } & \text { GbdR } \\ \text { PA5059 } & & \text { probable transcriptional regulator }\end{array}$

Translation, post-translational modification, degradation

PA2851 efp translation elongation factor $\mathrm{P}$

Transport of small molecules

$\begin{array}{lll}\text { PA1070 braG } & \begin{array}{l}\text { branched-chain amino acid transport protein } \\ \text { BraG } \\ \text { branched chain amino acid ABC transporter ATP } \\ \text { binding protein } \\ \text { probable binding protein component of ABC } \\ \text { transporter } \\ \text { probable ATP-binding component of ABC } \\ \text { transporter } \\ \text { pA4913 }\end{array} \\ \text { PA2329 } & \begin{array}{l}\text { transporter } \\ \text { PA4497 }\end{array}\end{array}$

- 4.8E-

2.903

- 7.6E-

$3.0 \quad 03$

7.2E-

6.9

8.7E-

$7.8 \quad 03$

7.7E-

$7.2 \quad 03$

9.9E-

$5.9 \quad 03$

$5.8 \mathrm{E}-$

$7.5 \quad 03$

6.5E-

$7.7 \quad 03$

4.4E-

$6.0 \quad 03$

7.0E-

6.303

8.1E-

$7.0 \quad 03$

9.0E-

6.303

9.6E-

$5.6 \quad 03$

$8.6 \mathrm{E}-$

$6.0 \quad 03$

$11.5 \quad 03$ 
Table S6: Regulation of biofilm-specific genes by AmpR. Significantly differentially regulated genes in PAO1 and PAO $\triangle a m p R$ in the presence and absence of B-lactam antibiotics. For sake of clarity, only significant fold changes (FC) and their corresponding corrected $p$-values are shown. Locus tag annotations are from the Pseudomonas Genome database (31).

\begin{tabular}{|c|c|c|c|c|c|c|}
\hline \multirow[t]{2}{*}{$\begin{array}{c}\text { Locus } \\
\text { Tag } \\
\end{array}$} & \multirow[t]{2}{*}{$\begin{array}{c}\text { Gene } \\
\text { Name } \\
\end{array}$} & \multirow[t]{2}{*}{ Product Name } & \multicolumn{2}{|c|}{$\begin{array}{c}\text { AmpR } \\
\text { dependent }\end{array}$} & \multicolumn{2}{|c|}{$\begin{array}{c}\text { AmpR, B-lactam } \\
\text { dependent }\end{array}$} \\
\hline & & & FC & $p$-value & FC & $p$-value \\
\hline \multicolumn{7}{|c|}{ Adaptation, Protection } \\
\hline PA4356 & xenB & xenobiotic reductase & 3.2 & $6.0 \mathrm{E}-03$ & & \\
\hline PA4671 & & $\begin{array}{l}\text { probable ribosomal protein } \mathrm{L} 25 \\
\text { serine/threonine protein kinase }\end{array}$ & & & -2.6 & $9.1 \mathrm{E}-03$ \\
\hline PA0074 & $p p k A$ & PpkA & -2.3 & $8.4 \mathrm{E}-03$ & & \\
\hline \multicolumn{7}{|c|}{ Amino acid biosynthesis and metabolism } \\
\hline PA2444 & glyA2 & serine hydroxymethyltransferase & -7.1 & $1.8 \mathrm{E}-03$ & & \\
\hline PA5415 & glyAl & $\begin{array}{l}\text { serine hydroxymethyltransferase } \\
\text { probable 2-isopropylmalate }\end{array}$ & -2.1 & $5.9 \mathrm{E}-03$ & & \\
\hline PA1217 & & synthase & & & 13.8 & $6.5 \mathrm{E}-03$ \\
\hline \multicolumn{7}{|c|}{$\begin{array}{l}\text { Carbon compound catabolism } \\
\text { phosphoenolpyruvate }\end{array}$} \\
\hline PA5192 & $p c k A$ & carboxykinase & & & -3.0 & $6.0 \mathrm{E}-04$ \\
\hline \multicolumn{7}{|c|}{ Cell wall / LPS / capsule } \\
\hline PA5322 & $\operatorname{alg} C$ & phosphomannomutase $\mathrm{AlgC}$ & -2.3 & 4.4E-03 & & \\
\hline \multicolumn{7}{|c|}{ Central intermediary metabolism } \\
\hline PA2445 & $g c v P 2$ & glycine cleavage system protein $\mathrm{P} 2$ & 14.3 & $5.0 \mathrm{E}-03$ & & \\
\hline \multicolumn{7}{|c|}{ Hypothetical, unclassified, unknown } \\
\hline PA0062 & & hypothetical protein & -4.9 & $2.9 \mathrm{E}-03$ & & \\
\hline PA0076 & & hypothetical protein & -2.5 & $3.3 \mathrm{E}-03$ & & \\
\hline PA0170 & & hypothetical protein & -3.5 & $4.2 \mathrm{E}-03$ & & \\
\hline PA0529 & & conserved hypothetical protein & 3.0 & $8.6 \mathrm{E}-03$ & & \\
\hline PA0737 & & hypothetical protein & -2.0 & 9.9E-03 & & \\
\hline PA1471 & & hypothetical protein & -6.5 & $2.8 \mathrm{E}-03$ & & \\
\hline PA2365 & & conserved hypothetical protein & 9.4 & $4.5 \mathrm{E}-03$ & & \\
\hline PA3484 & & hypothetical protein & -2.1 & $7.6 \mathrm{E}-03$ & & \\
\hline PA4154 & & conserved hypothetical protein & -3.8 & $6.0 \mathrm{E}-03$ & & \\
\hline PA4154 & & conserved hypothetical protein & 2.5 & 8.7E-03 & & \\
\hline PA5202 & & hypothetical protein & -2.3 & $6.9 \mathrm{E}-03$ & & \\
\hline PA0028 & & hypothetical protein & & & 2.5 & $8.5 \mathrm{E}-03$ \\
\hline PA0046 & & hypothetical protein & & & -3.4 & $9.1 \mathrm{E}-03$ \\
\hline PA0588 & & conserved hypothetical protein & & & 3.6 & $8.6 \mathrm{E}-03$ \\
\hline PA1216 & & hypothetical protein & & & 15.9 & 7.0E-03 \\
\hline PA2174 & & hypothetical protein & & & 5.3 & $9.1 \mathrm{E}-03$ \\
\hline PA3688 & & hypothetical protein & & & 4.9 & $9.1 \mathrm{E}-03$ \\
\hline PA4421 & & conserved hypothetical protein & & & 2.1 & 7.4E-03 \\
\hline PA4421 & & conserved hypothetical protein & & & 2.9 & 3.4E-03 \\
\hline PA5441 & & hypothetical protein & & & -2.0 & 7.1E-03 \\
\hline \multicolumn{7}{|c|}{ Membrane proteins } \\
\hline PA5182 & & hypothetical protein & -3.2 & $3.3 \mathrm{E}-03$ & & \\
\hline
\end{tabular}




\begin{tabular}{|c|c|c|c|c|c|c|}
\hline $\begin{array}{l}\text { PA5568 } \\
\text { PA3821 }\end{array}$ & $\sec D$ & $\begin{array}{l}\text { conserved hypothetical protein } \\
\text { secretion protein SecD }\end{array}$ & -2.0 & $6.1 \mathrm{E}-03$ & -3.2 & $6.3 \mathrm{E}-03$ \\
\hline \multicolumn{7}{|c|}{ Protein secretion/export apparatus } \\
\hline \multicolumn{7}{|l|}{$\begin{array}{l}\text { Putative } \\
\text { enzymes }\end{array}$} \\
\hline PA0853 & & probable oxidoreductase & -2.6 & 4.4E-03 & & \\
\hline PA2024 & & probable ring-cleaving dioxygenase & & & 6.1 & $2.5 \mathrm{E}-03$ \\
\hline \multicolumn{7}{|c|}{ Transcription, RNA processing and degradation } \\
\hline PA3744 & $\operatorname{rim} M$ & 16S rRNA processing protein & 6.2 & 4.7E-03 & & \\
\hline PA4262 & $r p l D$ & 50 S ribosomal protein L4 & & & -3.0 & $6.3 \mathrm{E}-03$ \\
\hline \multicolumn{7}{|c|}{ Transcriptional regulators } \\
\hline PA4781 & & $\begin{array}{l}\text { probable two-component response } \\
\text { regulator }\end{array}$ & & & 2.8 & $9.1 \mathrm{E}-03$ \\
\hline \multicolumn{7}{|c|}{ Translation, post-translational modification, degradation } \\
\hline PA2970 & $\operatorname{rpm} F$ & 50 S ribosomal protein $\mathrm{L} 32$ & & & -3.8 & 7.3E-03 \\
\hline PA4244 & rplo & $50 \mathrm{~S}$ ribosomal protein $\mathrm{L} 15$ & & & -2.6 & $6.0 \mathrm{E}-03$ \\
\hline PA4246 & rpsE & 30S ribosomal protein $\mathrm{S} 5$ & & & -2.4 & 4.4E-03 \\
\hline PA4247 & $r p l R$ & 50S ribosomal protein L18 & & & -2.9 & $7.5 \mathrm{E}-03$ \\
\hline PA4248 & $r p l F$ & 50S ribosomal protein L6 & & & -2.7 & $8.9 \mathrm{E}-03$ \\
\hline PA4261 & $r p l W$ & $50 \mathrm{~S}$ ribosomal protein $\mathrm{L} 23$ & & & -2.9 & $3.8 \mathrm{E}-03$ \\
\hline PA4272 & rplJ & $50 \mathrm{~S}$ ribosomal protein $\mathrm{L} 10$ & & & -3.7 & $6.3 \mathrm{E}-03$ \\
\hline PA4743 & $r b f A$ & ribosome-binding factor $\mathrm{A}$ & & & -2.8 & $9.3 \mathrm{E}-03$ \\
\hline
\end{tabular}


Table S7: Genes in RGPs that are AmpR-regulated. AmpR regulates genes that are part of the different RGPs both in the presence and absence of a B-lactam antibiotic, listed here with the corresponding fold changes (FC) and $p$-value. Locus tag annotations are from the Pseudomonas Genome database (31).

AmpR-Dependent

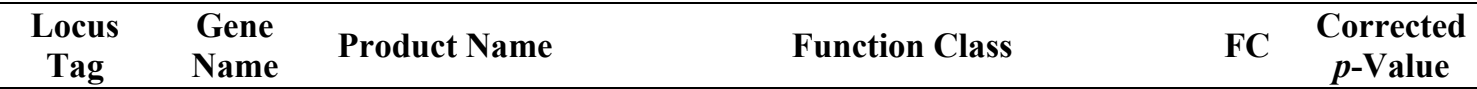

RGP01

PA0208 $\quad m d c A$

malonate decarboxylase alpha subunit

Carbon compound catabolism

$-2.4 \quad 3.91 \mathrm{E}-03$

RGP02

PA0263 hcpC secreted protein Hcp

\section{RGP03}

PA0614

PA0615

PA0616

PA0617

PA0618

PA0619

PA0620

PA0621

PA0622

PA0623

RGP04

PA0641

PA0643

RGP05

PA0729

PA0717

RGP09

PA1092 hypothetical protein

hypothetical protein

hypothetical protein

probable bacteriophage protein

probable bacteriophage protein

probable bacteriophage protein

probable bacteriophage protein

conserved hypothetical protein

probable bacteriophage protein

probable bacteriophage protein

probable bacteriophage protein

hypothetical protein

hypothetical protein

hypothetical protein of bacteriophage Pfl

fliC flagellin type B
Secreted Factors (toxins, enzymes, alginate)

$-7.7 \quad 4.66 \mathrm{E}-03$

Hypothetical, unclassified, unknown

$6.7 \quad 8.26 \mathrm{E}-03$

Hypothetical, unclassified, unknown

$6.5 \quad 5.78 \mathrm{E}-03$

Related to phage, transposon, or plasmid

$6.9 \quad 7.25 \mathrm{E}-03$

Related to phage, transposon, or plasmid

$7.8 \quad 8.66 \mathrm{E}-03$

Related to phage, transposon, or plasmid

$7.2 \quad 7.74 \mathrm{E}-03$

Related to phage, transposon, or plasmid

$5.9 \quad 9.87 \mathrm{E}-03$

Related to phage, transposon, or plasmid

$7.5 \quad 5.76 \mathrm{E}-03$

Related to phage, transposon, or plasmid

$7.7 \quad 6.53 \mathrm{E}-03$

Related to phage, transposon, or plasmid

$6.0 \quad 4.45 \mathrm{E}-03$

Related to phage, transposon, or plasmid

$6.3 \quad 7.01 \mathrm{E}-03$

Related to phage, transposon, or plasmid $5.69 .59 \mathrm{E}-03$ Related to phage, transposon, or plasmid

$6.0 \quad 8.59 \mathrm{E}-03$

Hypothetical, unclassified, unknown

$9.4 \quad 3.41 \mathrm{E}-03$

Hypothetical, unclassified, unknown

$3.5 \quad 3.44 \mathrm{E}-03$

Motility \& Attachment

$2.59 .63 \mathrm{E}-03$ 
RGP20

PA2030

hypothetical protein

Hypothetical, unclassified, unknown

$-4.1 \quad 8.89 \mathrm{E}-03$

Hypothetical, unclassified, unknown

$-3.2 \quad 8.70 \mathrm{E}-03$

PA2031

hypothetical protein

Hypothetical, unclassified,

$-4.8 \quad 5.91 \mathrm{E}-03$

PA2034

hypothetical protein unknown

Cell wall / LPS / capsule $\quad-3.2 \quad$ 8.70E-03

Cell wall / LPS / capsule ;

Transport of small

$-3.0 \quad 8.27 \mathrm{E}-03$

molecules

Hypothetical, unclassified, $\quad-3.1 \quad 4.94 \mathrm{E}-03$

unknown

$-3.0 \quad 3.18 \mathrm{E}-03$

Hypothetical, unclassified, unknown

Cell wall / LPS / capsule $\quad 3.7 \quad$ 8.81E-03

O-antigen chain length

PAB

$w z z$ regulator

RGP34

PA3499

hypothetical protein

Hypothetical, unclassified, unknown

$2.4 \quad 6.97 \mathrm{E}-03$

Hypothetical, unclassified, unknown

$5.7 \quad 3.92 \mathrm{E}-03$

Putative enzymes

- $24.62 \mathrm{E}-04$

PA3498

probable oxidoreductase

PA3506

probable decarboxylase

Putative enzymes

$4.8 \quad 5.76 \mathrm{E}-03$

Central intermediary metabolism

$-2.7 \quad 6.81 \mathrm{E}-03$

probable dehydrogenase

Putative enzymes

$-2.0 \quad 5.46 \mathrm{E}-03$

dehydrogenase

Putative enzymes

AmpR-B-lactam Dependent

\begin{tabular}{lllll}
\hline $\begin{array}{l}\text { Locus } \\
\text { Tag }\end{array}$ & $\begin{array}{c}\text { Gene } \\
\text { Name }\end{array}$ & Product Name & Function Class & $\begin{array}{c}\text { Corrected } \\
\boldsymbol{p} \text {-Value }\end{array}$ \\
\hline RGP04 & & & & \\
PA0644 & hypothetical protein & $\begin{array}{l}\text { Related to phage, } \\
\text { transposon, or plasmid }\end{array}$ & 5.0 & $9.73 \mathrm{E}-03$
\end{tabular}

RGP09 


\begin{tabular}{|c|c|c|c|c|c|}
\hline PA1088 & & hypothetical protein & $\begin{array}{l}\text { Hypothetical, unclassified, } \\
\text { unknown }\end{array}$ & 3.1 & $9.73 \mathrm{E}-03$ \\
\hline PA1090 & & hypothetical protein & $\begin{array}{l}\text { Hypothetical, unclassified, } \\
\text { unknown }\end{array}$ & 2.6 & $5.03 \mathrm{E}-03$ \\
\hline PA1091 & fgtA & $\begin{array}{l}\text { flagellar glycosyl transferase, } \\
\text { FgtA }\end{array}$ & $\begin{array}{l}\text { Hypothetical, unclassified, } \\
\text { unknown }\end{array}$ & 3.1 & $3.60 \mathrm{E}-03$ \\
\hline \multicolumn{6}{|l|}{ RGP20 } \\
\hline PA2024 & & $\begin{array}{l}\text { probable ring-cleaving } \\
\text { dioxygenase }\end{array}$ & Putative enzymes & 6.1 & $2.46 \mathrm{E}-03$ \\
\hline \multicolumn{6}{|l|}{ RGP23 } \\
\hline PA2226 & & hypothetical protein & $\begin{array}{l}\text { Hypothetical, unclassified, } \\
\text { unknown }\end{array}$ & 2.5 & $8.16 \mathrm{E}-03$ \\
\hline \multicolumn{6}{|l|}{ RGP37 } \\
\hline PA3866 & & pyocin protein & Adaptation, Protection & 2.1 & $9.34 \mathrm{E}-03$ \\
\hline \multicolumn{6}{|l|}{ RGP42 } \\
\hline PA4673 & & $\begin{array}{l}\text { conserved hypothetical } \\
\text { protein }\end{array}$ & $\begin{array}{l}\text { Hypothetical, unclassified, } \\
\text { unknown }\end{array}$ & -2.8 & $7.09 \mathrm{E}-03$ \\
\hline
\end{tabular}


Table S8: Exclusively AmpR-, and AmpR-B-lactam-dependent genes. The list of 219 genes, derived from the lists in Tables S3A and 4A, that are specifically dependent on AmpR, both without and with Blactam stress, and are not differentially regulated in any of the 20 other published transcriptome studies (see text for details). Genes are listed as either up- or down-regulated with the corresponding corrected $p$-values for the fold change (FC) observed. Locus tag annotations are from the Pseudomonas Genome database (31).

\begin{tabular}{|c|c|c|c|c|}
\hline \multicolumn{5}{|c|}{ AmpR-dependent; Downregulated } \\
\hline $\begin{array}{l}\text { Locus } \\
\text { Tag }\end{array}$ & $\begin{array}{l}\text { Gene } \\
\text { Name }\end{array}$ & Product Name & FC & $\begin{array}{l}\text { Corrected } p \text { - } \\
\text { value }\end{array}$ \\
\hline \multicolumn{5}{|c|}{ Adaptation, Protection } \\
\hline PA2703 & & hypothetical protein & -2.2 & $9.03 \mathrm{E}-03$ \\
\hline PA3109 & & hypothetical protein & -2.6 & $9.59 \mathrm{E}-03$ \\
\hline PA5 117 & typ $A$ & regulatory protein TypA & -3.6 & $8.06 \mathrm{E}-03$ \\
\hline \multicolumn{5}{|c|}{ Amino acid biosynthesis and metabolism } \\
\hline PA1337 & ans $B$ & glutaminase-asparaginase & -2.4 & $3.35 \mathrm{E}-03$ \\
\hline PA2443 & $s d a A$ & L-serine dehydratase & -10.6 & $4.02 \mathrm{E}-03$ \\
\hline PA5323 & $\arg B$ & acetylglutamate kinase & -2.6 & $4.45 \mathrm{E}-03$ \\
\hline \multicolumn{5}{|c|}{ Biosynthesis of cofactors, prosthetic groups and carriers } \\
\hline PA4664 & hemK & probable methyl transferase & -2.0 & $9.51 \mathrm{E}-03$ \\
\hline \multicolumn{5}{|c|}{ Carbon compound catabolism } \\
\hline PA0208 & $m d c A$ & malonate decarboxylase alpha subunit & -2.4 & $3.91 \mathrm{E}-03$ \\
\hline \multicolumn{5}{|c|}{ Cell division } \\
\hline PA0857 & bolA & morphogene protein BolA & -2.3 & $2.78 \mathrm{E}-03$ \\
\hline \multicolumn{5}{|c|}{$\begin{array}{l}\text { Cell wall / LPS / } \\
\text { capsule }\end{array}$} \\
\hline PA2232 & $p s l B$ & $\begin{array}{l}\text { probable phosphomannose isomerase/GDP- } \\
\text { mannose pyrophosphorylase }\end{array}$ & -3.2 & $8.70 \mathrm{E}-03$ \\
\hline PA2235 & pslE & hypothetical protein & -3.0 & $3.18 \mathrm{E}-03$ \\
\hline PA2236 & pslF & hypothetical protein & -2.8 & $6.78 \mathrm{E}-03$ \\
\hline PA2238 & pslH & hypothetical protein & -2.5 & 7.89E-03 \\
\hline PA3646 & $\operatorname{lpxD}$ & $\begin{array}{l}\text { UDP-3-O-[3-hydroxylauroyl] glucosamine N- } \\
\text { acyltransferase }\end{array}$ & -2.1 & $4.78 \mathrm{E}-03$ \\
\hline PA5077 & $m d o H$ & periplasmic glucans biosynthesis protein $\mathrm{MdoH}$ & -2.2 & $4.86 \mathrm{E}-03$ \\
\hline PA4625 & & hypothetical protein & -2.9 & $8.25 \mathrm{E}-03$ \\
\hline PA2234 & pslD & PslD & -3.0 & $8.27 \mathrm{E}-03$ \\
\hline \multicolumn{5}{|c|}{ Central intermediary metabolism } \\
\hline PA2023 & galU & UTP--glucose-1-phosphate uridylyltransferase & -2.5 & $4.75 \mathrm{E}-03$ \\
\hline PA4100 & & probable dehydrogenase & -2.7 & $6.81 \mathrm{E}-03$ \\
\hline PA2442 & $g c v T 2$ & glycine cleavage system protein $\mathrm{T} 2$ & -4.8 & $3.36 \mathrm{E}-03$ \\
\hline \multicolumn{5}{|c|}{ Energy metabolism } \\
\hline PA5553 & $\operatorname{atp} C$ & ATP synthase epsilon chain & -2.2 & $5.71 \mathrm{E}-03$ \\
\hline \multicolumn{5}{|c|}{ Fatty acid and phospholipid metabolism } \\
\hline PA0798 & pmtA & phospholipid methyltransferase & -2.3 & $5.91 \mathrm{E}-03$ \\
\hline \multicolumn{5}{|c|}{ Hypothetical, unclassified, unknown } \\
\hline PA0169 & & hypothetical protein & -3.3 & $2.95 \mathrm{E}-03$ \\
\hline PA0370 & & conserved hypothetical protein & -2.5 & $3.73 \mathrm{E}-03$ \\
\hline PA0371 & & hypothetical protein & -2.5 & $3.68 \mathrm{E}-03$ \\
\hline PA0713 & & hypothetical protein & -3.1 & $7.40 \mathrm{E}-03$ \\
\hline PA1605 & & hypothetical protein & -2.3 & $7.41 \mathrm{E}-03$ \\
\hline
\end{tabular}




\begin{tabular}{|c|c|c|c|c|}
\hline PA1732 & & conserved hypothetical protein & -2.6 & $6.70 \mathrm{E}-03$ \\
\hline PA1968 & & hypothetical protein & -2.5 & $6.60 \mathrm{E}-03$ \\
\hline PA1969 & & hypothetical protein & -2.5 & 7.41E-03 \\
\hline PA2222 & & hypothetical protein & -3.1 & $4.94 \mathrm{E}-03$ \\
\hline PA2242 & pslL & hypothetical protein & -2.7 & $3.41 \mathrm{E}-03$ \\
\hline PA2289 & & conserved hypothetical protein & -2.3 & $7.15 \mathrm{E}-03$ \\
\hline PA3042 & & hypothetical protein & -2.9 & $8.70 \mathrm{E}-03$ \\
\hline PA4182 & & hypothetical protein & -2.6 & $5.46 \mathrm{E}-03$ \\
\hline PA4204 & $\operatorname{ppgL}$ & periplasmic gluconolactonase, $\mathrm{PpgL}$ & -3.3 & $2.15 \mathrm{E}-03$ \\
\hline PA4384 & & hypothetical protein & -3.0 & $4.66 \mathrm{E}-03$ \\
\hline PA4458 & & conserved hypothetical protein & -2.0 & $2.15 \mathrm{E}-03$ \\
\hline PA4459 & & conserved hypothetical protein & -2.7 & $6.00 \mathrm{E}-03$ \\
\hline PA5023 & & conserved hypothetical protein & -2.2 & $4.66 \mathrm{E}-03$ \\
\hline PA5052 & & hypothetical protein & -2.0 & $8.60 \mathrm{E}-03$ \\
\hline PA5104 & & conserved hypothetical protein & -2.5 & $4.45 \mathrm{E}-03$ \\
\hline PA5463 & & hypothetical protein & -2.0 & 8.59E-03 \\
\hline PA0567 & & conserved hypothetical protein & -3.2 & $9.87 \mathrm{E}-03$ \\
\hline PA3791 & & hypothetical protein & -2.1 & $9.75 \mathrm{E}-03$ \\
\hline \multicolumn{5}{|c|}{ Membrane proteins } \\
\hline PA2763 & & hypothetical protein & -3.6 & 8.47E-03 \\
\hline PA0606 & & probable permease of $\mathrm{ABC}$ transporter & -2.4 & 4.43E-03 \\
\hline PA2811 & & probable permease of $\mathrm{ABC}-2$ transporter & -4.6 & $6.12 \mathrm{E}-03$ \\
\hline \multicolumn{5}{|c|}{ Nucleotide biosynthesis and metabolism } \\
\hline PA5496 & $n r d J b$ & $\begin{array}{l}\text { class II ribonucleotide-diphosphate reductase } \\
\text { subunit, NrdJb }\end{array}$ & -3.0 & $5.62 \mathrm{E}-03$ \\
\hline PA5497 & $n r d J a$ & $\begin{array}{l}\text { class II ribonucleotide-diphosphate reductase } \\
\text { subunit, NrdJa }\end{array}$ & -3.3 & $8.44 \mathrm{E}-03$ \\
\hline \multicolumn{5}{|c|}{ Protein secretion/export apparatus } \\
\hline PA0077 & $i c m F 1$ & IcmF1 & -3.0 & $8.66 \mathrm{E}-03$ \\
\hline PA5210 & & probable secretion pathway ATPase & -2.3 & $3.31 \mathrm{E}-03$ \\
\hline \multicolumn{5}{|c|}{ Putative enzymes } \\
\hline PA0507 & & probable acyl-CoA dehydrogenase & -3.8 & $6.26 \mathrm{E}-03$ \\
\hline PA0534 & & conserved hypothetical protein & -3.1 & 7.91E-03 \\
\hline PA2317 & & probable oxidoreductase & 2.9 & $2.78 \mathrm{E}-03$ \\
\hline PA3427 & & probable short-chain dehydrogenases & -3.8 & $8.59 \mathrm{E}-03$ \\
\hline PA3454 & & probable acyl-CoA thiolase & -3.9 & 4.33E-03 \\
\hline PA5181 & & probable oxidoreductase & -8.6 & $5.96 \mathrm{E}-03$ \\
\hline PA2237 & $\operatorname{pslG}$ & probable glycosyl hydrolase & -2.4 & $6.82 \mathrm{E}-03$ \\
\hline PA2239 & pslI & probable transferase & -3.0 & 4.02E-03 \\
\hline PA2702 & & hypothetical protein & -2.0 & 4.45E-03 \\
\hline \multicolumn{5}{|c|}{ Transcriptional regulators } \\
\hline PA0479 & & probable transcriptional regulator & -2.1 & $9.90 \mathrm{E}-03$ \\
\hline PA2281 & & probable transcriptional regulator & -2.1 & $9.89 \mathrm{E}-03$ \\
\hline PA5105 & hutC & histidine utilization repressor HutC & -3.1 & 8.27E-03 \\
\hline \multicolumn{5}{|c|}{ Translation, post-translational modification, degradation } \\
\hline PA0594 & surA & peptidyl-prolyl cis-trans isomerase SurA & -2.4 & $6.26 \mathrm{E}-03$ \\
\hline \multicolumn{5}{|c|}{ Transport of small molecules } \\
\hline PA2987 & & $\begin{array}{l}\text { probable ATP-binding component of ABC } \\
\text { transporter }\end{array}$ & -2.1 & $3.68 \mathrm{E}-03$ \\
\hline
\end{tabular}




\begin{tabular}{|c|c|c|c|c|}
\hline \multicolumn{5}{|c|}{ AmpR-dependent; Upregulated } \\
\hline $\begin{array}{l}\text { Locus } \\
\text { Tag }\end{array}$ & $\begin{array}{l}\text { Gene } \\
\text { Name }\end{array}$ & Product Name & FC & $\begin{array}{l}\text { Corrected } p \text { - } \\
\text { value }\end{array}$ \\
\hline \multicolumn{5}{|c|}{ Adaptation, Protection } \\
\hline PA4378 & inaA & InaA protein & 3.0 & $6.68 \mathrm{E}-03$ \\
\hline PA2652 & & probable chemotaxis transducer & 2.6 & $8.67 \mathrm{E}-03$ \\
\hline PA3348 & & probable chemotaxis protein methyltransferase & 2.2 & $3.64 \mathrm{E}-03$ \\
\hline PA4309 & $\operatorname{pct} A$ & chemotactic transducer PctA & 3.2 & $7.83 \mathrm{E}-03$ \\
\hline PA0923 & $\operatorname{din} B$ & DNA Polymerase IV, DinB & 4.5 & $6.05 \mathrm{E}-03$ \\
\hline \multicolumn{5}{|c|}{ Amino acid biosynthesis and metabolism } \\
\hline PA0245 & $\operatorname{aro} Q^{2}$ & 3-dehydroquinate dehydratase & 2.9 & $3.71 \mathrm{E}-03$ \\
\hline PA4565 & proB & glutamate 5-kinase & 2.9 & $5.03 \mathrm{E}-03$ \\
\hline \multicolumn{5}{|c|}{ Biosynthesis of cofactors, prosthetic groups and carriers } \\
\hline PA4919 & pncB1 & nicotinate phosphoribosyltransferase & 6.3 & $3.95 \mathrm{E}-03$ \\
\hline \multicolumn{5}{|c|}{ Carbon compound catabolism } \\
\hline PA0230 & pcaB & 3-carboxy-cis,cis-muconate cycloisomerase & 3.3 & $7.08 \mathrm{E}-03$ \\
\hline PA0231 & pcaD & beta-ketoadipate enol-lactone hydrolase & 3.7 & $5.16 \mathrm{E}-03$ \\
\hline PA0232 & pcaC & gamma-carboxymuconolactone decarboxylase & 4.1 & $8.44 \mathrm{E}-03$ \\
\hline PA2862 & $\operatorname{lip} A$ & lactonizing lipase precursor & 3.6 & $5.97 \mathrm{E}-03$ \\
\hline \multicolumn{5}{|c|}{$\begin{array}{l}\text { Cell wall / LPS / } \\
\text { capsule }\end{array}$} \\
\hline PA3160 & $w z z$ & O-antigen chain length regulator & 3.7 & $8.81 \mathrm{E}-03$ \\
\hline PA1082 & $f \lg G$ & flagellar basal-body rod protein FlgG & 2.9 & $8.52 \mathrm{E}-03$ \\
\hline \multicolumn{5}{|c|}{ Central intermediary metabolism } \\
\hline PA2813 & & probable glutathione S-transferase & 2.6 & $9.10 \mathrm{E}-03$ \\
\hline PA3629 & $a d h C$ & alcohol dehydrogenase class III & 3.2 & $4.98 \mathrm{E}-03$ \\
\hline \multicolumn{5}{|c|}{ DNA replication, recombination, modification and repair } \\
\hline PA0577 & $d n a G$ & DNA primase & 4.7 & $8.39 \mathrm{E}-03$ \\
\hline \multicolumn{5}{|c|}{ Hypothetical, unclassified, unknown } \\
\hline PA0124 & & hypothetical protein & 2.8 & $3.81 \mathrm{E}-03$ \\
\hline PA0729 & & hypothetical protein & 9.4 & $3.41 \mathrm{E}-03$ \\
\hline PA0911 & & hypothetical protein & 6.8 & $7.83 \mathrm{E}-03$ \\
\hline PA1016 & & hypothetical protein & 2.6 & $7.58 \mathrm{E}-03$ \\
\hline PA1164 & & conserved hypothetical protein & 2.1 & $3.66 \mathrm{E}-03$ \\
\hline PA1299 & & conserved hypothetical protein & 3.0 & $3.41 \mathrm{E}-03$ \\
\hline PA1439 & & conserved hypothetical protein & 2.2 & $7.28 \mathrm{E}-03$ \\
\hline PA1545 & & hypothetical protein & 2.7 & $6.65 \mathrm{E}-03$ \\
\hline PA1744 & & hypothetical protein & 3.1 & $5.62 \mathrm{E}-03$ \\
\hline PA2362 & & hypothetical protein & 2.4 & $9.87 \mathrm{E}-03$ \\
\hline PA2487 & & hypothetical protein & 11.6 & $2.26 \mathrm{E}-03$ \\
\hline PA2765 & & hypothetical protein & 14.6 & $1.60 \mathrm{E}-03$ \\
\hline PA2901 & & hypothetical protein & 16.5 & $4.43 \mathrm{E}-03$ \\
\hline PA3306 & & hypothetical protein & 14.9 & $2.90 \mathrm{E}-03$ \\
\hline PA3353 & & hypothetical protein & 2.3 & $6.40 \mathrm{E}-03$ \\
\hline PA3499 & & hypothetical protein & 2.4 & $6.97 \mathrm{E}-03$ \\
\hline PA3505 & & hypothetical protein & 5.7 & $3.92 \mathrm{E}-03$ \\
\hline PA3649 & & conserved hypothetical protein & 2.1 & $6.31 \mathrm{E}-03$ \\
\hline PA3741 & & hypothetical protein & 7.1 & $6.52 \mathrm{E}-03$ \\
\hline PA3951 & & conserved hypothetical protein & 2.1 & $8.33 \mathrm{E}-03$ \\
\hline
\end{tabular}




\begin{tabular}{|c|c|c|c|c|}
\hline PA4326 & & hypothetical protein & 3.1 & $7.63 \mathrm{E}-03$ \\
\hline PA4379 & & conserved hypothetical protein & 5.0 & $3.41 \mathrm{E}-03$ \\
\hline PA4918 & & hypothetical protein & 16.9 & $2.59 \mathrm{E}-03$ \\
\hline PA4921 & & hypothetical protein & 4.2 & $3.77 \mathrm{E}-03$ \\
\hline PA5292 & $p \operatorname{ch} P$ & phosphorylcholine phosphatase & 2.3 & $9.89 \mathrm{E}-03$ \\
\hline \multicolumn{5}{|c|}{ Membrane proteins } \\
\hline PA2988 & & conserved hypothetical protein & 2.2 & $5.73 \mathrm{E}-03$ \\
\hline PA3526 & & probable outer membrane protein precursor & 2.3 & $3.64 \mathrm{E}-03$ \\
\hline PA3575 & & hypothetical protein & 2.2 & $6.11 \mathrm{E}-03$ \\
\hline PA5561 & atpI & ATP synthase protein I & 2.6 & $5.05 \mathrm{E}-03$ \\
\hline PA0240 & & probable porin & 2.3 & $8.70 \mathrm{E}-03$ \\
\hline PA3228 & & $\begin{array}{l}\text { probable ATP-binding/permease fusion } \mathrm{ABC} \\
\text { transporter }\end{array}$ & 3.5 & $1.38 \mathrm{E}-03$ \\
\hline \multicolumn{5}{|c|}{ Motility \& Attachment } \\
\hline PA1099 & fleR & two-component response regulator & 3.0 & $7.63 \mathrm{E}-03$ \\
\hline \multicolumn{5}{|c|}{ Non-coding RNA gene } \\
\hline PA3621.1 & $r \operatorname{sm} Z$ & regulatory RNA $r s m Z$ & 4.8 & $9.25 \mathrm{E}-03$ \\
\hline \multicolumn{5}{|c|}{ Protein secretion/export apparatus } \\
\hline PA2863 & lipH & lipase modulator protein & 2.2 & $4.85 \mathrm{E}-03$ \\
\hline \multicolumn{5}{|c|}{ Putative enzymes } \\
\hline PA0242 & & hypothetical protein & 2.6 & 7.19E-03 \\
\hline PA0244 & & hypothetical protein & 2.9 & $4.52 \mathrm{E}-03$ \\
\hline PA1296 & & probable 2-hydroxyacid dehydrogenase & 3.2 & $3.44 \mathrm{E}-03$ \\
\hline PA3498 & & probable oxidoreductase & 2.4 & $7.51 \mathrm{E}-03$ \\
\hline PA3506 & & probable decarboxylase & 4.8 & $5.76 \mathrm{E}-03$ \\
\hline PA3628 & & probable esterase & 2.9 & $5.71 \mathrm{E}-03$ \\
\hline PA3817 & & probable methyltransferase & 4.2 & $5.32 \mathrm{E}-03$ \\
\hline PA5048 & & probable nuclease & 3.2 & $9.01 \mathrm{E}-03$ \\
\hline \multicolumn{5}{|c|}{ Secreted Factors (toxins, enzymes, alginate) } \\
\hline PA0843 & plcR & phospholipase accessory protein PlcR precursor & 2.2 & $2.16 \mathrm{E}-03$ \\
\hline \multicolumn{5}{|c|}{ Transcription, RNA processing and degradation } \\
\hline PA2840 & & probable ATP-dependent RNA helicase & 3.5 & 4.17E-04 \\
\hline PA2975 & $r l u C$ & ribosomal large subunit pseudouridine synthase $\mathrm{C}$ & 6.5 & $6.17 \mathrm{E}-03$ \\
\hline PA4275 & nusG & transcription antitermination protein NusG & 2.7 & $9.69 \mathrm{E}-03$ \\
\hline \multicolumn{5}{|c|}{ Transport of small molecules } \\
\hline PA1256 & & amino acid $\mathrm{ABC}$ transporter $\mathrm{ATP}$ binding protein & 2.3 & $8.26 \mathrm{E}-03$ \\
\hline PA5376 & & $\begin{array}{l}\text { probable ATP-binding component of } \mathrm{ABC} \\
\text { transporter }\end{array}$ & 2.2 & $6.12 \mathrm{E}-03$ \\
\hline \multicolumn{5}{|c|}{ Two-component regulatory systems } \\
\hline PA0757 & & probable two-component sensor & 2.3 & $3.77 \mathrm{E}-03$ \\
\hline PA1098 & fles & two-component sensor & 2.5 & $6.65 \mathrm{E}-03$ \\
\hline
\end{tabular}

\begin{tabular}{|c|c|c|c|c|}
\hline \multicolumn{5}{|c|}{ AmpR-ß-lactam dependent; Downregulated genes } \\
\hline $\begin{array}{l}\text { Locus } \\
\text { Tag } \\
\end{array}$ & $\begin{array}{l}\text { Gene } \\
\text { Name } \\
\end{array}$ & Product Name & FC & $\begin{array}{l}\text { Corrected } p \text { - } \\
\text { value }\end{array}$ \\
\hline \multicolumn{5}{|c|}{ Amino acid biosynthesis and metabolism } \\
\hline PA0390 & $\operatorname{met} X$ & homoserine $\mathrm{O}$-acetyltransferase & -2.5 & $6.29 \mathrm{E}-03$ \\
\hline
\end{tabular}




\begin{tabular}{|c|c|c|c|c|}
\hline PA1750 & & phospho-2-dehydro-3-deoxyheptonate aldolase & -2.4 & $8.53 \mathrm{E}-03$ \\
\hline PA4846 & $\operatorname{aro} Q 1$ & 3-dehydroquinate dehydratase & -2.3 & $6.79 \mathrm{E}-03$ \\
\hline PA0932 & cys $M$ & cysteine synthase B & -2.0 & $2.90 \mathrm{E}-03$ \\
\hline \multicolumn{5}{|c|}{ Biosynthesis of cofactors, prosthetic groups and carriers } \\
\hline PA0024 & hemF & coproporphyrinogen III oxidase, aerobic & -3.2 & $6.65 \mathrm{E}-03$ \\
\hline PA4280 & $\operatorname{bir} A$ & BirA bifunctional protein & -3.0 & $9.14 \mathrm{E}-03$ \\
\hline PA5118 & thiI & thiazole biosynthesis protein ThiI & -2.9 & $7.95 \mathrm{E}-03$ \\
\hline PA3652 & uppS & undecaprenyl pyrophosphate synthetase & -2.3 & $6.65 \mathrm{E}-03$ \\
\hline PA0342 & thy $A$ & thymidylate synthase & -2.1 & $8.99 \mathrm{E}-03$ \\
\hline \multicolumn{5}{|c|}{ Cell division } \\
\hline PA5563 & soj & chromosome partitioning protein Soj & -2.1 & $9.71 \mathrm{E}-03$ \\
\hline \multicolumn{5}{|c|}{$\begin{array}{l}\text { Cell wall / LPS / } \\
\text { capsule }\end{array}$} \\
\hline PA4450 & murA & $\begin{array}{l}\text { UDP-N-acetylglucosamine 1- } \\
\text { carboxyvinyltransferase }\end{array}$ & -2.1 & $9.73 \mathrm{E}-03$ \\
\hline PA4545 & comL & competence protein ComL & -2.1 & $7.95 \mathrm{E}-03$ \\
\hline PA4481 & $m r e B$ & rod shape-determining protein $\mathrm{MreB}$ & -4.4 & $6.51 \mathrm{E}-03$ \\
\hline \multicolumn{5}{|c|}{ Chaperones \& heat shock proteins } \\
\hline PA5193 & $y r f I$ & heat shock protein HSP33 & -2.5 & $6.65 \mathrm{E}-03$ \\
\hline \multicolumn{5}{|c|}{ DNA replication, recombination, modification and repair } \\
\hline PA3620 & mutS & DNA mismatch repair protein MutS & -2.6 & $9.22 \mathrm{E}-03$ \\
\hline \multicolumn{5}{|c|}{ Hypothetical, unclassified, unknown } \\
\hline PA3472 & & hypothetical protein & -5.4 & $8.53 \mathrm{E}-03$ \\
\hline PA4451 & & conserved hypothetical protein & -3.7 & $7.09 \mathrm{E}-03$ \\
\hline PA4438 & & conserved hypothetical protein & -3.4 & $6.65 \mathrm{E}-03$ \\
\hline PA2318 & & hypothetical protein & -3.1 & $8.53 \mathrm{E}-03$ \\
\hline PA3453 & & conserved hypothetical protein & -2.8 & $9.34 \mathrm{E}-03$ \\
\hline PA4004 & & conserved hypothetical protein & -2.7 & $6.65 \mathrm{E}-03$ \\
\hline PA4574 & & conserved hypothetical protein & -2.2 & $6.33 \mathrm{E}-03$ \\
\hline PA4492 & & conserved hypothetical protein & -2.2 & $8.57 \mathrm{E}-03$ \\
\hline PA3664 & & conserved hypothetical protein & -2.1 & $7.95 \mathrm{E}-03$ \\
\hline PA4460 & & conserved hypothetical protein & -2.1 & $8.44 \mathrm{E}-03$ \\
\hline PA2823 & & conserved hypothetical protein & -2.0 & $9.10 \mathrm{E}-03$ \\
\hline PA3483 & & hypothetical protein & -2.0 & $7.09 \mathrm{E}-03$ \\
\hline \multicolumn{5}{|c|}{ Membrane proteins } \\
\hline PA3828 & & conserved hypothetical protein & -2.7 & $9.24 \mathrm{E}-03$ \\
\hline PA3794 & & hypothetical protein & -2.3 & $4.37 \mathrm{E}-03$ \\
\hline PA4292 & & probable phosphate transporter & -2.4 & $9.10 \mathrm{E}-03$ \\
\hline \multicolumn{5}{|c|}{ Nucleotide biosynthesis and metabolism } \\
\hline PA5298 & & xanthine phosphoribosyltransferase & -3.2 & $9.75 \mathrm{E}-03$ \\
\hline PA5336 & $g m k$ & guanylate kinase & -3.0 & $8.86 \mathrm{E}-03$ \\
\hline PA3527 & pyrC & dihydroorotase & -2.5 & $8.09 \mathrm{E}-03$ \\
\hline PA0944 & $\operatorname{purN}$ & phosphoribosylaminoimidazole synthetase & -2.2 & $9.73 \mathrm{E}-03$ \\
\hline \multicolumn{5}{|c|}{ Putative enzymes } \\
\hline PA0386 & & probable oxidase & -2.4 & 7.33E-03 \\
\hline PA4389 & & probable short-chain dehydrogenase & -2.1 & $7.86 \mathrm{E}-03$ \\
\hline \multicolumn{5}{|c|}{ Transcription, RNA processing and degradation } \\
\hline PA0592 & $k s g A$ & rRNA (adenine-N6,N6)-dimethyltransferase & -2.6 & $8.56 \mathrm{E}-03$ \\
\hline PA4745 & nusA & $\mathrm{N}$ utilization substance protein $\mathrm{A}$ & -2.3 & 8.39E-03 \\
\hline
\end{tabular}




\begin{tabular}{|c|c|c|c|c|}
\hline PA4853 & fis & DNA-binding protein Fis & -3.3 & $6.65 \mathrm{E}-03$ \\
\hline PA3604 & & probable two-component response regulator & -2.2 & $6.96 \mathrm{E}-03$ \\
\hline PA0463 & creB & two-component response regulator $\mathrm{CreB}$ & -2.1 & $8.39 \mathrm{E}-03$ \\
\hline \multicolumn{5}{|c|}{ Translation, post-translational modification, degradation } \\
\hline PA3656 & $\operatorname{rps} B$ & $30 \mathrm{~S}$ ribosomal protein $\mathrm{S} 2$ & -4.0 & $9.34 \mathrm{E}-03$ \\
\hline PA4935 & $r p s F$ & $30 \mathrm{~S}$ ribosomal protein $\mathrm{S} 6$ & -3.7 & $6.74 \mathrm{E}-03$ \\
\hline PA5569 & rпpA & ribonuclease $\mathrm{P}$ protein component & -3.5 & $5.03 \mathrm{E}-03$ \\
\hline PA4253 & $r p l N$ & 50S ribosomal protein $\mathrm{L} 14$ & -3.0 & $9.10 \mathrm{E}-03$ \\
\hline PA4263 & rplC & 50S ribosomal protein $\mathrm{L} 3$ & -2.8 & $6.94 \mathrm{E}-03$ \\
\hline PA4257 & $r p s C$ & $30 \mathrm{~S}$ ribosomal protein $\mathrm{S} 3$ & -2.7 & $8.90 \mathrm{E}-03$ \\
\hline PA4239 & $r p s D$ & $30 \mathrm{~S}$ ribosomal protein $\mathrm{S} 4$ & -2.4 & $6.51 \mathrm{E}-03$ \\
\hline PA4254 & $r p s Q$ & $30 \mathrm{~S}$ ribosomal protein $\mathrm{S} 17$ & -2.4 & 4.37E-03 \\
\hline PA4251 & rplE & $50 \mathrm{~S}$ ribosomal protein $\mathrm{L} 5$ & -2.4 & $6.33 \mathrm{E}-03$ \\
\hline PA4665 & $\operatorname{prfA}$ & peptide chain release factor 1 & -2.2 & $6.65 \mathrm{E}-03$ \\
\hline PA4274 & $r p l K$ & $50 \mathrm{~S}$ ribosomal protein $\mathrm{L} 11$ & -2.0 & $5.03 \mathrm{E}-03$ \\
\hline PA3818 & & extragenic suppressor protein SuhB & -2.7 & $9.10 \mathrm{E}-03$ \\
\hline \multicolumn{5}{|c|}{ Transport of small molecules } \\
\hline PA2983 & & probable tolQ-type transport protein & -2.7 & $6.74 \mathrm{E}-03$ \\
\hline \multicolumn{5}{|c|}{ AmpR-ß-lactam dependent; Upregulated genes } \\
\hline $\begin{array}{l}\text { Locus } \\
\text { Tag } \\
\end{array}$ & $\begin{array}{l}\text { Gene } \\
\text { Name } \\
\end{array}$ & Product Name & FC & $\begin{array}{l}\text { Corrected } p \text { - } \\
\text { value }\end{array}$ \\
\hline \multicolumn{5}{|c|}{ Biosynthesis of cofactors, prosthetic groups and carriers } \\
\hline PA0502 & & probable biotin biosynthesis protein bioH & 2.3 & 7.53E-03 \\
\hline \multicolumn{5}{|c|}{ Hypothetical, unclassified, unknown } \\
\hline PA5191 & & hypothetical protein & 2.2 & $8.23 \mathrm{E}-03$ \\
\hline PA0959 & & hypothetical protein & 2.3 & 8.93E-03 \\
\hline PA1043 & & hypothetical protein & 2.3 & $6.65 \mathrm{E}-03$ \\
\hline PA2226 & & hypothetical protein & 2.5 & $8.16 \mathrm{E}-03$ \\
\hline PA1090 & & hypothetical protein & 2.6 & $5.03 \mathrm{E}-03$ \\
\hline PA4685 & & hypothetical protein & 2.6 & $5.03 \mathrm{E}-03$ \\
\hline PA1356 & & hypothetical protein & 2.6 & 7.87E-03 \\
\hline PA4608 & & hypothetical protein & 2.8 & $9.73 \mathrm{E}-03$ \\
\hline PA3089 & & hypothetical protein & 2.9 & $9.50 \mathrm{E}-03$ \\
\hline PA1091 & fgtA & flagellar glycosyl transferase, FgtA & 3.1 & $3.60 \mathrm{E}-03$ \\
\hline PA1088 & & hypothetical protein & 3.1 & $9.73 \mathrm{E}-03$ \\
\hline PA4362 & & hypothetical protein & 3.1 & $8.56 \mathrm{E}-03$ \\
\hline PA5101 & & hypothetical protein & 3.2 & $8.55 \mathrm{E}-03$ \\
\hline PA1135 & & conserved hypothetical protein & 3.8 & $5.57 \mathrm{E}-03$ \\
\hline PA2699 & & hypothetical protein & 3.9 & $9.10 \mathrm{E}-03$ \\
\hline PA0484 & & conserved hypothetical protein & 3.9 & $6.79 \mathrm{E}-03$ \\
\hline PA2374 & & hypothetical protein & 4.1 & $6.96 \mathrm{E}-03$ \\
\hline PA1728 & & hypothetical protein & 5.0 & $8.53 \mathrm{E}-03$ \\
\hline PA2381 & & hypothetical protein & 7.5 & $8.44 \mathrm{E}-03$ \\
\hline PA1874 & & hypothetical protein & 22.1 & $5.09 \mathrm{E}-03$ \\
\hline \multicolumn{5}{|c|}{ Membrane proteins } \\
\hline PA2072 & & conserved hypothetical protein & 2.2 & $9.80 \mathrm{E}-03$ \\
\hline PA1042 & & conserved hypothetical protein & 2.7 & $9.05 \mathrm{E}-03$ \\
\hline
\end{tabular}




\begin{tabular}{|c|c|c|c|}
\hline PA4614 & conductance mechanosensitive channel & 2.3 & $5.57 \mathrm{E}-03$ \\
\hline PA1119 & probable outer membrane protein precursor & 2.2 & $9.22 \mathrm{E}-03$ \\
\hline \multicolumn{4}{|c|}{ Protein secretion/export apparatus } \\
\hline PA1877 & probable secretion protein & 8.6 & $9.73 \mathrm{E}-03$ \\
\hline \multicolumn{4}{|l|}{ Putative enzymes } \\
\hline PA0838 & probable glutathione peroxidase & 2.4 & $9.34 \mathrm{E}-03$ \\
\hline PA0804 & probable oxidoreductase & 2.5 & $9.10 \mathrm{E}-03$ \\
\hline PA0704 & probable amidase & 3.6 & $8.56 \mathrm{E}-03$ \\
\hline \multicolumn{4}{|c|}{$\begin{array}{l}\text { Secreted Factors (toxins, enzymes, alginate) ; Protein secretion/export apparatus ; Transport of small } \\
\text { molecules }\end{array}$} \\
\hline PA1876 & $\begin{array}{l}\text { probable ATP-binding/permease fusion } \mathrm{ABC} \\
\text { transporter }\end{array}$ & 9.0 & $5.57 \mathrm{E}-03$ \\
\hline \multicolumn{4}{|c|}{ Transport of small molecules } \\
\hline PA3858 & probable amino acid-binding protein & 2.3 & 5.19E-03 \\
\hline
\end{tabular}


Table S9: Phenotypic microarray analysis of PAO1 and PAO $\triangle a m p R$. Phenotypic microarray analysis was performed using Biolog plates, as explained in the text. Phenotypes gained indicate negative regulation by AmpR of these phenotypes, whereas phenotypes lost are those that are positively regulated by AmpR.

\begin{tabular}{lll}
\multicolumn{2}{l}{ Phenotypes Gained } & \\
\hline Plate no. & Substrate & Nature of substrate \\
\hline PM05 & L-Histidine & Nutritional supplement \\
PM05 & L-Leucine & Nutritional supplement \\
PM05 & L-Serine & Nutritional supplement \\
PM05 & L-Citrulline & Nutritional supplement \\
PM05 & (-)Shikimic acid & Nutritional supplement \\
PM05 & Spermidine & Nutritional supplement \\
PM05 & Pyridoxal & Nutritional supplement \\
\hline
\end{tabular}

\begin{tabular}{lll} 
Phenotypes Lost & Nature of substrate \\
\hline Plate no. & Substrate & C-Source, amino acid \\
\hline PM02A & L-Pyroglutamic acid & N-Source, other \\
PM03B & Uric acid & wall, lactam \\
PM11C & Amoxicillin & toxic anion \\
PM11C & Potassium tellurite & wall, cephalosporin \\
PM11C & Cephalothin & wall, lactam \\
PM12B & Penicillin G & wall, lactam \\
PM12B & Oxacillin & protein synthesis, 30S ribosomal subunit, \\
PM12B & Paromomycin & aminoglycoside \\
PM12B & Vancomycin & wall \\
PM12B & Sisomicin & protein synthesis, 30S ribosomal subunit, \\
PM12B & Novobiocin & aminoglycoside \\
PM12B & 2,4-Diamino-6,7-diisopropylpteridine & DNA topoisomerase \\
folate antagonist, vibriostatic agent \\
PM12B & Tobramycin & protein synthesis, 30S ribosomal subunit, \\
& & aminoglycoside \\
PM12B & Spectinomycin & protein synthesis, 30S ribosomal subunit, \\
& & aminoglycoside \\
PM12B & Spiramycin & protein synthesis, 50S ribosomal subunit, \\
macrolide
\end{tabular}




\begin{tabular}{|c|c|c|}
\hline PM15B & 2- Nitroimidazole & $\begin{array}{l}\text { nitro compound, oxidizing agent, ribonucleotide } \\
\text { DP reductase inhibitor }\end{array}$ \\
\hline PM16A & Cefotaxime & wall, cephalosporin \\
\hline PM16A & Streptomycin & $\begin{array}{l}\text { protein synthesis, } 30 \mathrm{~S} \text { ribosomal subunit, } \\
\text { aminoglycoside }\end{array}$ \\
\hline PM16A & Chromium (III) chloride & toxic cation \\
\hline PM17A & 4-Aminopyridine & ion channel inhibitor, $\mathrm{K}+$ \\
\hline PM17A & Lithium chloride & toxic cation \\
\hline PM18C & Azathioprine & nucleic acid analog, purine \\
\hline PM18C & Poly-L-lysine & membrane, detergent, cationic \\
\hline PM18C & Pentachlorophenol & respiration, ionophore, $\mathrm{H}+$ \\
\hline PM18C & Sodium Bromate & toxic anion \\
\hline PM18C & Sodium periodate & toxic anion, oxidizing agent \\
\hline PM18C & Semicarbazide hydrochloride & amine oxidase inhibitor, carcinogen \\
\hline PM18C & 5-Fluoro-5`-deoxyuridine & nucleic acid analog, pyrimidine, DNA synthesis \\
\hline PM19 & Coumarin & DNA intercalator \\
\hline PM19 & Harmane & imidazoline binding sites, agonist \\
\hline PM19 & 7-Hydroxycoumarin & DNA intercalator \\
\hline PM19 & Phenethicilllin & wall, lactam \\
\hline PM19 & $\begin{array}{l}\text { Lauryl sulfobetaine (N-Dodecyl-N,N- } \\
\text { dimethyl-3- ammonio-1- } \\
\text { propanesulfonate) }\end{array}$ & membrane, detergent, zwitterionic \\
\hline PM20B & Amitriptyline & membrane, transport \\
\hline PM20B & Orphenadrine & anti-cholinergic \\
\hline PM20B & Ciprofloxacin & DNA topoisomerase \\
\hline PM20B & Oxytetracycline & $\begin{array}{l}\text { protein synthesis, } 30 \mathrm{~S} \text { ribosomal subunit, } \\
\text { tetracycline }\end{array}$ \\
\hline PM20B & Captan & fungicide, carbamate \\
\hline PM20B & Tolylfluanid & fungicide, phenylsulphamide \\
\hline
\end{tabular}


Table S10: Primers used in this work. All primers were designed as part of this study. qRT in the primer name indicates that the primer was designed for qPCR.

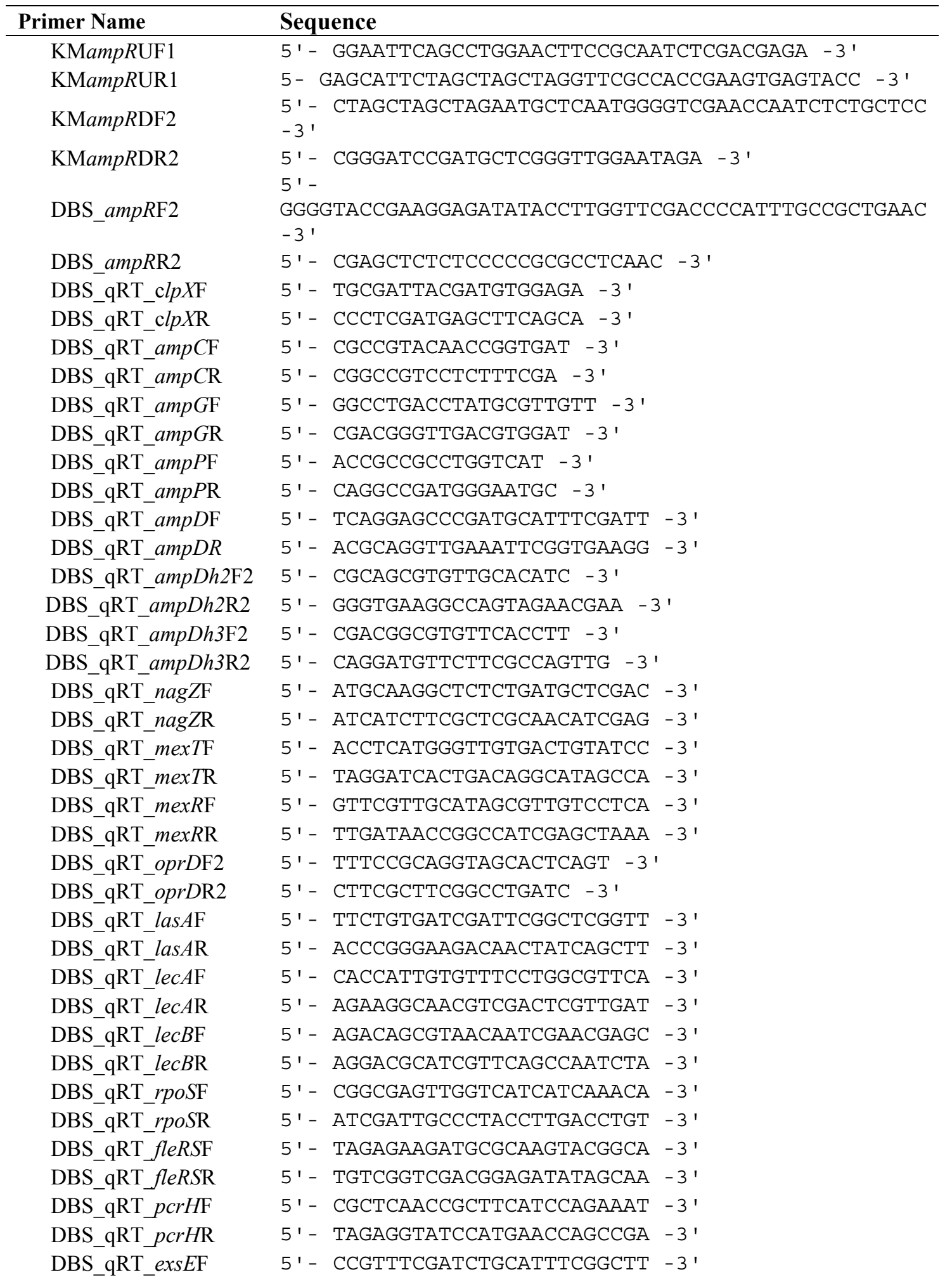




\begin{tabular}{|c|c|}
\hline DBS_qRT_exsER & $5^{\prime}-$ CAACTGACCCTCGAATGCTTCGA - 3' \\
\hline DBS_qRT_rsmZF2 & $5^{\prime}$ - GACGGTACTGGGTGTCAAAGG - $3^{\prime}$ \\
\hline DBS_qRT_rsmZR2 & 5' - TGGTAAATTTCCTCCCGGTGTA - 3' \\
\hline DBS_qRT_prtRF & $5^{\prime}$ - TCCCTGCACCCATGTGAAATCTCT - ' $^{\prime}$ \\
\hline DBS_qRT_prtRR & 5' - ATCGGCAATCTACAGACCGATGGA - 3 ' \\
\hline DBS_qRT_creBF & 5' - GCATATCCTGATCGTCGAAGATG - 3' \\
\hline DBS_qRT_creBR & 5' - GGCCTGCAGGGCGTAGA - 3' \\
\hline DBS_qRT_creDF & $5^{\prime}-$ CGCCATCGCCCTACTCAT - 3' \\
\hline DBS_qRT_creDR & $5^{\prime}-$ GGCGATCGCGGATCAG - $3^{\prime}$ \\
\hline DBS_qRT_prtNF & $5^{\prime}$ - CTGGTTTCGCAACCTGAACAAGGA - ${ }^{\prime}$ \\
\hline DBS_qRT_prtNR & 5' - TAGTCACAAGGCCATGCAGTTCGT - 3 ' \\
\hline DBS_qRT_algTF & $5^{\prime}$ - AACCCAGGAACAGGATCAGCAACT - ${ }^{\prime}$ \\
\hline DBS qRT $\operatorname{alg} T R$ & $5^{\prime}$ - AGATACTGGGATTGATCGTGCGGT - ' $^{\prime}$ \\
\hline DBS qRT $P A 3602 \mathrm{~F}$ & $5^{\prime}$ - ACGAATTCATCAGCCACTCGATGC - ' $^{\prime}$ \\
\hline DBS qRT $P A 3602 R$ & $5^{\prime}$ - TGTTCAACGTCTCGGCGATGAGTT - 3 ' \\
\hline DBS qRT mexEF & $5^{\prime}$ - TCATCGAACAACCGCTGAACGAGT - 3 ' \\
\hline DBS_qRT_mexER & 5 ' - ATGAAGGCGCACTGGTGAAGAAAG - 3 ' \\
\hline DBS_qRT_PA4121F & $5^{\prime}$ - ATCAAGACACCGAATACCCGCAAC - 3 ' \\
\hline DBS_qRT_PA4121R & $5^{\prime}$ - TACGTGATCGTCAACGAGTTCAGC - 3 ' \\
\hline DBS_qRT_popBF & $5^{\prime}$ - AACTCAAGCTGACCGAAATCGAGC - 3 ' \\
\hline DBS_qRT_popBR & $5^{\prime}$ - ACAACCAGCAGAAGATCAGGGAGT - 3 ' \\
\hline DBS_qRT_hcnAF & $5^{\prime}-$ TTGAACGTCAACACGATATCCAGCCC - ${ }^{\prime}$ \\
\hline DBS_qRT_hcnAR & $5^{\prime}-$ AGACCGTGCTCAACGTGCTCAAT - ${ }^{\prime}$ \\
\hline DBS_qRT $f l g C F$ & 5' - GTCGAGGAAATGGCCGACATGATT - 3 ' \\
\hline DBS_qRT $f l g C \mathrm{R}$ & $5^{\prime}$ - ACCGCCAAACAGATGATGCAGAAG - 3 ' \\
\hline DBS_qRT_PA2069F & $5^{\prime}$ - AAACCAGGTGCTGGGCATGTTCAA - ' $^{\prime}$ \\
\hline DBS_qRT_PA2069R & 5'- AACGCACCGAGTTCATGCCATT - 3' \\
\hline DBS_qRT_PA2331F & 5' - TTATATCCCGAATCTGCTCGGCGT - ' $^{\prime}$ \\
\hline DBS_qRT_PA2331R & $5^{\prime}-$ ATGGCTGCGACTTCTGCGTT - ' $^{\prime}$ \\
\hline
\end{tabular}


Supplementary information for Chapter 5 
Supplementary Table 1: Key to identifying AmpR-regulated genes. Using the data from Figure 5.2, AmpR-regulated genes, both in the presence and absence of $B$-lactam stress, were identified using this table. Condition 1: PAO1 uninduced vs. PAO1 induced; Condition II: PAO $\triangle a m p R$ uninduced vs. PAO $\triangle a m p R$ induced; Condition III: PAO1 uninduced vs. PAO $\triangle a m p R$ uninduced; Condition IV: PAO1 induced vs. PAO $\triangle a m p R$ induced. Out of the 1703 genes in Figure 5.2, 1623 genes were categorized here. Eighty genes in the following categories were omitted from further analysis for the following reasons: regulated in all four conditions [category $\mathrm{O}$ ( 24 genes)]; could not be assigned unambiguously [category $\mathrm{H}$ ( 44 genes), category K (1 gene), category M (4 genes), category N (7 genes)].

\begin{tabular}{|c|c|c|c|c|}
\hline Regulation & $\begin{array}{c}\text { Venn } \\
\text { Category }\end{array}$ & $\begin{array}{c}\text { \# Genes } \\
\text { Regulated } \\
\text { (Total in } \\
\text { Category) }\end{array}$ & Condition (Expression) & Regulation \\
\hline \multirow{10}{*}{$\begin{array}{c}\text { AmpR- } \\
\text { dependent, } \\
\text { B-lactam } \\
\text { independent } \\
(654)\end{array}$} & $\mathrm{B}$ & $135(281)$ & II (up) & Negative \\
\hline & $\mathrm{B}$ & $146(281)$ & II (down) & Positive \\
\hline & $\mathrm{C}$ & $132(171)$ & III (up) & Negative \\
\hline & $\mathrm{C}$ & $39(171)$ & III (down) & Positive \\
\hline & $\mathrm{J}$ & $70(185)$ & III (up), IV (up) & Negative \\
\hline & $\mathrm{J}$ & $115(185)$ & III (down), IV (down) & Positive \\
\hline & $\mathrm{M}$ & $2(12)$ & I (down), III (up), IV (up) & Negative \\
\hline & $\mathrm{M}$ & $6(12)$ & I (up), III (down), IV (down) & Positive \\
\hline & $\mathrm{N}$ & $2(27)$ & II (up), III (up), IV (up) & Negative \\
\hline & $\mathrm{N}$ & $7(27)$ & $\begin{array}{l}\text { II (down), III (down), IV } \\
\text { (down) }\end{array}$ & Positive \\
\hline \multirow{5}{*}{$\begin{array}{c}\text { AmpR-B- } \\
\text { lactam- } \\
\text { dependent } \\
(483)\end{array}$} & $\mathrm{D}$ & $239(392)$ & IV (up) & Negative \\
\hline & $\mathrm{D}$ & $153(392)$ & IV (down) & Positive \\
\hline & $\mathrm{H}$ & $72(116)$ & I (up), IV (down) & Positive \\
\hline & $\mathrm{L}$ & $11(26)$ & I (up), II (down), IV (down) & Positive \\
\hline & $\mathrm{L}$ & $8(26)$ & I (down), II (up), IV (up) & Negative \\
\hline \multirow{14}{*}{$\begin{array}{c}\text { AmpR- } \\
\text { independent, } \\
\text { B-lactam- } \\
\text { dependent } \\
\text { (486) }\end{array}$} & A & $26(108)$ & I (down) & Negative \\
\hline & A & $82(108)$ & I (up) & Positive \\
\hline & $\mathrm{G}$ & $49(65)$ & II (down), III (up) & Negative \\
\hline & $\mathrm{G}$ & $16(65)$ & II (up), III (down) & Positive \\
\hline & $\mathrm{F}$ & $16(47)$ & I (down), III (down) & Negative \\
\hline & $\mathrm{F}$ & $31(47)$ & I (up), III (up) & Positive \\
\hline & $\mathrm{I}$ & $84(198)$ & II (down), IV (down) & Negative \\
\hline & I & $114(198)$ & II (up), IV (up) & Positive \\
\hline & $\mathrm{E}$ & $19(50)$ & I (down), II (down) & Negative \\
\hline & $\mathrm{E}$ & $31(50)$ & I (up), II (up) & Positive \\
\hline & $\mathrm{L}$ & $6(26)$ & $\begin{array}{l}\text { I (down), II (down), IV } \\
\text { (down) }\end{array}$ & Negative \\
\hline & $\mathrm{L}$ & $1(26)$ & I (up), II (up), IV (up) & Positive \\
\hline & $\mathrm{N}$ & $8(27)$ & II (down), III (up), IV (down) & Negative \\
\hline & $\mathrm{N}$ & $3(27)$ & II (up), III (down), IV (up) & Positive \\
\hline
\end{tabular}


Supplementary Table 2: AmpR-dependent genes. The positively and negatively AmpR-regulated genes are classified based on information in Supplementary Table 1. Only genes that are $\geq 2.0$, Bonferroni correction $(<0.05)$ are listed. Functional categorization, gene ID and gene/product names are from the Pseudomonas Genome database (Winsor et al 2011). Lack of expression of a gene under any of the four conditions is denoted as NA.

\begin{tabular}{|c|c|c|c|c|c|c|c|c|c|c|}
\hline \multirow[b]{2}{*}{ Gene ID } & \multirow{2}{*}{$\begin{array}{l}\text { Gene } \\
\text { name }\end{array}$} & \multirow[b]{2}{*}{ Product Name } & \multicolumn{2}{|c|}{ Condition 1} & \multicolumn{2}{|c|}{ Condition 2} & \multicolumn{2}{|c|}{ Condition 3} & \multicolumn{2}{|c|}{ Condition 4} \\
\hline & & & FC & $\begin{array}{c}\text { Corrected } \\
p \text {-value }\end{array}$ & FC & $\begin{array}{c}\text { Corrected } \\
p \text {-value }\end{array}$ & FC & $\begin{array}{c}\text { Corrected } \\
p \text {-value }\end{array}$ & FC & $\begin{array}{c}\text { Corrected } \\
p \text {-value }\end{array}$ \\
\hline \multicolumn{11}{|c|}{ Genes positively regulated by AmpR (313) } \\
\hline \multicolumn{11}{|c|}{ Adaptation, Protection } \\
\hline PA1001 & $p h n A$ & anthranilate synthase component I & NA & NA & NA & NA & -20.0 & $8.86 \mathrm{E}-11$ & -81.2 & $0.00 \mathrm{E}+00$ \\
\hline PA2385 & $p v d Q$ & $\begin{array}{l}\text { 3-oxo-C12-homoserine lactone acylase } \\
\text { PvdQ }\end{array}$ & NA & NA & NA & NA & -11.2 & $1.10 \mathrm{E}-07$ & NA & NA \\
\hline PA2386 & $p v d A$ & L-ornithine N5-oxygenase & NA & NA & NA & NA & -5.3 & $0.00 \mathrm{E}+00$ & -7.2 & $6.05 \mathrm{E}-14$ \\
\hline PA2392 & $p v d P$ & PvdP & NA & NA & NA & NA & $\begin{array}{c}- \\
102.6\end{array}$ & $0.00 \mathrm{E}+00$ & -18.5 & $0.00 \mathrm{E}+00$ \\
\hline PA2394 & $p v d N$ & PvdN & NA & NA & NA & NA & -7.8 & $1.74 \mathrm{E}-08$ & -67.1 & $5.35 \mathrm{E}-14$ \\
\hline PA2395 & $p v d O$ & PvdO & NA & NA & NA & NA & -82.9 & $0.00 \mathrm{E}+00$ & -2.9 & $7.65 \mathrm{E}-06$ \\
\hline PA2411 & & probable thioesterase & NA & NA & NA & NA & -5.2 & $9.31 \mathrm{E}-14$ & -23.4 & $0.00 \mathrm{E}+00$ \\
\hline PA2413 & $p v d H$ & $\begin{array}{l}\text { L-2,4-diaminobutyrate:2-ketoglutarate } \\
\text { 4-aminotransferase, } \mathrm{PvdH}\end{array}$ & NA & NA & NA & NA & -3.5 & $5.94 \mathrm{E}-10$ & -4.1 & $1.05 \mathrm{E}-10$ \\
\hline PA2424 & $p v d L$ & PvdL & NA & NA & NA & NA & -5.2 & $1.80 \mathrm{E}-05$ & -7.1 & $1.19 \mathrm{E}-05$ \\
\hline PA2425 & $p v d G$ & PvdG & NA & NA & NA & NA & -7.6 & $5.66 \mathrm{E}-07$ & -37.6 & $2.58 \mathrm{E}-08$ \\
\hline PA2788 & & probable chemotaxis transducer & NA & NA & NA & NA & -12.4 & $2.08 \mathrm{E}-06$ & NA & NA \\
\hline PA2850 & $o h r$ & $\begin{array}{l}\text { organic hydroperoxide resistance } \\
\text { protein }\end{array}$ & NA & NA & 30.6 & $4.59 \mathrm{E}-08$ & NA & NA & NA & NA \\
\hline PA2867 & & probable chemotaxis transducer & NA & NA & -2.6 & $6.69 \mathrm{E}-08$ & NA & NA & NA & NA \\
\hline PA3327 & & $\begin{array}{l}\text { probable non-ribosomal peptide } \\
\text { synthetase }\end{array}$ & NA & NA & NA & NA & -22.3 & $0.00 \mathrm{E}+00$ & 113.0 & $1.03 \mathrm{E}-14$ \\
\hline PA3331 & & cytochrome P450 & NA & NA & NA & NA & -15.7 & $7.89 \mathrm{E}-14$ & $\begin{array}{c}- \\
160.0\end{array}$ & $0.00 \mathrm{E}+00$ \\
\hline PA3450 & & probable antioxidant protein & NA & NA & NA & NA & -4.3 & $8.61 \mathrm{E}-07$ & NA & NA \\
\hline
\end{tabular}




\begin{tabular}{|c|c|c|c|c|c|c|c|c|c|c|}
\hline PA3475 & pheC & cyclohexadienyl dehydratase precursor & NA & NA & NA & NA & -3.8 & $1.58 \mathrm{E}-06$ & -3.6 & 7.51E-09 \\
\hline PA4236 & katA & catalase & NA & NA & NA & NA & -2.1 & $1.58 \mathrm{E}-09$ & NA & NA \\
\hline PA4468 & $\operatorname{sod} M$ & superoxide dismutase & NA & NA & NA & NA & -34.8 & $3.26 \mathrm{E}-07$ & NA & NA \\
\hline PA4566 & $o b g$ & GTP-binding protein Obg & NA & NA & -2.2 & $1.38 \mathrm{E}-11$ & NA & NA & NA & NA \\
\hline \multicolumn{11}{|c|}{ Amino acid biosynthesis and metabolism } \\
\hline PA0292 & $\operatorname{aguA}$ & agmatine deiminase & NA & NA & -6.9 & $5.28 \mathrm{E}-12$ & NA & NA & NA & NA \\
\hline PA0297 & spuA & probable glutamine amidotransferase & NA & NA & -2.2 & 2.29E-06 & NA & NA & NA & NA \\
\hline PA1002 & $\operatorname{phnB}$ & anthranilate synthase component II & NA & NA & NA & NA & -31.4 & $1.36 \mathrm{E}-06$ & NA & NA \\
\hline PA1217 & & probable 2-isopropylmalate synthase & NA & NA & NA & NA & -39.7 & $3.85 \mathrm{E}-08$ & NA & NA \\
\hline PA1757 & $t h r H$ & homoserine kinase & NA & NA & -3.6 & $1.65 \mathrm{E}-07$ & NA & NA & NA & NA \\
\hline PA3121 & leuC & $\begin{array}{l}\text { 3-isopropylmalate dehydratase large } \\
\text { subunit }\end{array}$ & NA & NA & - & $5.82 \mathrm{E}-09$ & NA & NA & NA & NA \\
\hline PA3418 & $l d h$ & leucine dehydrogenase & NA & NA & NA & NA & -5.9 & $3.35 \mathrm{E}-07$ & NA & NA \\
\hline PA3736 & hom & homoserine dehydrogenase & NA & NA & -2.2 & $2.53 \mathrm{E}-06$ & NA & NA & NA & NA \\
\hline PA5092 & hutI & $\begin{array}{l}\text { imidazolone-5-propionate hydrolase } \\
\text { HutI }\end{array}$ & NA & NA & - & $5.35 \mathrm{E}-06$ & NA & NA & NA & NA \\
\hline \multicolumn{11}{|c|}{ Antibiotic Resistance and susceptibility } \\
\hline PA5471 & & hypothetical protein & NA & NA & -3.1 & $2.96 \mathrm{E}-12$ & NA & NA & NA & NA \\
\hline \multicolumn{11}{|c|}{ Biosynthesis of cofactors, prosthetic groups and carriers } \\
\hline PA0350 & folA & dihydrofolate reductase & NA & NA & -5.1 & $2.38 \mathrm{E}-08$ & NA & NA & NA & NA \\
\hline PA0582 & folB & dihydroneopterin aldolase & NA & NA & -3.6 & $1.11 \mathrm{E}-06$ & NA & NA & NA & NA \\
\hline PA0996 & $p q s A$ & probable coenzyme A ligase & NA & NA & NA & NA & -17.3 & $3.99 \mathrm{E}-15$ & $\begin{array}{c}- \\
101.7\end{array}$ & $0.00 \mathrm{E}+00$ \\
\hline PA0997 & $p q s B$ & $\mathrm{PqsB}$ & NA & NA & NA & NA & -25.2 & $4.83 \mathrm{E}-14$ & -8.4 & $0.00 \mathrm{E}+00$ \\
\hline PA0998 & $p q s C$ & $\mathrm{PqsC}$ & NA & NA & NA & NA & -81.3 & $7.75 \mathrm{E}-14$ & -89.2 & $0.00 \mathrm{E}+00$ \\
\hline PA0999 & $p q s D$ & $\begin{array}{l}\text { 3-oxoacyl-[acyl-carrier-protein] } \\
\text { synthase III }\end{array}$ & NA & NA & NA & NA & -5.9 & $1.24 \mathrm{E}-13$ & -18.5 & $0.00 \mathrm{E}+00$ \\
\hline PA1000 & $p q s E$ & Quinolone signal response protein & NA & NA & NA & NA & -8.2 & $0.00 \mathrm{E}+00$ & 190.3 & $0.00 \mathrm{E}+00$ \\
\hline
\end{tabular}




\begin{tabular}{|c|c|c|c|c|c|c|c|c|c|c|}
\hline $\begin{array}{l}\text { PA3627 } \\
\text { PA4055 }\end{array}$ & $\begin{array}{l}y g b B \\
r i b C \\
\end{array}$ & $\begin{array}{l}\text { 2C-methyl-D-erythritol } 2,4- \\
\text { cyclodiphosphate synthase } \\
\text { riboflavin synthase alpha chain }\end{array}$ & $\begin{array}{l}\text { NA } \\
\text { NA }\end{array}$ & $\begin{array}{l}\text { NA } \\
\text { NA }\end{array}$ & $\begin{array}{l}-2.4 \\
-2.7 \\
\end{array}$ & $\begin{array}{l}3.63 \mathrm{E}-07 \\
1.46 \mathrm{E}-05 \\
\end{array}$ & $\begin{array}{l}\text { NA } \\
\text { NA }\end{array}$ & $\begin{array}{l}\text { NA } \\
\text { NA }\end{array}$ & $\begin{array}{l}\text { NA } \\
\text { NA }\end{array}$ & $\begin{array}{l}\text { NA } \\
\text { NA }\end{array}$ \\
\hline \multicolumn{11}{|c|}{ Carbon compound catabolism } \\
\hline PA0211 & $m d c D$ & malonate decarboxylase beta subunit & NA & NA & 20.3 & $1.18 \mathrm{E}-05$ & NA & NA & NA & NA \\
\hline PA0810 & & probable haloacid dehalogenase & NA & NA & 25.0 & $9.50 \mathrm{E}-07$ & NA & NA & NA & NA \\
\hline PA2300 & chiC & chitinase & NA & NA & NA & NA & $\begin{array}{c}- \\
232.8\end{array}$ & $0.00 \mathrm{E}+00$ & -14.9 & $0.00 \mathrm{E}+00$ \\
\hline PA2507 & catA & catechol 1,2-dioxygenase & NA & NA & -8.0 & $5.38 \mathrm{E}-09$ & NA & NA & NA & NA \\
\hline PA2514 & antC & anthranilate dioxygenase reductase & NA & NA & -8.7 & $3.03 \mathrm{E}-09$ & -2.1 & $1.90 \mathrm{E}-05$ & -31.2 & $0.00 \mathrm{E}+00$ \\
\hline PA5418 & $\operatorname{sox} A$ & sarcosine oxidase alpha subunit & NA & NA & $\begin{array}{r}- \\
15.9\end{array}$ & $5.01 \mathrm{E}-07$ & NA & NA & NA & NA \\
\hline \multicolumn{11}{|c|}{ Cell wall / LPS / capsule ; Motility \& Attachment } \\
\hline PA1078 & $f \lg C$ & flagellar basal-body rod protein $\mathrm{FlgC}$ & NA & NA & -2.9 & $1.24 \mathrm{E}-06$ & NA & NA & NA & NA \\
\hline PA1094 & fliD & flagellar capping protein FliD & NA & NA & -2.0 & $2.20 \mathrm{E}-08$ & NA & NA & NA & NA \\
\hline \multicolumn{11}{|c|}{ Central intermediary metabolism } \\
\hline PA0654 & speD & $\begin{array}{l}\text { S-adenosylmethionine decarboxylase } \\
\text { proenzyme }\end{array}$ & NA & NA & -2.9 & $4.36 \mathrm{E}-12$ & NA & NA & NA & NA \\
\hline PA2193 & $h c n A$ & hydrogen cyanide synthase HcnA & NA & NA & NA & NA & $\begin{array}{c}- \\
150.1\end{array}$ & $6.88 \mathrm{E}-14$ & -9.7 & $0.00 \mathrm{E}+00$ \\
\hline PA2194 & $h c n B$ & hydrogen cyanide synthase HcnB & NA & NA & NA & NA & -4.5 & $7.21 \mathrm{E}-10$ & -5.0 & $5.09 \mathrm{E}-14$ \\
\hline PA2195 & henC & hydrogen cyanide synthase $\mathrm{HcnC}$ & NA & NA & NA & NA & -3.9 & $5.31 \mathrm{E}-10$ & -12.2 & $0.00 \mathrm{E}+00$ \\
\hline PA2393 & & probable dipeptidase precursor & NA & NA & NA & NA & -17.1 & $1.15 \mathrm{E}-10$ & -64.0 & $2.08 \mathrm{E}-13$ \\
\hline PA3629 & $a d h C$ & alcohol dehydrogenase class III & NA & NA & -2.3 & $2.79 \mathrm{E}-07$ & NA & NA & NA & NA \\
\hline PA4130 & & probable sulfite or nitrite reductase & NA & NA & NA & NA & -12.0 & $0.00 \mathrm{E}+00$ & -32.1 & $0.00 \mathrm{E}+00$ \\
\hline PA5058 & $\begin{array}{c}\text { phaC } \\
2\end{array}$ & $\begin{array}{l}\text { poly(3-hydroxyalkanoic acid) synthase } \\
2\end{array}$ & NA & NA & NA & NA & -6.9 & $1.10 \mathrm{E}-05$ & -25.6 & $5.40 \mathrm{E}-06$ \\
\hline PA5421 & $f d h A$ & $\begin{array}{l}\text { glutathione-independent formaldehyde } \\
\text { dehydrogenase }\end{array}$ & NA & NA & -4.7 & $1.87 \mathrm{E}-06$ & NA & NA & NA & NA \\
\hline
\end{tabular}




\begin{tabular}{|c|c|c|c|c|c|c|c|c|c|c|}
\hline \multicolumn{11}{|c|}{ Chemotaxis } \\
\hline PA0415 & $\operatorname{chpC}$ & probable chemotaxis protein & NA & NA & -2.4 & $1.26 \mathrm{E}-05$ & NA & NA & NA & NA \\
\hline \multicolumn{11}{|c|}{ DNA replication, recombination, modification and repair } \\
\hline PA3867 & & probable DNA invertase & NA & NA & 22.8 & $3.04 \mathrm{E}-06$ & NA & NA & NA & NA \\
\hline \multicolumn{11}{|c|}{ Energy metabolism } \\
\hline PA1175 & $n a p D$ & $\begin{array}{l}\text { NapD protein of periplasmic nitrate } \\
\text { reductase }\end{array}$ & NA & NA & NA & NA & -3.8 & $1.75 \mathrm{E}-05$ & -43.5 & $1.74 \mathrm{E}-09$ \\
\hline PA1320 & $c y o D$ & $\begin{array}{l}\text { cytochrome o ubiquinol oxidase subunit } \\
\text { IV }\end{array}$ & NA & NA & NA & NA & -35.2 & $2.73 \mathrm{E}-07$ & NA & NA \\
\hline PA3930 & $\operatorname{cioA}$ & cyanide insensitive terminal oxidase & NA & NA & NA & NA & -4.0 & $5.69 \mathrm{E}-10$ & -9.7 & $0.00 \mathrm{E}+00$ \\
\hline PA4133 & & $\begin{array}{l}\text { cytochrome c oxidase subunit (cbb3- } \\
\text { type) }\end{array}$ & NA & NA & NA & NA & -24.3 & $5.31 \mathrm{E}-14$ & -38.0 & $0.00 \mathrm{E}+00$ \\
\hline PA5490 & $c c 4$ & cytochrome c4 precursor & NA & NA & -2.1 & $0.00 \mathrm{E}+00$ & NA & NA & NA & NA \\
\hline \multicolumn{11}{|c|}{ Fatty acid and phospholipid metabolism } \\
\hline PA3333 & fabH2 & $\begin{array}{l}\text { 3-oxoacyl-[acyl-carrier-protein] } \\
\text { synthase III }\end{array}$ & NA & NA & NA & NA & $\begin{array}{c}- \\
147.6\end{array}$ & $3.15 \mathrm{E}-14$ & -37.0 & $0.00 \mathrm{E}+00$ \\
\hline PA3334 & & probable acyl carrier protein & 2.8 & $0.00 \mathrm{E}+00$ & NA & NA & $\begin{array}{c}- \\
118.2\end{array}$ & $0.00 \mathrm{E}+00$ & $\begin{array}{c}- \\
359.1\end{array}$ & $1.64 \mathrm{E}-13$ \\
\hline PA4050 & pgpA & phosphatidylglycerophosphatase A & NA & NA & -3.6 & $7.94 \mathrm{E}-09$ & NA & NA & NA & NA \\
\hline PA4351 & & probable acyltransferase & NA & NA & NA & NA & -30.4 & $2.03 \mathrm{E}-06$ & -27.7 & $2.09 \mathrm{E}-06$ \\
\hline \multicolumn{11}{|c|}{ Hypothetical, unclassified, unknown } \\
\hline PA0050 & & hypothetical protein & NA & NA & NA & NA & -4.5 & $0.00 \mathrm{E}+00$ & -4.4 & $8.02 \mathrm{E}-15$ \\
\hline PA0057 & & hypothetical protein & NA & NA & - & $1.11 \mathrm{E}-06$ & NA & NA & NA & NA \\
\hline PA0066 & & conserved hypothetical protein & NA & NA & -5.1 & $7.49 \mathrm{E}-08$ & NA & NA & NA & NA \\
\hline PA0122 & & conserved hypothetical protein & NA & NA & NA & NA & -14.7 & $1.07 \mathrm{E}-13$ & -42.9 & $1.48 \mathrm{E}-13$ \\
\hline PA0128 & & conserved hypothetical protein & NA & NA & -5.1 & $5.15 \mathrm{E}-12$ & NA & NA & NA & NA \\
\hline PA0160 & & hypothetical protein & NA & NA & $\begin{array}{c}- \\
47.1\end{array}$ & $5.36 \mathrm{E}-12$ & -2.4 & $1.52 \mathrm{E}-06$ & -77.2 & $5.35 \mathrm{E}-14$ \\
\hline PA0200 & & hypothetical protein & NA & NA & NA & NA & -4.0 & $0.00 \mathrm{E}+00$ & -9.7 & $0.00 \mathrm{E}+00$ \\
\hline
\end{tabular}




\begin{tabular}{|c|c|c|c|c|c|c|c|c|c|}
\hline PA0264 & hypothetical protein & NA & NA & $-\overline{10}$ & 7.91E-06 & NA & NA & NA & NA \\
\hline PA0284 & hypothetical protein & NA & NA & NA & NA & -64.6 & $8.56 \mathrm{E}-13$ & -39.2 & $1.19 \mathrm{E}-08$ \\
\hline PA0320 & conserved hypothetical protein & NA & NA & $3 \overline{7} .4$ & $1.11 \mathrm{E}-09$ & NA & NA & NA & NA \\
\hline PA0387 & conserved hypothetical protein & NA & NA & -2.7 & 4.49E-06 & NA & NA & NA & NA \\
\hline PA0433 & hypothetical protein & NA & NA & 20.1 & $1.26 \mathrm{E}-05$ & NA & NA & NA & NA \\
\hline PA0569 & hypothetical protein & NA & NA & NA & NA & -25.1 & $1.87 \mathrm{E}-05$ & NA & NA \\
\hline PA0570 & hypothetical protein & NA & NA & 25.2 & $8.47 \mathrm{E}-07$ & NA & NA & NA & NA \\
\hline PA0587 & conserved hypothetical protein & NA & NA & NA & NA & -4.9 & $1.59 \mathrm{E}-06$ & NA & NA \\
\hline PA0591 & conserved hypothetical protein & NA & NA & -3.6 & $2.87 \mathrm{E}-06$ & NA & NA & NA & NA \\
\hline PA0614 & hypothetical protein & NA & NA & -3.1 & $1.53 \mathrm{E}-13$ & NA & NA & NA & NA \\
\hline PA0671 & hypothetical protein & NA & NA & -3.3 & 7.38E-07 & NA & NA & NA & NA \\
\hline PA0719 & $\begin{array}{l}\text { hypothetical protein of bacteriophage } \\
\text { Pf1 }\end{array}$ & NA & NA & 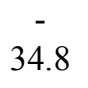 & 4.83E-09 & NA & NA & NA & NA \\
\hline PA0774 & conserved hypothetical protein & NA & NA & -4.0 & $5.47 \mathrm{E}-06$ & NA & NA & NA & NA \\
\hline PA0805 & hypothetical protein & NA & NA & -2.7 & $3.70 \mathrm{E}-13$ & NA & NA & NA & NA \\
\hline PA0812 & hypothetical protein & NA & NA & -8.7 & $1.81 \mathrm{E}-07$ & NA & NA & NA & NA \\
\hline PA0814 & conserved hypothetical protein & NA & NA & 30.2 & $5.78 \mathrm{E}-08$ & NA & NA & NA & NA \\
\hline PA0826 & hypothetical protein & NA & NA & $2 \overline{-} .4$ & 2.61E-07 & NA & NA & NA & NA \\
\hline PA0910 & hypothetical protein & NA & NA & -2.4 & $1.50 \mathrm{E}-14$ & NA & NA & NA & NA \\
\hline PA0911 & hypothetical protein & NA & NA & -3.1 & $6.56 \mathrm{E}-14$ & NA & NA & NA & NA \\
\hline PA0983 & conserved hypothetical protein & NA & NA & NA & NA & -73.9 & $2.82 \mathrm{E}-14$ & -49.9 & $9.97 \mathrm{E}-11$ \\
\hline PA1134 & hypothetical protein & NA & NA & 25.2 & 8.47E-07 & NA & NA & NA & NA \\
\hline PA1216 & hypothetical protein & NA & NA & NA & NA & -38.0 & $8.26 \mathrm{E}-08$ & -76.9 & $1.03 \mathrm{E}-14$ \\
\hline PA1299 & conserved hypothetical protein & NA & NA & -4.3 & $2.58 \mathrm{E}-07$ & NA & NA & NA & NA \\
\hline PA1371 & hypothetical protein & NA & NA & NA & NA & -2.6 & $1.79 \mathrm{E}-05$ & NA & NA \\
\hline
\end{tabular}




\begin{tabular}{|c|c|c|c|c|c|c|c|c|c|}
\hline PA1377 & conserved hypothetical protein & NA & NA & -2.9 & $1.22 \mathrm{E}-05$ & NA & NA & NA & NA \\
\hline PA1378 & hypothetical protein & NA & NA & NA & NA & -26.0 & $1.28 \mathrm{E}-05$ & NA & NA \\
\hline PA1533 & conserved hypothetical protein & NA & NA & NA & NA & -2.0 & $2.85 \mathrm{E}-07$ & NA & NA \\
\hline PA1539 & hypothetical protein & NA & NA & $\overline{25.8}$ & $6.10 \mathrm{E}-07$ & NA & NA & NA & NA \\
\hline PA1656 & hypothetical protein & NA & NA & NA & NA & -5.9 & 2.94E-09 & NA & NA \\
\hline PA1657 & conserved hypothetical protein & 2.7 & $2.58 \mathrm{E}-13$ & NA & NA & -79.2 & $1.86 \mathrm{E}-14$ & 226.7 & $0.00 \mathrm{E}+00$ \\
\hline PA1664 & hypothetical protein & NA & NA & NA & NA & -33.5 & $5.43 \mathrm{E}-07$ & -50.9 & $6.21 \mathrm{E}-11$ \\
\hline PA1888 & hypothetical protein & NA & NA & NA & NA & -28.5 & 4.59E-06 & NA & NA \\
\hline PA2031 & hypothetical protein & NA & NA & NA & NA & -27.8 & $6.11 \mathrm{E}-06$ & -28.2 & $1.73 \mathrm{E}-06$ \\
\hline PA2033 & hypothetical protein & NA & NA & NA & NA & -26.0 & $1.30 \mathrm{E}-05$ & NA & NA \\
\hline PA2066 & hypothetical protein & NA & NA & NA & NA & -40.7 & $2.57 \mathrm{E}-08$ & -33.7 & $1.43 \mathrm{E}-07$ \\
\hline PA2126 & conserved hypothetical protein & NA & NA & $\begin{array}{c}- \\
27.5\end{array}$ & $2.44 \mathrm{E}-07$ & NA & NA & NA & NA \\
\hline PA2274 & hypothetical protein & NA & NA & NA & NA & -37.5 & $1.00 \mathrm{E}-07$ & NA & NA \\
\hline PA2330 & hypothetical protein & NA & NA & NA & NA & -31.0 & $1.59 \mathrm{E}-06$ & NA & NA \\
\hline PA2362 & hypothetical protein & NA & NA & NA & NA & -34.3 & 3.99E-07 & -3.4 & 4.54E-06 \\
\hline PA2363 & hypothetical protein & NA & NA & NA & NA & -9.5 & $1.30 \mathrm{E}-05$ & -6.8 & $1.01 \mathrm{E}-05$ \\
\hline PA2366 & uricase PuuD & NA & NA & NA & NA & -55.7 & 3.99E-11 & -8.8 & $5.10 \mathrm{E}-07$ \\
\hline PA2367 & hypothetical protein & NA & NA & NA & NA & -8.1 & $3.77 \mathrm{E}-11$ & -57.7 & $2.93 \mathrm{E}-12$ \\
\hline PA2380 & hypothetical protein & NA & NA & 37.0 & $1.43 \mathrm{E}-09$ & NA & NA & NA & NA \\
\hline PA2412 & conserved hypothetical protein & NA & NA & NA & NA & -13.3 & $1.86 \mathrm{E}-14$ & -10.7 & $0.00 \mathrm{E}+00$ \\
\hline PA2427 & hypothetical protein & NA & NA & NA & NA & -55.5 & 4.23E-11 & NA & NA \\
\hline PA2605 & conserved hypothetical protein & NA & NA & -3.6 & 4.52E-06 & NA & NA & NA & NA \\
\hline PA2627 & conserved hypothetical protein & NA & NA & 28.2 & $1.70 \mathrm{E}-07$ & NA & NA & NA & NA \\
\hline PA2661 & hypothetical protein & NA & NA & 39.2 & $4.15 \mathrm{E}-10$ & NA & NA & NA & NA \\
\hline PA2669 & hypothetical protein & NA & NA & - & 3.67E-06 & NA & NA & NA & NA \\
\hline
\end{tabular}




\begin{tabular}{|c|c|}
\hline PA2679 & hypothetical protein \\
\hline PA2747 & hypothetical protein \\
\hline PA2781 & hypothetical protein \\
\hline PA2799 & hypothetical protein \\
\hline PA2941 & hypothetical protein \\
\hline PA2955 & hypothetical protein \\
\hline PA2980 & conserved hypothetical protein \\
\hline PA3008 & hypothetical protein \\
\hline PA3069 & hypothetical protein \\
\hline PA3088 & conserved hypothetical protein \\
\hline PA3089 & hypothetical protein \\
\hline PA3127 & hypothetical protein \\
\hline PA3199 & conserved hypothetical protein \\
\hline PA3209 & conserved hypothetical protein \\
\hline PA3273 & hypothetical protein \\
\hline PA3289 & hypothetical protein \\
\hline PA3329 & hypothetical protein \\
\hline PA3332 & conserved hypothetical protein \\
\hline PA3386 & conserved hypothetical protein \\
\hline PA3612 & conserved hypothetical protein \\
\hline PA3616 & conserved hypothetical protein \\
\hline PA3688 & hypothetical protein \\
\hline
\end{tabular}

\begin{tabular}{|c|c|c|c|c|c|c|c|}
\hline NA & NA & $\begin{array}{c}22.5 \\
- \\
23.3\end{array}$ & $2.38 \mathrm{E}-06$ & NA & NA & NA & NA \\
\hline NA & NA & NA & NA & -4.8 & 8.82E-09 & NA & NA \\
\hline NA & NA & $\begin{array}{c}- \\
25.6\end{array}$ & $6.75 \mathrm{E}-07$ & NA & NA & NA & NA \\
\hline NA & NA & NA & NA & -47.3 & $1.51 \mathrm{E}-09$ & -23.9 & $1.12 \mathrm{E}-05$ \\
\hline NA & NA & 20.4 & $1.13 \mathrm{E}-05$ & NA & NA & NA & NA \\
\hline NA & NA & -3.3 & 4.24E-06 & NA & NA & NA & NA \\
\hline NA & NA & -2.9 & $1.11 \mathrm{E}-07$ & NA & NA & NA & NA \\
\hline NA & NA & -2.3 & $1.13 \mathrm{E}-10$ & NA & NA & NA & NA \\
\hline NA & NA & NA & NA & -42.1 & $1.40 \mathrm{E}-08$ & -58.1 & $2.42 \mathrm{E}-12$ \\
\hline NA & NA & -3.6 & $1.62 \mathrm{E}-05$ & NA & NA & NA & NA \\
\hline NA & NA & NA & NA & -31.8 & $1.12 \mathrm{E}-06$ & NA & NA \\
\hline NA & NA & $\begin{array}{c}- \\
21.8\end{array}$ & $5.20 \mathrm{E}-06$ & NA & NA & NA & NA \\
\hline NA & NA & -4.3 & $1.68 \mathrm{E}-05$ & NA & NA & NA & NA \\
\hline NA & NA & 23.5 & $2.04 \mathrm{E}-06$ & NA & NA & NA & NA \\
\hline NA & NA & 21.9 & $4.91 \mathrm{E}-06$ & NA & NA & NA & NA \\
\hline NA & NA & NA & NA & -28.5 & $4.50 \mathrm{E}-06$ & NA & NA \\
\hline NA & NA & NA & NA & -14.7 & $0.00 \mathrm{E}+00$ & $\begin{array}{c}- \\
151.3\end{array}$ & $0.00 \mathrm{E}+00$ \\
\hline NA & NA & 30.8 & $4.11 \mathrm{E}-08$ & -6.8 & $0.00 \mathrm{E}+00$ & $\begin{array}{c}- \\
320.3\end{array}$ & $1.40 \mathrm{E}-13$ \\
\hline NA & NA & 23.9 & $1.66 \mathrm{E}-06$ & NA & NA & NA & NA \\
\hline NA & NA & NA & NA & -63.9 & $1.13 \mathrm{E}-12$ & -32.4 & $2.64 \mathrm{E}-07$ \\
\hline NA & NA & -3.1 & $1.13 \mathrm{E}-13$ & NA & NA & NA & NA \\
\hline NA & NA & NA & NA & -4.8 & $1.89 \mathrm{E}-07$ & - & $6.60 \mathrm{E}-14$ \\
\hline
\end{tabular}




\begin{tabular}{|c|c|c|c|c|c|c|c|c|c|c|}
\hline & & & & & & & & & 104.1 & \\
\hline PA3707 & $w s p B$ & hypothetical protein & NA & NA & -4.3 & $1.66 \mathrm{E}-06$ & NA & NA & NA & NA \\
\hline PA3784 & & hypothetical protein & NA & NA & NA & NA & -4.4 & $1.62 \mathrm{E}-11$ & -8.8 & $5.04 \mathrm{E}-14$ \\
\hline PA3785 & & conserved hypothetical protein & NA & NA & NA & NA & -3.0 & $7.44 \mathrm{E}-13$ & -3.6 & $3.86 \mathrm{E}-14$ \\
\hline PA3786 & & hypothetical protein & NA & NA & NA & NA & -2.1 & $1.56 \mathrm{E}-05$ & -2.9 & $4.52 \mathrm{E}-08$ \\
\hline PA3931 & & conserved hypothetical protein & NA & NA & NA & NA & -6.2 & $9.11 \mathrm{E}-10$ & -3.9 & $2.09 \mathrm{E}-07$ \\
\hline PA4018 & & hypothetical protein & NA & NA & 31.7 & $2.51 \mathrm{E}-08$ & NA & NA & NA & NA \\
\hline PA4129 & & hypothetical protein & NA & NA & $\overline{4} .8$ & $1.92 \mathrm{E}-11$ & -7.2 & $0.00 \mathrm{E}+00$ & $\begin{array}{c}- \\
440.6\end{array}$ & $1.03 \mathrm{E}-14$ \\
\hline PA4132 & & conserved hypothetical protein & NA & NA & NA & NA & -4.8 & $0.00 \mathrm{E}+00$ & -10.5 & $0.00 \mathrm{E}+00$ \\
\hline PA4134 & & hypothetical protein & NA & NA & NA & NA & 320.9 & $0.00 \mathrm{E}+00$ & 236.5 & $0.00 \mathrm{E}+00$ \\
\hline PA4220 & & hypothetical protein & NA & NA & $\mathrm{NA}$ & NA & -41.9 & $1.51 \mathrm{E}-08$ & -76.4 & $0.00 \mathrm{E}+00$ \\
\hline PA4294 & & hypothetical protein & NA & NA & $\mathrm{NA}$ & NA & -37.3 & $1.10 \mathrm{E}-07$ & -28.3 & $1.61 \mathrm{E}-06$ \\
\hline PA4336 & & conserved hypothetical protein & NA & NA & 29.9 & $6.69 \mathrm{E}-08$ & NA & NA & NA & NA \\
\hline PA4471 & & hypothetical protein & NA & NA & 22.1 & 4.37E-06 & NA & NA & NA & NA \\
\hline PA4536 & & hypothetical protein & 2.8 & $1.34 \mathrm{E}-05$ & NA & NA & -26.3 & $1.16 \mathrm{E}-05$ & -4.9 & $2.99 \mathrm{E}-09$ \\
\hline PA4607 & & hypothetical protein & NA & NA & $\mathrm{NA}$ & NA & -2.7 & $0.00 \mathrm{E}+00$ & -2.3 & $0.00 \mathrm{E}+00$ \\
\hline PA4634 & & hypothetical protein & NA & NA & 28.5 & $1.47 \mathrm{E}-07$ & NA & NA & NA & NA \\
\hline PA4702 & & hypothetical protein & NA & NA & NA & NA & -5.7 & $1.10 \mathrm{E}-09$ & NA & NA \\
\hline PA4800 & & hypothetical protein & NA & NA & $\overline{2} 1.9$ & $4.98 \mathrm{E}-06$ & NA & NA & NA & NA \\
\hline PA5139 & & hypothetical protein & NA & NA & $\begin{array}{r}- \\
41.0\end{array}$ & $1.49 \mathrm{E}-10$ & NA & NA & NA & NA \\
\hline PA5220 & & hypothetical protein & NA & NA & $\mathrm{NA}$ & NA & -10.5 & 7.39E-14 & -34.1 & $0.00 \mathrm{E}+00$ \\
\hline PA5275 & & conserved hypothetical protein & NA & NA & -3.6 & $5.51 \mathrm{E}-07$ & NA & NA & NA & NA \\
\hline PA5294 & & hypothetical protein & NA & NA & 29.9 & 7.04E-08 & NA & NA & NA & NA \\
\hline
\end{tabular}




\begin{tabular}{|c|c|c|c|c|c|c|c|c|c|c|}
\hline PA5343 & & hypothetical protein & NA & NA & -3.1 & $3.10 \mathrm{E}-06$ & NA & NA & NA & NA \\
\hline PA5359 & & hypothetical protein & NA & NA & NA & NA & -2.5 & $2.51 \mathrm{E}-10$ & -3.6 & $4.81 \mathrm{E}-14$ \\
\hline PA5381 & & hypothetical protein & NA & NA & -4.3 & $1.51 \mathrm{E}-08$ & NA & NA & NA & NA \\
\hline PA5414 & & hypothetical protein & NA & NA & -2.5 & $1.46 \mathrm{E}-06$ & NA & NA & NA & NA \\
\hline PA5543 & & hypothetical protein & NA & NA & 22.0 & 4.78E-06 & NA & NA & NA & NA \\
\hline \multicolumn{11}{|c|}{ Membrane proteins } \\
\hline PA1540 & & conserved hypothetical protein & NA & NA & $\overline{-}$ & 4.12E-07 & NA & NA & NA & NA \\
\hline PA2068 & & $\begin{array}{l}\text { probable major facilitator superfamily } \\
\text { (MFS) transporter }\end{array}$ & NA & NA & NA & NA & -87.3 & $4.55 \mathrm{E}-14$ & $\begin{array}{c}- \\
104.9\end{array}$ & $0.00 \mathrm{E}+00$ \\
\hline PA2331 & & hypothetical protein & NA & NA & NA & NA & -5.2 & $1.33 \mathrm{E}-05$ & -51.2 & $5.50 \mathrm{E}-11$ \\
\hline PA2568 & & hypothetical protein & NA & NA & 42.9 & $5.28 \mathrm{E}-11$ & NA & NA & NA & NA \\
\hline PA2653 & & probable transporter & NA & NA & $\overline{-}$ & $1.49 \mathrm{E}-05$ & NA & NA & NA & NA \\
\hline PA3234 & & probable sodium:solute symporter & NA & NA & -2.7 & $6.89 \mathrm{E}-07$ & NA & NA & NA & NA \\
\hline PA3275 & & conserved hypothetical protein & NA & NA & NA & NA & -43.0 & $9.54 \mathrm{E}-09$ & NA & NA \\
\hline PA3281 & & hypothetical protein & NA & NA & 22.2 & $4.12 \mathrm{E}-06$ & NA & NA & NA & NA \\
\hline PA3336 & & $\begin{array}{l}\text { probable major facilitator superfamily } \\
\text { (MFS) transporter }\end{array}$ & NA & NA & NA & NA & -7.0 & $1.47 \mathrm{E}-11$ & -16.6 & $5.35 \mathrm{E}-14$ \\
\hline PA3608 & $\operatorname{pot} B$ & polyamine transport protein PotB & NA & NA & $\overline{-}$ & $1.68 \mathrm{E}-05$ & NA & NA & NA & NA \\
\hline PA3788 & & hypothetical protein & NA & NA & NA & NA & -59.7 & $7.14 \mathrm{E}-12$ & -5.8 & $1.56 \mathrm{E}-13$ \\
\hline PA3794 & & hypothetical protein & NA & NA & 38.4 & $6.39 \mathrm{E}-10$ & NA & NA & NA & NA \\
\hline PA4205 & & hypothetical protein & NA & NA & NA & NA & 269.6 & $0.00 \mathrm{E}+00$ & $\begin{array}{c}- \\
257.1\end{array}$ & $0.00 \mathrm{E}+00$ \\
\hline PA4207 & $\operatorname{mexI}$ & $\begin{array}{l}\text { probable Resistance-Nodulation-Cell } \\
\text { Division (RND) efflux transporter }\end{array}$ & NA & NA & NA & NA & -11.5 & $0.00 \mathrm{E}+00$ & -10.2 & $5.04 \mathrm{E}-14$ \\
\hline PA4208 & opmD & $\begin{array}{l}\text { probable outer membrane protein } \\
\text { precursor }\end{array}$ & NA & NA & NA & NA & -10.1 & $6.22 \mathrm{E}-14$ & -33.1 & $0.00 \mathrm{E}+00$ \\
\hline
\end{tabular}




\begin{tabular}{|c|c|c|c|c|c|c|c|c|c|c|}
\hline PA4218 & ampP & AmpP & NA & NA & NA & NA & -81.6 & $0.00 \mathrm{E}+00$ & -12.7 & $1.46 \mathrm{E}-12$ \\
\hline PA4219 & & hypothetical protein & NA & NA & NA & NA & -49.9 & $5.03 \mathrm{E}-10$ & -42.4 & $2.86 \mathrm{E}-09$ \\
\hline PA4223 & & $\begin{array}{l}\text { probable ATP-binding component of } \\
\text { ABC transporter }\end{array}$ & NA & NA & NA & NA & -29.5 & $0.00 \mathrm{E}+00$ & -20.5 & $5.04 \mathrm{E}-14$ \\
\hline PA4317 & & hypothetical protein & NA & NA & -2.0 & $4.43 \mathrm{E}-08$ & NA & NA & NA & NA \\
\hline PA5097 & & probable amino acid permease & NA & NA & -8.0 & $2.80 \mathrm{E}-06$ & NA & NA & NA & NA \\
\hline PA5099 & & probable transporter & NA & NA & $\overline{2} 1.2$ & $6.98 \mathrm{E}-06$ & NA & NA & NA & NA \\
\hline PA5211 & & conserved hypothetical protein & NA & NA & NA & NA & -31.3 & $1.39 \mathrm{E}-06$ & NA & NA \\
\hline PA5219 & & hypothetical protein & NA & NA & NA & NA & -9.5 & $3.72 \mathrm{E}-06$ & -36.1 & $4.92 \mathrm{E}-08$ \\
\hline PA5469 & & conserved hypothetical protein & NA & NA & 22.9 & $2.90 \mathrm{E}-06$ & NA & NA & NA & NA \\
\hline \multicolumn{11}{|c|}{ Motility \& Attachment } \\
\hline PA4306 & $f l p$ & Type IVb pilin, Flp & NA & NA & $\begin{array}{c}- \\
60.0 \\
\end{array}$ & $0.00 \mathrm{E}+00$ & -5.2 & $0.00 \mathrm{E}+00$ & $\begin{array}{c}- \\
524.7 \\
\end{array}$ & $1.92 \mathrm{E}-13$ \\
\hline \multicolumn{11}{|c|}{ Non-coding RNA gene } \\
\hline $\begin{array}{l}\text { PA1030. } \\
1\end{array}$ & $P 8$ & P8 & 2.8 & $2.46 \mathrm{E}-06$ & NA & NA & -30.3 & $2.14 \mathrm{E}-06$ & -92.1 & $1.03 \mathrm{E}-14$ \\
\hline $\begin{array}{c}\text { PA2958. } \\
1\end{array}$ & $\operatorname{rgs} A$ & $\operatorname{RgsA}$ & NA & NA & -2.9 & $9.71 \mathrm{E}-14$ & -2.0 & $5.61 \mathrm{E}-13$ & -3.4 & $0.00 \mathrm{E}+00$ \\
\hline $\begin{array}{c}\text { PA4277. } \\
3\end{array}$ & & tRNA-Tyr & NA & NA & -2.0 & $0.00 \mathrm{E}+00$ & -5.5 & $0.00 \mathrm{E}+00$ & -8.4 & $0.00 \mathrm{E}+00$ \\
\hline $\begin{array}{l}\text { PA4581. } \\
\quad 1\end{array}$ & & tRNA-Arg & NA & NA & -3.1 & $3.62 \mathrm{E}-13$ & NA & NA & NA & NA \\
\hline $\begin{array}{c}\text { PA4704. } \\
\quad 1 \\
\end{array}$ & $\operatorname{prrF1}$ & regulatory RNA PrrF1 & NA & NA & NA & NA & -3.9 & $0.00 \mathrm{E}+00$ & NA & NA \\
\hline \multicolumn{11}{|c|}{ Protein secretion/export apparatus } \\
\hline PA1715 & $\operatorname{psc} B$ & type III export apparatus protein & NA & NA & 20.7 & $9.31 \mathrm{E}-06$ & NA & NA & NA & NA \\
\hline PA1719 & $p s c F$ & type III export protein PscF & NA & NA & NA & NA & -27.5 & $7.00 \mathrm{E}-06$ & -27.8 & $1.99 \mathrm{E}-06$ \\
\hline PA2674 & & $\begin{array}{l}\text { probable type II secretion system } \\
\text { protein }\end{array}$ & NA & NA & 32.0 & $2.20 \mathrm{E}-08$ & NA & NA & NA & NA \\
\hline
\end{tabular}




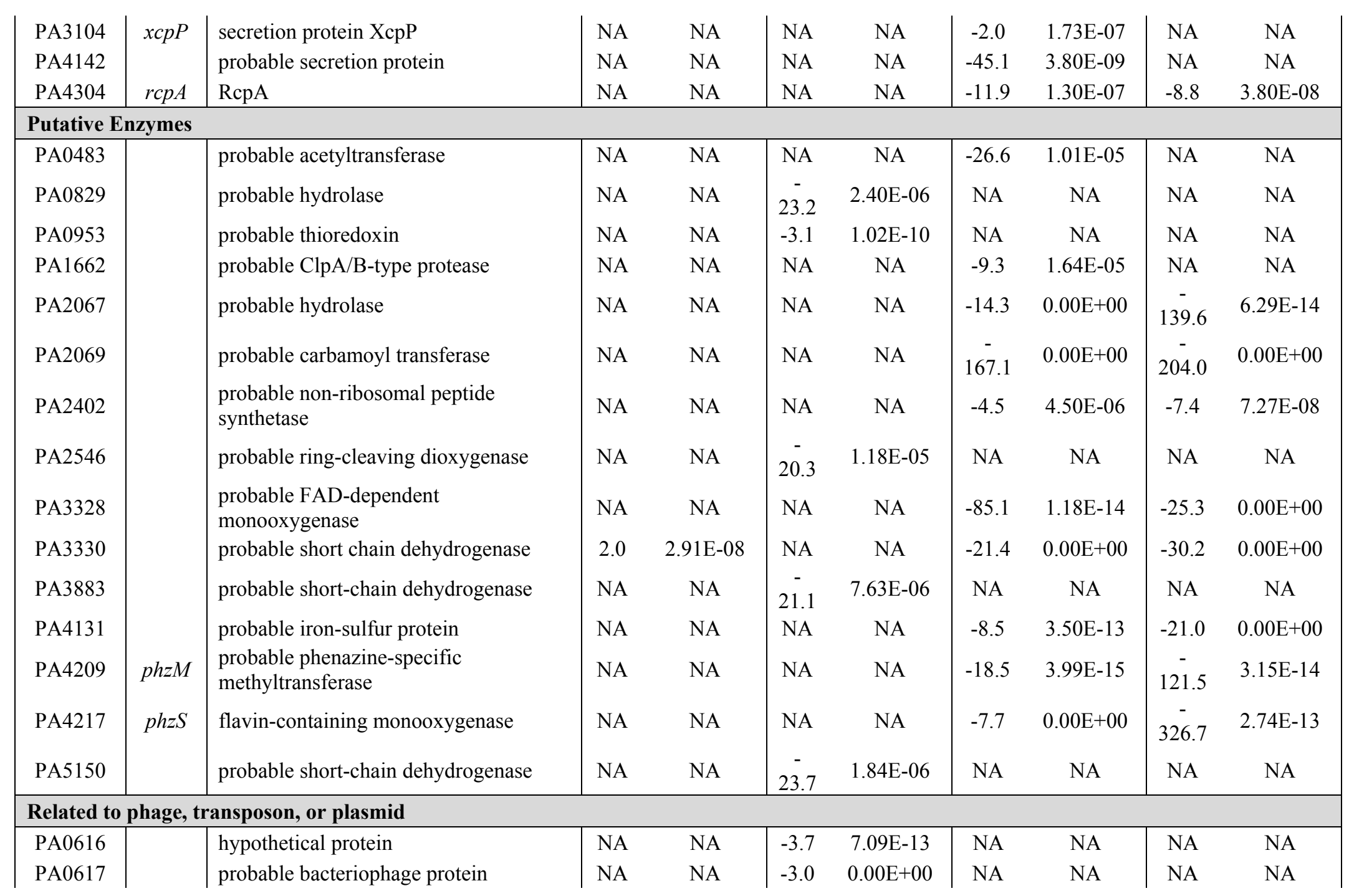




\begin{tabular}{|c|c|c|c|c|c|c|c|c|c|c|}
\hline PA0618 & & probable bacteriophage protein & NA & NA & -4.1 & $0.00 \mathrm{E}+00$ & NA & NA & NA & NA \\
\hline PA0619 & & probable bacteriophage protein & NA & NA & -4.0 & $0.00 \mathrm{E}+00$ & NA & NA & NA & NA \\
\hline PA0620 & & probable bacteriophage protein & NA & NA & -3.6 & $0.00 \mathrm{E}+00$ & NA & NA & NA & NA \\
\hline PA0622 & & probable bacteriophage protein & NA & NA & -3.5 & $0.00 \mathrm{E}+00$ & NA & NA & NA & NA \\
\hline PA0623 & & probable bacteriophage protein & NA & NA & -3.2 & $0.00 \mathrm{E}+00$ & NA & NA & NA & NA \\
\hline PA0624 & & hypothetical protein & NA & NA & -3.4 & $0.00 \mathrm{E}+00$ & NA & NA & NA & NA \\
\hline PA0625 & & hypothetical protein & NA & NA & -3.1 & $0.00 \mathrm{E}+00$ & NA & NA & NA & NA \\
\hline PA0626 & & hypothetical protein & NA & NA & -3.2 & $0.00 \mathrm{E}+00$ & NA & NA & NA & NA \\
\hline PA0627 & & conserved hypothetical protein & NA & NA & -3.3 & $6.47 \mathrm{E}-13$ & NA & NA & NA & NA \\
\hline PA0628 & & conserved hypothetical protein & NA & NA & -2.7 & $5.82 \mathrm{E}-13$ & NA & NA & NA & NA \\
\hline PA0629 & & conserved hypothetical protein & NA & NA & -3.5 & $0.00 \mathrm{E}+00$ & NA & NA & NA & NA \\
\hline PA0630 & & hypothetical protein & NA & NA & -3.0 & $3.78 \mathrm{E}-13$ & NA & NA & NA & NA \\
\hline PA0631 & & hypothetical protein & NA & NA & -3.0 & $2.27 \mathrm{E}-13$ & NA & NA & NA & NA \\
\hline PA0633 & & hypothetical protein & NA & NA & -3.9 & $0.00 \mathrm{E}+00$ & NA & NA & NA & NA \\
\hline PA0634 & & hypothetical protein & NA & NA & -3.8 & $0.00 \mathrm{E}+00$ & NA & NA & NA & NA \\
\hline PA0636 & & hypothetical protein & NA & NA & -3.0 & $6.56 \mathrm{E}-13$ & NA & NA & NA & NA \\
\hline PA0637 & & conserved hypothetical protein & NA & NA & -3.4 & $2.79 \mathrm{E}-13$ & NA & NA & NA & NA \\
\hline PA0639 & & conserved hypothetical protein & NA & NA & -2.5 & $4.52 \mathrm{E}-13$ & NA & NA & NA & NA \\
\hline PA0640 & & probable bacteriophage protein & NA & NA & -3.7 & $6.13 \mathrm{E}-13$ & NA & NA & NA & NA \\
\hline PA0641 & & probable bacteriophage protein & NA & NA & -3.2 & $0.00 \mathrm{E}+00$ & NA & NA & NA & NA \\
\hline PA0644 & & hypothetical protein & NA & NA & -2.7 & $1.04 \mathrm{E}-13$ & NA & NA & NA & NA \\
\hline PA0645 & & hypothetical protein & NA & NA & -4.4 & $0.00 \mathrm{E}+00$ & NA & NA & NA & NA \\
\hline PA0648 & & hypothetical protein & NA & NA & -2.8 & $0.00 \mathrm{E}+00$ & NA & NA & NA & NA \\
\hline \multicolumn{11}{|c|}{ Secreted Factors (toxins, enzymes, alginate) } \\
\hline PA0852 & $c b p D$ & chitin-binding protein $\mathrm{CbpD}$ precursor & NA & NA & NA & NA & -5.7 & $0.00 \mathrm{E}+00$ & -4.4 & $0.00 \mathrm{E}+00$ \\
\hline PA1871 & las $A$ & LasA protease precursor & NA & NA & NA & NA & -5.8 & $5.62 \mathrm{E}-11$ & -7.3 & $4.66 \mathrm{E}-12$ \\
\hline PA1899 & $p h z A 2$ & probable phenazine biosynthesis protein & NA & NA & NA & NA & $\begin{array}{c}- \\
203.0\end{array}$ & $0.00 \mathrm{E}+00$ & $\begin{array}{c}- \\
352.5\end{array}$ & $1.50 \mathrm{E}-14$ \\
\hline
\end{tabular}




\begin{tabular}{|c|c|c|c|c|c|c|c|c|c|c|}
\hline PA1900 & $p h z B 2$ & probable phenazine biosynthesis protein & NA & NA & NA & NA & $\begin{array}{c}- \\
270.6\end{array}$ & $0.00 \mathrm{E}+00$ & $\begin{array}{c}- \\
205.6\end{array}$ & $0.00 \mathrm{E}+00$ \\
\hline PA1901 & $p h z C 2$ & phenazine biosynthesis protein $\mathrm{PhzC}$ & NA & NA & NA & NA & -8.4 & $9.74 \mathrm{E}-14$ & $\begin{array}{c}- \\
188.7\end{array}$ & $0.00 \mathrm{E}+00$ \\
\hline PA1902 & phzD2 & phenazine biosynthesis protein $\mathrm{PhzD}$ & NA & NA & NA & NA & $\begin{array}{c}- \\
117.4\end{array}$ & $0.00 \mathrm{E}+00$ & $\begin{array}{c}- \\
115.1\end{array}$ & $0.00 \mathrm{E}+00$ \\
\hline PA1903 & phzE2 & phenazine biosynthesis protein PhzE & NA & NA & NA & NA & -91.6 & $0.00 \mathrm{E}+00$ & $\begin{array}{c}- \\
129.6\end{array}$ & $4.11 \mathrm{E}-14$ \\
\hline PA1904 & $p h z F 2$ & probable phenazine biosynthesis protein & 2.1 & $5.56 \mathrm{E}-08$ & NA & NA & -13.8 & $4.10 \mathrm{E}-13$ & -21.4 & $0.00 \mathrm{E}+00$ \\
\hline PA1905 & $p h z G 2$ & $\begin{array}{l}\text { probable pyridoxamine } 5 \text { '-phosphate } \\
\text { oxidase }\end{array}$ & NA & NA & NA & NA & $\begin{array}{c}- \\
113.1\end{array}$ & $0.00 \mathrm{E}+00$ & $\begin{array}{c}- \\
121.9\end{array}$ & $0.00 \mathrm{E}+00$ \\
\hline PA2396 & $p v d F$ & pyoverdine synthetase $\mathrm{F}$ & NA & NA & NA & NA & -4.4 & $5.82 \mathrm{E}-14$ & -5.8 & $2.73 \mathrm{E}-15$ \\
\hline PA2399 & $p v d D$ & pyoverdine synthetase D & NA & NA & NA & NA & -6.0 & 2.42E-09 & -4.0 & $1.33 \mathrm{E}-06$ \\
\hline PA2939 & & probable aminopeptidase & NA & NA & NA & NA & -6.7 & $0.00 \mathrm{E}+00$ & -8.1 & $0.00 \mathrm{E}+00$ \\
\hline PA3478 & $\operatorname{rhlB}$ & rhamnosyltransferase chain B & NA & NA & NA & NA & -22.6 & $0.00 \mathrm{E}+00$ & -28.2 & $0.00 \mathrm{E}+00$ \\
\hline PA3479 & rhlA & rhamnosyltransferase chain A & NA & NA & NA & NA & -18.1 & $0.00 \mathrm{E}+00$ & $\begin{array}{c}- \\
299.3\end{array}$ & $1.73 \mathrm{E}-13$ \\
\hline PA3724 & las $B$ & elastase LasB & NA & NA & NA & NA & -16.5 & $8.67 \mathrm{E}-14$ & -18.8 & $4.06 \mathrm{E}-13$ \\
\hline PA4175 & piv & protease IV & NA & NA & NA & NA & -3.0 & $5.56 \mathrm{E}-07$ & NA & NA \\
\hline PA4210 & phzAl & probable phenazine biosynthesis protein & NA & NA & NA & NA & $\begin{array}{c}- \\
169.2\end{array}$ & $0.00 \mathrm{E}+00$ & 220.3 & $0.00 \mathrm{E}+00$ \\
\hline PA4211 & phzB1 & probable phenazine biosynthesis protein & NA & NA & NA & NA & $\begin{array}{c}- \\
367.3\end{array}$ & $2.88 \mathrm{E}-13$ & $\begin{array}{c}- \\
381.9\end{array}$ & $2.58 \mathrm{E}-13$ \\
\hline PA4212 & $\operatorname{phz} C 1$ & phenazine biosynthesis protein $\mathrm{PhzC}$ & NA & NA & NA & NA & -41.3 & $0.00 \mathrm{E}+00$ & $\begin{array}{c}- \\
129.7\end{array}$ & $0.00 \mathrm{E}+00$ \\
\hline PA4213 & phzD1 & phenazine biosynthesis protein $\mathrm{PhzD}$ & NA & NA & NA & NA & $\begin{array}{c}- \\
109.8\end{array}$ & $5.95 \mathrm{E}-14$ & $\begin{array}{c}- \\
172.6\end{array}$ & $0.00 \mathrm{E}+00$ \\
\hline PA4214 & $p h z E 1$ & phenazine biosynthesis protein $\mathrm{PhzE}$ & NA & NA & NA & NA & -77.8 & $4.83 \mathrm{E}-14$ & -87.7 & $2.36 \mathrm{E}-14$ \\
\hline PA4215 & $p h z F 1$ & probable phenazine biosynthesis protein & NA & NA & NA & NA & -21.9 & 4.29E-14 & -15.6 & $0.00 \mathrm{E}+00$ \\
\hline PA4216 & $p h z G 1$ & $\begin{array}{l}\text { probable pyridoxamine } 5 \text { '-phosphate } \\
\text { oxidase }\end{array}$ & NA & NA & NA & NA & -10.9 & $9.50 \mathrm{E}-13$ & -12.7 & $0.00 \mathrm{E}+00$ \\
\hline
\end{tabular}




\begin{tabular}{|c|c|c|c|c|c|c|c|c|c|c|}
\hline PA4225 & $p c h F$ & pyochelin synthetase & NA & NA & NA & NA & -13.4 & $3.99 \mathrm{E}-15$ & -23.6 & $1.28 \mathrm{E}-14$ \\
\hline PA4226 & pchE & dihydroaeruginoic acid synthetase & NA & NA & NA & NA & -62.7 & $4.55 \mathrm{E}-14$ & -26.6 & $0.00 \mathrm{E}+00$ \\
\hline PA4228 & $\operatorname{pch} D$ & pyochelin biosynthesis protein PchD & NA & NA & NA & NA & -43.7 & 4.29E-14 & -25.3 & $0.00 \mathrm{E}+00$ \\
\hline PA4229 & $\operatorname{pchC}$ & pyochelin biosynthetic protein PchC & NA & NA & NA & NA & $\begin{array}{c}- \\
125.0\end{array}$ & $0.00 \mathrm{E}+00$ & -85.5 & $0.00 \mathrm{E}+00$ \\
\hline PA4230 & $\operatorname{pchB}$ & salicylate biosynthesis protein $\mathrm{PchB}$ & NA & NA & NA & NA & $\begin{array}{c}- \\
193.1\end{array}$ & $0.00 \mathrm{E}+00$ & $\begin{array}{c}- \\
187.8\end{array}$ & $0.00 \mathrm{E}+00$ \\
\hline PA4231 & pchA & $\begin{array}{l}\text { salicylate biosynthesis isochorismate } \\
\text { synthase }\end{array}$ & NA & NA & NA & NA & 125.5 & $0.00 \mathrm{E}+00$ & -95.4 & 4.64E-14 \\
\hline \multicolumn{11}{|c|}{ Transcription, RNA processing and degradation } \\
\hline PA3743 & $\operatorname{trm} D$ & tRNA (guanine-N1)-methyltransferase & NA & NA & -2.2 & $0.00 \mathrm{E}+00$ & NA & NA & NA & NA \\
\hline \multicolumn{11}{|c|}{ Transcriptional Regulators } \\
\hline PA1099 & fleR & two-component response regulator & NA & NA & $\begin{array}{c}- \\
13.0\end{array}$ & $3.96 \mathrm{E}-11$ & NA & NA & NA & NA \\
\hline PA1285 & & probable transcriptional regulator & NA & NA & NA & NA & -4.1 & $8.56 \mathrm{E}-13$ & NA & NA \\
\hline PA1397 & & $\begin{array}{l}\text { probable two-component response } \\
\text { regulator }\end{array}$ & NA & NA & 20.8 & $9.08 \mathrm{E}-06$ & NA & NA & NA & NA \\
\hline PA1456 & cheY & $\begin{array}{l}\text { two-component response regulator } \\
\text { CheY }\end{array}$ & NA & NA & -2.2 & $3.63 \mathrm{E}-07$ & NA & NA & NA & NA \\
\hline PA1799 & parR & $\begin{array}{l}\text { two-component response regulator, } \\
\text { ParR }\end{array}$ & NA & NA & 30.9 & $3.92 \mathrm{E}-08$ & NA & NA & NA & NA \\
\hline PA1859 & & probable transcriptional regulator & NA & NA & $\begin{array}{c}- \\
19.7\end{array}$ & $1.56 \mathrm{E}-05$ & NA & NA & NA & NA \\
\hline PA2312 & & probable transcriptional regulator & NA & NA & - & $3.45 \mathrm{E}-06$ & NA & NA & NA & NA \\
\hline PA2426 & $p v d S$ & sigma factor PvdS & NA & NA & NA & NA & -7.6 & 3.49E-09 & -38.2 & $1.93 \mathrm{E}-08$ \\
\hline PA2588 & & probable transcriptional regulator & NA & NA & NA & NA & -9.7 & $8.45 \mathrm{E}-14$ & -2.9 & 2.32E-06 \\
\hline PA2622 & $\operatorname{csp} D$ & cold-shock protein CspD & NA & NA & NA & NA & -2.1 & $1.99 \mathrm{E}-12$ & NA & NA \\
\hline PA2686 & $p f e R$ & two-component response regulator PfeR & NA & NA & NA & NA & -38.6 & $6.29 \mathrm{E}-08$ & NA & NA \\
\hline PA2834 & & probable transcriptional regulator & NA & NA & 22.8 & $3.04 \mathrm{E}-06$ & NA & NA & NA & NA \\
\hline
\end{tabular}




\begin{tabular}{|c|c|c|c|c|c|c|c|c|c|c|}
\hline PA3027 & & probable transcriptional regulator & NA & NA & $\begin{array}{c}- \\
34.0\end{array}$ & 7.49E-09 & NA & NA & NA & NA \\
\hline PA3423 & & probable transcriptional regulator & NA & NA & 23.5 & $2.04 \mathrm{E}-06$ & NA & NA & NA & NA \\
\hline PA3477 & $\operatorname{rhlR}$ & transcriptional regulator RhlR & NA & NA & NA & NA & -4.5 & $0.00 \mathrm{E}+00$ & -3.6 & $0.00 \mathrm{E}+00$ \\
\hline PA3622 & rpos & sigma factor RpoS & NA & NA & NA & NA & -2.3 & $7.89 \mathrm{E}-14$ & NA & NA \\
\hline PA3948 & $\operatorname{roc} A 1$ & $\begin{array}{l}\text { two-component response regulator } \\
\text { RocA1 }\end{array}$ & NA & NA & -4.3 & $1.68 \mathrm{E}-05$ & NA & NA & NA & NA \\
\hline PA4145 & & probable transcriptional regulator & NA & NA & 29.5 & $8.43 \mathrm{E}-08$ & NA & NA & NA & NA \\
\hline PA4296 & $\operatorname{pprB}$ & $\begin{array}{l}\text { two-component response regulator, } \\
\text { PprB }\end{array}$ & NA & NA & NA & NA & -3.5 & $1.60 \mathrm{E}-05$ & NA & NA \\
\hline PA4984 & & probable transcriptional regulator & NA & NA & 20.3 & $1.18 \mathrm{E}-05$ & NA & NA & NA & NA \\
\hline PA5059 & & probable transcriptional regulator & NA & NA & NA & NA & -49.7 & $5.31 \mathrm{E}-10$ & NA & NA \\
\hline PA5255 & $\operatorname{alg} Q$ & Alginate regulatory protein AlgQ & NA & NA & -2.1 & $7.66 \mathrm{E}-14$ & NA & NA & NA & NA \\
\hline \multicolumn{11}{|c|}{ Translation, post-translational modification, degradation } \\
\hline PA1712 & $\operatorname{exs} B$ & exoenzyme $\mathrm{S}$ synthesis protein $\mathrm{B}$ & NA & NA & NA & NA & -45.7 & $3.00 \mathrm{E}-09$ & NA & NA \\
\hline PA3326 & $\operatorname{clpP} 2$ & ClpP2 & NA & NA & NA & NA & -5.5 & $1.50 \mathrm{E}-14$ & -5.1 & $9.71 \mathrm{E}-14$ \\
\hline PA5018 & $m s r A$ & peptide methionine sulfoxide reductase & NA & NA & -2.4 & $1.68 \mathrm{E}-05$ & NA & NA & NA & NA \\
\hline PA5240 & $\operatorname{tr} x A$ & thioredoxin & NA & NA & -2.0 & $0.00 \mathrm{E}+00$ & NA & NA & NA & NA \\
\hline PA5316 & rpmB & 50S ribosomal protein L28 & NA & NA & -2.2 & $0.00 \mathrm{E}+00$ & NA & NA & NA & NA \\
\hline \multicolumn{11}{|c|}{ Transport of small molecules } \\
\hline PA2592 & & $\begin{array}{l}\text { probable periplasmic } \\
\text { spermidine/putrescine-binding protein }\end{array}$ & NA & NA & NA & NA & -3.4 & $4.14 \mathrm{E}-11$ & -2.8 & $8.45 \mathrm{E}-14$ \\
\hline PA2983 & & probable tolQ-type transport protein & NA & NA & -2.2 & $1.56 \mathrm{E}-05$ & NA & NA & NA & NA \\
\hline PA3373 & & conserved hypothetical protein & NA & NA & -5.1 & $1.15 \mathrm{E}-07$ & NA & NA & NA & NA \\
\hline PA4206 & mexH & $\begin{array}{l}\text { probable Resistance-Nodulation-Cell } \\
\text { Division (RND) efflux membrane } \\
\text { fusion protein precursor }\end{array}$ & NA & NA & NA & NA & -11.3 & $0.00 \mathrm{E}+00$ & -7.8 & $3.15 \mathrm{E}-14$ \\
\hline PA4221 & fptA & $\begin{array}{l}\mathrm{Fe}(\mathrm{III}) \text {-pyochelin outer membrane } \\
\text { receptor precursor }\end{array}$ & NA & NA & NA & NA & -25.4 & $3.99 \mathrm{E}-15$ & -29.2 & $0.00 \mathrm{E}+00$ \\
\hline
\end{tabular}




\begin{tabular}{|c|c|c|c|c|c|c|c|c|c|c|}
\hline PA4222 & & $\begin{array}{l}\text { probable ATP-binding component of } \\
\text { ABC transporter }\end{array}$ & NA & NA & NA & NA & -11.6 & $1.85 \mathrm{E}-10$ & -66.6 & $4.11 \mathrm{E}-14$ \\
\hline PA4224 & $\operatorname{pch} G$ & pyochelin biosynthetic protein PchG & NA & NA & NA & NA & -56.3 & $3.15 \mathrm{E}-11$ & -13.6 & $3.69 \mathrm{E}-14$ \\
\hline \multicolumn{11}{|c|}{ Two-component regulatory systems } \\
\hline PA0409 & pilH & twitching motility protein PilH & NA & NA & -3.5 & $4.93 \mathrm{E}-14$ & NA & NA & NA & NA \\
\hline
\end{tabular}

\begin{tabular}{|c|c|c|c|c|c|c|c|c|c|c|}
\hline \multicolumn{11}{|c|}{ Genes negatively regulated by AmpR (341) } \\
\hline \multicolumn{11}{|c|}{ Adaptation, Protection } \\
\hline PA0140 & ahpF & alkyl hydroperoxide reductase subunit $F$ & NA & NA & 3.0 & 4.76E-07 & NA & NA & NA & NA \\
\hline PA1008 & $b c p$ & bacterioferritin comigratory protein & NA & NA & NA & NA & 2.4 & 2.35E-09 & NA & NA \\
\hline PA1457 & cheZ & chemotaxis protein CheZ & NA & NA & 2.2 & $3.03 \mathrm{E}-07$ & NA & NA & NA & NA \\
\hline PA4356 & $x e n B$ & xenobiotic reductase & NA & NA & NA & NA & 10.5 & $0.00 \mathrm{E}+00$ & 4.4 & $2.93 \mathrm{E}-10$ \\
\hline PA4378 & inaA & InaA protein & NA & NA & 2.2 & $5.78 \mathrm{E}-06$ & NA & NA & NA & NA \\
\hline PA5117 & typA & regulatory protein TypA & NA & NA & NA & NA & 3.2 & $6.38 \mathrm{E}-09$ & 2.3 & $3.74 \mathrm{E}-07$ \\
\hline \multicolumn{11}{|c|}{ Amino acid biosynthesis and metabolism } \\
\hline PA0393 & proC & pyrroline-5-carboxylate reductase & NA & NA & NA & NA & 2.2 & $2.67 \mathrm{E}-06$ & 2.9 & $3.65 \mathrm{E}-09$ \\
\hline PA0904 & lys $C$ & aspartate kinase alpha and beta chain & NA & NA & NA & NA & 2.0 & $8.49 \mathrm{E}-07$ & NA & NA \\
\hline PA1004 & $\operatorname{nadA}$ & quinolinate synthetase A & NA & NA & NA & NA & 2.1 & $9.75 \mathrm{E}-06$ & NA & NA \\
\hline PA1010 & $\operatorname{dap} A$ & dihydrodipicolinate synthase & NA & NA & NA & NA & 2.0 & $2.49 \mathrm{E}-10$ & NA & NA \\
\hline PA1421 & gbuA & guanidinobutyrase & NA & NA & 28.5 & $3.92 \mathrm{E}-06$ & NA & NA & NA & NA \\
\hline PA1685 & mas $A$ & enolase-phosphatase E-1 & NA & NA & 3.1 & $2.66 \mathrm{E}-06$ & $\mathrm{NA}$ & NA & NA & NA \\
\hline PA1818 & $l d c A$ & $\begin{array}{l}\text { lysine-specific pyridoxal 5'-phosphate- } \\
\text { dependent carboxylase, LdcA }\end{array}$ & NA & NA & NA & NA & 2.2 & $2.51 \mathrm{E}-07$ & NA & NA \\
\hline PA1999 & $d h c A$ & $\begin{array}{l}\text { DhcA, dehydrocarnitine CoA } \\
\text { transferase, subunit A }\end{array}$ & NA & NA & 2.0 & $0.00 \mathrm{E}+00$ & NA & NA & NA & NA \\
\hline PA2629 & purB & adenylosuccinate lyase & NA & NA & NA & NA & 2.2 & $1.80 \mathrm{E}-05$ & NA & NA \\
\hline
\end{tabular}




\begin{tabular}{|c|c|c|c|c|c|c|c|c|c|c|}
\hline PA3167 & $\operatorname{ser} C$ & 3-phosphoserine aminotransferase & NA & NA & NA & NA & 2.2 & $6.38 \mathrm{E}-08$ & $\mathrm{NA}$ & NA \\
\hline PA5140 & hisF1 & $\begin{array}{l}\text { imidazoleglycerol-phosphate synthase, } \\
\text { cyclase subunit }\end{array}$ & NA & NA & 3.7 & $1.76 \mathrm{E}-06$ & NA & NA & NA & NA \\
\hline PA5203 & $g \operatorname{sh} A$ & glutamate--cysteine ligase & NA & NA & 2.4 & $1.37 \mathrm{E}-05$ & NA & NA & NA & NA \\
\hline PA5373 & bet $B$ & betaine aldehyde dehydrogenase & NA & NA & NA & NA & 3.4 & 4.44E-07 & 5.1 & $1.87 \mathrm{E}-09$ \\
\hline \multicolumn{11}{|c|}{ Biosynthesis of cofactors, prosthetic groups and carriers } \\
\hline PA0510 & & $\begin{array}{l}\text { probable uroporphyrin-III c- } \\
\text { methyltransferase }\end{array}$ & NA & NA & 48.9 & $6.85 \mathrm{E}-10$ & NA & NA & NA & NA \\
\hline PA0518 & nirM & cytochrome c-551 precursor & NA & NA & 43.5 & 7.17E-09 & NA & NA & NA & NA \\
\hline PA1986 & $p q q B$ & $\begin{array}{l}\text { pyrroloquinoline quinone biosynthesis } \\
\text { protein B }\end{array}$ & NA & NA & NA & NA & 7.4 & $5.76 \mathrm{E}-06$ & 29.9 & $9.02 \mathrm{E}-08$ \\
\hline PA3030 & mobA & $\begin{array}{l}\text { molybdopterin-guanine dinucleotide } \\
\text { biosynthesis protein MobA }\end{array}$ & NA & NA & 34.4 & $3.34 \mathrm{E}-07$ & NA & NA & NA & NA \\
\hline PA3812 & $i s c A$ & probable iron-binding protein IscA & NA & NA & 2.5 & $3.47 \mathrm{E}-14$ & NA & NA & NA & NA \\
\hline PA3917 & moaD & $\begin{array}{l}\text { molybdopterin converting factor, small } \\
\text { subunit }\end{array}$ & NA & NA & 2.8 & 4.77E-07 & NA & NA & NA & NA \\
\hline PA3976 & thiE & thiamin-phosphate pyrophosphorylase & NA & NA & NA & NA & 3.2 & $1.29 \mathrm{E}-07$ & NA & NA \\
\hline PA4044 & $d x s$ & 1-deoxyxylulose-5-phosphate synthase & NA & NA & NA & NA & 2.8 & $1.69 \mathrm{E}-07$ & NA & NA \\
\hline PA4053 & ribE & 6,7-dimethyl-8-ribityllumazine synthase & NA & NA & NA & NA & 3.0 & $0.00 \mathrm{E}+00$ & $\mathrm{NA}$ & NA \\
\hline PA4054 & $r i b B$ & $\begin{array}{l}\text { GTP cyclohydrolase II / 3,4-dihydroxy- } \\
\text { 2-butanone 4-phosphate synthase }\end{array}$ & NA & NA & NA & NA & 2.6 & $2.53 \mathrm{E}-07$ & NA & NA \\
\hline PA5259 & hemD & uroporphyrinogen-III synthetase & NA & NA & 4.1 & $0.00 \mathrm{E}+00$ & NA & NA & NA & NA \\
\hline \multicolumn{11}{|c|}{ Carbon compound catabolism } \\
\hline PA0210 & $m d c C$ & malonate decarboxylase delta subunit & NA & NA & NA & NA & 4.2 & $3.66 \mathrm{E}-07$ & 45.6 & $2.49 \mathrm{E}-11$ \\
\hline PA0231 & $p c a D$ & beta-ketoadipate enol-lactone hydrolase & NA & NA & 25.9 & $1.17 \mathrm{E}-05$ & NA & NA & NA & NA \\
\hline PA2008 & fahA & fumarylacetoacetase & NA & NA & NA & NA & 2.3 & $2.26 \mathrm{E}-13$ & NA & NA \\
\hline PA2011 & $\operatorname{liu} E$ & 3-hydroxy-3-methylglutaryl-CoA lyase & NA & NA & 2.9 & $1.98 \mathrm{E}-11$ & NA & NA & NA & NA \\
\hline PA2414 & & L-sorbosone dehydrogenase & NA & NA & 25.9 & $1.17 \mathrm{E}-05$ & NA & NA & $\mathrm{NA}$ & NA \\
\hline PA3569 & $m m s B$ & 3-hydroxyisobutyrate dehydrogenase & NA & NA & NA & NA & 2.0 & 7.71E-08 & 2.4 & $6.55 \mathrm{E}-13$ \\
\hline
\end{tabular}




\begin{tabular}{|c|c|c|c|c|c|c|c|c|c|c|}
\hline PA3636 & $k d s A$ & $\begin{array}{l}\text { 2-dehydro-3-deoxyphosphooctonate } \\
\text { aldolase }\end{array}$ & NA & NA & NA & NA & 2.0 & $1.74 \mathrm{E}-08$ & NA & NA \\
\hline \multicolumn{11}{|c|}{ Cell division } \\
\hline PA0857 & bolA & morphogene protein BolA & NA & NA & 2.1 & 7.84E-08 & NA & NA & NA & NA \\
\hline PA3245 & $\min E$ & $\begin{array}{l}\text { cell division topological specificity } \\
\text { factor MinE }\end{array}$ & NA & NA & 2.4 & $4.03 \mathrm{E}-14$ & NA & NA & NA & NA \\
\hline PA4413 & $f t s W$ & cell division protein FtsW & NA & NA & 2.5 & $5.64 \mathrm{E}-11$ & NA & NA & NA & NA \\
\hline \multicolumn{11}{|c|}{ Cell wall / LPS / capsule } \\
\hline PA1222 & & $\begin{array}{l}\text { probable membrane-bound lytic murein } \\
\text { transglycolase A }\end{array}$ & NA & NA & NA & NA & 3.1 & $1.42 \mathrm{E}-05$ & NA & NA \\
\hline PA3150 & $w b p G$ & LPS biosynthesis protein $\mathrm{WbpG}$ & NA & NA & 2.0 & $9.14 \mathrm{E}-13$ & NA & NA & NA & NA \\
\hline PA3552 & $\operatorname{arn} B$ & ArnB & NA & NA & NA & NA & 7.9 & $6.76 \mathrm{E}-10$ & NA & NA \\
\hline PA3666 & $d a p D$ & tetrahydrodipicolinate succinylase & NA & NA & NA & NA & 2.3 & $7.51 \mathrm{E}-07$ & NA & NA \\
\hline PA4001 & sltB1 & soluble lytic transglycosylase B & NA & NA & 2.0 & 4.15E-07 & NA & NA & NA & NA \\
\hline PA4545 & $\operatorname{comL}$ & competence protein ComL & NA & NA & NA & NA & 2.5 & $5.05 \mathrm{E}-10$ & NA & NA \\
\hline PA4624 & $c d r B$ & $\begin{array}{l}\text { cyclic diguanylate-regulated TPS } \\
\text { partner B, CdrB }\end{array}$ & NA & NA & 2.6 & $1.51 \mathrm{E}-07$ & NA & NA & NA & NA \\
\hline PA5077 & $m d o H$ & $\begin{array}{l}\text { periplasmic glucans biosynthesis } \\
\text { protein } \mathrm{MdoH}\end{array}$ & NA & NA & 2.1 & $7.64 \mathrm{E}-06$ & NA & NA & NA & NA \\
\hline PA5164 & $r m l C$ & $\begin{array}{l}\text { dTDP-4-dehydrorhamnose 3,5- } \\
\text { epimerase }\end{array}$ & NA & NA & 2.4 & $1.32 \mathrm{E}-07$ & NA & NA & NA & NA \\
\hline PA5452 & $w b p W$ & $\begin{array}{l}\text { phosphomannose isomerase/GDP- } \\
\text { mannose WbpW }\end{array}$ & NA & NA & 3.9 & $2.29 \mathrm{E}-06$ & NA & NA & NA & NA \\
\hline \multicolumn{11}{|c|}{ Central intermediary metabolism } \\
\hline PA0265 & $g a b D$ & succinate-semialdehyde dehydrogenase & NA & NA & NA & NA & 2.6 & $5.69 \mathrm{E}-10$ & 3.7 & $1.95 \mathrm{E}-14$ \\
\hline PA0266 & $g a b T$ & 4-aminobutyrate aminotransferase & NA & NA & NA & NA & 2.6 & $3.31 \mathrm{E}-11$ & 3.2 & 4.64E-14 \\
\hline PA2001 & ato $B$ & acetyl-CoA acetyltransferase & NA & NA & NA & NA & 2.3 & $1.50 \mathrm{E}-14$ & 2.4 & $4.11 \mathrm{E}-14$ \\
\hline PA2442 & gcvT2 & glycine cleavage system protein $\mathrm{T} 2$ & NA & NA & 2.1 & $1.05 \mathrm{E}-11$ & NA & NA & NA & NA \\
\hline PA2726 & & probable radical activating enzyme & NA & NA & NA & NA & 18.9 & $3.08 \mathrm{E}-13$ & NA & NA \\
\hline PA2813 & & probable glutathione S-transferase & NA & NA & NA & NA & 6.7 & $0.00 \mathrm{E}+00$ & 176.3 & $0.00 \mathrm{E}+00$ \\
\hline
\end{tabular}




\begin{tabular}{|c|c|c|c|c|c|c|c|c|c|c|}
\hline PA3181 & & $\begin{array}{l}\text { 2-keto-3-deoxy-6-phosphogluconate } \\
\text { aldolase }\end{array}$ & NA & NA & NA & NA & 4.7 & $6.71 \mathrm{E}-08$ & 103.2 & $0.00 \mathrm{E}+00$ \\
\hline PA3182 & $p g l$ & 6-phosphogluconolactonase & NA & NA & NA & NA & 4.2 & $3.98 \mathrm{E}-06$ & 2.8 & $3.58 \mathrm{E}-08$ \\
\hline PA4031 & ppa & inorganic pyrophosphatase & NA & $\mathrm{NA}$ & NA & NA & 2.0 & $0.00 \mathrm{E}+00$ & NA & NA \\
\hline PA5215 & gcvTl & glycine-cleavage system protein $\mathrm{T} 1$ & NA & NA & NA & NA & 2.1 & $2.26 \mathrm{E}-11$ & NA & NA \\
\hline PA5435 & & probable transcarboxylase subunit & NA & NA & NA & NA & 2.2 & 2.89E-09 & 2.7 & $1.03 \mathrm{E}-14$ \\
\hline PA5436 & & $\begin{array}{l}\text { probable biotin carboxylase subunit of a } \\
\text { transcarboxylase }\end{array}$ & NA & NA & NA & NA & 2.1 & $1.13 \mathrm{E}-07$ & NA & NA \\
\hline \multicolumn{11}{|c|}{ Chaperones \& heat shock proteins } \\
\hline PA2614 & lolA & periplasmic chaperone LolA & NA & NA & NA & NA & 2.3 & $1.29 \mathrm{E}-09$ & NA & NA \\
\hline PA3810 & $h s c A$ & heat shock protein HscA & NA & NA & 2.1 & $1.26 \mathrm{E}-06$ & NA & NA & NA & NA \\
\hline \multicolumn{11}{|c|}{ Chemotaxis ; Adaptation, Protection ; Motility \& Attachment } \\
\hline PA1445 & $\mathrm{fliO}$ & flagellar protein FliO & NA & NA & 6.9 & $6.54 \mathrm{E}-10$ & NA & NA & $\mathrm{NA}$ & NA \\
\hline \multicolumn{11}{|c|}{ DNA replication, recombination, modification and repair } \\
\hline PA3642 & $r n h B$ & ribonuclease HII & NA & NA & NA & NA & 2.8 & $6.84 \mathrm{E}-07$ & $\mathrm{NA}$ & NA \\
\hline PA3832 & holC & DNA polymerase III, chi subunit & NA & NA & 6.9 & $1.99 \mathrm{E}-10$ & NA & NA & NA & NA \\
\hline \multicolumn{11}{|c|}{ Energy metabolism } \\
\hline PA0362 & $f d x 1$ & ferredoxin $[4 \mathrm{Fe}-4 \mathrm{~S}]$ & NA & $\mathrm{NA}$ & NA & NA & 2.1 & $5.52 \mathrm{E}-06$ & 2.6 & $4.56 \mathrm{E}-09$ \\
\hline $\begin{array}{c}\text { PA1555. } \\
1\end{array}$ & $\operatorname{ccoQ} 2$ & $\begin{array}{l}\text { Cytochrome c oxidase, cbb3-type, } \\
\text { CcoQ subunit }\end{array}$ & NA & NA & NA & NA & 12.6 & $2.82 \mathrm{E}-14$ & 3.1 & $1.91 \mathrm{E}-09$ \\
\hline PA1581 & $s d h C$ & succinate dehydrogenase ( $\mathrm{C}$ subunit) & NA & NA & NA & NA & 2.5 & $0.00 \mathrm{E}+00$ & NA & NA \\
\hline PA1586 & $\operatorname{sucB}$ & $\begin{array}{l}\text { dihydrolipoamide succinyltransferase } \\
\text { (E2 subunit) }\end{array}$ & NA & NA & NA & NA & 2.1 & $0.00 \mathrm{E}+00$ & NA & NA \\
\hline PA1588 & sucC & succinyl-CoA synthetase beta chain & NA & NA & NA & NA & 2.4 & $3.00 \mathrm{E}-13$ & NA & NA \\
\hline PA1589 & $s u c D$ & succinyl-CoA synthetase alpha chain & NA & NA & NA & NA & 2.7 & $1.47 \mathrm{E}-13$ & 2.7 & $0.00 \mathrm{E}+00$ \\
\hline PA1787 & $\operatorname{acn} B$ & aconitate hydratase 2 & NA & NA & NA & NA & 2.0 & $4.01 \mathrm{E}-14$ & NA & NA \\
\hline PA2638 & пиов & NADH dehydrogenase I chain B & NA & $\mathrm{NA}$ & NA & NA & 2.0 & $2.02 \mathrm{E}-10$ & NA & NA \\
\hline PA2639 & пиоD & NADH dehydrogenase I chain C,D & NA & NA & NA & NA & 2.1 & $3.62 \mathrm{E}-07$ & 2.8 & $1.54 \mathrm{E}-10$ \\
\hline PA2640 & nuоE & NADH dehydrogenase I chain E & NA & NA & NA & NA & 2.0 & $1.37 \mathrm{E}-08$ & 2.2 & $2.07 \mathrm{E}-12$ \\
\hline PA2642 & nиоG & NADH dehydrogenase I chain G & NA & $\mathrm{NA}$ & NA & NA & 2.1 & 4.26E-06 & 2.6 & 4.66E-12 \\
\hline
\end{tabular}




\begin{tabular}{|c|c|c|c|c|c|c|c|c|c|c|}
\hline PA2643 & пион & NADH dehydrogenase I chain $\mathrm{H}$ & NA & NA & NA & NA & 2.4 & $3.61 \mathrm{E}-07$ & NA & NA \\
\hline PA2647 & nиоL & NADH dehydrogenase I chain L & NA & NA & NA & NA & 2.6 & $2.81 \mathrm{E}-08$ & NA & NA \\
\hline PA2953 & & $\begin{array}{l}\text { electron transfer flavoprotein- } \\
\text { ubiquinone oxidoreductase }\end{array}$ & NA & NA & NA & NA & 2.2 & $2.67 \mathrm{E}-07$ & 2.1 & $2.01 \mathrm{E}-08$ \\
\hline PA2994 & $n q r F$ & $\begin{array}{l}\mathrm{Na}+\text {-translocating NADH:quinone } \\
\text { oxidoreductase, subunit Nqr6 }\end{array}$ & NA & NA & NA & NA & 2.3 & $6.89 \mathrm{E}-07$ & 2.0 & $5.45 \mathrm{E}-06$ \\
\hline PA2996 & $n q r D$ & $\begin{array}{l}\mathrm{Na}+\text {-translocating NADH:uniquinone } \\
\text { oxidoreductase subunit Nqr4 }\end{array}$ & NA & NA & NA & NA & 3.0 & $8.48 \mathrm{E}-06$ & NA & NA \\
\hline PA3621 & $f d x A$ & ferredoxin I & NA & NA & NA & NA & 2.0 & 7.87E-09 & NA & NA \\
\hline PA4333 & & probable fumarase & NA & NA & NA & NA & 2.5 & 7.32E-09 & NA & NA \\
\hline PA4810 & $f d n I$ & $\begin{array}{l}\text { nitrate-inducible formate } \\
\text { dehydrogenase, gamma subunit }\end{array}$ & NA & NA & NA & NA & 15.8 & $7.75 \mathrm{E}-14$ & 3.1 & $5.18 \mathrm{E}-06$ \\
\hline PA5129 & $g r x$ & glutaredoxin & NA & NA & NA & NA & 2.1 & 4.09E-08 & 3.6 & $5.09 \mathrm{E}-14$ \\
\hline \multicolumn{11}{|c|}{ Fatty acid and phospholipid metabolism } \\
\hline PA2537 & & probable acyltransferase & NA & NA & 3.2 & $4.08 \mathrm{E}-06$ & NA & NA & NA & NA \\
\hline PA4339 & & probable phospholipase & NA & NA & 31.7 & $1.02 \mathrm{E}-06$ & NA & NA & NA & NA \\
\hline PA4848 & $\operatorname{accC}$ & biotin carboxylase & NA & NA & NA & NA & 2.0 & $2.71 \mathrm{E}-11$ & NA & NA \\
\hline \multicolumn{11}{|c|}{ Hypothetical, unclassified, unknown } \\
\hline PA0141 & & conserved hypothetical protein & NA & NA & 3.5 & $1.57 \mathrm{E}-06$ & NA & NA & NA & NA \\
\hline PA0309 & & hypothetical protein & NA & NA & NA & NA & 2.7 & $3.82 \mathrm{E}-06$ & NA & NA \\
\hline PA0315 & & hypothetical protein & NA & NA & 2.6 & $0.00 \mathrm{E}+00$ & NA & NA & NA & NA \\
\hline PA0370 & & conserved hypothetical protein & NA & NA & NA & NA & 3.2 & $3.40 \mathrm{E}-06$ & NA & NA \\
\hline PA0392 & & conserved hypothetical protein & NA & NA & NA & NA & 2.2 & $1.55 \mathrm{E}-06$ & NA & NA \\
\hline PA0442 & & hypothetical protein & NA & NA & NA & NA & 2.1 & $1.36 \mathrm{E}-05$ & 4.1 & $3.52 \mathrm{E}-14$ \\
\hline PA0490 & & hypothetical protein & NA & NA & 46.6 & $1.87 \mathrm{E}-09$ & NA & NA & NA & NA \\
\hline PA0754 & & hypothetical protein & NA & NA & 4.8 & $1.17 \mathrm{E}-05$ & NA & NA & NA & NA \\
\hline PA0760 & & conserved hypothetical protein & NA & NA & 2.8 & $5.59 \mathrm{E}-07$ & NA & NA & NA & NA \\
\hline PA0821 & & hypothetical protein & NA & NA & 37.7 & $8.26 \mathrm{E}-08$ & NA & NA & NA & NA \\
\hline PA0957 & & hypothetical protein & NA & NA & 33.5 & 4.77E-07 & NA & NA & NA & NA \\
\hline PA1006 & & conserved hypothetical protein & - & 4.96E-09 & NA & NA & 2.6 & $2.36 \mathrm{E}-06$ & 54.3 & $2.58 \mathrm{E}-13$ \\
\hline
\end{tabular}




\begin{tabular}{|c|c|c|c|c|c|c|c|c|c|c|}
\hline & & & 37.5 & & & & & & & \\
\hline PA1063 & & hypothetical protein & NA & NA & 54.3 & $6.55 \mathrm{E}-11$ & NA & NA & NA & NA \\
\hline PA1069 & & hypothetical protein & NA & NA & 2.0 & $3.96 \mathrm{E}-06$ & NA & NA & NA & NA \\
\hline PA1106 & & hypothetical protein & NA & NA & 4.1 & $6.47 \mathrm{E}-06$ & NA & NA & NA & NA \\
\hline PA1191 & & hypothetical protein & NA & NA & 34.6 & $3.12 \mathrm{E}-07$ & NA & NA & NA & NA \\
\hline PA1333 & & hypothetical protein & 68.5 & $0.00 \mathrm{E}+00$ & NA & NA & 2.1 & 3.33E-06 & 132.2 & $0.00 \mathrm{E}+00$ \\
\hline PA1469 & & hypothetical protein & NA & NA & NA & NA & 10.5 & $3.94 \mathrm{E}-06$ & NA & NA \\
\hline PA1638 & & conserved hypothetical protein & NA & NA & 45.2 & $3.41 \mathrm{E}-09$ & NA & NA & NA & NA \\
\hline PA1668 & & hypothetical protein & NA & NA & 31.5 & $1.12 \mathrm{E}-06$ & NA & NA & NA & NA \\
\hline PA1753 & & conserved hypothetical protein & NA & NA & 3.5 & $5.64 \mathrm{E}-06$ & NA & NA & NA & NA \\
\hline PA1755 & & hypothetical protein & NA & NA & NA & NA & 37.8 & $8.26 \mathrm{E}-10$ & 59.0 & $2.75 \mathrm{E}-14$ \\
\hline PA1766 & & hypothetical protein & NA & NA & NA & NA & 3.0 & $6.60 \mathrm{E}-09$ & NA & NA \\
\hline PA1768 & & hypothetical protein & NA & NA & NA & NA & 3.2 & $9.20 \mathrm{E}-09$ & NA & NA \\
\hline PA1789 & & hypothetical protein & NA & NA & 2.1 & $7.51 \mathrm{E}-06$ & NA & NA & NA & NA \\
\hline PA1814 & & hypothetical protein & NA & NA & 2.1 & $1.48 \mathrm{E}-05$ & NA & NA & NA & NA \\
\hline PA1830 & & hypothetical protein & NA & NA & 2.0 & $0.00 \mathrm{E}+00$ & NA & NA & NA & NA \\
\hline PA1831 & & hypothetical protein & NA & NA & NA & NA & 2.3 & 8.09E-14 & NA & NA \\
\hline PA1841 & & hypothetical protein & NA & NA & NA & NA & 3.7 & $9.54 \mathrm{E}-07$ & NA & NA \\
\hline PA1889 & & hypothetical protein & NA & NA & 3.0 & $8.33 \mathrm{E}-06$ & NA & NA & NA & NA \\
\hline PA1942 & & hypothetical protein & NA & NA & NA & NA & 63.1 & $5.73 \mathrm{E}-13$ & 355.5 & $8.45 \mathrm{E}-14$ \\
\hline PA1957 & & hypothetical protein & NA & NA & 38.9 & $5.04 \mathrm{E}-08$ & NA & NA & NA & NA \\
\hline PA1969 & & hypothetical protein & NA & NA & 2.2 & $2.21 \mathrm{E}-06$ & NA & NA & NA & NA \\
\hline PA1970 & & hypothetical protein & NA & NA & NA & NA & 54.8 & $5.95 \mathrm{E}-14$ & 114.1 & $5.09 \mathrm{E}-14$ \\
\hline PA2111 & & hypothetical protein & NA & NA & NA & NA & 2.0 & $0.00 \mathrm{E}+00$ & 2.2 & $0.00 \mathrm{E}+00$ \\
\hline PA2145 & & hypothetical protein & NA & NA & NA & NA & 32.9 & $1.19 \mathrm{E}-08$ & 51.5 & $1.15 \mathrm{E}-12$ \\
\hline PA2242 & pslL & hypothetical protein & NA & NA & 3.5 & $1.23 \mathrm{E}-05$ & NA & NA & NA & NA \\
\hline PA2251 & & hypothetical protein & NA & NA & 6.9 & $7.05 \mathrm{E}-06$ & NA & NA & NA & NA \\
\hline PA2311 & & hypothetical protein & NA & NA & NA & NA & 4.2 & $8.30 \mathrm{E}-07$ & 43.0 & $9.49 \mathrm{E}-11$ \\
\hline
\end{tabular}




\begin{tabular}{|c|c|c|c|c|c|c|c|c|c|}
\hline PA2345 & conserved hypothetical protein & NA & NA & 9.7 & $3.88 \mathrm{E}-06$ & NA & NA & NA & NA \\
\hline PA2485 & hypothetical protein & NA & NA & NA & NA & 3.8 & $0.00 \mathrm{E}+00$ & 7.7 & $2.07 \mathrm{E}-13$ \\
\hline PA2486 & hypothetical protein & NA & NA & 3.5 & $8.78 \mathrm{E}-14$ & 4.2 & $1.06 \mathrm{E}-05$ & 175.5 & $0.00 \mathrm{E}+00$ \\
\hline PA2651 & conserved hypothetical protein & NA & NA & 3.6 & $2.48 \mathrm{E}-07$ & NA & NA & NA & NA \\
\hline PA2655 & hypothetical protein & NA & NA & NA & NA & 42.5 & $5.82 \mathrm{E}-11$ & 3.1 & 4.39E-06 \\
\hline PA2713 & conserved hypothetical protein & NA & NA & 28.5 & $3.92 \mathrm{E}-06$ & NA & NA & NA & NA \\
\hline PA2753 & hypothetical protein & NA & NA & 2.8 & $4.26 \mathrm{E}-07$ & NA & NA & NA & NA \\
\hline PA2779 & hypothetical protein & NA & NA & NA & NA & 4.2 & $2.14 \mathrm{E}-10$ & 5.1 & $0.00 \mathrm{E}+00$ \\
\hline PA2819 & hypothetical protein & NA & NA & 5.5 & $5.95 \mathrm{E}-07$ & NA & NA & NA & NA \\
\hline PA2827 & conserved hypothetical protein & NA & NA & 3.2 & $3.90 \mathrm{E}-09$ & NA & NA & NA & NA \\
\hline PA2869 & hypothetical protein & NA & NA & NA & NA & 10.5 & $1.09 \mathrm{E}-08$ & NA & NA \\
\hline PA2901 & hypothetical protein & NA & NA & NA & NA & 2.6 & 4.59E-09 & NA & NA \\
\hline PA3012 & hypothetical protein & NA & NA & 54.7 & $5.42 \mathrm{E}-11$ & NA & NA & NA & NA \\
\hline PA3015 & hypothetical protein & NA & NA & 6.9 & 7.05E-06 & NA & NA & NA & NA \\
\hline PA3080 & hypothetical protein & NA & NA & NA & NA & 5.3 & $6.27 \mathrm{E}-06$ & 37.4 & $1.82 \mathrm{E}-09$ \\
\hline PA3110 & hypothetical protein & NA & NA & 2.8 & 3.97E-09 & NA & NA & NA & NA \\
\hline PA3229 & hypothetical protein & NA & NA & NA & NA & 76.8 & $0.00 \mathrm{E}+00$ & 794.6 & $3.08 \mathrm{E}-13$ \\
\hline PA3440 & conserved hypothetical protein & NA & NA & NA & NA & 2.5 & $6.26 \mathrm{E}-11$ & NA & NA \\
\hline PA3572 & hypothetical protein & NA & NA & NA & NA & 2.1 & $3.56 \mathrm{E}-08$ & NA & NA \\
\hline PA3580 & conserved hypothetical protein & NA & NA & 43.6 & $6.75 \mathrm{E}-09$ & NA & NA & NA & NA \\
\hline PA3674 & hypothetical protein & NA & NA & 3.5 & $3.21 \mathrm{E}-07$ & NA & NA & NA & NA \\
\hline PA3712 & hypothetical protein & NA & NA & 3.6 & $9.50 \mathrm{E}-11$ & NA & NA & NA & NA \\
\hline PA3741 & hypothetical protein & NA & NA & NA & NA & 3.5 & $1.54 \mathrm{E}-05$ & 64.3 & $6.69 \mathrm{E}-14$ \\
\hline PA3850 & hypothetical protein & NA & NA & NA & NA & 2.9 & $6.39 \mathrm{E}-11$ & NA & NA \\
\hline PA3869 & hypothetical protein & NA & NA & NA & NA & 34.2 & $5.91 \mathrm{E}-09$ & NA & NA \\
\hline PA3906 & hypothetical protein & NA & NA & NA & NA & 2.4 & $2.61 \mathrm{E}-06$ & NA & NA \\
\hline PA3962 & hypothetical protein & NA & NA & NA & NA & 3.4 & 3.42E-08 & 4.1 & $9.76 \mathrm{E}-08$ \\
\hline PA3967 & hypothetical protein & NA & NA & NA & NA & 3.5 & $6.29 \mathrm{E}-06$ & 34.8 & $6.97 \mathrm{E}-09$ \\
\hline PA3988 & hypothetical protein & NA & NA & NA & NA & 2.0 & $3.75 \mathrm{E}-06$ & NA & NA \\
\hline
\end{tabular}




\begin{tabular}{|c|c|c|c|c|c|c|c|c|c|c|}
\hline PA3998 & & conserved hypothetical protein & NA & NA & 2.0 & $0.00 \mathrm{E}+00$ & NA & NA & NA & NA \\
\hline PA4004 & & conserved hypothetical protein & NA & NA & NA & NA & 4.2 & $6.38 \mathrm{E}-07$ & NA & NA \\
\hline PA4045 & & conserved hypothetical protein & NA & NA & NA & NA & 3.4 & $3.54 \mathrm{E}-07$ & 4.1 & $4.86 \mathrm{E}-08$ \\
\hline PA4049 & & hypothetical protein & NA & NA & 4.1 & $1.08 \mathrm{E}-05$ & NA & NA & NA & NA \\
\hline PA4111 & & hypothetical protein & NA & NA & 5.5 & $3.62 \mathrm{E}-08$ & NA & NA & NA & NA \\
\hline PA4204 & $\operatorname{ppg} L$ & periplasmic gluconolactonase, $\mathrm{PpgL}$ & NA & NA & 3.2 & $1.57 \mathrm{E}-06$ & NA & NA & NA & NA \\
\hline PA4325 & & hypothetical protein & NA & NA & NA & NA & 2.6 & $2.08 \mathrm{E}-07$ & NA & NA \\
\hline PA4379 & & conserved hypothetical protein & NA & NA & NA & NA & 2.0 & $1.67 \mathrm{E}-09$ & NA & NA \\
\hline PA4405 & & hypothetical protein & NA & NA & NA & NA & 5.3 & $6.71 \mathrm{E}-08$ & NA & NA \\
\hline PA4438 & & conserved hypothetical protein & NA & NA & NA & NA & 8.7 & $0.00 \mathrm{E}+00$ & 2.7 & $3.97 \mathrm{E}-06$ \\
\hline PA4458 & & conserved hypothetical protein & NA & NA & 2.2 & $1.45 \mathrm{E}-07$ & NA & NA & NA & NA \\
\hline PA4460 & & conserved hypothetical protein & NA & NA & NA & NA & 2.2 & $1.74 \mathrm{E}-13$ & 2.1 & 4.34E-08 \\
\hline PA4487 & & conserved hypothetical protein & NA & NA & 2.9 & $6.16 \mathrm{E}-11$ & NA & NA & NA & NA \\
\hline PA4488 & & conserved hypothetical protein & NA & NA & 3.2 & $2.53 \mathrm{E}-07$ & NA & NA & NA & NA \\
\hline PA4531 & & hypothetical protein & NA & NA & 65.2 & $6.49 \mathrm{E}-13$ & NA & NA & NA & NA \\
\hline PA4603 & & hypothetical protein & NA & NA & 5.5 & $1.60 \mathrm{E}-08$ & NA & NA & NA & NA \\
\hline PA4608 & & hypothetical protein & NA & NA & 2.8 & 4.77E-07 & NA & NA & NA & NA \\
\hline PA4637 & & hypothetical protein & NA & NA & 2.3 & 4.11E-06 & NA & NA & NA & NA \\
\hline PA4643 & & hypothetical protein & NA & NA & NA & NA & 4.2 & $1.08 \mathrm{E}-06$ & NA & NA \\
\hline PA4661 & $\operatorname{pagL}$ & Lipid A 3-O-deacylase & NA & NA & NA & NA & 2.1 & $0.00 \mathrm{E}+00$ & 2.2 & $0.00 \mathrm{E}+00$ \\
\hline PA4673 & & conserved hypothetical protein & NA & NA & NA & NA & 2.4 & $1.17 \mathrm{E}-07$ & NA & NA \\
\hline PA4684 & & hypothetical protein & NA & NA & NA & NA & 3.5 & $1.82 \mathrm{E}-05$ & NA & NA \\
\hline PA4691 & & hypothetical protein & NA & NA & 33.7 & $4.45 \mathrm{E}-07$ & NA & NA & NA & NA \\
\hline PA4692 & & conserved hypothetical protein & NA & NA & NA & NA & 3.6 & $1.04 \mathrm{E}-05$ & NA & NA \\
\hline PA4718 & & hypothetical protein & NA & NA & NA & NA & 2.9 & $1.26 \mathrm{E}-05$ & NA & NA \\
\hline PA4866 & & conserved hypothetical protein & NA & NA & 26.4 & $9.58 \mathrm{E}-06$ & NA & NA & NA & NA \\
\hline PA4881 & & hypothetical protein & NA & NA & NA & NA & 192.1 & $2.82 \mathrm{E}-14$ & 200.1 & $0.00 \mathrm{E}+00$ \\
\hline PA5061 & & conserved hypothetical protein & NA & NA & 2.0 & $0.00 \mathrm{E}+00$ & NA & NA & NA & NA \\
\hline PA5137 & & hypothetical protein & NA & NA & NA & NA & 7.4 & $4.76 \mathrm{E}-07$ & NA & NA \\
\hline
\end{tabular}




\begin{tabular}{|c|c|c|c|c|c|c|c|c|c|c|}
\hline PA5184 & & hypothetical protein & NA & NA & NA & NA & 4.2 & $6.16 \mathrm{E}-06$ & NA & NA \\
\hline PA5209 & & hypothetical protein & NA & NA & NA & NA & 4.0 & $1.85 \mathrm{E}-06$ & NA & NA \\
\hline PA5222 & & hypothetical protein & NA & NA & 2.2 & 8.02E-07 & NA & NA & NA & NA \\
\hline PA5257 & & hypothetical protein & NA & NA & 2.1 & $9.47 \mathrm{E}-08$ & NA & NA & NA & NA \\
\hline PA5340 & & hypothetical protein & NA & NA & NA & NA & 3.4 & $9.35 \mathrm{E}-08$ & NA & NA \\
\hline PA5406 & & hypothetical protein & NA & NA & NA & NA & 2.9 & $1.06 \mathrm{E}-07$ & 3.1 & $1.01 \mathrm{E}-05$ \\
\hline PA5457 & & hypothetical protein & NA & NA & 8.3 & $5.46 \mathrm{E}-07$ & NA & NA & NA & NA \\
\hline PA5462 & & hypothetical protein & NA & NA & 5.5 & $7.92 \mathrm{E}-11$ & NA & NA & NA & NA \\
\hline PA5467 & & hypothetical protein & NA & NA & 37.7 & $8.26 \mathrm{E}-08$ & NA & NA & NA & NA \\
\hline PA5517 & & conserved hypothetical protein & NA & NA & 6.9 & $7.51 \mathrm{E}-08$ & NA & NA & NA & NA \\
\hline PA5532 & & hypothetical protein & NA & NA & 27.0 & $7.20 \mathrm{E}-06$ & NA & NA & NA & NA \\
\hline PA5537 & & hypothetical protein & NA & NA & 32.8 & $6.40 \mathrm{E}-07$ & NA & NA & NA & NA \\
\hline \multicolumn{11}{|c|}{ Membrane proteins } \\
\hline PA0291 & oprE & $\begin{array}{l}\text { Anaerobically-induced outer membrane } \\
\text { porin OprE precursor }\end{array}$ & NA & NA & NA & NA & 2.0 & 4.13E-07 & NA & NA \\
\hline PA0303 & $\operatorname{spu} G$ & polyamine transport protein PotH & NA & NA & NA & NA & 3.2 & $6.85 \mathrm{E}-07$ & 3.1 & $6.63 \mathrm{E}-10$ \\
\hline PA0334 & \multirow{10}{*}{ braD } & $\begin{array}{l}\text { probable major facilitator superfamily } \\
\text { (MFS) transporter }\end{array}$ & NA & NA & NA & NA & 14.7 & $1.45 \mathrm{E}-05$ & NA & NA \\
\hline PA0752 & & conserved hypothetical protein & NA & NA & 11.1 & 5.64E-06 & NA & NA & NA & NA \\
\hline PA0789 & & probable amino acid permease & NA & NA & NA & NA & 2.3 & $9.45 \mathrm{E}-08$ & NA & NA \\
\hline PA1048 & & $\begin{array}{l}\text { probable outer membrane protein } \\
\text { precursor }\end{array}$ & NA & NA & 2.6 & $0.00 \mathrm{E}+00$ & NA & NA & NA & NA \\
\hline PA1073 & & $\begin{array}{l}\text { branched-chain amino acid transport } \\
\text { protein BraD }\end{array}$ & NA & NA & NA & NA & 2.7 & 8.22E-08 & 4.1 & $4.46 \mathrm{E}-07$ \\
\hline PA1126 & & hypothetical protein & NA & NA & 6.9 & $8.15 \mathrm{E}-08$ & NA & NA & NA & NA \\
\hline PA1170 & & conserved hypothetical protein & NA & NA & NA & NA & 7.4 & $3.21 \mathrm{E}-07$ & NA & NA \\
\hline PA1577 & & hypothetical protein & NA & NA & NA & NA & 25.6 & $6.69 \mathrm{E}-07$ & NA & NA \\
\hline PA1767 & & hypothetical protein & NA & NA & 2.3 & $1.49 \mathrm{E}-08$ & NA & NA & NA & NA \\
\hline PA2114 & & $\begin{array}{l}\text { probable major facilitator superfamily } \\
\text { (MFS) transporter }\end{array}$ & NA & NA & NA & NA & 2.3 & $7.75 \mathrm{E}-14$ & NA & NA \\
\hline
\end{tabular}




\begin{tabular}{|c|c|c|c|c|c|c|c|c|c|c|}
\hline PA2241 & $p s l K$ & PslL & NA & NA & NA & NA & 5.8 & $1.80 \mathrm{E}-05$ & NA & NA \\
\hline PA2415 & & hypothetical protein & NA & NA & 32.1 & 8.49E-07 & NA & NA & NA & NA \\
\hline PA2495 & $\operatorname{oprN}$ & $\begin{array}{l}\text { Multidrug efflux outer membrane } \\
\text { protein OprN precursor }\end{array}$ & NA & NA & NA & NA & 345.7 & $0.00 \mathrm{E}+00$ & 496.8 & $3.36 \mathrm{E}-13$ \\
\hline PA2710 & & hypothetical protein & NA & NA & 5.5 & $1.24 \mathrm{E}-05$ & NA & NA & NA & NA \\
\hline PA2811 & & $\begin{array}{l}\text { probable permease of } \mathrm{ABC}-2 \\
\text { transporter }\end{array}$ & NA & NA & NA & NA & 3.2 & $1.11 \mathrm{E}-07$ & 2.5 & 7.47E-07 \\
\hline PA2868 & & hypothetical protein & NA & NA & NA & NA & 8.4 & $9.75 \mathrm{E}-09$ & NA & NA \\
\hline PA3041 & & hypothetical protein & NA & NA & 8.3 & $0.00 \mathrm{E}+00$ & NA & NA & NA & NA \\
\hline PA3145 & $w b p L$ & glycosyltransferase WbpL & NA & NA & 2.4 & $1.66 \mathrm{E}-12$ & NA & NA & NA & NA \\
\hline PA3602 & & conserved hypothetical protein & NA & NA & NA & NA & 3.8 & $1.11 \mathrm{E}-08$ & NA & NA \\
\hline PA3827 & & conserved hypothetical protein & NA & NA & 2.3 & 4.77E-07 & NA & NA & NA & NA \\
\hline PA4338 & & hypothetical protein & NA & NA & 5.1 & $8.11 \mathrm{E}-07$ & NA & NA & NA & NA \\
\hline PA4355 & & $\begin{array}{l}\text { probable major facilitator superfamily } \\
\text { (MFS) transporter }\end{array}$ & NA & NA & NA & NA & 8.4 & $1.07 \mathrm{E}-05$ & 10.3 & $8.55 \mathrm{E}-11$ \\
\hline PA4455 & & probable permease of $\mathrm{ABC}$ transporter & NA & NA & NA & NA & 2.3 & $6.35 \mathrm{E}-10$ & NA & NA \\
\hline PA4754 & & hypothetical protein & NA & NA & NA & NA & 2.3 & $2.50 \mathrm{E}-07$ & 2.8 & $1.21 \mathrm{E}-13$ \\
\hline PA5155 & & $\begin{array}{l}\text { amino acid } \\
\text { (lysine/arginine/ornithine/histidine/octo } \\
\text { pine) ABC transporter membrane } \\
\text { protein }\end{array}$ & NA & NA & NA & NA & 3.5 & $1.57 \mathrm{E}-09$ & 4.1 & 4.15E-09 \\
\hline PA5170 & $\operatorname{arcD}$ & arginine/ornithine antiporter & NA & NA & 2.1 & $8.49 \mathrm{E}-14$ & NA & NA & NA & NA \\
\hline PA5230 & & probable permease of $\mathrm{ABC}$ transporter & NA & NA & 2.4 & $8.24 \mathrm{E}-07$ & NA & NA & NA & NA \\
\hline PA5231 & & $\begin{array}{l}\text { probable ATP-binding/permease fusion } \\
\text { ABC transporter }\end{array}$ & NA & NA & 3.8 & $2.30 \mathrm{E}-11$ & NA & NA & NA & NA \\
\hline PA5430 & & hypothetical protein & NA & NA & NA & NA & 2.7 & $3.90 \mathrm{E}-06$ & NA & NA \\
\hline PA5479 & $g l t P$ & proton-glutamate symporter & NA & NA & NA & NA & 2.3 & $8.05 \mathrm{E}-08$ & NA & NA \\
\hline \multicolumn{11}{|c|}{ Motility \& Attachment } \\
\hline PA0171 & & hypothetical protein & NA & NA & NA & NA & 4.2 & $1.32 \mathrm{E}-09$ & NA & NA \\
\hline
\end{tabular}




\begin{tabular}{|c|c|c|c|c|c|c|c|c|c|c|}
\hline \multicolumn{11}{|c|}{ Non-coding RNA gene } \\
\hline $\begin{array}{c}\text { PA0887. } \\
1\end{array}$ & $P 7$ & P7 & NA & NA & NA & NA & 6.3 & $0.00 \mathrm{E}+00$ & 3.1 & $5.35 \mathrm{E}-14$ \\
\hline $\begin{array}{l}\text { PA1112. } \\
\quad 1\end{array}$ & & & NA & NA & NA & NA & 2.3 & $0.00 \mathrm{E}+00$ & NA & NA \\
\hline $\begin{array}{c}\text { PA3366. } \\
1\end{array}$ & amiL & AmiL & NA & NA & NA & NA & 2.5 & $1.82 \mathrm{E}-13$ & NA & NA \\
\hline $\begin{array}{c}\text { PA4406. } \\
1\end{array}$ & & & NA & NA & 2.5 & $0.00 \mathrm{E}+00$ & NA & NA & NA & NA \\
\hline $\begin{array}{c}\text { PA4541. } \\
1\end{array}$ & & tRNA-Lys & NA & NA & NA & NA & 2.7 & $0.00 \mathrm{E}+00$ & NA & NA \\
\hline \multicolumn{11}{|c|}{ Nucleotide biosynthesis and metabolism } \\
\hline PA0401 & & noncatalytic dihydroorotase-like protein & NA & NA & 2.6 & $6.88 \mathrm{E}-07$ & NA & NA & NA & NA \\
\hline PA0945 & purM & $\begin{array}{l}\text { phosphoribosylaminoimidazole } \\
\text { synthetase }\end{array}$ & NA & NA & NA & NA & 2.0 & $7.03 \mathrm{E}-06$ & 2.1 & $1.54 \mathrm{E}-06$ \\
\hline PA1013 & purC & $\begin{array}{l}\text { phosphoribosylaminoimidazole- } \\
\text { succinocarboxamide synthase }\end{array}$ & NA & NA & NA & NA & 2.0 & $2.77 \mathrm{E}-06$ & NA & NA \\
\hline PA2876 & pyrF & orotidine 5 '-phosphate decarboxylase & NA & NA & 6.9 & $9.97 \mathrm{E}-07$ & NA & NA & NA & NA \\
\hline PA2962 & tmk & thymidylate kinase & NA & NA & NA & NA & 3.4 & $1.11 \mathrm{E}-05$ & 3.8 & $2.65 \mathrm{E}-12$ \\
\hline PA4314 & purU1 & formyltetrahydrofolate deformylase & NA & NA & NA & NA & 2.0 & $1.09 \mathrm{E}-06$ & NA & NA \\
\hline PA4855 & $\operatorname{purD}$ & phosphoribosylamine--glycine ligase & NA & NA & NA & NA & 2.5 & $1.48 \mathrm{E}-05$ & NA & NA \\
\hline PA5336 & $g m k$ & guanylate kinase & NA & NA & NA & NA & 4.9 & $6.05 \mathrm{E}-07$ & NA & NA \\
\hline \multicolumn{11}{|c|}{ Protein secretion/export apparatus } \\
\hline PA0071 & $\operatorname{tag} R 1$ & TagR1 & NA & NA & 2.1 & $8.53 \mathrm{E}-09$ & NA & NA & NA & NA \\
\hline PA0091 & $\operatorname{vgr} G 1$ & VgrG1 & NA & NA & NA & NA & 3.2 & $9.81 \mathrm{E}-06$ & NA & NA \\
\hline PA3099 & $x c p V$ & general secretion pathway protein I & NA & NA & 4.1 & $1.69 \mathrm{E}-05$ & NA & NA & NA & NA \\
\hline PA3100 & $x c p U$ & $\begin{array}{l}\text { General secretion pathway outer } \\
\text { membrane protein } \mathrm{H} \text { precursor }\end{array}$ & NA & NA & 2.8 & $7.09 \mathrm{E}-07$ & NA & NA & NA & NA \\
\hline PA4559 & $\operatorname{lsp} A$ & prolipoprotein signal peptidase & NA & NA & NA & NA & 2.1 & $1.45 \mathrm{E}-05$ & NA & NA \\
\hline
\end{tabular}




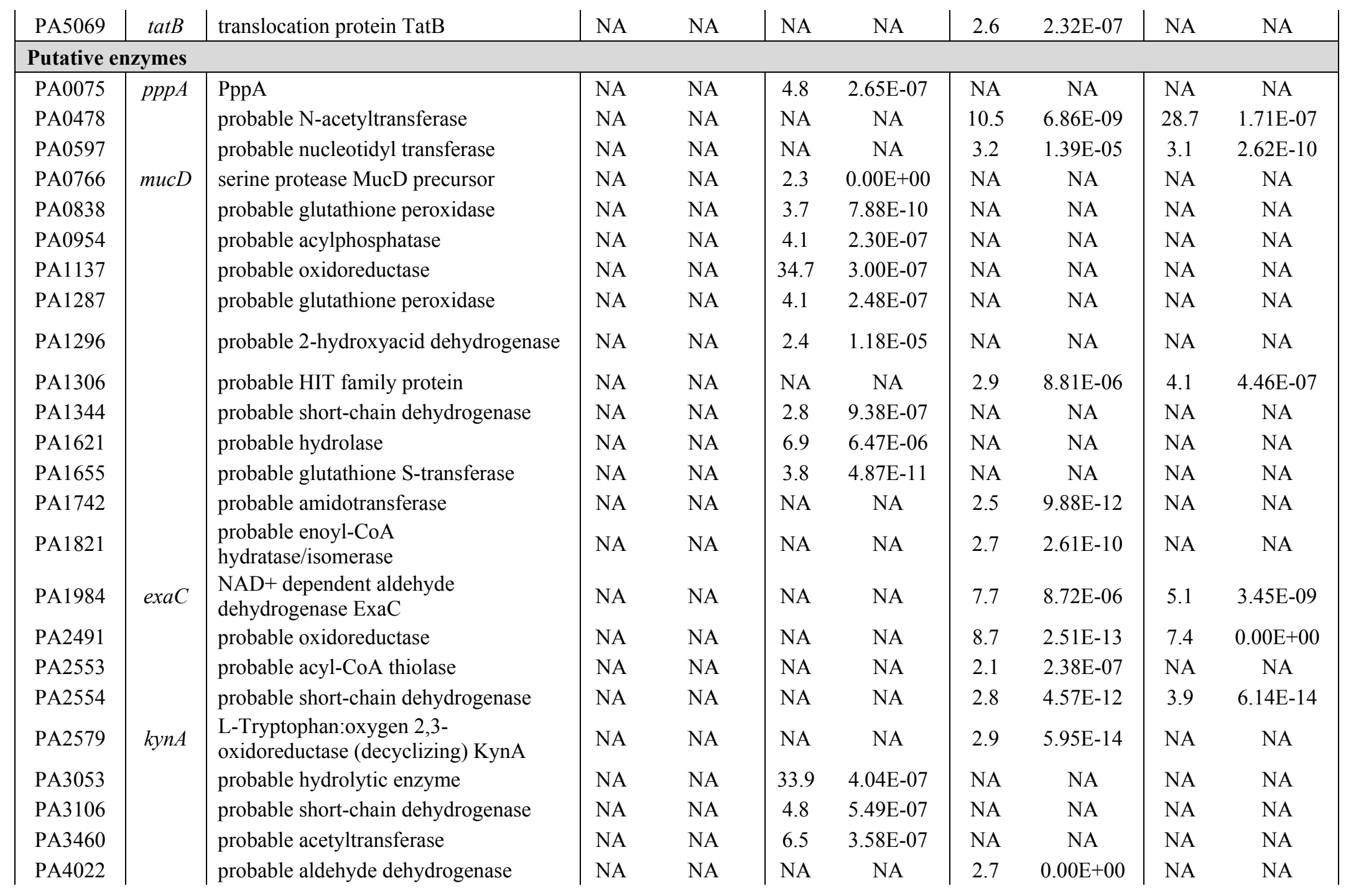




\begin{tabular}{|c|c|c|c|c|c|c|c|c|c|c|}
\hline $\begin{array}{l}\text { PA4576 } \\
\text { PA5432 } \\
\end{array}$ & & $\begin{array}{l}\text { probable ATP-dependent protease } \\
\text { probable acetyltransferase }\end{array}$ & $\begin{array}{l}\text { NA } \\
\text { NA }\end{array}$ & $\begin{array}{l}\text { NA } \\
\text { NA }\end{array}$ & $\begin{array}{l}\text { NA } \\
\text { NA }\end{array}$ & $\begin{array}{l}\text { NA } \\
\text { NA }\end{array}$ & $\begin{array}{c}2.3 \\
19.5\end{array}$ & $\begin{array}{l}2.40 \mathrm{E}-06 \\
1.87 \mathrm{E}-05 \\
\end{array}$ & $\begin{array}{l}\text { NA } \\
\text { NA }\end{array}$ & $\begin{array}{l}\text { NA } \\
\text { NA }\end{array}$ \\
\hline \multicolumn{11}{|c|}{ Secreted Factors (toxins, enzymes, alginate) } \\
\hline PA1844 & tsel & Tse1 & NA & NA & 29.4 & $2.66 \mathrm{E}-06$ & NA & NA & NA & NA \\
\hline \multicolumn{11}{|c|}{ Transcription, RNA processing and degradation } \\
\hline PA2626 & $\operatorname{trm} U$ & tRNA methyltransferase & NA & NA & NA & NA & 3.1 & $1.79 \mathrm{E}-10$ & NA & NA \\
\hline PA3861 & $r h l$ & ATP-dependent RNA helicase RhlB & NA & NA & NA & NA & 2.0 & $1.77 \mathrm{E}-05$ & NA & NA \\
\hline PA4238 & rроA & $\begin{array}{l}\text { DNA-directed RNA polymerase alpha } \\
\text { chain }\end{array}$ & NA & NA & NA & NA & 2.0 & $2.82 \mathrm{E}-14$ & NA & NA \\
\hline PA4262 & $r p l D$ & 50S ribosomal protein L4 & NA & NA & NA & NA & 2.2 & $0.00 \mathrm{E}+00$ & NA & NA \\
\hline \multicolumn{11}{|c|}{ Transcriptional regulators } \\
\hline PA0233 & & probable transcriptional regulator & NA & NA & 29.1 & $3.11 \mathrm{E}-06$ & NA & NA & NA & NA \\
\hline PA0448 & & probable transcriptional regulator & NA & NA & 3.3 & $2.81 \mathrm{E}-07$ & NA & NA & NA & NA \\
\hline PA0601 & & $\begin{array}{l}\text { probable two-component response } \\
\text { regulator }\end{array}$ & NA & NA & 5.5 & $1.68 \mathrm{E}-05$ & NA & NA & NA & NA \\
\hline PA0764 & тисв & $\begin{array}{l}\text { negative regulator for alginate } \\
\text { biosynthesis MucB }\end{array}$ & NA & NA & 2.3 & $0.00 \mathrm{E}+00$ & NA & NA & NA & NA \\
\hline PA1015 & & probable transcriptional regulator & NA & NA & NA & NA & 21.9 & $5.20 \mathrm{E}-06$ & NA & NA \\
\hline PA1850 & & probable transcriptional regulator & NA & NA & NA & NA & 21.8 & $5.43 \mathrm{E}-06$ & NA & NA \\
\hline PA2320 & gntR & transcriptional regulator GntR & NA & NA & 26.5 & 8.99E-06 & NA & NA & NA & NA \\
\hline PA3133 & & probable transcriptional regulator & NA & NA & 50.4 & $3.53 \mathrm{E}-10$ & NA & NA & NA & NA \\
\hline PA4174 & & probable transcriptional regulator & NA & NA & 29.6 & $2.46 \mathrm{E}-06$ & NA & NA & NA & NA \\
\hline PA5483 & $\operatorname{alg} B$ & $\begin{array}{l}\text { two-component response regulator } \\
\text { AlgB }\end{array}$ & NA & NA & 3.1 & $6.72 \mathrm{E}-07$ & NA & NA & NA & NA \\
\hline \multicolumn{11}{|c|}{ Translation, post-translational modification, degradation } \\
\hline PA0579 & $r p s U$ & 30S ribosomal protein S21 & NA & NA & NA & NA & 2.1 & $0.00 \mathrm{E}+00$ & $\mathrm{NA}$ & NA \\
\hline PA1796 & folD & $\begin{array}{l}\text { 5,10-methylene-tetrahydrofolate } \\
\text { dehydrogenase / cyclohydrolase }\end{array}$ & NA & NA & NA & NA & 2.4 & $9.36 \mathrm{E}-06$ & NA & NA \\
\hline PA2740 & pheS & $\begin{array}{l}\text { phenylalanyl-tRNA synthetase, alpha- } \\
\text { subunit }\end{array}$ & NA & NA & NA & NA & 2.2 & $5.58 \mathrm{E}-06$ & NA & NA \\
\hline
\end{tabular}




\begin{tabular}{|c|c|c|c|c|c|c|c|c|c|c|}
\hline PA2742 & rpmI & 50S ribosomal protein L35 & NA & NA & NA & NA & 2.0 & $2.93 \mathrm{E}-13$ & NA & NA \\
\hline PA3655 & tsf & elongation factor Ts & NA & NA & NA & NA & 2.0 & $0.00 \mathrm{E}+00$ & NA & NA \\
\hline PA3656 & $r p s B$ & $30 \mathrm{~S}$ ribosomal protein $\mathrm{S} 2$ & NA & NA & NA & NA & 2.2 & $0.00 \mathrm{E}+00$ & NA & NA \\
\hline PA3818 & & extragenic suppressor protein SuhB & NA & NA & NA & NA & 2.3 & $1.16 \mathrm{E}-06$ & NA & NA \\
\hline PA4237 & $r p l Q$ & 50S ribosomal protein L17 & NA & NA & NA & NA & 3.3 & $6.55 \mathrm{E}-13$ & NA & NA \\
\hline PA4244 & rplO & 50S ribosomal protein L15 & NA & NA & NA & NA & 2.3 & $5.02 \mathrm{E}-14$ & NA & NA \\
\hline PA4245 & rpmD & $50 \mathrm{~S}$ ribosomal protein L30 & NA & NA & NA & NA & 2.0 & $1.99 \mathrm{E}-13$ & NA & NA \\
\hline PA4246 & $r p s E$ & 30S ribosomal protein $\mathrm{S} 5$ & NA & NA & NA & NA & 2.0 & $1.86 \mathrm{E}-13$ & NA & NA \\
\hline PA4248 & $r p l F$ & 50S ribosomal protein L6 & NA & NA & NA & NA & 2.5 & $2.48 \mathrm{E}-13$ & NA & NA \\
\hline PA4250 & $r p s N$ & 30S ribosomal protein $\mathrm{S} 14$ & NA & NA & NA & NA & 2.3 & $4.15 \mathrm{E}-13$ & NA & NA \\
\hline PA4256 & $r p l P$ & 50S ribosomal protein L16 & NA & NA & NA & NA & 2.0 & $3.49 \mathrm{E}-13$ & NA & NA \\
\hline PA4257 & $r p s C$ & $30 \mathrm{~S}$ ribosomal protein $\mathrm{S} 3$ & NA & NA & NA & NA & 2.2 & $4.21 \mathrm{E}-13$ & NA & NA \\
\hline PA4258 & rplV & 50S ribosomal protein L22 & NA & NA & NA & NA & 2.2 & $8.86 \mathrm{E}-14$ & NA & NA \\
\hline PA4260 & rplB & 50S ribosomal protein L2 & NA & NA & NA & NA & 2.0 & $2.77 \mathrm{E}-13$ & NA & NA \\
\hline PA4267 & $r p s G$ & 30 S ribosomal protein $\mathrm{S} 7$ & NA & NA & NA & NA & 2.1 & $0.00 \mathrm{E}+00$ & NA & NA \\
\hline PA4268 & rps $L$ & $30 \mathrm{~S}$ ribosomal protein $\mathrm{S} 12$ & NA & NA & NA & NA & 2.0 & $3.30 \mathrm{E}-13$ & NA & NA \\
\hline PA4272 & rplJ & 50S ribosomal protein L10 & NA & NA & NA & NA & 2.1 & $4.21 \mathrm{E}-13$ & NA & NA \\
\hline PA4273 & rplA & $50 \mathrm{~S}$ ribosomal protein $\mathrm{L} 1$ & NA & NA & NA & NA & 2.1 & $3.57 \mathrm{E}-13$ & NA & NA \\
\hline PA4558 & & $\begin{array}{l}\text { probable peptidyl-prolyl cis-trans } \\
\text { isomerase, FkbP-type }\end{array}$ & NA & NA & NA & NA & 2.1 & $1.80 \mathrm{E}-05$ & NA & NA \\
\hline PA4567 & $\operatorname{rpm} A$ & 50S ribosomal protein L27 & NA & NA & NA & NA & 2.0 & $4.01 \mathrm{E}-14$ & NA & NA \\
\hline PA4665 & prfA & peptide chain release factor 1 & NA & NA & NA & NA & 2.7 & $6.05 \mathrm{E}-07$ & NA & NA \\
\hline PA4743 & $r b f A$ & ribosome-binding factor $\mathrm{A}$ & NA & NA & NA & NA & 3.0 & $2.94 \mathrm{E}-12$ & NA & NA \\
\hline PA4932 & rplI & $50 \mathrm{~S}$ ribosomal protein L9 & NA & NA & NA & NA & 2.3 & $4.08 \mathrm{E}-13$ & NA & NA \\
\hline PA5254 & & $\begin{array}{l}\text { probable peptidyl-prolyl cis-trans } \\
\text { isomerase, FkbP-type }\end{array}$ & NA & NA & NA & NA & 4.2 & $7.23 \mathrm{E}-07$ & NA & NA \\
\hline \multicolumn{11}{|c|}{ Transport of small molecules } \\
\hline PA0866 & $\operatorname{aroP} 2$ & $\begin{array}{l}\text { aromatic amino acid transport protein } \\
\text { AroP2 }\end{array}$ & NA & NA & NA & NA & 2.6 & $1.47 \mathrm{E}-11$ & 2.7 & $3.52 \mathrm{E}-14$ \\
\hline
\end{tabular}




\begin{tabular}{|c|c|c|c|c|c|c|c|c|c|c|}
\hline PA1071 & braF & $\begin{array}{l}\text { branched-chain amino acid transport } \\
\text { protein BraF }\end{array}$ & NA & NA & NA & NA & 3.0 & $1.38 \mathrm{E}-06$ & NA & NA \\
\hline PA1074 & braC & $\begin{array}{l}\text { branched-chain amino acid transport } \\
\text { protein BraC }\end{array}$ & NA & NA & NA & NA & 2.2 & $8.45 \mathrm{E}-14$ & NA & NA \\
\hline PA1862 & $\bmod B$ & molybdenum transport protein ModB & NA & NA & NA & NA & 6.3 & $2.88 \mathrm{E}-06$ & 39.8 & $5.06 \mathrm{E}-10$ \\
\hline PA2041 & & probable amino acid permease & NA & NA & NA & NA & 7.0 & $1.27 \mathrm{E}-05$ & 5.1 & 4.72E-07 \\
\hline PA2113 & opdO & pyroglutamate porin OpdO & NA & NA & NA & NA & 2.4 & $1.50 \mathrm{E}-14$ & 2.2 & $0.00 \mathrm{E}+00$ \\
\hline PA2408 & & $\begin{array}{l}\text { probable ATP-binding component of } \\
\text { ABC transporter }\end{array}$ & NA & NA & 8.3 & $7.48 \mathrm{E}-08$ & NA & NA & NA & NA \\
\hline PA2493 & $\operatorname{mex} E$ & $\begin{array}{l}\text { Resistance-Nodulation-Cell Division } \\
\text { (RND) multidrug efflux membrane } \\
\text { fusion protein MexE precursor }\end{array}$ & NA & NA & NA & NA & 298.8 & $0.00 \mathrm{E}+00$ & 113.0 & $2.70 \mathrm{E}-13$ \\
\hline PA2494 & $\operatorname{mexF}$ & $\begin{array}{l}\text { Resistance-Nodulation-Cell Division } \\
\text { (RND) multidrug efflux transporter } \\
\text { MexF }\end{array}$ & NA & NA & NA & NA & 578.6 & $1.82 \mathrm{E}-13$ & 547.2 & $0.00 \mathrm{E}+00$ \\
\hline PA2760 & oprQ & OprQ & NA & NA & NA & NA & 2.7 & $3.26 \mathrm{E}-13$ & NA & NA \\
\hline PA2812 & & $\begin{array}{l}\text { probable ATP-binding component of } \\
\text { ABC transporter }\end{array}$ & NA & NA & NA & NA & 5.4 & $2.82 \mathrm{E}-14$ & 4.4 & $1.95 \mathrm{E}-14$ \\
\hline PA3187 & & $\begin{array}{l}\text { probable ATP-binding component of } \\
\text { ABC transporter }\end{array}$ & NA & NA & 6.9 & $1.79 \mathrm{E}-11$ & NA & NA & NA & NA \\
\hline PA3531 & bfrB & bacterioferritin & NA & NA & NA & NA & 2.1 & $5.09 \mathrm{E}-09$ & NA & NA \\
\hline PA3838 & & $\begin{array}{l}\text { probable ATP-binding component of } \\
\text { ABC transporter }\end{array}$ & NA & NA & NA & NA & 5.9 & $1.89 \mathrm{E}-11$ & 77.5 & $4.11 \mathrm{E}-14$ \\
\hline PA4064 & & $\begin{array}{l}\text { probable ATP-binding component of } \\
\mathrm{ABC} \text { transporter }\end{array}$ & NA & NA & 29.1 & $3.04 \mathrm{E}-06$ & NA & NA & NA & NA \\
\hline PA4466 & & probable phosphoryl carrier protein & NA & NA & 2.1 & 4.31E-06 & 4.2 & $8.63 \mathrm{E}-08$ & 3.1 & $1.60 \mathrm{E}-12$ \\
\hline PA4500 & & $\begin{array}{l}\text { probable binding protein component of } \\
\text { ABC transporter }\end{array}$ & NA & NA & NA & NA & 2.0 & 8.61E-06 & NA & NA \\
\hline PA4514 & & $\begin{array}{l}\text { probable outer membrane receptor for } \\
\text { iron transport }\end{array}$ & NA & NA & 5.5 & $4.82 \mathrm{E}-06$ & NA & NA & NA & NA \\
\hline PA4590 & pra & protein activator & NA & NA & 2.8 & $1.17 \mathrm{E}-05$ & NA & NA & NA & NA \\
\hline
\end{tabular}




\begin{tabular}{|c|c|c|c|c|c|c|c|c|c|}
\hline PA5076 & $\begin{array}{l}\text { probable binding protein component of } \\
\text { ABC transporter }\end{array}$ & NA & NA & NA & NA & 2.5 & $2.42 \mathrm{E}-09$ & NA & NA \\
\hline PA5152 & $\begin{array}{l}\text { probable ATP-binding component of } \\
\text { ABC transporter }\end{array}$ & NA & NA & NA & NA & 3.1 & $3.78 \mathrm{E}-12$ & 2.5 & $8.06 \mathrm{E}-13$ \\
\hline PA5217 & $\begin{array}{l}\text { probable binding protein component of } \\
A B C \text { iron transporter }\end{array}$ & NA & NA & 2.7 & $2.60 \mathrm{E}-09$ & NA & NA & NA & NA \\
\hline
\end{tabular}


Supplementary Table 3: AmpR-B-lactam-dependent genes. The genes that are either positively or negatively regulated by AmpR when exposed to $\beta$-lactam stress are classified based on information in Supplementary Table 1. Only genes that are $\geq 2.0$, Bonferroni correction $(<0.05)$ is listed. Functional categorization, gene ID and gene/product names are from the Pseudomonas Genome database (27). Lack of expression of a gene under any of the four conditions is denoted as NA.

\begin{tabular}{|c|c|c|c|c|c|c|c|c|c|c|}
\hline \multirow[b]{2}{*}{ Gene ID } & \multirow{2}{*}{$\begin{array}{l}\text { Gene } \\
\text { name }\end{array}$} & \multirow[b]{2}{*}{ Product Name } & \multicolumn{2}{|c|}{ Condition 1} & \multicolumn{2}{|c|}{ Condition 2} & \multicolumn{2}{|c|}{ Condition 3} & \multicolumn{2}{|c|}{ Condition 4} \\
\hline & & & FC & $\begin{array}{c}\text { Corrected } \\
p \text {-value }\end{array}$ & FC & $\begin{array}{c}\text { Corrected } \\
p \text {-value }\end{array}$ & FC & $\begin{array}{c}\text { Corrected } \\
p \text {-value }\end{array}$ & FC & $\begin{array}{c}\text { Corrected } \\
p \text {-value }\end{array}$ \\
\hline \multicolumn{11}{|c|}{ Genes positively regulated by AmpR upon b-lactam exposure (236) } \\
\hline \multicolumn{11}{|c|}{ Adaptation, Protection } \\
\hline PA0176 & aer2 & aerotaxis transducer Aer2 & NA & NA & NA & NA & NA & NA & -5.2 & $4.53 \mathrm{E}-07$ \\
\hline PA0177 & & $\begin{array}{l}\text { probable purine-binding } \\
\text { chemotaxis protein }\end{array}$ & NA & NA & NA & NA & NA & NA & -73.9 & 4.27E-14 \\
\hline PA0848 & & $\begin{array}{l}\text { probable alkyl hydroperoxide } \\
\text { reductase }\end{array}$ & NA & NA & NA & NA & NA & NA & -4.9 & $3.48 \mathrm{E}-07$ \\
\hline PA1561 & aer & aerotaxis receptor Aer & NA & NA & NA & NA & NA & NA & -2.8 & $2.21 \mathrm{E}-07$ \\
\hline PA2400 & $p v d J$ & PvdJ & NA & NA & NA & NA & NA & NA & -4.4 & $8.65 \mathrm{E}-06$ \\
\hline PA2703 & $t s i 2$ & Tsi2 & NA & NA & NA & NA & NA & NA & -30.7 & $5.57 \mathrm{E}-07$ \\
\hline PA2826 & & probable glutathione peroxidase & 4.3 & $6.54 \mathrm{E}-09$ & NA & NA & NA & NA & -2.9 & $1.13 \mathrm{E}-06$ \\
\hline PA4110 & $\operatorname{ampC}$ & beta-lactamase precursor & 14.2 & $1.11 \mathrm{E}-13$ & NA & NA & NA & NA & -24.3 & $1.50 \mathrm{E}-14$ \\
\hline PA4915 & & probable chemotaxis transducer & NA & NA & NA & NA & NA & NA & -12.7 & $7.54 \mathrm{E}-10$ \\
\hline \multicolumn{11}{|c|}{ Amino acid biosynthesis and metabolism } \\
\hline PA0407 & $g s h B$ & glutathione synthetase & NA & NA & NA & NA & NA & NA & -4.4 & $1.72 \mathrm{E}-07$ \\
\hline PA0546 & metK & methionine adenosyltransferase & NA & NA & NA & NA & NA & NA & -2.2 & $1.95 \mathrm{E}-13$ \\
\hline PA3537 & $\arg F$ & $\begin{array}{l}\text { ornithine carbamoyltransferase, } \\
\text { anabolic }\end{array}$ & 3.8 & $5.55 \mathrm{E}-06$ & NA & NA & NA & NA & -3.9 & $1.92 \mathrm{E}-06$ \\
\hline PA4519 & speC & ornithine decarboxylase & NA & NA & NA & NA & NA & NA & -2.9 & 4.17E-08 \\
\hline \multicolumn{11}{|c|}{ Biosynthesis of cofactors, prosthetic groups and carriers } \\
\hline PA0672 & hemO & heme oxygenase & NA & NA & NA & NA & NA & NA & -3.4 & $1.93 \mathrm{E}-07$ \\
\hline PA1049 & $p d x H$ & $\begin{array}{l}\text { pyridoxine } 5 \text { '-phosphate } \\
\text { oxidase }\end{array}$ & NA & NA & NA & NA & NA & NA & -2.6 & 8.07E-06 \\
\hline
\end{tabular}




\begin{tabular}{|c|c|c|c|c|c|c|c|c|c|c|}
\hline PA3397 & $f p r$ & ferredoxin--NADP + reductase & NA & NA & NA & NA & NA & NA & -2.8 & $0.00 \mathrm{E}+00$ \\
\hline \multicolumn{11}{|c|}{ Carbon compound catabolism } \\
\hline PA2509 & catB & muconate cycloisomerase I & NA & NA & NA & NA & NA & NA & -44.8 & $9.76 \mathrm{E}-10$ \\
\hline PA5350 & rubA2 & Rubredoxin 2 & 2.1 & $1.44 \mathrm{E}-05$ & NA & NA & NA & NA & -2.9 & $3.28 \mathrm{E}-09$ \\
\hline PA5351 & rubAl & Rubredoxin 1 & 2.5 & $0.00 \mathrm{E}+00$ & NA & NA & NA & NA & -2.3 & $6.60 \mathrm{E}-14$ \\
\hline \multicolumn{11}{|c|}{ Cell division } \\
\hline PA1462 & & $\begin{array}{l}\text { probable plasmid partitioning } \\
\text { protein }\end{array}$ & 2.1 & $2.17 \mathrm{E}-07$ & NA & NA & NA & NA & -3.4 & $0.00 \mathrm{E}+00$ \\
\hline \multicolumn{11}{|c|}{ Cell wall / LPS / capsule } \\
\hline PA1077 & $\operatorname{flg} B$ & $\begin{array}{l}\text { flagellar basal-body rod protein } \\
\text { FlgB }\end{array}$ & NA & NA & NA & NA & NA & NA & -88.0 & $2.36 \mathrm{E}-14$ \\
\hline PA1079 & $f \lg D$ & $\begin{array}{l}\text { flagellar basal-body rod } \\
\text { modification protein FlgD }\end{array}$ & NA & NA & NA & NA & NA & NA & -2.9 & 8.59E-07 \\
\hline PA1101 & $f l i F$ & $\begin{array}{l}\text { Flagella M-ring outer } \\
\text { membrane protein precursor }\end{array}$ & NA & NA & NA & NA & NA & NA & -2.7 & $2.92 \mathrm{E}-09$ \\
\hline PA1130 & $\operatorname{rhlC}$ & rhamnosyltransferase 2 & NA & NA & NA & NA & NA & NA & -36.7 & $3.72 \mathrm{E}-08$ \\
\hline \multicolumn{11}{|c|}{ Central intermediary metabolism } \\
\hline PA0710 & gloA2 & lactoylglutathione lyase & NA & NA & NA & NA & NA & NA & -36.3 & $4.53 \mathrm{E}-08$ \\
\hline PA4443 & cys $D$ & ATP sulfurylase small subunit & NA & NA & NA & NA & NA & NA & -2.6 & 2.74E-09 \\
\hline PA4865 & ureA & urease gamma subunit & 5.7 & 4.49E-06 & NA & NA & NA & NA & -47.4 & $3.02 \mathrm{E}-10$ \\
\hline PA4868 & ureC & urease alpha subunit & NA & NA & NA & NA & NA & NA & -33.8 & $1.40 \mathrm{E}-07$ \\
\hline \multicolumn{11}{|c|}{ Chemotaxis, Adaptation, Protection } \\
\hline PA0178 & & probable two-component sensor & NA & NA & NA & NA & NA & NA & -5.2 & $1.88 \mathrm{E}-07$ \\
\hline \multicolumn{11}{|c|}{ DNA replication, recombination, modification and repair } \\
\hline PA4042 & $x s e B$ & $\begin{array}{l}\text { exodeoxyribonuclease VII } \\
\text { small subunit }\end{array}$ & 2.8 & $3.91 \mathrm{E}-06$ & NA & NA & NA & NA & -2.9 & $1.13 \mathrm{E}-06$ \\
\hline \multicolumn{11}{|c|}{ Energy metabolism } \\
\hline PA1317 & $c y o A$ & $\begin{array}{l}\text { cytochrome o ubiquinol } \\
\text { oxidase subunit II }\end{array}$ & NA & NA & NA & NA & NA & NA & -43.3 & $1.96 \mathrm{E}-09$ \\
\hline PA1318 & $c y o B$ & $\begin{array}{l}\text { cytochrome o ubiquinol } \\
\text { oxidase subunit I }\end{array}$ & NA & NA & NA & NA & NA & NA & -5.8 & $4.86 \mathrm{E}-06$ \\
\hline
\end{tabular}




\begin{tabular}{|c|c|c|c|c|c|c|c|c|c|c|}
\hline PA1319 & cyoC & $\begin{array}{l}\text { cytochrome o ubiquinol } \\
\text { oxidase subunit III }\end{array}$ & NA & NA & NA & NA & NA & NA & -34.2 & $1.15 \mathrm{E}-07$ \\
\hline PA1883 & & $\begin{array}{l}\text { probable NADH- } \\
\text { ubiquinone/plastoquinone } \\
\text { oxidoreductase }\end{array}$ & NA & NA & NA & NA & NA & NA & -36.0 & $5.12 \mathrm{E}-08$ \\
\hline PA1983 & $\operatorname{exaB}$ & cytochrome c550 & 32.8 & $4.68 \mathrm{E}-07$ & NA & NA & NA & NA & -32.8 & $2.16 \mathrm{E}-07$ \\
\hline PA2646 & пиок & $\begin{array}{l}\text { NADH dehydrogenase I chain } \\
\text { K }\end{array}$ & 2.4 & 4.37E-06 & -3.6 & $1.65 \mathrm{E}-07$ & NA & NA & -4.9 & $4.71 \mathrm{E}-13$ \\
\hline PA2998 & $n q r B$ & $\begin{array}{l}\mathrm{Na}+\text {-translocating } \\
\text { NADH:ubiquinone } \\
\text { oxidoreductase subunit Nrq2 }\end{array}$ & NA & NA & NA & NA & NA & NA & -2.4 & $1.05 \mathrm{E}-05$ \\
\hline \multicolumn{11}{|c|}{ Fatty acid and phospholipid metabolism } \\
\hline PA2856 & tes $A$ & $\begin{array}{l}\text { acyl-CoA thioesterase I } \\
\text { precursor }\end{array}$ & NA & NA & NA & NA & NA & NA & -2.4 & $8.32 \mathrm{E}-07$ \\
\hline PA3223 & azoR3 & AzoR3, azoreductase 3 & NA & NA & NA & NA & NA & $\mathrm{NA}$ & -5.8 & 9.35E-09 \\
\hline \multicolumn{11}{|c|}{ Hypothetical, unclassified, unknown } \\
\hline PA0015 & & hypothetical protein & 6.6 & $1.37 \mathrm{E}-13$ & NA & NA & NA & NA & -3.4 & $3.93 \mathrm{E}-13$ \\
\hline PA0028 & & hypothetical protein & NA & NA & NA & NA & NA & NA & -47.6 & $2.73 \mathrm{E}-10$ \\
\hline PA0052 & & hypothetical protein & NA & NA & NA & NA & NA & NA & -33.5 & $1.59 \mathrm{E}-07$ \\
\hline PA0055 & & hypothetical protein & NA & NA & NA & NA & NA & NA & -4.9 & $2.57 \mathrm{E}-08$ \\
\hline PA0065 & & hypothetical protein & 5.7 & $2.08 \mathrm{E}-10$ & NA & NA & NA & NA & -2.6 & $1.08 \mathrm{E}-05$ \\
\hline PA0115 & & conserved hypothetical protein & 7.1 & $1.10 \mathrm{E}-10$ & NA & NA & NA & NA & -4.9 & 3.38E-09 \\
\hline PA0144 & & hypothetical protein & 2.8 & $1.28 \mathrm{E}-05$ & NA & NA & NA & NA & -6.8 & $5.10 \mathrm{E}-11$ \\
\hline PA0187 & & hypothetical protein & 35.2 & $1.64 \mathrm{E}-07$ & NA & NA & NA & NA & -35.2 & 7.32E-08 \\
\hline PA0255 & & conserved hypothetical protein & 5.1 & $1.24 \mathrm{E}-10$ & NA & NA & NA & NA & -2.9 & $5.10 \mathrm{E}-07$ \\
\hline PA0271 & & hypothetical protein & NA & NA & NA & NA & NA & NA & -25.2 & $6.47 \mathrm{E}-06$ \\
\hline PA0346 & & hypothetical protein & NA & NA & NA & NA & NA & NA & -39.6 & $1.04 \mathrm{E}-08$ \\
\hline PA0384 & & hypothetical protein & 27.8 & 4.08E-06 & NA & NA & NA & NA & -27.8 & $1.99 \mathrm{E}-06$ \\
\hline PA0419 & & conserved hypothetical protein & NA & NA & NA & NA & NA & NA & -5.8 & $6.94 \mathrm{E}-08$ \\
\hline PA0431 & & hypothetical protein & NA & NA & NA & NA & NA & NA & -8.8 & $0.00 \mathrm{E}+00$ \\
\hline PA0481 & & hypothetical protein & 7.1 & $7.01 \mathrm{E}-11$ & NA & NA & NA & NA & -80.9 & $5.39 \mathrm{E}-15$ \\
\hline
\end{tabular}




\begin{tabular}{|c|c|c|c|c|c|c|c|c|c|}
\hline PA0484 & conserved hypothetical protein & NA & NA & NA & NA & NA & NA & -69.6 & $2.18 \mathrm{E}-14$ \\
\hline PA0486 & conserved hypothetical protein & NA & NA & NA & NA & NA & NA & -36.8 & $3.55 \mathrm{E}-08$ \\
\hline PA0526 & hypothetical protein & 36.8 & $8.06 \mathrm{E}-08$ & NA & NA & NA & NA & -36.8 & $3.55 \mathrm{E}-08$ \\
\hline PA0563 & conserved hypothetical protein & NA & NA & NA & NA & NA & NA & -2.2 & $2.73 \mathrm{E}-15$ \\
\hline PA0581 & conserved hypothetical protein & NA & NA & NA & NA & NA & NA & -5.8 & $9.72 \mathrm{E}-10$ \\
\hline PA0588 & conserved hypothetical protein & NA & NA & NA & NA & NA & NA & -2.4 & $3.27 \mathrm{E}-12$ \\
\hline PA0664 & hypothetical protein & 2.1 & $2.02 \mathrm{E}-07$ & NA & NA & NA & NA & -2.1 & $3.21 \mathrm{E}-08$ \\
\hline PA0734 & hypothetical protein & 4.3 & 5.13E-08 & NA & NA & NA & NA & -2.9 & 4.72E-06 \\
\hline PA0823 & hypothetical protein & 30.7 & $1.18 \mathrm{E}-06$ & NA & NA & NA & NA & -30.7 & $5.57 \mathrm{E}-07$ \\
\hline PA0874 & hypothetical protein & NA & NA & NA & NA & NA & NA & -26.9 & $3.04 \mathrm{E}-06$ \\
\hline PA0938 & hypothetical protein & NA & NA & NA & NA & NA & NA & -2.2 & $0.00 \mathrm{E}+00$ \\
\hline PA0980 & hypothetical protein & NA & NA & NA & NA & NA & NA & -2.9 & $7.65 \mathrm{E}-06$ \\
\hline PA1036 & hypothetical protein & NA & NA & NA & NA & NA & NA & -2.6 & $1.47 \mathrm{E}-06$ \\
\hline PA1040 & hypothetical protein & NA & NA & NA & NA & NA & NA & -28.8 & $1.28 \mathrm{E}-06$ \\
\hline PA1043 & hypothetical protein & NA & NA & NA & NA & NA & NA & -3.4 & $9.38 \mathrm{E}-06$ \\
\hline PA1117 & hypothetical protein & 14.2 & $1.62 \mathrm{E}-13$ & NA & NA & NA & NA & -4.9 & $6.65 \mathrm{E}-11$ \\
\hline PA1133 & hypothetical protein & 40.9 & $1.30 \mathrm{E}-08$ & NA & NA & NA & NA & -40.9 & 5.61E-09 \\
\hline PA1154 & conserved hypothetical protein & NA & NA & NA & NA & NA & NA & -26.9 & $3.04 \mathrm{E}-06$ \\
\hline PA1198 & conserved hypothetical protein & NA & NA & NA & NA & NA & NA & -2.4 & $0.00 \mathrm{E}+00$ \\
\hline PA1215 & hypothetical protein & NA & NA & NA & NA & NA & NA & -27.9 & $1.95 \mathrm{E}-06$ \\
\hline PA1244 & hypothetical protein & NA & NA & NA & NA & NA & NA & -2.8 & $0.00 \mathrm{E}+00$ \\
\hline PA1358 & hypothetical protein & 72.0 & $0.00 \mathrm{E}+00$ & NA & NA & NA & NA & -3.9 & 2.93E-07 \\
\hline PA1369 & hypothetical protein & NA & NA & NA & NA & NA & NA & -2.3 & $8.58 \mathrm{E}-07$ \\
\hline PA1394 & hypothetical protein & 4.3 & $1.57 \mathrm{E}-08$ & 34.4 & $6.00 \mathrm{E}-09$ & NA & NA & -84.5 & 4.11E-14 \\
\hline PA1440 & hypothetical protein & NA & NA & NA & NA & NA & NA & -2.1 & $1.05 \mathrm{E}-07$ \\
\hline PA1531 & hypothetical protein & NA & NA & NA & NA & NA & NA & -41.4 & 4.50E-09 \\
\hline PA1545 & hypothetical protein & NA & NA & NA & NA & NA & NA & -2.3 & $2.06 \mathrm{E}-09$ \\
\hline PA1550 & hypothetical protein & NA & NA & NA & NA & NA & NA & -2.9 & $1.16 \mathrm{E}-08$ \\
\hline
\end{tabular}




\begin{tabular}{|c|c|c|c|c|c|c|c|c|c|}
\hline PA1575 & hypothetical protein & NA & NA & NA & NA & NA & NA & -26.0 & 4.49E-06 \\
\hline PA1728 & hypothetical protein & 3.2 & $9.16 \mathrm{E}-13$ & NA & NA & NA & NA & -2.6 & $3.96 \mathrm{E}-10$ \\
\hline PA1745 & hypothetical protein & NA & NA & NA & NA & NA & NA & -29.4 & $1.00 \mathrm{E}-06$ \\
\hline PA1874 & hypothetical protein & NA & NA & NA & NA & NA & NA & -12.7 & $7.98 \mathrm{E}-07$ \\
\hline PA1967 & hypothetical protein & NA & NA & NA & NA & NA & NA & -57.3 & $3.39 \mathrm{E}-12$ \\
\hline PA2036 & hypothetical protein & 8.5 & $2.50 \mathrm{E}-06$ & NA & NA & NA & NA & -41.3 & 4.80E-09 \\
\hline PA2045 & conserved hypothetical protein & 55.0 & 2.39E-11 & NA & NA & NA & NA & -55.0 & $9.82 \mathrm{E}-12$ \\
\hline PA2198 & hypothetical protein & 8.5 & 3.67E-09 & NA & NA & NA & NA & -62.5 & $3.43 \mathrm{E}-13$ \\
\hline PA2288 & hypothetical protein & 2.2 & $1.42 \mathrm{E}-13$ & -3.4 & $6.77 \mathrm{E}-14$ & NA & NA & -5.5 & $0.00 \mathrm{E}+00$ \\
\hline PA2365 & conserved hypothetical protein & NA & NA & NA & NA & NA & NA & -4.9 & $8.50 \mathrm{E}-08$ \\
\hline PA2372 & hypothetical protein & NA & NA & NA & NA & NA & NA & -3.4 & $1.03 \mathrm{E}-07$ \\
\hline PA2406 & hypothetical protein & NA & NA & NA & NA & NA & NA & -25.6 & $5.40 \mathrm{E}-06$ \\
\hline PA2410 & hypothetical protein & 2.8 & $1.67 \mathrm{E}-05$ & NA & NA & NA & NA & -3.2 & $1.12 \mathrm{E}-06$ \\
\hline PA2423 & hypothetical protein & NA & NA & NA & NA & NA & NA & -3.2 & $1.48 \mathrm{E}-07$ \\
\hline PA2434 & hypothetical protein & NA & NA & NA & NA & NA & NA & -26.3 & $3.95 \mathrm{E}-06$ \\
\hline PA2487 & hypothetical protein & 26.3 & $8.04 \mathrm{E}-06$ & NA & NA & NA & NA & -26.3 & $3.95 \mathrm{E}-06$ \\
\hline PA2496 & hypothetical protein & NA & NA & NA & NA & NA & NA & -27.8 & $1.99 \mathrm{E}-06$ \\
\hline PA2504 & hypothetical protein & NA & NA & NA & NA & NA & NA & -81.4 & $0.00 \mathrm{E}+00$ \\
\hline PA2564 & hypothetical protein & NA & NA & NA & NA & NA & NA & -60.7 & $7.49 \mathrm{E}-13$ \\
\hline PA2565 & hypothetical protein & NA & NA & NA & NA & NA & NA & -33.7 & $1.43 \mathrm{E}-07$ \\
\hline PA2566 & conserved hypothetical protein & NA & NA & NA & NA & NA & NA & -42.3 & 2.99E-09 \\
\hline PA2706 & hypothetical protein & 4.3 & $8.85 \mathrm{E}-06$ & $\begin{array}{c}- \\
22.1\end{array}$ & $4.37 \mathrm{E}-06$ & NA & NA & -54.4 & $1.30 \mathrm{E}-11$ \\
\hline PA2721 & hypothetical protein & NA & NA & NA & NA & NA & NA & -44.9 & $9.43 \mathrm{E}-10$ \\
\hline PA3198 & conserved hypothetical protein & NA & NA & NA & NA & NA & NA & -3.4 & 4.14E-06 \\
\hline PA3288 & hypothetical protein & NA & NA & NA & NA & NA & NA & -4.9 & $2.32 \mathrm{E}-08$ \\
\hline PA3325 & conserved hypothetical protein & NA & NA & NA & NA & NA & NA & -32.4 & $2.64 \mathrm{E}-07$ \\
\hline PA3335 & hypothetical protein & NA & NA & NA & NA & NA & NA & -76.3 & $2.18 \mathrm{E}-14$ \\
\hline PA3390 & hypothetical protein & 5.7 & 4.49E-06 & NA & NA & NA & NA & -47.4 & $3.02 \mathrm{E}-10$ \\
\hline
\end{tabular}




\begin{tabular}{|c|c|c|c|c|c|c|c|c|c|}
\hline PA3446 & conserved hypothetical protein & 2.6 & $2.03 \mathrm{E}-06$ & NA & NA & NA & NA & -4.4 & $1.99 \mathrm{E}-11$ \\
\hline PA3520 & hypothetical protein & 73.7 & $0.00 \mathrm{E}+00$ & NA & NA & NA & $\mathrm{NA}$ & -73.7 & $0.00 \mathrm{E}+00$ \\
\hline PA3623 & conserved hypothetical protein & NA & NA & NA & NA & NA & NA & -2.3 & $0.00 \mathrm{E}+00$ \\
\hline PA3684 & hypothetical protein & 2.6 & $2.08 \mathrm{E}-10$ & NA & NA & NA & NA & -4.4 & $0.00 \mathrm{E}+00$ \\
\hline PA3698 & hypothetical protein & 5.1 & $4.23 \mathrm{E}-13$ & NA & NA & NA & NA & -2.9 & $1.73 \mathrm{E}-08$ \\
\hline PA3752 & hypothetical protein & NA & NA & NA & NA & NA & NA & -36.8 & $3.55 \mathrm{E}-08$ \\
\hline PA3755 & conserved hypothetical protein & NA & NA & NA & NA & NA & NA & -2.1 & $1.67 \mathrm{E}-06$ \\
\hline PA3793 & hypothetical protein & 2.0 & $1.28 \mathrm{E}-05$ & -6.5 & $9.93 \mathrm{E}-14$ & NA & NA & -6.8 & $2.58 \mathrm{E}-14$ \\
\hline PA3797 & conserved hypothetical protein & 2.6 & $3.75 \mathrm{E}-07$ & NA & NA & NA & NA & -2.5 & $3.94 \mathrm{E}-07$ \\
\hline PA3808 & conserved hypothetical protein & 2.5 & 2.72E-13 & NA & NA & NA & NA & -2.3 & $7.31 \mathrm{E}-12$ \\
\hline PA3905 & hypothetical protein & 2.5 & $3.24 \mathrm{E}-10$ & NA & NA & NA & NA & -13.6 & $0.00 \mathrm{E}+00$ \\
\hline PA3911 & conserved hypothetical protein & NA & NA & NA & NA & NA & NA & -27.8 & $1.99 \mathrm{E}-06$ \\
\hline PA3928 & hypothetical protein & 84.0 & $5.05 \mathrm{E}-14$ & NA & NA & NA & NA & -84.0 & $5.24 \mathrm{E}-14$ \\
\hline PA4009 & hypothetical protein & NA & NA & NA & NA & NA & NA & -33.5 & $1.59 \mathrm{E}-07$ \\
\hline PA4027 & hypothetical protein & NA & NA & NA & NA & NA & NA & -24.1 & $1.05 \mathrm{E}-05$ \\
\hline PA4059 & hypothetical protein & NA & NA & NA & NA & NA & NA & -38.0 & $2.11 \mathrm{E}-08$ \\
\hline PA4093 & hypothetical protein & NA & NA & NA & NA & NA & NA & -34.4 & $1.03 \mathrm{E}-07$ \\
\hline PA4183 & hypothetical protein & NA & NA & NA & NA & NA & NA & -30.7 & $5.57 \mathrm{E}-07$ \\
\hline PA4200 & hypothetical protein & NA & NA & NA & NA & NA & NA & -24.9 & $7.55 \mathrm{E}-06$ \\
\hline PA4327 & hypothetical protein & NA & NA & NA & NA & NA & NA & -35.6 & $6.13 \mathrm{E}-08$ \\
\hline PA4337 & hypothetical protein & 8.5 & $1.63 \mathrm{E}-05$ & NA & NA & NA & NA & -35.2 & 7.32E-08 \\
\hline PA4349 & hypothetical protein & NA & NA & NA & NA & NA & NA & -40.0 & 8.38E-09 \\
\hline PA4352 & conserved hypothetical protein & NA & NA & NA & NA & NA & NA & -2.3 & $3.69 \mathrm{E}-14$ \\
\hline PA4357 & conserved hypothetical protein & 29.6 & $1.94 \mathrm{E}-06$ & NA & NA & NA & NA & -29.6 & $9.24 \mathrm{E}-07$ \\
\hline PA4362 & hypothetical protein & NA & NA & NA & NA & NA & NA & -3.9 & $1.00 \mathrm{E}-05$ \\
\hline PA4364 & hypothetical protein & 35.7 & $1.30 \mathrm{E}-07$ & NA & NA & NA & NA & -35.7 & 5.77E-08 \\
\hline PA4530 & conserved hypothetical protein & NA & NA & NA & NA & NA & NA & -71.5 & $0.00 \mathrm{E}+00$ \\
\hline PA4533 & hypothetical protein & NA & NA & NA & NA & NA & NA & -4.9 & $6.98 \mathrm{E}-09$ \\
\hline PA4535 & hypothetical protein & 2.5 & $9.73 \mathrm{E}-11$ & NA & NA & NA & NA & -4.1 & $0.00 \mathrm{E}+00$ \\
\hline
\end{tabular}




\begin{tabular}{|c|c|c|c|c|c|c|c|c|c|}
\hline PA4582 & conserved hypothetical protein & NA & NA & NA & NA & NA & NA & -2.3 & $2.62 \mathrm{E}-11$ \\
\hline PA4583 & conserved hypothetical protein & NA & NA & NA & NA & NA & NA & -3.8 & $3.69 \mathrm{E}-14$ \\
\hline PA4610 & hypothetical protein & 17.0 & $0.00 \mathrm{E}+00$ & NA & NA & NA & NA & -5.8 & $1.82 \mathrm{E}-12$ \\
\hline PA4635 & conserved hypothetical protein & 2.8 & $6.39 \mathrm{E}-07$ & $\begin{array}{c}- \\
37.3\end{array}$ & $1.21 \mathrm{E}-09$ & NA & NA & -101.9 & $2.36 \mathrm{E}-14$ \\
\hline PA4648 & hypothetical protein & NA & NA & NA & NA & NA & NA & -26.2 & $4.21 \mathrm{E}-06$ \\
\hline PA4650 & hypothetical protein & NA & NA & NA & NA & NA & NA & -26.5 & $3.71 \mathrm{E}-06$ \\
\hline PA4677 & hypothetical protein & NA & NA & NA & NA & NA & NA & -5.4 & $5.23 \mathrm{E}-08$ \\
\hline PA4679 & hypothetical protein & 2.4 & $1.88 \mathrm{E}-05$ & NA & NA & NA & NA & -2.4 & $5.13 \mathrm{E}-06$ \\
\hline PA4714 & conserved hypothetical protein & NA & NA & NA & NA & NA & NA & -5.8 & $4.40 \mathrm{E}-12$ \\
\hline PA4736 & hypothetical protein & 2.5 & $1.02 \mathrm{E}-10$ & NA & NA & NA & NA & -3.9 & $0.00 \mathrm{E}+00$ \\
\hline PA4790 & conserved hypothetical protein & 67.0 & $2.68 \mathrm{E}-14$ & NA & NA & NA & NA & -6.8 & $2.25 \mathrm{E}-09$ \\
\hline PA4830 & hypothetical protein & NA & NA & NA & NA & NA & NA & -53.2 & $2.26 \mathrm{E}-11$ \\
\hline PA4840 & conserved hypothetical protein & 2.2 & $1.83 \mathrm{E}-09$ & -2.2 & $1.19 \mathrm{E}-05$ & NA & NA & -3.6 & $0.00 \mathrm{E}+00$ \\
\hline PA4916 & hypothetical protein & NA & NA & NA & NA & NA & NA & -3.9 & $8.92 \mathrm{E}-12$ \\
\hline PA5026 & hypothetical protein & NA & NA & NA & NA & NA & NA & -31.7 & $3.54 \mathrm{E}-07$ \\
\hline PA5101 & hypothetical protein & NA & NA & NA & NA & NA & NA & -27.0 & $2.91 \mathrm{E}-06$ \\
\hline PA5123 & hypothetical protein & NA & NA & NA & NA & NA & NA & -37.6 & $2.53 \mathrm{E}-08$ \\
\hline PA5185 & conserved hypothetical protein & 3.5 & $4.36 \mathrm{E}-07$ & NA & NA & NA & NA & -4.9 & $2.31 \mathrm{E}-09$ \\
\hline PA5286 & conserved hypothetical protein & NA & NA & NA & NA & NA & NA & -2.1 & $2.92 \mathrm{E}-10$ \\
\hline PA5333 & conserved hypothetical protein & 2.8 & $6.42 \mathrm{E}-09$ & NA & NA & NA & NA & -2.3 & $7.31 \mathrm{E}-07$ \\
\hline PA5339 & conserved hypothetical protein & NA & NA & NA & NA & NA & NA & -2.1 & $3.47 \mathrm{E}-10$ \\
\hline PA5405 & hypothetical protein & 30.7 & $1.18 \mathrm{E}-06$ & NA & NA & NA & NA & -30.7 & $5.57 \mathrm{E}-07$ \\
\hline PA5423 & hypothetical protein & NA & NA & NA & NA & NA & NA & -2.2 & $9.04 \mathrm{E}-06$ \\
\hline \multicolumn{10}{|c|}{ Membrane proteins } \\
\hline PA0007 & hypothetical protein & NA & NA & NA & NA & NA & NA & -25.2 & $6.36 \mathrm{E}-06$ \\
\hline PA1118 & hypothetical protein & 3.1 & $1.26 \mathrm{E}-08$ & NA & NA & NA & NA & -5.4 & $1.09 \mathrm{E}-13$ \\
\hline PA1131 & $\begin{array}{l}\text { probable major facilitator } \\
\text { superfamily (MFS) transporter }\end{array}$ & NA & NA & NA & NA & NA & NA & -62.3 & $3.57 \mathrm{E}-13$ \\
\hline
\end{tabular}




\begin{tabular}{|c|c|c|c|c|c|c|c|c|c|c|}
\hline PA1297 & \multirow{20}{*}{$a m b A$} & probable metal transporter & 8.5 & $1.12 \mathrm{E}-06$ & NA & NA & NA & NA & -43.9 & $1.46 \mathrm{E}-09$ \\
\hline PA1541 & & probable drug efflux transporter & NA & NA & NA & NA & NA & NA & -58.4 & $2.11 \mathrm{E}-12$ \\
\hline PA1547 & & hypothetical protein & 4.3 & $1.46 \mathrm{E}-06$ & NA & NA & NA & NA & -5.8 & $2.81 \mathrm{E}-08$ \\
\hline PA1650 & & probable transporter & NA & NA & NA & NA & NA & NA & -24.1 & $1.05 \mathrm{E}-05$ \\
\hline PA1825 & & hypothetical protein & NA & NA & NA & NA & NA & NA & -5.8 & $1.02 \mathrm{E}-08$ \\
\hline PA2306 & & AmbA & NA & NA & NA & NA & NA & NA & -4.4 & $5.03 \mathrm{E}-11$ \\
\hline PA2327 & & $\begin{array}{l}\text { probable permease of } \mathrm{ABC} \\
\text { transporter }\end{array}$ & NA & NA & NA & NA & NA & NA & -28.2 & $1.73 \mathrm{E}-06$ \\
\hline PA2409 & & $\begin{array}{l}\text { probable permease of } \mathrm{ABC} \\
\text { transporter }\end{array}$ & NA & NA & NA & NA & NA & NA & -8.8 & $7.14 \mathrm{E}-11$ \\
\hline PA2501 & & hypothetical protein & 3.5 & $1.11 \mathrm{E}-13$ & NA & NA & NA & NA & -4.9 & $5.59 \mathrm{E}-14$ \\
\hline PA2663 & & $\begin{array}{l}\text { psl and pyoverdine operon } \\
\text { regulator, PpyR }\end{array}$ & NA & NA & NA & NA & NA & NA & -27.8 & $1.99 \mathrm{E}-06$ \\
\hline PA3235 & & conserved hypothetical protein & NA & NA & NA & NA & NA & NA & -2.9 & $7.64 \mathrm{E}-10$ \\
\hline PA3370 & & hypothetical protein & 48.9 & $3.87 \mathrm{E}-10$ & NA & NA & NA & NA & -48.9 & $1.59 \mathrm{E}-10$ \\
\hline PA3432 & & hypothetical protein & NA & NA & NA & NA & NA & NA & -36.8 & $3.55 \mathrm{E}-08$ \\
\hline PA3789 & & hypothetical protein & NA & NA & NA & NA & NA & NA & -30.4 & $6.24 \mathrm{E}-07$ \\
\hline PA3897 & & hypothetical protein & 23.9 & $2.37 \mathrm{E}-05$ & NA & NA & NA & NA & -23.9 & $1.17 \mathrm{E}-05$ \\
\hline PA3920 & & $\begin{array}{l}\text { probable metal transporting } \mathrm{P} \text { - } \\
\text { type ATPase }\end{array}$ & 4.8 & $1.92 \mathrm{E}-07$ & NA & NA & NA & NA & -10.7 & $8.43 \mathrm{E}-11$ \\
\hline PA3966 & & hypothetical protein & NA & NA & NA & NA & NA & NA & -2.4 & $2.22 \mathrm{E}-09$ \\
\hline PA4046 & & hypothetical protein & NA & NA & NA & NA & NA & NA & -3.9 & $6.00 \mathrm{E}-07$ \\
\hline PA4507 & & hypothetical protein & NA & NA & NA & NA & NA & NA & -34.0 & $1.24 \mathrm{E}-07$ \\
\hline PA5132 & & hypothetical protein & 11.3 & $6.08 \mathrm{E}-06$ & 21.4 & $6.46 \mathrm{E}-06$ & NA & NA & -35.1 & 7.75E-08 \\
\hline \multicolumn{11}{|c|}{ Motility \& Attachment } \\
\hline PA1461 & $m o t D$ & MotD & 2.4 & $2.67 \mathrm{E}-06$ & NA & NA & NA & NA & -2.4 & $6.34 \mathrm{E}-07$ \\
\hline PA3361 & $\operatorname{lec} B$ & fucose-binding lectin PA-IIL & NA & NA & NA & NA & NA & NA & -4.9 & $1.13 \mathrm{E}-11$ \\
\hline PA4086 & сирB1 & $\begin{array}{l}\text { probable fimbrial subunit } \\
\text { CupB1 }\end{array}$ & 25.2 & $1.31 \mathrm{E}-05$ & NA & NA & NA & NA & -25.2 & $6.47 \mathrm{E}-06$ \\
\hline PA4305 & $r c p C$ & $\mathrm{RcpC}$ & NA & NA & NA & NA & NA & NA & -8.8 & $8.24 \mathrm{E}-11$ \\
\hline
\end{tabular}




\begin{tabular}{|c|c|c|c|c|c|c|c|c|c|c|}
\hline $\begin{array}{l}\text { PA4551 } \\
\text { PA4555 }\end{array}$ & $\begin{array}{l}\text { pilV } \\
\text { pilY2 }\end{array}$ & $\begin{array}{l}\text { type } 4 \text { fimbrial biogenesis } \\
\text { protein PilV } \\
\text { type } 4 \text { fimbrial biogenesis } \\
\text { protein PilY2 }\end{array}$ & $\begin{array}{l}\text { NA } \\
\text { NA }\end{array}$ & $\begin{array}{l}\text { NA } \\
\text { NA }\end{array}$ & $\begin{array}{l}\text { NA } \\
\text { NA }\end{array}$ & $\begin{array}{l}\text { NA } \\
\text { NA }\end{array}$ & NA & NA & $\begin{array}{l}-25.7 \\
-61.9\end{array}$ & $\begin{array}{l}5.09 \mathrm{E}-06 \\
4.31 \mathrm{E}-13\end{array}$ \\
\hline \multicolumn{11}{|c|}{ Non-coding RNA gene } \\
\hline PA2819.3 & & tRNA-Glu & 2.8 & $1.77 \mathrm{E}-06$ & $\begin{array}{c}- \\
57.6 \\
\end{array}$ & $0.00 \mathrm{E}+00$ & NA & NA & -94.5 & $0.00 \mathrm{E}+00$ \\
\hline \multicolumn{11}{|c|}{ Nucleotide biosynthesis and metabolism } \\
\hline PA0143 & nuh & purine nucleosidase Nuh & NA & NA & NA & NA & NA & NA & -2.2 & $2.19 \mathrm{E}-06$ \\
\hline PA0849 & $\operatorname{trx} B 2$ & thioredoxin reductase 2 & NA & NA & NA & NA & NA & NA & -4.2 & $4.39 \mathrm{E}-14$ \\
\hline \multicolumn{11}{|c|}{ Protein secretion/export apparatus } \\
\hline PA1692 & & $\begin{array}{l}\text { probable translocation protein } \\
\text { in type III secretion }\end{array}$ & NA & NA & NA & NA & NA & NA & -26.9 & $3.04 \mathrm{E}-06$ \\
\hline PA1705 & $\operatorname{pcr} G$ & regulator in type III secretion & 24.2 & $2.05 \mathrm{E}-05$ & NA & NA & NA & NA & -24.2 & $1.01 \mathrm{E}-05$ \\
\hline PA1713 & $\operatorname{exs} A$ & transcriptional regulator ExsA & NA & NA & NA & NA & NA & NA & -5.8 & $6.00 \mathrm{E}-07$ \\
\hline PA1875 & & $\begin{array}{l}\text { probable outer membrane } \\
\text { protein precursor }\end{array}$ & NA & NA & NA & NA & NA & NA & -28.1 & $1.78 \mathrm{E}-06$ \\
\hline PA3095 & $x c p Z$ & $\begin{array}{l}\text { general secretion pathway } \\
\text { protein } \mathrm{M}\end{array}$ & NA & NA & NA & NA & NA & NA & -6.8 & $5.40 \mathrm{E}-13$ \\
\hline PA3098 & $x c p W$ & $\begin{array}{l}\text { general secretion pathway } \\
\text { protein } \mathrm{J}\end{array}$ & NA & NA & NA & NA & NA & NA & -7.8 & $1.18 \mathrm{E}-11$ \\
\hline \multicolumn{11}{|c|}{ Putative enzymes } \\
\hline PA0534 & & conserved hypothetical protein & NA & NA & NA & NA & NA & NA & -2.9 & $2.05 \mathrm{E}-07$ \\
\hline PA0817 & & $\begin{array}{l}\text { probable ring-cleaving } \\
\text { dioxygenase }\end{array}$ & 3.3 & $2.41 \mathrm{E}-09$ & NA & NA & NA & NA & -2.3 & $4.01 \mathrm{E}-06$ \\
\hline PA1914 & & conserved hypothetical protein & NA & NA & NA & NA & NA & NA & -29.3 & $1.05 \mathrm{E}-06$ \\
\hline PA2024 & & $\begin{array}{l}\text { probable ring-cleaving } \\
\text { dioxygenase }\end{array}$ & 11.3 & $8.90 \mathrm{E}-11$ & 20.7 & $9.31 \mathrm{E}-06$ & NA & NA & -67.9 & $5.04 \mathrm{E}-14$ \\
\hline PA2271 & & probable acetyltransferase & 8.5 & $2.17 \mathrm{E}-06$ & NA & NA & NA & NA & -41.8 & $3.86 \mathrm{E}-09$ \\
\hline PA2379 & & probable oxidoreductase & NA & NA & NA & NA & NA & NA & -5.8 & $1.41 \mathrm{E}-11$ \\
\hline PA3295 & & probable HIT family protein & 2.8 & $1.04 \mathrm{E}-06$ & NA & NA & NA & NA & -2.9 & $2.70 \mathrm{E}-07$ \\
\hline PA4026 & & probable acetyltransferase & NA & NA & NA & NA & NA & NA & -5.8 & $8.72 \mathrm{E}-12$ \\
\hline
\end{tabular}




\begin{tabular}{|c|c|c|c|c|c|c|c|c|c|c|}
\hline \multicolumn{11}{|c|}{ Transcription, RNA processing and degradation } \\
\hline PA5197 & $\operatorname{rim} K$ & $\begin{array}{l}\text { ribosomal protein } \mathrm{S} 6 \\
\text { modification protein }\end{array}$ & NA & NA & NA & NA & NA & NA & -3.9 & $1.63 \mathrm{E}-06$ \\
\hline \multicolumn{11}{|c|}{ Transcriptional regulators } \\
\hline PA0175 & \multirow{9}{*}{$\begin{array}{l}\text { fliA } \\
\text { copR }\end{array}$} & $\begin{array}{l}\text { probable chemotaxis protein } \\
\text { methyltransferase }\end{array}$ & NA & NA & NA & NA & NA & NA & -25.6 & $5.51 \mathrm{E}-06$ \\
\hline PA0547 & & $\begin{array}{l}\text { probable transcriptional } \\
\text { regulator }\end{array}$ & 2.0 & $4.43 \mathrm{E}-12$ & NA & NA & NA & NA & -2.7 & $1.75 \mathrm{E}-14$ \\
\hline PA1109 & & $\begin{array}{l}\text { probable transcriptional } \\
\text { regulator }\end{array}$ & NA & NA & NA & NA & NA & NA & -27.6 & $2.19 \mathrm{E}-06$ \\
\hline PA1359 & & $\begin{array}{l}\text { probable transcriptional } \\
\text { regulator }\end{array}$ & 8.5 & $1.40 \mathrm{E}-09$ & NA & NA & NA & NA & -5.8 & $1.41 \mathrm{E}-08$ \\
\hline PA1455 & & sigma factor FliA & NA & NA & NA & NA & NA & NA & -2.9 & $2.85 \mathrm{E}-10$ \\
\hline PA2809 & & $\begin{array}{l}\text { two-component response } \\
\text { regulator, CopR }\end{array}$ & NA & NA & NA & NA & NA & NA & -31.6 & $3.64 \mathrm{E}-07$ \\
\hline PA2877 & & $\begin{array}{l}\text { probable transcriptional } \\
\text { regulator }\end{array}$ & NA & NA & NA & NA & NA & NA & -24.1 & $1.05 \mathrm{E}-05$ \\
\hline PA3034 & & $\begin{array}{l}\text { probable transcriptional } \\
\text { regulator }\end{array}$ & 6.4 & $0.00 \mathrm{E}+00$ & NA & NA & NA & NA & -4.4 & $4.09 \mathrm{E}-12$ \\
\hline PA3077 & & $\begin{array}{l}\text { probable two-component } \\
\text { response regulator }\end{array}$ & 8.5 & $2.20 \mathrm{E}-09$ & NA & NA & NA & NA & -5.8 & $2.10 \mathrm{E}-08$ \\
\hline PA3351 & \multirow[t]{7}{*}{$f \lg M$} & FlgM & 2.0 & $0.00 \mathrm{E}+00$ & NA & NA & NA & NA & -2.4 & $4.81 \mathrm{E}-14$ \\
\hline PA3630 & & $\begin{array}{l}\text { probable transcriptional } \\
\text { regulator }\end{array}$ & NA & NA & NA & NA & NA & NA & -31.6 & $3.70 \mathrm{E}-07$ \\
\hline PA4070 & & $\begin{array}{l}\text { probable transcriptional } \\
\text { regulator }\end{array}$ & NA & NA & NA & NA & NA & NA & -30.2 & $6.91 \mathrm{E}-07$ \\
\hline PA4077 & & $\begin{array}{l}\text { probable transcriptional } \\
\text { regulator }\end{array}$ & 34.7 & $2.02 \mathrm{E}-07$ & NA & NA & NA & NA & -34.7 & $9.20 \mathrm{E}-08$ \\
\hline PA4135 & & $\begin{array}{l}\text { probable transcriptional } \\
\text { regulator }\end{array}$ & 2.2 & $1.47 \mathrm{E}-05$ & NA & NA & NA & NA & -3.4 & $4.26 \mathrm{E}-10$ \\
\hline PA4203 & & $\begin{array}{l}\text { probable transcriptional } \\
\text { regulator }\end{array}$ & NA & NA & NA & NA & NA & NA & -33.6 & $1.50 \mathrm{E}-07$ \\
\hline PA4987 & & $\begin{array}{l}\text { probable transcriptional } \\
\text { regulator }\end{array}$ & NA & NA & NA & NA & NA & NA & -4.9 & $3.48 \mathrm{E}-07$ \\
\hline
\end{tabular}




\begin{tabular}{|c|c|c|c|c|c|c|c|c|c|c|}
\hline \multicolumn{11}{|c|}{ Translation, post-translational modification, degradation } \\
\hline PA1165 & $p c p S$ & $\begin{array}{l}\text { PcpS } \\
\text { serine/threonine }\end{array}$ & NA & NA & NA & NA & NA & NA & -39.4 & $1.11 \mathrm{E}-08$ \\
\hline PA1670 & stpl & $\begin{array}{l}\text { phosphoprotein phosphatase } \\
\text { Stp1 }\end{array}$ & NA & NA & NA & NA & NA & NA & -4.9 & $4.29 \mathrm{E}-06$ \\
\hline PA2371 & & probable ClpA/B-type protease & NA & NA & NA & NA & NA & NA & -28.2 & $1.73 \mathrm{E}-06$ \\
\hline PA3227 & ppiA & $\begin{array}{l}\text { peptidyl-prolyl cis-trans } \\
\text { isomerase A }\end{array}$ & NA & NA & NA & NA & NA & NA & -2.9 & $6.82 \mathrm{E}-06$ \\
\hline PA3601 & & conserved hypothetical protein & NA & NA & NA & NA & NA & NA & -27.2 & $2.65 \mathrm{E}-06$ \\
\hline PA3717 & & $\begin{array}{l}\text { probable peptidyl-prolyl cis- } \\
\text { trans isomerase, FkbP-type }\end{array}$ & 11.3 & $2.72 \mathrm{E}-13$ & NA & NA & NA & NA & -3.9 & $2.65 \mathrm{E}-08$ \\
\hline PA4482 & gatC & $\begin{array}{l}\text { Glu-tRNA(Gln) } \\
\text { amidotransferase subunit C }\end{array}$ & NA & NA & NA & NA & NA & NA & -4.9 & $8.36 \mathrm{E}-14$ \\
\hline \multicolumn{11}{|c|}{ Transport of small molecules } \\
\hline PA0487 & & $\begin{array}{l}\text { probable molybdenum transport } \\
\text { regulator }\end{array}$ & 8.5 & $2.16 \mathrm{E}-08$ & NA & NA & NA & NA & -5.8 & $1.46 \mathrm{E}-07$ \\
\hline PA0958 & oprD & $\begin{array}{l}\text { Basic amino acid, basic peptide } \\
\text { and imipenem outer membrane } \\
\text { porin OprD precursor }\end{array}$ & NA & NA & NA & NA & NA & NA & -3.0 & $2.52 \mathrm{E}-13$ \\
\hline PA3407 & hasAp & $\begin{array}{l}\text { heme acquisition protein } \\
\text { HasAp }\end{array}$ & NA & NA & NA & NA & NA & NA & -34.9 & $8.55 \mathrm{E}-08$ \\
\hline PA3753 & & conserved hypothetical protein & 2.2 & $6.17 \mathrm{E}-07$ & $\begin{array}{c}- \\
75.1\end{array}$ & $0.00 \mathrm{E}+00$ & NA & NA & -150.5 & $0.00 \mathrm{E}+00$ \\
\hline
\end{tabular}




\begin{tabular}{|c|c|c|c|c|c|c|c|c|c|c|}
\hline \multicolumn{11}{|c|}{ Genes negatively regulated by AmpR upon B-lactam exposure (247) } \\
\hline \multirow[b]{2}{*}{ Gene ID } & \multirow[b]{2}{*}{$\begin{array}{l}\text { Gene } \\
\text { name }\end{array}$} & \multirow[b]{2}{*}{ Product Name } & \multicolumn{2}{|c|}{ Condition 1} & \multicolumn{2}{|c|}{ Condition 2} & \multicolumn{2}{|c|}{ Condition 3} & \multicolumn{2}{|c|}{ Condition 4} \\
\hline & & & $\begin{array}{c}\text { Fold } \\
\text { Change }\end{array}$ & $\begin{array}{c}\text { Corrected } \\
p \text {-value }\end{array}$ & $\begin{array}{l}\text { Fold } \\
\text { Cha } \\
\text { nge }\end{array}$ & $\begin{array}{l}\text { Corrected } \\
p \text {-value }\end{array}$ & $\begin{array}{c}\text { Fold } \\
\text { Change }\end{array}$ & $\begin{array}{l}\text { Corrected } \\
p \text {-value }\end{array}$ & $\begin{array}{c}\text { Fold } \\
\text { Change }\end{array}$ & $\begin{array}{c}\text { Corrected } \\
p \text {-value }\end{array}$ \\
\hline \multicolumn{11}{|c|}{ Adaptation, Protection } \\
\hline PA3553 & $\operatorname{arnC}$ & ArnC & NA & NA & NA & NA & NA & NA & 53.7 & $3.65 \mathrm{E}-13$ \\
\hline PA3624 & pcm & $\begin{array}{l}\text { L-isoaspartate protein } \\
\text { carboxylmethyltransferase type } \\
\text { II }\end{array}$ & NA & NA & NA & NA & NA & NA & 2.0 & 4.42E-08 \\
\hline PA4671 & & probable ribosomal protein $\mathrm{L} 25$ & NA & NA & NA & NA & NA & NA & 2.2 & $0.00 \mathrm{E}+00$ \\
\hline \multicolumn{11}{|c|}{ Amino acid biosynthesis and metabolism } \\
\hline PA5278 & dapF & diaminopimelate epimerase & NA & NA & NA & NA & NA & NA & 2.0 & $2.02 \mathrm{E}-06$ \\
\hline PA2446 & $g c v H 2$ & $\begin{array}{l}\text { glycine cleavage system protein } \\
\mathrm{H} 2\end{array}$ & NA & NA & NA & NA & NA & NA & 2.1 & $4.91 \mathrm{E}-14$ \\
\hline PA5413 & ltaA & $\begin{array}{l}\text { low specificity l-threonine } \\
\text { aldolase }\end{array}$ & NA & NA & NA & NA & NA & NA & 2.3 & 2.24E-06 \\
\hline PA2247 & $b k d A 1$ & $\begin{array}{l}\text { 2-oxoisovalerate } \\
\text { dehydrogenase (alpha subunit) }\end{array}$ & NA & NA & NA & NA & NA & NA & 2.4 & $5.09 \mathrm{E}-14$ \\
\hline PA3117 & asd & $\begin{array}{l}\text { aspartate semialdehyde } \\
\text { dehydrogenase }\end{array}$ & NA & NA & NA & NA & NA & NA & 2.4 & $1.73 \mathrm{E}-11$ \\
\hline PA5067 & hisE & $\begin{array}{l}\text { phosphoribosyl-ATP } \\
\text { pyrophosphohydrolase }\end{array}$ & NA & NA & NA & NA & NA & NA & 3.6 & $4.64 \mathrm{E}-14$ \\
\hline PA5417 & $\operatorname{sox} D$ & sarcosine oxidase delta subunit & NA & NA & NA & NA & NA & NA & 21.3 & $7.88 \mathrm{E}-06$ \\
\hline PA3570 & $m m s A$ & $\begin{array}{l}\text { methylmalonate-semialdehyde } \\
\text { dehydrogenase }\end{array}$ & NA & NA & NA & NA & NA & NA & 2.3 & $6.69 \mathrm{E}-14$ \\
\hline PA2000 & $d h c B$ & $\begin{array}{l}\text { DhcB, dehydrocarnitine CoA } \\
\text { transferase, subunit B }\end{array}$ & NA & NA & NA & NA & NA & NA & 2.7 & $0.00 \mathrm{E}+00$ \\
\hline PA2250 & $\operatorname{lpdV}$ & lipoamide dehydrogenase-Val & NA & NA & NA & NA & NA & NA & 2.0 & $0.00 \mathrm{E}+00$ \\
\hline PA4758 & $\operatorname{car} A$ & $\begin{array}{l}\text { carbamoyl-phosphate synthase } \\
\text { small chain }\end{array}$ & NA & NA & NA & NA & NA & NA & 2.2 & $1.07 \mathrm{E}-08$ \\
\hline PA0402 & pyrB & aspartate carbamoyltransferase & NA & NA & NA & NA & NA & NA & 2.6 & $4.58 \mathrm{E}-07$ \\
\hline
\end{tabular}




\begin{tabular}{|c|c|c|c|c|c|c|c|c|c|c|}
\hline \multicolumn{11}{|c|}{ Antibiotic resistance and susceptibility ; Membrane proteins } \\
\hline PA0157 & triB & $\begin{array}{l}\text { Resistance-Nodulation-Cell } \\
\text { Division (RND) triclosan efflux } \\
\text { membrane fusion protein, TriB }\end{array}$ & NA & NA & NA & NA & NA & NA & 4.1 & $3.03 \mathrm{E}-06$ \\
\hline \multicolumn{11}{|l|}{ 、 } \\
\hline PA0551 & epd & $\begin{array}{l}\text { D-erythrose 4-phosphate } \\
\text { dehydrogenase }\end{array}$ & NA & NA & NA & NA & NA & NA & 2.5 & $3.97 \mathrm{E}-09$ \\
\hline PA4529 & $c o a E$ & dephosphocoenzyme A kinase & NA & NA & NA & NA & NA & NA & 2.6 & $1.21 \mathrm{E}-07$ \\
\hline PA1275 & $\operatorname{cobD}$ & $\begin{array}{l}\text { cobalamin biosynthetic protein } \\
\text { CobD }\end{array}$ & NA & NA & NA & NA & NA & NA & 6.2 & $6.55 \mathrm{E}-07$ \\
\hline PA2905 & $c o b H$ & precorrin isomerase $\mathrm{CobH}$ & NA & NA & NA & NA & NA & NA & 65.5 & $5.09 \mathrm{E}-14$ \\
\hline PA0024 & hemF & $\begin{array}{l}\text { coproporphyrinogen III } \\
\text { oxidase, aerobic }\end{array}$ & -2.6 & $1.80 \mathrm{E}-05$ & 2.8 & $5.65 \mathrm{E}-06$ & NA & NA & 3.1 & $7.66 \mathrm{E}-08$ \\
\hline PA5320 & $\operatorname{coaC}$ & $\begin{array}{l}\text { Phosphopantothenoylcysteine } \\
\text { synthase/(R)-4'-phospho-N- } \\
\text { pantothenoylcysteine } \\
\text { decarboxylase }\end{array}$ & NA & NA & NA & NA & NA & NA & 3.9 & $3.13 \mathrm{E}-09$ \\
\hline \multicolumn{11}{|c|}{ Carbon compound catabolism } \\
\hline PA2013 & $\operatorname{liu} C$ & $\begin{array}{l}\text { putative 3-methylglutaconyl- } \\
\text { CoA hydratase }\end{array}$ & NA & NA & NA & NA & NA & NA & 2.2 & $3.74 \mathrm{E}-06$ \\
\hline PA2007 & maiA & maleylacetoacetate isomerase & NA & NA & NA & NA & NA & NA & 2.6 & $0.00 \mathrm{E}+00$ \\
\hline PA1311 & $\operatorname{phnX}$ & $\begin{array}{l}\text { 2-phosphonoacetaldehyde } \\
\text { hydrolase }\end{array}$ & NA & NA & NA & NA & NA & NA & 24.8 & $1.30 \mathrm{E}-06$ \\
\hline PA2862 & $\operatorname{lip} A$ & lactonizing lipase precursor & NA & NA & NA & NA & NA & NA & 21.9 & $5.72 \mathrm{E}-06$ \\
\hline \multicolumn{11}{|c|}{ Cell division } \\
\hline PA4408 & $f t s A$ & cell division protein FtsA & NA & NA & NA & NA & NA & NA & 2.2 & $1.49 \mathrm{E}-12$ \\
\hline PA5563 & soj & $\begin{array}{l}\text { chromosome partitioning } \\
\text { protein Soj }\end{array}$ & NA & NA & NA & NA & NA & NA & 3.9 & $0.00 \mathrm{E}+00$ \\
\hline \multicolumn{11}{|c|}{ Cell wall / LPS / capsule } \\
\hline PA3643 & $\operatorname{lpxB}$ & lipid A-disaccharide synthase & NA & NA & NA & NA & NA & NA & 2.0 & $7.76 \mathrm{E}-07$ \\
\hline PA5162 & $r m l D$ & $\begin{array}{l}\text { dTDP-4-dehydrorhamnose } \\
\text { reductase }\end{array}$ & NA & NA & NA & NA & NA & NA & 2.5 & $4.76 \mathrm{E}-11$ \\
\hline
\end{tabular}




\begin{tabular}{|c|c|c|c|c|c|c|c|c|c|c|}
\hline PA2236 & pslF & PslF & NA & NA & NA & NA & NA & NA & 3.6 & $2.60 \mathrm{E}-08$ \\
\hline PA2238 & pslH & PslH & NA & NA & NA & NA & NA & NA & 5.6 & $5.14 \mathrm{E}-09$ \\
\hline \multicolumn{11}{|c|}{ Central intermediary metabolism } \\
\hline PA5046 & & malic enzyme & NA & NA & NA & NA & NA & NA & 2.2 & $0.00 \mathrm{E}+00$ \\
\hline PA2023 & galU & $\begin{array}{l}\text { UTP--glucose-1-phosphate } \\
\text { uridylyltransferase }\end{array}$ & NA & NA & NA & NA & NA & NA & 2.2 & $2.02 \mathrm{E}-11$ \\
\hline PA0102 & & probable carbonic anhydrase & NA & NA & NA & NA & NA & NA & 2.2 & $1.22 \mathrm{E}-06$ \\
\hline PA4956 & $\operatorname{rhdA}$ & $\begin{array}{l}\text { thiosulfate:cyanide } \\
\text { sulfurtransferase }\end{array}$ & NA & NA & NA & NA & NA & NA & 3.4 & $3.14 \mathrm{E}-08$ \\
\hline PA1780 & nirD & $\begin{array}{l}\text { assimilatory nitrite reductase } \\
\text { small subunit }\end{array}$ & NA & NA & NA & NA & NA & NA & 20.9 & $9.60 \mathrm{E}-06$ \\
\hline PA0555 & $f d a$ & $\begin{array}{l}\text { fructose-1,6-bisphosphate } \\
\text { aldolase }\end{array}$ & NA & NA & NA & NA & NA & NA & 2.3 & 4.39E-14 \\
\hline PA1614 & gpsA & $\begin{array}{l}\text { glycerol-3-phosphate } \\
\text { dehydrogenase, biosynthetic }\end{array}$ & NA & NA & NA & NA & NA & NA & 5.1 & $3.36 \mathrm{E}-13$ \\
\hline \multicolumn{11}{|c|}{ Chemotaxis } \\
\hline PA1459 & & probable methyltransferase & NA & NA & NA & NA & NA & NA & 2.8 & $2.68 \mathrm{E}-10$ \\
\hline PA1448 & fliR & $\begin{array}{l}\text { flagellar biosynthetic protein } \\
\text { FliR }\end{array}$ & NA & NA & NA & NA & NA & NA & 26.4 & $5.58 \mathrm{E}-07$ \\
\hline PA0410 & pilI & twitching motility protein PilI & NA & NA & NA & NA & NA & NA & 3.1 & $7.57 \mathrm{E}-07$ \\
\hline \multicolumn{11}{|c|}{ DNA replication, recombination, modification and repair } \\
\hline PA5147 & mut $Y$ & $\begin{array}{l}\text { A / G specific adenine } \\
\text { glycosylase }\end{array}$ & NA & NA & NA & NA & NA & NA & 2.4 & $9.25 \mathrm{E}-06$ \\
\hline PA0357 & mutM & $\begin{array}{l}\text { formamidopyrimidine-DNA } \\
\text { glycosylase }\end{array}$ & NA & NA & NA & NA & NA & NA & 2.6 & $5.28 \mathrm{E}-06$ \\
\hline PA5345 & $\operatorname{rec} G$ & $\begin{array}{l}\text { ATP-dependent DNA helicase } \\
\text { RecG }\end{array}$ & NA & NA & NA & NA & NA & NA & 2.9 & $9.51 \mathrm{E}-06$ \\
\hline PA5280 & sss & site-specific recombinase Sss & NA & NA & NA & NA & NA & NA & 6.2 & $4.40 \mathrm{E}-07$ \\
\hline PA3738 & xerD & integrase/recombinase XerD & NA & NA & NA & NA & NA & NA & 22.9 & $3.50 \mathrm{E}-06$ \\
\hline \multicolumn{11}{|c|}{ Energy metabolism } \\
\hline PA2637 & пиол & $\begin{array}{l}\text { NADH dehydrogenase I chain } \\
\text { A }\end{array}$ & NA & NA & NA & NA & NA & NA & 2.1 & $2.36 \mathrm{E}-14$ \\
\hline
\end{tabular}




\begin{tabular}{|c|c|c|c|c|c|c|c|c|c|c|}
\hline PA5491 & & probable cytochrome & NA & NA & NA & NA & NA & NA & 2.2 & $0.00 \mathrm{E}+00$ \\
\hline PA4571 & & probable cytochrome c & NA & NA & NA & NA & NA & NA & 2.3 & $2.66 \mathrm{E}-06$ \\
\hline PA0794 & & probable aconitate hydratase & NA & NA & NA & NA & NA & NA & 2.3 & $1.06 \mathrm{E}-05$ \\
\hline PA3809 & $f d x 2$ & ferredoxin $[2 \mathrm{Fe}-2 \mathrm{~S}]$ & NA & NA & NA & NA & NA & NA & 2.3 & $5.04 \mathrm{E}-10$ \\
\hline PA2641 & nuoF & $\begin{array}{l}\text { NADH dehydrogenase I chain } \\
\text { F }\end{array}$ & NA & NA & NA & NA & NA & NA & 2.7 & $1.73 \mathrm{E}-10$ \\
\hline PA2649 & nuoN & $\begin{array}{l}\text { NADH dehydrogenase I chain } \\
\mathrm{N}\end{array}$ & NA & NA & NA & NA & NA & NA & 4.0 & $1.27 \mathrm{E}-13$ \\
\hline PA3195 & gap $A$ & $\begin{array}{l}\text { glyceraldehyde 3-phosphate } \\
\text { dehydrogenase }\end{array}$ & NA & NA & NA & NA & NA & NA & 2.8 & 4.03E-06 \\
\hline PA3194 & edd & phosphogluconate dehydratase & NA & NA & NA & NA & NA & NA & 2.9 & $2.17 \mathrm{E}-06$ \\
\hline PA1557 & $\operatorname{ccoN} 2$ & $\begin{array}{l}\text { Cytochrome c oxidase, cbb3- } \\
\text { type, CcoN subunit }\end{array}$ & NA & NA & NA & NA & NA & NA & 2.1 & 7.13E-06 \\
\hline PA1555 & ccoP2 & $\begin{array}{l}\text { Cytochrome c oxidase, cbb3- } \\
\text { type, CcoP subunit }\end{array}$ & NA & NA & NA & NA & NA & NA & 2.2 & $3.75 \mathrm{E}-07$ \\
\hline \multicolumn{11}{|c|}{ Fatty acid and phospholipid metabolism } \\
\hline PA2969 & $p l s X$ & $\begin{array}{l}\text { fatty acid biosynthesis protein } \\
\text { PlsX }\end{array}$ & NA & NA & NA & NA & NA & NA & 2.7 & $2.65 \mathrm{E}-08$ \\
\hline PA0447 & $g c d H$ & glutaryl-CoA dehydrogenase & NA & NA & NA & NA & NA & NA & 3.1 & $0.00 \mathrm{E}+00$ \\
\hline PA0796 & $\operatorname{prpB}$ & $\begin{array}{l}\text { carboxyphosphonoenolpyruvate } \\
\text { phosphonomutase }\end{array}$ & NA & NA & NA & NA & NA & NA & 2.2 & $9.11 \mathrm{E}-07$ \\
\hline \multicolumn{11}{|c|}{ Hypothetical, unclassified, unknown } \\
\hline PA0100 & & hypothetical protein & NA & NA & NA & NA & NA & NA & 2.0 & $6.80 \mathrm{E}-06$ \\
\hline PA0394 & & conserved hypothetical protein & NA & NA & NA & NA & NA & NA & 2.0 & $2.88 \mathrm{E}-08$ \\
\hline PA4000 & & hypothetical protein & NA & NA & NA & NA & NA & NA & 2.0 & $2.30 \mathrm{E}-12$ \\
\hline PA0317 & & hypothetical protein & NA & NA & NA & NA & NA & NA & 2.0 & $3.84 \mathrm{E}-06$ \\
\hline PA4454 & & conserved hypothetical protein & NA & NA & NA & NA & NA & NA & 2.1 & $2.63 \mathrm{E}-12$ \\
\hline PA3801 & & conserved hypothetical protein & NA & NA & NA & NA & NA & NA & 2.1 & $3.67 \mathrm{E}-13$ \\
\hline PA3980 & & conserved hypothetical protein & NA & NA & NA & NA & NA & NA & 2.1 & 4.74E-06 \\
\hline PA5526 & & hypothetical protein & NA & NA & NA & NA & NA & NA & 2.2 & $0.00 \mathrm{E}+00$ \\
\hline PA0446 & & conserved hypothetical protein & NA & NA & NA & NA & NA & NA & 2.2 & $1.19 \mathrm{E}-10$ \\
\hline
\end{tabular}




\begin{tabular}{|c|c|c|c|c|c|c|c|c|c|c|}
\hline PA3214 & & hypothetical protein & NA & NA & NA & NA & NA & NA & 2.3 & $9.52 \mathrm{E}-06$ \\
\hline PA5446 & & hypothetical protein & NA & NA & NA & NA & NA & NA & 2.3 & $4.39 \mathrm{E}-14$ \\
\hline PA2112 & & conserved hypothetical protein & NA & NA & NA & NA & NA & NA & 2.4 & $0.00 \mathrm{E}+00$ \\
\hline PA5492 & & conserved hypothetical protein & NA & NA & NA & NA & NA & NA & 2.4 & 7.77E-09 \\
\hline PA3263 & & conserved hypothetical protein & NA & NA & NA & NA & NA & NA & 2.4 & $1.64 \mathrm{E}-06$ \\
\hline PA3731 & & conserved hypothetical protein & NA & NA & NA & NA & NA & NA & 2.4 & $2.71 \mathrm{E}-08$ \\
\hline PA2110 & & hypothetical protein & NA & NA & NA & NA & NA & NA & 2.5 & $0.00 \mathrm{E}+00$ \\
\hline PA2894 & & hypothetical protein & NA & NA & NA & NA & NA & NA & 2.6 & $8.23 \mathrm{E}-06$ \\
\hline PA2733 & & conserved hypothetical protein & NA & NA & NA & NA & NA & NA & 2.6 & $1.07 \mathrm{E}-08$ \\
\hline PA3345 & & hypothetical protein & NA & NA & NA & NA & NA & NA & 2.6 & $8.61 \mathrm{E}-07$ \\
\hline PA2959 & & conserved hypothetical protein & NA & NA & NA & NA & NA & NA & 2.6 & 7.67E-11 \\
\hline PA2080 & $k y n U$ & kynureninase KynU & NA & NA & NA & NA & NA & NA & 2.7 & $2.41 \mathrm{E}-08$ \\
\hline PA0388 & & hypothetical protein & NA & NA & NA & NA & NA & NA & 2.7 & $1.56 \mathrm{E}-09$ \\
\hline PA0542 & & conserved hypothetical protein & NA & NA & NA & NA & NA & NA & 2.7 & $1.56 \mathrm{E}-09$ \\
\hline PA4697 & & hypothetical protein & NA & NA & NA & NA & NA & NA & 2.7 & $6.53 \mathrm{E}-09$ \\
\hline PA0598 & & hypothetical protein & NA & NA & NA & NA & NA & NA & 2.7 & $9.96 \mathrm{E}-06$ \\
\hline PA5303 & & conserved hypothetical protein & NA & NA & NA & NA & NA & NA & 2.7 & $5.35 \mathrm{E}-14$ \\
\hline PA0462 & & hypothetical protein & NA & NA & NA & NA & NA & NA & 2.8 & $2.70 \mathrm{E}-08$ \\
\hline PA5422 & & hypothetical protein & NA & NA & NA & NA & NA & NA & 2.9 & $5.19 \mathrm{E}-08$ \\
\hline PA1114 & & hypothetical protein & NA & NA & NA & NA & NA & NA & 3.1 & $1.10 \mathrm{E}-05$ \\
\hline PA4322 & & conserved hypothetical protein & NA & NA & NA & NA & NA & NA & 3.1 & $1.10 \mathrm{E}-05$ \\
\hline PA4335 & & hypothetical protein & NA & NA & NA & NA & NA & NA & 3.1 & $4.39 \mathrm{E}-06$ \\
\hline PA4841 & & conserved hypothetical protein & NA & NA & NA & NA & NA & NA & 3.1 & 7.57E-07 \\
\hline PA0385 & & hypothetical protein & NA & NA & NA & NA & NA & NA & 3.1 & $7.51 \mathrm{E}-06$ \\
\hline PA1639 & & hypothetical protein & NA & NA & NA & NA & NA & NA & 3.1 & $8.98 \mathrm{E}-09$ \\
\hline PA3284 & & hypothetical protein & NA & NA & NA & NA & NA & NA & 3.1 & $5.46 \mathrm{E}-06$ \\
\hline PA4605 & & conserved hypothetical protein & NA & NA & NA & NA & NA & NA & 3.1 & $1.07 \mathrm{E}-08$ \\
\hline PA0553 & & hypothetical protein & NA & NA & NA & NA & NA & NA & 3.1 & $8.20 \mathrm{E}-09$ \\
\hline PA1034 & & hypothetical protein & NA & NA & NA & NA & NA & NA & 3.1 & $8.20 \mathrm{E}-09$ \\
\hline
\end{tabular}




\begin{tabular}{|c|c|c|c|c|c|c|c|c|c|}
\hline PA4746 & conserved hypothetical protein & NA & NA & NA & NA & NA & NA & 3.3 & $0.00 \mathrm{E}+00$ \\
\hline PA3200 & conserved hypothetical protein & NA & NA & NA & NA & NA & NA & 3.6 & $6.76 \mathrm{E}-06$ \\
\hline PA5459 & hypothetical protein & NA & NA & NA & NA & NA & NA & 3.6 & $3.42 \mathrm{E}-06$ \\
\hline PA4321 & hypothetical protein & NA & NA & NA & NA & NA & NA & 3.8 & $1.29 \mathrm{E}-06$ \\
\hline PA3084 & hypothetical protein & NA & NA & NA & NA & NA & NA & 3.8 & $6.37 \mathrm{E}-10$ \\
\hline PA2986 & conserved hypothetical protein & NA & NA & NA & NA & NA & NA & 3.9 & $1.22 \mathrm{E}-08$ \\
\hline PA0568 & hypothetical protein & NA & NA & NA & NA & NA & NA & 4.1 & $4.06 \mathrm{E}-07$ \\
\hline PA1514 & ureidoglycolate hydrolaseYbbT & NA & NA & NA & NA & NA & NA & 4.1 & $1.66 \mathrm{E}-06$ \\
\hline PA1837 & hypothetical protein & NA & NA & NA & NA & NA & NA & 4.1 & $1.22 \mathrm{E}-06$ \\
\hline PA4062 & hypothetical protein & NA & NA & NA & NA & NA & NA & 4.1 & 7.19E-09 \\
\hline PA0389 & hypothetical protein & NA & NA & NA & NA & NA & NA & 4.8 & $4.91 \mathrm{E}-14$ \\
\hline PA4579 & hypothetical protein & NA & NA & NA & NA & NA & NA & 4.8 & $5.72 \mathrm{E}-07$ \\
\hline PA2230 & hypothetical protein & NA & NA & NA & NA & NA & NA & 5.1 & $3.56 \mathrm{E}-07$ \\
\hline PA2441 & hypothetical protein & NA & NA & NA & NA & NA & NA & 5.1 & $1.76 \mathrm{E}-10$ \\
\hline PA2632 & hypothetical protein & NA & NA & NA & NA & NA & NA & 5.1 & $1.10 \mathrm{E}-07$ \\
\hline PA0022 & conserved hypothetical protein & NA & NA & NA & NA & NA & NA & 6.2 & $5.95 \mathrm{E}-11$ \\
\hline PA3670 & hypothetical protein & NA & NA & NA & NA & NA & NA & 6.7 & $8.82 \mathrm{E}-08$ \\
\hline PA5145 & hypothetical protein & NA & NA & NA & NA & NA & NA & 7.2 & $2.60 \mathrm{E}-07$ \\
\hline PA1193 & hypothetical protein & NA & NA & NA & NA & NA & NA & 7.2 & $2.12 \mathrm{E}-11$ \\
\hline PA3680 & conserved hypothetical protein & NA & NA & NA & NA & NA & NA & 7.2 & $6.79 \mathrm{E}-10$ \\
\hline PA4617 & conserved hypothetical protein & NA & NA & NA & NA & NA & NA & 11.3 & $1.67 \mathrm{E}-12$ \\
\hline PA2670 & hypothetical protein & NA & NA & NA & NA & NA & NA & 20.8 & $1.02 \mathrm{E}-05$ \\
\hline PA0977 & hypothetical protein & NA & NA & NA & NA & NA & NA & 21.1 & $8.70 \mathrm{E}-06$ \\
\hline PA1123 & hypothetical protein & NA & NA & NA & NA & NA & NA & 21.3 & $7.88 \mathrm{E}-06$ \\
\hline PA4182 & hypothetical protein & NA & NA & NA & NA & NA & NA & 21.4 & $7.49 \mathrm{E}-06$ \\
\hline PA2874 & hypothetical protein & NA & NA & NA & NA & NA & NA & 21.5 & $7.10 \mathrm{E}-06$ \\
\hline PA2422 & hypothetical protein & NA & NA & NA & NA & NA & NA & 21.7 & $6.39 \mathrm{E}-06$ \\
\hline PA1517 & conserved hypothetical protein & NA & NA & NA & NA & NA & NA & 22.1 & $5.14 \mathrm{E}-06$ \\
\hline PA0104 & hypothetical protein & NA & NA & NA & NA & NA & NA & 22.9 & $3.43 \mathrm{E}-06$ \\
\hline
\end{tabular}




\begin{tabular}{|c|c|c|c|c|c|c|c|c|c|}
\hline PA1489 & hypothetical protein & NA & NA & NA & NA & NA & NA & 23.0 & 3.33E-06 \\
\hline PA0709 & hypothetical protein & NA & NA & NA & NA & NA & NA & 23.0 & $3.23 \mathrm{E}-06$ \\
\hline PA5407 & hypothetical protein & NA & NA & NA & NA & NA & NA & 23.5 & $2.52 \mathrm{E}-06$ \\
\hline PA3066 & hypothetical protein & NA & NA & NA & NA & NA & NA & 23.9 & $2.09 \mathrm{E}-06$ \\
\hline PA3283 & conserved hypothetical protein & NA & NA & NA & NA & NA & NA & 24.0 & $1.96 \mathrm{E}-06$ \\
\hline PA1139 & hypothetical protein & NA & NA & NA & NA & NA & NA & 24.2 & $1.79 \mathrm{E}-06$ \\
\hline PA2292 & hypothetical protein & NA & NA & NA & NA & NA & NA & 24.3 & $1.72 \mathrm{E}-06$ \\
\hline PA4985 & hypothetical protein & NA & NA & NA & NA & NA & NA & 25.1 & $1.13 \mathrm{E}-06$ \\
\hline PA3991 & hypothetical protein & NA & NA & NA & NA & NA & NA & 25.9 & $7.26 \mathrm{E}-07$ \\
\hline PA0711 & hypothetical protein & NA & NA & NA & NA & NA & NA & 27.0 & $4.15 \mathrm{E}-07$ \\
\hline PA1234 & hypothetical protein & NA & NA & NA & NA & NA & NA & 27.2 & $3.81 \mathrm{E}-07$ \\
\hline PA2625 & conserved hypothetical protein & NA & NA & NA & NA & NA & NA & 29.1 & $1.42 \mathrm{E}-07$ \\
\hline PA1761 & hypothetical protein & NA & NA & NA & NA & NA & NA & 31.2 & $4.51 \mathrm{E}-08$ \\
\hline PA4169 & conserved hypothetical protein & NA & NA & NA & NA & NA & NA & 31.9 & $3.22 \mathrm{E}-08$ \\
\hline PA5408 & hypothetical protein & NA & NA & NA & NA & NA & NA & 38.7 & $9.39 \mathrm{E}-10$ \\
\hline PA1135 & conserved hypothetical protein & NA & NA & NA & NA & NA & NA & 39.1 & $7.60 \mathrm{E}-10$ \\
\hline PA1593 & hypothetical protein & NA & NA & NA & NA & NA & NA & 43.3 & $8.24 \mathrm{E}-11$ \\
\hline PA2264 & conserved hypothetical protein & NA & NA & NA & NA & NA & NA & 47.7 & $8.25 \mathrm{E}-12$ \\
\hline PA1641 & hypothetical protein & NA & NA & NA & NA & NA & NA & 49.1 & $4.05 \mathrm{E}-12$ \\
\hline PA5533 & hypothetical protein & NA & NA & NA & NA & NA & NA & 55.6 & $1.27 \mathrm{E}-13$ \\
\hline PA4753 & conserved hypothetical protein & -30.0 & 2.19E-07 & 6.9 & $5.20 \mathrm{E}-14$ & NA & NA & 108.6 & $0.00 \mathrm{E}+00$ \\
\hline PA1518 & conserved hypothetical protein & -24.8 & $2.96 \mathrm{E}-06$ & 53.9 & 7.87E-11 & NA & NA & 53.9 & $3.13 \mathrm{E}-13$ \\
\hline PA0469 & hypothetical protein & -24.8 & $2.99 \mathrm{E}-06$ & 4.8 & $2.38 \mathrm{E}-06$ & NA & NA & 55.8 & $1.40 \mathrm{E}-13$ \\
\hline PA0541 & hypothetical protein & -4.9 & 4.55E-09 & 2.2 & $1.32 \mathrm{E}-05$ & NA & NA & 8.2 & $4.39 \mathrm{E}-14$ \\
\hline PA3767 & conserved hypothetical protein & -4.2 & $4.40 \mathrm{E}-06$ & 3.2 & $7.00 \mathrm{E}-07$ & NA & NA & 7.2 & $1.03 \mathrm{E}-13$ \\
\hline PA4612 & conserved hypothetical protein & NA & NA & NA & NA & NA & NA & 24.8 & $1.30 \mathrm{E}-06$ \\
\hline PA0718 & $\begin{array}{l}\text { hypothetical protein of } \\
\text { bacteriophage Pf1 }\end{array}$ & NA & NA & NA & NA & NA & NA & 23.5 & $2.52 \mathrm{E}-06$ \\
\hline
\end{tabular}




\begin{tabular}{|c|c|c|c|c|c|c|c|c|c|c|}
\hline \multicolumn{11}{|c|}{ Membrane proteins } \\
\hline PA3819 & & conserved hypothetical protein & NA & NA & NA & NA & NA & NA & 2.1 & $0.00 \mathrm{E}+00$ \\
\hline PA1053 & & conserved hypothetical protein & NA & NA & NA & NA & NA & NA & 2.2 & $5.59 \mathrm{E}-14$ \\
\hline PA5568 & & conserved hypothetical protein & NA & NA & NA & NA & NA & NA & 2.3 & $2.98 \mathrm{E}-09$ \\
\hline PA5250 & & conserved hypothetical protein & NA & NA & NA & NA & NA & NA & 3.8 & $2.05 \mathrm{E}-10$ \\
\hline PA2004 & & conserved hypothetical protein & NA & NA & NA & NA & NA & NA & 4.8 & 5.77E-09 \\
\hline PA2929 & & hypothetical protein & NA & NA & NA & NA & NA & NA & 5.1 & $9.48 \mathrm{E}-08$ \\
\hline PA3362 & & hypothetical protein & NA & NA & NA & NA & NA & NA & 5.1 & 5.35E-09 \\
\hline PA3748 & & conserved hypothetical protein & NA & NA & NA & NA & NA & NA & 7.2 & 2.09E-06 \\
\hline PA2751 & & conserved hypothetical protein & NA & NA & NA & NA & NA & NA & 22.8 & $3.64 \mathrm{E}-06$ \\
\hline PA2936 & & hypothetical protein & NA & NA & NA & NA & NA & NA & 23.5 & $2.52 \mathrm{E}-06$ \\
\hline PA2784 & & hypothetical protein & NA & NA & NA & NA & NA & NA & 24.5 & $1.50 \mathrm{E}-06$ \\
\hline PA2712 & & hypothetical protein & NA & NA & NA & NA & NA & NA & 31.7 & $3.60 \mathrm{E}-08$ \\
\hline PA1055 & & conserved hypothetical protein & -27.9 & $6.43 \mathrm{E}-07$ & 5.5 & $1.75 \mathrm{E}-09$ & NA & NA & 80.8 & $2.36 \mathrm{E}-14$ \\
\hline PA0070 & $\operatorname{tag} Q 1$ & TagQ1 & NA & NA & NA & NA & NA & NA & 2.2 & $4.11 \mathrm{E}-14$ \\
\hline PA0984 & & colicin immunity protein & NA & NA & NA & NA & NA & NA & 3.1 & $8.30 \mathrm{E}-06$ \\
\hline PA1911 & femR & sigma factor regulator, FemR & NA & NA & NA & NA & NA & NA & 21.6 & $6.86 \mathrm{E}-06$ \\
\hline PA4233 & & $\begin{array}{l}\text { probable major facilitator } \\
\text { superfamily (MFS) transporter }\end{array}$ & NA & NA & NA & NA & NA & NA & 2.1 & $8.92 \mathrm{E}-07$ \\
\hline PA1341 & & $\begin{array}{l}\text { amino acid } \mathrm{ABC} \text { transporter } \\
\text { membrane protein }\end{array}$ & NA & NA & NA & NA & NA & NA & 2.3 & $8.39 \mathrm{E}-06$ \\
\hline PA4503 & & $\begin{array}{l}\text { probable permease of } \mathrm{ABC} \\
\text { transporter }\end{array}$ & NA & NA & NA & NA & NA & NA & 2.6 & 4.97E-07 \\
\hline PA4765 & omlA & $\begin{array}{l}\text { Outer membrane lipoprotein } \\
\text { OmlA precursor }\end{array}$ & NA & NA & NA & NA & NA & NA & 3.1 & $7.54 \mathrm{E}-13$ \\
\hline PA5504 & & $\begin{array}{l}\text { D-methionine } \mathrm{ABC} \text { transporter } \\
\text { membrane protein }\end{array}$ & NA & NA & NA & NA & NA & NA & 3.1 & $2.86 \mathrm{E}-10$ \\
\hline PA2533 & & $\begin{array}{l}\text { probable sodium:alanine } \\
\text { symporter }\end{array}$ & NA & NA & NA & NA & NA & NA & 3.6 & $1.95 \mathrm{E}-07$ \\
\hline PA0890 & aotM & $\begin{array}{l}\text { arginine/ornithine transport } \\
\text { protein AotM }\end{array}$ & NA & NA & NA & NA & NA & NA & 3.6 & $1.85 \mathrm{E}-13$ \\
\hline
\end{tabular}




\begin{tabular}{|c|c|c|c|c|c|c|c|c|c|c|}
\hline PA4707 & \multirow{6}{*}{$g l p M$} & $\begin{array}{l}\text { probable permease of } \mathrm{ABC} \\
\text { transporter }\end{array}$ & NA & NA & NA & NA & NA & NA & 10.3 & $1.26 \mathrm{E}-12$ \\
\hline PA5216 & & $\begin{array}{l}\text { probable permease of } \mathrm{ABC} \text { iron } \\
\text { transporter }\end{array}$ & NA & NA & NA & NA & NA & NA & 12.3 & $6.54 \mathrm{E}-10$ \\
\hline PA3585 & & membrane protein GlpM & NA & NA & NA & NA & NA & NA & 20.7 & $1.05 \mathrm{E}-05$ \\
\hline PA0786 & & probable transporter & NA & NA & NA & NA & NA & NA & 22.4 & 4.59E-06 \\
\hline PA3888 & & $\begin{array}{l}\text { probable permease of } \mathrm{ABC} \\
\text { transporter }\end{array}$ & NA & NA & NA & NA & NA & NA & 37.9 & $1.43 \mathrm{E}-09$ \\
\hline PA4990 & & $\begin{array}{l}\text { SMR multidrug efflux } \\
\text { transporter }\end{array}$ & NA & NA & NA & NA & NA & NA & 20.6 & $1.16 \mathrm{E}-05$ \\
\hline \multicolumn{11}{|c|}{ Motility \& Attachment } \\
\hline PA0406 & tonB3 & TonB3 & NA & NA & NA & NA & NA & NA & 6.2 & 8.79E-07 \\
\hline \multicolumn{11}{|c|}{ Non-coding RNA gene } \\
\hline PA3368.1 & & tRNA-Arg & NA & NA & NA & NA & NA & NA & 2.1 & $0.00 \mathrm{E}+00$ \\
\hline PA3133.2 & & tRNA-Ala & NA & NA & NA & NA & NA & NA & 2.4 & $0.00 \mathrm{E}+00$ \\
\hline \multicolumn{11}{|c|}{ Nucleotide biosynthesis and metabolism } \\
\hline PA0944 & $\operatorname{purN}$ & $\begin{array}{l}\text { phosphoribosylaminoimidazole } \\
\text { synthetase }\end{array}$ & NA & NA & NA & NA & NA & NA & 2.6 & $4.02 \mathrm{E}-10$ \\
\hline PA3527 & pyrC & dihydroorotase & NA & NA & NA & NA & NA & NA & 3.6 & 2.67E-09 \\
\hline \multicolumn{11}{|c|}{ Protein secretion/export apparatus } \\
\hline PA0086 & $\operatorname{tag} J 1$ & TagJ1 & NA & NA & NA & NA & NA & NA & 2.2 & $7.28 \mathrm{E}-06$ \\
\hline PA0089 & $\operatorname{tss} G 1$ & TssG1 & NA & NA & NA & NA & NA & NA & 2.6 & 9.13E-09 \\
\hline PA0077 & tssM1 & TssM1 & NA & NA & NA & NA & NA & NA & 2.6 & $1.82 \mathrm{E}-07$ \\
\hline PA0080 & tss $J 1$ & TssJ1 & -25.4 & $2.22 \mathrm{E}-06$ & 5.5 & $1.88 \mathrm{E}-13$ & NA & NA & 117.7 & $2.75 \mathrm{E}-14$ \\
\hline PA1718 & $p s c E$ & type III export protein PscE & NA & NA & NA & NA & NA & NA & 33.5 & $1.37 \mathrm{E}-08$ \\
\hline PA1699 & pcrl & Pcr1 & NA & NA & NA & NA & NA & NA & 24.5 & $1.50 \mathrm{E}-06$ \\
\hline \multicolumn{11}{|c|}{ Putative enzymes } \\
\hline PA2631 & & probable acetyl transferase & NA & NA & NA & NA & NA & NA & 2.1 & $5.03 \mathrm{E}-06$ \\
\hline PA5312 & & probable aldehyde & NA & NA & NA & NA & NA & NA & 2.1 & 4.94E-08 \\
\hline
\end{tabular}




\begin{tabular}{|c|c|c|c|c|c|c|c|c|c|c|}
\hline PA0506 & & $\begin{array}{l}\text { dehydrogenase } \\
\text { probable acyl-CoA } \\
\text { dehydrogenase }\end{array}$ & NA & NA & NA & NA & NA & NA & 2.4 & $2.13 \mathrm{E}-11$ \\
\hline PA0318 & & conserved hypothetical protein & NA & NA & NA & NA & NA & NA & 3.1 & $1.01 \mathrm{E}-05$ \\
\hline PA5521 & & $\begin{array}{l}\text { probable short-chain } \\
\text { dehydrogenase }\end{array}$ & NA & NA & NA & NA & NA & NA & 3.1 & $2.40 \mathrm{E}-11$ \\
\hline PA0130 & & $\begin{array}{l}\text { probable aldehyde } \\
\text { dehydrogenase }\end{array}$ & NA & NA & NA & NA & NA & NA & 3.3 & $4.83 \mathrm{E}-07$ \\
\hline PA1047 & & probable esterase & NA & NA & NA & NA & NA & NA & 3.4 & $5.13 \mathrm{E}-11$ \\
\hline PA5313 & & $\begin{array}{l}\text { probable pyridoxal-dependent } \\
\text { aminotransferase }\end{array}$ & NA & NA & NA & NA & NA & NA & 3.7 & $1.32 \mathrm{E}-09$ \\
\hline PA0840 & & probable oxidoreductase & NA & NA & NA & NA & NA & NA & 4.6 & $3.10 \mathrm{E}-07$ \\
\hline PA2040 & & probable glutamine synthetase & NA & NA & NA & NA & NA & NA & 5.5 & $4.76 \mathrm{E}-11$ \\
\hline PA3427 & & $\begin{array}{l}\text { probable short-chain } \\
\text { dehydrogenases }\end{array}$ & $\mathrm{NA}$ & NA & NA & NA & NA & NA & 7.2 & $1.16 \mathrm{E}-08$ \\
\hline PA1537 & & $\begin{array}{l}\text { probable short-chain } \\
\text { dehydrogenase }\end{array}$ & $\mathrm{NA}$ & NA & NA & NA & NA & NA & 23.1 & $3.10 \mathrm{E}-06$ \\
\hline PA2550 & & $\begin{array}{l}\text { probable acyl-CoA } \\
\text { dehydrogenase }\end{array}$ & NA & NA & NA & NA & NA & NA & 33.4 & $1.48 \mathrm{E}-08$ \\
\hline PA5020 & & $\begin{array}{l}\text { probable acyl-CoA } \\
\text { dehydrogenase }\end{array}$ & NA & NA & NA & NA & NA & NA & 38.0 & $1.36 \mathrm{E}-09$ \\
\hline PA0656 & & probable HIT family protein & NA & NA & NA & NA & NA & NA & 40.4 & $3.82 \mathrm{E}-10$ \\
\hline PA3554 & $\operatorname{arnA}$ & ArnA & NA & NA & NA & NA & NA & NA & 3.5 & $3.74 \mathrm{E}-06$ \\
\hline PA0705 & $\operatorname{mig} A$ & $\begin{array}{l}\text { alpha-1,6-rhamnosyltransferase } \\
\text { MigA }\end{array}$ & NA & NA & NA & NA & NA & NA & 3.1 & $3.10 \mathrm{E}-06$ \\
\hline PA2237 & $p s l G$ & PslG & NA & NA & NA & NA & NA & NA & 3.1 & $6.60 \mathrm{E}-07$ \\
\hline \multicolumn{11}{|c|}{ Secreted Factors (toxins, enzymes, alginate) } \\
\hline PA0085 & hcpl & Hcp1 & NA & NA & NA & NA & NA & NA & 2.1 & $0.00 \mathrm{E}+00$ \\
\hline PA2702 & tse 2 & Tse2 & NA & NA & NA & NA & NA & NA & 4.1 & $6.90 \mathrm{E}-07$ \\
\hline \multicolumn{11}{|c|}{ Transcription, RNA processing and degradation } \\
\hline PA3823 & $\operatorname{tg} t$ & $\begin{array}{l}\text { queuine tRNA- } \\
\text { ribosyltransferase }\end{array}$ & NA & NA & NA & NA & NA & NA & 2.1 & $2.94 \mathrm{E}-06$ \\
\hline
\end{tabular}




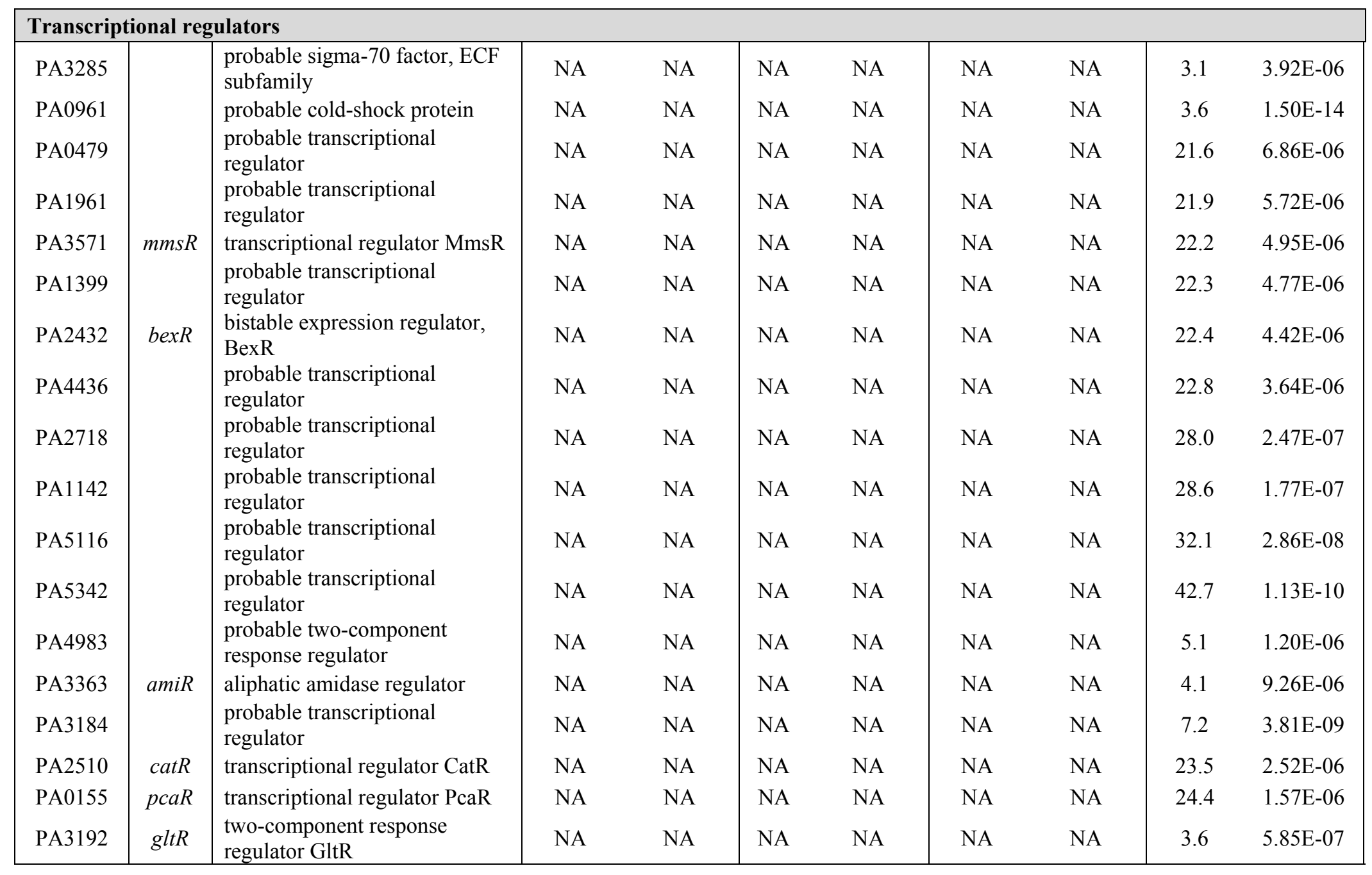




\begin{tabular}{|c|c|c|c|c|c|c|c|c|c|c|}
\hline PA3701 & $\operatorname{prfB}$ & peptide chain release factor 2 & NA & NA & NA & NA & NA & NA & 2.0 & $4.71 \mathrm{E}-07$ \\
\hline PA4498 & & probable metallopeptidase & NA & NA & NA & NA & NA & NA & 2.2 & $1.08 \mathrm{E}-05$ \\
\hline PA3262 & & $\begin{array}{l}\text { probable peptidyl-prolyl cis- } \\
\text { trans isomerase, FkbP-type }\end{array}$ & NA & NA & NA & NA & NA & NA & 2.5 & $3.86 \mathrm{E}-14$ \\
\hline PA0538 & $d s b B$ & $\begin{array}{l}\text { disulfide bond formation } \\
\text { protein }\end{array}$ & NA & NA & NA & NA & NA & NA & 4.1 & $1.66 \mathrm{E}-06$ \\
\hline PA4572 & $f k l B$ & $\begin{array}{l}\text { peptidyl-prolyl cis-trans } \\
\text { isomerase FklB }\end{array}$ & NA & NA & NA & NA & NA & NA & 4.1 & $2.75 \mathrm{E}-14$ \\
\hline \multicolumn{11}{|c|}{ Transport of small molecules } \\
\hline PA5074 & & $\begin{array}{l}\text { probable ATP-binding } \\
\text { component of ABC transporter }\end{array}$ & NA & NA & NA & NA & NA & NA & 2.7 & 7.03E-06 \\
\hline PA1964 & & $\begin{array}{l}\text { probable ATP-binding } \\
\text { component of ABC transporter }\end{array}$ & NA & NA & NA & NA & NA & NA & 5.1 & $2.66 \mathrm{E}-06$ \\
\hline PA3188 & & $\begin{array}{l}\text { probable permease of } \mathrm{ABC} \\
\text { sugar transporter }\end{array}$ & NA & NA & NA & NA & NA & NA & 8.2 & $5.42 \mathrm{E}-11$ \\
\hline PA1183 & $d c t A$ & $\begin{array}{l}\text { C4-dicarboxylate transport } \\
\text { protein }\end{array}$ & NA & NA & NA & NA & NA & NA & 20.9 & $9.85 \mathrm{E}-06$ \\
\hline PA0295 & & $\begin{array}{l}\text { probable periplasmic } \\
\text { polyamine binding protein }\end{array}$ & NA & NA & NA & NA & NA & NA & 25.8 & 7.82E-07 \\
\hline PA3372 & & conserved hypothetical protein & NA & NA & NA & NA & NA & NA & 35.5 & 4.90E-09 \\
\hline PA0950 & & probable arsenate reductase & NA & NA & NA & NA & NA & NA & 2.1 & 4.84E-07 \\
\hline PA3153 & $w z x$ & O-antigen translocase & NA & NA & NA & NA & NA & NA & 2.4 & 5.39E-15 \\
\hline
\end{tabular}


Table 4: B-lactam-dependent genes. The genes that are either positively or negatively regulated when exposed to B-lactam stress in the absence of AmpR are classified based on information in Supplementary Table 1. Only genes that are expressed $\geq 2.0$, FDR-corrected (Bonferroni, $<0.05$ ) are listed. Functional categorization, gene ID and gene/product names are from the Pseudomonas Genome database (27). Lack of expression of a gene under any of the four conditions is denoted as NA.

\begin{tabular}{|c|c|c|c|c|c|c|c|c|c|c|}
\hline \multirow[b]{2}{*}{$\begin{array}{c}\text { Gene } \\
\text { ID }\end{array}$} & \multirow[b]{2}{*}{$\begin{array}{l}\text { Gene } \\
\text { name }\end{array}$} & \multirow[b]{2}{*}{ Product Name } & \multicolumn{2}{|c|}{ Condition 1} & \multicolumn{2}{|c|}{ Condition 2} & \multicolumn{2}{|c|}{ Condition 3} & \multicolumn{2}{|c|}{ Condition 4} \\
\hline & & & FC & $\begin{array}{c}\text { Corrected } \\
p \text {-value }\end{array}$ & FC & $\begin{array}{c}\text { Corrected } \\
p \text {-value }\end{array}$ & FC & $\begin{array}{c}\text { Corrected } \\
p \text {-value }\end{array}$ & FC & $\begin{array}{c}\text { Corrected } \\
p \text {-value }\end{array}$ \\
\hline \multicolumn{11}{|c|}{ Genes positively regulated in an AmpR-independent, B-lactam-dependent manner (278) } \\
\hline \multicolumn{11}{|c|}{ Adaptation, Protection } \\
\hline PA0059 & osm C & $\begin{array}{l}\text { osmotically inducible protein } \\
\text { OsmC }\end{array}$ & NA & NA & 9.7 & $0.00 \mathrm{E}+00$ & NA & NA & 3.6 & $1.57 \mathrm{E}-10$ \\
\hline \multicolumn{11}{|c|}{ Amino acid biosynthesis and metabolism } \\
\hline PA0025 & aroE & shikimate dehydrogenase & NA & NA & 4.8 & $1.43 \mathrm{E}-06$ & NA & NA & 3.6 & $2.88 \mathrm{E}-06$ \\
\hline PA0896 & aruF & $\begin{array}{l}\text { arginine/ornithine } \\
\text { succinyltransferase AI subunit }\end{array}$ & 2.0 & $2.91 \mathrm{E}-10$ & NA & NA & NA & NA & NA & NA \\
\hline PA1338 & $g g t$ & $\begin{array}{l}\text { gamma-glutamyltranspeptidase } \\
\text { precursor }\end{array}$ & NA & NA & 2.6 & $9.04 \mathrm{E}-07$ & NA & NA & 3.6 & $2.19 \mathrm{E}-11$ \\
\hline PA2443 & $s d a A$ & L-serine dehydratase & NA & NA & 2.3 & $1.95 \mathrm{E}-10$ & NA & NA & 2.2 & $4.10 \mathrm{E}-11$ \\
\hline PA2612 & $\operatorname{ser} S$ & seryl-tRNA synthetase & NA & NA & 2.1 & $5.43 \mathrm{E}-07$ & NA & NA & 3.0 & $1.27 \mathrm{E}-13$ \\
\hline PA3107 & met $Z$ & $\begin{array}{l}\text { o-succinylhomoserine } \\
\text { sulfhydrylase }\end{array}$ & NA & NA & 2.2 & 2.03E-07 & NA & NA & 2.3 & 5.30E-09 \\
\hline PA3997 & $\operatorname{lip} B$ & lipoate-protein ligase B & 2.0 & $2.60 \mathrm{E}-06$ & NA & NA & NA & NA & NA & NA \\
\hline PA4449 & hisG & ATP-phosphoribosyltransferase & NA & NA & 2.2 & 8.79E-09 & NA & NA & 6.5 & $0.00 \mathrm{E}+00$ \\
\hline PA4602 & glyA3 & $\begin{array}{l}\text { serine } \\
\text { hydroxymethyltransferase }\end{array}$ & 2.0 & $1.64 \mathrm{E}-07$ & NA & NA & 2.4 & $9.05 \mathrm{E}-13$ & NA & NA \\
\hline PA4731 & $\operatorname{pan} D$ & $\begin{array}{l}\text { aspartate 1-decarboxylase } \\
\text { precursor }\end{array}$ & 2.8 & $2.48 \mathrm{E}-05$ & 71.8 & $1.50 \mathrm{E}-14$ & NA & NA & NA & NA \\
\hline PA4846 & $\operatorname{aroQ1}$ & 3-dehydroquinate dehydratase & NA & NA & 3.0 & $0.00 \mathrm{E}+00$ & NA & NA & 4.2 & $0.00 \mathrm{E}+00$ \\
\hline PA5015 & aceE & pyruvate dehydrogenase & NA & NA & 2.0 & 4.46E-08 & NA & NA & 2.6 & $0.00 \mathrm{E}+00$ \\
\hline PA5171 & $\operatorname{arcA}$ & arginine deiminase & 2.1 & $2.39 \mathrm{E}-11$ & 2.2 & $6.77 \mathrm{E}-14$ & NA & NA & NA & NA \\
\hline PA5172 & $\operatorname{arcB}$ & ornithine carbamoyltransferase, & NA & NA & 2.4 & $0.00 \mathrm{E}+00$ & NA & NA & 2.0 & $3.52 \mathrm{E}-14$ \\
\hline
\end{tabular}




\begin{tabular}{|c|c|c|c|c|c|c|c|c|c|c|}
\hline PA5173 & $\operatorname{arcc}$ & $\begin{array}{l}\text { catabolic } \\
\text { carbamate kinase }\end{array}$ & 2.3 & $1.20 \mathrm{E}-08$ & NA & NA & NA & NA & NA & NA \\
\hline PA5323 & $\arg B$ & acetylglutamate kinase & NA & NA & 2.1 & $2.58 \mathrm{E}-10$ & NA & NA & 2.1 & $1.24 \mathrm{E}-11$ \\
\hline \multicolumn{11}{|c|}{ Biosynthesis of cofactors, prosthetic groups and carriers } \\
\hline PA0583 & & hypothetical protein & 5.7 & $1.13 \mathrm{E}-06$ & NA & NA & NA & NA & NA & NA \\
\hline PA3029 & moaB2 & $\begin{array}{l}\text { molybdopterin biosynthetic } \\
\text { protein B2 }\end{array}$ & 3.1 & $6.26 \mathrm{E}-10$ & NA & NA & 2.8 & $1.25 \mathrm{E}-07$ & NA & NA \\
\hline PA4047 & $\operatorname{ribA}$ & GTP cyclohydrolase II & 3.1 & 7.10E-11 & NA & NA & 3.0 & $5.42 \mathrm{E}-10$ & NA & NA \\
\hline PA4728 & folK & $\begin{array}{l}\text { 2-amino-4-hydroxy-6- } \\
\text { hydroxymethyldihydropteridine } \\
\text { pyrophosphokinase }\end{array}$ & NA & NA & 8.3 & $1.01 \mathrm{E}-11$ & NA & NA & 84.0 & $3.86 \mathrm{E}-14$ \\
\hline \multicolumn{11}{|c|}{ Carbon compound catabolism } \\
\hline PA0232 & pcaC & $\begin{array}{l}\text { gamma-carboxymuconolactone } \\
\text { decarboxylase }\end{array}$ & NA & NA & 6.9 & $4.48 \mathrm{E}-11$ & NA & NA & 5.1 & $2.50 \mathrm{E}-11$ \\
\hline PA4127 & $h p c G$ & $\begin{array}{l}\text { 2-oxo-hept-3-ene-1,7-dioate } \\
\text { hydratase }\end{array}$ & 26.8 & $6.45 \mathrm{E}-06$ & NA & NA & NA & NA & NA & NA \\
\hline \multicolumn{11}{|c|}{ Cell division } \\
\hline PA4419 & $f t s L$ & cell division protein FtsL & NA & NA & 2.4 & $1.98 \mathrm{E}-08$ & NA & NA & 2.4 & $1.23 \mathrm{E}-09$ \\
\hline PA5564 & $\operatorname{gid} B$ & $\begin{array}{l}\text { glucose inhibited division } \\
\text { protein B }\end{array}$ & NA & NA & 5.5 & $2.19 \mathrm{E}-14$ & NA & NA & 2.5 & $4.45 \mathrm{E}-08$ \\
\hline \multicolumn{11}{|c|}{ Cell wall / LPS / capsule } \\
\hline PA2232 & $p s l B$ & PslB & NA & NA & 2.5 & 4.91E-09 & NA & NA & 2.6 & $2.02 \mathrm{E}-11$ \\
\hline PA2235 & pslE & PslE & NA & NA & 2.4 & $3.42 \mathrm{E}-07$ & NA & NA & 2.3 & $1.12 \mathrm{E}-07$ \\
\hline PA3999 & dacC & D-ala-D-ala-carboxypeptidase & NA & NA & 2.2 & $8.78 \mathrm{E}-14$ & NA & NA & 2.0 & $1.51 \mathrm{E}-13$ \\
\hline PA4415 & $\operatorname{mraY}$ & $\begin{array}{l}\text { phospho-N-acetylmuramoyl- } \\
\text { pentapeptide-transferase }\end{array}$ & 2.0 & 4.46E-07 & NA & NA & NA & NA & NA & NA \\
\hline PA4668 & & $\begin{array}{l}\text { probable lipoprotein } \\
\text { localization protein LolB }\end{array}$ & 3.6 & $3.65 \mathrm{E}-09$ & NA & NA & NA & NA & NA & NA \\
\hline PA5163 & rmlA & $\begin{array}{l}\text { glucose-1-phosphate } \\
\text { thymidylyltransferase }\end{array}$ & NA & NA & 2.5 & $5.49 \mathrm{E}-12$ & NA & NA & 2.1 & $1.71 \mathrm{E}-10$ \\
\hline PA5276 & $\operatorname{lppL}$ & Lipopeptide LppL precursor & 5.7 & $0.00 \mathrm{E}+00$ & NA & NA & 4.2 & $4.55 \mathrm{E}-14$ & NA & NA \\
\hline
\end{tabular}




\begin{tabular}{|c|c|c|c|c|c|c|c|c|c|c|}
\hline PA5322 & $\operatorname{alg} C$ & phosphomannomutase AlgC & NA & NA & 2.6 & $1.06 \mathrm{E}-13$ & NA & NA & 3.3 & $0.00 \mathrm{E}+00$ \\
\hline \multicolumn{11}{|c|}{ Central intermediary metabolism } \\
\hline PA3524 & gloA1 & lactoylglutathione lyase & 2.0 & 5.32E-07 & NA & NA & 2.0 & $4.42 \mathrm{E}-06$ & NA & NA \\
\hline PA4880 & & probable bacterioferritin & 26.9 & $6.17 \mathrm{E}-06$ & NA & NA & NA & NA & NA & NA \\
\hline \multicolumn{11}{|c|}{ Chemotaxis ; Adaptation, Protection ; Motility \& Attachment } \\
\hline PA1446 & fliP & $\begin{array}{l}\text { flagellar biosynthetic protein } \\
\text { FliP }\end{array}$ & 2.3 & $4.37 \mathrm{E}-06$ & NA & NA & NA & NA & NA & NA \\
\hline \multicolumn{11}{|c|}{ DNA replication, recombination, modification and repair } \\
\hline PA0002 & dnaN & DNA polymerase III, beta chain & 2.1 & $9.60 \mathrm{E}-12$ & NA & NA & NA & NA & NA & NA \\
\hline PA0720 & & $\begin{array}{l}\text { helix destabilizing protein of } \\
\text { bacteriophage Pf1 }\end{array}$ & 8.5 & $1.98 \mathrm{E}-07$ & NA & NA & NA & NA & NA & NA \\
\hline PA0965 & $\operatorname{ruv} C$ & $\begin{array}{l}\text { Holliday junction resolvase } \\
\text { RuvC }\end{array}$ & 2.8 & $3.41 \mathrm{E}-08$ & NA & NA & NA & NA & NA & NA \\
\hline PA1534 & reck & recombination protein $\mathrm{RecR}$ & 4.7 & $9.40 \mathrm{E}-07$ & NA & NA & NA & NA & NA & NA \\
\hline PA1815 & $\operatorname{rnh} A$ & ribonuclease $\mathrm{H}$ & 2.8 & $1.80 \mathrm{E}-11$ & NA & NA & NA & NA & NA & NA \\
\hline PA2961 & holB & $\begin{array}{l}\text { DNA polymerase III, delta } \\
\text { prime subunit }\end{array}$ & NA & NA & 10.4 & $0.00 \mathrm{E}+00$ & NA & NA & 2.6 & $3.54 \mathrm{E}-07$ \\
\hline \multicolumn{11}{|c|}{ Energy metabolism } \\
\hline PA1562 & acnA & $\begin{array}{l}\text { aconitate hydratase } 1 \\
\mathrm{Na}+\text {-translocating }\end{array}$ & NA & NA & 4.0 & $1.28 \mathrm{E}-12$ & NA & NA & 3.0 & $4.48 \mathrm{E}-11$ \\
\hline PA2995 & $n q r E$ & $\begin{array}{l}\text { NADH:quinone oxidoreductase } \\
\text { subunit Nqr5 } \\
\text { Na+-translocating }\end{array}$ & NA & NA & 2.2 & 8.00E-07 & NA & NA & 3.3 & $2.68 \mathrm{E}-13$ \\
\hline PA2997 & $n q r C$ & $\begin{array}{l}\text { NADH:ubiquinone } \\
\text { oxidoreductase subunit Nrq3 }\end{array}$ & 2.5 & $4.15 \mathrm{E}-07$ & NA & NA & NA & NA & NA & NA \\
\hline PA3183 & $z w f$ & $\begin{array}{l}\text { glucose-6-phosphate } 1- \\
\text { dehydrogenase }\end{array}$ & 2.7 & $1.40 \mathrm{E}-06$ & 2.8 & $3.20 \mathrm{E}-07$ & NA & NA & NA & NA \\
\hline PA4975 & & $\begin{array}{l}\mathrm{NAD}(\mathrm{P}) \mathrm{H} \text { quinone } \\
\text { oxidoreductase }\end{array}$ & 5.7 & $2.50 \mathrm{E}-05$ & NA & NA & NA & NA & NA & NA \\
\hline \multicolumn{11}{|c|}{ Fatty acid and phospholipid metabolism } \\
\hline PA1806 & $f a b I$ & $\begin{array}{l}\text { NADH-dependent enoyl-ACP } \\
\text { reductase }\end{array}$ & 3.6 & $2.85 \mathrm{E}-07$ & NA & NA & NA & NA & NA & NA \\
\hline
\end{tabular}




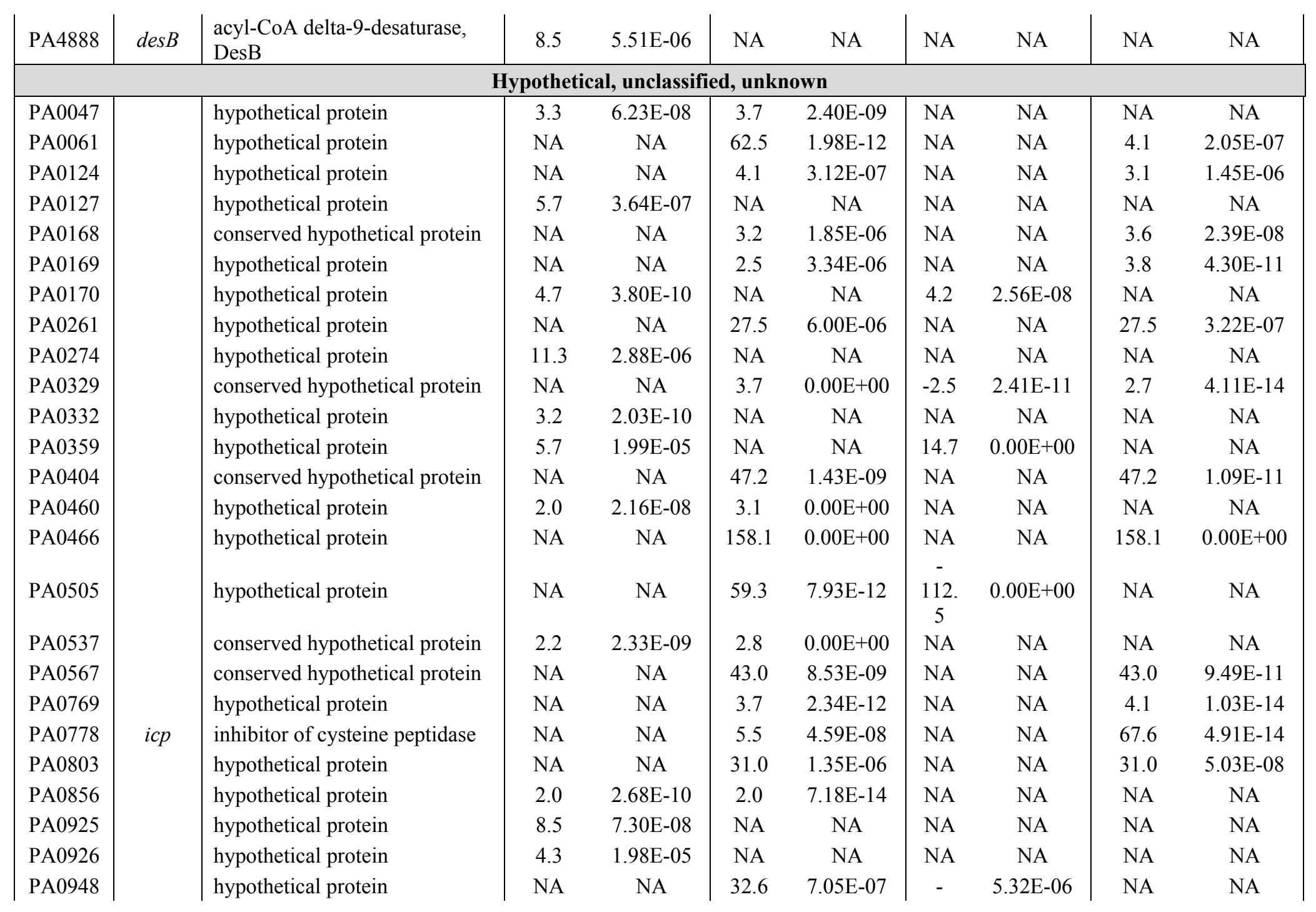




\begin{tabular}{|c|c|c|c|c|c|c|c|c|c|c|}
\hline & & & & & & & 28.1 & & & \\
\hline PA0952 & & hypothetical protein & NA & NA & 35.6 & $1.99 \mathrm{E}-07$ & NA & NA & 35.6 & 4.55E-09 \\
\hline PA1324 & & hypothetical protein & NA & NA & 3.5 & $8.70 \mathrm{E}-11$ & NA & NA & 5.1 & 4.64E-14 \\
\hline PA1343 & & hypothetical protein & 8.5 & 4.44E-07 & NA & NA & 10.5 & $3.29 \mathrm{E}-09$ & NA & NA \\
\hline PA1395 & & hypothetical protein & 8.5 & $1.04 \mathrm{E}-07$ & NA & NA & NA & NA & NA & NA \\
\hline PA1439 & & conserved hypothetical protein & 5.7 & $1.26 \mathrm{E}-08$ & NA & NA & NA & NA & NA & NA \\
\hline PA1471 & & hypothetical protein & NA & NA & 5.5 & $1.00 \mathrm{E}-10$ & NA & NA & 4.1 & $1.84 \mathrm{E}-10$ \\
\hline PA1560 & & hypothetical protein & NA & NA & 2.8 & $2.53 \mathrm{E}-06$ & NA & NA & 6.2 & $6.14 \mathrm{E}-14$ \\
\hline PA1592 & & hypothetical protein & NA & NA & 4.2 & $0.00 \mathrm{E}+00$ & NA & NA & 3.5 & $0.00 \mathrm{E}+00$ \\
\hline PA1744 & & hypothetical protein & 32.8 & $4.68 \mathrm{E}-07$ & NA & NA & 60.0 & $0.00 \mathrm{E}+00$ & NA & NA \\
\hline PA1747 & & hypothetical protein & 76.0 & $1.55 \mathrm{E}-13$ & 36.2 & $1.57 \mathrm{E}-07$ & NA & NA & NA & NA \\
\hline PA1774 & $\operatorname{crf} X$ & CrfX protein & 3.3 & $0.00 \mathrm{E}+00$ & NA & NA & NA & NA & NA & NA \\
\hline PA1817 & & hypothetical protein & 36.8 & $8.06 \mathrm{E}-08$ & NA & NA & 22.5 & $3.81 \mathrm{E}-06$ & NA & NA \\
\hline PA1855 & & hypothetical protein & NA & NA & 30.8 & $1.47 \mathrm{E}-06$ & NA & NA & 30.8 & $5.61 \mathrm{E}-08$ \\
\hline PA1879 & & hypothetical protein & 25.9 & $9.68 \mathrm{E}-06$ & NA & NA & NA & NA & NA & NA \\
\hline PA1892 & & hypothetical protein & 38.9 & $3.23 \mathrm{E}-08$ & 37.1 & $1.08 \mathrm{E}-07$ & NA & NA & NA & NA \\
\hline PA1963 & & hypothetical protein & 53.8 & $4.28 \mathrm{E}-11$ & 51.3 & $2.44 \mathrm{E}-10$ & NA & NA & NA & NA \\
\hline PA2021 & & hypothetical protein & NA & NA & 30.4 & $1.76 \mathrm{E}-06$ & NA & NA & 30.4 & $6.97 \mathrm{E}-08$ \\
\hline PA2029 & & hypothetical protein & NA & NA & 4.1 & $7.35 \mathrm{E}-07$ & NA & NA & 68.4 & $5.59 \mathrm{E}-14$ \\
\hline PA2106 & & hypothetical protein & 4.7 & $1.23 \mathrm{E}-05$ & 3.7 & $9.34 \mathrm{E}-07$ & NA & NA & NA & NA \\
\hline PA2127 & & conserved hypothetical protein & 4.5 & $6.61 \mathrm{E}-07$ & NA & NA & NA & NA & NA & NA \\
\hline PA2174 & & hypothetical protein & NA & NA & 44.3 & $5.00 \mathrm{E}-09$ & NA & NA & 44.3 & $5.00 \mathrm{E}-11$ \\
\hline PA2226 & & hypothetical protein & NA & NA & 27.3 & $6.44 \mathrm{E}-06$ & $\begin{array}{c}- \\
28.3\end{array}$ & $4.97 \mathrm{E}-06$ & NA & NA \\
\hline PA2293 & & hypothetical protein & NA & NA & 28.3 & $4.21 \mathrm{E}-06$ & NA & NA & 28.3 & $2.05 \mathrm{E}-07$ \\
\hline PA2368 & & hypothetical protein & 50.6 & $1.81 \mathrm{E}-10$ & NA & NA & NA & NA & NA & NA \\
\hline PA2484 & & conserved hypothetical protein & NA & NA & 3.2 & $3.05 \mathrm{E}-06$ & NA & NA & 3.6 & 4.57E-08 \\
\hline PA2560 & & hypothetical protein & 2.4 & $3.23 \mathrm{E}-12$ & 3.2 & $2.25 \mathrm{E}-12$ & NA & NA & NA & NA \\
\hline PA2598 & & hypothetical protein & 26.5 & 7.31E-06 & NA & NA & NA & NA & NA & NA \\
\hline
\end{tabular}




\begin{tabular}{|c|c|c|c|c|c|c|c|c|c|}
\hline PA2607 & conserved hypothetical protein & 4.3 & $3.08 \mathrm{E}-07$ & NA & NA & NA & NA & NA & NA \\
\hline PA2658 & hypothetical protein & NA & NA & 4.1 & $1.41 \mathrm{E}-06$ & -3.8 & $1.07 \mathrm{E}-05$ & NA & NA \\
\hline PA2730 & hypothetical protein & NA & NA & 5.8 & $0.00 \mathrm{E}+00$ & -3.3 & $5.40 \mathrm{E}-07$ & NA & NA \\
\hline PA2736 & hypothetical protein & 2.0 & $2.48 \mathrm{E}-05$ & 2.8 & $1.12 \mathrm{E}-09$ & NA & NA & NA & NA \\
\hline PA2737 & conserved hypothetical protein & NA & NA & 2.4 & $1.68 \mathrm{E}-09$ & -2.7 & $1.02 \mathrm{E}-12$ & NA & NA \\
\hline PA2792 & hypothetical protein & NA & NA & 4.1 & $6.88 \mathrm{E}-07$ & NA & NA & 6.2 & $2.66 \mathrm{E}-10$ \\
\hline PA2797 & hypothetical protein & 3.1 & $1.55 \mathrm{E}-13$ & 2.1 & $9.12 \mathrm{E}-07$ & NA & NA & NA & NA \\
\hline PA2864 & conserved hypothetical protein & NA & NA & 5.5 & $1.45 \mathrm{E}-07$ & NA & NA & 4.1 & $1.86 \mathrm{E}-07$ \\
\hline PA2872 & hypothetical protein & 7.1 & $1.37 \mathrm{E}-06$ & NA & NA & NA & NA & NA & NA \\
\hline PA2883 & hypothetical protein & 2.8 & $6.09 \mathrm{E}-06$ & 2.8 & $1.58 \mathrm{E}-05$ & NA & NA & NA & NA \\
\hline PA2935 & hypothetical protein & NA & NA & 28.5 & $3.92 \mathrm{E}-06$ & NA & NA & 28.5 & $1.88 \mathrm{E}-07$ \\
\hline PA2956 & conserved hypothetical protein & 7.1 & $8.86 \mathrm{E}-11$ & NA & NA & NA & NA & NA & NA \\
\hline PA2972 & conserved hypothetical protein & NA & NA & 5.5 & $6.36 \mathrm{E}-06$ & NA & NA & 47.3 & $1.05 \mathrm{E}-11$ \\
\hline PA3010 & hypothetical protein & 3.6 & $8.86 \mathrm{E}-11$ & NA & NA & NA & NA & NA & NA \\
\hline PA3018 & hypothetical protein & 14.2 & $7.00 \mathrm{E}-09$ & 49.8 & $4.60 \mathrm{E}-10$ & NA & NA & NA & NA \\
\hline PA3022 & hypothetical protein & NA & NA & 2.8 & $1.46 \mathrm{E}-09$ & -2.9 & $1.20 \mathrm{E}-10$ & NA & NA \\
\hline PA3031 & hypothetical protein & NA & NA & 3.2 & $1.79 \mathrm{E}-13$ & NA & NA & 3.1 & $6.14 \mathrm{E}-14$ \\
\hline PA3040 & conserved hypothetical protein & NA & NA & 4.8 & $0.00 \mathrm{E}+00$ & -3.3 & $5.85 \mathrm{E}-08$ & 2.4 & $1.08 \mathrm{E}-08$ \\
\hline PA3042 & hypothetical protein & NA & NA & 2.8 & 4.56E-08 & NA & NA & 3.1 & $1.06 \mathrm{E}-10$ \\
\hline PA3051 & hypothetical protein & 5.7 & $1.85 \mathrm{E}-06$ & NA & NA & NA & NA & NA & NA \\
\hline PA3056 & hypothetical protein & 2.8 & $3.07 \mathrm{E}-06$ & 5.5 & $5.48 \mathrm{E}-07$ & NA & NA & NA & NA \\
\hline PA3057 & hypothetical protein & NA & $\mathrm{NA}$ & 29.6 & $2.46 \mathrm{E}-06$ & NA & NA & 29.6 & $1.05 \mathrm{E}-07$ \\
\hline PA3072 & hypothetical protein & 58.0 & $6.07 \mathrm{E}-12$ & NA & NA & 35.4 & $3.06 \mathrm{E}-09$ & NA & NA \\
\hline PA3123 & conserved hypothetical protein & NA & NA & 3.2 & $1.34 \mathrm{E}-09$ & -2.7 & 1.19E-06 & 2.4 & $1.02 \mathrm{E}-07$ \\
\hline PA3203 & hypothetical protein & 2.4 & $1.84 \mathrm{E}-06$ & NA & NA & NA & NA & NA & NA \\
\hline PA3274 & hypothetical protein & 24.9 & $1.47 \mathrm{E}-05$ & NA & NA & NA & NA & NA & NA \\
\hline PA3291 & hypothetical protein & NA & NA & 37.0 & $1.12 \mathrm{E}-07$ & NA & NA & 37.0 & $2.25 \mathrm{E}-09$ \\
\hline PA3307 & hypothetical protein & NA & NA & 2.3 & $1.30 \mathrm{E}-05$ & NA & NA & 2.6 & $1.41 \mathrm{E}-07$ \\
\hline PA3338 & hypothetical protein & 5.7 & $2.74 \mathrm{E}-06$ & NA & NA & NA & NA & NA & NA \\
\hline
\end{tabular}




\begin{tabular}{|c|c|c|c|c|c|c|c|c|c|c|}
\hline PA3412 & & hypothetical protein & NA & NA & 25.3 & $1.47 \mathrm{E}-05$ & NA & NA & 25.3 & $9.73 \mathrm{E}-07$ \\
\hline PA3425 & & hypothetical protein & NA & NA & 39.7 & 3.64E-08 & NA & NA & 39.7 & $5.53 \mathrm{E}-10$ \\
\hline PA3470 & & hypothetical protein & 4.3 & 2.17E-09 & NA & NA & 3.2 & $1.71 \mathrm{E}-05$ & NA & NA \\
\hline PA3501 & & hypothetical protein & 32.8 & $4.68 \mathrm{E}-07$ & 62.5 & $1.98 \mathrm{E}-12$ & NA & NA & NA & NA \\
\hline PA3626 & & conserved hypothetical protein & 2.5 & $7.56 \mathrm{E}-06$ & NA & NA & NA & NA & NA & NA \\
\hline PA3668 & & conserved hypothetical protein & 4.3 & $1.84 \mathrm{E}-05$ & NA & NA & NA & NA & NA & NA \\
\hline PA3691 & & hypothetical protein & NA & NA & 8.8 & $0.00 \mathrm{E}+00$ & NA & NA & 5.9 & $2.01 \mathrm{E}-13$ \\
\hline PA3716 & & hypothetical protein & 2.1 & $1.01 \mathrm{E}-05$ & NA & NA & NA & NA & NA & NA \\
\hline PA3762 & & hypothetical protein & 2.4 & $6.16 \mathrm{E}-06$ & NA & NA & NA & NA & NA & NA \\
\hline PA3952 & & hypothetical protein & NA & NA & 2.8 & $5.60 \mathrm{E}-09$ & NA & NA & 2.5 & $6.26 \mathrm{E}-09$ \\
\hline PA3982 & & conserved hypothetical protein & NA & NA & 3.7 & $7.18 \mathrm{E}-14$ & NA & NA & 2.7 & $4.11 \mathrm{E}-14$ \\
\hline PA3986 & & hypothetical protein & NA & NA & 63.4 & $1.35 \mathrm{E}-12$ & $\begin{array}{c}- \\
27.4\end{array}$ & $7.38 \mathrm{E}-06$ & NA & NA \\
\hline PA4028 & & hypothetical protein & NA & NA & 61.7 & $2.79 \mathrm{E}-12$ & NA & NA & 61.7 & $0.00 \mathrm{E}+00$ \\
\hline PA4033 & $m u c E$ & MucE & NA & NA & 50.7 & $3.12 \mathrm{E}-10$ & NA & NA & 50.7 & $1.72 \mathrm{E}-12$ \\
\hline PA4090 & & hypothetical protein & NA & NA & 47.0 & $1.53 \mathrm{E}-09$ & $\begin{array}{c}- \\
65.0\end{array}$ & $7.36 \mathrm{E}-13$ & NA & NA \\
\hline PA4116 & bphO & heme oxygenase, BphO & 2.1 & $2.95 \mathrm{E}-06$ & NA & NA & NA & NA & NA & NA \\
\hline PA4154 & & conserved hypothetical protein & 14.2 & 4.24E-09 & 3.5 & $1.92 \mathrm{E}-08$ & NA & NA & NA & NA \\
\hline PA4346 & & hypothetical protein & NA & NA & 4.1 & $1.34 \mathrm{E}-11$ & NA & NA & 2.1 & $8.80 \mathrm{E}-06$ \\
\hline PA4388 & & hypothetical protein & NA & NA & 37.2 & $1.01 \mathrm{E}-07$ & 28.9 & $3.82 \mathrm{E}-06$ & NA & NA \\
\hline PA4392 & & conserved hypothetical protein & NA & NA & 5.5 & $1.84 \mathrm{E}-08$ & NA & NA & 71.3 & $4.91 \mathrm{E}-14$ \\
\hline PA4422 & & conserved hypothetical protein & NA & NA & 3.5 & $7.56 \mathrm{E}-07$ & NA & NA & 2.6 & $8.55 \mathrm{E}-06$ \\
\hline PA4537 & & hypothetical protein & 42.0 & 8.09E-09 & NA & NA & 25.6 & $6.69 \mathrm{E}-07$ & NA & NA \\
\hline PA4570 & & hypothetical protein & 31.9 & $6.88 \mathrm{E}-07$ & NA & NA & 19.5 & $1.87 \mathrm{E}-05$ & NA & NA \\
\hline PA4699 & & hypothetical protein & 2.2 & $1.77 \mathrm{E}-07$ & 2.6 & $1.11 \mathrm{E}-08$ & NA & NA & NA & NA \\
\hline PA4870 & & conserved hypothetical protein & 5.7 & $8.94 \mathrm{E}-07$ & NA & NA & NA & NA & NA & NA \\
\hline PA4965 & & hypothetical protein & NA & NA & 2.2 & $2.39 \mathrm{E}-06$ & NA & NA & 2.8 & $8.32 \mathrm{E}-11$ \\
\hline PA5062 & & conserved hypothetical protein & 26.0 & $9.13 \mathrm{E}-06$ & NA & NA & 31.7 & $2.33 \mathrm{E}-08$ & NA & NA \\
\hline
\end{tabular}




\begin{tabular}{|c|c|c|c|c|c|c|c|c|c|}
\hline PA5078 & conserved hypothetical protein & NA & NA & 2.2 & $1.37 \mathrm{E}-08$ & NA & NA & 2.0 & $1.37 \mathrm{E}-08$ \\
\hline PA5108 & hypothetical protein & 2.1 & $3.93 \mathrm{E}-10$ & 4.6 & $8.07 \mathrm{E}-14$ & NA & NA & NA & NA \\
\hline PA5120 & hypothetical protein & NA & NA & 50.7 & $3.12 \mathrm{E}-10$ & NA & NA & 50.7 & $1.72 \mathrm{E}-12$ \\
\hline PA5148 & conserved hypothetical protein & NA & NA & 3.8 & $6.31 \mathrm{E}-14$ & NA & NA & 2.8 & $2.75 \mathrm{E}-14$ \\
\hline PA5178 & conserved hypothetical protein & NA & NA & 4.6 & $0.00 \mathrm{E}+00$ & NA & NA & 2.9 & $0.00 \mathrm{E}+00$ \\
\hline PA5208 & conserved hypothetical protein & 2.8 & $1.57 \mathrm{E}-06$ & NA & NA & NA & NA & NA & NA \\
\hline PA5212 & hypothetical protein & 3.8 & $1.39 \mathrm{E}-14$ & 2.8 & $1.06 \mathrm{E}-12$ & NA & NA & NA & NA \\
\hline PA5229 & conserved hypothetical protein & NA & NA & 3.2 & $4.80 \mathrm{E}-08$ & NA & NA & 2.4 & $1.57 \mathrm{E}-06$ \\
\hline PA5258 & hypothetical protein & 2.0 & $9.23 \mathrm{E}-06$ & NA & NA & NA & NA & NA & NA \\
\hline PA5271 & hypothetical protein & NA & NA & 4.1 & $8.78 \mathrm{E}-14$ & NA & NA & 2.1 & 7.40E-08 \\
\hline PA5279 & conserved hypothetical protein & 2.4 & $1.88 \mathrm{E}-05$ & NA & NA & NA & NA & NA & NA \\
\hline PA5330 & hypothetical protein & 2.2 & $5.08 \mathrm{E}-06$ & NA & NA & NA & NA & NA & NA \\
\hline PA5456 & hypothetical protein & 3.8 & $2.79 \mathrm{E}-06$ & NA & NA & NA & NA & NA & NA \\
\hline PA5461 & hypothetical protein & 2.0 & $1.62 \mathrm{E}-13$ & NA & NA & NA & NA & NA & NA \\
\hline PA5494 & hypothetical protein & 2.6 & $0.00 \mathrm{E}+00$ & NA & NA & NA & NA & NA & NA \\
\hline PA5519 & conserved hypothetical protein & NA & NA & 60.3 & $4.92 \mathrm{E}-12$ & NA & NA & 5.1 & $2.58 \mathrm{E}-08$ \\
\hline PA5545 & conserved hypothetical protein & NA & NA & 3.7 & $6.90 \mathrm{E}-10$ & NA & NA & 2.7 & $1.84 \mathrm{E}-08$ \\
\hline PA5551 & hypothetical protein & NA & NA & 26.8 & 7.87E-06 & 41.7 & $1.65 \mathrm{E}-08$ & NA & NA \\
\hline
\end{tabular}

\begin{tabular}{|c|c|c|c|c|c|c|c|c|c|c|}
\hline \multicolumn{11}{|c|}{ Membrane proteins } \\
\hline PA0013 & \multirow{4}{*}{ creD } & conserved hypothetical protein & 5.7 & 4.07E-08 & NA & NA & NA & NA & NA & NA \\
\hline PA0340 & & conserved hypothetical protein & NA & NA & 4.8 & $1.01 \mathrm{E}-06$ & NA & NA & 3.6 & 2.09E-06 \\
\hline PA0465 & & inner membrane protein $\mathrm{CreD}$ & 9.0 & $0.00 \mathrm{E}+00$ & 27.7 & $0.00 \mathrm{E}+00$ & NA & NA & 2.2 & $8.64 \mathrm{E}-10$ \\
\hline PA0833 & & hypothetical protein & 2.5 & $0.00 \mathrm{E}+00$ & 2.9 & $2.19 \mathrm{E}-14$ & NA & NA & NA & NA \\
\hline PA1119 & \multirow[t]{3}{*}{$y f i B$} & YfiB & NA & NA & 2.3 & $1.23 \mathrm{E}-06$ & NA & NA & 2.1 & $2.83 \mathrm{E}-06$ \\
\hline PA1199 & & probable lipoprotein & 3.2 & $1.11 \mathrm{E}-13$ & NA & NA & 2.1 & $1.07 \mathrm{E}-05$ & NA & NA \\
\hline PA1288 & & $\begin{array}{l}\text { probable outer membrane } \\
\text { protein precursor }\end{array}$ & 2.0 & $5.81 \mathrm{E}-10$ & NA & NA & 2.3 & $0.00 \mathrm{E}+00$ & NA & NA \\
\hline
\end{tabular}




\begin{tabular}{|c|c|c|c|c|c|c|c|c|c|c|}
\hline PA1496 & \multirow{10}{*}{$\operatorname{cmaX}$} & probable potassium channel & 7.1 & 4.79E-06 & NA & NA & NA & NA & NA & NA \\
\hline PA1676 & & hypothetical protein & NA & NA & 3.5 & $9.17 \mathrm{E}-09$ & NA & NA & 5.1 & $7.91 \mathrm{E}-14$ \\
\hline PA1773 & & CmaX protein & 2.1 & $1.30 \mathrm{E}-07$ & NA & NA & NA & NA & NA & NA \\
\hline PA2757 & & hypothetical protein & NA & NA & 30.8 & $1.47 \mathrm{E}-06$ & NA & NA & 30.8 & $5.61 \mathrm{E}-08$ \\
\hline PA2763 & & hypothetical protein & NA & NA & 65.2 & $6.49 \mathrm{E}-13$ & NA & NA & 65.2 & $4.64 \mathrm{E}-14$ \\
\hline PA2775 & & hypothetical protein & 3.1 & $4.06 \mathrm{E}-12$ & NA & NA & 2.6 & $1.39 \mathrm{E}-07$ & NA & NA \\
\hline PA2777 & & conserved hypothetical protein & NA & NA & 3.3 & $5.77 \mathrm{E}-07$ & NA & NA & 3.1 & $1.46 \mathrm{E}-07$ \\
\hline PA2900 & & $\begin{array}{l}\text { probable outer membrane } \\
\text { protein precursor }\end{array}$ & 2.5 & $2.11 \mathrm{E}-06$ & NA & NA & NA & NA & NA & NA \\
\hline PA3278 & & hypothetical protein & 4.5 & $0.00 \mathrm{E}+00$ & NA & NA & NA & NA & NA & NA \\
\hline PA3369 & & hypothetical protein & NA & NA & 49.1 & $6.39 \mathrm{E}-10$ & $\begin{array}{c}- \\
76.2\end{array}$ & $0.00 \mathrm{E}+00$ & NA & NA \\
\hline PA3558 & $\operatorname{arnF}$ & ArnF & NA & NA & 33.1 & 5.79E-07 & NA & NA & 33.1 & $1.77 \mathrm{E}-08$ \\
\hline PA3692 & \multirow{3}{*}{$l p t F$} & Lipotoxon F, LptF & NA & NA & 6.8 & $0.00 \mathrm{E}+00$ & NA & NA & 10.2 & $1.74 \mathrm{E}-13$ \\
\hline PA3890 & & $\begin{array}{l}\text { probable permease of } \mathrm{ABC} \\
\text { transporter }\end{array}$ & NA & NA & 51.6 & $2.10 \mathrm{E}-10$ & NA & NA & 51.6 & $1.06 \mathrm{E}-12$ \\
\hline PA4014 & & hypothetical protein & 7.1 & $9.95 \mathrm{E}-12$ & NA & NA & 6.3 & $5.86 \mathrm{E}-10$ & NA & NA \\
\hline PA4034 & \multirow[t]{2}{*}{ aqpZ } & aquaporin $\mathrm{Z}$ & NA & NA & 11.1 & $4.03 \mathrm{E}-14$ & NA & NA & 4.1 & $0.00 \mathrm{E}+00$ \\
\hline PA4940 & & conserved hypothetical protein & 2.8 & $1.92 \mathrm{E}-05$ & NA & NA & 3.2 & $1.42 \mathrm{E}-06$ & NA & NA \\
\hline PA5107 & \multirow[t]{6}{*}{$b l c$} & outer membrane lipoprotein Blc & 2.6 & $6.54 \mathrm{E}-09$ & 2.9 & $1.70 \mathrm{E}-13$ & NA & NA & NA & NA \\
\hline PA5154 & & $\begin{array}{l}\text { probable permease of } \mathrm{ABC} \\
\text { transporter }\end{array}$ & 3.5 & 7.84E-09 & NA & NA & 3.2 & $5.67 \mathrm{E}-07$ & NA & NA \\
\hline PA5167 & & $\begin{array}{l}\text { probable c4-dicarboxylate- } \\
\text { binding protein }\end{array}$ & NA & NA & 3.7 & $2.80 \mathrm{E}-14$ & NA & NA & 12.3 & $0.00 \mathrm{E}+00$ \\
\hline PA5182 & & hypothetical protein & NA & NA & 2.6 & 8.07E-14 & NA & NA & 3.6 & $0.00 \mathrm{E}+00$ \\
\hline PA5183 & & hypothetical protein & NA & NA & 2.8 & $1.95 \mathrm{E}-10$ & NA & NA & 3.4 & $0.00 \mathrm{E}+00$ \\
\hline PA5424 & & conserved hypothetical protein & 2.1 & $1.37 \mathrm{E}-10$ & 2.8 & $5.20 \mathrm{E}-14$ & NA & NA & NA & NA \\
\hline \multicolumn{11}{|c|}{ Non-coding RNA gene } \\
\hline $\begin{array}{c}\text { PA4272 } \\
.1\end{array}$ & $P 27$ & $\mathrm{P} 27$ & 2.8 & $0.00 \mathrm{E}+00$ & NA & NA & 3.2 & $1.50 \mathrm{E}-14$ & NA & NA \\
\hline PA4276 & & tRNA-Trp & NA & NA & 2.1 & $4.62 \mathrm{E}-14$ & -2.0 & $3.12 \mathrm{E}-13$ & NA & NA \\
\hline
\end{tabular}




\begin{tabular}{|c|c|c|c|c|c|c|c|c|c|c|}
\hline \\
\hline $\begin{array}{l}\text { PA4704 } \\
\quad .2\end{array}$ & $\operatorname{prrF2}$ & regulatory RNA PrrF2 & NA & NA & 2.6 & $1.15 \mathrm{E}-13$ & -4.8 & $0.00 \mathrm{E}+00$ & NA & NA \\
\hline $\begin{array}{l}\text { PA4758 } \\
\quad .1\end{array}$ & P32 & P32 & 2.8 & $1.45 \mathrm{E}-12$ & NA & NA & 2.1 & $7.59 \mathrm{E}-06$ & NA & NA \\
\hline $\begin{array}{l}\text { PA5181 } \\
.1\end{array}$ & P34 & P34 & NA & NA & 2.8 & $9.88 \mathrm{E}-07$ & NA & NA & 3.1 & $7.15 \mathrm{E}-09$ \\
\hline \multicolumn{11}{|c|}{ Nucleotide biosynthesis and metabolism } \\
\hline PA5426 & purE & $\begin{array}{l}\text { phosphoribosylaminoimidazole } \\
\text { carboxylase, catalytic subunit }\end{array}$ & 2.8 & $8.16 \mathrm{E}-08$ & NA & NA & NA & NA & NA & NA \\
\hline \multicolumn{11}{|c|}{ Protein secretion/export apparatus } \\
\hline PA0076 & $\operatorname{tag} F 1$ & TagF1 & 4.3 & $1.75 \mathrm{E}-09$ & 3.7 & $3.28 \mathrm{E}-07$ & NA & NA & NA & NA \\
\hline PA0081 & fhal & Fhal & NA & NA & 3.4 & $7.04 \mathrm{E}-10$ & NA & NA & 2.5 & $3.90 \mathrm{E}-08$ \\
\hline PA0088 & $\operatorname{tss} F 1$ & TssF1 & NA & NA & 2.5 & $2.35 \mathrm{E}-07$ & NA & NA & 5.1 & $1.95 \mathrm{E}-14$ \\
\hline PA3221 & $\operatorname{csaA}$ & CsaA protein & 11.3 & $2.41 \mathrm{E}-13$ & NA & NA & 6.3 & $2.16 \mathrm{E}-06$ & NA & NA \\
\hline \multicolumn{11}{|c|}{ Putative enzymes } \\
\hline PA0745 & & $\begin{array}{l}\text { probable enoyl-CoA } \\
\text { hydratase/isomerase }\end{array}$ & NA & NA & 2.1 & $4.49 \mathrm{E}-13$ & NA & NA & 2.3 & $1.95 \mathrm{E}-14$ \\
\hline PA1023 & & $\begin{array}{l}\text { probable short-chain } \\
\text { dehydrogenase }\end{array}$ & 11.3 & $2.19 \mathrm{E}-05$ & NA & NA & NA & NA & NA & NA \\
\hline PA1615 & & probable lipase & 2.5 & 2.64E-09 & 3.3 & $0.00 \mathrm{E}+00$ & NA & NA & NA & NA \\
\hline PA1683 & & probable sugar aldolase & NA & NA & 3.7 & 7.49E-08 & NA & NA & 2.7 & $8.51 \mathrm{E}-07$ \\
\hline PA1828 & & $\begin{array}{l}\text { probable short-chain } \\
\text { dehydrogenase }\end{array}$ & NA & NA & 2.4 & $1.13 \mathrm{E}-06$ & NA & NA & 3.6 & $2.47 \mathrm{E}-12$ \\
\hline PA1832 & & probable protease & 2.4 & $8.27 \mathrm{E}-07$ & NA & NA & NA & NA & NA & NA \\
\hline PA2119 & & $\begin{array}{l}\text { alcohol dehydrogenase (Zn- } \\
\text { dependent) }\end{array}$ & 3.1 & $3.80 \mathrm{E}-10$ & NA & NA & NA & NA & NA & NA \\
\hline PA3302 & & conserved hypothetical protein & 2.5 & $5.67 \mathrm{E}-07$ & NA & NA & 2.3 & $1.09 \mathrm{E}-05$ & NA & NA \\
\hline PA3568 & & probable acetyl-coa synthetase & NA & NA & 2.3 & $6.74 \mathrm{E}-06$ & NA & NA & 3.4 & $3.25 \mathrm{E}-11$ \\
\hline PA4128 & & conserved hypothetical protein & 11.3 & $5.08 \mathrm{E}-06$ & NA & NA & NA & NA & NA & NA \\
\hline PA4615 & & probable oxidoreductase & 3.1 & $1.11 \mathrm{E}-07$ & NA & NA & NA & NA & NA & NA \\
\hline
\end{tabular}




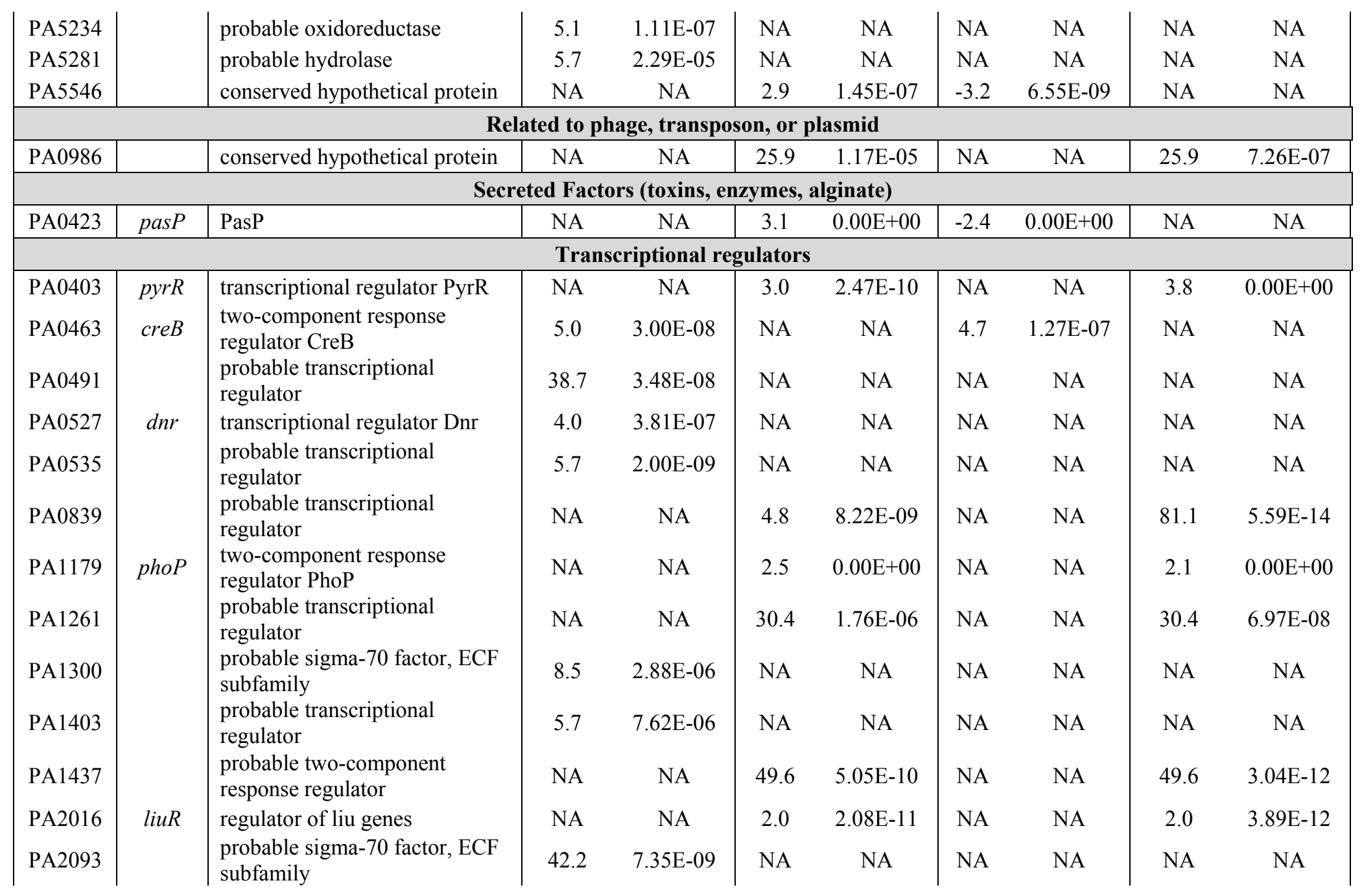




\begin{tabular}{|c|c|c|c|c|c|c|c|c|c|c|}
\hline PA2798 & & $\begin{array}{l}\text { probable two-component } \\
\text { response regulator }\end{array}$ & 2.1 & $6.55 \mathrm{E}-09$ & NA & NA & NA & NA & NA & NA \\
\hline PA2885 & atuR & putative repressor of $a t u$ genes & 4.0 & $4.51 \mathrm{E}-08$ & NA & NA & NA & NA & NA & NA \\
\hline PA2957 & & $\begin{array}{l}\text { probable transcriptional } \\
\text { regulator }\end{array}$ & 2.6 & $1.03 \mathrm{E}-08$ & NA & NA & NA & NA & NA & NA \\
\hline PA3458 & & $\begin{array}{l}\text { probable transcriptional } \\
\text { regulator }\end{array}$ & 4.7 & $2.74 \mathrm{E}-08$ & NA & NA & NA & NA & NA & NA \\
\hline PA3899 & & $\begin{array}{l}\text { probable sigma-70 factor, ECF } \\
\text { subfamily }\end{array}$ & 4.7 & $9.09 \mathrm{E}-08$ & 26.8 & $7.87 \mathrm{E}-06$ & NA & NA & NA & NA \\
\hline PA4853 & fis & DNA-binding protein Fis & 2.6 & $1.63 \mathrm{E}-12$ & NA & NA & 2.5 & $4.18 \mathrm{E}-11$ & NA & NA \\
\hline PA5261 & $\operatorname{alg} R$ & $\begin{array}{l}\text { alginate biosynthesis regulatory } \\
\text { protein AlgR }\end{array}$ & NA & NA & 2.9 & $0.00 \mathrm{E}+00$ & NA & NA & 2.1 & $1.35 \mathrm{E}-13$ \\
\hline PA5403 & & $\begin{array}{l}\text { probable transcriptional } \\
\text { regulator }\end{array}$ & 34.7 & $2.02 \mathrm{E}-07$ & NA & NA & 21.2 & 7.63E-06 & NA & NA \\
\hline PA5550 & $\operatorname{glm} R$ & GlmR transcriptional regulator & 3.8 & $6.16 \mathrm{E}-07$ & NA & NA & NA & NA & NA & NA \\
\hline \multicolumn{11}{|c|}{ Translation, post-translational modification, degradation } \\
\hline PA0019 & def & polypeptide deformylase & NA & NA & 2.1 & $7.20 \mathrm{E}-08$ & NA & NA & 2.6 & $4.60 \mathrm{E}-13$ \\
\hline PA0090 & clpV1 & ClpV1 & NA & NA & 2.1 & $1.99 \mathrm{E}-06$ & NA & NA & 2.8 & 7.68E-11 \\
\hline PA0355 & $p f p I$ & protease PfpI & NA & NA & 25.3 & $1.47 \mathrm{E}-05$ & NA & NA & 25.3 & $9.73 \mathrm{E}-07$ \\
\hline PA0767 & lepA & GTP-binding protein LepA & 2.0 & $1.04 \mathrm{E}-05$ & NA & NA & NA & NA & NA & NA \\
\hline PA0837 & slyD & $\begin{array}{l}\text { peptidyl-prolyl cis-trans } \\
\text { isomerase SlyD }\end{array}$ & 2.4 & $6.71 \mathrm{E}-11$ & NA & NA & 2.1 & $1.05 \mathrm{E}-07$ & NA & NA \\
\hline PA1996 & ppiC1 & $\begin{array}{l}\text { peptidyl-prolyl cis-trans } \\
\text { isomerase } \mathrm{C} 1\end{array}$ & 5.7 & $1.60 \mathrm{E}-06$ & NA & NA & NA & NA & NA & NA \\
\hline PA2755 & eco & ecotin precursor & NA & NA & 2.4 & $2.42 \mathrm{E}-10$ & NA & NA & 2.1 & $5.98 \mathrm{E}-09$ \\
\hline PA2970 & rpmF & 50S ribosomal protein L32 & 2.2 & $6.56 \mathrm{E}-13$ & NA & NA & NA & NA & NA & NA \\
\hline PA3114 & $\operatorname{tru} A$ & tRNA-pseudouridine synthase I & NA & NA & 4.1 & $3.03 \mathrm{E}-09$ & NA & NA & 4.1 & 7.91E-11 \\
\hline PA3600 & & conserved hypothetical protein & NA & NA & 44.7 & 4.14E-09 & NA & NA & 44.7 & $3.99 \mathrm{E}-11$ \\
\hline PA4255 & rpmC & 50S ribosomal protein L29 & 2.0 & $0.00 \mathrm{E}+00$ & NA & NA & 2.4 & $3.12 \mathrm{E}-13$ & NA & NA \\
\hline PA5256 & $d s b H$ & $\begin{array}{l}\text { disulfide bond formation } \\
\text { protein }\end{array}$ & NA & NA & 8.3 & $1.17 \mathrm{E}-11$ & NA & NA & 3.1 & $2.20 \mathrm{E}-07$ \\
\hline PA5569 & $\operatorname{rnp} A$ & ribonuclease $\mathrm{P}$ protein comp. & 2.4 & $1.42 \mathrm{E}-13$ & NA & NA & 2.1 & $2.75 \mathrm{E}-12$ & NA & NA \\
\hline
\end{tabular}




\begin{tabular}{|c|c|c|c|c|c|c|c|c|c|c|}
\hline \multicolumn{11}{|c|}{ Transport of small molecules } \\
\hline PA0970 & tolR & TolR protein & 2.3 & $5.18 \mathrm{E}-10$ & 2.3 & $1.76 \mathrm{E}-07$ & NA & NA & NA & NA \\
\hline PA1339 & & $\begin{array}{l}\text { amino acid } \mathrm{ABC} \text { transporter } \\
\mathrm{ATP} \text { binding protein }\end{array}$ & 2.4 & $1.84 \mathrm{E}-07$ & 2.3 & $7.82 \mathrm{E}-07$ & NA & NA & NA & NA \\
\hline PA1493 & cys $P$ & $\begin{array}{l}\text { sulfate-binding protein of } \mathrm{ABC} \\
\text { transporter }\end{array}$ & 3.0 & $6.40 \mathrm{E}-11$ & NA & NA & NA & NA & NA & NA \\
\hline PA2291 & & $\begin{array}{l}\text { probable glucose-sensitive } \\
\text { porin }\end{array}$ & NA & NA & 6.2 & $4.93 \mathrm{E}-06$ & NA & NA & 9.2 & $2.65 \mathrm{E}-08$ \\
\hline PA3186 & $o p r B$ & $\begin{array}{l}\text { Glucose/carbohydrate outer } \\
\text { membrane porin OprB } \\
\text { precursor }\end{array}$ & NA & NA & 2.8 & $2.47 \mathrm{E}-12$ & NA & NA & 5.9 & $0.00 \mathrm{E}+00$ \\
\hline PA3189 & & $\begin{array}{l}\text { probable permease of } \mathrm{ABC} \\
\text { sugar transporter }\end{array}$ & NA & NA & 9.0 & $1.28 \mathrm{E}-13$ & NA & NA & 2.7 & $5.17 \mathrm{E}-07$ \\
\hline PA3190 & & $\begin{array}{l}\text { probable binding protein } \\
\text { component of ABC sugar } \\
\text { transporter }\end{array}$ & NA & NA & 4.2 & $8.78 \mathrm{E}-14$ & NA & NA & 2.8 & $0.00 \mathrm{E}+00$ \\
\hline PA3441 & & $\begin{array}{l}\text { probable molybdopterin- } \\
\text { binding protein }\end{array}$ & 33.3 & 3.87E-07 & 31.7 & $1.02 \mathrm{E}-06$ & NA & NA & NA & NA \\
\hline PA4235 & $b f r A$ & bacterioferritin & 2.4 & $8.15 \mathrm{E}-08$ & NA & NA & NA & NA & NA & NA \\
\hline PA4502 & & $\begin{array}{l}\text { probable binding protein } \\
\text { component of } \mathrm{ABC} \text { transporter }\end{array}$ & NA & NA & 2.4 & $2.09 \mathrm{E}-06$ & NA & NA & 2.5 & 5.39E-08 \\
\hline PA4506 & & $\begin{array}{l}\text { probable ATP-binding } \\
\text { component of ABC dipeptide } \\
\text { transporter }\end{array}$ & 3.8 & $1.08 \mathrm{E}-05$ & NA & NA & NA & NA & NA & NA \\
\hline \multicolumn{11}{|c|}{ Two-component regulatory systems } \\
\hline PA3879 & narL & $\begin{array}{l}\text { two-component response } \\
\text { regulator NarL }\end{array}$ & 7.1 & $1.47 \mathrm{E}-07$ & NA & NA & NA & NA & NA & NA \\
\hline PA5484 & $\operatorname{kin} B$ & KinB & NA & NA & 2.8 & $1.13 \mathrm{E}-05$ & NA & NA & 2.8 & 9.37E-07 \\
\hline
\end{tabular}




\begin{tabular}{|c|c|c|c|c|c|c|c|c|c|c|}
\hline \multicolumn{11}{|c|}{ Genes negatively regulated in an AmpR-independent, ß-lactam-dependent manner (208) } \\
\hline \multirow[b]{2}{*}{$\begin{array}{l}\text { Gene } \\
\text { ID }\end{array}$} & \multirow[b]{2}{*}{$\begin{array}{l}\text { Gene } \\
\text { name }\end{array}$} & \multirow[b]{2}{*}{ Product Name } & \multicolumn{2}{|c|}{ Condition 1} & \multicolumn{2}{|c|}{ Condition 2} & \multicolumn{2}{|c|}{ Condition 3} & \multicolumn{2}{|c|}{ Condition 4} \\
\hline & & & $\begin{array}{c}\text { Fold } \\
\text { Chang } \\
\text { e }\end{array}$ & $\begin{array}{c}\text { Corrected } \\
p \text {-value }\end{array}$ & $\begin{array}{l}\text { Fold } \\
\text { Chan } \\
\text { ge } \\
\end{array}$ & $\begin{array}{c}\text { Corrected } \\
p \text {-value }\end{array}$ & $\begin{array}{l}\text { Fold } \\
\text { Cha } \\
\text { nge }\end{array}$ & $\begin{array}{c}\text { Corrected } \\
p \text {-value }\end{array}$ & $\begin{array}{c}\text { Fold } \\
\text { Change }\end{array}$ & $\begin{array}{c}\text { Corrected } \\
p \text {-value }\end{array}$ \\
\hline \multicolumn{11}{|c|}{ Adaptation, Protection } \\
\hline PA1150 & pys 2 & pyocin S2 & NA & NA & -4.4 & $0.00 \mathrm{E}+00$ & NA & NA & -3.8 & $0.00 \mathrm{E}+00$ \\
\hline PA1151 & imm2 & pyocin S2 immunity protein & NA & NA & -3.1 & $0.00 \mathrm{E}+00$ & NA & NA & -3.2 & $2.47 \mathrm{E}-13$ \\
\hline PA3007 & $\operatorname{lex} A$ & repressor protein LexA & NA & NA & -4.0 & $0.00 \mathrm{E}+00$ & NA & NA & -2.6 & $3.15 \mathrm{E}-14$ \\
\hline PA3866 & & pyocin protein & NA & NA & -3.3 & $0.00 \mathrm{E}+00$ & NA & NA & -2.4 & 4.27E-14 \\
\hline \multicolumn{11}{|c|}{ Amino acid biosynthesis and metabolism } \\
\hline PA0009 & $\operatorname{gly} Q$ & $\begin{array}{l}\text { glycyl-tRNA synthetase alpha } \\
\text { chain }\end{array}$ & NA & NA & -2.0 & 3.89E-07 & 2.4 & $9.54 \mathrm{E}-10$ & NA & NA \\
\hline PA4565 & proB & glutamate 5-kinase & NA & NA & -2.1 & $2.16 \mathrm{E}-10$ & 2.1 & 8.47E-10 & NA & NA \\
\hline PA5098 & hutH & histidine ammonia-lyase & NA & NA & -14.5 & $9.80 \mathrm{E}-12$ & 3.8 & $1.55 \mathrm{E}-06$ & NA & NA \\
\hline PA5100 & hutU & urocanase & NA & NA & -13.7 & $0.00 \mathrm{E}+00$ & NA & NA & -5.4 & $3.61 \mathrm{E}-06$ \\
\hline PA5143 & $h i s B$ & $\begin{array}{l}\text { imidazoleglycerol-phosphate } \\
\text { dehydratase }\end{array}$ & -123.3 & $0.00 \mathrm{E}+00$ & -10.1 & $1.50 \mathrm{E}-14$ & NA & NA & NA & NA \\
\hline \multicolumn{11}{|c|}{ Antibiotic resistance and susceptibility } \\
\hline PA0807 & $\begin{array}{l}\text { ampDh } \\
3\end{array}$ & AmpDh3 & -2.1 & $0.00 \mathrm{E}+00$ & -4.5 & $0.00 \mathrm{E}+00$ & NA & NA & NA & NA \\
\hline PA3719 & $\operatorname{armR}$ & antirepressor for MexR, ArmR & -29.2 & $3.39 \mathrm{E}-07$ & -54.1 & $1.22 \mathrm{E}-13$ & NA & NA & NA & NA \\
\hline \multicolumn{11}{|c|}{ Biosynthesis of cofactors, prosthetic groups and carriers } \\
\hline PA0381 & thiG & $\begin{array}{l}\text { thiamine biosynthesis protein, } \\
\text { thiazole moiety }\end{array}$ & NA & NA & -8.7 & $1.21 \mathrm{E}-11$ & NA & NA & -7.8 & $1.57 \mathrm{E}-10$ \\
\hline PA0512 & & conserved hypothetical protein & NA & NA & -25.5 & $7.28 \mathrm{E}-07$ & 25.5 & $7.21 \mathrm{E}-07$ & NA & NA \\
\hline PA1272 & $\operatorname{cobO}$ & $\begin{array}{l}\text { cob(I)alamin } \\
\text { adenosyltransferase }\end{array}$ & NA & NA & -35.8 & $2.73 \mathrm{E}-09$ & NA & NA & -46.9 & $3.73 \mathrm{E}-10$ \\
\hline PA1985 & $p q q A$ & $\begin{array}{l}\text { pyrroloquinoline quinone } \\
\text { biosynthesis protein } \mathrm{A}\end{array}$ & -32.8 & $5.34 \mathrm{E}-08$ & -60.8 & $0.00 \mathrm{E}+00$ & NA & NA & NA & NA \\
\hline
\end{tabular}




\begin{tabular}{|c|c|c|c|c|c|c|c|c|c|c|}
\hline $\begin{array}{l}\text { PA3633 } \\
\text { PA4891 }\end{array}$ & $\begin{array}{l}y g b P \\
\text { ureE }\end{array}$ & $\begin{array}{l}\text { 4-diphosphocytidyl-2-C- } \\
\text { methylerythritol synthase } \\
\text { urease accessory protein UreE }\end{array}$ & $\begin{array}{l}\text { NA } \\
\text { NA }\end{array}$ & $\begin{array}{l}\text { NA } \\
\text { NA }\end{array}$ & $\begin{array}{l}-3.3 \\
-26.1 \\
\end{array}$ & $\begin{array}{l}1.42 \mathrm{E}-05 \\
5.32 \mathrm{E}-07 \\
\end{array}$ & $\begin{array}{l}\text { NA } \\
26.1 \\
\end{array}$ & $\begin{array}{c}\text { NA } \\
5.25 \mathrm{E}-07\end{array}$ & $\begin{array}{l}-3.4 \\
\text { NA }\end{array}$ & $\begin{array}{c}1.87 \mathrm{E}-06 \\
\text { NA }\end{array}$ \\
\hline \multicolumn{11}{|c|}{ Carbon compound catabolism } \\
\hline PA0887 & $\operatorname{acs} A$ & acetyl-coenzyme A synthetase & NA & NA & -2.6 & $0.00 \mathrm{E}+00$ & 2.1 & $2.74 \mathrm{E}-13$ & NA & NA \\
\hline PA2508 & catC & muconolactone delta-isomerase & NA & NA & -45.2 & $1.57 \mathrm{E}-11$ & NA & NA & -24.7 & $8.15 \mathrm{E}-06$ \\
\hline PA2512 & antA & $\begin{array}{l}\text { anthranilate dioxygenase large } \\
\text { subunit }\end{array}$ & NA & NA & -5.6 & $1.42 \mathrm{E}-13$ & NA & NA & -12.7 & $0.00 \mathrm{E}+00$ \\
\hline PA2513 & antB & $\begin{array}{l}\text { anthranilate dioxygenase small } \\
\text { subunit }\end{array}$ & NA & NA & -89.0 & $0.00 \mathrm{E}+00$ & NA & NA & -233.6 & $0.00 \mathrm{E}+00$ \\
\hline PA4125 & $h p c D$ & $\begin{array}{l}\text { 5-carboxymethyl-2- } \\
\text { hydroxymuconate isomerase }\end{array}$ & NA & NA & -22.3 & $4.01 \mathrm{E}-06$ & 22.3 & 4.16E-06 & NA & NA \\
\hline \multicolumn{11}{|c|}{ Central intermediary metabolism } \\
\hline PA4864 & ureD & urease accessory protein & NA & NA & -20.8 & 8.97E-06 & 20.8 & $9.37 \mathrm{E}-06$ & NA & NA \\
\hline \multicolumn{11}{|c|}{ DNA replication, recombination, modification and repair } \\
\hline PA3617 & recA & RecA protein & NA & NA & -2.9 & $0.00 \mathrm{E}+00$ & NA & NA & -2.1 & $4.64 \mathrm{E}-14$ \\
\hline PA4232 & $s s b$ & $\begin{array}{l}\text { single-stranded DNA-binding } \\
\text { protein }\end{array}$ & NA & NA & -2.2 & $6.98 \mathrm{E}-14$ & 2.2 & $6.88 \mathrm{E}-14$ & NA & NA \\
\hline PA4763 & $\operatorname{rec} N$ & DNA repair protein $\mathrm{RecN}$ & NA & NA & -5.5 & $0.00 \mathrm{E}+00$ & NA & NA & -4.4 & $0.00 \mathrm{E}+00$ \\
\hline \multicolumn{11}{|c|}{ Energy metabolism } \\
\hline PA0523 & norC & nitric-oxide reductase subunit $\mathrm{C}$ & NA & NA & -19.9 & $1.46 \mathrm{E}-05$ & 19.9 & $1.53 \mathrm{E}-05$ & NA & NA \\
\hline PA1172 & napC & $\begin{array}{l}\text { cytochrome c-type protein } \\
\text { NapC }\end{array}$ & NA & NA & -36.7 & $1.67 \mathrm{E}-09$ & NA & NA & -48.1 & $2.21 \mathrm{E}-10$ \\
\hline PA3929 & $\operatorname{cioB}$ & $\begin{array}{l}\text { cyanide insensitive terminal } \\
\text { oxidase }\end{array}$ & -2.2 & $1.53 \mathrm{E}-06$ & NA & NA & -6.7 & $2.82 \mathrm{E}-14$ & NA & NA \\
\hline \multicolumn{11}{|c|}{ Hypothetical, unclassified, unknown } \\
\hline PA0014 & & hypothetical protein & -26.0 & $1.69 \mathrm{E}-06$ & -32.1 & $2.07 \mathrm{E}-08$ & NA & NA & NA & NA \\
\hline PA0020 & & hypothetical protein & NA & NA & -2.0 & $4.45 \mathrm{E}-06$ & 2.4 & $3.67 \mathrm{E}-08$ & NA & NA \\
\hline PA0045 & & hypothetical protein & -3.2 & $1.43 \mathrm{E}-05$ & NA & NA & NA & NA & NA & NA \\
\hline PA0053 & & hypothetical protein & NA & NA & $\begin{array}{c}- \\
103.1\end{array}$ & $4.03 \mathrm{E}-14$ & NA & NA & -28.2 & $1.73 \mathrm{E}-06$ \\
\hline
\end{tabular}




\begin{tabular}{|c|c|c|c|c|c|c|c|c|c|}
\hline PA0116 & hypothetical protein & NA & NA & -55.4 & $6.31 \mathrm{E}-14$ & NA & NA & -45.5 & $7.30 \mathrm{E}-10$ \\
\hline PA0161 & hypothetical protein & NA & NA & -4.3 & $2.49 \mathrm{E}-14$ & NA & NA & -2.9 & $5.14 \mathrm{E}-10$ \\
\hline PA0250 & conserved hypothetical protein & -3.0 & $1.96 \mathrm{E}-07$ & NA & NA & -2.7 & $6.91 \mathrm{E}-06$ & NA & NA \\
\hline PA0277 & conserved hypothetical protein & NA & NA & -4.1 & $3.07 \mathrm{E}-11$ & 3.6 & $5.10 \mathrm{E}-10$ & NA & NA \\
\hline PA0449 & hypothetical protein & -6.4 & $2.39 \mathrm{E}-11$ & -2.7 & $1.38 \mathrm{E}-06$ & NA & NA & NA & NA \\
\hline PA0468 & hypothetical protein & NA & NA & -5.8 & $7.61 \mathrm{E}-06$ & NA & NA & -5.8 & $3.09 \mathrm{E}-06$ \\
\hline PA0540 & hypothetical protein & -30.5 & $1.70 \mathrm{E}-07$ & NA & NA & NA & NA & NA & NA \\
\hline PA0578 & conserved hypothetical protein & NA & NA & 107.1 & $0.00 \mathrm{E}+00$ & NA & NA & -111.7 & 4.06E-14 \\
\hline PA0613 & hypothetical protein & NA & NA & -7.4 & $0.00 \mathrm{E}+00$ & NA & NA & -3.0 & $2.85 \mathrm{E}-13$ \\
\hline PA0615 & hypothetical protein & NA & NA & -3.2 & $5.03 \mathrm{E}-13$ & NA & NA & -2.3 & $7.47 \mathrm{E}-14$ \\
\hline PA0808 & hypothetical protein & -2.1 & $1.17 \mathrm{E}-05$ & -10.8 & $0.00 \mathrm{E}+00$ & NA & NA & -3.9 & $2.85 \mathrm{E}-06$ \\
\hline PA0819 & hypothetical protein & NA & NA & -29.8 & 7.27E-08 & NA & NA & -24.4 & $9.09 \mathrm{E}-06$ \\
\hline PA0907 & hypothetical protein & NA & NA & -3.8 & $0.00 \mathrm{E}+00$ & NA & NA & -2.9 & $0.00 \mathrm{E}+00$ \\
\hline PA0908 & hypothetical protein & -2.4 & $0.00 \mathrm{E}+00$ & -3.0 & $0.00 \mathrm{E}+00$ & NA & NA & NA & NA \\
\hline PA0922 & hypothetical protein & NA & NA & -6.1 & $0.00 \mathrm{E}+00$ & NA & NA & -3.9 & $0.00 \mathrm{E}+00$ \\
\hline PA0940 & hypothetical protein & NA & NA & -34.4 & $6.00 \mathrm{E}-09$ & 34.4 & $5.49 \mathrm{E}-09$ & NA & NA \\
\hline PA0947 & conserved hypothetical protein & NA & NA & -3.6 & $1.43 \mathrm{E}-12$ & NA & NA & -3.7 & $1.59 \mathrm{E}-13$ \\
\hline PA0959 & hypothetical protein & NA & NA & -2.7 & $8.59 \mathrm{E}-10$ & NA & NA & -3.5 & $3.15 \mathrm{E}-14$ \\
\hline PA1030 & hypothetical protein & NA & NA & -4.3 & $1.94 \mathrm{E}-12$ & NA & NA & -3.6 & $2.22 \mathrm{E}-09$ \\
\hline PA1035 & hypothetical protein & -3.9 & $8.85 \mathrm{E}-06$ & NA & NA & NA & NA & NA & NA \\
\hline PA1039 & conserved hypothetical protein & NA & NA & -27.7 & $2.22 \mathrm{E}-07$ & NA & NA & -45.5 & $7.30 \mathrm{E}-10$ \\
\hline PA1095 & hypothetical protein & NA & NA & -3.6 & $3.47 \mathrm{E}-14$ & NA & NA & -2.7 & $6.05 \mathrm{E}-14$ \\
\hline PA1152 & hypothetical protein & NA & NA & -8.0 & $2.80 \mathrm{E}-14$ & NA & NA & -9.7 & $0.00 \mathrm{E}+00$ \\
\hline PA1168 & hypothetical protein & -36.8 & 7.05E-09 & -27.3 & $2.79 \mathrm{E}-07$ & NA & NA & NA & NA \\
\hline PA1314 & hypothetical protein & NA & NA & -22.6 & $3.34 \mathrm{E}-06$ & 22.6 & $3.46 \mathrm{E}-06$ & NA & NA \\
\hline PA1332 & hypothetical protein & NA & NA & -47.6 & $4.11 \mathrm{E}-12$ & 4.2 & $5.43 \mathrm{E}-06$ & NA & NA \\
\hline PA1362 & hypothetical protein & NA & NA & -27.0 & $3.18 \mathrm{E}-07$ & 27.0 & $3.14 \mathrm{E}-07$ & NA & NA \\
\hline PA1402 & hypothetical protein & NA & NA & -19.7 & $1.56 \mathrm{E}-05$ & 19.7 & $1.64 \mathrm{E}-05$ & NA & NA \\
\hline
\end{tabular}




\begin{tabular}{|c|c|c|c|c|c|c|c|c|c|}
\hline PA1536 & conserved hypothetical protein & -21.9 & $1.33 \mathrm{E}-05$ & NA & NA & NA & NA & NA & NA \\
\hline PA1574 & conserved hypothetical protein & NA & NA & -4.0 & $2.49 \mathrm{E}-14$ & NA & NA & -2.4 & 3.07E-07 \\
\hline PA1679 & hypothetical protein & NA & NA & -22.5 & $3.67 \mathrm{E}-06$ & NA & NA & -64.5 & $1.32 \mathrm{E}-13$ \\
\hline PA1743 & hypothetical protein & NA & NA & -36.5 & $1.84 \mathrm{E}-09$ & 36.5 & $1.65 \mathrm{E}-09$ & NA & NA \\
\hline PA1751 & hypothetical protein & NA & NA & -46.4 & $8.13 \mathrm{E}-12$ & NA & NA & -38.0 & $2.11 \mathrm{E}-08$ \\
\hline PA1842 & hypothetical protein & NA & NA & -24.1 & $1.48 \mathrm{E}-06$ & NA & NA & -39.6 & $1.04 \mathrm{E}-08$ \\
\hline PA1870 & hypothetical protein & -22.2 & $1.13 \mathrm{E}-05$ & NA & NA & NA & NA & NA & NA \\
\hline PA1936 & hypothetical protein & NA & NA & -21.8 & $5.20 \mathrm{E}-06$ & NA & NA & -35.7 & $5.77 \mathrm{E}-08$ \\
\hline PA2146 & conserved hypothetical protein & NA & NA & -26.1 & $5.32 \mathrm{E}-07$ & 26.1 & $5.25 \mathrm{E}-07$ & NA & NA \\
\hline PA2381 & hypothetical protein & -5.3 & $9.84 \mathrm{E}-14$ & NA & NA & -7.1 & $2.21 \mathrm{E}-14$ & NA & NA \\
\hline PA2405 & hypothetical protein & NA & NA & -26.5 & $4.12 \mathrm{E}-07$ & NA & NA & -43.5 & $1.74 \mathrm{E}-09$ \\
\hline PA2436 & hypothetical protein & NA & NA & -20.1 & $1.26 \mathrm{E}-05$ & 20.1 & $1.33 \mathrm{E}-05$ & NA & NA \\
\hline PA2450 & hypothetical protein & NA & NA & -33.4 & $1.01 \mathrm{E}-08$ & NA & NA & -46.9 & $3.73 \mathrm{E}-10$ \\
\hline PA2506 & hypothetical protein & -21.6 & $1.54 \mathrm{E}-05$ & NA & NA & NA & NA & NA & NA \\
\hline PA2569 & hypothetical protein & NA & NA & -3.6 & $6.60 \mathrm{E}-13$ & 3.0 & $1.10 \mathrm{E}-10$ & -2.4 & $3.79 \mathrm{E}-06$ \\
\hline PA2606 & conserved hypothetical protein & NA & NA & -48.7 & $2.31 \mathrm{E}-12$ & NA & NA & -59.9 & $1.10 \mathrm{E}-12$ \\
\hline PA2660 & hypothetical protein & NA & NA & -28.3 & $1.62 \mathrm{E}-07$ & NA & NA & -46.4 & 4.73E-10 \\
\hline PA2780 & hypothetical protein & NA & NA & -3.6 & 7.89E-07 & 5.3 & $5.83 \mathrm{E}-09$ & NA & NA \\
\hline PA3033 & hypothetical protein & NA & NA & -3.6 & $3.20 \mathrm{E}-08$ & 2.6 & 8.07E-06 & NA & NA \\
\hline PA3085 & hypothetical protein & NA & NA & -3.6 & $1.20 \mathrm{E}-08$ & 5.3 & $1.71 \mathrm{E}-11$ & NA & NA \\
\hline PA3140 & hypothetical protein & NA & NA & -92.9 & $0.00 \mathrm{E}+00$ & 14.7 & $0.00 \mathrm{E}+00$ & NA & NA \\
\hline PA3178 & hypothetical protein & NA & NA & -69.5 & $1.04 \mathrm{E}-13$ & 3.2 & $1.75 \mathrm{E}-06$ & -57.0 & $4.01 \mathrm{E}-12$ \\
\hline PA3237 & hypothetical protein & -21.3 & $1.79 \mathrm{E}-05$ & -19.7 & $1.56 \mathrm{E}-05$ & NA & NA & NA & NA \\
\hline PA3250 & hypothetical protein & NA & NA & -20.7 & $9.46 \mathrm{E}-06$ & NA & NA & -40.7 & $6.23 \mathrm{E}-09$ \\
\hline PA3352 & hypothetical protein & NA & NA & -2.6 & $4.03 \mathrm{E}-14$ & NA & NA & -2.5 & $1.03 \mathrm{E}-14$ \\
\hline PA3413 & conserved hypothetical protein & NA & NA & -2.3 & $2.49 \mathrm{E}-14$ & 2.7 & $6.88 \mathrm{E}-14$ & NA & NA \\
\hline PA3414 & hypothetical protein & NA & NA & -5.8 & $0.00 \mathrm{E}+00$ & NA & NA & -5.8 & $0.00 \mathrm{E}+00$ \\
\hline PA3435 & conserved hypothetical protein & -2.7 & $2.76 \mathrm{E}-08$ & NA & NA & NA & NA & NA & NA \\
\hline PA3451 & hypothetical protein & -45.0 & $1.05 \mathrm{E}-10$ & -20.9 & $8.60 \mathrm{E}-06$ & NA & NA & NA & NA \\
\hline
\end{tabular}




\begin{tabular}{|c|c|c|c|c|c|c|c|c|c|c|}
\hline PA3463 & & conserved hypothetical protein & -30.7 & $1.57 \mathrm{E}-07$ & -56.9 & $0.00 \mathrm{E}+00$ & NA & NA & NA & NA \\
\hline PA3472 & & hypothetical protein & NA & NA & -4.3 & $1.24 \mathrm{E}-10$ & 3.2 & 5.44E-08 & NA & NA \\
\hline PA3492 & & conserved hypothetical protein & NA & NA & -29.6 & $7.90 \mathrm{E}-08$ & 14.7 & $1.72 \mathrm{E}-06$ & NA & NA \\
\hline PA3530 & & conserved hypothetical protein & -4.2 & $3.94 \mathrm{E}-14$ & NA & NA & -2.9 & $2.10 \mathrm{E}-08$ & NA & NA \\
\hline PA3536 & & hypothetical protein & NA & NA & -74.9 & $0.00 \mathrm{E}+00$ & NA & NA & -61.4 & $5.51 \mathrm{E}-13$ \\
\hline PA3577 & & hypothetical protein & -20.7 & $2.38 \mathrm{E}-05$ & NA & NA & NA & NA & NA & NA \\
\hline PA3611 & & hypothetical protein & NA & NA & -2.4 & $1.01 \mathrm{E}-06$ & 2.3 & $2.15 \mathrm{E}-06$ & NA & NA \\
\hline PA3634 & & conserved hypothetical protein & NA & NA & -3.6 & 4.57E-08 & 5.3 & $1.07 \mathrm{E}-10$ & NA & NA \\
\hline PA3662 & & hypothetical protein & NA & NA & -3.6 & $1.16 \mathrm{E}-14$ & 3.5 & 5.95E-14 & NA & NA \\
\hline PA3705 & wspD & hypothetical protein & -30.8 & $1.47 \mathrm{E}-07$ & NA & NA & NA & NA & NA & NA \\
\hline PA3722 & & hypothetical protein & NA & NA & -4.7 & $0.00 \mathrm{E}+00$ & NA & NA & -5.8 & $0.00 \mathrm{E}+00$ \\
\hline PA3787 & & conserved hypothetical protein & -3.1 & $3.93 \mathrm{E}-06$ & NA & NA & -4.1 & 4.10E-07 & NA & NA \\
\hline PA3822 & & conserved hypothetical protein & NA & NA & -2.7 & $0.00 \mathrm{E}+00$ & 2.5 & $1.50 \mathrm{E}-14$ & NA & NA \\
\hline PA3951 & & conserved hypothetical protein & -23.4 & $6.01 \mathrm{E}-06$ & NA & NA & NA & NA & NA & NA \\
\hline PA3990 & & conserved hypothetical protein & NA & NA & -85.9 & $0.00 \mathrm{E}+00$ & NA & NA & -46.9 & $3.73 \mathrm{E}-10$ \\
\hline PA4029 & & conserved hypothetical protein & -5.3 & $3.49 \mathrm{E}-07$ & NA & NA & NA & NA & NA & NA \\
\hline PA4298 & & hypothetical protein & -24.9 & $2.88 \mathrm{E}-06$ & NA & NA & NA & NA & NA & NA \\
\hline PA4573 & & hypothetical protein & NA & NA & -42.9 & $5.28 \mathrm{E}-11$ & NA & NA & -46.9 & $3.73 \mathrm{E}-10$ \\
\hline PA4574 & & conserved hypothetical protein & NA & NA & -6.5 & $1.69 \mathrm{E}-12$ & 3.8 & $8.31 \mathrm{E}-09$ & -4.9 & $1.37 \mathrm{E}-08$ \\
\hline PA4575 & & hypothetical protein & -34.6 & $2.25 \mathrm{E}-08$ & NA & NA & $\begin{array}{c}- \\
34.6\end{array}$ & $3.55 \mathrm{E}-07$ & NA & NA \\
\hline PA4580 & & conserved hypothetical protein & NA & NA & -8.0 & $2.80 \mathrm{E}-14$ & 2.9 & $3.73 \mathrm{E}-07$ & -5.8 & $5.62 \mathrm{E}-10$ \\
\hline PA4674 & & conserved hypothetical protein & NA & NA & -3.6 & $3.79 \mathrm{E}-14$ & NA & NA & -3.4 & $1.36 \mathrm{E}-13$ \\
\hline PA4682 & & hypothetical protein & -24.8 & $2.95 \mathrm{E}-06$ & NA & NA & NA & NA & NA & NA \\
\hline PA4739 & & conserved hypothetical protein & -2.1 & $5.81 \mathrm{E}-10$ & -4.3 & $7.18 \mathrm{E}-14$ & NA & NA & -2.9 & $5.40 \mathrm{E}-09$ \\
\hline PA4782 & & hypothetical protein & NA & NA & -2.5 & $2.81 \mathrm{E}-08$ & 14.7 & $9.31 \mathrm{E}-14$ & NA & NA \\
\hline PA4793 & & hypothetical protein & NA & NA & -2.4 & $3.60 \mathrm{E}-06$ & 2.3 & $7.35 \mathrm{E}-06$ & -2.2 & $1.20 \mathrm{E}-05$ \\
\hline PA4794 & & hypothetical protein & NA & NA & -36.3 & $2.08 \mathrm{E}-09$ & NA & NA & -29.7 & 8.54E-07 \\
\hline PA4826 & & hypothetical protein & -2.8 & $0.00 \mathrm{E}+00$ & -10.8 & $0.00 \mathrm{E}+00$ & NA & NA & -2.9 & $2.70 \mathrm{E}-07$ \\
\hline
\end{tabular}




\begin{tabular}{|c|c|c|c|c|c|c|c|c|c|c|}
\hline PA4917 & & hypothetical protein & NA & NA & -3.8 & $0.00 \mathrm{E}+00$ & NA & NA & -5.8 & $0.00 \mathrm{E}+00$ \\
\hline PA4918 & & hypothetical protein & NA & NA & -3.7 & $0.00 \mathrm{E}+00$ & 2.4 & $0.00 \mathrm{E}+00$ & NA & NA \\
\hline PA5126 & & hypothetical protein & NA & NA & -46.8 & $6.33 \mathrm{E}-12$ & NA & NA & -61.4 & $5.51 \mathrm{E}-13$ \\
\hline PA5227 & & conserved hypothetical protein & -2.6 & $1.34 \mathrm{E}-07$ & NA & NA & -2.4 & $6.82 \mathrm{E}-06$ & NA & NA \\
\hline PA5246 & & conserved hypothetical protein & NA & NA & -27.7 & $2.22 \mathrm{E}-07$ & 27.7 & $2.15 \mathrm{E}-07$ & NA & NA \\
\hline PA5247 & & conserved hypothetical protein & NA & NA & -81.6 & 8.97E-14 & NA & NA & -89.2 & $5.59 \mathrm{E}-14$ \\
\hline PA5269 & & hypothetical protein & NA & NA & -2.9 & $1.72 \mathrm{E}-05$ & NA & NA & -2.9 & 5.34E-06 \\
\hline PA5289 & & hypothetical protein & NA & NA & -67.1 & $8.97 \mathrm{E}-14$ & NA & NA & -55.0 & $9.82 \mathrm{E}-12$ \\
\hline PA5397 & & hypothetical protein & -26.7 & $1.17 \mathrm{E}-06$ & -24.7 & $1.06 \mathrm{E}-06$ & NA & NA & NA & NA \\
\hline PA5402 & & hypothetical protein & -37.3 & 5.49E-09 & NA & NA & NA & NA & NA & NA \\
\hline PA5465 & & hypothetical protein & NA & NA & -3.6 & $1.11 \mathrm{E}-10$ & 3.5 & $2.07 \mathrm{E}-10$ & NA & NA \\
\hline PA5527 & & hypothetical protein & NA & NA & -3.4 & $8.49 \mathrm{E}-14$ & 2.5 & $1.54 \mathrm{E}-09$ & NA & NA \\
\hline \multicolumn{11}{|c|}{ Membrane proteins } \\
\hline PA0109 & \multirow{5}{*}{ putP } & hypothetical protein & NA & NA & -41.7 & $1.03 \mathrm{E}-10$ & NA & NA & -34.2 & $1.15 \mathrm{E}-07$ \\
\hline PA0215 & & malonate transporter MadL & NA & NA & -43.3 & $4.46 \mathrm{E}-11$ & NA & NA & -70.9 & $2.73 \mathrm{E}-15$ \\
\hline PA0738 & & conserved hypothetical protein & NA & NA & -35.6 & $3.01 \mathrm{E}-09$ & 35.6 & $2.73 \mathrm{E}-09$ & NA & NA \\
\hline PA0783 & & sodium/proline symporter PutP & NA & NA & -3.0 & $2.25 \mathrm{E}-06$ & 2.8 & $9.14 \mathrm{E}-06$ & NA & NA \\
\hline PA0811 & & $\begin{array}{l}\text { probable major facilitator } \\
\text { superfamily (MFS) transporter }\end{array}$ & NA & NA & -10.1 & $2.57 \mathrm{E}-09$ & NA & NA & -6.8 & 4.92E-06 \\
\hline PA0985 & \multirow[t]{2}{*}{ pyoS5 } & pyocin S5 & NA & NA & -5.0 & $0.00 \mathrm{E}+00$ & NA & NA & -3.2 & $6.05 \mathrm{E}-14$ \\
\hline PA1042 & & conserved hypothetical protein & NA & NA & -43.8 & $3.32 \mathrm{E}-11$ & NA & NA & -47.9 & $2.46 \mathrm{E}-10$ \\
\hline PA1072 & \multirow[t]{6}{*}{ braE } & $\begin{array}{l}\text { branched-chain amino acid } \\
\text { transport protein BraE }\end{array}$ & NA & NA & -2.4 & $1.19 \mathrm{E}-05$ & 3.4 & $6.21 \mathrm{E}-09$ & NA & NA \\
\hline PA1513 & & hypothetical protein & -24.0 & 4.42E-06 & NA & NA & NA & NA & NA & NA \\
\hline PA1958 & & probable transporter & NA & NA & -30.4 & $5.15 \mathrm{E}-08$ & 8.4 & $9.24 \mathrm{E}-06$ & -24.9 & 7.27E-06 \\
\hline PA2558 & & probable transport protein & -27.3 & $8.74 \mathrm{E}-07$ & NA & NA & $\begin{array}{c}- \\
27.3\end{array}$ & $7.61 \mathrm{E}-06$ & NA & NA \\
\hline PA2576 & & hypothetical protein & NA & NA & -43.8 & $3.32 \mathrm{E}-11$ & 9.5 & $3.00 \mathrm{E}-08$ & NA & NA \\
\hline PA3231 & & hypothetical protein & NA & NA & -27.5 & $2.44 \mathrm{E}-07$ & 27.5 & $2.37 \mathrm{E}-07$ & NA & NA \\
\hline
\end{tabular}




\begin{tabular}{|c|c|c|c|c|c|c|c|c|c|c|}
\hline PA3526 & & $\begin{array}{l}\text { probable outer membrane } \\
\text { protein precursor }\end{array}$ & NA & NA & -5.1 & $1.00 \mathrm{E}-08$ & NA & NA & -5.4 & $5.44 \mathrm{E}-10$ \\
\hline PA3647 & & $\begin{array}{l}\text { probable outer membrane } \\
\text { protein precursor }\end{array}$ & -2.0 & 2.74E-09 & NA & NA & NA & NA & NA & NA \\
\hline PA3747 & & conserved hypothetical protein & -2.7 & $1.34 \mathrm{E}-06$ & NA & NA & NA & NA & NA & NA \\
\hline PA3837 & & $\begin{array}{l}\text { probable permease of } \mathrm{ABC} \\
\text { transporter }\end{array}$ & NA & NA & -4.0 & 1.99E-06 & NA & NA & -3.9 & $1.31 \mathrm{E}-06$ \\
\hline PA5095 & & $\begin{array}{l}\text { probable permease of } \mathrm{ABC} \\
\text { transporter }\end{array}$ & -27.7 & $6.91 \mathrm{E}-07$ & NA & NA & NA & NA & NA & NA \\
\hline PA5482 & & hypothetical protein & NA & NA & -54.1 & $1.22 \mathrm{E}-13$ & NA & NA & -133.0 & $1.95 \mathrm{E}-14$ \\
\hline \multicolumn{11}{|c|}{ Motility \& Attachment } \\
\hline PA0992 & cupC1 & fimbrial subunit $\mathrm{CupC1}$ & NA & NA & -21.3 & $6.93 \mathrm{E}-06$ & NA & NA & -34.9 & $8.55 \mathrm{E}-08$ \\
\hline PA1080 & $f l g E$ & flagellar hook protein FlgE & NA & NA & -3.1 & $1.98 \mathrm{E}-07$ & NA & NA & -2.9 & $5.90 \mathrm{E}-07$ \\
\hline PA1081 & $f \lg F$ & $\begin{array}{l}\text { flagellar basal-body rod protein } \\
\text { FlgF }\end{array}$ & NA & NA & -3.9 & $3.21 \mathrm{E}-10$ & 4.2 & $5.07 \mathrm{E}-11$ & NA & NA \\
\hline PA1083 & $f l g H$ & $\begin{array}{l}\text { flagellar L-ring protein } \\
\text { precursor FlgH }\end{array}$ & NA & NA & -10.8 & $5.76 \mathrm{E}-14$ & 2.4 & $4.26 \mathrm{E}-06$ & NA & NA \\
\hline PA1087 & $f \lg L$ & $\begin{array}{l}\text { flagellar hook-associated } \\
\text { protein type } 3 \mathrm{FlgL}\end{array}$ & NA & NA & -12.3 & $3.71 \mathrm{E}-11$ & NA & NA & -9.7 & $8.51 \mathrm{E}-09$ \\
\hline PA1092 & $f l i C$ & flagellin type B & NA & NA & -2.5 & $0.00 \mathrm{E}+00$ & NA & NA & -2.3 & $0.00 \mathrm{E}+00$ \\
\hline PA1 100 & fliE & $\begin{array}{l}\text { flagellar hook-basal body } \\
\text { complex protein FliE }\end{array}$ & -3.2 & $9.42 \mathrm{E}-11$ & -2.4 & $3.91 \mathrm{E}-08$ & NA & NA & NA & NA \\
\hline PA1107 & & conserved hypothetical protein & -21.7 & $1.44 \mathrm{E}-05$ & NA & NA & NA & NA & NA & NA \\
\hline PA1447 & fliQ & $\begin{array}{l}\text { flagellar biosynthetic protein } \\
\text { FliQ }\end{array}$ & -26.3 & $1.46 \mathrm{E}-06$ & -64.9 & $0.00 \mathrm{E}+00$ & NA & NA & NA & NA \\
\hline PA4299 & $\operatorname{tad} D$ & TadD & -25.6 & $2.01 \mathrm{E}-06$ & NA & NA & $\overline{-}$ & $1.52 \mathrm{E}-05$ & NA & NA \\
\hline \multicolumn{11}{|c|}{ Non-coding RNA gene } \\
\hline $\begin{array}{l}\text { PA0668 } \\
.5\end{array}$ & & 5S ribosomal RNA & -2.3 & $0.00 \mathrm{E}+00$ & NA & NA & NA & NA & NA & NA \\
\hline $\begin{array}{l}\text { PA0836 } \\
.1\end{array}$ & P5 & P5 & -33.3 & 4.23E-08 & NA & NA & - & $5.98 \mathrm{E}-07$ & NA & NA \\
\hline PA4451 & P35 & P35 & -38.1 & 3.64E-09 & NA & NA & - & 7.76E-08 & NA & NA \\
\hline
\end{tabular}




\begin{tabular}{|c|c|c|c|c|c|c|c|c|c|}
\hline $\begin{array}{c}.1 \\
\text { PA5471 } \\
.1 \\
\end{array}$ & PA5471 leader peptide & -56.3 & $3.33 \mathrm{E}-13$ & NA & NA & $\begin{array}{c}38.1 \\
- \\
56.3 \\
\end{array}$ & $3.15 \mathrm{E}-11$ & NA & NA \\
\hline \multicolumn{10}{|c|}{ Putative enzymes } \\
\hline PA0249 & probable acetyltransferase & NA & NA & -29.4 & $8.89 \mathrm{E}-08$ & NA & NA & -32.1 & $2.92 \mathrm{E}-07$ \\
\hline PA2580 & conserved hypothetical protein & NA & NA & -22.2 & $4.12 \mathrm{E}-06$ & NA & NA & -24.3 & $9.60 \mathrm{E}-06$ \\
\hline PA3172 & probable hydrolase & NA & NA & -4.0 & 4.04E-08 & 3.3 & $6.66 \mathrm{E}-07$ & -3.4 & $1.20 \mathrm{E}-06$ \\
\hline PA3968 & $\begin{array}{l}\text { probable pseudouridine } \\
\text { synthase }\end{array}$ & -4.6 & $1.16 \mathrm{E}-06$ & NA & NA & -6.2 & $6.36 \mathrm{E}-07$ & NA & NA \\
\hline PA4114 & lysine decarboxylase & -4.2 & $1.76 \mathrm{E}-06$ & NA & NA & NA & NA & NA & NA \\
\hline PA4709 & $\begin{array}{l}\text { probable hemin degrading } \\
\text { factor }\end{array}$ & -39.9 & $1.44 \mathrm{E}-09$ & NA & NA & NA & NA & NA & NA \\
\hline PA5445 & $\begin{array}{l}\text { probable coenzyme A } \\
\text { transferase }\end{array}$ & NA & NA & -4.2 & $5.04 \mathrm{E}-10$ & 2.5 & 5.91E-06 & NA & NA \\
\hline \multicolumn{10}{|c|}{ Related to phage, transposon, or plasmid } \\
\hline PA0621 & conserved hypothetical protein & -2.0 & $9.38 \mathrm{E}-13$ & -4.3 & $0.00 \mathrm{E}+00$ & NA & NA & -2.1 & $2.44 \mathrm{E}-13$ \\
\hline PA0632 & hypothetical protein & -2.1 & $0.00 \mathrm{E}+00$ & -5.7 & $0.00 \mathrm{E}+00$ & NA & NA & -2.2 & $0.00 \mathrm{E}+00$ \\
\hline PA0635 & hypothetical protein & -2.1 & $0.00 \mathrm{E}+00$ & -3.4 & $0.00 \mathrm{E}+00$ & NA & NA & NA & NA \\
\hline PA0638 & probable bacteriophage protein & -2.3 & $5.88 \mathrm{E}-13$ & -3.4 & $1.90 \mathrm{E}-13$ & NA & NA & NA & NA \\
\hline PA0642 & hypothetical protein & NA & NA & -3.8 & $5.73 \mathrm{E}-13$ & NA & NA & -2.0 & $0.00 \mathrm{E}+00$ \\
\hline PA0643 & hypothetical protein & NA & NA & -3.7 & $6.06 \mathrm{E}-13$ & NA & NA & -2.2 & $0.00 \mathrm{E}+00$ \\
\hline PA0646 & hypothetical protein & NA & NA & -3.7 & $7.28 \mathrm{E}-13$ & NA & NA & -2.1 & $0.00 \mathrm{E}+00$ \\
\hline PA0647 & hypothetical protein & -2.4 & $5.93 \mathrm{E}-13$ & -5.4 & $1.24 \mathrm{E}-13$ & NA & NA & NA & NA \\
\hline PA0909 & hypothetical protein & NA & NA & -6.7 & $0.00 \mathrm{E}+00$ & NA & NA & -4.2 & $0.00 \mathrm{E}+00$ \\
\hline \multicolumn{10}{|c|}{ Transcription, RNA processing and degradation } \\
\hline PA0733 & $\begin{array}{l}\text { probable pseudouridylate } \\
\text { synthase }\end{array}$ & NA & NA & -31.6 & $2.70 \mathrm{E}-08$ & 10.5 & 2.31E-06 & NA & NA \\
\hline \multicolumn{10}{|c|}{ Transcriptional regulators } \\
\hline PA0367 & $\begin{array}{l}\text { probable transcriptional } \\
\text { regulator }\end{array}$ & -4.6 & $5.84 \mathrm{E}-06$ & -3.6 & $3.31 \mathrm{E}-07$ & NA & NA & NA & NA \\
\hline
\end{tabular}




\begin{tabular}{|c|c|c|c|c|c|c|c|c|c|c|}
\hline PA0424 & $\operatorname{mexR}$ & $\begin{array}{l}\text { multidrug resistance operon } \\
\text { repressor MexR }\end{array}$ & NA & NA & -59.2 & $0.00 \mathrm{E}+00$ & NA & NA & -97.1 & $4.11 \mathrm{E}-14$ \\
\hline PA0610 & $\operatorname{prtN}$ & transcriptional regulator $\operatorname{PrtN}$ & -2.2 & $1.50 \mathrm{E}-13$ & -9.9 & $0.00 \mathrm{E}+00$ & NA & NA & -3.4 & $0.00 \mathrm{E}+00$ \\
\hline PA0611 & prtR & transcriptional regulator PrtR & NA & NA & -4.2 & $0.00 \mathrm{E}+00$ & NA & NA & -2.3 & $6.21 \mathrm{E}-09$ \\
\hline PA0612 & $p \operatorname{tr} B$ & repressor, PtrB & NA & NA & -5.1 & $0.00 \mathrm{E}+00$ & NA & NA & -2.1 & $3.14 \mathrm{E}-13$ \\
\hline PA0708 & & $\begin{array}{l}\text { probable transcriptional } \\
\text { regulator }\end{array}$ & NA & NA & -20.5 & $1.05 \mathrm{E}-05$ & NA & NA & -25.2 & $6.47 \mathrm{E}-06$ \\
\hline PA0831 & oruR & transcriptional regulator OruR & -6.7 & $7.91 \mathrm{E}-07$ & NA & NA & NA & NA & NA & NA \\
\hline PA0906 & & $\begin{array}{l}\text { probable transcriptional } \\
\text { regulator }\end{array}$ & NA & NA & -3.9 & $1.50 \mathrm{E}-14$ & 2.3 & 4.71E-09 & NA & NA \\
\hline PA1603 & & $\begin{array}{l}\text { probable transcriptional } \\
\text { regulator }\end{array}$ & NA & NA & -41.4 & $1.21 \mathrm{E}-10$ & NA & NA & -34.0 & $1.28 \mathrm{E}-07$ \\
\hline PA2277 & $\operatorname{ars} R$ & ArsR protein & -26.9 & $1.05 \mathrm{E}-06$ & NA & NA & NA & NA & NA & NA \\
\hline PA2332 & & $\begin{array}{l}\text { probable transcriptional } \\
\text { regulator }\end{array}$ & -20.9 & $2.22 \mathrm{E}-05$ & NA & NA & NA & NA & NA & NA \\
\hline PA2387 & $f p v I$ & FpvI & NA & NA & -27.4 & $2.66 \mathrm{E}-07$ & NA & NA & -29.9 & $7.84 \mathrm{E}-07$ \\
\hline PA2511 & & $\begin{array}{l}\text { probable transcriptional } \\
\text { regulator }\end{array}$ & NA & NA & -26.2 & $4.88 \mathrm{E}-07$ & NA & NA & -64.5 & $1.37 \mathrm{E}-13$ \\
\hline PA3067 & & $\begin{array}{l}\text { probable transcriptional } \\
\text { regulator }\end{array}$ & NA & NA & -39.5 & $3.57 \mathrm{E}-10$ & NA & NA & -32.4 & $2.64 \mathrm{E}-07$ \\
\hline PA3220 & & $\begin{array}{l}\text { probable transcriptional } \\
\text { regulator }\end{array}$ & NA & NA & -45.8 & $1.10 \mathrm{E}-11$ & NA & NA & -37.6 & $2.58 \mathrm{E}-08$ \\
\hline PA3341 & & $\begin{array}{l}\text { probable transcriptional } \\
\text { regulator }\end{array}$ & -2.6 & 8.89E-08 & NA & NA & -2.6 & 4.28E-07 & NA & NA \\
\hline PA4109 & $\operatorname{ampR}$ & transcriptional regulator AmpR & -6.7 & $0.00 \mathrm{E}+00$ & -6.4 & $0.00 \mathrm{E}+00$ & NA & NA & NA & NA \\
\hline PA4227 & $p c h R$ & transcriptional regulator $\mathrm{PchR}$ & -7.4 & 8.51E-09 & NA & NA & -5.0 & $2.15 \mathrm{E}-06$ & NA & NA \\
\hline PA4764 & fur & ferric uptake regulation protein & NA & NA & -2.2 & $0.00 \mathrm{E}+00$ & NA & NA & -2.6 & $0.00 \mathrm{E}+00$ \\
\hline PA5308 & $\operatorname{lrp}$ & $\begin{array}{l}\text { leucine-responsive regulatory } \\
\text { protein }\end{array}$ & -4.6 & $1.25 \mathrm{E}-07$ & NA & NA & NA & NA & NA & NA \\
\hline PA5374 & betI & transcriptional regulator BetI & NA & NA & -5.8 & $3.89 \mathrm{E}-15$ & 3.9 & $0.00 \mathrm{E}+00$ & NA & NA \\
\hline \multicolumn{11}{|c|}{ Translation, post-translational modification, degradation } \\
\hline PA2618 & & hypothetical protein & NA & NA & -30.9 & $3.92 \mathrm{E}-08$ & NA & NA & -30.4 & $6.24 \mathrm{E}-07$ \\
\hline
\end{tabular}




\begin{tabular}{|c|c|c|c|c|c|c|c|c|c|c|}
\hline PA3049 & $r m f$ & ribosome modulation factor & -2.3 & $0.00 \mathrm{E}+00$ & NA & NA & -2.5 & $0.00 \mathrm{E}+00$ & NA & NA \\
\hline PA5049 & rpmE & 50S ribosomal protein L31 & NA & NA & -2.1 & $0.00 \mathrm{E}+00$ & 2.1 & $0.00 \mathrm{E}+00$ & NA & NA \\
\hline PA5315 & $\operatorname{rpm} G$ & $50 \mathrm{~S}$ ribosomal protein $\mathrm{L} 33$ & NA & NA & -3.3 & $0.00 \mathrm{E}+00$ & 2.2 & $0.00 \mathrm{E}+00$ & -2.3 & $0.00 \mathrm{E}+00$ \\
\hline PA5470 & & $\begin{array}{l}\text { probable peptide chain release } \\
\text { factor }\end{array}$ & NA & NA & -12.3 & $0.00 \mathrm{E}+00$ & NA & NA & -4.9 & 4.93E-07 \\
\hline \multicolumn{11}{|c|}{ Transport of small molecules } \\
\hline PA0892 & $\operatorname{aot} P$ & $\begin{array}{l}\text { arginine/ornithine transport } \\
\text { protein AotP }\end{array}$ & NA & NA & -2.1 & $1.40 \mathrm{E}-08$ & 2.5 & $1.36 \mathrm{E}-11$ & NA & NA \\
\hline PA3790 & oprC & $\begin{array}{l}\text { Putative copper transport outer } \\
\text { membrane porin OprC } \\
\text { precursor }\end{array}$ & NA & NA & -4.0 & $9.12 \mathrm{E}-07$ & NA & NA & -4.3 & 7.52E-08 \\
\hline
\end{tabular}


Table 5: Functional enrichment of genes: AmpR-, AmpR-ß-lactam- and ß-lactam-dependent genes (Appendix; Supplementary information for Chapter 5; Tables 2, 3 and 4) were tested for enrichment of functional categories using GOEAST (518). There was no significant enrichment in the AmpR-ß-lactam-dependent and $\beta$-lactam-dependent gene sets.

AmpR +ve
\begin{tabular}{|c|l|l|c|c|c|c|}
\hline GOID & \multicolumn{1}{|c|}{ Ontology } & \multicolumn{1}{|c|}{ Term } & $\begin{array}{c}\text { \# in } \\
\text { list }\end{array}$ & $\begin{array}{c}\# \text { in } \\
\text { genome }\end{array}$ & $\begin{array}{c}\text { Log- } \\
\text { odd } \\
\text { ratio }\end{array}$ & $p$-value \\
\hline GO:0050896 & biological_process & response to stimulus & 25 & 208 & 0.989 & $4.51 \mathrm{E}-02$ \\
\hline GO:0006518 & biological_process & peptide metabolic process & 8 & 15 & 3.138 & $2.27 \mathrm{E}-04$ \\
\hline GO:0009237 & biological_process & siderophore metabolic process & 8 & 9 & 3.875 & $1.51 \mathrm{E}-06$ \\
\hline GO:0019184 & biological_process & nonribosomal peptide biosynthetic process & 8 & 12 & 3.46 & $2.85 \mathrm{E}-05$ \\
\hline GO:0019290 & biological_process & siderophore biosynthetic process & 8 & 9 & 3.875 & $1.51 \mathrm{E}-06$ \\
\hline GO:0019748 & biological_process & secondary metabolic process & 8 & 10 & 3.723 & $3.58 \mathrm{E}-06$ \\
\hline GO:0043043 & biological_process & peptide biosynthetic process & 8 & 13 & 3.345 & $5.87 \mathrm{E}-05$ \\
\hline GO:0043603 & biological_process & cellular amide metabolic process & 8 & 21 & 2.653 & $4.12 \mathrm{E}-03$ \\
\hline GO:0043604 & biological_process & amide biosynthetic process & 8 & 17 & 2.958 & $6.79 \mathrm{E}-04$ \\
\hline GO:0044550 & biological_process & secondary metabolite biosynthetic process & 8 & 10 & 3.723 & $3.58 \mathrm{E}-06$ \\
\hline GO:0016999 & biological_process & antibiotic metabolic process & 4 & 6 & 3.46 & $3.31 \mathrm{E}-02$ \\
\hline GO:0017000 & biological_process & antibiotic biosynthetic process & 4 & 6 & 3.46 & $3.31 \mathrm{E}-02$ \\
\hline GO:0017144 & biological_process & drug metabolic process & 4 & 6 & 3.46 & $3.31 \mathrm{E}-02$ \\
\hline
\end{tabular}




\begin{tabular}{|c|c|c|c|c|c|c|}
\hline GOID & Ontology & Term & $\begin{array}{l}\# \text { in } \\
\text { list }\end{array}$ & $\begin{array}{l}\# \text { in } \\
\text { genome }\end{array}$ & $\begin{array}{c}\text { Log- } \\
\text { odd } \\
\text { ratio }\end{array}$ & $p$-value \\
\hline GO:0005737 & cellular_component & cytoplasm & 14 & 48 & 1.714 & $1.85 \mathrm{E}-02$ \\
\hline GO:0005840 & cellular_component & ribosome & 13 & 31 & 2.238 & $1.36 \mathrm{E}-03$ \\
\hline GO:0030529 & cellular_component & ribonucleoprotein complex & 13 & 32 & 2.193 & $1.42 \mathrm{E}-03$ \\
\hline GO:0043226 & cellular_component & organelle & 13 & 48 & 1.608 & $5.60 \mathrm{E}-02$ \\
\hline GO:0043228 & cellular_component & non-membrane-bounded organelle & 13 & 48 & 1.608 & $5.60 \mathrm{E}-02$ \\
\hline GO:0043229 & cellular_component & intracellular organelle & 13 & 48 & 1.608 & $5.60 \mathrm{E}-02$ \\
\hline GO:0043232 & cellular_component & $\begin{array}{l}\text { intracellular non-membrane-bounded } \\
\text { organelle }\end{array}$ & 13 & 48 & 1.608 & $5.60 \mathrm{E}-02$ \\
\hline GO:0044391 & cellular_component & ribosomal subunit & 13 & 31 & 2.238 & $1.36 \mathrm{E}-03$ \\
\hline GO:0044422 & cellular_component & organelle part & 13 & 42 & 1.8 & $1.85 \mathrm{E}-02$ \\
\hline GO:0044444 & cellular_component & cytoplasmic part & 14 & 44 & 1.84 & $8.04 \mathrm{E}-03$ \\
\hline GO:0044446 & cellular_component & intracellular organelle part & 13 & 33 & 2.148 & $1.62 \mathrm{E}-03$ \\
\hline GO:0006119 & biological_process & oxidative phosphorylation & 6 & 12 & 2.492 & $8.07 \mathrm{E}-02$ \\
\hline GO:0016310 & biological_process & phosphorylation & 6 & 12 & 2.492 & $8.07 \mathrm{E}-02$ \\
\hline GO:0015934 & cellular_component & large ribosomal subunit & 9 & 18 & 2.492 & $5.63 \mathrm{E}-03$ \\
\hline
\end{tabular}


BIBLIOGRAPHY 
1. Palleroni, N.J. (1992) In Belows, A., Truper, H. G., Dworkin, M., Harder, W. and Schleifer, K. H. (eds.), The prokaryotes, a handbook on the biology of bacteria, ecophysiology, isolation, identification and applications. 2 ed. Springer-Verlag, New York, Vol. III, pp. 3086-3103.

2. Palleroni, N.J. and Moore, R.B.E. (2004) In Ramos, J.-L. (ed.), Pseudomonas: Genomics, lifestyle and molecular architecture. Kluwer Academic/Plenum, New York, Vol. 1, pp. 3-44.

3. Palleroni, N.J. (2005) In Brenner, D. J., Kreig, N. R. and Staley, J. T. (eds.), The Proteobacteria, Part B, the Gammaproteobacteria. 2nd ed. Springer, New York, Vol. 2, pp. 323-379.

4. Palleroni, N.J. (1984) In Kreig, N. R. and Holt, J. G. (eds.), Bergey's manual of systematic bacteriology William \& Wilkins, Baltimore, MD, USA, Vol. 1, pp. 141-199.

5. Palleroni, N.J. (2010) The Pseudomonas story. Environ Microbiol., 12, 13771383.

6. Stanier, R.Y., Palleroni, N.J. and Doudoroff, M. (1966) The aerobic pseudomonads: a taxonomic study. J Gen Microbiol., 43, 159-271.

7. Holloway, B.W. and Morgan, A.F. (1986) Genome organization in Pseudomonas. Annu Rev Microbiol, 40, 79-105.

8. Grassme, H., Jin, J., Wilker, B., von Kurthy, G., Wick, W., Weller, M., Moroy, T. and Gulbins, E. (2006) Regulation of pulmonary Pseudomonas aeruginosa infection by the transcriptional repressor Gfi1. Cell Microbiol, 8, 1096-1105.

9. Gregory, A.D., Hogue, L.A., Ferkol, T.W. and Link, D.C. (2007) Regulation of systemic and local neutrophil responses by G-CSF during pulmonary Pseudomonas aeruginosa infection. Blood, 109, 3235-3243.

10. Rodriguez, C.N., Molina, N., Garcia, A., Nino, C.R., Rodriguez, A.J. and Meijomil, P. (2002) Comparative study of antimicrobial resistance of Pseudomonas aeruginosa strains isolated from urinary tract infection in patients from Caracas and Lima. Int J Antimicrob Agents, 20, 476-477. 
11. Shigemura, K., Arakawa, S., Sakai, Y., Kinoshita, S., Tanaka, K. and Fujisawa, M. (2006) Complicated urinary tract infection caused by Pseudomonas aeruginosa in a single institution (1999-2003). Int J Urol, 13, 538-542.

12. Sobczyk, D., Krynicki, T., Blumczynski, A., Zaniew, M., Kroll, P., Siwinska, A. and Zachwieja, J. (2006) New, successful treatment of urinary tract infection caused by Pseudomonas aeruginosa. Przegl Lek, 63, 140-141.

13. Takeyama, K., Kunishima, Y., Matsukawa, M., Takahashi, S., Hirose, T., Kobayashi, N., Kobayashi, I. and Tsukamoto, T. (2002) Multidrug-resistant Pseudomonas aeruginosa isolated from the urine of patients with urinary tract infection. J Infect Chemother, 8, 59-63.

14. Dale, R.M., Schnell, G. and Wong, J.P. (2004) Therapeutic efficacy of "nubiotics" against burn wound infection by Pseudomonas aeruginosa. Antimicrob Agents Chemother, 48, 2918-2923.

15. Estahbanati, H.K., Kashani, P.P. and Ghanaatpisheh, F. (2002) Frequency of Pseudomonas aeruginosa serotypes in burn wound infections and their resistance to antibiotics. Burns, 28, 340-348.

16. McVay, C.S., Velasquez, M. and Fralick, J.A. (2007) Phage therapy of Pseudomonas aeruginosa infection in a mouse burn wound model. Antimicrob Agents Chemother, 51, 1934-1938.

17. Johnstone, I.E. (1977) Pseudomonas aeruginosa and wound infections. Nurs Times, 73, suppl 55.

18. Secher, I., Hermes, I., Pre, S., Carreau, F. and Bahuet, F. (2005) Surgical wound infections due to Pseudomonas aeruginosa in orthopedic surgery. Med Mal Infect, 35, 149-154.

19. Vento, S., Cainelli, F. and Temesgen, Z. (2008) Lung infections after cancer chemotherapy. Lancet Oncol., 9, 982-992.

20. Martinez-Solano, L., Macia, M.D., Fajardo, A., Oliver, A. and Martinez, J.L. (2008) Chronic Pseudomonas aeruginosa infection in chronic obstructive pulmonary disease. Clin Infect Dis., 47, 1526-1533. 
21. Angrill, J., Agusti, C., de Celis, R., Rano, A., Gonzalez, J., Sole, T., Xaubet, A., Rodriguez-Roisin, R. and Torres, A. (2002) Bacterial colonisation in patients with bronchiectasis: microbiological pattern and risk factors. Thorax, 57, 15-19.

22. Manfredi, R., Nanetti, A., Ferri, M. and Chiodo, F. (2000) Pseudomonas spp. complications in patients with HIV disease: an eight-year clinical and microbiological survey. Eur J Epidemiol, 16, 111-118.

23. Armour, A.D., Shankowsky, H.A., Swanson, T., Lee, J. and Tredget, E.E. (2007) The impact of nosocomially-acquired resistant Pseudomonas aeruginosa infection in a burn unit. J Trauma, 63, 164-171.

24. Marra, A.R., Bar, K., Bearman, G.M., Wenzel, R.P. and Edmond, M.B. (2006) Systemic inflammatory response syndrome in adult patients with nosocomial bloodstream infection due to Pseudomonas aeruginosa. J Infect, 53, 30-35.

25. Stover, C.K., Pham, X.Q., Erwin, A.L., Mizoguchi, S.D., Warrener, P., Hickey, M.J., Brinkman, F.S., Hufnagle, W.O., Kowalik, D.J., Lagrou, M. et al. (2000) Complete genome sequence of Pseudomonas aeruginosa PA01, an opportunistic pathogen. Nature, 406, 959-964.

26. Holloway, B.W. (1955) Genetic recombination in Pseudomonas aeruginosa. $J$ Gen Microbiol., 13, 572-581.

27. Winsor, G.L., Lam, D.K., Fleming, L., Lo, R., Whiteside, M.D., Yu, N.Y., Hancock, R.E. and Brinkman, F.S. (2011) Pseudomonas Genome Database: improved comparative analysis and population genomics capability for Pseudomonas genomes. Nucleic Acids Res, 39, D596-600.

28. Goodman, A.L. and Lory, S. (2004) Analysis of regulatory networks in Pseudomonas aeruginosa by genomewide transcriptional profiling. Curr Opin Microbiol, 7, 39-44.

29. Wolfgang, M.C., Lee, V.T., Gilmore, M.E. and Lory, S. (2003) Coordinate regulation of bacterial virulence genes by a novel adenylate cyclase-dependent signaling pathway. Dev Cell, 4, 253-263. 
30. Rahme, L.G., Stevens, E.J., Wolfort, S.F., Shao, J., Tompkins, R.G. and Ausubel, F.M. (1995) Common virulence factors for bacterial pathogenicity in plants and animals. Science, 268, 1899-1902.

31. Pier, G.B., Matthews, W.J., Jr. and Eardley, D.D. (1983) Immunochemical characterization of the mucoid exopolysaccharide of Pseudomonas aeruginosa. $J$ Infect Dis, 147, 494-503.

32. Jones, A.M., Govan, J.R., Doherty, C.J., Dodd, M.E., Isalska, B.J., Stanbridge, T.N. and Webb, A.K. (2001) Spread of a multiresistant strain of Pseudomonas aeruginosa in an adult cystic fibrosis clinic. Lancet, 358, 557-558.

33. Mathee, K., Narasimhan, G., Valdes, C., Qiu, X., Matewish, J.M., Koehrsen, M., Rokas, A., Yandava, C.N., Engels, R., Zeng, E. et al. (2008) Dynamics of Pseudomonas aeruginosa genome evolution. Proc Natl Acad Sci U S A, 105, 3100-3105.

34. Brazas, M.D. and Hancock, R.E. (2005) Ciprofloxacin induction of a susceptibility determinant in Pseudomonas aeruginosa. Antimicrob Agents Chemother., 49, 3222-3227.

35. Fernandez, L., Breidenstein, E.B. and Hancock, R.E. (2011) Creeping baselines and adaptive resistance to antibiotics. Drug Resist Updat., 14, 1-21.

36. Calabrese, E.J. and Baldwin, L.A. (2002) Defining hormesis. Hum Exp Toxicol., 21, 91-97.

37. Linares, J.F., Gustafsson, I., Baquero, F. and Martinez, J.L. (2006) Antibiotics as intermicrobial signaling agents instead of weapons. PNAS, 103, 19484-19489.

38. Horinouchi, S. (2007) Mining and polishing of the treasure trove in the bacterial genus Streptomyces. Biosci Biotechnol Biochem., 71, 283-299.

39. Dubern, J.F. and Diggle, S.P. (2008) Quorum sensing by 2-alkyl-4-quinolones in Pseudomonas aeruginosa and other bacterial species. Mol Biosyst., 4, 882-888.

40. Kaufmann, G.F., Sartorio, R., Lee, S.H., Rogers, C.J., Meijler, M.M., Moss, J.A., Clapham, B., Brogan, A.P., Dickerson, T.J. and Janda, K.D. (2005) Revisiting 
quorum sensing: Discovery of additional chemical and biological functions for 3oxo-N-acylhomoserine lactones. PNAS, 102, 309-314.

41. Tamaoki, J., Kadota, J. and Takizawa, H. (2004) Clinical implications of the immunomodulatory effects of macrolides. Am J Med., 117 Suppl 9A, 5S-11S.

42. Yuhas, Y., Berent, E., Cohen, R. and Ashkenazi, S. (2009) Roles of NF-kappaB activation and peroxisome proliferator-activated receptor gamma inhibition in the effect of rifampin on inducible nitric oxide synthase transcription in human lung epithelial cells. Antimicrob Agents Chemother., 53, 1539-1545.

43. Hall, B.G. and Barlow, M. (2004) Evolution of the serine beta-lactamases: past, present and future. Drug Resist Updat., 7, 111-123.

44. Hawkey, P.M. and Jones, A.M. (2009) The changing epidemiology of resistance. J Antimicrob Chemother., 64 Suppl 1, i3-10.

45. Baquero, F. (2001) Low-level antibacterial resistance: a gateway to clinical resistance. Drug Resist Updat., 4, 93-105.

46. Kislak, J.W., Razavi, L.M., Daly, A.K. and Finland, M. (1965) Susceptibility of pneumococci to nine antibiotics. Am J Med Sci., 250, 261-268.

47. Anderson, G.G., Moreau-Marquis, S., Stanton, B.A. and O'Toole, G.A. (2008) In vitro analysis of tobramycin-treated Pseudomonas aeruginosa biofilms on cystic fibrosis-derived airway epithelial cells. Infect Immun, 76, 1423-1433.

48. Masecar, B.L., Celesk, R.A. and Robillard, N.J. (1990) Analysis of acquired ciprofloxacin resistance in a clinical strain of Pseudomonas aeruginosa. Antimicrob Agents Chemother., 34, 281-286.

49. Huczko, E., Conetta, B., Bonner, D., Valera, L., Stickle, T., Macko, A. and FungTomc, J. (2000) Susceptibility of bacterial isolates to gatifloxacin and ciprofloxacin from clinical trials 1997-1998. Int J Antimicrob Agents, 16, 401-405.

50. Fujimura, T., Anan, N., Sugimori, G., Watanabe, T., Jinushi, Y., Yoshida, I. and Yamano, Y. (2009) Susceptibility of Pseudomonas aeruginosa clinical isolates in 
Japan to doripenem and other antipseudomonal agents. Int J Antimicrob Agents, 34, 523-528.

51. Dibb, W.L., Asphaug Kjellevold, V. and Digranes, A. (1983) Pseudomonas aeruginosa and Acinetobacter calcoaceticus: in vitro susceptibility of 150 clinical isolates to five beta-lactam antibiotics and tobramycin. Chemotherapy, 29, 332336.

52. Stratton, C.W., Weeks, L.S. and Tausk, F. (1987) Beta-lactamase induction and aminoglycoside susceptibility in Pseudomonas aeruginosa. $J$ Antimicrob Chemother., 19, 21-25.

53. MacLeod, D.L., Nelson, L.E., Shawar, R.M., Lin, B.B., Lockwood, L.G., Dirk, J.E., Miller, G.H., Burns, J.L. and Garber, R.L. (2000) Aminoglycoside-resistance mechanisms for cystic fibrosis Pseudomonas aeruginosa isolates are unchanged by long-term, intermittent, inhaled tobramycin treatment. J Infect Dis., 181, 11801184.

54. Hancock, R.E. (1998) Resistance mechanisms in Pseudomonas aeruginosa and other nonfermentative gram-negative bacteria. Clin Infect Dis., 27, S93-99.

55. Li, X.Z., Barre, N. and Poole, K. (2000) Influence of the MexA-MexB-oprM multidrug efflux system on expression of the MexC-MexD-oprJ and MexEMexF-oprN multidrug efflux systems in Pseudomonas aeruginosa. J Antimicrob Chemother., 46, 885-893.

56. Li, X.Z., Zhang, L. and Poole, K. (2000) Interplay between the MexA-MexBOprM multidrug efflux system and the outer membrane barrier in the multiple antibiotic resistance of Pseudomonas aeruginosa. J Antimicrob Chemother., 45, 433-436.

57. Li, X.Z., Nikaido, H. and Poole, K. (1995) Role of mexA-mexB-oprM in antibiotic efflux in Pseudomonas aeruginosa. Antimicrob Agents Chemother., 39, 19481953.

58. Masuda, N., Gotoh, N., Ishii, C., Sakagawa, E., Ohya, S. and Nishino, T. (1999) Interplay between chromosomal beta-lactamase and the MexAB-OprM efflux system in intrinsic resistance to beta-lactams in Pseudomonas aeruginosa. Antimicrob Agents Chemother, 43, 400-402. 
59. Alvarez-Ortega, C., Wiegand, I., Olivares, J., Hancock, R.E. and Martinez, J.L. (2010) Genetic determinants involved in the susceptibility of Pseudomonas aeruginosa to beta-lactam antibiotics. Antimicrob Agents Chemother., 54, 41594167.

60. Dotsch, A., Becker, T., Pommerenke, C., Magnowska, Z., Jansch, L. and Haussler, S. (2009) Genomewide identification of genetic determinants of antimicrobial drug resistance in Pseudomonas aeruginosa. Antimicrob Agents Chemother., 53, 2522-2531.

61. Fajardo, A., Martinez-Martin, N., Mercadillo, M., Galan, J.C., Ghysels, B., Matthijs, S., Cornelis, P., Wiehlmann, L., Tummler, B., Baquero, F. et al. (2008) The neglected intrinsic resistome of bacterial pathogens. PLOS ONE, 3, e1619.

62. Breidenstein, E.B., Khaira, B.K., Wiegand, I., Overhage, J. and Hancock, R.E. (2008) Complex ciprofloxacin resistome revealed by screening a Pseudomonas aeruginosa mutant library for altered susceptibility. Antimicrob Agents Chemother., 52, 4486-4491.

63. Vakulenko, S.B. and Mobashery, S. (2003) Versatility of aminoglycosides and prospects for their future. Clin Microbiol Rev., 16, 430-450.

64. Sacha, P., Wieczorek, P., Hauschild, T., Zorawski, M., Olszanska, D. and Tryniszewska, E. (2008) Metallo-beta-lactamases of Pseudomonas aeruginosa--a novel mechanism resistance to beta-lactam antibiotics. Folia Histochem Cytobiol., 46, 137-142.

65. Walsh, T.R. (2005) The emergence and implications of metallo-beta-lactamases in Gram-negative bacteria. Clin Microbiol Infect., 11 Suppl 6, 2-9.

66. Driffield, K., Miller, K., Bostock, J.M., O'Neill, A.J. and Chopra, I. (2008) Increased mutability of Pseudomonas aeruginosa in biofilms. J Antimicrob Chemother, 61, 1053-1056.

67. Tanimoto, K., Tomita, H., Fujimoto, S., Okuzumi, K. and Ike, Y. (2008) Fluoroquinolone enhances the mutation frequency for meropenem-selected carbapenem resistance in Pseudomonas aeruginosa, but use of the high-potency drug doripenem inhibits mutant formation. Antimicrob Agents Chemother., 52, 3795-3800. 
68. Wiegand, I., Marr, A.K., Breidenstein, E.B., Schurek, K.N., Taylor, P. and Hancock, R.E. (2008) Mutator genes giving rise to decreased antibiotic susceptibility in Pseudomonas aeruginosa. Antimicrob Agents Chemother., 52, 3810-3813.

69. Walsh, F. and Amyes, S.G. (2007) Carbapenem resistance in clinical isolates of Pseudomonas aeruginosa. J Chemother., 19, 376-381.

70. Sobel, M.L., Neshat, S. and Poole, K. (2005) Mutations in PA2491 (mexS) promote MexT-dependent mexEF-opr $N$ expression and multidrug resistance in a clinical strain of Pseudomonas aeruginosa. J Bacteriol., 187, 1246-1253.

71. Stickland, H.G., Davenport, P.W., Lilley, K.S., Griffin, J.L. and Welch, M. (2010) Mutation of $n f x B$ causes global changes in the physiology and metabolism of Pseudomonas aeruginosa. J Proteome Res., 9, 2957-2967.

72. Muller, C., Plesiat, P. and Jeannot, K. (2011) A two-component regulatory system interconnects resistance to polymyxins, aminoglycosides, fluoroquinolones, and beta-lactams in Pseudomonas aeruginosa. Antimicrob Agents Chemother., 55, 1211-1221.

73. Juan, C., Moya, B., Perez, J.L. and Oliver, A. (2006) Stepwise upregulation of the Pseudomonas aeruginosa chromosomal cephalosporinase conferring high-level beta-lactam resistance involves three AmpD homologues. Antimicrob Agents Chemother., 50, 1780-1787.

74. Schmidtke, A.J. and Hanson, N.D. (2008) Role of ampD homologs in overproduction of AmpC in clinical isolates of Pseudomonas aeruginosa. Antimicrob Agents Chemother., 52, 3922-3927.

75. Xavier, D.E., Picao, R.C., Girardello, R., Fehlberg, L.C. and Gales, A.C. (2010) Efflux pumps expression and its association with porin down-regulation and betalactamase production among Pseudomonas aeruginosa causing bloodstream infections in Brazil. BMC Microbiol., 10, 217.

76. Quale, J., Bratu, S., Gupta, J. and Landman, D. (2006) Interplay of efflux system, ampC, and oprD expression in carbapenem resistance of Pseudomonas aeruginosa clinical isolates. Antimicrob Agents Chemother., 50, 1633-1641. 
77. Hooper, D.C. (2001) Emerging mechanisms of fluoroquinolone resistance. Emerg Infect Dis., 7, 337-341.

78. Dunham, S.A., McPherson, C.J. and Miller, A.A. (2010) The relative contribution of efflux and target gene mutations to fluoroquinolone resistance in recent clinical isolates of Pseudomonas aeruginosa. Eur J Clin Microbiol Infect Dis., 29, 279288.

79. Lister, P.D., Wolter, D.J. and Hanson, N.D. (2009) Antibacterial-resistant Pseudomonas aeruginosa: clinical impact and complex regulation of chromosomally encoded resistance mechanisms. Clin Microbiol Rev, 22, 582-610.

80. Mawer, S.L. and Greenwood, D. (1978) Specific and non-specific resistance to aminoglycosides in Escherichia coli. J Clin Pathol., 31, 12-15.

81. Barber, M. and Waterworth, P.M. (1966) Activity of gentamicin against Pseudomonas and hospital Staphylococci. Br Med J, 1, 203-205.

82. Fernandez, L., Jenssen, H., Bains, M., Wiegand, I., Gooderham, W.J. and Hancock, R.E. (2012) The two-component system CprRS senses cationic peptides and triggers adaptive resistance in Pseudomonas aeruginosa independently of ParRS. Antimicrob Agents Chemother.

83. Fauvart, M., De Groote, V.N. and Michiels, J. (2011) Role of persister cells in chronic infections: clinical relevance and perspectives on anti-persister therapies. J Med Microbiol., 60, 699-709.

84. Foundation. (2011). Cystic Fibrosis Foundation, Bethesda, Maryland.

85. Chen, H.Y., Yuan, M. and Livermore, D.M. (1995) Mechanisms of resistance to beta-lactam antibiotics amongst Pseudomonas aeruginosa isolates collected in the UK in 1993. $J$ Med Microbiol, 43, 300-309.

86. Bonfiglio, G., Laksai, Y., Franchino, L., Amicosante, G. and Nicoletti, G. (1998) Mechanisms of beta-lactam resistance amongst Pseudomonas aeruginosa isolated in an Italian survey. J Antimicrob Chemother, 42, 697-702. 
87. Ciofu, O., Beveridge, T.J., Kadurugamuwa, J., Walther-Rasmussen, J. and Hoiby, N. (2000) Chromosomal beta-lactamase is packaged into membrane vesicles and secreted from Pseudomonas aeruginosa. J Antimicrob Chemother., 45, 9-13.

88. Hengzhuang, W., Ciofu, O., Yang, L., Wu, H., Song, Z., Oliver, A. and Hoiby, N. (2012) High beta-lactamase levels change the pharmacodynamics of beta-lactam antibiotics in Pseudomonas aeruginosa biofilms. Antimicrob Agents Chemother.

89. Hengzhuang, W., Wu, H., Ciofu, O., Song, Z. and Hoiby, N. (2011) Pharmacokinetics/pharmacodynamics of colistin and imipenem on mucoid and nonmucoid Pseudomonas aeruginosa biofilms. Antimicrob Agents Chemother., 55, 4469-4474.

90. Giwercman, B., Lambert, P.A., Rosdahl, V.T., Shand, G.H. and Hoiby, N. (1990) Rapid emergence of resistance in Pseudomonas aeruginosa in cystic fibrosis patients due to in-vivo selection of stable partially derepressed beta-lactamase producing strains. $J$ Antimicrob Chemother, 26, 247-259.

91. Campbell, J.I., Ciofu, O. and Hoiby, N. (1997) Pseudomonas aeruginosa isolates from patients with cystic fibrosis have different beta-lactamase expression phenotypes but are homogeneous in the ampC-ampR genetic region. Antimicrob Agents Chemother, 41, 1380-1384.

92. Bagge, N., Ciofu, O., Hentzer, M., Campbell, J.I., Givskov, M. and Hoiby, N. (2002) Constitutive high expression of chromosomal beta-lactamase in Pseudomonas aeruginosa caused by a new insertion sequence (IS1669) located in ampD. Antimicrob Agents Chemother, 46, 3406-3411.

93. Hennessey, T.D. (1967) Inducible beta-lactamase in Enterobacter. $J$ Gen Microbiol, 49, 277-285.

94. Hanson, N.D. and Sanders, C.C. (1999) Regulation of inducible AmpC betalactamase expression among Enterobacteriaceae. Curr Pharm Des, 5, 881-894.

95. Lindberg, F. and Normark, S. (1986) Contribution of chromosomal betalactamases to beta-lactam resistance in enterobacteria. Rev Infect Dis, 8 Suppl 3, S292-304. 
96. Normark, S., Lindquist, S. and Lindberg, F. (1986) Chromosomal beta-lactam resistance in enterobacteria. Scand J Infect Dis Suppl, 49, 38-45.

97. Poirel, L., Guibert, M., Girlich, D., Naas, T. and Nordmann, P. (1999) Cloning, sequence analyses, expression, and distribution of ampC-ampR from Morganella morganii clinical isolates. Antimicrob Agents Chemother, 43, 769-776.

98. Lindquist, S., Lindberg, F. and Normark, S. (1989) Binding of the Citrobacter freundii AmpR regulator to a single DNA site provides both autoregulation and activation of the inducible $a m p C$ beta-lactamase gene. $J$ Bacteriol, 171, 37463753.

99. Lindberg, F., Lindquist, S. and Normark, S. (1988) Genetic basis of induction and overproduction of chromosomal class I beta-lactamase in nonfastidious Gramnegative bacilli. Rev Infect Dis, 10, 782-785.

100. Honore, N., Nicolas, M.H. and Cole, S.T. (1986) Inducible cephalosporinase production in clinical isolates of Enterobacter cloacae is controlled by a regulatory gene that has been deleted from Escherichia coli. EMBO J, 5, 37093714.

101. Lindberg, F., Lindquist, S. and Normark, S. (1987) Inactivation of the ampD gene causes semiconstitutive overproduction of the inducible Citrobacter freundii betalactamase. J Bacteriol, 169, 1923-1928.

102. Lodge, J.M., Minchin, S.D., Piddock, L.J. and Busby, J.W. (1990) Cloning, sequencing and analysis of the structural gene and regulatory region of the Pseudomonas aeruginosa chromosomal ampC beta-lactamase. Biochem J, 272, 627-631.

103. Schell, M.A. (1993) Molecular biology of the LysR family of transcriptional regulators. Annu Rev Microbiol, 47, 597-626.

104. Maddocks, S.E. and Oyston, P.C. (2008) Structure and function of the LysR-type transcriptional regulator (LTTR) family proteins. Microbiology, 154, 3609-3623.

105. Kong, K.F., Jayawardena, S.R., Indulkar, S.D., Del Puerto, A., Koh, C.L., Hoiby, N. and Mathee, K. (2005) Pseudomonas aeruginosa AmpR is a global transcriptional factor that regulates expression of $\mathrm{AmpC}$ and PoxB beta- 
lactamases, proteases, quorum sensing, and other virulence factors. Antimicrob Agents Chemother, 49, 4567-4575.

106. Kong, K.F., Jayawardena, S.R., Del Puerto, A., Wiehlmann, L., Laabs, U., Tummler, B. and Mathee, K. (2005) Characterization of poxB, a chromosomalencoded Pseudomonas aeruginosa oxacillinase. Gene, 358, 82-92.

107. Vasil, M.L. (2007) How we learnt about iron acquisition in Pseudomonas aeruginosa: a series of very fortunate events. Biometals, 20, 587-601.

108. Cornelis, P., and Matthijs, S. (2007) Pseudomonas siderophores and their biological significance Springer Berlin Heidelberg, Berlin.

109. Van Delden, C. and Iglewski, B.H. (1998) Cell-to-cell signaling and Pseudomonas aeruginosa infections. Emerg Infect Dis, 4, 551-560.

110. Kessler, E. (1995) Beta-lytic endopeptidases. Methods Enzymol, 248, 740-756.

111. Preston, M.J., Seed, P.C., Toder, D.S., Iglewski, B.H., Ohman, D.E., Gustin, J.K., Goldberg, J.B. and Pier, G.B. (1997) Contribution of proteases and LasR to the virulence of Pseudomonas aeruginosa during corneal infections. Infect Immun, 65, 3086-3090.

112. Scheurwater, E.M. and Burrows, L.L. (2011) Maintaining network security: how macromolecular structures cross the peptidoglycan layer. FEMS Microbiol Lett., 318, 1-9.

113. Vollmer, W., Blanot, D. and de Pedro, M.A. (2008) Peptidoglycan structure and architecture. FEMS Microbiol Rev., 32, 149-167.

114. Vollmer, W. (2008) Structural variation in the glycan strands of bacterial peptidoglycan. FEMS Microbiol Rev., 32, 287-306.

115. Barreteau, H., Kovac, A., Boniface, A., Sova, M., Gobec, S. and Blanot, D. (2008) Cytoplasmic steps of peptidoglycan biosynthesis. FEMS Microbiol Rev., 32, 168-207. 
116. Bouhss, A., Trunkfield, A.E., Bugg, T.D. and Mengin-Lecreulx, D. (2008) The biosynthesis of peptidoglycan lipid-linked intermediates. FEMS Microbiol Rev., 32, 208-233.

117. Sauvage, E., Kerff, F., Terrak, M., Ayala, J.A. and Charlier, P. (2008) The penicillin-binding proteins: structure and role in peptidoglycan biosynthesis. FEMS Microbiol Rev., 32, 234-258.

118. Goodell, E.W. and Schwarz, U. (1985) Release of cell wall peptides into culture medium by exponentially growing Escherichia coli. J Bacteriol., 162, 391-397.

119. Reith, J. and Mayer, C. (2011) Peptidoglycan turnover and recycling in Grampositive bacteria. Appl Microbiol Biotechnol., 92, 1-11.

120. Park, J.T. and Uehara, T. (2008) How bacteria consume their own exoskeletons (turnover and recycling of cell wall peptidoglycan). Microbiol Mol Biol Rev., 72, 211-227, table of contents.

121. Jacobs, C., Huang, L.J., Bartowsky, E., Normark, S. and Park, J.T. (1994) Bacterial cell wall recycling provides cytosolic muropeptides as effectors for betalactamase induction. EMBO J, 13, 4684-4694.

122. Cheng, Q., Li, H., Merdek, K. and Park, J.T. (2000) Molecular characterization of the beta-N-acetylglucosaminidase of Escherichia coli and its role in cell wall recycling. J Bacteriol., 182, 4836-4840.

123. Asgarali, A., Stubbs, K.A., Oliver, A., Vocadlo, D.J. and Mark, B.L. (2009) Inactivation of the glycoside hydrolase NagZ attenuates antipseudomonal betalactam resistance in Pseudomonas aeruginosa. Antimicrob Agents Chemother., 53, 2274-2282.

124. Zamorano, L., Reeve, T.M., Deng, L., Juan, C., Moya, B., Cabot, G., Vocadlo, D.J., Mark, B.L. and Oliver, A. (2010) NagZ inactivation prevents and reverts beta-lactam resistance, driven by AmpD and PBP 4 mutations, in Pseudomonas aeruginosa. Antimic Agents Chemother., 54, 3557-3563.

125. Lindquist, S., Galleni, M., Lindberg, F. and Normark, S. (1989) Signalling proteins in enterobacterial $\mathrm{AmpC}$ beta-lactamase regulation. Mol Microbiol, 3, 1091-1102. 
126. Ruiz, N. (2008) Bioinformatics identification of MurJ (MviN) as the peptidoglycan lipid II flippase in Escherichia coli. PNAS, 105, 15553-15557.

127. Kong, K.F., Aguila, A., Schneper, L. and Mathee, K. (2010) Pseudomonas aeruginosa beta-lactamase induction requires two permeases, AmpG and AmpP. BMC Microbiol., 10, 328.

128. Kellam, P. (2000) Host-pathogen studies in the post-genomic era. Genome Biol, 1, reviews 1009.1001-1009.1004.

129. Liang, P. and Pardee, A.B. (1995) Recent advances in differential display. Curr Opin Immunol, 7, 274-280.

130. Liang, P. and Pardee, A.B. (1992) Differential display of eukaryotic messenger RNA by means of the polymerase chain reaction. Science, 257, 967-971.

131. Welsh, J., Chada, K., Dalal, S.S., Cheng, R., Ralph, D. and McClelland, M. (1992) Arbitrarily primed PCR fingerprinting of RNA. Nucleic Acids Res, 20, 4965-4970.

132. Vos, P., Hogers, R., Bleeker, M., Reijans, M., van de Lee, T., Hornes, M., Frijters, A., Pot, J., Peleman, J., Kuiper, M. et al. (1995) AFLP: a new technique for DNA fingerprinting. Nucleic Acids Res, 23, 4407-4414.

133. Bachem, C.W., van der Hoeven, R.S., de Bruijn, S.M., Vreugdenhil, D., Zabeau, M. and Visser, R.G. (1996) Visualization of differential gene expression using a novel method of RNA fingerprinting based on AFLP: analysis of gene expression during potato tuber development. Plant J, 9, 745-753.

134. Brugmans, B., Fernandez del Carmen, A., Bachem, C.W., van Os, H., van Eck, H.J. and Visser, R.G. (2002) A novel method for the construction of genome wide transcriptome maps. Plant J, 31, 211-222.

135. Kamoun, S., Hraber, P., Sobral, B., Nuss, D. and Govers, F. (1999) Initial assessment of gene diversity for the oomycete pathogen Phytophthora infestans based on expressed sequences. Fungal Genet Biol, 28, 94-106. 
136. Velculescu, V.E., Zhang, L., Zhou, W., Vogelstein, J., Basrai, M.A., Bassett, D.E., Jr., Hieter, P., Vogelstein, B. and Kinzler, K.W. (1997) Characterization of the yeast transcriptome. Cell, 88, 243-251.

137. Saha, S., Sparks, A.B., Rago, C., Akmaev, V., Wang, C.J., Vogelstein, B., Kinzler, K.W. and Velculescu, V.E. (2002) Using the transcriptome to annotate the genome. Nat Biotechnol, 20, 508-512.

138. Matsumura, H., Ito, A., Saitoh, H., Winter, P., Kahl, G., Reuter, M., Kruger, D.H. and Terauchi, R. (2005) SuperSAGE. Cell Microbiol, 7, 11-18.

139. Matsumura, H., Reich, S., Ito, A., Saitoh, H., Kamoun, S., Winter, P., Kahl, G., Reuter, M., Kruger, D.H. and Terauchi, R. (2003) Gene expression analysis of plant host-pathogen interactions by SuperSAGE. Proc Natl Acad Sci US A, 100, 15718-15723.

140. Brenner, S., Johnson, M., Bridgham, J., Golda, G., Lloyd, D.H., Johnson, D., Luo, S., McCurdy, S., Foy, M., Ewan, M. et al. (2000) Gene expression analysis by massively parallel signature sequencing (MPSS) on microbead arrays. Nat Biotechnol, 18, 630-634.

141. Fleischmann, R.D., Adams, M.D., White, O., Clayton, R.A., Kirkness, E.F., Kerlavage, A.R., Bult, C.J., Tomb, J.F., Dougherty, B.A., Merrick, J.M. et al. (1995) Whole-genome random sequencing and assembly of Haemophilus influenzae Rd. Science, 269, 496-512.

142. Lee, D.G., Urbach, J.M., Wu, G., Liberati, N.T., Feinbaum, R.L., Miyata, S., Diggins, L.T., He, J., Saucier, M., Deziel, E. et al. (2006) Genomic analysis reveals that Pseudomonas aeruginosa virulence is combinatorial. Genome Biol, 7, R90.

143. Ochsner, U.A., Vasil, A.I. and Vasil, M.L. (1995) Role of the ferric uptake regulator of Pseudomonas aeruginosa in the regulation of siderophores and exotoxin A expression: purification and activity on iron-regulated promoters. $J$ Bacteriol, 177, 7194-7201.

144. Ochsner, U.A., Wilderman, P.J., Vasil, A.I. and Vasil, M.L. (2002) GeneChip expression analysis of the iron starvation response in Pseudomonas aeruginosa: 
identification of novel pyoverdine biosynthesis genes. Mol Microbiol., 45, 12771287.

145. Palma, M., Worgall, S. and Quadri, L.E. (2003) Transcriptome analysis of the Pseudomonas aeruginosa response to iron. Arch Microbiol, 180, 374-379.

146. Zheng, P., Sun, J., Geffers, R. and Zeng, A.P. (2007) Functional characterization of the gene PA2384 in large-scale gene regulation in response to iron starvation in Pseudomonas aeruginosa. J Biotechnol, 132, 342-352.

147. Palma, M., DeLuca, D., Worgall, S. and Quadri, L.E. (2004) Transcriptome analysis of the response of Pseudomonas aeruginosa to hydrogen peroxide. $J$ Bacteriol, 186, 248-252.

148. Salunkhe, P., Topfer, T., Buer, J. and Tummler, B. (2005) Genome-wide transcriptional profiling of the steady-state response of Pseudomonas aeruginosa to hydrogen peroxide. $J$ Bacteriol, 187, 2565-2572.

149. Salunkhe P., v.G.F., Weihlmann L., Lauber J., Buer J., Tummler B. (2002) GeneChip expression analysis of the response of Pseudomonas aeruginosa to Paraquat-induced superoxide stress. Genome Letters, 1, 165-174.

150. Small, D.A., Chang, W., Toghrol, F. and Bentley, W.E. (2007) Toxicogenomic analysis of sodium hypochlorite antimicrobial mechanisms in Pseudomonas aeruginosa. Appl Microbiol Biotechnol, 74, 176-185.

151. Small, D.A., Chang, W., Toghrol, F. and Bentley, W.E. (2007) Comparative global transcription analysis of sodium hypochlorite, peracetic acid, and hydrogen peroxide on Pseudomonas aeruginosa. Appl Microbiol Biotechnol, 76, 1093-1105.

152. Chang, W., Small, D.A., Toghrol, F. and Bentley, W.E. (2005) Microarray analysis of Pseudomonas aeruginosa reveals induction of pyocin genes in response to hydrogen peroxide. BMC Genomics, $\mathbf{6}, 115$.

153. Nde, C.W., Jang, H.J., Toghrol, F. and Bentley, W.E. (2008) Toxicogenomic response of Pseudomonas aeruginosa to ortho-phenylphenol. BMC Genomics, 9, 473. 
154. Muller, J.F., Stevens, A.M., Craig, J. and Love, N.G. (2007) Transcriptome analysis reveals that multidrug efflux genes are upregulated to protect Pseudomonas aeruginosa from pentachlorophenol stress. Appl Environ Microbiol, 73, 4550-4558.

155. Escher, B.I., Hunziker, R., and Schwarzenbach, R.P. (1999) Kinetic model to describe the intrinsic coupling activity of substitutued phenols in energy transducing membranes. Environmental Science and Technology, 33, 560-570.

156. Frieden, E., Osaki, S. and Kobayashi, H. (1965) Copper proteins and oxygen. Correlations between structure and function of the copper oxidases. J Gen Physiol, 49, Suppl:213-252.

157. Teitzel, G.M., Geddie, A., De Long, S.K., Kirisits, M.J., Whiteley, M. and Parsek, M.R. (2006) Survival and growth in the presence of elevated copper: transcriptional profiling of copper-stressed Pseudomonas aeruginosa. J Bacteriol, 188, $7242-7256$.

158. Frangipani, E., Slaveykova, V.I., Reimmann, C. and Haas, D. (2008) Adaptation of aerobically growing Pseudomonas aeruginosa to copper starvation. J Bacteriol, 190, 6706-6717.

159. Chace, K.V., Leahy, D.S., Martin, R., Carubelli, R., Flux, M. and Sachdev, G.P. (1983) Respiratory mucous secretions in patients with cystic fibrosis: relationship between levels of highly sulfated mucin component and severity of the disease. Clin Chim Acta, 132, 143-155.

160. Xia, B., Royall, J.A., Damera, G., Sachdev, G.P. and Cummings, R.D. (2005) Altered O-glycosylation and sulfation of airway mucins associated with cystic fibrosis. Glycobiology, 15, 747-775.

161. Quadroni, M., James, P., Dainese-Hatt, P. and Kertesz, M.A. (1999) Proteome mapping, mass spectrometric sequencing and reverse transcription-PCR for characterization of the sulfate starvation-induced response in Pseudomonas aeruginosa PAO1. Eur J Biochem, 266, 986-996.

162. Tralau, T., Vuilleumier, S., Thibault, C., Campbell, B.J., Hart, C.A. and Kertesz, M.A. (2007) Transcriptomic analysis of the sulfate starvation response of Pseudomonas aeruginosa. J Bacteriol, 189, 6743-6750. 
163. Aspedon, A., Palmer, K. and Whiteley, M. (2006) Microarray analysis of the osmotic stress response in Pseudomonas aeruginosa. J Bacteriol, 188, 2721-2725.

164. Garay-Arroyo A, C.-F.J., Garciarrubio A, Covarrubias AA. (2000) Highly hydrophilic proteins in prokaryotes and eukaryotes are common during conditions of water deficit. Journal of Biological Chemistry, 275, 5668-5574.

165. Waters, C.M. and Bassler, B.L. (2005) Quorum sensing: cell-to-cell communication in bacteria. Annu Rev Cell Dev Biol, 21, 319-346.

166. Juhas, M., Eberl, L. and Tummler, B. (2005) Quorum sensing: the power of cooperation in the world of Pseudomonas. Environ Microbiol, 7, 459-471.

167. Wagner, V.E., Bushnell, D., Passador, L., Brooks, A.I. and Iglewski, B.H. (2003) Microarray analysis of Pseudomonas aeruginosa quorum-sensing regulons: effects of growth phase and environment. $J$ Bacteriol, 185, 2080-2095.

168. Schuster, M., Lostroh, C.P., Ogi, T. and Greenberg, E.P. (2003) Identification, timing, and signal specificity of Pseudomonas aeruginosa quorum-controlled genes: a transcriptome analysis. J Bacteriol, 185, 2066-2079.

169. Hentzer, M., Wu, H., Andersen, J.B., Riedel, K., Rasmussen, T.B., Bagge, N., Kumar, N., Schembri, M.A., Song, Z., Kristoffersen, P. et al. (2003) Attenuation of Pseudomonas aeruginosa virulence by quorum sensing inhibitors. Embo J, 22, 3803-3815.

170. Schuster, M. and Greenberg, E.P. (2006) A network of networks: quorum-sensing gene regulation in Pseudomonas aeruginosa. Int J Med Microbiol, 296, 73-81.

171. Costerton, J.W., Stewart, P.S. and Greenberg, E.P. (1999) Bacterial biofilms: a common cause of persistent infections. Science, 284, 1318-1322.

172. Drenkard, E. (2003) Antimicrobial resistance of Pseudomonas aeruginosa biofilms. Microbes Infect, 5, 1213-1219.

173. Whiteley, M., Bangera, M.G., Bumgarner, R.E., Parsek, M.R., Teitzel, G.M., Lory, S. and Greenberg, E.P. (2001) Gene expression in Pseudomonas aeruginosa biofilms. Nature, 413, 860-864. 
174. Bagge, N., Schuster, M., Hentzer, M., Ciofu, O., Givskov, M., Greenberg, E.P. and Hoiby, N. (2004) Pseudomonas aeruginosa biofilms exposed to imipenem exhibit changes in global gene expression and beta-lactamase and alginate production. Antimicrob Agents Chemother, 48, 1175-1187.

175. Govan, J.R. and Deretic, V. (1996) Microbial pathogenesis in cystic fibrosis: mucoid Pseudomonas aeruginosa and Burkholderia cepacia. Microbiol Rev, 60, 539-574.

176. Firoved, A.M., Boucher, J.C. and Deretic, V. (2002) Global genomic analysis of $\mathrm{AlgU}$ (sigma(E))-dependent promoters (sigmulon) in Pseudomonas aeruginosa and implications for inflammatory processes in cystic fibrosis. $J$ Bacteriol, 184, 1057-1064.

177. Firoved, A.M. and Deretic, V. (2003) Microarray analysis of global gene expression in mucoid Pseudomonas aeruginosa. J Bacteriol, 185, 1071-1081.

178. Feldman, M., Bryan, R., Rajan, S., Scheffler, L., Brunnert, S., Tang, H. and Prince, A. (1998) Role of flagella in pathogenesis of Pseudomonas aeruginosa pulmonary infection. Infect Immun, 66, 43-51.

179. Tart, A.H., Wolfgang, M.C. and Wozniak, D.J. (2005) The alternative sigma factor AlgT represses Pseudomonas aeruginosa flagellum biosynthesis by inhibiting expression of fleQ.J Bacteriol, 187, 7955-7962.

180. Dasgupta, N., Wolfgang, M.C., Goodman, A.L., Arora, S.K., Jyot, J., Lory, S. and Ramphal, R. (2003) A four-tiered transcriptional regulatory circuit controls flagellar biogenesis in Pseudomonas aeruginosa. Mol Microbiol, 50, 809-824.

181. Kipnis, E., Sawa, T. and Wiener-Kronish, J. (2006) Targeting mechanisms of Pseudomonas aeruginosa pathogenesis. Med Mal Infect, 36, 78-91.

182. Knirel, Y.A., Bystrova, O.V., Kocharova, N.A., Zahringer, U. and Pier, G.B. (2006) Conserved and variable structural features in the lipopolysaccharide of Pseudomonas aeruginosa. J Endotoxin Res, 12, 324-336.

183. Goldberg J.B., Hatano K and Pier G.B. (1993) Synthesis of lipopolysaccharide O side chains by Pseudomonas aeruginosa PAO1 requires the enzyme phosphomannomutase. J Bacteriol., 175, 1605-1611. 
184. Sabra, W.H.L., and A.-P. Zeng. (2003) Alterations in the formation of lipopolysaccharide and membrane vesicles on the surface of Pseudomonas aeruginosa PAO1 under oxygen stress conditions. Microbiology, 149, 2789-2795.

185. Worlitzsch D, Tarran R, Ulrich M, Schwab U, Cekici A, Meyer K.C., Birrer P, Bellon G, Berger J, Weiss T et al. (2003) Effects of reduced mucus oxygen concentration in airway Pseudomonas infections of cystic fibrosis patients. $J$ Clin Invest., 109, 317-325.

186. Hassett, D.J. (1996) Anaerobic production of alginate by Pseudomonas aeruginosa: alginate restricts diffusion of oxygen. $J$ Bacteriol, 178, 7322-7325.

187. Firoved, A.M., Ornatowski, W. and Deretic, V. (2004) Microarray analysis reveals induction of lipoprotein genes in mucoid Pseudomonas aeruginosa: implications for inflammation in cystic fibrosis. Infect Immun, 72, 5012-5018.

188. Burrowes, E., Baysse, C., Adams, C. and O'Gara, F. (2006) Influence of the regulatory protein RsmA on cellular functions in Pseudomonas aeruginosa PAO1, as revealed by transcriptome analysis. Microbiology, 152, 405-418.

189. Burrowes, E., Abbas, A., O'Neill, A., Adams, C. and O'Gara, F. (2005) Characterisation of the regulatory RNA RsmB from Pseudomonas aeruginosa PAO1. Res Microbiol, 156, 7-16.

190. Pessi, G., Williams, F., Hindle, Z., Heurlier, K., Holden, M.T., Camara, M., Haas, D. and Williams, P. (2001) The global posttranscriptional regulator RsmA modulates production of virulence determinants and N-acylhomoserine lactones in Pseudomonas aeruginosa. J Bacteriol, 183, 6676-6683.

191. Ichikawa, J.K., Norris, A., Bangera, M.G., Geiss, G.K., van 't Wout, A.B., Bumgarner, R.E. and Lory, S. (2000) Interaction of Pseudomonas aeruginosa with epithelial cells: identification of differentially regulated genes by expression microarray analysis of human cDNAs. Proc Natl Acad Sci U S A, 97, 9659-9664.

192. Frisk, A., Schurr, J.R., Wang, G., Bertucci, D.C., Marrero, L., Hwang, S.H., Hassett, D.J. and Schurr, M.J. (2004) Transcriptome analysis of Pseudomonas aeruginosa after interaction with human airway epithelial cells. Infect Immun, $\mathbf{7 2}$, 5433-5438. 
193. Chugani, S. and Greenberg, E.P. (2007) The influence of human respiratory epithelia on Pseudomonas aeruginosa gene expression. Microb Pathog, 42, 29-35.

194. Jander, G., Rahme, L.G. and Ausubel, F.M. (2000) Positive correlation between virulence of Pseudomonas aeruginosa mutants in mice and insects. $J$ Bacteriol, 182, 3843-3845.

195. Mahajan-Miklos, S., Tan, M.W., Rahme, L.G. and Ausubel, F.M. (1999) Molecular mechanisms of bacterial virulence elucidated using a Pseudomonas aeruginosa-Caenorhabditis elegans pathogenesis model. Cell, 96, 47-56.

196. Rahme, L.G., Tan, M.W., Le, L., Wong, S.M., Tompkins, R.G., Calderwood, S.B. and Ausubel, F.M. (1997) Use of model plant hosts to identify Pseudomonas aeruginosa virulence factors. Proc Natl Acad Sci U S A, 94, 13245-13250.

197. Mahar, P., Padiglione, A.A., Cleland, H., Paul, E., Hinrichs, M. and Wasiak, J. (2010) Pseudomonas aeruginosa bacteraemia in burns patients: Risk factors and outcomes. Burns, 36, 1228-1233.

198. Melendez, J.H., Frankel, Y.M., An, A.T., Williams, L., Price, L.B., Wang, N.Y., Lazarus, G.S. and Zenilman, J.M. (2010) Real-time PCR assays compared to culture-based approaches for identification of aerobic bacteria in chronic wounds. Clin Microbiol Infect, 16, 1762-1769.

199. Hassett, D.J., Korfhagen, T.R., Irvin, R.T., Schurr, M.J., Sauer, K., Lau, G.W., Sutton, M.D., Yu, H. and Hoiby, N. (2010) Pseudomonas aeruginosa biofilm infections in cystic fibrosis: insights into pathogenic processes and treatment strategies. Expert Opin Ther Targets, 14, 117-130.

200. Murray, T.S., Egan, M. and Kazmierczak, B.I. (2007) Pseudomonas aeruginosa chronic colonization in cystic fibrosis patients. Curr Opin Pediatr, 19, 83-88.

201. Kerr, K.G. and Snelling, A.M. (2009) Pseudomonas aeruginosa: a formidable and ever-present adversary. $J$ Hosp Infect, 73, 338-344.

202. Cohen, J. (2002) The immunopathogenesis of sepsis. Nature, 420, 885-891. 
203. Rocchetta, H.L., Burrows, L.L. and Lam, J.S. (1999) Genetics of O-antigen biosynthesis in Pseudomonas aeruginosa. Microbiol Mol Biol Rev, 63, 523-553.

204. Senf, F., Tommassen, J. and Koster, M. (2008) Polar secretion of proteins via the Xcp type II secretion system in Pseudomonas aeruginosa. Microbiology, 154, 3025-3032.

205. Rosenau, F. and Jaeger, K. (2000) Bacterial lipases from Pseudomonas: regulation of gene expression and mechanisms of secretion. Biochimie, 82, 10231032.

206. Hauser, A.R. (2009) The type III secretion system of Pseudomonas aeruginosa: infection by injection. Nat Rev Microbiol, 7, 654-665.

207. Filloux, A., Hachani, A. and Bleves, S. (2008) The bacterial type VI secretion machine: yet another player for protein transport across membranes. Microbiology, 154, 1570-1583.

208. Ng, W.L. and Bassler, B.L. (2009) Bacterial quorum-sensing network architectures. Annu Rev Genet, 43, 197-222.

209. Ventre, I., Goodman, A.L., Vallet-Gely, I., Vasseur, P., Soscia, C., Molin, S., Bleves, S., Lazdunski, A., Lory, S. and Filloux, A. (2006) Multiple sensors control reciprocal expression of Pseudomonas aeruginosa regulatory RNA and virulence genes. Proc Natl Acad Sci U S A, 103, 171-176.

210. Goodman, A.L., Kulasekara, B., Rietsch, A., Boyd, D., Smith, R.S. and Lory, S. (2004) A signaling network reciprocally regulates genes associated with acute infection and chronic persistence in Pseudomonas aeruginosa. Dev Cell, 7, 745754.

211. Laskowski, M.A., Osborn, E. and Kazmierczak, B.I. (2004) A novel sensor kinase-response regulator hybrid regulates type III secretion and is required for virulence in Pseudomonas aeruginosa. Mol Microbiol, 54, 1090-1103.

212. Zolfaghar, I., Angus, A.A., Kang, P.J., To, A., Evans, D.J. and Fleiszig, S.M. (2005) Mutation of retS, encoding a putative hybrid two-component regulatory protein in Pseudomonas aeruginosa, attenuates multiple virulence mechanisms. Microbes Infect, 7, 1305-1316. 
213. Buelow, D.R. and Raivio, T.L. (2010) Three (and more) component regulatory systems - auxiliary regulators of bacterial histidine kinases. Mol Microbiol, 75, 547-566.

214. Klockgether, J., Munder, A., Neugebauer, J., Davenport, C.F., Stanke, F., Larbig, K.D., Heeb, S., Schock, U., Pohl, T.M., Wiehlmann, L. et al. (2010) Genome diversity of Pseudomonas aeruginosa PAO1 laboratory strains. J Bacteriol, 192, 1113-1121.

215. Madan Babu, M., Teichmann, S.A. and Aravind, L. (2006) Evolutionary dynamics of prokaryotic transcriptional regulatory networks. $J$ Mol Biol, 358, 614-633.

216. Larkin, M.A., Blackshields, G., Brown, N.P., Chenna, R., McGettigan, P.A., McWilliam, H., Valentin, F., Wallace, I.M., Wilm, A., Lopez, R. et al. (2007) Clustal W and Clustal X version 2.0. Bioinformatics, 23, 2947-2948.

217. Nei, M. and Kumar, S. (2000) Molecular evolution and phylogenetics. Oxford University Press, New York.

218. Eck, R.V. and Dayhoff, M.O. (1966), National Biomedical Research Foundation, Silver Spring, Maryland.

219. Tamura, K., Dudley, J., Nei, M. and Kumar, S. (2007) MEGA4: Molecular Evolutionary Genetics Analysis (MEGA) software version 4.0. Molecular Biology and Evolution, 24, 1596-1599.

220. Heroven, A.K. and Dersch, P. (2006) RovM, a novel LysR-type regulator of the virulence activator gene rovA, controls cell invasion, virulence and motility of Yersinia pseudotuberculosis. Mol Microbiol, 62, 1469-1483.

221. Hernandez-Lucas, I., Gallego-Hernandez, A.L., Encarnacion, S., Fernandez-Mora, M., Martinez-Batallar, A.G., Salgado, H., Oropeza, R. and Calva, E. (2008) The LysR-type transcriptional regulator LeuO controls expression of several genes in Salmonella enterica serovar Typhi. J Bacteriol, 190, 1658-1670.

222. Stragier, P., Borne, F., Richaud, F., Richaud, C. and Patte, J.C. (1983) Regulatory pattern of the Escherichia coli lys A gene: expression of chromosomal lysA-lacZ fusions. J Bacteriol, 156, 1198-1203. 
223. Cao, H., Krishnan, G., Goumnerov, B., Tsongalis, J., Tompkins, R. and Rahme, L.G. (2001) A quorum sensing-associated virulence gene of Pseudomonas aeruginosa encodes a LysR-like transcription regulator with a unique selfregulatory mechanism. Proc Natl Acad Sci U S A, 98, 14613-14618.

224. Deziel, E., Gopalan, S., Tampakaki, A.P., Lepine, F., Padfield, K.E., Saucier, M., Xiao, G. and Rahme, L.G. (2005) The contribution of MvfR to Pseudomonas aeruginosa pathogenesis and quorum sensing circuitry regulation: multiple quorum sensing-regulated genes are modulated without affecting lasRI, rhlRI or the production of N-acyl-L-homoserine lactones. Mol Microbiol, 55, 998-1014.

225. Balasubramanian, D., Schneper, L., Merighi, M., Smith, R., Narasimhan, G., Lory, S. and Mathee, K. (2012) The regulatory repertoire of Pseudomonas aeruginosa AmpC ß-lactamase regulator AmpR includes virulence genes. PLOS ONE, 7, e34067.

226. Balasubramanian, D., Kong, K.F., Jayawardena, S.R., Leal, S.M., Sautter, R.T. and Mathee, K. (2011) Co-regulation of B-lactam resistance, alginate production and quorum sensing in Pseudomonas aeruginosa. J Med Microbiol, 60, 147-156.

227. Ochsner, U.A., Vasil, M.L., Alsabbagh, E., Parvatiyar, K. and Hassett, D.J. (2000) Role of the Pseudomonas aeruginosa oxyR-rec $G$ operon in oxidative stress defense and DNA repair: OxyR-dependent regulation of $k a t B-a n k B$, ahpB, and ahpC-ahpF. J Bacteriol, 182, 4533-4544.

228. Lau, G.W., Britigan, B.E. and Hassett, D.J. (2005) Pseudomonas aeruginosa OxyR is required for full virulence in rodent and insect models of infection and for resistance to human neutrophils. Infect Immun, 73, 2550-2553.

229. Melstrom, K.A., Jr., Kozlowski, R., Hassett, D.J., Suzuki, H., Bates, D.M., Gamelli, R.L. and Shankar, R. (2007) Cytotoxicity of Pseudomonas secreted exotoxins requires OxyR expression. $J$ Surg Res, 143, 50-57.

230. Vinckx, T., Wei, Q., Matthijs, S. and Cornelis, P. (2010) The Pseudomonas aeruginosa oxidative stress regulator OxyR influences production of pyocyanin and rhamnolipids: protective role of pyocyanin. Microbiology, 156, 678-686. 
231. Vinckx, T., Matthijs, S. and Cornelis, P. (2008) Loss of the oxidative stress regulator OxyR in Pseudomonas aeruginosa PAO1 impairs growth under ironlimited conditions. FEMS Microbiol Lett, 288, 258-265.

232. Turner, K.H., Vallet-Gely, I. and Dove, S.L. (2009) Epigenetic control of virulence gene expression in Pseudomonas aeruginosa by a LysR-type transcription regulator. PLoS Genet, 5, e1000779.

233. Jin, Y., Yang, H., Qiao, M. and Jin, S. (2010) MexT regulates type III secretion system through MexS and PtrC in Pseudomonas aeruginosa.J Bacteriol.

234. Yeung, A.T., Torfs, E.C., Jamshidi, F., Bains, M., Wiegand, I., Hancock, R.E. and Overhage, J. (2009) Swarming of Pseudomonas aeruginosa is controlled by a broad spectrum of transcriptional regulators, including MetR. $J$ Bacteriol, 191, 5592-5602.

235. Molina-Henares, A.J., Krell, T., Eugenia Guazzaroni, M., Segura, A. and Ramos, J.L. (2006) Members of the IclR family of bacterial transcriptional regulators function as activators and/or repressors. FEMS Microbiol Rev, 30, 157-186.

236. Krell, T., Molina-Henares, A.J. and Ramos, J.L. (2006) The IclR family of transcriptional activators and repressors can be defined by a single profile. Protein Sci, 15, 1207-1213.

237. Zhang, R.G., Kim, Y., Skarina, T., Beasley, S., Laskowski, R., Arrowsmith, C., Edwards, A., Joachimiak, A. and Savchenko, A. (2002) Crystal structure of Thermotoga maritima 0065 , a member of the IclR transcriptional factor family. $J$ Biol Chem, 277, 19183-19190.

238. Romero-Steiner, S., Parales, R.E., Harwood, C.S. and Houghton, J.E. (1994) Characterization of the pcaR regulatory gene from Pseudomonas putida, which is required for the complete degradation of p-hydroxybenzoate. J Bacteriol, 176, 5771-5779.

239. Smith, C.P. and Chater, K.F. (1988) Structure and regulation of controlling sequences for the Streptomyces coelicolor glycerol operon. J Mol Biol, 204, 569580 . 
240. Nguyen, C.C. and Saier, M.H., Jr. (1995) Phylogenetic, structural and functional analyses of the LacI-GalR family of bacterial transcription factors. FEBS Lett, 377, 98-102.

241. Swint-Kruse, L. and Matthews, K.S. (2009) Allostery in the LacI/GalR family: variations on a theme. Curr Opin Microbiol, 12, 129-137.

242. Fukami-Kobayashi, K., Tateno, Y. and Nishikawa, K. (2003) Parallel evolution of ligand specificity between LacI/GalR family repressors and periplasmic sugarbinding proteins. Mol Biol Evol, 20, 267-277.

243. Chauvaux, S., Paulsen, I.T. and Saier, M.H., Jr. (1998) CcpB, a novel transcription factor implicated in catabolite repression in Bacillus subtilis. $J$ Bacteriol, 180, 491-497.

244. Maskell, D.J., Szabo, M.J., Deadman, M.E. and Moxon, E.R. (1992) The gal locus from Haemophilus influenzae: cloning, sequencing and the use of gal mutants to study lipopolysaccharide. Mol Microbiol, 6, 3051-3063.

245. Wilson, C.J., Zhan, H., Swint-Kruse, L. and Matthews, K.S. (2007) The lactose repressor system: paradigms for regulation, allosteric behavior and protein folding. Cell Mol Life Sci, 64, 3-16.

246. Vartak, N.B., Reizer, J., Reizer, A., Gripp, J.T., Groisman, E.A., Wu, L.F., Tomich, J.M. and Saier, M.H., Jr. (1991) Sequence and evolution of the FruR protein of Salmonella typhimurium: a pleiotropic transcriptional regulatory protein possessing both activator and repressor functions which is homologous to the periplasmic ribose-binding protein. Res Microbiol, 142, 951-963.

247. Stickland, H.G., Davenport, P.W., Lilley, K.S., Griffin, J.L. and Welch, M. (2010) Mutation of $n f x B$ causes global changes in the physiology and metabolism of Pseudomonas aeruginosa. J Proteome Res, 9, 2957-2967.

248. Colmer, J.A. and Hamood, A.N. (1999) Expression of $p t x R$ and its effect on toxA and regA expression during the growth cycle of Pseudomonas aeruginosa strain PAO1. Can J Microbiol, 45, 1008-1016. 
249. Colmer-Hamood, J.A., Aramaki, H., Gaines, J.M. and Hamood, A.N. (2006) Transcriptional analysis of the Pseudomonas aeruginosa toxA regulatory gene ptxR. Can J Microbiol, 52, 343-356.

250. Swanson, B.L., Hager, P., Phibbs, P., Jr., Ochsner, U., Vasil, M.L. and Hamood, A.N. (2000) Characterization of the 2-ketogluconate utilization operon in Pseudomonas aeruginosa PAO1. Mol Microbiol, 37, 561-573.

251. Buck, D. and Guest, J.R. (1989) Overexpression and site-directed mutagenesis of the succinyl-CoA synthetase of Escherichia coli and nucleotide sequence of a gene (g30) that is adjacent to the suc operon. Biochem J, 260, 737-747.

252. Haydon, D.J. and Guest, J.R. (1991) A new family of bacterial regulatory proteins. FEMS Microbiol Lett, 63, 291-295.

253. Rigali, S., Schlicht, M., Hoskisson, P., Nothaft, H., Merzbacher, M., Joris, B. and Titgemeyer, F. (2004) Extending the classification of bacterial transcription factors beyond the helix-turn-helix motif as an alternative approach to discover new cis/trans relationships. Nucleic Acids Res, 32, 3418-3426.

254. Rigali, S., Derouaux, A., Giannotta, F. and Dusart, J. (2002) Subdivision of the helix-turn-helix GntR family of bacterial regulators in the FadR, HutC, MocR, and YtrA subfamilies. $J$ Biol Chem, 277, 12507-12515.

255. Peekhaus, N. and Conway, T. (1998) Positive and negative transcriptional regulation of the Escherichia coli gluconate regulon gene gntT by GntR and the cyclic AMP (cAMP)-cAMP receptor protein complex. J Bacteriol, 180, 17771785 .

256. Xu, Y., Heath, R.J., Li, Z., Rock, C.O. and White, S.W. (2001) The FadR-DNA complex. Transcriptional control of fatty acid metabolism in Escherichia coli. $J$ Biol Chem, 276, 17373-17379.

257. van Aalten, D.M., DiRusso, C.C. and Knudsen, J. (2001) The structural basis of acyl coenzyme A-dependent regulation of the transcription factor FadR. EMBO J, 20, 2041-2050. 
258. Wang, J., Lory, S., Ramphal, R. and Jin, S. (1996) Isolation and characterization of Pseudomonas aeruginosa genes inducible by respiratory mucus derived from cystic fibrosis patients. Mol Microbiol, 22, 1005-1012.

259. Engebrecht, J., Nealson, K. and Silverman, M. (1983) Bacterial bioluminescence: isolation and genetic analysis of functions from Vibrio fischeri. Cell, 32, 773-781.

260. Engebrecht, J. and Silverman, M. (1984) Identification of genes and gene products necessary for bacterial bioluminescence. Proc Natl Acad Sci U S A, 81, 4154-4158.

261. Engebrecht, J. and Silverman, M. (1987) Nucleotide sequence of the regulatory locus controlling expression of bacterial genes for bioluminescence. Nucleic Acids Res, 15, 10455-10467.

262. Fuqua, W.C., Winans, S.C. and Greenberg, E.P. (1994) Quorum sensing in bacteria: the LuxR-LuxI family of cell density-responsive transcriptional regulators. J Bacteriol, 176, 269-275.

263. Nasser, W. and Reverchon, S. (2007) New insights into the regulatory mechanisms of the LuxR family of quorum sensing regulators. Anal Bioanal Chem, 387, 381-390.

264. Ducros, V.M., Lewis, R.J., Verma, C.S., Dodson, E.J., Leonard, G., Turkenburg, J.P., Murshudov, G.N., Wilkinson, A.J. and Brannigan, J.A. (2001) Crystal structure of GerE, the ultimate transcriptional regulator of spore formation in Bacillus subtilis. J Mol Biol, 306, 759-771.

265. Birck, C., Malfois, M., Svergun, D. and Samama, J. (2002) Insights into signal transduction revealed by the low resolution structure of the FixJ response regulator. J Mol Biol, 321, 447-457.

266. Da Re, S., Bertagnoli, S., Fourment, J., Reyrat, J.M. and Kahn, D. (1994) Intramolecular signal transduction within the FixJ transcriptional activator: in vitro evidence for the inhibitory effect of the phosphorylatable regulatory domain. Nucleic Acids Res, 22, 1555-1561. 
267. David, M., Daveran, M.L., Batut, J., Dedieu, A., Domergue, O., Ghai, J., Hertig, C., Boistard, P. and Kahn, D. (1988) Cascade regulation of nif gene expression in Rhizobium meliloti. Cell, 54, 671-683.

268. Kahn, D. and Ditta, G. (1991) Modular structure of FixJ: homology of the transcriptional activator domain with the -35 binding domain of sigma factors. Mol Microbiol, 5, 987-997.

269. Raibaud, O. and Richet, E. (1987) Maltotriose is the inducer of the maltose regulon of Escherichia coli. J Bacteriol, 169, 3059-3061.

270. Richet, E. and Raibaud, O. (1989) MalT, the regulatory protein of the Escherichia coli maltose system, is an ATP-dependent transcriptional activator. EMBO J, 8 , 981-987.

271. Ducros, V.M., Brannigan, J.A., Lewis, R.J. and Wilkinson, A.J. (1998) Bacillus subtilis regulatory protein GerE. Acta Crystallogr D Biol Crystallogr, 54, 14531455.

272. de Bruijn, I. and Raaijmakers, J.M. (2009) Diversity and functional analysis of LuxR-type transcriptional regulators of cyclic lipopeptide biosynthesis in Pseudomonas fluorescens. Appl Environ Microbiol, 75, 4753-4761.

273. Benkert, B., Quack, N., Schreiber, K., Jaensch, L., Jahn, D. and Schobert, M. (2008) Nitrate-responsive NarX-NarL represses arginine-mediated induction of the Pseudomonas aeruginosa arginine fermentation $\operatorname{arcDABC}$ operon. Microbiology, 154, 3053-3060.

274. Kulasekara, H.D., Ventre, I., Kulasekara, B.R., Lazdunski, A., Filloux, A. and Lory, S. (2005) A novel two-component system controls the expression of Pseudomonas aeruginosa fimbrial cup genes. Mol Microbiol, 55, 368-380.

275. Petrova, O.E. and Sauer, K. (2009) A novel signaling network essential for regulating Pseudomonas aeruginosa biofilm development. PLoS Pathog, 5, e1000668.

276. Petrova, O.E. and Sauer, K. (2010) The novel two-component regulatory system BfiSR regulates biofilm development directly through CafA by its control over the small RNA rsmZ. J Bacteriol. 
277. Mern, D.S., Ha, S.W., Khodaverdi, V., Gliese, N. and Gorisch, H. (2010) A complex regulatory network controls aerobic ethanol oxidation in Pseudomonas aeruginosa: indication of four levels of sensor kinases and response regulators. Microbiology, 156, 1505-1516.

278. Reimmann, C., Beyeler, M., Latifi, A., Winteler, H., Foglino, M., Lazdunski, A. and Haas, D. (1997) The global activator GacA of Pseudomonas aeruginosa PAO positively controls the production of the autoinducer N-butyryl-homoserine lactone and the formation of the virulence factors pyocyanin, cyanide, and lipase. Mol Microbiol, 24, 309-319.

279. Brint, J. and Ohman, D. (1995) Synthesis of multiple exoproducts in Pseudomonas aeruginosa is under the control of RhlR-RhlI, another set of regulators in strain PAO1 with homology to the autoinducer-responsive LuxRLuxI family. J Bacteriol, 177, 7155-7163.

280. Chugani, S.A., Whiteley, M., Lee, K.M., D'Argenio, D., Manoil, C. and Greenberg, E.P. (2001) QscR, a modulator of quorum-sensing signal synthesis and virulence in Pseudomonas aeruginosa. Proc Natl Acad Sci U S A, 98, 27522757.

281. Gambello, M.J. and Iglewski, B.H. (1991) Cloning and characterization of the Pseudomonas aeruginosa lasR gene, a transcriptional activator of elastase expression. J Bacteriol, 173, 3000-3009.

282. Juhas, M., Wiehlmann, L., Huber, B., Jordan, D., Lauber, J., Salunkhe, P., Limpert, A.S., von Gotz, F., Steinmetz, I., Eberl, L. et al. (2004) Global regulation of quorum sensing and virulence by VqsR in Pseudomonas aeruginosa. Microbiology, 150, 831-841.

283. Morett, E. and Segovia, L. (1993) The sigma 54 bacterial enhancer-binding protein family: mechanism of action and phylogenetic relationship of their functional domains. J Bacteriol, 175, 6067-6074.

284. Austin, S. and Dixon, R. (1992) The prokaryotic enhancer binding protein NTRC has an ATPase activity which is phosphorylation and DNA dependent. EMBO J, 11, 2219-2228. 
285. Sigrist, C.J., Cerutti, L., de Castro, E., Langendijk-Genevaux, P.S., Bulliard, V., Bairoch, A. and Hulo, N. (2010) PROSITE, a protein domain database for functional characterization and annotation. Nucleic Acids Res, 38, D161-166.

286. Magasanik, B. (1993) The regulation of nitrogen utilization in enteric bacteria. $J$ Cell Biochem, 51, 34-40.

287. Nishijyo, T., Haas, D. and Itoh, Y. (2001) The CbrA-CbrB two-component regulatory system controls the utilization of multiple carbon and nitrogen sources in Pseudomonas aeruginosa. Mol Microbiol, 40, 917-931.

288. Kuchma, S.L., Connolly, J.P. and O'Toole, G.A. (2005) A three-component regulatory system regulates biofilm maturation and type III secretion in Pseudomonas aeruginosa. J Bacteriol, 187, 1441-1454.

289. Hoiby, N., Bjarnsholt, T., Givskov, M., Molin, S. and Ciofu, O. (2010) Antibiotic resistance of bacterial biofilms. Int J Antimicrob Agents, 35, 322-332.

290. Brown, N.L., Stoyanov, J.V., Kidd, S.P. and Hobman, J.L. (2003) The MerR family of transcriptional regulators. FEMS Microbiol Rev, 27, 145-163.

291. Heldwein, E.E. and Brennan, R.G. (2001) Crystal structure of the transcription activator BmrR bound to DNA and a drug. Nature, 409, 378-382.

292. Helmann, J.D., Wang, Y., Mahler, I. and Walsh, C.T. (1989) Homologous metalloregulatory proteins from both Gram-positive and gram-negative bacteria control transcription of mercury resistance operons. J Bacteriol, 171, 222-229.

293. Kobayashi, K. and Tagawa, S. (2004) Activation of SoxR-dependent transcription in Pseudomonas aeruginosa. J Biochem, 136, 607-615.

294. Amabile-Cuevas, C.F. and Demple, B. (1991) Molecular characterization of the soxRS genes of Escherichia coli: two genes control a superoxide stress regulon. Nucleic Acids Res, 19, 4479-4484.

295. Wu, J. and Weiss, B. (1991) Two divergently transcribed genes, soxR and soxS, control a superoxide response regulon of Escherichia coli. J Bacteriol, 173, 28642871. 
296. Thaden, J.T., Lory, S. and Gardner, T.S. (2010) Quorum-sensing regulation of a copper toxicity system in Pseudomonas aeruginosa.J Bacteriol, 192, 2557-2568.

297. Lee, S.W., Glickmann, E. and Cooksey, D.A. (2001) Chromosomal locus for cadmium resistance in Pseudomonas putida consisting of a cadmium-transporting ATPase and a MerR family response regulator. Appl Environ Microbiol, 67, 1437-1444.

298. Aramaki, H., Yagi, N. and Suzuki, M. (1995) Residues important for the function of a multihelical DNA binding domain in the new transcription factor family of Cam and Tet repressors. Protein Eng, 8, 1259-1266.

299. Ramos, J.L., Martinez-Bueno, M., Molina-Henares, A.J., Teran, W., Watanabe, K., Zhang, X., Gallegos, M.T., Brennan, R. and Tobes, R. (2005) The TetR family of transcriptional repressors. Microbiol Mol Biol Rev, 69, 326-356.

300. Orth, P., Schnappinger, D., Hillen, W., Saenger, W. and Hinrichs, W. (2000) Structural basis of gene regulation by the tetracycline inducible Tet repressoroperator system. Nat Struct Biol, 7, 215-219.

301. Hinrichs, W., Kisker, C., Duvel, M., Muller, A., Tovar, K., Hillen, W. and Saenger, W. (1994) Structure of the Tet repressor-tetracycline complex and regulation of antibiotic resistance. Science, 264, 418-420.

302. Engohang-Ndong, J., Baillat, D., Aumercier, M., Bellefontaine, F., Besra, G.S., Locht, C. and Baulard, A.R. (2004) EthR, a repressor of the TetR/CamR family implicated in ethionamide resistance in mycobacteria, octamerizes cooperatively on its operator. Mol Microbiol, 51, 175-188.

303. Grkovic, S., Brown, M.H. and Skurray, R.A. (2002) Regulation of bacterial drug export systems. Microbiol Mol Biol Rev, 66, 671-701, table of contents.

304. Masuda, N., Sakagawa, E., Ohya, S., Gotoh, N., Tsujimoto, H. and Nishino, T. (2000) Substrate specificities of MexAB-OprM, MexCD-OprJ, and MexXYoprM efflux pumps in Pseudomonas aeruginosa. Antimicrob Agents Chemother, 44, 3322-3327.

305. Cao, L., Srikumar, R. and Poole, K. (2004) MexAB-OprM hyperexpression in NalC-type multidrug-resistant Pseudomonas aeruginosa: identification and 
characterization of the $\mathrm{nalC}$ gene encoding a repressor of PA3720-PA3719. Mol Microbiol, 53, 1423-1436.

306. Morita, Y., Cao, L., Gould, V.C., Avison, M.B. and Poole, K. (2006) nalD encodes a second repressor of the mexAB-oprM multidrug efflux operon of Pseudomonas aeruginosa. J Bacteriol, 188, 8649-8654.

307. Shen, D.K., Filopon, D., Kuhn, L., Polack, B. and Toussaint, B. (2006) PsrA is a positive transcriptional regulator of the type III secretion system in Pseudomonas aeruginosa. Infect Immun, 74, 1121-1129.

308. Hogardt, M., Roeder, M., Schreff, A.M., Eberl, L. and Heesemann, J. (2004) Expression of Pseudomonas aeruginosa exoS is controlled by quorum sensing and RpoS. Microbiology, 150, 843-851.

309. MacEachran, D.P., Stanton, B.A. and O'Toole, G.A. (2008) Cif is negatively regulated by the TetR family repressor CifR. Infect Immun, 76, 3197-3206.

310. MacEachran, D.P., Ye, S., Bomberger, J.M., Hogan, D.A., Swiatecka-Urban, A., Stanton, B.A. and O'Toole, G.A. (2007) The Pseudomonas aeruginosa secreted protein PA2934 decreases apical membrane expression of the cystic fibrosis transmembrane conductance regulator. Infect Immun, 75, 3902-3912.

311. Lamark, T., Kaasen, I., Eshoo, M.W., Falkenberg, P., McDougall, J. and Strom, A.R. (1991) DNA sequence and analysis of the bet genes encoding the osmoregulatory choline-glycine betaine pathway of Escherichia coli. Mol Microbiol, 5, 1049-1064.

312. Nakada, Y., Jiang, Y., Nishijyo, T., Itoh, Y. and Lu, C.D. (2001) Molecular characterization and regulation of the aguBA operon, responsible for agmatine utilization in Pseudomonas aeruginosa PAO1. J Bacteriol, 183, 6517-6524.

313. Zhu, K., Choi, K.H., Schweizer, H.P., Rock, C.O. and Zhang, Y.M. (2006) Two aerobic pathways for the formation of unsaturated fatty acids in Pseudomonas aeruginosa. Mol Microbiol, 60, 260-273.

314. Forster-Fromme, K., Hoschle, B., Mack, C., Bott, M., Armbruster, W. and Jendrossek, D. (2006) Identification of genes and proteins necessary for 
catabolism of acyclic terpenes and leucine/isovalerate in Pseudomonas aeruginosa. Appl Environ Microbiol, 72, 4819-4828.

315. Greenblatt, J. and Schleif, R. (1971) Arabinose C protein: regulation of the arabinose operon in vitro. Nat New Biol, 233, 166-170.

316. Schleif, R. (1969) An L-arabinose binding protein and arabinose permeation in Escherichia coli. J Mol Biol, 46, 185-196.

317. Gallegos, M.T., Schleif, R., Bairoch, A., Hofmann, K. and Ramos, J.L. (1997) Arac/XylS family of transcriptional regulators. Microbiol Mol Biol Rev, 61, 393410.

318. Martin, R.G. and Rosner, J.L. (2001) The AraC transcriptional activators. Curr Opin Microbiol, 4, 132-137.

319. Martin, R.G., Gillette, W.K., Rhee, S. and Rosner, J.L. (1999) Structural requirements for marbox function in transcriptional activation of mar/sox/rob regulon promoters in Escherichia coli: sequence, orientation and spatial relationship to the core promoter. Mol Microbiol, 34, 431-441.

320. Johnson, C.M. and Schleif, R.F. (2000) Cooperative action of the catabolite activator protein and AraC in vitro at the araFGH promoter. $J$ Bacteriol, 182, 1995-2000.

321. Heinrichs, D.E. and Poole, K. (1996) PchR, a regulator of ferripyochelin receptor gene (fptA) expression in Pseudomonas aeruginosa, functions both as an activator and as a repressor. $J$ Bacteriol, 178, 2586-2592.

322. Michel, L., Gonzalez, N., Jagdeep, S., Nguyen-Ngoc, T. and Reimmann, C. (2005) PchR-box recognition by the AraC-type regulator PchR of Pseudomonas aeruginosa requires the siderophore pyochelin as an effector. Mol Microbiol, 58, 495-509.

323. Frank, D.W. and Iglewski, B.H. (1991) Cloning and sequence analysis of a transregulatory locus required for exoenzyme S synthesis in Pseudomonas aeruginosa. $J$ Bacteriol, 173, 6460-6468. 
324. Yahr, T.L. and Frank, D.W. (1994) Transcriptional organization of the transregulatory locus which controls exoenzyme S synthesis in Pseudomonas aeruginosa. J Bacteriol, 176, 3832-3838.

325. Yahr, T.L. and Wolfgang, M.C. (2006) Transcriptional regulation of the Pseudomonas aeruginosa type III secretion system. Mol Microbiol, 62, 631-640.

326. Steele, M.I., Lorenz, D., Hatter, K., Park, A. and Sokatch, J.R. (1992) Characterization of the mmsAB operon of Pseudomonas aeruginosa PAO encoding methylmalonate-semialdehyde dehydrogenase and 3-hydroxyisobutyrate dehydrogenase. J Biol Chem, 267, 13585-13592.

327. Guarente, L., Roberts, T.M. and Ptashne, M. (1980) A technique for expressing eukaryotic genes in bacteria. Science, 209, 1428-1430.

328. Aggarwal, A.K., Rodgers, D.W., Drottar, M., Ptashne, M. and Harrison, S.C. (1988) Recognition of a DNA operator by the repressor of phage 434: a view at high resolution. Science, 242, 899-907.

329. Anderson, W.F. and Cygler, M. (1985) Computer modeling studies of the structure of a repressor. Biosystems, 18, 3-14.

330. Matsui, H., Sano, Y., Ishihara, H. and Shinomiya, T. (1993) Regulation of pyocin genes in Pseudomonas aeruginosa by positive (prtN) and negative (prtR) regulatory genes. $J$ Bacteriol, 175, 1257-1263.

331. Wu, W. and Jin, S. (2005) PtrB of Pseudomonas aeruginosa suppresses the type III secretion system under the stress of DNA damage. J Bacteriol, 187, 6058-6068.

332. Winsor, G.L., Van Rossum, T., Lo, R., Khaira, B., Whiteside, M.D., Hancock, R.E. and Brinkman, F.S. (2009) Pseudomonas Genome Database: facilitating user-friendly, comprehensive comparisons of microbial genomes. Nucleic Acids Res, 37, D483-488.

333. Black, D.S., Kelly, A.J., Mardis, M.J. and Moyed, H.S. (1991) Structure and organization of hip, an operon that affects lethality due to inhibition of peptidoglycan or DNA synthesis. J Bacteriol, 173, 5732-5739. 
334. Black, D.S., Irwin, B. and Moyed, H.S. (1994) Autoregulation of hip, an operon that affects lethality due to inhibition of peptidoglycan or DNA synthesis. $J$ Bacteriol, 176, 4081-4091.

335. Korch, S.B. and Hill, T.M. (2006) Ectopic overexpression of wild-type and mutant hipA genes in Escherichia coli: effects on macromolecular synthesis and persister formation. $J$ Bacteriol, 188, 3826-3836.

336. Yokoyama, K., Ishijima, S.A., Clowney, L., Koike, H., Aramaki, H., Tanaka, C., Makino, K. and Suzuki, M. (2006) Feast/famine regulatory proteins (FFRPs): Escherichia coli Lrp, AsnC and related archaeal transcription factors. FEMS Microbiol Rev, 30, 89-108.

337. Leonard, P.M., Smits, S.H., Sedelnikova, S.E., Brinkman, A.B., de Vos, W.M., van der Oost, J., Rice, D.W. and Rafferty, J.B. (2001) Crystal structure of the Lrplike transcriptional regulator from the archaeon Pyrococcus furiosus. EMBO J, 20, 990-997.

338. Brinkman, A.B., Ettema, T.J., de Vos, W.M. and van der Oost, J. (2003) The Lrp family of transcriptional regulators. Mol Microbiol, 48, 287-294.

339. Kawasaki, S., Arai, H., Kodama, T. and Igarashi, Y. (1997) Gene cluster for dissimilatory nitrite reductase (nir) from Pseudomonas aeruginosa: sequencing and identification of a locus for heme d1 biosynthesis. $J$ Bacteriol, 179, 235-242.

340. Madhusudhan, K.T., Lorenz, D. and Sokatch, J.R. (1993) The $b k d R$ gene of Pseudomonas putida is required for expression of the bkd operon and encodes a protein related to Lrp of Escherichia coli. J Bacteriol, 175, 3934-3940.

341. Chou, H.T., Kwon, D.H., Hegazy, M. and Lu, C.D. (2008) Transcriptome analysis of agmatine and putrescine catabolism in Pseudomonas aeruginosa PAO1. $J$ Bacteriol, 190, 1966-1975.

342. Busenlehner, L.S., Pennella, M.A. and Giedroc, D.P. (2003) The SmtB/ArsR family of metalloregulatory transcriptional repressors: Structural insights into prokaryotic metal resistance. FEMS Microbiol Rev, 27, 131-143.

343. Osman, D. and Cavet, J.S. (2010) Bacterial metal-sensing proteins exemplified by ArsR-SmtB family repressors. Nat Prod Rep, 27, 668-680. 
344. Eicken, C., Pennella, M.A., Chen, X., Koshlap, K.M., VanZile, M.L., Sacchettini, J.C. and Giedroc, D.P. (2003) A metal-ligand-mediated intersubunit allosteric switch in related SmtB/ArsR zinc sensor proteins. J Mol Biol, 333, 683-695.

345. Cai, J., Salmon, K. and DuBow, M.S. (1998) A chromosomal ars operon homologue of Pseudomonas aeruginosa confers increased resistance to arsenic and antimony in Escherichia coli. Microbiology, 144 ( Pt 10), 2705-2713.

346. Drury, L.S. and Buxton, R.S. (1988) Identification and sequencing of the Escherichia coli cet gene which codes for an inner membrane protein, mutation of which causes tolerance to colicin E2. Mol Microbiol, 2, 109-119.

347. Avison, M.B., Niumsup, P., Nurmahomed, K., Walsh, T.R. and Bennett, P.M. (2004) Role of the 'cre/blr-tag' DNA sequence in regulation of gene expression by the Aeromonas hydrophila beta-lactamase regulator, BlrA. J Antimic Chemoth, 53, 197-202.

348. Moya, B., Dotsch, A., Juan, C., Blazquez, J., Zamorano, L., Haussler, S. and Oliver, A. (2009) Beta-lactam resistance response triggered by inactivation of a nonessential penicillin-binding protein. PLoS Pathog, 5, e1000353.

349. Calero, S., Garriga, X. and Barbe, J. (1993) Analysis of the DNA damagemediated induction of Pseudomonas putida and Pseudomonas aeruginosa lexA genes. FEMS Microbiol Lett, 110, 65-70.

350. van der Lelie, D., Schwuchow, T., Schwidetzky, U., Wuertz, S., Baeyens, W., Mergeay, M. and Nies, D.H. (1997) Two-component regulatory system involved in transcriptional control of heavy-metal homoeostasis in Alcaligenes eutrophus. Mol Microbiol, 23, 493-503.

351. Jensen, L.J., Kuhn, M., Stark, M., Chaffron, S., Creevey, C., Muller, J., Doerks, T., Julien, P., Roth, A., Simonovic, M. et al. (2009) STRING 8--a global view on proteins and their functional interactions in 630 organisms. Nucleic Acids Res, 37, D412-416.

352. Korner, H., Sofia, H.J. and Zumft, W.G. (2003) Phylogeny of the bacterial superfamily of Crp-Fnr transcription regulators: exploiting the metabolic spectrum by controlling alternative gene programs. FEMS Microbiol Rev, 27, 559-592. 
353. Shannon, P., Markiel, A., Ozier, O., Baliga, N.S., Wang, J.T., Ramage, D., Amin, N., Schwikowski, B. and Ideker, T. (2003) Cytoscape: a software environment for integrated models of biomolecular interaction networks. Genome Res, 13, 24982504.

354. Hu, Z., Hung, J.H., Wang, Y., Chang, Y.C., Huang, C.L., Huyck, M. and DeLisi, C. (2009) VisANT 3.5: multi-scale network visualization, analysis and inference based on the gene ontology. Nucleic Acids Res, 37, W115-121.

355. Davinic, M., Carty, N.L., Colmer-Hamood, J.A., San Francisco, M. and Hamood, A.N. (2009) Role of Vfr in regulating exotoxin A production by Pseudomonas aeruginosa. Microbiology, 155, 2265-2273.

356. Fuchs, E.L., Brutinel, E.D., Jones, A.K., Fulcher, N.B., Urbanowski, M.L., Yahr, T.L. and Wolfgang, M.C. (2010) The Pseudomonas aeruginosa Vfr regulator controls global virulence factor expression through cyclic AMP-dependent and independent mechanisms. $J$ Bacteriol, 192, 3553-3564.

357. Jones, A.K., Fulcher, N.B., Balzer, G.J., Urbanowski, M.L., Pritchett, C.L., Schurr, M.J., Yahr, T.L. and Wolfgang, M.C. (2010) Activation of the Pseudomonas aeruginosa $\mathrm{AlgU}$ regulon through mucA mutation inhibits cyclic AMP/Vfr signaling. J Bacteriol, 192, 5709-5717.

358. Ariza, R.R., Cohen, S.P., Bachhawat, N., Levy, S.B. and Demple, B. (1994) Repressor mutations in the $\operatorname{marR} A B$ operon that activate oxidative stress genes and multiple antibiotic resistance in Escherichia coli. J Bacteriol, 176, 143-148.

359. Pearson, J.P., Gray, K.M., Passador, L., Tucker, K.D., Eberhard, A., Iglewski, B.H. and Greenberg, E.P. (1994) Structure of the autoinducer required for expression of Pseudomonas aeruginosa virulence genes. Proc Natl Acad Sci U S $A, 91,197-201$.

360. Pearson, J.P., Passador, L., Iglewski, B.H. and Greenberg, E.P. (1995) A second $\mathrm{N}$-acylhomoserine lactone signal produced by Pseudomonas aeruginosa. Proc Natl Acad Sci U S A, 92, 1490-1494.

361. Pesci, E.C., Milbank, J.B., Pearson, J.P., McKnight, S., Kende, A.S., Greenberg, E.P. and Iglewski, B.H. (1999) Quinolone signaling in the cell-to-cell 
communication system of Pseudomonas aeruginosa. Proc Natl Acad Sci U S A, 96, 11229-11234.

362. Ochsner, U.A. and Reiser, J. (1995) Autoinducer-mediated regulation of rhamnolipid biosurfactant synthesis in Pseudomonas aeruginosa. Proc Natl Acad Sci U S A, 92, 6424-6428.

363. Latifi, A., Foglino, M., Tanaka, K., Williams, P. and Lazdunski, A. (1996) A hierarchical quorum-sensing cascade in Pseudomonas aeruginosa links the transcriptional activators LasR and RhIR (VsmR) to expression of the stationaryphase sigma factor RpoS. Mol Microbiol, 21, 1137-1146.

364. Pesci, E.C., Pearson, J.P., Seed, P.C. and Iglewski, B.H. (1997) Regulation of las and $r h l$ quorum sensing in Pseudomonas aeruginosa. J Bacteriol, 179, 3127-3132.

365. Gilbert, K.B., Kim, T.H., Gupta, R., Greenberg, E.P. and Schuster, M. (2009) Global position analysis of the Pseudomonas aeruginosa quorum-sensing transcription factor LasR. Mol Microbiol, 73, 1072-1085.

366. Schuster, M. and Greenberg, E.P. (2007) Early activation of quorum sensing in Pseudomonas aeruginosa reveals the architecture of a complex regulon. BMC Genomics, 8, 287.

367. Wagner, V.E., Gillis, R.J. and Iglewski, B.H. (2004) Transcriptome analysis of quorum-sensing regulation and virulence factor expression in Pseudomonas aeruginosa. Vaccine, 22 Suppl 1, S15-20.

368. Schuster, M., Hawkins, A.C., Harwood, C.S. and Greenberg, E.P. (2004) The Pseudomonas aeruginosa RpoS regulon and its relationship to quorum sensing. Mol Microbiol, 51, 973-985.

369. Albus, A.M., Pesci, E.C., Runyen-Janecky, L.J., West, S.E. and Iglewski, B.H. (1997) Vfr controls quorum sensing in Pseudomonas aeruginosa. J Bacteriol, 179, 3928-3935.

370. Medina, G., Juarez, K., Diaz, R. and Soberon-Chavez, G. (2003) Transcriptional regulation of Pseudomonas aeruginosa rhlR, encoding a quorum-sensing regulatory protein. Microbiology, 149, 3073-3081. 
371. Whitchurch, C.B., Beatson, S.A., Comolli, J.C., Jakobsen, T., Sargent, J.L., Bertrand, J.J., West, J., Klausen, M., Waite, L.L., Kang, P.J. et al. (2005) Pseudomonas aeruginosa fimL regulates multiple virulence functions by intersecting with Vfr-modulated pathways. Mol Microbiol, 55, 1357-1378.

372. Rampioni, G., Bertani, I., Zennaro, E., Polticelli, F., Venturi, V. and Leoni, L. (2006) The quorum-sensing negative regulator RsaL of Pseudomonas aeruginosa binds to the lasI promoter. J Bacteriol, 188, 815-819.

373. Rampioni, G., Schuster, M., Greenberg, E.P., Bertani, I., Grasso, M., Venturi, V., Zennaro, E. and Leoni, L. (2007) RsaL provides quorum sensing homeostasis and functions as a global regulator of gene expression in Pseudomonas aeruginosa. Mol Microbiol, 66, 1557-1565.

374. Pessi, G. and Haas, D. (2000) Transcriptional control of the hydrogen cyanide biosynthetic genes $h c n A B C$ by the anaerobic regulator Anr and the quorumsensing regulators LasR and RhIR in Pseudomonas aeruginosa. J Bacteriol, 182, 6940-6949.

375. Dekimpe, V. and Deziel, E. (2009) Revisiting the quorum-sensing hierarchy in Pseudomonas aeruginosa: the transcriptional regulator RhlR regulates LasRspecific factors. Microbiology, 155, 712-723.

376. Duan, K. and Surette, M.G. (2007) Environmental regulation of Pseudomonas aeruginosa PAO1 Las and Rhl quorum-sensing systems. J Bacteriol, 189, 48274836.

377. Deziel, E., Lepine, F., Milot, S., He, J., Mindrinos, M.N., Tompkins, R.G. and Rahme, L.G. (2004) Analysis of Pseudomonas aeruginosa 4-hydroxy-2alkylquinolines (HAQs) reveals a role for 4-hydroxy-2-heptylquinoline in cell-tocell communication. Proc Natl Acad Sci U S A, 101, 1339-1344.

378. Diggle, S.P., Matthijs, S., Wright, V.J., Fletcher, M.P., Chhabra, S.R., Lamont, I.L., Kong, X., Hider, R.C., Cornelis, P., Camara, M. et al. (2007) The Pseudomonas aeruginosa 4-quinolone signal molecules HHQ and PQS play multifunctional roles in quorum sensing and iron entrapment. Chem Biol, 14, 8796. 
379. Diggle, S.P., Winzer, K., Chhabra, S.R., Worrall, K.E., Camara, M. and Williams, P. (2003) The Pseudomonas aeruginosa quinolone signal molecule overcomes the cell density-dependency of the quorum sensing hierarchy, regulates rhl-dependent genes at the onset of stationary phase and can be produced in the absence of LasR. Mol Microbiol, 50, 29-43.

380. Gallagher, L.A., McKnight, S.L., Kuznetsova, M.S., Pesci, E.C. and Manoil, C. (2002) Functions required for extracellular quinolone signaling by Pseudomonas aeruginosa. J Bacteriol, 184, 6472-6480.

381. Xiao, G., Deziel, E., He, J., Lepine, F., Lesic, B., Castonguay, M.H., Milot, S., Tampakaki, A.P., Stachel, S.E. and Rahme, L.G. (2006) MvfR, a key Pseudomonas aeruginosa pathogenicity LTTR-class regulatory protein, has dual ligands. Mol Microbiol, 62, 1689-1699.

382. Wade, D.S., Calfee, M.W., Rocha, E.R., Ling, E.A., Engstrom, E., Coleman, J.P. and Pesci, E.C. (2005) Regulation of Pseudomonas quinolone signal synthesis in Pseudomonas aeruginosa. J Bacteriol, 187, 4372-4380.

383. Liang, H., Li, L., Dong, Z., Surette, M.G. and Duan, K. (2008) The YebC family protein PA0964 negatively regulates the Pseudomonas aeruginosa quinolone signal system and pyocyanin production. $J$ Bacteriol, 190, 6217-6227.

384. Bredenbruch, F., Geffers, R., Nimtz, M., Buer, J. and Haussler, S. (2006) The Pseudomonas aeruginosa quinolone signal (PQS) has an iron-chelating activity. Environ Microbiol, 8, 1318-1329.

385. Lequette, Y., Lee, J.H., Ledgham, F., Lazdunski, A. and Greenberg, E.P. (2006) A distinct QscR regulon in the Pseudomonas aeruginosa quorum-sensing circuit. J Bacteriol, 188, 3365-3370.

386. Ledgham, F., Ventre, I., Soscia, C., Foglino, M., Sturgis, J.N. and Lazdunski, A. (2003) Interactions of the quorum sensing regulator QscR: interaction with itself and the other regulators of Pseudomonas aeruginosa LasR and RhlR. Mol Microbiol, 48, 3365-3370.

387. Dong, Y.H., Zhang, X.F., Soo, H.M., Greenberg, E.P. and Zhang, L.H. (2005) The two-component response regulator PprB modulates quorum-sensing signal 
production and global gene expression in Pseudomonas aeruginosa. Mol Microbiol, 56, 1287-1301.

388. Liang, H., Li, L., Kong, W., Shen, L. and Duan, K. (2009) Identification of a novel regulator of the quorum-sensing systems in Pseudomonas aeruginosa. FEMS Microbiol Lett, 293, 196-204.

389. Kay, E., Humair, B., Denervaud, V., Riedel, K., Spahr, S., Eberl, L., Valverde, C. and Haas, D. (2006) Two GacA-dependent small RNAs modulate the quorumsensing response in Pseudomonas aeruginosa. J Bacteriol, 188, 6026-6033.

390. Brencic, A., McFarland, K.A., McManus, H.R., Castang, S., Mogno, I., Dove, S.L. and Lory, S. (2009) The GacS/GacA signal transduction system of Pseudomonas aeruginosa acts exclusively through its control over the transcription of the RsmY and RsmZ regulatory small RNAs. Mol Microbiol, 73, 434-445.

391. Heeb, S. and Haas, D. (2001) Regulatory roles of the GacS/GacA two-component system in plant-associated and other Gram-negative bacteria. Mol Plant Microbe Interact, 14, 1351-1363.

392. Siehnel, R., Traxler, B., An, D.D., Parsek, M.R., Schaefer, A.L. and Singh, P.K. (2010) A unique regulator controls the activation threshold of quorum-regulated genes in Pseudomonas aeruginosa. Proc Natl Acad Sci U S A, 107, 7916-7921.

393. Greenway, D.L. and England, R.R. (1999) ppGpp accumulation in Pseudomonas aeruginosa and Pseudomonas fluorescens subjected to nutrient limitation and biocide exposure. Lett Appl Microbiol, 29, 298-302.

394. Baysse, C., Cullinane, M., Denervaud, V., Burrowes, E., Dow, J.M., Morrissey, J.P., Tam, L., Trevors, J.T. and O'Gara, F. (2005) Modulation of quorum sensing in Pseudomonas aeruginosa through alteration of membrane properties. Microbiology, 151, 2529-2542.

395. van Delden, C., Comte, R. and Bally, A.M. (2001) Stringent response activates quorum sensing and modulates cell density-dependent gene expression in Pseudomonas aeruginosa. J Bacteriol, 183, 5376-5384. 
396. Chugani, S. and Greenberg, E.P. (2010) LuxR homolog-independent gene regulation by acyl-homoserine lactones in Pseudomonas aeruginosa. Proc Natl Acad Sci U S A, 107, 10673-10678.

397. Coleman, J.P., Hudson, L.L., McKnight, S.L., Farrow, J.M., 3rd, Calfee, M.W., Lindsey, C.A. and Pesci, E.C. (2008) Pseudomonas aeruginosa PqsA is an anthranilate-coenzyme A ligase. J Bacteriol, 190, 1247-1255.

398. Martinez-Nunez, M.A., Perez-Rueda, E., Gutierrez-Rios, R.M. and Merino, E. (2010) New insights into the regulatory networks of paralogous genes in bacteria. Microbiology, 156, 14-22.

399. Alon, U. (2007) Network motifs: theory and experimental approaches. Nat Rev Genet, 8, 450-461.

400. Balasubramanian, D. and Mathee, K. (2009) Comparative transcriptome analyses of Pseudomonas aeruginosa. Hum Genomics, 3, 349-361.

401. Obritsch, M.D., Fish, D.N., MacLaren, R. and Jung, R. (2004) National surveillance of antimicrobial resistance in Pseudomonas aeruginosa isolates obtained from intensive care unit patients from 1993 to 2002. Antimicrob Agents Chemother, 48, 4606-4610.

402. Bouza, E., Burillo, A. and Munoz, P. (2002) Catheter-related infections: diagnosis and intravascular treatment. Clin Microbiol Infect, 8, 265-274.

403. Gallagher, P.G. and Watanakunakorn, C. (1989) Pseudomonas bacteremia in a community teaching hospital, 1980-1984. Rev Infect Dis, 11, 846-852.

404. Collin, B.A., Leather, H.L., Wingard, J.R. and Ramphal, R. (2001) Evolution, incidence, and susceptibility of bacterial bloodstream isolates from 519 bone marrow transplant patients. Clin Infect Dis, 33, 947-953.

405. Davies, J.C. (2002) Pseudomonas aeruginosa in cystic fibrosis: pathogenesis and persistence. Paediatr Respir Rev, 3, 128-134. 
406. Kong, K.F., Aguila, A., Schneper, L. and Mathee, K. (2010) Pseudomonas aeruginosa beta-lactamase induction requires two permeases, AmpG and AmpP. BMC Microbiol, 10, 328.

407. Proenca, R., Niu, W.W., Cacalano, G. and Prince, A. (1993) The Pseudomonas cepacia 249 chromosomal penicillinase is a member of the AmpC family of chromosomal beta-lactamases. Antimicrob Agents Chemother, 37, 667-674.

408. Seoane, A., Francia, M.V. and Garcia Lobo, J.M. (1992) Nucleotide sequence of the ampC-ampR region from the chromosome of Yersinia enterocolitica. Antimicrob Agents Chemother, 36, 1049-1052.

409. Okazaki, A. and Avison, M.B. (2008) Induction of L1 and L2 beta-lactamase production in Stenotrophomonas maltophilia is dependent on an AmpR-type regulator. Antimicrob Agents Chemother, 52, 1525-1528.

410. Jacobs, C., Frere, J.M. and Normark, S. (1997) Cytosolic intermediates for cell wall biosynthesis and degradation control inducible beta-lactam resistance in Gram-negative bacteria. Cell, 88, 823-832.

411. Lindberg, F., Lindquist, S. and Normark, S. (1986) Induction of chromosomal beta-lactamase expression in Enterobacteria. J Antimicrob Chemother, 18 Suppl C, 43-50.

412. Adonizio, A., Leal, S.M., Jr., Ausubel, F.M. and Mathee, K. (2008) Attenuation of Pseudomonas aeruginosa virulence by medicinal plants in a Caenorhabditis elegans model system. J Med Microbiol, 57, 809-813.

413. Tan, M.W., Rahme, L.G., Sternberg, J.A., Tompkins, R.G. and Ausubel, F.M. (1999) Pseudomonas aeruginosa killing of Caenorhabditis elegans used to identify P. aeruginosa virulence factors. Proc Natl Acad Sci U S A, 96, 24082413.

414. Kong, K.F., Schneper, L. and Mathee, K. (2010) Beta-lactam antibiotics: from antibiosis to resistance and bacteriology. APMIS, 118, 1-36.

415. Lodge, J., Busby, S. and Piddock, L. (1993) Investigation of the Pseudomonas aeruginosa ampR gene and its role at the chromosomal ampC beta-lactamase promoter. FEMS Microbiol Lett, 111, 315-320. 
416. Olsson, O., Bergstrom, S., Lindberg, F.P. and Normark, S. (1983) ampC betalactamase hyperproduction in Escherichia coli: natural ampicillin resistance generated by horizontal chromosomal DNA transfer from Shigella. Proc Natl Acad Sci US A, 80, 7556-7560.

417. Zhang, Y., Bao, Q., Gagnon, L.A., Huletsky, A., Oliver, A., Jin, S. and Langaee, T. (2010) ampG gene of Pseudomonas aeruginosa and its role in beta-lactamase expression. Antimicrob Agents Chemother, 54, 4772-4779.

418. Schmidtke, A.J. and Hanson, N.D. (2008) Role of ampD homologs in overproduction of AmpC in clinical isolates of Pseudomonas aeruginosa. Antimicrob Agents Chemother, 52, 3922-3927.

419. Zamorano, L., Reeve, T.M., Deng, L., Juan, C., Moya, B., Cabot, G., Vocadlo, D.J., Mark, B.L. and Oliver, A. (2010) NagZ inactivation prevents and reverts beta-lactam resistance, driven by AmpD and PBP 4 mutations, in Pseudomonas aeruginosa. Antimicrob Agents Chemother, 54, 3557-3563.

420. Asgarali, A., Stubbs, K.A., Oliver, A., Vocadlo, D.J. and Mark, B.L. (2009) Inactivation of the glycoside hydrolase NagZ attenuates antipseudomonal betalactam resistance in Pseudomonas aeruginosa. Antimicrob Agents Chemother, 53, 2274-2282.

421. Mao, F., Dam, P., Chou, J., Olman, V. and Xu, Y. (2009) DOOR: a database for prokaryotic operons. Nucleic Acids Res, 37, D459-463.

422. Dam, P., Olman, V., Harris, K., Su, Z. and Xu, Y. (2007) Operon prediction using both genome-specific and general genomic information. Nucleic Acids Res, 35, 288-298.

423. Turatsinze, J.V., Thomas-Chollier, M., Defrance, M. and van Helden, J. (2008) Using RSAT to scan genome sequences for transcription factor binding sites and cis-regulatory modules. Nature Protoc., 3, 1578-1588.

424. Crooks, G.E., Hon, G., Chandonia, J.M. and Brenner, S.E. (2004) WebLogo: a sequence logo generator. Genome Res., 14, 1188-1190.

425. Balasubramanian, D., Kong, K.F., Jayawardena, S.R., Leal, S.M., Sautter, R.T. and Mathee, K. (2011) Co-regulation of $\{$ beta $\}$-lactam resistance, alginate 
production and quorum sensing in Pseudomonas aeruginosa. J Med Microbiol, 60, 147-156.

426. Nikaido, H. and Takatsuka, Y. (2009) Mechanisms of RND multidrug efflux pumps. Biochim Biophys Acta, 1794, 769-781.

427. Kohler, T., Michea-Hamzehpour, M., Henze, U., Gotoh, N., Curty, L.K. and Pechere, J.C. (1997) Characterization of MexE-MexF-OprN, a positively regulated multidrug efflux system of Pseudomonas aeruginosa. Mol Microbiol, 23, 345-354.

428. Kohler, T., Epp, S.F., Curty, L.K. and Pechere, J.C. (1999) Characterization of MexT, the regulator of the MexE-MexF-OprN multidrug efflux system of Pseudomonas aeruginosa. J Bacteriol, 181, 6300-6305.

429. Trias, J. and Nikaido, H. (1990) Outer membrane protein D2 catalyzes facilitated diffusion of carbapenems and penems through the outer membrane of Pseudomonas aeruginosa. Antimicrob Agents Chemother, 34, 52-57.

430. Ochs, M.M., McCusker, M.P., Bains, M. and Hancock, R.E. (1999) Negative regulation of the Pseudomonas aeruginosa outer membrane porin OprD selective for imipenem and basic amino acids. Antimicrob Agents Chemother, 43, 10851090.

431. Kohler, T., Epp, S.F., Curty, L.K. and Pechere, J.C. (1999) Characterization of MexT, the regulator of the MexE-MexF-OprN multidrug efflux system of Pseudomonas aeruginosa. Journal of Bacteriology, 181, 6300-6305.

432. Westfall, L.W., Carty, N.L., Layland, N., Kuan, P., Colmer-Hamood, J.A. and Hamood, A.N. (2006) mvaT mutation modifies the expression of the Pseudomonas aeruginosa multidrug efflux operon mexEF-oprN. FEMS Microbiol Lett, 255, 247-254.

433. CLSI. (2006) Methods for dilution antimicrobial susceptibility tests for bacteria that grow aerobically; Approved standards- 7th edition. Clinical and Laboratory Standards Institute, $\mathbf{2 6}$. 
434. Zeng, E., Mathee, K. and Narasimhan, G. (2007) IEM: An Algorithm for Iterative Enhancement of Motifs Using Comparative Genomics Data. Comput Syst Bioinformatics Conf, 6, 227-235.

435. Poole, K., Krebes, K., McNally, C. and Neshat, S. (1993) Multiple antibiotic resistance in Pseudomonas aeruginosa: evidence for involvement of an efflux operon. J Bacteriol, 175, 7363-7372.

436. Nakae, T., Nakajima, A., Ono, T., Saito, K. and Yoneyama, H. (1999) Resistance to beta-lactam antibiotics in Pseudomonas aeruginosa due to interplay between the MexAB-OprM efflux pump and beta-lactamase. Antimicrob Agents Chemother, 43, 1301-1303.

437. Kohler, T., Buckling, A. and van Delden, C. (2009) Cooperation and virulence of clinical Pseudomonas aeruginosa populations. Proc Natl Acad Sci U S A, 106, 6339-6344.

438. Caldwell, C.C., Chen, Y., Goetzmann, H.S., Hao, Y., Borchers, M.T., Hassett, D.J., Young, L.R., Mavrodi, D., Thomashow, L. and Lau, G.W. (2009) Pseudomonas aeruginosa exotoxin pyocyanin causes cystic fibrosis airway pathogenesis. Am J Pathol, 175, 2473-2488.

439. Lau, G.W., Hassett, D.J., Ran, H. and Kong, F. (2004) The role of pyocyanin in Pseudomonas aeruginosa infection. Trends Mol Med, 10, 599-606.

440. Aendekerk, S., Diggle, S.P., Song, Z., Hoiby, N., Cornelis, P., Williams, P. and Camara, M. (2005) The MexGHI-OpmD multidrug efflux pump controls growth, antibiotic susceptibility and virulence in Pseudomonas aeruginosa via 4quinolone-dependent cell-to-cell communication. Microbiology, 151, 1113-1125.

441. Tamura, Y., Suzuki, S., Kijima, M., Takahashi, T. and Nakamura, M. (1992) Effect of proteolytic enzyme on experimental infection of mice with Pseudomonas aeruginosa. J Vet Med Sci, 54, 597-599.

442. Tamura, Y., Suzuki, S. and Sawada, T. (1992) Role of elastase as a virulence factor in experimental Pseudomonas aeruginosa infection in mice. Microb Pathog, 12, 237-244. 
443. Liu, P.V. (1974) Extracellular toxins of Pseudomonas aeruginosa. J Infect Dis, 130, S94-99.

444. Kamath, S., Kapatral, V. and Chakrabarty, A.M. (1998) Cellular function of elastase in Pseudomonas aeruginosa: role in the cleavage of nucleoside diphosphate kinase and in alginate synthesis. Mol Microbiol, 30, 933-941.

445. Matsumoto, K. (2004) Role of bacterial proteases in pseudomonal and serratial keratitis. Biol Chem, 385, 1007-1016.

446. Gallagher, L.A. and Manoil, C. (2001) Pseudomonas aeruginosa PAO1 kills Caenorhabditis elegans by cyanide poisoning. J Bacteriol, 183, 6207-6214.

447. Kirkeby, S., Hansen, A.K., d'Apice, A. and Moe, D. (2006) The galactophilic lectin (PA-IL, gene LecA) from Pseudomonas aeruginosa. Its binding requirements and the localization of lectin receptors in various mouse tissues. Microb Pathog, 40, 191-197.

448. Diggle, S.P., Stacey, R.E., Dodd, C., Camara, M., Williams, P. and Winzer, K. (2006) The galactophilic lectin, LecA, contributes to biofilm development in Pseudomonas aeruginosa. Environ Microbiol, 8, 1095-1104.

449. Winzer, K., Falconer, C., Garber, N.C., Diggle, S.P., Camara, M. and Williams, P. (2000) The Pseudomonas aeruginosa lectins PA-IL and PA-IIL are controlled by quorum sensing and by RpoS. J Bacteriol, 182, 6401-6411.

450. Wolfgang, M.C., Jyot, J., Goodman, A.L., Ramphal, R. and Lory, S. (2004) Pseudomonas aeruginosa regulates flagellin expression as part of a global response to airway fluid from cystic fibrosis patients. Proc Natl Acad Sci U S A, 101, 6664-6668.

451. Harmsen, M., Yang, L., Pamp, S.J. and Tolker-Nielsen, T. (2010) An update on Pseudomonas aeruginosa biofilm formation, tolerance, and dispersal. FEMS Immunol Med Microbiol, 59, 253-268.

452. Klausen, M., Aaes-Jorgensen, A., Molin, S. and Tolker-Nielsen, T. (2003) Involvement of bacterial migration in the development of complex multicellular structures in Pseudomonas aeruginosa biofilms. Mol Microbiol, 50, 61-68. 
453. Friedman, L. and Kolter, R. (2004) Genes involved in matrix formation in Pseudomonas aeruginosa PA14 biofilms. Mol Microbiol, 51, 675-690.

454. Friedman, L. and Kolter, R. (2004) Two genetic loci produce distinct carbohydrate-rich structural components of the Pseudomonas aeruginosa biofilm matrix. J Bacteriol, 186, 4457-4465.

455. Zhang, L. and Mah, T.F. (2008) Involvement of a novel efflux system in biofilmspecific resistance to antibiotics. J Bacteriol, 190, 4447-4452.

456. Journet, L., Hughes, K.T. and Cornelis, G.R. (2005) Type III secretion: a secretory pathway serving both motility and virulence. Mol Membr Biol, 22, 4150 .

457. Holder, I.A., Neely, A.N. and Frank, D.W. (2001) Type III secretion/intoxication system important in virulence of Pseudomonas aeruginosa infections in burns. Burns, 27, 129-130.

458. Brencic, A. and Lory, S. (2009) Determination of the regulon and identification of novel mRNA targets of Pseudomonas aeruginosa RsmA. Mol Microbiol, 72, 612632.

459. Mougous, J.D., Cuff, M.E., Raunser, S., Shen, A., Zhou, M., Gifford, C.A., Goodman, A.L., Joachimiak, G., Ordonez, C.L., Lory, S. et al. (2006) A virulence locus of Pseudomonas aeruginosa encodes a protein secretion apparatus. Science, 312, 1526-1530.

460. Michel-Briand, Y. and Baysse, C. (2002) The pyocins of Pseudomonas aeruginosa. Biochimie, 84, 499-510.

461. Potvin, E., Sanschagrin, F. and Levesque, R.C. (2008) Sigma factors in Pseudomonas aeruginosa. FEMS Microbiol Rev, 32, 38-55.

462. Dong, T. and Schellhorn, H.E. (2010) Role of RpoS in virulence of pathogens. Infect Immun, 78, 887-897. 
463. Suh, S.J., Silo-Suh, L., Woods, D.E., Hassett, D.J., West, S.E. and Ohman, D.E. (1999) Effect of rpoS mutation on the stress response and expression of virulence factors in Pseudomonas aeruginosa. J Bacteriol, 181, 3890-3897.

464. Sonnleitner, E., Hagens, S., Rosenau, F., Wilhelm, S., Habel, A., Jager, K.E. and Blasi, U. (2003) Reduced virulence of a $h f q$ mutant of Pseudomonas aeruginosa O1. Microb Pathog, 35, 217-228.

465. DeVries, C.A. and Ohman, D.E. (1994) Mucoid-to-nonmucoid conversion in alginate-producing Pseudomonas aeruginosa often results from spontaneous mutations in $\operatorname{alg} T$, encoding a putative alternate sigma factor, and shows evidence for autoregulation. $J$ Bacteriol, 176, 6677-6687.

466. Martin, D.W., Schurr, M.J., Mudd, M.H., Govan, J.R., Holloway, B.W. and Deretic, V. (1993) Mechanism of conversion to mucoidy in Pseudomonas aeruginosa infecting cystic fibrosis patients. Proc Natl Acad Sci U S A, 90, 83778381.

467. Wood, L.F. and Ohman, D.E. (2009) Use of cell wall stress to characterize sigma $22(\mathrm{AlgT} / \mathrm{U})$ activation by regulated proteolysis and its regulon in Pseudomonas aeruginosa. Mol Microbiol, 72, 183-201.

468. Avison, M.B., Niumsup, P., Nurmahomed, K., Walsh, T.R. and Bennett, P.M. (2004) Role of the 'cre/blr-tag' DNA sequence in regulation of gene expression by the Aeromonas hydrophila beta-lactamase regulator, BlrA. J Antimicrob Chemoth, 53, 197-202.

469. Avison, M.B., Horton, R.E., Walsh, T.R. and Bennett, P.M. (2001) Escherichia coli $\mathrm{CreBC}$ is a global regulator of gene expression that responds to growth in minimal media. J Biol Chem, 276, 26955-26961.

470. Maseda, H., Saito, K., Nakajima, A. and Nakae, T. (2000) Variation of the mexT gene, a regulator of the MexEF-OprN efflux pump expression in wild-type strains of Pseudomonas aeruginosa. FEMS Microbiol Lett, 192, 107-112.

471. Michea-Hamzehpour, M., Auckenthaler, R., Regamey, P. and Pechere, J.C. (1987) Resistance occurring after fluoroquinolone therapy of experimental Pseudomonas aeruginosa peritonitis. Antimicrob Agents Chemother, 31, 18031808. 
472. Maseda, H., Uwate, M. and Nakae, T. (2010) Transcriptional regulation of the mexEF-oprN multidrug efflux pump operon by MexT and an unidentified repressor in $n f x C$-type mutant of Pseudomonas aeruginosa. FEMS Microbiol. Lett., 311, 36-43.

473. Kumar, A. and Schweizer, H.P. (2011) Evidence of MexT-independent overexpression of MexEF-OprN multidrug efflux pump of Pseudomonas aeruginosa in presence of metabolic stress. PloS one, 6, e26520.

474. Li, X.Z., Barre, N. and Poole, K. (2000) Influence of the MexA-MexB-oprM multidrug efflux system on expression of the MexC-MexD-oprJ and MexEMexF-oprN multidrug efflux systems in Pseudomonas aeruginosa. J Antimicrob Chemother, 46, 885-893.

475. Vettoretti, L., Plesiat, P., Muller, C., El Garch, F., Phan, G., Attree, I., Ducruix, A. and Llanes, C. (2009) Efflux unbalance in Pseudomonas aeruginosa isolates from cystic fibrosis patients. Antimicrob Agents Chemother, 53, 1987-1997.

476. De Kievit, T.R., Parkins, M.D., Gillis, R.J., Srikumar, R., Ceri, H., Poole, K., Iglewski, B.H. and Storey, D.G. (2001) Multidrug efflux pumps: expression patterns and contribution to antibiotic resistance in Pseudomonas aeruginosa biofilms. Antimicrob Agents Chemother, 45, 1761-1770.

477. Sivaneson, M., Mikkelsen, H., Ventre, I., Bordi, C. and Filloux, A. (2011) Twocomponent regulatory systems in Pseudomonas aeruginosa: an intricate network mediating fimbrial and efflux pump gene expression. Mol Microbiol, 79, 13531366.

478. Yeung, A.T., Bains, M. and Hancock, R.E. (2011) The sensor kinase CbrA is a global regulator that modulates metabolism, virulence, and antibiotic resistance in Pseudomonas aeruginosa. J Bacteriol, 193, 918-931.

479. Dietrich, L.E., Teal, T.K., Price-Whelan, A. and Newman, D.K. (2008) Redoxactive antibiotics control gene expression and community behavior in divergent bacteria. Science, 321, 1203-1206.

480. Carterson, A.J., Morici, L.A., Jackson, D.W., Frisk, A., Lizewski, S.E., Jupiter, R., Simpson, K., Kunz, D.A., Davis, S.H., Schurr, J.R. et al. (2004) The 
transcriptional regulator AlgR controls cyanide production in Pseudomonas aeruginosa. J Bacteriol, 186, 6837-6844.

481. Wozniak, D.J. and Ohman, D.E. (1994) Transcriptional analysis of the Pseudomonas aeruginosa genes $\operatorname{alg} R, \operatorname{alg} B$, and $\operatorname{alg} D$ reveals a hierarchy of alginate gene expression which is modulated by algT. $J$ Bacteriol, 176, 6007-6014.

482. Martin, D.W., Schurr, M.J., Yu, H. and Deretic, V. (1994) Analysis of promoters controlled by the putative sigma factor AlgU regulating conversion to mucoidy in Pseudomonas aeruginosa: relationship to sigma E and stress response. J Bacteriol, 176, 6688-6696.

483. Heeb, S., Valverde, C., Gigot-Bonnefoy, C. and Haas, D. (2005) Role of the stress sigma factor RpoS in GacA/RsmA-controlled secondary metabolism and resistance to oxidative stress in Pseudomonas fluorescens CHA0. FEMS Microbiol Lett, 243, 251-258.

484. Laughlin, R.S., Musch, M.W., Hollbrook, C.J., Rocha, F.M., Chang, E.B. and Alverdy, J.C. (2000) The key role of Pseudomonas aeruginosa PA-I lectin on experimental gut-derived sepsis. Ann Surg, 232, 133-142.

485. Nakayama, K., Takashima, K., Ishihara, H., Shinomiya, T., Kageyama, M., Kanaya, S., Ohnishi, M., Murata, T., Mori, H. and Hayashi, T. (2000) The R-type pyocin of Pseudomonas aeruginosa is related to P2 phage, and the F-type is related to lambda phage. Mol Microbiol, 38, 213-231.

486. Brazas, M.D. and Hancock, R.E. (2005) Ciprofloxacin induction of a susceptibility determinant in Pseudomonas aeruginosa. Antimicrob Agents Chemother, 49, 3222-3227.

487. Bakkal, S., Robinson, S.M., Ordonez, C.L., Waltz, D.A. and Riley, M.A. (2010) Role of bacteriocins in mediating interactions of bacterial isolates taken from cystic fibrosis patients. Microbiology, 156, 2058-2067.

488. Qiu, X., Gurkar, A.U. and Lory, S. (2006) Interstrain transfer of the large pathogenicity island (PAPI-1) of Pseudomonas aeruginosa. Proc Natl Acad Sci U $S A, \mathbf{1 0 3}, 19830-19835$. 
489. Heeb, S., Blumer, C. and Haas, D. (2002) Regulatory RNA as mediator in GacA/RsmA-dependent global control of exoproduct formation in Pseudomonas fluorescens CHA0. J Bacteriol, 184, 1046-1056.

490. Valverde, C., Heeb, S., Keel, C. and Haas, D. (2003) RsmY, a small regulatory RNA, is required in concert with RsmZ for GacA-dependent expression of biocontrol traits in Pseudomonas fluorescens CHA0. Mol Microbiol, 50, 13611379.

491. Liu, M.Y., Gui, G., Wei, B., Preston, J.F., 3rd, Oakford, L., Yuksel, U., Giedroc, D.P. and Romeo, T. (1997) The RNA molecule CsrB binds to the global regulatory protein CsrA and antagonizes its activity in Escherichia coli. J Biol Chem, 272, 17502-17510.

492. Mulcahy, H., O'Callaghan, J., O'Grady, E.P., Macia, M.D., Borrell, N., Gomez, C., Casey, P.G., Hill, C., Adams, C., Gahan, C.G. et al. (2008) Pseudomonas aeruginosa RsmA plays an important role during murine infection by influencing colonization, virulence, persistence, and pulmonary inflammation. Infect Immun, 76, 632-638.

493. Mulcahy, H., O'Callaghan, J., O'Grady, E.P., Adams, C. and O'Gara, F. (2006) The posttranscriptional regulator RsmA plays a role in the interaction between Pseudomonas aeruginosa and human airway epithelial cells by positively regulating the type III secretion system. Infect Immun, 74, 3012-3015.

494. Nicas, T.I. and Iglewski, B.H. (1985) The contribution of exoproducts to virulence of Pseudomonas aeruginosa. Can J Microbiol, 31, 387-392.

495. Rietsch, A., Vallet-Gely, I., Dove, S.L. and Mekalanos, J.J. (2005) ExsE, a secreted regulator of type III secretion genes in Pseudomonas aeruginosa. Proc Natl Acad Sci U S A, 102, 8006-8011.

496. Furste, J.P., Pansegrau, W., Frank, R., Blocker, H., Scholz, P., Bagdasarian, M. and Lanka, E. (1986) Molecular cloning of the plasmid RP4 primase region in a multi-host-range tacP expression vector. Gene, 48, 119-131.

497. Horton, R.M., Cai, Z.L., Ho, S.N. and Pease, L.R. (1990) Gene splicing by overlap extension: tailor-made genes using the polymerase chain reaction. Biotechniques, 8, 528-535. 
498. Schweizer, H.P. and Hoang, T.T. (1995) An improved system for gene replacement and xylE fusion analysis in Pseudomonas aeruginosa. Gene, 158, 1522.

499. Kessler, B., de Lorenzo, V. and Timmis, K.N. (1992) A general system to integrate lac $Z$ fusions into the chromosomes of Gram-negative eubacteria: regulation of the Pm promoter of the TOL plasmid studied with all controlling elements in monocopy. Mol Gen Genet, 233, 293-301.

500. Choi, K.H., Kumar, A. and Schweizer, H.P. (2006) A 10-min method for preparation of highly electrocompetent Pseudomonas aeruginosa cells: application for DNA fragment transfer between chromosomes and plasmid transformation. J Microbiol Methods, 64, 391-397.

501. Deziel, E., Comeau, Y. and Villemur, R. (2001) Initiation of biofilm formation by Pseudomonas aeruginosa 57RP correlates with emergence of hyperpiliated and highly adherent phenotypic variants deficient in swimming, swarming, and twitching motilities. J Bacteriol, 183, 1195-1204.

502. Kessler, E., Safrin, M., Olson, J.C. and Ohman, D.E. (1993) Secreted LasA of Pseudomonas aeruginosa is a staphylolytic protease. J Biol Chem, 268, 75037508.

503. Ohman, D.E., Cryz, S.J. and Iglewski, B.H. (1980) Isolation and characterization of Pseudomonas aeruginosa PAO mutant that produces altered elastase. $J$ Bacteriol, 142, 836-842.

504. Essar, D.W., Eberly, L., Hadero, A. and Crawford, I.P. (1990) Identification and characterization of genes for a second anthranilate synthase in Pseudomonas aeruginosa: interchangeability of the two anthranilate synthases and evolutionary implications. J Bacteriol, 172, 884-900.

505. Morrison, A.J., Jr. and Wenzel, R.P. (1984) Epidemiology of infections due to Pseudomonas aeruginosa. Rev Infect Dis., 6, S627-642.

506. Pollack, M. (1995) In Mandell, G. L., Dolan, R. and Bennett, J. E. (eds.), Principles and practices of infectious diseases. Churchill Livingston, New York, pp. 1820-2003. 
507. Pier, G.B. (2007) Pseudomonas aeruginosa lipopolysaccharide: a major virulence factor, initiator of inflammation and target for effective immunity. Int $J$ Med Microbiol., 297, 277-295.

508. Craig, L., Pique, M.E. and Tainer, J.A. (2004) Type IV pilus structure and bacterial pathogenicity. Nat Rev Microbiol., 2, 363-378.

509. Kuang, Z., Hao, Y., Hwang, S., Zhang, S., Kim, E., Akinbi, H.T., Schurr, M.J., Irvin, R.T., Hassett, D.J. and Lau, G.W. (2011) The Pseudomonas aeruginosa flagellum confers resistance to pulmonary surfactant protein-A by impacting the production of exoproteases through quorum-sensing. Mol Microbiol., 79, 12201235.

510. Riedel, K., Hentzer, M., Geisenberger, O., Huber, B., Steidle, A., Wu, H., Hoiby, N., Givskov, M., Molin, S. and Eberl, L. (2001) N-acylhomoserine-lactonemediated communication between Pseudomonas aeruginosa and Burkholderia cepacia in mixed biofilms. Microbiology, 147, 3249-3262.

511. Balasubramanian, D., Schneper, L., Kumari, H. and Mathee, K. (2013) A dynamic and intricate regulatory network determines Pseudomonas aeruginosa virulence. Nucleic Acids Res., 41, 1-20.

512. Jimenez, P.N., Koch, G., Thompson, J.A., Xavier, K.B., Cool, R.H. and Quax, W.J. (2012) The multiple signaling systems regulating virulence in Pseudomonas aeruginosa. Microbiol Mol Biol Rev., 76, 46-65.

513. Piddock, L.J. (2006) Multidrug-resistance efflux pumps - not just for resistance. Nat Rev Microbiol., 4, 629-636.

514. Fernandez, L. and Hancock, R.E. (2012) Adaptive and mutational resistance: role of porins and efflux pumps in drug resistance. Clin Microbiol Rev., 25, 661-681.

515. Fothergill, J.L., Winstanley, C. and James, C.E. (2012) Novel therapeutic strategies to counter Pseudomonas aeruginosa infections. Expert Rev Anti Infect Ther., 10, 219-235.

516. (2004) National Nosocomial Infections Surveillance (NNIS) System Report, data summary from January 1992 through June 2004, issued October 2004. Am J Infect Control, 32, 470-485. 
517. Llamas, M.A., Mooij, M.J., Sparrius, M., Vandenbroucke-Grauls, C.M., Ratledge, C. and Bitter, W. (2008) Characterization of five novel Pseudomonas aeruginosa cell-surface signalling systems. Mol Microbiol, 67, 458-472.

518. Zheng, Q. and Wang, X.J. (2008) GOEAST: a web-based software toolkit for Gene Ontology enrichment analysis. Nucleic Acids Res., 36, W358-363.

519. Livny, J., Brencic, A., Lory, S. and Waldor, M.K. (2006) Identification of 17 Pseudomonas aeruginosa sRNAs and prediction of sRNA-encoding genes in 10 diverse pathogens using the bioinformatic tool sRNAPredict2. Nucleic Acids Res., 34, 3484-3493.

520. Cornelis, P. (2010) Iron uptake and metabolism in pseudomonads. Appl Environ Microbiol., 86, 1637-1645.

521. Hohnadel, D. and Meyer, J.M. (1988) Specificity of pyoverdine-mediated iron uptake among fluorescent Pseudomonas strains. J Bacteriol., 170, 4865-4873.

522. Liu, P.V. and Shokrani, F. (1978) Biological activities of pyochelins: ironchelating agents of Pseudomonas aeruginosa. Infect Immun., 22, 878-890.

523. Poole, K., Young, L. and Neshat, S. (1990) Enterobactin-mediated iron transport in Pseudomonas aeruginosa. J Bacteriol., 172, 6991-6996.

524. Meyer, J.M. (2000) Pyoverdines: pigments, siderophores and potential taxonomic markers of fluorescent Pseudomonas species. Arch Microbiol., 174, 135-142.

525. Miyazaki, H., Kato, H., Nakazawa, T. and Tsuda, M. (1995) A positive regulatory gene, $p v d S$, for expression of pyoverdin biosynthetic genes in Pseudomonas aeruginosa PAO. Mol Gen Genet., 248, 17-24.

526. Wilderman, P.J., Vasil, A.I., Johnson, Z., Wilson, M.J., Cunliffe, H.E., Lamont, I.L. and Vasil, M.L. (2001) Characterization of an endoprotease (PrpL) encoded by a PvdS-regulated gene in Pseudomonas aeruginosa. Infect Immun., 69, 53855394. 
527. Dean, C.R., Neshat, S. and Poole, K. (1996) PfeR, an enterobactin-responsive activator of ferric enterobactin receptor gene expression in Pseudomonas aeruginosa. J Bacteriol., 178, 5361-5369.

528. Meyer, J.M. and Abdallah, M.A. (1978) The fluorescent pigment of Pseudomonas fluorescens: biosynthesis, purification and physicochemical properties. J Gen Microbiol., 107, 319-328.

529. Marshall, B., Stintzi, A., Gilmour, C., Meyer, J.M. and Poole, K. (2009) Citratemediated iron uptake in Pseudomonas aeruginosa: involvement of the citrateinducible FecA receptor and the FeoB ferrous iron transporter. Microbiology, 155, 305-315.

530. Genevaux, P., Georgopoulos, C. and Kelley, W.L. (2007) The Hsp70 chaperone machines of Escherichia coli: a paradigm for the repartition of chaperone functions. Mol Microbiol., 66, 840-857.

531. Meyer, A.S. and Baker, T.A. (2011) Proteolysis in the Escherichia coli heat shock response: a player at many levels. Curr Opin Microbiol., 14, 194-199.

532. Harrison, C. (2003) GrpE, a nucleotide exchange factor for DnaK. Cell Stress Chaperones., 8, 218-224.

533. Farr, S.B., Touati, D. and Kogoma, T. (1988) Effects of oxygen stress on membrane functions in Escherichia coli: role of HPI catalase. J Bacteriol., 170, 1837-1842.

534. Moody, C.S. and Hassan, H.M. (1982) Mutagenicity of oxygen free radicals. $P N A S, 79,2855-2859$.

535. Lee, J.S., Heo, Y.J., Lee, J.K. and Cho, Y.H. (2005) KatA, the major catalase, is critical for osmoprotection and virulence in Pseudomonas aeruginosa PA14. Infect Immun., 73, 4399-4403.

536. Gonzalez, N., Heeb, S., Valverde, C., Kay, E., Reimmann, C., Junier, T. and Haas, D. (2008) Genome-wide search reveals a novel GacA-regulated small RNA in Pseudomonas species. BMC Genomics, 9, 167. 
537. Nakayama, K., Takashima, K., Ishihara, H., Shinomiya, T., Kageyama, M., Kanaya, S., Ohnishi, M., Murata, T., Mori, H. and Hayashi, T. (2000) The R-type pyocin of Pseudomonas aeruginosa is related to P2 phage, and the F-type is related to lambda phage. Mol Microbiol., 38, 213-231.

538. Weidner, U., Geier, S., Ptock, A., Friedrich, T., Leif, H. and Weiss, H. (1993) The gene locus of the proton-translocating NADH: ubiquinone oxidoreductase in Escherichia coli. Organization of the 14 genes and relationship between the derived proteins and subunits of mitochondrial complex I. J Mol Biol., 233, 109122.

539. Heo, Y.J., Chung, I.Y., Cho, W.J., Lee, B.Y., Kim, J.H., Choi, K.H., Lee, J.W., Hassett, D.J. and Cho, Y.H. (2010) The major catalase gene (katA) of Pseudomonas aeruginosa PA14 is under both positive and negative control of the global transactivator OxyR in response to hydrogen peroxide. J Bacteriol., 192, 381-390.

540. Brown, S.M., Howell, M.L., Vasil, M.L., Anderson, A.J. and Hassett, D.J. (1995) Cloning and characterization of the katB gene of Pseudomonas aeruginosa encoding a hydrogen peroxide-inducible catalase: purification of KatB, cellular localization, and demonstration that it is essential for optimal resistance to hydrogen peroxide. $J$ Bacteriol, 177, 6536-6544.

541. Recinos, D.A., Sekedat, M.D., Hernandez, A., Cohen, T.S., Sakhtah, H., Prince, A.S., Price-Whelan, A. and Dietrich, L.E. (2012) Redundant phenazine operons in Pseudomonas aeruginosa exhibit environment-dependent expression and differential roles in pathogenicity. PNAS.

542. McKnight, S.L., Iglewski, B.H. and Pesci, E.C. (2000) The Pseudomonas quinolone signal regulates $r h l$ quorum sensing in Pseudomonas aeruginosa. $j$ Bacteriol., 182, 2702-2708.

543. Bredenbruch, F., Nimtz, M., Wray, V., Morr, M., Muller, R. and Haussler, S. (2005) Biosynthetic pathway of Pseudomonas aeruginosa 4-hydroxy-2alkylquinolines. $J$ Bacteriol., 187, 3630-3635.

544. Xiao, G., He, J. and Rahme, L.G. (2006) Mutation analysis of the Pseudomonas aeruginosa mvfR and pqsABCDE gene promoters demonstrates complex quorumsensing circuitry. Microbiology, 152, 1679-1686. 
545. Kay, E., Humair, B., Denervaud, V., Riedel, K., Spahr, S., Eberl, L., Valverde, C. and Haas, D. (2006) Two GacA-dependent small RNAs modulate the quorumsensing response in Pseudomonas aeruginosa. J Bacteriol., 188, 6026-6033.

546. O'Callaghan, J., Reen, F.J., Adams, C. and O'Gara, F. (2011) Low oxygen induces the type III secretion system in Pseudomonas aeruginosa via modulation of the small RNAs $r s m Z$ and $r s m Y$. Microbiology, 157, 3417-3428.

547. Petrova, O.E. and Sauer, K. (2010) The novel two-component regulatory system BfiSR regulates biofilm development by controlling the small RNA rsmZ through CafA. J Bacteriol., 192, 5275-5288.

548. Heeb, S., Blumer, C. and Haas, D. (2002) Regulatory RNA as mediator in GacA/RsmA-dependent global control of exoproduct formation in Pseudomonas fluorescens CHA0. J Bacteriol., 184, 1046-1056.

549. Valverde, C., Heeb, S., Keel, C. and Haas, D. (2003) RsmY, a small regulatory RNA, is required in concert with RsmZ for GacA-dependent expression of biocontrol traits in Pseudomonas fluorescens CHA0. Mol Microbiol., 50, 13611379 .

550. Bartowsky, E. and Normark, S. (1993) Interactions of wild-type and mutant AmpR of Citrobacter freundii with target DNA. Mol Microbiol., 10, 555-565.

551. Sirbu, A., Kerr, G., Crane, M. and Ruskin, H.J. (2012) RNA-Seq vs dual- and single-channel microarray data: sensitivity analysis for differential expression and clustering. PLoS ONE, 7, e50986.

552. Kogenaru, S., Qing, Y., Guo, Y. and Wang, N. (2012) RNA-seq and microarray complement each other in transcriptome profiling. BMC Genomics, 13, 629.

553. Nookaew, I., Papini, M., Pornputtapong, N., Scalcinati, G., Fagerberg, L., Uhlen, M. and Nielsen, J. (2012) A comprehensive comparison of RNA-Seq-based transcriptome analysis from reads to differential gene expression and crosscomparison with microarrays: a case study in Saccharomyces cerevisiae. Nucleic Acids Res., 40, 10084-10097. 
554. Lim, C.K., Hassan, K.A., Tetu, S.G., Loper, J.E. and Paulsen, I.T. (2012) The effect of iron limitation on the transcriptome and proteome of Pseudomonas fluorescens Pf-5. PLOS ONE, 7, e39139.

555. Nie, L., Wu, G. and Zhang, W. (2006) Correlation of mRNA expression and protein abundance affected by multiple sequence features related to translational efficiency in Desulfovibrio vulgaris: a quantitative analysis. Genetics, 174, 22292243.

556. Nie, L., Wu, G. and Zhang, W. (2006) Correlation between mRNA and protein abundance in Desulfovibrio vulgaris: a multiple regression to identify sources of variations. Biochem Biophy Res Commun., 339, 603-610.

557. Yahr, T.L., Mende-Mueller, L.M., Friese, M.B. and Frank, D.W. (1997) Identification of type III secreted products of the Pseudomonas aeruginosa exoenzyme S regulon. J Bacteriol., 179, 7165-7168.

558. Abe, K., Watabe, S., Emori, Y., Watanabe, M. and Arai, S. (1989) An ice nucleation active gene of Erwinia ananas. Sequence similarity to those of Pseudomonas species and regions required for ice nucleation activity. FEBS Lett., 258, 297-300.

559. Rosner, J.L. and Slonczewski, J.L. (1994) Dual regulation of inaA by the multiple antibiotic resistance (mar) and superoxide (soxRS) stress response systems of Escherichia coli. J Bacteriol., 176, 6262-6269.

560. Balasubramanian, D., Murugapiran, S.K., Silva-Herzog, E., Schneper, L., Yang, X., Tatke, G., Narasimhan, G. and Mathee, K. (2013) In M, M. B. (ed.), Bacterial Gene Regulation and Transcriptional Networks. Caiser Academic Press, United Kingdom.

561. Fuchs, E.L., Brutinel, E.D., Jones, A.K., Fulcher, N.B., Urbanowski, M.L., Yahr, T.L. and Wolfgang, M.C. (2010) The Pseudomonas aeruginosa Vfr regulator controls global virulence factor expression through cyclic AMP-dependent and independent mechanisms. J Bacteriol., 192, 3553-3564.

562. Ferrell, E., Carty, N.L., Colmer-Hamood, J.A., Hamood, A.N. and West, S.E. (2008) Regulation of Pseudomonas aeruginosa ptxR by Vfr. Microbiology, 154, 431-439. 
563. Ozsolak, F. and Milos, P.M. (2011) RNA sequencing: advances, challenges and opportunities. Nat Rev Genet., 12, 87-98.

564. Smith, D.J., Lamont, I.L., Anderson, G.J. and Reid, D.W. (2012) Targeting iron uptake to control Pseudomonas aeruginosa infections in cystic fibrosis. Eur Respir $J$.

565. Reid, D.W., Carroll, V., O'May, C., Champion, A. and Kirov, S.M. (2007) Increased airway iron as a potential factor in the persistence of Pseudomonas aeruginosa infection in cystic fibrosis. Eur Respir J., 30, 286-292.

566. Meyer, J.M., Neely, A., Stintzi, A., Georges, C. and Holder, I.A. (1996) Pyoverdin is essential for virulence of Pseudomonas aeruginosa. Infect Immun., 64, 518-523.

567. Martin, L.W., Reid, D.W., Sharples, K.J. and Lamont, I.L. (2011) Pseudomonas siderophores in the sputum of patients with cystic fibrosis. Biometals, 24, 10591067.

568. Stites, S.W., Walters, B., O'Brien-Ladner, A.R., Bailey, K. and Wesselius, L.J. (1998) Increased iron and ferritin content of sputum from patients with cystic fibrosis or chronic bronchitis. Chest, 114, 814-819.

569. Mathee, K., Ciofu, O., Sternberg, C., Lindum, P.W., Campbell, J.I., Jensen, P., Johnsen, A.H., Givskov, M., Ohman, D.E., Molin, S. et al. (1999) Mucoid conversion of Pseudomonas aeruginosa by hydrogen peroxide: a mechanism for virulence activation in the cystic fibrosis lung. Microbiology, 145, 1349-1357.

570. Mathee, K., McPherson, C.J. and Ohman, D.E. (1997) Posttranslational control of the $\operatorname{alg} T(\operatorname{alg} U)$-encoded sigma22 for expression of the alginate regulon in Pseudomonas aeruginosa and localization of its antagonist proteins MucA and MucB (AlgN). J Bacteriol, 179, 3711-3720.

571. Coggan, K.A. and Wolfgang, M.C. (2012) Global regulatory pathways and crosstalk control Pseudomonas aeruginosa environmental lifestyle and virulence phenotype. Curr Issues Mol Biol., 14, 47-70. 
572. Lamb, J.R., Patel, H., Montminy, T., Wagner, V.E. and Iglewski, B.H. (2003) Functional domains of the RhlR transcriptional regulator of Pseudomonas aeruginosa. J Bacteriol., 185, 7129-7139.

573. Juhas, M., Wiehlmann, L., Salunkhe, P., Lauber, J., Buer, J. and Tummler, B. (2005) GeneChip expression analysis of the VqsR regulon of Pseudomonas aeruginosa TB. FEMS Microbiol Lett, 242, 287-295.

574. Visca, P., Leoni, L., Wilson, M.J. and Lamont, I.L. (2002) Iron transport and regulation, cell signalling and genomics: lessons from Escherichia coli and Pseudomonas. Mol Microbiol., 45, 1177-1190.

575. Oglesby, A.G., Farrow, J.M., 3rd, Lee, J.H., Tomaras, A.P., Greenberg, E.P., Pesci, E.C. and Vasil, M.L. (2008) The influence of iron on Pseudomonas aeruginosa physiology: a regulatory link between iron and quorum sensing. $J$ Biol Chem., 283, 15558-15567.

576. Mashburn, L.M. and Whiteley, M. (2005) Membrane vesicles traffic signals and facilitate group activities in a prokaryote. Nature, 437, 422-425.

577. Folkesson, A., Jelsbak, L., Yang, L., Johansen, H.K., Ciofu, O., Hoiby, N. and Molin, S. (2012) Adaptation of Pseudomonas aeruginosa to the cystic fibrosis airway: an evolutionary perspective. Nat Rev Microbiol., 10, 841-851.

578. Feliziani, S., Lujan, A.M., Moyano, A.J., Sola, C., Bocco, J.L., Montanaro, P., Canigia, L.F., Argarana, C.E. and Smania, A.M. (2010) Mucoidy, quorum sensing, mismatch repair and antibiotic resistance in Pseudomonas aeruginosa from cystic fibrosis chronic airways infections. PLOS ONE, 5.

579. Oliver, A. and Mena, A. (2010) Bacterial hypermutation in cystic fibrosis, not only for antibiotic resistance. Clin Microbiol Infect., 16, 798-808.

580. Mena, A., Smith, E.E., Burns, J.L., Speert, D.P., Moskowitz, S.M., Perez, J.L. and Oliver, A. (2008) Genetic adaptation of Pseudomonas aeruginosa to the airways of cystic fibrosis patients is catalyzed by hypermutation. J Bacteriol., 190, 79107917.

581. Ciofu, O., Mandsberg, L.F., Bjarnsholt, T., Wassermann, T. and Hoiby, N. (2010) Genetic adaptation of Pseudomonas aeruginosa during chronic lung infection of 
patients with cystic fibrosis: strong and weak mutators with heterogeneous genetic backgrounds emerge in mucA and/or lasR mutants. Microbiology, 156, 1108-1119.

582. Damron, F.H. and Goldberg, J.B. (2012) Proteolytic regulation of alginate overproduction in Pseudomonas aeruginosa. Mol. Microbiol., 84, 595-607.

583. Venturi, V. (2006) Regulation of quorum sensing in Pseudomonas. FEMS Microbiol Rev., 30, 274-291.

584. Ciofu, O., Mandsberg, L.F., Wang, H. and Hoiby, N. (2012) Phenotypes selected during chronic lung infection in cystic fibrosis patients: implications for the treatment of Pseudomonas aeruginosa biofilm infections. FEMS Immunol Med Microbiol., 65, 215-225.

585. Cabot, G., Ocampo-Sosa, A.A., Dominguez, M.A., Gago, J.F., Juan, C., Tubau, F., Rodriguez, C., Moya, B., Pena, C., Martinez-Martinez, L. et al. (2012) Genetic markers of widespread extensively drug-resistant Pseudomonas aeruginosa highrisk clones. Antimicrob Agents Chemother., 56, 6349-6357.

586. Ng, W.L., Perez, L., Cong, J., Semmelhack, M.F. and Bassler, B.L. (2012) Broad spectrum pro-quorum-sensing molecules as inhibitors of virulence in vibrios. PLoS Pathog., 8, e1002767.

587. Moqtaderi, Z. and Struhl, K. (2008) Expanding the repertoire of plasmids for PCR-mediated epitope tagging in yeast. Yeast, 25, 287-292.

588. Hoang, T.T., Kutchma, A.J., Becher, A. and Schweizer, H.P. (2000) Integrationproficient plasmids for Pseudomonas aeruginosa: site-specific integration and use for engineering of reporter and expression strains. Plasmid, 43, 59-72.

589. Yoder-Himes, D.R., Chain, P.S., Zhu, Y., Wurtzel, O., Rubin, E.M., Tiedje, J.M. and Sorek, R. (2009) Mapping the Burkholderia cenocepacia niche response via high-throughput sequencing. Proc Natl Acad Sci U S A., 106, 3976-3981.

590. Mizuno, T. (1997) Compilation of all genes encoding two-component phosphotransfer signal transducers in the genome of Escherichia coli. DNA Res., 4, 161-168. 
591. Fabret, C., Feher, V.A. and Hoch, J.A. (1999) Two-component signal transduction in Bacillus subtilis: how one organism sees its world. J Bacteriol., 181, 1975-1983.

592. Gooderham, W.J. and Hancock, R.E. (2009) Regulation of virulence and antibiotic resistance by two-component regulatory systems in Pseudomonas aeruginosa. FEMS Microbiol Rev, 33, 279-294.

593. Njoroge, J. and Sperandio, V. (2009) Jamming bacterial communication: new approaches for the treatment of infectious diseases. EMBO Mol Med, 1, 201-210.

594. Burns, J.L., Gibson, R.L., McNamara, S., Yim, D., Emerson, J., Rosenfeld, M., Hiatt, P., McCoy, K., Castile, R., Smith, A.L. et al. (2001) Longitudinal assessment of Pseudomonas aeruginosa in young children with cystic fibrosis. $J$ Infect Dis., 183, 444-452.

595. Hassett, D.J., Sutton, M.D., Schurr, M.J., Herr, A.B., Caldwell, C.C. and Matu, J.O. (2009) Pseudomonas aeruginosa hypoxic or anaerobic biofilm infections within cystic fibrosis airways. Trends Microbiol, 17, 130-138.

596. Potvin, E., Sanschagrin, F. and Levesque, R.C. (2008) Sigma factors in Pseudomonas aeruginosa. FEMS Microbiol Rev., 32, 38-55.

597. Galan-Vasquez, E., Luna, B. and Martinez-Antonio, A. (2011) The Regulatory Network of Pseudomonas aeruginosa. Microb Inform Exp, 1, 3.

598. Girard, G. and Bloemberg, G.V. (2008) Central role of quorum sensing in regulating the production of pathogenicity factors in Pseudomonas aeruginosa. Future Microbiol., 3, 97-106.

599. De Kievit, T.R., Gillis, R., Marx, S., Brown, C. and Iglewski, B.H. (2001) Quorum-sensing genes in Pseudomonas aeruginosa biofilms: their role and expression patterns. Appl Environ Microbiol., 67, 1865-1873.

600. Wong, A., Rodrigue, N. and Kassen, R. (2012) Genomics of adaptation during experimental evolution of the opportunistic pathogen Pseudomonas aeruginosa. PLOS Genet., 8, e1002928. 
601. Behrends, V., Ryall, B., Zlosnik, J.E., Speert, D.P., Bundy, J.G. and Williams, H.D. (2012) Metabolic adaptations of Pseudomonas aeruginosa during cystic fibrosis chronic lung infections. Environ Microbiol.

602. Chung, J.C., Becq, J., Fraser, L., Schulz-Trieglaff, O., Bond, N.J., Foweraker, J., Bruce, K.D., Smith, G.P. and Welch, M. (2012) Genomic variation among contemporary Pseudomonas aeruginosa isolates from chronically infected cystic fibrosis patients. $J$ Bacteriol., 194, 4857-4866.

603. Cramer, N., Wiehlmann, L. and Tummler, B. (2010) Clonal epidemiology of Pseudomonas aeruginosa in cystic fibrosis. Int J Med Microbiol., 300, 526-533.

604. Hogardt, M. and Heesemann, J. (2012) Microevolution of Pseudomonas aeruginosa to a chronic pathogen of the cystic fibrosis lung. Curr Top Microbiol Immunol.

605. Rodriguez-Martinez, J.M., Poirel, L. and Nordmann, P. (2009) Molecular epidemiology and mechanisms of carbapenem resistance in Pseudomonas aeruginosa. Antimicrob Agents Chemother., 53, 4783-4788.

606. Suarez, C., Pena, C., Arch, O., Dominguez, M.A., Tubau, F., Juan, C., Gavalda, L., Sora, M., Oliver, A., Pujol, M. et al. (2011) A large sustained endemic outbreak of multiresistant Pseudomonas aeruginosa: a new epidemiological scenario for nosocomial acquisition. BMC Infect Dis., 11, 272.

607. Deplano, A., Denis, O., Poirel, L., Hocquet, D., Nonhoff, C., Byl, B., Nordmann, P., Vincent, J.L. and Struelens, M.J. (2005) Molecular characterization of an epidemic clone of panantibiotic-resistant Pseudomonas aeruginosa. J Clin Microbiol., 43, 1198-1204.

608. Mesaros, N., Nordmann, P., Plesiat, P., Roussel-Delvallez, M., Van Eldere, J., Glupczynski, Y., Van Laethem, Y., Jacobs, F., Lebecque, P., Malfroot, A. et al. (2007) Pseudomonas aeruginosa: resistance and therapeutic options at the turn of the new millennium. Clin Microbiol Infect., 13, 560-578.

609. Pena, C., Suarez, C., Gozalo, M., Murillas, J., Almirante, B., Pomar, V., Aguilar, M., Granados, A., Calbo, E., Rodriguez-Bano, J. et al. (2012) Prospective multicenter study of the impact of carbapenem resistance on mortality in 
Pseudomonas aeruginosa bloodstream infections. Antimicrob Agents Chemother., 56, 1265-1272.

610. Woodford, N., Turton, J.F. and Livermore, D.M. (2011) Multiresistant Gramnegative bacteria: the role of high-risk clones in the dissemination of antibiotic resistance. FEMS Microbiol Rev., 35, 736-755.

611. Kuga, A., Okamoto, R. and Inoue, M. (2000) ampR gene mutations that greatly increase class C beta-lactamase activity in Enterobacter cloacae. Antimicrob Agents Chemother., 44, 561-567.

612. Balcewich, M.D., Reeve, T.M., Orlikow, E.A., Donald, L.J., Vocadlo, D.J. and Mark, B.L. (2010) Crystal structure of the AmpR effector binding domain provides insight into the molecular regulation of inducible ampc beta-lactamase. $J$ Mol Biol, 400, 998-1010.

613. Babu, M.M., Teichmann, S.A. and Aravind, L. (2006) Evolutionary dynamics of prokaryotic transcriptional regulatory networks. J Mol Biol., 358, 614-633.

614. Ong, C.T. and Corces, V.G. (2011) Enhancer function: new insights into the regulation of tissue-specific gene expression. Nat Rev Genet., 12, 283-293.

615. Levine, M. (2010) Transcriptional enhancers in animal development and evolution. Curr Biol., 20, R754-763.

616. Bulger, M. and Groudine, M. (2011) Functional and mechanistic diversity of distal transcription enhancers. Cell, 144, 327-339.

617. Ishihama, A. (2010) Prokaryotic genome regulation: multifactor promoters, multitarget regulators and hierarchic networks. FEMS Microbiol Rev., 34, 628645 .

618. Storz, G., Vogel, J. and Wassarman, K.M. (2011) Regulation by small RNAs in bacteria: expanding frontiers. Mol Cell, 43, 880-891.

619. Waters, L.S. and Storz, G. (2009) Regulatory RNAs in bacteria. Cell, 136, 615628. 
620. Reitzer, L.J. and Magasanik, B. (1986) Transcription of $g \ln A$ in E. coli is stimulated by activator bound to sites far from the promoter. Cell, 45, 785-792.

621. Popham, D.L., Szeto, D., Keener, J. and Kustu, S. (1989) Function of a bacterial activator protein that binds to transcriptional enhancers. Science, 243, 629-635.

622. Buck, M., Gallegos, M.T., Studholme, D.J., Guo, Y. and Gralla, J.D. (2000) The bacterial enhancer-dependent sigma $(54)(\operatorname{sigma}(\mathrm{N}))$ transcription factor. $J$ Bacteriol., 182, 4129-4136.

623. Nealson, K.H., Platt, T. and Hastings, J.W. (1970) Cellular control of the synthesis and activity of the bacterial luminescent system. J Bacteriol., 104, 313322.

624. Stevens, A.M., Schuster, M. and Rumbaugh, K.P. (2012) Working together for the common good: cell-cell communication in bacteria. J Bacteriol., 194, 21312141 .

625. Williams, P. and Camara, M. (2009) Quorum sensing and environmental adaptation in Pseudomonas aeruginosa: a tale of regulatory networks and multifunctional signal molecules. Curr Opin Microbiol., 12, 182-191.

626. Passador, L., Cook, J.M., Gambello, M.J., Rust, L. and Iglewski, B.H. (1993) Expression of Pseudomonas aeruginosa virulence genes requires cell-to-cell communication. Science, 260, 1127-1130.

627. Brint, J.M. and Ohman, D.E. (1995) Synthesis of multiple exoproducts in Pseudomonas aeruginosa is under the control of RhlR-RhlI, another set of regulators in strain PAO1 with homology to the autoinducer-responsive LuxRLuxI family. J Bacteriol, 177, 7155-7163.

628. Latifi, A., Winson, M.K., Foglino, M., Bycroft, B.W., Stewart, G.S., Lazdunski, A. and Williams, P. (1995) Multiple homologues of LuxR and LuxI control expression of virulence determinants and secondary metabolites through quorum sensing in Pseudomonas aeruginosa PAO1. Mol Microbiol, 17, 333-343.

629. Kiratisin, P., Tucker, K.D. and Passador, L. (2002) LasR, a transcriptional activator of Pseudomonas aeruginosa virulence genes, functions as a multimer. $J$ Bacteriol, 184, 4912-4919. 
630. Lamb, J.R., Patel, H., Montminy, T., Wagner, V.E. and Iglewski, B.H. (2003) Functional domains of the RhlR transcriptional regulator of Pseudomonas aeruginosa. J Bacteriol, 185, 7129-7139.

631. Schuster, M., Urbanowski, M.L. and Greenberg, E.P. (2004) Promoter specificity in Pseudomonas aeruginosa quorum sensing revealed by DNA binding of purified LasR. Proc Natl Acad Sci U S A, 101, 15833-15839.

632. Schertzer, J.W. and Whiteley, M. (2012) A bilayer-couple model of bacterial outer membrane vesicle biogenesis. MBio, 3.

633. Wagner, V.E., Li, L.L., Isabella, V.M. and Iglewski, B.H. (2007) Analysis of the hierarchy of quorum-sensing regulation in Pseudomonas aeruginosa. Anal Bioanal Chem., 387, 469-479.

634. McGrath, S., Wade, D.S. and Pesci, E.C. (2004) Dueling quorum sensing systems in Pseudomonas aeruginosa control the production of the Pseudomonas quinolone signal (PQS). FEMS Microbiol Lett, 230, 27-34.

635. Castang, S., McManus, H.R., Turner, K.H. and Dove, S.L. (2008) H-NS family members function coordinately in an opportunistic pathogen. Proc Natl Acad Sci US A, 105, 18947-18952.

636. Rampioni, G., Schuster, M., Greenberg, E.P., Zennaro, E. and Leoni, L. (2009) Contribution of the RsaL global regulator to Pseudomonas aeruginosa virulence and biofilm formation. FEMS Microbiol Lett, 301, 210-217.

637. Wei, Q., Le Minh, P.N., Dotsch, A., Hildebrand, F., Panmanee, W., Elfarash, A., Schulz, S., Plaisance, S., Charlier, D., Hassett, D. et al. (2012) Global regulation of gene expression by OxyR in an important human opportunistic pathogen. Nucleic Acids Res, 40, 4320-4333.

638. Dieppois, G., Ducret, V., Caille, O. and Perron, K. (2012) The Transcriptional Regulator CzcR Modulates Antibiotic Resistance and Quorum Sensing in Pseudomonas aeruginosa. PLoS One, 7, e38148.

639. Perron, K., Caille, O., Rossier, C., Van Delden, C., Dumas, J.L. and Kohler, T. (2004) CzcR-CzcS, a two-component system involved in heavy metal and carbapenem resistance in Pseudomonas aeruginosa. J Biol Chem, 279, 8761-8768. 
640. Li, L.L., Malone, J.E. and Iglewski, B.H. (2007) Regulation of the Pseudomonas aeruginosa quorum-sensing regulator VqsR. J Bacteriol, 189, 4367-4374.

641. Liang, H., Deng, X., Ji, Q., Sun, F., Shen, T. and He, C. (2012) The Pseudomonas aeruginosa Global Regulator VqsR Directly Inhibits QscR To Control QuorumSensing and Virulence Gene Expression. J Bacteriol, 194, 3098-3108.

642. Lee, J.H., Lequette, Y. and Greenberg, E.P. (2006) Activity of purified QscR, a Pseudomonas aeruginosa orphan quorum-sensing transcription factor. Mol Microbiol, 59, 602-609.

643. Ledgham, F., Ventre, I., Soscia, C., Foglino, M., Sturgis, J.N. and Lazdunski, A. (2003) Interactions of the quorum sensing regulator QscR: interaction with itself and the other regulators of Pseudomonas aeruginosa LasR and RhlR. Mol Microbiol, 48, 199-210.

644. Dong, Y.H., Zhang, X.F., Xu, J.L., Tan, A.T. and Zhang, L.H. (2005) VqsM, a novel AraC-type global regulator of quorum-sensing signalling and virulence in Pseudomonas aeruginosa. Mol Microbiol, 58, 552-564.

645. Farrow, J.M., 3rd and Pesci, E.C. (2007) Two distinct pathways supply anthranilate as a precursor of the Pseudomonas quinolone signal. J Bacteriol., 189, 3425-3433.

646. Siehnel, R., Traxler, B., An, D.D., Parsek, M.R., Schaefer, A.L. and Singh, P.K. (2010) A unique regulator controls the activation threshold of quorum-regulated genes in Pseudomonas aeruginosa. Proc Natl Acad Sci U S A, 107, 7916-7921.

647. Sonnleitner, E., Schuster, M., Sorger-Domenigg, T., Greenberg, E.P. and Blasi, U. (2006) Hfq-dependent alterations of the transcriptome profile and effects on quorum sensing in Pseudomonas aeruginosa. Mol Microbiol, 59, 1542-1558.

648. Rodrigue, A., Quentin, Y., Lazdunski, A., Mejean, V. and Foglino, M. (2000) Two-component systems in Pseudomonas aeruginosa: why so many? Trends Microbiol., 8, 498-504.

649. Raghavan, V. and Groisman, E.A. (2010) Orphan and hybrid two-component system proteins in health and disease. Curr Opin Microbiol, 13, 226-231. 
650. Stock, A.M., Robinson, V.L. and Goudreau, P.N. (2000) Two-component signal transduction. Апnu Rev Biochem, 69, 183-215.

651. Laub, M.T. and Goulian, M. (2007) Specificity in two-component signal transduction pathways. Annu Rev Genet., 41, 121-145.

652. Mascher, T., Helmann, J.D. and Unden, G. (2006) Stimulus perception in bacterial signal-transducing histidine kinases. Microbiol Mol Biol Rev, 70, 910938.

653. Gao, R. and Stock, A.M. (2010) Molecular strategies for phosphorylationmediated regulation of response regulator activity. Curr Opin Microbiol, 13, 160167.

654. Mitrophanov, A.Y. and Groisman, E.A. (2008) Signal integration in bacterial twocomponent regulatory systems. Genes Dev, 22, 2601-2611.

655. Kitten, T., Kinscherf, T.G., McEvoy, J.L. and Willis, D.K. (1998) A newly identified regulator is required for virulence and toxin production in Pseudomonas syringae. Mol Microbiol, 28, 917-929.

656. Coleman, F.T., Mueschenborn, S., Meluleni, G., Ray, C., Carey, V.J., Vargas, S.O., Cannon, C.L., Ausubel, F.M. and Pier, G.B. (2003) Hypersusceptibility of cystic fibrosis mice to chronic Pseudomonas aeruginosa oropharyngeal colonization and lung infection. Proc Natl Acad Sci U S A, 100, 1949-1954.

657. Rahme, L.G., Ausubel, F.M., Cao, H., Drenkard, E., Goumnerov, B.C., Lau, G.W., Mahajan-Miklos, S., Plotnikova, J., Tan, M.W., Tsongalis, J. et al. (2000) Plants and animals share functionally common bacterial virulence factors. Proc Natl Acad Sci U S A, 97, 8815-8821.

658. Hrabak, E.M. and Willis, D.K. (1992) The lemA gene required for pathogenicity of Pseudomonas syringae pv. syringae on bean is a member of a family of twocomponent regulators. J Bacteriol, 174, 3011-3020.

659. Goodman, A.L., Merighi, M., Hyodo, M., Ventre, I., Filloux, A. and Lory, S. (2009) Direct interaction between sensor kinase proteins mediates acute and chronic disease phenotypes in a bacterial pathogen. Genes Dev, 23, 249-259. 
660. Linares, J.F., Gustafsson, I., Baquero, F. and Martinez, J.L. (2006) Antibiotics as intermicrobial signaling agents instead of weapons. Proc Natl Acad Sci U S A, 103, 19484-19489.

661. McPhee, J.B., Bains, M., Winsor, G., Lewenza, S., Kwasnicka, A., Brazas, M.D., Brinkman, F.S. and Hancock, R.E. (2006) Contribution of the PhoP-PhoQ and PmrA-PmrB two-component regulatory systems to $\mathrm{Mg} 2+$-induced gene regulation in Pseudomonas aeruginosa. J Bacteriol, 188, 3995-4006.

662. Macfarlane, E.L., Kwasnicka, A. and Hancock, R.E. (2000) Role of Pseudomonas aeruginosa PhoP-phoQ in resistance to antimicrobial cationic peptides and aminoglycosides. Microbiology, 146 ( Pt 10), 2543-2554.

663. Gooderham, W.J., Gellatly, S.L., Sanschagrin, F., McPhee, J.B., Bains, M., Cosseau, C., Levesque, R.C. and Hancock, R.E. (2009) The sensor kinase PhoQ mediates virulence in Pseudomonas aeruginosa. Microbiology, 155, 699-711.

664. McPhee, J.B., Lewenza, S. and Hancock, R.E. (2003) Cationic antimicrobial peptides activate a two-component regulatory system, PmrA-PmrB, that regulates resistance to polymyxin B and cationic antimicrobial peptides in Pseudomonas aeruginosa. Mol Microbiol, 50, 205-217.

665. Kwon, D.H. and Lu, C.D. (2006) Polyamines induce resistance to cationic peptide, aminoglycoside, and quinolone antibiotics in Pseudomonas aeruginosa PAO1. Antimicrob Agents Chemother, 50, 1615-1622.

666. Kwon, D.H. and Lu, C.D. (2007) Polyamine effects on antibiotic susceptibility in bacteria. Antimicrob Agents Chemother, 51, 2070-2077.

667. Flemming, H.C. and Wingender, J. (2010) The biofilm matrix. Nat Rev Microbiol, 8, 623-633.

668. Donlan, R.M. and Costerton, J.W. (2002) Biofilms: survival mechanisms of clinically relevant microorganisms. Clin Microbiol Rev, 15, 167-193.

669. Lopez, D., Vlamakis, H. and Kolter, R. (2010) Biofilms. Cold Spring Harb Perspect Biol, 2, a000398. 
670. Stapper, A.P., Narasimhan, G., Ohman, D.E., Barakat, J., Hentzer, M., Molin, S., Kharazmi, A., Hoiby, N. and Mathee, K. (2004) Alginate production affects Pseudomonas aeruginosa biofilm development and architecture, but is not essential for biofilm formation. J Med Microbiol, 53, 679-690.

671. Klausen, M., Heydorn, A., Ragas, P., Lambertsen, L., Aaes-Jorgensen, A., Molin, S. and Tolker-Nielsen, T. (2003) Biofilm formation by Pseudomonas aeruginosa wild type, flagella and type IV pili mutants. Mol Microbiol, 48, 1511-1524.

672. Caiazza, N.C. and O'Toole, G.A. (2004) SadB is required for the transition from reversible to irreversible attachment during biofilm formation by Pseudomonas aeruginosa PA14. J Bacteriol, 186, 4476-4485.

673. Merritt, J.H., Brothers, K.M., Kuchma, S.L. and O'Toole, G.A. (2007) SadC reciprocally influences biofilm formation and swarming motility via modulation of exopolysaccharide production and flagellar function. $J$ Bacteriol, 189, 81548164.

674. Vallet, I., Olson, J.W., Lory, S., Lazdunski, A. and Filloux, A. (2001) The chaperone/usher pathways of Pseudomonas aeruginosa: identification of fimbrial gene clusters (cup) and their involvement in biofilm formation. Proc Natl Acad Sci U S A, 98, 6911-6916.

675. Vallet, I., Diggle, S.P., Stacey, R.E., Camara, M., Ventre, I., Lory, S., Lazdunski, A., Williams, P. and Filloux, A. (2004) Biofilm formation in Pseudomonas aeruginosa: fimbrial cup gene clusters are controlled by the transcriptional regulator MvaT. J Bacteriol, 186, 2880-2890.

676. Vallet-Gely, I., Donovan, K.E., Fang, R., Joung, J.K. and Dove, S.L. (2005) Repression of phase-variable cup gene expression by H-NS-like proteins in Pseudomonas aeruginosa. Proc Natl Acad Sci U S A, 102, 11082-11087.

677. Merkel, T.J., Barros, C. and Stibitz, S. (1998) Characterization of the $b v g R$ locus of Bordetella pertussis. J Bacteriol, 180, 1682-1690.

678. Mikkelsen, H., Ball, G., Giraud, C. and Filloux, A. (2009) Expression of Pseudomonas aeruginosa CupD fimbrial genes is antagonistically controlled by RcsB and the EAL-containing PvrR response regulators. PLoS One, 4, e6018. 
679. Hickman, J.W., Tifrea, D.F. and Harwood, C.S. (2005) A chemosensory system that regulates biofilm formation through modulation of cyclic diguanylate levels. Proc Natl Acad Sci U S A, 102, 14422-14427.

680. Guvener, Z.T. and Harwood, C.S. (2007) Subcellular location characteristics of the Pseudomonas aeruginosa GGDEF protein, WspR, indicate that it produces cyclic-di-GMP in response to growth on surfaces. Mol Microbiol, 66, 1459-1473.

681. Choy, W.K., Zhou, L., Syn, C.K., Zhang, L.H. and Swarup, S. (2004) MorA defines a new class of regulators affecting flagellar development and biofilm formation in diverse Pseudomonas species. J Bacteriol, 186, 7221-7228.

682. Ueda, A. and Wood, T.K. (2009) Connecting quorum sensing, c-di-GMP, pel polysaccharide, and biofilm formation in Pseudomonas aeruginosa through tyrosine phosphatase TpbA (PA3885). PLoS Pathog, 5, e1000483.

683. Hickman, J.W. and Harwood, C.S. (2008) Identification of FleQ from Pseudomonas aeruginosa as a c-di-GMP-responsive transcription factor. $\mathrm{Mol}$ Microbiol, 69, 376-389.

684. Attila, C., Ueda, A. and Wood, T.K. (2008) PA2663 (PpyR) increases biofilm formation in Pseudomonas aeruginosa PAO1 through the psl operon and stimulates virulence and quorum-sensing phenotypes. Appl Microbiol Biotechnol, 78, 293-307.

685. D'Argenio, D.A., Calfee, M.W., Rainey, P.B. and Pesci, E.C. (2002) Autolysis and autoaggregation in Pseudomonas aeruginosa colony morphology mutants. $J$ Bacteriol, 184, 6481-6489.

686. Allesen-Holm, M., Barken, K.B., Yang, L., Klausen, M., Webb, J.S., Kjelleberg, S., Molin, S., Givskov, M. and Tolker-Nielsen, T. (2006) A characterization of DNA release in Pseudomonas aeruginosa cultures and biofilms. Mol Microbiol, 59, 1114-1128.

687. Heurlier, K., Denervaud, V., Haenni, M., Guy, L., Krishnapillai, V. and Haas, D. (2005) Quorum-sensing-negative (lasR) mutants of Pseudomonas aeruginosa avoid cell lysis and death. $J$ Bacteriol, 187, 4875-4883. 
688. Whitchurch, C.B., Tolker-Nielsen, T., Ragas, P.C. and Mattick, J.S. (2002) Extracellular DNA required for bacterial biofilm formation. Science, 295, 1487.

689. Dominiak, D.M., Nielsen, J.L. and Nielsen, P.H. (2010) Extracellular DNA is abundant and important for microcolony strength in mixed microbial biofilms. Environ Microbiol.

690. Pamp, S.J. and Tolker-Nielsen, T. (2007) Multiple roles of biosurfactants in structural biofilm development by Pseudomonas aeruginosa. J Bacteriol, 189, 2531-2539.

691. Davey, M.E., Caiazza, N.C. and O'Toole, G.A. (2003) Rhamnolipid surfactant production affects biofilm architecture in Pseudomonas aeruginosa PAO1. $J$ Bacteriol, 185, 1027-1036.

692. Petrova, O.E. and Sauer, K. (2011) SagS contributes to the motile-sessile switch and acts in concert with BfiSR to enable Pseudomonas aeruginosa biofilm formation. J Bacteriol., 193, 6614-6628.

693. Janjua, H.A., Segata, N., Bernabo, P., Tamburini, S., Ellen, A. and Jousson, O. (2012) Clinical populations of Pseudomonas aeruginosa isolated from acute infections show a wide virulence range partially correlated with population structure and virulence gene expression. Microbiology, 158, 2089-2098.

694. Storey, D.G., Ujack, E.E., Rabin, H.R. and Mitchell, I. (1998) Pseudomonas aeruginosa las $R$ transcription correlates with the transcription of las $A$, las $B$, and toxA in chronic lung infections associated with cystic fibrosis. Infect Immun, 66, 2521-2528.

695. Singh, P.K., Schaefer, A.L., Parsek, M.R., Moninger, T.O., Welsh, M.J. and Greenberg, E.P. (2000) Quorum-sensing signals indicate that cystic fibrosis lungs are infected with bacterial biofilms. Nature, 407, 762-764.

696. Wu, H., Song, Z., Givskov, M., Doring, G., Worlitzsch, D., Mathee, K., Rygaard, J. and Hoiby, N. (2001) Pseudomonas aeruginosa mutations in lasI and rhlI quorum sensing systems result in milder chronic lung infection. Microbiology, 147, 1105-1113. 
697. Bjarnsholt, T., Jensen, P.O., Burmolle, M., Hentzer, M., Haagensen, J.A., Hougen, H.P., Calum, H., Madsen, K.G., Moser, C., Molin, S. et al. (2005) Pseudomonas aeruginosa tolerance to tobramycin, hydrogen peroxide and polymorphonuclear leukocytes is quorum-sensing dependent. Microbiology, 151, 373-383.

698. Purevdorj, B., Costerton, J.W. and Stoodley, P. (2002) Influence of hydrodynamics and cell signaling on the structure and behavior of Pseudomonas aeruginosa biofilms. Appl Environ Microbiol., 68, 4457-4464.

699. Heydorn, A., Ersboll, B., Kato, J., Hentzer, M., Parsek, M.R., Tolker-Nielsen, T., Givskov, M. and Molin, S. (2002) Statistical analysis of Pseudomonas aeruginosa biofilm development: impact of mutations in genes involved in twitching motility, cell-to-cell signaling, and stationary-phase sigma factor expression. Appl Environ Microbiol., 68, 2008-2017.

700. Smith, E.E., Buckley, D.G., Wu, Z., Saenphimmachak, C., Hoffman, L.R., D'Argenio, D.A., Miller, S.I., Ramsey, B.W., Speert, D.P., Moskowitz, S.M. et al. (2006) Genetic adaptation by Pseudomonas aeruginosa to the airways of cystic fibrosis patients. PNAS, 103, 8487-8492.

701. Van Delden, C., Pesci, E.C., Pearson, J.P. and Iglewski, B.H. (1998) Starvation selection restores elastase and rhamnolipid production in a Pseudomonas aeruginosa quorum-sensing mutant. Infect Immun., 66, 4499-4502.

702. Kirisits, M.J. and Parsek, M.R. (2006) Does Pseudomonas aeruginosa use intercellular signalling to build biofilm communities? Cell Microbiol., 8, 18411849.

703. Lewis, K. (2008) Multidrug tolerance of biofilms and persister cells. Curr Top Microbiol Immunol., 322, 107-131.

704. Keren, I., Kaldalu, N., Spoering, A., Wang, Y. and Lewis, K. (2004) Persister cells and tolerance to antimicrobials. FEMS Microbiol Lett., 230, 13-18.

705. Keren, I., Shah, D., Spoering, A., Kaldalu, N. and Lewis, K. (2004) Specialized persister cells and the mechanism of multidrug tolerance in Escherichia coli. $J$ Bacteriol., 186, 8172-8180. 
706. De Groote, V.N., Verstraeten, N., Fauvart, M., Kint, C.I., Verbeeck, A.M., Beullens, S., Cornelis, P. and Michiels, J. (2009) Novel persistence genes in Pseudomonas aeruginosa identified by high-throughput screening. FEMS Microbiol Lett., 297, 73-79.

707. Lewis, K. (2010) Persister cells. Annu Rev Microbiol., 64, 357-372.

708. Pressler, T., Bohmova, C., Conway, S., Dumcius, S., Hjelte, L., Hoiby, N., Kollberg, H., Tummler, B. and Vavrova, V. (2011) Chronic Pseudomonas aeruginosa infection definition: EuroCareCF Working Group report. J Cyst Fibros., 10, S75-78.

709. de Jong, P.A., Nakano, Y., Lequin, M.H., Mayo, J.R., Woods, R., Pare, P.D. and Tiddens, H.A. (2004) Progressive damage on high resolution computed tomography despite stable lung function in cystic fibrosis. Eur Respir J, 23, 93-97.

710. Meyer, K.C. and Sharma, A. (1997) Regional variability of lung inflammation in cystic fibrosis. Am J Respir Crit Care Med, 156, 1536-1540.

711. Tiddens, H.A. (2002) Detecting early structural lung damage in cystic fibrosis. Pediatr Pulmonol, 34, 228-231.

712. Worlitzsch, D., Tarran, R., Ulrich, M., Schwab, U., Cekici, A., Meyer, K.C., Birrer, P., Bellon, G., Berger, J., Weiss, T. et al. (2002) Effects of reduced mucus oxygen concentration in airway Pseudomonas infections of cystic fibrosis patients. J Clin Invest, 109, 317-325.

713. Doggett, R.G., Harrison, G.M. and Carter, R.E. (1971) Mucoid Pseudomonas aeruginosa in patients with chronic illnesses. Lancet, 1, 236-237.

714. Hoiby, N. (1974) Pseudomonas aeruginosa infection in cystic fibrosis. Relationship between mucoid strains of Pseudomonas aeruginosa and the humoral immune response. Acta Pathol Microbiol Scand [B] Microbiol Immunol, 82, 551-558.

715. Evans, L.R. and Linker, A. (1973) Production and characterization of the slime polysaccharide of Pseudomonas aeruginosa.J Bacteriol, 116, 915-924. 
716. Pedersen, S.S., Espersen, F., Hoiby, N. and Jensen, T. (1990) Immunoglobulin A and immunoglobulin $\mathrm{G}$ antibody responses to alginates from Pseudomonas aeruginosa in patients with cystic fibrosis. J Clin Microbiol, 28, 747-755.

717. Pier, G.B., Coleman, F., Grout, M., Franklin, M. and Ohman, D.E. (2001) Role of alginate $\mathrm{O}$ acetylation in resistance of mucoid Pseudomonas aeruginosa to opsonic phagocytosis. Infect Immun, 69, 1895-1901.

718. Simpson, J.A., Smith, S.E. and Dean, R.T. (1988) Alginate inhibition of the uptake of Pseudomonas aeruginosa by macrophages. J Gen Microbiol, 134, 2936.

719. Govan, J.R. and Fyfe, J.A. (1978) Mucoid Pseudomonas aeruginosa and cystic fibrosis: resistance of the mucoid from to carbenicillin, flucloxacillin and tobramycin and the isolation of mucoid variants in vitro. J Antimicrob Chemother, 4, 233-240.

720. Speert, D.P., Farmer, S.W., Campbell, M.E., Musser, J.M., Selander, R.K. and Kuo, S. (1990) Conversion of Pseudomonas aeruginosa to the phenotype characteristic of strains from patients with cystic fibrosis. $J$ Clin Microbiol, 28, 188-194.

721. Terry, J.M., Pina, S.E. and Mattingly, S.J. (1991) Environmental conditions which influence mucoid conversion Pseudomonas aeruginosa PAO1. Infect Immun, 59, $471-477$

722. Martin, D.W., Holloway, B.W. and Deretic, V. (1993) Characterization of a locus determining the mucoid status of Pseudomonas aeruginosa: AlgU shows sequence similarities with a Bacillus sigma factor. J Bacteriol, 175, 1153-1164.

723. Martin, D.W., Schurr, M.J., Mudd, M.H. and Deretic, V. (1993) Differentiation of Pseudomonas aeruginosa into the alginate-producing form: inactivation of mисB causes conversion to mucoidy. Mol Microbiol, 9, 497-506.

724. Mathee, K., Kharazami, A.A. \& Hoiby, N. (2002) In McLean, R. J. C. (ed.), Molecular Ecology of Biofilms. Horizon, Norfolk, pp. 23-55. 
725. Goldberg, J.B., Gorman, W.L., Flynn, J.L. and Ohman, D.E. (1993) A mutation in $\operatorname{alg} N$ permits trans activation of alginate production by algT in Pseudomonas species. J Bacteriol, 175, 1303-1308.

726. Ohman, D. (2009) In Rehm, B. H. A. (ed.), Alginates: Biology and Applications. Springer Berlin / Heidelberg, Vol. 13, pp. 117-133.

727. Damron, F.H. and Yu, H.D. (2011) Pseudomonas aeruginosa MucD regulates the alginate pathway through activation of MucA degradation via MucP proteolytic activity. J Bacteriol, 193, 286-291.

728. Wozniak, D.J., Sprinkle, A.B. and Baynham, P.J. (2003) Control of Pseudomonas aeruginosa algZ expression by the alternative sigma factor AlgT. $J$ Bacteriol, 185, 7297-7300.

729. Chitnis, C.E. and Ohman, D.E. (1993) Genetic analysis of the alginate biosynthetic gene cluster of Pseudomonas aeruginosa shows evidence of an operonic structure. Mol Microbiol, 8, 583-593.

730. Deretic, V., Gill, J.F. and Chakrabarty, A.M. (1987) Gene algD coding for GDPmannose dehydrogenase is transcriptionally activated in mucoid Pseudomonas aeruginosa. J Bacteriol, 169, 351-358.

731. Leech, A.J., Sprinkle, A., Wood, L., Wozniak, D.J. and Ohman, D.E. (2008) The NtrC family regulator $\mathrm{AlgB}$, which controls alginate biosynthesis in mucoid Pseudomonas aeruginosa, binds directly to the algD promoter. J Bacteriol, 190, 581-589.

732. Kato, J. and Chakrabarty, A.M. (1991) Purification of the regulatory protein AlgR 1 and its binding in the far upstream region of the $\operatorname{alg} D$ promoter in Pseudomonas aeruginosa. Proc Natl Acad Sci U S A, 88, 1760-1764.

733. Mohr, C.D., Leveau, J.H., Krieg, D.P., Hibler, N.S. and Deretic, V. (1992) AlgRbinding sites within the algD promoter make up a set of inverted repeats separated by a large intervening segment of DNA. J Bacteriol, 174, 6624-6633.

734. Mohr, C.D., Martin, D.W., Konyecsni, W.M., Govan, J.R., Lory, S. and Deretic, V. (1990) Role of the far-upstream sites of the $\operatorname{alg} D$ promoter and the $\operatorname{alg} R$ and 
rpoN genes in environmental modulation of mucoidy in Pseudomonas aeruginosa. J Bacteriol, 172, 6576-6580.

735. Baynham, P.J., Brown, A.L., Hall, L.L. and Wozniak, D.J. (1999) Pseudomonas aeruginosa AlgZ, a ribbon-helix-helix DNA-binding protein, is essential for alginate synthesis and $\operatorname{alg} D$ transcriptional activation. Mol Microbiol, 33, 10691080 .

736. Damron, F.H., Qiu, D. and Yu, H.D. (2009) The Pseudomonas aeruginosa sensor kinase KinB negatively controls alginate production through AlgW-dependent MucA proteolysis. J Bacteriol, 191, 2285-2295.

737. Wood, L.F., Leech, A.J. and Ohman, D.E. (2006) Cell wall-inhibitory antibiotics activate the alginate biosynthesis operon in Pseudomonas aeruginosa: Roles of sigma (AlgT) and the AlgW and Prc proteases. Mol Microbiol, 62, 412-426.

738. Ma, S., Selvaraj, U., Ohman, D.E., Quarless, R., Hassett, D.J. and Wozniak, D.J. (1998) Phosphorylation-independent activity of the response regulators AlgB and AlgR in promoting alginate biosynthesis in mucoid Pseudomonas aeruginosa. $J$ Bacteriol, 180, 956-968.

739. Zegans, M.E., Wozniak, D., Griffin, E., Toutain-Kidd, C.M., Hammond, J.H., Garfoot, A. and Lam, J.S. (2012) Pseudomonas aeruginosa exopolysaccharide Psl promotes resistance to the biofilm inhibitor polysorbate 80. Antimicrob Agents Chemother.

740. Fujiwara, S., Zielinski, N.A. and Chakrabarty, A.M. (1993) Enhancer-like activity of A1gR1-binding site in alginate gene activation: positional, orientational, and sequence specificity. J Bacteriol, 175, 5452-5459.

741. Ye, R.W., Zielinski, N.A. and Chakrabarty, A.M. (1994) Purification and characterization of phosphomannomutase/phosphoglucomutase from Pseudomonas aeruginosa involved in biosynthesis of both alginate and lipopolysaccharide. J Bacteriol, 176, 4851-4857.

742. Zielinski, N.A., Maharaj, R., Roychoudhury, S., Danganan, C.E., Hendrickson, W. and Chakrabarty, A.M. (1992) Alginate synthesis in Pseudomonas aeruginosa: environmental regulation of the algC promoter. J Bacteriol, 174, 7680-7688. 
743. Morici, L.A., Carterson, A.J., Wagner, V.E., Frisk, A., Schurr, J.R., Honer zu Bentrup, K., Hassett, D.J., Iglewski, B.H., Sauer, K. and Schurr, M.J. (2007) Pseudomonas aeruginosa AlgR represses the Rhl quorum-sensing system in a biofilm-specific manner. J Bacteriol, 189, 7752-7764.

744. Belete, B., Lu, H. and Wozniak, D.J. (2008) Pseudomonas aeruginosa AlgR regulates type IV pilus biosynthesis by activating transcription of the fimUpilVWXY1Y2E operon. J Bacteriol, 190, 2023-2030.

745. Lizewski, S.E., Lundberg, D.S. and Schurr, M.J. (2002) The transcriptional regulator AlgR is essential for Pseudomonas aeruginosa pathogenesis. Infect Immun, 70, 6083-6093.

746. Cody, W.L., Pritchett, C.L., Jones, A.K., Carterson, A.J., Jackson, D., Frisk, A., Wolfgang, M.C. and Schurr, M.J. (2009) Pseudomonas aeruginosa AlgR controls cyanide production in an AlgZ-dependent manner. J Bacteriol, 191, 2993-3002.

747. Jones, A.K., Fulcher, N.B., Balzer, G.J., Urbanowski, M.L., Pritchett, C.L., Schurr, M.J., Yahr, T.L. and Wolfgang, M.C. (2010) Activation of the Pseudomonas aeruginosa AlgU regulon through mucA mutation inhibits cyclic AMP/Vfr signaling. J Bacteriol., 192, 5709-5717.

748. Lizewski, S.E., Schurr, J.R., Jackson, D.W., Frisk, A., Carterson, A.J. and Schurr, M.J. (2004) Identification of AlgR-regulated genes in Pseudomonas aeruginosa by use of microarray analysis. $J$ Bacteriol, 186, 5672-5684.

749. Remminghorst, U. and Rehm, B.H. (2006) Alg44, a unique protein required for alginate biosynthesis in Pseudomonas aeruginosa. FEBS Lett, 580, 3883-3888.

750. Hay, I.D., Remminghorst, U. and Rehm, B.H. (2009) MucR, a novel membraneassociated regulator of alginate biosynthesis in Pseudomonas aeruginosa. Appl Environ Microbiol, 75, 1110-1120.

751. Merighi, M., Lee, V.T., Hyodo, M., Hayakawa, Y. and Lory, S. (2007) The second messenger bis-(3'-5')-cyclic-GMP and its PilZ domain-containing receptor Alg44 are required for alginate biosynthesis in Pseudomonas aeruginosa. Mol Microbiol, 65, 876-895. 
752. Visca, P., Imperi, F. and Lamont, I.L. (2007) Pyoverdine siderophores: from biogenesis to biosignificance. Trends Microbiol, 15, 22-30.

753. Poole, K. and McKay, G.A. (2003) Iron acquisition and its control in Pseudomonas aeruginosa: many roads lead to Rome. Front Biosci, 8, d661-686.

754. Ochsner, U.A., Johnson, Z. and Vasil, M.L. (2000) Genetics and regulation of two distinct haem-uptake systems, phu and has, in Pseudomonas aeruginosa. Microbiology, 146 ( Pt 1), 185-198.

755. Touati, D. (2000) Iron and oxidative stress in bacteria. Arch Biochem Biophys, 373, 1-6.

756. Vasil, M.L. and Ochsner, U.A. (1999) The response of Pseudomonas aeruginosa to iron: genetics, biochemistry and virulence. Mol Microbiol, 34, 399-413.

757. Pohl, E., Haller, J.C., Mijovilovich, A., Meyer-Klaucke, W., Garman, E. and Vasil, M.L. (2003) Architecture of a protein central to iron homeostasis: crystal structure and spectroscopic analysis of the ferric uptake regulator. Mol Microbiol, 47, 903-915.

758. Wilderman, P.J., Sowa, N.A., FitzGerald, D.J., FitzGerald, P.C., Gottesman, S., Ochsner, U.A. and Vasil, M.L. (2004) Identification of tandem duplicate regulatory small RNAs in Pseudomonas aeruginosa involved in iron homeostasis. Proc Natl Acad Sci U S A, 101, 9792-9797.

759. Cornelis, P., Matthijs, S. and Van Oeffelen, L. (2009) Iron uptake regulation in Pseudomonas aeruginosa. Biometals, 22, 15-22.

760. Ochsner, U.A. and Vasil, M.L. (1996) Gene repression by the ferric uptake regulator in Pseudomonas aeruginosa: cycle selection of iron-regulated genes. Proc Natl Acad Sci U S A, 93, 4409-4414.

761. Wilderman, P.J., Vasil, A.I., Johnson, Z., Wilson, M.J., Cunliffe, H.E., Lamont, I.L. and Vasil, M.L. (2001) Characterization of an endoprotease (PrpL) encoded by a PvdS-regulated gene in Pseudomonas aeruginosa. Infect Immun, 69, 53855394. 
762. Ochsner, U.A., Johnson, Z., Lamont, I.L., Cunliffe, H.E. and Vasil, M.L. (1996) Exotoxin A production in Pseudomonas aeruginosa requires the iron-regulated $p v d S$ gene encoding an alternative sigma factor. Mol Microbiol, 21, 1019-1028.

763. Gaines, J.M., Carty, N.L., Tiburzi, F., Davinic, M., Visca, P., Colmer-Hamood, J.A. and Hamood, A.N. (2007) Regulation of the Pseudomonas aeruginosa toxA, regA and $p t x R$ genes by the iron-starvation sigma factor $\mathrm{PvdS}$ under reduced levels of oxygen. Microbiology, 153, 4219-4233.

764. Xiong, Y.Q., Vasil, M.L., Johnson, Z., Ochsner, U.A. and Bayer, A.S. (2000) The oxygen- and iron-dependent sigma factor $p v d S$ of Pseudomonas aeruginosa is an important virulence factor in experimental infective endocarditis. J Infect Dis, 181, 1020-1026.

765. Singh, P.K., Parsek, M.R., Greenberg, E.P. and Welsh, M.J. (2002) A component of innate immunity prevents bacterial biofilm development. Nature, 417, 552-555.

766. Singh, P.K. (2004) Iron sequestration by human lactoferrin stimulates $P$. aeruginosa surface motility and blocks biofilm formation. Biometals, 17, 267-270.

767. Banin, E., Vasil, M.L. and Greenberg, E.P. (2005) Iron and Pseudomonas aeruginosa biofilm formation. Proc Natl Acad Sci U S A, 102, 11076-11081.

768. Yang, L., Barken, K.B., Skindersoe, M.E., Christensen, A.B., Givskov, M. and Tolker-Nielsen, T. (2007) Effects of iron on DNA release and biofilm development by Pseudomonas aeruginosa. Microbiology, 153, 1318-1328.

769. Bollinger, N., Hassett, D.J., Iglewski, B.H., Costerton, J.W. and McDermott, T.R. (2001) Gene expression in Pseudomonas aeruginosa: evidence of iron override effects on quorum sensing and biofilm-specific gene regulation. $J$ Bacteriol, 183, 1990-1996.

770. Kim, E.J., Wang, W., Deckwer, W.D. and Zeng, A.P. (2005) Expression of the quorum-sensing regulatory protein LasR is strongly affected by iron and oxygen concentrations in cultures of Pseudomonas aeruginosa irrespective of cell density. Microbiology, 151, 1127-1138. 
771. Visca, P., Leoni, L., Wilson, M.J. and Lamont, I.L. (2002) Iron transport and regulation, cell signalling and genomics: lessons from Escherichia coli and Pseudomonas. Mol Microbiol, 45, 1177-1190.

772. Hazan, R., He, J., Xiao, G., Dekimpe, V., Apidianakis, Y., Lesic, B., Astrakas, C., Deziel, E., Lepine, F. and Rahme, L.G. (2010) Homeostatic interplay between bacterial cell-cell signaling and iron in virulence. PLoS Pathog, 6, e1000810.

773. Oglesby, A.G., Farrow, J.M., 3rd, Lee, J.H., Tomaras, A.P., Greenberg, E.P., Pesci, E.C. and Vasil, M.L. (2008) The influence of iron on Pseudomonas aeruginosa physiology: a regulatory link between iron and quorum sensing. $J$ Biol Chem, 283, 15558-15567.

774. Bleves, S., Viarre, V., Salacha, R., Michel, G.P., Filloux, A. and Voulhoux, R. (2010) Protein secretion systems in Pseudomonas aeruginosa: A wealth of pathogenic weapons. Int J Med Microbiol, 300, 534-543.

775. Guzzo, J., Pages, J.M., Duong, F., Lazdunski, A. and Murgier, M. (1991) Pseudomonas aeruginosa alkaline protease: evidence for secretion genes and study of secretion mechanism. J Bacteriol, 173, 5290-5297.

776. Wandersman, C. and Delepelaire, P. (2004) Bacterial iron sources: from siderophores to hemophores. Annu Rev Microbiol, 58, 611-647.

777. Hood, R.D., Singh, P., Hsu, F., Guvener, T., Carl, M.A., Trinidad, R.R., Silverman, J.M., Ohlson, B.B., Hicks, K.G., Plemel, R.L. et al. (2010) A type VI secretion system of Pseudomonas aeruginosa targets a toxin to bacteria. Cell Host Microbe, 7, 25-37.

778. Moscoso, J.A., Mikkelsen, H., Heeb, S., Williams, P. and Filloux, A. (2011) The Pseudomonas aeruginosa sensor RetS switches type III and type VI secretion via c-di-GMP signalling. Environ Microbiol., 13, 3128-3138.

779. Pearson, J.P., Pesci, E.C. and Iglewski, B.H. (1997) Roles of Pseudomonas aeruginosa las and rhl quorum-sensing systems in control of elastase and rhamnolipid biosynthesis genes. $J$ Bacteriol, 179, 5756-5767. 
780. Chapon-Herve, V., Akrim, M., Latifi, A., Williams, P., Lazdunski, A. and Bally, M. (1997) Regulation of the $x c p$ secretion pathway by multiple quorum-sensing modulons in Pseudomonas aeruginosa. Mol Microbiol, 24, 1169-1178.

781. Lazdunski, A.M., Ventre, I. and Sturgis, J.N. (2004) Regulatory circuits and communication in Gram-negative bacteria. Nat Rev Microbiol, 2, 581-592.

782. Filloux, A., Bally, M., Soscia, C., Murgier, M. and Lazdunski, A. (1988) Phosphate regulation in Pseudomonas aeruginosa: cloning of the alkaline phosphatase gene and identification of $p h o B$ - and phoR-like genes. Mol Gen Genet, 212, 510-513.

783. Ball, G., Durand, E., Lazdunski, A. and Filloux, A. (2002) A novel type II secretion system in Pseudomonas aeruginosa. Mol Microbiol, 43, 475-485.

784. Llamas, M.A., van der Sar, A., Chu, B.C., Sparrius, M., Vogel, H.J. and Bitter, W. (2009) A Novel extracytoplasmic function (ECF) sigma factor regulates virulence in Pseudomonas aeruginosa. PLoS Pathog, 5, e1000572.

785. Lesic, B., Starkey, M., He, J., Hazan, R. and Rahme, L.G. (2009) Quorum sensing differentially regulates Pseudomonas aeruginosa type VI secretion locus I and homologous loci II and III, which are required for pathogenesis. Microbiology, 155, 2845-2855.

786. Frank, D.W. (1997) The exoenzyme S regulon of Pseudomonas aeruginosa. Mol Microbiol, 26, 621-629.

787. Vallis, A.J., Yahr, T.L., Barbieri, J.T. and Frank, D.W. (1999) Regulation of ExoS production and secretion by Pseudomonas aeruginosa in response to tissue culture conditions. Infect Immun, 67, 914-920.

788. Hovey, A.K. and Frank, D.W. (1995) Analyses of the DNA-binding and transcriptional activation properties of ExsA, the transcriptional activator of the Pseudomonas aeruginosa exoenzyme S regulon. J Bacteriol, 177, 4427-4436.

789. Dasgupta, N., Lykken, G.L., Wolfgang, M.C. and Yahr, T.L. (2004) A novel antianti-activator mechanism regulates expression of the Pseudomonas aeruginosa type III secretion system. Mol Microbiol, 53, 297-308. 
790. Hornef, M.W., Roggenkamp, A., Geiger, A.M., Hogardt, M., Jacobi, C.A. and Heesemann, J. (2000) Triggering the ExoS regulon of Pseudomonas aeruginosa: A GFP-reporter analysis of exoenzyme (Exo) S, ExoT and ExoU synthesis. Microb Pathog, 29, 329-343.

791. Rietsch, A. and Mekalanos, J.J. (2006) Metabolic regulation of type III secretion gene expression in Pseudomonas aeruginosa. Mol Microbiol, 59, 807-820.

792. Dacheux, D., Epaulard, O., de Groot, A., Guery, B., Leberre, R., Attree, I., Polack, B. and Toussaint, B. (2002) Activation of the Pseudomonas aeruginosa type III secretion system requires an intact pyruvate dehydrogenase $a c e A B$ operon. Infect Immun, 70, 3973-3977.

793. Wu, W., Badrane, H., Arora, S., Baker, H.V. and Jin, S. (2004) MucA-mediated coordination of type III secretion and alginate synthesis in Pseudomonas aeruginosa. J Bacteriol, 186, 7575-7585.

794. Jin, Y., Yang, H., Qiao, M. and Jun, S. (2011) MexT Regulates the Type III Secretion System through MexS and PtrC in Pseudomonas aeruginosa. $J$ Bacteriol, 193, 399-410.

795. Dubnau, D. and Losick, R. (2006) Bistability in bacteria. Mol Microbiol, 61, 564572.

796. Storz, G., Altuvia, S. and Wassarman, K.M. (2005) An abundance of RNA regulators. Аnпи Rev Biochem., 74, 199-217.

797. Papenfort, K. and Vogel, J. (2009) Multiple target regulation by small noncoding RNAs rewires gene expression at the post-transcriptional level. Res Microbiol., 160, 278-287.

798. Gottesman, S. (2004) The small RNA regulators of Escherichia coli: roles and mechanisms. Anпu Rev Microbiol., 58, 303-328.

799. Ferrara, S., Brugnoli, M., De Bonis, A., Righetti, F., Delvillani, F., Deho, G., Horner, D., Briani, F. and Bertoni, G. (2012) Comparative profiling of Pseudomonas aeruginosa strains reveals differential expression of novel unique and conserved small RNAs. PLoS ONE, 7, e36553. 
800. Heurlier, K., Williams, F., Heeb, S., Dormond, C., Pessi, G., Singer, D., Camara, M., Williams, P. and Haas, D. (2004) Positive control of swarming, rhamnolipid synthesis, and lipase production by the posttranscriptional RsmA/RsmZ system in Pseudomonas aeruginosa PAO1. J Bacteriol., 186, 2936-2945.

801. Kay, E., Dubuis, C. and Haas, D. (2005) Three small RNAs jointly ensure secondary metabolism and biocontrol in Pseudomonas fluorescens CHA0. PNAS, 102, 17136-17141.

802. Heeb, S., Kuehne, S.A., Bycroft, M., Crivii, S., Allen, M.D., Haas, D., Camara, M. and Williams, P. (2006) Functional analysis of the post-transcriptional regulator RsmA reveals a novel RNA-binding site. J Mol Biol., 355, 1026-1036.

803. Bordi, C., Lamy, M.C., Ventre, I., Termine, E., Hachani, A., Fillet, S., Roche, B., Bleves, S., Mejean, V., Lazdunski, A. et al. (2010) Regulatory RNAs and the $\mathrm{HptB} /$ RetS signalling pathways fine-tune Pseudomonas aeruginosa pathogenesis. Mol Microbiol., 76, 1427-1443.

804. Hsu, J.L., Chen, H.C., Peng, H.L. and Chang, H.Y. (2008) Characterization of the histidine-containing phosphotransfer protein B-mediated multistep phosphorelay system in Pseudomonas aeruginosa PAO1. J Biol Chem., 283, 9933-9944.

805. Lapouge, K., Schubert, M., Allain, F.H. and Haas, D. (2008) Gac/Rsm signal transduction pathway of gamma-proteobacteria: from RNA recognition to regulation of social behaviour. Mol Microbiol., 67, 241-253.

806. Sorger-Domenigg, T., Sonnleitner, E., Kaberdin, V.R. and Blasi, U. (2007) Distinct and overlapping binding sites of Pseudomonas aeruginosa $\mathrm{Hfq}$ and RsmA proteins on the non-coding RNA RsmY. Biochem Biophys Res Commun, 352, 769-773.

807. Moreno, R., Marzi, S., Romby, P. and Rojo, F. (2009) The Crc global regulator binds to an unpaired A-rich motif at the Pseudomonas putida alkS mRNA coding sequence and inhibits translation initiation. Nucleic Acids Res., 37, 7678-7690.

808. Moreno, R. and Rojo, F. (2008) The target for the Pseudomonas putida Crc global regulator in the benzoate degradation pathway is the BenR transcriptional regulator. J Bacteriol., 190, 1539-1545. 
809. Moreno, R., Ruiz-Manzano, A., Yuste, L. and Rojo, F. (2007) The Pseudomonas putida Crc global regulator is an RNA binding protein that inhibits translation of the AlkS transcriptional regulator. Mol Microbiol., 64, 665-675.

810. Sonnleitner, E., Abdou, L. and Haas, D. (2009) Small RNA as global regulator of carbon catabolite repression in Pseudomonas aeruginosa. PNAS, 106, 2186621871.

811. Yeung, A.T., Bains, M. and Hancock, R.E. (2011) The sensor kinase CbrA is a global regulator that modulates metabolism, virulence, and antibiotic resistance in Pseudomonas aeruginosa. J Bacteriol., 193, 918-931.

812. Abdou, L., Chou, H.T., Haas, D. and Lu, C.D. (2011) Promoter recognition and activation by the global response regulator CbrB in Pseudomonas aeruginosa. $J$ Bacteriol., 193, 2784-2792.

813. Li, W. and Lu, C.D. (2007) Regulation of carbon and nitrogen utilization by CbrAB and NtrBC two-component systems in Pseudomonas aeruginosa. $J$ Bacteriol, 189, 5413-5420.

814. Nishijyo, T., Haas, D. and Itoh, Y. (2001) The CbrA-CbrB two-component regulatory system controls the utilization of multiple carbon and nitrogen sources in Pseudomonas aeruginosa. Mol Microbiol., 40, 917-931.

815. Rietsch, A., Wolfgang, M.C. and Mekalanos, J.J. (2004) Effect of metabolic imbalance on expression of type III secretion genes in Pseudomonas aeruginosa. Infect Immun., 72, 1383-1390.

816. Linares, J.F., Moreno, R., Fajardo, A., Martinez-Solano, L., Escalante, R., Rojo, F. and Martinez, J.L. (2010) The global regulator Crc modulates metabolism, susceptibility to antibiotics and virulence in Pseudomonas aeruginosa. Environ Microbiol., 12, 3196-3212.

817. O'Toole, G.A., Gibbs, K.A., Hager, P.W., Phibbs, P.V., Jr. and Kolter, R. (2000) The global carbon metabolism regulator $\mathrm{Crc}$ is a component of a signal transduction pathway required for biofilm development by Pseudomonas aeruginosa. J Bacteriol., 182, 425-431. 
818. Sonnleitner, E., Romeo, A. and Blasi, U. (2012) Small regulatory RNAs in Pseudomonas aeruginosa. RNA Biol., 9.

819. Sonnleitner, E., Gonzalez, N., Sorger-Domenigg, T., Heeb, S., Richter, A.S., Backofen, R., Williams, P., Huttenhofer, A., Haas, D. and Blasi, U. (2011) The small RNA PhrS stimulates synthesis of the Pseudomonas aeruginosa quinolone signal. Mol. Microbiol., 80, 868-885.

820. Moll, I., Afonyushkin, T., Vytvytska, O., Kaberdin, V.R. and Blasi, U. (2003) Coincident Hfq binding and RNase E cleavage sites on mRNA and small regulatory RNAs. Rna, 9, 1308-1314.

821. Masse, E. and Gottesman, S. (2002) A small RNA regulates the expression of genes involved in iron metabolism in Escherichia coli. PNAS, 99, 4620-4625.

822. Oglesby-Sherrouse, A.G. and Vasil, M.L. (2010) Characterization of a hemeregulated non-coding RNA encoded by the prrF locus of Pseudomonas aeruginosa. PLoS ONE, 5, e9930.

823. Bornholdt, S. (2008) Boolean network models of cellular regulation: prospects and limitations. J R Soc Interface., 5 Suppl 1, S85-94.

824. Kauffman, S.A. (1969) Metabolic stability and epigenesis in randomly constructed genetic nets. $J$ Theor Biol., 22, 437-467.

825. Thomas, R. (1973) Boolean formalization of genetic control circuits. $J$ Theor Biol., 42, 563-585.

826. Shmulevich, I., Dougherty, E.R. and Zhang, W. (2002) Gene perturbation and intervention in probabilistic Boolean networks. Bioinformatics, 18, 1319-1331.

827. Shmulevich, I., Dougherty, E.R., Kim, S. and Zhang, W. (2002) Probabilistic Boolean Networks: a rule-based uncertainty model for gene regulatory networks. Bioinformatics, 18, 261-274.

828. Liang, J. and Han, J. (2012) Stochastic Boolean networks: An efficient approach to modeling gene regulatory networks. BMC Syst Biol., 6, 113. 
829. Babu, M.M. (2010) Early Career Research Award Lecture. Structure, evolution and dynamics of transcriptional regulatory networks. Biochem Soc Trans., 38, 1155-1178.

830. Babu, M.M., Lang, B. and Aravind, L. (2009) Methods to reconstruct and compare transcriptional regulatory networks. Methods Mol Biol., 541, 163-180.

831. Oestreicher, C. (2007) A history of chaos theory. Dialogues Clin Neurosci., 9, 279-289.

832. Goldbeter, A. (1996) Biochemical oscillations and cellular rythms. Cambridge University Press, Cambridge, UK.

833. Novak, B. and Tyson, J.J. (2008) Design principles of biochemical oscillators. Nat Rev Mol Cell Biol., 9, 981-991.

834. Leloup, J.C. and Goldbeter, A. (1999) Chaos and birhythmicity in a model for circadian oscillations of the PER and TIM proteins in drosophila. $J$ Theor Biol., 198, 445-459.

835. Suguna, C., Chowdhury, K.K. and Sinha, S. (1999) Minimal model for complex dynamics in cellular processes. Phys Rev E Stat Phys Plasmas Fluids Relat Interdiscip Topics, 60, 5943-5949.

836. Zhang, Z., Ye, W., Qian, Y., Zheng, Z., Huang, X. and Hu, G. (2012) Chaotic motifs in gene regulatory networks. PLoS ONE, 7, e39355.

837. Trosko, J.E. and Ruch, R.J. (1998) Cell-cell communication in carcinogenesis. Front Biosci., 3, d208-236.

838. Thomas, R. and D'Ari, R. (1990) Biological Feedback. CRC Press, Boca Raton, USA.

839. Dietz, H., Pfeifle, D. and Wiedemann, B. (1997) The signal molecule for betalactamase induction in Enterobacter cloacae is the anhydromuramyl-pentapeptide. Antimicrob Agents Chemother, 41, 2113-2120. 
840. Kong, K.-F. (2005), Florida International University, Miami.

841. French C., K.-M.E., Ward J.M. (1996) Development of a simple method for the recovery of recombinant proteins from the Escherichia coli periplasm. Enzyme Microb Technol, 19, 332-338.

842. Mathee, K. and Howe, M.M. (1993) Bacteriophage Mu Mor protein requires sigma 70 to activate the Mu middle promoter. J Bacteriol, 175, 5314-5323.

843. Dotsch, A., Eckweiler, D., Schniederjans, M., Zimmermann, A., Jensen, V., Scharfe, M., Geffers, R. and Haussler, S. (2012) The Pseudomonas aeruginosa transcriptome in planktonic cultures and static biofilms using RNA sequencing. PLOS ONE, 7, e31092.

844. Gomez-Lozano, M., Marvig, R.L., Molin, S. and Long, K.S. (2012) Genome-wide identification of novel small RNAs in Pseudomonas aeruginosa. Environ Microbiol, 14, 2006-2016.

845. Wiedenheft, B., Sternberg, S.H. and Doudna, J.A. (2012) RNA-guided genetic silencing systems in bacteria and archaea. Nature, 482, 331-338.

846. Sonnleitner, E., Sorger-Domenigg, T., Madej, M.J., Findeiss, S., Hackermuller, J., Huttenhofer, A., Stadler, P.F., Blasi, U. and Moll, I. (2008) Detection of small RNAs in Pseudomonas aeruginosa by RNomics and structure-based bioinformatic tools. Microbiology, 154, 3175-3187. 
VITA

\section{DEEPAK BALASUBRAMANIAN}

1995-1998

$1998-2000$

2000-2006

2007-2013
University of Madras

B.S. Microbiology

Madurai Kamaraj University

M.S. Microbiology

GangaGen Biotechnologies Pvt. Ltd.

Associate Scientist

Florida International University

Doctoral Candidate in Biology

\section{PUBLICATIONS}

D. Balasubramanian, L. Schneper, H. Kumari and K. Mathee. 2013. A dynamic and intricate regulatory network determines Pseudomonas aeruginosa virulence. Nucleic Acids Research. 41(1): 1-20; doi: 10.1093/nar/gks1039

D. Balasubramanian, S.K. Murugapiran, E. Silva-Herzog, L. Schneper, X. Yang, G. Tatke, G. Narasimhan and K. Mathee. 2013. Transcriptional regulatory network in Pseudomonas aeruginosa. In Bacterial Gene Regulation. 195-248. M. Babu (Ed.), Horizon Press.

D. Balasubramanian, L. Schneper, M. Merighi, R. Smith, G. Narasimhan, S. Lory, and K. Mathee. 2012. The regulatory repertoire of Pseudomonas aeruginosa AmpC Blactamase regulator AmpR includes virulence genes. PLOS ONE. 7(3):e34067.

D. Balasubramanian, K.F. Kong, S. Jayawardena, S. Leal, R. Sautter, and K. Mathee. 2011. Coregulation of B-lactam resistance, alginate production, and quorum sensing in Pseudomonas aeruginosa. Journal of Medical Microbiology 60(2):147-156. 
D. Balasubramanian, and K. Mathee. 2009 Comparative transcriptome analyses of Pseudomonas aeruginosa. Human Genomics. 3(4):349-61.

\section{AWARDS AND HONORS}

2013 Dissertation Year Fellowship, University Graduate School, Florida International University.

2011 Student Traineeship Award (BALASU11H0), Cystic Fibrosis Foundation.

2011 NIH MBRS RISE BRI Student Summer Research Award, Florida International University.

2009 Student Traineeship Award (BALASU08H0), Cystic Fibrosis Foundation.

2000 Gold medal for Academic Excellence during Master's degree 